\title{
Herkunft, Migrationsformen und Verbleib von Haupt- und Spurenelementen in Sicker- und Porenwässern des ehemaligen Braunkohletagebaus Zwenkau/Cospuden
}

Zur experimentellen und rechnerischen Speziation in Sicker- und Porenwässern

\author{
Dissertation \\ zur Erlangung des Doktorgrades \\ der Mathematisch-Naturwissenschaftlichen Fakultäten \\ der Georg-August-Universität zu Göttingen
}

vorgelegt von

Kathrin Brüschke

aus Heidelberg

Göttingen 2001 
D 7

Referent: Prof. Dr. H. Heinrichs

Korreferent: Prof. Dr. J. Hoefs

Tag der mündlichen Prüfung: 02. Mai 2001 


\section{Inhalt}

\section{Einleitung}

1.1. Allgemeines

1.1.1. Geschichte und wirtschaftliche Bedeutung der Braunkohlenutzung 7

\begin{tabular}{|lr}
\hline 1.1.2. Umweltfolgen der Braunkohlenutzung & 9 \\
\hline
\end{tabular}

$\begin{array}{ll}1.2 . \text { Zielsetzung dieser Arbeit } & 16\end{array}$

\begin{tabular}{ll}
\hline 2. Beschreibung der Standorte & 19 \\
\hline
\end{tabular}

\begin{tabular}{lr}
\hline 2.1. Cospuden/Zwenkau 19 \\
\hline
\end{tabular}

\begin{tabular}{ll}
\hline 2.2. Lausitz & 24 \\
\hline
\end{tabular}

\begin{tabular}{ll}
\hline 3. Methodik 26 \\
\hline
\end{tabular}

\begin{tabular}{ll}
\hline 3.1. Experimentelles & 26 \\
\hline
\end{tabular}

3.1.1. Probennahmen und Präparation 26

\begin{tabular}{lr}
3.1 .2 . Gewinnung von Porenlösungen 30 \\
\hline 3.13 Rehand
\end{tabular}

\begin{tabular}{ll}
\hline 3.1.3. Behandlung der Wasserproben & 31 \\
\hline
\end{tabular}

\begin{tabular}{ll}
\hline 3.1 .4 . Verwendete Materialien 31 \\
\hline 3.15 Sneziation
\end{tabular}

\begin{tabular}{ll}
\hline 3.1.5. Speziation & 32 \\
\hline
\end{tabular}

\begin{tabular}{lr}
3.2. Analytik 33 \\
\hline
\end{tabular}

\begin{tabular}{ll}
\hline 3.2 .1 . Wasserproben 33 \\
\hline 3.2. Fectoffen
\end{tabular}

\begin{tabular}{lr}
\hline 3.2 .2 . Feststoffe & 34 \\
\hline
\end{tabular}

\begin{tabular}{lr}
\hline 3.2 .3 . Software & 34 \\
\hline
\end{tabular}

\begin{tabular}{lr}
\hline 3.3. Modellierung & 34 \\
\hline
\end{tabular}

\begin{tabular}{ll}
\hline 3.4. Fehlerbetrachtung & 35 \\
\hline
\end{tabular}

4. Analyse von Sicker- und Porenwässern sowie von Bohrkernmaterial 36

4.1. Mineralbestand in Cospuden 36

\begin{tabular}{ll}
\hline 4.2. Verwitterungszustand und Elementmassenbilanz & 42 \\
\hline
\end{tabular}

4.3. Untersuchungen an Poren-, Grund- und Oberflächenwässern aus Cospuden 58

\begin{tabular}{|lr}
\hline 4.3.1. Räumliches Bild der Wasserqualität & 58 \\
\hline
\end{tabular} 
\begin{tabular}{|cc|}
4.3 .2$. Zeitliche Entwicklung der Wasserqualität & 64 \\
\hline
\end{tabular}

\begin{tabular}{|lr}
\hline 4.3 .2 .1$. See & 64 \\
\hline
\end{tabular}

\begin{tabular}{lr}
\hline $4.3 .2 .2 . \mathrm{CML}$ & 68 \\
\hline
\end{tabular}

\begin{tabular}{|lr}
\hline 4.3 .2 .3$. Grundwassermessstellen(RCO) & 73 \\
\hline
\end{tabular}

\begin{tabular}{ll}
\hline 4.4. Vergleich der Messungen am UFZ und aus dieser Arbeit & 79 \\
\hline
\end{tabular}

\begin{tabular}{lr}
\hline 4.5. Tiefenprofile & 81 \\
\hline
\end{tabular}

\begin{tabular}{lr}
\hline 4.5.1. Cospuden & 81 \\
\hline
\end{tabular}

4.5.1.1. Bohrkern des Grundwasserpegels RCO4 81

\begin{tabular}{|lr}
\hline 4.5.1.2. Multilevelbrunnen CML & 91 \\
\hline
\end{tabular}

4.5.1.3. Oberflächennahe Bohrung 98

\begin{tabular}{ll}
\hline 4.5.2. Lausitz 102 \\
\hline
\end{tabular}

\begin{tabular}{lr}
\hline 4.5 .2 .1 . Bohrkern 5-6 m & 103 \\
\hline
\end{tabular}

\begin{tabular}{lc}
\hline 4.5 .2 .2 . Bohrkern $21-22 \mathrm{~m}$ & 107 \\
\hline
\end{tabular}

\begin{tabular}{ll}
\hline 5. Speziation & 112
\end{tabular}

\begin{tabular}{ll}
\hline 5.1. Einführung & 112 \\
\hline
\end{tabular}

\begin{tabular}{ll}
\hline $5.1 .1 . \mathrm{DOC}$ & 115 \\
\hline
\end{tabular}

\begin{tabular}{lr}
\hline 5.1.2. Adsorption & 122 \\
\hline
\end{tabular}

\begin{tabular}{ll}
\hline 5.1.3. Kolloide und Partikel & 125 \\
\hline
\end{tabular}

\begin{tabular}{ll}
\hline 5.1.4. Redoxchemie 126 \\
\hline
\end{tabular}

5.2. Experimentelle und rechnerische Verfahren zur Speziation 128

\begin{tabular}{ll}
\hline 5.2 .1 . Experimentelle Speziationsverfahren & 128 \\
\hline
\end{tabular}

\begin{tabular}{ll}
\hline 5.2.2. Computerprogramme zur Speziationsberechnung 130 \\
\hline
\end{tabular}

5.3. Statistische Betrachtung der experimentellen Daten 132

5.4. Vergleich der Daten aus Experiment und Rechnung 144

\begin{tabular}{ll}
\hline 5.4.1. Experimentelle Klassifizierung der Proben & 145 \\
\hline
\end{tabular}

\begin{tabular}{ll} 
5.4.2. Berechnung ohne Berücksichtigung der organischen Komponenten & 147 \\
\hline
\end{tabular}

\begin{tabular}{lc} 
5.4.3. Verhalten des DOC bei der Speziation & 162 \\
\hline
\end{tabular}

5.4.4. Speziation unter Berücksichtigung der organischen Komponenten und

anorganischer Kolloide 165

\begin{tabular}{ll}
\hline 5.4.5. Diskussion der Speziationsergebnisse & 190 \\
\hline
\end{tabular}

\begin{tabular}{ll}
\hline 6. Zusammenfassung und Ausblick & 193 \\
\hline
\end{tabular} 
8. Anhang

8.1. Abkürzungen

8.2. Tabellenanhang 


\section{Einleitung}

\subsection{Allgemeines}

\subsubsection{Geschichte und wirtschaftliche Bedeutung der Braunkohlenutzung}

Braunkohle gewann im Vergleich $\mathrm{zu}$ anderen Bergbauprodukten erst relativ spät an Bedeutung - dann aber wurde sie für fast ein Jahrhundert zu einem der wichtigsten Energieträger Deutschlands.

Seit Ende des 17. Jahrhunderts wurde Braunkohle in geringem Umfang für den Hausbrand genutzt. Im Verlauf des 18. Jahrhunderts fand sie verstärkt industrielle Verwendung, z.B. in Salinen. Doch erst mit der Entwicklung neuer Fördertechniken um die Mitte des 19. Jahrhunderts wurde der großflächige Tagebau möglich, der gegenüber dem Tiefbau klare Vorteile bei der Ausnutzung der vorhandenen Braunkohlelager eröffnete (WILSDORF 1987).

Während im Tiefbau bis zu $50 \%$ der Braunkohle beim Abbau verloren gingen, betrugen die Verluste im Tagebau lediglich $8-12 \%$. Die Entwicklung von Großbaggern und Förderbrücken ermöglichte die Abtragung riesiger Flächen.

In Mitteleuropa entstanden vier große Braunkohletagebauregionen in Nordböhmen, im Mitteldeutschen Braunkohlerevier, in der Lausitz und im Rheinischen Revier. Abb. 1.1 zeigt die Lage dieser Reviere und die Fördermengen in den 70er Jahren.

Besonders in der DDR avancierte Braunkohle zu dem Energieträger überhaupt, war aber auch als Grundstoff für die Petrochemie von großer Bedeutung. 1988 wurden $90 \%$ des Energiebedarfs der DDR durch Braunkohle gedeckt; die Förderung erreichte $310 \mathrm{mio}$ t/Jahr (GELLER et al. 1998).

Mit dem Ende der DDR verlor ab 1990 auch die Braunkohle ihre wirtschaftliche Bedeutung, zumal der vergleichsweise hohe Schwefelgehalt der ostdeutschen Braunkohle Umweltprobleme sowohl durch den Abbau als auch durch die Verfeuerung zur Folge hatte. 

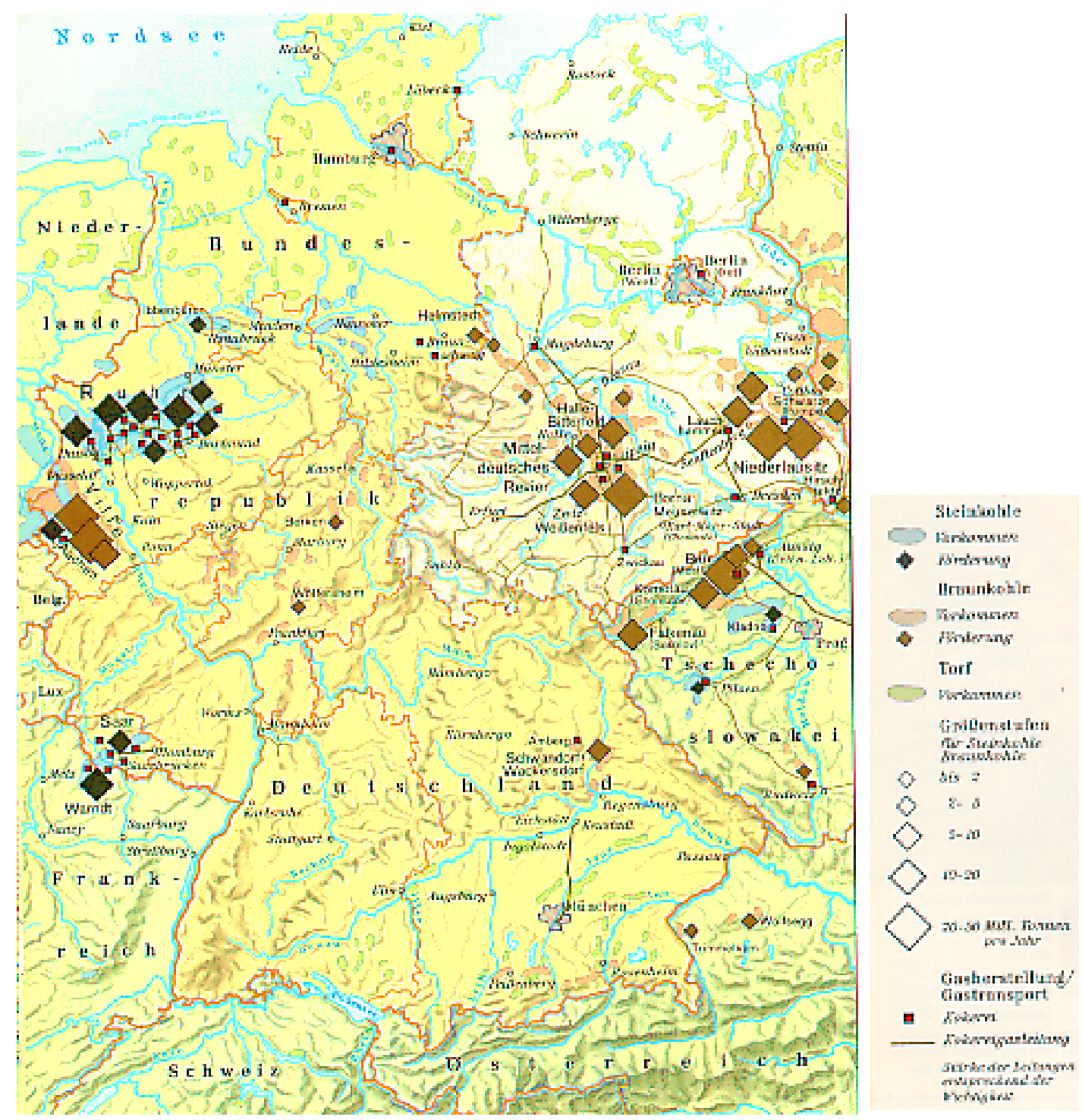

Abb. 1.1: Braunkohlegewinnung in Mitteleuropa (Diercke-Weltatlas 1974).

\begin{tabular}{|l|l|l|}
\hline Jahr & Kohleförderung (mio t) & Bemerkungen \\
\hline $\mathbf{1 8 8 5}$ & ca. 15,0 & davon $75 \%$ aus Tiefbau \\
\hline $\mathbf{1 9 1 3}$ & ca. 19,2 & davon $30 \%$ aus Tiefbau \\
\hline $\mathbf{1 9 3 4}$ & ca. 91,1 & davon $12 \%$ aus Tiefbau \\
\hline $\mathbf{1 9 6 3}$ & ca. 145,5 & - \\
\hline $\mathbf{1 9 8 5}$ & ca. 115,4 & 20 Tagebaue \\
\hline $\mathbf{1 9 8 9}$ & ca. 105,6 & 20 Tagebaue \\
\hline $\mathbf{1 9 9 0}$ & ca. 80,9 & 19 Tagebaue \\
\hline $\mathbf{1 9 9 1}$ & ca. 50,9 & 11 Tagebaue \\
\hline $\mathbf{1 9 9 2}$ & ca. 36,6 & 9 Tagebaue \\
\hline $\mathbf{1 9 9 3}$ & ca. 28,5 & 5 Tagebaue \\
\hline $\mathbf{1 9 9 4}$ & ca. 17,5 & 3 Tagebaue \\
\hline
\end{tabular}

Tab. 1.2.: Entwicklung der Kohleförderung im Mitteldeutschen Revier. 
Tab. 1.2. zeigt die Entwicklung der Kohleförderung im gesamten Mitteldeutschen Revier. Deutlich wird erkennbar, wie die Kohleförderung mit dem aufkommenden Tagebau (und damit dem sinkenden Anteil des Tiefbaus) anstieg und nach dem Ende der DDR sehr schnell wieder abnahm.

Ein großer Teil der Tagebaue in Ostdeutschland wurde seit 1990 stillgelegt; im Mitteldeutschen Revier sind lediglich die Gruben Schleenhain und Profen noch für einen längerfristigen Abbau vorgesehen.

1998 wurden in der Bundesrepublik Deutschland noch 166 mio t Braunkohle gefördert (SAUS und SCHIFFER 1999), davon $59 \%$ im Rheinischen Revier, $30 \%$ in der Lausitz, 8 \% im Mitteldeutschen Revier und $3 \%$ in Helmstedt und Bayern. Zeitgleich befindet sich eine große Anzahl von Tagebauen in der Rekultivierung; Ziel ist dabei die Wiederherstellung des regionalen Wasserhaushalts (GLÄßER 1995, LMBV 1995, BERUFSVERBAND DEUTSCHER GEOLOGEN 1996), die Absicherung der Abbruchkanten und die Nutzbarmachung als Trinkwasserreservoir, Naherholungsgebiet oder Fischgewässer (BERKNER 1995, KLAPPER 1995, Broll et al. 2000). Für das Mitteldeutsche und das Lausitzer Revier liegt die Verantwortung für die Sanierung seit 1994/95 bei der Lausitzer und Mitteldeutschen BergbauVerwaltungsgesellschaft mbH (LMBV).

\subsubsection{Umweltfolgen der Braunkohlenutzung}

Die Folgen der intensiven Braunkohlenutzung wurden in der Öffentlichkeit der Bundesrepublik Deutschland im letzten Jahrzehnt vor allem im Zusammenhang mit der Erschließung des Tagebaus Garzweiler II im Rheinischen Braunkohlerevier erörtert. Im Mittelpunkt der Kritik standen dabei der große Landschaftsverbrauch durch den Tagebau, die sozialen Folgen durch die Verlegung ganzer Ortschaften, aber auch die weiträumigen Konsequenzen der Grundwasserabsenkung im Vorfeld des Tagebaus, die sich bis jenseits der niederländischen Grenze und auf zahlreiche auch weit entfernt liegende Naturlandschaften auswirken werden. 


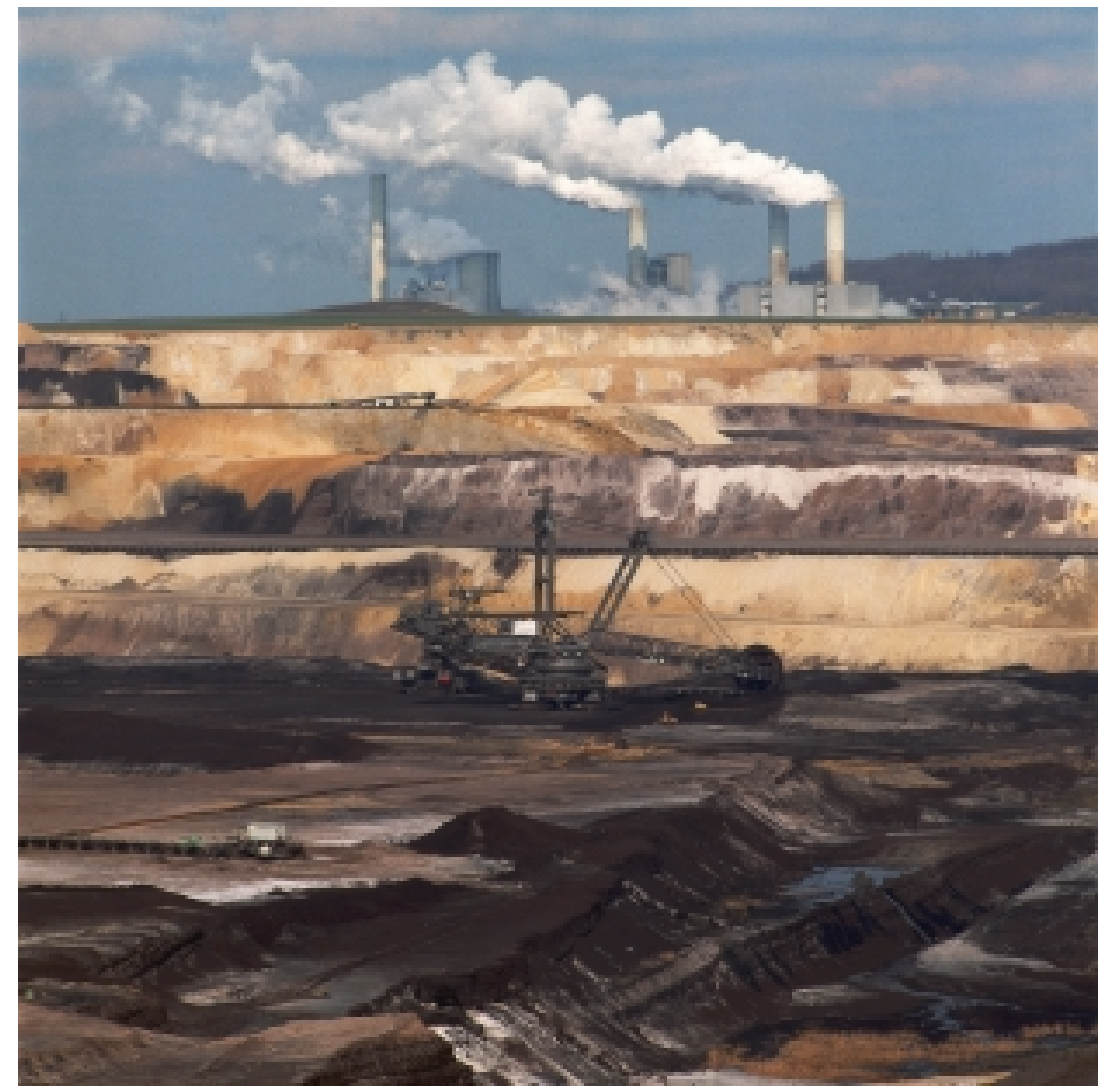

Abb. 2.2: Braunkohleabbau in Garzweiler (Quelle: http://www.rwe.com/1.6_RWE_Presse/Bilder-undGrafiken/Stammhaeuser/RWE_Rheinbraun/RWE_Rheinbraun_index.jsp, 08.01.2001).

Während die gewaltigen Landschaftsveränderungen („Mondlandschaften“, Abb. 2.2.) durch den Tagebau auf den ersten Blick wahrnehmbar sind, trat ein anderes Problem dabei in der öffentlichen Diskussion in den Hintergrund, das jedoch sowohl in der Lausitz und auch im Mitteldeutschen Braunkohlerevier große Aufmerksamkeit verdient. Wegen der vergleichsweise hohen Pyritgehalte in diesen Regionen - 1-8 \% in Cospuden/Zwenkau (BELlmann et al. 1984, KitTsteiner 1998, KitTsteiner et al. 2000) und 0,4-3 \% (BozAU 2000) bzw. 1,4 \% (LANDESUMWELTAMT BRANDENBURG 1995) in der Lausitz - müssen hier die Folgen der Pyritverwitterung aufmerksam beobachtet werden, um frühzeitig auf eine zu starke Versauerung und Kontamination von Boden und Wässern reagieren zu können. Im Rheinischen Braunkohlerevier ist das Problem wegen der niedrigeren Pyritgehalte von 0,4 1,6 \% (WERNERY-SEIDEL 1958, WiSOTZKY 1994, KITTSTEINER 1998) nicht ganz so groß; trotzdem wurden auch hier umfangreiche Programme entwickelt, um die Versauerung zu bremsen.

Durch die Absenkung des Grundwassers und die großflächigen und häufig unselektiven Abraumbewegungen im Zuge des Tagebaus kommt eisendisulfidhaltiges Sediment in Kontakt mit Sauerstoff. Dadurch wird eine Kette von Oxidationsprozessen in Gang gesetzt. Die 
einzelnen Reaktionen und ihre Mechanismen waren und sind Gegenstand umfangreicher Untersuchungen (SINGER und STUMm 1970, Moses et al. 1987, WILLIAMSON und RimstidT 1994, NichOlsON 1994, EGGLESTON et al. 1996). Ausführliche Übersichtsdarstellungen liefern u.a. WisotzKy (1994, 1996, 1998, 2000) und PeIFFER (1997). Der Prozess lässt sich nach SiNGER und STUMM (1970) zusammenfassen in den Formeln:

[1] $2 \mathrm{FeS}_{2}+7 \mathrm{O}_{2}+2 \mathrm{H}_{2} \mathrm{O} \rightarrow 2 \mathrm{Fe}^{2+}+4 \mathrm{SO}_{4}{ }^{2-}+4 \mathrm{H}^{+}$ Oxidation von Pyrit durch Luftsauerstoff

[2] $\mathrm{FeS}_{2}+14 \mathrm{Fe}^{3+}+8 \mathrm{H}_{2} \mathrm{O} \rightarrow 15 \mathrm{Fe}^{2+}+2 \mathrm{SO}_{4}{ }^{2-}+16 \mathrm{H}^{+}$ Oxidation von Pyrit durch Fe

[3] $\quad 4 \mathrm{Fe}^{2+}+\mathrm{O}_{2}+4 \mathrm{H}^{+} \rightarrow 4 \mathrm{Fe}^{3+}+2 \mathrm{H}_{2} \mathrm{O}$ Oxidation von $\mathrm{Fe}^{I I}$ durch Luftsauerstoff unterhalb von $\mathrm{pH} 3$

$4 \mathrm{Fe}^{2+}+\mathrm{O}_{2}+10 \mathrm{H}_{2} \mathrm{O} \rightarrow 4 \mathrm{Fe}(\mathrm{OH})_{3} \downarrow+8 \mathrm{H}^{+}$ Oxidation von Fe $e^{I I}$ durch Luftsauerstoff oberhalb von $\mathrm{pH} 3$

[5] $4 \mathrm{FeS}_{2}+15 \mathrm{O}_{2}+14 \mathrm{H}_{2} \mathrm{O} \rightarrow 4 \mathrm{Fe}(\mathrm{OH})_{3} \downarrow+8 \mathrm{SO}_{4}{ }^{2-}+16 \mathrm{H}^{+}$ Summenformel

Diese wenigen Formeln sollen jedoch nicht darüber hinwegtäuschen, das zahlreiche Teilschritte beteiligt sind, die wiederum beeinflusst werden durch Faktoren wie die spezifische Oberfläche des Pyrits, den Sauerstoffzutritt, den $\mathrm{pH}$, die Temperatur und den Wassergehalt (Singer und Stumm 1970, Nordstrom 1982, VAn BREEMEN et al. 1984, BOREK 1994, WISOTZKY 1994) und die Beteiligung von Mikroorganismen (AlPERS und BLOWES 1994; GOULD et al. 1994), aber auch indirekt durch sekundär gebildete Mineralphasen (CRAVOTTA 1994). Die Kinetik der einzelnen Teilreaktionen im Verhältnis zueinander bestimmt letztlich das Gesamtgeschehen, das somit zahlreichen Einflüssen unterliegt und nur schwer rechnerisch zu erfassen ist.

Wie die Summenformel zeigt, werden je Mol Pyrit 4 Mol Wasserstoffionen in Form von Schwefelsäure freigesetzt. Die Folge ist eine starke Versauerung der Wässer. Versauerte Sicker- und Porenwässer reagieren mit den umgebenden Mineralphasen, führen teilweise zu deren Auflösung und setzen damit nicht nur aus dem Pyrit, sondern auch aus anderen Mineralen große Mengen an Haupt- und Spurenelementen frei. 


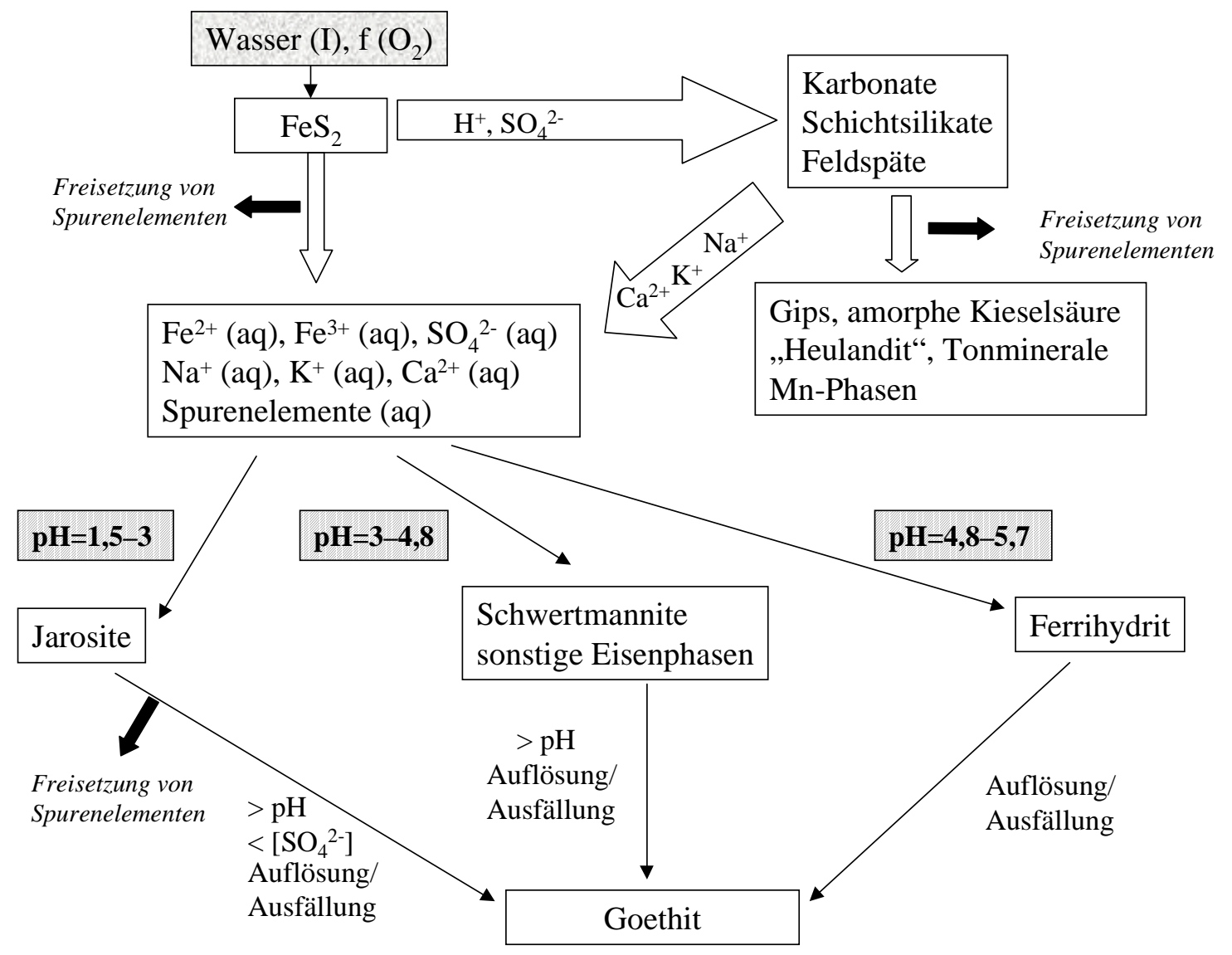

Abb. 1.3.: Fließbild der Pyritoxidation und der Folgereaktionen im Abraum (stark modifiziert nach CESNOVAR und Pentinghaus (1999) bzw. Bigham und Murad (1997)).

Abb. 1.3. zeigt die beteiligten Prozesse, die für die Freisetzung, aber auch die Bindung von Elementen verantwortlich sind. Die entstandene Schwefelsäure reagiert zunächst mit Karbonaten; dabei entsteht vor allem Gips. Sowohl durch den Angriff der Schwefelsäure auf Silikate als auch auf karbonatische Minerale werden die darin festgelegten Spurenelemente in die Lösung abgegeben, und verschiedene Sekundärphasen wie amorphe Kieselsäure und Schichtsilikate bilden sich. Das Eisen wird je nach den chemischen Bedingungen in Form verschiedener sekundärer Eisenphasen gebunden. Die Mineralogie der Sekundärphasen der Pyritoxidation war in den letzten Jahren Gegenstand gründlicher Untersuchungen (z.B. Bigham und Murad 1997, Bigham et al. 1992). Bei pH-Werten unterhalb von 3 in Anwesenheit von $\mathrm{K}^{+}$-Ionen und bei erhöhten Sulfatkonzentrationen bilden sich vorrangig Jarosite; sie machen in der Lausitz den Hauptanteil der sekundär gebildeten Eisenphasen aus (PoHLMANN et al. 2000). pH-Werte zwischen 3 und 4 können die Rahmenbedingungen für die Bildung von Schwertmannit bereitstellen, dessen Vorkommen nicht nur in sauren Minenwässern, sondern auch in der Natur beobachtet wird (SCHWERTMANN und FOJT (1996), 
SCHWERTMANN et al. 1995). pH-Werte oberhalb von 5 führen zur Enstehung von Ferrihydrit. Ändern sich die Umweltbedingungen, z.B. durch einen Anstieg des pH-Wertes oder ein Absinken der Schwefelkonzentration, gehen die Phasen wieder in Lösung. In der Folge bildet sich in der Regel Goethit als stabile Eisenphase. Besonders Jarosite können dabei als temporäres Reservoir für zahlreiche Spurenelemente dienen, die in die Kristallstruktur eingebunden und bei der Auflösung wieder freigesetzt werden.

Zwar kommt es im Rahmen von Neutralisationsreaktionen bzw. nach Oxidation von $\mathrm{Fe}^{\mathrm{II}} \mathrm{zu}$ $\mathrm{Fe}^{\mathrm{III}} \mathrm{zu}$ Ausfällungen von Eisenoxyhydroxiden, die adsorptive Oberflächen bereitstellen bzw. zu Mitfällungen anderer Elemente führen, doch können diese Prozesse in der Regel die Belastung mit Haupt- und Spurenelementen nicht völlig ausgleichen (GERMAIN et al. 1994, KIMBALL et al. 1994, WEBSTER et al. 1994).

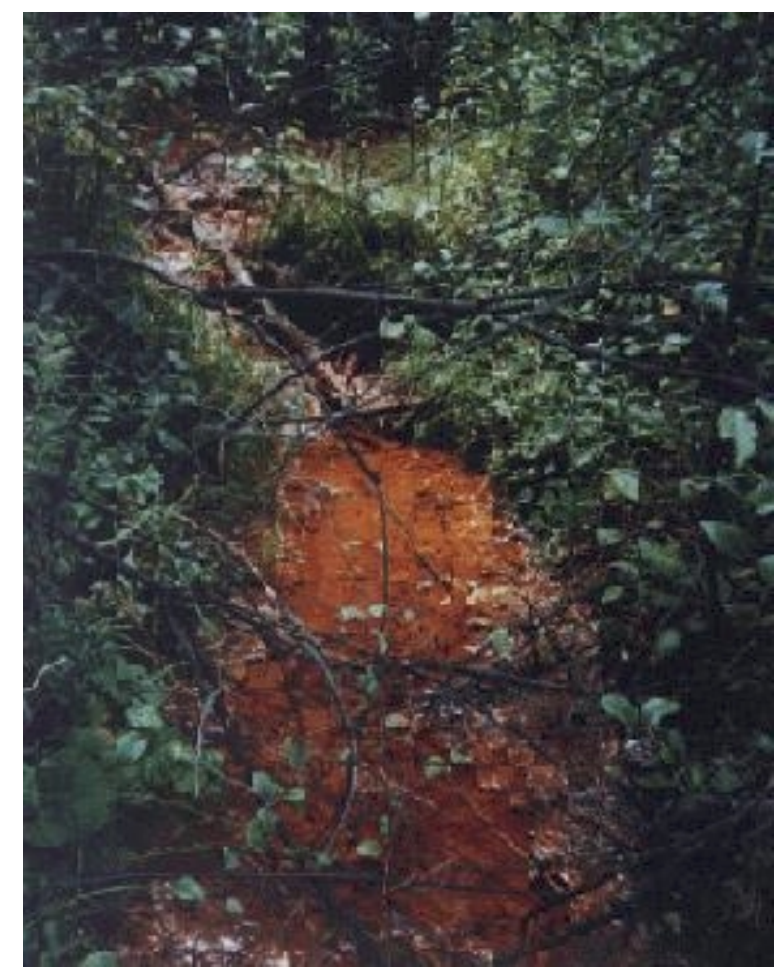

Abb 1.4.: Eisenhydroxidniederschläge in der Umgebung saurer Minenwässer in Pennsylvania (Quelle: http://ctcnet.net/scrip/picsum.htm, Stand 09.01.2001).

Übersichten über die Ausfällung und die Mineralogie von Eisenhydroxidniederschlägen finden sich u.a. bei Fortin et al. (1993), Bigham et al. (1996) und Bigham und Murad (1997). Die Problematik saurer Minenwässer (,acid mine drainage“, AMD) wurde an zahlreichen Beispielen untersucht (LONGMIRE et al. 1990, BLOWES und JAMBOR 1990, KWong und LaWrence 1998, Hemm et al. 1999, IgARAShi 1999). Detaillierte Studien beschäftigen sich z.B. mit der Entwicklung von Modellen zur Vorhersage, welche Stoffe 
freigesetzt und in der Folge wieder adsorptiv oder durch Fällung gebunden werden, so z.B. STRÖMBERG und BANWART (1994), SimMS et al. (2000). Eine Studie zur Umwandlung von Schwertmannit zu Goethit im Sediment eines sauren Restsees präsentierten PEIFFER et al. (2000).

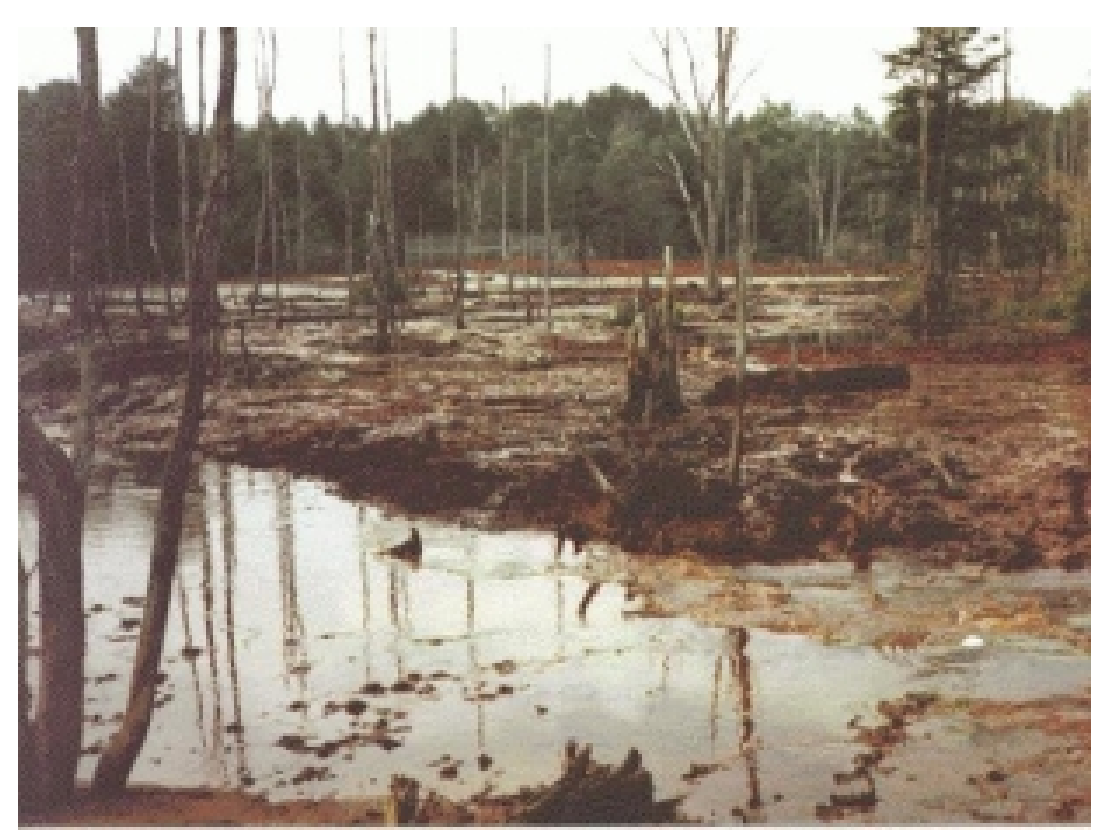

Abb. 1.5.: Schäden durch saure Minenwässer aus dem Tiefbau in Pennsylvania (Quelle: http://ctcnet.net/scrip/picsum.htm, Stand 09.01.2001).

Die Folgen der Versauerung von Gewässern wurden im Umfeld der Problematik der Pyritoxidation, aber auch im Zusammenhang mit der Belastung durch „Sauren Regen“ untersucht (GELlER et al. 1998, HeMm et al. 1999, PEIFFER et al. 1999, Abb. 1.5.). Eine Übersicht über die Auswirkungen von pH-Änderungen in Seen sowohl in biologischer als auch chemischer Hinsicht lieferte z.B. ein Versuch in Wisconsin, USA, bei dem ein See kontrolliert versauert und die Folgen sowie seine Regeneration hinsichtlich chemischer und biologischer Fragestellungen beobachtet wurden (FrOST et al. 1999). In der jüngeren Vergangenheit gab es zahlreiche Versuche, die Folgen der Pyritoxidation zu bekämpfen. So wurden schon während der Verkippung Kraftwerksasche oder andere basische Substanzen dem Abraum zugesetzt, um die gebildete Säure direkt am Ort ihrer Entstehung zu binden. In einem anderen Projekt sollte ein bereits versauerter See durch Zusatz von Soda im großen Rahmen neutralisiert werden in der Hoffnung, dass diese Maßnahme ausreichen würde, um einen natürlichen Regenerationsprozess zu initiieren. Einen Überblick über Maßnahmen zur Eindämmung oder Regeneration von sauren Minenwässern bieten FöRSTNER (1995) und ALLAN (1995). Ein häufig eingesetztes Verfahren zur Regeneration saurer Minenwässer ist 
der Einsatz von künstlichen Feuchtgebieten mit Kalksteinbetten, über die die versauerten Wässer geleitet werden (KLEINMANN et al. 1998, Abb. 1.6.).
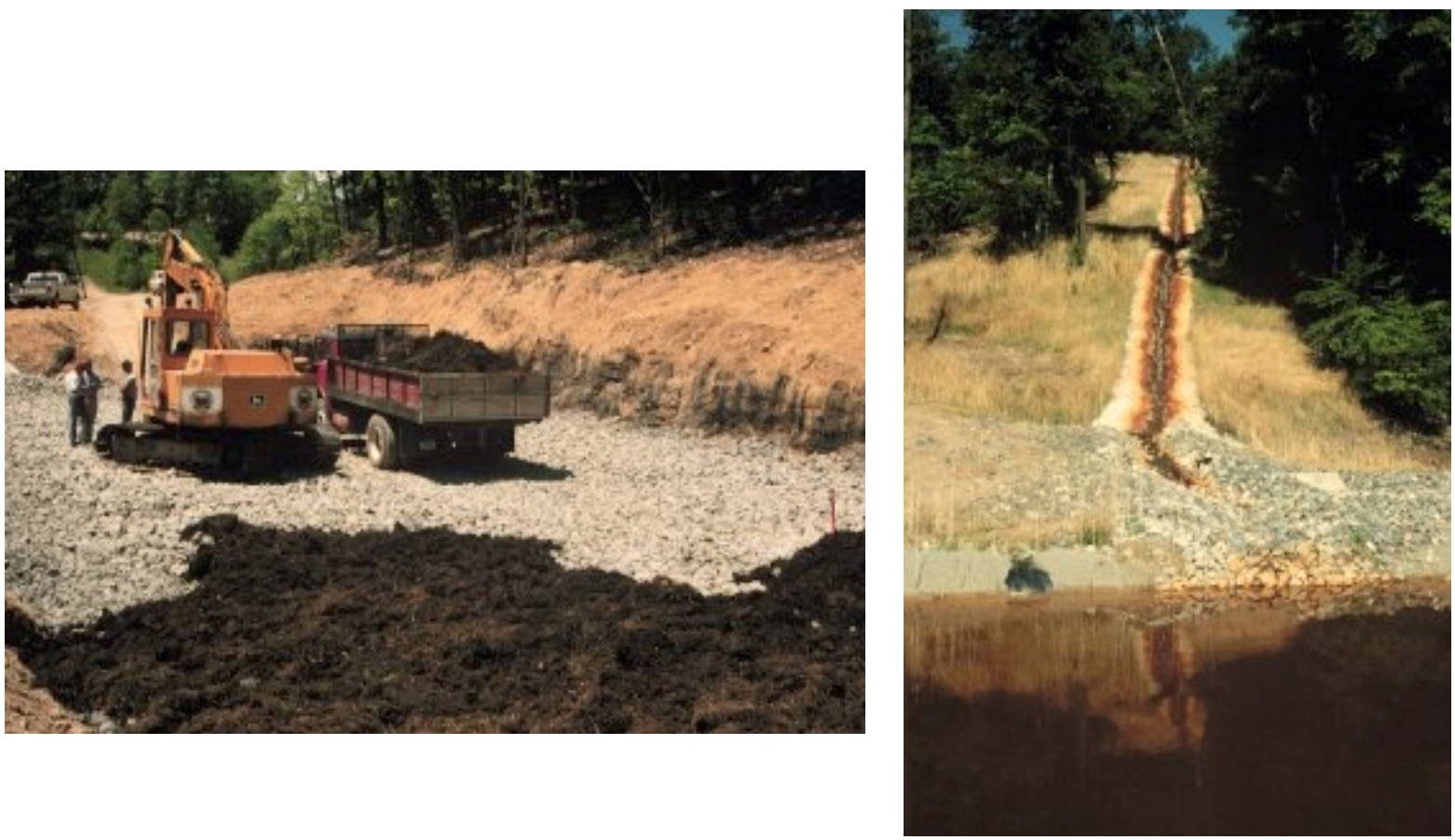

Abb. 1.6.: Regeneration von Minenwässern durch Kalksteinbetten (Quelle: http://www.wvu.edu/ agexten/landrec/passtrt/passtrt.htm, Stand 09.01.2001).

Allerdings muss man dabei auch darauf achten, nicht im Zuge der Sanierungsmaßnahmen bereits gebundene Stoffe durch pH-Wert-Änderungen o.ä. wieder freizusetzen (ROSE und ELLiOTT (2000)). Dies gilt vor allem dann, wenn pflanzliches Material zugesetzt wird als Substrat für sulfatreduzierende Bakterien, die auf biologische Weise für eine Säurebindung sorgen sollen; bei der Verrottung des organischen Materials werden Komplexbildner freigesetzt, die u.U. Schadstoffe mobilisieren und über weite Strecken in Gebiete transportieren, die ansonsten nicht von den Folgen des Bergbaus betroffen wären. Einzelne Stimmen fordern, die sauren Seen sich selbst zu überlassen (SCHUlZ und WIEGLEB (2000)); eine Neutralisierung sei in der Regel sehr kostenaufwendig, ohne dass ein nachhaltiger Erfolg garantiert werden könne. Ein solches Vorgehen erscheint aber nur begrenzt sinnvoll, da die sauren Seen nur artenarme Ökosysteme ausbilden können (PACKROFF et al. 2000), für wirtschaftliche Nutzung wie auch für Naturschutz nur begrenzt zur Verfügung stehen und die Gefahr eines weiteren Eintrags von Schadstoffen in Oberflächenwässer und Grundwasserleiter durch die sauren Wässer besteht. 


\subsection{Zielsetzung dieser Arbeit}

Die vorliegende Arbeit ist Teil eines $5 \frac{1}{2}$-jährigen Projektes „Transportprozesse und Migrationsformen von Spurenmetallen in Porenlösungen von Sicker- und Grundwasserleitern“ am Geochemischen Institut der Georg-August-Universität Göttingen im Rahmen des DFG-Schwerpunktprogramms 546 „Geochemische Prozesse mit Langzeitfolgen im anthropogen beeinflussten Sickerwasser und Grundwasser“, Arbeitskreis Bergbaufolgen. Die grundlegende Überlegung ist, dass die absoluten Gehalte von Haupt- und Spurenelementen in Sicker- und Porenwässern nicht genug über die tatsächliche Umweltrelevanz aussagen. Hierfür ist vielmehr auch $\mathrm{zu}$ berücksichtigen, welche Migrationsformen jeweils vorliegen - kommt es bei einer pH-Änderung zur Ausfällung der betreffenden Elemente? Werden sie leicht in der Kippe an Mineraloberflächen adsorbiert, oder sind sie hochmobil und können langfristig in Oberflächen- und Grundwässer der Umgebung gelangen? Sind sie gar organisch gebunden und damit für Organismen um vieles leichter aufzunehmen als anorganische Komplexe?

Um diese Fragen zu beantworten, wurde im Rahmen des Projekts in einer ersten Promotion (PleßOw 1998, PleßOw und HeinRICHS 2000a, b) ein Speziationsverfahren entwickelt, das die Bestimmung von Migrationsformen zahlreicher Elemente auch in den kleinen Probenmengen erlauben sollte, wie sie durch Pressen aus Bohrkernen gewonnen werden. Im Rahmen der Arbeit von PLEßOW (1998) wurden außerdem bereits zahlreiche Proben gemessen. Begleitend untersuchte Kittsteiner im Rahmen seiner Diplomarbeit (KITTSTEINER 1998, KitTSTEINER et al. 2000) Proben aus dem unverritzten Anstehenden des Tagebaus Zwenkau, um das Gesamtkontaminationspotential des Kippenmaterials zu bestimmen. In einer weiteren Diplomarbeit (BRINKMANN 2000) wurde das Kippenmaterial chemisch und mineralogisch analysiert, um Aufschluss über verbliebene Pyritgehalte und etwaige Sekundärminerale zu gewinnen. Tab. 1.3. gibt eine Übersicht über die im Rahmen des Projekts durchgeführten Untersuchungen. 


\begin{tabular}{|c|c|c|c|}
\hline Ziele & $\begin{array}{l}\text { Untersuchungen und } \\
\text { Berechnungen }\end{array}$ & Instrumentelle Methoden & Arbeit \\
\hline $\begin{array}{l}\text { Herkunft von } \\
\text { Spurenmetallen }\end{array}$ & $\begin{array}{l}\text { Mineralanalysen, geochemische } \\
\text { Massenbilanzen, } \\
\text { Reservoirgrößen }\end{array}$ & $\begin{array}{l}\text { Phasentrennungen, } \\
\text { Röntgendiffraktometrie, IR- } \\
\text { Spektrometrie, } \\
\text { Elektronenstrahlmikrosonde, } \\
\text { Laser-ICPMS, } \\
\text { Röntgenfluoreszenz, ICPMS, } \\
\text { ICPOES }\end{array}$ & $\begin{array}{l}\text { Kittsteiner } \\
(1997), \\
\text { vorliegende } \\
\text { Arbeit }\end{array}$ \\
\hline $\begin{array}{l}\text { Migrationsformen } \\
\text { von Spurenmetallen }\end{array}$ & $\begin{array}{l}\text { Ionenaustauschbilanzen, } \\
\text { Zustands- bzw. Bindungsformen, } \\
\text { Untersuchungen synthetischer } \\
\text { Lösungen mit definierten } \\
\text { Zusatzstoffen, zeitliche und } \\
\text { räumliche Beobachtung der } \\
\text { Situation im Tagebau, } \\
\text { computergestützte } \\
\text { Speziationsberechnungen }\end{array}$ & $\begin{array}{l}\text { ICPMS, ICPOES, AAS, DOC- } \\
\text { Analyse, C- und S-Bestimmung, } \\
\text { Ultrafiltration, Kationen - und } \\
\text { Anionenaustausch } \\
\text { Computermodell MINTEQA2 }\end{array}$ & $\begin{array}{l}\text { Pleßow (1998), } \\
\text { vorliegende } \\
\text { Arbeit }\end{array}$ \\
\hline $\begin{array}{l}\text { Verbleib von } \\
\text { Spurenmetallen }\end{array}$ & Mineralanalysen & $\begin{array}{l}\text { IR-Spektrometrie, } \\
\text { Röntgendiffraktometrie, } \\
\text { Phasentrennungen, ICPMS, } \\
\text { Röntgenfluoreszenz }\end{array}$ & $\begin{array}{l}\text { Brinkmann } \\
(2000), \\
\text { vorliegende } \\
\text { Arbeit }\end{array}$ \\
\hline
\end{tabular}

Tab. 1.3.: Arbeiten im Rahmen des $5^{1} \frac{2}{2}$ jährigen Projektes „Transportprozesse und Migrationsformen von Spurenmetallen in Porenlösungen von Sicker- und Grundwasserleitern“.

Ziel der vorliegenden Arbeit war es, Poren-, Sicker- und Oberflächenwässer zu analysieren und auf der Basis des von PlEßOw (1998) entwickelten Speziationsverfahrens Aussagen über die Migrationsformen der enthaltenen Elemente zu machen. Untersucht wurden dabei neben den Hauptelementen vor allem Li, Be, Sc, Ti, V, Cr, Mn, Fe, Co, Ni, Cu, Zn, As, Rb, Sr, Y, $\mathrm{Zr}$, Mo, Cd, Sb, Cs, Ba, La, die Lanthanoide, Hf, Tl, Pb, Bi, Th und U. Unter Einbeziehung der von PLEßOW (1998) untersuchten Proben konnte dabei die zeitliche Entwicklung über fast fünf Jahre von Beginn der Flutung des Restlochs Cospuden bis zu ihrem Abschluss im Jahr 1999 beobachtet werden. Berücksichtigt man zusätzlich die Arbeiten von KITTSTEINER (1998) und BRINKMANN (2000), so sollte es letztlich möglich sein, Herkunft, Transport und Verbleib der untersuchten Elemente zu erfassen und auf dieser Basis Aussagen über die Zukunft der Wässer im Bereich Cospuden zu machen. Im Idealfall lassen sich die hierbei gewonnenen Erkenntnisse dann auch auf andere ehemalige Tagebaue, die noch vor der Flutung stehen, übertragen, und ggf. kann durch entsprechende Maßnahmen einer Versauerung und Kontamination von Sicker- und Grundwässern vorgebeugt werden.

Entgegen der ursprünglichen Planung war eine vergleichende Studie zwischen Porenwässern aus Cospuden und aus Garzweiler nicht möglich, da aus den Sedimentproben aus Garzweiler keine ausreichenden Wassermengen gewonnen werden konnten. Allerdings führte Pleßow (PleßOw 1998, PleßOw und HeinRICHS 2000a) einige Analysen von Sickerwässern aus einem Großlysimeter durch. Die Säule war mit Sedimentmaterial mit typischem Pyritgehalt befüllt. 
Eine automatisierte Beregnungsanlage simulierte die äußeren Bedingungen im Braunkohletagebau Garzweiler I. Die beobachteten Elementkonzentrationen lagen dabei in etwa im Bereich der Werte, die in oberflächennahen Sickerwässern im Bereich Cospuden zu Beginn der Flutung gemessen wurden.

Um einen Vergleich zwischen unterschiedlich geprägten Standorten durchführen zu können, wurden im Rahmen dieser Arbeit außer Proben aus Cospuden auch zwei Bohrkerne aus dem Bereich des Restsees 111 im Lausitzer Braunkohlegebiet untersucht.

In Ergänzung $\mathrm{zu}$ den experimentellen Untersuchungen der Wasserproben wurden Speziationsberechnungen mit dem Computerprogramm MINTEQA2 durchgeführt. Aus dem Vergleich der Ergebnisse können Rückschlüsse gezogen werden auf den Einfluss organischer Komponenten und die Kontrolle der Löslichkeit durch Präzipitation und/oder Adsorption.

Die vorliegende Arbeit gliedert sich damit im wesentlichen in drei große Bereiche: Die Beschreibung der Standorte und die Auswertung der absolut gemessenen Elementgehalte; hier sind insbesondere die zeitliche Entwicklung, das räumliche Profil sowie die Tiefenprofile der Sickerwässer aus drei Bohrkernen in Cospuden von Interesse. Im zweiten Teil der Arbeit werden die Ergebnisse der experimentellen Speziation ausgewertet und diskutiert. Im dritten Abschnitt schließlich werden die Resultate der Berechnungen mit dem Programm MINTEQA2 vorgestellt und in Zusammenhang mit den experimentellen Daten gestellt. Hier wird auch ein Vergleich gezogen zu den Resultaten der Untersuchung von Sickerwässern, die aus zwei Bohrkernen aus der Lausitz (Restloch 111) gewonnen wurden. 


\section{Beschreibung der Standorte}

\subsection{Cospuden/Zwenkau}

Die Braunkohletagebaue Cospuden und Zwenkau liegen im Mitteldeutschen Braunkohlerevier etwa $10 \mathrm{~km}$ südwestlich der Stadtgrenze von Leipzig (Abb. 2.1.) und grenzen unmittelbar aneinander. Ein Artikel zur Entstehung und Geologie der ostdeutschen Braunkohlereviere findet sich bei SCHRECK und GLÄßER (1998), ausführliche Angaben zur Hydrogeologie vor, während und nach dem Kohleabbau machen GLÄßER (1995) und die LMBV (1995).

Bereits für das Jahr 1743 sind in dieser Gegend erste Versuche zur Braunkohlegewinnung belegt, die jedoch wegen des hohen Grundwasserspiegels scheiterten. Erst mit dem Aufkommen der Dampfmaschine konnten die Bergwerke ausreichend entwässert werden. Das Braunkohlewerk Zwenkau war bereits 1870 im Tiefbau in Betrieb. Dennoch blieb die Entwässerung der limitierende Faktor für die Kohlegewinnung im Leipzig-Bornaer Raum. 1921 begann die Erschließung von Zwenkau (bis 1969 "Tagebau Böhlen") als Tagebau. Seit Anfang der 30er Jahre wurden durch neue Pumptechniken große Eingriffe in den Grundwasserhaushalt des Gebietes möglich.

Während der Betrieb im unmittelbar benachbarten Tagebau Zwenkau noch bis ins Jahr 1999 fortdauerte, wurde die Nutzung des später erschlossenen Tagebaus Cospuden, der vor allem zur Deckung des Bedarfs an Braunkohle in der Petrochemie gedacht war, nach nur 11 Jahren im Jahr 1992 eingestellt. Bis zu diesem Zeitpunkt waren in Cospuden 85 mio m³ Abraum auf einer Fläche von 3,1 km² bewegt und 32 mio. t Kohle gefördert worden. In Zwenkau wurden sogar 565 mio t Kohle gewonnen; dazu mußten 1400 mio m³ Abraum auf einer Fläche von $27,5 \mathrm{~km}^{2}$ umgelagert werden (BERKNER 2000). Der Abraum setzt sich zusammen aus Kiesen und Sanden der Grundwasserleiter, Ton- und Lehmschichten und Resten von Braunkohle (LMBV 1995). 

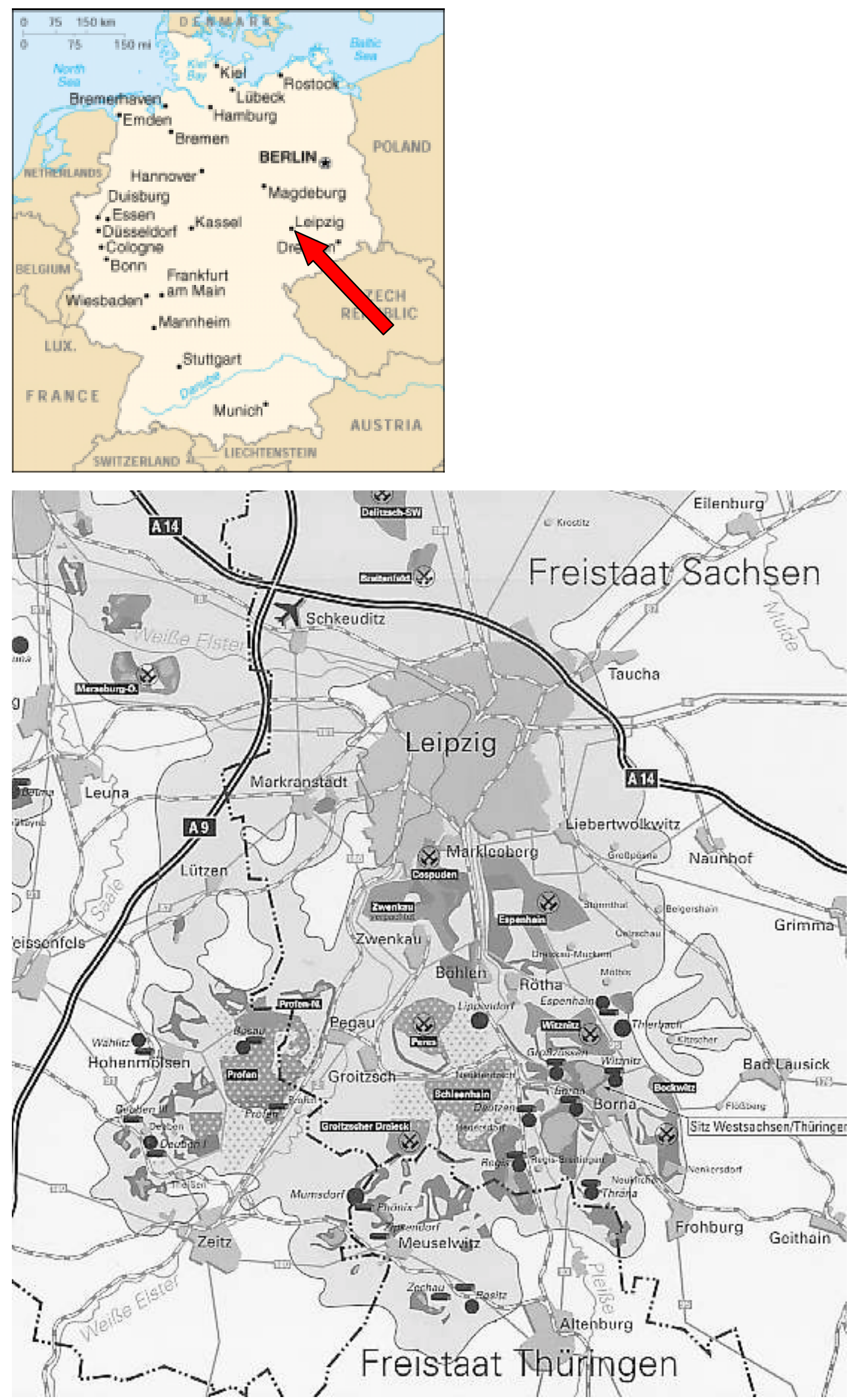

Abb. 2.1.: Geographische Lage des ehemaligen Tagebaus Cospuden. 


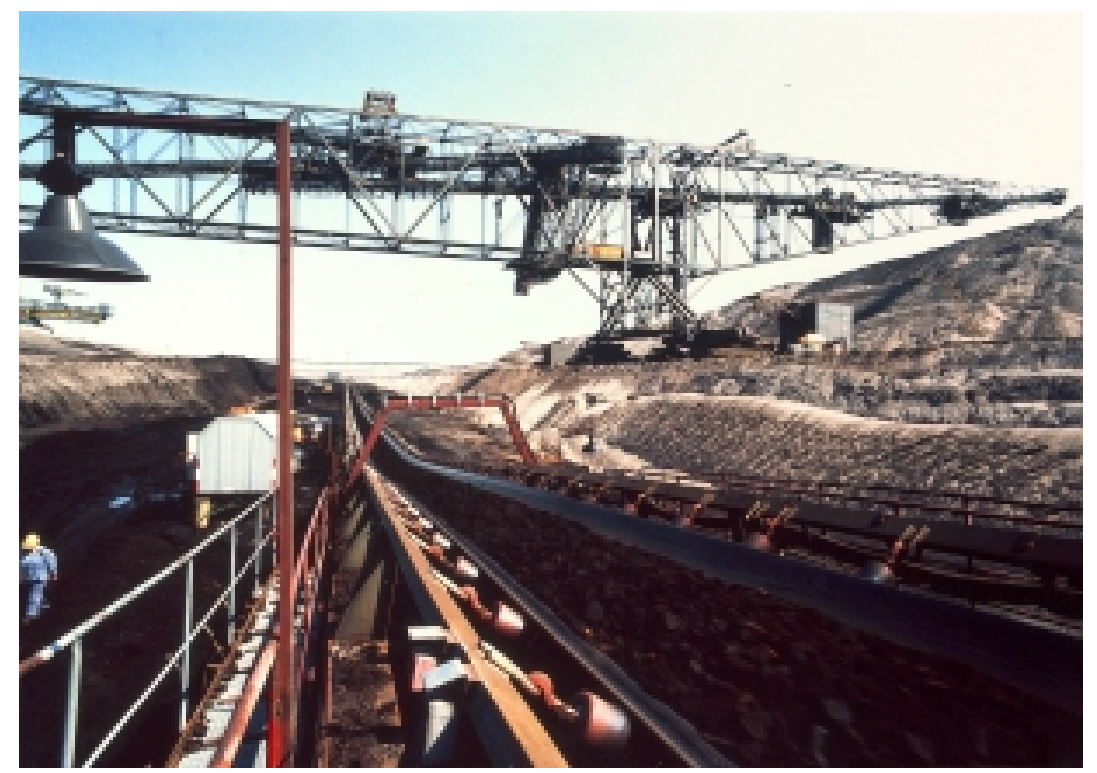

Abb. 2.2.: Förderbrücke im Lausitzer Braunkohlerevier (Quelle: http://www.rwe.com/1.6_RWE_Presse/Bilderund-Grafiken/Stammhaeuser/RWE_Rheinbraun/RWE_Rheinbraun_index.jsp, Stand 09.01.2001.

Durch Einsatz der Förderbrückentechnik (Abb. 2.2.) kam es in Cospuden zu einer stark inhomogenen Aufschüttung der Kippe mit einer Rippenstruktur, die die Charakterisierung der hydrogeologischen Verhältnisse stark erschwert. Die natürlichen Fließbedingungen wurden durch das Einbringen der Kippe, die eine geringere Transmissivität besitzt als das natürlich vorliegende Material, und durch das Ausräumen der Grundwasserleiter verändert (LMBV 1995). Da bei der Aufschüttung nicht auf eine Abdeckung der pyrithaltigen Gesteine mit inertem Kippenmaterial geachtet wurde, die einen Kontakt des Materials mit Luftsauerstoff hätte vermindern können, kam es im Kippenbereich zu einer ausgeprägten Pyritoxidation, die sich teilweise sogar in starken Temperaturanstiegen niederschlugen (NITZSCHE et al. 1999). Um wenigstens die weitere Oxidation $\mathrm{zu}$ bremsen, wurde versucht, durch eine schnelle Einleitung von Wasser in das Restloch (ab 1993) den weiteren Kontakt von Eisendisulfiden mit der Luft zu unterbinden; außerdem war allein aus dem Grundwasserzufluss nicht mit einer Füllung des Restlochs in einem akzeptablen Zeitraum zu rechnen. Das verwendete Wasser stammte vor allem aus der Sümpfung anderer Tagebaue (z.B. Profen) und aus der Elster. Zusätzlich strömte Grundwasser in das Gebiet ein (POKRANDT und ZEH (1999)). Durch die schnelle Flutung wurde zusätzlich eine Stabilisierung der Haldenböschungen erreicht. Im Jahr 2000 hat der See mit max. 54 m Wassertiefe seine Flutungsgrenze (110 m über NN) erreicht. Im stationären Zustand fließt dem See aus angrenzenden Grundwasserleitern eine Wassermenge von ca. $3 \mathrm{~m} \mathrm{~m}^{3} / \mathrm{min}$ zu. Die Verteilung der Zuflüsse, der Zehrung und der Abflüsse zeigt Abb. 2.3. (nach LMBV 1995). 


\section{Restloch Cospuden - Zu- und Abflüsse}

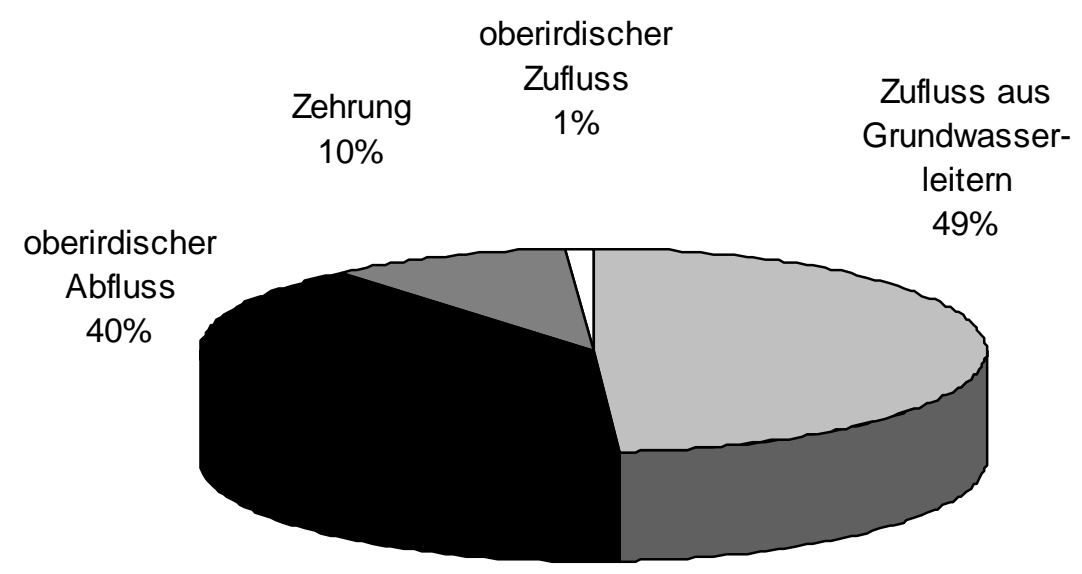

Abb. 2.3.: Wasserbilanz des Restlochs Cospuden im quasistationären Zustand.

Umfangreiche Planierungsarbeiten waren notwendig, um die Böschungen des Sees standsicher $\mathrm{zu}$ gestalten und die für Naturschutz und Wassersport benötigten Flachwasserzonen und Uferbereiche zu schaffen.

Die Umgebung wird derzeit rekultiviert und bepflanzt (Abb. 2.4.-2.6.); es liegen umfangreiche Pläne für die Nutzung als Naherholungsgebiet und Landschaftssee (LMBV (1995), TRETTIN und GLÄSER (1995)) vor. Der gesamte südliche Teil und kleinere Bereiche im Nordosten und Nordwesten sollen dem Natur- und Landschaftsschutz vorbehalten bleiben, während der nördliche Teil touristisch zu erschließen ist. Kleine Strände für Wasser- und Angelsport sollen im westlichen und östlichen Uferbereich im Wechsel mit Vegetationsflächen den künftigen See landschaftlich attraktiv und abwechslungsreich machen. Erste Badegäste haben sich in den vergangenen Sommern bereits eingefunden. 


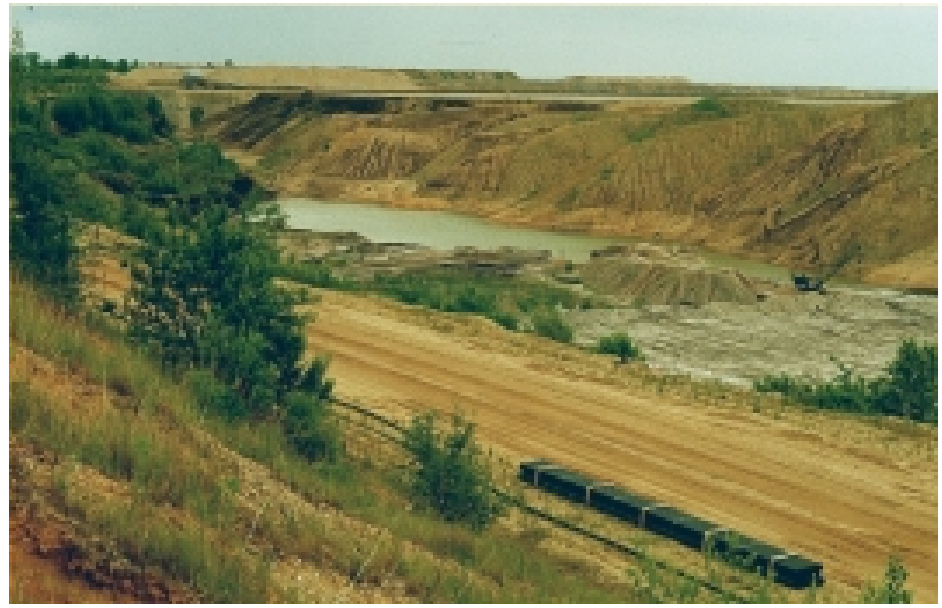

Abb. 2.4.: Planierungs- und Drainage-Arbeiten im Restloch Cospuden 1997 (Bild: Pleßow).

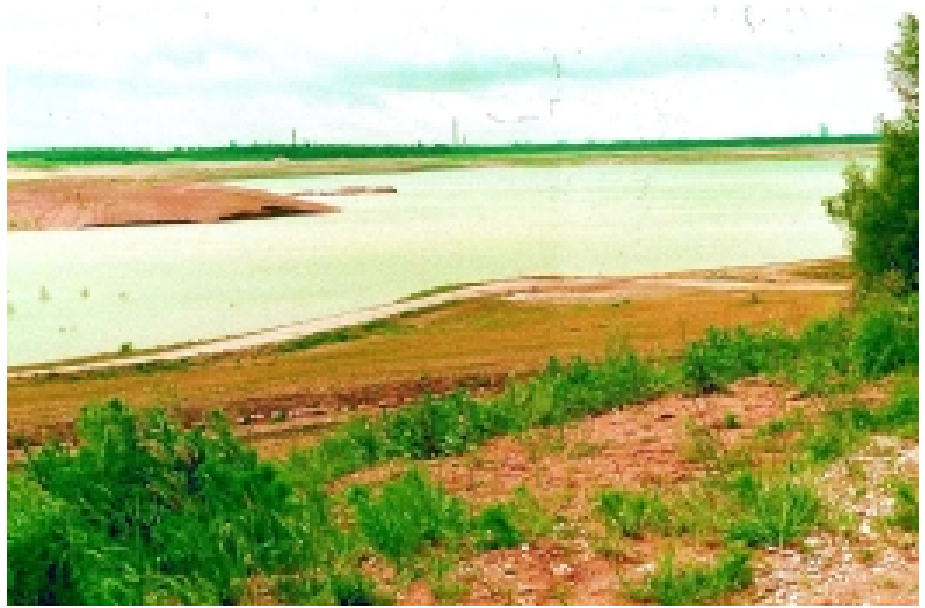

Abb. 2.5.: Blick über den Restsee Cospuden in Richtung Nordosten während der Flutung im Jahr 1997; im Hintergrund erkennbar das Universitäts-Hochhaus Leipzig (Bild: Pleßow).

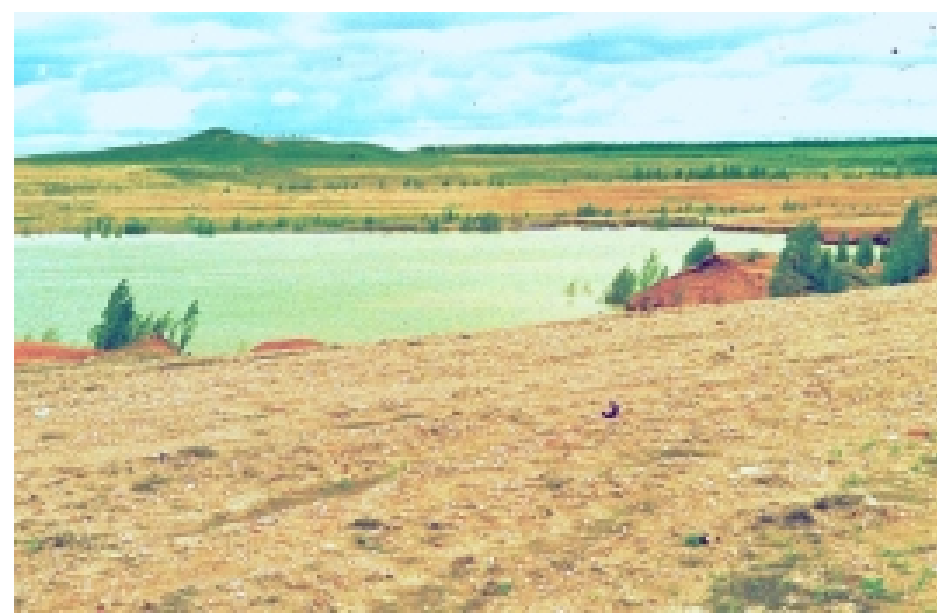

Abb. 2.6..: Restsee Cospuden während der Flutung im Jahr 1997 (Bild: Pleßow). 


\subsection{Lausitz}

In der Lausitz sind mehr als 100 Seen von der Versauerung betroffen. Die im Rahmen der vorliegenden Arbeit untersuchten zwei Bohrkerne stammen aus dem Einzugsgebiet des Restlochs 111 in der Lausitz. Restloch 111 gehört zum ehemaligen Tagebau Plessa, dessen geographische Lage in Abb. 2.7. dargestellt ist. In dieser Gegend wurde von etwa 1925 bis in die fünfziger Jahre hinein Braunkohle gewonnen. Dabei kam die erste Abraumförderbrücke der Welt zum Einsatz, was zu einer Rippenstruktur der Kippe und ähnlichen Problemen bei der hydrogeologischen und geologischen Beschreibung führte, wie sie im Fall Cospuden beschrieben wurden. Nach der Einstellung des Tagebaus wurde das Restloch durch zuströmendes Grundwasser geflutet. Ost- und Südufer des Sees sowie Teile des Westufers bestehen aus Kippensedimenten. Der See umfasst heute drei miteinander verbundene Teilbecken, die insgesamt eine Längsausdehnung von etwa 900 m in Nord-Süd-Richtung und eine Breite von 120 bis 140 m einnehmen. Im mittleren Teilbecken erreicht der See seine maximale Tiefe mit bis zu 10,2 m. Das nördliche und südliche Teilbecken sind dagegen nur bis zu ca. $7 \mathrm{~m}$ tief. Der See ist fast vollständig von Kiefern- und Birkenwäldern umgeben.

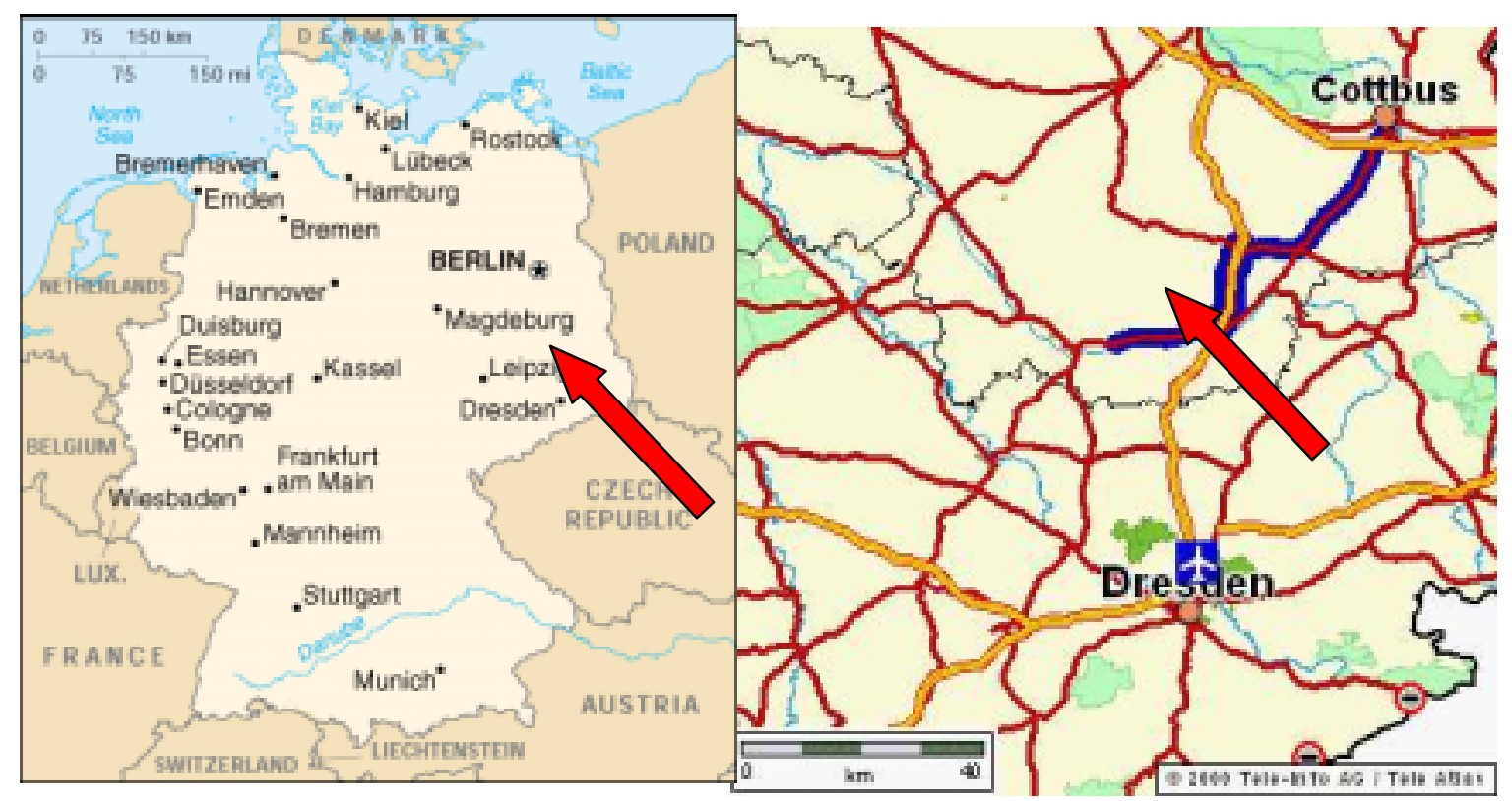

Abb. 2.7.: Geographische Lage des Restsees 111.

Da große Teile des Seeufers aus Kippensedimenten bestehen, die in unmittelbarer Nähe zum See kaum Pflanzenbewuchs zeigen, werden durch Erosion jährlich ca. 3000 t Kippensedimente in den See gespült. Der durchschnittliche Schwefelgehalt der Sedimente beträgt rund 7 Massen-\% und setzt sich zusammen aus löslicher Schwefelsäure, Gips, Jarosit und kleineren Anteilen von Pyrit und organischem Schwefel (BOZAU et al. 2000). 
Zwar beläuft sich der Pyritgehalt der Braunkohle in diesem Gebiet nur auf ca. 0,2 bis etwas über 3 Massen-\%. Da die Böden jedoch über eine überaus niedrige Säureneutralisationskapazität verfügen und auch die tertiären Sedimente Pyritgehalte aufweisen, die teilweise über denen der Braunkohle liegen, stellt die Versauerung infolge der Pyritoxidation ein ernstzunehmendes Problem dar. Daran hat sich seit der Flutung des Restlochs wenig geändert. Das Wasser des Sees weist einen pH-Wert von 2,6 - 3,1 auf; die Sulfatkonzentrationen liegen typischerweise bei $1,3 \mathrm{~g} / \mathrm{l}$, die Eisengehalte betragen $150 \mathrm{mg} / \mathrm{l}$ (BozAU et al. 2000). Auch das Grundwasser weist hohe Gehalte an Sulfat, Eisen und Aluminium auf. Zwischen dem See und den umgebenden Wässern findet ein lebhafter Austausch statt, wobei die Hauptflussrichtung von Südwest nach Nordost verläuft. Im See selbst kommt es kaum zu Neutralisationreaktionen, während am Ausfluss Sulfatreduktion zu einer Regeneration des belasteten Wassers führt (KNÖLLER 1999). Um auch für den See ein Regenerationskonzept zu entwickeln, werden dort durch das UFZ Halle-Leipzig GmbH umfangreiche hydrogeologische und hydrogeochemische Untersuchungen durchgeführt (BozAU et al. 2000). 


\section{Methodik}

\subsection{Experimentelles}

\subsubsection{Probennahmen und Präparation}

Im Rahmen der vorliegenden Arbeit wurden insgesamt 68 Poren- und Sickerwasserproben untersucht. 55 davon stammen aus dem Bereich des ehemaligen Tagebaus Cospuden. Bei den Proben RCO4 17 m, 20 m, 22 m, 26 m, 36 m, 40 m, 44 m, 48 m und 52 m handelt es sich um Bohrkernmaterial aus der Bohrung des Grundwasserpegels RCO4 vom November 1995, die unter Argon tiefgekühlt gelagert und im Mai 2000 zur Ergänzung des Tiefenprofils der Porenwässer von RCO4 untersucht wurden (weitere Daten des Tiefenprofils der Porenwässer stammen aus der Arbeit von PleßOW (1998)). Zusätzlich wurden im Rahmen der vorliegenden Arbeit von 25 Proben aus RCO4 mineralogische und elementaranalytische Untersuchungen der Festphasen durchgeführt.

Aus dem Bohrkernmaterial des Multilevelbrunnens CML, der im August 1998 angelegt wurde, stellte das UFZ Halle-Leipzig GmbH (H.R. Gläser und J. Richert) insgesamt acht Proben zur Verfügung.

Im November 1998 wurde in Cospuden in Zusammenarbeit mit H. R. Gläser (UFZ HalleLeipzig GmbH) und J.-U. Brinkmann (Geochemisches Institut der Universität Göttingen) mit einem Rammkernbohrer ein oberflächennaher Bohrkern aus der Absetzerkippe entnommen (stratigraphische Tiefe: 1,85-3,78 m). Der Bohrkern wurde vor Ort unter Argon eingefroren. Die Festphasen aus dem Bohrkern von CML sowie der oberflächennahen Bohrung wurden von Brinkmann untersucht (BRINKMANN 2000).

Wasserproben aus dem Multilevelbrunnen CML und den RCO-Pegeln wurden im Oktober 1998, Januar 1999, August 1999 und Januar 2000 gewonnen. Eine genaue Beschreibung der Technik der RCO-Messstellen findet sich bei NiTSCHE et al. (1999). Dort werden auch die Lage der Grundwasserleiter und der jeweilige Grundwasserentnahmeraum erläutert.

Eine Übersicht über die im Rahmen dieser Arbeit untersuchten Proben zeigt Tabelle 3.1. Wasserproben und Bohrkerne wurden von der Probennahme bis zur Bearbeitung kühl und dunkel gelagert; soweit möglich wurde das Material bei $-30^{\circ} \mathrm{C}$ unter einer ArgonInertgasatmosphäre eingefroren. Abb. 3.1. gibt Aufschluss über die Lage der einzelnen Probenahmepunkte im Bereich Cospuden. 


\begin{tabular}{|c|c|c|c|}
\hline Datum & Beprobung durch & Probenbezeichnungen & Anmerkungen \\
\hline November 1995 & UFZ Halle-Leipzig GmbH & 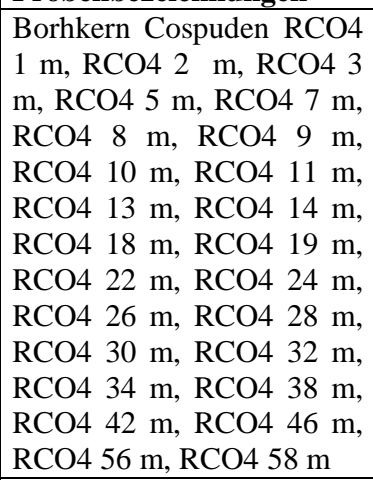 & Festphasen; bearbeitet 1999 - 2001 \\
\hline November 1995 & UFZ Halle-Leipzig GmbH & $\begin{array}{l}\text { Bohrkern Cospuden RCO4 } \\
17 \text { m; RCO4 } 20 \mathrm{~m} ; \text { RCO4 } \\
22 \mathrm{~m} \text {; RCO4 } 26 \mathrm{~m} ; \mathrm{RCO} 4 \\
36 \mathrm{~m} \text {; RCO4 } 44 \mathrm{~m} ; \mathrm{RCO} 4 \\
48 \mathrm{~m} \text {; RCO4 } 52 \mathrm{~m}\end{array}$ & Porenlösungen; bearbeitet im Mai 2000 \\
\hline August 1998 & UFZ Halle-Leipzig GmbH & $\begin{array}{l}\text { Bohrkern Cospuden CML } \\
\text { 24 m; CML 26 m; CML } 32 \\
\text { m; CML } 36 \text { m; CML 37,5 } \\
\text { m }\end{array}$ & Porenlösungen; bearbeitet im Oktober 1998 \\
\hline August 1998 & UFZ Halle-Leipzig GmbH & $\begin{array}{l}\text { Bohrkern Cospuden CML } \\
24 \text { m; CML } 26 \text { m; CML } 32 \\
\text { m; CML } 36 \text { m; CML 37,5 } \\
\text { m }\end{array}$ & Festphasen (BRINKMANN 2000) \\
\hline August 1998 & UFZ Halle-Leipzig GmbH & $\begin{array}{l}\text { Bohrkern Cospuden CML } \\
\text { 21-22 m; CML 39-40 m }\end{array}$ & Porenlösungen; bearbeitet im Mai 2000 \\
\hline Oktober 1998 & $\begin{array}{l}\text { Wasser und Boden Labor } \\
\text { GmbH Dresden }\end{array}$ & \begin{tabular}{lr}
\multicolumn{2}{l}{ Grundwassermesstellen } \\
Cospuden & RCO4; \\
RCO10A; & RCO12; \\
RCO13; RCO14 & \\
\end{tabular} & $\begin{array}{l}\text { Wasserproben; bearbeitet im November } \\
1998\end{array}$ \\
\hline November 1998 & \begin{tabular}{|l|} 
K. Brüschke \\
J.-U. Brinkmann \\
H.-R. Gläser \\
\end{tabular} & $\begin{array}{l}\text { oberflächennahe Bohrung } \\
\text { Cospuden } 1 \mathrm{~m}, 2 \mathrm{~m}, 3 \mathrm{~m} ; \\
\text { See }\end{array}$ & $\begin{array}{l}\text { Porenlösungen; bearbeitet im August } 1999 \\
\text { Wasserprobe }\end{array}$ \\
\hline November 1998 & $\begin{array}{l}\text { K. Brüschke } \\
\text { J.-U. Brinkmann } \\
\text { H.-R. Gläser } \\
\end{array}$ & $\begin{array}{l}\text { oberflächennahe Bohrung } \\
\text { Cospuden } 1 \mathrm{~m}, 2 \mathrm{~m}, 3 \mathrm{~m}\end{array}$ & Festphasen (BRINKMANN 2000) \\
\hline Januar 1999 & UFZ Halle-Leipzig GmbH & $\begin{array}{l}\text { Multilevelbrunnen } \\
\text { Cospuden CML 20 m; } \\
\text { CML 27,5 m; CML 31,5 m; } \\
\text { CML 39,5 m; CML 43,5 m }\end{array}$ & $\begin{array}{l}\text { Wasserproben; bearbeitet im April - Juni } \\
1999\end{array}$ \\
\hline August 1999 & $\begin{array}{l}\text { K Brüschke in } \\
\text { Zusammenarbeit mit UFZ } \\
\text { Halle-Leipzig GmbH }\end{array}$ & $\begin{array}{l}\text { Multilevelbrunnen } \\
\text { Cospuden CML 20 m; } \\
\text { CML 27,5 m; CML } 31,5 \mathrm{~m} \text {; } \\
\text { CML 39,5 m; CML 43,5 m; } \\
\text { Grundwassermessstellen } \\
\text { Cospuden RCO3; RCO4; } \\
\text { RCO12; RCO18; See; } \\
\text { See/Sediment } \\
\end{array}$ & 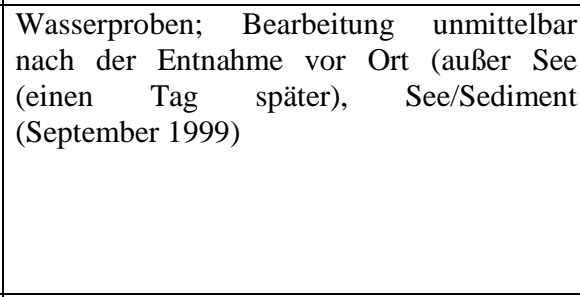 \\
\hline August 1999 & UFZ Halle-Leipzig GmbH & $\begin{array}{l}\text { Grundwassermessstellen } \\
\text { Cospuden RCO15; RCO17 }\end{array}$ & $\begin{array}{l}\text { Wasserproben; bearbeitet im Dezember } \\
1999\end{array}$ \\
\hline Oktober 1999 & UFZ Halle-Leipzig GmbH & \begin{tabular}{|l|} 
Bohrkern Lausitz 5-6 m \\
Bohrkern Lausitz 21-22 m \\
\end{tabular} & $\begin{array}{l}\text { Porenlösungen; bearbeitet im April } 2000 \\
\text { bearbeitet im November/Dezember } 1999\end{array}$ \\
\hline Januar 2000 & UFZ Halle-Leipzig GmbH & $\begin{array}{l}\text { Grundwassermessstellen } \\
\text { Cospuden RCO3; RCO4; } \\
\text { RCO12; RCO15; RCO 17; } \\
\text { RCO18; Multilevelbrunnen } \\
\text { Cospuden CML 20 m; } \\
\text { CML27,5 m; CML 31,5 m; } \\
\text { CML 39,5 m; CML 43,5 m; }\end{array}$ & $\begin{array}{l}\text { Wasserproben; bearbeitet im September } \\
2000\end{array}$ \\
\hline
\end{tabular}

Tab. 3.1.: Übersicht über die im Rahmen der vorliegenden Arbeit untersuchten Proben. 


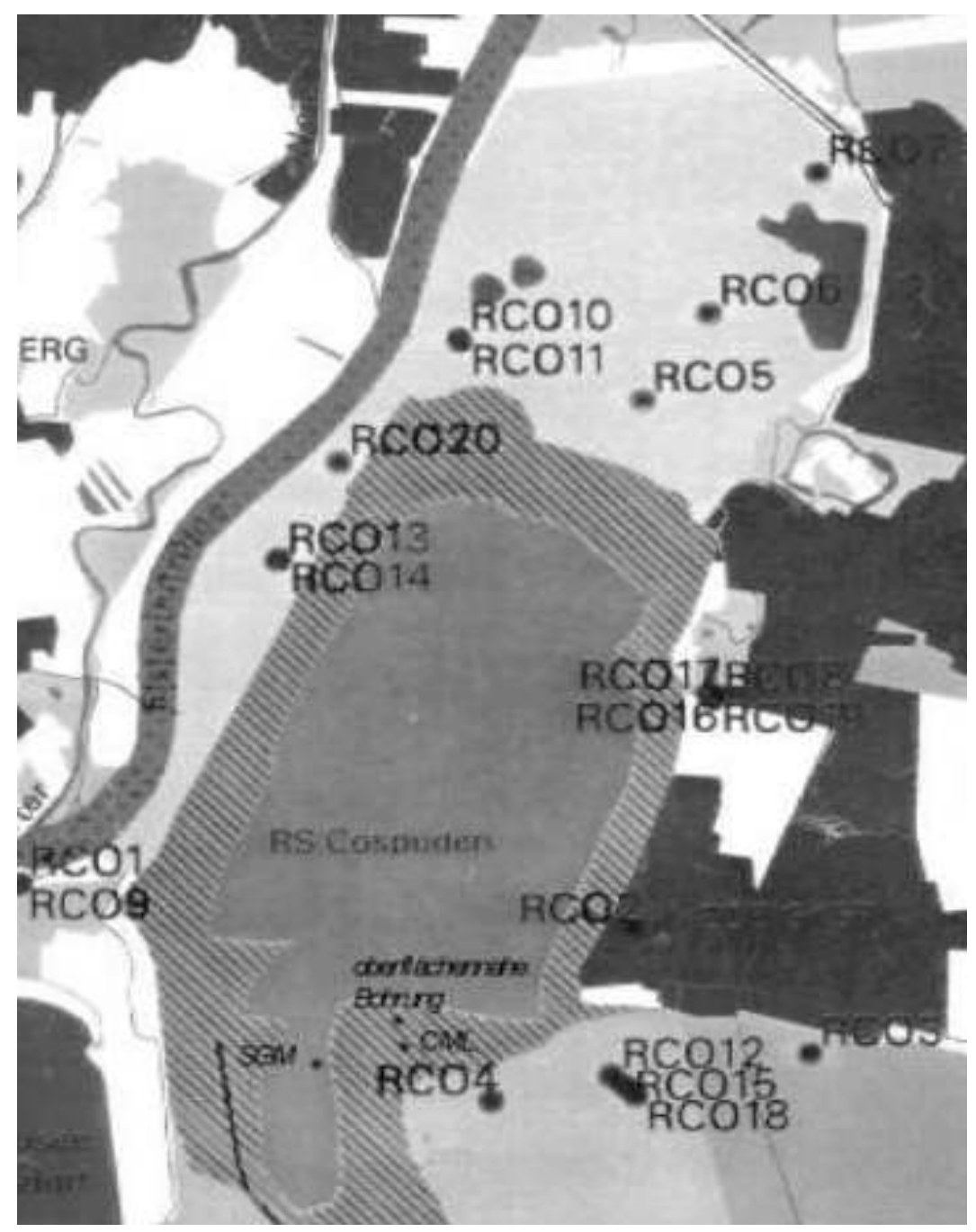

Abb. 3.1.: Lage der Probenahmepunkte im Raum des ehemaligen Tagebaus Cospuden.

Der ehemalige Sicker- und Grundwasserbrunnen SGM, der Multilevelbrunnen CML sowie die Grundwassermessstellen RCO4, RCO12, RCO15 und RCO18 liegen im Bereich der Kippe. RCO3 befindet sich in einem Waldgebiet in der Nähe. Die Pegel RCO10, RCO13 und RCO14, aus denen im Rahmen dieser Arbeit ebenfalls Wasserproben untersucht wurden, liegen im Tagebauvorfeld, aus dem der Mutterboden abgetragen wurde.

Aus der unmittelbaren Umgebung des Restlochs 111 im ehemaligen Tagebau Plessa im Lausitzer Braunkohlerevier wurden von Dr. E. Bozau (UFZ Halle-Leipzig GmbH) zwei Bohrkerne (21 - $22 \mathrm{~m}$ und $5-6 \mathrm{~m}$ ) aus Trockenbohrungen zur Verfügung gestellt. 
Der Bohrkern 5 -6 m stammt aus einer Bohrung am Ostufer des Sees; das Grundwasser liegt dort in ca. 4,5 m Tiefe über der Geländeoberkante (GOK). Die Bohrung 21 - $22 \mathrm{~m}$ dagegen wurde am Südufer der Kippe durchgeführt. Der Grundwasserleiter dort liegt in ca. $21 \mathrm{~m}$ Tiefe über GOK. Das Material wurde unter Argon tiefgefroren gelagert und im Mai 2000 bearbeitet. Die Aufteilung des Materials erfolgte nach einem Längsschnitt durch den Bohrkern nach optischen Gesichtspunkten und ist in Tab. 3.2. dargestellt.

\begin{tabular}{|l|l|}
\hline Bohrkern 5-6 m & Beschreibung \\
\hline $\mathbf{5 , 0 0 - 5 , 2 1 ~} \mathbf{~ m}$ & dunkelbraun/schwarz mit hellbraunen Sprenkeln, locker \\
\hline $\mathbf{5 , 2 1 - 5 , 3 0 ~} \mathbf{m}$ & hellbraun/weiß, gipshaltig \\
\hline $\mathbf{5 , 3 0 - 5 , 7 5} \mathbf{~ m}$ & dunkelbraun/schwarz porös, mit Gips gemischt \\
\hline $\mathbf{5 , 7 5 - 5 , 9 3 ~} \mathbf{m}$ & weiß, stark gipshaltig \\
\hline $\mathbf{5 , 9 3 - 6 , 0 0 ~} \mathbf{m}$ & dunkelbraun, schwarz \\
\hline
\end{tabular}

\begin{tabular}{|l|l|}
\hline Bohrkern 21-22 $\mathbf{~ m}$ & Beschreibung \\
\hline $\mathbf{2 1 , 0 7 - 2 1 , 1 3 ~} \mathbf{~ m}$ & sehr dunkel, nass \\
\hline $\mathbf{2 1 , 1 3 - 2 1 , 7 0 ~} \mathbf{m}$ & hellbraun, sandig, trockener \\
\hline $\mathbf{2 1 , 7 0 - 2 1 , 8 0 ~} \mathbf{m}$ & dunkel, feucht \\
\hline $\mathbf{2 1 , 8 0 - 2 1 , 9 0 ~} \mathbf{m}$ & schwarz, nass \\
\hline $\mathbf{2 1 , 9 0 - 2 2 , 0 0 ~} \mathbf{m}$ & braun, sandig \\
\hline
\end{tabular}

Tab. 3.2.: Aufteilung der beiden Kerne aus den Trockenbohrungen am Restsee 111.

Insgesamt waren die Proben sehr viel wasserhaltiger als das Material aus Cospuden, so dass eine relativ kleinräumige Differenzierung möglich war. 


\subsubsection{Gewinnung von Porenlösungen}

Um das Porenwasser aus Bohrkernmaterial zu gewinnen, wurde eine Presse benutzt, die in Abb. 3.2. schematisch dargestellt ist. Einzelheiten sind bei HeINRICHS et al. (1996), BöTTCHER et al. (1997) und PLEßOw (1998) beschrieben.

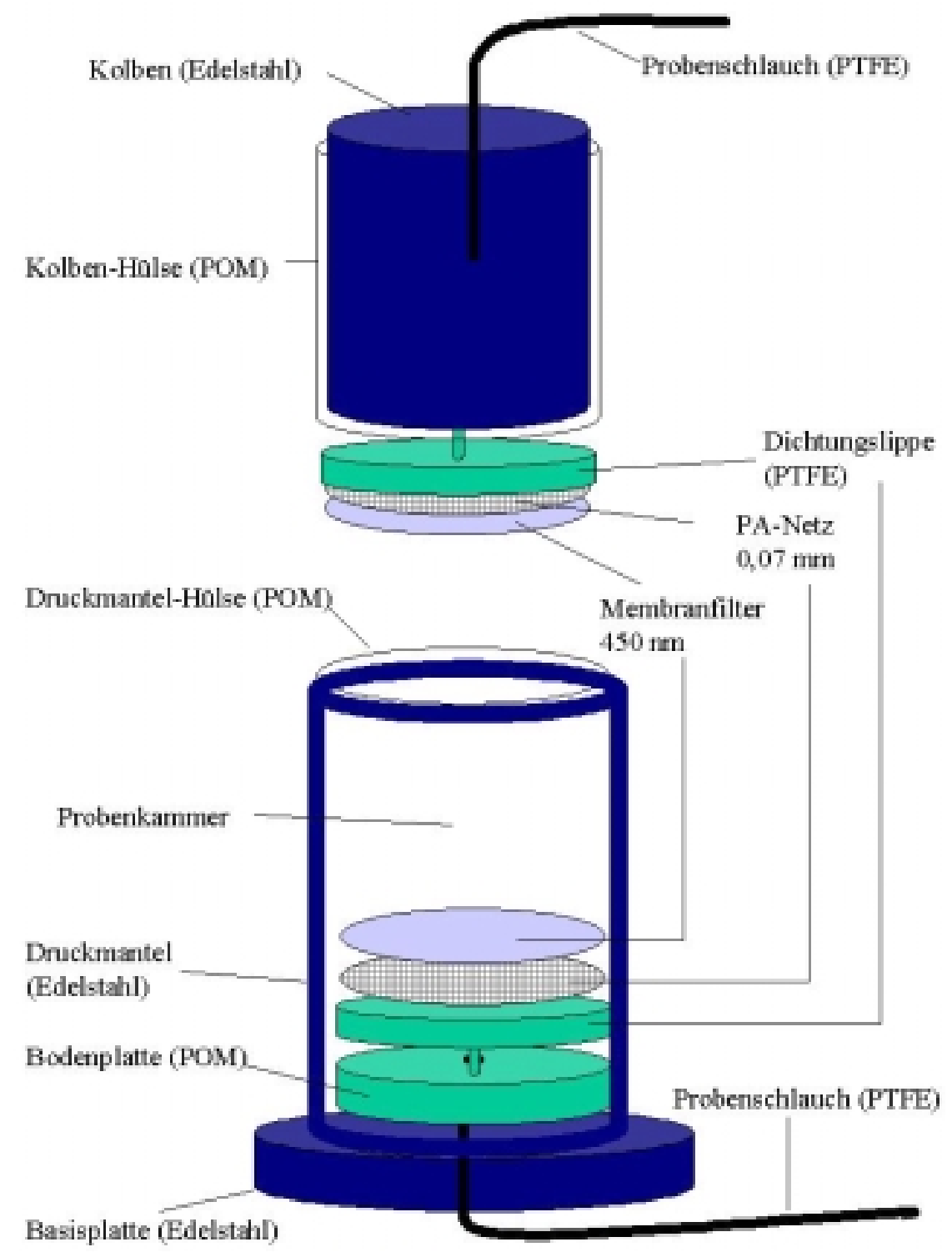

Abb 3.2.: Presse zur Gewinnung von Porenlösungen.

Gegenüber anderen Methoden wie z.B. der Zentrifugation lassen sich mit der Presse auch aus weichem tonigen oder gipshaltigen Material noch ausreichende Wassermengen für die Untersuchung gewinnen. Allerdings ist das Verfahren sehr zeitaufwendig, und bei stark tonoder gipshaltigem Material mussten bis zu 12 Portionen à ca. $200 \mathrm{~g}$ gepresst werden. Je nach Charakter des Probenmaterials kann ein einzelner Pressvorgang bis zu sechs Stunden dauern; 
die Gewinnung von $150 \mathrm{ml}$, die für das Speziationsverfahren benötigt werden, kann mehrere Tage in Anspruch nehmen. Dabei wird der Druck in jedem einzelnen Pressgang möglichst gleichmäßig bis auf $130 \mathrm{MPa}$ gesteigert.

Um das Probenmaterial keinem Kontakt mit Sauerstoff auszusetzen, wurden die Pressköpfe in einer mit Argon gespülten Arbeitsbox gefüllt und zusammengebaut. Die gewonnene Lösung wurde durch Probenschläuche in eine mit Argon gespülte Flasche geleitet, die sich in einem argongefüllten Kunststoffbeutel befand.

\subsubsection{Behandlung der Wasserproben}

Alle Wasserproben wurden durch eine Druckfiltration (0,5 - 1,2 MPa Ar in Druckbehältern aus PTFE; Cellulosenitrat-Filtermembranen 450 nm Porenweite von Sartorius, Göttingen) von ungelösten Partikeln befreit. Im Anschluss an ihre Bearbeitung (Ultrafiltration, Kationenoder Anionenaustausch) mussten sie für die Analyse durch Zusatz von Salpetersäure bis zu einem $\mathrm{HNO}_{3}$-Gehalt von $2 \%$ stabilisiert werden. Ein Aliquot von ca. $20 \mathrm{ml}$ wurde jeweils ohne Säurezusatz für die Bestimmung des DOC aufbewahrt. Die Lagerung der Proben erfolgte in PE-Flaschen dunkel bei ca. $4^{\circ} \mathrm{C}$.

\subsubsection{Verwendete Materialien}

Das zum Herstellen von Lösungen und Verdünnungen verwendete Wasser wurde mit einer Seralpur Pro $90 \mathrm{CN}$ Wasseraufbereitungsanlage behandelt. Die verwendeten Säuren $\left(\mathrm{HNO}_{3}\right.$ $65 \%$; $\mathrm{HCl} 22 \%$ ) entsprachen dem Reinheitsgrad suprapur oder konnten in vergleichbarer Qualität durch Destillation unterhalb des jeweiligen Siedepunktes in Suprasil-Quarz-Destillen bzw. Teflon-Apparaturen gewonnen werden. Die zur Regeneration der Ionentauscher eingesetzte Natronlauge wurde aus $1 \mathrm{~N}$-Maßlösung oder aus $\mathrm{NaOH}-\mathrm{Plätzchen,} \mathrm{beide} \mathrm{in} \mathrm{der}$ Qualität p.a. von Merck, Darmstadt, hergestellt. Die KCl-Lösung zur Aufbewahrung gebrauchter Ionentauscher ließ sich aus Kaliumchlorid p.a. bzw. reinst von Merck, Darmstadt, herstellen. Zur Lagerung der Proben wurden fabrikneue PE-Flaschen verwendet. Auf ein vorheriges Spülen oder sonstige Maßnahmen zur Vorbereitung wurde verzichtet, um Verdünnungen oder sonstige Kontamination der Proben zu vermeiden.

Zur Untersuchung der Wirkung organischer Zusätze kamen Harnstoff p.a., L-Threonin für biochem. Zwecke (beide von Merck, Darmstadt) und R-(+)-Cystein zur Synthese der Firma Schuchardt zum Einsatz. 


\subsubsection{Speziation}

Die Speziation der Proben erfolgte nach dem von Pleßow (PlEßOW 1998, PleßOw und HEINRICHS 2000b) entwickelten Schema (Abb. 3.3.). Für die Ultrafiltration wurden 1 kDMembranen von Pall/Filtron (Typ Omega) verwendet. Vor der Verwendung lagerten die Membranen dreimal jeweils eine Stunde in reinem Wasser. Nach dem Einbau in die handelsübliche Rührzelle der Firma Amicon (Typ 8200) wurde die Membran mit $50 \mathrm{ml}$ Wasser gespült, ehe die Probe filtriert werden konnte. Die ersten $2 \mathrm{ml}$ des erhaltenen Filtrates wurden verworfen, um Verdünnungen durch das Spülwasser zu vermeiden. Auf eine Wiederverwendung der Membranen mussten wegen der Kontaminationsgefahr verzichtet werden.

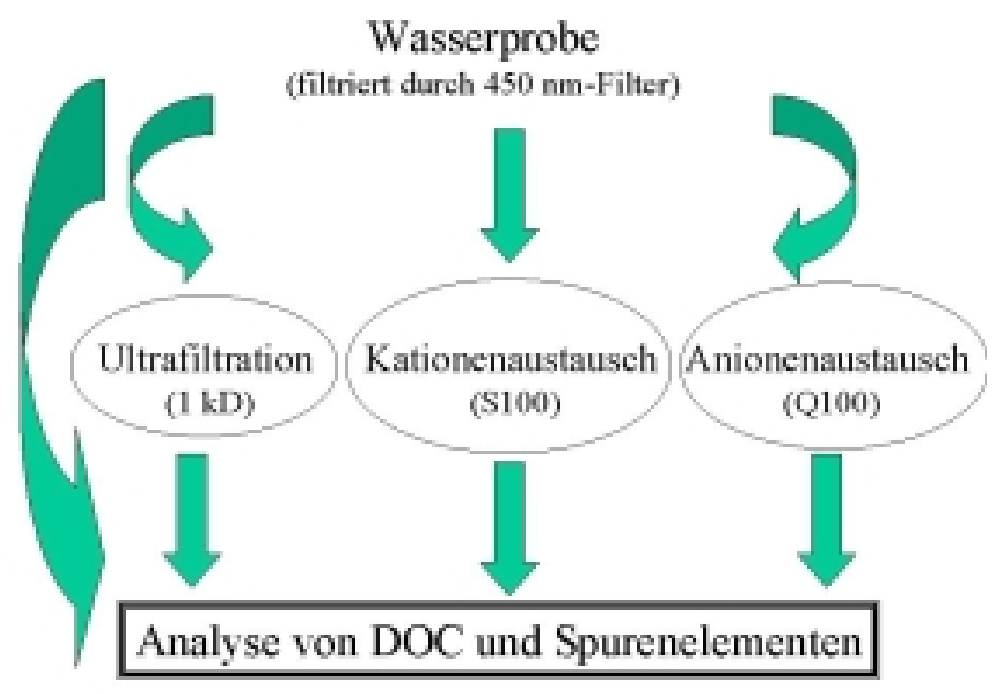

Abb. 3.3.: Schema des Speziationsverfahrens; ausführliche Erläuterungen s. Kap.5.

Der Kationenaustausch erfolgte über Sartobind-Module S100 der Firma Sartorius. Die Module wurden vorbereitet bzw. regeneriert, indem zunächst $250 \mathrm{ml} 1 \mathrm{~N}-\mathrm{HCl}$ und anschließend Wasser bis zur Neutralität (ca. $250 \mathrm{ml}$ ) darübergeleitet wurden.

Für den Anionenaustausch kamen Sartobind-Module Q100 der Firma Sartorius zum Einsatz. Anfangs wurden sie nach den Angaben von PleßOW (1998) mit $250 \mathrm{ml} 1 \mathrm{~N}-\mathrm{NaOH}$ und 250 ml Wasser präpariert. Durch die Veränderung des $\mathrm{pH}$-Wertes bei der Behandlung der Proben kam es jedoch zu Problemen bis hin zur Bildung deutlich sichtbarer EisenhydroxidNiederschläge im Austauschermodul. Deswegen wurde das Verfahren modifiziert: Die Module wurden mit $250 \mathrm{ml} 1 \mathrm{~N}-\mathrm{NaOH}, 250 \mathrm{ml}$ Wasser, dann $250 \mathrm{ml} 1 \mathrm{~N}-\mathrm{HCl}$ und Wasser bis zur Neutralität vorbereitet, um einen Austausch gegen $\mathrm{Cl}^{-}-$statt gegen $\mathrm{OH}^{-}$-Ionen 
durchzuführen. Im Tabellenanhang (Tab. A.7. und Tab. A.8.) ist für jede Probe vermerkt, gegen welches Anion ausgetauscht wurde.

Zum Pumpen diente eine peristaltische Pumpe (Desaga STA 131900; gespült wurde bei einer Durchflussgeschwindigkeit von $50 \mathrm{ml} / \mathrm{min}$. Der Ionenaustausch erfolgte bei einer Pumpgeschwindigkeit von $5 \mathrm{ml} / \mathrm{min}$. Von den Probenlösungen wurden beim Austausch jeweils die ersten $\mathrm{ml}$ verworfen, um Kontaminationen und Verdünnungseffekte zu vermeiden.

Gebrauchte Ionentauschereinheiten lagerten bis zur nächsten Benutzung in Kaliumchloridlösung $(1 \mathrm{~mol} / \mathrm{l})$ bei $+5^{\circ} \mathrm{C}$ und Dunkelheit.

\subsection{Analytik}

\subsubsection{Wasserproben}

Die Wasserproben wurden atomemissionsspektrometrisch und massenspektrometrisch untersucht.

Für die ICP-OES-Messungen kam ein Emissionsspektrometer Perkin Elmer Optima 3300 DV mit Probenautomat FS90 zum Einsatz. Ausführliche Angaben zur Methode finden sich bei HeINRICHS et al. (1985), HeINRICHS und HERMANn (1990) und SKoog und LEARy (1996). Die ICP-MS-Messungen erfolgten an dem Massenspektrometer VG Plasma Quad 2+ STE. Zur Kalibration wurden Maßlösungen und Mehrelementstandardlösungen der Bernd Kraft GmbH (Duisburg-Neumühl) sowie von Merck (Darmstadt) verwendet.

Die Bestimmung der DOC-Gehalte führte Frau K. Schmidt im Institut für Bodenwissenschaft der Universität Göttingen durch.

Die Messung der pH-Werte erfolgte mittels Einstabmesskette (Sentron 1001 pH bzw. WTW $\mathrm{pH}$ 330). Das Redoxpotential wurde über eine Platinelektrode mit einer Silber/SilberchloridReferenz-Elektrode (WTW pH 96 mit Hamilton Special Sensor) bestimmt. Die Sauerstoffmessung ließ sich mit Hilfe eines WTW OXI 191-Geräts mit Oxical-S-Elektrode bestimmen. Die elektrische Leitfähigkeit wurde mit einem WTW LF 90 KLE-Gerät gemessen. Angaben zu den Verfahren und deren Grundlagen finden sich bei KöLLING (1986), Schwedt (1992), Skoog und Leary (1996), Funk und Schär (1996), SigG (2000) sowie KÖLLING (2000). 


\subsubsection{Feststoffe}

Die Messung des Gesamt-Kohlenstoff- und Schwefel-Gehaltes wurde an einem $\mathrm{CO}_{2} / \mathrm{SO}_{2}$-IRAnalysator (Eltra CS 100/1000 RF) nach Verbrennung der Probe im Sauerstoffstrom eines Induktionsofens durchgeführt. Eine ausführliche Beschreibung der in Anlehnung an BRUMSACK (1981) und MELCHERS (1994) verwendeten Methode zur Unterscheidung von Sulfat-S und Sulfid-S sowie Carbonat-C und organischem C gibt BRINKMANN (2000). Die Hauptelemente ( $\mathrm{Si}, \mathrm{Ti}, \mathrm{Al}, \mathrm{Fe}, \mathrm{Mn}, \mathrm{Mg}, \mathrm{Ca}, \mathrm{Na}, \mathrm{K}$ und P) und 15 Spurenelemente (Nb, Zr, Y, $\mathrm{Sr}, \mathrm{Rb}, \mathrm{Pb}, \mathrm{Ga}, \mathrm{Zn}, \mathrm{Cu}, \mathrm{Ni}, \mathrm{Co}, \mathrm{Cr}, \mathrm{V}, \mathrm{Ba}, \mathrm{Sc})$ wurden mit einem wellenlängendispersiven Röntgenfluoreszenz-Spektrometer (Philips PW1480) bestimmt. Dazu wurden aus den Proben Schmelztabletten hergestellt; näheres s. HARTMANN (1994). Vollaufschlüsse der Proben wurden in Druckaufschlussblöcken (HEINRICHS 1989/90) mit $\mathrm{HClO}_{4}$ und $\mathrm{HF}$ hergestellt und massenspektrometrisch untersucht.

Zusätzliche Mineralphasenbestimmungen mit Röntgendiffraktometrie wurden nur an ausgewählten Proben durchgeführt. Hier bestand Verdacht auf neu gebildete Eisensulfatphasen. Ansonsten wurden alle Mineralphasenuntersuchungen im Rahmen der Arbeiten von KITTSTEINER (1998) und BRINKMANN (2000) erstellt.

\subsubsection{Software}

Alle Berechnungen, auch die der Korrelationskoeffizienten, und die Erstellung der Graphiken wurden mit Microsoft Excel 95 bzw. Microsoft Excel 2000 durchgeführt.

\subsection{Modellierung}

Das Programm MINTEQA2 wird vertrieben durch die U.S. Environmental Protection Agency (EPA) und erlaubt u.a. die Berechnung der Verteilung von Oxidationsstufen, die Speziation der Spurenmetalle und die Ermittlung der adsorbierten Anteile an verschiedenen Oberflächen (HydroGeologic und Allison 1999, HydroGeologic 1999, Allison et al. 1991). Zwar handelt es sich bei MINTEQA2 um ein Gleichgewichtsmodell, während unsere Proben vielfach einer kontinuierlichen Beeinflussung von außen unterliegen (z.B. Transport von Porenwässern, Austausch mit Mineraloberflächen); doch dürfte zumindest in den sich langsam bewegenden Porenwässern Verhältnisse herrschen, die als quasistationär betrachtet werden können. Es sollte jedoch immer berücksichtigt werden, dass einzelne Prozesse auch einer kinetischen Kontrolle unterliegen können und dann mit MINTEQA2 nicht korrekt erfasst werden (KöLLING und SCHULZ 1992). 
In seiner neuesten Version enthält das Programm auch eine Datenbank mit Parametern für DOC als Summenparameter. Der zugrundeliegende DOC entstammt einem Flusswasser und enthält wenige Bindungsstellen ( 1 meq/g $\quad$ C) mit vergleichsweise niedrigen Komplexbildungskonstanten (SUSETYO et al. 1991).

\subsection{Fehlerbetrachtung}

Zur Kontrolle über die Genauigkeit der Analysen wurden jeweils Institutsstandards und internationales Referenzmaterial mitgemessen (TW, BB, GD bzw. SLRS-3, J1K-1). Für einige Elemente wurden Doppelmessungen sowohl mit der ICP-MS als auch mit der ICP-OES durchgeführt. Dabei kam es teilweise zu großen Abweichungen zwischen den beiden Messwerten; welche Werte verwendet wurden, konnte nach der Güte der mitgemessenen Standards entschieden werden. Der relative Gesamtfehler der Analysen betrug bei den ICPMS- und ICP-OES-Messungen maximal $20 \%$, stieg jedoch im Bereich der Bestimmungsgrenzen auf bis zu $50 \%$ an. Lagen die Fehler darüber, wurden die Werte verworfen. Durch die hohen Konzentrationen in den Lösungen können unerkannt gebliebende Matrixeffekte nicht ausgeschlossen werden.

Insgesamt kann man davon ausgehen, dass die Analysenfehler in der Regel deutlich geringer lagen, als jene, die durch die Inhomogenität und die Bearbeitung des Probenmaterials entstanden. Deshalb wird auf eine detaillierte rechnerische Darstellung der Analysenfehler verzichtet.

Als systematische Fehler kommen bei den Porenwässern und Festphasen vor allem Inhomogenitäten in Frage; auf eine Homogenisierung der Proben wurde wegen der geringen Mengen und der Gefahr eines Kontakts mit Sauerstoff verzichtet. Bei den Wasserproben traten teilweise während der Lagerung beobachtbare Veränderungen auf. Dies wird in Kap. 4 diskutiert. Zu möglichen Fehlerquellen bei der Probennahme gibt BARCELONA (1990) eine ausführliche Darstellung.

Fehlerquellen im Speziationsverfahren bei der Ultrafiltration (BUFFLE et al. 1992) und beim Ionenaustausch wurden soweit wie möglich minimiert. So wurden die Lösungen bei der Ultrafiltration gerührt, um Coagulationseffekte auf der Membran zu verhindern. Der Anionenaustausch wurde auf $\mathrm{Cl}^{-}$als Austauschion umgestellt, um Fehler durch pH-WertÄnderungen auszuschließen. 


\section{Analyse von Sicker- und Porenwässern sowie von Bohrkernmaterial}

\subsection{Mineralbestand in Cospuden}

Abb. 4.1. zeigt die geologischen Schichten im unverritzten Material des Tagebaus Zwenkau Cospuden nach BELLMANN et al. (1984). Nach oben werden die Sedimente abgeschlossen durch eine 3 - 15 m mächtige Deckschicht unbekannter Zusammensetzung. Sie war bislang nicht Gegenstand einer eingehenden Analyse; bekannt ist nur, dass es sich um sehr quarzreiche Sedimente mit stark wechselnden karbonatischen Nebengemengeteilen handelt. Betrachtet man nun die Ergebnisse der Mineralphasenanalyse von BRINKMANN (2000) sowie die mineralogischen Daten des Tiefenprofils RCO4, fallen die extrem hohen Quarzanteile im Haldenmaterial auf, die aus einer reinen gleichverteilten Mischung der von KITTSTEINER (1998) untersuchten Sedimente, wie sie bisher in den Arbeiten von KITTSTEINER (1998) und PLEßOW (1998) angenommen wurde, nicht erklärt werden können. Vielmehr ist davon auszugehen, dass diese Sedimente mit Material aus der Deckschicht durchmischt wurden. Da genaue Informationen über die Zusammensetzung dieser Deckschicht fehlen, müssen einige Annahmen gemacht werden: Bekannt ist, dass es sich um ein sehr quarzreiches Sediment handelt. Der Pyritgehalt liegt nach BELLMANN et al. (1984) in der Randfazies unterhalb der Nachweisgrenze; die Beckenfazies wurde nicht analysiert. Wir nehmen für unsere Berechnungen an, dass der Quarzgehalt der Deckschicht bei $95 \%$ lag (dies entspricht den Quarzgehalten einiger tieferliegender quarzreicher Schichten nach BELLMANN et al. (1984)) und der Pyritgehalt max. $1 \%$ betrug. 


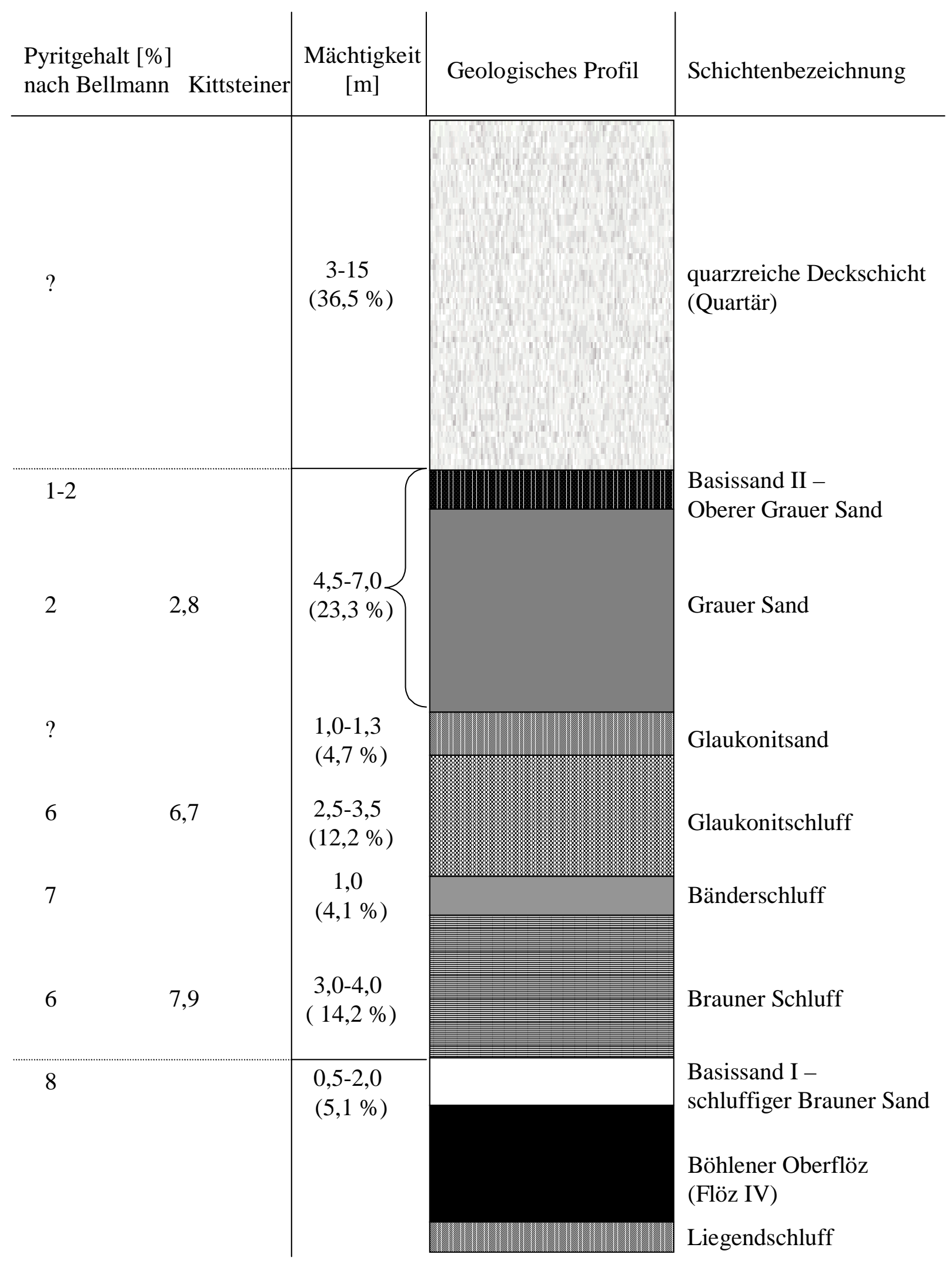


Außerdem ist das Verhältnis der Mächtigkeit der von KITTSTEINER (1998) untersuchten Schichten nicht 1:1:1, sondern 1,9:1,2:1, was sich auch in der Zusammensetzung des Kippensediments widerspiegeln sollte. Eine Überprüfung erlaubt die Berechnung des daraus resultierenden Quarzgehalts des Haldenmaterials und der Vergleich mit den gemessenen Quarzanteilen. Bei einem 1,9:1,2:1-Gemisch aus Grauem Sand, Braunem Sand und Glaukonitschluff ergibt sich für den durchschnittlichen Quarzgehalt der Sedimente unterhalb der Deckschicht ein Wert von 62,6\%.

Wie aus Abb. 4.1. zu entnehmen ist, macht die quarzreiche Deckschicht etwa 36,5\% des Sedimentprofils aus. Unter der Annahme, dass ihr Quarzgehalt bei etwa $95 \%$ liegt, erhält man für den durchschnittlichen Quarzgehalt der gesamten Sedimente einen Wert von $74 \%$. Dieser liegt im Bereich der gemessenen Quarzanteile aus der Arbeit von BRINKMANN (2000) und dem Tiefenprofil RCO4. Man kann also bei der Modellierung der Gesamtsedimente von einer Zusammensetzung der Kippensedimente aus 36,5 \% Deckschicht, 29,4 \% Grauem Sand, 18,6 \% Braunem Sand und 15,5\% Glaukonitschluff ausgehen, wenn die übrigen Sedimente vernachlässigt werden. Der Graue Sand ist das quarzreichste der von KITTSTEINER (1998) untersuchten Sedimente. Ohne Berücksichtigung des Pyritanteils enthält er 76 \% Quarz. Um die Deckschicht zu berechnen, wurde der Pyrit zunächst nicht berücksichtigt; die

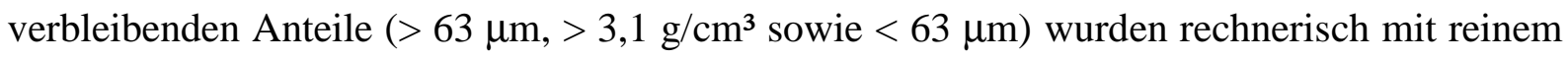
$\mathrm{SiO}_{2}$ auf einen Quarzgehalt von $95 \%$ und $4 \%$ an sonstigen Mineralien „verdünnt“ gemäß dem Dreisatz:

$\mathrm{x} * 0,76+\mathrm{y}=0,95 \quad$ (für den Quarzgehalt)

$0,24 * \mathrm{x}=0,04 \quad$ (für die übrigen Phasen ohne Pyrit).

Anschließend wurde wieder $1 \%$ Pyrit eingerechnet. Man erhielt damit $82 \%$ reinen Quarz als $\mathrm{SiO}_{2}, 17 \%$ Grauen Sand (ohne Pyrit) und $1 \%$ Pyrit. Die Zusammensetzung des Pyrits in der Deckschicht soll der des Pyrits aus dem Grauen Sand entsprechen.

Wie bereits in der Einleitung dargestellt, verändert sich im Zuge der Pyritoxidation der Mineralbestand des Tagebaus. Einige Mineralphasen werden durch Verwitterung direkt oder indirekt angegriffen und zersetzt, während andere Phasen sich sekundär vorübergehend oder dauerhaft bilden. Nach Cesnovar und Pentinghaus (1999) befindet sich in 18 bis $20 \mathrm{~m}$ Tiefe in der Kippe eine Oxidationszone, in der keine Pyrite mehr vorliegen. Dagegen entdeckten sie Gips, Jarosit und Heulandit als Mineralneubildungen. In den darüber- und darunterliegenden Schichten findet sich Siderit als Sekundärphase. Auch die obersten Kippenschichten wurden durch Cesnovar und Pentinghaus (1999) untersucht. Die Pyrite waren dort innerhalb eines Jahres bis in eine Tiefe von mindestens 2 m oxidiert. Mit einer 
Verzögerung von etwa 18 Monaten nach der Freisetzung von $\mathrm{K}^{+}$-Ionen aus Kalifeldspat, Illit und Glaukonit folgte dann die Bildung von Jarositen.

Auch WIEGAND und KRÜGER (1999) beschäftigten sich mit der Mineralogie der Kippensedimente im Bereich Zwenkau/Cospuden am Beispiel einer Schüttungsrippe. Sie stellten fest, dass die Pyritverwitterung in verschiedenen Kippensegmenten unterschiedlich schnell verlief und die Versauerung in Gegenwart quartärer Sedimente durch karbonatische Minerale gepuffert wurde. Gips, Kaolinit und Illit konnten in allen Proben gefunden werden. Pyrit wurde nur bis zu einem Lagerungsalter von einem Jahr sicher nachgewiesen; bis zu einem Alter von drei Jahren gibt es noch Anzeichen für sein Vorhandensein. Ältere oberflächennahe Proben waren vollständig oxidiert. In verdichteten und feinen Sedimenten jedoch wurde auch nach drei Jahren noch in zwei Metern Tiefe das Auftreten von Pyrit beobachtet.

WIEGAND und KRÜGER (1999) fanden Anzeichen für ein gleichzeitiges Vorliegen von Pyrit und Jarosit; aufgrund der langsamen parallel ablaufenden Prozesse können verschiedene Mineralphasen kurzzeitig nebeneinander auftreten. Gips, Kaolinit und Illit waren an der Oberfläche durch Regeneinwirkung und Verwitterung abgereichert. Karbonat wurde in den untersuchten Proben nicht gefunden, obwohl es Belege gibt, dass ursprünglich karbonathaltiges Substrat in den Sedimenten vorhanden war. Es wurde anscheinend durch die Säurebildung vollständig aufgelöst.

Die Ergebnisse von BRINKMANN (2000) bestätigen diese Beobachtungen. Danach dominieren Kies- und Sandfraktionen das Haldenmaterial. Insgesamt ist das Material sehr heterogen und durch die Schüttung stark durchmischt. Nur Spatenproben von der Oberfläche zeigen Anzeichen einer fluviatilen Sortierung. Die Hauptmineralphase aller untersuchten Proben ist Quarz; daneben werden in allen Proben Kaolinit, Illit, Chlorit, Feldspäte und Braunkohlereste gefunden. In allen Proben aus dem Multilevelbrunnen CML und in zwei Proben aus der oberflächennahen Bohrung tritt Montmorillonit auf; in zwei anderen Proben aus der oberflächennahen Bohrung wurden Wechsellagerungsminerale nachgewiesen. In 32 und $38 \mathrm{~m}$ Tiefe des Multilevelbrunnen-Materials liegen größere Mengen von Calcit und Dolomit vor. Pyrit und Gips werden in fast allen Proben aus dem Multilevelbrunnen und wenigen oberflächennahen Proben gefunden. Die Pyrite liegen in Form nierig-traubiger Konkretionen vor; Quarzkörner sind darin eingeschlossen, und die Oberflächen weist deutliche Verwitterungsspuren auf. Als Akzessorien werden in den verschiedenen Proben Glaukonit, Biotit, Muskovit, Rutil, Apatit, Turmalin und Zirkon gefunden. In der Probe CML 24-24,5 m treten im RDA-Spektrum zwei Peaks auf, die einem K-Jarosit zugeordnet werden können. 
Abb. 4.2. zeigt die Verteilungen der wichtigsten Mineralphasen in den Proben der unverritzten Sedimente nach BELLMANN et al. (1984) und KITTSTEINER (1998) in der Gewichtung des Anteils der Schichten am Gesamtsediment und unter Berücksichtigung der quarzreichen Deckschicht im Vergleich zu den von BRINKMANN (2000) untersuchten Proben aus dem Haldenmaterial. Dabei wurden die Haldensedimente einfach gemittelt.

Der durchschnittliche Quarzgehalt ist in den Haldensedimenten gegenüber dem unverritzten Material gleichgeblieben. Die Spuren der Pyritverwitterung sind an der deutlichen Abnahme des Pyritanteils und dem Auftreten von Gips zu erkennen, der in den unverritzten Sedimenten nicht gefunden wurde. Die Gehalte an Gips betragen dabei in einzelnen Schichten bis zu $2 \%$ und mehr, und zwar vor allem im Umfeld der Oxidationszone, wo er durch den Angriff von Schwefelsäure auf Calcit und andere Minerale gebildet wurde. Die Anteile von karbonatischen Mineralen wie Dolomit und Calcit liegen insgesamt sehr niedrig. Die beobachtete Zunahme zeigt, dass entweder die untersuchten Proben nicht repräsentativ für das Modell sind oder die unbekannte Deckschicht karbonatische Mineralphasen enthalten hat. Zwar wurden diese Minerale in den oberen Schichten der Halde durch die versauerten Wässer aus der Pyritoxidation praktisch vollständig zersetzt; in 32-33 m und 36-37 m Tiefe im Profil des Multilevelbrunnens CML wurden jedoch noch Gehalte von mehr als $10 \%$ Calcit und Dolomit festgestellt (BRINKMANN 2000). Der Verdacht, dass die Calcit- und Dolomitgehalte in den Ausgangssedimenten höher gelegen haben müssen, wird durch die Elementmassenbilanz im nachfolgenden Kapitel bestätigt. Durch die Durchmischung der verschiedenen Schichten wurden Braunkohlereste in den Sedimenten verteilt (WIEGAND und KRÜGER 1999); der organisch gebundene Kohlenstoff macht einen großen Teil des Gesamtkohlenstoffgehalts im Haldenmaterial aus.

Betrachtet man anstelle der einfachen Mittelwerte aus den Haldensedimenten von Brinkmann (2000) die Gehalte von Gips, Pyrit und Calcit, die sich aus der nach Tab. 4.4. gewichteten Berechnung über die Sulfatgehalte, die Sulfidschwefel- bzw. die KarbonatKohlenstoffanteile der Proben von BRINKMANN (2000) und des Bohrkerns RCO4 (vorliegende Arbeit) ergeben, bleibt es bei einem Pyritgehalt von $1 \%$ im Haldenmaterial. Auch für den Gipsgehalt erhält man $1 \%$, während der Anteil an Calcit mit $2 \%$ etwas niedriger liegt, als es sich nur aus den Proben von BRINKMANN (2000) ergibt. 


\section{unverritzte Sedimente \\ (nach Bellmann 1984 und Kittsteiner 1998)}

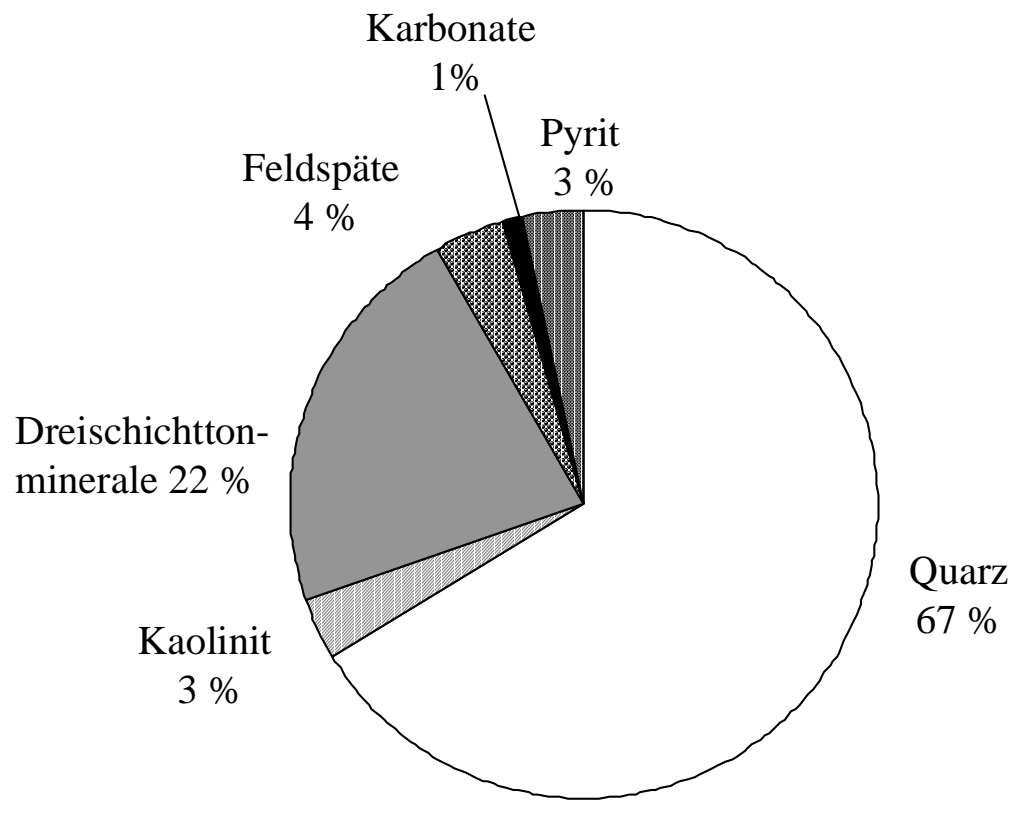

Haldensedimente (nach Brinkmann 2000)

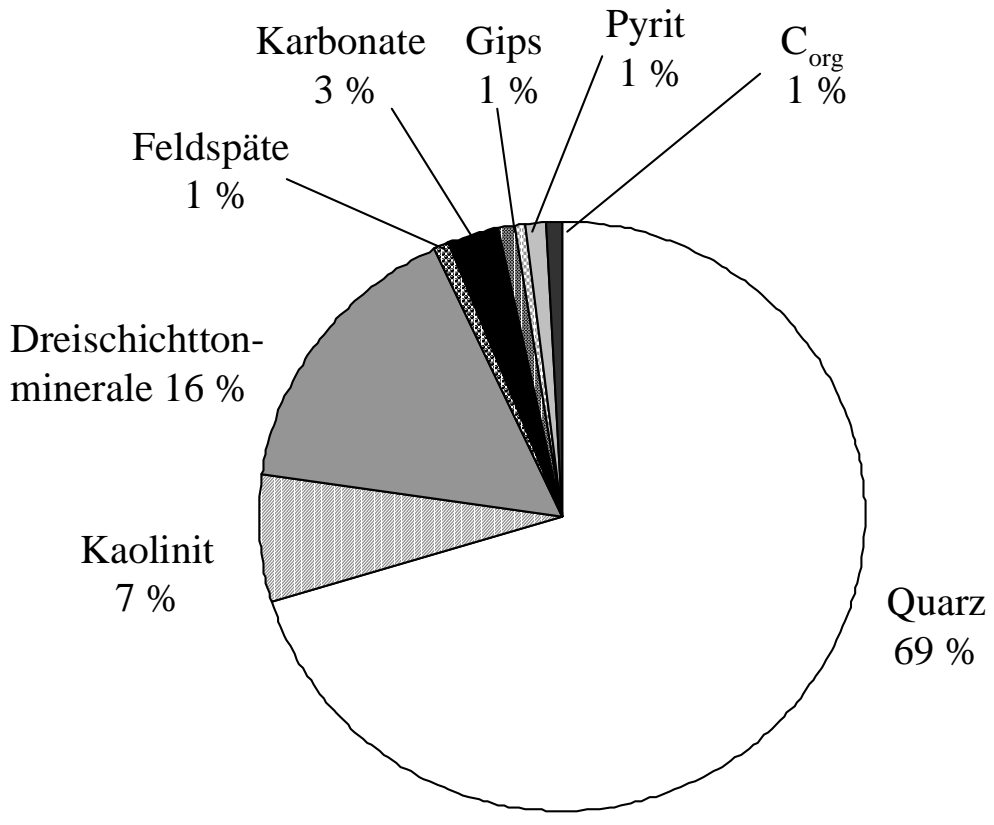

Abb. 4.2.: Mineralphasenzusammensetzung der unverritzten Sedimente nach BELLMANN et al.(1984) und KITTSTEINER (1998) und der Haldensedimente nach BRINKMANN (2000). 
Abgenommen hat der Gehalt an Dreischichttonmineralen, der sich zusammensetzt aus Illit > Montmorillonit > Chlorit. Wechsellagerungsminerale konnten nur in wenigen Fällen nachgewiesen werden. Auch Feldspäte wie Albit und Mikrolin wurden im Zuge der Verwitterung abgebaut, während die Kaolinitgehalte deutlich zunahmen. Zusätzlich wurden kleine Mengen an Rutil und Zirkon im Haldenmaterial gefunden. Sekundärminerale wie Jarosite liegen im Kippenmaterial allenfalls in akzessorischen Mengen vor. Bei der Röntgendiffraktometrie treten in allen Proben Peaks auf, die nicht eindeutig identifiziert werden konnten und eventuell zu Sekundärmineralen aus der Pyritverwitterung gehören, also etwa Jarosite, Schwertmannit, Ferrihydrit und Goethit. Eine Mineralphasentrennung in größerem Maßstab konnte jedoch nicht durchgeführt werden. Unter dem Binokluar fanden sich nierig-traubige Pyrit-Konkretionen mit eingeschlossenen Quarz-Körnern und deutlichen Verwitterungsspuren an der Oberfläche. Nach Berechnungen von BRINKMANN (2000) zeigt der Vergleich der in den Bohrkernen gemessenen Werte für Pyrit mit denen aus dem unverritzten Material einen Verwitterungsgrad von $94 \%$ gemäß der oberflächennahen Bohrung vom November 1998 bzw. ca. 80 \% im Durchschnitt der CML-Proben. In einer Braunkohlenprobe aus dem Kippenbereich fand BRINKMANN (2000) aber auch im Sommer 1998 noch einen Pyritgehalt von ca. 6,1\%.

\subsection{Verwitterungszustand und Elementmassenbilanz}

Bereits in den Arbeiten von KitTsteiner (1998) und PleßOW (1998) wurden Berechnungen zum Oxidationszustand des Kippenmaterials durchgeführt. Als Anhaltspunkt diente dabei der $\mathrm{pH}$ der untersuchten Sickerwässer; er lag bei 3,5, was einem Oxidationsgrad von 0,02 \% des ursprünglichen Pyritgehalts entspricht. Aus dem Vergleich der gemessenen Elementkonzentrationen in Sickerwässern mit den Mengen, die bei der Pyritverwitterung hätten freigesetzt werden können, folgerte PLEßOW (1998), dass ein Großteil der im Sickerwasser angereicherten Elemente nicht aus Pyrit, sondern auch aus anderen Mineralphasen stammen müsse, die durch saure Sicker- und Porenwässer angegriffen wurden. Berechnungen dieser Art können immer nur Überschlagsrechnungen sein, weil sie sich zum einen auf Messwerte stützen, die nur an einzelnen Probennahmestellen ermittelt wurden und eventuell kein repräsentatives Bild liefern. Zum anderen wurde als Bezugsgröße das Gesamtwasserdefizit der Tagebaue Zwenkau und Cospuden zugrunde gelegt.

Erstens aber umfasst dieses nicht nur den ehemaligen Tagebau Cospuden, sondern schließt auch den bis vor kurzem noch aktiven Bereich von Zwenkau mit ein, der noch nicht geflutet ist. Das Restloch Cospuden macht nur einen geringen Teil dieses Gesamtwasserdefizits aus, 
während große Teile der Kippe auch heute, nach Flutung des Sees, noch nicht wassergesättigt sind; so ist es schwierig, zuverlässige Annahmen über das Gesamtwasservolumen und damit über die absoluten Elementmengen im Tagebau zu machen. Die folgenden Berechnungen nehmen hinsichtlich des Gesamtwasserdefizits, aber auch der Abraummengen, Bezug auf den Gesamtkomplex der Tagebaue Cospuden und Zwenkau, um einen Vergleich mit den Daten von KitTSTEINER (1998) und PleßOW (1998) zu ermöglichen.

Zweitens werden solche Berechnungen mit fortschreitender Zeit immer schwieriger, weil andere Prozesse als die Pyritoxidation zunehmend die Elementkonzentrationen in den Sickerwässern beeinflussen. Ausfällungen und Auflösungen von Mineralphasen, Verdünnung durch zuströmendes Wasser, Austragung von Elementen durch Abfluss über Oberflächenwässer und Grundwasserleiter, mikrobielle Aktivitäten u.a. verändern die Elementkonzentrationen in See- und Kippenwässern. Während der $\mathrm{pH}-$ Wert und die Eisenkonzentration dabei vielen verschiedenen Einflüssen durch Fällungsreaktionen bzw. Reaktionen mit basischem Material unterliegen, erscheint der Sulfatgehalt als vergleichsweise konservative Größe, die als Basis für Berechnungen herangezogen werden kann.

Für den August 1999 wurde eine neue Überschlagsrechnung durchgeführt: Das Volumen des Sees beträgt rund 107 mio. $\mathrm{m}^{3}$ (BERKNER 2000); sein Sulfatgehalt liegt bei 0,99 g/l (vorliegende Arbeit). Das Gesamtwasserdefizit der beiden Tagebaue beträgt rund 1,6*10 12 (KITTSTEINER 1998), ohne den See also rund $1,49 * 10^{12}$ l. Der gemittelte Sulfatgehalt aus den Proben vom August 1999, die sowohl Kippenpegel als auch Messstellen außerhalb der Kippe einschließen sowie verschiedene Tiefen umfassen, ist 2,11 g/l. Für das gesamte Wasser ergibt sich damit abzüglich des Untergrunds aus dem Flutungswasser (PLEßOW 1998) eine Sulfatkonzentration von $1,23 \mathrm{~g} / \mathrm{l}$ bzw. eine Gesamtmenge von $2,05^{*} 10^{10}$ mol Sulfat, das aus der Pyritverwitterung stammen sollte. Zur Berechnung der ursprünglich vorhandenen Pyritmenge führte bereits KITTSTEINER (1998) Überschlagsrechnungen durch. Sie beruhten auf der Annahme, beim Cospudener Kippenmaterial handele es sich um ein 1:1:1-Gemisch der von ihm untersuchten Zwenkauer Sedimente Grauer Sand, Brauner Sand und Glaukonitschluff. Unberücksichtigt blieb in dieser Rechnung jedoch die bereits oben dargestellte quarzreiche Deckschicht. Bezieht man diese in die Rechnung mit ein, ergibt sich der durchschnittliche Pyritgehalt aus den gewichteten Gehalten von Pyrit in Grauem Sand (2,8 $\%)$, Braunem Sand (6,7\%), Glaukonitschluff (7,9\%) und quarzreicher Deckschicht (1\%) zu 3,66\%. Er liegt damit deutlich unter dem von KITTSTEINER (1998) angenommenen Wert und im Bereich der Werte, die man bei einer analogen Rechnung mit den Daten von BELLMANN (1984) erhält; aus ihnen resultiert ein durchschnittlicher Pyritanteil von 3,3-4,0\%. 
Die Gesamtabraummenge in Cospuden und Zwenkau betrug nach BERKNER (2000) rund 1490 mio $\mathrm{m}^{3}$. GLÄßER et al. (1998) ermittelten für das Abraummaterial eine Trockendichte von durchschnittlich 1,47 kg/l und eine Wassersättigung von 23,8\%. Daraus ergibt sich eine durchschnittliche Dichte des Materials von 1,36 kg/l und somit eine Menge von 2026 mio t Haldenmaterial. Im unverwitterten Tagebaumaterial lagen demnach ursprünglich rund 74,2 mio t Pyrit vor. Damit resultiert aus dieser Rechnung ein Verwitterungsgrad von 1,7 \% des ursprünglich vorhandenen Pyrits, wenn nur das Sulfat einbezogen wird, das in den Wässern vorgefunden wurde. Berücksichtigt man, dass im Kippenmaterial beträchtliche Mengen Gips auftraten, die im unverritzten Sediment nicht anzutreffen waren, wird der Wert sogar noch größer. Nach BRINKMANN (2000) lagen die Gipsgehalte in der Kippe zwischen 0,1 \% in Proben aus der oberflächennahen Bohrung und bis $\mathrm{zu} 2 \% \mathrm{im}$ Material aus dem Multilevelbrunnen CML. Geht man im Mittel von nur 0,5\% Gips in der Halde aus, so ergeben sich zusätzlich 10,1 mio t Gips oder $5,63 * 10^{10}$ mol Sulfat. Der Verwitterungsgrad des Pyrits läge danach bei 4,6\%.

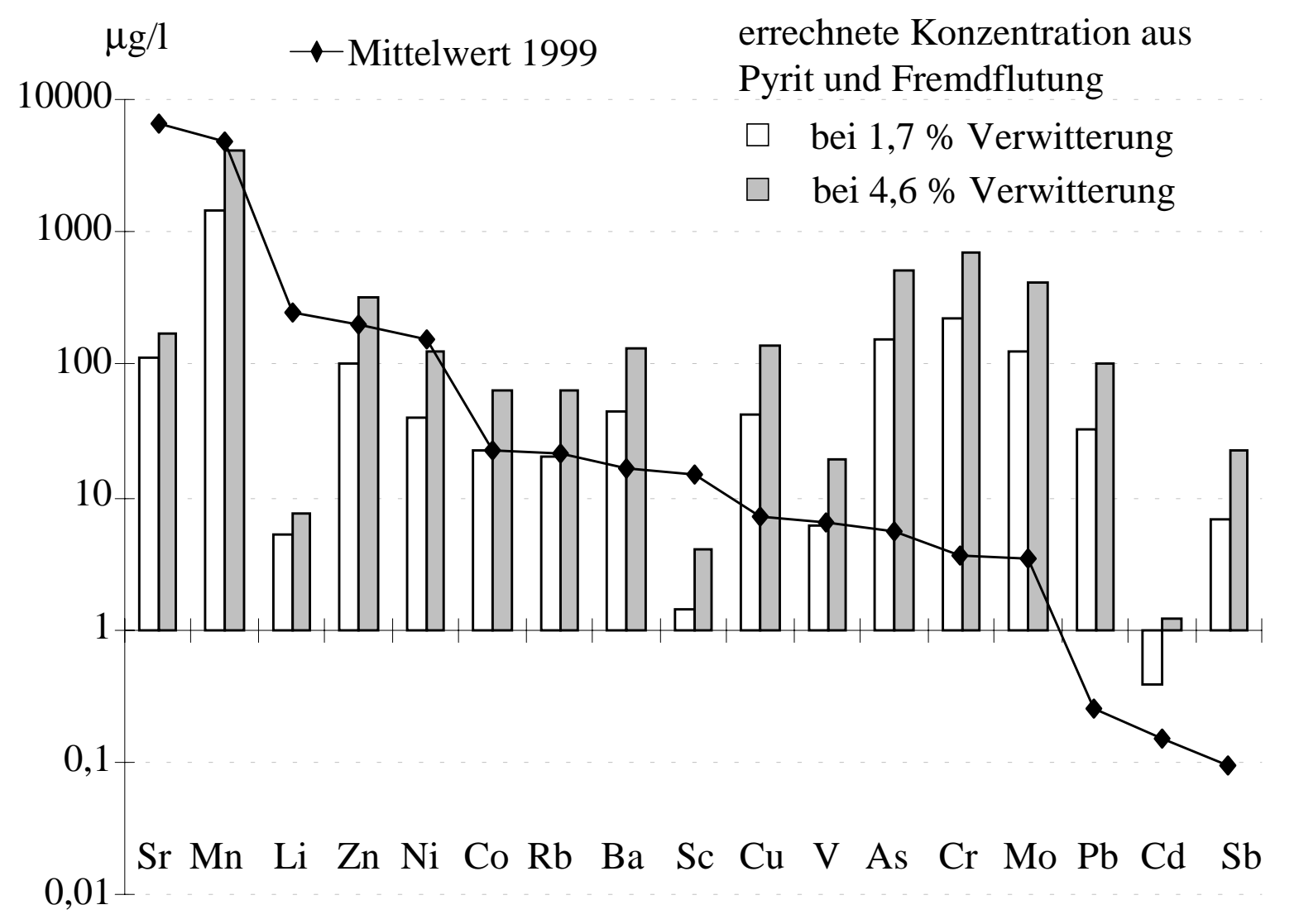

Abb. 4.3.: Mittelwerte in den Proben vom August $1999 \mathrm{im}$ Vergleich mit den Elementkonzentrationen, die sich bei Auflösung von 1,7 \% des Pyrits bzw. 4,6 \% des Pyrits (gewichteter Mittelwert aus den Elementkonzentrationen im Pyrit nach KITTSTEINER (1998) unter Berücksichtigung der Deckschicht), bezogen auf das Gesamtwasserdefizit, unter Berücksichtigung der Hintergrundkonzentration aus dem Flutungswasser ergeben. 
Abb. 4.3. zeigt die gemessenen Mittelwerte einiger Elemente in den Proben vom August 1999 und die Elementkonzentrationen, die in den Wässern - unter Berücksichtigung der Hintergrundkonzentration aus dem Flutungswasser - aus der Pyritverwitterung zu erwarten wären. Für die Elementgehalte im Pyrit der quarzreichen Deckschicht wurde der Pyrit aus dem Grauen Sand (KITTSTEINER 1998) zugrunde gelegt, da unser Modell für die Deckschicht auf diesem Sediment beruht; Einzelheiten dazu werden unten erläutert.

In Übereinstimmung mit den Berechnungen von PlEßOW (1998) und KiTTSTEINER (1998), die Verwitterungsszenarien für $0,02 \%$ und $20 \%$ des Pyrits zugrunde legten, liegen die Konzentrationen von $\mathrm{As}$, $\mathrm{Sb}$ und $\mathrm{Mo}$, die ursprünglich zu großen Teilen pyritgebunden vorlagen, für beide Szenarien deutlich unter den aus der Verwitterung und der Fremdflutung zu erwartenden Werten. Dasselbe gilt für $\mathrm{Cu}, \mathrm{Pb}$ und $\mathrm{Cr}$, in geringerem Maße auch für $\mathrm{Ba}$ und $\mathrm{Cd}$. Li und Sr sowie Sc sind dagegen stärker konzentriert, als die reine Pyritverwitterung auch bei 4,6 \% des Materials zuzüglich der Fremdflutung erwarten ließe; die Mn-Konzentration liegt für 1,7\% Verwitterung oberhalb des zu erwartenden Wertes. Auch Zn und Ni verhalten sich ähnlich. Für $\mathrm{Co}, \mathrm{Rb}$ und $\mathrm{V}$ liegen berechnete und gemessene Werte bei 1,7 \% Verwitterung in einer ähnlichen Größenordnung. Besonders die Abweichungen bei Li und Sr, die nach KITTSTEINER (1998) kaum pyritgebunden vorlagen, weisen deutlich darauf hin, dass neben dem Pyrit noch andere Elementquellen zum Tragen gekommen sind. Die sauren Wässer aus der Pyritoxidation greifen auch andere Mineralphasen an und führen zur Freisetzung von Haupt- und Spurenelementen.

Betrachtet man den Pyrit als einzige relevante Eisenquelle, ergibt sich für den berechneten Verwitterungszustand eine theoretische Fe-Konzentration von rund $346 \mathrm{mg} / \mathrm{l}$ bei $1,7 \%$ Verwitterung bzw. $995 \mathrm{mg} / \mathrm{l}$ bei $4,6 \%$. Gemessen wurde eine durchschnittliche Eisenkonzentration von $259 \mathrm{mg} / \mathrm{l}$. Die Differenz kann durch die Ausfällung von Eisenhydroxidphasen und z.B. durch die temporäre Bildung von Eisensulfaten entstehen. Auf den Seesedimenten sind amorphe Eisenhydroxide in Form gelblicher oder roter Niederschläge deutlich zu sehen. Andererseits ist davon auszugehen, dass es bereits bei der Sümpfung des Tagebaus zu großen Elementaustragungen gekommen ist. Zusätzlich kann es im Zuge der Flutung zu einem Kontakt zwischen den Kippenwässern und wasserleitenden Schichten gekommen sein, über die ein weiterer Teil des Elementgehalts aus dem Tagebau ausgetragen wurde.

Wie bereits oben erwähnt, basiert die Berechnung der Elementgehalte der quarzreichen Deckschicht auf einem Modell, das auf den Werten des Grauen Sandes beruht. Damit wurden die Elementgehalte für das Modell der Deckschicht berechnet, die in Tab. 4.1. in Auszügen, in 
Tab. A.15. im Anhang vollständig wiedergegeben sind. Zur Kontrolle wurden die Elementgehalte einer quarzreichen Schicht aus Cospuden, die von BELLMANN et al. (1984) als „Weiße und Braune Sandfolge“ untersucht wurden, gegenübergestellt:

\begin{tabular}{|l|l|l|}
\hline Element & Elementgehalt Modell $[\mathbf{m g} / \mathbf{k g}]$ & Elementgehalt aus BELLMANN et al. (1984) \\
\hline $\mathrm{Mn}$ & 39 & 55 \\
\hline $\mathrm{Cu}$ & 1,6 & 8 \\
$\mathrm{~Pb}$ & 1,7 & 9 \\
\hline $\mathrm{Cr}$ & 26 & 25 \\
\hline $\mathrm{Ni}$ & 2,3 & 13 \\
\hline $\mathrm{Co}$ & 2,0 & 6 \\
\hline $\mathrm{Zr}$ & 38 & 75 \\
\hline $\mathrm{Ti}$ & 710 & 1200 \\
\hline $\mathrm{Ba}$ & 54 & 250 \\
\hline $\mathrm{V}$ & 9,5 & $\mathrm{nn}$ \\
\hline
\end{tabular}

Tab. 4.1.: Berechnete Spurenelementgehalte der quarzreichen Deckschicht.

Der Vergleich mit den Daten von BELLMANN et al. (1984) fällt nicht für alle Elemente befriedigend aus; generell scheint die Verwendung dieses Modells jedoch gerechtfertigt.

Die Gesamtgehalte der Pyritfraktion an Spurenelementen für den Gesamtkomplex Zwenkau/Cospuden zeigt Tab. 4.2.. Die Pyritfraktion wurde aus dem gewichteten Mittelwert der Sedimente Grauer Sand, Brauner Sand und Glaukonitschluff und des Modells für die quarzreiche Deckschicht berechnet. Zusammen mit den Elementfrachten, die durch das Flutungswasser $\left(1,6^{*} 10^{12} 1\right)$ in den Tagebau gebracht wurden, ergibt sich die Ausgangsmenge der Elemente, die nur bei Berücksichtigung von Pyritverwitterung im Tagebau zur Verfügung stehen. Dem sind die Gesamtgehalte in den Wässern im Jahr 1999 gegenübergestellt. Sie resultieren aus dem Mittelwert der Elementgehalte in den Wasserproben aus dem August 1999, multipliziert mit dem Gesamtwasserdefizit.

\begin{tabular}{|c|c|c|c|c|}
\hline & $\begin{array}{l}\text { Gesamtgehalt in } \\
\text { der Pyritfraktion } \\
\text { [t] } \\
\text { (74,2 mio t Pyrit) } \\
\text { A }\end{array}$ & $\begin{array}{c}\text { Gesamtgehalt aus } \\
\text { Fremdflutung } \\
{[\text { t] }} \\
\text { B } \\
\end{array}$ & $\begin{array}{c}\text { Gesamtgehalt } \\
\text { im Wasser im } \\
\text { Jahr } 1999[\mathrm{t}] \\
\mathrm{C} \\
\end{array}$ & $\begin{array}{l}\text { Gesamtgehalt im Wasser bezogen auf } \\
\text { Gesamtgehalt im Pyrit unter } \\
\text { Berücksichtigung der Fremdflutung [\%] } \\
\text { D }(\%)=((\mathrm{C}-\mathrm{B}) / \mathrm{A}) * 100\end{array}$ \\
\hline $\mathrm{Mn}$ & 117625 & 4960 & 7719 & 2,3 \\
\hline $\mathrm{Cr}$ & 20933 & 9,3 & 5,7 & $-0,02$ \\
\hline As & 14699 & 1,2 & 9,0 & 0,05 \\
\hline Mo & 12029 & 0,912 & 5,6 & 0,04 \\
\hline $\mathrm{Ni}$ & 3512 & 64 & 242 & 5,1 \\
\hline $\mathrm{Zn}$ & 9372 & 48 & 326 & 3,0 \\
\hline $\mathrm{Cu}$ & 4023 & 0,70 & 11,6 & 0,27 \\
\hline $\mathrm{Ba}$ & 3878 & 56 & 26 & $-0,77$ \\
\hline $\mathrm{Pb}$ & 3066 & 1,2 & 0,4 & $-0,03$ \\
\hline $\mathrm{Sr}$ & 2582 & 2080 & 10570 & 329 \\
\hline $\mathrm{Co}$ & 1848 & 37 & 36 & $-0,05$ \\
\hline $\mathrm{Rb}$ & 1818 & 14 & 34 & 1,1 \\
\hline $\mathrm{Sb}$ & 637 & 0,21 & \begin{tabular}{|l|}
0,15 \\
\end{tabular} & $-0,01$ \\
\hline $\mathrm{V}$ & 576 & 3,0 & 10,5 & 1,3 \\
\hline $\mathrm{Li}$ & 99 & 98 & 392 & 297 \\
\hline $\mathrm{Cd}$ & 35 & 0,37 & 0,2 & $-0,49$ \\
\hline
\end{tabular}

Tab. 4.2.:Verwitterungsszenario für Pyrit in Abhängigkeit verschiedener Elemente. 
Die Prozentzahl in der letzten Spalte gibt an, wie groß der Verwitterungsgrad des Pyrits der gemessenen Elementkonzentration nach sein müsste, wenn man außer der Fremdflutung keine anderen Elementquellen berïcksichtigt. Man sieht deutlich, dass die Werte weit differieren. Für Sr und Li müssen andere Elementquellen als Pyrit existieren; für die anderen Elemente sind die Differenzen in den Werten ebenfalls auf unterschiedliche Quellen bzw. im Fall von $\mathrm{Cr}, \mathrm{Ba}, \mathrm{Pb}, \mathrm{Co}, \mathrm{Sb}$ und $\mathrm{Cd}$, für die ein negativer Verwitterungsgrad berechnet wird, auf Senken zurückzuführen. Einem berechneten Verwitterungsgrad zwischen 1,7 und 4,6\% entsprechen einige Elemente wie $\mathrm{Mn}, \mathrm{Ni}, \mathrm{Zn}, \mathrm{Rb}$ und $\mathrm{V}$; gerade die pyritgebundenen Elemente Mo, As und Sb liegen aber weit darunter.

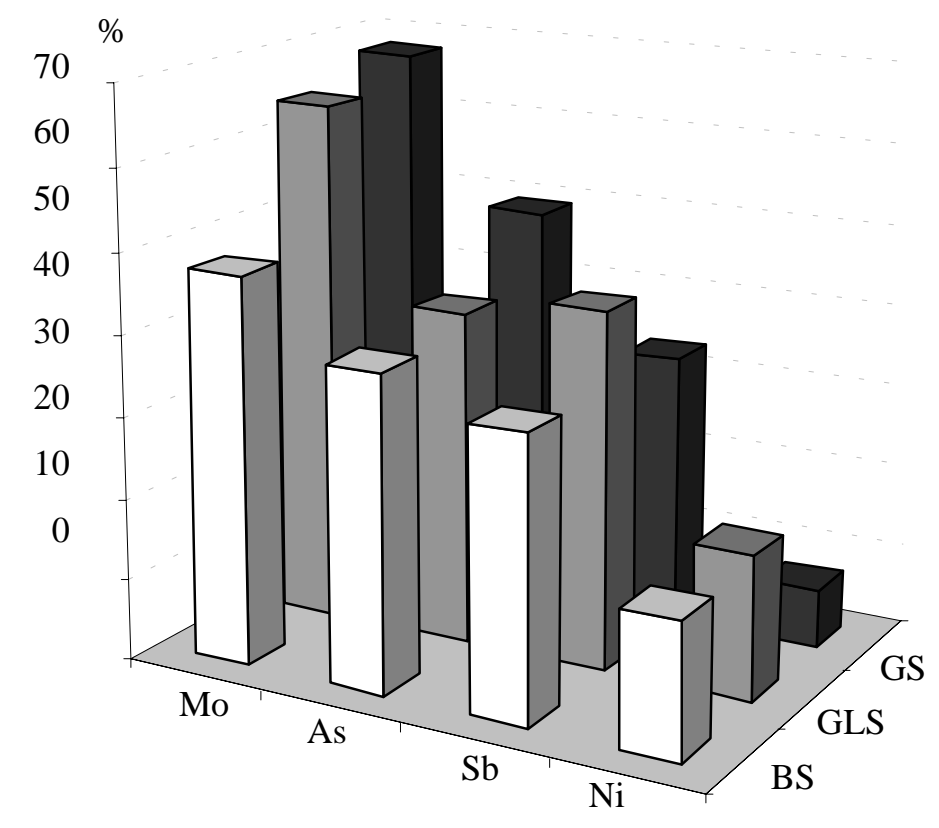

Abb. 4.4.: Anteile des pyritgebundenen Mo, As, Sb und Ni am Gesamtgehalt dieser Elemente in den Sedimenten nach KITTSTEINER (1998); GS = Grauer Sand, BS = Brauner Sand, GLS = Glaukonitschluff

Große Anteile der Elementgesamtmasse von Mo (47,5 - 68 \%), As (38,6 - 49,5 \%), Sb (33,3 - 43,7 \%) und Ni (7,1 - 17,7 \%) lagen nach KITTSTEINER (1998) ursprünglich pyritgebunden vor (Abb. 4.4.). Gerade bei den ersten drei Elementen deuten die Konzentrationen in den Wässern aber nach Tab. 4.2. auf einen niedrigeren Verwitterungsgrad des Pyrits hin als die berechneten 1,6 \%. Demnach sind diese Elemente entweder nicht vollständig in die Wässer abgegeben worden, sondern adsorptiv, durch Präzipitation oder durch Mitfällungen in den Festphasen der Halde zurückgehalten worden, oder sie sind bereits während der Sümpfung oder in der Flutungsphase aus dem Gebiet des Tagebaus ausgetragen worden. 
Wenn es nicht zu Austragungen in größerem Umfang gekommen ist, müssten die Anteile an Elementen, die aus dem Pyrit stammen und nicht im Wasser auftauchen, in den Mineralphasen der Kippe zu finden sein.

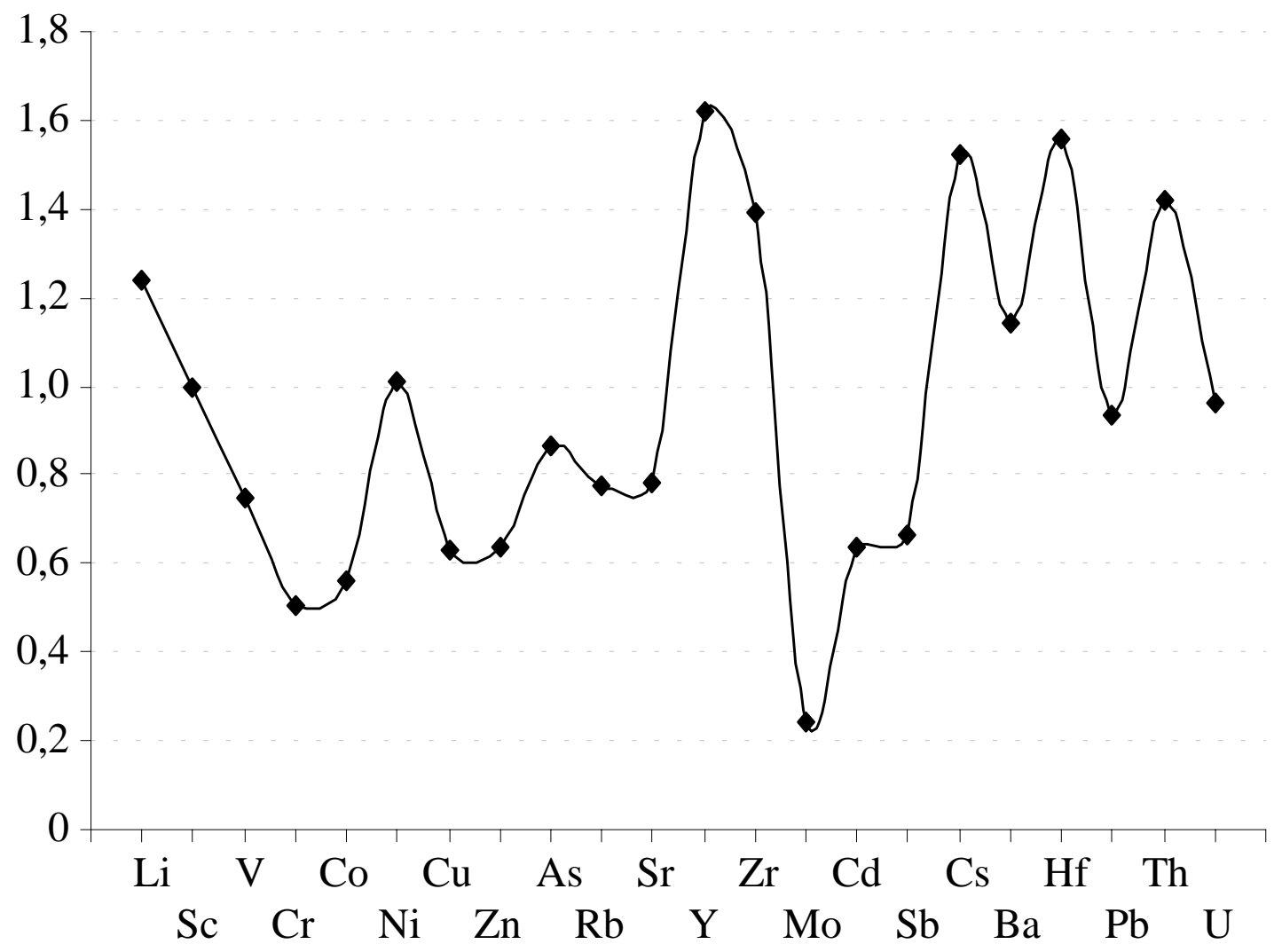

Abb. 4.5.: Elementanreicherungsfaktoren aus den Mittelwerten der Elementkonzentrationen der Haldensedimente (Ermittlung der Werte analog Tab. 4.4.) gegenüber den gewichteten Mittelwerten der Elementkonzentrationen der unverritzten Sedimente aus Zwenkau (einschließlich der Deckschicht).

Die Abb. 4.5. zeigt die Elementanreicherungsfaktoren der Haldensedimente gegenüber den unverritzten Sedimenten über alle Mineralfraktionen einschließlich der Deckschicht. Stark abgereichert sind Mo (-76 \%), Cr (- $50 \%)$, Co (- $44 \%), \mathrm{Cu}(-37 \%), \mathrm{Zn}(-36 \%), \mathrm{Cd}(-36$ $\%), \mathrm{Sb}(-34 \%)$ und V (- $23 \%)$. Nur wenig oder gar nicht verändert waren Li (+ $24 \%), \mathrm{Sc}(0$ $\%)$, Ni (+ $1 \%)$, As (- $14 \%), \mathrm{Rb}(-22 \%), \mathrm{Sr}(-22 \%), \mathrm{Ba}(+14 \%), \mathrm{Pb}(-7 \%)$ und U (- $3 \%)$. Dagegen waren Y $(+64 \%), \mathrm{Zr}(+39 \%)$, Cs $(+52 \%)$, Hf $(+56 \%)$ und Th $(+42 \%)$ angereichert.

Die größte Abreicherung betrifft das Mo, das zu großen Anteilen pyritgebunden vorliegt. Es kann in Lösung gegangen und aus dem Tagebau ausgeschwemmt worden sein. Die deutliche Anreicherung von z.B. Zr, Y, Th und Hf spricht für die Bindung an stabile akzessorische Mineralphasen, die nicht repräsentativ beprobt werden konnten. Diese Elemente sind in sehr konservativen Mineralen wie z.B. Zirkon, Rutil oder Turmalin gebunden, die die meisten 
Veränderungen im Tagebau durch Verwitterung oder Lösungsprozesse unbeeinflusst überstehen.

Für die REEs kann die Anreicherung nur in Bezug auf eine Mischung der von KITTSTEINER (1998) untersuchten Elemente ohne Berücksichtigung der Deckschicht berechnet werden, da die nötigen Daten zur Modellierung der Elementgehalte in der Deckschicht nicht vorliegen. Abb. 4.6. gibt die Anreicherungsfaktoren wieder:

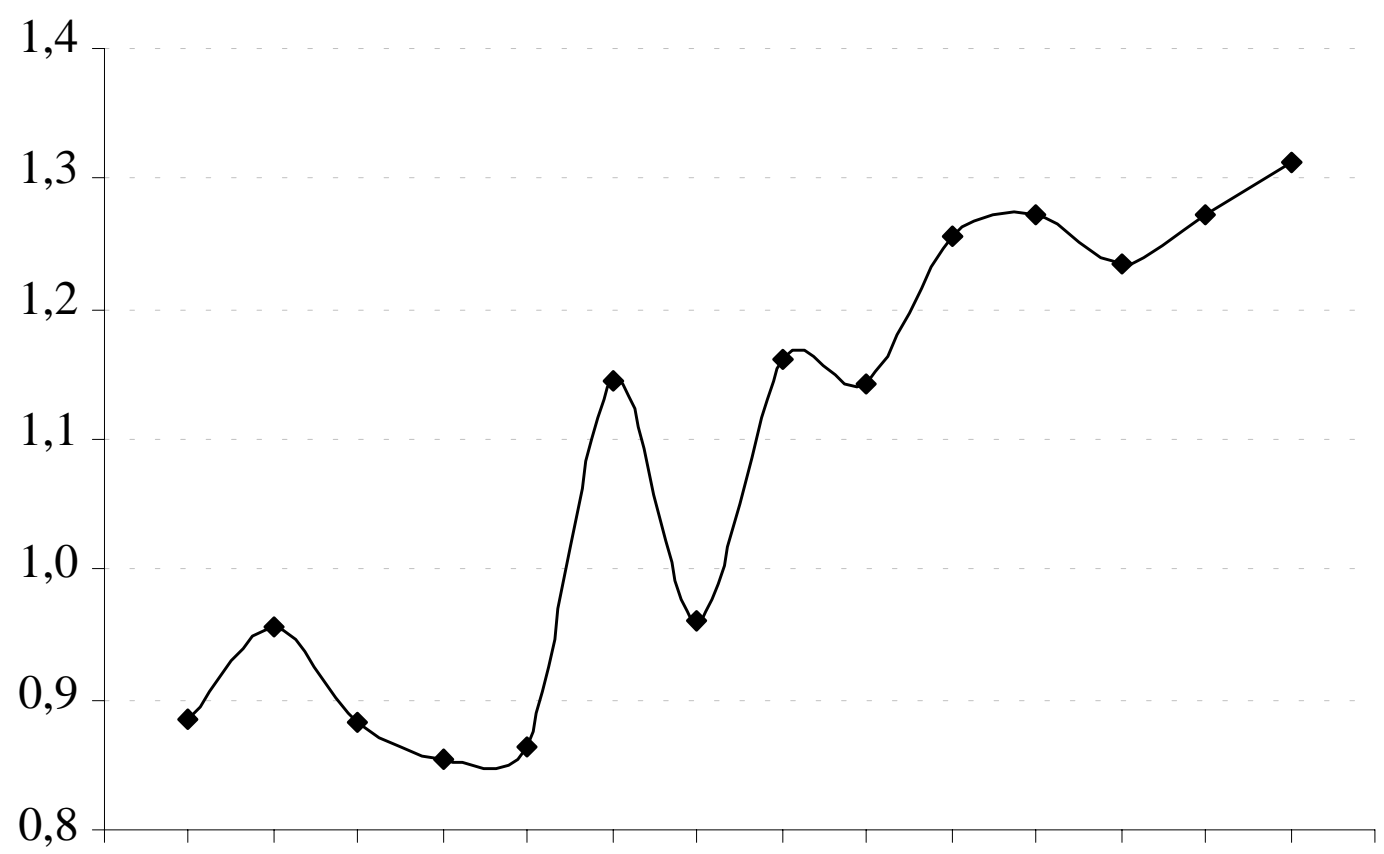

La Ce Pr Nd Sm Eu Gd Tb Dy Ho Er Tm Yb Lu

Abb. 4.6.: Anreicherungsfaktoren der REEs in den Haldensedimenten (gewichteter Mittelwert der Proben von BRINKMANN (2000)) gegenüber den unveritzten Sedimenten (gewichtet, aber ohne Berücksichtigung der Deckschicht).

Die Grafik zeigt einen Trend, nach dem die leichteren REEs abgereichert wurden, während die schweren Vertreter der Elementgruppe leicht angereichert sind. In diesem Verhalten spiegelt sich die mit dem Elementgewicht variierende Tendenz zur Adsorption an Oberflächen bzw. Komplexierung durch Huminstoffe wider. Die Tendenz der dreiwertigen Lanthanid-Ionen zum Ionenaustausch wächst mit zunehmendem Ionenradius, also vom Luthetium zum Lanthan hin. In umgekehrter Richtung, also vom Lanthan zum Luthetium nimmt die Neigung der Lanthanide zur Bildung anionischer Komplexe zu (Holleman 1985). Der beobachtete Trend der stärkeren Anreicherung in den Festphasen vom Lanthan zum Luthetium hin ist durch den letztgenannten Effekt zu erklären, der offenbar die Wirkung 
adsorptiver Oberflächen, die wie Kationenaustauscher wirken können, stark überwiegt. Die leichteren Lanthanide werden bevorzugt an Komplexbildner mit Citratcharakter gebunden und in Lösung gebracht, während die schwereren Vertreter der Gruppe in den Festphasen zurückbleiben.

Im Rahmen seiner Untersuchungen analysierte BRINKMANN (2000) auch die Elementgehalte einer pyrithaltigen Braunkohleprobe aus der Halde. Abb. 4.7. zeigt die Elementanreicherungsfaktoren der Braunkohle für einige Spurenelemente gegenüber den unverritzten Sedimenten (gewichtet, unter Berücksichtigung der Deckschicht).

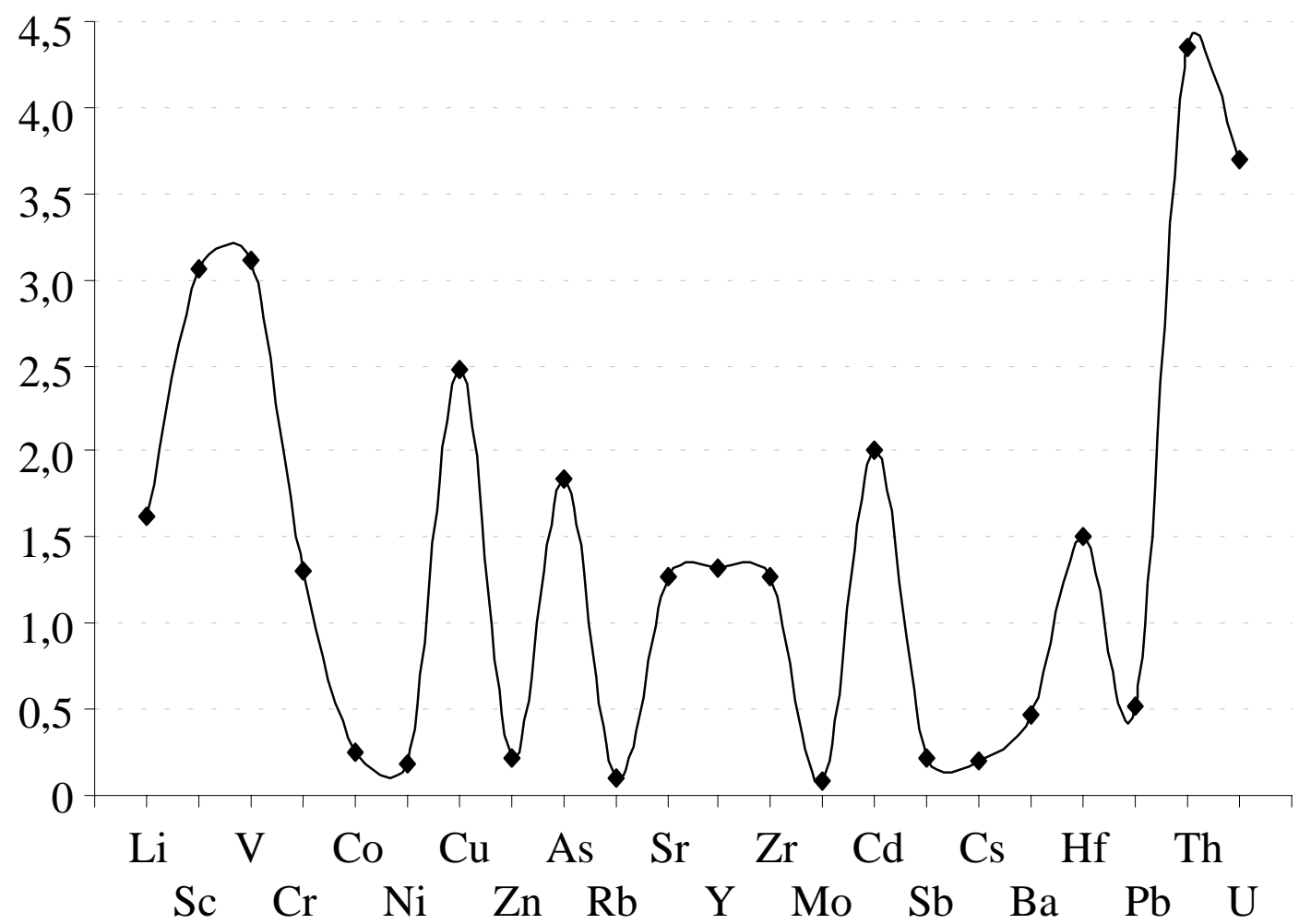

Abb. 4.7.: Elementanreicherungsfaktoren in Braunkohle aus der Halde gegenüber den unverritzten Sedimenten (gewichtet, unter Berücksichtigung der Deckschicht).

Die Anreicherungen in der Braunkohle gegenüber dem unverritzten Sediment sind signifikant. Besonders auffällig sind dabei $\mathrm{Cu}$, As und $\mathrm{Cd}$ mit Faktoren um 2 und $\mathrm{Sc}, \mathrm{V}$, Th und $\mathrm{U}$ mit Faktoren > 3. Die Spurenelemente müssen dabei nicht notwendigerweise in der Braunkohle gebunden sein. Mehrere Modelle erklären die Unterschiede der Elementverteilung in der Braunkohle gegenüber dem umgebenden Sediment (MASON und MOORE 1985). Bestimmte Elemente können beim Wachstum von Pflanzen des Braunkohle-Moores bevorzugt aufgenommen und im Humus akkumuliert werden. Während der Inkohlungsphase beeinflussen Fällungsprozesse, Adsorption und chemische Bindung die 
Spurenelementverteilung in der Kohle. Durch ihre adsorptive Oberfläche kann die Kohle auch Elemente aus Sicker- und Porenwässern binden. Die Kohlereste im Sedimente stellen daher ein siginfikantes Reservoir für einige Spurenelemente innerhalb des Kippenmaterials dar.

Abb. 4.8. zeigt die Anreicherungsfaktoren der REEs in der Braunkohle gegenüber den unverritzten Sedimenten (gewichtet, ohne Berücksichtigung der Deckschicht).

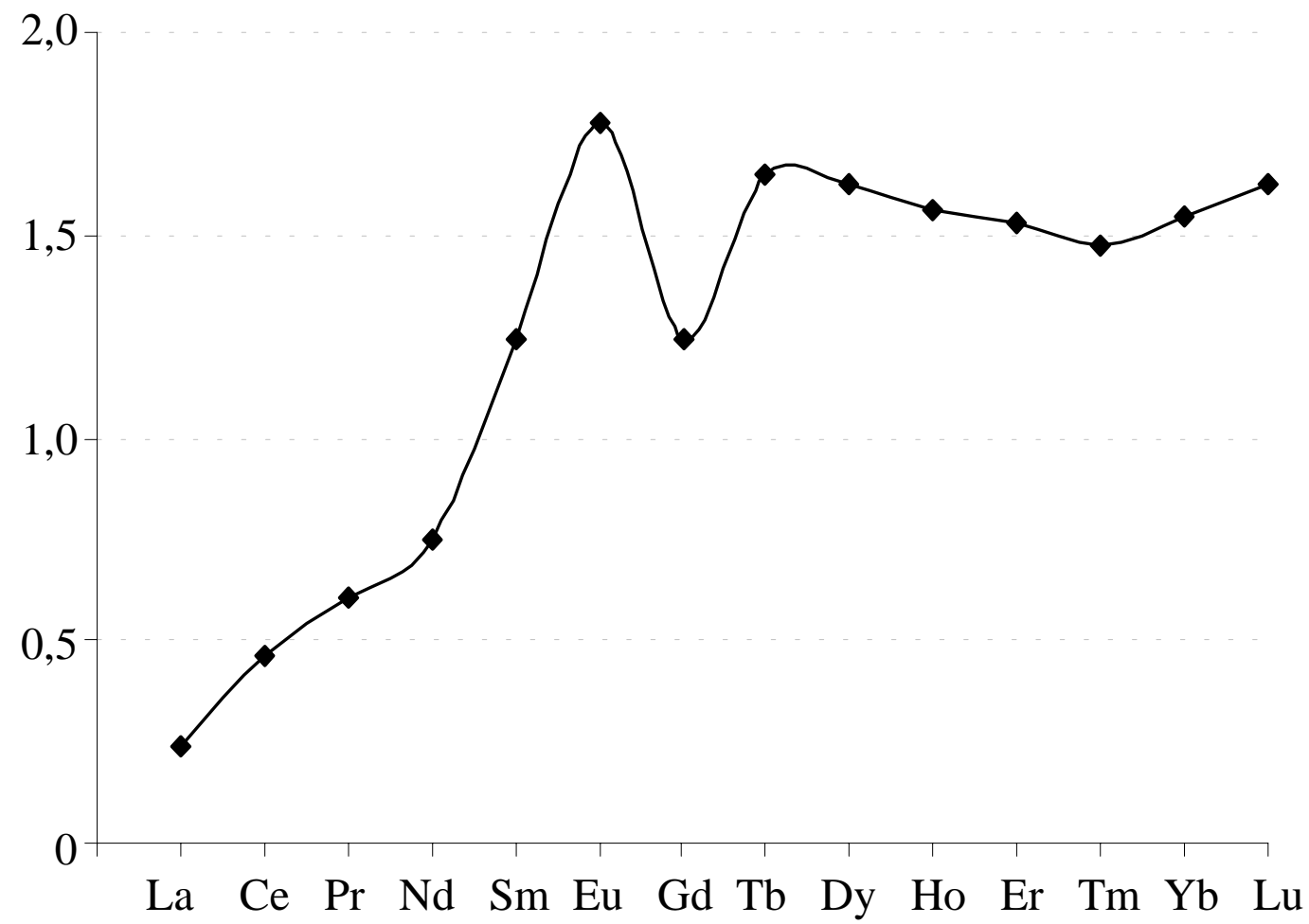

Abb. 4.8.: Elementanreicherungsfaktoren der REEs in der Braunkohle aus dem Haldensediment gegenüber den unverritzten Sedimenten (gewichtet, ohne Berücksichtigung der Deckschicht).

Für die REEs zeigt sich eine ähnliche Tendenz wie in den sonstigen Haldensedimenten: Die leichteren Elemente sind abgereichert, die schwereren Elemente werden bevorzugt an die Braunkohle gebunden und zeigen Anreicherungsfaktoren um 1,5.

In welchen Mineralphasen die verbliebenen Spurenelemente gebunden sind, konnte nicht eindeutig geklärt werden; jedoch zeigte sich ein Trend zu einer vorrangigen Bindung in Proben mit höherem Anteil an schluffigem und tonigem Material, während Proben mit hohen Quarzgehalten geringe Spurenelementgehalte aufwiesen.

Mit den Ergebnissen von KitTsteiner (1998), PleßOW (1998), BRinKMANN (2000) und aus dieser Arbeit kann ein Versuch unternommen werden, eine Bilanz der Gesamtelementmengen aufzustellen.

Tab. 4.3. zeigt die Ergebnisse für die Hauptelemente. Die Elementgehalte des unverritzten Sediments wurden wie oben beschrieben aus den gewichteten Anteilen von Grauem und 
Braunem Sand und Glaukonitschluff errechnet; soweit eine entsprechende Datenbasis vorhanden war (s. Tab. A.15. im Anhang), wurde die quarzreiche Deckschicht nach dem oben beschriebenen Modell berechnet. Für die Hauptelemente wurde die Deckschicht ausgehend vom Grauen Sand, umgerechnet auf einen $\mathrm{SiO}_{2}$-Anteil von $95 \%$, modelliert. Dazu kommt der Anteil an Elementen, die über das Fremdflutungswasser in den ehemaligen Tagebau gebracht wurden. Der Elementgehalt der Festphasen in der Halde wurde aus dem Mittelwert der Proben des Bohrkerns von RCO4 (soweit bekannt; s. Tab. A.13. und A.14. im Anhang) und dem Mittelwert der Daten aus der Arbeit von BrinKMANN (2000) ermittelt. Dabei wurde die Probe RCO4 $58 \mathrm{~m}$ ausgespart, weil sie nicht mehr das Haldensediment erfasst, sondern aus der darunterliegenden Kohleschicht stammt. Um zu berücksichtigen, dass von einigen Tiefen mehrere Werte vorliegen bzw. die genommenen Proben enger beisammenliegen, wurden die vorliegenden Werte jeweils für $5 \mathrm{~m}$ des Profils zusammengefasst und dann gemittelt, wie Tab. 4.4. verdeutlicht.

\begin{tabular}{|l|l|}
\hline Tiefe [m] & berücksichtigte Proben \\
\hline $1-5$ & RCO4 $1 \mathrm{~m}$, RCO4 $2 \mathrm{~m}$, RCO4 $3 \mathrm{~m}$, RCO4 $5 \mathrm{~m}$, oberflächennahe Bohrung 1,85-3,78 m \\
\hline $6-10$ & RCO4 $7 \mathrm{~m}$, RCO4 $8 \mathrm{~m}$, RCO4 $9 \mathrm{~m}$, RCO4 $10 \mathrm{~m}$ \\
\hline $11-15$ & RCO4 $11 \mathrm{~m}$, RCO4 $13 \mathrm{~m}$, RCO4 $14 \mathrm{~m}$ \\
\hline $16-20$ & RCO4 $18 \mathrm{~m}$, RCO4 $19 \mathrm{~m}$ \\
\hline $21-25$ & RCO4 $22 \mathrm{~m}$, RCO4 24 m, CML 24-24,5 m \\
\hline $26-30$ & RCO4 26 m, RCO4 28 m, RCO4 30 m, CML 26-27 m \\
\hline $31-35$ & RCO4 $32 \mathrm{~m}$, RCO4 34 m, CML 32-33 m \\
\hline $36-40$ & CML 36-37 m, CML 37,5-38 m \\
\hline $41-45$ & RCO4 42 m \\
\hline $46-50$ & RCO4 $46 \mathrm{~m}$ \\
\hline $51-56$ & RCO4 $56 \mathrm{~m}$ \\
\hline
\end{tabular}

Tab. 4.4.: Ermittlung der Haldenzusammensetzung; für jede Tiefe wurde der Mittelwert der aufgeführten Proben berechnet und dann die Haldenzusammensetzung über das gesamte Profil berechnet.

Für den Gesamtelementgehalt der Wässer wurden die im August 1999 ermittelten Werte gemittelt und auf das Gesamtwasserdefizit hochgerechnet. Sie stammen aus Proben aus verschiedenen Bereichen und Tiefen und spiegeln damit die Verhältnisse im gesamten Tagebau möglichst vollständig wieder. Da diese Daten auf Stichproben beruhen, können sie nur Tendenzen hinsichtlich Anreicherung oder Abreicherung wiedergeben; eine qualitative Aussage auf dieser Basis sollte aber möglich sein. 


\begin{tabular}{|c|c|c|c|c|c|c|c|}
\hline Element & \begin{tabular}{l} 
Element- \\
gehalt in \\
allen \\
Festphasen \\
im \\
unverritzten \\
Sediment \\
$\quad$ [mio t] \\
\multicolumn{1}{c}{ A }
\end{tabular} & $\begin{array}{l}\text { Element- } \\
\text { gehalt der } \\
\text { Fremd- } \\
\text { flutung } \\
\quad \text { [t] }\end{array}$ & 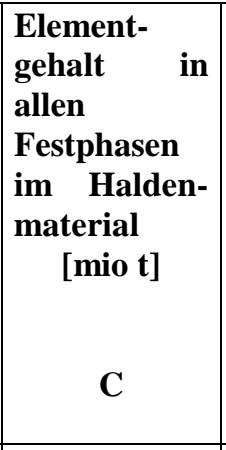 & $\begin{array}{l}\text { Element- } \\
\text { gehalt der } \\
\text { Wässer } \\
\text { (Sommer } \\
\text { 1999) } \quad \text { [t] }\end{array}$ & $\begin{array}{l}\text { Geamt- } \\
\text { element- } \\
\text { gehalt aus } \\
\text { Festphasen } \\
\text { vor dem } \\
\text { Tagebau } \\
\text { und Fremd- } \\
\text { flutung } \\
\quad \text { [mio t] } \\
\quad \text { A+B }\end{array}$ & \begin{tabular}{|l} 
Gesamt- \\
element- \\
gehalt aus \\
Festphasen \\
und \\
Wässern des \\
ehemaligen \\
Tagebaus \\
$\quad$ [mio t] \\
$\quad$ C+D
\end{tabular} & $\begin{array}{c}((\mathbf{C}+\mathbf{D})- \\
(\mathbf{A}+\mathbf{B})) \\
/(\mathbf{A}+\mathbf{B})\end{array}$ \\
\hline $\mathrm{SiO}_{2}$ & 1628 & $\mathrm{nb}$ & 1650 & $\mathrm{nb}$ & 1628 & 1650 & $+1,4 \%$ \\
\hline $\mathrm{TiO}_{2}$ & 9,9 & $\mathrm{nb}$ & 9,7 & $\mathrm{nb}$ & 9,9 & 9,7 & $-2,1 \%$ \\
\hline $\mathrm{Al}_{2} \mathrm{O}_{3}$ & 118 & 78,6 & 103 & 2621 & 118 & 103 & $-13 \%$ \\
\hline $\begin{array}{l}\Sigma \mathrm{Fe} \text { als } \\
\mathrm{Fe}_{2} \mathrm{O}_{3}\end{array}$ & 101 & 48,0 & 49 & 537063 & 101 & 50 & $-49 \%$ \\
\hline $\mathrm{MnO}$ & 0,28 & 6403 & 0,43 & 9143 & 0,28 & 0,43 & $+54 \%$ \\
\hline $\mathrm{MgO}$ & 12 & 140598 & 14 & 618963 & 12 & 15 & $+25 \%$ \\
\hline $\mathrm{CaO}$ & 9,0 & 604449 & 25 & 1051090 & 9,6 & 26 & $+170 \%$ \\
\hline $\mathrm{Na}_{2} \mathrm{O}$ & 3,5 & 94887 & 8,9 & 1575090 & 3,6 & 10,5 & $+192 \%$ \\
\hline $\mathrm{K}_{2} \mathrm{O}$ & 24 & 21210 & 20 & 39083 & 24 & 20 & $-17 \%$ \\
\hline $\mathrm{P}_{2} \mathrm{O}_{5}$ & 1,3 & $\mathrm{nb}$ & 1,4 & $\mathrm{nb}$ & 1,3 & 1,4 & $+7,7 \%$ \\
\hline $\begin{array}{l}\Sigma \mathrm{S} \\
\mathrm{SO}_{4}^{2-}\end{array}$ & 122 & $1,3^{*} 10^{6}$ & 44,7 & $2,0 * 10^{6}$ & 123 & 47 & $-62 \%$ \\
\hline
\end{tabular}

Tab. 4.5.: Elementmassenbilanz für die Hauptelemente; Vergleich der unverritzten Sedimente mit dem Haldenmaterial.

Bei den Hauptelementen (Tab. 4.5.) fällt auf, dass für $\mathrm{Si}, \mathrm{Ti}, \mathrm{Al}, \mathrm{K}$ und $\mathrm{P}$ keine signifikanten Veränderungen aufgetreten sind. Diese Elemente sind teilweise in den Wässern des Tagebaus gelöst; der größte Teil ist aber in den Festphasen verblieben, wo er in Form der ursprünglichen Minerale (vor allem Si in Form von Quarz), in Sekundärmineralen oder adsorptiv gebunden vorliegt.

Rechnet man den ermittelten Überschuss an $\mathrm{Ca}$ in Calcit um, der ursprünglich im unverritzten Sediment vorhanden war, so erhält man 29,27 mio t Calcit. Demnach waren im ursprünglichen Sediment 1,4 \% mehr Calcit bezogen auf das Gesamtmaterial vorhanden, als bei unserer Berechnung vorausgesetzt wurde. Nach den Daten von KITTSTEINER (1999) und BELLMANN et al. (1984) lagen im unverritzten Sediment lediglich $1 \%$ Calcit und Dolomit vor; wie bereits erläutert, erscheint dieser Wert aber eher als zu niedrig. Da karbonatische Gesteine durch die in der Pyritverwitterung entstandene Schwefelsäure sehr schnell aufgelöst werden, könnte der absolute Calcitanteil ursprünglich sogar noch höher gelegen haben. Eine vergleichbare Rechnung für Na ergibt, dass ursprünglich 2,9\% Albit mehr vorgelegen haben müssen als gemessen. 


\begin{tabular}{|c|c|c|c|c|c|c|c|}
\hline Element & \begin{tabular}{|l} 
Element- \\
gehalt in \\
allen \\
Festphasen \\
im \\
unverritzten \\
Sediment \\
[t] \\
A
\end{tabular} & \begin{tabular}{|l|} 
Element- \\
gehalt der \\
Fremd- \\
flutung \\
[t]
\end{tabular} & \begin{tabular}{|l} 
Element- \\
gehalt in \\
allen \\
Festphasen \\
im Halden- \\
material \\
[t] \\
C \\
\end{tabular} & 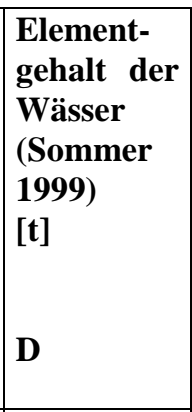 & 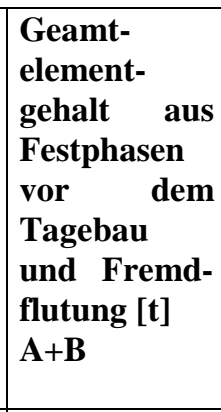 & 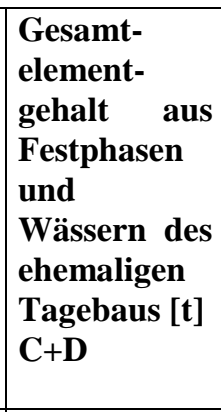 & $\begin{array}{l}((\mathbf{C}+\mathbf{D})- \\
(\mathbf{A}+\mathbf{B})) \\
/(\mathbf{A}+\mathbf{B})\end{array}$ \\
\hline $\mathbf{L i}$ & 42157 & 97,6 & 53588 & 392 & 42255 & 53980 & $28,0 \%$ \\
\hline Be & 16781 & $\mathrm{nq}$ & 2191 & 2,25 & 16781 & 2193 & $-86,9 \%$ \\
\hline Sc & 12259 & 4,5 & 12082 & 23 & 12264 & 12105 & $-1,3 \%$ \\
\hline $\mathbf{V}$ & 111354 & 3,0 & 83334 & 11 & 111357 & 83345 & $-25,0 \%$ \\
\hline $\mathrm{Cr}$ & \begin{tabular}{|l|l}
186437 \\
\end{tabular} & 9,3 & \begin{tabular}{|l|l}
93553 \\
\end{tabular} & \begin{tabular}{|l|}
5,7 \\
\end{tabular} & \begin{tabular}{|l|l}
186446 \\
\end{tabular} & \begin{tabular}{|l|l}
93559 \\
\end{tabular} & $-50,2 \%$ \\
\hline Co & \begin{tabular}{|l}
14814 \\
\end{tabular} & 37 & 8254 & 36 & $\mid$\begin{tabular}{|l}
14851 \\
\end{tabular} & \begin{tabular}{|l|}
8290 \\
\end{tabular} & $-44,2 \%$ \\
\hline $\mathrm{Ni}$ & 31710 & 64 & 32878 & 242 & 31774 & 33120 & $4,2 \%$ \\
\hline $\mathbf{C u}$ & \begin{tabular}{|l|}
16387 \\
\end{tabular} & 0,70 & \begin{tabular}{|l|l}
10273 \\
\end{tabular} & 12 & \begin{tabular}{|l|l}
16388 \\
\end{tabular} & 10285 & $-37,2 \%$ \\
\hline Zn & \begin{tabular}{|l}
74480 \\
\end{tabular} & 48 & 47622 & 236 & 74528 & \begin{tabular}{|l}
47858 \\
\end{tabular} & $-35,8 \%$ \\
\hline As & 31496 & 1,2 & 27863 & 9,0 & 31497 & 27872 & $-11,5 \%$ \\
\hline $\mathbf{R b}$ & 99558 & 14 & $\overline{77003}$ & 34 & 99572 & 77037 & $-22,6 \%$ \\
\hline $\mathrm{Sr}$ & \begin{tabular}{|l|l}
147049 \\
\end{tabular} & 2080 & 114528 & \begin{tabular}{|l}
10570 \\
\end{tabular} & \begin{tabular}{|l|l}
149129 \\
\end{tabular} & \begin{tabular}{|l|l}
125098 \\
\end{tabular} & $-16,1 \%$ \\
\hline $\mathbf{Y}$ & 15480 & 0,30 & 24920 & 142 & 15480 & 25062 & $61,9 \%$ \\
\hline $\mathbf{Z r}$ & 268090 & $\mathrm{nq}$ & 371802 & 34 & 268090 & 371836 & $38,7 \%$ \\
\hline $\mathrm{Nb}$ & 39482 & $\mathrm{nq}$ & 21731 & 1,8 & 39482 & 21733 & $-45,0$ \\
\hline Mo & 27638 & 0,91 & 6804 & 5,6 & \begin{tabular}{|l|l|}
27639 \\
\end{tabular} & \begin{tabular}{|l|l|}
6810 \\
\end{tabular} & $-75,4 \%$ \\
\hline Cd & 497 & 0,37 & 328 & 0,20 & \begin{tabular}{|l|l|}
497 \\
\end{tabular} & 328 & $-34,0 \%$ \\
\hline Sn & 4230 & $\mathrm{nq}$ & $\overline{4574}$ & 0,92 & \begin{tabular}{|l|}
4230 \\
\end{tabular} & 4575 & $8,2 \%$ \\
\hline $\mathbf{S b}$ & 1741 & 0,21 & 1161 & 0,15 & 1741 & 1161 & $-33,3 \%$ \\
\hline Cs & 5006 & $\mathrm{nq}$ & $\overline{7775}$ & 0,85 & \begin{tabular}{|l}
5006 \\
\end{tabular} & 7776 & $55,3 \%$ \\
\hline Ba & 344795 & 56 & $\overline{393560}$ & 26 & \begin{tabular}{|l|l}
344795 \\
\end{tabular} & 393586 & $14,2 \%$ \\
\hline La & 42694 & $\mathrm{nq}$ & 37684 & 50 & \begin{tabular}{|l|l|}
426994 \\
\end{tabular} & \begin{tabular}{|l|l|}
37734 \\
\end{tabular} & $-11,6 \%$ \\
\hline $\mathrm{Ce}$ & 82572 & $\mathrm{nq}$ & 79318 & 54 & 82572 & \begin{tabular}{|l|l|}
79372 \\
\end{tabular} & $-3,9 \%$ \\
\hline Pr & $\mid 10357$ & $\mathrm{nq}$ & $\overline{9026}$ & \begin{tabular}{|l|}
9,9 \\
\end{tabular} & $\mid 10357$ & \begin{tabular}{|l|}
9036 \\
\end{tabular} & $-12,8 \%$ \\
\hline Nd & 40866 & $\mathrm{nq}$ & 34594 & 9,3 & 40866 & 34603 & $-15,3 \%$ \\
\hline Sm & \begin{tabular}{|l|l|}
7437 \\
\end{tabular} & $\mathrm{nq}$ & $\underline{6509}$ & 0,96 & \begin{tabular}{|l|l|}
7437 \\
\end{tabular} & 6510 & $-12,5 \%$ \\
\hline Eu & 1250 & $\mathrm{nq}$ & $\overline{1443}$ & 0,54 & 1250 & 1444 & $15,5 \%$ \\
\hline Gd & 5337 & $\mathrm{nq}$ & 5144 & 4,3 & \begin{tabular}{|l|l|}
5337 \\
\end{tabular} & \begin{tabular}{|l|l|}
5148 \\
\end{tabular} & $-3,5 \%$ \\
\hline Tb & 754 & $\mathrm{nq}$ & $\underline{862}$ & 1,4 & 754 & 863 & $14,5 \%$ \\
\hline Dy & 4245 & $\mathrm{nq}$ & $\overline{4955}$ & 2,2 & 4245 & 4957 & $16,8 \%$ \\
\hline Ho & \begin{tabular}{|l|l|}
789 \\
\end{tabular} & $\mathrm{nq}$ & 996 & 1,9 & \begin{tabular}{|l|}
789 \\
\end{tabular} & 998 & $26,5 \%$ \\
\hline Er & 2261 & $\mathrm{nq}$ & 2856 & 2,4 & 2261 & 2858 & $26,4 \%$ \\
\hline Tm & 335 & $\mathrm{nq}$ & 432 & 0,36 & 335 & \begin{tabular}{|l}
432 \\
\end{tabular} & $29,0 \%$ \\
\hline $\mathbf{Y b}$ & 2224 & $\mathrm{nq}$ & $2 \overline{2801}$ & \begin{tabular}{|l|l|}
0,67 \\
\end{tabular} & 2224 & 2802 & $26,0 \%$ \\
\hline Lu & 330 & $\mathrm{nq}$ & 425 & 0,28 & 330 & 425 & $28,8 \%$ \\
\hline Hf & 6856 & $\mathrm{nq}$ & 10834 & $\mathrm{nq}$ & 6856 & 10834 & $58,0 \%$ \\
\hline Tl & 723 & $\mathrm{nq}$ & 676 & \begin{tabular}{|l|l|}
0,27 \\
\end{tabular} & 723 & \begin{tabular}{|l|}
674 \\
\end{tabular} & $-6,8 \%$ \\
\hline $\mathbf{P b}$ & 18260 & 1,2 & \begin{tabular}{|l|l|}
17069 \\
\end{tabular} & 0,40 & \begin{tabular}{|l|l|}
18261 \\
\end{tabular} & \begin{tabular}{|l|l|}
17069 \\
\end{tabular} & $-6,5 \%$ \\
\hline Bi & 191 & $\mathrm{nq}$ & $n b$ & $\mathrm{nb}$ & $\mid 191$ & nb & $\mathrm{nb}$ \\
\hline Th & \begin{tabular}{|l|l|}
8637 \\
\end{tabular} & 1,3 & 12328 & nb & \begin{tabular}{|l|l|}
8638 \\
\end{tabular} & 12328 & $42,7 \%$ \\
\hline $\mathbf{U}$ & 5930 & 1,0 & $\underline{\overline{5673}}$ & 9,5 & \begin{tabular}{|l}
5931 \\
\end{tabular} & 5683 & $-4,2 \%$ \\
\hline
\end{tabular}

Tab. 4.6.: Elementmassenbilanz für die Spurenelemente; Vergleich der unverritzten Sedimente mit dem Haldenmaterial; kursiv dargestellte Elemente wurden ohne Berücksichtigung der Deckschicht berechnet, bei unterstrichenen Werten wurde das Haldenmaterial nur aus Werten von BRINKMANN (2000) ermittelt. 
Ähnlich erklärt sich die Abweichung beim Mn. Einige karbonatische Minerale wie z.B. Siderit $\left(\mathrm{FeCO}_{3}\right)$ enthalten $\mathrm{Mn}$. Geht man von einem Gehalt von nur $2 \% \mathrm{Mn}$ in Siderit aus, entspricht das ,überschüssige“ $\mathrm{MnO}$ einem Anteil von weniger als 0,3\% Siderit im unverritzten Sediment. Das Vorkommen von Siderit in pyritführenden Sedimenten ist bekannt, der Nachweis in diesem Konzentrationsbereich gelingt selten.

Diese Ergebnisse zeigen, dass es sich bei der Gesamtelementbilanz auf der Basis der ursprünglichen Daten nur um die Ermittlung eines Trends, nicht um eine exakte Berechnung von An- und Abreicherungen handelt. Letztere würde eine erheblich größere Probenzahl sowohl aus dem unverritzten Material als auch aus der Halde voraussetzen.

Eisen wurde insgesamt sehr stark abgereichert. Der oxidierte Pyrit konnte in unseren Proben nicht in Form von Eisenoxiden oder Eisensulfaten wiedergefunden werden. Parallel nahm auch der Gesamtschwefelgehalt um über $60 \% \mathrm{ab}$.

Tab. 4.6. gibt die Verhältnisse für die Spurenelemente wieder. Keine oder nur geringe Abweichungen zeigen Sc, Ni, As, Sr, Sn, Ba, La, Ce, Pr, Nd, Sm, Eu, Gd, Tb, Dy, Pb, Tl und U. Zwischen 20 und $40 \%$ betrug die Abreicherung bei V, Cu, Zn, Rb, Cd und Sb. Starke Abreicherungen oberhalb von $40 \%$ wiesen dagegen $\mathrm{Be}, \mathrm{Cr}, \mathrm{Co}, \mathrm{Nb}$ und $\mathrm{Mo}$ auf, während $\mathrm{Li}$, $\mathrm{Ho}, \mathrm{Er}, \mathrm{Tm}, \mathrm{Yb}$ und Lu schwach angereichert wurden. Hohe Anreicherungsraten hatten $\mathrm{Y}, \mathrm{Zr}$, Cs, Hf und Th. Abb. 4.9. zeigt die Anreicherungsfaktoren für Elemente, bei denen die Gesamtgehalte im Haldenmaterial um mehr als $20 \%$ von denen in den unverritzten Sedimenten abwichen: 


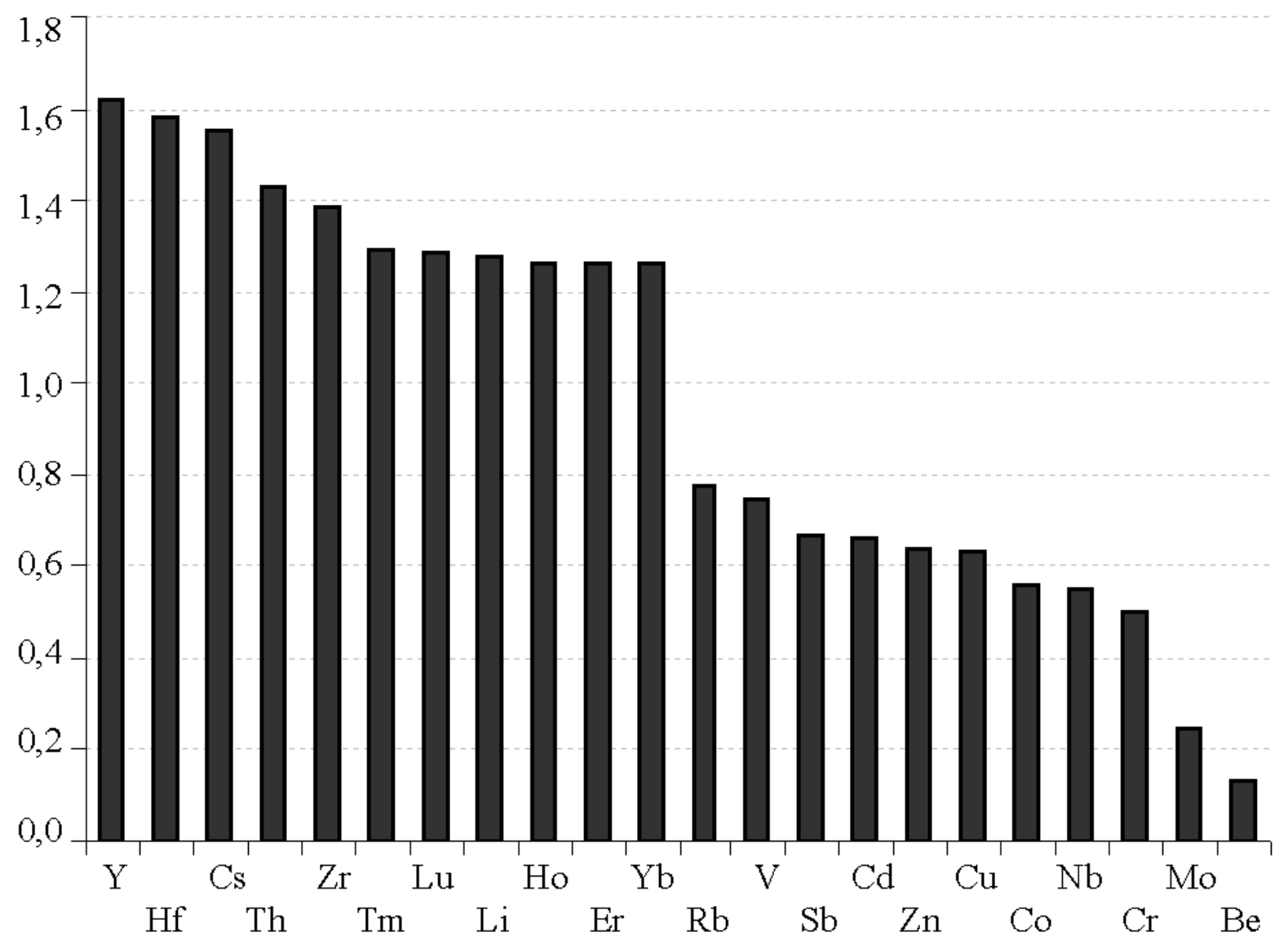

Abb. 4.9.: Elementanreicherungsfaktoren nach dem Tagebau gegenüber den unverritzten Sedimenten zzgl. Flutungswasser; berücksichtigt wurden nur Elemente, bei denen die Abweichung mehr als $20 \%$ betrug.

Bei den am stärksten abgereicherten Elementen finden sich die $\mathrm{zu}$ großen Teilen pyritgebundenen Elemente Mo und Sb, aber auch einige Elemente der Nebengruppenmetalle.

Den beobachteten Trends zufolge scheinen sich die meisten Elemente nach wie vor im Bereich des Tagebaus zu befinden. Einige Elemente wie Be, Mo, Fe, Cr, Nb, Co, Cu, Zn, Sb, Cd und Sulfat sind mit mehr als $20 \%$ abgereichert und können durch Austragungen aus dem Tagebau entfernt worden sein.

Aus den dargestellten Rechnungen ergibt sich ein Gesamtbild für den Zustand des Materials im Tagebau verglichen mit den unverritzten Sedimenten. Nach Abschätzung über die Mineralphasenzusammensetzung (Abb. 4.2.) mit und ohne Einbeziehung der Deckschicht sind $2-2,7 \%$ Pyrit (absolut) verwittert. Entsprechend sind rund $19-25$ mio t Fe auf diese Weise freigesetzt worden. In der Elementmassenbilanz (Tab. 4.5.) fehlen $49 \%$ Fe oder 49 mio $\mathrm{t}$ als $\mathrm{Fe}_{2} \mathrm{O}_{3}$ und $61 \%$ oder 76 mio t Sulfat; das entspricht $6,13^{*} 10^{11} \mathrm{~mol} \mathrm{Fe}$ und $7,92 * 10^{11}$ mol Sulfat, die aus dem Tagebau ausgetragen wurden. Doch auch andere Mineralphasen wurden durch die bei der Pyritoxidation entstandene Säure angegriffen. Dadurch wurden zahlreiche Elemente freigesetzt. Während die Hauptelemente mit Ausnahme des Eisens im 
Wesentlichen im Kippenmaterial verblieben sind, gingen viele Spurenelemente zunächst in Lösung.

Die durch die Pyritverwitterung und in der Folge durch den Säurekontakt aus anderen Mineralphasen freigesetzten Elemente können in mehreren Formen vorliegen. Unsere Untersuchung schließt die gelösten Spezies in den Wässern und die Minerale ein. Bei der Aufbereitung der Proben zur RFA-Analyse und zur ICP-MS-Untersuchung sollten auch adsorbierte Elementanteile nicht verlorengegangen sein. Zwar ist der Pyrit zu großen Teilen verschwunden, doch im Rahmen dieser Untersuchungen wurden keine Mengen von Sekundärmineralen gefunden, die den Verbleib des freigesetzten und nicht in der Lösung befindlichen Eisens erklären könnten. Studien anderer Arbeitsgruppen (CESNOVAR und Pentinghaus 1997, 1999) gehen davon aus, dass sich in etwa 18 m Tiefe in der Kippe eine mächtige Schicht von Jarosit befindet, in der das freigesetzte Eisen gebunden wurde. Die von BRINKMANN (2000) und im Rahmen der vorliegenden Arbeit untersuchten Proben inklusive des Bohrkerns RCO4, der bis auf die Sohle der Kippe reicht, geben keinen Anhaltspunkt für eine solche Schicht von eisenhaltigen Sekundärmineralen. Entweder befindet sich das Eisen nicht mehr in der Kippe, sondern ist gemeinsam mit anderen Elementen, die ebenfalls stark abgereichert wurden, aus der Kippe ausgeschwemmt worden, oder in der Kippe befinden sich noch große Lager von Eisen, die temporär oder bleibend in sekundären Mineralphasen festgelegt sind und sich in unseren Proben aufgrund der Inhomogenität der Kippe nicht zeigen. Ein Teil der gelösten Spurenelemente wurde an sekundär gebildeten Mineralphasen gebunden, andere, wie $\mathrm{Zr}$ oder Y, sind in extrem resistenten Phasen wie Zirkon gebunden und blieben daher durch die Verwitterungsprozesse unbeeinflusst. Für die übrigen abgereicherten Elemente, müssen, soweit die Abreicherungen nicht auf Verdünnungseffekte bei der Kippenaufschüttung zurückgehen, entweder noch Senken in Form von Adsorptionsoberflächen oder Sekundärmineralien vorhanden sein, die bei den Probennahmen nicht erfasst wurden, oder man muss davon ausgehen, dass in der Folge der Sümpfung bzw. der Flutung und der langsamen Aufsättigung der Kippe bereits eine nicht unerhebliche Elementfracht über Grundwasserleiter aus dem Bereich des ehemaligen Tagebaus ausgeschwemmt wurde. 


\subsection{Untersuchungen an Poren-, Grund- und Oberflächenwässern aus Cospuden}

In diesem Abschnitt sollen die Absolutgehalte in den untersuchten Proben nach verschiedenen Kriterien geordnet werden. Zunächst soll ein räumliches Bild der Wasserqualität entworfen werden, das zeigt, in welchen Bereichen des ehemaligen Tagebaus sich die Pyritverwitterung auf die Wässer zu Beginn der Flutung besonders stark auswirkte. Zum zweiten zeigt die Beobachtung von Proben aus dem See, aus dem Mulitlevelbrunnen CML und aus den verschiedenen RCO-Pegeln über die letzten 5 Jahre die zeitliche Entwicklung der Wasserqualität und der Spurenelementsignaturen in Cospuden sowie eventuell die Infiltrationswege des Wassers aus dem See in die umliegenden Sedimente bzw. aus der Kippe in den See.

\subsubsection{Räumliches Bild der Wasserqualität}

Wie Abb. 3.1. in Kap. 3 zeigt, befinden sich die Probenahmestellen in unterschiedlicher Lage zur Kippe und in verschiedenen Grundwasserleitern. Anhand eines Vergleichs der Porenwasserbeschaffenheit verschiedener Proben und der Proben aus den Grundwassermesspegeln (RCO) soll zunächst die anfängliche Wasserqualität an verschiedenen Standorten vor bzw. im Frühstadium der Flutung verglichen werden.

Für die an der Oberfläche austretenden Sickerwässer sowie einige Standgewässer im Bereich des ehemaligen Tagebaus während der Flutung fertigte PLEßOW (1998) bereits Analysen an. Danach zeigten die Sicker- und Standgewässer im Kippenbereich deutliche Auswirkungen der Pyritverwitterung; niedrige $\mathrm{pH}-$ Werte von 1,5 bis 3,5 gingen einher mit Sulfatgehalten von 1 $\mathrm{g} / \mathrm{l}$ bis über $30 \mathrm{~g} / \mathrm{l}$ und erhöhten Konzentrationen zahlreicher Elemente. An der Westböschung des Restsees austretende Sickerwässer dagegen wiesen bei pH-Werten oberhalb von 5 und niedrigen Sulfatgehalten von $233 \mathrm{mg} / \mathrm{l}$ kaum Anreicherungen auf; sie schienen von der Pyritoxidation unbeeinflusst.

Untersuchungen an Porenwässern (RCO4, RCO10, RCO13 und RCO16 aus der Arbeit von PLEßOW (1998), RCO4, CML und die oberflächennahe Bohrung aus der vorliegenden Arbeit) bestätigen diesen Eindruck. Zwar waren auch in den Porenwässern aus den Proben im Tagebauvorfeld einige Elemente, vor allem die Alkali- und Erdalkalimetalle, gegenüber unbelasteten Grundwässern angereichert, dies entspricht aber dem allgemeinen Befund für Porenwässer. Im Vergleich zwischen den Porenwässern aus der Kippe und denen aus dem unverritzten Anstehenden des Vorfeldes liegt in den Kippenwässern eine deutlich stärkere 
Belastung vor, die in der Oxidationszone ihren Höhepunkt erreicht. Die Tiefenprofile der Bohrkerne aus dem Kippenbereich werden weiter unten ausführlich diskutiert.

Abb. 4.10. zeigt einige Schlüsselparameter wie $\mathrm{pH}$, elektrische Leitfähigkeit, Sulfatgehalt und Eisengehalt für Wasserproben aus verschiedenen Grundwassermessstellen, die im Sommer 1998 (drei Jahre nach Beginn der Flutung des Restsees) entnommen wurden.

RCO13 und RCO14 erfassen zwei verschiedene Grundwasserleiter im westlichen Tagebauvorfeld, RCO10 liegt im nördlichen Tagebauvorfeld, RCO12 und RCO4 befinden sich im Kippenbereich (Abb. 3.1.). Die Messstelle RCO14 liegt im Grundwasserleiter 1, der einen erheblichen Teil des Zustroms (im Jahr 1999 ca. 1/5 des Grundwasserzustroms) zum Restsee lieferte. Die Messpegel RCO10 und RCO13 im Grundwasserleiter 2 geben den Grundwasserzustrom zum Restsee aus dem westlichen Bereich wieder. RCO4 und RCO12 erfassen den Wasserzustrom aus dem Kippenbereich bis ca. Oktober 1998 (NITSCHE et al. 1999). Die pH-Werte liegen ungefähr zwischen 6 und 7; auch die Wässer aus den beiden Pegeln im Kippenbereich sind nicht merklich versauert. Trotzdem sind die Folgen der Pyritoxidation deutlich erkennbar, und dies nicht nur an den Sulfatkonzentrationen. So beträgt die Leitfähigkeit in beiden Kippenpegelproben mehr als $3 \mathrm{mS} / \mathrm{cm}$ und liegt damit in Größenordnungen, die sonst in unseren Untersuchungen nur in Porenwässern erreicht wurden. Dafür sind die hohen Konzentrationen an Haupt- und Spurenelementen verantwortlich. Nicht nur die Fe-Werte liegen um mehrere Zehnerpotenzen über den Gehalten in den nicht in der Kippe gelegenen Pegeln, auch die Werte von $\mathrm{Li}, \mathrm{Na}, \mathrm{Mg}, \mathrm{K}, \mathrm{Ca}, \mathrm{Mn}$ und $\mathrm{Sr}$ sind in RCO4 und RCO12 um Größenordnungen höher als in den übrigen Proben. Für die Übergangsmetalle dagegen lässt sich das nicht beobachten. 

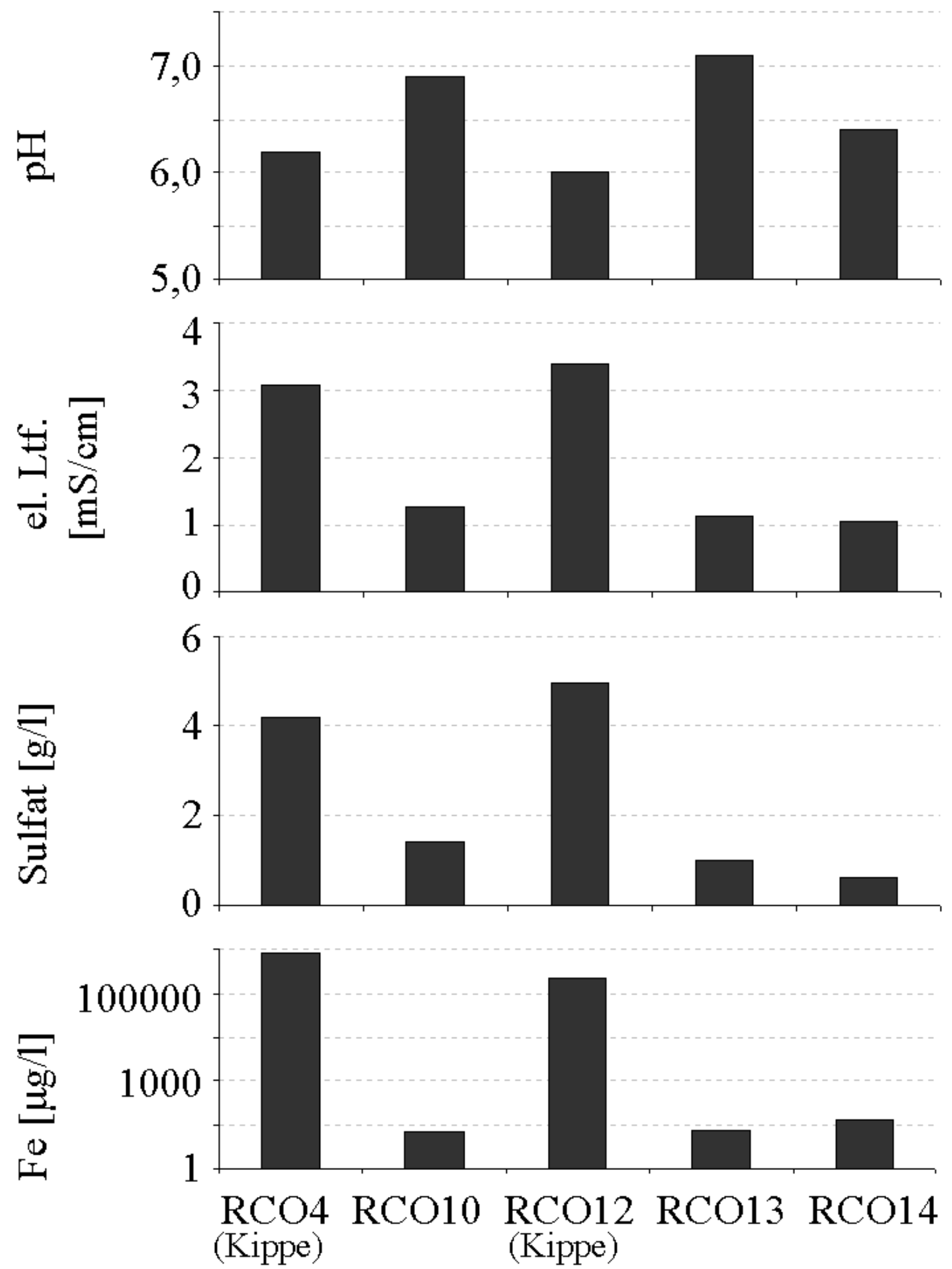

Abb. 4.10.: Darstellung einiger Parameter in Abhängigkeit der Probennahmestelle in Cospuden; Probennahme vom August 1998 (zur Lage der Grundwassermesspegel s. Abb. 3.1. in Kap. 3). 
Abb. 4.11. zeigt einige Elementgehalte. Im Vergleich mit Grundwasser aus Leipzig (PLEßOW 1998) sind die meisten Hauptelemente in den Kippenpegeln 1998 angereichert. Bei den Spurenelementen Cr und V dagegen sind Abreicherungen zu beobachten. Das Grundwasser aus Leipzig zeigt gegenüber unbelasteten Grundwässern aus Niedersachsen und Hessen (Pleßow et al. 1997) Konzentrationen, die für die meisten Elemente etwa das Fünffache betragen. Eisengehalte von ca. $3 \mathrm{mg} / \mathrm{l}$ und Aluminiumkonzentrationen von 2,2 mg/l lassen eventuell darauf schließen, dass die untersuchten Grundwasserproben aus den Wasserwerken Naunhof und Belgershain bereits unter dem Einfluss der Sulfidoxidation aus Tagebauen und Halden südlich von Leipzig stehen. Insgesamt sind die Konzentrationen jedoch nicht so hoch, dass in jedem Fall von signifikanten anthropogenen Einflüssen auszugehen ist. Um die Wasserqualität der Proben aus Cospuden zu bemessen, soll trotz dieser Zweifel Bezug auf das Grundwasser aus Leipzig genommen werden, denn nur so ist es möglich zu beurteilen, ob die Wässer aus Cospuden gegenüber Grundwässern aus der Region ein besonderes Kontaminationspotential besitzen. Gegenüber Leipziger Grundwasser zeigen alle Pegel, auch die im Tagebau-Vorfeld, deutlich erhöhte Gehalte an Haupt- und Spurenelementen.

Eine leichte Verarmung an $\mathrm{Ba}$ in den Wässern aus den Kippenpegeln gegenüber den anderen Proben kann darauf zurückgeführt werden, dass durch die Pyritoxidation Sulfat freigesetzt und dadurch das Löslichkeitsprodukt von $\mathrm{BaSO}_{4}$ überschritten wird (s. dazu Sättigungsindizes in Tab. A.16. im Anhang); die Ausfällung ist die Folge. Auch der in diesen Wässern bestimmte Sauerstoffgehalt von nur $12 \%$ (RCO4) bzw. $34 \%(\mathrm{RCO} 12)$ gegenüber $56 \%$ (RCO10), $68 \%$ (RCO13) bzw. $43 \%$ (RCO14) (eigene Messungen) deutet darauf hin, dass dort Pyritoxidation abläuft und den Wässern dadurch Sauerstoff entzogen wird. Die DOCGehalte sind in den Kippenpegeln deutlich erhöht.

Die REEs liegen in allen Proben unterhalb der Nachweisgrenze, auch in denen aus dem Kippenbereich. Entweder waren sie in Mineralphasen gebunden, die von den Folgen der Pyritoxidation nicht betroffen waren, oder sie sind durch Adsorption oder Sekundärmineralisation bereits wieder aus den Wässern entfernt worden. 

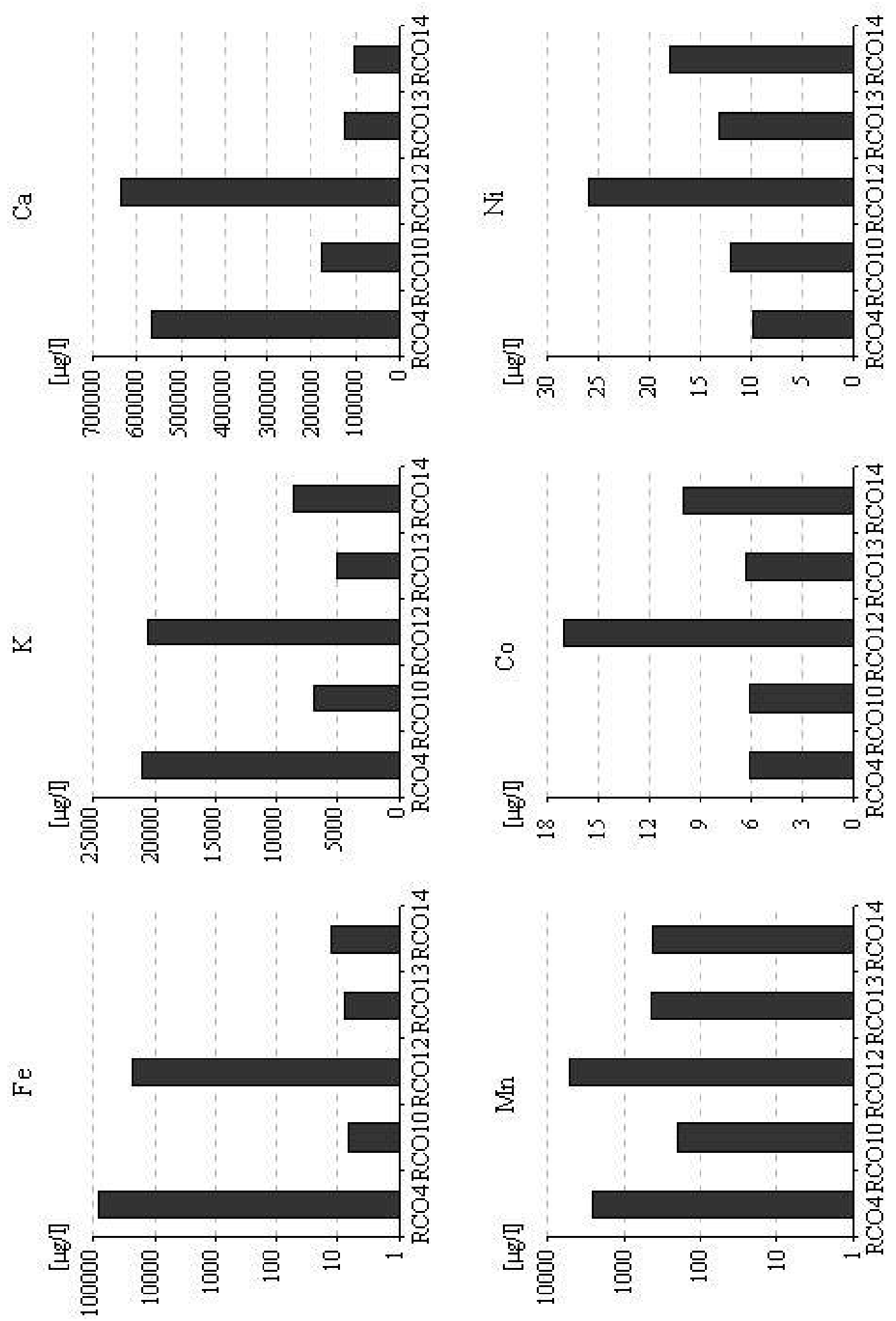

Abb. 4.11.: Konzentrationen einiger Elemente in Wässern aus den Messpegeln in Cospuden (Probennahme August 1998. 

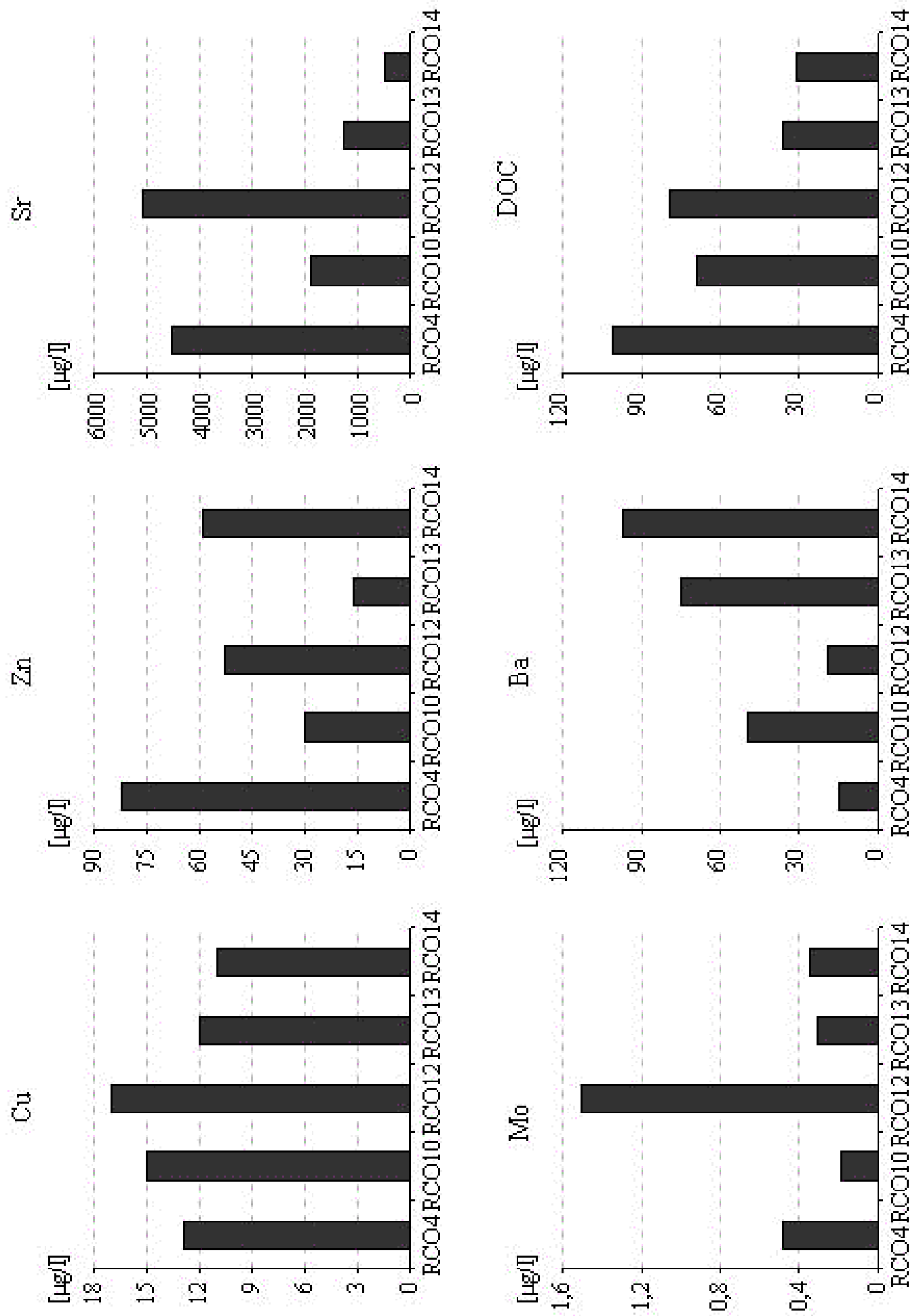

Abb. 4.11. (Forts.): Konzentrationen einiger Elemente in Wässern aus den Messpegeln in Cospuden (Probennahme August 1998. 


\subsubsection{Zeitliche Entwicklung der Wasserqualität}

Nach NITSCHE et al. (1999) stellt sich die Hydrogeologie des ehemaligen Tagebaus Cospuden folgendermaßen dar: Bis März 1999 infiltrierten die Grundwasserleiter 1, 2 und 3 in den Restseekörper. Seit Oktober 1998 zeigten bis zum Frühjahr 1999 zunächst nur die Kippengrundwasserstellen RCO3 und RCO4 eine Infiltration von Restseewasser in den Kippenkörper, die schließlich alle weiteren Kippengrundwassermesstellen erreicht haben sollte.

\subsubsection{See}

Der pH-Wert des Sees ist im Verlauf der Flutung zwischen Juni 1998 (PLEßOW 1998) und August 1999 von $\mathrm{pH}=4,3$ auf Werte um 7 angestiegen. Gleichzeitig sind die Konzentrationen der meisten Elemente kontinuierlich zurückgegangen; besonders auffällig ist dies bei Al (110 $\mu \mathrm{g} / \mathrm{l}$ auf $0,05 \mu \mathrm{g} / \mathrm{l}), \mathrm{Sc}(35 \mu \mathrm{g} / \mathrm{l}$ auf $1,9 \mu \mathrm{g} / \mathrm{l}), \mathrm{Mn}(7800 \mu \mathrm{g} / \mathrm{l}$ auf $753 \mu \mathrm{g} / \mathrm{l})$, Co $(300 \mu \mathrm{g} / \mathrm{l}$ auf $15 \mu \mathrm{g} / \mathrm{l}), \mathrm{Ni}(492 \mu \mathrm{g} / \mathrm{l}$ auf $52 \mu \mathrm{g} / \mathrm{l}), \mathrm{Zn}(1100 \mu \mathrm{g} / \mathrm{l}$ auf $10 \mu \mathrm{g} / \mathrm{l}), \mathrm{Y}(30 \mu \mathrm{g} / \mathrm{l}$ auf $<0,1 \mu \mathrm{g} / \mathrm{l}), \mathrm{La}$ $(31 \mu \mathrm{g} / \mathrm{l}$ auf $<0,1 \mu \mathrm{g} / \mathrm{l})$ und Ce $(52 \mu \mathrm{g} / \mathrm{l}$ auf $<0,1 \mu \mathrm{g} / \mathrm{l})$. Zwar weist der See im August 1999 gegenüber unbelasteten Grundwässern (PLEßOW 1998) erhebliche Elementanreicherungen auf; der sinnvollere Vergleich bezieht sich jedoch auf regionale Wässer, die, wie bereits dargestellt, gegenüber unbelasteten Grundwässern aus Niedersachsen und Hessen deutlich höhere Elementkonzentrationen aufweisen. Gegenüber dem Grundwasser aus Leipzig (PLEßOW 1998) waren im August 1999 nur noch wenige Elemente angereichert. Anreicherungsfaktoren > 1 lagen bei Li, Na, Mg, K, Ca, Mn, Co, Ni, Sr, Sn, Tl, U und Sulfat vor. Einen Anreicherungsfaktor > 10 zeigte lediglich Zr. Zahlreiche Elemente waren gegenüber dem Grundwasser aus Leipzig abgereichert: Faktoren zwischen 0,5 und 1 wurden für $\mathrm{Cu}$, As und $\mathrm{Rb}$ gefunden; unter 0,5 lagen sie bei $\mathrm{Al}, \mathrm{Sc}, \mathrm{V}, \mathrm{Cr}, \mathrm{Fe}, \mathrm{Zn}, \mathrm{Mo}, \mathrm{Cd}, \mathrm{Ba}, \mathrm{Nd}$, $\mathrm{Sm}$ und $\mathrm{Pb}$.

Bei einigen Elementen kam es von Juni 1998 bis zum November 1998 zu einem starken Anstieg der im See gemessenen Werte; bis August 1999 sanken sie wieder ab. Darunter fallen die Elemente $\mathrm{K}, \mathrm{Cu}, \mathrm{Rb}, \mathrm{Zr}$ und $\mathrm{Ba}$ sowie das Sulfat. Abb. 4.12. zeigt ihren Konzentrationsverlauf. 

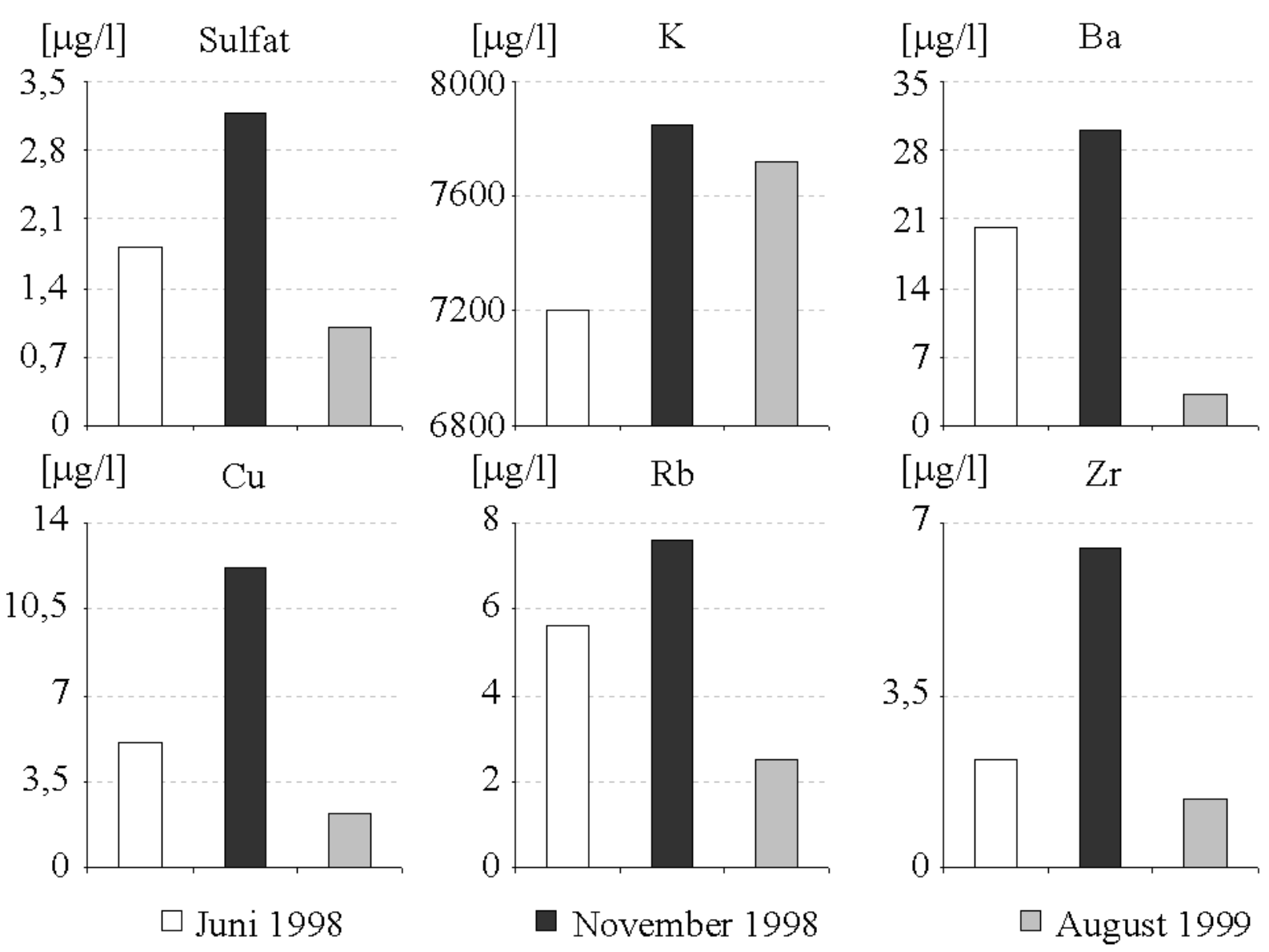

Abb. 4.12.: Konzentrationsverlauf von Sulfat, $K, B a, C u, R b$ und $\mathrm{Zr}$ im Seewasser in Cospuden zwischen Juni 1998 und August 1999.

Ursache für dieses Verhalten kann ein Eintrag der Elemente in gelöster Form aus der Kippe zwischen Juni und November 1998 in den See sein; dort werden in der Folge die Löslichkeitsprodukte einiger Festphasen wie etwa $\mathrm{BaSO}_{4}$ erreicht, die Festphasen fallen aus, und Elemente wie $\mathrm{Cu}$ oder $\mathrm{Zr}$ werden adsorptiv gebunden und so aus dem Seewasser entfernt. Einige Elementkonzentrationen im Seewasser zeigen zwischen dem Beginn im Juni 1998 und November 1998 einen starken Rückgang, um bis August 1999 wieder leicht anzusteigen. Dies trifft zu auf $\mathrm{Ca}, \mathrm{Fe}, \mathrm{As}, \mathrm{Sr}, \mathrm{Nb}, \mathrm{Pb}$ und $\mathrm{U}$; ihre zeitliche Entwicklung ist in Abb. 4.13. dargestellt.

Diese Elemente werden zunächst aus der Kippe oder durch das Flutungswasser in den See eingetragen. Dort kommt es zur Übersättigung; beispielsweise kann durch den Eintrag von Sulfat aus der Kippe das Löslichkeitsprodukt von Gips überschritten worden sein. Durch die Sedimentation von Gips verringerte sich die Ca-Konzentration im Seewasser. Fe, das als Fe ${ }^{\text {II }}$ in den See gelangt, wird durch den Kontakt mit Sauerstoff $\mathrm{zu} \mathrm{Fe}^{\mathrm{III}}$ oxidiert und sedimentiert in Form amorpher Fe-Oxyhydroxide, eventuell auch als Schwertmannit. 

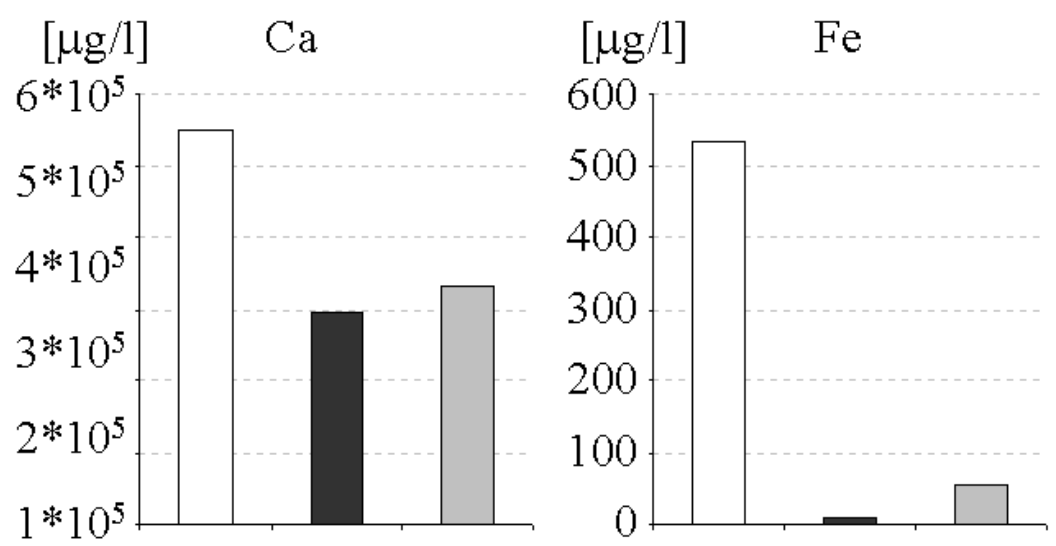

$[\mu \mathrm{g} / 1] \quad$ As
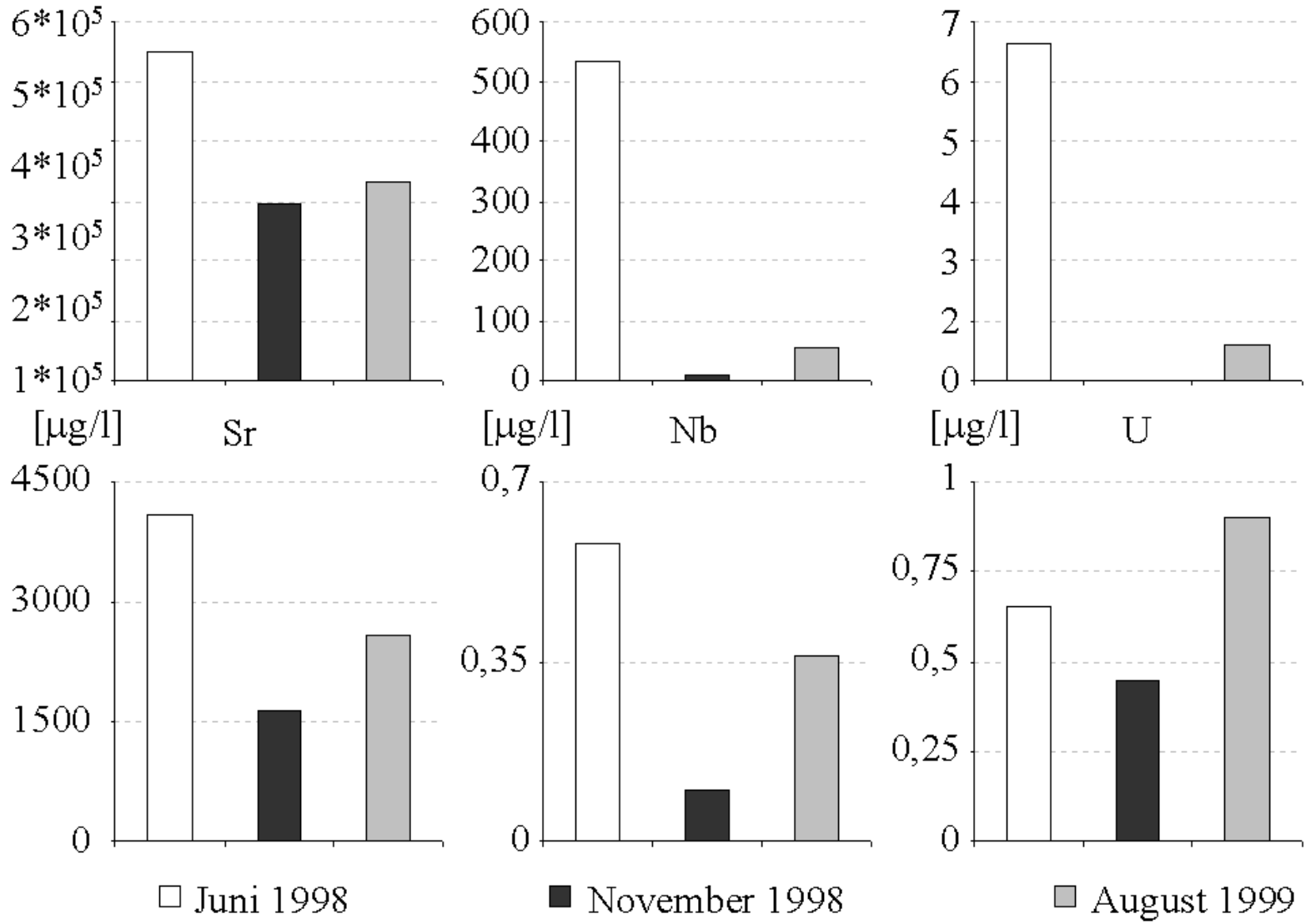

Abb. 4.13.: Konzentrationsverlauf von $\mathrm{Ca}, \mathrm{Fe}, \mathrm{As}, \mathrm{Sr}, \mathrm{Nb}$ und $\mathrm{U}$ im Seewasser in Cospuden zwischen Juni 1998 und August 1999

Schon leichte $\mathrm{pH}-$ Wert-Änderungen, aber auch bakterielle Prozesse wie die Reduktion von $\mathrm{Fe}^{\mathrm{III}} \mathrm{zu} \mathrm{Fe} \mathrm{Fe}^{\mathrm{II}}$ können dazu führen, das die vorübergehend gebildeten Eisenphasen wieder in Lösung gehen.

Einen Beitrag zu diesem Verlauf der Elementkonzentrationen können auch Temperaturunterschiede leisten. In den Sommermonaten erwärmt sich das Seewasser, dadurch erhöht sich die Löslichkeit. Im November lagen die Temperaturen bei der Probennahme unterhalb des Gefrierpunktes, der Multilevelbrunnen war eingefroren und konnte nicht beprobt werden. Im gegenüber den Sommermonaten kalten Seewasser verringert sich die Löslichkeit, mehr Festphasen sedimentieren und die gelösten Elementkonzentrationen sinken. Auch die Redoxverhältnisse in der Wassersäule von Seen unterliegen jahreszeitlichen Schwankungen und beeinflussen damit das Konzentrationsprofil von redoxsensitiven Elementen wie Fe oder Mn.

Geht man davon aus, dass sich das Seewasser zu späteren Zeitpunkten im Wesentlichen aus dem Seewasser des Jahres 1998 und Wasser aus der Fremdflutung zusammensetzt, müsste 
sich das Mischungsverhältnis der beiden Komponenten aus den gemessenen Konzentrationen der Elemente nach dem folgenden Gleichungssystem berechnen lassen:

$\mathrm{C}_{\mathrm{j}} * \mathrm{~S}_{\mathrm{j}}=\mathrm{C}_{\mathrm{a}} * \mathrm{~S}_{\mathrm{a}}+\mathrm{C}_{\mathrm{f}} * \mathrm{~S}_{\mathrm{f}}$

$\mathrm{S}_{\mathrm{a}}+\mathrm{S}_{\mathrm{f}}=\mathrm{S}_{\mathrm{j}}$

dabei ist $\quad \mathrm{C}_{\mathrm{j}}=$ Konzentration im Seewasser aktuell

$\mathrm{C}_{\mathrm{a}}=$ Konzentration im ursprünglichen Seewasser

$\mathrm{C}_{\mathrm{f}}=$ Konzentration im Flutungswasser

$\mathrm{S}_{\mathrm{j}}=$ Volumen des Seewassers im aktuell

$\mathrm{S}_{\mathrm{a}}=$ Volumen des ursprünglichen Seewassers

$\mathrm{S}_{\mathrm{f}}=$ Volumen des Flutungswassers

Daraus ergibt sich für den Anteil des ursprünglichen Seewassers am aktuellen Seewasser:

$\mathrm{S}_{\mathrm{a}} / \mathrm{S}_{\mathrm{j}}=\left(\mathrm{C}_{\mathrm{j}}-\mathrm{C}_{\mathrm{f}}\right) /\left(\mathrm{C}_{\mathrm{a}}-\mathrm{C}_{\mathrm{f}}\right)$

Diese Rechnung wurde sowohl für den November 1998 in Bezug auf das Seewasser vom Juni 1988 als auch für den August 1999 in Bezug auf das Seewasser vom November 1998 durchgeführt. Tab. 4.7. zeigt die Ergebnisse:

\begin{tabular}{|l|l|l|}
\hline Element & $\begin{array}{l}\text { Anteil Seewasser Juni 1998 am } \\
\text { Seewasser vom November 1998 }\end{array}$ & $\begin{array}{l}\text { Anteil Seewasser November 1998 } \\
\text { am Seewasser August 1999 }\end{array}$ \\
\hline Sulfat & 2,39 & 0,08 \\
\hline $\mathbf{L i}$ & 0,14 & 0,29 \\
\hline $\mathbf{N a}$ & 0,71 & $-0,64$ \\
\hline $\mathbf{M g}$ & 0,46 & 0,63 \\
\hline $\mathbf{A l}$ & 0,00 & 1,48 \\
\hline $\mathbf{K}$ & 0,83 & 1,04 \\
\hline $\mathbf{C a}$ & 0,09 & 2,55 \\
\hline $\mathbf{S c}$ & 0,09 & $-0,32$ \\
\hline $\mathbf{V}$ & $-0,75$ & 1,00 \\
\hline $\mathbf{C r}$ & 1,31 & $-0,69$ \\
\hline $\mathbf{M n}$ & $-0,29$ & 1,70 \\
\hline $\mathbf{F e}$ & $-0,03$ & $-2,57$ \\
\hline $\mathbf{C o}$ & 0,06 & $-0,49$ \\
\hline $\mathbf{N i}$ & 0,13 & 0,20 \\
\hline $\mathbf{C u}$ & 2,52 & 0,15 \\
\hline $\mathbf{Z n}$ & 0,05 & $-0,38$ \\
\hline $\mathbf{R b}$ & 0,33 & 6,10 \\
\hline $\mathbf{S r}$ & 0,12 & 3,81 \\
\hline $\mathbf{M o}$ & $-1,29$ & 1,07 \\
\hline $\mathbf{B a}$ & 0,33 & 6,34 \\
\hline $\mathbf{U}$ & $-9,00$ & $-1,50$ \\
\hline
\end{tabular}

Tab. 4.7.: Anteil des Seewassers an der Gesamtwassermenge, berechnet nach verschiedenen Elementkonzentrationen.

Es fällt auf, dass die Koeffizienten je nach Element sehr uneinheitlich sind. Werte größer 1 bedeuten, dass noch andere Quellen als die beiden Wässer existiert haben müssen; so können Sickerwässer aus der Kippe in den See gelangt sein, oder es ist im See selbst durch 
Mineralauflösungen oder -umbildungen zu einer Freisetzung von Elementen gekommen. Werte unter 0 besagen, dass Elementkonzentrationen durch Austragung, Ausfällungen oder Verdünnung mit unbelasteten Wässern niedriger liegen, als es bei einer reinen Mischung aus dem Seewasser und dem Flutungswasser zu erwarten wäre. Das häufige Auftreten von Werten $>1$ bzw. < 0 sowie die starken Unterschiede zwischen den berechneten Seewasseranteilen für verschiedene Elemente zeigen, dass neben der Verdünnung durch das Flutungswasser noch zahlreiche andere Faktoren die Elementkonzentrationen im Seewasser beeinflussen. Die Entwicklung der Elementkonzentrationen in den Wässern spiegeln dabei auch die zeitliche Abfolge des Geschehens in der Kippe wieder. So kam es zwischen Juni und November 1998 zu einem starken Eintrag von Sulfat in das Seewasser. Auf Sulfat als direktes Produkt der Pyritoxidation folgten dann bis zum August 1999 Elemente wie Al, K, Ca und Mn, die beim Angriff der gebildeten Schwefelsäure auf karbonatische und silikatische Minerale freigesetzt wurden. Bereits in der ersten Phase bis zum November 1998 wurden $\mathrm{Cr}$ und $\mathrm{Cu}$ ebenfalls in den See eingetragen; in der zweiten Phase folgten $\mathrm{V}, \mathrm{Ni}, \mathrm{Cu}, \mathrm{Rb}, \mathrm{Sr}, \mathrm{Mo}$ und $\mathrm{Ba}$, die vermutlich ebenfalls bei der Reaktion von versauerten Wässern mit anderen Mineralphasen in die Kippenwässer abgeben wurden. Gleichzeitig stieg der pH-Wert an, $\mathrm{Fe}^{\mathrm{II}}$ wurde oxidiert, und es bildeten sich Eisenhydroxidniederschläge, die zu einer Verarmung an Eisen führten. Im Zuge dieser Sedimentation nahmen auch die Konzentrationen von $\mathrm{Na}, \mathrm{Sc}, \mathrm{Cr}, \mathrm{Co}$, Zn und $\mathrm{U}$ weitaus stärker $\mathrm{ab}$, als es der reine Mischungsprozess nahelegen würde. Der Seewasserchemismus ist offensichtlich entscheidend geprägt von Wechselwirkungen mit den Kippenwässern.

\subsubsection{2. $C M L$}

An den Proben aus dem Multilevelbrunnen CML und aus den RCO-Pegeln, die im Rahmen dieser Arbeit untersucht wurden, führte auch das UFZ Messungen der wichtigsten chemischen Parameter durch. Dabei ergaben sich teilweise Abweichungen, die auf die Lagerung der Proben zwischen Entnahme und Analyse im Rahmen dieser Arbeit zurückzuführen waren. Im Folgenden werden zunächst die Resultate vorgestellt, die sich aus den Messungen für die vorliegende Arbeit ergaben. Anschließend wird anhand eines Vergleichs zwischen diesen und den am UFZ bestimmten Daten diskutiert, wie sich die Situation im Tagebau entwickelt hat und welche Implikationen sich aus den Abweichungen der Messwerte ergeben.

Abb. 4.14. zeigt den Verlauf von pH-Wert, elektrischer Leitfähigkeit, Sulfat- und Eisenkonzentration im Multilevelbrunnen CML im Januar 1999, August 1999 und Januar 2000. Bei den pH-Werten scheint sich im Lauf der Zeit in einer Tiefe von $30 \mathrm{~m}$ eine 
Versauerungsfront auszubilden. Gegenüber den Porenwässern aus dem Bohrkern ist zum Januar 1999 zunächst ein starker Anstieg der pH-Werte zu verzeichnen, dann bildet sich wieder ein Tiefenprofil aus, das dem in den Porenwässern (s. Kap. 4.5.) auftretenden pHProfil in der Tendenz recht gut zu entsprechen scheint. Allerdings sind die Schwankungen mit der Tiefe weniger ausgeprägt. Die elektrische Leitfähigkeit stieg von Januar 1999 bis August 1999 stark an, sank in der Folgezeit jedoch etwas ab. Über das Tiefenprofil zeigte sich dabei jeweils ein Anstieg von 20 m Tiefe bis zu einem Maximum in $32 \mathrm{~m}$ Tiefe; unterhalb davon sanken die Werte für die Leitfähigkeit wieder ab. Für die Sulfatkonzentrationen war zwischen Januar 1999 und August 1999 ebenfalls eine starke Zunahme zu verzeichnen. Die Werte verharrten bis zum Januar 2000 auf hohem Niveau. Die Tiefenprofile vom August 1999 und Januar 2000 sind praktisch deckungsgleich mit einem ausgeprägten Maximum in etwa $30 \mathrm{~m}$ Tiefe. Ein ähnliches Verhalten zeigen die Eisenkonzentrationen. In 20 m, 24 m, 39,5 m und 43,5m Tiefe stiegen die Konzentrationen zwischen Januar 1999 und August 1999 an, während sie in 32 m Tiefe praktisch konstant blieben. Zum Januar 2000 sanken die Konzentrationen in den beiden tiefsten Proben wieder ab, während sie in den höher gelegenen Wässer gleich blieben.

Die Ergebnisse der Parameter pH-Wert, Leitfähigkeit und Sulfat- und Eisenkonzentration weisen daraufhin, dass sich zwischen Januar 1999 und August 1999 in den Wässern des Multilevelbrunnens eine Oxidationsfront in etwa $30 \mathrm{~m}$ Tiefe ausbildete, die bis zum Januar 2000 erhalten blieb. Allerdings wurden einige der Proben vor der Analyse über einen längeren Zeitraum gelagert; dabei kam es zu Veränderungen der Wässer. Bei der Beurteilung, ob die hier dargestellten Ergebnisse tatsächlich den Gegebenheiten im natürlichen Umfeld der Kippe entsprechen, müssen diese Veränderungen berücksichtigt werden. Darauf wird weiter unten noch ausführlicher eingegangen.

Abb. 4.15. zeigt die gemessenen Konzentrationen einiger Elemente. Für die meisten Elemente war zwischen Januar und August 1999 ein Anstieg zu verzeichnen; bis zum Januar 2000 sanken die Konzentrationen dann wieder ab. Das Niveau war allerdings höher als bei den Proben vom Januar 1999, wobei das Konzentrationsmaximum im Profil erhalten blieb. Diese Beobachtung trifft für $\mathrm{Al}, \mathrm{Mn}, \mathrm{Fe}, \mathrm{Ni}, \mathrm{Zn}, \mathrm{Y}, \mathrm{Zr}$ und die REEs zu. Einen weitgehend ähnlichen Verlauf zeigte V. 

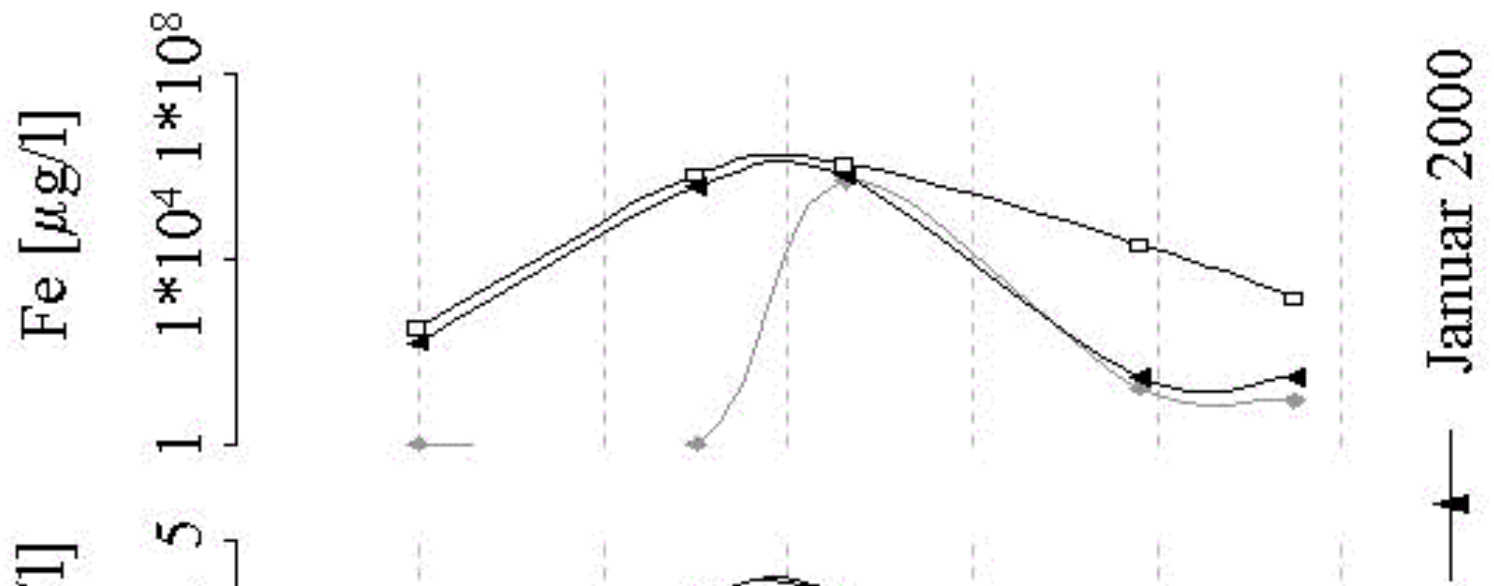

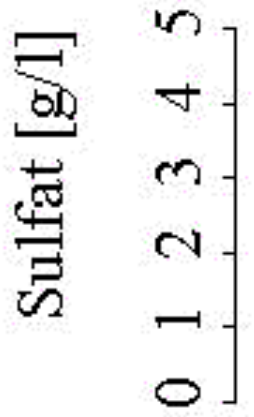

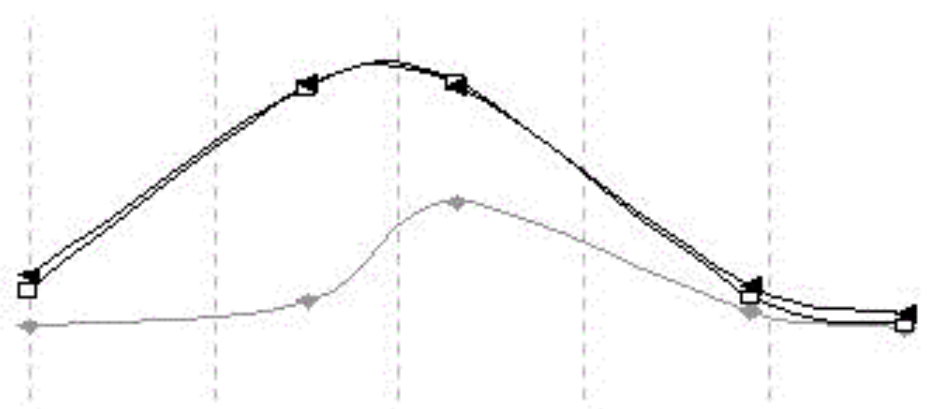

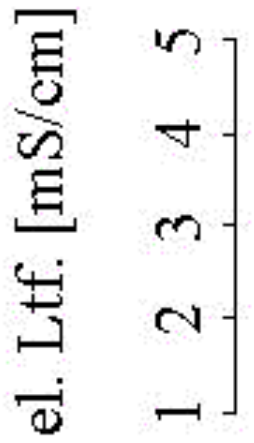
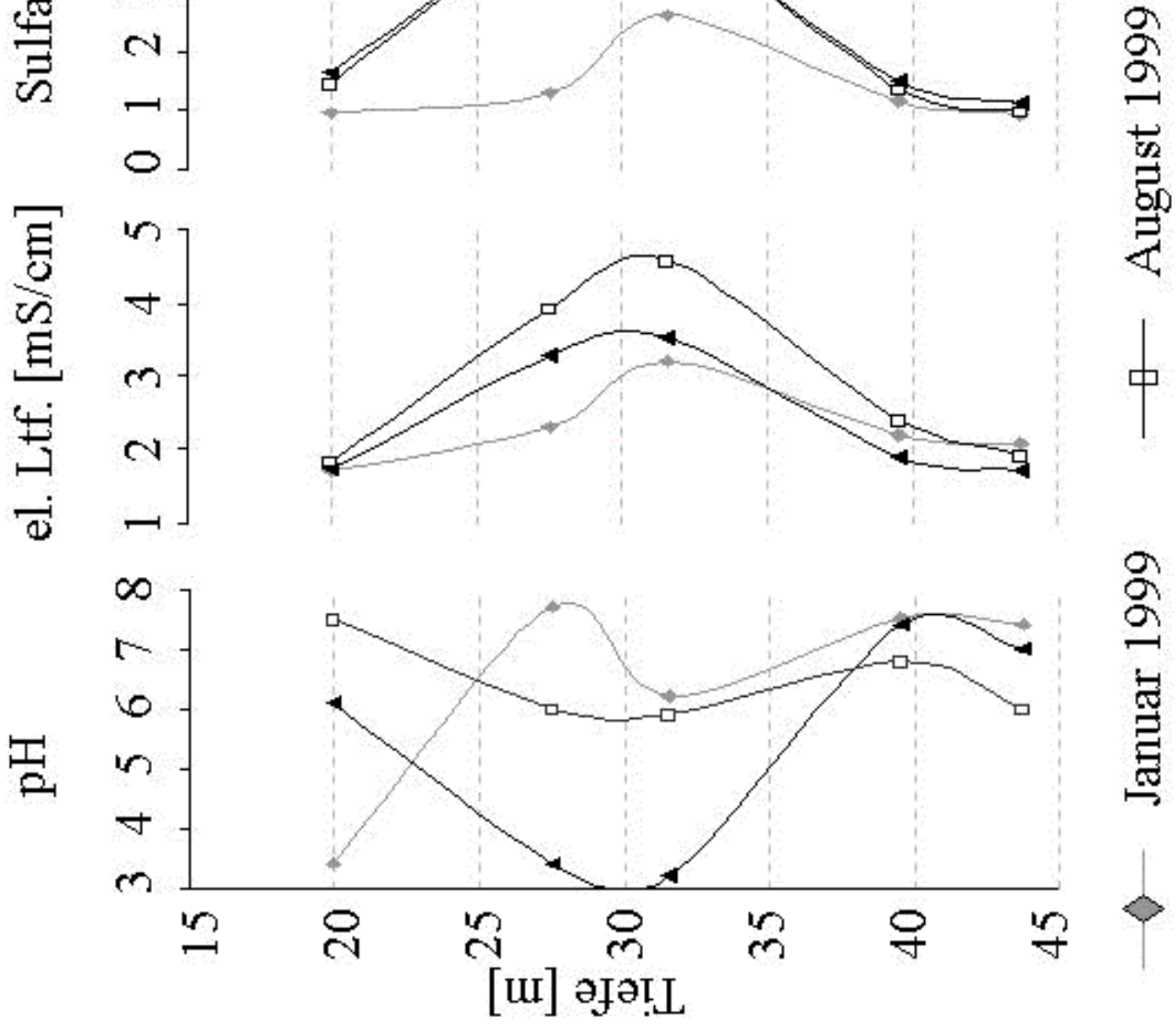

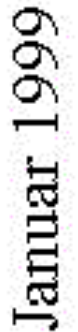

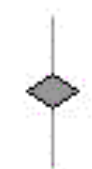

Abb. 4.14.: Zeitliche Entwicklung der Profile von pH, elektrischer Leitfähigkeit, Sulfat- und Eisenkonzentration in Wasserproben aus dem Multilevelbrunnen in Cospuden. 

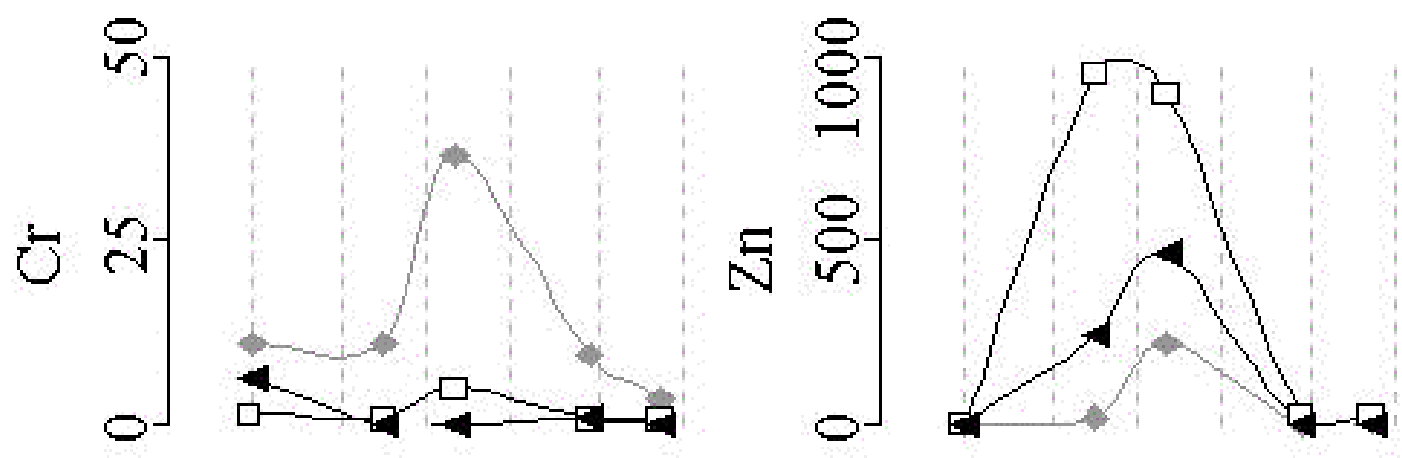

9
8
慙
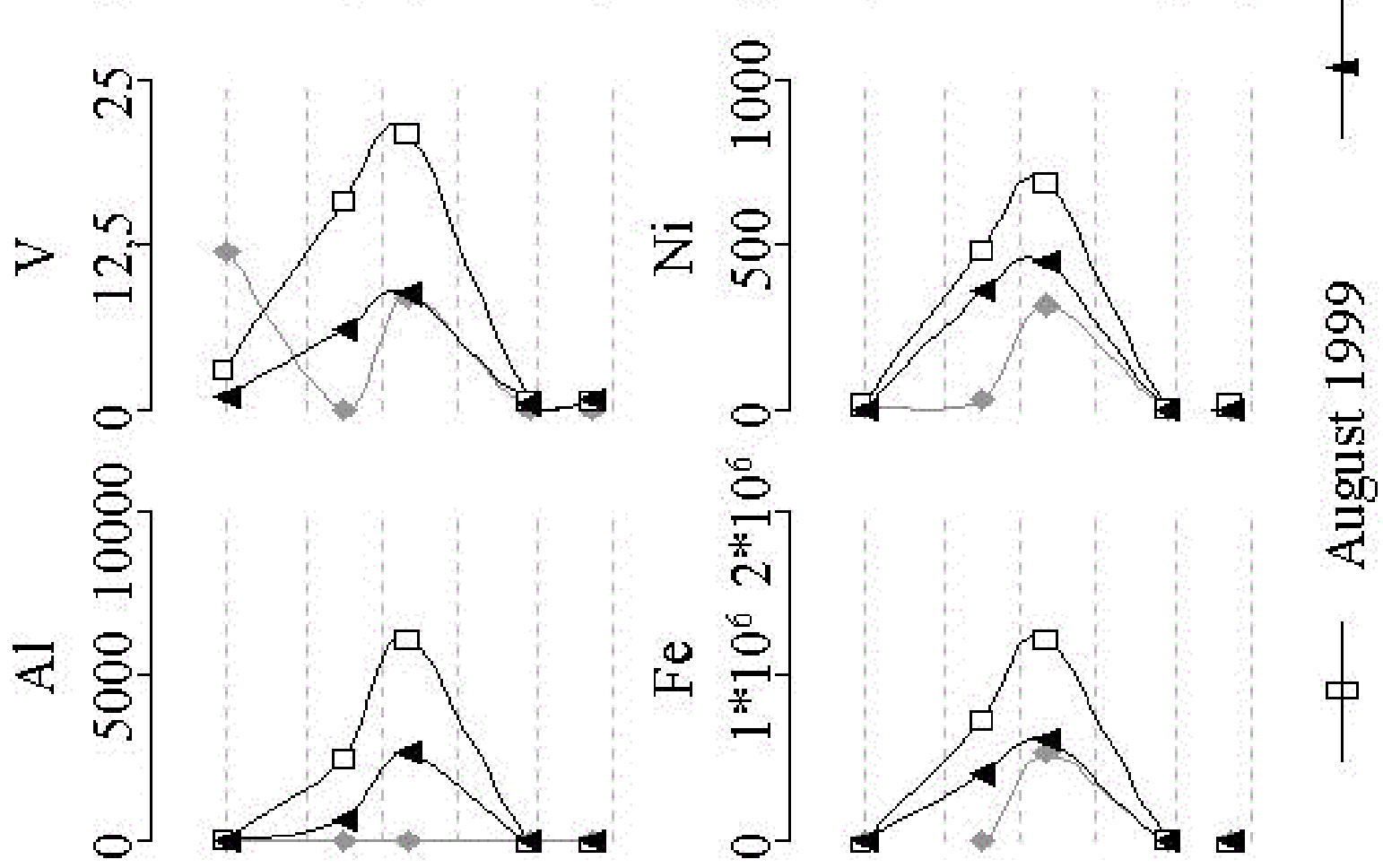

$\frac{\Omega}{2}$
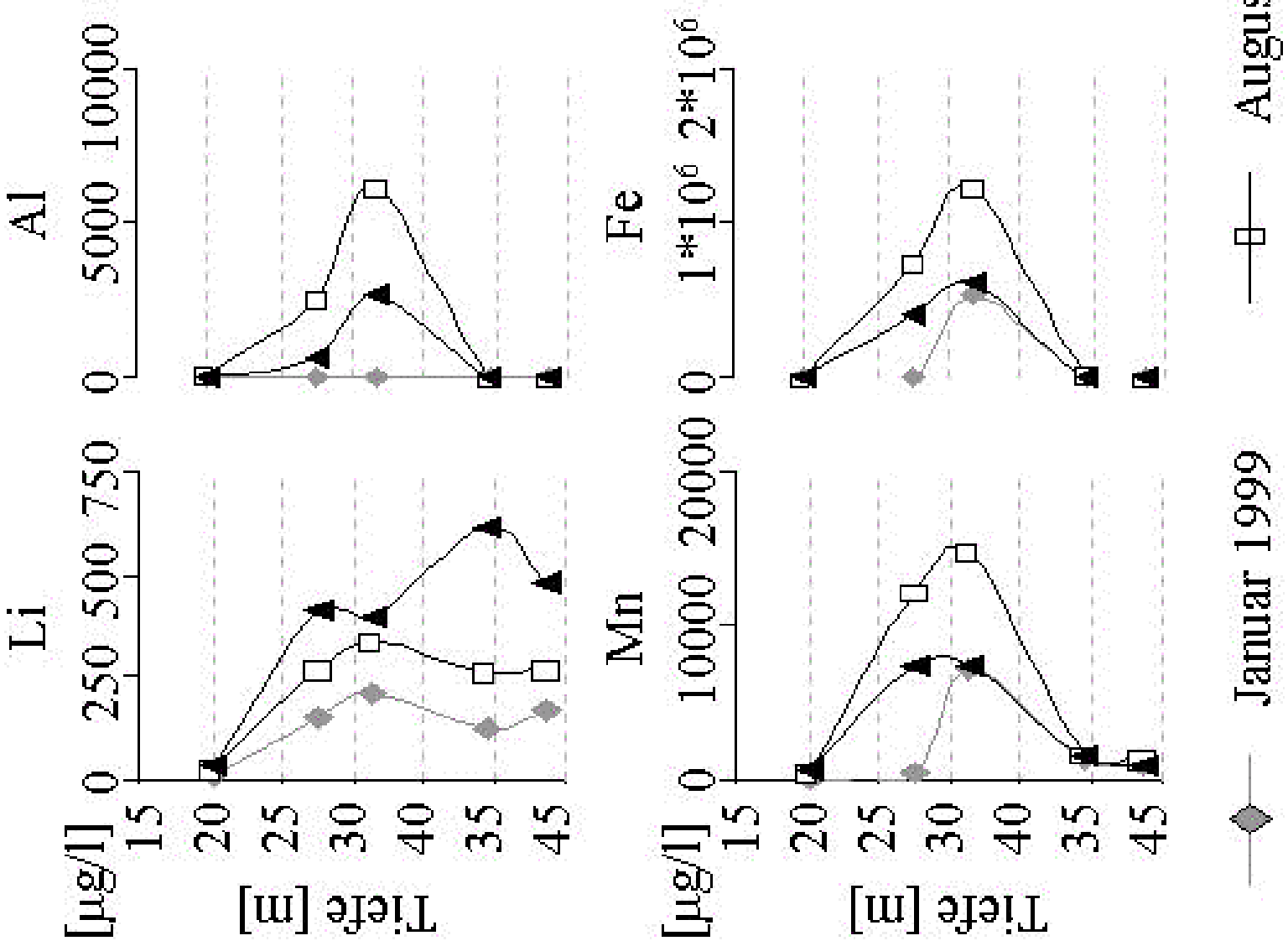

Abb. 4.15.: Zeitliche Entwicklung einiger Elementkonzentrationen im Tiefenprofil der Wässer des Multilevelbrunnens CML in Cospuden. 

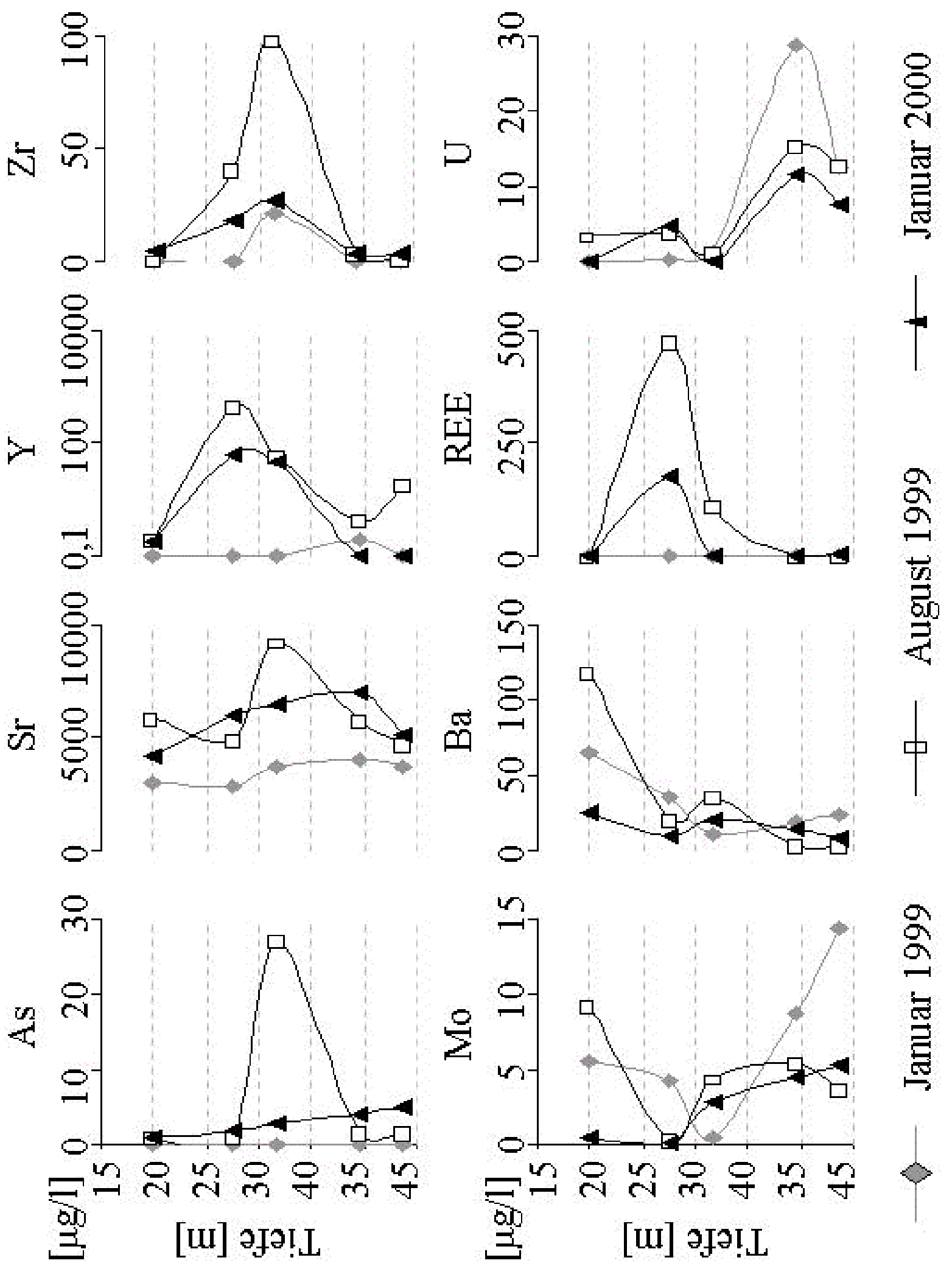

Abb. 4.15. (Forts.): Zeitliche Entwicklung einiger Elementkonzentrationen im Tiefenprofil der Wässer des Multilevelbrunnens CML in Cospuden. 
Nur wenige Elemente verhielten sich abweichend. So stieg die Konzentration von Li vom Januar 1999 zum Januar 2000 kontinuierlich an. Cr dagegen wurde seit dem Januar 1999 bis zum Januar 2000 fast vollständig aus den Wässern entfernt. As und Sr zeigten im August 1999 ein ausgeprägtes Profil mit einem Maximum in 31,5 m Tiefe, das in den beiden anderen Proben vom Januar 1999 und Januar 2000 so jeweils nicht zu finden war. Ba und Mo schließlich ähneln sich im Verlauf der Kurven sehr stark. Beide waren in nur 20 m Tiefe am höchsten konzentriert. In größeren Tiefen sanken die Werte dann ab.

\subsubsection{Grundwassermessstellen (RCO)}

Das Gesamtbild der Proben aus den Grundwasserpegeln zeigt für den Medianwert vieler Elemente einen Anstieg, der bis zum Januar 2000 andauerte. Abb. 4.16. stellt die Elementanreicherungsfaktoren aller untersuchten Elemente im Median der Werte aus den Grundwasserpegeln gegenüber dem Median aus unbelasteten Grundwässern (PLEßOW et al. 1997) dar.

Beim Vergleich der Anreicherungsfaktoren zwischen Medianwerten aus den zur Verfügung stehenden RCO-Proben und denen unbelasteter Grundwässer sieht man, dass noch zwischen August 1999 und Januar 2000 eine Zunahme diverser Elementkonzentrationen zu beobachten war. Darunter fallen Li, Be und Ni. Insbesondere gilt das für die REEs, Sb, Tl und Th, die in den Proben vom Oktober 1998 praktisch vollständig unterhalb der Bestimmungsgrenze lagen. Ihre Anreicherungsfaktoren liegen im Januar 2000 bei 10-40. Auch die Elementanreicherungsfaktoren von $\mathrm{Rb}, \mathrm{Y}$ und $\mathrm{Pb}$ stiegen kontinuierlich an. $\mathrm{Rb}$ und $\mathrm{Y}$ waren im Januar 2000 zwei- bzw. dreifach angereichert, während die Pb-Konzentration immer noch unterhalb der unbelasteter Grundwässer lag. Die Elementanreicherungsfaktoren von V, Cr, Mn, As, Sr und Mo stiegen zwischen Oktober 1998 und August 1999 an. Bis zum Januar 2000 pendelten sie sich auf einen mittleren Wert ein. Schließlich lagen sie zwischen 1,9 (Mo) und 188 (Sr). Die Elementanreicherungsfaktoren von $\mathrm{Cu}, \mathrm{Zn}, \mathrm{Zr}$ und Ba sanken von Oktober 1998 bis Januar 2000 kontinuierlich ab. Die Gehalte von Zn und Ba waren schließlich geringer als in unbelasteten Grundwässern. Die Konzentration von Sc, die vom Oktober 1998 zum August 1999 zugenommen hatte, lag im Januar 2000 weit unterhalb der unbelasteter Grundwässer. Die Elementanreicherungsfaktoren von Co und U sanken vom Oktober 1998 zum August 1999 zunächst ab und stiegen dann wieder an. Die Konzentration von Co lag im Januar 2000 am höchsten mit einem Elementanreicherungsfaktor von 47, während U einen Elementanreicherungsfaktor von 82 aufwies. 


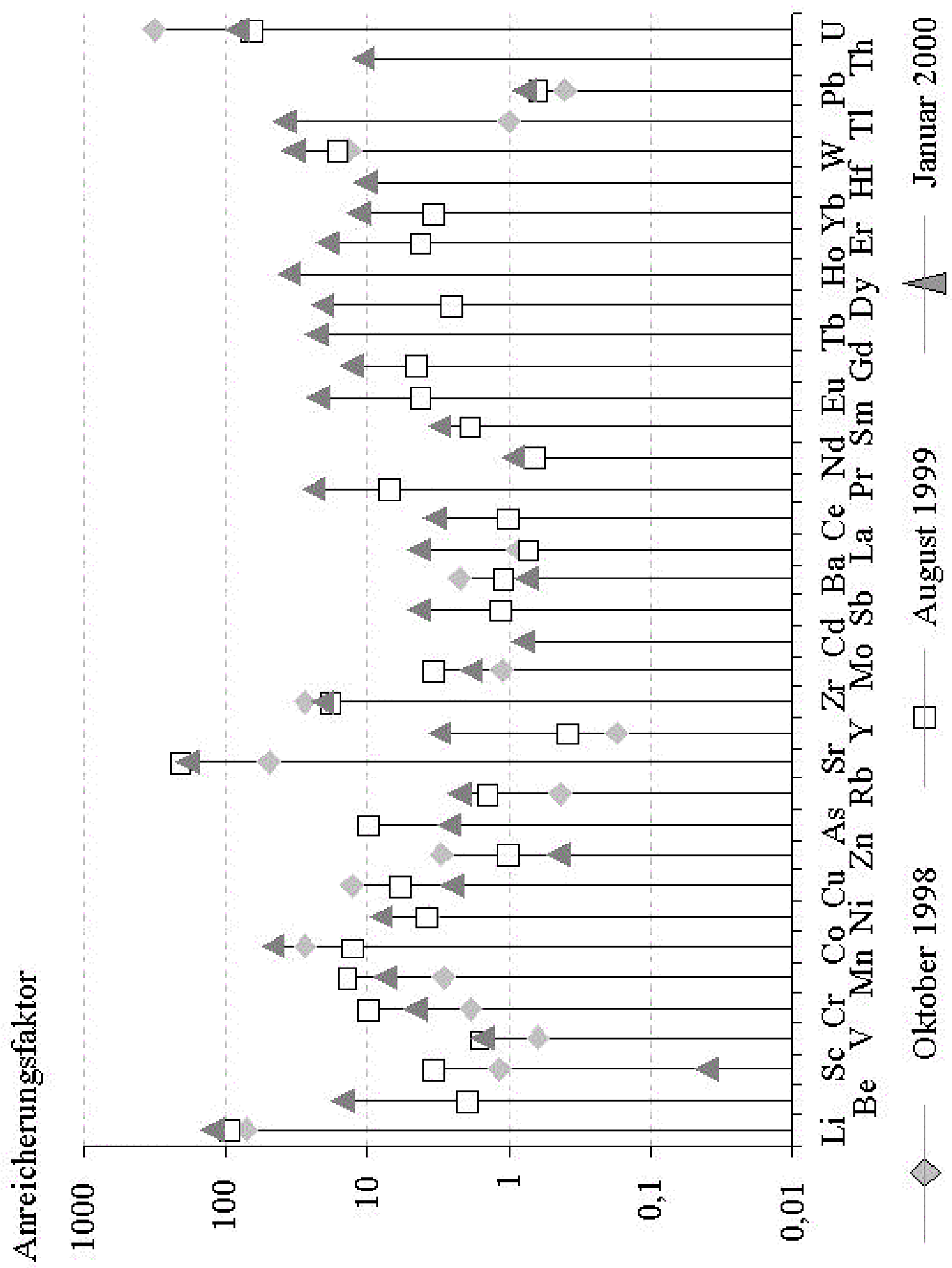

Abb.4.16.: Elementanreicherungsfaktoren für die Medianwerte der Konzentrationen in den Grundwasserpegeln in Cospuden gegenüber dem Median unbelasteter Grundwässer (PLEßOW 1998, PLEßOW et al. 1997). 
Insgesamt lagen die Medianwerte der Konzentrationen in den Grundwasserpegeln aus Cospuden im Januar 2000 für fast alle Elemente über denen unbelasteter Grundwässer. Nur $\mathrm{Sc}, \mathrm{Zn}, \mathrm{Cd}, \mathrm{Ba}, \mathrm{Nd}$ und $\mathrm{Pb}$ waren demgegenüber abgereichert.

Im Vergleich zu Leipziger Grundwasser (PLEßOw 1998) stellt sich die Situation etwas anders dar. Elementanreicherungsfaktoren < 1 zeigen Sc, V, Cr, Cu, Zn, As, Y, Mo, Cd, Ba, Ce, Nd, $\mathrm{Sm}$, und Pb. Die Elementanreicherungsfaktoren bei Li, Be, Mn, Co, Ni, Rb, La, Pr, Eu, Gd, Tb, Dy, Ho, Er, Yb, Hf, W und U lagen zwischen 1 und 10. Größer als 10 waren die Faktoren nur bei $\mathrm{Sr}(\mathrm{EF}=17), \mathrm{Zr}(\mathrm{EF}=50), \mathrm{Tl}(\mathrm{EF}=13)$ und $\mathrm{Th}(\mathrm{EF}=11)$.

Der pH-Wert ist in den Messtellen RCO4, RCO12, RCO15 und RCO18 im Kippenbereich im Untersuchungszeitraum angestiegen und bewegt sich dort überall im Bereich nahe 7. Auch der Pegel RCO17 auf einer bebauten, dem Tagebau nahegelegenen Fläche zeigt einen pHWert um 6,5, der im Untersuchungszeitraum nahezu unverändert geblieben ist. Auffällig ist dagegen die Entwicklung des pH-Wertes im Pegel RCO3 in einem ungestörten Gelände nahe der Tagebaukippe, für den ein pH-Wert von 2,5 bestimmt wurde. Eine Übersicht über die Entwicklung des pH-Werts, der Leitfähigkeit und der Sulfatkonzentration gibt Abb. 4.17..

$\mathrm{pH}$
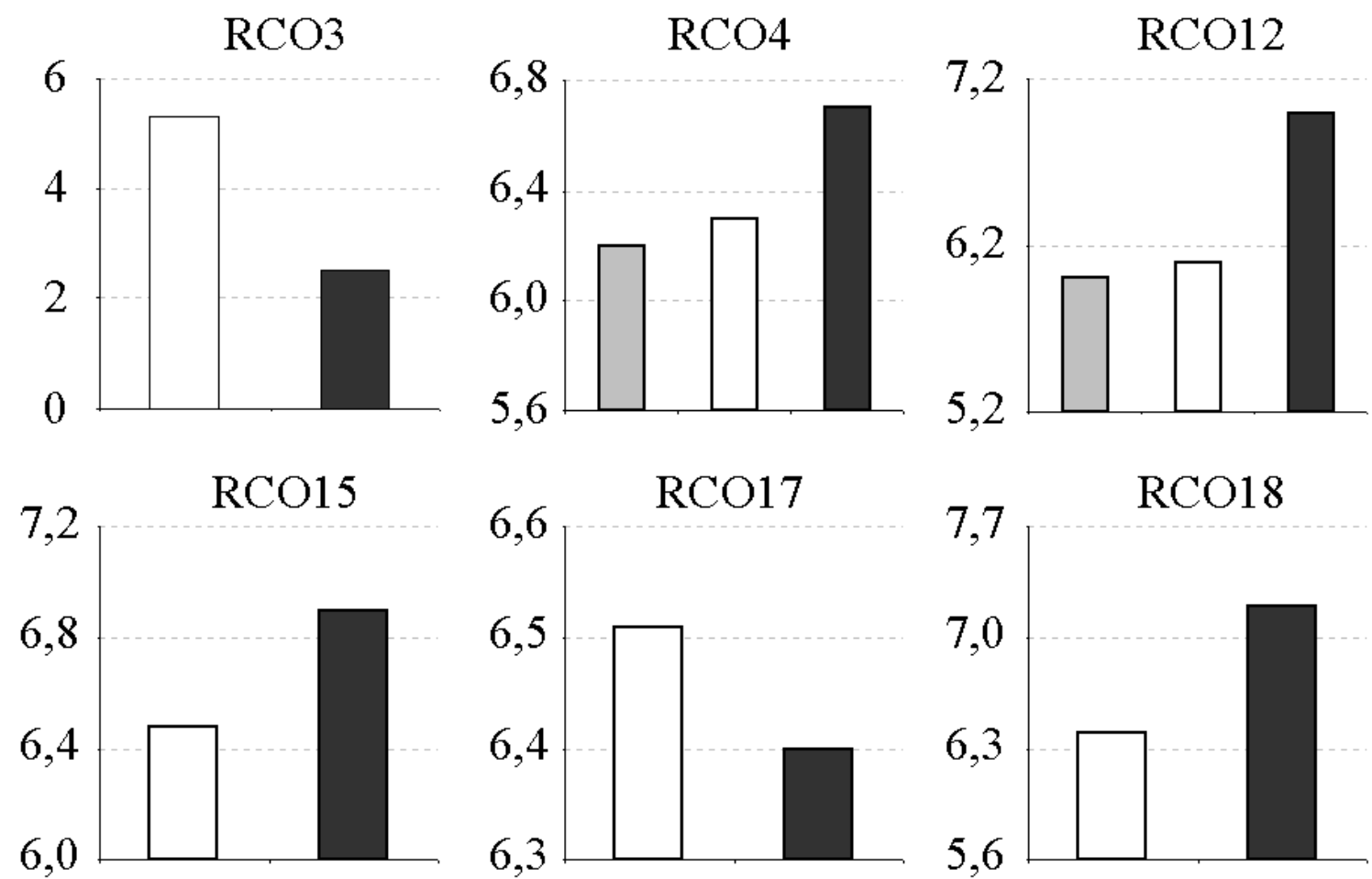

Oktober 1998

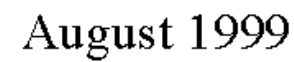

Januar 2000

Abb. 4.17.: Zeitliche Entwicklung des pH-Wertes in den Grundwasserpegeln. 


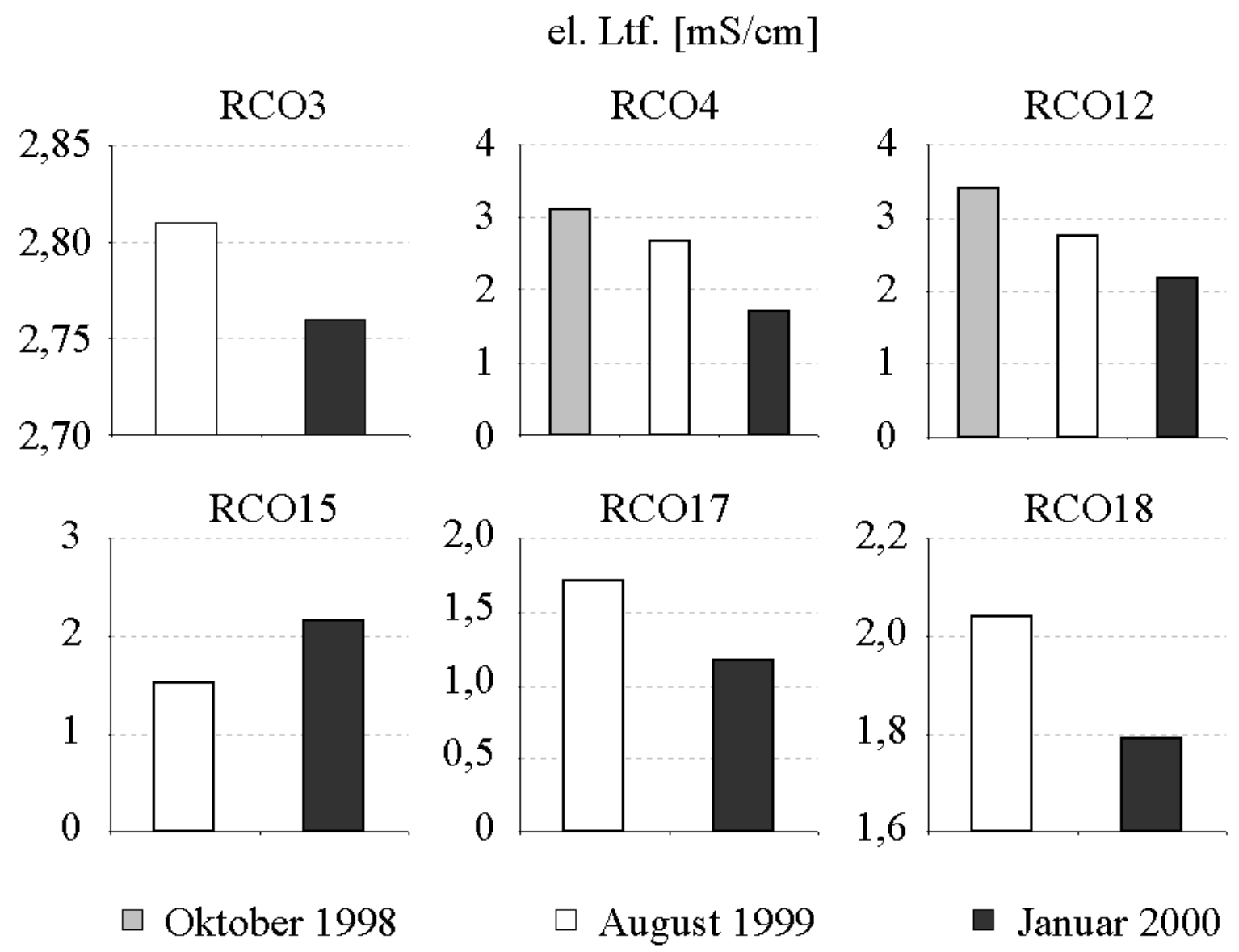

Sulfat [g/l]
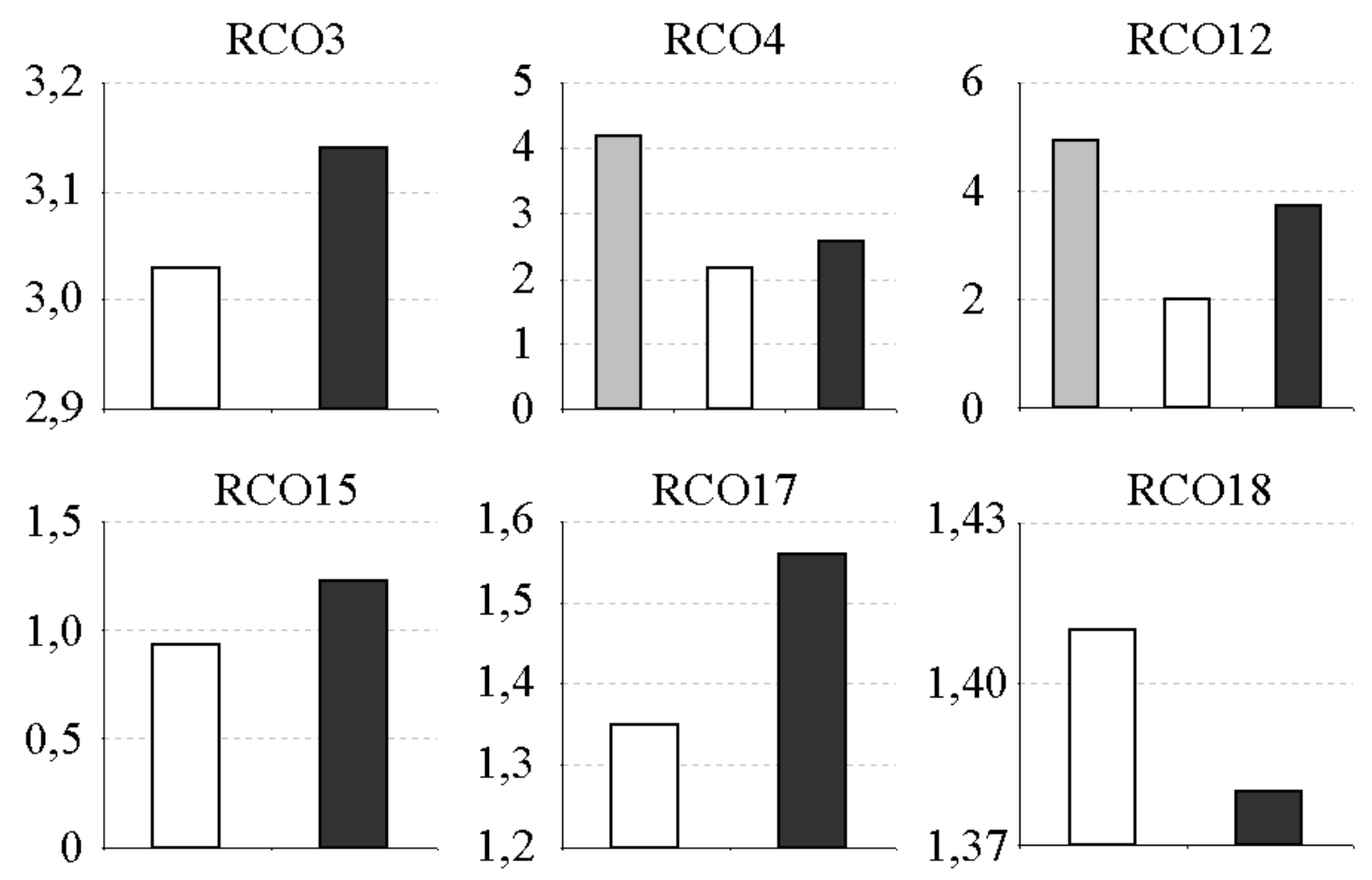

$\square$ Oktober 1998

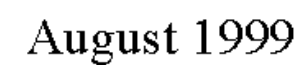

- Januar 2000

Abb. 4.17. (Forts.): Zeitliche Entwicklung von Leitfähigkeit und Sulfatgehalt in den Grundwasserpegeln. 
Bis auf die Proben RCO3 und RCO17 sind die pH-Werte leicht angestiegen und schwanken um 6,8. Die Proben RCO3 und RCO17 sind während der Lagerung versauert (s.u.). Mit der Abnahme der Leitfähigkeit korrespondieren sinkende Eisenkonzentrationen und abnehmende Gehalte zahlreicher Elemente. Dies ist auf Fällungsprozesse während der Lagerung zurückzuführen; bei Analysen, die zeitnäher zur Probennahme durchgeführt werden, treten diese Effekte nicht auf (s.u.).

Die elektrische Leitfähigkeit kann als Indikator für die absolute Ionenfracht der Wässer gelten. Sie ist in den Proben aus RCO4, RCO12, RCO17 und RCO18 gesunken. Dies kann zum einen auf Verdünnungseffekten beruhen, zum anderen auf der Bildung von Ausfällungen bei gleichbleibendem bzw. steigendem $\mathrm{pH}$.

Während sowohl in RCO3 als auch in RCO4 die Sulfatkonzentration zwischen August 1999 und Januar 2000 keine drastischen Veränderungen zeigte, stieg der Wert in RCO12, der zwischen Oktober 1998 und August 1999 von 4,95 auf 2,02 g/l erheblich abgesunken war, zwischen August 1999 und Januar 2000 wieder auf 3,72 g/l an.

\section{$[\mu \mathrm{g} / 1]$}

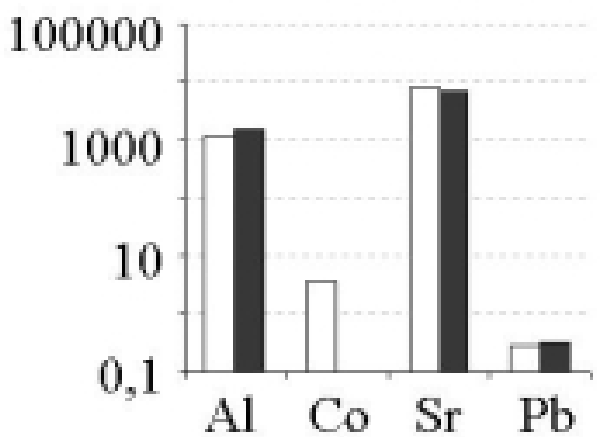

$[\mu \mathrm{g} / 1] \quad \mathrm{RCO} 15$

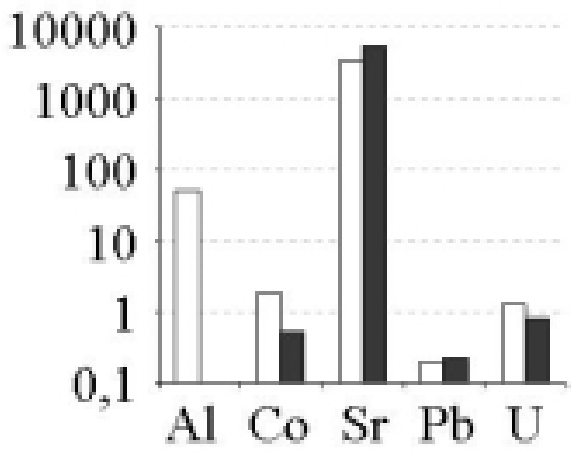

Oktober 1998

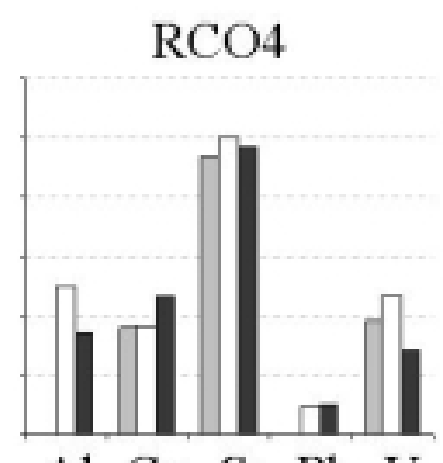

$\mathrm{Al} \mathrm{Co} \mathrm{Sr} \mathrm{Pb} \mathrm{U}$ $\mathrm{RCO} 17$

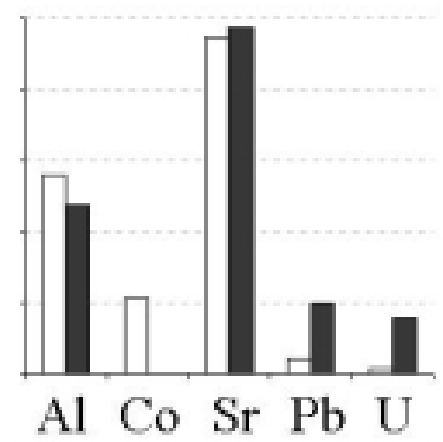

August 1999
$\mathrm{RCO} 12$

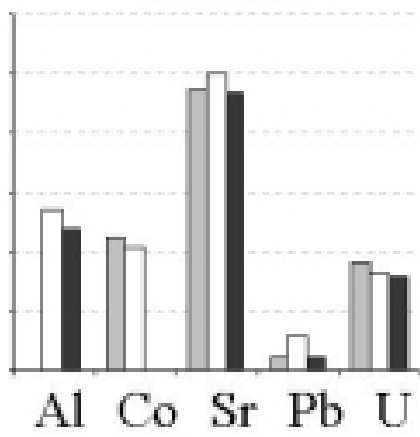

$\mathrm{RCO} 18$

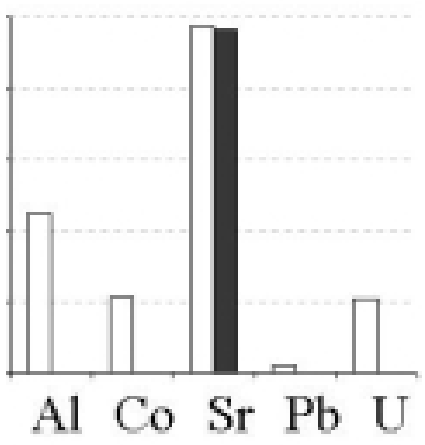

Januar 2000

Abb. 4.18.: Zeitliche Entwicklung einiger Elemente in den Grundwasserpegeln in Cospuden. 
Die Konzentrationsentwicklung einiger Elemente in den Grundwasserpegeln ist in Abb. 4.18. dargestellt. Dass Fällungsprozesse nicht genügen, um durch Copräzipitation oder Adsorption die Elementgehalte in allen Fällen zu verringern, zeigen die Daten für $\mathrm{Sr}, \mathrm{Pb}$ oder zum Teil für die Übergangsmetalle, die, einmal in Lösung, zu erheblichen Teilen dort verbleiben. In RCO3 ist ausserdem ein starker Anstieg zahlreicher Element-Konzentrationen zu verzeichnen, die trotz der Bildung von Eisenhydroxid-Niederschlägen während der Lagerung in der Lösung verblieben. So stieg die Al-Konzentration leicht von 1143 auf $1457 \mu \mathrm{g} / \mathrm{l}$, As nahm von 0,92 auf 4,1 $\mu \mathrm{g} / \mathrm{l} \mathrm{zu}$. Co dagegen sank von 3,8 mg/l unter die Bestimmungsgrenze, und auch der Sr-Gehalt ging zurück, wie Abb. 4.18. zeigt. Ba blieb mit 24 bzw. $25 \mu \mathrm{g} / 1$ praktisch gleich (Tab. A.1.). Gleiches gilt für $\mathrm{Pb}$, während die REE-Gehalte stark zunahmen, z.B. La von 7,4 auf $46 \mu \mathrm{g} / \mathrm{l}$, Ce 2,1 auf $50 \mu \mathrm{g} / \mathrm{l}$, Pr von 0,69 auf 4,3 $\mu \mathrm{g} / \mathrm{l}$, Nd von 2,2 auf 15 $\mu \mathrm{g} / \mathrm{l}$, Sm von 0,55 auf 1,7 $\mu \mathrm{g} / \mathrm{l}$, Gd von 0,5 auf 3,2 $\mu \mathrm{g} / \mathrm{l}$, Tb von $<0,1$ auf $0,59 \mu \mathrm{g} / \mathrm{l}$, Dy von 0,44 auf $2,3 \mu \mathrm{g} / \mathrm{l}$ und Er von 0,25 auf $1,4 \mu \mathrm{g} / \mathrm{l}$.

In RCO4 entwickelten sich die Konzentrationen der einzelnen Elemente ebenfalls sehr unterschiedlich. Während die Hauptelemente ziemlich gleich blieben, stiegen die Konzentrationen von Al, Sr und U vom Oktober 1998 zum August 1999 zunächst an, sanken aber bis Januar 2000 wieder. Der Gehalt von Co stieg kontinuierlich an. Auch die Werte von $\mathrm{Pb}$ nahmen zu (Abb. 4.18.). Die REEs lagen zu allen Probenahmeterminen unterhalb der Nachweisgrenze (Tab. A.1.).

In RCO12 stieg die Al-Konzentration ebenso wie die Fe-Konzentration zunächst bis zum August 1999 an, ging dann jedoch wieder zurück. Auch der Sr-Gehalt stieg von 5076 auf $10047 \mu \mathrm{g} / \mathrm{l}$ an und fiel dann auf $4583 \mu \mathrm{g} / \mathrm{l}$. Ähnlich verlief die Entwicklung der PbKonzentration. Insgesamt blieben die meisten Elementgehalte zwischen Oktober 1998 und August 1999 in der gleichen Größenordnung und sanken dann zum Januar 2000 erheblich ab, so etwa bei Co und U (Abb. 4.18., Tab. A.1.).

In RCO15 stiegen die Konzentrationen der Alkali- und Erdalkalimetalle zwischen August 1999 und Januar 2000 stark an (Tab. A.1.). Auch die Sulfatkonzentration nahm von 0,93 auf $1,22 \mathrm{~g} / 1$ leicht zu. Bei den Übergangsmetallen war teilweise eine Zunahme zu beobachten, so bei $\mathrm{Cu}$ von 2,6 auf $20 \mu \mathrm{g} / \mathrm{l}$ und bei $\mathrm{Zn}$ von 2,6 auf $12 \mu \mathrm{g} / \mathrm{l}$. Auch der Gehalt an Sr wuchs von 3260 auf 5108 mg/l. Die Konzentrationen einiger anderer Elemente wie Al, Co und U sanken dagegen, während die Pb-Konzentration konstant blieb (Abb. 4.18.). Die REE-Werte lagen um die Nachweisgrenze (Tab. A.1.).

In RCO17 nahmen zwischen dem August 1999 und dem Januar 2000 die Werte der meisten Alkali- und Erdalkalimetalle leicht zu. Der Mn-Gehalt ging von 1025 auf 8,3 $\mu \mathrm{g} / 1$ stark 
zurïck; auch Al sank von 61 auf $24 \mu \mathrm{g} / \mathrm{l}$ und Co von 1,2 $\mu \mathrm{g} / \mathrm{l}$ unterhalb die Bestimmungsgrenze, während der Fe-Gehalt von 71 auf $126 \mu \mathrm{g} / \mathrm{l}$ anstieg. Die meisten anderen Elemente blieben mit ihren Konzentrationen in der gleichen Größenordnung. Nur die REEs stiegen deutlich an und lagen im Januar 2000 alle oberhalb der Nachweisgrenze (Tab. A.1.). Einen deutlichen Anstieg zeigten auch $\mathrm{Pb}$ und $\mathrm{U}$, während die Sr-Konzentration nur leicht zunahm (Abb. 4.18.).

In RCO18 blieb der Sulfatgehalt weitgehend gleich. Auch die Alkali- und Erdalkalielemente veränderten sich nicht erheblich. Während die Mn-Konzentration konstant blieb, sank der FeGehalt von 3230 auf $25 \mu \mathrm{g} / \mathrm{l} \mathrm{ab}$. Auch Al, die Übergangsmetalle, Sr, Pb und U nahmen in ihren Werten ab (Abb. 4.18., Tab. A.1.).

Bevor aus den dargestellten Daten Schlüsse auf die Wasserströme im Bereich Cospuden gezogen werden können, muss zunächst geklärt werden, welche Rolle die Lagerung für die Zusammensetzung der Wasserproben gespielt hat.

\subsection{Vergleich der Messungen am UFZ und aus dieser Arbeit}

Wie bereits in der Fehlerbetrachtung dargestellt, können Prozesse wie Ausfällungen und Redoxreaktionen während der Lagerung die Proben beeinflussen. Für einige Proben, die nicht direkt im Anschluss an die Entnahme in Cospuden bearbeitet und konserviert werden konnten, liegen zusätzlich zu unseren später ermittelten Daten Messungen vom UFZ vor (RICHERT, persönliche Mitteilung). Dort wurden pH und Eh während der Entnahme erfasst, außerdem wurden auch die Gehalte einiger Hauptelemente bestimmt. Bei einigen Proben ergaben sich dabei signifikante Abweichungen. Aus dieser Beobachtung lassen sich einige Schlussfolgerungen für die Bewertung der zuvor dargestellten Daten ziehen:

1) CML-Proben

Betroffen sind besonders die Proben CML 20 m vom Januar 1999 und CML 27,5 m und CML 31,5 m vom Januar 2000. In diesen drei Proben sanken die gemessenen pH-Werte während der Lagerung drastisch; in CML 20 m vom Januar 1999 wurde im UFZ ein pH-Wert von 10,8 gemessen, unsere Probe hatte einen $\mathrm{pH}-$ Wert von 3,5. In CML 27,5 m wurde ein $\mathrm{pH}-$ Wert 5,8 bzw. 3,4 gemessen und in CML 31,5 m ein pH-Wert von 5,8 bzw. 3,2. Obwohl der Sauerstoffzutritt unterbunden und die Proben dunkel und kühl gelagert wurden, kam es anscheinend zur Oxidation von $\mathrm{Fe}^{\mathrm{II}}$ zu Fe $\mathrm{Fe}^{\mathrm{III}}$. In den Probenbehältern hatten sich bis zum Zeitpunkt der Untersuchung amorphe Eisenhydroxidniederschläge gebildet. Damit einhergehend weichen auch die Werte für die Leitfähigkeit, für die Fe-Konzentration sowie für einige andere Hauptelemente in unseren Proben gegenüber denen vom UFZ nach unten 
ab. Ohne dass eine Versauerung zu beobachten war, sanken auch die Leitfähigkeit und die Eisenkonzentration in den Proben CML 39,5 m und CML 43,5 m vom August 1999 und in den Proben vom Januar 2000. Dort fielen Eisenhydroxide aus, die vorher in übersättigter Lösung oder kolloidal vorlagen. Bei den Proben vom August 1999 ist diese Beobachtung überraschend, denn sie wurden direkt nach der Entnahme vor Ort im Tagebau bearbeitet und stabilisiert. Aus dem Vergleich der Daten dieser Arbeit und des UFZ Halle-Leipzig ergibt sich der Schluss, dass sich im Bereich des CML-Brunnens in einer Tiefe von ca . 25 - $30 \mathrm{~m}$ eine Zone ausgebildet hat, in der zwar keine auffällige Versauerung zu beobachten ist, in der jedoch hohe Konzentrationen an $\mathrm{Fe}^{\mathrm{II}}$ vorliegen, die bei Sauerstoffkontakt schnell oxidieren und zur Versauerung führen.

2) Proben aus den Grundwasserpegeln (RCO)

Bei den Proben aus den Grundwasserpegeln kam es nur im Fall der Probe RCO3 vom Januar 2000 zu einer Versauerung während der Lagerung. In den Grundwasserproben vom Oktober 1998 und vom Januar 2000 bildeten sich Eisenhydroxidniederschläge, ohne das eine Veränderung des $\mathrm{pH}$-Wertes auftrat. Diese Beobachtungen müssen insbesondere in der Folge bei den Betrachtungen zur Speziation der Lösungen berücksichtigt werden.

Die Beobachtungen von NITSCHE et al. (1999) werden durch unsere Messungen bestätigt. Der Pegel RCO17 zeigt leichte Anzeichen einer sich nähernden Versauerungsfront; dafür sprechen der etwas gestiegene Sulfat-Gehalt, der schwach abgesunkene pH-Wert und die Zunahme der Konzentrationen an $\mathrm{Ca}$ und $\mathrm{Na}$. Auch der Fe-Gehalt stieg leicht an, und die REE-Konzentrationen wuchsen deutlich. Für die übrigen Grundwasserpegel stellten NITSCHE et al. (1999) keine deutlichen Veränderungen zwischen 1995 und 1999 fest.

Insgesamt erscheint die Entwicklung der pH-Werte und der Gesamtionenfrachten in den Wasserproben aus den Pegeln selbst im Kippenbereich beruhigend. Entwarnung kann trotzdem nicht gegeben werden, denn die Untersuchungen an CML zeigen, dass es in der Kippe weiterhin eine Oxidationszone mit niedrigem $\mathrm{pH}-$ Wert gibt. Aus Pyrit und anderen Mineralphasen werden so auch in Zukunft noch Haupt- und Spurenelemente in beträchtlichem Umfang freigesetzt werden, die den experimentellen Ergebnissen zufolge auch bei einem Anstieg der pH-Werte nicht vollständig aus den Wässern entfernt werden. Ob die so belasteten Wässer eine Gefahr für Grund- und Oberflächenwässer der Umgebung darstellen, hängt zum einen von den komplizierten hydrogeologischen Gegebenheiten ab; andererseits ist dafür auch die Mobilität der untersuchten Elemente ausschlaggebend, über die Untersuchungen zur Elementspeziation Aufschluss geben, die in den folgenden Kapiteln dargestellt werden. 


\subsection{Tiefenprofile}

\subsubsection{Cospuden}

Anhand des Materials aus drei Bohrungen konnten Tiefenprofile sowohl der Porenwässer als auch der Festphasen erstellt werden. Die Bohrung RCO4 stammt aus dem Sommer 1995; der Multilevelbrunnen CML wurde im August 1998 angelegt. Der oberflächennahe Bohrkern wurde im November 1999 entnommen.

\subsubsection{Bohrkern des Grundwasserpegels RCO4}

Bei der Bohrung zur Anlage des Grundwasserpegels RCO4 im November 1995 wurde Material aus 25 verschiedenen Tiefen gewonnen, wobei die Proben von 5 - 8 m und von $11-$ $19 \mathrm{~m}$ zur Untersuchung der Porenwässer zusammengefasst wurden, um ausreichende Probenmengen zu gewinnen. Mit 58 m Tiefe reicht die Bohrung bis zur Basis der Kippe. 20 Proben wurden hinsichtlich der Zusammensetzung ihrer Porenwässer untersucht; von 25 Proben liegt eine eine Analyse der chemischen Zusammensetzung der Festphasen vor. Im Rahmen der vorliegenden Arbeit wurden Porenlösungen aus den Tiefen 17 m, 20 m, 22 m, 26 m, 36 m, 44 m, $48 \mathrm{~m}$ und $52 \mathrm{~m}$ analysiert, um das Porenwasserprofil von PLEßOW (1998) zu ergänzen. Die Festphasenanalysen wurden im Rahmen der vorliegenden Arbeit erstellt.

Die Bohrung weist hinsichtlich der Mineralogie ihrer Festphasen kein ausgeprägtes Tiefenprofil auf. Die Festphasen sind vom Quarz geprägt; Gips, Calcit und Dolomit treten überwiegend als akzessorische Mineralphasen bis in Tiefen von rund $20 \mathrm{~m}$ und mehr auf. Parallel mit der Tiefe steigen die Konzentrationen von Carbonatkohlenstoff, $\mathrm{CaO}$ und $\mathrm{MgO}$ an. Eine generelle Zunahme ist mit der Tiefe auch für $\mathrm{SO}_{3} \mathrm{zu}$ beobachten. Gips, Calcit und Dolomit können in der unteren Hälfte der Kippe deutlich über $1 \%$ liegen und sind hier Nebengemengeteile (Abb. 4.19. und 4.20.). 


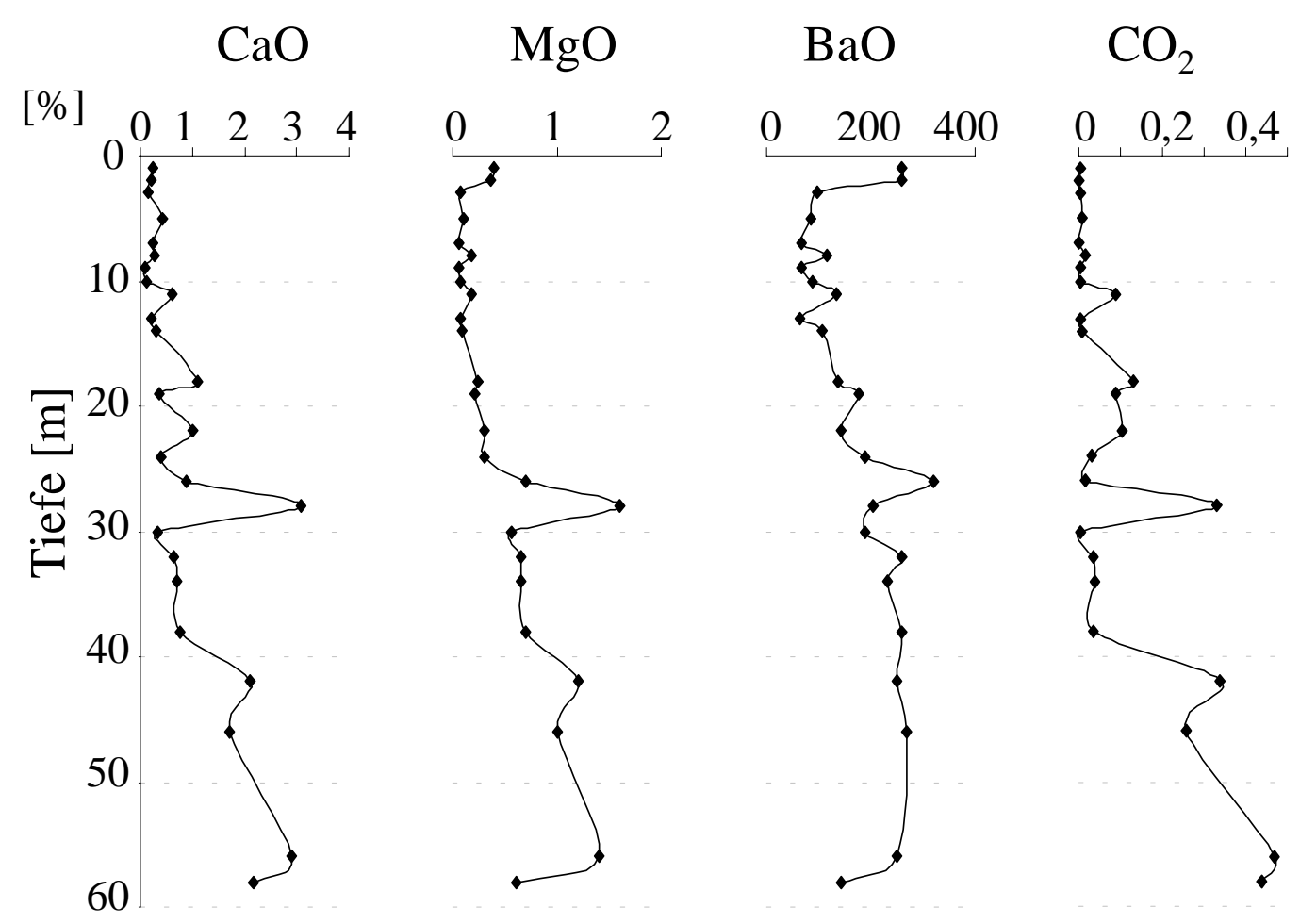

Abb. 4.19.: Tiefenprofil der Gehalte (in Massen-\%) an Calciumoxid, Magnesiumoxid, Bariumoxid und Karbonat-Kohlenstoff $\left(\right.$ als $\left.\mathrm{CO}_{2}\right)$ in den Festphasen des Bohrkerns RCO4.

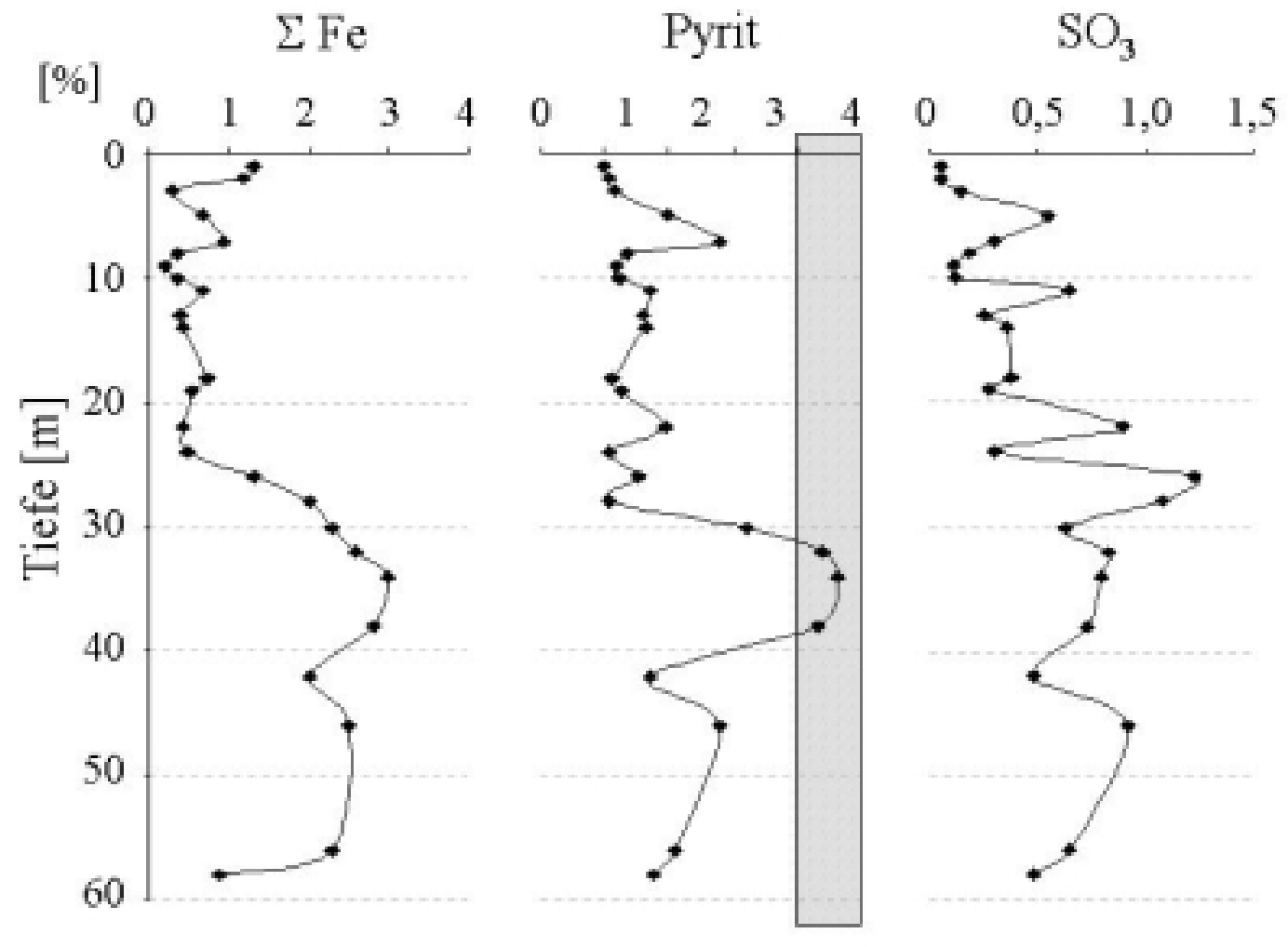

$\mathrm{SO}_{3}$

Abb. 4.20.: Tiefenprofil der Gehalte (in Massen-\%) an Gesamteisen, Pyrit und Sulfat-Schwefel als in den Festphasen des Bohrkerns RCO4; grau unterlegt: ursprünglicher durchschnittlicher Pyritgehalt nach KITTSTEINER (1998) bzw. BELLMANN et al (1984). 
Die Pyritgehalte erreichen ihr Maximum in der Tiefe zwischen 32 und $38 \mathrm{~m}$ mit bis zu 3,6 \%. Der Sulfatschwefelgehalt in der Festphase beträgt maximal 0,5\%. Gegenüber den Pyritgehalten der unverritzten Sedimente von 2 - 8 Massen-\% ist eine deutliche Abnahme festzustellen, ohne dass der Verbleib der Pyrite oder ihrer Oxidationsprodukte derzeit festgestellt werden kann.

Die Spurenelementkonzentrationen in den Sedimenten liegen in der Regel im Bereich der Spurenelementgehalte der mittleren kontinentalen Oberkruste (Abb. 4.21.). So wurden in den Bohrkernproben nur 1-9 ppm $\mathrm{Cu}$, 4-46 ppm $\mathrm{Zn}$ und 1-15 ppm Pb gefunden. Insgesamt lagen die Konzentrationen an Spurenmetallen in den bisher untersuchten Sedimenten aus dem Tagebauvorfeld deutlich höher als in den Festphasen dieses Tiefenprofils. Die Sedimente in der Halde stellen damit kein auffälliges Spurenelementreservoir dar. Die meisten der untersuchten Spurenelemente zeigen ein deutliches Tiefenprofil, das jedoch nicht für alle Elemente gleich ist. Bei $\mathrm{Cr}, \mathrm{Co}, \mathrm{Ni}, \mathrm{Cu}, \mathrm{Zn}, \mathrm{Rb}$ und $\mathrm{Sr}$ fällt die Konzentration von der Oberfläche bis in etwa 20 m Tiefe etwas ab und erreicht dann bei $28 \mathrm{~m}$ Tiefe ein bei Cr, Co, $\mathrm{Ni}, \mathrm{Cu}$ und $\mathrm{Zn}$ sehr ausgeprägtes Maximum. Ein weiteres Maximum liegt bei diesen Elementen zwischen 40 und 50 m Tiefe vor, während die Konzentrationen in 58 m Tiefe stark zurückgehen. Anders liegen die Verhältnisse bei $\mathrm{Zr}, \mathrm{Nb}$ und $\mathrm{Pb}$, die in $28 \mathrm{~m}$ Tiefe ein lokales Minimum aufweisen und nur bei ca. $18 \mathrm{~m}$ ein schwaches Maximum zeigen. Einige Elemente weisen im Tiefenprofil ein deutliches Maximum zwischen 25 und 30 m Tiefe auf. Dort liegen auch bei einigen der untersuchten Festphasen Maxima. Befindet sich dort eine Oxidationszone, so ist es dort vermutlich zu Auflösungen und Ausfällungen von Elementen gekommen. Im Zuge dessen kann es zur Mitfällung oder zur Adsorption von gelösten Spurenelementen an Mineraloberflächen - z.B. unverwittertem Pyrit oder ausgefallenem Gips - und damit zu einer Anreicherung gegenüber den umgebenden Festphasen gekommen sein. $\mathrm{Ob}$ sich in dieser Tiefe eine Oxidationszone befindet, darüber kann die Analyse der Porenwässer Aufschluss geben. 

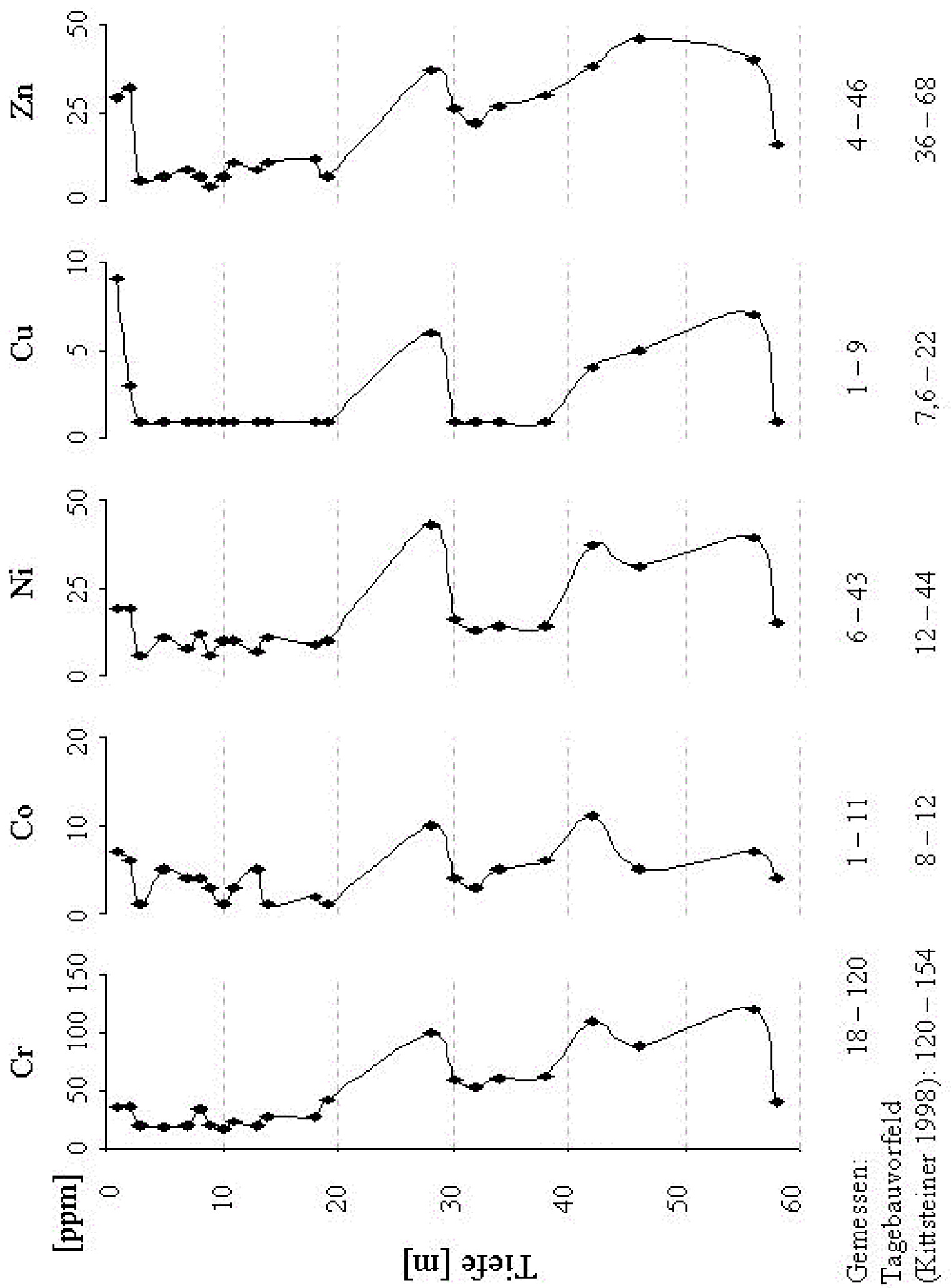

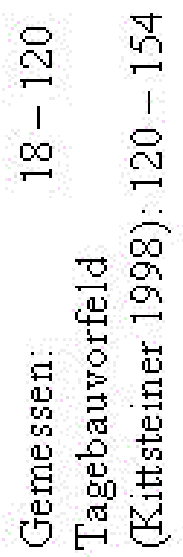

Abb. 4.21.: Tiefenprofile von Spurenelementgehalten der Festphasen des Bohrkerns RCO4. 

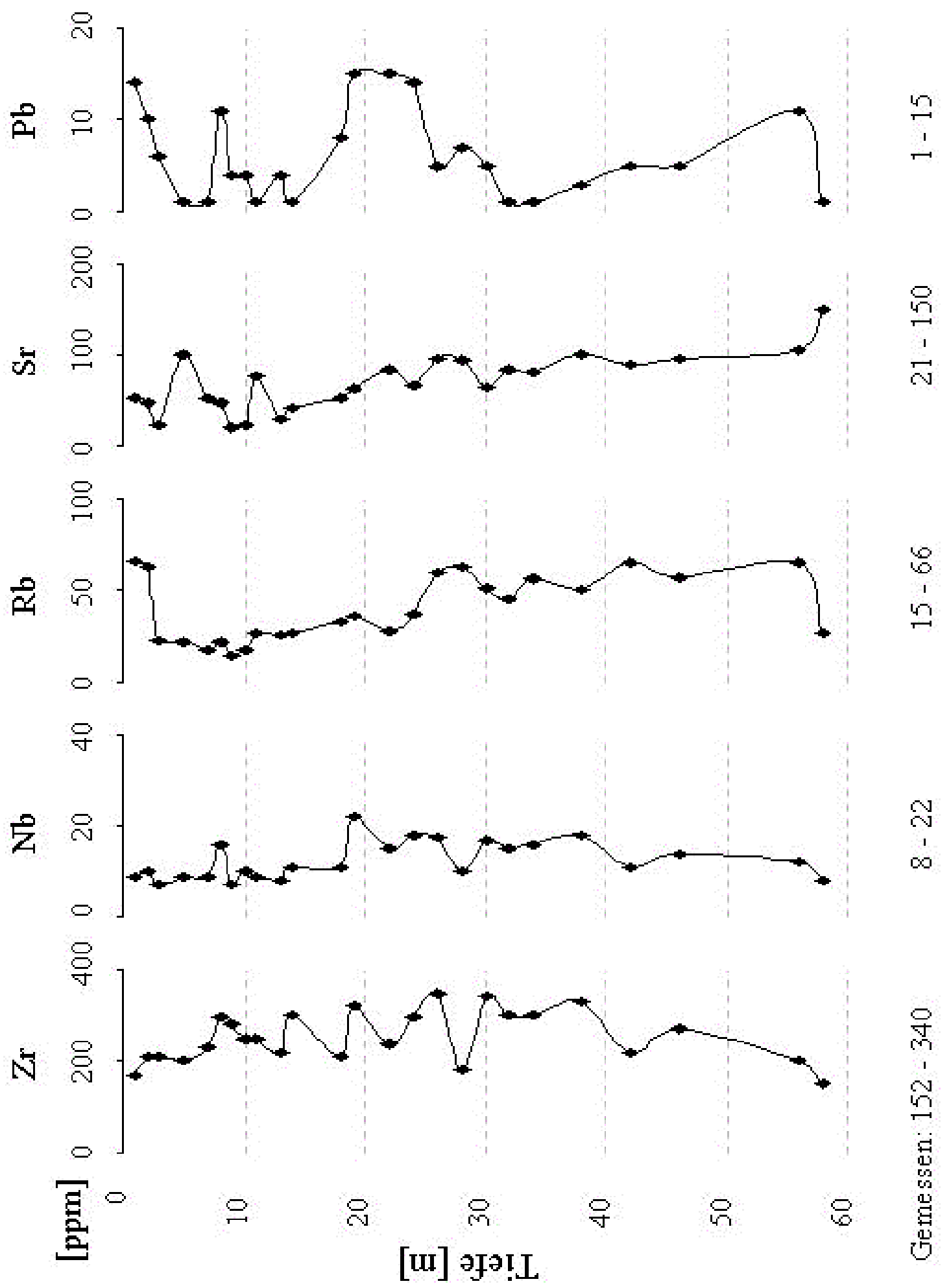

Abb. 4.21. (Forts.): Tiefenprofile von Spurenelementgehalten der Festphasen des Bohrkerns RCO4. 


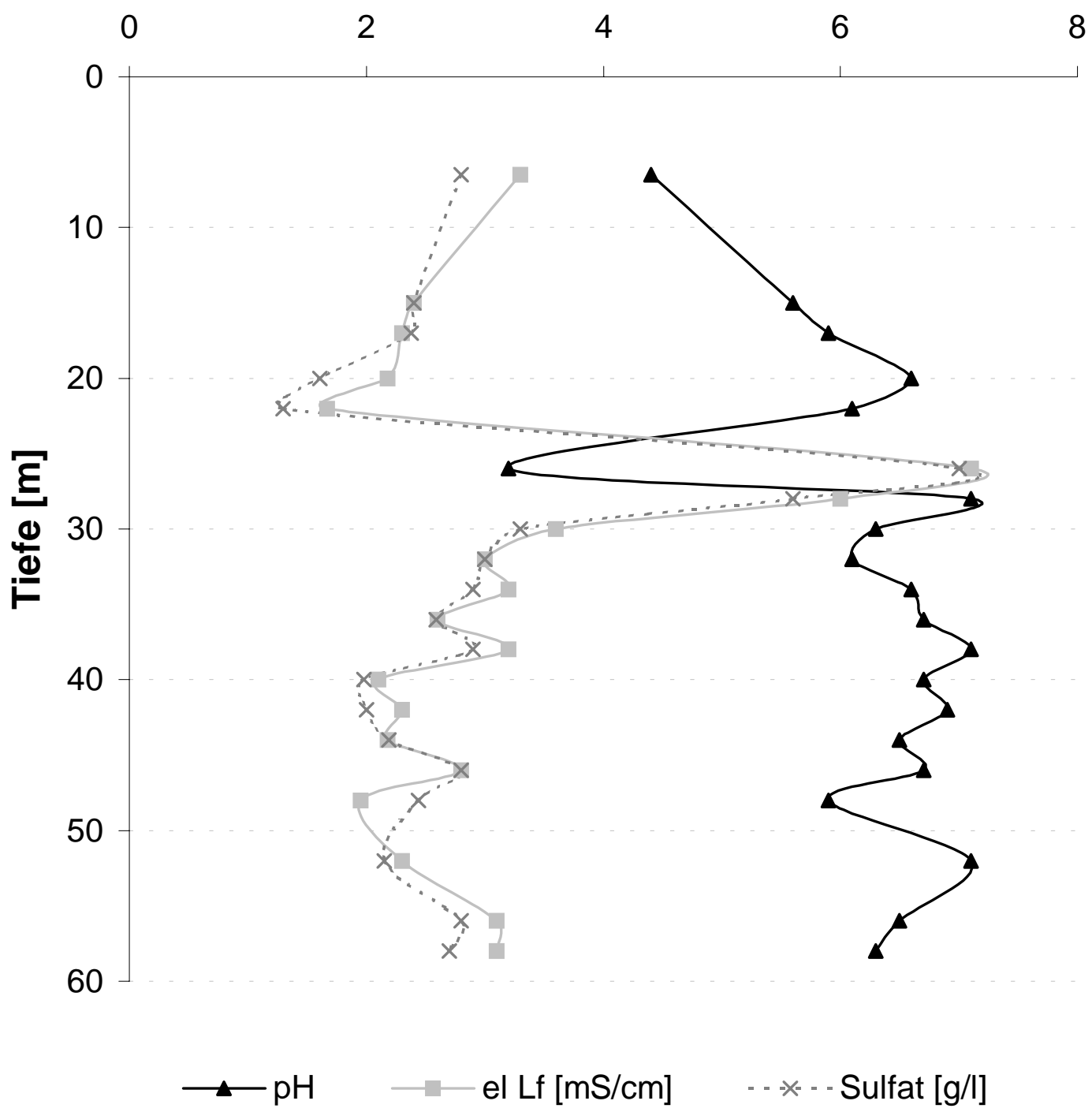

Abb. 4.22.: Tiefenprofil von pH, elektrischer Leitfähigkeit und Sulfatkonzentration in Porenwässern des Bohrkerns RCO4.

Abb. 4.22. zeigt den Verlauf von $\mathrm{pH}$, elektrischer Leitfähigkeit und Sulfatkonzentration in den Porenwässern des Bohrkerns RCO4. Deutlich weist der Kurvenverlauf in einer Tiefe von $26 \mathrm{~m}$ eine Zone mit niedrigem $\mathrm{pH}$ und hohen Konzentrationen von Ionen auf, was sich in der stark erhöhten Leitfähigkeit zeigt.

Ebenso wie für Sulfat, das in dieser Tiefe eine Konzentration von $7 \mathrm{~g} / \mathrm{l}$ erreicht, liegt für zahlreiche Elemente in den Porenwässern das Konzentrationsmaximum in dieser Tiefe. Das gilt z.B. für Li $(1170 \mu \mathrm{g} / \mathrm{l})$, Ni $(3510 \mu \mathrm{g} / \mathrm{l}), \mathrm{Cu}(128 \mu \mathrm{g} / \mathrm{l}), \mathrm{U}(28 \mu \mathrm{g} / \mathrm{l})$, die REEs $(4760 \mu \mathrm{g} / \mathrm{l})$, Zn $(12215 \mu \mathrm{g} / \mathrm{l})$, Co $(1310 \mu \mathrm{g} / \mathrm{l}), \mathrm{Pb}(5,6 \mu \mathrm{g} / \mathrm{l}), \mathrm{Cd}(41 \mu \mathrm{g} / \mathrm{l})$, As $(141 \mu \mathrm{g} / \mathrm{l})$ und $\mathrm{Cr}(49 \mu \mathrm{g} / \mathrm{l})$. Abb. 4.23. zeigt beispielhaft die Tiefenprofile für diese Elemente; die Daten weiterer Elemente sind im Anhang in Tab. A.1. aufgeführt. Auch Eisen ist in 26 m Tiefe am stärksten 
konzentriert (876 mg/l); dicht unter der Oberfläche $(10 \mathrm{mg} / \mathrm{l})$ sowie in $48 \mathrm{~m}$ Tiefe $(21 \mathrm{mg} / \mathrm{l})$ liegen auffällige lokale Maxima vor. Für $\mathrm{Sb}$ und Mo, die nach der Arbeit von KITTSTEINER (1998) in den unverritzten Sedimenten aus Zwenkau zu großen Teilen pyritgebunden vorlagen, findet sich in $26 \mathrm{~m}$ Tiefe jedoch kein Maximum, sondern sogar ein lokales Minimum. Hier liegen die Maximalkonzentrationen erst in Tiefen von $36 \mathrm{~m}$ (Mo, $28 \mu \mathrm{g} / \mathrm{l})$ bzw. $42 \mathrm{~m}(\mathrm{Sb}, 13 \mu \mathrm{g} / \mathrm{l})$ vor. Sieht man von den Extremwerten in $26 \mathrm{~m}$ Tiefe ab, so liegen die Gehalte für Fe, Al, Cu, Ba, Y, La und REE in den Porenwässern des Bohrkerns in den übrigen Tiefen im Rahmen der Werte für unbelastete Leipziger Grundwässer oder unterschreiten sie sogar. Die Konzentrationen von $\mathrm{Li}, \mathrm{V}, \mathrm{Cr}, \mathrm{As}, \mathrm{Mn}, \mathrm{Co}, \mathrm{Ni}, \mathrm{Rb}, \mathrm{Sr}$, Mo und $\mathrm{Sb}$ sind dagegen deutlich höher als im unbelasteten Grundwasser.

Betrachtet man das Bild der Tiefenprofile für Porenwässer des Bohrkerns RCO4, so kann man folgende Feststellungen treffen: In einer Tiefe von etwa $26 \mathrm{~m}$ befindet sich eine ausgeprägte Oxidationszone, geprägt durch niedrigen $\mathrm{pH}$ und hohe Elementkonzentrationen. Diese entstehen wahrscheinlich durch die Verwitterung durch Pyrit; die dadurch versauerten Wässer greifen auch andere Mineralphasen an und führen so $\mathrm{zu}$ einer deutlichen Anhebung zahlreicher Elementkonzentrationen. Die signifikant pyritgebundenen Elemente Sb und Mo erreichen entgegen den Erwartungen ihre Maximalkonzentrationen erst in größeren Tiefen; entweder gibt es dort noch wesentlichere Quellen für $\mathrm{Sb}$ und Mo als die Verwitterung von Pyrit, oder sie werden in der Oxidationszone sofort nach der Freisetzung adsorptiv oder durch Sekundärmineralbildung gebunden. Das lokale Maximum von Gips weist darauf hin, dass es in $28 \mathrm{~m}$ Tiefe bereits zu einer Ausfällung von Sekundärphasen gekommen ist. Einige Spurenelemente weisen dort ebenfalls Maxima auf, die darauf zurückgehen können, dass sie an die Gipsoberfläche adsorbiert und damit gegenüber den umgebenden Festphasen angereichert wurden. Gips kann außerdem erhebliche Verunreinigungen von Fe und vielen Spurenelementen aufweisen. Das Auftreten von geringen Mengen an Fe-Sulfaten, die unterhalb der Nachweisgrenze der RDA liegen, wäre ebenfalls denkbar. 

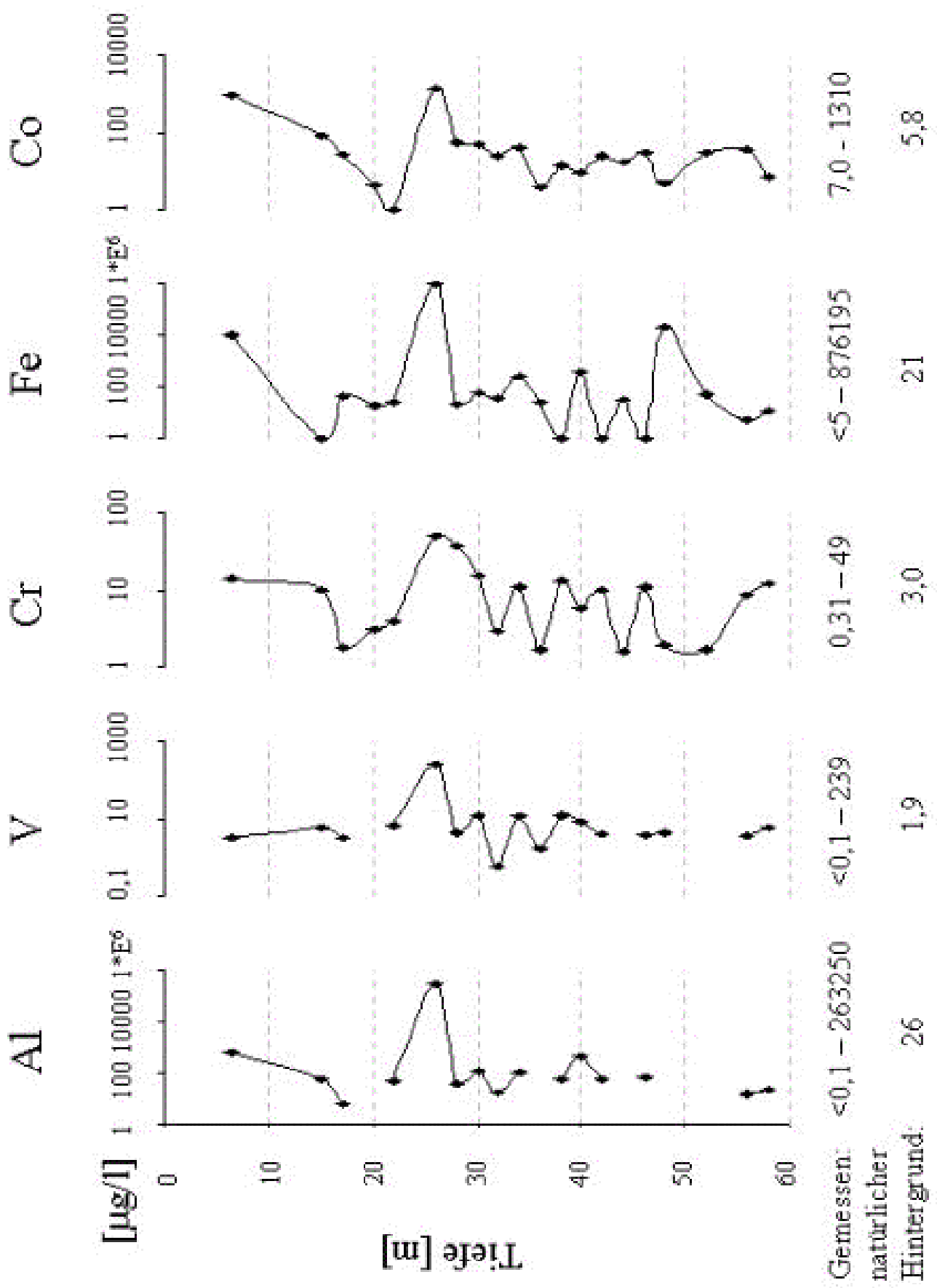


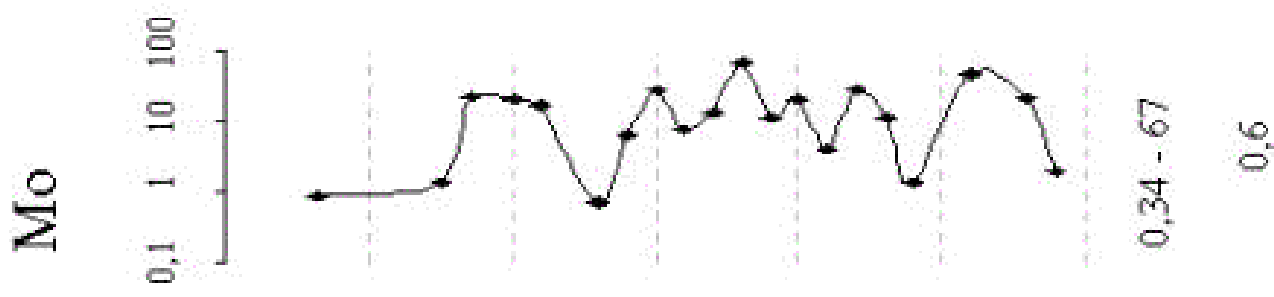
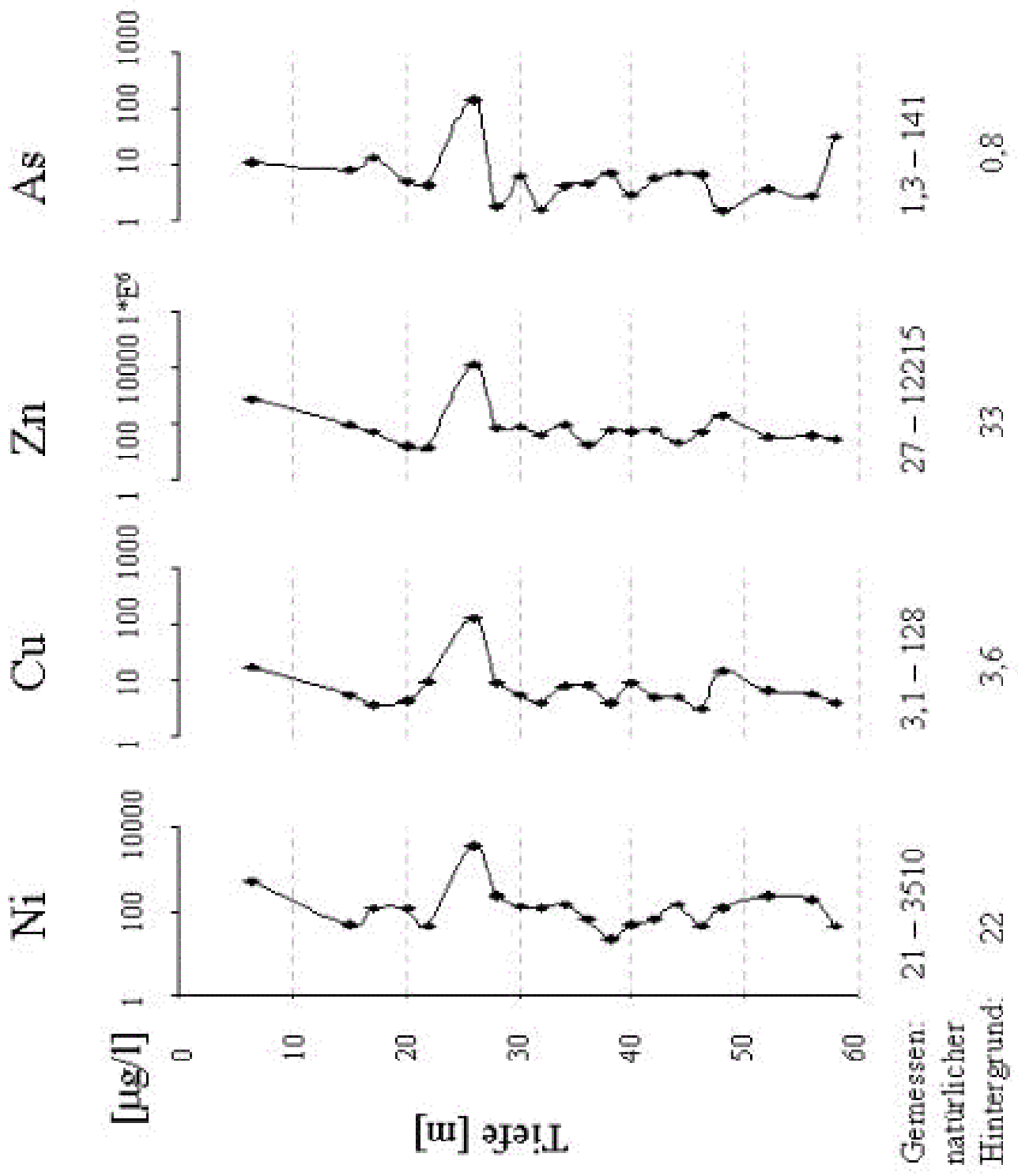

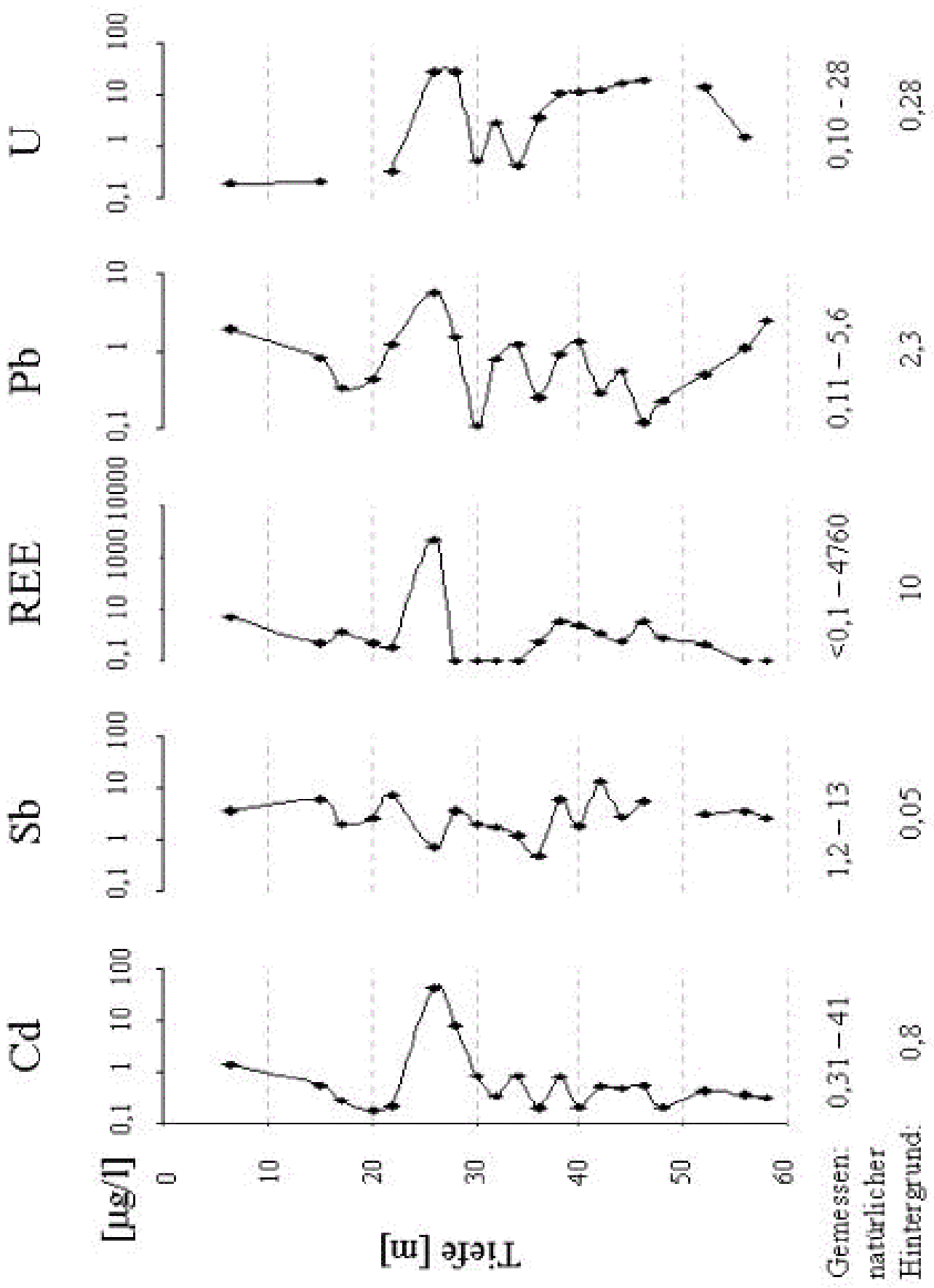


\subsubsection{Multilevelbrunnen CML}

Aus dem drei Jahre nach RCO4 angelegten Multilevelbrunnen CML wurde ebenfalls Bohrkernmaterial zur Untersuchung gewonnen. Dabei wurden 5 Proben zwischen 24 und 38 m Tiefe analysiert; die Untersuchungen der Sicker- und Porenwässer wurden im Rahmen dieser Arbeit vorgenommen. Die Analysen hinsichtlich des Gehalts an verschiedenen mineralischen Festphasen und ihrer elementaren Zusammensetzung stammen von BRINKMANN (2000). Abb. 4.24. und 4.25. zeigen den Verlauf der Kurven für Gips, Calcit, Pyrit und den Sulfatgehalt in den Festphasen.

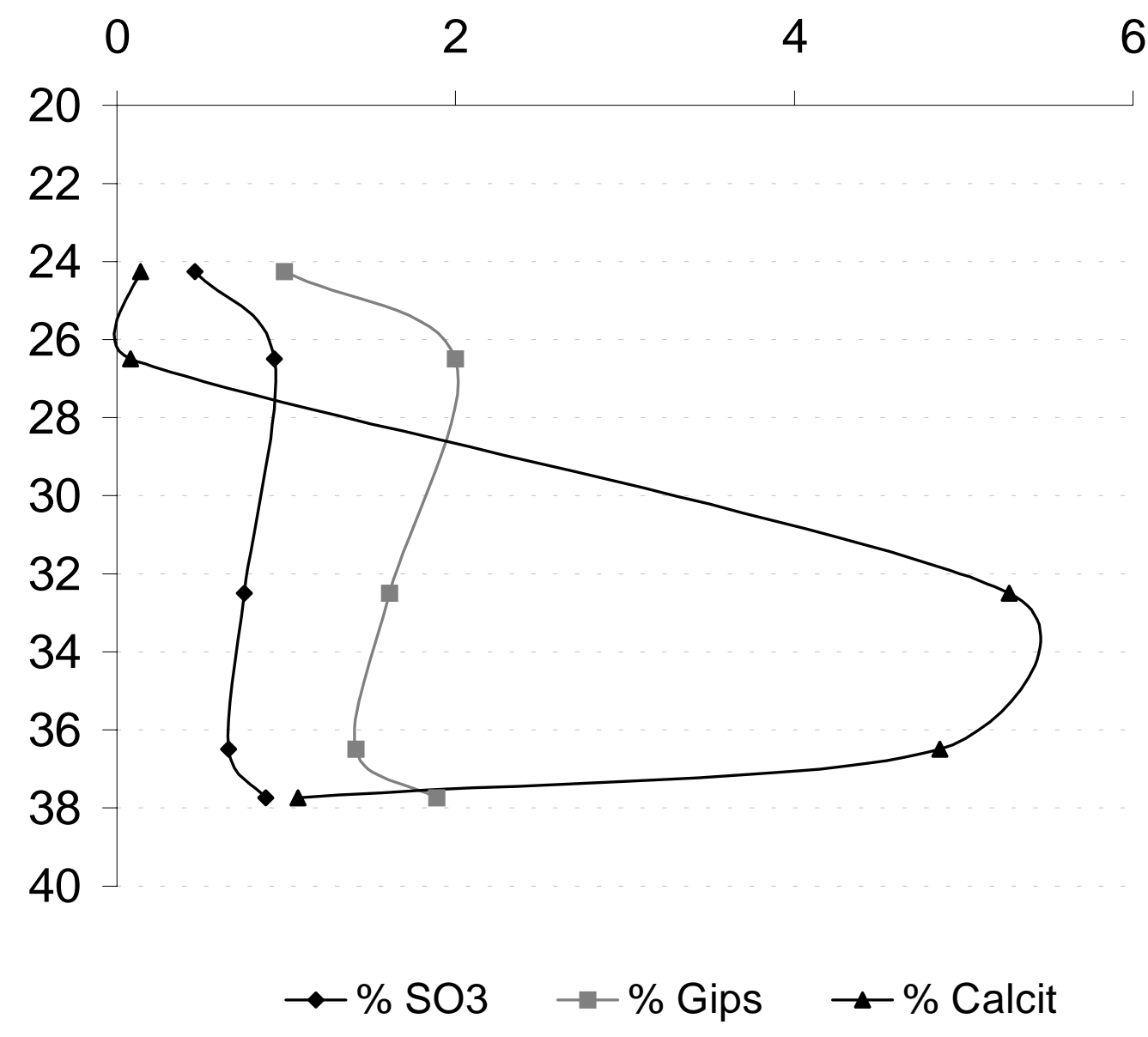

Abb. 4.24.: Tiefenprofil der Gehalte an Sulfat-Schwefel, Gips und Calcit in den Festphasen im Bohrkern des Multilevelbrunnens CML. 


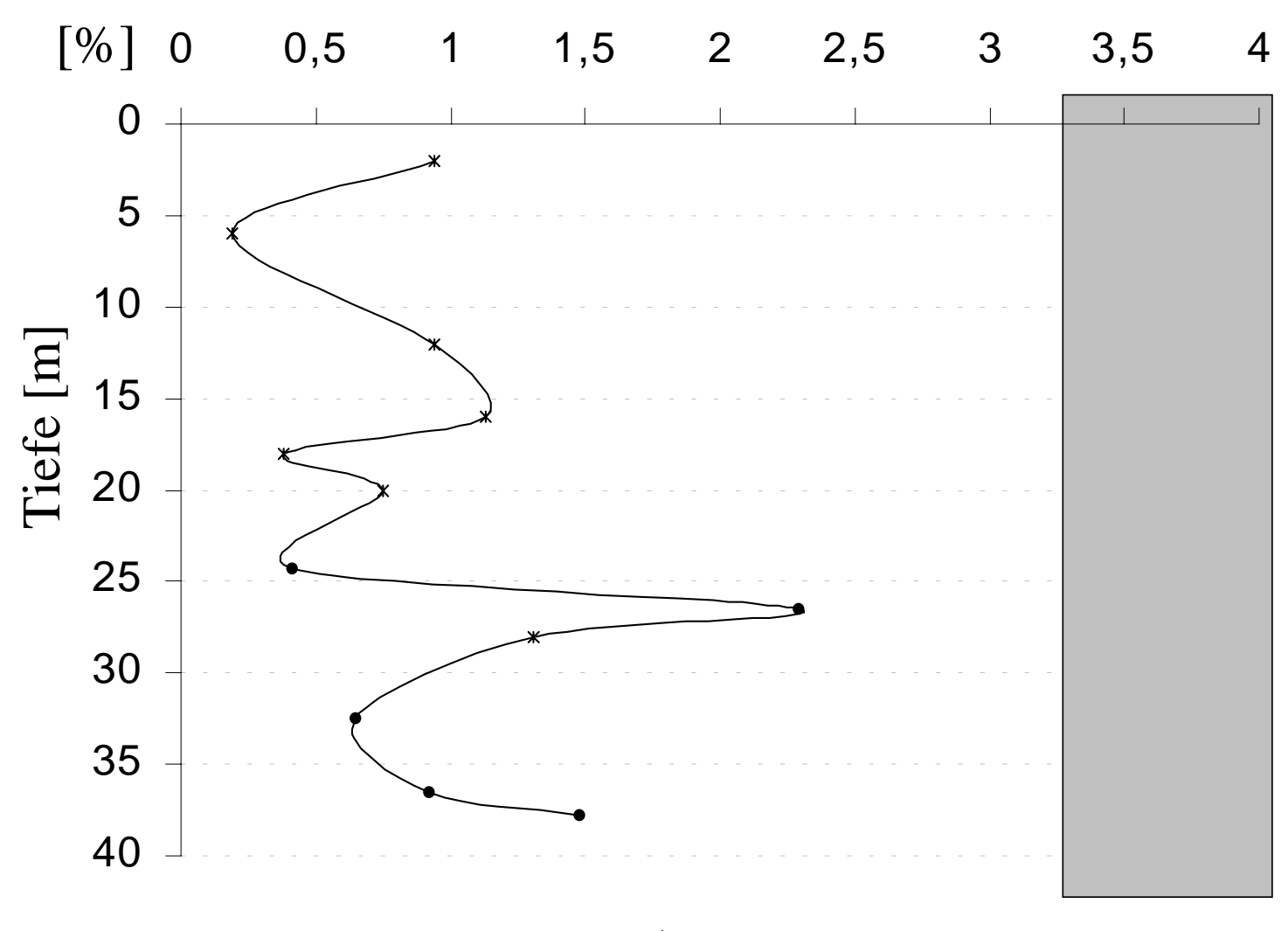

- Werte aus Brinkmann 2000 * Werte aus Nitzsche et al. 1999

Abb. 4.25.: Pyritgehalte in Sedimentproben aus CML und SGM; graue Markierung: Pyritgehalte in den unverritzten Sedimenten nach KITTSTEINER (1998) und BELLMANN et al. (1984).

In der Probe aus 26 - 27 m liegen die höchsten Pyritgehalte des Profils mit 2,3\% vor. Gleichzeitig findet sich dort auch ein leichtes Maximum des Gipsgehaltes mit $2 \%$. Calcit ist dagegen in diesem Bereich nur mit 0,08\% vertreten, steigt aber in den tieferen Proben auf Werte um $5 \%$ an. Davon abgesehen zeigen die Proben keine Auffälligkeiten: Sie sind von Quarz dominiert und haben $\mathrm{SiO}_{2}$-Gehalte zwischen 72 - $92 \%$, während alle anderen Hauptelemente (als Oxide berechnet) Gehalte unter $8 \%$ aufweisen (BRINKMANN 2000). Im Bereich zwischen 32 und 33 m sowie zwischen 36 und 37 m liegen erhöhte Werte von $\mathrm{MgO}$, $\mathrm{CaO}$ und $\mathrm{CO}_{2}$ vor, die auf einen relativ hohen Carbonatgehalt in diesen Tiefen hinweisen. Im gleichen Tiefenabschnitt ist auch $\mathrm{K}_{2} \mathrm{O}$ etwas angereichert, ein Indiz für einen höheren IllitGehalt. 


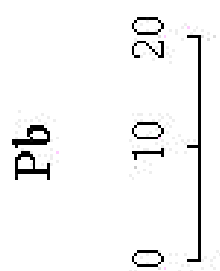

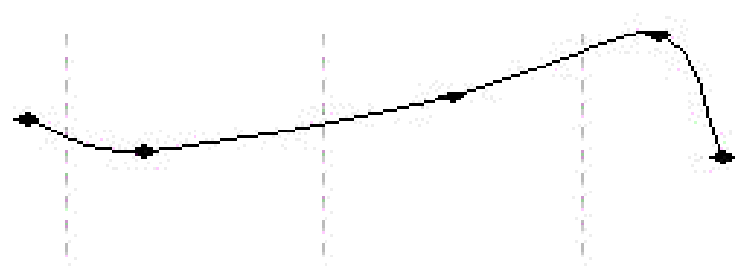

只 志

$\begin{array}{cc}1 & 1 \\ \infty & \infty\end{array}$

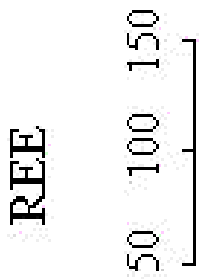

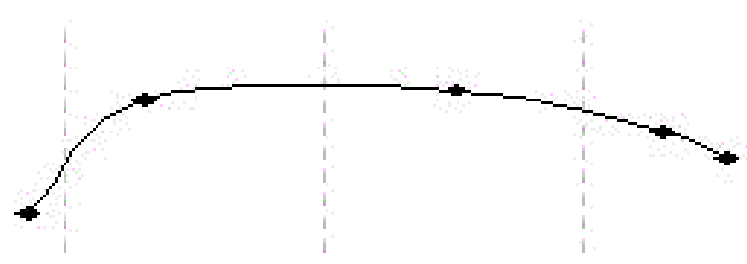

\begin{tabular}{ll}
$\stackrel{0}{\sigma}$ & \multicolumn{2}{c}{} \\
1 & 1 \\
$\infty$ & 6
\end{tabular}

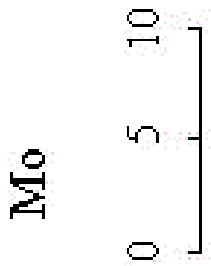

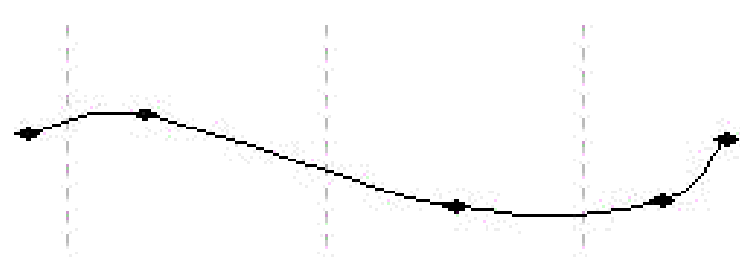

$\cdots m$

i 1

$\stackrel{\square}{\circ}$

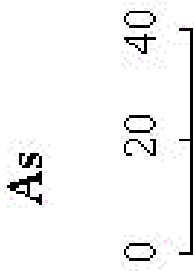

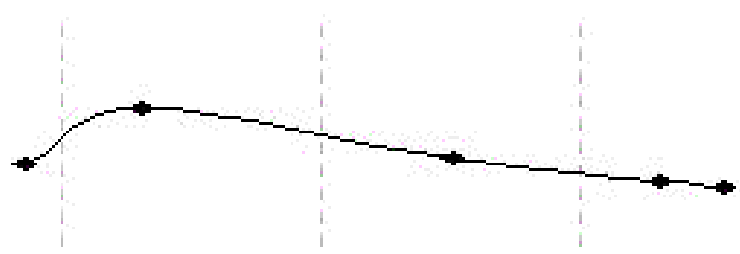

$\begin{array}{cc}\infty & m \\ 0 & m \\ 1 & \cdots \\ 0 & \end{array}$

$\left.\begin{array}{cc} & \circ \\ 0 & \wp \\ 0 & \\ & \circ\end{array}\right]$

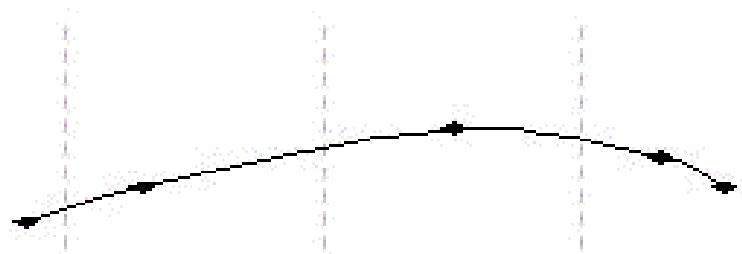

$\stackrel{\varrho}{\varrho}$

m 1

m

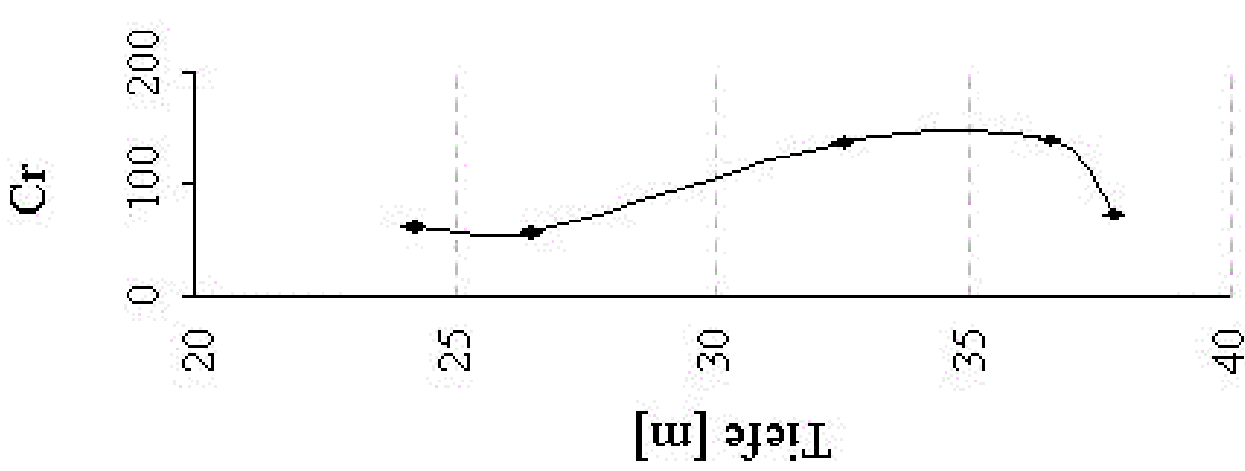

蒫

$\stackrel{\circ}{\stackrel{9}{\Xi}}$

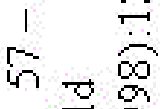

造

- $\stackrel{5}{\circ}$

范䒠

要

岁焉

获田

Abb. 4.26.: Tiefenprofile einiger Spurenelemente in den Festphasen des Bohrkerns aus CML. 
Abb. 4.26. zeigt exemplarisch die Kurvenverläufe einiger Spurenelemente in den Festphasen des Bohrkerns von CML. Ausgeprägte Änderungen über die Tiefenprofile sind nicht erkennbar. Die Spurenelementgehalte liegen im Bereich der Werte, die im Tagebauvorfeld gefunden wurden. Nur Mo scheint gegenüber dem unverritzten Material abgereichert zu sein. 7 Proben aus Tiefen zwischen 21 und 40 m wurden im Rahmen der vorliegenden Arbeit hinsichtlich der Zusammensetzung ihrer Porenwässer untersucht. Abb. 4.27. zeigt die Tiefenprofile für den pH-Wert und die elektrische Leitfähigkeit. Deutlich ist auch hier in $26 \mathrm{~m}$ Tiefe eine Zone mit niedrigem pH $(2,9)$ und hoher Leitfähigkeit $(8,3 \mathrm{mS} / \mathrm{cm})$ zu erkennen.

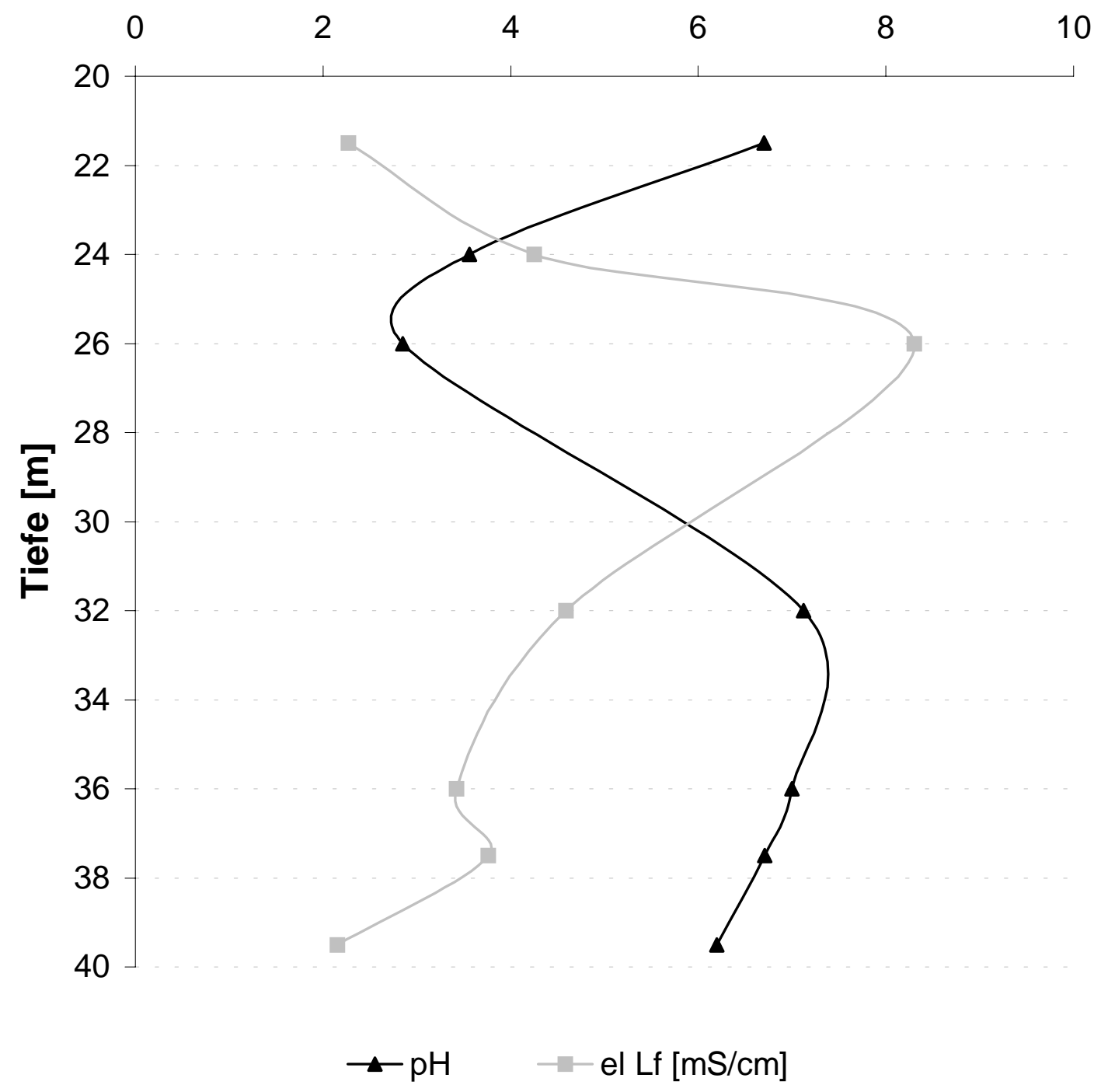

Abb. 4.27.: Tiefenprofil von pH-Wert und elektrischer Leitfähigkeit in den Porenwässern des Bohrkerns CML.

In Abb. 4.28. sind die Profile einiger Haupt- und Spurenelemente in Sickerwässern des Bohrkerns CML dargestellt. Für Be, Al, V, Cr, Ni, Zn, As, Sr und Y ist ein deutliches Maximum im Bereich von 24 - $26 \mathrm{~m}$ festzustellen. Fe und Co weisen im gleichen Tiefenbereich ein leichtes Maximum auf, wobei in der Abbildung die logarithmische 
Auftragung zu berücksichtigen ist. Ba dagegen hat ein Minimum bei $26 \mathrm{~m}$. Das Maximum von Mo liegt dagegen deutlich unterhalb dieses Bereichs, nämlich bei $36 \mathrm{~m}$ (in der Abbildung nicht dargestellt, s. Tab. A.1. im Anhang). Berücksichtigt man die geringen Werte in der Festphase gegenüber den Gehalten im Tagebauvorfeld, scheint es, dass Mo mit dem Sickerwasser aus der Oxidationszone in tiefere Schichten ausgetragen wurde.

Insgesamt lässt sich feststellen, dass ebenso wie bei der drei Jahre früher gewonnenen Probe RCO4 auch im Bohrkern von CML in $24-26$ m eine Oxidationszone mit niedrigem pH und hohen Konzentrationen von gelösten Elementen vorliegt. Gleichzeitig findet sich hier in der Festphase von CML auch der höchste Pyritanteil, während im Bohrkern RCO4 das Maximum des Pyritgehalts erst bei 36 m Tiefe liegt. Das Maximum für Sulfat in der Festphase liegt bei RCO4 in 30 m Tiefe, im Bohrkern von CML lag dagegen ein leichtes Maximum bei etwa 26 $\mathrm{m}$ Tiefe. Im Bohrkern RCO4 scheinen viele Spurenelemente im Zuge der Pyritoxidation in der Tiefe um 26 m Tiefe freigesetzt und dann in tiefere Schichten ausgetragen worden zu sein; in den Wässern dieses Bohrkerns dagegen sind die meisten Elemente in 26 m Tiefe, dort, wo auch der $\mathrm{pH}-$ Wert am niedrigsten ist, stark angereichert. Dort liegen auch im Bohrkern CML der niedrigste $\mathrm{pH}-$ Wert und gleichzeitig eine stark erhöhte Leitfähigkeit. Im Bohrkern CML sind in den Festphasen in 26 m Tiefe die Elemente As, Mo, die REEs und U angereichert; man kann vermuten, dass das durch die Pyritoxidation freigesetzte Sulfat dort teilweise in Form von Gips und Bariumsulfat ausfällt. Die meisten anderen Elemente sind eher abgereichert; sie wurden vermutlich durch die versauerten Wässer in tiefer gelegene Schichten transportiert. Im übrigen aber zeigen sich die gleichen Hinweise für das Vorliegen einer Pyritverwitterungszone wie im Bohrkern RCO4. Die Oxidation scheint auch im Jahr 1998 noch unvermindert anzudauern. 

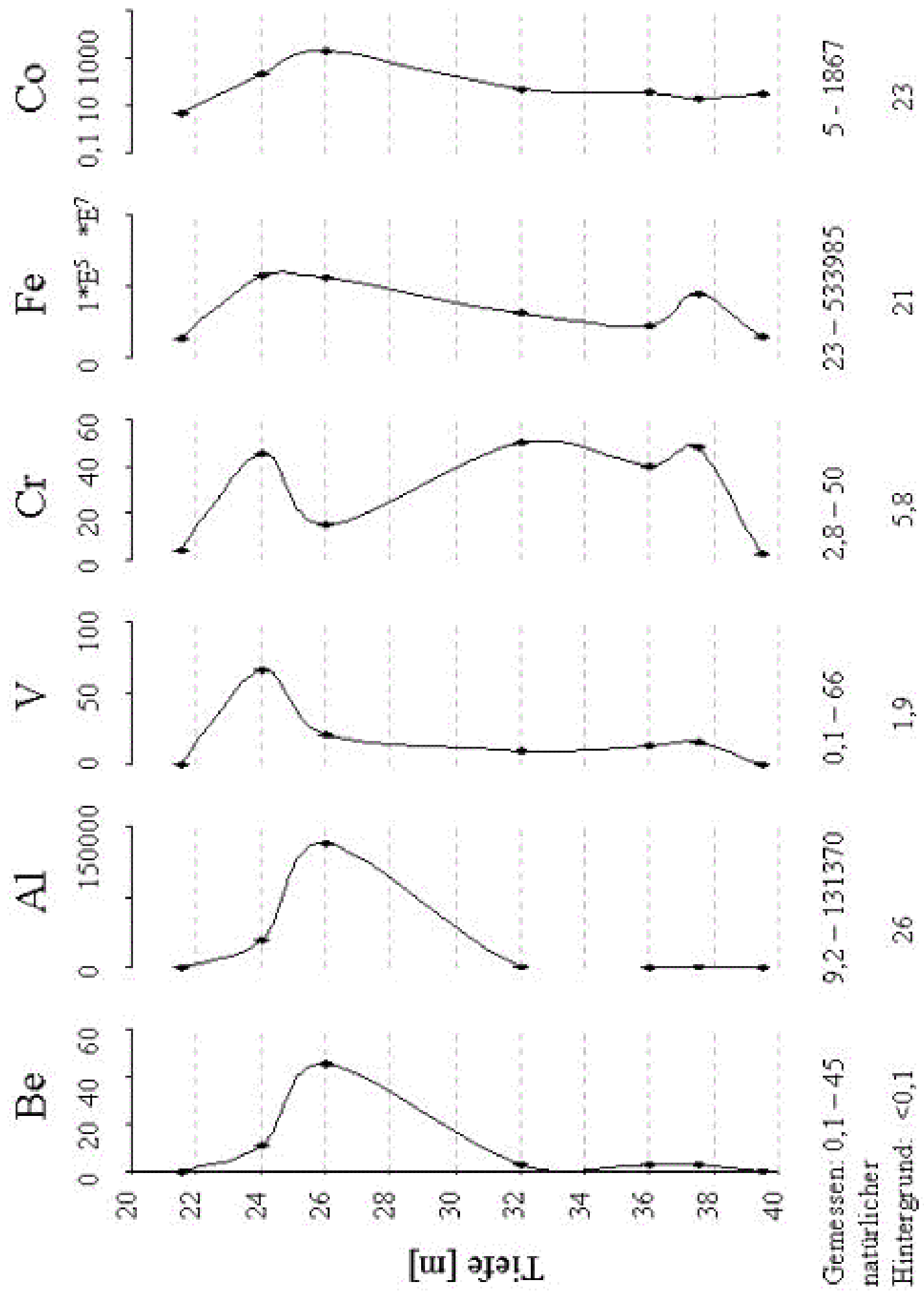

Abb. 4.28.: Tiefenprofile von Elementkonzentrationen in Porenwässern des Bohrkerns des Multilevelbrunnens $C M L$. 

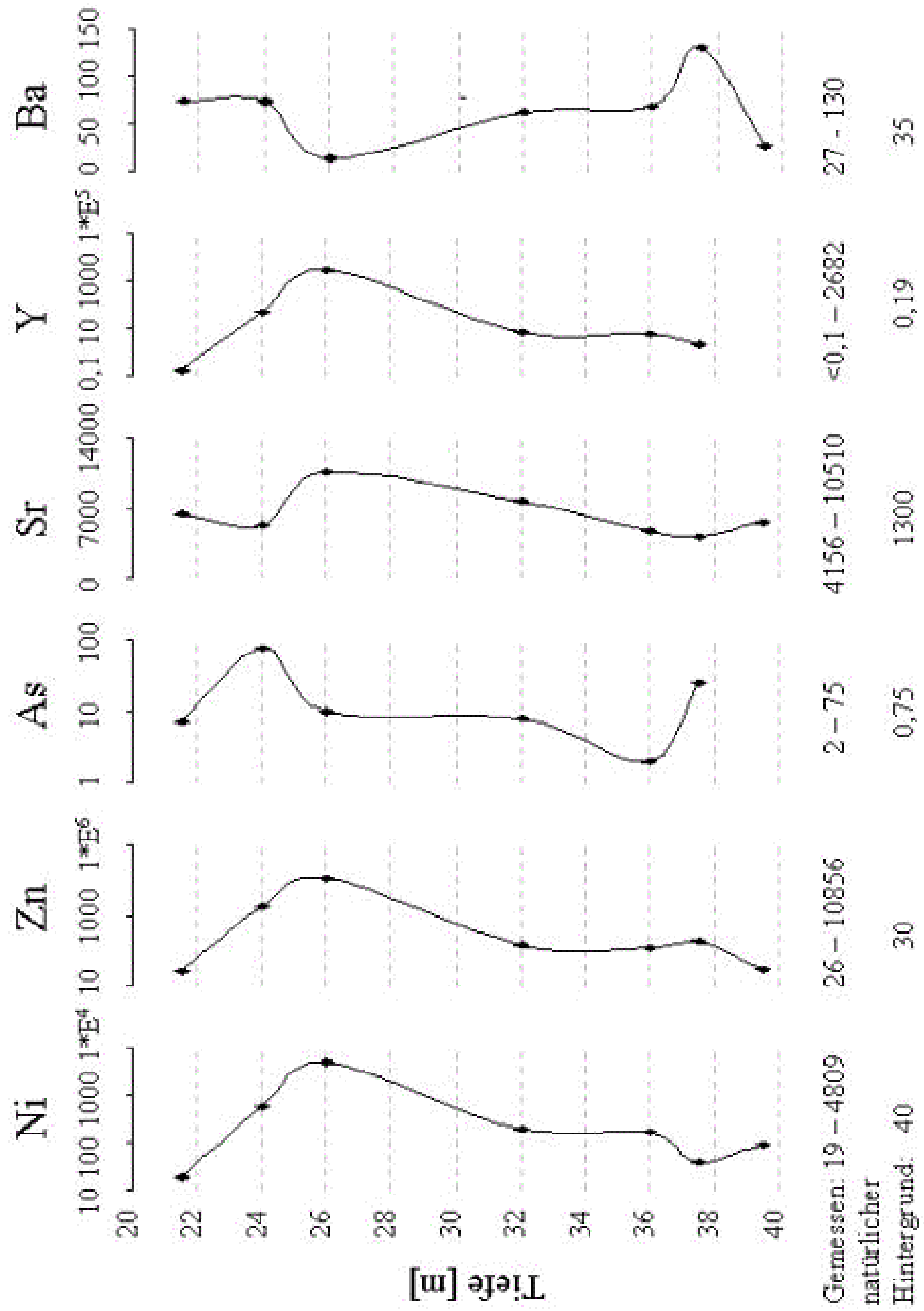

Abb. 4.28. (Forts.): Tiefenprofile von Elementkonzentrationen in Porenwässern des Bohrkerns des Multilevelbrunnens CML. 


\subsubsection{Oberflächennahe Bohrung}

Mit einem Handbohrer wurde im November 1999 eine oberflächennahe Bohrung durchgeführt. Dabei wurde ein drei Meter langes Rohr in einer Auswaschungsrinne im Kippenbereich eingesetzt, um die nachträglich aufgebrachte Deckschicht der Kippe zu umgehen und den tatsächlichen Kippenkörper oberflächennah zu untersuchen. Der Bohrkern reichte von 0 -1,93 m (1,94 - 3,64 m strat. Teufe). Für die Untersuchung der Porenwässer wurde er gedrittelt, für die Analyse der Festphasen wurden die Proben nochmals aufgeteilt, um ein differenziertes Profil zu gewinnen. Die Porenwasseranalysen wurden im Rahmen der vorliegenden Arbeit durchgeführt, die Festphasenanalysen stammen von BRINKMANN (2000).

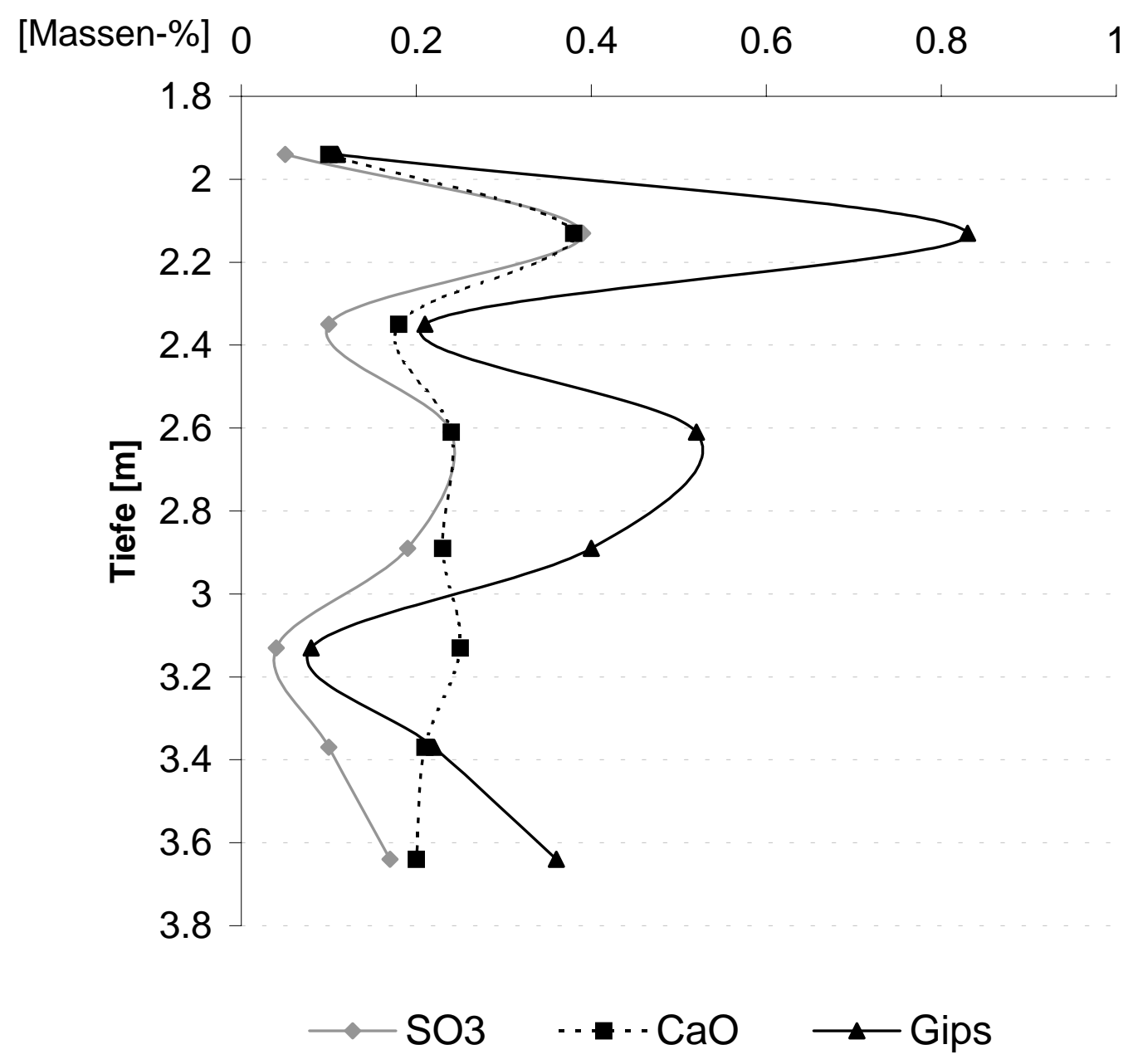

Abb. 4.29.: Tiefenprofil der Gehalte Gips, $\mathrm{CaO}$ und $\mathrm{SO}_{3}$ in den Festphasen im Kern der oberflächennahen Bohrung. 
$\left.\begin{array}{ll}\omega & 0 \\ \pi & 0 \\ 0\end{array}\right]$

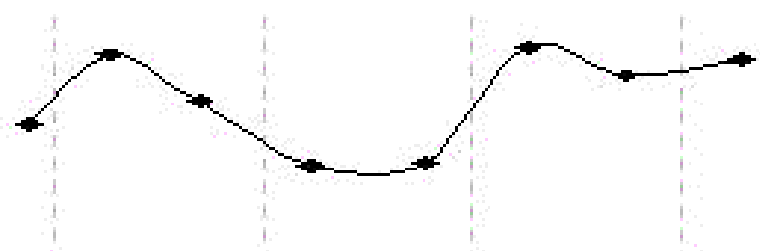

0
1
0
$n$
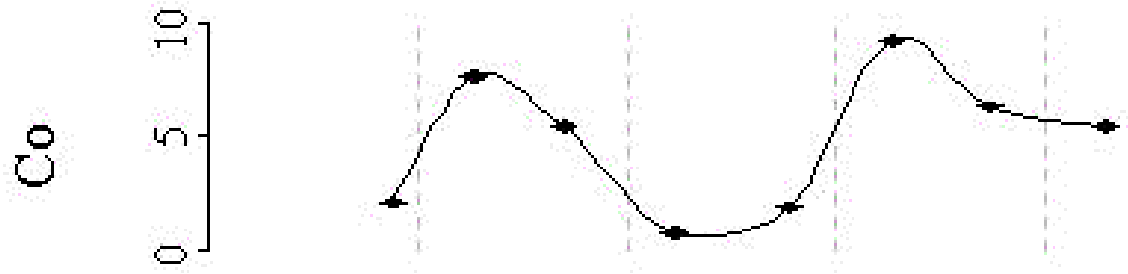

$m$
0
0

is

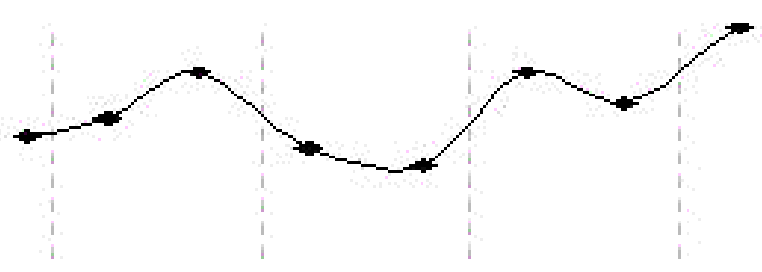

ถึ

$\stackrel{1}{\circ}$

$\left.p \quad \begin{array}{l}\Omega_{6} \\ \circ\end{array}\right]$

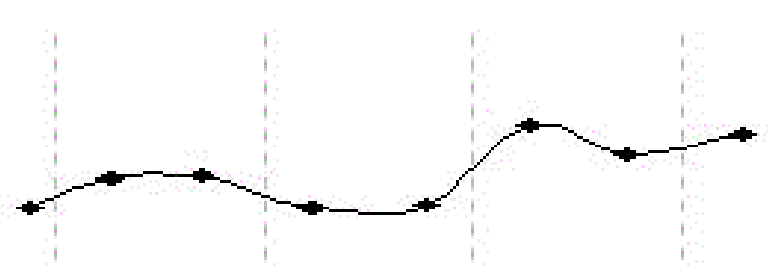

$\stackrel{\infty}{1}$

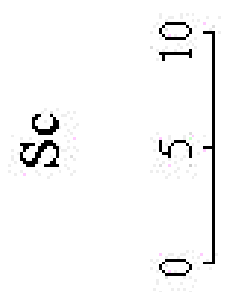

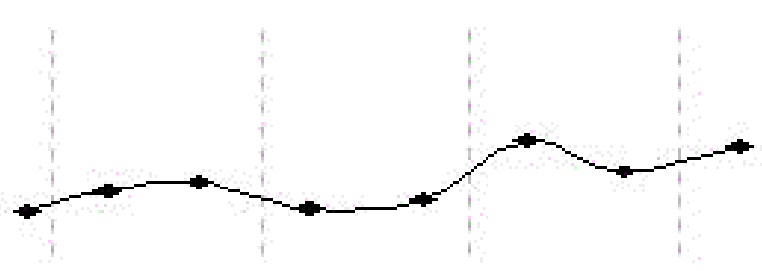

$\infty$
1
1
-

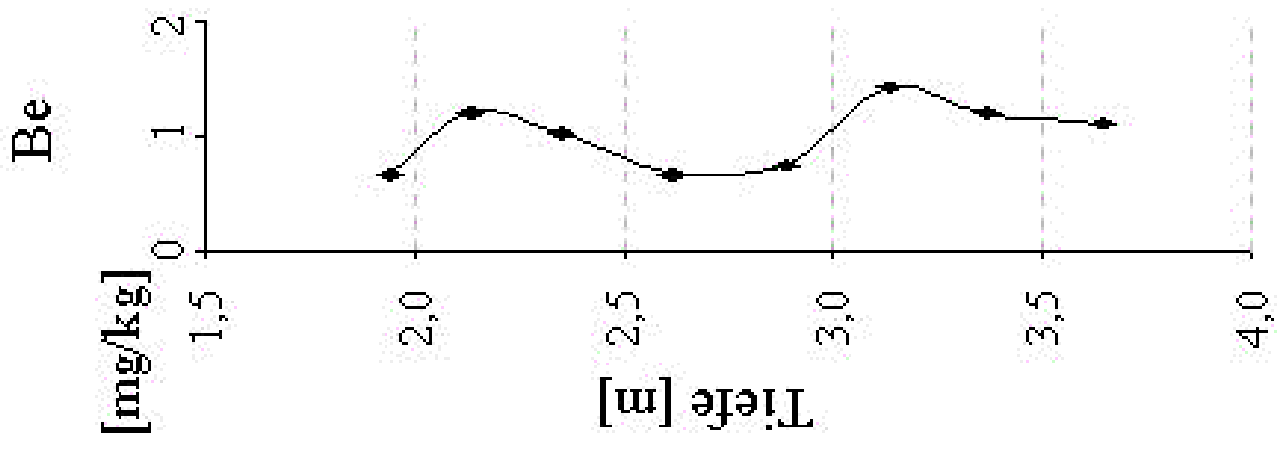

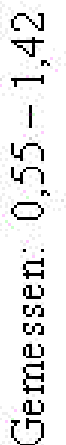

Abb. 4.30.: Tiefenprofile einiger Spurenelemente in den Festphasen der oberflächennahen Bohrung. 


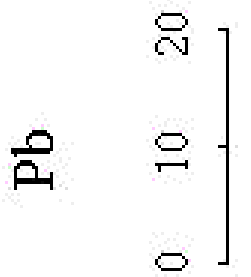

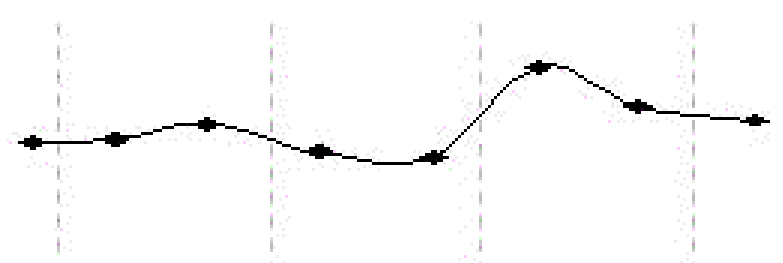

$\infty$

n

$\frac{1}{\infty}$

$\left[\begin{array}{l}- \\ 6 \\ 0\end{array}\right]$

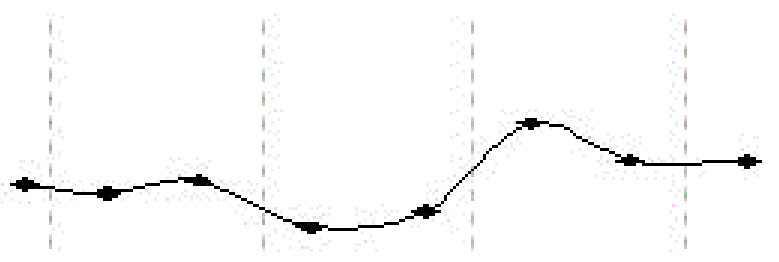

$m$
1
0
$\infty$
0

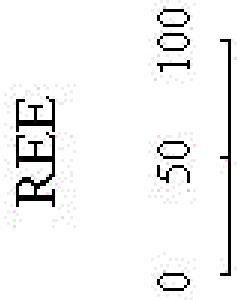

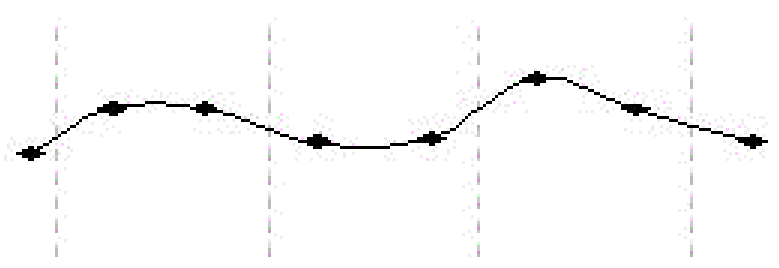

$\infty$

1

m

$\left.\begin{array}{cc}n & n \\ 0 & 0 \\ 0\end{array}\right]$

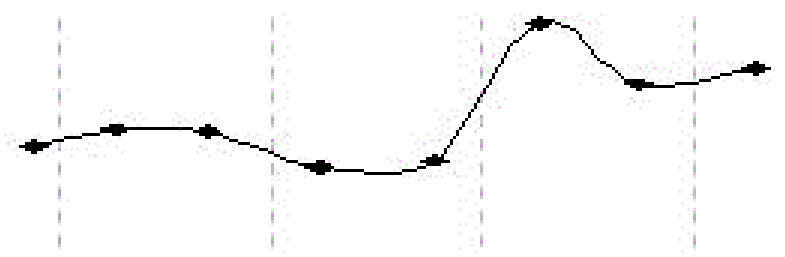

5

0

$m$

$\left.\begin{array}{ll}0 & \nabla \\ 2 & 0 \\ & 0\end{array}\right]$

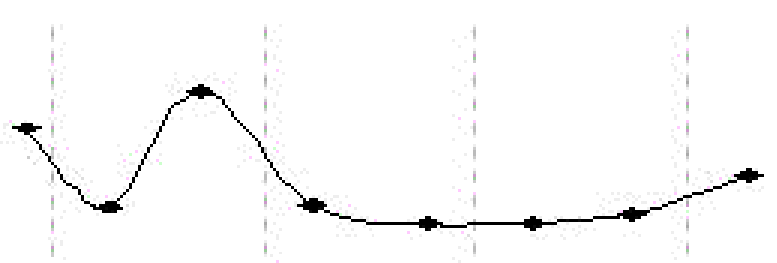

$a$

$\infty$

1

$\stackrel{\infty}{\circ}$

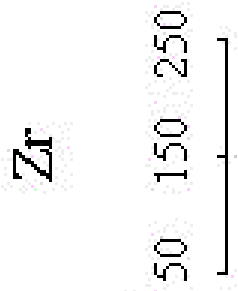

की

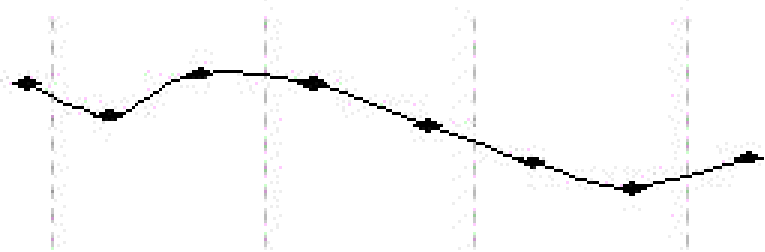

$\stackrel{\infty}{\curvearrowright}$

I

g

$\stackrel{\circ}{\circ}$

$m$

咅 
Die Analyse der C- und S-Gehalte im Bohrkern zeigt eine deutliche Varianz im Tiefenprofil (Abb. 4.29.). Die gemessenen Werte liegen dabei für Sulfatschwefel als $\mathrm{SO}_{3}$ im Bereich zwischen 0,04 und 0,39\%, für Sulfid zwischen 0,01 und 0,6 \% (BRINKMANN 2000). In 2,02 m bis 2,23 m lagen die höchsten Gehalte an $\mathrm{SO}_{3}$ - und Sulfidschwefel. Darunter, zwischen 2,46 $\mathrm{m}$ und 2,76 m, findet sich ein weniger ausgeprägtes lokales Maximum. Unterhalb von 3,40 m steigen die Werte wieder an. Die Tiefenprofile einiger Elemente zeigt exemplarisch Abb. 4.25.. Etwas erhöhte Gehalte an vielen Elementen werden dabei in einer Tiefe von 3,01 - 3,25 m festgestellt. Für einige Elemente ist auch noch ein kleineres lokales Maximum bei 2,02 2,23 $\mathrm{m} \mathrm{zu}$ sehen. Ob diese Effekte jedoch tatsächlich auf lokale Differenzierungen zurückgehen oder durch Heterogenität in der Schüttung entstehen, ist ohne Berücksichtigung der Porenwasserkonzentrationen schwer zu beurteilen. Von diesen konnte aber kein so differenziertes Tiefenprofil erstellt werden wie von den Festphasen, da zur Gewinnung ausreichender Mengen von Porenwasser mehrere Proben zusammengefasst werden mussten. So konnte der Bohrkern insgesamt nur in drei Teilmengen aufgespalten werden.

Die pH-Werte der Porenwässer lagen mit 3,5 - 4,9 im sauren Bereich; am stärksten von der Versauerung betroffen war dabei die oberste Schicht des entnommenen Bohrkerns. Erwartungsgemäß wurde hier auch eine stark erhöhte elektrische Leitfähigkeit von 3.24 $\mathrm{mS} / \mathrm{cm}$ festgestellt, die auf die hohen Ionenkonzentrationen in den Wässern zurückzuführen ist. So erreicht die Aluminiumkonzentration $7820 \mu \mathrm{g} / \mathrm{l}$. Alle Lanthanide lagen über der Nachweisgrenze, teilweise wurden deutlich über $10 \mu \mathrm{g} / \mathrm{l}$ gemessen, so bei $\mathrm{La}, \mathrm{Ce}, \mathrm{Pr}, \mathrm{Nd}, \mathrm{Gd}$ und Dy. Ca, Ba und einige andere Elemente sind dagegen im mittleren Abschnitt vermehrt vertreten; möglicherweise kommt es im oberen und unteren Bereich zur Ausfällung übersättigter Festphasen. In der mittleren Schicht des entnommenen Bohrkerns gehen die gemessenen Konzentrationen der überwiegenden Zahl von Elementen deutlich zurück, was durch den höheren pH-Wert erklärt werden kann (Tab. A.1. im Anhang).

Die pH-Werte in den Sickerwässern der oberflächennahen Bohrung sind mit denen in den oberen Schichten des Bohrkerns RCO4 vergleichbar. Die anderen Elementgehalte unterscheiden sich jedoch in der obersten Schicht der Kippe von denen, die in RCO4 5-8 m gemessen wurden. Während $\mathrm{Fe}$ und $\mathrm{Mn}$ deutlich niedriger liegen, ist $\mathrm{Al}$ weit höher konzentriert. Auch die Lanthanidwerte liegen deutlich über denen, die in den übrigen Bohrkernen mit Ausnahme der Tiefen um 26 m, wo wir die Pyritoxidationszone vermuten, festgestellt wurden. Nach den Erkenntnissen von BRINKMANN (2000) ist die Pyritoxidation in den obersten Schichten der Kippe nahezu quantitativ abgeschlossen. Die Pyritgehalte schwanken in den Bohrkernproben zwischen 0,01 und 1,1\% und liegen im Mittel bei 0,34 \%. 


\subsubsection{Lausitz}

Die beiden Bohrkerne aus dem Gebiet des Restlochs 111 in der Lausitz wurden in 6 bzw. 7 Proben aufgeteilt und im Rahmen dieser Arbeit hinsichtlich der Zusammensetzung ihrer Porenwässer untersucht. Insgesamt sind diese Wässer mit pH-Werten zwischen 2,7 und 3,9 deutlich saurer als die Wässer aus dem Cospudener Bereich. Dementsprechend hoch sind die Konzentrationen zahlreicher Elemente, z.B. Li bis $438 \mu \mathrm{g} / \mathrm{l}$, Be bis $124 \mu \mathrm{g} / \mathrm{l}$, Al bis $368 \mathrm{mg} / \mathrm{l}$, $\mathrm{V}$ bis $218 \mu \mathrm{g} / \mathrm{l}$, Mn bis $10 \mathrm{mg} / \mathrm{l}$, Fe bis $470 \mathrm{mg} / \mathrm{l}$, Co bis 3,0 mg/l, Ni bis 1,8 mg/l, Zn bis 9 $\mathrm{mg} / \mathrm{l}$, As bis $594 \mu \mathrm{g} / \mathrm{l}, \mathrm{Rb}$ bis $590 \mu \mathrm{g} / \mathrm{l}$, Ba bis $159 \mu \mathrm{g} / \mathrm{l}$, La bis $540 \mu \mathrm{g} / \mathrm{l}$, Ce bis 1,2 mg/l und $\mathrm{Pb}$ bis $159 \mu \mathrm{g} / \mathrm{l}$. Die Sulfatgehalte betragen zwischen 2,2 und 3,7 g/l. Für viele Elemente liegen damit in beiden Bohrkernen und unterschiedlichen Tiefen Werte vor, die in Cospuden nur in den am stärksten belasteten Wässern (Sickerwasser, Standgewässer aus der Arbeit von PlEßOW (1998)) erreicht werden. Die Konzentrationen von Be, Al, As, Ba, Rb liegen teilweise sogar noch oberhalb der höchsten in Cospuden gemessenen Konzentrationen. Die Gehalte an $\mathrm{Ca}, \mathrm{Mg}$, Mn, Sulfat, Al und Fe entsprechen in etwa den Ergebnissen, die SCHÖPKE et al. (1999) an Tagebauseen des Gebiets Schlabendorf-Nord in der Niederlausitz gemessen haben. Festphasenanalysen für die hier diskutierten Bohrkerne wurden von anderen Arbeitskreisen durchgeführt (BOZAU et al., in Vorbereitung). 


\subsubsection{Bohrkern 5-6 m}

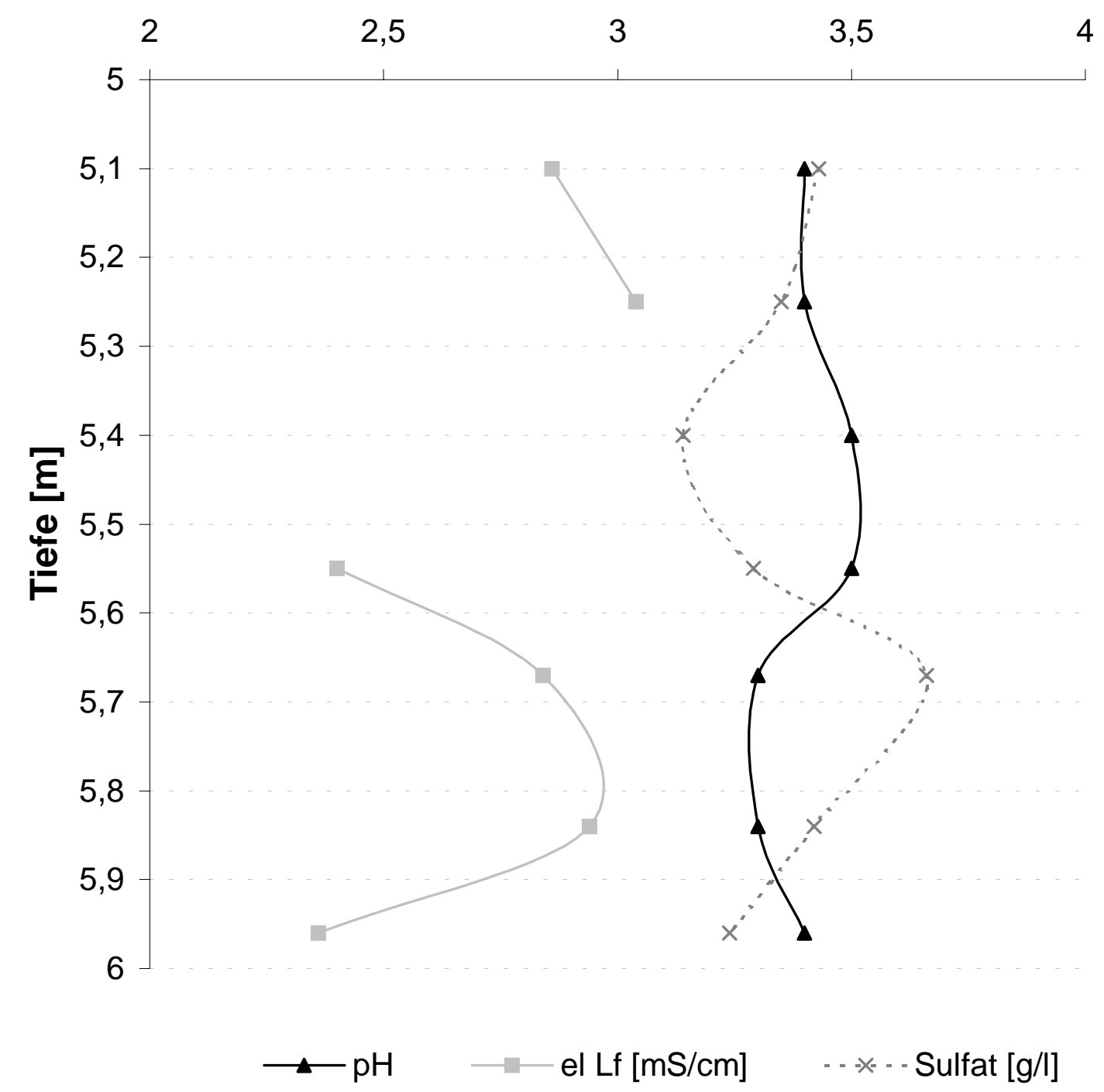

Abb. 4.31.: Tiefenprofil von $\mathrm{pH}$, elektrischer Leitfähigkeit und Sulfatkonzentration in den Porenwässern des Bohrkerns 5-6 m aus der Lausitz

Abb. 4.31. zeigt das Tiefenprofil des Bohrkerns 5-6 m hinsichtlich $\mathrm{pH}$, Sulfat und elektrischer Leitfähigkeit. Die pH-Werte liegen in einem engen Bereich zwischen 3,3 und 3,5. Hohe Sulfatkonzentrationen - alle Werte in diesem Profil liegen oberhalb von $3 \mathrm{~g} / \mathrm{l}-$ in den Porenwässern decken sich mit der optischen Feststellung, dass große Mengen von Gips in den Proben bereits ausgefallen sind. Deutliche Korrelationen zwischen pH-Wert und Elementkonzentrationen lassen sich nicht feststellen. Abb. 4.32. zeigt den Konzentrationsverlauf einiger Elemente in den Porenwässern des Bohrkerns. Auffällig ist die gute Korrelation $(\mathrm{r}=0,88)$ für Fe und As. 

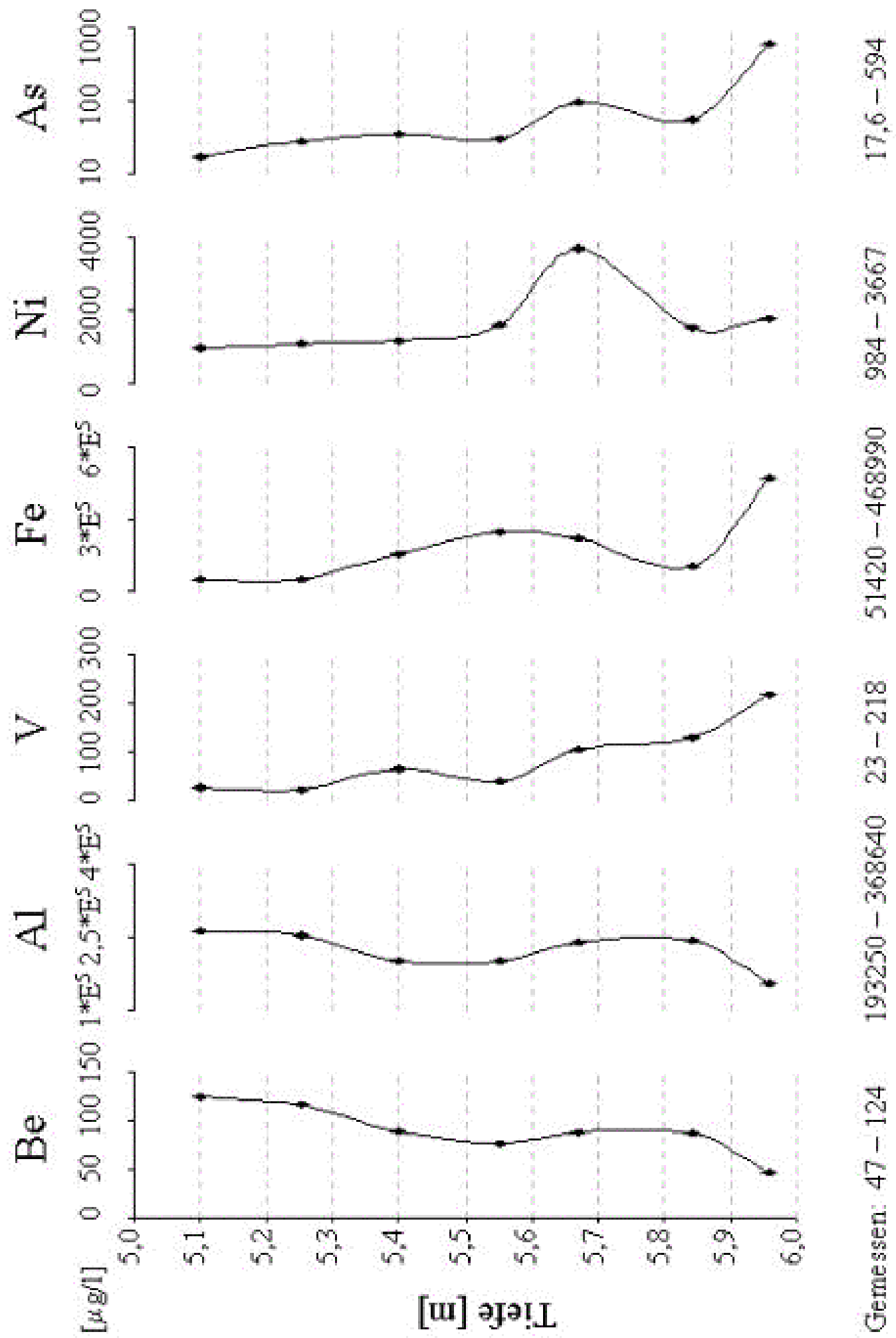

Abb. 4.32.: Tiefenprofil einiger Elementkonzentrationen in den Porenwässern des Bohrkerns 5 - $6 m$ aus der Lausitz. 
$\left[\begin{array}{l}8 \\ 8 \\ 0\end{array}\right]$

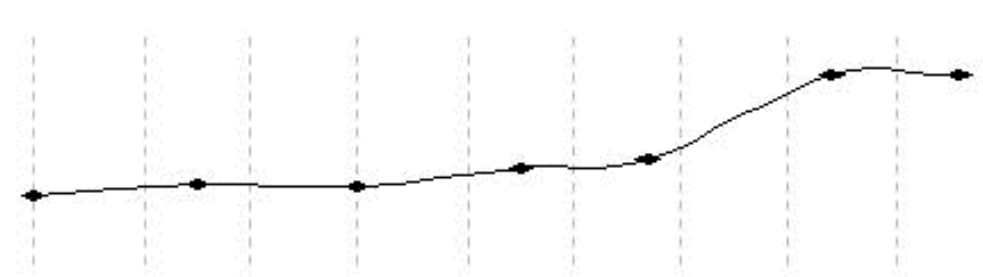

$\frac{9}{n}$

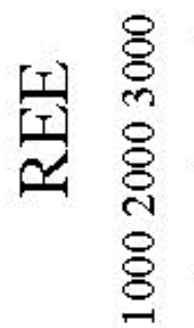

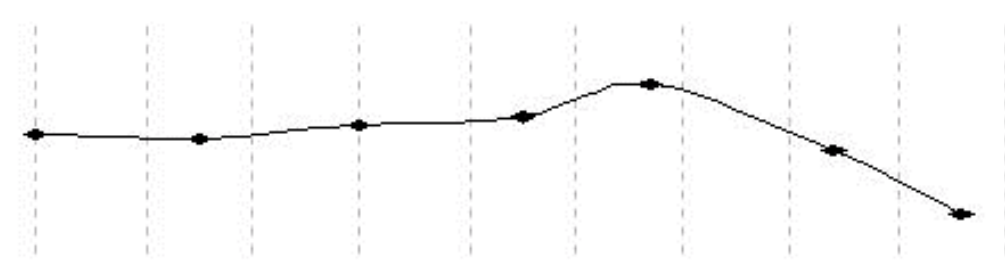

$m$
$m$
0
1
$\dot{m}$
$m$

$\left.\begin{array}{cc}0 & 0 \\ 0 & 0 \\ 0 & 0 \\ 0 & 0\end{array}\right]$

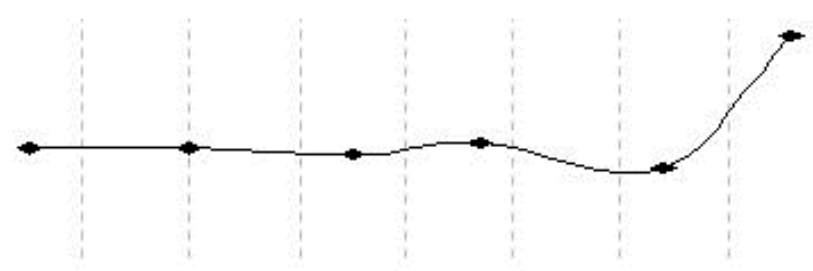

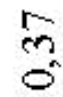

$\frac{1}{8}$

$\left.\sum_{1} \begin{array}{l}8 \\ -1\end{array}\right]$

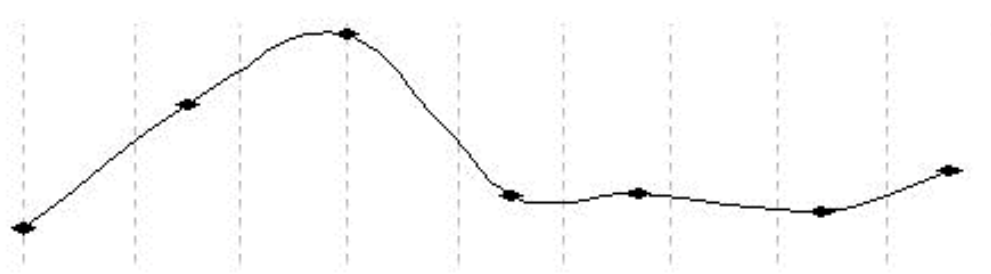

$\infty$
1
0
0
0

$\left.\begin{array}{c}8 \\ 8 \\ 0\end{array}\right]$

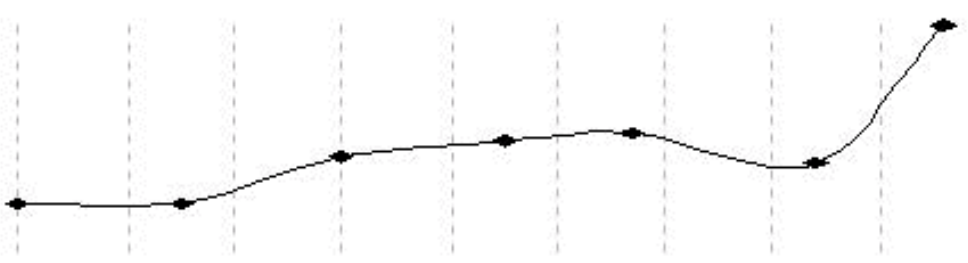

$\infty$

त)

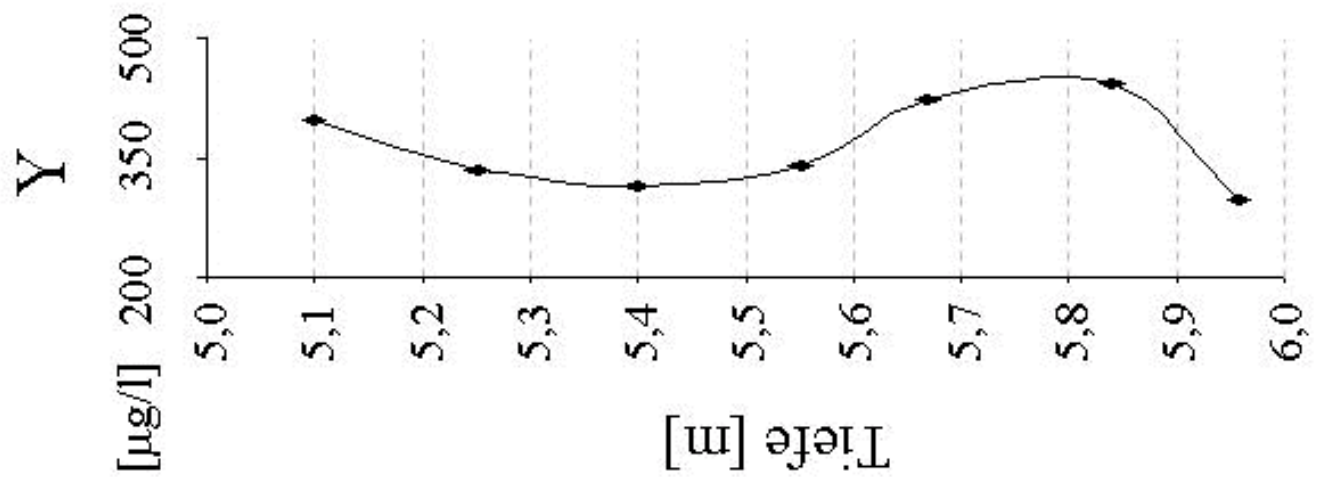

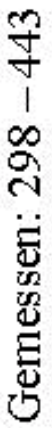

Abb. 4.32. (Forts.): Tiefenprofil einiger Elementkonzentrationen in den Porenwässern des Bohrkerns $5-6 m$ aus der Lausitz. 
Beide Konzentrationen steigen in der am tiefsten gelegenen Probe zwischen 5,93 und 6,00 m an (Abb. 4.31.). As liegt häufig pyritgebunden vor; eventuell lässt sich diese Beobachtung daher durch die Auflösung von Pyrit in dieser Tiefe erklären. Bemerkenswert ist, dass selbst bei diesem kleinräumigen Tiefenprofil signifikante Unterschiede in den Elementkonzentrationen auftreten, die sich mit den optisch wahrnehmbaren Abgrenzungen decken. So waren in den Proben bis 5,93 m Tiefe große Mengen an Gips erkennbar, während die darunter liegende Probe 5,93 - 6,00 m dunkelbraun bis schwarz ohne größere Gipseinsprengsel war. In dieser Probe stiegen die Konzentrationen von V, Fe, As, Zr, Sb, Ba, $\mathrm{W}$ und $\mathrm{Pb}$ auffällig an. Möglicherweise werden diese Elemente in den übrigen Proben an Gips oder andere Fällungsprodukte gebunden. Für viele andere Elemente wie $\mathrm{Mn}, \mathrm{Na}, \mathrm{K}, \mathrm{Mg}$ sind keine ausgeprägten Profile erkennbar.

Ein Zusammenhang zwischen den Konzentrationen von Fe und Sulfat lässt sich nicht herstellen. Im Unterschied $\mathrm{zu}$ den Ergebnissen des Bohrkerns 21 - $22 \mathrm{~m}$ steht die Sulfatkonzentration auch nicht im Verhältnis zu den Konzentrationen von Ba oder Ca. 


\subsubsection{Bohrkern 21-22 m}

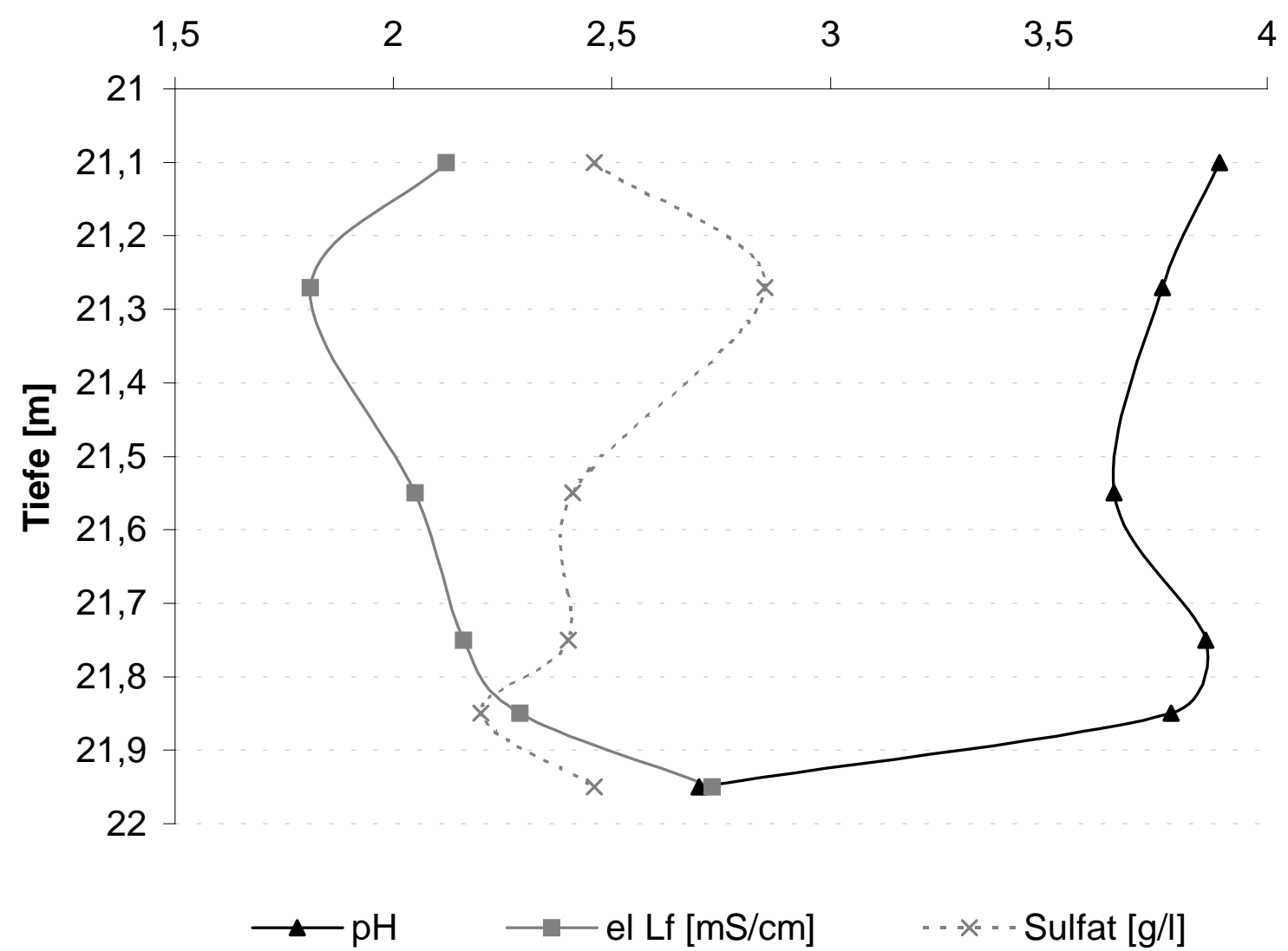

Abb. 4.33.: Tiefenprofil für $\mathrm{pH}$-Wert, elektrische Leitfähigkeit und Sulfatkonzentration in den Porenwässern des Bohrkerns 21-22 m aus der Lausitz.

Abb. 4.33. zeigt das Tiefenprofil von $\mathrm{pH}$, Leitfähigkeit und Sulfatkonzentration in den Porenwässern des Bohrkerns 21 - $22 \mathrm{~m}$. Hier schwanken die pH-Werte etwas stärker zwischen 2,7 und 3,9. Dafür liegen die Sulfatgehalte deutlich niedriger - zwischen 2,20 und 2,85 g/l - als in dem höher gelegenen Bohrkern, obwohl auch in den Festphasen dieses Kerns optisch Gips wahrgenommen wurde.

Unterhalb von 21,90 m nimmt der $\mathrm{pH}-$ Wert auf 2,7 ab. Gleichzeitig steigt die elektrische Leitfähigkeit an auf 2,73 mS/cm. Gemeinsam mit dem leicht über dem Mittelwert liegenden Sulfatwert von 2,46 g/l ist dies ein Indiz für eine lokale Säurequelle, beispielsweise aus der Verwitterung von Pyrit. 

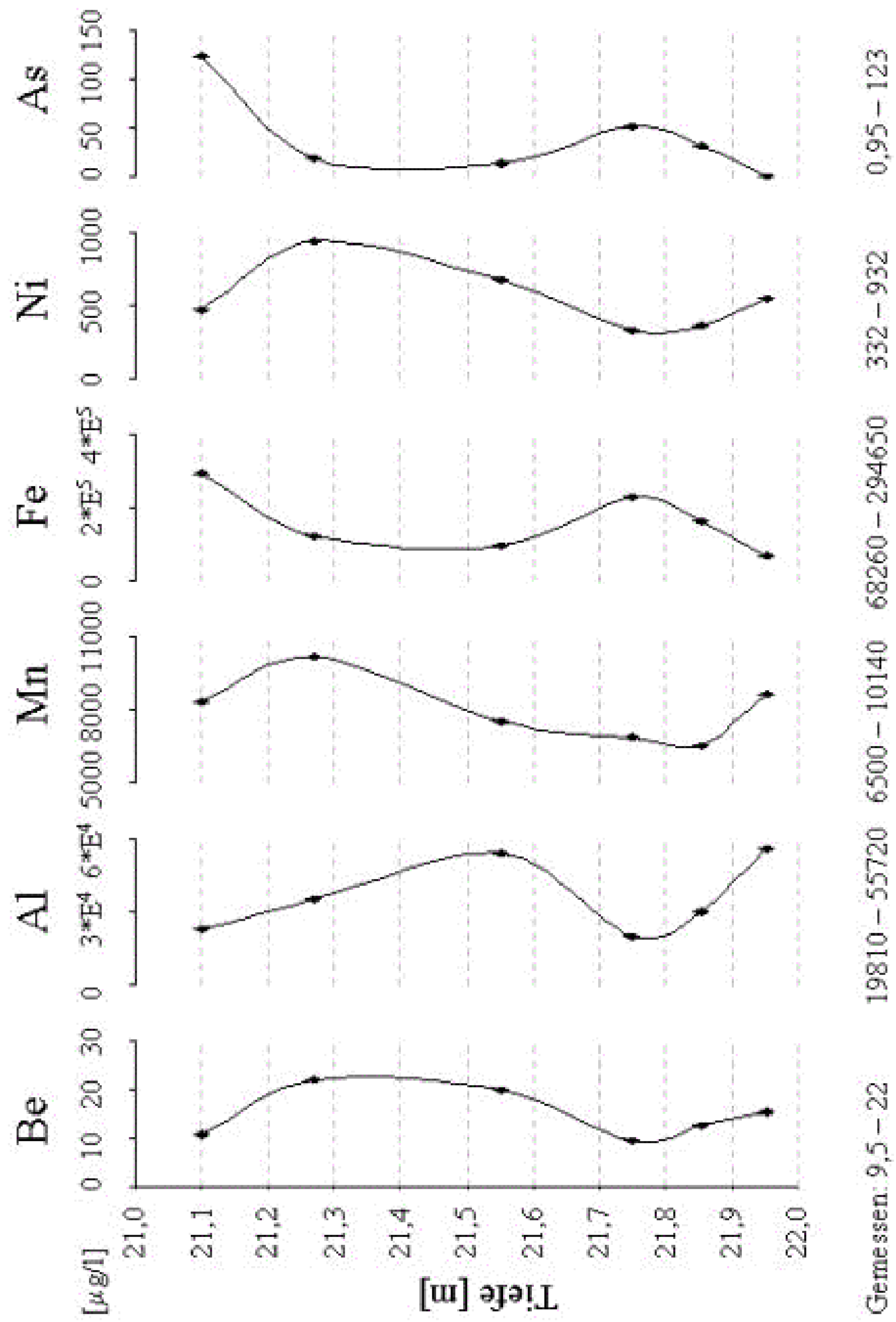

Abb. 4.34: Tiefenprofil von Elementkonzentrationen in Porenwässern des Bohrkerns 21 - 22 m aus der Lausitz. 

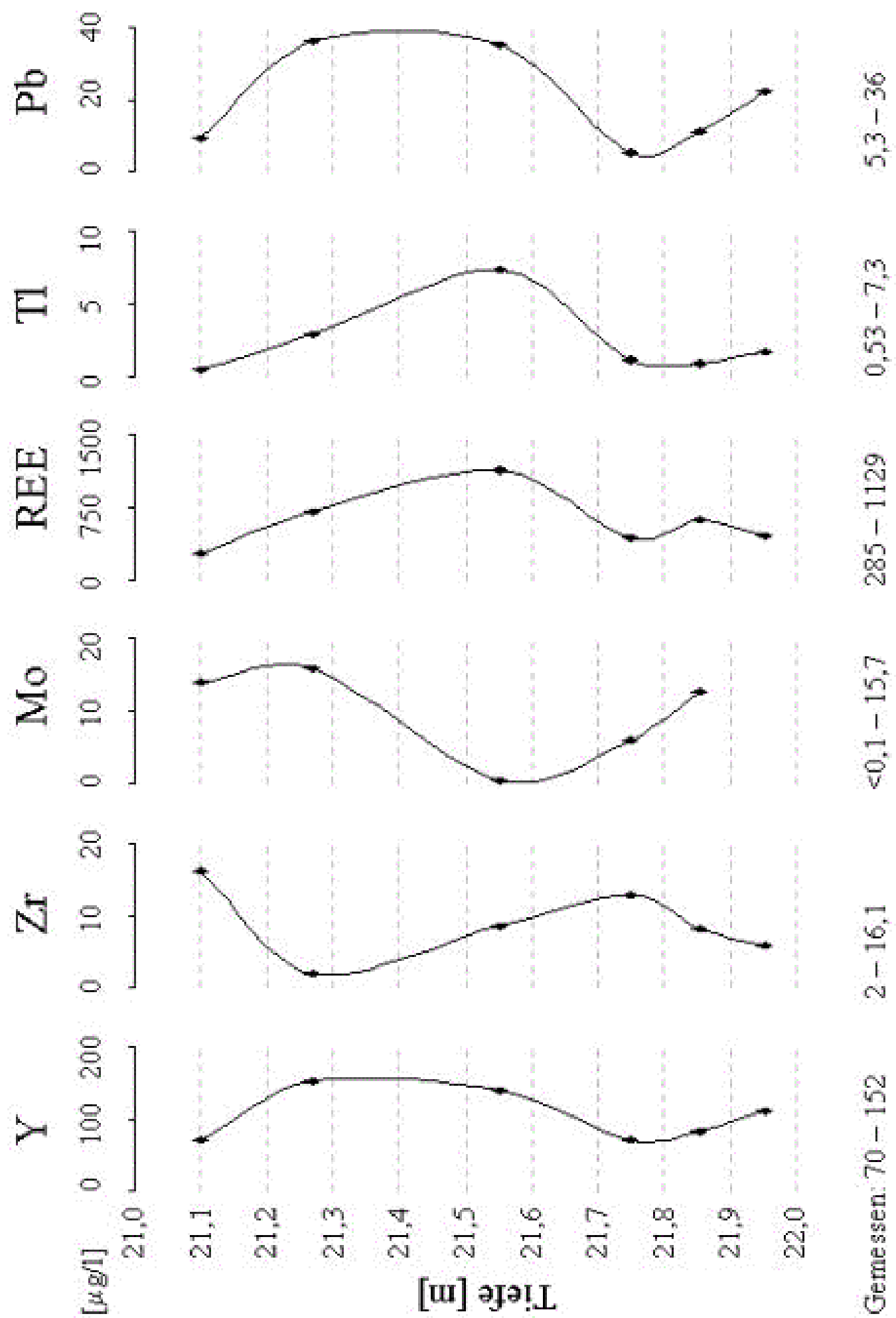

Abb. 4.34. (Forts.): Tiefenprofil von Elementkonzentrationen in Porenwässern des Bohrkerns $21-22 \mathrm{~m}$ aus der Lausitz. 
Die Tiefenprofile der Elementkonzentrationen (Abb. 4.34.) im Bohrkern Lausitz $21-22 \mathrm{~m}$ sind ebenfalls schwierig zu interpretieren. Während $\mathrm{Be}, \mathrm{Al}, \mathrm{Mn}, \mathrm{Ni}, \mathrm{Y}$ und $\mathrm{Pb}$ in den Proben zwischen 21,13 und 21,70 m stärker angereichert sind, zwischen 21,70 und 21,80 m ein Minimum erreichen und unterhalb davon wieder ansteigen, verlaufen die Konzentrationprofile von $\mathrm{Fe}$, As und $\mathrm{Zr}$ gerade entgegengesetzt und korrelieren miteinander $\left(\mathrm{r}_{\mathrm{Fe}-\mathrm{As}}=0,952, \mathrm{Abb} .4 .35\right.$; $\left.\mathrm{r}_{\mathrm{Fe}-\mathrm{Zr}}=0,841\right)$.

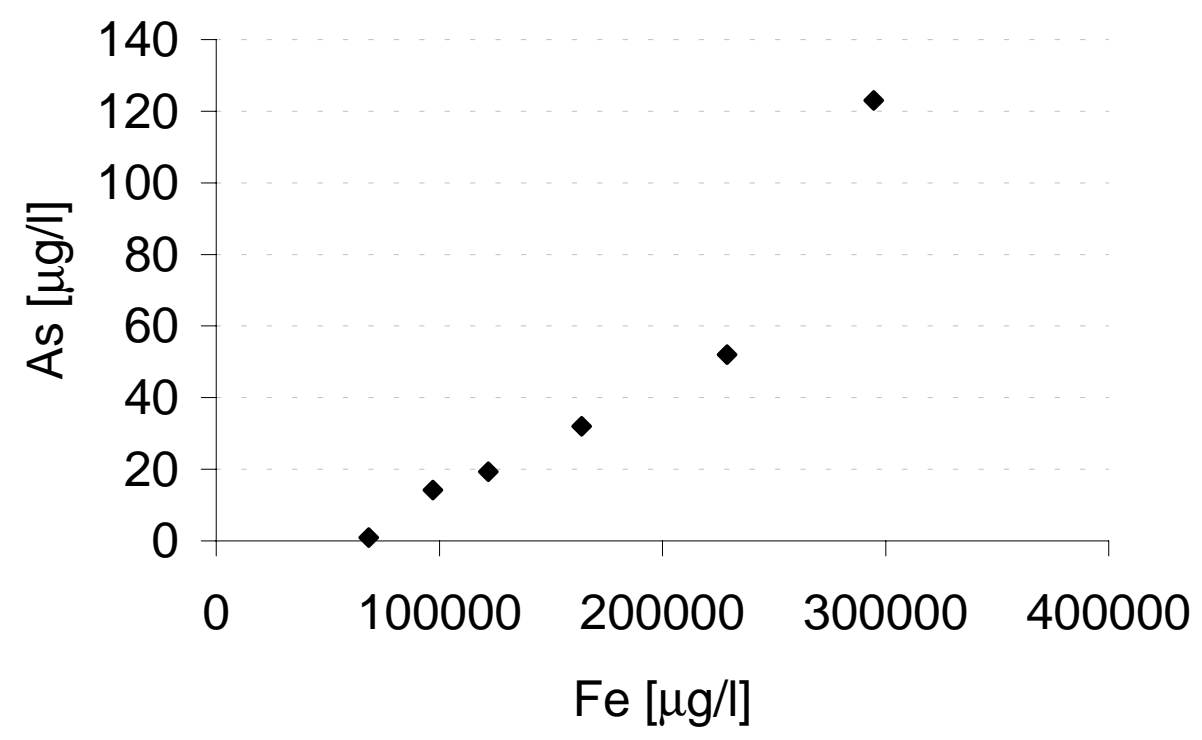

Abb. 4.35.: Korrelation zwischen den Konzentrationen von As und Fe in den Porenwässern des Bohrkerns 21 $22 m$ aus der Lausitz.

Auch der pH-Wert verhält sich ähnlich. Die Alkali- und Erdalkalimetalle sowie $\mathrm{Cu}$ und $\mathrm{Zn}$ verhalten sich dagegen wie die erstgenannten Elemente, während der Verlauf des Profils von Co dem der REEs gleicht (nicht in der Abbildung dargestellt; Werte s. Tabelle A.1. im Anhang). Mehrere Elemente korrelieren stark mit Sulfat, was darauf hindeutet, dass ihre Konzentrationen durch die Ausbildung sulfathaltiger Festphasen adsorptiv reguliert werden Tab. 4.8.).

\begin{tabular}{|l|l|}
\hline Element & Korrelationskoeffizient r Element-Sulfat \\
\hline $\mathrm{Li}$ & 0,922 \\
\hline $\mathrm{Na}$ & 0,829 \\
\hline $\mathrm{Ca}$ & 0,884 \\
\hline $\mathrm{Mn}$ & 0,933 \\
\hline $\mathrm{Ni}$ & 0,861 \\
\hline $\mathrm{Ba}$ & 0,902 \\
\hline
\end{tabular}

Tab. 4.8.: Korrelation zwischen Sulfatkonzentration und anderen Elementkonzentrationen in Porenwässern des Bohrkerns Lausitz $21-22 \mathrm{~m}$. 
Trotz der niedrigeren Leitfähigkeit und der niedrigeren Sulfatgehalte liegen die Elementgehalte in diesem Bohrkern deutlich höher als in den Porenwässern aus dem Bohrkern 5 -6 m. Die DOC-Konzentration ist jedoch ist weit niedriger als in dem höher gelegenen Bohrkern; dies entspricht den Erwartungen (s. Kap. 5). Mit den vergleichsweise wenigen Proben lässt sich natürlich kein so differenziertes Tiefenprofil erstellen, wie dies für den Bereich Cospuden möglich war. Interessant wird daher vor allem der Vergleich zwischen dem Verhalten der gelösten Spurenelemente in den Wässern aus Cospuden und den Porenwässern aus der Lausitz sein. 


\section{Speziation}

\subsection{Einführung}

Viele Parameter nehmen Einfluss auf Absolutgehalt und Lösungsform gelöster Spezies. In Abb. 5.1. sind die wichtigsten Vorgänge dargestellt, durch die die Konzentrationen gelöster Ionen kontrolliert werden.

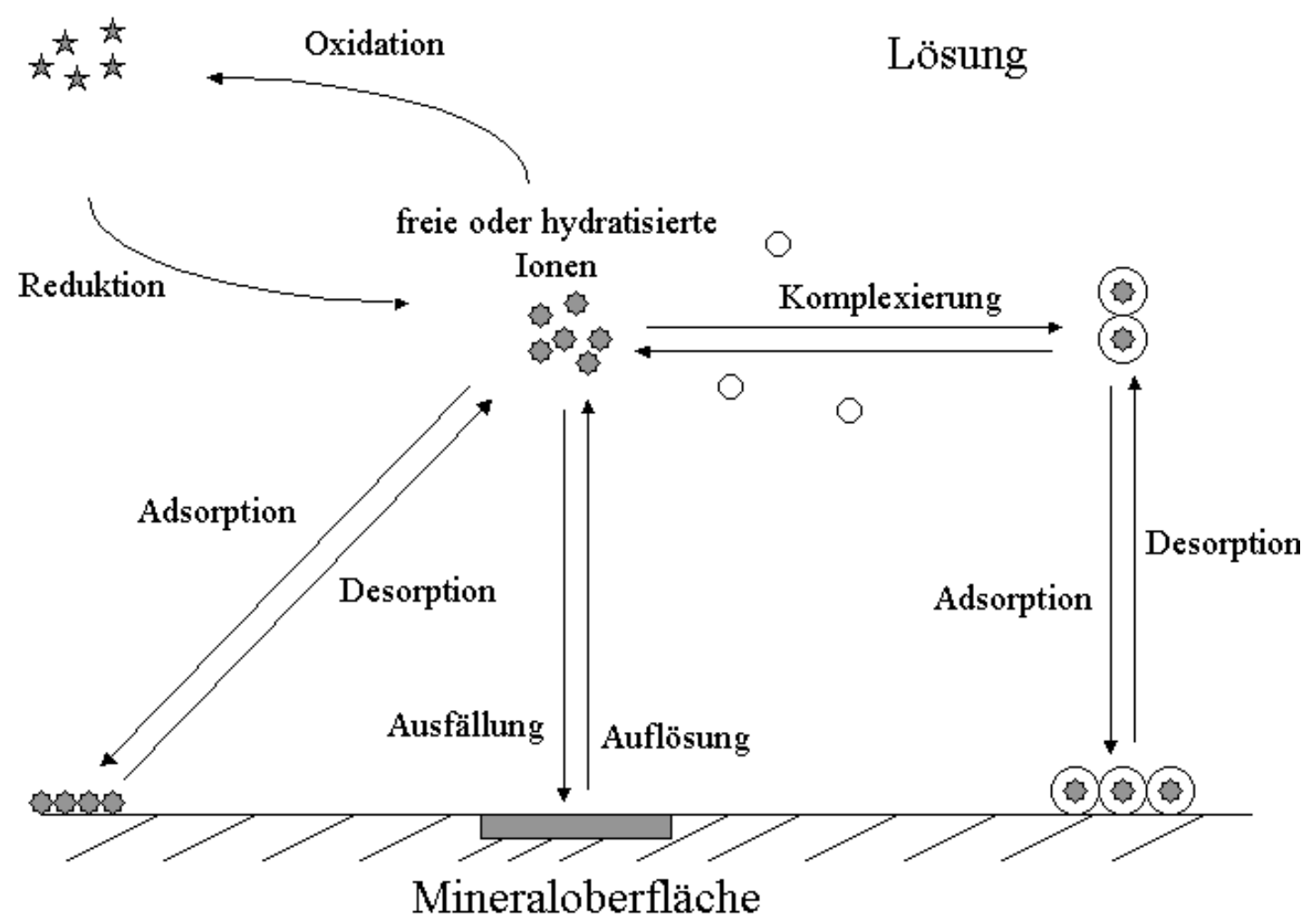

Abb. 5.1.: Kontrollmechanismen für den Absolutgehalt gelöster Ionen

Der Absolutgehalt einer gelösten Substanz unterliegt in erster Linie der Kontrolle durch Ausfällungs- oder Adsorptionsvorgänge (CHAPMAN et al. 1983). Besonders wichtig ist hier der Fe- und Mn-Kreislauf in Seen, der durch Redox- und Fällungs- bzw. Auflösungsvorgänge gesteuert wird (NORDSTROM 1979, SHOLKOVITZ 1985, BALISTRIERI 1992, BALISTRIERI et al. 1994). Die entstehenden Fe- oder Mn-Hydroxid-Oberflächen bieten Adsorptionsflächen für zahlreiche andere Elemente und kontrollieren damit wiederum deren Konzentrationen in der Lösung (CALMANO et al. 1994).

Gelöste Kationen existieren als freie oder hydratisierte Ionen, können aber auch von Sulfat oder anderen Anionen (LANGMUIR 1979, KREMLING 1983) oder Bestandteilen des DOC 
komplexiert werden. Dies wiederum kann die Adsorption an Partikeln oder Mineraloberflächen begünstigen oder behindern. Die Komplexliganden beeinflussen aber auch die Löslichkeit, indem sie freie Kationen binden, und fördern damit die Bildung übersättigter Lösungen. Zusätzlich unterliegen manche Elemente Oxidations- oder Reduktionsvorgängen, die vom Vorhandensein eines Redoxpartners, vom pH oder durch die Beteiligung von Mikroorganismen beeinflusst werden. Auch die Adsorption an Oberflächen kann eine Rolle für das Redoxverhalten von Ionen spielen (WEHRLI 1990, STUMM 1992). Die Konzentration eines Elements unterliegt damit der Kontrolle eines komplexen Geflechts von Gleichgewichtsreaktionen. Diese können ihrerseits wiederum kinetisch oder thermodynamisch kontrolliert sein. Schon die Betrachtung der Absolutgehalte von Elementen in Wässern bedarf also der Berücksichtigung zahlreicher komplizierter Vorgänge, deren Zusammenwirken nur schwierig zu erfassen ist.

Doch nicht nur die Absolutgehalte sind entscheidend für die Einschätzung, ob ein kontaminiertes Wasser als bedenklich einzustufen ist oder nicht. Wichtig ist auch, inwieweit sich ein lokal angereichertes Element in den Wässern verbreiten kann. Das hängt u.a. vom Lösungsverhalten des untersuchten Elements ab. Außerdem entscheidet die Bindungsform auch über die Toxizität des Elements; bekannteste Beispiele sind $\mathrm{Cr}$, das nur in der Oxidationsstufe +VI von toxikologischer Bedeutung ist, oder Quecksilber, das organisch gebunden weit toxischer ist als anorganisch gebundenes Quecksilber, dessen Verbindungen im Körper kaum resorbiert werden (MERKEL und SPERLING 1998). Für die Einschätzung belasteter Wässer ist daher auch wichtig, wie die fraglichen Elemente gebunden sind, ob sie eine signifikante Mobilität im Wasser aufweisen und ob sie in toxischer Form vorliegen.

Generell werden die Eigenschaften von Elementen durch ihre Stellung im Periodensystem geprägt. Diese gibt auch im Fall der Speziation erste Anhaltspunkte für die zu erwartenden Bindungsformen der gelösten Stoffe. 


\begin{tabular}{|c|c|c|c|c|c|c|c|c|c|c|c|c|c|c|c|c|c|}
\hline $\mathrm{H}$ & & & & & & & & & & & & & & & & & $\mathrm{He}$ \\
\hline $\mathrm{Li}_{\mathrm{i}}$ & $\mathrm{Be}$ & & & & & & & & & & & $B$ & C & N & 0 & $F$ & $\mathrm{Ne}$ \\
\hline $\mathrm{Na}$ & $\mathrm{Mg}$ & & & & & & & & & & & $\mathrm{Al}$ & $\mathrm{Si}$ & $\bar{P}$ & $\bar{S}$ & $\mathrm{Cl}$ & $\mathrm{Ar}$ \\
\hline $\mathrm{K}$ & $\mathrm{Ca}$ & $\mathrm{Sc}$ & $\mathrm{Ti}$ & $\mathrm{V}$ & et: & $1 \mathrm{~m} n$ & $E E$ & co & Ni: & $\mathrm{Cu}$ & $\mathrm{Zn}$ & $\mathrm{Ga}$ & $\mathrm{Ge}$ & As & $\mathrm{Se}$ & $\mathrm{Br}$ & $\mathrm{Kr}$ \\
\hline $\mathrm{Rb}$ & $\mathrm{Sr}$ & $\mathrm{Y}$ & $\mathrm{Zr}$ & $\mathrm{Nb}$ & $\mathrm{Mo}$ & $T_{c}$ & $\mathrm{Ru}$ & $\mathrm{Rh}$ & $\mathrm{Pd}$ & Ag & C्त्त: & $\operatorname{In}$ & $\mathrm{Sn}$ & $\mathrm{Sb}$ & $\mathrm{Te}$ & I & $\mathrm{Xe}$ \\
\hline Cs & $\mathrm{Ba}$ & $\mathrm{La}$ & $\mathrm{Hf}$ & $\mathrm{Ta}$ & $W$ & $\operatorname{Re}$ & Os & Ir & $\mathrm{Pt}$ & $\mathrm{Au}_{\mathrm{H}}$ & IHIg & 71 & $P b:$ & $\mathrm{B}_{1}$ & $\mathrm{Po}$ & At & $\mathrm{Rn}$ \\
\hline $\mathrm{Fr}$ & $\mathrm{Ra}$ & $\mathrm{Ac}$ & \begin{tabular}{|l|l|}
$\mathrm{Rf}$ \\
\end{tabular} & $\mathrm{Db}$ & $\mathrm{Sg}$ & $\mathrm{Bh}$ & \begin{tabular}{l|l}
$\mathrm{Hs}$ \\
\end{tabular} & \begin{tabular}{l|l}
$\mathrm{Mt}$ & $\mathrm{T}$ \\
\end{tabular} & Uun & Uuu T & Uud & Uut & Uuq & Uup T & Uuh T & Uus & Uuo \\
\hline & & & $\mathrm{Ce}$ & $\mathrm{Pr}$ & $\mathrm{Nd}$ & $\mathrm{Pm}$ & $\mathrm{Sm}$ & $\mathrm{Eu}$ & $\mathrm{Gd}$ & $\mathrm{Tb}$ & Dy & Ho & $\mathrm{Er}$ & $\operatorname{Tm}$ & $\mathrm{Yb}$ & $\mathrm{Lu}$ & \\
\hline & & & $\mathrm{Th}$ & $\mathrm{Pa}$ & $\mathbb{8}$ & $\mathrm{Np}$ & $\mathrm{Pu}$ & Am & $\mathrm{Cm}$ & $\mathrm{Bk}$ & $\mathrm{Cf}$ & $E_{s}$ & Fm & $\mathrm{Md}$ & No & $\mathrm{Lr}$ & \\
\hline & & & & & & & & & & & $D+$ & सा16 & & & & & \\
\hline & & $a^{c}-$ Typ & $\mathrm{h}$ & & nen, & sc & wach & te elek & ktros & tatiso & che $\mathrm{B}$ & Bind & ungen & $\mathrm{n}, \mathrm{Ub}$ & rit & angsf & form \\
\hline & & b-Typ & : wei & iche I & Ioner & n, star & rke & koval & lente & Bind & Aunge & & & & & & \\
\hline & 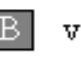 & wollstä & and & hyd & rol & sierte & $\mathrm{SP}_{\mathrm{F}}$ & & & & & & & & & & \\
\hline & & & & & & & & & & & & & & & & & \\
\hline
\end{tabular}

Abb. 5.2.: Periodensystem der Elemente und Bindungseigenschaften in wässriger Lösung (modifiziert nach Turner et al. 1981).

Nach der Klassifizierung im Periodensystem nach TURNER et al. (1981) (Abb. 5.2.) lassen sich für einige Gruppen Trends für ihr Verhalten in wässrigen Lösungen formulieren. Alkaliund Erdalkalimetalle treten in der Regel als einfache Kationen in einer Hydrathülle auf. Einige Haupt- und Nebengruppenelemente neigen dagegen stark zur Hydrolyse und liegen häufig als Oxoanionen vor, so z.B. As, Sn, Sb, V und Mo. Übergangsmetalle mit Elektronen in d-Orbitalen, z.B. $\mathrm{Fe}^{\mathrm{II}}$, $\mathrm{Co}^{\mathrm{II}}, \mathrm{Ni}^{\mathrm{II}}$, neigen zur Bildung von stabilen Komplexverbindungen mit Elektronendonatoren wie Stickstoffverbindungen, Carbonylen und schwefel- oder sauerstoffhaltigen funktionellen Gruppen organischer Verbindungen. Die Elemente, die in obigem Schema als „diverse Übergangsformen“ klassifiziert sind, unterscheiden sich in ihrem Verhalten sehr stark je nach ihrem Oxidationszustand: Während $\mathrm{Cr}^{\mathrm{III}} \mathrm{zu}$ Komplexbildung neigt, liegt $\mathrm{Cr}^{\mathrm{VI}}$ praktisch ausschließlich in hydrolysierter Form vor. Die Lanthanoide weisen untereinander sehr große Ähnlichkeiten auf, weil ihre Elektronenkonfiguration in den äußeren Orbitalen identisch ist und sich nur durch die Besetzung der 4f-Orbitale voneinander unterscheidet. In der Regel liegen sie in der Oxidationsstufe $+\mathrm{III}$ vor; $\mathrm{Ce}^{\mathrm{IV}}$ und $\mathrm{Eu}^{\mathrm{II}}$ bilden in wässrigen Lösungen die einzigen Ausnahmen (GREENWOOD und EARNSHAW 1980). 
Das bekannteste Konzept zur Beschreibung von Komplexbildung ist das Pearson-Konzept von harten und weichen Säuren und Basen (HSAB). Harte Säuren sind dabei Kationen mit hoher Ladung, kleinem Ionenradius und geringer Polarisierbarkeit, die Bindungen mit eher ionischem Charakter ausbilden. Weiche Säuren haben einen größeren Ionenradius, sind besser polarisierbar und formen Bindungen mit kovalentem Charakter. Entsprechendes gilt für die Anionen oder Basen. Bevorzugt bilden sich Komplexe zwischen harten Kationen und harten Anionen bzw. weichen Kationen und weichen Anionen (ausführliche Erläuterungen s. z. B. LANGMUIR (1997) und BUFFLE (1988)). Lanthanide werden nach Pearson als harte Kationen klassifiziert. Sie bevorzugen $-\mathrm{O}$ als Komplexpartner; ihre Komplexe zeichnen sich durch hohe Koordinationszahlen und durch die Einlagerung von Wassermolekülen aus, die eine genaue Bestimmung der Komplexstöchiometrie sehr erschweren. Trotz ihres Charakters als harte Kationen, die anorganische Komplexbildner bevorzugen sollten, belegen experimentelle Untersuchungen, dass die Komplexierung durch Humate eine große Rolle bei der Speziation der Lanthanide spielt (TAKAHASHI et al. 1999).

\subsubsection{DOC}

Das Konzept von Pearson beschreibt nicht nur die Bildung anorganischer Komplexe in wässrigen Lösungen, sondern kann auch auf die Interaktion von organischen und anorganischen Ionen bzw. Molekülen angewendet werden. In organischen Molekülen dienen O-, S- und N-Atome als Elektronendonatoren für Komplexe. Die Verfügbarkeit ihrer freien Elektronenpaare für die Ausbildung kovalenter oder elektrostatischer Bindungen hängt sehr stark von der Struktur des organischen Moleküls ab. Ein weiterer Faktor, der entscheidend die Komplexstabilität bestimmt, ist der Chelateffekt: Kann ein organisches Molekül zwei oder mehr Bindungsstellen zur Verfügung stellen, die mit dem Kation eine stabile Ringstruktur ausbilden können (besonders Fünf- und Sechsringe), wird die Beständigkeit der Komplexe um Größenordnungen erhöht. Auch andere sterische Faktoren kommen bei der Komplexbildung zum Tragen, so etwa die Ausbildung von „Taschen“ in Proteinstrukturen, in die sich die Kationen einlagern können (BUFFLE 1988).

Im Rahmen der Arbeit von PlEßOw (1998) und der vorliegenden Arbeit wurden Vorversuche durchgeführt, um die Komplexstabilität der untersuchten Kationen mit verschiedenen Liganden und ihre Auswirkungen auf das Verhalten der Elemente im Speziationsschema zu ermitteln. Dazu wurde eine Testlösung mit $\mathrm{pH}=1,4$ und je $5 \mathrm{mg} / \mathrm{l} \mathrm{Na}, \mathrm{Mg}, \mathrm{Ca}$, je $2 \mathrm{mg} / \mathrm{l} \mathrm{Al}$, $\mathrm{Fe}, \mathrm{Zn}$, je $1 \mathrm{mg} / \mathrm{l} \mathrm{K}$, Sr sowie 57 weiteren Elementen mit je 20-100 $\mu \mathrm{g} / \mathrm{l}$ mit jeweils 30g/l $\mathrm{D}(+)$-Glucose-Monohydrat (für biochemische Zwecke), Glycin (p.a.), Metaweinsäure (zur 
Synthese), tri-Natrium-Citrat-Hydrat (p.a.), 4-Hydroxybenzoesäure (zur Synthese), Harnstoff (p.a.), Alanin, Cystein und Threonin sowie 6,9 g/l Fulvosäure (isoliert durch Frau Dr. A. Krüger (Universität Leipzig) aus einem Hochmoor) versetzt. Die entsprechenden Molekülstrukturen sind in Abb. 5.3. dargestellt.

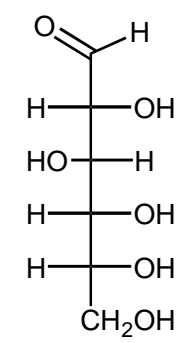

Glucose

(<smiles>NCC(=O)[O-]</smiles>

Glycin<smiles>O=C([O-])CC(O)(CC(=O)[O-])C(=O)O</smiles>

Citrat<smiles>O=C([O-])C(O)C(=O)O</smiles>

Tartrat<smiles>NC(N)=O</smiles><smiles>O=C(O)c1ccc(O)cc1</smiles>

Harnstoff

\section{4-Hydroxybenzoesäure}

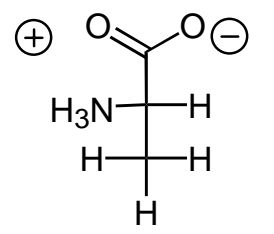

Alanin<smiles>[CH]C(O)C(N)C(=O)[O-]</smiles>

Threonin<smiles>NC(C(=O)[O-])C([NH3+])[O-]</smiles>

Cystein

Funktionelle Gruppen an Fulvosäuren und Huminstoffen

\begin{tabular}{|c|c|c|c|c|}
\hline Amino- & $-\mathrm{NH}_{2}$ & Carbonsäure- & $-\mathrm{COOH}$ & Thiol- \\
\hline Hydroxy- & $-\mathrm{OH}$ & Amid- & $-\mathrm{CONH}_{2}$ & \\
\hline
\end{tabular}

Abb. 5.3.: Untersuchte organische Komplexbildner und funktionelle Gruppe

Glucose, Citrat, Tartrat, 4-Hydroxybenzoesäure und Glycin wurden ebenso wie Fulvosäure aus einem Moorsee und Huminstoffeluate aus Cospuden im Rahmen der Arbeit von PLEßOW (1998) untersucht und werden dort ausführlich diskutiert. Im Rahmen der vorliegenden Arbeit wurden zusätzlich Testlösungen mit Harnstoff (p.a.), Alanin, Cystein und Threonin dem Speziationsschema unterworfen.

In Ergänzung zu den bei PLEßOW (1998) diskutierten Effekten der organischen Zusatzstoffe waren dabei folgende Phänomene zu beobachten: Harnstoff wirkte sich auf das Verhalten zahlreicher Elemente in der Ultrafiltration aus, und zwar dahingehend, dass die Rückhalteraten teilweise erhöht wurden, wie Abb. 5.6. zeigt (die Ergebnisse für alle Zusatzstoffe gibt Tab. A.9. im Anhang wieder). 


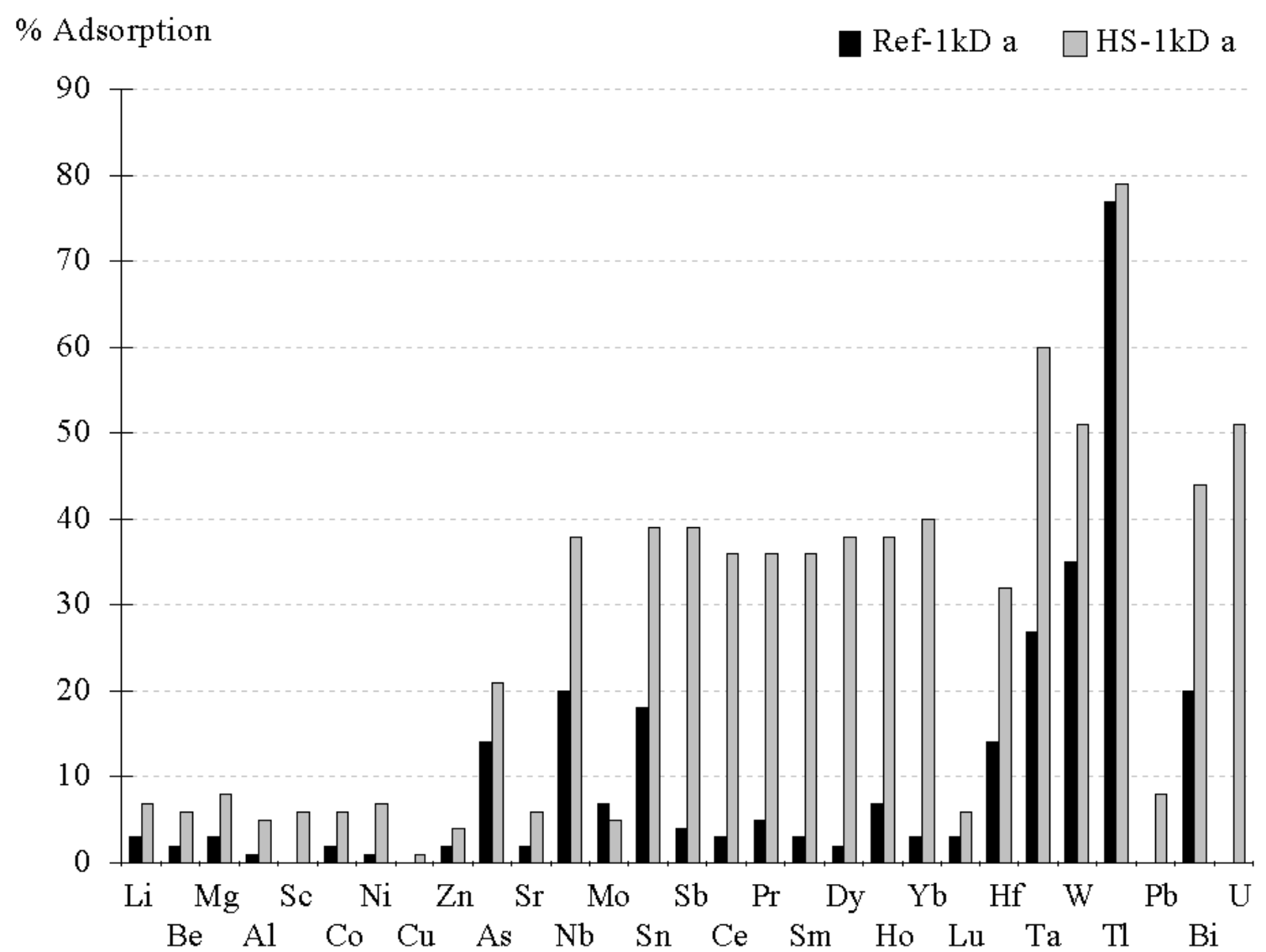

Abb. 5.6.: Auswirkungen des Zusatzes von Harnstoff auf die Ergebnisse der Ultrafiltration über eine $1 \mathrm{kD}$ Membran; Ref = Referenzlösung ohne organischen Zusatz; HS = Referenzlösung mit Harnstoff;

Ähnliche Beobachtungen machte PLEßOW (1998) bei den von ihm untersuchten organischen Zusätzen nur bei Weinsäure und Fulvosäure. Bei dem niedrigen pH-Wert der Testlösung ist davon auszugehen, dass die Stickstoffatome des Harnstoffmoleküls protoniert vorliegen und Anionen komplexieren können. Möglicherweise bilden sich dadurch Kolloide, die zurückgehalten werden. In den natürlichen Proben können Amino- und Amidstrukturen im DOC Sulfat komplexieren und damit zur Ausbildung kolloidaler Strukturen führen.

Beim Kationenaustausch zeigten die Aminosäuren Glycin, Alanin und Cystein in ihren Auswirkungen auf das Verhalten der gelösten Spurenmetalle die gleichen Trends, nämlich verringerte Rückhalteraten bei kationisch vorliegenden Elementen. Der Effekt von Cystein ist jedoch weitaus größer als der der beiden anderen Aminosäuren - ein Indiz für die Bedeutung von schwefelhaltigen funktionellen Gruppen bei der Komplexierung von Spurenelementen durch organische Substanzen. Eine Übersicht über die Komplexbildungskonstanten und Eigenschaften der Aminosäuren findet sich bei WILKINSON (1997). 
Abb. 5.7. zeigt exemplarisch die unterschiedlichen Effekte verschiedener Zusätze beim Kationenaustausch am Beispiel von Sb, W und Tl (die vollständigen Ergebnisse sind in Tab. A.10. im Anhang aufgeführt).

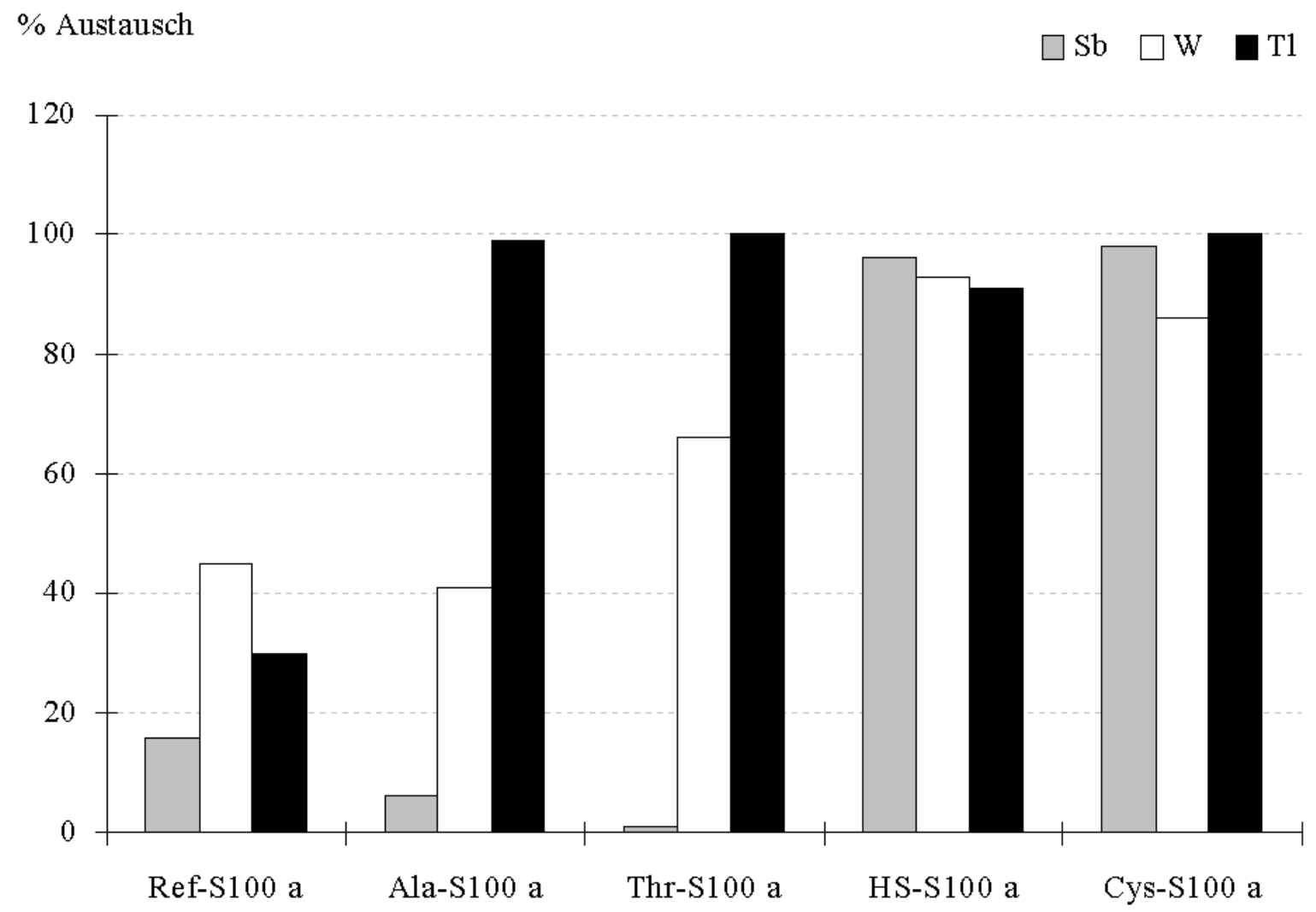

Abb. 5.7.: Effekt verschiedener organischer Zusätze beim Kationenaustausch am Beispiel von Antimon, Wolfram und Thallium; Ref = Referenzlösung ohne organischen Zusatz; Ala = Alanin, Thr $=$ Threonin, HS = Harnstoff, Cys $=$ Cystein.

In der Referenzlösung ohne organischen Zusatz wurden alle drei Elemente nur zu geringen Anteilen beim Kationenaustausch zurückgehalten. Organische Komplexbildner verändern dieses Verhalten teilweise drastisch. Tl wird in Anwesenheit von Alanin, Threonin, Harnstoff oder Cystein fast vollständig ausgetauscht; vermutlich wird es durch die negativ geladenen Gruppen der zwitterionischen Aminosäuren bzw. am Carbonyl-Sauerstoffatom im Harnstoff gebunden; die organischen Moleküle wiederum verfügen über kationisch geladene bzw. polarisierte Gruppen, die zu ihrer Bindung an den Ionentauscher führen. Ähnlich sind die Ergebnisse beim Wolfram. Dort ist allerdings ein differenzierterer Effekt zu beobachten, denn während Alanin keinen sichtbaren Einfluss auf die Austauschrate ausübt, erhöhen Threonin und noch mehr das schwefelhaltige Cystein die Austauschrate erheblich. Am größten sind die Auswirkungen von Harnstoff, der zu einem nahezu vollständigen Austausch von W am 
Kationenaustauscher führt. Sb, das in hydrolisierter Form als Anion in der Lösung vorliegt, bleibt durch die Anwesenheit von Alanin oder Threonin nahezu unbeeinflusst. Mit Harnstoff oder Cystein jedoch steigt auch hier die Austauschrate auf fast $100 \%$. Wahrscheinlich ist Cystein als Komplexligand stark genug, das Sb aus seinem Hydroxidionenkomplex zu lösen und als Kation in eine stabile Komplexstruktur einzubinden. Beim Harnstoff kann $\mathrm{Sb}$ in anionischer Form an eine der beiden $\mathrm{NH}_{3}{ }^{+}$-Gruppen gebunden werden; die andere steht weiterhin für die Bindung an den Kationenaustauscher zur Verfügung.

Beim Anionenaustausch waren keine Auffälligkeiten zu beobachten; die Resultate sind im Anhang in Tab. A.11. und A.12. dargestellt.

Die Verhältnisse in natürlichen Wässern sind weit komplizierter als die in synthetischen Lösungen mit einer einzigen, klar definierten organischen Substanz, die zudem nur einfache Funktionalitäten umfasst. In natürlichen Wässern liegt dagegen ein komplexes Gemisch unterschiedlichster Moleküle vor. Ihre Trennung und Analytik erfordert große Mengen Materials, ist extrem aufwendig und in der Regel für die Betrachtung wenig sinnvoll. Stattdessen werden die organischen Substanzen normalerweise als Summenparameter erfasst. Ein Maß für die Menge an gelösten Organika ist der gelöste organische gebundene Kohlenstoff DOC (=dissolved organic carbon). Eine sehr umfassende Beschreibung der natürlichen gelösten organischen Substanz findet man z.B. bei THURMAN (1985) und bei RANVILLE und MACALADY (1997).

Die DOC-Gehalte in verschiedenen Wässern und Böden variieren sehr stark. Generell nimmt er in Böden von oben nach unten ab. Einsickerndes Wasser trägt den DOC aus verrottendem Pflanzenmaterial und bakteriellen Stoffwechselvorgängen aus der Humusschicht in die oberste Bodenschicht, wo er mit Kationen wie Fe und Al Komplexe bildet. In tonreichen Horizonten wird ein großer Teil des DOC an Mineraloberflächen adsorbiert. In noch tieferen Bereichen wird der DOC bakteriell zu $\mathrm{CO}_{2}$ oxidiert oder zu Methan reduziert. CRONAN und AIKEN (1985) geben für Porenwässer in A- und oberen B-Horizonten DOC-Gehalte von 10 $30 \mathrm{mg} \mathrm{C} / \mathrm{l}$ an; in unteren B- und in C-Horizonten liegen die Konzentrationen nur noch bei 2 $5 \mathrm{mg}$ C/l. In 100 Grundwässern aus den USA fanden LEENHEER et al. (1974) einen Medianwert von $0,7 \mathrm{mg} \mathrm{C} / \mathrm{l}$ mit einer Bandbreite von 0,2 bis $15 \mathrm{mg} \mathrm{C} / \mathrm{l}$. In Oberflächenwässern liegen die DOC-Gehalte deutlich höher; nach THURMAN (1985) reichen sie von $2 \mathrm{mg} \mathrm{C/1}$ in arktischen und alpinen Gewässern bis über $25 \mathrm{mg}$ C/l in Sumpf- und Moorgewässern.

Abb. 5.8. zeigt die Größenordnungen der Durchmesser verschiedener organischer Bestandteile, die in Wässern gefunden werden. 


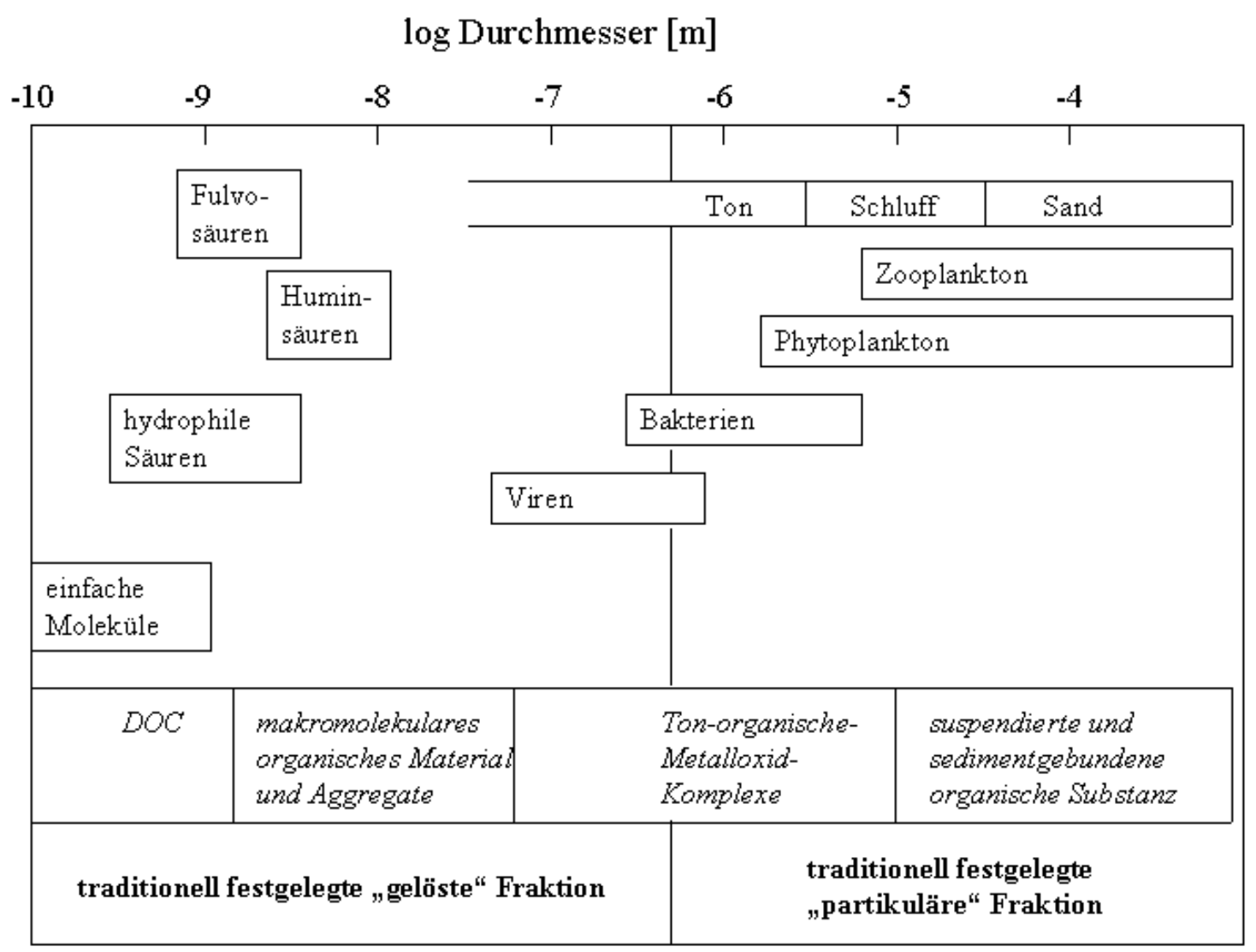

Abb. 5.8.: Das Kontinuum von partikulärem und gelöstem organischen Kohlenstoff in natürlichen Wässern (nach THURMAN 1985).

Die Größenangabe der organischen Stoffe nach dem Durchmesser ist bei Filtrationen unüblich. Stattdessen werden in der Regel die Größenverhältnisse als Gewichte in Dalton bzw. Kilodalton $(\mathrm{kD})$ angegeben. Dabei entspricht eine Masse von $1 \mathrm{kD}$ in etwa einem Moleküldurchmesser von $1 \mathrm{~nm}$. Ein exakter Bezug zwischen Molekülmasse und -gewicht ist allerdings kaum herzustellen; so stellen die Porengrößen bei der Ultrafiltration auch keine scharfen Trenngrenzen dar, denn ob ein Molekül eine Pore passieren kann, hängt auch von seiner Form ab.

Der DOC besteht aus einer weiten Bandbreite von Substanzen; ihr Molekulargewicht liegt nicht weit über $1 \mathrm{kD}$, und sie besitzen eine große Zahl polarer und/oder ionisierbarer funktioneller Gruppen.

Entgegen dem Schema von ThuRMAN (1985) in Abb. 5.8. werden dem DOC üblicherweise auch die kleineren Huminstoffe bis 10000 Dalton zugerechnet, zumal die Filtration in der Regel keine scharfe Bestimmung von Ausschlussgrößen zulässt.

Der DOC besteht danach aus Huminstoffen, also Humin- und Fulvosäuren, daneben aus kleineren organischen Molekülen wie Bruchstücken von Proteinen und Kohlehydraten, aus 
Aminosäuren, kleinen organischen Säuren und Zuckern. Abb. 5.9. zeigt die Zusammensetzung des DOC für ein durchschnittliches Flusswasser. Die Fulvo- und Huminsäuren haben ein Molekulargewicht zwischen 500 und 1000 bzw. 1000 und 5000 Dalton. Fulvosäuren werden als gelöste Substanz klassifiziert, während Huminsäuren an der Grenze zwischen gelöster und kolloidaler Substanz liegen. Ein umfangreiches Bild zur Isolierung, Charakterisierung, strukturellen und komplexierenden Eigenschaften und zur Umweltrelevanz dieser Stoffe vermitteln GAFFNEY et al. (1996).

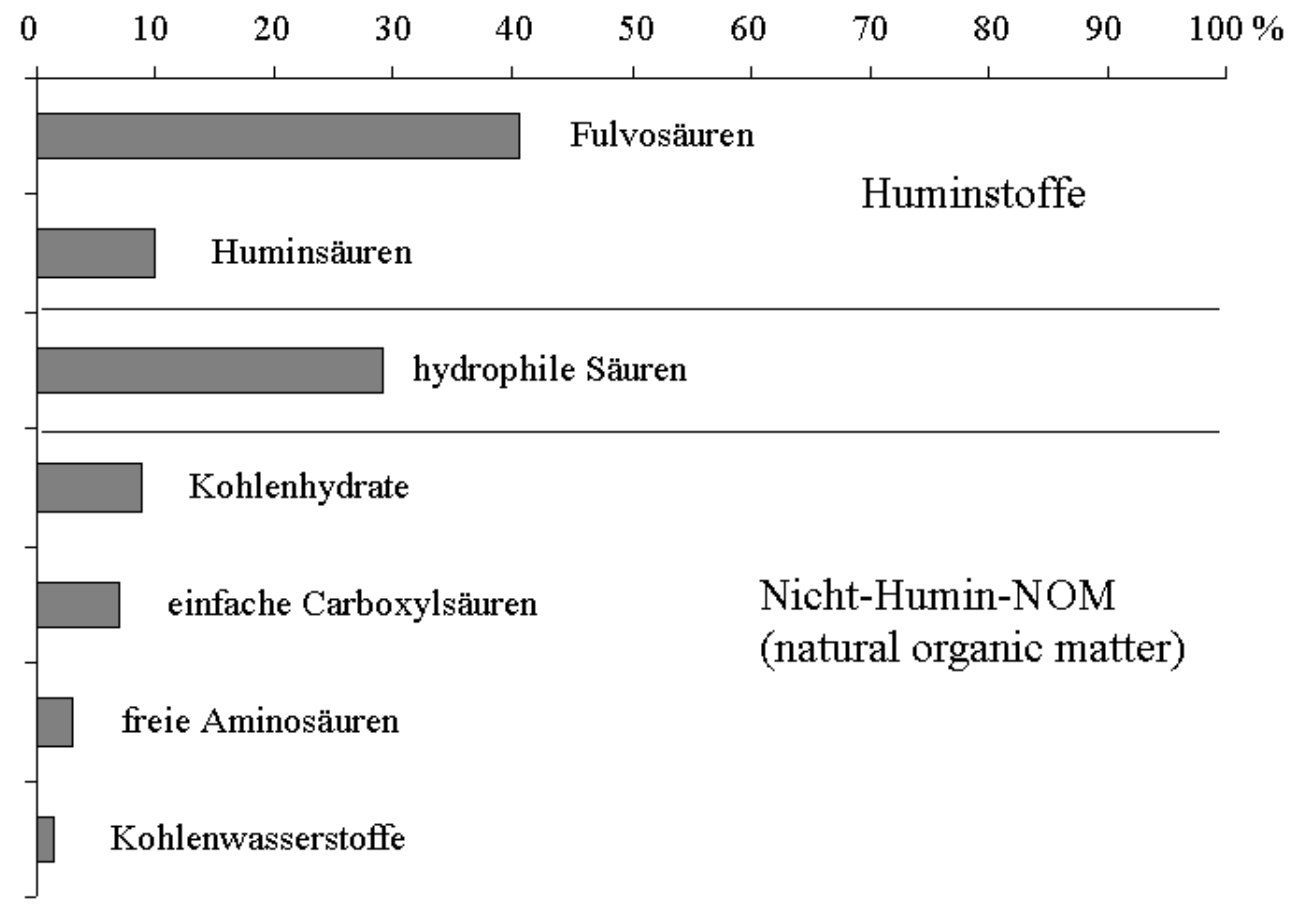

Abb. 5.9.: Histogramm des DOC für ein durchschnittliches Flusswasser mit einem DOC-Gehalt von $5 \mathrm{mg} / \mathrm{l}$ (nach THURMAN 1985).

Die wichtigsten komplexbildenden Bestandteile des DOC sind neben den Humin- und Fulvosäuren hydrophile Säuren, Pigmente und Aminosäuren. Kationen werden durch die funktionellen Gruppen dieser Moleküle gebunden, und zwar durch die verschiedenen Gruppen in der Reihe $-\mathrm{O}^{-}>-\mathrm{NH}_{2}>-\mathrm{N}=\mathrm{N}->-\mathrm{COO}^{-}>-\mathrm{O}->\mathrm{C}=\mathrm{O}$ mit abnehmender Stärke. Aminosäuren bilden etwa $3-5 \%$ des DOC. Ihre Komplexbildungskonstanten sind ähnlich hoch wie die von Huminsäuren.

Die Zusammensetzung des DOC unterscheidet sich nicht nur nach verschiedenen klimatischen Zonen und Standorten, sondern auch nach jahreszeitlichen Aspekten (CURTIS 1998). Zahlreiche Studien befassen sich damit, Huminsäuren und DOC wenigstens durch Summenparameter zu beschreiben (z.B. ABBT-Braun et al. 1990). De NOBILI und ForNASIER 
(1996) arbeiteten einen Zusammenhang zwischen molekularer Größe und elektrophoretischer Mobilität von Huminstoffen heraus. Versuche, auch die Wechselwirkungen von gelösten Metallionen mit komplizierten natürlichen Organika $\mathrm{zu}$ beschreiben, finden sich in der Literatur z.B. bei Perdue (1998), VAN HeES und Lundström (2000), LundeGARD und Kharaka (1990), Wells et. al. (1998) und Vogl und Heumann (1997). Die Komplexierung durch den DOC beeinflusst nicht nur die Adsorptionsraten an Oberflächen und Partikeln, sondern auch die allgemeine Reaktivität der Metallionen (HERING und MoREL 1990). Bei neutralem $\mathrm{pH}$ gilt für die Bindungsstärke von Metallkationen mit pedogenen (von Landpflanzen und Mikroorganismen stammenden) Huminstoffen die Reihe $\mathrm{Pb}^{2+}>\mathrm{Cu}^{2+}>\mathrm{Ni}^{2+}>\mathrm{Co}^{2+}>\mathrm{Zn}^{2+}>\mathrm{Cd}^{2+}>\mathrm{Fe}^{2+}>\mathrm{Mn}^{2+}>\mathrm{Mg}^{2+}$, bei $\mathrm{pH}=3$ dagegen $\mathrm{Fe}^{3+}>\mathrm{Al}^{3+}>\mathrm{Cu}^{2+}>\mathrm{Ni}^{2+}>\mathrm{Co}^{2+}>\mathrm{Pb}^{2+}>\mathrm{Ca}^{2+}>\mathrm{Zn}^{2+}>\mathrm{Mn}^{2+}>\mathrm{Mg}^{2+}$.

Für aquogene Huminstoffe fanden MANTOURA et al. (1978) folgende Abfolge: $\mathrm{Hg}^{2+}>\mathrm{Cu}^{2+}>\mathrm{Ni}^{2+}>\mathrm{Co}^{2+}>\mathrm{Mn}^{2+}>\mathrm{Cd}^{2+}>\mathrm{Ca}^{2+}>\mathrm{Mg}^{2+}$.

Obwohl sich diverse Studien mit der Interaktion zwischen DOC und einzelnen Kationen beschäftigen (z.B. Reuter und Perdue 1977, van DEN BERG und Kramer 1979, MATthess und Pekdeger 1980, Frimmel und Geywitz 1983, McKnight et al. 1983, Gruhn et al. 1985, Frimmel und Christman 1988, De Witt et al. 1991, Stone 1997, Frimmel und ABBT-BRAUn 1997), ist die Komplexierung anorganischer Ionen durch DOC und deren Auswirkungen auf das Verhalten der anorganischen Ionen noch kaum geklärt.

\subsubsection{Adsorption}

Wie bereits in der Einführung angesprochen, ist neben der Ausfällung oder Copräzipitation von Elementen die Adsorption der wichtigste Kontrollmechanismus für Elementkonzentrationen in wässrigen Lösungen. Als Adsorptionsflächen kommen dabei vor allem Minerale in Frage, die aufgrund von Störungen im Kristallgitter freie HydroxidGruppen auf der Oberfläche tragen, an denen sich Kationen aus der Lösung anlagern können (LANGMUIR 1997).

Zur Adsorption von Elementen an verschiedenen Oberflächen gibt es zahlreiche Studien, jedoch in der Regel mit sehr eingeschränkten Parametern; meist werden nur wenige Elemente (z.B. Zn bei JAMES und MACNAUGHTON 1977 und TESSIER et al. 1989) an der gut definierten Oberfläche eines bestimmten Minerals beobachtet. Eine gute Einführung gibt STUMM (1992). Als Minerale mit adsorptiven Oberflächen werden z.B. Eisenhydroxide, Manganhydroxide (LAXEN und Sholkovitz 1981, Routh und IKramudDin 1996, LAXEN 1984/85, TeSSIER et 
al. 1985, JOHNSON 1986, LIENEMANN et al. 1997), aber auch sulfidische Mineralphasen (JEAN und BANCROFt 1986, Fuge et al. 1994) wie Pyrit (AL et al. 1997) diskutiert. In der Bodenpassage, aber auch in den heterogen geschütteten Kippen in ehemaligen Tagebauen bieten Tonmineraloberflächen eine Vielzahl an Bindungs- oder Einlagerungsmöglichkeiten für anorganische Kationen. Auch Braunkohlereste im Kippensediment nehmen durch ihre große Oberfläche und ihr Bindungsvermögen für organische Substanzen Einfluss auf die Zusammensetzung der Sickerwässer.

Die Anwesenheit organischer Komplexbildner kann das Adsorptionsverhalten verschiedener Elemente stark beeinflussen (SINGH et al. 1996, DAVIS 1984), indem die Elemente als Komplexe in der Lösung stabilisiert werden oder aber die Adsorption durch die organische Substanz gefördert wird.

Vier mögliche Modelle werden für den Einfluss organischer Komplexbildner auf das Adsorptionsverhalten anorganischer Ionen diskutiert: Das „Liganden-Modell“ geht davon aus, dass die Metalladsorption an Oberflächen durch adsorbierte Huminstoffe gefördert wird; entweder werden die Metall dabei vor oder nach der Adsorption des Huminstoffs an die Oberfläche durch den Huminstoff komplexiert. Das zweite Modell besagt, dass die Interaktionen zwischen Metallen und Huminstoffen stärker sind, wenn die Huminstoffe adsorbiert sind als bei freien Huminstoffen. Modell 3 postuliert, die anorganische Oberfläche bzw. die elektrische Doppelschicht auf ihr werde durch die Anwesenheit adsorbierter Huminstoffe so verändert, dass sie leichter Metallionen adsorptiv binden kann. Das vierte Modell schließlich formuliert, dass Huminstoff-Metall-Komplexe leichter aus der Lösung adsorbiert werden als rein anorganische Spezies. Der Einfluss von Huminstoffen auf die Adsorption von $\mathrm{Cd}, \mathrm{Cu}$, $\mathrm{Ni}$ und $\mathrm{Pb}$ an Eisenhydroxid-Oberflächen wurde z.B. von LAXEN (1985) untersucht. Er ermittelte für $\mathrm{Cd}, \mathrm{Cu}$ und $\mathrm{Ni}$, dass die Copräzipitation in Anwesenheit von Huminsäuren gefördert wurde. Dabei beschrieb ein „ligand like“-Adsorptionsmodell das Verhalten der Elemente am besten. Ein theoretisches Modell für die Koadsorption von anorganischen Ionen und organischen Molekülen auf Quarz findet man bei REA und PARKS (1990). Eine umfassende Arbeit über die Adsorption von $\mathrm{As}, \mathrm{Cd}$, $\mathrm{Ni}$ und $\mathrm{Pb}$ an Quarz, Kaolinit, Feldspat, Calciumcarbonat, $\mathrm{Mn}$ - und Fe-Hydroxiden in Abhängigkeit von der Anwesenheit von Huminsäuren lieferte HodEl (1994). Er fand heraus, dass die Huminstoffe die Adsorption der anorganischen Kationen stark verringern und ihre Austragung aus den Versuchssäulen erleichtern. Arsen zeigt zudem eine stark erhöhte Mobilität, wenn es als Anion vorliegt. HodEL (1994) konnte weiterhin nachweisen, dass der Einfluss der Huminstoffe (Förderung der Festlegung an der Mineraloberfläche oder erhöhte Mobilität 
durch Komplexierung in wässriger Lösung) auch von der Konzentration des jeweiligen Elementes in der Lösung abhängt.

Die umfassendste Studie zum Einfluss von Huminstoffen auf das Adsorptionsverhalten zahlreicher Elemente stammt von TAKAHASHI et al. (1999). Sie beschreiben das Adsorptionsverhalten von 32 Elementen an Kaolinit und Silicagel unter dem Einfluss von Huminsäuren und vergleichen die Ergebnisse mit den berechneten Resultaten aus einem Gleichgewichtsmodell. Sie ermittelten dabei, dass sich die untersuchten Elemente in sechs verschiedene Gruppen aufteilen lassen. Danach werden REEs, Y und Sc als eine Gruppe am stärksten durch die Anwesenheit von Huminsäuren beeinflusst. Sowohl in der wässrigen Phase als auch bei der Adsorption an pseudokolloidalen Partikeln aus anorganischem Kern und organischem Mantel spielen organische Komplexbildner eine entscheidende Rolle. Sc wird von Humaten etwas weniger beeinflusst als Y und die REE. Bei der zweiten Gruppe dagegen, den Erdalkalimetallen, stehen die Bildung von Humatkomplexen und die Adsorption an Mineraloberflächen im Wettstreit. Bei hohen Konzentrationen von Huminsäuren bilden Sr und $\mathrm{Ba}$ Humatkomplexe; sonst liegen sie in Wasser als freie Kationen vor. Die zweiwertigen Übergangsmetalle Mn, Co und $\mathrm{Zn}$ als dritte Gruppe können gleichermaßen Humatkomplexe bilden, adsorbiert werden oder als anorganische Komplexe vorliegen. In größerem Ausmaß beeinflusst die Humatkomplexierung dagegen die vierte Gruppe, nämlich die trivalenten Übergangsmetalle $\mathrm{Fe}$ und $\mathrm{Cr}$ bei niedrigem $\mathrm{pH}$, während bei höheren $\mathrm{pH}$-Werten Adsorption und Hydrolyse an Bedeutung gewinnen. Die Verteilung der Elemente der fünften Gruppe, Ru, $\mathrm{Rh}$ und Pt, wird indirekt vom Vorhandensein von Huminstoffen kontrolliert; sie verhindern die Adsorption an anorganischen Oberflächen, während die Komplexbildung für diese Elemente keine Rolle zu spielen scheint. Für $\mathrm{Ga}, \mathrm{Zr}$ und $\mathrm{Hf}$ als sechste Gruppe sind Hydrolyse und Adsorption die entscheidenden Faktoren bei der Speziation. Alkalimetalle interagieren kaum mit Huminsäure. Oxoanionen von As und Se werden wiederum indirekt beeinflusst, indem durch einen organischen Überzug auf Partikeln die Adsorption der Anionen verhindert wird.

Die Adsorption an Mn- und Fe-Oxidoberflächen in Anwesenheit organischer Substanz untersuchten TESSIER et al. (1996). Die Studie ergab, dass bei höheren pH-Werten die organische Substanz für die Adsorption von $\mathrm{Ca}, \mathrm{Cd}, \mathrm{Cu}, \mathrm{Mg}, \mathrm{Ni}, \mathrm{Pb}$ und $\mathrm{Zn}$ keine Rolle spielte; die Kationen wurden direkt an die freien OH-Gruppen der Hydroxide gebunden. Bei $\mathrm{pH}-$ Werten unterhalb von 5 dagegen wurden die Metalle an die organischen Überzüge der Minerale gebunden. 
Die kurze Literaturübersicht zeigt bereits, wieviele verschiedene Parameter für das Adsorptionsverhalten von Metallen eine Rolle spielen und wie komplex die beteiligten Vorgänge sind. In Kapitel 5.3. wird darauf Bezug genommen, wenn es um die Kontrolle der absoluten Elementkonzentrationen und die Speziation der Spurenmetalle in unseren Proben geht.

\subsubsection{Kolloide und Partikel}

Nicht nur die Adsorption an größeren Oberflächen ist für die Speziation von großer Bedeutung, auch die adsorptive Bindung an kolloidale oder subkolloidale Partikel wirkt sich auf das beobachtete Verhalten der Elemente aus (LuOMA und DAVIS 1983, BENOIT et al. 1994). Kolloide sind sehr kleine Partikel, die in Lösung stabil sind, nicht sedimentieren und sich durch eine hohe Dichte an geladenen Gruppen auf ihrer Oberfläche auszeichnen. Die wichtigsten anorganischen Komponenten, die zur Kolloidbildung neigen, sind die Hydroxide von Mangan und Eisen, aus denen bei der Hydrolyse kolloidale Polymere entstehen, bevor sie als amorphe oder kristalline Sedimente aus der Lösung ausfallen. Kontrolliert werden diese Prozesse vor allem durch den $\mathrm{pH}$ und das Redoxmilieu. Andere Elemente, die wie $\mathrm{Pb}, \mathrm{Ac}$ oder Th ebenfalls zur Eigenkolloidbildung neigen, liegen in der Regel in $\mathrm{zu}$ geringen Konzentrationen vor, als dass sie eine große Rolle spielen könnten. Sie werden adsorptiv an bereits vorhandene Kolloide gebunden. Kolloidale und subkolloidale organische Liganden haben dagegen ein große Bedeutung für die Speziation von gelösten Spurenmetallen, die nach DUFFY et al. (1989) zur $20-91 \%$ an organische Liganden gebunden sind. Die Anwesenheit von anorganische Kationen fördert die Tendenz von Fulvo- und Huminsäuren zur Aggregation und Kolloidbildung.

Bislang liegen vor allem Studien vor, die sich mit der Rolle von schwebenden oder sinkenden Partikeln in Meerwasser befassen (HONEYMAN 1992). BALISTRIERI et al. (1981) schildern, dass sich die Bindung zwischen Partikeln und Metallionen durch ähnliche Komplexbildungskonstanten beschreiben lässt wie die Interaktion zwischen Metallionen und organischer Substanz. Sie postulierten daher, dass in natürlichen Meerwässern auftretende Partikel durch eine Hülle von Huminsäuren umschlossen seien, die die adsorptiven Eigenschaften des Teilchens bestimmt. Die Modelle dafür wurden oben bei der Adsorption an anorganischen Oberflächen bereits beschrieben. SANTSCHI et al. (1997) sowie MURPHY und ZACHARA (1995) und BENOIT (1995) richten ihr Augenmerk ebenfalls auf die Rolle der organischen Moleküle für die Verteilung von Ionen zwischen Kolloiden, Partikeln und Lösung. Weitere Arbeiten, die sich mit der Rolle von Partikeln bei der Entfernung von 
Metallionen aus Wasser befassen, finden sich z.B. bei SiGg (1987) und SiGG et al. (1987). LOFTS und TIPPING (1998) entwickelten ein Modell zur Berechnung der Komplexbildung zwischen natürlichen Partikeln und anorganischen Ionen anhand eines Gleichgewichtsmodells. Eine Studie über die Differenzierung von Metallionen ausgehend von der Partikelgröße in Flusswässern erstellten Douglas et al. (1999). Auch kolloidal vorliegende Partikel, die sich nicht ohne weiteres aus der Lösung abtrennen lassen, können als Adsorbens wirken (MOREL und GSCHWEND 1987, DuPRÉ et al. 1999). Dafür kommen in den von uns beobachteten Wässern vor allem Eisenhydroxidpartikel in Frage. Eine Charakterisierung dieser Teilchen findet sich z.B. bei MiLl (1980), bei BufFLE et al. (1989) und bei DAVISON (1992). Auch ihre Struktur und Größe unterliegt wiederum Einflüssen durch die organischen Substanzen in den Wässern (LIANG und MORGAN 1990).

\subsubsection{Redoxchemie}

Ein weiterer wichtiger Faktor im Verhalten anorganischer Stoffe in Wässern ist ihre Redoxchemie. Wie bereits in der Einführung dieses Kapitels beschrieben, hängt das Verhalten einiger Elemente entscheidend von ihrem Redoxzustand ab; zu nennen sind hier insbesondere $\mathrm{Fe}^{\mathrm{II} / \mathrm{III}}, \mathrm{Mn}^{\mathrm{II} / \mathrm{IV}}, \mathrm{Cu}^{\mathrm{I} / \mathrm{II}}, \mathrm{Cr}^{\mathrm{III} / \mathrm{VI}}, \mathrm{Co}^{\mathrm{II} / \mathrm{III}}, \mathrm{V}^{\mathrm{III} / \mathrm{IV} / \mathrm{V}}, \mathrm{Sb}^{\mathrm{III} / \mathrm{V}}, \mathrm{Sn}^{\mathrm{II} / \mathrm{IV}}, \mathrm{As}^{\mathrm{III} / \mathrm{V}}, \mathrm{U}^{\mathrm{IV} / \mathrm{VI}}$ u.a. (DAVISON 1985, LANGMUIR 1997, SigG 2000). pH-Eh-Diagramme bieten eine Möglichkeit, die Stabilität verschiedener gelöster und fester Phasen anhand dieser beiden Parameter vorherzusagen (KÖLling et al. 2000, WISOTZKY 2000). Doch im Unterschied zu synthetischen Lösungen, in denen sich die Redoxverhältnisse bei Kenntnis der Elementkonzentrationen noch mit einigermaßen großer Sicherheit erfassen lassen, sind die Gegebenheiten in natürlichen Wässern weit komplizierter. Häufig liegt dort nicht ein dominierendes Redoxpaar vor, sondern eine Vielzahl von Redoxpaaren führt zu einem Mischpotential. Viele Redoxprozesse brauchen sehr lange, bis es zu einer Gleichgewichtseinstellung kommt. Unter diesen Voraussetzungen sind Redoxpotentiale analytisch kaum exakt bestimmbar (KöLLING 2000). Auch beeinflussen nicht nur Mineraloberflächen die stattfindenden Redoxprozesse; Pflanzen und Mikroorganismen greifen in das Geschehen ein, indem sie Reaktionen katalysieren oder überhaupt erst ermöglichen, die andernfalls nicht ablaufen würden. Der wichtigste pflanzliche Redoxprozess ist die Photosynthese; die Umkehrreaktion der Photosynthese ist die Atmung, die vor allem in tierischen Organismen, aber auch in Pflanzen abläuft (DREVER 1997). Steht kein molekularer Sauerstoff zur Verfügung, kommen außerdem Verrottungsprozesse dazu. Jeder Reduktionsprozess benötigt einen Elektronenlieferanten; umgekehrt müssen die Elektronen, die bei einer Oxidationsreaktion frei werden, durch einen Akzeptor aufgenommen 
werden. Im Kontext der vorliegenden Arbeit sind vor allem zwei Prozesse als Antagonisten der Pyritoxidation von großem Interesse, die die Redoxchemie der untersuchten Wässer bestimmen: die Reduktion dreiwertigen Eisens und die Sulfatreduktion (BOULEGUE und MICHARD 1979). Beide Vorgänge beruhen auf mikrobieller Aktivität in anoxischen Wässern, wie sie in tieferen Schichten von Seen, aber auch im Boden auftreten. Die Summenformeln lauten:

$\mathrm{C}_{\text {org }}+4 \mathrm{Fe}(\mathrm{OH})_{3}+8 \mathrm{H}^{+} \rightarrow \mathrm{CO}_{2}+4 \mathrm{Fe}^{2+}+10 \mathrm{H}_{2} \mathrm{O}$

und

$\mathrm{SO}_{4}{ }^{2-}+2 \mathrm{C}_{\text {org }}+2 \mathrm{H}_{2} \mathrm{O} \rightarrow \mathrm{H}_{2} \mathrm{~S}+2 \mathrm{HCO}_{3}^{-}$

In Folge der beiden Reaktionen kann es zur Neubildung von Pyriten oder anderen Metallsulfiden kommen; bei der Eisenreduktion wird zugleich Säure gebunden. Beide Reduktionsvorgänge sind auf das Vorhandensein abbaubarer organischer Substanz angewiesen. HERZSPRUNG et al. (1998) belegten, dass der für versauerte Weichwasserseen beschriebene Prozess der Photoreduktion von $\mathrm{Fe}^{\mathrm{III}} \mathrm{zu} \mathrm{Fe}^{\mathrm{II}}$ bei gleichzeitiger Oxidation des DOC auch für saure Tagebauseen gültig ist. PACKROFF et al. (2000) ermittelten am Restsee 111 in der Lausitz, dass in den oberflächennahen Schichten pyritversauerter Seen unter Einwirkung von Sonnenlicht etwa $1-5 \%$ des sehr hohen Gesamtgehalts an $\mathrm{Fe}^{\mathrm{III}}$ in $\mathrm{Fe}^{\mathrm{II}}$ umgewandelt wird, wobei ein beträchtlicher Anteil des DOC $\mathrm{zu} \mathrm{CO}_{2}$ abgebaut wird und in die Atmosphäre entweicht. Dadurch werden die DOC-Konzentrationen in den Wässern soweit verringert, dass sich weitere Mikroorganismen nur sehr schwer ansiedeln können. Um dies auszugleichen und die bakterielle Säurebindung und Sulfatreduktion zu fördern, werden teilweise in pyrithaltiges Kippenmaterial bzw. versauerte Seen organische Materialien (Rindenmulch, Laub o.ä.) eingebracht, um ausreichend Substrat für Bakterien bereitzustellen. Im Schwarzwald werden Erzbergbauhalden mit Klärschlamm abgedeckt (HoDEL 1994). In Kanada wurde jedoch beobachtet, dass es zu unerwünschten Nebeneffekten kommt, denn die organischen Materialien setzen beim Abbau Komplexbildner frei. Dadurch werden Schwermetalle komplexiert und mobilisiert, und die ökotoxischen Kippeninventare verteilen sich weit über das unmittelbar betroffene Areal hinaus. 


\subsection{Experimentelle und rechnerische Verfahren zur Speziation}

\subsubsection{Experimentelle Speziationsverfahren}

Die Mobilität von Metallen bzw. Halbmetallen, ihre Bioverfügbarkeit und physiologische Wirkung hängen eng mit den Zustands- bzw. Bindungsformen, den Metallspezies, zusammen. Die verschiedenen stabilen Metallspezies besitzen unterschiedliche Massen und überwiegend charakteristische Größen, die als Grundlage zur Fraktionierung, z.B. durch Ultrafiltration, herangezogen werden können. In der Literatur finden sich zahlreiche detaillierte Arbeiten zur Elementspeziation. Allerdings beschäftigen diese sich häufig nur mit einzelnen Elementen (z.B. VAN LUIK und JURINAK 1979, SUNDA 1979, Boughriet et al. 1992, ERICKSON und Helz 2000, TAILLEFERT et al. 2000) oder synthetischen Wässern mit einfacher Zusammensetzung.

Natürliche Wässer stellen als hochkomplexe Systeme weit höhere Anforderungen an den analytischen Prozess. In zahlreichen Publikationen sind Speziationsschemata zur Untersuchung von Wasserproben beschrieben worden, die keine exakte Charakterisierung einzelner Verbindungen zum Ziel haben, sondern lediglich die Zuordnung zu verschiedenen Speziesgruppen. Mehr ist mit vertretbarem Aufwand nicht $\mathrm{zu}$ erreichen, denn alle physikalischen Trennmethoden sind massive Eingriffe in bestehende Systeme. Dabei können eine Reihe von Fehlern auftreten, wie z.B. Koagulation, Adsorption am Filtermaterial, Mitreißeffekte, Veränderung der Porengröße durch Verstopfung, Kontamination etc.. Diese möglichen Fehler werden in der Literatur sehr kontrovers diskutiert.

Den instrumentellen Konzentrationsbestimmungen geht eine Trennung der einzelnen Spezies in einzelne Fraktionen voraus. Die Ultrafiltration mit Membranfiltern unterschiedlicher Poren- und damit Ausschlussgrößen kann Hinweise darauf geben, ob Anteile der Metalle kolloidal gelöst und an Makromoleküle assoziiert sind oder molekular-dispers in niedermolekularen Spezies vorliegen.

Zur Unterteilung in kationische, anionische und neutrale Spezies können diverse Ionenaustauscher eingesetzt werden.

Experimentelle Verfahren zur Elementspeziation finden sich in der Literatur in zahlreichen Arbeiten und nach unterschiedlichen Methoden. Eine Übersicht über einige gängige Verfahren findet man bei MACH et al. (1996), BouRg (1995), FLORENCE und BATLEY (1980), Buffle (1981, 1988), SCHWEdT (1981,1997), SAUER (1990) und SAUER und LIESER (1994, 1995). Die geläufigste Speziationsmethode ist die sequentielle Extraktion von Sedimenten 
oder Wässern. Sie benötigt jedoch sehr große Probenmengen. Ein Verfahren zur Untersuchung kleinerer Probenmengen, wie z.B. Porenwässer, beschreiben BRÜGMANN et al. (1994), die Meer- und Porenwasser aus dem Ostseegebiet untersuchten. Das Schema ist unserem Verfahren ähnlich; es besteht aus drei konsekutiven Schritten: Chromatographische Abtrennung von Kolloiden, Anionenaustausch und Kationenaustausch.

Die teilweise sehr sauren, sulfathaltigen Wässer aus Cospuden und der Lausitz stellen hohe Anforderungen an das verwendete Material. Das von uns eingesetzte Speziationsschema entwickelte PLEßOW (1998) nach umfangreichen Vorversuchen; es ist in Abb. 5.10. dargestellt.

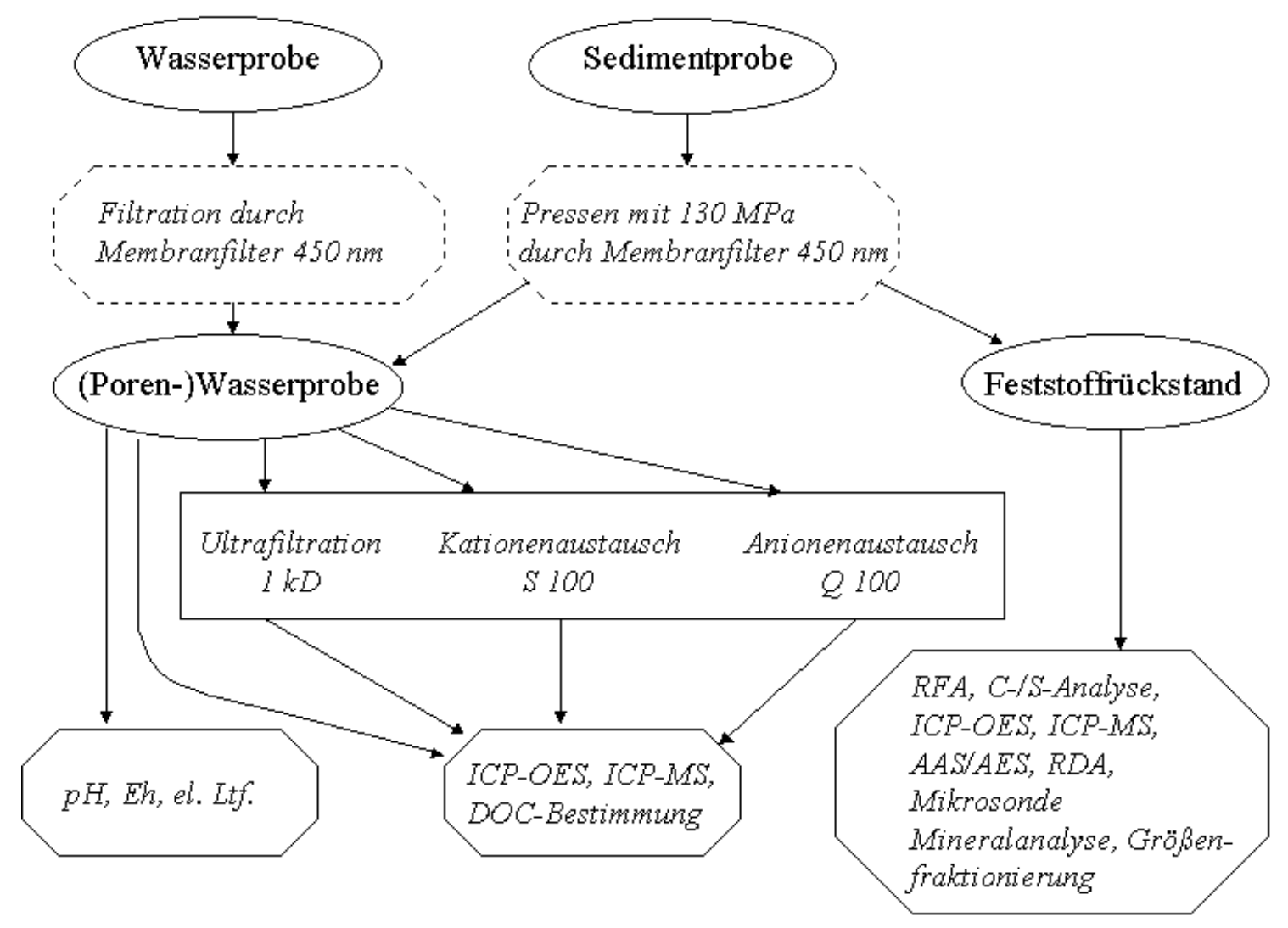

Abb. 5.10.: Speziationsschema zur operationell definierten Trennung von Gruppen unterschiedlicher Migrationsformen gelöster Elemente in Wässern und Porenlösungen nach PLEßOW (1998).

Für die zu erwartenden Lösungszusammensetzungen erwiesen sich eine einstufige Ultrafiltration bei $1 \mathrm{kD}$ und parallel dazu jeweils ein Kationen- sowie Anionenaustausch als geeignet. Leider sind die Mengen an isolierten Kolloiden aus der Ultrafiltration aus 20 bis 100 ml Probenvolumen viel zu gering, um mittels GC, NMR, IR in Kombination mit der ICP-MS und ICP-OES charakterisiert werden zu können (MARLEY et al. 1992).

Ein für diese Anwendung neuartiges Ionenaustauschverfahren ermöglicht eine sehr schnelle Arbeitsweise, so dass unerwünschte Veränderungen in der Probe weitgehend vermieden 
werden können. Dafür wurden regenerierbare Membranadsorbereinheiten mit einer Membranfläche von $100 \mathrm{~cm}^{2}$ eingesetzt, die ausreichende Kapazitäten zur Behandlung von 50 $\mathrm{ml}$ Probenvolumen haben. Der entscheidende Vorteil dieses neuartigen Ionenaustauschverfahrens liegt in der außerordentlich kurzen Austauschzeit von $50 \mathrm{ml}$ in ca. 10 Minuten. Gegenüber dem herkömmlichen Batch-Verfahren, das außerdem noch eine zusätzliche Filtration erfordert, wird so die Gefahr unerwünschter Nebenreaktionen oder Gleichgewichtsveränderungen minimiert. Die Auswirkungen der sich mit dem Ionenaustausch zwangsläufig ändernden Wasserchemie in den Lösungen sind am geringsten, wenn der Kationenaustausch gegen $\mathrm{H}^{+}$und der Anionenaustausch gegen $\mathrm{Cl}^{-}$erfolgt.

Zusätzlich zur Analyse der Wässer wurden phasen- und elementaranalytische Untersuchungen der Mineralphasen durchgeführt.

Adsorptionseffekte sollten ebenfalls praktisch untersucht werden; Vorversuche mit Aktivkohle scheiterten jedoch an hohen Blindwerten (PLEßOW 1998). Versuche zur Adsorption an Kaolinit und Quarz, die im Rahmen dieser Arbeit durchgeführt wurden, führten zu Problemen: Zum einen kam es ebenfalls teilweise zu hohen Blindwerten, zum anderen erwies es sich in den Vorversuchen als extrem schwierig, konstante $\mathrm{pH}-$ Werte einzuhalten. Diese Schwierigkeiten in den Griff zu bekommen hätte den zeitlichen Rahmen dieser Arbeit gesprengt. Auf die Fortsetzung experimenteller Versuche zur Adsorption wurde daher verzichtet.

Um wenigstens quantitative Aussagen über die Speziation der wichtigsten Elemente in den einzelnen Proben treffen $\mathrm{zu}$ können, wurden die Ergebnisse des experimentellen Speziationsverfahrens mit denen der Berechnung mit Hilfe des Programms MINTEQA2 verglichen.

\subsubsection{Computerprogramme zur Speziationsberechnung}

Wegen der großen analytischen Schwierigkeiten bei der experimentellen Speziation von Spurenmetallen wurden in den letzten Jahrzehnten zahlreiche Programme zur computergestützten Speziationsberechnung entwickelt, die kontinuierlich erweitert und verbessert werden (SMITH und JAFFÉ 1998). Einen Überblick über die Entwicklung der ersten Programme findet man bei NORDSTROM et al. (1979). SCHULZ (1992) schildert einige grundsätzliche Überlegungen zu hydrogeochemischen Modellierungen. In der Literatur finden sich zahlreiche Arbeiten, in denen Computermodellierungen mit Hilfe von MINTEQA oder PHREEQE durchgeführt wurden, um Speziationen zu ermitteln oder Reaktionswege zu modellieren (z.B. Herbert 1994, Foos 1997, Shum und Lavkulich 1999, Pavlik und 
RunNElls 1990, SimPSon et al. 1998, NordSTROM et al. 1992). ChOI und West (1997) nutzten MINTEQA2 zur Modellierung von Apatit als Fällungsmittel zur Behandlung von sauren Minenwässern. Eine vergleichende Übersicht $\mathrm{zu}$ analytischen vs. rechnerischen Verfahren gibt NORDSTROM (1996). Jedoch existieren nur wenige Berichte darüber, dass einund dieselben Proben experimentell und rechnerisch untersucht und die Ergebnisse verglichen wurden. Eine vergleichende Studie der Berechnungen von Säulenexperimenten mit den Programmen MINTEQA2, PHREEQC und HYDROGEOCHEM (HGC) veröffentlichten BROWN et al. (2000). 


\subsection{Statistische Betrachtung der experimentellen Daten}

Zwar sollte eine statistische Betrachtung der gemessenen Daten mit großer Vorsicht erfolgen, denn viele relevante Parameter (Zeit und Ort der Probennahme, Sicker- oder Porenwasser, Art und Menge des DOC) lassen sich darin kaum angemessen erfassen. Trotzdem kann eine kurze statistische Übersicht ersten Aufschluss geben über Faktoren, die die Speziation verschiedener Proben stark beeinflussen.

Tab. A.18. und A.19. im Anhang zeigen eine Übersicht über die experimentellen Ergebnisse der Ultrafiltration für alle Proben und alle Elemente, deren Konzentrationen nach dem Durchlauf noch oberhalb der Nachweisgrenze lagen.

Es zeigt sich dabei deutlich, dass sich ein Großteil der Proben aus Cospuden nach einem von zwei Mustern verhält: Entweder wird bei der Ultrafiltration kaum etwas zurückgehalten, oder fast alle Elemente werden zu 90 - $100 \%$ abfiltriert. Ein Beispiel für Proben nahezu ohne Abtrennung von Elementen bei der Ultrafiltration sind die Proben aus der oberflächenahen Bohrung, wo fast alle Elemente zu über $80 \%$ im Filtrat wiedergefunden werden. In den Standgewässern aus der Arbeit von PLEßOW (1998) werden dagegen fast alle Elemente zu über $90 \%$ abgetrennt.

Grund hierfür können drei Faktoren sein: 1) Adsorption, 2) die Bindung an Bestandteile des DOC oder 3) die Bildung anorganischer Kolloide (zu unerwünschten Effekten bei der Ultrafiltration durch Koagulation etc. s.u.a. BUFFLE und NEWMAN 1992; Literaturübersicht bei Pleßow 1998 und Pleßow und HeinRichs 2000b).

In den Vorversuchen hatte PLEßOW (1998) eine starke Zunahme der Rückhalteraten insbesondere für die Selten-Erd-Elemente mit steigendem $\mathrm{pH}-$ Wert beobachtet und diese auf Adsorptionseffekte an der Membran zurückgeführt.

Die Zusammenfassung der Daten aus der Arbeit von PLEßOW (1998) und der vorliegenden Arbeit bestätigt diese Beobachtung für die natürlichen Proben jedoch nicht; bei keinem der untersuchten Elemente steht die Rückhalterate direkt im Zusammenhang mit dem pH-Wert, wie die Korrelationskoeffizienten in Tab. 5.1. exemplarisch belegen. 


\begin{tabular}{|l|l|l|l|}
\hline Element & $\begin{array}{l}\text { Adsorptionsverluste in \% bei } \\
\mathbf{p H = 1 , 4} \text { (PLEßOW 1998) }\end{array}$ & $\begin{array}{l}\text { Adsorptionsverluste in \% bei } \\
\mathbf{p H = 5 , 6} \text { (PLEßOW 1998) }\end{array}$ & $\begin{array}{l}\text { Korrelationskoeffizient } \\
\mathbf{r}_{\mathbf{p H}-\text { Element }}\end{array}$ \\
\hline $\mathbf{A l}$ & 4 & 16 & $-0,053$ \\
\hline $\mathbf{N b}$ & 25 & 98 & 0,555 \\
\hline $\mathbf{M o}$ & 3 & 47 & 0,361 \\
\hline Sb & 9 & 47 & 0,176 \\
\hline La & 3 & 13 & 0,149 \\
\hline $\mathbf{C e}$ & 2 & 51 & 0,197 \\
\hline Eu & 2 & 62 & 0,319 \\
\hline Gd & 3 & 52 & 0,563 \\
\hline $\mathbf{U}$ & 2 & 96 & 0,350 \\
\hline
\end{tabular}

Tab.5.1.: Ultrafiltration in Abhängigkeit vom pH; Daten aus der Arbeit von PLEßOW (1998) für synthetische Lösungen und Korrelation zwischen $p H$ und Rückhalterate für alle natürlichen Proben aus der Arbeit von PLEßOW (1998) und dieser Arbeit..

Bei den von PLEßOW (1998) untersuchten Proben handelte es sich um synthetische Lösungen, in denen keine Komplexbildner enthalten waren. Möglicherweise werden Adsorptionseffekte in den natürlichen Wässern jedoch durch die Bildung von Komplexen verhindert, die klein genug sind, die Ultrafiltrationsmembran zu passieren. Es kann allerdings auch sein, dass es sich bei dem von PLEßOW (1998) beobachteten Phänomen nicht um Adsorptionseffekte handelte, sondern es zur Bildung anorganischer Eisenoxihydroxid-Partikel kam, die wegen ihrer Größe bei der Ultrafiltration zurückgehalten wurden und an denen andere Elemente adsorbiert wurden. Ein Hinweis darauf könnte jedenfalls die Rückhalterate von $43 \%$ aus einer synthetischen Lösung für Eisen bei $\mathrm{pH}=5,8$ sein (PLEßOW 1998).

Für die vollständige Abtrennung zahlreicher Elemente aus einer großen Anzahl von Proben könnte die Komplexbildung durch organische Substanzen verantwortlich sein. Für die Porenwässer aus dem Bohrkern von RCO4 wurde versucht, die theoretisch besetzten Bindungsstellen je Gramm DOC für organische Moleküle größer als 1 kD zu berechnen. Dazu wurde aus den Daten von PLEßOW (1998) linear extrapoliert, welche Anteile der Spurenelemente aufgrund des $\mathrm{pH}$ und der Sulfatkonzentration an der Ultrafiltrationsmembran zurückgehalten wurden. Diese Menge wurde von den experimentell bestimmten Rückhalteraten abgezogen. Der verbliebene Anteil zurückgehaltener Spurenelemente wurde in Mol umgerechnet und auf den zurückgehaltenen DOC bezogen. Die Ergebnisse liegen zwischen 0.11 und 1.62 mol besetzter Bindungsstellen je Gramm DOC; geht man davon aus, dass nicht alle Bindungsstellen besetzt werden, sollte die absolute Zahl möglicher Bindungsstellen sogar noch höher liegen. Diese Werte liegen aber um mehrere Zehnerpotenzen über denen, die in der Literatur für Fulvo- und Huminsäuren angegeben werden (z.B. LOFTS und TIPPING 1998). Sie entsprechen sogar einer Bindungsstellendichte 
von mehr als 1 je Kohlenstoffatom, was nicht möglich ist. Der DOC kann also nicht für die hohen Rückhalteraten zahlreicher Elemente verantwortlich sein.

Demnach handelt es sich bei einem großen Teil der zurückgehaltenen Kolloide um anorganische Komplexe bzw. um suspendierte Partikel, an deren Oberfläche andere Elemente gebunden werden (s. dazu Kap. 5.1.).

Untermauert wird dies durch die Feststellung, dass die Rückhalteraten zahlreicher Elemente gut oder sehr gut mit denen für Ca, Sulfat bzw. Ba korrelieren (Tab. 5.2., Abb. 5.11.):

\begin{tabular}{|l|l|l|l|}
\hline Element & $\begin{array}{l}\text { Korrelationskoeffizient } \\
\mathbf{r}_{\text {Element-Ba }}\end{array}$ & $\begin{array}{l}\text { Korrlationskoeffizient } \\
\mathbf{r}_{\text {Element-Sulfat }}\end{array}$ & $\begin{array}{l}\text { Korrelationskoeffizient } \\
\mathbf{r}_{\text {Element-Ca }}\end{array}$ \\
\hline Sulfat & 0,873 & - & 0,888 \\
\hline $\mathbf{B e}$ & 0,752 & 0,879 & 0,905 \\
\hline $\mathbf{C o}$ & 0,858 & 0,861 & 0,942 \\
\hline $\mathbf{N i}$ & 0,801 & 0,822 & 0,889 \\
\hline $\mathbf{C u}$ & 0,799 & 0,740 & 0,883 \\
\hline $\mathbf{M o}$ & 0,813 & 0,800 & 0,827 \\
\hline $\mathbf{H f}$ & 0,838 & 0,381 & 0,843 \\
\hline $\mathbf{T l}$ & 0,934 & 0,842 & 0,834 \\
\hline $\mathbf{P b}$ & 0,784 & 0,792 & 0,843 \\
\hline $\mathbf{R E E}$ & $0,427-0,733$ & $0,555-0,742$ & $0,547-0,760$ \\
\hline
\end{tabular}

Tab.5.2.: Korrelationskoeffizienten für die Rückhalteraten ausgewählter Elemente mit den Rückhalteraten von $B a$, Sulfat und Ca in allen Proben aus Cospuden bei der Ultrafiltration.

Demnach liegen in vielen Proben kolloidales Ba- oder Ca-Sulfat, vermutlich aber auch Eisenoxihydroxid- oder Manganhydroxid-Kolloide vor, auf denen wiederum andere Elemente wie Co, Ni u.a. adsorbiert werden. Dass die Korrelation zwischen Ca, Ba und Sulfat und den REEs nur um 0,5 liegt, kann darin begründet sein, dass die Adsorption an den Oberflächen konkurriert mit der Komplexierung durch DOC-Bestandteile oder gelöstes Sulfat. TAKAHASHI et al. (1999) haben untersucht, wie sich die Anwesenheit von Huminsäuren auf das Adsorptionsverhalten verschiedener Elemente an Kaolinit- und Silica-Oberflächen auswirkt und welchen Einfluss der pH dabei ausübt. Für die Selten-Erd-Elemente stellten sie dabei fest, dass in Anwesenheit von Huminsäuren die Adsorption an Kaolinit bei pH-Werten unterhalb von 4 gefördert wurde, während bei pH-Werten oberhalb von 4 die gelöste Fraktion aufgrund der Bildung von Humatkomplexen anstieg. Ohne Huminsäuren wurden die Kationen oberhalb von pH 5 auf der Kaolinit-Oberfläche adsorbiert. Da die meisten Proben pH-Werte oberhalb von 4 aufwiesen, stützen die Ergebnisse von TAKAHASHI et al. (1999) unsere These, dass Selten-Erd-Elemente mit DOC Komplexe bilden und deswegen nicht an der Oberfläche von $\mathrm{Ca}-$ und Ba-Sulfat-Partikeln gebunden werden. 

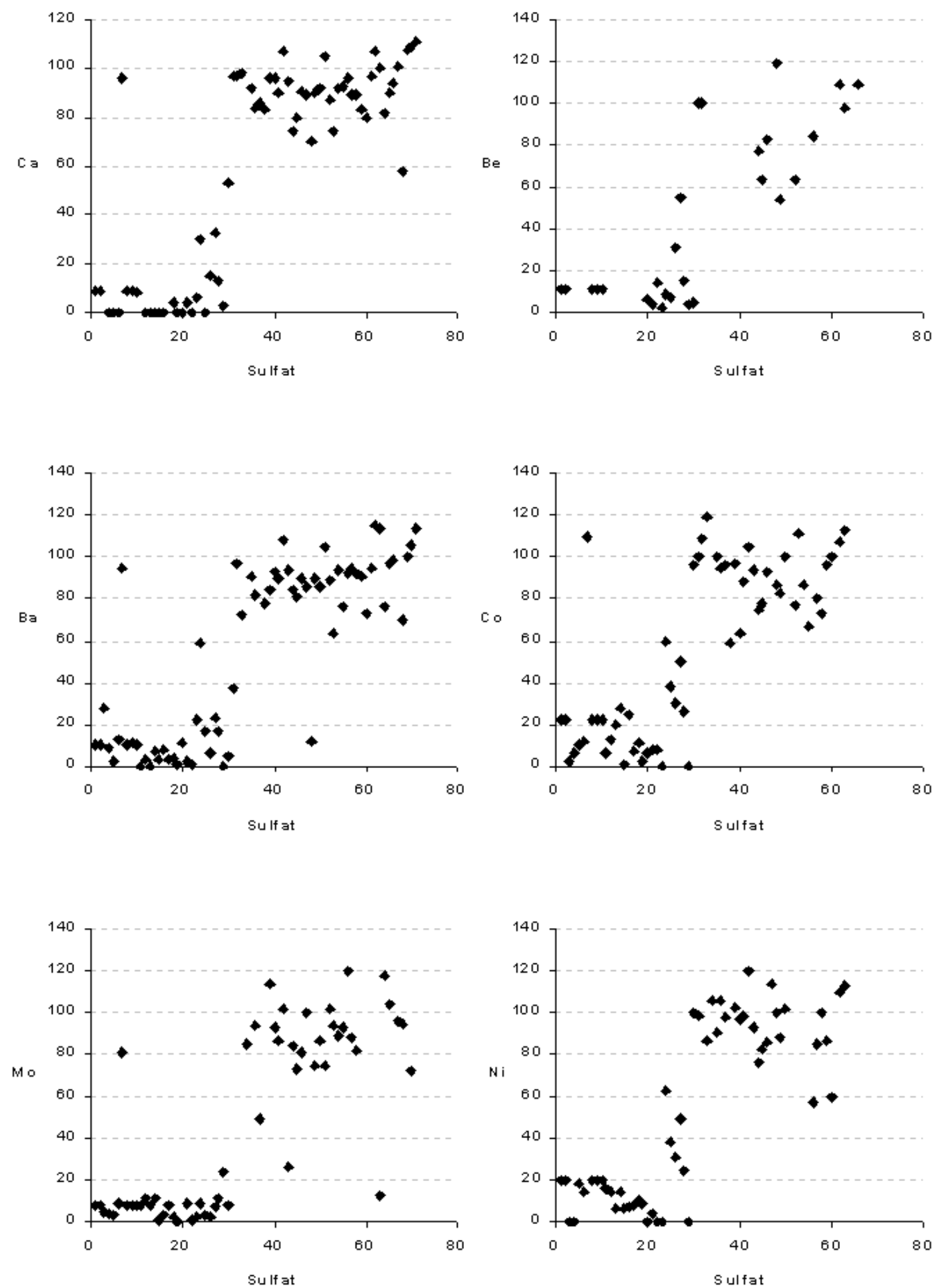

Abb. 5.11.: Rückhalteraten in der Ultrafiltration aus allen Proben aus der Arbeit von PLEßOW (1998) und dieser Arbeit für einige Elemente gegenüber Sulfat. 


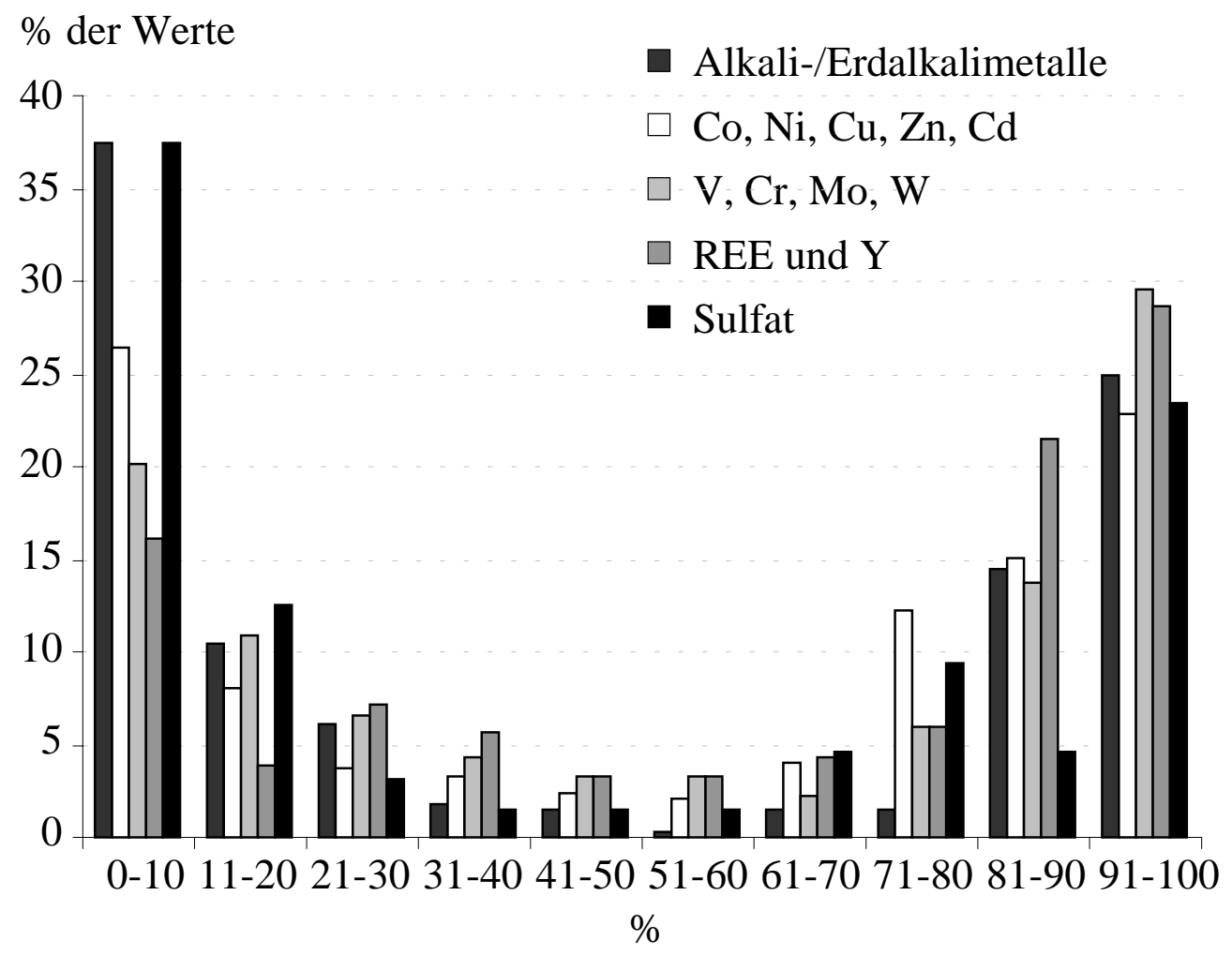

40
35
30
25
20
15
10
5
0
40 der Werte
60
50
$0-1011-2$
0

60

50

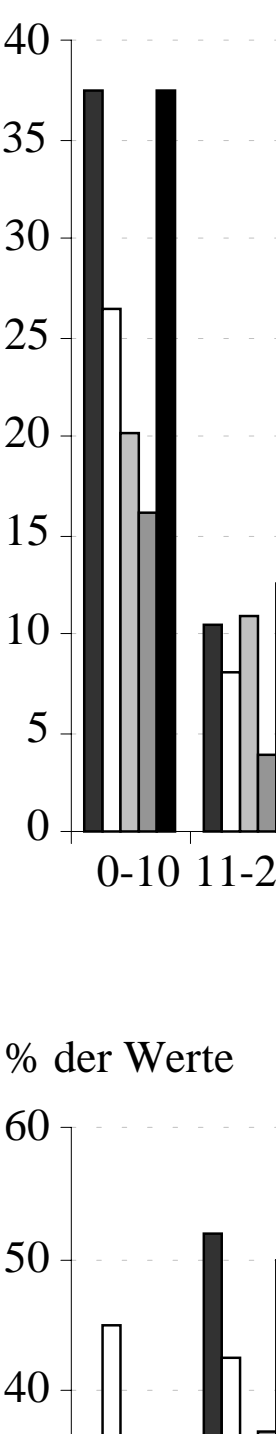

40

40
30

20

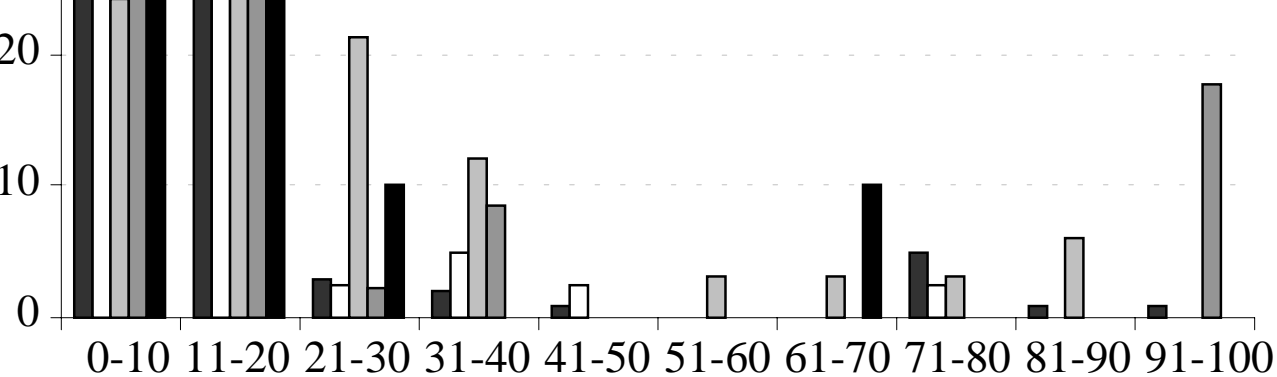

$\%$
- Alkali-/Erdalkalimetalle

$\square \mathrm{Co}, \mathrm{Ni}, \mathrm{Cu}, \mathrm{Zn}, \mathrm{Cd}$

$\square \mathrm{V}, \mathrm{Cr}, \mathrm{Mo}, \mathrm{W}$

$\square$ REE und $\mathrm{Y}$

- Sulfat

Abb. 5.12.: Übersicht zur Ultrafiltration am Beispiel einiger Elemente; Anteil der gemessenen Rückhalteraten in verschiedenen Prozentbereichen. Obere Abbildung: Proben aus Cospuden, untere Abbildung: Proben aus der Lausitz. 
Abb. 5.12. zeigt in einem Überblick, wie sich die gemessenen Werte für verschiedene Elementgruppen in Proben aus Cospuden hinsichtlich der Rückhalteraten von Elementen bei der Ultrafiltration aufteilen. Zum Vergleich ist darunter eine entsprechende Auftragung für die Proben aus der Lausitz abgebildet.

Bei den Proben aus der Lausitz zeigt sich eine weniger systematische Verteilung der Adsorptionsraten als bei den Proben aus Cospuden. Allerdings lassen sich bei 13 Proben nur sehr begrenzt statistische Überlegungen anstellen. Während in Cospuden 37,5\% der gemesssenen Austauschanteile für Alkali- und Erdalkalimetalle zwischen 0 und $10 \%$ liegen, sind weitere 24,9\% der gemessenen Werte zwischen 91 und $100 \%$ zu finden. Der Rest verteilt sich auf mittlere Austauschraten. In den Proben aus der Lausitz liegen insgesamt $87 \%$ der gemessenen Austauschraten für Alkali- und Erdalkalimetalle zwischen 0 und $20 \%$. Ähnliche Beobachtungen lassen sich für alle abgebildeten Elementgruppen feststellen: In den Proben aus Cospuden liegen die meisten gemessenen Austauschraten entweder in sehr niedrigen oder sehr hohen Bereichen, während die mittleren Werte kaum vertreten sind. In der Lausitz dagegen passieren die meisten Elemente in fast allen Proben ungehindert die Membran; nur die REEs zeigen noch einmal einen größeren Anteil an gemessenen Werten im Bereich der Austauschraten zwischen 90 und $100 \%$ mit 17,7 \% der gemessenen Werte, und Ba wird in vielen Proben fast vollständig zurückgehalten. Der DOC wird in den Proben aus der Lausitz in keinem Fall vollständig abgetrennt, stattdessen passiert er in 5 Fällen völlig ungehindert die Ultrafiltrationsmembran. Durch den relativ niedrigen $\mathrm{pH}-$ Wert dieser Proben ist keine starke Adsorption der Elemente auf der Membran zu erwarten. Der Vergleich zwischen den Auftragungen lässt darauf schließen, dass in den Proben aus der Lausitz im Gegensatz zu den Proben aus Cospuden offenbar weder organische noch anorganische Kolloide in größerem Umfang vorliegen. 

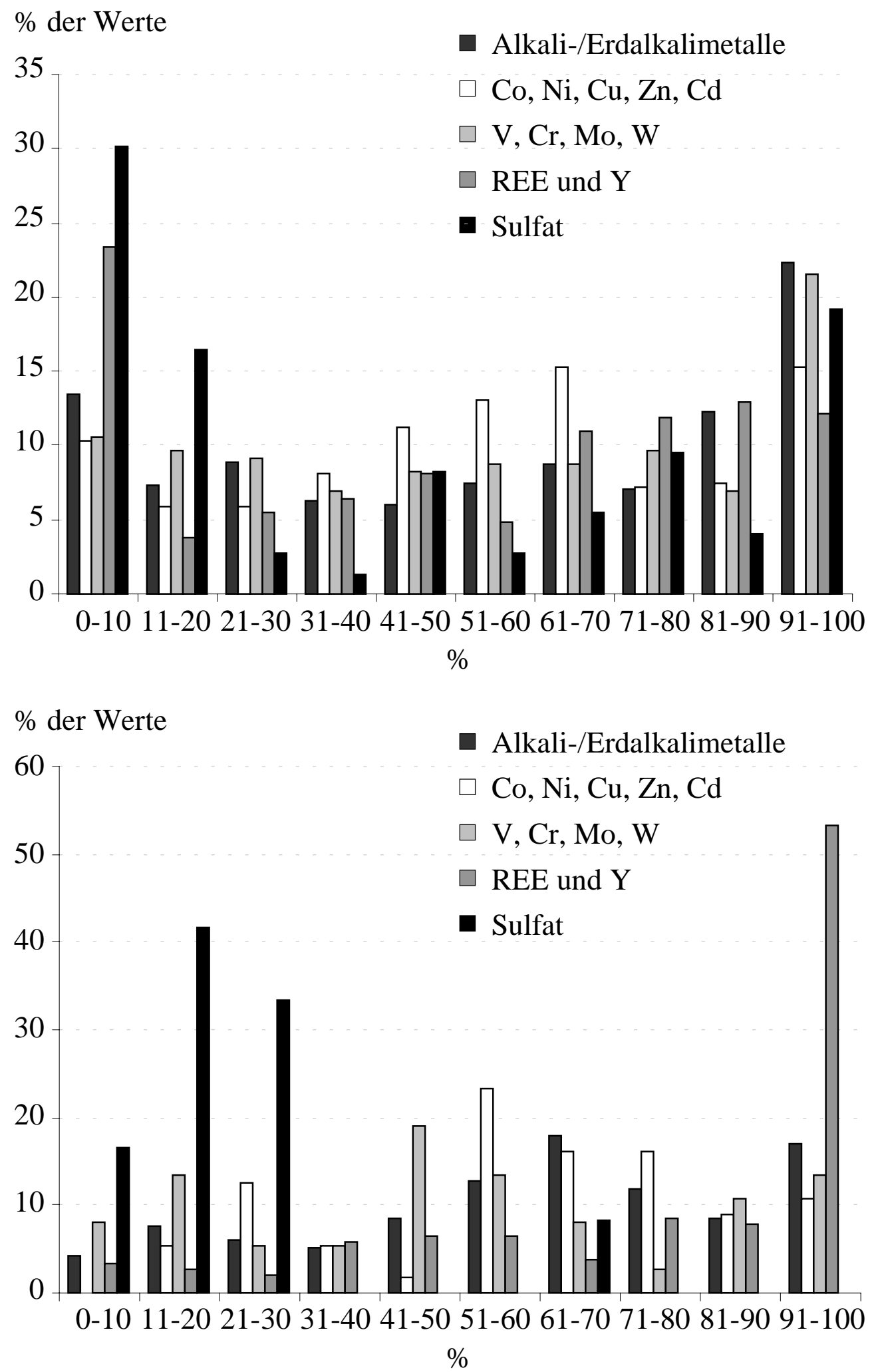

Abb. 5.13.: Übersicht zum Kationenaustausch am Beispiel einiger Elemente; Anteil der gemessenen Rückhalteraten in verschiedenen Prozentbereichen. Obere Abbildung: Proben aus Cospuden, untere Abbildung: Proben aus der Lausitz. 
Tab A.20 und A.21. im Anhang geben eine vergleichbare Übersicht für den Kationenaustausch. Ohne Komplexbildung wäre für die meisten Ionen zu erwarten, dass sie vollständig ausgetauscht werden, während die wenigen Elemente, die in wässriger Lösung anionisch vorliegen können (Mo, U, Cr, Sb, Sn) den Ionentauscher ungehindert passieren sollten.

Abb. 5.13. zeigt, dass das Bild weit komplexer ist: Zwar werden in den Proben aus Cospuden einige Elemente tatsächlich in zahlreichen Proben vollständig ausgetauscht (z.B. Li, K, Ca, $\mathrm{Co}, \mathrm{Rb}, \mathrm{Sr}, \mathrm{Ba}, \mathrm{La}, \mathrm{Ce})$, doch für alle diese Elemente gibt es eine fast gleich große Anzahl an Proben, bei denen der Austausch nur zu geringen Anteilen erfolgt. So liegen von den gemessenen Austauschraten der Alkali- und Erdalkalimetalle nur 13,5 \% zwischen 0 und 10 $\%$, von den Übergangsmetallen nur $10 \%$ und von den REEs $23 \%$. Doch auch zwischen 90 und $100 \%$ Austauschrate liegen $22 \%$ der gemessenen Werte der Alkali- und Erdalkalimetalle, 15,3 \% der Werte bei den Übergangsmetallen und 12,2 \% der REE- und YWerte. Gründe hierfür können die Komplexierung durch Sulfat oder durch organische Komplexbildner sein. In diesem Zusammenhang ist das Verhalten von Sulfat sehr auffällig, das eigentlich nicht durch den Kationenaustauscher zurückgehalten werden sollte. Dennoch wird in 15 der insgesamt 66 Proben das Sulfat vollständig durch den Kationenaustausch aus der Probe entfernt; insgesamt liegen $30 \%$ der gemessenen Werte zwischen 0 und $10 \%$ Austauschrate. Ein Teil des Sulfates wird vermutlich mit Kationen positiv geladene Komplexe bilden, z.B. $\mathrm{AlSO}_{4}{ }^{+}$oder Lanthansulfatkomplexe.

Außerdem kann es sich um Komplexierung durch organische Substanzen handeln, die auch über Bindungsstellen für Anionen, z.B. in Form von Aminogruppen, verfügen. Allerdings zeigt auch hier wieder eine Überschlagsrechnung mit den zurückgehaltenen Sulfatanteilen in RCO4 5-8 m und 11-19 m, dass die Komplexbildung durch Kationen und DOC als Erklärung für die hohen Austauschraten nicht ausreicht. In RCO4 5-8 m werden 21,3 mmol Sulfat zurückgehalten, in 11-19 m 18,2 mmol. Dem stehen aber nur 0,023 mmol bzw. 0,002 mmol Aluminium gegenüber, die ausgetauscht werden und theoretisch mit Sulfat als kationischer Komplex vorliegen könnten. In 5-8 m bleiben 11,4 mmol, in 11-19 m 14,6 mmol DOC auf dem Ionentauscher. Diese Mengen reichen keinesfalls, um für die Rückhalteraten des Sulfats verantwortlich zu sein. Die Werte von V, Cr, Mo und W verteilen sich relativ gleichmäßig über das gesamte Spektrum der Austauschraten; die maximale Anzahl aber liegt ziwschen 91 und $100 \%$. Ähnlich wie beim Sulfat muss dies auf Komplexierungen oder Adsorptionseffekte zurückzuführen sein. 
Für die Proben aus der Lausitz (Tab. A.21. im Anhang, Abb. 5.13. unten) lässt sich, wie oben erwähnt, wegen der vergleichsweise geringen Anzahl an Proben nur eingeschränkt eine statistische Betrachtung anstellen. Dennoch sieht man Unterschiede zu den Proben aus Cospuden. So fällt ins Auge, dass in keiner der Proben aus der Lausitz das Sulfat durch den Kationenaustausch vollständig abgetrennt wurde. Die maximale Rückhalterate liegt hier bei $69 \%$, sonst aber in der Regel unterhalb von $30 \%$. Auch der DOC wies kaum kationischen Charakter auf. Der größte Anteil der gemessenen Werte bei den Alkali- und Erdalkalimetallen lag mit knapp $18 \%$ zwischen 61 und $70 \%$ Rückhalterate. Bei den Nebengruppenelementen fanden sich 23,2 \% der gemessenen Werte zwischen 51 und $60 \%$. Al und $\mathrm{Pb}$ (ohne Abbildung) wurden ebenfalls fast in allen Proben nahezu vollständig ausgetauscht. V, Cr, Mo und W verteilten sich gleichmäßig über den gesamten Bereich der gemessenen Austauschraten. Die REEs wurden in den meisten Proben vollständig durch den Kationenaustauscher zurückgehalten; 53,3 \% der gemessenen Werte lagen zwischen 91 und $100 \%$. Anscheinend lag hier kaum eine Bindung an den DOC vor, oder die Bindung war so schwach, dass die Komplexe durch den Kationenaustauscher gespalten werden konnten. Abgesehen von den REEs, die alle gut miteinander korrelieren, gibt es bei den Proben aus Cospuden nur bei wenigen Elementen Korrelationen mit $r>0,8$, die aber eher Zufallscharakter tragen als wirkliche Aussagen über paralleles Verhalten beinhalten. Die Elemente in den Lausitzer Proben korrelieren weit besser, gerade im Bereich der Nebengruppenmetalle. Allerdings ist wegen der geringen Probenzahl eine Aussage über die Signifikanz dieser Korrelationen schwierig zu treffen.

Insgesamt verhalten sich in den Proben aus der Lausitz die Elemente erwartungsgemäß, indem die kationisch vorliegenden Elemente zu großen Teilen ausgetauscht werden, während Sulfat in der Regel ungehindert passiert. Das Verhalten der Elemente in den Proben aus Cospuden dagegen lässt sich offensichtlich nicht nur durch die Effekte der Hydrolyse erklären, sondern wird von anderen Faktoren mitbestimmt; dafür kommen vor allem Einflüsse aus dem DOC und Adsorptionsvorgänge in Frage. 

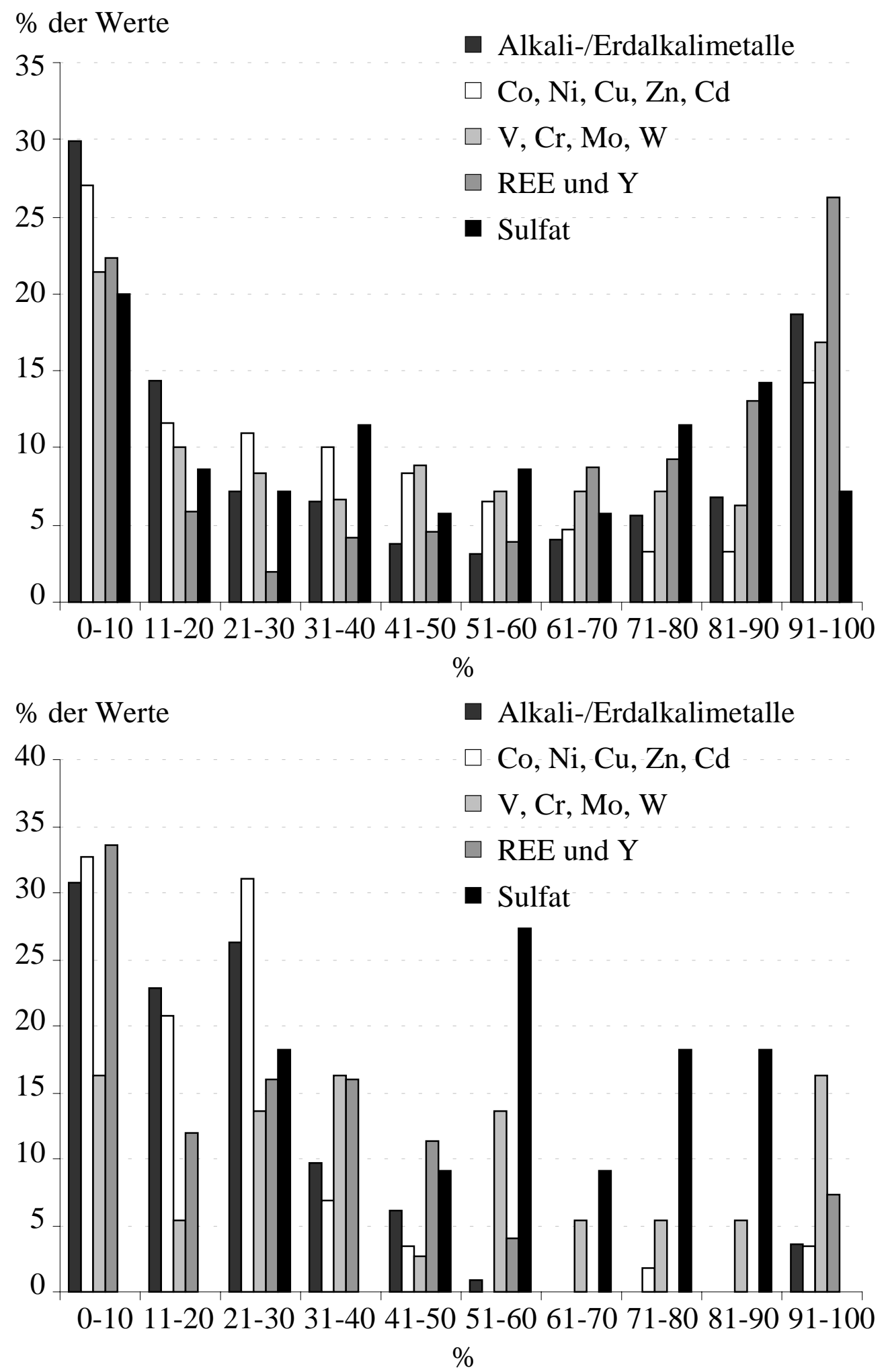

Abb. 5.14.: Übersicht zum Anionenaustausch am Beispiel einiger Elemente; Anteil der gemessenen Rückhalteraten in verschiedenen Prozentbereichen. Obere Abbildung: Proben aus Cospuden, untere Abbildung: Proben aus der Lausitz. 
Tab. A.22. und A.23. im Anhang und Abb. 5.14. schließlich geben einen Überblick über die Ergebnisse des Anionenaustausches. Entgegen den Erwartungen wurde Sulfat in den Proben aus Cospuden nur in wenigen Proben vollständig ausgetauscht; lediglich $7 \%$ der gemessenen Werte liegen zwischen 90 und $100 \%$ Rückhalterate. In 13 von insgesamt 68 berücksichtigten Proben aus Cospuden dagegen passierte es den Austauscher ungehindert. Ähnlich wie bei den Ergebnissen des Kationenaustausches lässt diese Beobachtung den Schluss zu, dass Sulfat in anorganischen oder organischen Komplexen vorliegt und für den Anionenaustausch weitgehend nicht zur Verfügung steht. Das Verhalten des DOC in den verschiedenen Proben ist sehr unterschiedlich und nicht systematisch darzustellen.

Bei einigen Elementen zeigt sich im Anionenaustausch wieder ein ähnliches, nämlich überwiegend gegensätzliches Verhalten wie bei der Ultrafiltration: Entweder, sie werden vollständig ausgetauscht oder passieren vollständig. So liegen $30 \%$ der gemessenen Werte der Alkali- und Erdalkalimetalle bei Rückhalteraten zwischen 0 und $10 \%$, aber auch 18,6\% zwischen 91 und $100 \%$. Für die Übergangsmetalle sind $27 \%$ aller gemessenen Werte kleiner als $10 \%$, während 14,2 \% der Werte größer als $90 \%$ sind. Ähnliche Verteilungen zeigen sich auch bei den REEs. Dass diese Elemente, die man eigentlich alle als Kationen erwarten würde, in vielen Proben komplett beim Anionenaustausch zurückgehalten werden, kann teilweise wiederum an Komplexierungseffekten, aber auch am Vorhandensein adsorptiver Kolloide liegen.

Andererseits muss jedoch auch berücksichtigt werden, dass in der Übersicht 39 Proben enthalten sind, bei denen der Austausch gegen $\mathrm{OH}^{-}$erfolgte und es zu Niederschlagsbildungen im Ionentauscher kam (hier sei besonders darauf hingewiesen, dass auch Eisen in mehreren Proben vollständig auf dem Ionentauscher zurückblieb). Auffällige Korrelationen sind allerdings nicht festzustellen. Die Rückhalteraten sind nicht in allen Fällen einem tatsächlichen Austausch zuzurechnen.

Bei den Proben aus der Lausitz kann diese Überlegung entfallen, denn dort wurde grundsätzlich gegen $\mathrm{Cl}^{-}$ausgetauscht. Sulfat wurde zwar in keiner Probe vollständig ausgetauscht, in vielen aber in recht großen Anteilen; $27 \%$ der Proben liegen zwischen 51 und $60 \%$, jeweils $18 \%$ der Proben im Bereich zwischen 71 und $80 \%$ bzw 81 und $90 \%$. Der DOC passierte ebenso wie beim Kationenaustausch in vielen Proben den Austauscher fast ungehindert und weist damit auch nur wenig anionischen Charakter auf. Wie auch ohne Berücksichtigung von Komplexen zu erwarten, passieren die Alkali- und Erdalkalimetalle, die REEs und $\mathrm{Y}$ und auch Mn, Fe, und Zn zu großen Teilen den Austauscher nahezu ungehindert; bei den Übergangsmetallen liegen fast alle Werte unterhalb von $30 \%$. U wird dagegen in 11 
von insgesamt 21 Proben nahezu quantitativ ausgetauscht. Dass sich bei den REEs die Proben über fast das gesamt Spektrum der Rückhalteraten verteilen, kann an der Komplexierung durch Sulfat oder organische Komplexbildner liegen. Überwiegend zeigen sie aber doch kationischen Charakter.

Die statistische Übersicht der Proben lässt auf deutliche Unterschiede zwischen der Speziation in den Wässern aus Cospuden und aus der Lausitz schließen. In den Proben aus Cospuden verhält sich nur ein Teil der Elemente so, wie es in wässriger Lösung zu erwarten wäre. Eine ebenso große Zahl der gemessenen Werte weicht aber von diesem beobachteten Verhalten ab. Die Ergebnisse der Ultrafiltration weisen darauf hin, dass - vor allem anorganische Kolloide großen Einfluss nehmen und zahlreiche Elemente an sich binden. Daneben können Komplexbildungen zwischen Kationen, Sulfat und DOC die Beobachtungen beim Kationenund Anionenaustausch erklären.

In den Ergebnissen der Proben aus der Lausitz dagegen finden sich keine Anhaltspunkte für eine Beteiligung anorganischer oder organischer Kolloide an der Speziation. Der DOC weist nur wenige Funktionalitäten auf und nimmt keinen nennenswerten Einfluss auf das Verhalten der Elemente in der Lösung.

Insgesamt kann die statistische Betrachtung aus den o.g. Gründen und in einem so komplexen System nur erste Anhaltspunkte liefern. Zumindest lässt sie aber deutliche qualitative Unterschiede zwischen den beiden Untersuchungsgebieten erkennen. Für eine genaue Analyse muss dagegen jede Probe einzeln betrachtet werden. Inwieweit die eben aufgestellten Thesen zutreffend sind, wird dann im Einzelnen zu prüfen sein. 


\subsection{Vergleich der Daten aus Experiment und Rechnung}

Mit Hilfe des von uns verwendeten Speziationsschemas gelingt zwar experimentell die Unterscheidung zwischen kationisch, anionisch oder neutral vorliegenden Elementspezies, wobei es sich darum genau handelt, ist allerdings auf diesem Weg nicht zu ermitteln. In Frage kommen neben freien auch hydratisierte Ionen, Oxoanionen, Sulfatkomplexe oder organisch gebundene Spezies (alle übrigen Anionen sind in den Proben im Verhältnis zum Sulfat in so geringen Mengen enthalten, dass ihr Einfluss vernachlässigt werden kann). Parallel zu den experimentellen Verfahren gibt es in der Literatur verschiedene Ansätze, die Speziation von Elementen in Wässern rechnerisch zu ermitteln (TURNER et al. 1981). PLEßOW (persönliche Mitteilung) unternahm daher erste Versuche zu flankierenden Berechnungen, indem er $\mathrm{pH}$ Eh-Diagramme (s. z.B. STUMm 1996, KöLLING et al. 2000) mit Hilfe des Programms HSC Chemistry erstellte. Dabei stieß er jedoch auf große Schwierigkeiten, die zum einen daher rühren, dass die gemessenen Eh-Werte nicht in jedem Fall als zuverlässig betrachtet werden können (MACALADY 1990), zum anderen blieben aber auch zahlreiche wichtige Parameter im System unberücksichtigt, so z.B. die organischen Komplexbildner. Besonders diese beeinflussen jedoch das Verhalten der Elemente stark, was ihre Stabilisierung in Lösung und ihre Mobilität betrifft. Der DOC unserer Proben entzieht sich einer differenzierten Analytik, da dafür sehr große Probenmengen benötigt werden, die im Falle der Sicker- und Porenwässer nicht zur Verfügung stehen (MARLEY et al. 1992).

Um dennoch Aussagen über Bedeutung und Art des Einflusses organischer Komplexe auf das Verhalten der gelösten Spurenmetalle machen zu können, wurden zusätzlich zu den experimentellen Ergebnissen Berechnungen mit dem Speziationsprogramm MINTEQA2 angestellt.

Durch die Kombination beider Methoden wird uns eine differenzierte Betrachtung der einzelnen Elemente und der prozentualen Anteile ihrer jeweiligen Spezies an der Gesamtkonzentration möglich. Die Datenbank bietet die Grundlage für die Bestimmung der Sulfat- und anderer Anionenkomplexe (wobei letztere für unsere Proben keine Rolle spielen, da alle anderen Anionen gegenüber Sulfat in vernachlässigbaren Konzentrationen vorliegen), die Bestimmung der Oxidationszustände im jeweiligen Redoxmilieu und die Berücksichtigung organischer Komplexbildner (dies allerdings bislang nicht in großem Umfang, s.u.). 


\subsubsection{Experimentelle Klassifizierung der Proben}

Um eine sinnvolle Anwendung des Programms MINTEQA2 auf die Proben zu ermöglichen, werden sie zunächst anhand der optischen Beobachtungen und der Ergebnisse der Ultrafiltration eingeordnet. Ausschlaggebend ist dabei der Zustand der Proben bei der Messung.. Abb. 5.15. zeigt das Schema, nach dem sich die Proben klassifizieren lassen.

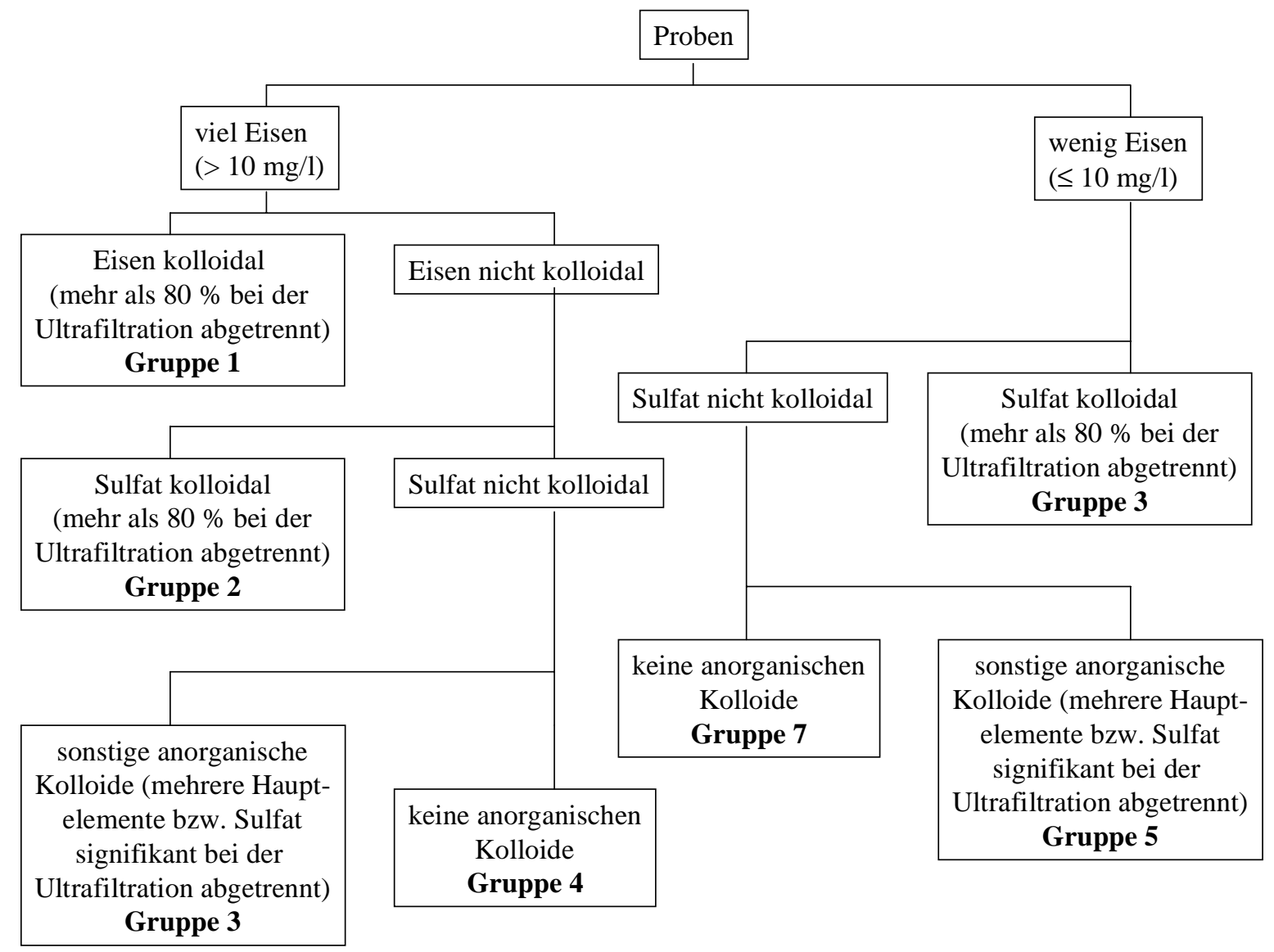

Abb. 5.15.: Klassifikationsschema der Proben nach den Ergebnissen der Ultrafiltration

Daraus ergeben sich mehrere Gruppen; die einzelnen Gruppen weisen die folgenden Merkmale auf:

Gruppe 1:

In diesen Proben liegen große Mengen von Eisen (> $10 \mathrm{mg} / \mathrm{l}$ ) in kolloidaler Form vor (> $80 \%$ Rückhalterate für Fe bei der Ultrafiltration); vermutlich befinden sich die Proben zum großen Teil noch nicht im Gleichgewicht. Eisenhydroxide bilden sich und formen Kolloide In manchen Proben hat die Sedimentation schon eingesetzt, und während der Lagerung haben sich in den Proben orange-rote Eisenhydroxid-Niederschläge gebildet. In anderen Proben steht der Fällungsprozess noch am Anfang. Das Eisen liegt bereits in kolloidaler Form vor, optisch wahrnehmbare Ausfällungen treten jedoch noch nicht auf. Auf den Eisenkolloiden in 
der Lösung lagern sich Spurenelemente ab, die mit den Kolloiden bei der Ultrafiltration zurückgehalten werden. In diese Gruppe fallen z.B. fast alle Proben aus eisenreichen Standgewässern aus der Frühphase der Flutung.

Gruppe 2:

Diese Proben sind eisenreich (> $10 \mathrm{mg} / \mathrm{l}$ ), das Eisen ist allerdings durch die Ultrafiltration nur in geringen Anteilen zu entfernen. Dafür ist in der Regel der niedrige pH der Lösungen verantwortlich. Übersättigte Sulfatphasen liegen jedoch in der Lösung vor. Hohe Rückhalteraten für Ca zeigen in vielen Proben, dass es sich um kolloidales $\mathrm{CaSO}_{4}$ handelt. In dieser Gruppe liegen einige Proben aus der Lausitz sowie zwei Proben von Standgewässern bzw. die Porenlösung aus dem Seesediment aus der Arbeit von PlEßOw (1998).

Gruppe 3:

In diesen Proben liegt nur wenig ,gelöstes“ Eisen vor; teilweise haben sich schon vor der Probennahme Niederschläge gebildet, in anderen Proben ist das Eisen während der Lagerung sedimentiert. Viele Spurenelemente wurden auf den ausgefallenen Eisenphasen adsorbiert; diese Proben sind deshalb in der Regel arm an gelösten Spurenelementen. Kolloidale Sulfatphasen, z.B. Gips oder $\mathrm{AlOHSO}_{4}$, befinden sich in der Lösung und werden bei der Ultrafiltration abgetrennt; bei einigen Proben zeigt die sehr hohe Rate für abgetrenntes $\mathrm{Ca}$, dass es sich in der Hauptsache um kolloidales $\mathrm{CaSO}_{4}$ handelt. Einige Spurenelemente lagern sich auf ihrer Oberfläche an und werden mit den Kolloiden bei der Ultrafiltration aus der Lösung entfernt. In dieser Gruppe findet man z.B. das Wasser der Fremdflutung, das Sickerwasser und die Seewasserprobe aus der Arbeit von PLEßOw (1998).

\section{Gruppe 4:}

Die eisenreichen Proben dieser Gruppe weisen einen niedrigen pH-Wert auf; weder Eisennoch Sulfatkolloide lassen sich bei der Ultrafiltration zu mehr als $80 \%$ abtrennen, allerdings deuten die hohen Rückhalteraten (60 - $70 \%$ ) für mehrere Hauptelemente und/oder Sulfat bei der Ultrafiltration darauf hin, dass Kolloide eine Rolle spielen.

\section{Gruppe 5:}

In Proben dieser Gruppe liegen nur wenig Eisen und keine vollständig abtrennbaren kolloidalen Sulfatphasen vor. Insgesamt allerdings deuten hohe Rückhalteraten $(60-70 \%)$ für mehrere Hauptelemente und/oder Sulfat bei der Ultrafiltration darauf hin, dass Kolloide für die Speziation eine Rolle spielen. 


\section{Gruppe 6:}

Diese Proben sind eisenreich; anorganische Kolloide fallen bei der Ultrafiltration nicht auf. In der Regel sind auch die Konzentrationen von Übergangsmetallen und Spurenelementen sowie den REEs hoch. In diese Gruppe fallen vor allem Proben aus der Lausitz. Dort begünstigen niedrige $\mathrm{pH}-$ Werte und u.U. ein reduzierendes Milieu die Stabilität hochkonzentrierter Lösungen.

\section{Gruppe 7:}

In diesen eisenarmen Proben liegen keine auffälligen Mengen anorganischer Kolloide vor. Die pH-Werte sind im neutralen oder leicht sauren Bereich. Die meisten Elemente weisen nur geringe Gehalte auf, weil durch vorangegangene Fällungen viele Übergangsmetalle, Spurenelemente und REEs bereits im Vorfeld aus den Wässern entfernt wurden.

Die Tab. A.24. im Anhang zeigt, inwieweit sich die Proben den einzelnen Gruppen zuordnen lassen und welche Kriterien zu Überprüfung dieser Zuordnung angewendet wurden. Für die meisten Proben stimmen die untersuchten Kriterien mit den Annahmen überein, die für die jeweilige Gruppe gemacht wurden. Wo dies nicht der Fall ist, muss davon ausgegangen werden, dass die Probe sehr weit vom Gleichgewichtszustand entfernt ist oder organische Komplexbildner ihr Verhalten beeinflussen. Darauf wird noch näher einzugehen sein.

Die Einteilung der Proben nach diesem Klassifikationsschema leistet eine wichtige Hilfe bei der Bewertung der Ergebnisse aus der rechnerischen und der experimentellen Speziation.

\subsubsection{Berechnung ohne Berücksichtigung der organischen Komponenten}

Zunächst wurde für alle Proben eine Berechnung ohne organische Zusatzstoffe durchgeführt. Dafür wurden die pH-Werte, die Konzentrationen aller in der Datenbank berücksichtigen Elemente bzw. von Sulfat und (soweit gemessen) das Redoxpotential in MINTEQA2 eingegeben. Da die Verteilung der Redoxspezies nicht berechenbar war, wenn alle Parameter gleichzeitig berücksichtigt wurden, mussten dafür eigene Rechnungen mit weniger Variablen durchgeführt werden, ehe alle Elemente gleichzeitig berechnet werden konnten. Präzipitation wurde zunächst nicht zugelassen; auch Adsorptionsvorgänge blieben für den Anfang unberücksichtigt. Die Ergebnisse sind in Tab. A.25. im Anhang aufgeführt.

Wie nicht anders zu erwarten, traten für die Alkali- und Erdalkalimetalle zwischen den verschiedenen Proben kaum Unterschiede im Rechenergebnis auf. Li, Na und $\mathrm{K}$ wären danach in allen Proben praktisch ausschließlich als freies Kation und nur zu sehr geringem Teil als anionischer Sulfatkomplex zu erwarten. Ca, Mn und Sr bilden etwas bereitwilliger Sulfatkomplexe aus, liegen aber immer noch zum größten Teil als freies Kation vor. Auch Co, 
das in allen Proben nach der Rechnung als Co ${ }^{\text {III }}$ vorliegt, und Ni verteilen sich ausschließlich auf die freie kationische und die Sulfatspezies, ohne dass in den verschiedenen Proben große Unterschiede im Verhältnis der beiden Lösungsformen bestehen sollten. Dabei lässt sich über den tatsächlichen Oxidationszustand von Co nur schwer eine Aussage machen; in Salzen ist das $\mathrm{Co}^{\mathrm{II}}$-Kation die stabilere Spezies, während in Komplexen $\mathrm{Co}^{\mathrm{III}}$ stabiler ist. Organische Komplexe können eine solche Stabilisierung bewirken.

Komplizierter als für die nicht hydrolyseempfindlichen Elemente sind die Ergebnisse für Elemente, die wie $\mathrm{Be}, \mathrm{Al}$ und $\mathrm{Pb}$ zur Hydrolyse neigen und ebenso Hydroxo- oder AquoKomplexe ausbilden wie solche mit Sulfat. Hier schwanken die Verteilungen je nach pH deutlich stärker. Abb. 5.16. - 5.20. zeigen dies am Beispiel einiger Elemente in Anwesenheit von Sulfat bzw. von Kationenkonzentrationen, die in etwa denen der Wässer aus Cospuden entsprechen, und in Abhängigkeit vom pH-Wert. Die pH-Werte der untersuchten Proben aus Cospuden und aus der Lausitz liegen in Bereichen zwischen 2 und 8. Al liegt nach dem Diagramm nur bei $\mathrm{pH}-$ Werten unterhalb von 3 in nennenswerten Mengen als freies Kation vor. Im sauren Bereich bis zu einem $\mathrm{pH}-$ Wert von ca. 5 dominiert das $\mathrm{AlSO}_{4}{ }^{+}$-Kation. Ein geringer, ziemlich konstanter Anteil von Al-Ionen bildet mit jeweils zwei Sulfatmolekülen einfach negativ geladene Komplexe. Zwischen $\mathrm{pH}=5$ und $\mathrm{pH}=7$ liegt ein komplexes Gemisch von hydratisierten und sulfatgebundenen Aluminiumspezies vor, das sehr sensibel auch auf leichte $\mathrm{pH}-$ Wert-Änderungen reagiert. Oberhalb von $\mathrm{pH}=7$ liegt $\mathrm{Al}(\mathrm{OH})_{4}{ }^{-}$praktisch als einzige Spezies vor. 


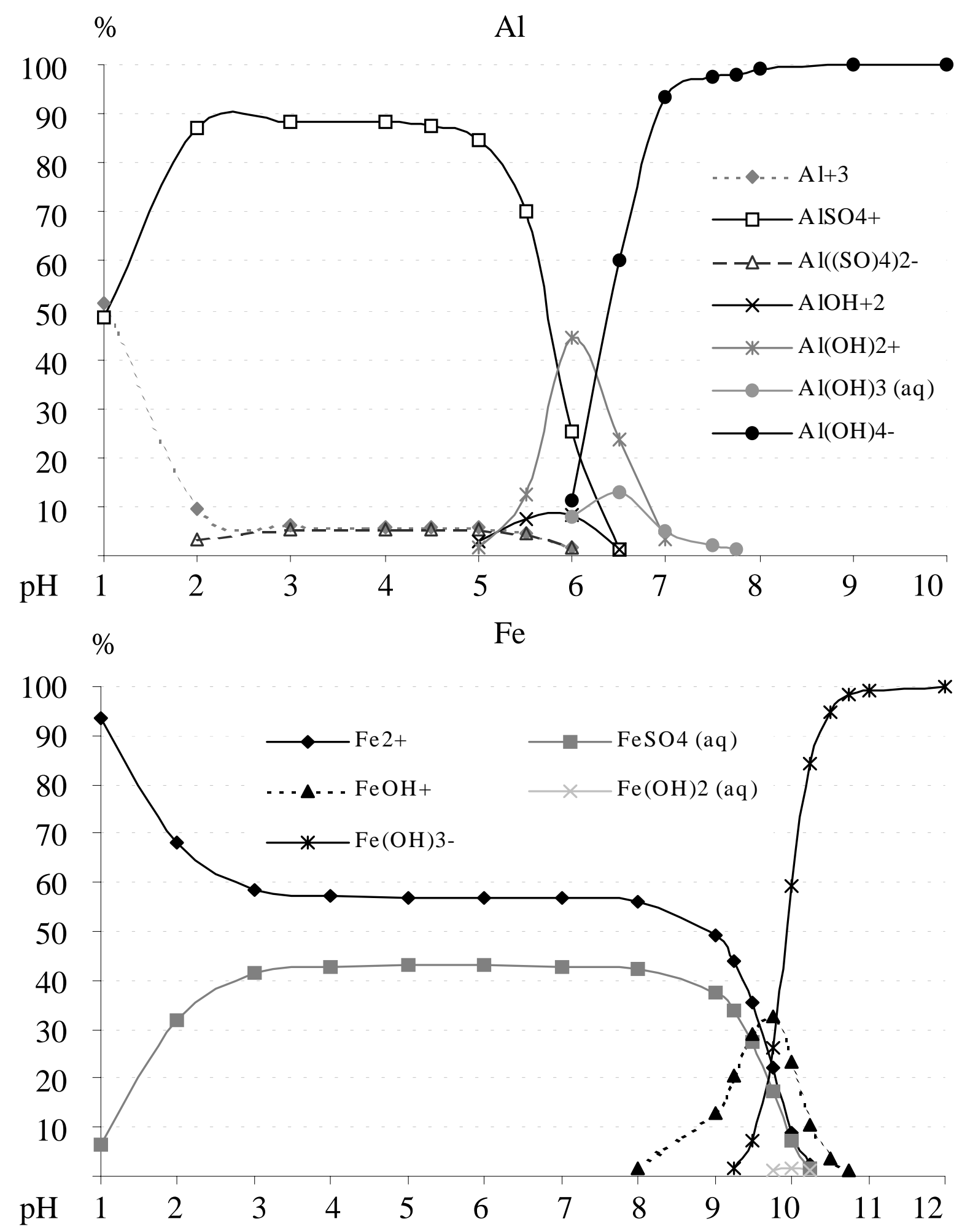

Abb. 5.16.: Speziationsdiagramme von Al und Fe in wässriger Lösung, berechnet mit MINTEQA2; $25^{\circ} \mathrm{C}$; für Al: $\left[\mathrm{Al}^{3+}\right]=1 \mathrm{mg} / \mathrm{l},\left[\mathrm{SO}_{4}^{2-}\right]=1 \mathrm{~g} / \mathrm{l}$; für $\mathrm{Fe}:\left[\mathrm{Fe}^{2+}\right]=100 \mathrm{mg} / \mathrm{l},\left[\mathrm{SO}_{4}{ }^{2-}\right]=1 \mathrm{~g} / \mathrm{l}$, keine Redoxreaktion zugelassen. 

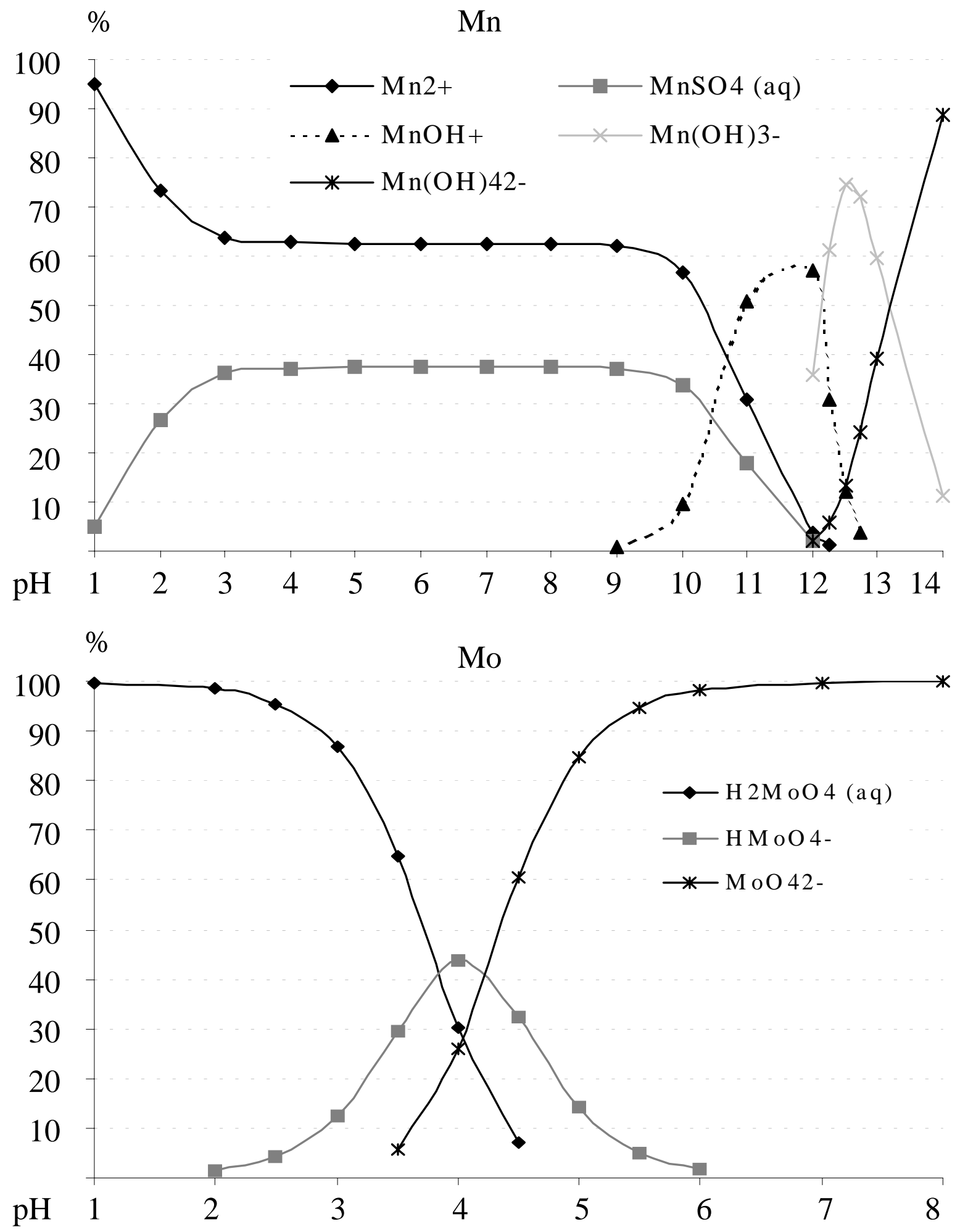

Abb. 5.17.: Speziationsdiagramme von Mn und Mo in wässriger Lösung, berechnet mit MINTEQA2; $25^{\circ} \mathrm{C}$; für $\mathrm{Mn}:[\mathrm{Mn}]=1 \mathrm{mg} / \mathrm{l},\left[\mathrm{SO}_{4}{ }^{2-}\right]=1 \mathrm{~g} / \mathrm{l}$; für $\mathrm{Mo}:\left[\mathrm{MoO}_{4}{ }^{2-}\right]=1,7 \mu \mathrm{g} / \mathrm{l}\left[\mathrm{Fe}^{2+}\right]=100 \mathrm{mg} / \mathrm{l},[\mathrm{Na}]^{+}=100 \mathrm{mg} / \mathrm{l}$; keine Redoxreaktion zugelassen. 


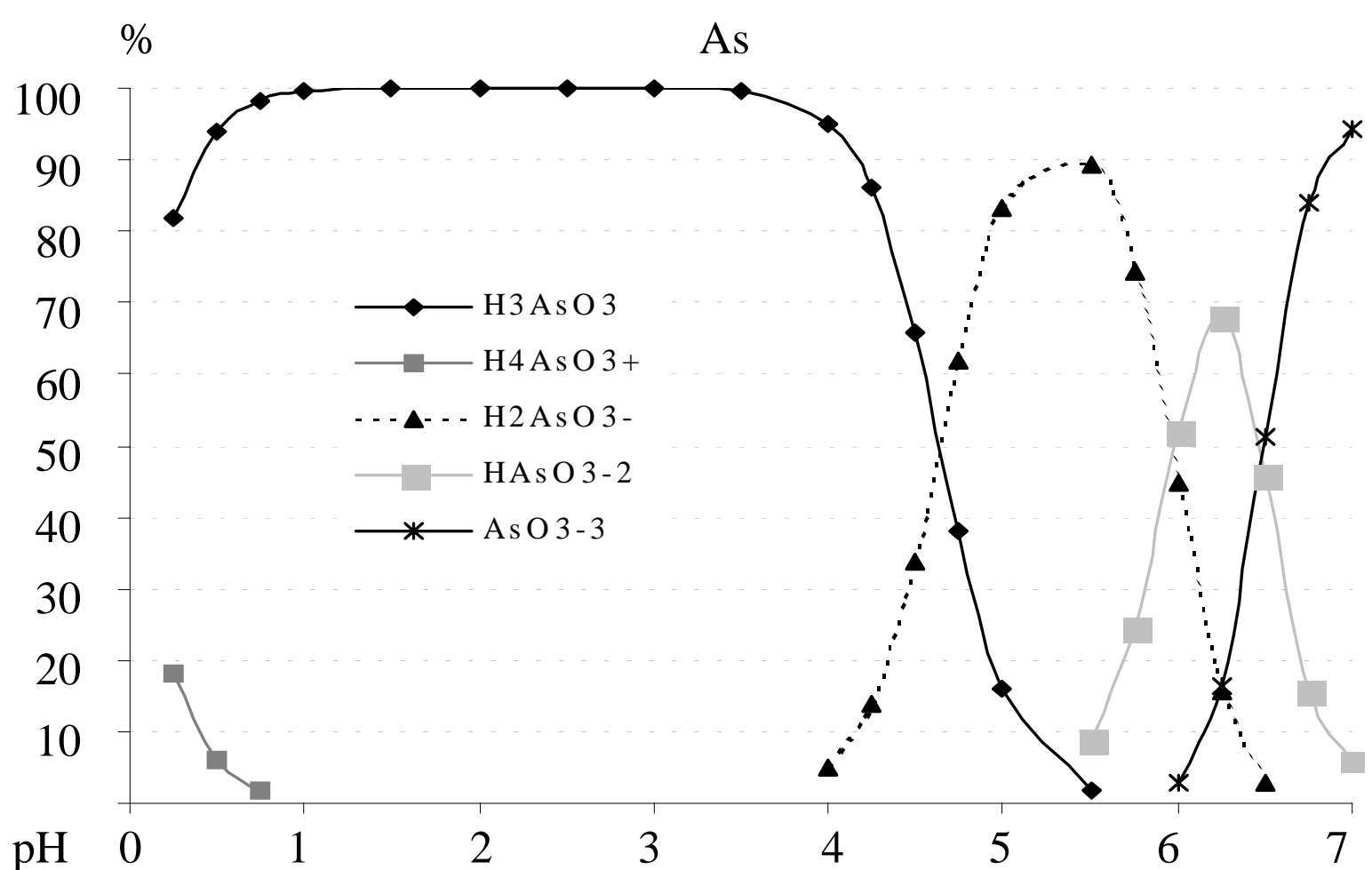

$\%$

$\mathrm{Sb}$

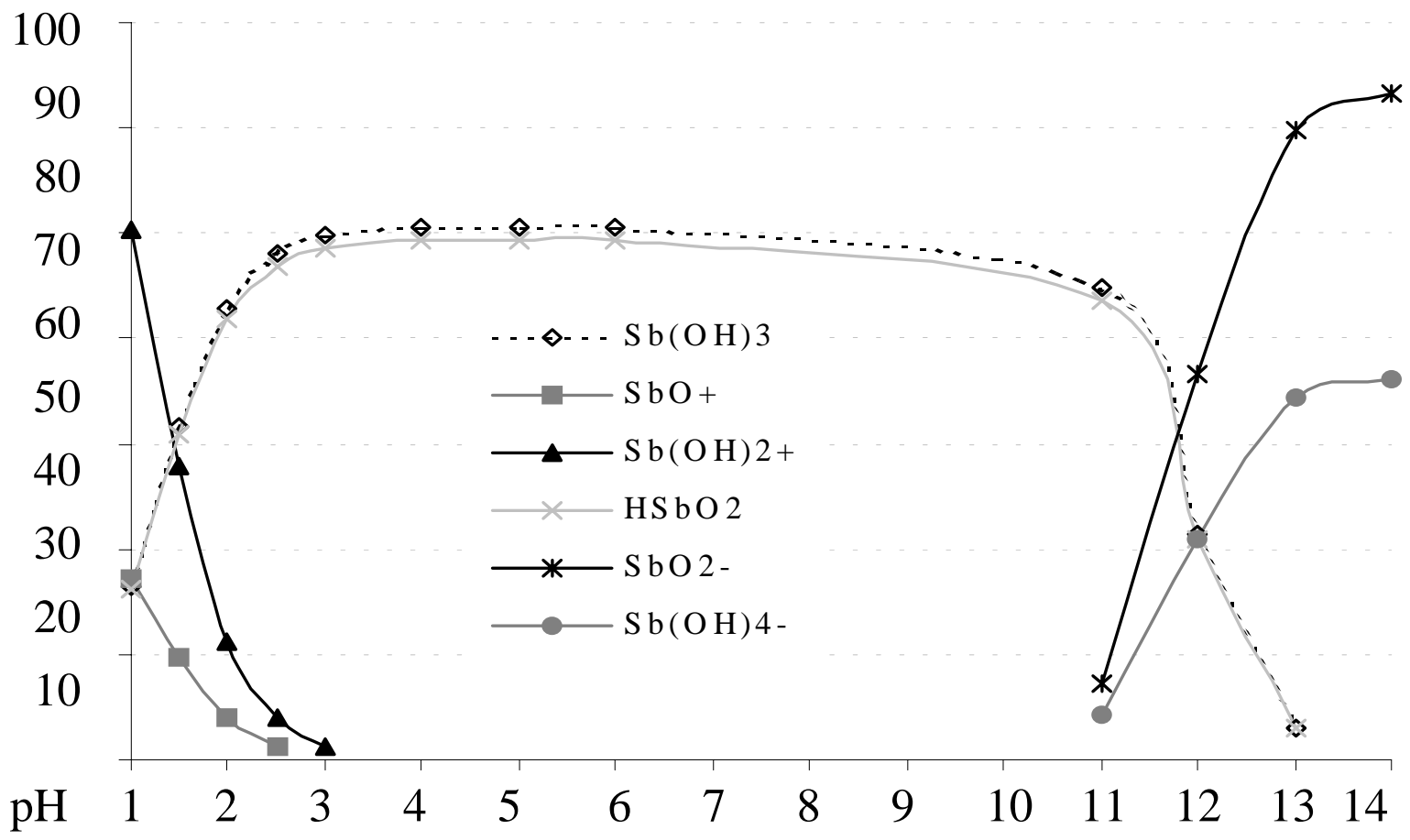

Abb. 5.18.: Speziationsdiagramme von As und Sb in wässriger Lösung, berechnet mit MINTEQA2; $25^{\circ} \mathrm{C}$; für As: $\left[\mathrm{H}_{3} \mathrm{AsO}_{3}\right]=1,7 \mu \mathrm{g} / l$; für $\mathrm{Sb}:\left[\mathrm{Sb}(\mathrm{OH})_{3}\right]=10 \mu \mathrm{g} /$; keine Redoxreaktion zugelassen. 
Die Speziationsdiagramme von zweiwertigem Eisen (Abb. 5.16. unten) und zweiwertigem Mangan (Abb. 5.17. oben) sind sich recht ähnlich. Bis zu einem pH-Wert von etwa 8 bei Eisen bzw. 9 bei Mangan liegen vor allem freie Kationen und zu einem geringeren Anteil neutrale Sulfatkomplexe vor. Erst bei pH-Werten, die deutlich oberhalb der Werte in den untersuchten Proben liegen, setzt die Hydrolyse der Kationen ein. Als Lösungsform in den untersuchten Proben aus Cospuden und aus der Lausitz sind daher vor allem freie Kationen und neutrale Sulfatkomplexe zu erwarten; auch eine Bindung an organische Spezies kommt für $\mathrm{Fe}^{\mathrm{II}}$ in Betracht. $\mathrm{Fe}^{\mathrm{III}}$ ist nur bei niedrigen $\mathrm{pH}-$ Werten in Lösung stabil. Für Mo kommen nur neutrale oder anionische Spezies in Betracht; deshalb wurde in der Rechnung nicht Sulfat, sondern $\mathrm{Fe}^{2+}$ und $\mathrm{Na}^{+}$berücksichtigt, um eventuelle Komplexe von Mo-Anionen mit Kationen in der Lösung zu erfassen. Das Diagramm in Abb. 5.17. zeigt, dass bis zu einem $\mathrm{pH}-$ Wert von ca. 3 praktisch ausschließlich das neutrale $\mathrm{H}_{2} \mathrm{MoO}_{4}$ (aq) vorliegt. Zwischen $\mathrm{pH}$ 3 und pH 5 befinden sich $\mathrm{H}_{2} \mathrm{MoO}_{4}, \mathrm{HMoO}_{4}{ }^{-}$und $\mathrm{MoO}_{4}{ }^{2-}$ im Gleichgewicht, während oberhalb von $\mathrm{pH}=5$ das $\mathrm{MoO}_{4}{ }^{2-}$-Anion dominiert.

Abb. 5.18. zeigt außerdem die Speziationsdiagramme der häufig pyritgebundenen Elemente As und $\mathrm{Sb}$. Beide treten über den gesamten berechneten $\mathrm{pH}$-Bereich in hydrolisierter Form auf. Während $\mathrm{Sb}$ zwischen $\mathrm{pH}=3$ und $\mathrm{pH}=11 \mathrm{zu}$ fast gleichen Teilen als neutrales $\mathrm{Sb}(\mathrm{OH})_{3}$ und neutrales $\mathrm{HSbO}_{2}$ vorliegt, wird die Arsenige Säure $\mathrm{HAsO}_{3}$ bei $\mathrm{pH}$-Werten oberhalb von 4 nach und nach deprotoniert und liegt zwischen $\mathrm{pH}=4$ und $\mathrm{pH}=7$ als Gemisch verschiedener anionischer Spezies in der wässrigen Lösung vor.

Am komplexesten sind die Verhältnisse bei Ionen, die in verschiedenen Proben in unterschiedlichen Redoxstufen vorliegen; hier sind vor allem $\mathrm{Cr}, \mathrm{V}, \mathrm{Fe}, \mathrm{Cu}$ und $\mathrm{U}$ zu nennen. Ähnlich wie bei den Sättigungsindices liefert das Speziationsmodell auch bei den Redoxstufen jedoch nur Anhaltspunkte. Ein Beispiel dafür ist $\mathrm{Cu}$, für das das Modell häufig die Oxidationsstufe $+\mathrm{I}$ ergibt. De facto ist $\mathrm{Cu}^{\mathrm{I}}$ in wässriger Lösung jedoch nicht stabil, sondern disproportioniert in $\mathrm{Cu}^{0}$ und $\mathrm{Cu}^{\mathrm{II}}$, so dass entgegen den berechneten Ergebnissen das $\mathrm{Cu}$ im Modell als $\mathrm{Cu}^{\mathrm{II}}$ eingesetzt werden muss. Über die Schwierigkeiten bei der Einbindung von Redoxpotentialen in Grundwassermodelle berichtet SCHÄFER (2000).

In Abb. 5.19. und Abb. 5.20. sind die berechneten Diagramme von $\mathrm{Cr}$ und $\mathrm{V}$ als Beispiel für die Speziation redoxempfindlicher Elemente dargestellt. Oben ist jeweils das Bild für ein Redoxpotential $=250 \mathrm{mV}$, also ein wenig reduzierendes Milieu, wiedergegeben, während unten das Diagramm für ein Redoxpotential $=400 \mathrm{mV}$, also ein eher reduzierendes Milieu dargestellt ist. Diese Redoxpotentiale entsprechen in etwa denen, die in vielen Proben aus Cospuden bzw. aus der Lausitz festgestellt wurden. 


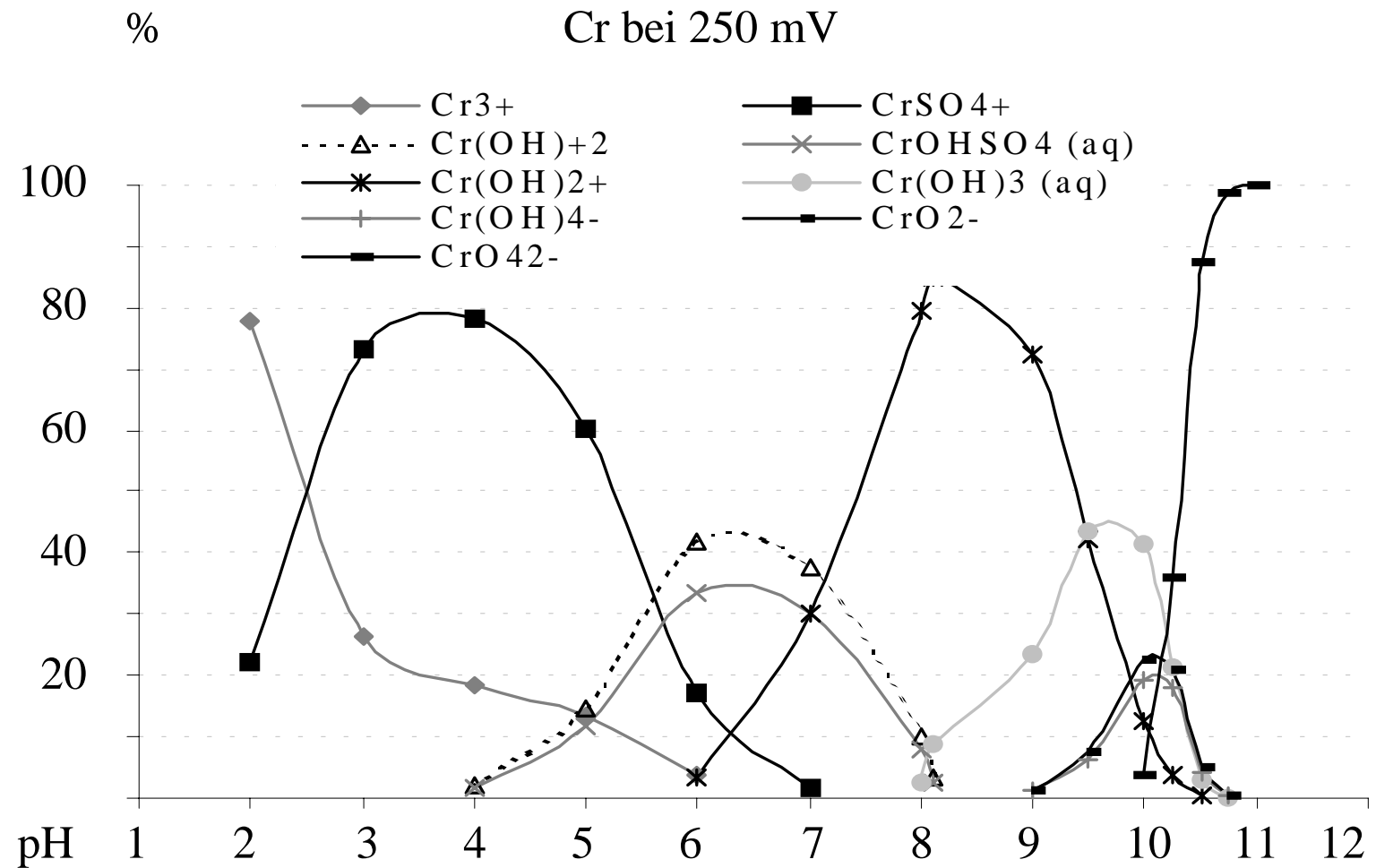

$\%$

Cr bei $400 \mathrm{mV}$

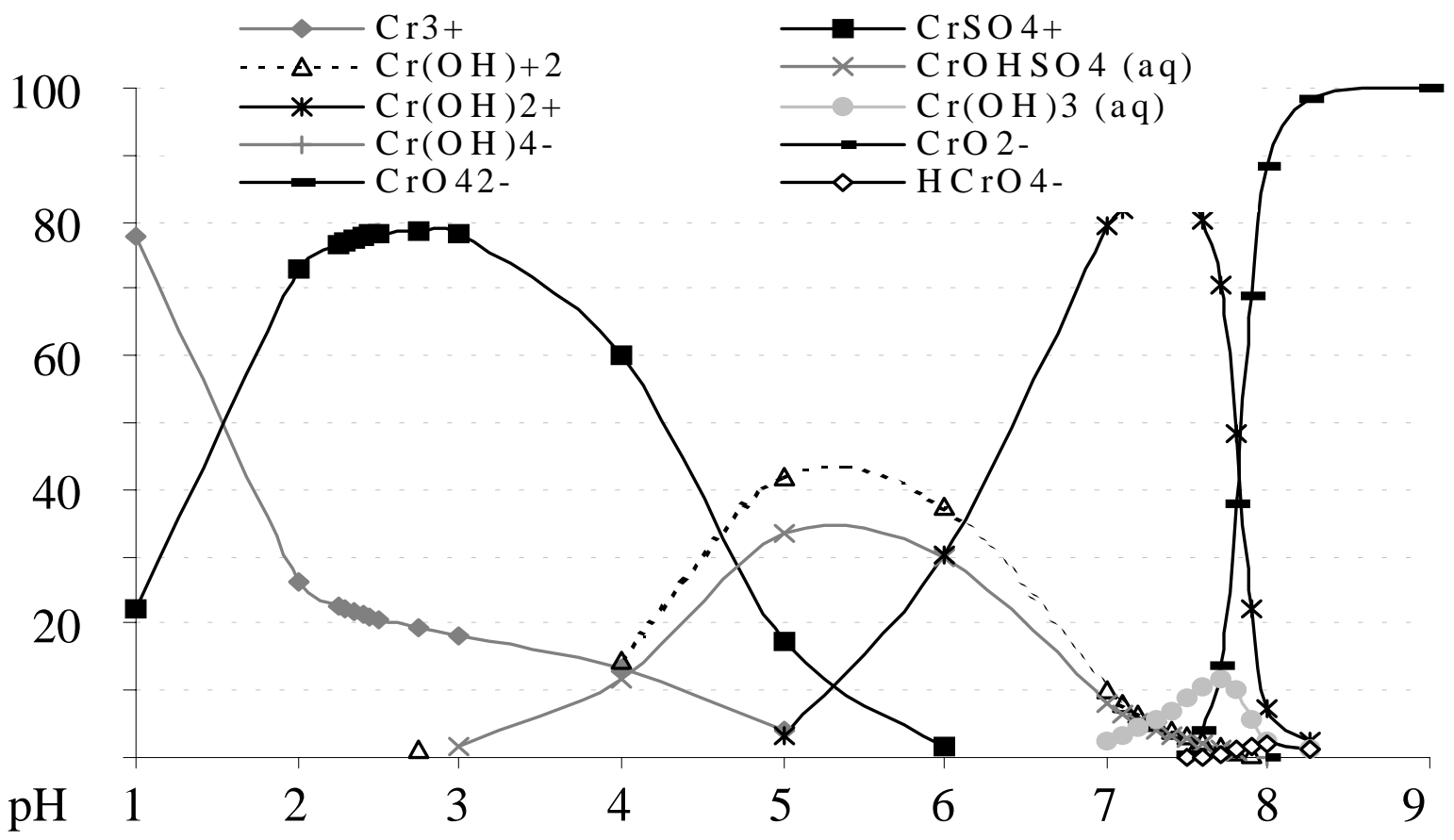

Abb. 5.19.: Speziationsdiagramme von $\mathrm{Cr}$ in wässriger Lösung, berechnet mit MINTEQA2; $25^{\circ} \mathrm{C} ;\left[\mathrm{Cr}^{3+}\right]=10$ $\mu \mathrm{g} / \mathrm{l},\left[\mathrm{SO}_{4}{ }^{2-}\right]=1 \mathrm{mg} /$; Redoxpotential oben $250 \mathrm{mV}$, unten $400 \mathrm{mV}$. 
$\%$
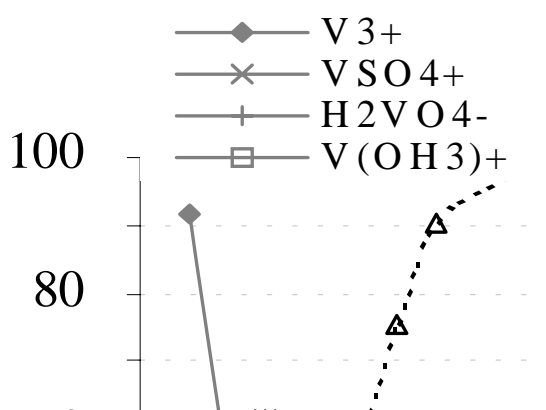

60

40

20

$\mathrm{pH}$

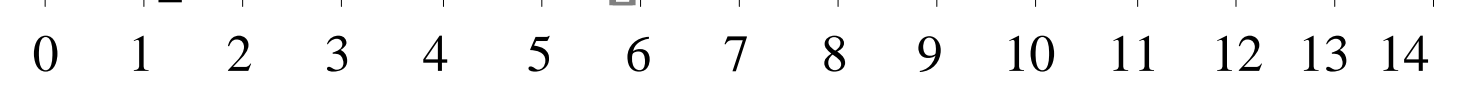
$\%$

$\mathrm{V}$ bei $250 \mathrm{mV}$

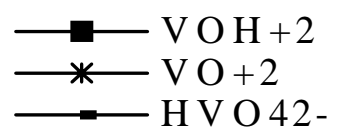

$$
\begin{array}{r}
\cdots \Delta-\operatorname{V}(\mathrm{OH}) 3(\mathrm{aq}) \\
\square \\
\hline
\end{array}
$$
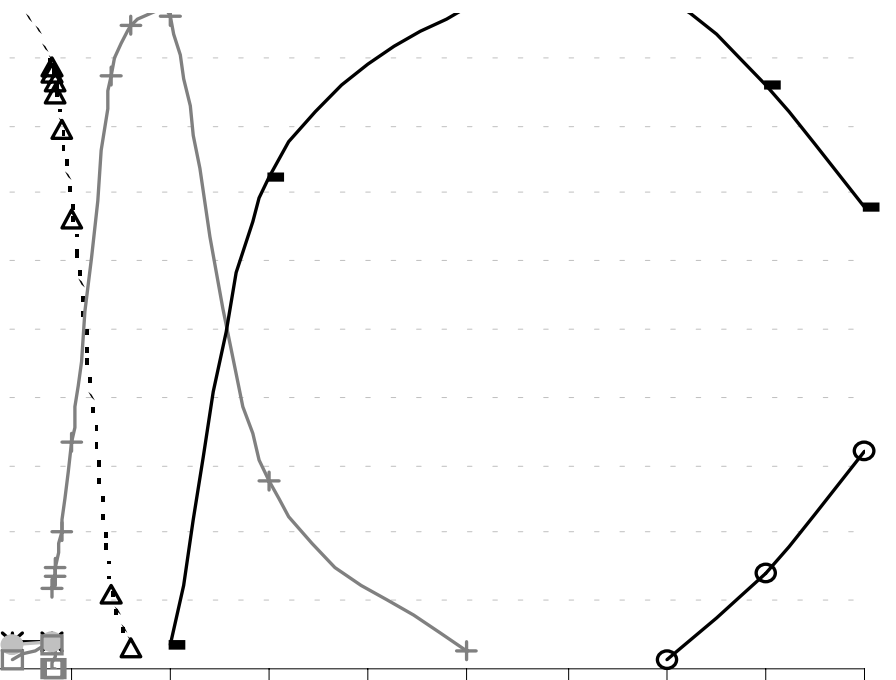

$\mathrm{V}$ bei $400 \mathrm{mV}$

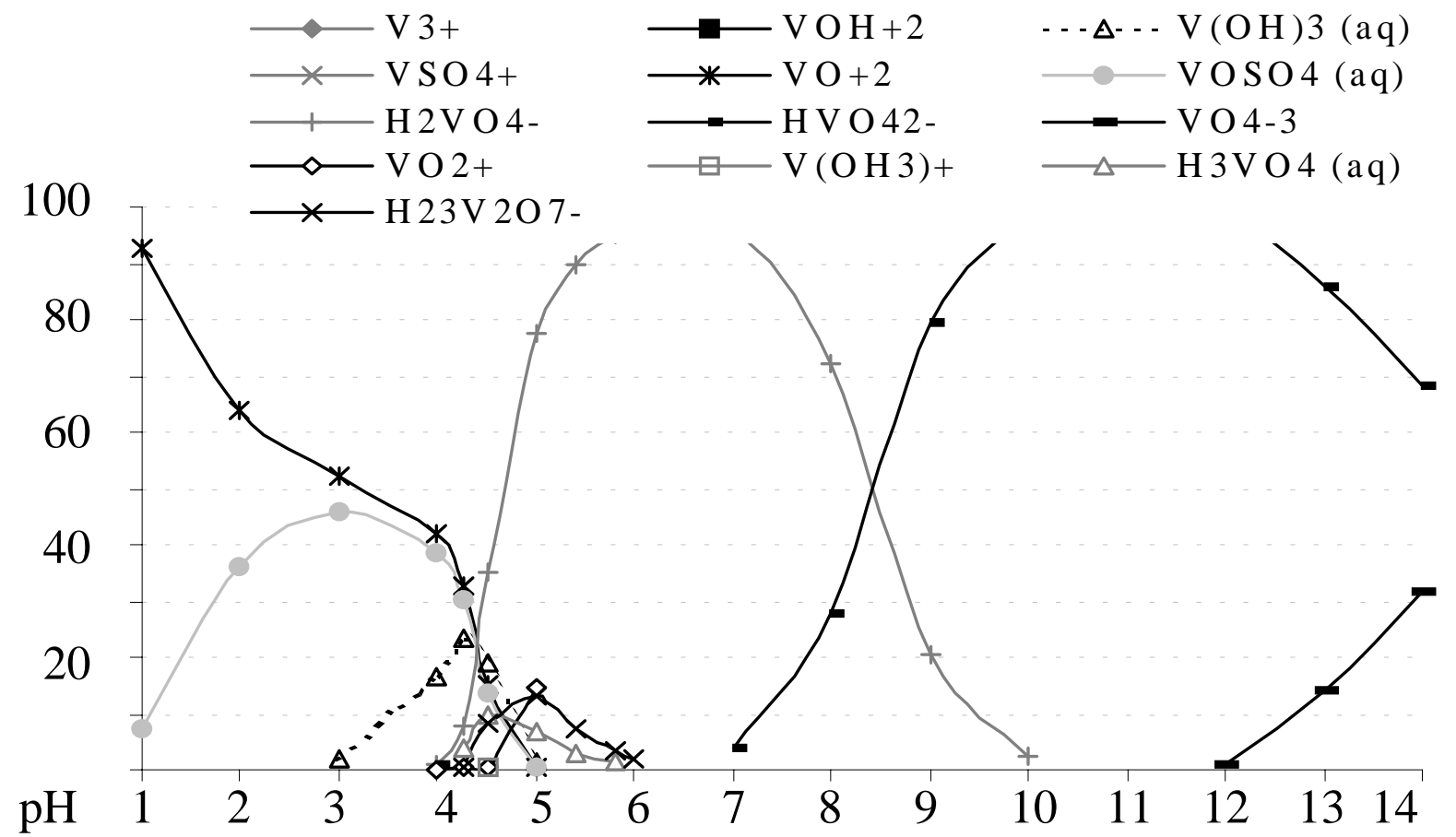

Abb. 5.20.: Speziationsdiagramme von $V$ in wässriger Lösung, berechnet mit MINTEQA2; $25^{\circ} \mathrm{C} ;\left[\mathrm{V}^{3+}\right]=10$ $\mu \mathrm{g} / \mathrm{l},\left[\mathrm{SO}_{4}{ }^{2-}\right]=1 \mathrm{mg} / \mathrm{l}$; Redoxpotential oben $250 \mathrm{mV}$, unten $400 \mathrm{mV}$. 
Während die Abweichungen zwischen den beiden Diagrammen bei Cr nur gering sind, zeigt V eine erheblich größere Veränderung in Abhängigkeit vom Redoxpotential.

Bei Cr liegt im pH-Bereich zwischen 3 und 8 bei beiden Redoxpotentialen ein Gemisch verschiedener positiv geladener oder neutraler hydrolisierter und sulfatgebundener Spezies vor; bei niedrigeren $\mathrm{pH}$-Werten dominieren freie Kationen und ein einfacher positiv geladener Sulfatkomplex, während bei $250 \mathrm{mV}$ erst bei pH-Werten oberhalb von 9, bei $400 \mathrm{mV}$ bei pHWerten oberhalb von 8 anionische Spezies auftreten. V tritt bei einem Redoxpotential von 400 $\mathrm{mV}$ im pH-Bereich zwischen 4 und 8 vor allem als $\mathrm{H}_{2} \mathrm{VO}_{4}{ }^{-}$auf; unterhalb von 4 liegt ein Gemisch von neutralen Aquo- und Sulfatkomplexen und $\mathrm{VO}_{2}{ }^{+}$vor. Bei $250 \mathrm{mV}$ dominiert zwischen $\mathrm{pH}=3$ und $6 \mathrm{~V}(\mathrm{OH})_{3}(\mathrm{aq})$; zwischen 6 und 7 ist $\mathrm{H}_{2} \mathrm{VO}_{4}{ }^{-}$die Hauptspezies, oberhalb von 7 kommt $\mathrm{HVO}_{4}{ }^{2-}$ dazu.

Vergleicht man jedoch die Ergebnisse der Berechnung mit denen der experimentellen Speziation, so erkennt man, dass sie teilweise weit auseinanderklaffen. Gründe dafür können z.B. Übersättigung der Lösungen und der Einfluss komplexierender DOC-Komponenten sein. Ein Anhaltspunkt für die Übersättigung von Lösungen sind die Sättigungsindizes verschiedener Minerale, die von MINTEQA automatisch mitberechnet werden. Ein Sättigungsindex größer als 0 bedeutet, dass die entsprechende Festphase der Thermodynamik zufolge ausfallen müsste. Das heißt allerdings nicht automatisch, dass das Mineral tatsächlich ausfällt, denn darüber entscheiden auch die kinetischen Gegebenheiten. Bei stark übersättigten Lösungen ist allerdings davon auszugehen, dass die Speziationsberechnungen für das entsprechende Element nicht korrekt sind. Tab. A.16. im Anhang gibt die berechneten Sättigungsindizes für die wichtigsten Mineralphasen an, die sich ausbilden können; dazu gehören Eisen- und Manganhydroxide, Gips und Baryt. Ein Vergleich mit den gemessenen Festphasenanteilen aus den Tiefenprofilen von RCO4 zeigt, dass mit den berechnten Sättigungsindizes des Profils kein Zusammenhang erkennbar ist.

Trägt man jedoch die Sättigungsindizes verschiedener Festphasen eines Elements gegen den pH-Wert auf, ermöglicht die Auftragung Rückschlüsse darüber, welche Festphase die Konzentration des jeweiligen Elements in den Lösungen kontrolliert (SHUM und LAVKULICH 1999): Sättigungsindizes um 0 (in der Praxis -1 < SI < 1) lassen darauf schließen, dass das gelöste Element im Gleichgewicht mit der entsprechenden Festphase steht. Die Abb. 5.21. 5.24. zeigen beispielhaft solche Auftragungen für einige Elemente in den Proben aus Cospuden und den Proben aus der Lausitz. 

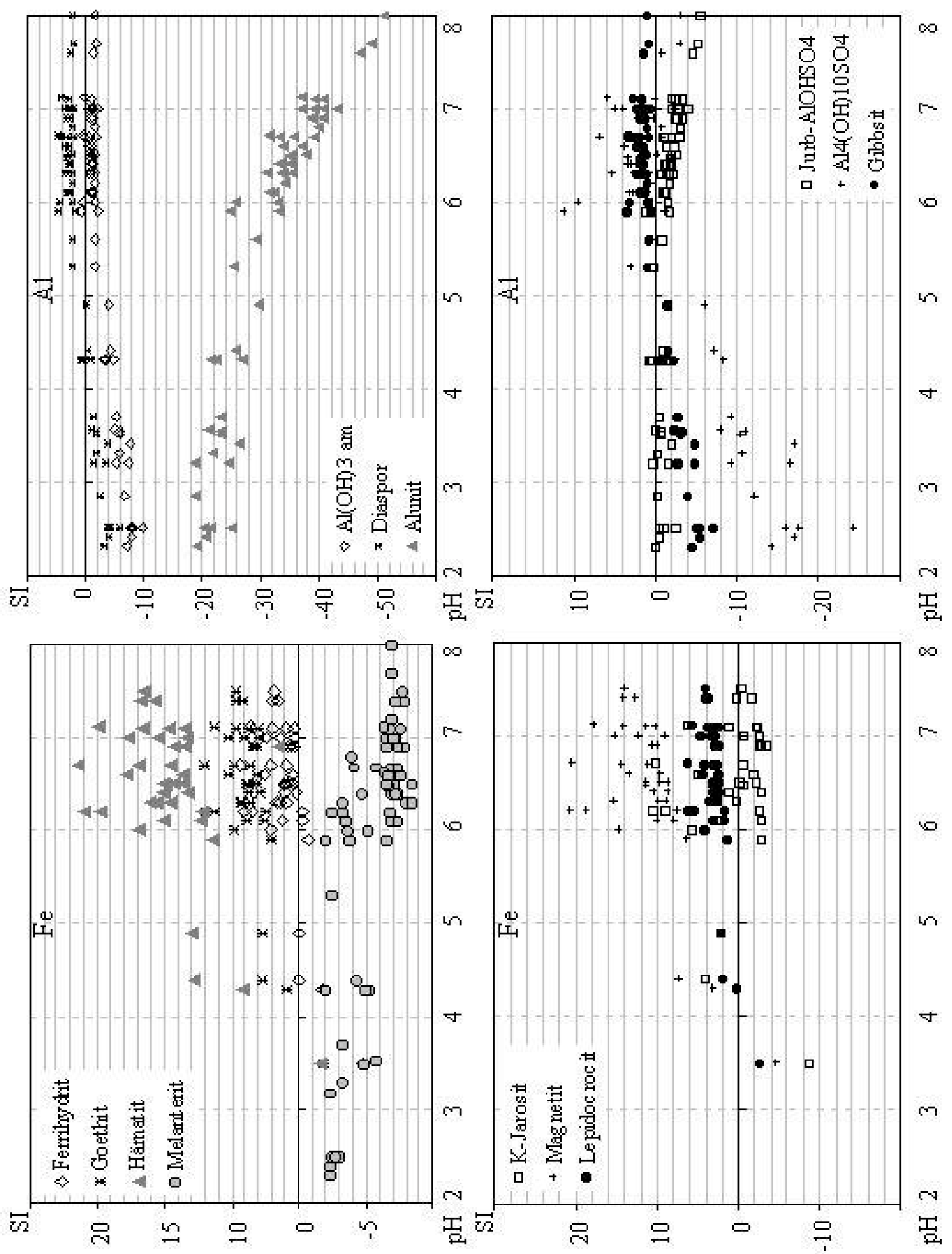

Abb. 5.21.: Sättigungsindizes verschiedener Festphasen in Proben aus Cospuden, aufgetragen gegen den $\mathrm{pH}$ Wert. 
In Abb. 5.21. sind links in zwei Diagrammen die Sättigungsindizes der wichtigsten Eisenphasen (Shum und LAvKULICH 1999) gegen den $\mathrm{pH}$ für Proben aus Cospuden aufgetragen. Schwertmannit ist in der Datenbank von MINTEQA nicht enthalten und konnte aufgrund des Mangels an thermodynamischen Daten nicht berücksichtigt werden. Melanterit scheint danach keine Rolle für die Kontrolle der Eisenkonzentration in der Lösung zu spielen. Dagegen sind alle anderen Eisenphasen, also Ferrihydrit, Goethit, Hämatit, K-Jarosit, Magentit und Lepidocrocit, in den meisten Lösungen entweder im Gleichgewicht mit der Lösung oder übersättigt; mit steigendem $\mathrm{pH}$-Wert sind Hämatit und Magnetit offensichtlich die stabilsten Phasen.

Auf der rechten Seite der Abb. 5.21. sind in zwei Diagrammen die wichtigsten Al-Festphasen dargestellt. Demzufolge hat Alunit über den gesamten pH-Bereich keinen Einfluss auf die AlKonzentration in der Lösung. Bei pH-Werten unterhalb von ca. 4 ist sind die Lösungen hinsichtlich amorphem $\mathrm{Al}(\mathrm{OH})_{3}$ nicht gesättigt; auch Gibbsit und $\mathrm{Al}_{4}(\mathrm{OH})_{10} \mathrm{SO}_{4}$ sind untersättigt. In diesem pH-Bereich scheinen ein Al-OH-Sulfat vom Jurbanit-Typ und Diaspor die entscheidenden Festphasen zu sein. Bei höheren pH-Werten stehen amorphes $\mathrm{Al}(\mathrm{OH})_{3}$, Diaspor, $\mathrm{Al}(\mathrm{OH}) \mathrm{SO}_{4}$ und Gibbsit im Gleichgewicht mit der Lösung oder sind übersättigt; bei pH-Werten oberhalb von etwa 6,5 ist $\mathrm{AlOHSO}_{4}$ untersättigt.

Wie Tab. A.16. im Anhang zeigt, sind in fast allen Proben aus Cospuden Eisen- oder Aluminiumphasen übersättigt. Das hat großen Einfluss auf die Speziation, wie noch erörtert werden wird.

Abb. 5.22. zeigt die Sättigungsindizes einiger weiterer wichtiger Festphasen für Elemente in den Wässern aus Cospuden. Mn ist danach nur in wenigen Lösungen hinsichtlich Pyrolusit übersättigt. In acht Proben liegt Mn nach den Sättigungindizes im Gleichgewicht mit Manganit, wobei keine $\mathrm{pH}$-Abhängigkeit zu erkennen ist.

Die Sättigung von $\mathrm{Cr}$ ist dagegen offensichtlich stark pH-abhängig. Bis zu einem pH-Wert von etwa 6 liegen keine Festphasen im Gleichgewicht mit dem gelösten Cr; oberhalb davon stehen zunächst amorphes $\mathrm{Cr}(\mathrm{OH})_{3}$, bei höheren pH-Werten auch $\mathrm{Cr}_{2} \mathrm{O}_{3}, \mathrm{Cr}(\mathrm{OH})_{3}$ und $\mathrm{FeCr}_{2} \mathrm{O}_{4}$ im Gleichgewicht mit der Lösung oder sind leicht übersättigt. $\mathrm{Cr}_{2} \mathrm{O}_{3}$ ist dabei die stabilste der in Frage kommenden Festphasen.

Die Kupferkonzentration unterliegt bei pH-Werten oberhalb von 4,5 der Kontrolle durch Kupferferrit; bei pH-Werten oberhalb von 7 kommt auch Tenorit als entscheidende Festphase in Betracht. 

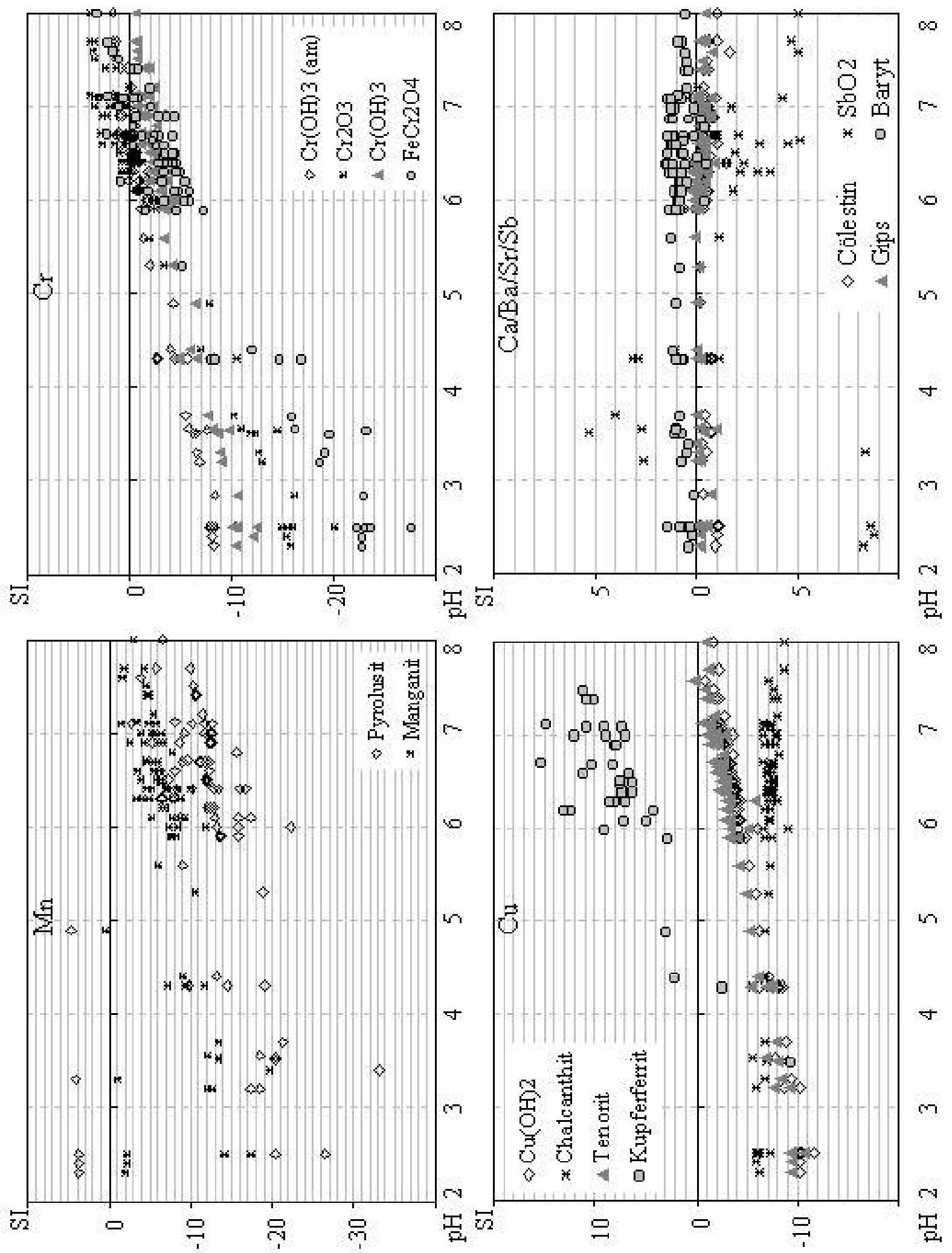

Abb. 5.22.: Sättigungsindizes verschiedener Festphasen in Proben aus Cospuden, aufgetragen gegen den pHWert. 
Fast alle Proben über den gesamten pH-Bereich stehen im Gleichgewicht mit Gips oder sind leicht übersättigt; Baryt ist in einem Großteil der Proben übersättigt. Auch die Sättigungsindizes von Cölestin bewegen sich in fast allen Proben zwischen -1 und +1 , so dass davon auszugehen ist, dass auch $\mathrm{Sr}$ in vielen Proben übersättigt ist. Die Sb-Konzentration wird bei pH-Werten zwischen 3 und 4,5 teilweise von $\mathrm{SbO}_{2}$ kontrolliert.

Abb. 5.23. und 5.24. zeigen vergleichbare Sättigungsdiagramme für die Proben aus der Lausitz. Die pH-Werte lagen in diesen Proben alle deutlich im sauren Bereich. Trotz der hohen Eisenkonzentrationen spielen Eisenfestphasen den Sättigungindizes zufolge keine Rolle für die Kontrolle der Eisenkonzentration. Mit Ausnahme der Probe Lausitz 21,90 m stehen aber alle Wässer im Gleichgewicht mit Diaspor. Fast alle Proben sind im Hinblick auf $\mathrm{AlOHSO}_{4}$ gesättigt oder leicht übersättigt. Alunit, amorphes $\mathrm{Al}(\mathrm{OH})_{3}, \mathrm{Al}_{4}(\mathrm{OH})_{10} \mathrm{SO}_{4}$ und Gibbsit sind dagegen in allen Proben deutlich untersättigt. Auch Mn steht in keiner erkennbaren Wechselwirkung mit seinen Festphasen Pyrolusit und Manganit. Keine der Proben zeigt eine Übersättigung hinsichtlich einer der betrachteten Festphasen von Cr oder $\mathrm{Cu}$. Alle Lösungen stehen dagegen offensichtlich im Gleichgewicht mit Gips und Cölestin und sind hinsichtlich Baryt übersättigt. $\mathrm{SbO}_{2}$ ist außerdem in fünf Proben übersättigt.

Ähnliche Diagramme wie die hier gezeigten ließen sich theoretisch für eine Reihe weiterer Elemente aufstellen. Dabei wären allerdings neben dem pH-Wert in vielen Fällen zusätzlich die Konzentrationen weiterer Elemente $\mathrm{zu}$ berücksichtigen; so bildet das anionisch auftretende As theoretisch mit fast allen vorhandenen Kationen Festphasen, wobei die Sättigungsindizes nicht in erster Linie vom $\mathrm{pH}-$ Wert, sondern von den Kationenkonzentrationen abhängig sind. Ähnliches gilt für den Einfluss der Sulfatkonzentration auf die Sättigung der Festphasen z.B. bei Al. So fanden andere Arbeitsgruppen bei ähnlichen Studien an sauren Minenwässern beispielsweise eine Abhängigkeit der Al-Konzentration von Alunit; (SHUM und LAVKULICH 1999). In den hier dargestellten Proben spielt dieses Mineral keine Rolle für die Al-Gehalte der Lösungen. 

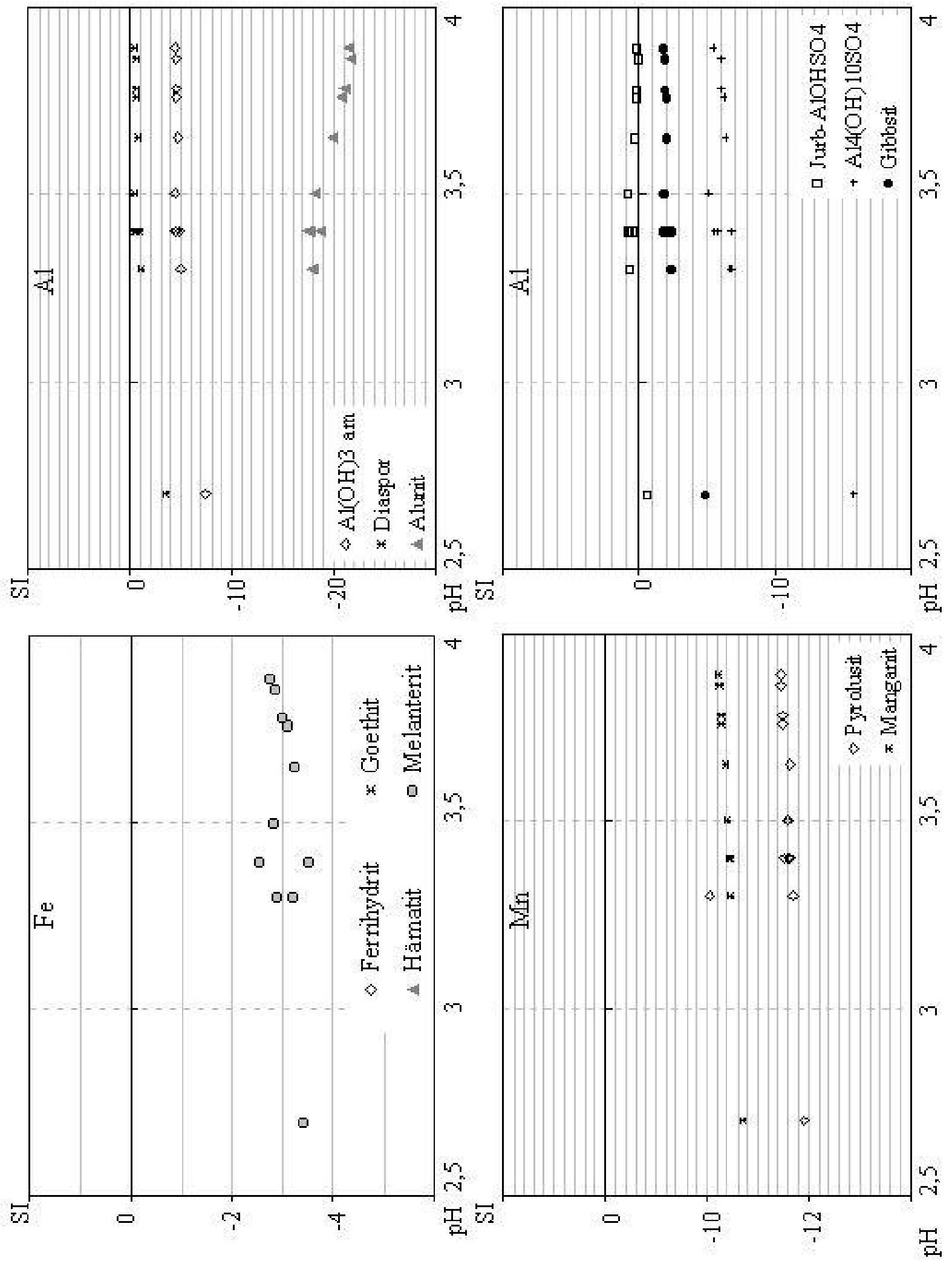

Abb. 5.23.: Sättigungsindizes verschiedener Festphasen in Proben aus der Lausitz, aufgetragen gegen den pHWert. 


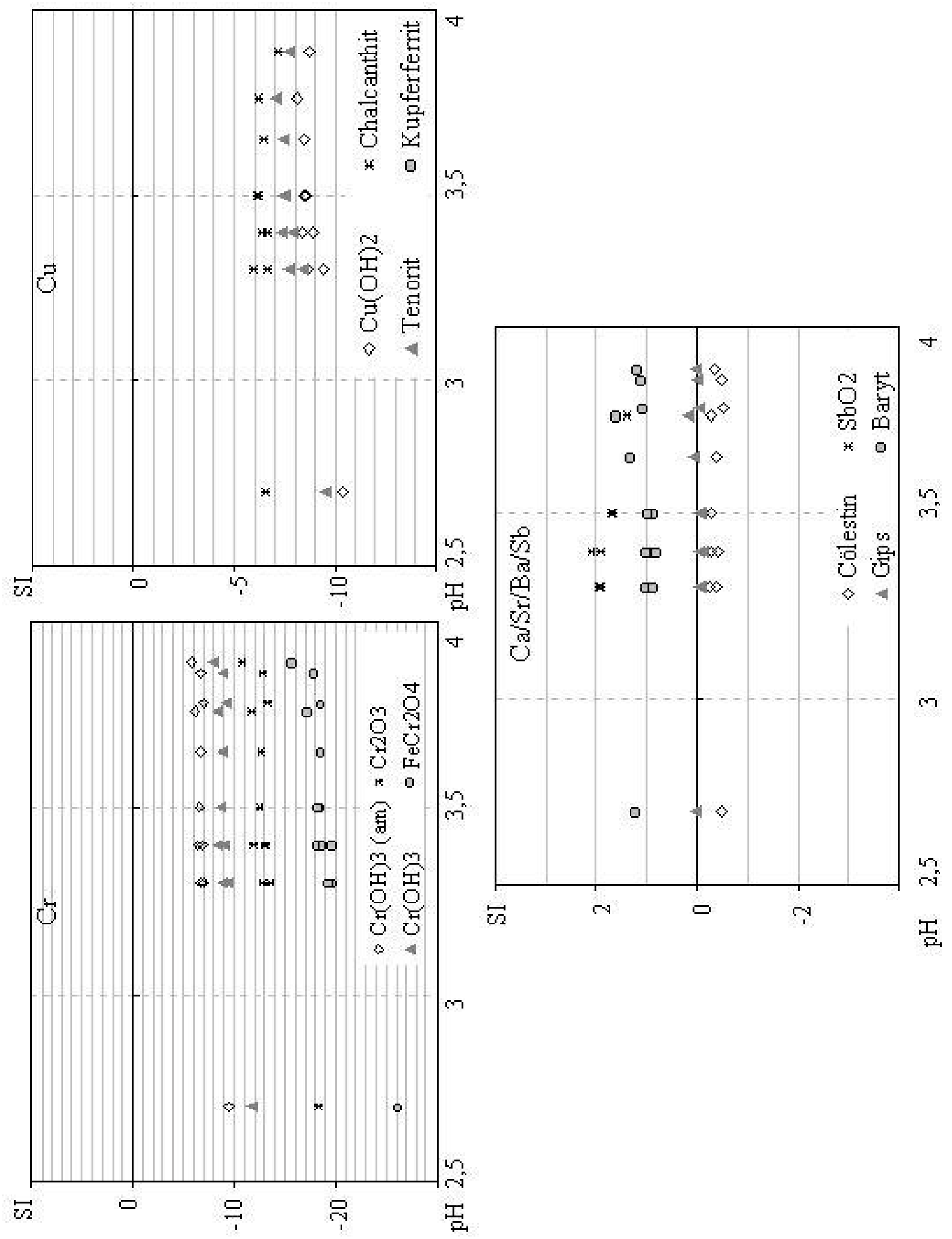

Abb. 5.24.: Sättigungsindizes verschiedener Festphasen in Proben aus der Lausitz, aufgetragen gegen den pHWert. 
Damit eine sinnvolle Speziationsberechnung möglich ist, ist es notwendig, die Proben zunächst nach optischen Gesichtspunkten und den Ergebnissen der Ultrafiltration zu ordnen, um Verfälschungen der experimentellen Ergebnisse durch kolloidale Phasen zu erkennen. Dabei erhält man verschiedene Arten von Wasserproben; einige sind geprägt durch das Vorhandensein von Eisenniederschlägen oder kolloidalen Eisenphasen in der Lösung, bei anderen ist das Eisen bereits ausgefallen. Als nächstes muss die Überlegung angestellt werden, inwieweit die Lösungen sich im Gleichgewicht befinden oder - z.B. im Fall starker Übersättigung - sehr weit davon entfernt sind.

Bei einer ganzen Anzahl von Proben war die Bildung von Eisenhydroxidniederschlägen schon vor oder während der Speziation deutlich zu sehen. Betrachtet man die Speziationsdaten, lässt sich unter diesem Gesichtspunkt bereits eine Gruppe von Proben festlegen, die aus der weiteren Betrachtung ausgenommen werden muss. Es handelt sich dabei um Proben, in denen offensichtlich kolloidale Eisenniederschläge vorliegen, auf deren Oberfläche zahlreiche Spurenelemente adsorbiert wurden. Diese Proben zeichnen sich dadurch aus, dass sowohl bei der Ultrafiltration wie auch beim Kationen- und Anionenaustausch das Eisen sowie viele andere Elemente vollständig zurückgehalten werden. Dies basiert jedoch nicht auf Ionenaustausch, sondern auf der Filtration von Partikeln an den Membranen. Betroffen davon sind vor allem die Standgewässer aus der Frühzeit der Flutung, die von PLEßOW (1998) untersucht wurden. Dies erscheint auch plausibel; die Wässer weisen trotz Sauerstoffkontakt extrem hohe Eisenwerte auf, die nur durch das Vorliegen kolloidalen Eisens erklärt werden können. Auch optisch ist das an der rotbraunen Färbung der Wässer zu erkennen.

Nicht alle Proben, in denen sich schon vor der Untersuchung Eisenniederschläge bildeten, zeigen das beschriebene Verhalten. Bei einigen Proben, aus denen ursprünglich schon Niederschläge ausgefallen waren, traten sogar nach dem Kationenaustausch noch Niederschlagsbildungen von rostroten Eisenhydroxidniederschlägen auf. Für eine zuverlässige Speziation muss daher unbedingt auch der optische Eindruck der Proben sowie ein u.U. auftretender $\mathrm{H}_{2} \mathrm{~S}$-Geruch berücksichtigt werden.

\subsubsection{Verhalten des DOC bei der Speziation}

Wie bereits erwähnt, handelt es sich beim DOC um einen Summenparameter, der für ein hochkomplexes Gemisch verschiedenster organischer Komponenten steht, die auf unterschiedliche Weise mit anorganischen Bestandteilen der Wässer wechselwirken.

Eine zusätzliche Schwierigkeit bei der Beurteilung organischer Einflüsse entsteht dadurch, dass sich der DOC in verschiedenen Proben und zu verschiedenen Zeiten stark unterscheidet. 
Ein Zusammenhang zwischen $\mathrm{pH}$ und Absolutgehalt an DOC ist nicht erkennbar (Korrelationskoeffizient $\mathrm{r}=0.25$ ); demnach unterscheiden sich auch die Anteile von Huminund Fulvosäuren am Gesamt-DOC, denn diese beiden Komponenten sind in ihrem Lösungsverhalten stark pH-abhängig. Auch bei pH-Werten oberhalb von 7 werden bis zu 63 $\mathrm{mg} / \mathrm{l}$ DOC gefunden.

Da vom Ultrafilter nur in wenigen Fällen größere Mengen des DOC zurückgehalten werden, ist davon auszugehen, dass es sich in der Regel um verhältnismäßig niedermolekulare Verbindungen handelt.

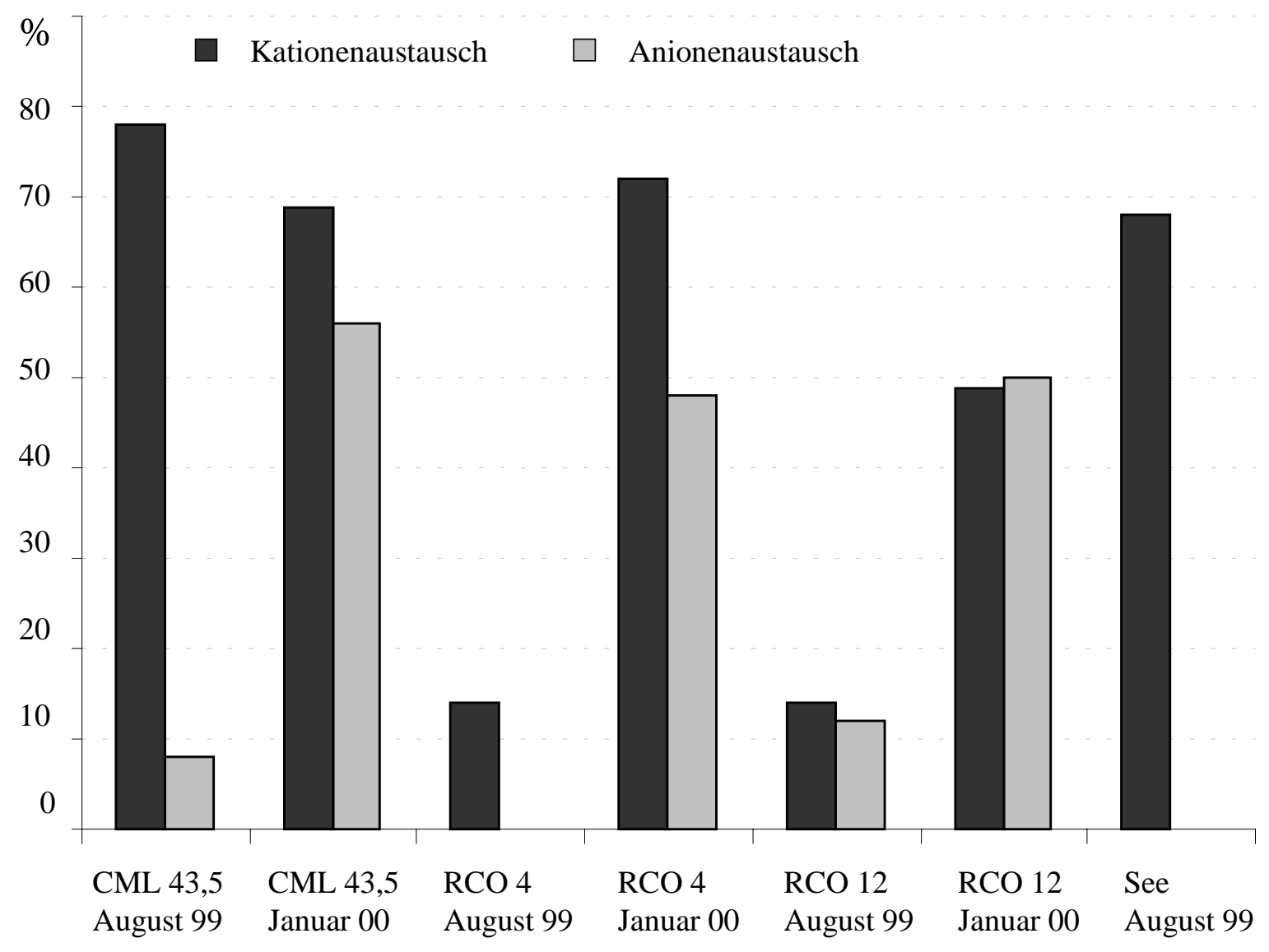

Abb. 5.25.: Verhalten von DOC beim Ionenaustausch in verschiedenen Proben aus Cospuden; zurückgehaltener Anteil der Ausgangskonzentration in \%.

Abb. 5.25. zeigt das Verhalten des DOC beim Ionenaustausch bei Proben von verschiedenen Standorten und zu verschiedenen Zeitpunkten an einigen Beispielen. Auf den ersten Blick sind die großen Unterschiede zwischen den einzelnen Proben festzustellen. Generell fällt bei den Proben aus Cospuden auf, das der DOC häufig große Austauschraten aufweist. Ganz anders sieht es bei den Proben aus der Lausitz aus; hier sind die Austauschraten sowohl bei Anionen als auch bei Kationen sehr gering. In Cospuden dagegen liegt die Summe von 
Kationen- und Anionenaustausch häufig bei über $100 \%$, wie etwa bei den Proben CML 43,5 m Januar 2000, RCO4 Januar 2000 und RCO12 Januar 2000 (Abb. 5.25.) (s.a. BRÜSCHKE et al. (1999)). Das bedeutet, dass ein Teil des DOC aus Molekülen besteht, die sowohl anionische als auch kationische funktionelle Gruppen aufweisen. Der DOC ist damit nicht nur ein Faktor für die Speziation der kationischen Elemente, sondern kann auch das Verhalten von Anionen, z.B. Sulfat, beeinflussen. Die hohen Austauschraten deuten außerdem darauf hin, dass es sich um hochfunktionalisierte Substanzen mit zahlreichen Bindungsstellen handelt. Der DOC kann aber fast ausschließlich kationischen oder anionischen Charakter haben; in Abb. 5.25. sind die Proben CML 43,5 m August 1999 und See August 1999 praktisch nur vom Kationenaustausch betroffen. Auch neutrale Moleküle können Bestandteile des DOC sein; in der Probe RCO4 August 1999 (Abb. 5.25.) werden nur geringe Anteile des DOC beim Kationenaustausch zurückgehalten, vom Anionenaustausch bleibt der DOC völlig unbeeinflusst; fast $90 \%$ des DOC haben neutralen Charakter.

Diese Beobachtungen für den DOC aus Cospuden im Vergleich zu den Proben aus der Lausitz decken sich mit den Ergebnissen der statistischen Auswertung; dort verhalten sich ja die meisten Elemente in den Proben aus der Lausitz so, wie es ohne starke Einflüsse des DOC zu erwarten wäre, während die Proben aus Cospuden starke Abweichungen zeigen.

Wegen der Durchmischung des Kippenmaterials mit Braunkohleresten ist anzunehmen, dass große Anteile des DOC braunkohletypische Strukturen aufweisen. Im Zuge der Inkohlung kommt es zu einem Verlust leicht abbaubarer Kohlenstoffspezies wie Polysaccharide (HATCHER et al. 1989); typisch für Braunkohle-NMR-Spektren ist ein hoher Anteil aromatischen und alkylgebundenen Kohlenstoffs (MEILER und MEUSINGER 1991). Außerdem enthält Braunkohle geringe Anteile an Ligninstrukturen. Unter der Annahme, dass die Braunkohle die Zusammensetzung des DOC in Cospuden prägt, ist analog zu dieser ein relativ hoher Schwefelanteil anzunehmen, der dieses Verhalten erklären könnte. Im Untersuchungsgebiet der Lausitz, dass bereits Pflanzenbewuchs besitzt, stammt der DOC vermutlich sowohl aus der Braunkohle als auch aus rezenten Ablagerungen durch die Vegetation (KÖGEL-KNABNER und RUMPEL 2000). Zusätzlich können organische Substanzen aus den Abgasen der Braunkohleverstromung über die Luft in das Untersuchungsgebiet gelangen (RUMPEL et al. 1998). 


\subsubsection{Speziation unter Berücksichtigung der organischen Komponenten und anorganischer Kolloide}

Geht man von der Einordnung der Proben nach dem dargestellten Klassifikationsschema aus, lassen sich generell zwei Arten von Proben festlegen: Die Proben der Gruppen 6 und 7 sind offenbar frei von signifikanten Mengen an anorganischen Kolloiden. In allen anderen Proben liegen anorganische Kolloide vor, die das Speziationsergebnis verfälschen können. Außerdem ist für viele dieser Proben davon auszugehen, dass sie sich keineswegs im Gleichgewicht befinden, sondern Fällungsprozesse ablaufen.

Wir diskutieren die Speziation der einzelnen Proben zunächst anhand der dargestellten Klassifikation (Tab. A.24. im Anhang) und gehen dann auf einige interessante Elemente ein, bevor exemplarisch gezeigt werden soll, wie eine möglichst realitätsnahe, alle relevanten Parameter berücksichtigende Speziationsberechnung aussehen kann. Dabei dienen zur Orientierung in erster Linie die experimentellen und berechneten Ergebnisse von Sulfat und die Übergangsmetalle $\mathrm{Co}, \mathrm{Ni}, \mathrm{Cu}$ und $\mathrm{Zn}$. Bei den Ergebnissen der Alkalimetalle ist zu berücksichtigen, dass der Austausch dieser Elemente am Kationentauscher in synthetischen, nicht hochkonzentrierten Lösungen nicht vollständig verläuft (PLEßOW 1998).

Gruppe 1:

Die Proben Standgewässer 2 PL und WP, Standgewässer 3 WP, Kippe SW PL, SGM 24,5 m und SGM 48,5 $\mathrm{m}$ aus der Arbeit von PLEßOW (1998) enthalten nach den Daten der Ultrafiltration größere Mengen an Eisenkolloiden. Dies schlägt sich deutlich in den Resultaten der Speziation nieder: Viele Spurenelemente werden in den Standgewässerproben und SGM 48,5 m sowohl beim Kationen- wie beim Anionenaustausch quantitativ aus den Proben entfernt. Dies ist nicht auf einen Ionenaustausch zurückzuführen, sondern auf mechanische Filtration der Eisenkolloide durch das Trägermaterial. In den Proben aus dem Standgewässer 2 werden jedoch die REEs nur zu sehr geringen Anteilen ausgetauscht. Eventuell treten hier DOC-Komponenten in Konkurrenz zu den Oberlächen der Eisenkolloide; neutrale oder schwach geladene organo-REE-Komplexe können die Ionentauscher passieren.

Gruppe 2 und Gruppe 3:

Große Mengen nicht ultrafiltrierbaren Eisens, aber Sulfatkolloide in größeren Mengen liegen in den Proben Kippe W und See Südufer PL aus der Arbeit von PLEßOW (1998) vor; geringe Eisenmengen und Sulfatkolloide fanden sich in einigen Porenlösungen (genaue Aufstellung s. Tab. A.24. im Anhang) sowie in den Proben Fremdflutung, Sickerwasser, Standgewässer 1 und See Südufer WP aus der Arbeit von PLEßOW (1998). 
Ca wurde bei der Ultrafiltration aus allen Proben der Gruppen 2 und 3 zu mehr als $80 \%$ abgetrennt. Bei den Sulfatkolloiden handelt es sich also wahrscheinlich zu großen Teilen um $\mathrm{CaSO}_{4}$. Auch die Proben RCO13 Oktober 1998 (s.u.) und See Südufer WP AP (67 \% Ca bei der Ultrafiltration abgetrennt) können der Gruppe 3 zugerechnet werden. Wie auch in den Proben der Gruppe 1 ist die experimentelle Rückhalterate beim Kationenaustausch von $\mathrm{Ba}$ in allen Proben erstaunlich niedrig; parallel zu $\mathrm{CaSO}_{4}$ liegt vermutlich in praktisch allen Proben auch $\mathrm{BaSO}_{4}$ vor. Dafür werden signifikante Anteile von Sulfat in allen Proben im Kationenaustauscher zurückgehalten. Davon abgesehen sind die Auswirkungen der Sulfatkolloide nicht mit denen der Eisenkolloide vergleichbar. Zwar werden Li, Na und K häufig mit $\mathrm{Ca}$ zusammen beim Anionenaustausch zurückgehalten. Doch die Ergebnisse für die Übergangsmetalle harmonieren in einigen Proben sowohl für den Kationen- wie für den Anionenanteil sehr gut (RCO4 11-19 m, RCO4 28 m, RCO4 30 m, RCO4 56 m, Standgewässer 1 AP, See Südufer AP). Leichte Abweichungen beim Anionenaustausch, wo die gemessenen Rückhalteraten höher liegen als die berechneten Raten, zeigen die Proben RCO4 58 m, RCO13 15 m, Kippe West AP und Sickerwasser. Das kann durch große Mengen $\mathrm{CaSO}_{4}$ verursacht werden, das die Oberfläche des Anionentauschers blockiert.

In vier Proben aus der Gruppe 3 und einer Probe aus der Gruppe 2 stimmten für $\mathrm{Cu}$, Co und Zn zwar die Ergebnisse der anionischen Anteile überein, die Resultate der kationischen Anteile lagen jedoch im Experiment unter den berechneten Anteilen. In diesen Proben RCO16 29 m AP, RCO10 18,5 m AP, Fremdflutung AP, See Südufer PL AP und RCO4 34 m - wurde entweder die Oberfläche des Kationenaustauschers von kolloidalen Sulfaten für Kationen blockiert, oder organische Komponenten komplexierten die Kationen und verhinderten so ihren Austausch.

In der Probe RCO13 Oktober 1998 findet sich kein Anhaltspunkt, in welcher Form das Sulfat ausgefallen sein kann; alle Hauptelemente zeigen nur geringe Rückhalteraten bei der Ultrafiltration. Soweit Daten für die Speziation vorliegen, stimmen rechnerisch und experimentell bestimmte Ergebnisse gut überein.

\section{Gruppe 4 und Gruppe 5:}

Die insgesamt sieben eindeutig zuzuordnenden Proben der Gruppe 5 zeigen keine vollständige Abtrennung eines anorganischen Kolloids bei der Ultrafiltration. Die Daten weisen aber auf das Vorhandensein signifikanter Mengen anorganischer Kolloide hin. Zwei weitere Proben, bei denen die UF-Daten unvollständig sind, wurden aufgrund der sonstigen Ergebnisse der Gruppe 5 zugeordnet (s. Tab. A.24. im Anhang). Zur Gruppe 4 war nur die 
Probe Lausitz 21,40 m eindeutig zuzuordnen, vermutlich gehört aber z.B. die Probe Standgewässer 3 PL AP auch in diese Kategorie.

Unter den Sulfatkolloiden spielen vor allem $\mathrm{CaSO}_{4}$ (Standgewässer 3 Porenlösung, RCO13 30 m AP, RCO4 32 m, RCO10 Oktober 1998) und teilweise Mn (RCO12 Januar 2000) eine Rolle. Die Verhältnisse gestalten sich ähnlich wie in den Gruppen 2 und 3. Die Ergebnisse des Kationenaustausches der Übergangsmetalle stimmt in der Probe RCO4 $32 \mathrm{~m}$ sehr gut überein. $\mathrm{Zu}$ niedrig liegen die Raten beim experimentellen Kationenaustausch in den Proben Lausitz 21,40 m, Standgewässer 3 PL, RCO13 30 m. RCO10 vom Oktober 1998 zeigt Abweichungen nach oben.

Gruppe 6 und Gruppe 7:

Hierbei handelt es sich um eine große Anzahl Proben, die ein sehr komplexes Bild liefern. Ausgehend von den Resultaten der Ultrafiltration sollte es sich hierbei nur noch um Wässer handeln, in denen Kolloide allenfalls eine untergeordnete Rolle spielen. Für die Speziation sollten danach ausschließlich Faktoren wie pH-Wert, Redoxpotential, Elementkonzentrationen und organische Komponenten relevant sein. Die DOC-Daten aus der Ultrafiltration (soweit sie vorliegen) liefern keine Anhaltspunkte für das Vorliegen organischer Kolloide in einer dieser Proben.

Der Vergleich der Ergebnisse aus Rechnung und Experiment (Tab. A.25. im Anhang) für die Proben dieser Gruppe zeigt jedoch, dass die Verhältnisse keineswegs so einfach sind. Als Indikator für verschiedene Sorten von Proben innerhalb dieser Gruppe soll das Verhalten des Sulfats dienen; in Abb. 5.26. sind die berechneten und die experimentellen Ergebnisse der Speziation für Sulfat nur für die Proben dieser beiden Gruppen einander gegenübergestellt. 


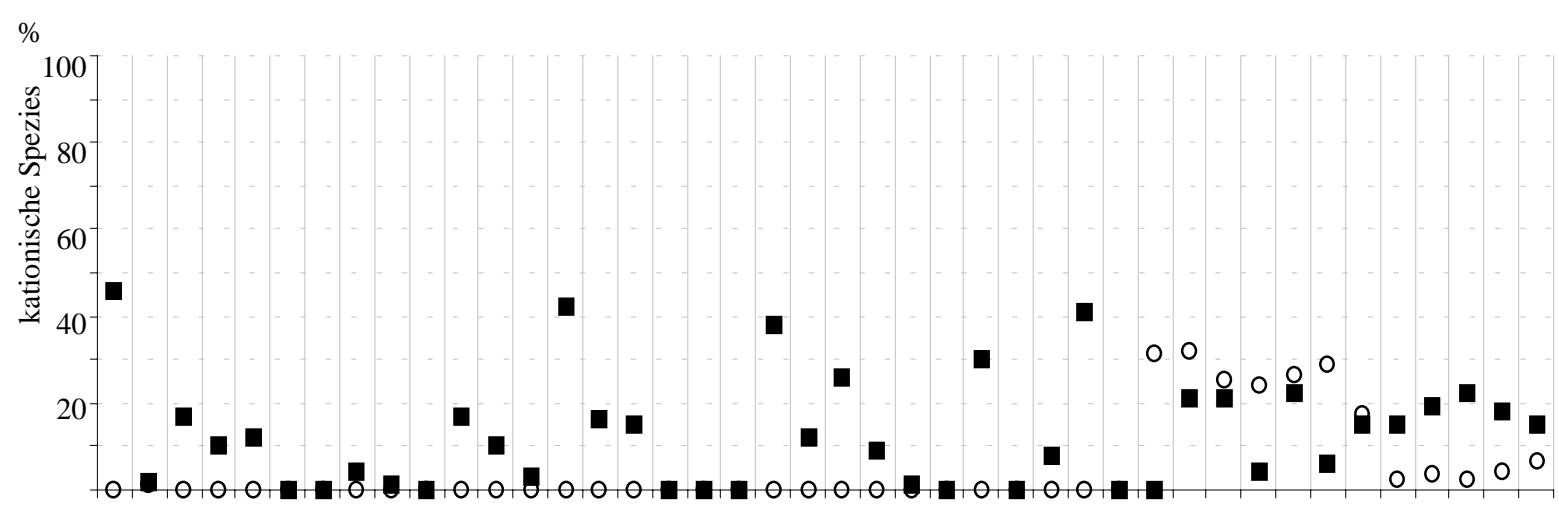

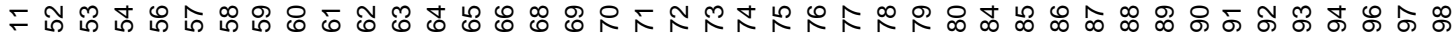
$\%$

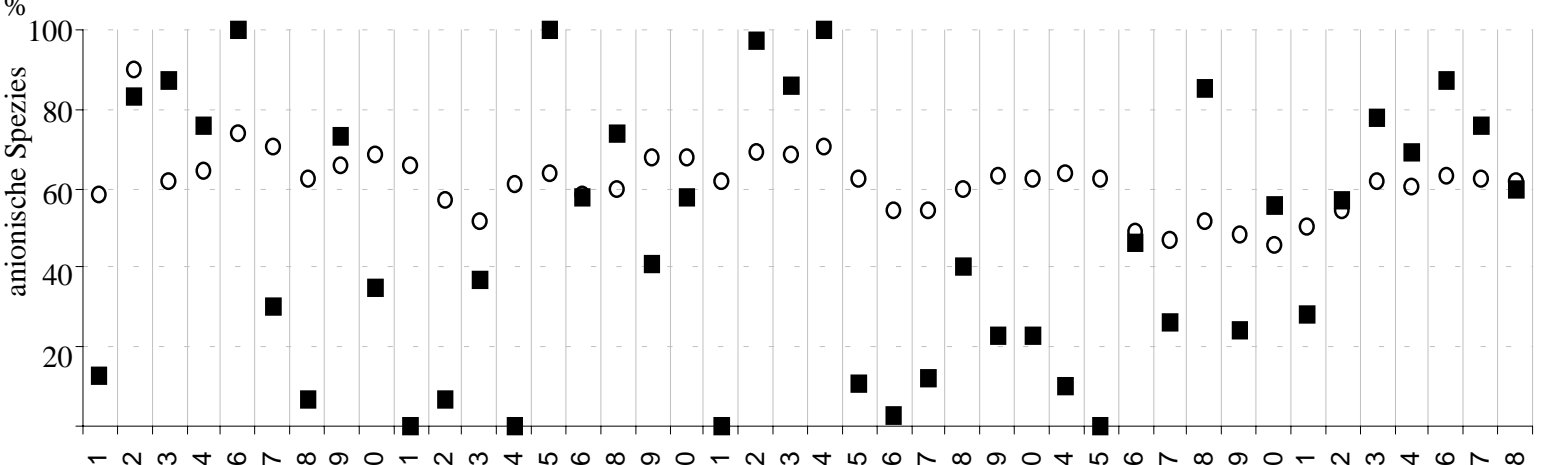

experimentell

$\bigcirc$ berechnet

Abb. 5.26.: Experimentelle und berechnete Sulfatspeziation in Proben der Gruppen 6 und 7; Zuordnung der Nummern s. Tab. A.24 im Anhang.

Bei der Diskussion der Ergebnisse aus Experiment und Rechnung gilt als gute Übereinstimmung, wenn die Abweichung zwischen den Resultaten bis zu $20 \%$ absolut beträgt; bis zu $20 \%$ beträgt der analytische Fehler bei den Speziationsexperimenten. Die Nummern hinter den Probenbezeichnungen im Text beziehen sich auf die Nummern in den Abbildungen; eine vollständige Aufzählung der Proben mit Zuordnung der Nummern ist in Tab. A.24. im Anhang enthalten. Die Gegenüberstellung der Ergebnisse aus Experiment und Rechnung ist vollständig in Tab. A.25. im Anhang wiedergegeben.

In fünf Proben aus den Gruppen 6 und 7 liegt definitiv keine Übereinstimmung zwischen den berechneten und den experimentellen Ergebnissen des Ionenaustauschs bei Sulfat vor (Abb. 5.26.). Dabei handelt es sich um die Proben RCO4 36 m (11), CML 43,5 m August 1999 (65), See August 1999 (72), See Sediment PL August 1999 (74) und RCO17 Januar 2000 (84). Trotzdem stimmen in vielen Proben vor allem die Ergebnisse für die Alkali- und Erdalkalimetalle gut überein (Tab. A.25. im Anhang). Die Ergebnisse des experimentellen Kationenaustausches liegen eher zu hoch. 
In 18 Proben wurde Sulfat zwar im Kationenaustausch erwartungsgemäß kaum abgetrennt jedoch lagen auch die Rückhalteraten im Anionenaustausch in der Regel niedriger, als nach der Rechnung zu erwarten war (Abb. 5.26.). Betrachtet man die Ergebnisse der Übergangsmetalle, kommen nach den Größenordnungen der Abweichungen in vielen Proben organische Einflüsse in Frage (Aufstellung der Proben s. Tab. A.25. im Anhang). Damit lässt sich jedoch die Abweichung beim Sulfat im Anionenaustausch in der beobachteten Größenordnung nicht erklären. Möglicherweise liegen in diesen Lösungen subkolloidale Sulfatkomplexe von $\mathrm{Ca}$ oder anderen Erdalkalimetallen vor, deren Einfluss auf die Spurenelemente nur schwer zu erfassen ist.

In zwei weiteren Proben liegen mit großer Sicherheit $\mathrm{CaSO}_{4}-$ Kolloide vor. Sie wurden bei der Ultrafiltration nicht abgetrennt, es handelt sich also wohl um sehr kleine Strukturen. Trotzdem deutet der Vergleich der Daten aus Berechnung und Experiment für die Proben See 1998 (55) und RCO17 Januar 2000 (84) darauf hin, dass Sulfat und Ca nicht vollständig gelöst vorliegen. Ähnlich wie in den Proben mit nachgewiesenen Sulfatkolloiden liegen die experimentellen Daten für den Kationenaustausch bei Sulfat zu hoch. Im Anionenaustausch wird in RCO17 Januar 2000 (84) und See 1998 (55) zuwenig Sulfat abgetrennt (Abb. 5.26.). In beiden Proben werden mehr als $80 \%$ des Ca beim Anionenaustausch zurückgehalten. Die Rückhalteraten der Übergangsmetalle liegen beim Anionenaustausch ebenfalls sehr hoch (Tab. A.7. im Anhang).

Übereinstimmungen zwischen berechneten und experimentell bestimmten Sulfatanteilen bei der Speziation zeigen insgesamt 15 Proben aus den Gruppen 6 und 7; in Abb. 5.25. fallen dabei die Symbole für die experimentellen und die berechneten Werte direkt aufeinander.

Vier Proben aus der Gruppe 7 stimmen bis auf wenige Ausnahmen für alle Arten von Elementen gut mit der theoretischen Berechnung überein, nämlich die Proben Lausitz 21,90 m (98), Lausitz 5,21 m (87), Lausitz 5,60 m (90), und Lausitz 5,93 m (92) (Tab. A.25. im Anhang).

Bei drei weiteren Proben aus der Lausitz, nämlich Lausitz 5,30 m (88), 21,13 m (94) und 21,80 m (97), zeigen die meisten Elemente eine leichte Abweichung nach oben bei den experimentellen Ergebnissen des Anionenaustausches gegenüber den berechneten Resultaten. Diese Abweichungen liegen jedoch bei den Übergangsmetallen unter $30 \%$. Auch CML 39,5 m vom Januar 1999 (59) zeigt bei den vorhandenen Daten gute Übereinstimmung zwischen Rechnung und Experiment (Tab. A.25.).

Die übrigen Proben ergaben für die Kationen ein sehr uneinheitliches Bild. Die Daten der Übergangsmetalle beim Kationenaustausch stimmten in der Regel zwischen Rechnung und 
Experiment überein. Im Anionenaustausch wurden teilweise Rückhalteraten um $30 \%$ beobachtet (November $19981 \mathrm{~m}$ (52), November 19983 m (54), CML 21-22 m PL (40), Lausitz 21,07 m (93), RCO3 August 1999 (66)). Dies liegt im Rahmen dessen, was bei Einflussnahme organischer Komplexbildner zu erwarten wäre. Auch die Abweichungen beim Kationenaustausch für komplexbildende Elemente liegen bei einigen Proben in einem Bereich, der eine Beteiligung organischer Komponenten als realistisch erscheinen lässt (so bei RCO4 20 m (4), RCO4 22 m (5), RCO4 52 m (18), RCO 17 August 1999 (70), CML 31,5 m August 1999 (63), RCO12 August 1999 (68)) (Tab. A.25., Abb. 5.27. - 5.32.).

Ausgehend von diesen Erkenntnissen kann der Versuch unternommen werden, Speziationsberechnungen unter Berücksichtigung organischer und kolloidaler Einflüsse durchzuführen, die die realen Verhältnisse der Lösung möglichst genau erfassen.

Zunächst muss anhand der Daten aus der Ultrafiltration und aus dem Vergleich von berechnetem und experimentell beobachtetem Verhalten überprüft werden, ob in der Lösung größere Mengen Kolloide vorliegen. Die Sättigungsindizes für die Festphasen sollten für jedes einzelne Element betrachtet werden. Liegt ein Sättigungsindex über Null, ist dies bei der Betrachtung des entsprechenden Elements zu berücksichtigen; das Element könnte dann eventuell kolloidal vorliegen oder durch die Übersättigung soweit vom thermodynamischen Gleichgewicht entfernt sein, dass die Rechenergebnisse, die sich ja auf thermodynamische Gleichgewichte beziehen, für dieses Element in der Proben nicht verwertbar sind.

Beispielhaft sollen hier einige Proben der Gruppen 6 und 7 aus Cospuden diskutiert werden, in denen die Resultate aus Rechnung und Experimten für die Sulfatspeziation übereinstimmten.

Die Probe November 19981 m (52) zeigt vor allem bei den Hauptelementen zahlreiche Abweichungen. Kolloide wurden bei der Ultrafiltration nicht abgetrennt. Trotzdem legt die Betrachtung der Ergebnisse beim Ba nahe, dass kolloidaler Baryt in der Lösung vorlag. Der Sättigungsindex von Baryt liegt in dieser Probe bei 0,915. Beim Anionenaustausch wurde Ba quantitativ abgetrennt. Zusätzlich sind mehrere Sn- und Sb-haltige Festphasen stark übersättigt.

Ebenso ließen sich in CML 31,5 m August 1999 (63) bei der Ultrafiltration keine Kolloide nachweisen. Doch die Sättigungsindizes (Tab. A.16. im Anhang) zeigen, dass Al-Sulfate stark übersättigt sind; die Abweichungen zwischen Rechnung und Experiment bei der AlSpeziation sind also vielleicht nicht auf organische Al-Komplexe zurückzuführen, sondern auf kleine kolloidale oder subkolloidale Strukturen, die durch adsorptive Oberflächen auch andere Elemente beeinflussen können. 
Die Abb. 5.27 bis 5.38. zeigen Übersichten für die Speziation einiger interessanter Elemente in Proben aus Cospuden und aus der Lausitz.

Nur in sechs Proben (RCO10 18,5 m (22), RCO13 15 m (24), Lausitz 5,21 m (87), Lausitz 5,30 m (88), Lausitz 5,60 m (90) und Lausitz 5,93 m (92)) stimmen für Al die Ergebnisse von Kationen- und Anionenaustausch aus Rechnung und Experiment überein (Abb. 5.27.). In den übrigen Wässern klaffen die Ergebnisse vielfach auseinander, auch in den Proben aus den Gruppen 6 und 7. In einigen Fällen können dafür kolloidale oder subkolloidale Al-Phasen verantwortlich gemacht werden, z.B. in den kolloidgeprägten Oberflächenwässern. In einigen anderen Proben jedoch kann auch der Einfluss organischer Komplexbildner nicht ausgeschlossen werden, etwa in den Proben CML 43,5 m August 1999 (65) oder RCO12 August 1999 (68) (Tab. A.25. im Anhang). Hier deuten die Abweichungen - beim Kationenaustausch zu niedrige experimentell festgestellte Raten, beim Anionenaustausch höhere Austauschraten als berechnet, aber kein vollständiger „Austausch“, der auf Kolloidbildung schließen lässt - auf die Beteiligung negativ geladener organischer Komponenten hin. Auch die weitgehende Übereinstimmung der Ergebnisse der Sulfatspeziation in diesen beiden Proben spricht für den Einfluss von Komplexbildnern und gegen ein Vorliegen kolloidaler Spezies (Abb. 5.38.).

Die Fe-Speziation aus Experiment und Rechnung stimmte nur in fünf Proben sowohl beim Kationen- als auch beim Anionenaustausch überein (Abb. 5.28.). Dies waren RCO4 $38 \mathrm{~m}$ (12), Lausitz 21,90 m (98), Lausitz 5,21 m (87), Lausitz 5,60 m (90) und Lausitz 5,93 m (92). In zahlreichen Proben zeigt sich im weitgehenden oder gar vollständigen Austausch des Eisens am Anionentauscher der Effekt der pH-Änderung beim Austausch gegen $\mathrm{OH}^{-}$. Doch auch bei vielen Proben, in denen gegen Chlorid ausgetauscht wurde, liegen die Werte im Anionenaustausch deutlich über den Erwartungen aus der Rechnungen, etwa bei Bohrung November 19983 m (54), CML 43,5 m August 1999 (65) und RCO17 Januar 2000 (84). Dafür wurden in einem Großteil der Proben zu geringe Raten beim Kationenaustausch festgestellt. Der Einfluss organischer Komplexbildner kann dabei vor allem in Proben aus dem August 1999 aus dem CML-Brunnen und aus RCO-Pegeln als wahrscheinlich angesehen werden. Dort wurde keine Kolloidbildung festgestellt, und die Abweichungen zwischen berechneten und experimentellen Austauschraten bewegen sich in Größenordnungen, die den Einfluss von DOC-Komponenten nicht notwendig überschreiten (Tab. A.25. im Anhang). 


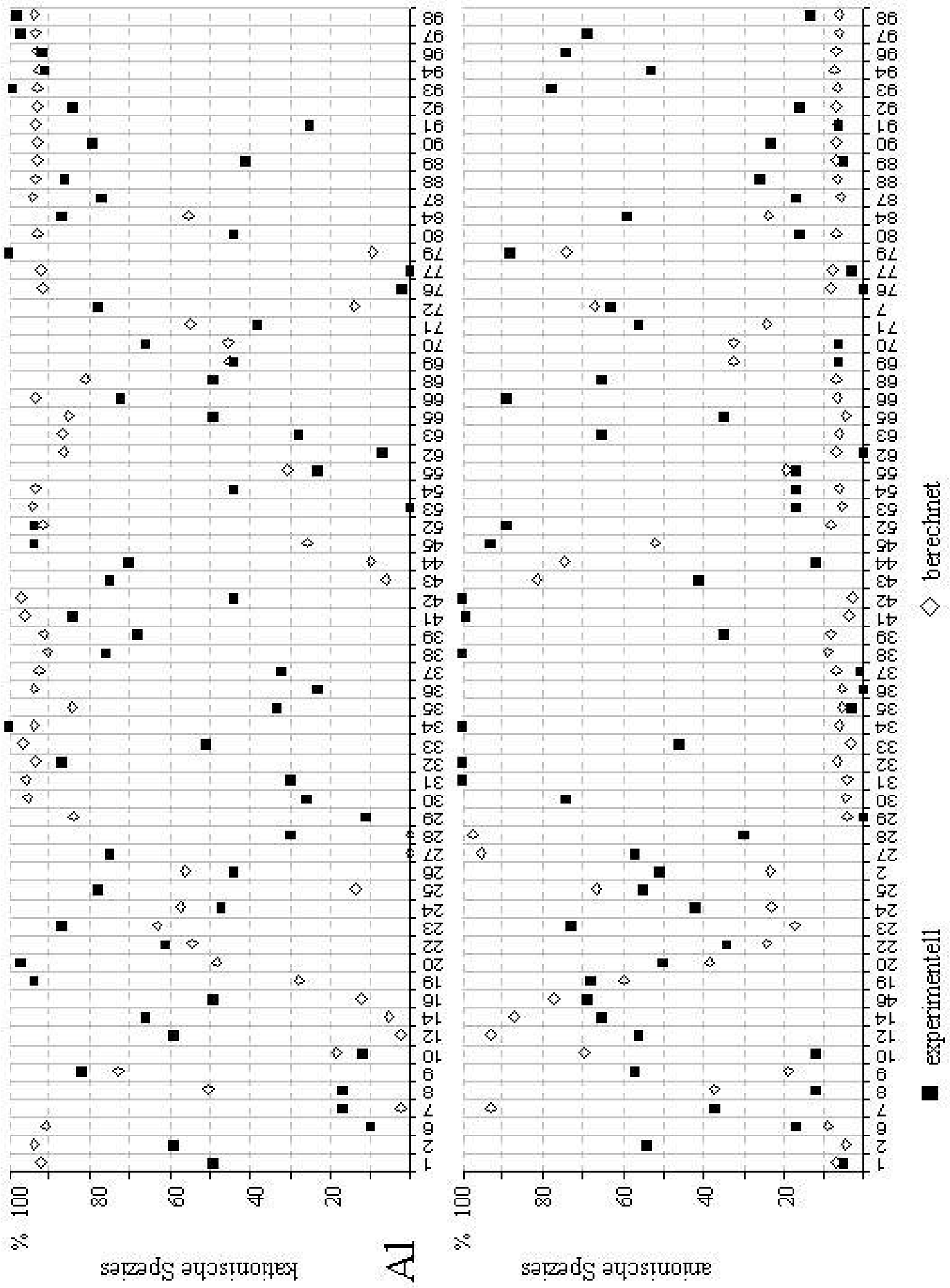

Abb. 5.27.: Experimentelle und berechnete Speziation von Al in Proben aus Cospuden und aus der Lausitz. 


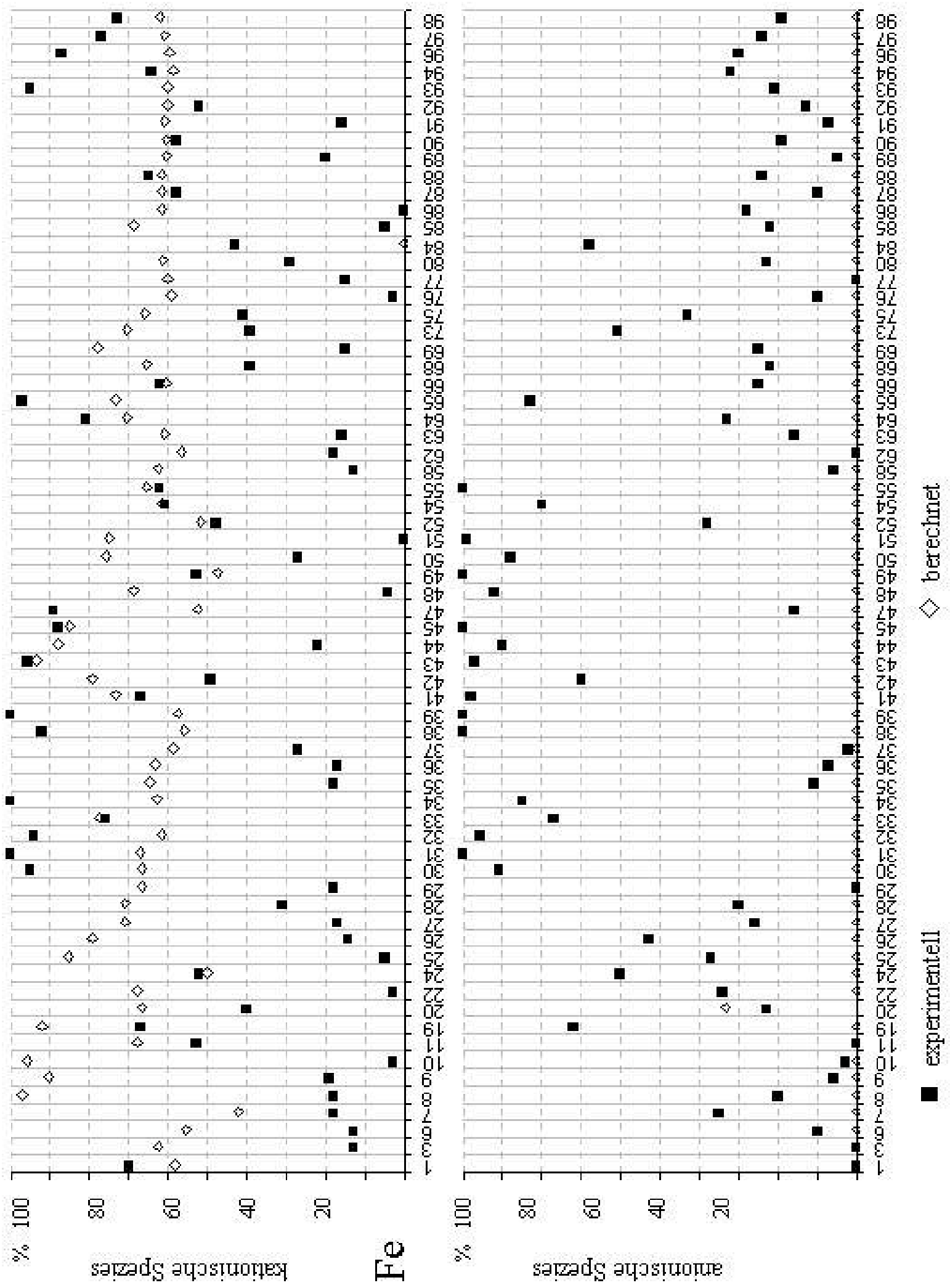

Abb. 5.28.: Experimentelle und berechnete Speziation von Fe in Proben aus Cospuden und aus der Lausitz. 


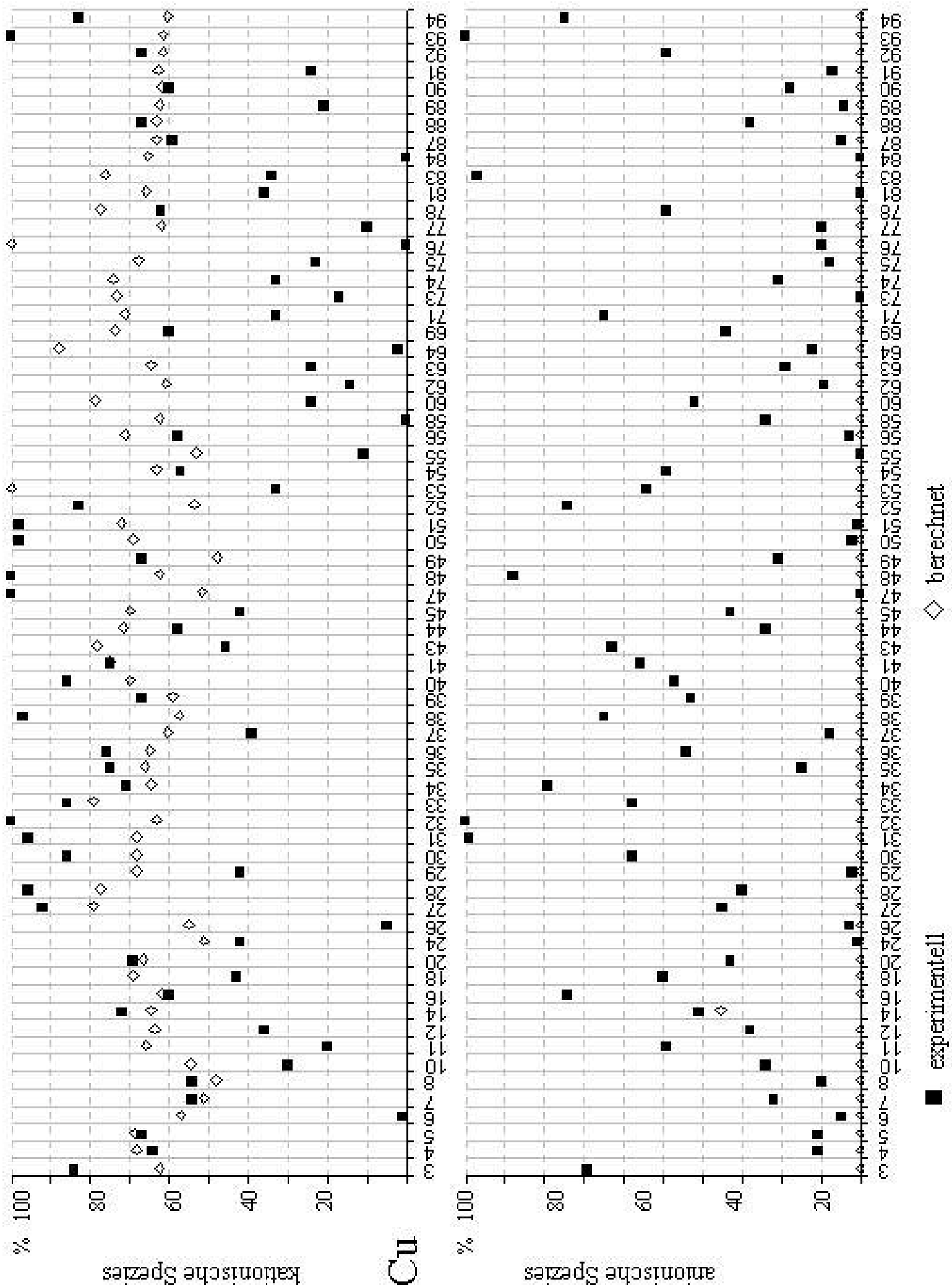

Abb. 5.29.: Experimentelle und berechnete Speziation von $\mathrm{Cu}$ in Proben aus Cospuden und aus der Lausitz. 


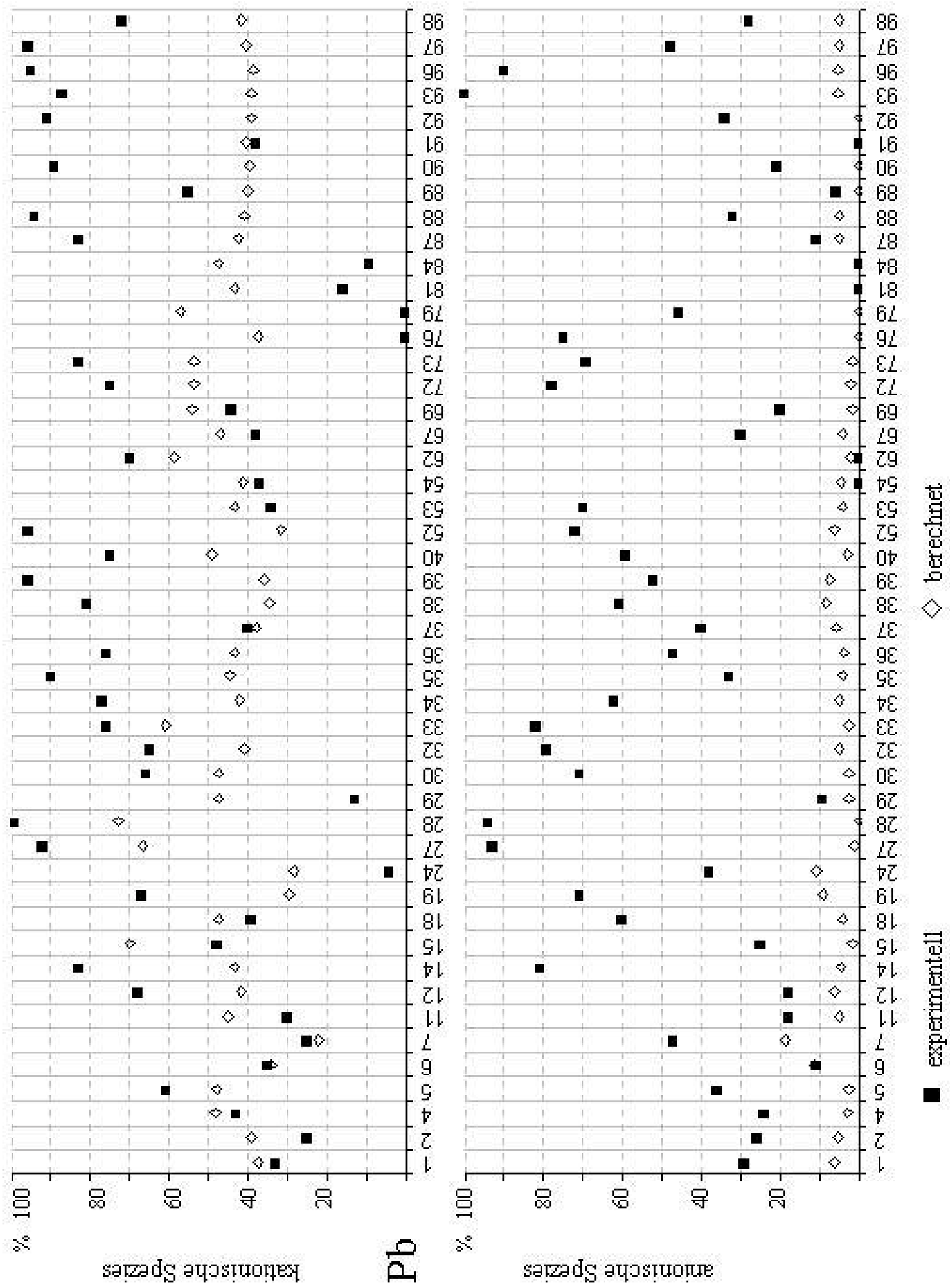

Abb. 5.30.: Experimentelle und berechnete Speziation von Pb in Proben aus Cospuden und aus der Lausitz. 
Bei $\mathrm{Cu}$ (Abb. 5.29.) zeigt eine deutlich größere Anzahl von Proben eine gute Übereinstimmung der Ergebnisse. Bei insgesamt zehn Proben betrug der Unterschied zwischen Rechnung und Experiment weder für den Kationen- noch für den Anionenaustausch mehr als 20 \%. Dazu zählen die Proben RCO4 20 m (4), RCO4 22 m (5), RCO4 28 m (7), RCO4 42 m (14), Sickerwasser WP AP (28), Kippe West PL AP (35), CML 20 m Januar 2000 (75), Lausitz 5,30 m (88) und Lausitz 5,60 m (90). Bemerkenswert ist, dass diese Aussage auch auf die drei Proben RCO4 30 m (8); RCO13 15 m (24) und RCO12 Oktober 1998 (49) zutrifft, die hinsichtlich Kupferferrit deutlich übersättigt waren (Tab. A.16. im Anhang). In 16 weiteren Proben stimmten die Ergebnisse für den Kationenaustausch bei $\mathrm{Cu}$ gut überein, während die gemessenen Austauschraten im Anionenaustausch deutlich zu hoch waren. Für einen Teil der Proben kann dies auf pH-Wert-Änderungen beim Anionenaustausch gegen $\mathrm{OH}^{-}$beruhen.

Auffällig sind 19 weitere Proben, in denen $\mathrm{Cu}$ im Anionenaustausch erwartungsgemäß nicht zurückgehalten wurde, aber die Ergebnisse des Kationenaustauschs auseinanderklafften. $\mathrm{Zu}$ diesen Proben gehören sowohl Porenlösungen als auch Wasserproben aus dem Oktober 1998 aus den RCO-Grundwasserpegeln sowie Proben aus dem CML-Brunnen und RCO-Pegeln aus dem August 1999 und dem Januar 2000. Möglicherweise wird der neutrale $\mathrm{CuSO}_{4}-\mathrm{Komplex}$ am Kationenaustauscher in diesen Proben gespalten. Möglich ist auch eine Beteiligung organischer Komplexbildner, die die Kationen in neutralen Komplexen binden.

$\mathrm{Pb}$ verhielt sich in insgesamt acht Proben im Experiment so, wie die Berechnung erwarten ließ (Abb. 5.30.). Dazu zählen neben sechs Proben aus den Gruppen 6 und 7 zwei Proben aus dem Bohrkern RCO4 (11-19 m (2) und 20 m (4)), die der Gruppe 3 zugerechnet wurden. In den übrigen Lösungen lagen die Austauschraten am Anionentauscher häufig sehr hoch. Bis auf wenige Ausnahmen überschritten auch die Austauschraten am Kationenaustauscher die erwarteten Werte. Letzteres geht möglicherweise auf eine Spaltung der neutralen $\mathrm{PbSO}_{4}$ Komplexe am Kationentauscher zurück; doch bildet $\mathrm{Pb}$ auch mit organischen Molekülen bereitwillig Komplexe, so dass die beobachteten Abweichungen teilweise auf DOC-Einfluss zurückzuführen sein können. 


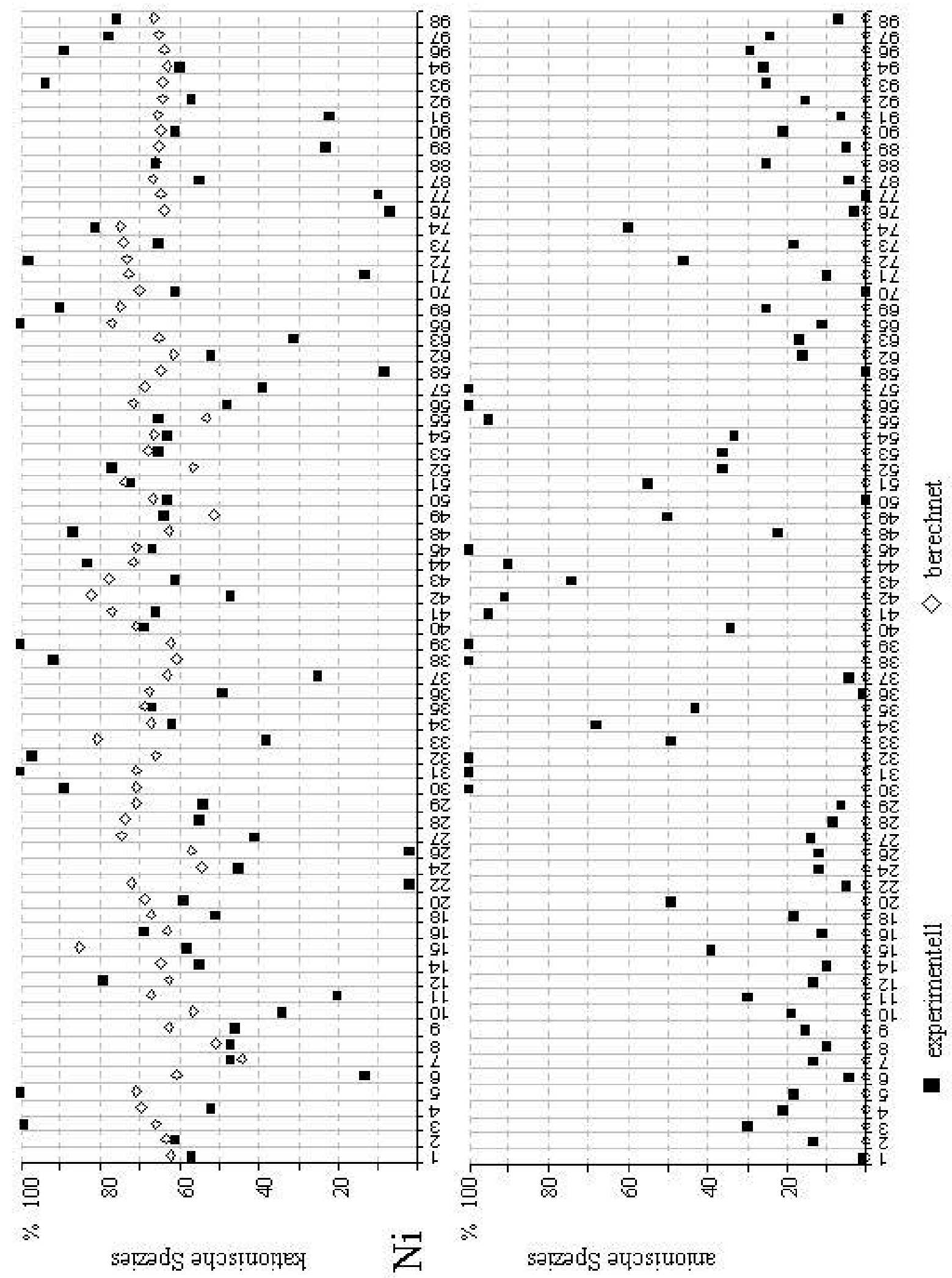

Abb. 5.31.: Experimentelle und berechnete Speziation von Ni in Proben aus Cospuden und aus der Lausitz. 


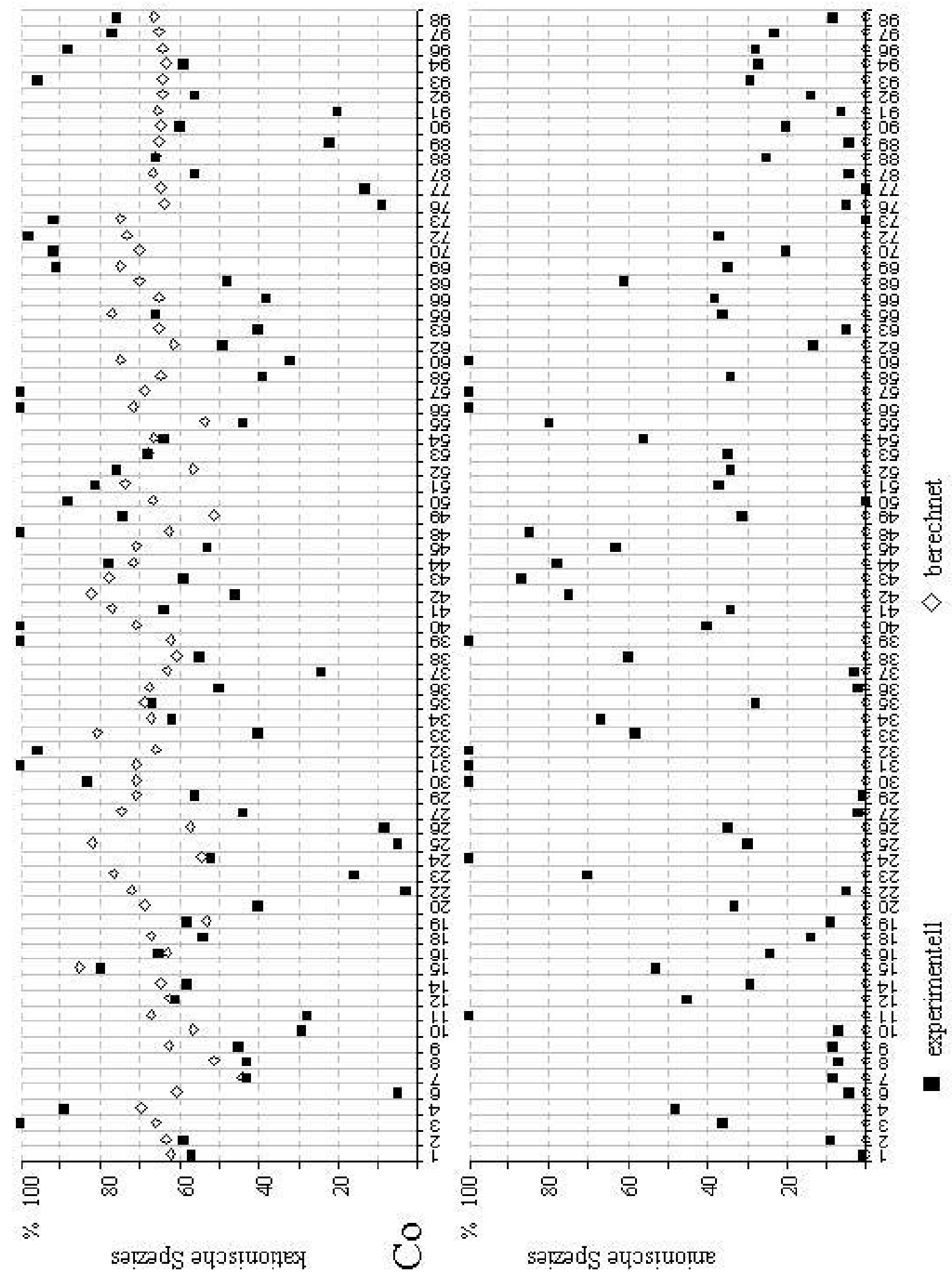

Abb. 5.32.: Experimentelle und berechnete Speziation von Co in Proben aus Cospuden und aus der Lausitz. 
$\mathrm{Ni}$ und $\mathrm{Co}$ (Abb. 5.31. und 5.32.) ähneln sich in ihrer Chemie so stark, dass die Speziationsberechnungen für beide Elemente praktisch keine Abweichungen ergeben. Auch im Experiment verhielten sich Ni und Co in der Regel ähnlich. In 21 Proben ergaben sich für $\mathrm{Ni}$ im Experiment die aus der Berechnung erwarteten Austauschraten bei Kationen- und Anionenaustausch. In 16 dieser Proben deckten sich auch die Resultate für Co, z.B. in RCO 4 5-8 m (1), 11-19 m (2), 30 m (8) und 32 m (9), Standgewässer 1 WP AP (29), RCO13 Oktober 1998 (50) und in Lausitz 5,21 m (87), 5,60 m (90), 5,93m (92), 21,80 m (97) und 21,90 m (98).

Starke Abweichungen, die auf kolloidalen Einfluss schließen ließen, zeigten neben einigen kolloidhaltigen Proben auch die Proben CML 20 m (56) und CML 27,5 m (57) vom Januar 1999 sowie die Probe See Sediment PL August 1999 (74) (Abb. 5.31. und 5.32.). Festphasen für die beiden Elemente wurden durch MINTEQA in diesen Proben nicht ermittelt (Tab. A.16 im Anhang). Die übrigen hier diskutierten Kationen zeigten den Effekt nicht (Tab. A.25. im Anhang). Eine mögliche Erklärung wäre das Vorkommen eines sehr selektiven organischen Komplexbildners.

Für Mo (Abb. 5.33.) weichen die Ergebnisse von Experiment und Rechnung sehr weit voneinander ab. Theoretisch wäre Mo in fast allen Proben mit Ausnahme der Standgewässer und der sauren Wässer aus der Lausitz vollständig als $\mathrm{MoO}_{4}{ }^{-} \mathrm{zu}$ erwarten. Die gemessenen Austauschraten liegen jedoch für den Anionenaustausch häufig sehr niedrig, während hohe Austauschraten im Kationenaustausch auftraten. Nur in den Proben CML 27,5 m (57) und 43,5 m (60) aus dem Januar 1999, CML 39,5 m (64) aus dem August 1999 und CML 39,5 m Januar 2000 (78) wurde eine Übereinstimmung zwischen rechnerischer und experimenteller Speziation festgestellt. Bei den geringen Konzentrationen von Mo, die in den meisten Proben beobachtet wurden, und der Neigung des Mo, z.B. mit stickstoffhaltigen Verbindungen Komplexe zu bilden (HoLLEMAN 1985), ist eine Beteiligung organischer Komplexbildner an der Speziation nicht auszuschließen.

Ähnlich wie bei Mo sind auch bei As kaum Zusammenhänge zwischen den Ergebnissen der Rechnung und denen des Experiments festzustellen (Abb. 5.34.). Nur in einer einzigen Probe (CML 36 m PL (44)) liegt As erwartungsgemäß vollständig in anionischer Form vor. In einer Vielzahl anderer Proben sind hohe Austauschraten beim Kationentausch und niedrige Raten beim Anionenaustausch zu beobachten, die auch in Proben ohne Kolloide (z.B. Proben aus Cospuden) auftreten. Ebenso wie beim Mo kann über die Beteiligung organischer Komplexbildner nur spekuliert werden. 


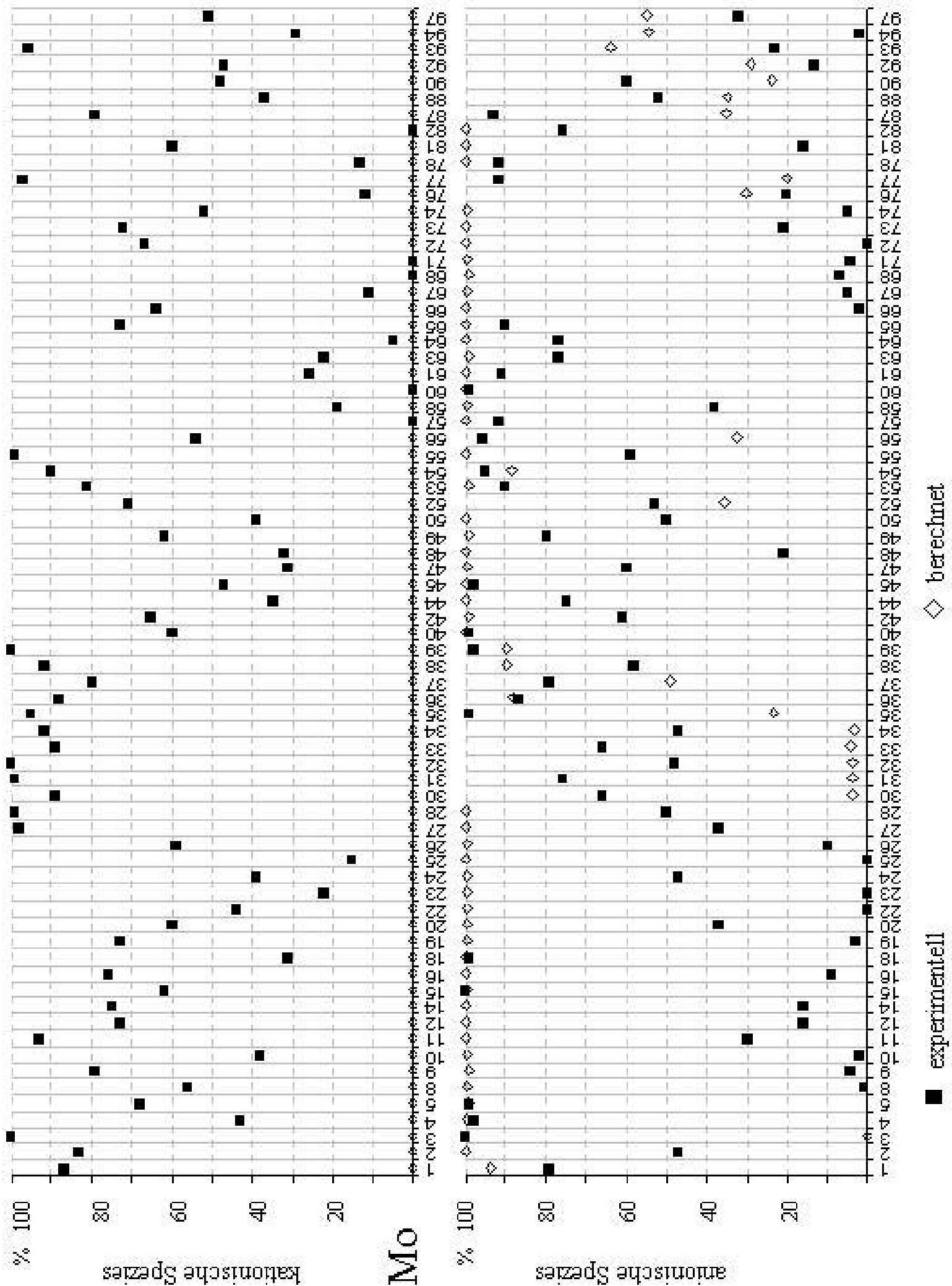

Abb. 5.33.: Experimentelle und berechnete Speziation von Mo in Proben aus Cospuden und aus der Lausitz. 


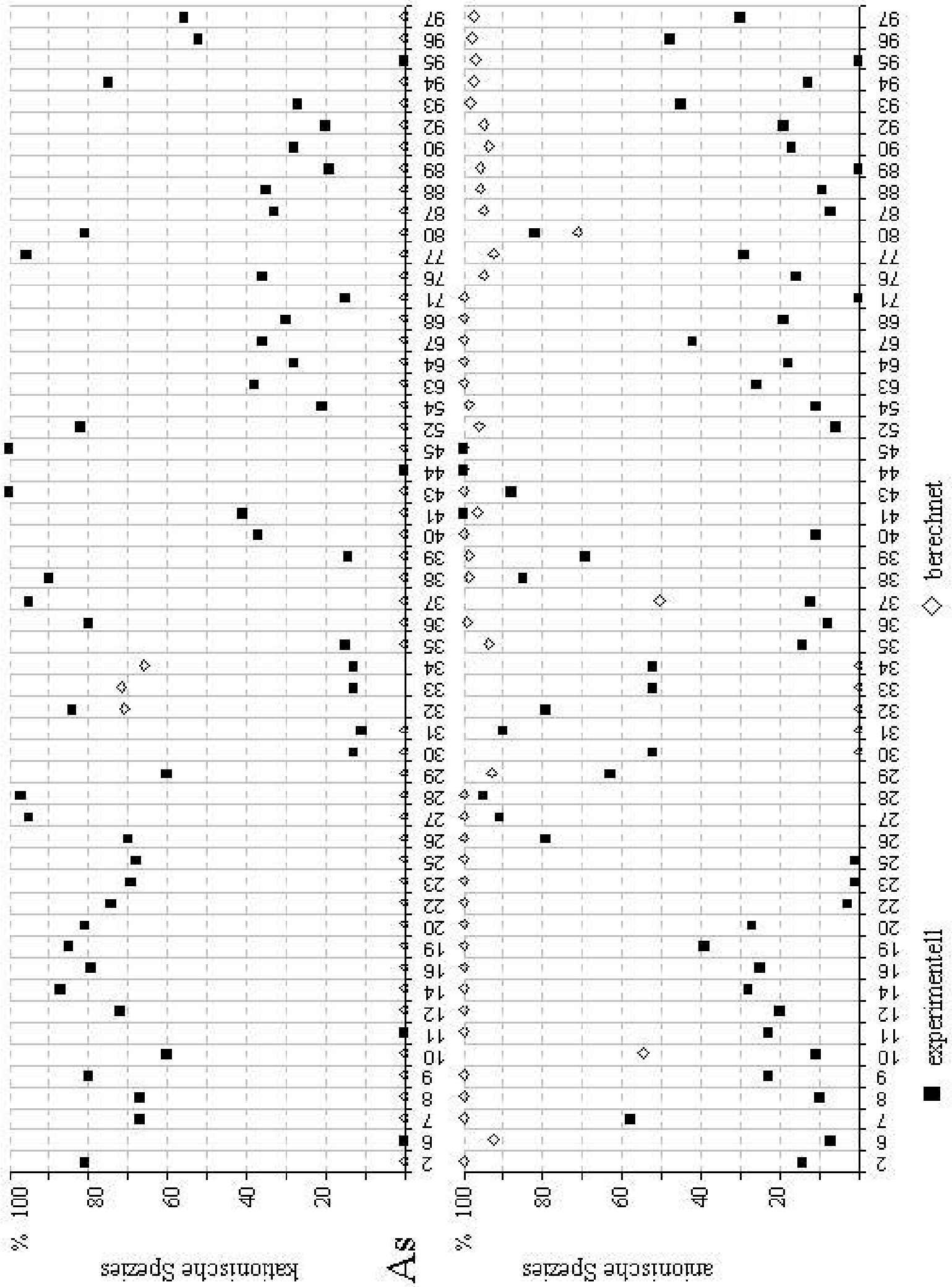

Abb. 5.34.: Experimentelle und berechnete Speziation von As in Proben aus Cospuden und aus der Lausitz. 


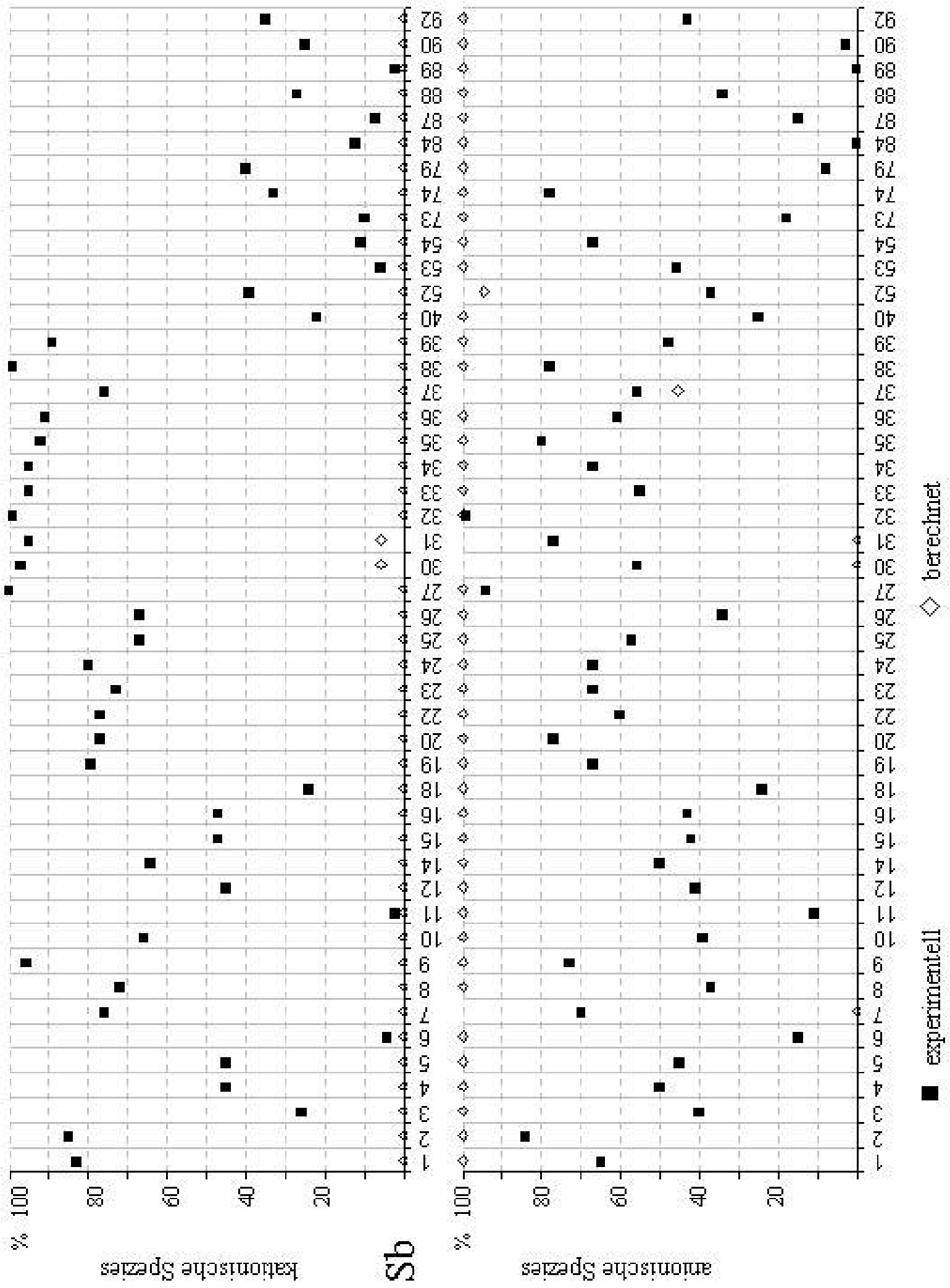

Abb. 5.35.: Experimentelle und berechnete Speziation von Sb in Proben aus Cospuden und aus der Lausitz. 
Nach der Berechnung sollte Sb (Abb. 5.35.) in allen Proben in anionischer Form vorliegen. Dies konnte in keiner einzigen Probe beobachtet werden. Stattdessen erstrecken sich die Ergebnisse sowohl aus dem Kationen- als auch aus dem Anionenaustausch über das gesamte Spektrum der möglichen Austauschraten. In den stark kolloidal geprägten Standgewässern wurde $\mathrm{Sb}$ beim Kationenaustausch entfernt, in der Ultrafiltration blieben Mo, As und $\mathrm{Sb}$ praktisch vollständig auf dem Filter zurück, so dass für diese Proben davon auszugehen ist, dass diese Elemente in Kolloide eingebunden oder an ihnen adsorbiert wurden. In Proben aus den Gruppen 6 und 7 lagen offenbar große Teile des Sb in neutraler Form vor. Wie bei Mo und As weisen diese Ergebnisse nicht auf die Beteiligung von Festphasen hin; entweder sind anorganische Komplexe beteiligt, die im Programm MINTEQA nicht berücksichtigt sind, oder organische Moleküle komplexieren das Sb.

$\mathrm{Cr}$ und $\mathrm{V}$ sind Elemente mit einem sehr komplexen und redoxsensitiven Lösungsverhalten. In Abb. 5.36. sind Ergebnisse der Speziation von Cr dargestellt. Nur drei Proben zeigen eine gute Übereinstimmung sowohl der negativen als auch der positiv geladenen Spezies. Der Rechnung zufolge wäre $\mathrm{Cr}$ in allen Proben mit Ausnahme von CML 20 m (75) und CML 39,5 m (78) vom Januar 2000 und Lausitz 5,50 m (89), 5,60 m (90) und 5,75 m (91) vollständig in Form positiv geladener Spezies zu erwarten. Trotzdem liegen in vielen Proben die Austauschraten beim Kationenaustausch um ca. 50 \%, während am Anionenaustauscher die Austauschraten vieler Proben zwischen 20 und $90 \%$ liegen. Diese Beobachtung spricht nicht für eine starke Beteiligung aktiver Oberflächen oder übersättigter Cr-Festphasen. Denkbare Erklärungen wären die hohe Redoxsensibilität und pH-Abhängigkeit der rechnerischen Speziation, durch die schon kleine analytische Fehler zu einer starken Verfälschung der Ergebnisse führen, oder die Beteiligung organischer Komplexbildner an der Cr-Speziation.

Noch komplexer sind die Resultate des V (Abb. 5.37.), denn hier variieren bereits die Rechenergebnisse zwischen den einzelnen Proben sehr stark. Übereinstimmungen zwischen berechneten und experimentell ermittelten Ergebnissen wurden nur in den beiden Proben RCO13 30 m (25) und See WP AP (36) gefunden. Ähnlich wie bei Cr sind die Ursachen für die Unterschiede kaum zu ermitteln; in Frage kommen leichte Abweichungen bei der Ermittlung von $\mathrm{pH}-$ Wert und Redoxpotential ebenso wie organische Komplexbildner.

Abb. 5.38. zeigt die vollständige Übersicht der Sulfatspeziation für alle Proben, für die die entsprechenden Ergebnisse vollständig vorlagen. Deutlich sind die Oberflächenwässer und Porenlösungen mit kolloidalen Sulfatphasen an den sehr hohen „Austauschraten“ beim Kationenaustausch zu erkennen (das schließt auch Proben der Gruppe 1 mit ein, in denen neben Eisenkolloiden sehr häufig auch Sulfatkolloide vorlagen). 


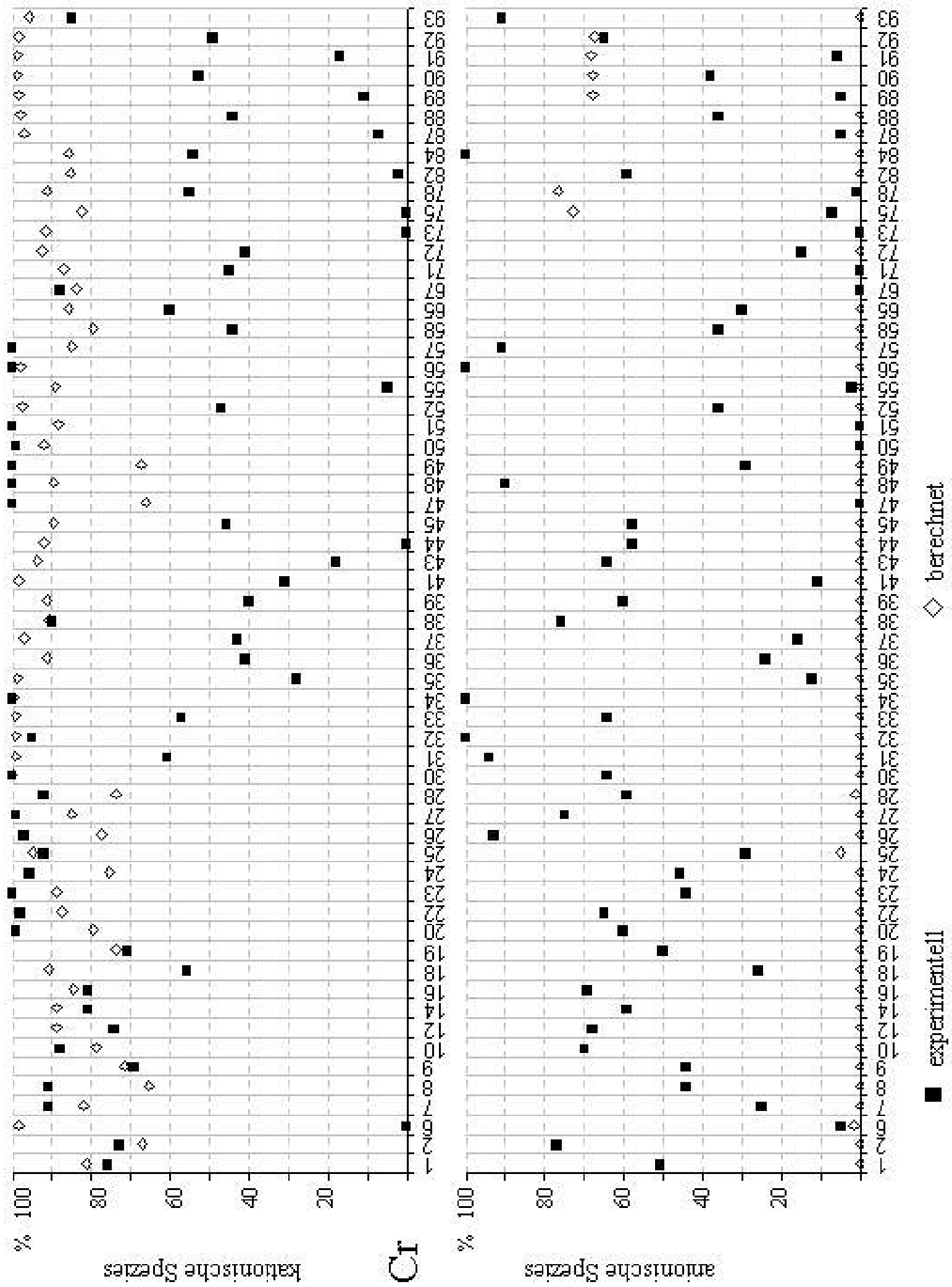

Abb. 5.36.: Experimentelle und berechnete Speziation von Cr in Proben aus Cospuden und aus der Lausitz. 


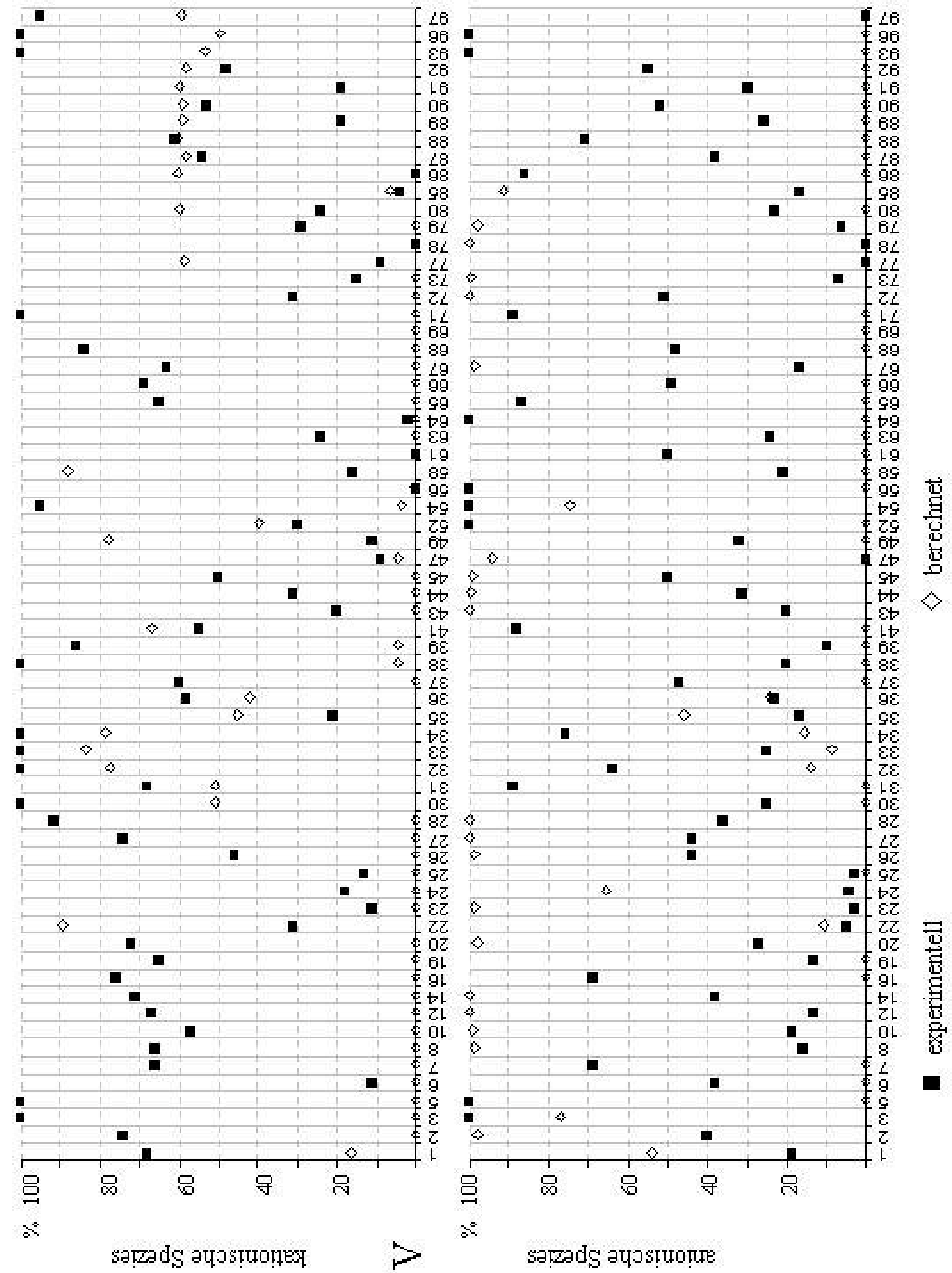

Abb. 5.37.: Experimentelle und berechnete Speziation von V in Proben aus Cospuden und aus der Lausitz. 


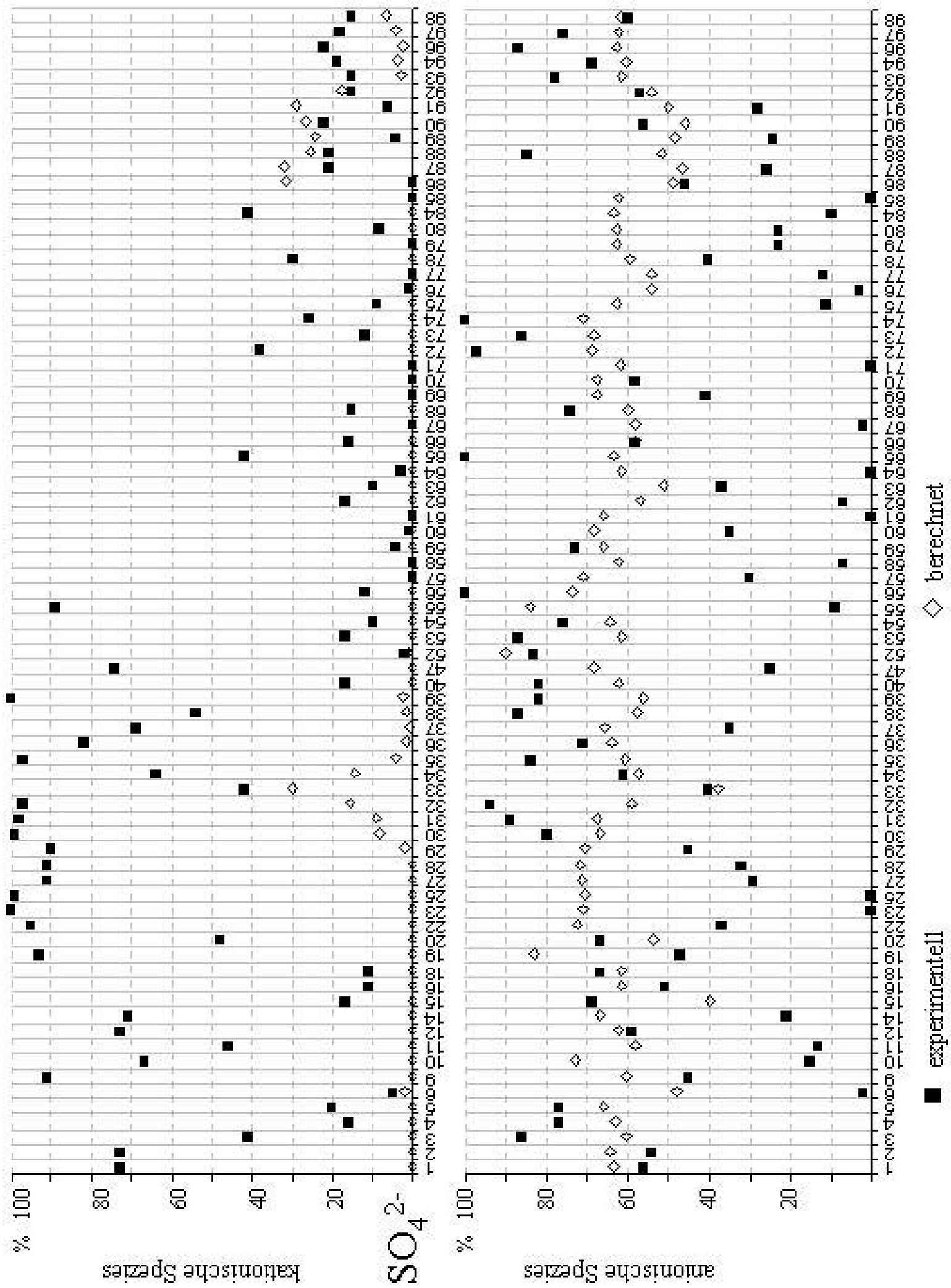

Abb. 5.38.: Experimentelle und berechnete Speziation von Sulfat in Proben aus Cospuden und aus der Lausitz. 
Gute Übereinstimmungen zeigten vor allem die Proben aus der Lausitz, aber auch einige andere Proben der Gruppen 6 und 7. In Proben anderer Gruppen wurde nur in vier Fällen gute Übereinstimmung beobachtet, nämlich in den Proben RCO4 20 m (4), RCO4 46 m (16), RCO4 52 m (18) und Standgewässer 3 PL AP (32). In diesen vier Proben liegen nach den Ergebnissen der Ultrafiltration kolloidale Phasen vor. Die Übereinstimmungen in diesen vier Proben dürften daher eher Zufallscharakter haben.

Die dargestellten Beispiele zeigen, mit welcher Vorsicht die Ergebnisse aus der Speziation ausgewertet werden müssen. Einflüsse von subkolloidalen Strukturen lassen sich in den natürlichen Wässerns selten ganz ausschließen, zumal nach den Ergebnissen der Berechnung fast in jeder der untersuchten Proben einzelne Festphasen übersättigt sind. Umgekehrt ist nicht jede Abweichung zwischen berechneten und experimentellen Ergebnissen, die nicht auf Kolloide zurückzuführen ist, mit organischen Komplexbildnern zu erklären. Oft zeigen schon Überschlagsrechnungen, dass die Abweichungen zumindest nicht ausschließlich auf dem Einfluss des DOC beruhen können. Eine Möglichkeit dazu ist, die theoretisch benötigte Anzahl von Bindungsstellen je g C am DOC zu berechnen, die mindestens notwendig wären, um bei vollständiger Bindung eines Elements an den DOC die Abweichungen zwischen experimentellen und berechneten Resultaten zu verursachen. Liegt diese über $5 \mathrm{mmol} / \mathrm{g} \mathrm{C}$, müssen andere Prozesse als nur die organische Komplexbildung an der Speziation der Proben beteiligt sein.

Dennoch ist es - unter Berücksichtigung dieser Umstände - für viele Proben durchaus sinnvoll, Speziationsberechnungen durchzuführen, die Aufschluss über die Natur und den Einfluss der organischen Komponenten geben können. Dies soll an einigen Proben exemplarisch durchgeführt werden.

Um den DOC zu modellieren, werden kleine, definierte organische Moleküle genutzt, die in der Datenbank enthalten sind und für die die Komplexbildungskonstanten mit möglichst vielen relevanten Elementen bekannt sind. Diese Kriterien werden von Citrat erfüllt. In der Literatur wird berichtet, dass die Bindungseigenschaften von Huminsäuren vor allem durch aliphatische Carboxylgruppen bestimmt werden und daher Citrat und Malonat als Modelle für die Komplexierung durch Huminsäuren geeignet erscheinen (TOWN und POWELL 1993).

Als Beispiel wurde die Probe RCO4 5-8m ausgewählt. Diese Probe enthält zwar Kolloide, doch handelt es sich dabei vor allem um $\mathrm{CaSO}_{4}$, das die Übergangsmetalle nur in geringem Maße adsorptiv bindet. Der Vergleich der Daten aus Experiment und Rechnung weist nicht auf einen kolloidalen Einfluss hin, allerdings weisen die Resultate der sehr leicht organisch komplexierbaren Elemente $\mathrm{Al}$ und $\mathrm{Pb}$ deutliche Abweichungen auf. 
Zunächst wurde der DOC aus der Datenbank von MINTEQA2 für eine Berechnung der Probe RCO4 5-8m zugrundegelegt. Die Ergebnisse sind in der Tab. 5.3. dargestellt.

\begin{tabular}{|c|c|c|c|c|}
\hline Element & Spezies & $\%$ ohne/mit Citrat & Summe ohne/mit Citrat & $\begin{array}{l}\text { zurückgehalten am } \\
\text { Austauscher }\end{array}$ \\
\hline $\mathbf{F e}$ & $\begin{array}{l}\mathrm{Fe}^{2+} \\
\mathrm{FeSO}_{4}(\mathrm{aq})\end{array}$ & $\begin{array}{l}56,6 \% / 56,3 \% \\
43,4 \% / 43,2 \%\end{array}$ & $\begin{array}{l}\text { kationisch } 56,6 \% / 56,3 \% \\
\text { neutral } 43,4 \% / 43,2 \%\end{array}$ & Kationen $70 \%$ \\
\hline $\mathbf{N i}$ & $\begin{array}{l}\mathrm{Ni}^{2+} \\
\mathrm{NiSO}_{4}(\mathrm{aq}) \\
\mathrm{Ni}[\text { Citrate] }\end{array}$ & $\begin{array}{l}61,5 \% / 60,7 \% \\
38,3 \% / 37,8 \% \\
-/ 1,1 \%\end{array}$ & $\begin{array}{l}\text { kationisch } 61,5 \% / 60,7 \% \\
\text { neutral } 38,3 \% / 37,8 \% \\
\text { org.-geb. - / 1,1\% }\end{array}$ & $\begin{array}{l}\text { Kationen } 60 \% \\
\text { Anionen } 1 \%\end{array}$ \\
\hline $\mathbf{C u}$ & $\begin{array}{l}\mathrm{Cu}^{2+} \\
\mathrm{CuSO}_{4}(\mathrm{aq}) \\
\mathrm{Cu}(\mathrm{II}) \mathrm{DOM} \\
\mathrm{Cu}(\mathrm{II})[\text { Citrate] }\end{array}$ & $\begin{array}{l}56,6 \% / 51,2 \% \\
40,5 \% / 36,6 \% \\
2,9 \% / 2,7 \% \\
/ 9,0 \%\end{array}$ & $\begin{array}{l}\text { kationisch } 56,6 \% / 51,2 \% \\
\text { neutral } 40,5 \% / 36,6 \% \\
\text { org-geb } 2,9 \% / 11,7 \%\end{array}$ & $\begin{array}{l}\text { Kationen } 50 \% \\
\text { Anionen } \quad 6 \%\end{array}$ \\
\hline $\mathbf{Z n}$ & $\begin{array}{l}\mathrm{Zn}^{2+} \\
\mathrm{Zn}\left(\mathrm{SO}_{4}\right)_{2}^{2-} \\
\mathrm{ZnSO}_{4}(\mathrm{aq})\end{array}$ & $\begin{array}{l}55,6 \% / 55,3 \% \\
6,3 \% / 6,2 \% \\
38 \% / 37,8 \%\end{array}$ & $\begin{array}{l}\text { kationisch } 55,6 \% / 55,3 \% \\
\text { anionisch } 6,3 \% / 6,2 \% \\
\text { neutral } 38 \% / 37,8 \%\end{array}$ & $\begin{array}{l}\text { Kationen } 68 \% \\
\text { Anionen } 1 \%\end{array}$ \\
\hline Cd & $\begin{array}{l}\mathrm{Cd}^{2+} \\
\mathrm{Cd}\left(\mathrm{SO}_{4}\right)_{2}^{2-} \\
\mathrm{CdSO}_{4}(\mathrm{aq})\end{array}$ & $\begin{array}{l}52,1 \% / 52 \% \\
9,8 \% / 9,8 \% \\
38,1 \% / 38,1 \%\end{array}$ & $\begin{array}{l}\text { kationisch } 52,1 \% / 52 \% \\
\text { anionisch } 9,8 \% / 9,8 \% \\
\text { neutral } 38,1 \% / 38,1 \%\end{array}$ & $\begin{array}{l}\text { Kationen } 68 \% \\
\text { Anionen } 11 \%\end{array}$ \\
\hline Al & $\begin{array}{l}\mathrm{Al}^{3+} \\
\mathrm{AlSO}_{4}^{+} \\
\mathrm{Al}\left(\mathrm{SO}_{4}\right)^{2-} \\
\mathrm{Al}[\text { Citrate] } \\
\text { AlH[Citrate] }\end{array}$ & $\begin{array}{l}8,3 \% / 4,2 \% \\
83,8 \% / 42,4 \% \\
6,9 \% / 3,5 \% \\
/ 47,6 \% \\
-/ 1,8 \%\end{array}$ & $\begin{array}{l}\text { kationisch } 92,1 \% / 46,6 \% \\
\text { anionisch }\end{array}$ & $\begin{array}{l}\text { Kationen } 49 \% \\
\text { Anionen } 5 \%\end{array}$ \\
\hline
\end{tabular}

Tab. 5.3.: Vergleich der experimentellen Ergebnisse mit den Resultaten aus MINTEQA2 für Fe, $\mathrm{Ni}, \mathrm{Cu}, \mathrm{Zn}, \mathrm{Cd}$ und Al.

In der Probe RCO4 5-8 m herrscht ein anoxisches Milieu (pH 4,4; Eh = 95 mV). Das gelöste Eisen liegt zu $100 \%$ als $\mathrm{Fe}^{\mathrm{II}}$ vor. Dagegen stehen ein- und zweiwertiges Kupfer theoretisch im Gleichgewicht miteinander; das Programm ermittelt $65 \%$ an ein- und $35 \%$ an zweiwertigem Kupfer. Allerdings berücksichtigt das Programm nicht die unterschiedliche Hydratationsenthalpie der $\mathrm{Cu}$-Ionen, die dafür verantwortlich ist, dass das $\mathrm{Cu}^{\mathrm{I}}$ in wässriger Lösung nicht stabil ist, sondern in $\mathrm{Cu}^{0}$ und $\mathrm{Cu}^{\mathrm{II}}$ disproportioniert (HoLLEMAN 1985). Daher wird in der Berechnung von $\mathrm{Cu}^{\mathrm{II}}$ ausgegangen.

Es zeigt sich im Vergleich der gemessenen mit den berechneten Daten, dass der weitaus größte Teil der untersuchten Elemente in dieser Probe nicht unter dem Einfluss organischer Komplexbildner steht (BRÜSCHKE et al. 2000). Cd, Zn, Ni und Fe entsprechen in ihrem gemessenen Verhalten den theoretischen Berechnungen; wie die vorher genannten Erdalkalimetalle liegen sie in hohem Maße als neutrale und anionische Sulfatkomplexe vor, was den Austausch an negativ geladenen Mineraloberflächen erschweren und die Mobilität der Metalle erhöhen könnte; die teilweise gegenüber den berechneten etwas höheren 
gemessenen kationischen Anteile sind wahrscheinlich auf die Spaltung eines Teils der Sulfatkomplexe am Ionentauscher zurückzuführen.

Am gravierendsten ist der Einfluss organischer Komplexbildner auf das einzige dreiwertige Kation Aluminium. Ohne die Berücksichtigung von Citrat liegt es zu $92 \%$ als freies bzw. sulfatisiertes Kation vor. Fügt man bei der Berechnung lediglich $5 \mathrm{mg} / \mathrm{l}$ Citrat zu, sinkt dieser Anteil schlagartig auf $47 \%$, während der organisch gebundene Anteil $49 \%$ beträgt. Die 5 mg/l Citrat "simulieren" bezogen auf die enthaltenen $12 \mathrm{mg} / \mathrm{l}$ DOC $0,2 \mathrm{mM} / \mathrm{g} \mathrm{C}$ an "citratartigen" Bindungsstellen, d.h. mit relativ hohen Komplexbildungskonstanten und einer großen Affinität für dreiwertige Kationen, von der die zweiwertigen Kationen weitgehend ausgeschlossen bleiben. Diese Dichte an Bindungsstellen ist durchaus realistisch. Je nach Ladung des Gesamtkomplexes aus Al und DOC muss davon ausgegangen werden, dass die Bindung an den DOC die Mobilität des Aluminiums stark erhöht und es nicht mehr so leicht an Mineraloberflächen adsorbiert wird, wie es beim freien bzw. sulfatisierten Kation mit großer Ladungsdichte und vergleichsweise geringer sterischer Ausdehnung der Fall ist.

\begin{tabular}{|c|c|c|c|c|}
\hline Element & Spezies & \% ohne/mit Citrat & Summe ohne/mit Citrat & $\begin{array}{l}\text { zurückgehalten am } \\
\text { Austauscher }\end{array}$ \\
\hline $\mathbf{C a}$ & $\begin{array}{l}\mathrm{Ca}^{2+} \\
\mathrm{Ca} \mathrm{SO}_{4}(\mathrm{aq})\end{array}$ & $\begin{array}{l}58,3 \% / 58,2 \% \\
41,7 \% / 36,2 \% \\
\end{array}$ & $\begin{array}{l}\text { kationisch 58,3 \% / 58,2\% } \\
\text { neutral 41,7 \% / 36,2\% }\end{array}$ & $\begin{array}{l}\text { Kationen } ~ 56 \% \\
\text { Anionen } ~ 86 \%\end{array}$ \\
\hline Mg & $\begin{array}{l}\mathrm{Mg}^{2+} \\
\mathrm{MgSO}_{4}(\mathrm{aq})\end{array}$ & $\begin{array}{l}63,8 \% / 63,7 \% \\
36,2 \% 736,25\end{array}$ & $\begin{array}{l}\text { kationisch 63,8 \% / 63,7 \% } \\
\text { neutral 36,2\% / 36,2\% }\end{array}$ & $\begin{array}{l}\text { Kationen } ~ 67 \% \\
\text { Anionen } 63 \%\end{array}$ \\
\hline $\mathbf{P b}$ & $\begin{array}{l}\mathrm{Pb}^{2+} \\
\mathrm{Pb}\left(\mathrm{SO}_{4}\right)_{2}{ }^{2-} \\
\mathrm{PbSO}_{4}(\mathrm{aq}) \\
\mathrm{PbDOM} \\
\mathrm{Pb}[\text { Citrate] }\end{array}$ & $\begin{array}{l}35,6 \% / 34,5 \% \\
6,2 \% / 6,2 \\
54,5 \% / 52,8 \% \\
3,7 \% / 3,6 \% \\
-/ 3,0 \%\end{array}$ & $\begin{array}{l}\text { kationisch 35,6 \% / 34,5\% } \\
\text { anionisch 6,2\% / 6,0\% } \\
\text { neutral 54,5\% / 52,8 \% } \\
\text { org.-geb. 3,7 5 / 6,6\% }\end{array}$ & $\begin{array}{l}\text { Kationen } ~ 33 \% \\
\text { Anionen } 29 \%\end{array}$ \\
\hline
\end{tabular}

Tab. 5.4.: Vergleich der experimentellen Ergebnisse mit Resultaten aus MINTEQA2 für Ca, Mg und Pb

Tab. 5.4. zeigt die Ergebnisse von Experiment und Rechnung für $\mathrm{Ca}, \mathrm{Mg}$ und $\mathrm{Pb}$. Die kationischen Anteile von $\mathrm{Mg}$ und $\mathrm{Ca}$ entsprechen sich in Messung und Berechnung genau. Die festgestellte hohe Rückhalterate von $\mathrm{Mg}$, $\mathrm{Ca}$ und z.B. auch beim $\mathrm{Ba}$ am Anionenaustauscher ist nicht auf einen Ionenaustausch zurückzuführen, sondern auf die Ausfällung der Elemente als Hydroxid bzw. Sulfat durch die pH-Änderung beim Durchlaufen der $\mathrm{OH}^{-}$-beladenen Austauschereinheit. Ein ähnlicher Effekt mag in schwachem Maße auch beim Blei zum Tragen kommen. Hier zeigt sich deutlich, warum der seit 1998 praktizierte Austausch gegen $\mathrm{Cl}^{-}$statt $\mathrm{OH}^{-}$als Austauschion trotz analytischer Schwierigkeiten sinnvoll ist.

In der Probe RCO4 5-8 m wird beispielhaft deutlich, wie durch die Kombination experimenteller und rechnerischer Verfahren die Bestimmung der Migrationsformen in den 
Wässern des ehemaligen Tagebaus Cospuden erfolgen kann. Dieser vielversprechende Ansatz kann sowohl auf andere Elemente erweitert als auch hinsichtlich der organischen Komponenten verfeinert werden. So dominieren im DOC anderer Proben u.U. nicht "citratähnliche", sondern andersartige Bindungsstellen, die beispielsweise durch Amine oder Thiole simuliert werden können und andere Elemente beeinflussen. Durch geeignete Erweiterungen der Datenbanken wird ein Vergleich zwischen dem DOC verschiedener Wässer möglich. Unter der Voraussetzung, dass entsprechende Literaturdaten vorhanden sind, ist die Datenbank mit relativ geringem Aufwand zu erweitern. Damit eröffnet sich uns die Möglichkeit, sowohl für Tiefenprofile als auch zeitlich und räumlich an verschiedenen Pegeln die Entwicklung der Migrationsformen mit fortschreitender Pyritoxidation und beim Eindringen des Seewassers in die Kippe zu verfolgen.

Außerdem lässt sich das Verfahren auf weitere Elemente ausweiten; eine Datenbank für MINTEQA2, die die Selten-Erd-Elemente berücksichtigt, existiert bereits (TURNER et al. 1993), war aber für diese Arbeit nicht zugänglich.

Auch für die Proben, in denen Kolloide vorliegen, ist eine Speziationsberechnung zumindest theoretisch möglich. Voraussetzung ist, dass die Menge der Kolloide ungefähr bekannt ist. Über die Retentionsrate bei der Ultrafiltration und den Ausgangsgehalt des Elements lässt sie sich abschätzen. Weiterhin werden Daten für die Oberflächenstruktur der Kolloide benötigt; existieren keine solchen Werte, kann über Daten für Mineraloberflächen zumindest eine Abschätzung getroffen werden. Die Kolloide können dann als adsorptive Oberfläche in der Rechnung berücksichtigt werden.

\subsubsection{Diskussion der Speziationsergebnisse}

Die zuvor dargestellten Resultate zeigen, wie das beschriebene Speziationsverfahren in Kombination mit einem rechnerischen Modell zur Beschreibung natürlicher Wässer eingesetzt werden kann. Im Rahmen dieser Arbeit konnte dazu nur ein Überblick gegeben werden; Redoxpotentiale wurden beispielsweise nicht angemessen überprüft und berücksichtigt, woraus sich die großen Abweichungen der Ergebnisse aus Rechnung und Experiment bei den redoxsensitiven Elementen wie $\mathrm{Cr}$ oder $\mathrm{V}$ erklären. Auch die Modellierung organischer Komponenten oder anorganischer Kolloide konnte nur in groben Zügen dargestellt werden. Jedoch zeigt sich, dass mit dem beschriebenen Verfahren grundsätzlich eine ziemlich exakte Beschreibung von Lösungen möglich ist, wenn alle relevanten Parameter bekannt sind und in die Speziationsrechnung einbezogen werden. 
Auch ohne dieses aufwendige Vorgehen liefert das Verfahren durch den Vergleich von Rechnung und Experiment wertvolle Erkenntnisse über die Lösungen, die sich bei Anwendung nur einer Methode nicht gewinnen lassen. Die rein experimentelle Speziation liefert Anhaltspunkte, inwieweit die Elemente kolloidal vorliegen oder einem Ionenaustausch zugänglich sind. Doch ist die experimentelle Speziation in mancherlei Hinsicht fehlerbehaftet. Beispielsweise kann nicht unterschieden werden, ob die Rückhalteraten bei der Ultrafiltration nur auf Kolloide zurückzuführen sind oder auch auf Adsorption von Elementen an der Membran. Ebenso kann beim Ionenaustausch durch suspendierte Feststoffe die Oberfläche blockiert werden, oder es werden Elemente mechanisch am Trägermaterial abfiltriert. Besonders die Proben, bei denen der Anionenaustausch gegen $\mathrm{OH}^{-}$durchgeführt wurde, müssen mit großer Vorsicht interpretiert werden.

Ein rein rechnerisches Vorgehen ohne Einbeziehung experimenteller Beobachtungen kann gar nicht alle relevanten Parameter wie Kolloidbildung oder organische Komplexierung angemessen erfassen. Erst die Kombination beider Wege ermöglicht es, eine große Anzahl von Parametern in die Betrachtung einzubeziehen. So wurden offensichtlich nicht alle Kolloide tatsächlich durch die experimentelle Ultrafiltration erfasst. Vielmehr lagen in vielen Proben kleine kolloidale oder subkolloidale Strukturen vor, die sich auf die Elementspeziation gravierend auswirkten. Das Vorhandensein von Kolloiden bedeutet aber nicht in jedem Fall, dass in naher Zukunft eine Niederschlagsbildung auftritt und die Elemente damit aus der Lösung entfernt werden. Auch Proben, in denen sich schon während der Lagerung Niederschläge gebildet hatten sowie Sicker- und Seewässer, die schon über längere Zeit Luftkontakt hatten und in denen Niederschläge zu beobachten waren, zeigten noch hohe Elementkonzentrationen, die anscheinend in Form stabiler subkolloidaler oder kolloidaler Strukturen in den Wässern vorlagen. Inwieweit Bestandteile des DOC für die Stabilisierung solcher Kolloide eine Rolle spielen, kann analytisch kaum festgestellt werden. Da der DOC aus Cospuden in vielen Fällen aber einen hohen Anteil an Funktionalitäten aufweist, wie durch das Speziationsschema gezeigt werden konnte, und die DOC-Konzentrationen in zahlreichen Proben sehr hoch sind, ist davon auszugehen, dass der DOC kolloidale Partikel überzieht und ihre Sedimentation behindert.

In den Wässern des Bohrkerns RCO4 liegen zum großen Teil Sulfatkolloide vor. Davon ist auch das Verhalten der meisten Elemente geprägt. Bis auf die Probe RCO4 26 m waren die Proben an Eisen verarmt; das hatte auch eine Verarmung vieler anderer Elemente zur Folge.

Die Standgewässer weisen große Mengen kolloidal gelösten Eisens auf. Auch andere Elemente sind darin stark angereichert. Den Ultrafiltrationsraten zufolge sind die 
Übergangsmetalle an den Eisenkolloiden adsorbiert. Die REEs wurden in der Ultrafiltration ebenfalls weitgehend entfernt. Bis auf Standgewässer 3 WP passierten die REEs nahezu ungehindert den Kationentauscher; beim Anionenaustausch wurden sie dagegen quantitativ entfernt. Diese Beobachtungen sprechen insgesamt dafür, dass die REEs ebenfalls an den Eisenkolloiden adsorbiert waren. Dadurch wurde der Austausch am Kationenaustauscher verhindert, während im Anionenaustausch gegen $\mathrm{OH}^{-}$große Mengen Eisens vermutlich im Anionenaustauscher aufgrund der pH-Wert-Änderung ausfielen und die REEs mit aufhielten. In den Proben aus der oberflächennahen Bohrung dagegen wurde das Verhalten der REEs nicht so eindeutig von anorganischen Kolloiden beeinflusst. Bei der Ultrafiltration wurden sie nur moderat zurückgehalten (Rückhalteraten um $30 \%$ ), während der Kationenaustausch in den meisten Fällen praktisch quantitativ verlief. Auch im Anionenaustausch wurden jedoch hohe Rückhalteraten oberhalb von $70 \%$ erzielt, die die Rückhalteraten der Übergangsmetalle deutlich übertrafen. Eventuell ist hier eine Auswirkung einer Komplexierung der REEs durch negativ geladene DOC-Bestandteile zu sehen.

In den meisten Wasserproben, die zwischen 1998 und 2000 in Cospuden entnommen wurden, konnten bei der Ultrafiltration keine anorganischen Kolloide festgestellt werden. Für die REEs liegen hier nur sehr wenige Daten vor, weil ihre Gehalte in den meisten Proben unterhalb oder so dicht um die Bestimmungsgrenze lagen, dass eine Speziation nicht möglich war. Andere Spurenlemente wie etwa Mo oder As zeigen ein sehr variables Verhalten, dass auf Komplexierungen oder Adsorptionseffekte zurückzuführen sein kann.

In den Wässern aus der Lausitz lassen sich in den meisten Proben keine Einflüsse von anorganischen Kolloiden oder Komplexbildnern beobachten. Die REEs verhalten sich in den Proben erwartungsgemäß. Nur in Lausitz 21,07 m und Lausitz 21,13 m werden sie bei der Ultrafiltration abgetrennt, während sie beim Anionenaustausch passieren; hier sind sie offensichtlich an neutrale Kolloide gebunden. 


\section{Zusammenfassung und Ausblick}

Die Auswirkungen des Braunkohletagebaus zeigen sich nicht nur an den oberflächlich wahrnehmbaren Landschaftsveränderungen. Durch die gewaltigen Erdverschiebungen gelangen oxidationsempfindliche Minerale an die Oberfläche und in Kontakt mit Sauerstoff. Pyrite und andere Festphasen werden oxdidiert; die Folge ist die Entstehung saurer Sickerwässer, die ihrerseits andere Mineralphasen angreifen. Im Zuge dieser Verwitterungsprozesse werden große Mengen von Haupt- und Spurenelementen in Lösung gebracht. Inwieweit die kontaminierten Wässer im Kippenbereich eine Gefahr für Mensch und Umwelt darstellen, lässt sich nur beantworten, wenn zum einen die Absolutgehalte der Elemente beobachtet werden und ermittelt wird, welcher Kontrolle diese unterliegen; zum anderen ist für die Verweildauer gelöster Phasen in den Wässern, für ihre Mobilität und ihre Toxizität von entscheidender Bedeutung, in welcher Lösungsform die Elemente vorliegen.

Die vorliegende Arbeit bildet den Abschluss eines 5 1/2-jährigen Forschungsprojekts zur Untersuchung von Herkunft, Migration und Verbleib gelöster Haupt- und Spurenelemente in den Wässern des ehemaligen Tagebaus Cospuden im Mitteldeutschen Braunkohlerevier. Im Rahmen dieser Arbeit wurden außerdem Porenlösungen aus zwei Bohrkernen aus dem Gebiet des Restlochs 111 in der Lausitz untersucht, um einen Vergleich zwischen verschiedenen Standorten zu ermöglichen.

Die Zusammensetzung der Festphasen weist zumindest in den oberen Schichten des Haldenmaterials auf eine weit fortgeschrittene Pyritverwitterung hin; die Pyritgehalte, die im Ausgangsmaterial im Mittel 3,6 \% betrugen, sanken auf Durchschnittswerte unterhalb von 1 Massen-\%. Gleichzeitig bildete sich Gips.

Elementmassenbilanzen waren auf der Basis der vorliegenden Proben nur qualitativ möglich. Es zeigte sich, dass die meisten Elemente im Bereich des ehemaligen Tagebaus Cospuden verblieben waren. Anreicherungen von $\mathrm{Ca}$ und $\mathrm{Na}$ deuteten darauf hin, dass die ursprünglichen Gehalte an Karbonaten und Albit in den unverritzten Sedimenten höher gelegen haben müssen als in den Arbeiten von KitTSTEINER (1998) und BELLMANN et al. (1984) angegeben. Starke Abreicherungen wurden gefunden für Fe (-49\%), Be (-87\%), Cr ($50 \%)$, Co (-44\%), Nb (-45\%) und Mo (-75\%); diese Elemente wurden u.U. bereits im Zuge der Sümpfung oder durch Kontakt zwischen den Wässern des Tagebaus und umliegenden Grundwasserleitung nach der Flutung aus dem Gebiet von Cospuden ausgetragen. Auch für die Sulfatbilanz lässt sich die gleich Feststellung treffen. Der Gesamtschwefelgehalt im Tagebau hat um $62 \%$ abgenommen. 
Grundwasserpegel im Kippenbereich zeigten im Jahr 1998 eine leichte Versauerung und erhöhte Konzentrationen an gelösten Haupt- und Spurenelementen; die elektrische Leitfähigkeit überschritt in Proben aus dem Kippenbereich $3 \mathrm{mS} / \mathrm{cm}$, während sie in anderen Pegeln nur knapp oberhalb von $1 \mathrm{mS} / \mathrm{cm}$ lag.

Die Konzentrationen zahlreicher Elemente, die im November 1998 im Seewasser noch stark erhöht waren, sanken bis zum August 1999 auf Werte, die nicht auffällig höher sind als die Gehalte in Grundwasser aus Leipzig. Die Seewasserchemie war dabei vor allem durch den Eintrag von Wässern aus dem Kippenbereich und die Sedimentation von Festphasen geprägt; der Seegrund zeigte deutliche Ablagerungen braunroter Eisenphasen.

Proben aus dem CML-Brunnen zeigen, dass sich seit Januar 1999 ein Tiefenprofil in den Porenwässern ausgebildet hat. Dabei nahmen viele Elementkonzentrationen, z.B. Al, Mn, Fe, Ni, Zn, Y, Zr und die REEs, von Januar bis August 1999 erheblich zu, sanken dann aber wieder auf ein mittleres Niveau. In ca. $30 \mathrm{~m}$ Tiefe fand sich eine Zone mit hohen Konzentrationen an $\mathrm{Fe}^{\mathrm{II}}$, die bei Kontakt mit Sauerstoff zu einer schnellen Versauerung der Proben führte. Trotz der Bildung von Niederschlägen bei der Lagerung von Proben aus CML blieben die Konzentrationen einiger Elemente in der Lösung sehr hoch. Eine ähnliche Charakteristik zeigte eine Probe aus dem Grundwassermesspegel RCO3, der in der Nähe der Kippe im Tagebauvorfeld liegt.

In den übrigen Pegeln stieg der pH-Wert über den Beobachtungszeitraum auf Werte um 7 bzw. blieb nahezu unverändert im neutralen Bereich. Parallel sanken in den meisten Pegeln die elektrische Leitfähigkeit, ein Indiz für abnehmende Elementkonzentrationen in den Wässern.

Anhand der chemischen Analyse von Festphasen und Porenwässern aus den Bohrkernen des Grundwassermesspegels RCO4, des Multilevelbrunnens CML und einer oberflächennahen Bohrung konnten Tiefenprofile für Elementkonzentrationen in Festphasen und Porenwässern erstellt werden. Dabei ließen die Ergebnisse der Untersuchungen an den Bohrkernen RCO4 und CML darauf schließen, dass in einer Tiefe von etwa $26 \mathrm{~m}$ in der Halde eine Oxidationszone vorliegt, in der die Oxidation zwischen 1995 und 1998 unvermindert andauerte. Als Sekundärmineral trat vor allem Gips auf. Der Verbleib des aus der Pyritoxidation stammenden Eisens konnte jedoch nicht ermittelt werden, obwohl die Proben des Bohrkerns RCO4 mit einer maximalen Tiefe von 58 m den gesamten Querschnitt der Kippe abdeckten.

In der oberflächennahen Bohrung war der Pyrit weitgehend verwittert. Niedrige pH-Werte und hohe Spurenelementkonzentrationen in den Porenwässern zeugten von der Fortdauer der 
sekundären Prozesse, die durch die sauren Sickerwässer aus der Pyritoxidation in Gang gesetzt wurden.

Die Porenlösungen aus den beiden Bohrkernen aus der Lausitz wiesen niedrige pH-Werte zwischen 2,5 und 4 auf und zeigten Elementkonzentrationen, die teilweise die höchsten in Cospuden gemessenen Werte deutlich überschritten.

Da für die Beurteilung des Gefahrenpotentials aus der Kippe nicht nur die Absolutgehalte berücksichtigt werden sollten, sondern auch die Migrationsformen, wurden im Rahmen der vorliegenden Arbeit auf der Basis des Speziationsschemas von PLEßOW (1998) umfangreiche Untersuchungen an Sicker- und Grundwasserproben des ehemaligen Tagebaus Cospuden südwestlich von Leipzig und an Porenwässern aus dem Bereich des Restlochs 111 in der Niederlausitz vorgenommen. Die Speziation von Elementen aus natürlichen Wässern erfordert die Berücksichtigung zahlreicher Parameter; anorganische Kolloide, adsorptive Mineraloberflächen und organische Komplexbildner sind ebenso in Betracht zu ziehen wie anorganische Komplexliganden, das Redoxpotential und der pH-Wert. Mikrobielle Prozesse können zusätzlich einen Großteil der genannten Parameter beeinflussen.

Das Speziationsschema aus Ultrafiltration, Kationen- und Anionenaustausch ist geeignet für kleine Probenmengen, wie sie beispielsweise bei der Gewinnung von Porenwässern aus Bohrkernproben anfallen. Wegen der komplizierten Situation in natürlichen Wässern, gerade in den hochbelasteten sauren Minenwässern aus pyritreichen ehemaligen Tagebauen, reicht ein Verfahren allein aber kaum aus, um tragfähige Aussagen über die Elementspeziation zu machen. Hier empfiehlt sich die Kombination mehrerer Methoden, z.B. ein Vergleich von experimentellen und rechnerischen Ergebnissen. Daher wurde für alle untersuchten Proben eine Berechnung der Elementspeziation mit dem Programm MINTEQA2 durchgeführt.

Das im Rahmen dieser Arbeit angewandte Speziationsschema ermöglicht im Zusammenspiel mit Speziationsberechnungen im Prinzip für viele Elemente eine genauere Eingrenzung der vorliegenden Migrationsformen. Allerdings muss für ein exaktes Vorgehen jedes Element in jeder einzelnen Probe unter Berücksichtigung der sonstigen Parameter untersucht werden; dieser Aufwand ist bei großen Probenmengen kaum zu bewältigen. Deshalb empfiehlt sich eine schrittweise Einteilung der Proben nach folgendem Schema:

1) Liegen bei der Ultrafiltration erkennbare Kolloide vor? Hohe Eisengehalte in der Probe und hohe Rückhalteraten für Eisen bei der Ultrafiltration bedeuten, dass kolloidale Eisenhydroxid-Phasen in den Wässern vorliegen. Solche Phasen binden Übergangsmetalle und teilweise andere Spurenelemente in ihre Struktur ein oder adsorbieren sie an ihrer Oberfläche und prägen damit deren Verhalten in der Speziation; im experimentellen 
Speziationsschema zeigt sich dies nicht nur in hohen Ultrafiltrationsraten für viele Elemente, sondern auch in einem vollständigen vermeintlichen "Austausch" beim Kationen- und Anionenaustausch; tatsächlich handelt es sich dabei um eine Filtration der Kolloide am Trägermaterial der Ionenaustauscher. Speziationsberechnungen ohne Berücksichtigung dieser Phasen liefern in der Regel falsche Resultate; die Einbeziehung solcher Kolloide in die Berechnung ist prinzipiell möglich, wenn die vorliegenden Kolloide hinsichtlich ihrer Oberfläche charakterisiert werden können. Andere kolloidale Phasen wie $\mathrm{CaSO}_{4}$ beeinflussen Spurenelemente nach den Beobachtungen dieser Arbeit nicht so stark; u.U. sind auch in Anwesenheit solcher Kolloide Speziationsberechnungen möglich und liefern sinnvolle Ergebnisse. Große Mengen kolloidaler Sulfatphasen können jedoch die Oberflächen der Ionentauscher blockieren und damit die experimentellen Speziationsergebnisse verfälschen.

2) Finden sich bei der Ultrafiltration keine Hinweise für das Vorliegen kolloidaler Phasen, müssen die Ergebnisse aus Rechnung und Experiment genau verglichen werden. Finden sich Hinweise auf kolloidale Strukturen, die bei der Ultrafiltration nicht erfasst wurden? Dazu gehören beispielsweise sehr hohe Austauschraten bei Elementen, die dem Austausch nicht zugänglich sein sollten, oder umgekehrt stark herabgesetzte Austauschraten bei Elementen, die eigentlich in Lösung ionisch vorliegen sollten. Ein Beispiel hierfür ist Ba. Den Rechnungen zufolge sollte $\mathrm{Ba}$ in allen Proben zu $100 \%$ als zweiwertiges, nicht komplexiertes Kation vorliegen. Trotzdem zeigen einige Proben hohe Rückhalteraten beim Anionenaustausch. Je nach Größe der Strukturen war der Kationenaustausch gleichzeitig erniedrigt, weil $\mathrm{Ba}$ in neutrale Strukturen eingebunden wurde, die aber zum großen Teil klein genug waren, um den Ionenaustauscher zu passieren. Baryt war in praktisch allen Wässern übersättigt. Insgesamt fiel die Abgrenzung zwischen anorganischen Kolloiden und einfachen Komplexen sehr schwer; die Trenngrenze von 1 $\mathrm{kD}$ bei der Ultrafiltration scheint hier willkürlich, da in der Realität von einem Kontinuum ausgegangen werden muss, das vom einfachen Metall-Sulfatkomplex über Komplexe aus mehreren Ionen bis hin zu Kolloiden und Partikeln reicht.

3) Sind keine kolloidalen Einflüsse feststellbar, müssen weitere mögliche Erklärungen für Abweichungen zwischen Rechnung und Experiment in Augenschein genommen werden. Dazu gehört für redoxempfindliche Elemente wie $\mathrm{Cr}$ oder $\mathrm{V}$, die schon auf kleine Abweichungen im pH-Wert und Redoxpotential sehr empfindlich mit Veränderungen ihrer Lösungsformen reagieren, die Betrachtung dieser Parameter. 
4) Werden organische Komplexbildner als mögliche Ursache für unterschiedliche Resultate aus Experiment und Speziationsrechnung vermutet, lässt sich diese Möglichkeit zunächst mittels einer einfachen Überschlagsrechnung prüfen. Übersteigt die Zahl der Bindungsstellen je $\mathrm{g} / \mathrm{C}$, die bei vollständiger Bindung eines Elements an den DOC notwendig wären, um die Abweichung zu erklären, die literaturbekannten Gehalte von Bindungsstellen im DOC um ein Vielfaches, können organische Komplexbildner nicht die Ursache sein. Erscheint die Zahl jedoch realistisch, kann durch Einsetzen von Modellsubstanzen wie Citrat oder Amidstrukturen in der Speziationsrechnung der Charakter des DOC sowohl hinsichtlich der Art als auch der Dichte seiner funktionellen Gruppen ermittelt werden.

In allen Oberflächenwässern aus der Arbeit von PLEßOW (1998) und der vorliegenden Arbeit sowie in zahlreichen Porenlösungen liegen anorganische Kolloide vor, die das Verhalten praktisch aller Elemente in diesen Proben prägen. Teilweise handelt es sich dabei um Eisenkolloide, aber auch $\mathrm{CaSO}_{4}$ tritt in zahlreichen Wässern kolloidal auf.

Waren keine Kolloide oder nur $\mathrm{CaSO}_{4}$-Kolloide feststellbar, zeigten die Proben teilweise bei den Übergangsmetallen $\mathrm{Cu}, \mathrm{Co}, \mathrm{Ni}$ und $\mathrm{Zn}$ sowie bei $\mathrm{Pb}$ eine gute Übereinstimmung zwischen berechneten und experimentellen Daten. Mo, As und Sb jedoch verhielten sich in fast allen Proben entgegen den Erwartungen, ohne dass dafür Kolloide verantwortlich gemacht werden konnten; hier kommt dem Einfluss organischer Komplexbildner vermutlich eine entscheidende Rolle zu. Berechnungen dazu waren nicht möglich, weil MINTEQA keine Daten für die Komplexierung dieser Elemente enthält. Prinzipiell ist die Erweiterung der Datenbank aber ohne großen Aufwand möglich, wenn entsprechende Komplexbildungskonstanten bekannt sind. Auch $\mathrm{Cr}$ und $\mathrm{V}$ zeigten große Abweichungen vom berechneten Verhalten; inwieweit dies auf organische Komplexbildner oder auf Abweichungen beim gemessenen Redoxpotential zurückgeht, konnte nicht ermittelt werden. Die Alkali- und Erdalkalimetalle waren in ihrem Verhalten nur von Kolloiden oder einfachen Sulfatkomplexen beeinflusst.

In den Proben aus der Lausitz deutete nichts auf Kolloide hin; durch ihre niedrigen pH-Werte und hohen Redoxpotentiale konnten auch hohe Konzentrationen zahlreicher Elemente als echte Lösungen vorliegen. Ein nennenswerter Einfluss des DOC ist in diesen Proben nicht zu erwarten; der DOC verfügt offenbar nur über wenige Funktionalitäten und wurde beim Ionenaustausch kaum zurückgehalten. In den Wässern aus Cospuden dagegen lagen sehr unterschiedliche DOC-Gemische vor; in vielen Proben zeigte der DOC ein hochgradig amphoteres Verhalten, was auf eine große Zahl potentieller Bindungsstellen sowohl für 
Kationen als auch Anionen hinweist. Untersuchungen mit harnstoffhaltigen synthetischen Lösungen deuten darauf hin, dass Sulfat mit stickstoffhaltigen organischen Strukturen Kolloide ausbilden kann, an die eventuell auch andere Elemente gebunden werden.

In keiner Probe waren Phänomene zu beobachten, die auf ein Vorliegen organischer Kolloide ohne den Einfluss anorganischer Kolloide hinwiesen (d.h. hohe Rückhalterate für DOC bei der Ultrafiltration bei gleichzeitig niedrigen Rückhalteraten für Eisen und Sulfat). Trotzdem kann der Einfluss organischer Komplexbildner insbesondere auf Übergangsmetalle und REEs nicht ausgeschlossen werden. Beispielhaft wurde an einer Probe gezeigt, wie Citrat als Modell für organische Komplexbildner in Speziationsrechnungen eingebunden werden kann.

Eine interessante Frage, die im Rahmen dieser Arbeit nicht diskutiert werden konnte, ist, inwieweit der teilweise in großen Mengen vorliegende und hochfunktionalisierte DOC zur Stabilisierung anorganischer Kolloide beiträgt. Denkbar ist, dass er sie mit einer organischen Schicht überzieht und ihre Sedimentation behindert. Eventuell kämen dann nicht nur einfache organische Komplexe von Ionen als Transportformen in Frage, wie sie für Mo, As und $\mathrm{Sb}$ vermutet werden, sondern auch anorganische Kolloide auf der Basis von Eisen- oder $\mathrm{CaSO}_{4}{ }^{-}$ Phasen, an die zahlreiche andere Elemente gebunden sind und die durch organische Überzüge in der Lösung gehalten werden.

Die Ergebnisse der vorliegenden Arbeit zeigen, dass im Haldenmaterial in Cospuden weiterhin Oxidationsprozesse ablaufen und ein hohes Versauerungspotential vorliegt. Fraglich ist, inwieweit nach Abschluss der Flutung die natürliche Säureneutralisationskapazität in Kippe und Seewasser ausreicht, um den See dauerhaft vor Versauerung durch den Eintrag saurer und hochkonzentrierter Kippenwässer zu schützen. Bislang sind die eingetragenen Elemente zum großen Teil durch Sedimentationsprozesse aus den Wässern entfernt worden.

In der Lausitz liegen sehr hohe Konzentrationen vieler Elemente in nichtkomplexierter und nichtkolloidaler Form in den Porenwässern vor. Die meisten Elemente waren in Form einfacher ionischer oder neutraler Komplexe gelöst, so dass sie zumindest für die Adsorption an reaktiven Mineraloberflächen zugänglich sind. Ist jedoch das Potential an adsorptiven Oberflächen erschöpft oder kommen die Wässer in Kontakt mit DOC-reicheren Wässern, ist zu befürchten, dass die hohen Elementkonzentrationen mobil genug sind, um sich auch über das eigentliche Areal des Restlochs 111 hinaus auszubreiten. 


\section{Literatur}

Abbt-Braun, G., Frimmel, F.H. und Schulten, H.-R. (1990): Strukturelle Charakterisierung isolierter aquatischer Huminstoffe - Anwendbarkeit, Grenzen und Vergleich ausgewählter Methoden. Vom Wasser 74, S. 325-338.

Al, T.A., Blowes, D.W., Martin, C.J., Cabri, L.J. and Jambor, J.L. (1997): Aqueous geochemistry and analysis of pyrite surfaces in sulfide-rich mine tailings. Geochim. Cosmochim. Acta 61 (12), p. 2353-2366.

Allan, R.J. (1995): Impact of mining activities on the terrestrial and aquatic environment with emphasis on mitigation and remedial measures. In: Allan, R., Förstner, U. and Salomons, W. (Hrsg.): Heavy metals: problems and solutions. Springer, Berlin, p. 119-140.

Allison, J.D., Brown, D.S. and Novo-Gradac, K.J. (1991): MINTEQA2/PRODEFA2, A geochemical assessment model for environmental systems: Version 3.0 User's manual. EPA/600/3-91/021.

Alpers, C.N. and Blowes, D.W. (Hrsg.) (1994): Environmental geochemistry of sulfide oxidation. ACS symposium series 550, Washington, 659 p.

Balistrieri, L.S., Murray, J.W. and Paul, B. (1994): The geochemical cycling of trace elements in a biogenic meromitic lake. Geochim. Cosmochim. Acta 58 (19), p. 3993-4008.

Balistrieri, L.S. (1992): The cycling of iron and manganese in the water column of Lake Sammamish, Washington. Limnol. Oceanogr. 37 (3), p. 510-528.

Balistrieri, L., Brewer, P.G. and Murray, J.W. (1981): Scavenging residence times of trace metals and surface chemistry of sinking particles in the deep ocean. Deep-Sea Res. 28A, p. 101-121.

Barcelona, M.J. (1990): Uncertainties in ground water chemistry and sampling procedures. In: Melchior, D.C. and Bassett, R.L. (Hrsg.): Chemical modeling of aqueous systems II, ACS symposium series 416, Washington, p. 310-320.

Bellmann, H.-J., Rösler, H.J. und Starke, R. (1984): Faziesdifferenzierung und Tonmineralbestand der oligozänen Schichten in der Leipziger Bucht. Zeitschrift für geologische Wissenschaften 12 (4), S. 409-418.

Benoit, G. (1995): Evidence of the particle concentration effect for lead and other metals in fresh waters based on ultraclean technique analyses. Geochim. Cosmochim. Acta 59 (13), p. 2677-2687.

Benoit, G., Oktay-Marshall, S.D., Cantu, A., Hood, E.M., Coleman, C.H., Corapcioglu, M.O. and Santschi, P. (1994): Partitioning of $\mathrm{Cu}, \mathrm{Pb}, \mathrm{Ag}, \mathrm{Zn}, \mathrm{Fe}, \mathrm{Al}$, and $\mathrm{Mn}$ between filterretained particles, colloids, and solution in six Texas estuaries. Mar. Chem. 45, p. 307-336. 
Berkner, A. (2000): Die Mitteldeutsche Straße der Braunkohle. URL: http://www.braunkohlenstrasse.de.

Berkner, A. (1995): Von der Braunkohlen- zur Seenlandschaft - Chancen und Konflikte bei der Gestaltung und Folgenutzung von Tagebaurestseen in Westsachsen. UFZ-Bericht 4/95, S. 4-13.

Berufsverband Deutscher Geologen (BDG) (1996): Die Wiederherstellung des Wasserhaushalts im ehemaligen mittel- und ostdeutschen Braunkohlenbergbau. BDG Mitteilungsblatt Nr. 68, S. 25-26.

Bigham, J.M. and Murad, E. (1997): Mineralogy of ochre deposits formed by the oxidation of iron sulfide minerals. Advances in GeoEcology 30, p. 193-225.

Bigham, J.M., Schwertmann, U., Traina, S.J., Winland, R.L. and Wolf, M. (1996): Schwertmannite and the chemical modeling of iron in acid sulfate waters. Geochim. Cosmochim. Acta 60 (12), p. 2111-2121.

Bigham, J.M., Schwertmann, U. and Carlson, L. (1992): Mineralogy of precipitates formed by the biogeochemical oxidation of Fe(II) in mine drainage. Catena Suppl. 21, p. 219-232.

Blowes, D.W. and Jambor, J.L. (1990): The pore-water geochemistry and the mineralogy of the vadose zone of sulfide tailings, Waite Amulet, Quebec, Canada. Appl. Geochem. 5, p. 327-346.

Böttcher, G., Brumsack, H.-J., Heinrichs, H. and Pohlmann, M. (1997): A new high-pressure squeezing technique for pore fluid extraction from terrestrial soils. Water, Air and Soil Pollution 94, p. 289-296.

Borek, S.L. (1994): Effect of humidity on pyrite oxidation. In: Alpers, C.N. and Blowes, D.W. (Hrsg.): Environmental chemistry of sulfide oxidation. ACS symposium series 550, Washington, p. 31-44.

Boughriet, A., Ouddane, B., Fischer, J.C., Wartel, M. and Leman, G. (1992): Variability of dissolved $\mathrm{Mn}$ and $\mathrm{Zn}$ in the Seine estuary and chemical speciation of these metals in suspended matter. Wat. Res. 26 (10), p. 1359-1378.

Boulegue, J. and Michard, G. (1979): Sulfur speciation and redox processes in reducing environments. In: Chemical modeling in aqueous systems. Speciation, sorption, solubility, and kinetics. ACS symposium series 93, Washington, p.25-50.

Bourg, A.C.M. (1995): Speciation of heavy metals in soils and groundwater and implications for their natural and provoked mobility. In: Allan, R., Förstner, U. and Salomons, W. (Hrsg.): Heavy metals: problems and solutions. Springer, Berlin, p. 19-31. 
Bozau, E., Knöller, K. and Strauch, G. (2000): Hydrogeological and geochemical investigations of an acidic mining lake as a basis for biotechnological remediation. In: Silico et al. (2000) (Hrsg.): Groundwater. Past achievements and future challenges. Balkema, Rotterdam, p. 1099-1102.

Brinkmann, J.-U. (2000): Geochemisch-mineralogische Untersuchungen an Haldensedimenten aus dem Braunkohlentagebau Zwenkau/Cospuden. Diplomarbeit, Geochemisches Institut Göttingen.

Broll, G., Dunger, W., Keplin, B. und Topp, W. (Hrsg.): Rekultivierung in Bergbaufolgelandschaften: Bodenorganismen, bodenökologische Prozesse und Standortentwicklung. Springer, Berlin, 306 S..

Brown, J.G., Bassett, R.L. and Glynn, P.D. (2000): Reactive transport of metal contaminants in alluvium - model comparison and column simulation. Appl. Geochem. 15, p. 35-49.

Brügmann, L., Hallberg, R. and Larsson, C. (1998): Trace metal speciation in sea and pore water of the Gotland Deep, Baltic Sea, 1994. Appl. Geochem. 13, p. 359-368.

Brüschke, K., Pleßow, A., Kittsteiner, M. und Heinrichs, H. (1999): Herkunft und Lösungsverhalten von Spurenelementen in Sicker- und Porenwässern im Tagebaubereich Zwenkau/Cospuden. UFZ-Bericht 25/99, S. 106-115.

Brüschke, K., Pleßow, A. and Heinrichs, H. (2000): Transport and fate of solutes in acid mine drainage at Cospuden (Germany). In: Applied Mineralogy in Research, Economy, Technology, Ecology and Culture. Proceedings of the sixth ICAM, Balkema, Rotterdamm, p.479-482.

Brumsack, H.J. (1981): A simple method for the determination of sulfide- and sulfate-sulfur in geological materials by using different temperatures of decomposition. Fresenius Zeitschrift für Analytische Chemie 307, S. 206-207.

Buffle, J., Perret, D. and Newman, M. (1992): The use of filtration and ultrafiltration for size fractionation of aquatic particles, colloids, and macromolecules. In: Buffle, J. and Van Leeuwen, H.P. (Hrsg.): Environmental Particles, Lewis Publishers, Chelsea, p. 171-230.

Buffle, J., DeVitre, R.R., Perret, D. and Leppard, G.G. (1989): Physico-chemical characteristics of a colloidal iron phosphate species formed at the oxic-anoxic interface of a eutrophic lake. Geochim. Cosmochim. Acta 53, p. 399-408.

Buffle, J. (1988): Complexation reactions in aquatic systems: an analytical approach. Ellis Horwood Limited, Chichester, 692 p.

Buffle, J. (1981): Speciation of trace elements in natural waters. Trends in Analytical Chemistry 4, p. 90-95. 
Calmano, W., Förstner, U. and Hong, J. (1994): Mobilization and scavenging of heavy metals following resuspension of anoxic sediments from the Elbe River. In: Alpers, C.N. and Blowes, D.W. (Hrsg): Environmental geochemistry of sulfide oxidation. ACS symposium series 550, Washington, p. 298-321.

Cesnovar, R. und Pentinghaus, H. (1999): Mineralogische Signaturen des Abraums in Cospuden und Zwenkau: Folgen der Flutung des Tagebaurestlochs. UFZ-Bericht 25/99, S. 127-133.

Cesnovar, R. und Pentinghaus, H. (1997): Anthropogener und geogener Einfluss auf die regionale Hydrogeochemie durch Öffnung der Braunkohlelagerstätten: Mineralogische Signatur des Abraums in Cospuden. In: Deutsche Mineralogische Gesellschaft (Hrsg.): Berichte der Deutschen Mineralogischen Gesellschaft, Beiheft zum Eur. J. Mineral. 9 (1), S. 71.

Chapman, B.M., Jones, D.R. and Jung, R.F. (1983): Processes controlling metal ion attenuation in acid mine drainage streams. Geochim. Cosmochim. Acta 47, p. 1957-1973.

Choi, J.-C. and West, T.R. (1997): Application of MINTEQA2 to the evaluation of apatite as a precipitant for acid mine drainage treatment. Environm. Engineer. Geosc. III (2), p. $217-$ 223.

Cravotta, C.A. (1994): Secondary iron-sulfate minerals as sources of sulfate and acidity: Geochemical evolution of acidic ground water at a reclaimed surface coal mine in Pennsylvania. In: Alpers, C.N. und Blowes, D.W. (Hrsg.): Environmental geochemistry of sulfide oxidation. ACS symposium 550, Washington, p. 345-364.

Cronan, C.S. and Aiken, G.R. (1985): Chemistry and transport of soluble humic substances in forested watersheds of the Adirondack Park, New York. Geochim. Cosmochim. Acta 49, p. 1697-1705.

Curtis, P.J. (1998): Climatic and hydrologic control of DOM concentration and quality in lakes. In: : Hessen, D.O. and Tranvik, L.J. (Hrsg.): Aquatic humic substances: ecology and biochemistry. Springer, Berlin, p. 93-105.

Davis, J.A. (1984): Complexation of trace metals by adsorbed natural organic matter. Geochim. Cosmochim. Acta 48, p. 679-691.

Davison, W. (1992): Iron particles in freshwater. In: Buffle, J. and Van Leeuwen, H.P. (Hrsg.): Environmental Particles, Lewis Publishers, Chelsea, p. 315-355.

Davison, W. (1985): Conceptual models for transport at a redox boundary. In: Stumm, W. (Hrsg.): Chemical processes in lakes. Wiley, New York, p. 31-53. 
De Nobili, M. and Fornasier, F. (1996): Assessment of the effect of molecular size on the electrophoretic mobility of humic substances. Eur. J. of Soil Sc. 47, p. 223-229.

De Witt, J.V.M., Nederlof, M.M., van Riemsduk, W.H. and Koopal, L.K. (1991): Determination on $\mathrm{H}^{+}$and metal ion affinity distributions for humic substances. Water, Air and Soil Pollution 57-58, p. 339-349.

Diercke-Weltatlas (1974), Auflage 1983/83, Westermann, Braunschweig, S. 66-67.

Donahoe, R.J. and Liu, C. (1998): Pore water geochemistry near the sediment-water interface of a zoned, freshwater wetland in the southeastern United States. Environ. Geol. 33 (2/3), p. $143-153$.

Douglas, G.B., Hart, B.T., Beckett, R., Gray, C.M. and Oliver, R.L. (1999): Geochemistry of suspended particulate matter (SPM) in the Murray-Darling river system: A conceptual isotopic/geochemical model for the fractionation of major, trace and reare earth elements. Aquatic Geochem. 5, p. 167-194.

Drever, J. (1997): The geochemistry of natural waters: surface and groundwater environments. Prentice Hall, London, p. 159-174.

Duffy, S.J., Hay, G.W., Micklethwaite, R.K. and van Loon, G.W. (1989): Distribution and classification of metal species in soil leachates. Science of the Total Environment 87/88, p. 189-197.

Dupré, B., Viers, J., Dandurand, J.-L., Polve, M., Bénézeth, P., Vervier, P. and Braun, J.-J. (1999): Major and trace elements associated with colloids in organic-rich river waters: ultrafiltration of natural and spiked solutions. Chem. Geol. 160, p. 63-80.

Eggleston, C.M., Erhardt, J.-J. and Stumm, W. (1996): Surface structural controls on pyrite oxidation kinetics: An XPS-UPS, STM, and modeling study. American Mineralogist 81, p. 1036-1056.

Erickson, B.E. and Helz, G.R. (2000): Molybdenum (VI) speciation in sulfidic waters: Stability and lability of thiomolybdates. Geochim. Cosmochim. Acta 64 (7), p. 1149-1158.

Florence, T.M. and Batley, G.E. (1980): Chemical speciation in natural waters. CRC Critical Reviews in Analytical Chemistry 9 (3), p. 219-296.

Fortin, D., Leppard, G.G. and Tessier, A. (1993): Characteristics of lacustrine diagenetic iron oxyhydroxides. Geochim. Cosmochim. Acta 57, p. 4391-4404.

Förstner, U. (1995): Contaminated aquatic sediments and waste sites: Geochemical engineering solutions. In: Allan, R., Förstner, U. and Salomons, W. (Hrsg.): Heavy metals: problems and solutions. Springer, Berlin, p. 237-256. 
Foos, A. (1997): Geochemical modeling of coal mine drainage, Summit County, Ohio. Environ. Geol. 31 (3/4), p. 205-210.

Frimmel, F.H. and Abbt-Braun, G. (Hrsg.) (1997) Symposium on refractory organic substances in the environment: ROSE - Abstracts of orals and poster papers. Veröffentlichungen des Lehrstuhls für Wasserchemie und DVGW-Forschungsstelle am Engler-Bunte-Institut der Universität Karlsruhe 35, 253 S.

Frimmel, F.H. and Christman, F.H. (Hrsg.) (1988): Humic substances and their role in the environment. Dahlem Workshop Reports Life Sciences Research Report 41, Springer, Berlin etc., $271 \mathrm{p}$.

Frimmel, F.H. und Geywitz, J. (1983): Zur koordinativen Bindung von Metallionen an Gewässerhuminstoffe. Fresenius Zeitung für Analytische Chemie 316, S. 582-588.

Frost, T.M., Montz, P.K., Kratz, T.K., Badillo, T., Brezonik, P.L., Gonzalez, M.J., Rada, R.G., Watras, C.J., Webster, K.E., Wiener, J.G., Williamson, C.E. and Morris, D.P. (1999): Multiple stresses from a single agent: Diverse responses to the experimental acidification of Little Rock Lake, Wisconsin. Limnol. Oceaonogr. 44 (3, Teil 2), p. 784-794.

Fuge, R., Pearce, F.M., Perace, N.J.G. and Perkins, W.T. (1994): Acid mine drainage in Wales and influence of ochre precipitation on water chemistry. In: Alpers, C.N. and Blowes, D.W. (Hrsg): Environmental geochemistry of sulfide oxidation. ACS symposium series 550, Washington, p. 261-274.

Funk, W. und Schär, P. (1996): Praktikerwissen Analysenmesstechnik - pH, Redox, LF, $\mathrm{O}_{2}-$ Messprinzip, Anwendung, Geräte, Problemlösung. Dr. Bruno Lange GmbH Berlin, 98 S.

Gaffney, J.S., Marley, N.A. and Clark, S.B. (Hrsg.) (1996): Humic and fulvic acids: isolation, structure, and environmental role. ACS Symposium Series 651, Washington, 331 p.

Geller, W., Klapper, H. and Schultze, M. (1998): Natural and anthropogenic sulfuric acidification of lakes. In: Geller, W., Klapper, H. and Salomons, W. (Hrsg.): Acidic mining lakes: acid mine drainage, limnology, and reclamation. Springer, Berlin, p. 3-13.

Germain, M.D. , Tassé, E. and Bergeron, M. (1994): Limit to self-neutralization in acid mine tailings. In: Alpers, C.N. and Blowes, D.W. (Hrsg): Environmental geochemistry of sulfide oxidation. ACS symposium series 550, Washington, p. 365-379.

Gläßer, W. (1995): Der Einfluß des Braunkohlenbergbaus auf Grund- und Oberflächenwasser. Hydrogeologische Untersuchungen in Mitteldeutschland. Geowissenschaften 13 (8-9), S. 291-296. 
Gläßer, W. und Wohnlich, S. (1998): Hydrogeochemische Prozesse in Braunkohletagebaukippen Mitteldeutschlands. Zwischenbericht zum DFG-Projekt GI 233/1-3 und Wo 396/3-3. S. 19-20.

Gould, W.D., Béchard, G. and Lortie, L. (1994): The nature and role of microorganisms in the tailings environment. In: Jambor, J.L. and Blowes, D.W.: Short course handbook on environmental geochemistry of sulfide mine-waste. Vol. 22, 7, Mineralogical Association of Canada, Waterloo Ontario, p. 185-200.

Greenwood, N.N. und Earnshaw, A. (1990): Chemie der Elemente. Verlag Chemie, Weinheim, $1707 \mathrm{~S}$.

Gruhn, A., Matthess, G., Pekdeger, A und Scholtis, A. (1985): Die Rolle der gelösten organischen Substanz beim Transport von Schwermetallen. Zeitschrift der deutschen geologischen Gesellschaft 136, S. 417-427.

Hartmann, G. (1994): Late-medieval glass manufacture in the Eichsfeld region (Thuringia, Germany). Chem. Erde 54, p. 103-128.

Hatcher, P.G., Wilson, M.A., Vasallo, A.M. and Lerch, H.E. (1989): Studies of angiospermous woods in Australian brown coals by nuclear magnetic resonance and analytical pyrolysis: new insights into early coalification. International Journal of Coal Geology 13, p. 99-126.

Heinrichs, H., Böttcher, G., Brumsack, H.-J. and Pohlmann, M. (1996): Squeezed soil-pore solutes - a comparison to lysimeter samples and percolation experiments. Water, Air and Soil Pollution 89, p. 189-204.

Heinrichs, H. und Hermann, A.G. (1990): Praktikum der Analytischen Geochemie. Springer, Berlin, $669 \mathrm{~S}$.

Heinrichs, H. (1989/90): Aufschlußverfahren in der Analytischen Geochemie (Teil 1/2); Sonderdruck aus LaborPraxis 12/89 und 1+2/90, Vogel-Verlag, $10 \mathrm{~S}$.

Heinrichs, H., König, N. und Schultz, R. (1985): Atom-Absorptions- und Emissionsspektrometrische Bestimmungsmethoden für Haupt- und Spurenelemente in Porenlösungen aus Waldökosystem-Untersuchungen. Berichte des Forschungszentrums Waldökosysteme/Waldsterben der Universität Göttingen 8, S. 1-92.

Hemm, M., Jannack, K. und Nixdorf, B. (1999): Entwicklungsstadien des Tagebausees Gräbendorf (Niederlausitz) vom Restloch über den Zustand des sauren Sees zu einem neutralen Gewässer. Deutsche Gesellschaft für Limnologie, Tagungsbericht 1998 (Klagenfurt), S. 254-258. 
Herbert, R.B. jr (1994): Metal transport in groundwater contaminated by acid mine drainage. Nordic Hydrology 25, p. 193-212.

Hering, J.G. and Morel, F.M.M. (1990): The kinetics of trace metal complexation: implications for metal reactivity in natural waters. In: Stumm, W. (Hrsg.): Aquatic chemical kinetics: reaction rates of processes in natural waters. Wiley, New York, p. 145171.

Herzsprung, P., Friese, K. Packroff, G., Schimmele, M., Wendt-Potthoff, K. and Winkler, M. (1998): Vertical and annual distribution of ferric and ferrous iron in acidic mining lakes. Acta hydrochim. hydrobiol. 26, p. 253-262.

Hodel, M. (1994): Untersuchungen zur Festlegung und Mobilisierung der Elemente As, Cd, $\mathrm{Ni}$ und $\mathrm{Pb}$ an ausgewählten Festphasen unter besonderer Berücksichtigung des Einflusses von Huminstoffen. Karlsruher Geochemische Hefte 5, 129 S.

Holleman, A.F. und Wiberg, E. (1985): Lehrbuch der Anorganischen Chemie. Walter de Gruyter Berlin, 1451 S..

Honeyman, B.D. (1992): The role of particles and colloids in the transport of radionucleides and trace metals in oceans. In: Buffle, J. and Van Leeuwen, H.P. (Hrsg.): Environmental Particles, Lewis Publishers, Chelsea, p. 379-421.

HydroGeoLogic, Inc. and Allison Geoscience Consultants, Inc. (1999): MINTEQA2/PRODEFA2, A geochemical assessment model for environmental systems: User manual supplement for version 4.0. US Environmental Protection Agency.

HydroGeoLogic, Inc. (1999): Diffuse-Layer sorption reactions for use in MINTEQA2 for HWIR Metals and Metalloids. US Environmental Protection Agency.

Igarashi, T. and Oyama, Takahiro (1999): Deterioration of water quality in a reservoir receiving pyrite-bearing rock drainage and its geochemical modeling. Engineering Geol. 55 , p. $45-55$.

James, R.O. and MacNaughton, M.G. (1977): The adsorption of aqueous heavy metals on inorganic minerals. Geochim. Cosmochim. Acta 41, p. 1549-1555.

Jean, G.E. and Bancroft, G.M. (1986): Heavy metal adsorption by sulphide mineral surfaces. Geochim. Cosmochim. Acta 50, p. 1455-1463.

Johnson, C.A. (1986) : The regulation of trace element concentrations in river and estuarine waters contaminated with acid mine drainage: The adsorption of $\mathrm{Cu}$ and $\mathrm{Zn}$ on amorphous Fe oxyhydroxides. Geochim. Cosmochim. Acta 50, p. 2433-2438.

Kimball, B.A., Brosheats, R.E., McKnight, D.M. and Bencala, K.E. (1994): Effects of instream $\mathrm{pH}$ modification on transport of sulfide-oxidation products. In: Alpers, C.N. and 
Blowes, D.W. (Hrsg): Environmental geochemistry of sulfide oxidation. ACS symposium series 550, Washington, p. 224-243.

Kittsteiner, M. (1998): Mineralogisch-geochemische Charakterisierung und Elementmassenbilanzierung pyrithaltiger Sedimente aus den Braunkohletagebauen Zwenkau und Garzweiler. Diplomarbeit, Geochemisches Institut Göttingen.

Kittsteiner, M., Pleßow, A. und Heinrichs, H. (2000): Pyrithaltige Sedimente aus dem Braunkohlentagebau Zwenkau/Sachsen als Reservoir von Spurenelementen und deren umweltrelevante Freisetzung. In Wippermann, T. (Hrsg.): Bergbau und Umwelt Langfristige geochemische Einflüsse. Bd. 6, Springer, Berlin, S. 25-40.

Klapper, H. (1995): Ökotechnologisch nutzbare Naturpotentiale zur Verbesserung der Wasserbeschaffenheit in Bergbaurestseen. UFZ-Bericht 4/95, S. 14-25.

Kleinmann, R.L.P., Hedin, R.S. and Nairn, R.W. (1998): Treatment of mine drainage by anoxic limestone drains and constructed wetlands. In: Geller, W., Klapper, H. and Salomons, W. (Hrsg.): Acidic mining lakes: acid mine drainage, limnology, and reclamation. Springer, Berlin, p. 303-319.

Kögel-Knabner, I. und Rumpel, C. (2000): Bestimmung und Charakterisierung der organischen Substanz in braunkohlehaltigen aschemeliorierten Kippenböden unter Wald. In: Broll, G., Dunger, W., Keplin, B. und Topp, W. (Hrsg.): Rekultivierung in Bergbaufolgelandschaften: Bodenorganismen, bodenökologische Prozesse und Standortentwicklung. Springer, Berlin, 306 S..

Kölling, M., Ebert, M. and Schulz, H.D. (2000): A novel approach to the presentation of pE/pH-diagrams. In: Schüring, J., Fischer, W.R. and Duijnisveld, W.H.M. (Hrsg.): Redox: fundamentals, processes, and applications. Springer, Berlin, p. 55-63.

Kölling, M. (2000): Comparison of different methods for redox potential determination in natural waters. In: Schüring, J., Fischer, W.R. and Duijnisveld, W.H.M. (Hrsg.): Redox: fundamentals, processes, and applications. Springer, Berlin, p. 42-53.

Kölling, M. and Schulz, H.D. (1992): Description of geochemical environments with thermodynamic equilibrium models. In: Matthess, G., Frimmel, F., Hirsch, P., Schulz, H.D. and Usdowski, H.E. (Hrsg.): Progress in hydrogeochemistry. Springer, Berlin, p. 484498.

Kölling, M. (1986): Vergleich verschiedener Methoden zur Bestimmung des Redoxpotentials natürlicher Wässer. Meyniana 38, S. 1-19.

Kremling, K. (1983): The behavior of $\mathrm{Zn}, \mathrm{Cd}, \mathrm{Cu}, \mathrm{Ni}, \mathrm{Co}, \mathrm{Fe}$, and $\mathrm{Mn}$ in anoxic baltic waters. Mar. Chem. 13, p. 87-108. 
Kwong, Y.T.J. and Lawrence, J.R. (1998): Acid gegneration and metal immobilization in the vicinity of a naturally acidic lake in Central Yukon Territory, Canada. In: Geller, W., Klapper, H. and Salomons, W. (Hrsg.): Acidic mining lakes: acid mine drainage, limnology, and reclamation. Springer, Berlin, p. 65-86.

Landesumweltamt Brandenburg (1995): Wasserbeschaffenheit in Tagebaurestseen. Studien und Tagungsberichte Band 6, Potsdam.

Langmuir, D. (1997): Aqueous environmental geochemistry. Prentice-Hall, Upper Saddle River, 600 p.

Langmuir, D. (1979): Techniques of estimating thermodynamic properties for some aqueous complexes of chemical interest. In: Chemical modeling in aqueous systems. Speciation, sorption, solubility, and kinetics. ACS symposium series 93, Washington, p. 353-387.

Lausitzer und Mitteldeutsche Bergbauverwaltungsgesellschaft mbH (LMBV) (Hrsg.) (1995): Rehabilitierung des Wasserhaushaltes im Braunkohlenrevier Mitteldeutschland. Broschüre, $88 \mathrm{~S}$.

Laxen, D.P.H. (1985): Trace metal adsorption/coprecipitation on hydrous ferric oxide under realistic conditions. Water Res. 19 (10), p. 1229-1236.

Laxen, D.P.H. (1984/85): Adsorption of $\mathrm{Cd}, \mathrm{Pb}$ and $\mathrm{Cu}$ during the precipitation of hydrous ferric oxide in a natural water. Chem. Geol. 47, p. 321-332.

Laxen, D.P.H. and Sholkovitz, E.R. (1981): Adsorption (co-precipitation) of trace metals at natural concentrations on hydrous ferric oxide samples. Environm. Techn. Letters 2, p. 561-568.

Leenheer, J.A., Malcolm, R.L., McKinley, P.W. and Eccles, L.A. (1974): Occurence of dissolved organic carbon in selected groundwater samples in the United States. Us Geol. Surv. J. Res. 2, p. 361-369.

Liang, L. and Morgan, J.J. (1990): Coagulation of iron oxide particles in the presence of organic materials. In: Melchior, D.C. and Bassett, R.L. (Hrsg.): Chemical modeling of aqueous systems II, ACS symposium series 416, Washington, p. 291-308.

Lienemann, C.-P., Taillefert, M., Perret, D. and Gaillard, J.-F. (1997): Association of cobalt and manganese in aquatic systems: Chemical and microscopic evidence. Geochim. Cosmochim. Acta. 61 (7), p. 1437-1446.

Lofts, S. and Tipping, E. (1998): An assemblage model for cation binding by natural particulate matter. Geochim. Cosmochim. Acta 62 (15), p. 2609-2625.

Longmire, P., Brookins, D.G. and Thomson, B.M. (1990): Hydrogeochemical interactions and evolution of acidic solutions in soil. In: Melchior, D.C. and Bassett, R.L. (Hrsg.): 
Chemical modeling of aqueous systems II, ACS symposium series 416, Washington, p. 155-168.

Lundegard, P.D. and Kharaka, Y.K. (1990): Geochemistry of organic acids in subsurface waters. Field data, experimental data, and models. In: Melchior, D.C. and Bassett, R.L. (Hrsg.): Chemical modeling of aqueous systems II, ACS symposium series 416, Washington, p. 169-189.

Luoma, S.N. and Davis, J.A. (1983): Requirements for modeling trace metal partitioning in oxidized estuarine sediments. Mar. Chem. 12, p. 159-181.

Macalady, D.L., Langmuir, D., Grundl, T. and Elzerman, A. (1990): Use of model-generated $\mathrm{Fe}^{3+}$ ion activities to compute $\mathrm{Eh}$ and ferric oxyhydroxide solubilities in anaerobic systems. In: Melchior, D.C. and Bassett, R.L. (Hrsg.): Chemical modeling of aqueous systems II, ACS symposium series 416, Washington, p. 350-367.

Mach, H.M., Nott, B., Scott, J.W., Maddalone, R.F. and Whiddon, N.T. (1996): Metal speciation: Survey of environmental methods of analysis. Water, Air and Soil Pollution 90, p. 269-279.

Mantoura, R.F.C., Dickson, A. and Riley, J.P. (1978): The complexation of metals with humic materials in natural waters. Estuarine and Coastal Marine Sci. 6, p. 387-408.

Marley, N.A., Gaffney, J.S., Orlandi, K.A., Picel, K.C. and Choppin, G.R. (1992) Chemical characterization of size fractionated humic and fulvic materials in aqueous samples. Sci. Tot. Environ. 113, p. 159-177.

Mason, B. und Moore, C.B. (1985): Grundzüge der Geochemie; F. Enke Verlag, Stuttgart, $340 \mathrm{~S}$.

Matthess G. und Pekdeger, A. (1980): Chemisch-biochemische Umsetzungen bei der Grundwasserneubildung. Gas- und Wasserfach 121. S. 214-219.

McKnight, D.M., Thurman, E.M., Wershaw, R.L. and Westall, J.C. (1983): Complexation of copper by aquatic humic substances from different environments. Sci Total Environ. 28, p. 65-76.

Meiler, W. and Meusinger, R. (1991): NMR of coals and coal products. Annual Reports of NMR Spectroscopy 23, p. 376-410.

Melchers, K. (1994): Karbonatgehalte im Bereich Spät-/Postglazial Attersee (Salzkammergut, Österreich). Diplomarbeit, Institut für Geologie und Dynamik der Lithossphäre, GeorgAugust-Universität Göttingen, $82 \mathrm{~S}$. 
Merkel, B. und Sperling, B. (1998): Hydrogeochemische Stoffsysteme Teil II. DVWKSchriftenreihe 117, Wirtschafts- und Verlagsgesellschaft Gas und Wasser mbH, Bonn, 397 S.

Mill, A.J.B. (1980): Colloidal and macromolecular forms of iron in natural waters 1: A review. Environm. Techn. Letters I, p. 97-108.

Morel, F.M.M. and Gschwend, P.M. (1987): The role of colloids in the partitioning of solutes in natural waters. In: Stumm, W. (Hrsg.): Aquatic surface chemistry. Wiley, p. 405-422.

Moses, C.O., Nordstrom, D.K., Herman, J.S. und Mills, A.A. (1987): Aqueous pyrite oxidation by dissolved oxygen und ferric iron. Geochim. Cosmochim. Acta 51, p.15611571.

Murphy, E.M. and Zachara, J.M. (1995): The role of sorbed humic substances on the distribution of organic and inorganic contaminants in groundwater. Geoderma 67, p. 103124.

Nicholson, R.V. (1994): Iron-sulfide oxidation mechanisms: Laboratory studies. In: Jambor, J.L. and Blowes, D.W. (Hrsg.): Short course handbook on the environmental geochemistry of sulfide mine wastes. Mineralogical Association of Canada, Waterloo Ontario 22 (6), p. 163-183.

Nitsche, C., Guderitz, I. und Neumann, V. (1999): Hydrochemie der Grundwässer im Bereich des Tagebaus Cospuden und Methodik der Probennahme. UFZ-Bericht 25/99, S. 71- 91.

Nitzsche, H.-M., Gläser, H.R. und Schreck, M. (1999): Faseroptische Temperaturmessungen und gasisotopische Untersuchungen auf der Innenflurkippe des Tagebaurestlochs Cospuden. UFZ-Bericht Nr. 25/99, S. 153-161.

Nordstrom, D.K. (1996): Trace metal speciation in natural waters: Computational vs. analytical. Water, Air and Soil Pollution 90, p. 257-267.

Nordstrom, D.K., McNutt, R.H:, Puigdomènech, I., Smellie, J.A.T. and Wolf, M. (1992): Ground water chemistry and geochemical modeling of water-rock interactions at the Osamu Utsumi mine and the Morro do Ferro analogue study sites, Pocos de Caldas, Minas Gerais, Brazil. J. Geochem. Explor. 45, p. 249-287.

Nordstrom, D.K. (1982): Aqueous pyrite oxidation and the consequent formation of secondary iron minerals. In: Kittrick, J.A. (Hrsg.): Acid sulfate weathering. Soil Sci. Soc. Am. Special Publ. 10, p. 35-37.

Nordstrom, D.K. (1979): Redox equilibria of iron in acid mine waters. In: Jenne, E.A. (Hrsg.): Chemical modeling in aqueous systems. Speciation, sorption, solubility, and kinetics. ACS symposium series 93, Washington, p. 51-79. 
Nordstrom, D.K., Plummer, L.N., Wigley, T.M.L., Wolery, T.J., Ball, J.W., Jenne, E.A., Bassett, R.L., Crerar, D.A., Florence, T.M., Fritz, B., Hoffman, M., Holdren, G.R., Lafon, G.M., Mattigod, S.V., McDuff, R.E:, Morel, F., Reddy, M.M., Sposip, G. and Thrailkill, J. (1979): A comparison of computerized chemical models for equilibrium models for equilibrium calculations in aqueous systems. In: Jenne, E.A. (Hrsg.): Chemical modeling in aqueous systems. Speciation, sorption, solubility, and kinetics. ACS symposium series 93, Washington, p. 857-892.

Packroff, G., Blaschke, W., Herzsprung, P., Meier, J., Schimmele, M. und Wollmann, K. (2000): Limnologie und Gewässerchemie von ausgewählten, geogen schwefelsauren Tagebauseen der Niederlausitz. In: Wiegleb, G., Bröring, U., Mrzljak, J. und Schulz, F. (Hrsg.): Naturschutz in Bergbaufolgelandschaften: Landschaftsanalyse und Leitbildentwicklung. Physica-Verlag, Heidelberg, S. 315-330.

Pavlik, H.F. and Runnells, D.D. (1990): Reconstruction of reaction pathways in a rock-fluid system using MINTEQ. In: Melchior, D.C. and Bassett, R.L. (Hrsg.): Chemical modeling of aqueous systems II, ACS symposium series 416, Washington, p. 140-153.

Perdue, E.M. (1998): Chemical composition, structure, and metal binding properties. In: Hessen, D.O. and Tranvik, L.J. (Hrsg.): Aquatic humic substances: ecology and biochemistry. Springer, Berlin, p. 41-61.

Peiffer, S. (1999): Schwefelsaure Restlochseen des Braunkohletagebaus - ein neuer Seentyp? Ein geochemischer Klassifizierungsversuch. Deutsche Gesellschaft für Limnologie, Tagungsbericht 1998 (Klagenfurt), S. 259-263.

Peiffer, S., Peine, A. and Tritschler, A. (2000): Transformation of schwertmannite to goethite in the sediment of an acidic mining lake - an IR spectroscopical study. In: Applied Mineralogy in Research, Economy, Technology, Ecology and Culture. Proceedings of the sixth ICAM, Balkema, Rotterdamm. p.625-627.

Peiffer, S. (1997): Umweltgeochemische Bedeutung der Bildung und Oxidation von Pyrit in Gewässersedimenten. Bayreuther Forum Ökologie 47, 104 S..

Pleßow, A. (1998): Verfahren zur Bestimmung der Migrationsformen von Spurenelementen in Sickerwässern und Porenlösungen sulfidhaltiger Abraumhalden. Verlag für Wissenschaft und Forschung Berlin. 158 S..

Pleßow, A., Bielert, U., Heinrichs, H. und Steiner, I. (1997): Problematik der Grundwasserversauerung und das Lösungsverhalten von Spurenstoffen. In: Matschullat, J., Tobschalld, H.J. und Voigt, H.-J. (Hrsg.): Geochemie und Umwelt - Relevante Prozesse in Atmo-, Pedo- und Hydrosphäre. Springer, Berlin, 442 S.. 
Pleßow, A. und Heinrichs, H. (2000a): Einfluß der Pyritoxidation auf das Lösungsverhalten von Spurenelementen in Poren-, Sicker- und Grundwässern einer Abraumhalde (Zwenkau/Sachsen). In: Wippermann, T. (Hrsg.): Bergbau und Umwelt - Langfristige geochemische Einflüsse. Geowissenschaften und Umwelt Bd. 6, Springer, Berlin, S. 41-56.

Pleßow, A. and Heinrichs, H. (2000b): Speciation of trace elements in acidic pore waters from waste rock dumps by ultrafiltration and ion exchange combined with ICPMS and ICPOES. Aquatic Geochem. 6, p. 347-366.

Pokrandt, K.-H. und Zeh, E. (1999): Flutungskonzept Südraum Leipzig. UFZ-Bericht 25/99, S. 6-17.

Pohlmann, M., Göttlicher, J., Bozau, E. and Strauch, G. (2000): The fate of sulfur in lignite mining dumps and its role for an in situ-remediation of acidic mining lakes. In: Applied Mineralogy in Research, Economy, Technology, Ecology and Culture. Proceedings of the sixth ICAM, Balkema, Rotterdamm. p.629-630.

Ranville, J.F. and Macalady, D.L. (1997): Natural organic matter in catchments. In: Saether, O.M. and de Caritat, P. (Hrsg.): Geochemical processes, weathering and groundwater recharge in catchments. Balkema, Rotterdam, p. 264-303.

Rea, R.L. and Parks, G.A. (1990): Numerical simulation of coadsorption of ionic surfactants with inorganic ions on quartz. In: Melchior, D.C. and Bassett, R.L. (Hrsg.): Chemical modeling of aqueous systems II, ACS symposium series 416, Washington, p. 260-271.

Reuter, J.H. and Perdue, E.M. (1977): Importance of heavy metal-organic matter interactions in natural waters. Geochim. Cosmochim. Acta 41, p. 325-334.

Rose, S. and Elliott, W.C. (2000): The effects of pH regulation upon the release of sulfate from ferric precipitates formed in acid mine drainage. Appl. Geochem. 15, p. 27-34.

Routh, J. and Ikramuddin, M. (1996): Trace-element geochemistry of Onion Creek near Van Stone lead-zinc mine (Washington, USA) - Chemical analysis and geochemical modeling. Chem. Geol. 133, p. 211-224.

Rumpel, C., Knicker, H., Kögel-Knabner, I. and Hüttl, R.F. (1998); Airborne contamination of forest soils by lignite derived materials: its detection and its impact on the composition of organic matter in soils. Water, Air and Soil Pollution 105, p. 481-492.

Santschi, P.H., Lenhart, J.J. and Honeyman, B.D. (1997): Heterogenous processes affecting trace contaminant distribution in estuaries: The role of natural organic matter. Mar. Chem. 58 , p. 99-125.

Sauer, C. und Lieser, K.H. (1995): Bestimmung und Speziation von etwa 20 Spurenelementen in Rohwässern der Trinkwasseraufbereitung. Vom Wasser 84, S. 1-18. 
Sauer, C. und Lieser, K.H. (1994): Methoden der Speziation und Entwicklung eines erweiterten Speziationsschemas für die Bestimmung von etwa 20 Spurenelementen in Wasser. Vom Wasser 83, S. 23-41.

Sauer, C. (1990): Chemische Form (Speziation) von Metallen in natürlichen Wässern. Dissertation im Fachbereich Chemie der Technischen Hochschule Darmstadt, 186 S.

Saus, T. und Schiffer, H.-W. (1999): Braunkohle in Europa. Rheinbraun informiert. Broschüre, Rheinbraun AG, 27 S.

Schäfer, W. (2000): Implementation of redox reactions in groundwater models. In: Schüring, J., Fischer, W.R. and Duijnisveld, W.H.M. (Hrsg.): Redox: fundamentals, processes, and applications. Springer, Berlin, p. 111-119.

Schöpke, R., Koch, R. und Pietsch, W. (1999): Chemisch bedingte Beschaffenheitsänderungen des Sicker- und Grundwassers. In: Hüttl, R.F. (Hrsg.): Rekultivierung von Bergbaufolgelandschaften: das Beispiel des Lausitzer Braunkohlereviers. De Gruyter, Berlin, S. 185-195.

Schreck, P. and Gläßer, W. (1998): Regional geology of the lignite mining districts in Eastern Germany. In: Geller, W., Klapper, H. and Salomons, W. (Hrsg.): Acidic mining lakes: acid mine drainage, limnology, and reclamation. Springer, Berlin, p. 13-21.

Schulz, F. und Wiegleb, G. (2000): Die Niederlausitzer Bergbaufolgelandschaft - Probleme und Chancen. In: Wiegleb, G., Bröring, U., Mrzljak, J. und Schulz, F. (Hrsg.): Naturschutz in Bergbaufolgelandschaften: Landschaftsanalyse und Leitbildentwicklung. PhysicaVerlag, Heidelberg, S. 3-23.

Schulz, H.D. (1992): Hydrogeochemical and geochemical-hydraulic models and model concepts. In: Matthess, G., Frimmel, F., Hirsch, P., Schulz, H.D. and Usdowski, H.E. (Hrsg.): Progress in hydrogeochemistry. Springer, Berlin. p. 417-483.

Schwedt, G. (1997): Elementspeziesanalytik. Chemie in unserer Zeit 4, S. 183-187.

Schwedt, G. (1992): Taschenatlas der Analytik. Thieme, Stuttgart, 233 S.

Schwedt, G. (1981): Methoden zur Bestimmung von Element-Spezies in natürlichen Wässern. In: Bock, R., Fresenius, W., Günzler, H. Huber, W. und Tölg, G. (Hrsg.): Analytiker Taschenbuch Bd 2, Springer, Berlin, S. 255-266.

Schwertmann, U. und Fojt, B. (1996): Schwertmannit - ein neues Mineral und seine Geschichte. Lapis 3, S. 33-34.

Schwertmann, U., Bigham, J.M. and Murad, E. (1995): The first occurence of schwertmannite in a natural stream environment. Eur. J. Min. 7, p. 547-552. 
Sholkovitz, E.R. (1985): Redox-related geochemistry in lakes: alkali metals, alkaline-earth elements, and ${ }^{137}$ Cs. In: Stumm, W. (Hrsg.): Chemical processes in lakes. Wiley, New York, p.119-142.

Shum, M. and Lavkulich, L. (1999): Speciation and solubility relationships of $\mathrm{Al}, \mathrm{Cu}$ and $\mathrm{Fe}$ in solutions associated with sulfuric acid leached mine waste rock. Environm. Geology 38 (1), p. 59-68.

Sigg, L. (2000): Redox potential measurements in natural waters: Significance, concepts and problems. In: Schüring, J., Fischer, W.R. and Duijnisveld, W.H.M. (Hrsg.): Redox: fundamentals, processes, and applications. Springer, Berlin, p. 1-12.

Sigg, L., Sturm, M. and Kistler, D. (1987): Vertical transport of heavy metals by settling particles in Lake Zurich. Limnol. Oceanogr. 32 (1), p. 112-130.

Sigg, L. (1987): Surface chemical aspects of the distribution and fate of metal ions in lakes. In: Stumm, W. (Hrsg.): Aquatic surface chemistry. Wiley, p. 319-350.

Simms, P-H., Yanful, E.K., St-Arnaud, L. and Aubé, B. (2000): A laboratory evaluation of metal release and transport in flooded pre-oxidized mine tailings. Appl. Geochem. 15, p. 1245-1263.

Simpson, S.L., Apte, S.C., Hortle, K.G. and Richards, D.G. (1998): An evaluation of copper remobilization from mine tailings in sulfidic environments. J. of Geochem. Explor. 63, p. 203-215.

Singer, P.C. and Stumm, W. (1970): Acidic mine drainage: The rate-determining step. Science 167, p. 1121-1123.

Singh, J., Huang, P.M., Hammer, U.T. and Liaw, W.K. (1996): Influence of citric acid and glycine on the adsorption of mercury (II) by kaolinite under various $\mathrm{pH}$-conditions. Clays and Clay Minerals 44 (1), p. 41-48.

Skoog, D.A. und Leary, J.J. (1996): Instrumentelle Analytik - Grundlagen, Geräte, Anwendungen. Springer, Berlin, $898 \mathrm{~S}$.

Smith, S.L. and Jaffé, P.R. (1998): Modeling the transport and reaction of trace metals in water-saturated soils and sediments. Water Resources Res. 34 (11), p. 3135-3147.

Stabel, H.-H. (1985): Mechanims controlling the sedimentation sequence of various elements in prealpine lakes. In: Stumm, W. (Hrsg.): Chemical processes in lakes. Wiley, New York, p.143-167.

Stone, A.T. (1997): Reactions of extracellular organic ligands with dissolved metal ions and mineral surfaces. Reviews in Mineralogy 35, p. 310-344. 
Strömberg, B. and Banwart, S. (1994): Kinetic modeling of geochemical processes at the Aitik mining waste rock site in northern Sweden. Appl. Geochem. 9, p. 583-595.

Stumm, W. (1996): Aquatic chemistry: chemical equilibria and rates in natural waters. Wiley, New York, 455-465.

Stumm, W. (1992): Chemistry of the solid-water interface: processes at the mineral-water and particle-water interface in natural systems. Wiley, New York, 428 p.

Sunda, W.G. and Hanson, P.J. (1979): Chemical speciation of copper in river water. In: Chemical modeling in aqueous systems. Speciation, sorption, solubility, and kinetics. ACS symposium series 93, American Chemical Society, Washington, p. 148-180.

Susetyo, W., Carreira, L.A., Azarraga, L.V. and Grimm, D.M. (1991): Fluroescence techniques for metal-humic interactions. Fresenius J. Anal. Chem. 339, p. 624-635.

Taillefert, M., Lienemann, C.-P., Gaillard, J.-F. and Perret, D. (2000): Speciation, reactivity, and cycling of $\mathrm{Fe}$ and $\mathrm{Pb}$ in a meromitic lake. Geochim. Cosmochim. Acta 64 (2), p. 169183.

Takahashi, Y., Minai, Y., Ambe, S., Maikde, Y. and Ambe, F. (1999): Comparison of adsorption behavior of multiple inorganic ions on kaolinite and silica in the presence of humic acid using the multitracer technique. Geochim. Cosmochim. Acta 63 (6), p. 815 836.

Tessier, A., Carignan, R., Dubreuil, B. and Rapin, F. (1989): Partitioning of zinc between the water column and the oxic sediments in lakes. Geochim. Cosmochim. Acta 53, p. 15111522 .

Tessier, A., Fortin, D. , Belzile, N., DeVitre, R.R. and Leppard, G.G. (1996): Metal sorption to diagenetic iron and manganese oxyhydroxides and associated organic matter: Narrowing the gap between field and laboratory measurements. Geochim. Cosmochim. Acta 60 (3), p. 387-404.

Tessier, A., Rapin, F. and Carignan, R. (1985): Trace metal in oxic lake sediments: possible adsorption onto iron oxyhydroxides. Geochim. Cosmochim. Acta 49, p. 183-194.

Thurman, E.M. (1985): Organic geochemistry of natural waters. Nijhoff/Junk Publishers Dordrecht, Boston 1985. 497 p.

Town, R.M. and Powell, H.K.J. (1993): Ion-selective electrode potentiometric studies on the complexation of copper(II) by soil-derived humic and fulvic acids. Anal. Chim. Acta 279, p. 221-233.

Trettin, R. und Gläser, H.R. (1995): Hydrochemische Entwicklung bei der Flutung des Tagebaurestloches Cospuden. UFZ-Bericht 4/95, S. 103-114. 
Turner, D.R., Griffin, T. and Dietrich, T.B. (1993) Radionucleide sorption modeling using the MINTEQA2 speciation code. Mat. res. soc. symp. proc. 294, p. 783-789

Turner, D.R., Whitfield, M. and Dickson, A.G. (1981): The equilibrium speciation of dissolved components in freshwater and seawater at $25^{\circ} \mathrm{C}$ and 1 atm pressure. Geochim. Cosmochim. Acta 45, p. 855-881.

Van Breemen, N., Driscoll, C.T. and Mulder, J. (1984): Acidic deposition and internal proton sources in acidification of soils and waters. Nature 307, p. 599-604.

Van den Berg, C.M.G. and Kramer, J.R. (1979): Conditional stability constants for copper ions with ligands in natural waters. In: Chemical modeling in aqueous systems. Speciation, sorption, solubility, and kinetics. ACS symposium series 93, American Chemical Society, Washington, p.115-132.

Van Hees, P.A.W. and Lundström, U.S. (2000): Equilibrium models of aluminium and iron complexation with different organic acids in soil solution. Geoderma 94, p. 201-221.

Van Luik, A.E. and Jurinak, J.J. (1979): Equilibrium chemistry of heavy metals in concentrated electrolyte solution. In: Chemical modeling in aqueous systems. Speciation, sorption, solubility, and kinetics. ACS symposium series 93, American Chemical Society, Washington, p. 653-710.

Vogl, J. and Heumann, K.G. (1997): Determination of heavy metal complexes with humic substances by HPLC/ICP-MS coupling using on-line isotope dilution technique. Fresenius J. anal. Chem. 359, p. 438-441.

Webster, J.G., Nordstrom, D.K. and Smith, K.S. (1994): Transport and natural attenuation of $\mathrm{Cu}, \mathrm{Zn}, \mathrm{As}$, and $\mathrm{Fe}$ in the acid mine drainage of Leviathan and Bryant Creeks. In: Alpers, C.N. and Blowes, D.W. (Hrsg): Environmental geochemistry of sulfide oxidation. ACS symposium series 550, American Chemical Society, Washington, p. 244-260.

Wehrli, B. (1990): Redox reactions of metal ions at mineral surfaces. In: Stumm, W. (Hrsg.): Aquatic chemical kinetics: reaction rates of processes in natural waters. Wiley, New York, p. 311-336.

Wells, M.L., Kozelka, P.B. and Bruland, K.W. (1998): The complexation of "dissolved" Cu, $\mathrm{Zn}, \mathrm{Cd}$ and $\mathrm{Pb}$ by soluble and colloidal organic matter in Narragansett Bay, RI. Marine Chemistry 62, p. 203-217.

Wernery-Seidel, U. (1958): Zur Sedimentgeographie der Neurather Sande im Bereich der Tagebaue Frimmersdorf-Süd und Neurath. Fortschr. Geol. Rheinld. u. Westfl. 1, Krefeld. 
Wiegand, U. und Krüger, G. (1999): Geochemische und mineralogische Charakterisierung von Kippensedimenten des Tagebaus Zwenkau - zeitabhängige Betrachtungen. UFZBericht 26/99, S. 117-126.

Wilkinson, G. (1997): Comprehensive coordination chemistry Vol. 2, Pergamon, New York, p. $740-750$.

Williamson, M. and Rimstidt, J.D. (1994): The kinetics and electromchemical ratedetermining step of aqueous pyrite oxidation. Geochim. Cosmochim. Acta 58, p. 54435454.

Wilsdorf, H.(1987): Kulturgeschichte des Bergbaus. ein illustrierter Streifzug durch Zeiten und Kontinente. Verlag Glückauf GmbH Essen, S. 281f., $303 f$.

Wisotzky, F. (2000): Redox reactions, multi-component stability diagrams and isotopic investigations in sulfur- and iron-dominated groundwater systems. In: Schüring, J., Fischer, W.R. and Duijnisveld, W.H.M. (Hrsg.): Redox: fundamentals, processes, and applications. Springer, Berlin, p. 175-188.

Wisotzky, F. (1998): Chemical reactions in aquifers influenced by sulfide oxidation and in sulfide oxidation zones. In: Geller, W., Klapper, H. and Salomons, W. (Hrsg.): Acidic mining lakes: acid mine drainage, limnology, and reclamation. Springer, Berlin, p. 223233.

Wisotzky, F. (1996): Hydrogeochemische Reaktionen im Sicker- und Grundwasserbereich von Barunkohletagebaukippen. Grundwasser 3-4, S. 129-136.

Wisotzky, F. (1994): Untersuchungen zur Pyritoxidation in Sedimenten des Rheinischen Braunkohlenreviers und deren Auswirkungen auf die Chemie des Grundwassers. Besondere Mitteilungen zum Deutschen Gewässerkundlichen Jahrbuch 58, Landesumweltamt Nordrhein-Westfalen, Essen, 153 S.. 


\section{Anhang}

\subsection{Abkürzungen}

AAS Atomabsorptionsspektrometrie

AMD sauere Minenwässer (Acid Mine Drainage)

AP Daten aus der Arbeit von PleßOW (1998)

CML Cospuden Multi-Level-Brunnen

DOC gelöster organisch gebundender Kohlenstoff (Dissolved Organic Carbon)

el. Ltf. elektrische Leitfähigkeit

GC Gaschromatographie

ICP-MS Massenspektrometrie mit induktiv gekoppeltem Plasma (Inductively Coupled Plasma Mass Spectrometry)

ICP-OES Atomemissionsspektrometrie mit induktiv gekoppeltem Plasma (Inductively Coupled Plasma Optical Emission Spectrometry)

IR Infrarot

KB Daten aus der vorliegenden Arbeit

LMBV Lausitzer und Mitteldeutsche Bergbau-Verwaltungsgesellschaft ( $\mathrm{mbH})$

$\mathrm{nb}$ nicht bestimmt

NMR Kernresonanzspektrometrie (Nuclear Resonance Spectrometry)

nq nicht quantifizierbar

PE Polyethylen

PL Porenlösung

PTFE Polytetrafluorethylen

RCO Grundwassermessentnahmestelle

REE Selten-Erd-Elemente (Rare Earth Elements)

RFA Röntgenfluoreszenzanalyse

SGM Sicker- und Grundwasser-Messeinrichtung

UFZ Umweltforschungszentrum Halle-Leipzig GmbH

WP Wasserprobe 


\subsection{Tabellenanhang}

Tabelle A.1.: Analysendaten der Porenwässer und Wasserproben aus Cospuden.

\begin{tabular}{|c|c|c|c|c|c|c|c|c|c|}
\hline & & RCO4 17 m & RCO4 20 m & RCO4 22 m & $\mathrm{RCO426} \mathrm{m}$ & RCO436 m & RCO4 40 m & RCO4 44 m & RCO4 48 m \\
\hline & & Porenlösung & Porenlösung & Porenlösung & Porenlösung & Porenlösung & Porenlösung & Porenlösung & Porenlösung \\
\hline & & Nov 1995 & \begin{tabular}{|l|} 
Nov 1995 \\
\end{tabular} & \begin{tabular}{|l|l|} 
Nov 1995 \\
\end{tabular} & Nov 1995 & \begin{tabular}{|l|} 
Nov 1995 \\
\end{tabular} & \begin{tabular}{|l|} 
Nov 1995 \\
\end{tabular} & \begin{tabular}{|l|} 
Nov 1995 \\
\end{tabular} & \begin{tabular}{|l|} 
Nov 1995 \\
\end{tabular} \\
\hline pH & & 5,9 & 6,6 & 6,1 & 3,2 & 6,7 & 6,7 & 6,5 & 5,9 \\
\hline el Lf & {$[\mathrm{mS} / \mathrm{cm}]$} & 2,3 & 2,2 & 1,7 & 7,1 & 2,6 & 2,1 & 2,2 & 2,0 \\
\hline Redox & {$[\mathrm{mV}]$} & 280 & 250 & 270 & 440 & 240 & 250 & 250 & 280 \\
\hline Sulfat & {$[\mathrm{g} / \mathrm{l}]$} & 2,4 & 1,6 & 1,3 & 7,0 & 2,6 & 2,0 & 2,2 & 2,4 \\
\hline DOC & {$[\mathrm{mg} / \mathrm{l}]$} & 18,9 & 25,0 & 24,9 & 54,4 & 22,8 & 30,8 & 51,2 & 21,4 \\
\hline $\mathbf{L i}$ & {$[\mu \mathrm{g} / \mathrm{l}]$} & 170 & 110 & 82 & 1200 & 460 & 71 & 29 & 240 \\
\hline $\mathrm{Be}$ & {$[\mu \mathrm{g} / \mathrm{l}]$} & $<0,1$ & $<0,1$ & $<0,1$ & 29 & $<0,1$ & $<0,1$ & $<0,1$ & $<0,1$ \\
\hline $\mathrm{Na}$ & {$[\mu \mathrm{g} / \mathrm{l}]$} & 87000 & 85000 & 85000 & 47000 & 150000 & 99000 & 110000 & 120000 \\
\hline Mg & {$[\mu \mathrm{g} / \mathrm{l}]$} & 180000 & 130000 & 110000 & 1100000 & 380000 & 210000 & 2100000 & 250000 \\
\hline Al & {$[\mu \mathrm{g} / \mathrm{l}]$} & 6,5 & $k 1$ & 46 & 260000 & $k 1$ & 420 & $<1$ & < 1 \\
\hline $\mathbf{K}$ & {$[\mu \mathrm{g} / \mathrm{l}]$} & 29000 & 21000 & 15000 & 9600 & 39000 & 26000 & 20000 & 35000 \\
\hline $\mathbf{C a}$ & {$[\mu \mathrm{g} / \mathrm{l}]$} & 780000 & 550000 & 410000 & 530000 & 680000 & 610000 & 700000 & 710000 \\
\hline Sc & {$[\mu \mathrm{g} / \mathrm{l}]$} & $<0,1$ & $k 0,1$ & 2,9 & 7,3 & 4,1 & 2,7 & 4,8 & 5,4 \\
\hline $\mathbf{T i}$ & {$[\mu \mathrm{g} / \mathrm{l}]$} & $<1$ & $k 1$ & $k 1$ & 27 & < 1 & 32 & $<1$ & $<1$ \\
\hline $\mathbf{V}$ & {$[\mu \mathrm{g} / \mathrm{l}]$} & 3,2 & $<0,1$ & 6,5 & 240 & 1,7 & 8,2 & $<0,1$ & 4,2 \\
\hline $\mathrm{Cr}$ & {$[\mu \mathrm{g} / \mathrm{l}]$} & 1,8 & 3,1 & 3,9 & 49 & 1,7 & 5,7 & 1,6 & 1,9 \\
\hline Mn & {$[\mu \mathrm{g} / \mathrm{I}]$} & 3700 & 2000 & 1100 & 48000 & 3000 & 6000 & 9300 & 3300 \\
\hline $\mathrm{Fe}$ & {$[\mu \mathrm{g} / \mathrm{I}]$} & 44 & 19 & 26 & 876200 & 26 & 370 & 33 & 21270 \\
\hline Co & {$[\mu \mathrm{g} / \mathrm{l}]$} & 26 & 4,5 & $k 0,1$ & 1300 & 3,8 & 9,1 & 17 & 4,8 \\
\hline $\mathbf{N i}$ & {$[\mu \mathrm{g} / \mathrm{I}]$} & 120 & 120 & 44 & 3500 & 65 & 47 & 140 & 120 \\
\hline $\mathbf{C u}$ & {$[\mu \mathrm{g} / \mathrm{l}]$} & 3,6 & 4,3 & 9,6 & 130 & 8,2 & 9,3 & 5,0 & 15 \\
\hline Zn & {$[\mu \mathrm{g} / \mathrm{l}]$} & 47 & 16 & 15 & 12000 & 17 & 52 & 21 & 190 \\
\hline As & {$[\mu \mathrm{g} / \mathrm{l}]$} & 13 & 5,0 & 4,4 & 140 & 4,5 & 2,9 & 6,8 & 1,5 \\
\hline $\mathbf{R b}$ & {$[\mu \mathrm{g} / \mathrm{l}]$} & 50 & 17 & 13 & 49 & 37 & 11 & 12 & 33 \\
\hline $\mathbf{S r}$ & {$[\mu \mathrm{g} / \mathrm{l}]$} & 7200 & 5200 & 3800 & 5800 & 10000 & 3800 & 3700 & 4900 \\
\hline $\mathbf{Y}$ & {$[\mu \mathrm{g} / \mathrm{l}]$} & 0,41 & 0,14 & 0,12 & 700 & 0,21 & 0,47 & 0,27 & 0,27 \\
\hline $\mathbf{Z r}$ & {$[\mu \mathrm{g} / \mathrm{l}]$} & 2,2 & 0,15 & 2,5 & 56 & 2,7 & 2,7 & 2,3 & 3,7 \\
\hline $\mathbf{N b}$ & {$[\mu \mathrm{g} / \mathrm{l}]$} & 0,96 & 0,35 & 0,16 & 0,73 & 0,29 & 0,32 & 0,45 & $<0,1$ \\
\hline Mo & {$[\mu \mathrm{g} / \mathrm{ll}]$} & 22 & 20 & 17 & 0,71 & 67 & 20 & 28 & 1,4 \\
\hline $\mathbf{C d}$ & {$[\mu \mathrm{g} / \mathrm{ll}]$} & 0,27 & 0,18 & 0,22 & 41 & 0,20 & 0,21 & 0,48 & 0,21 \\
\hline Sn & {$[\mu \mathrm{g} / \mathrm{l}]$} & $<0,1$ & $<0,1$ & 0,11 & 0,83 & 0,18 & 0,40 & 1,0 & $<0,1$ \\
\hline $\mathbf{S b}$ & {$[\mu \mathrm{g} / \mathrm{l}]$} & 2,0 & 2,6 & 7,3 & 0,72 & 0,47 & 1,8 & 2,7 & $<0,1$ \\
\hline Cs & {$[\mu \mathrm{g} / \mathrm{l}]$} & 0,34 & 0,27 & 0,21 & 0,69 & 0,32 & 0,13 & 0,24 & 0,25 \\
\hline Ba & {$[\mu \mathrm{g} / \mathrm{l}]$} & 92 & 92 & 74 & 21 & 25 & 53 & 71 & 29 \\
\hline La & {$[\mu \mathrm{g} / \mathrm{l}]$} & 0,46 & 0,18 & 0,13 & 980 & 0,16 & 0,53 & 0,21 & 0,28 \\
\hline Ce & {$[\mu \mathrm{g} / \mathrm{l}]$} & 0,55 & 0,22 & 0,19 & 2200 & 0,23 & 1,0 & 0,23 & 0,31 \\
\hline $\mathbf{P r}$ & {$[\mu \mathrm{g} / \mathrm{l}]$} & $<0,1$ & $<0,1$ & $<0,1$ & 230 & $<0,1$ & 0,13 & $<0,1$ & $<0,1$ \\
\hline Nd & {$[\mu \mathrm{g} / \mathrm{l}]$} & 0,29 & 0,10 & $<0,1$ & 850 & 0,12 & 0,55 & 0,15 & 0,15 \\
\hline $\mathbf{S m}$ & {$[\mu \mathrm{g} / \mathrm{I}]$} & $<0,1$ & $<0,1$ & $<0,1$ & 130 & $<0,1$ & 0,11 & $<0,1$ & $<0,1$ \\
\hline Eu & {$[\mu \mathrm{g} / \mathrm{l}]$} & $<0,1$ & $<0,1$ & $<0,1$ & 35 & $<0,1$ & $<0,1$ & $<0,1$ & $<0,1$ \\
\hline$\overline{\text { Gd }}$ & {$[\mu \mathrm{g} / \mathrm{l}]$} & $<0,1$ & $<0,1$ & $<0,1$ & 120 & $<0,1$ & $<0,1$ & $<0,1$ & $<0,1$ \\
\hline Tb & {$[\mu \mathrm{g} / \mathrm{l}]$} & $<0,1$ & $<0,1$ & $<0,1$ & 22 & $<0,1$ & $<0,1$ & $<0,1$ & $<0,1$ \\
\hline Dy & {$[\mu \mathrm{g} / \mathrm{I}]$} & $<0,1$ & $<0,1$ & $<0,1$ & 97 & $<0,1$ & $<0,1$ & $<0,1$ & $<0,1$ \\
\hline Ho & {$[\mu \mathrm{g} / \mathrm{l}]$} & $<0,1$ & $<0,1$ & $<0,1$ & 22 & $<0,1$ & $<0,1$ & $<0,1$ & $<0,1$ \\
\hline $\mathbf{E r}$ & {$[\mu \mathrm{g} / \mathrm{I}]$} & $<0,1$ & $<0,1$ & $<0,1$ & 46 & $\mid<0,1$ & $<0,1$ & $<0,1$ & $<0,1$ \\
\hline Tm & {$[\mu \mathrm{g} / \mathrm{l}]$} & $<0,1$ & $<0,1$ & $<0,1$ & 8,0 & $<0,1$ & $<0,1$ & $<0,1$ & $<0,1$ \\
\hline $\mathbf{Y b}$ & {$[\mu \mathrm{g} / \mathrm{l}]$} & $<0,1$ & $<0,1$ & $<0,1$ & 38 & $<0,1$ & $<0,1$ & $<0,1$ & $<0,1$ \\
\hline Lu & {$[\mu \mathrm{g} / \mathrm{l}]$} & $<0,1$ & $<0,1$ & $<0,1$ & 6,2 & $<0,1$ & $<0,1$ & $<0,1$ & $<0,1$ \\
\hline Hf & {$[\mu \mathrm{g} / \mathrm{I}]$} & $<0,1$ & $<0,1$ & $<0,1$ & 0,43 & $<0,1$ & $<0,1$ & $<0,1$ & $<0,1$ \\
\hline Ta & {$[\mu \mathrm{g} / \mathrm{I}]$} & $<0,1$ & $<0,1$ & $<0,1$ & 3,2 & $<0,1$ & $<0,1$ & 0,47 & $<0,1$ \\
\hline W & {$[\mu \mathrm{g} / \mathrm{I}]$} & 1,1 & 0,73 & 0,48 & 0,52 & 0,16 & 0,19 & 0,33 & 0,10 \\
\hline $\mathrm{Hg}$ & {$[\mu \mathrm{g} / \mathrm{l}]$} & nb & nb & $n b$ & nb & nb & $n b$ & nb & nb \\
\hline $\mathbf{T l}$ & {$[\mu \mathrm{g} / \mathrm{l}]$} & 0,36 & 0,32 & 0,20 & 1,0 & 0,16 & 0,19 & 0,23 & 0,14 \\
\hline $\mathbf{P b}$ & {$[\mu \mathrm{g} / \mathrm{l}]$} & 0,33 & 0,44 & 1,2 & 5,6 & 0,25 & 1,3 & 0,54 & 0,23 \\
\hline$\overline{B i}$ & {$[\mu \mathrm{g} / \mathrm{l}]$} & nb & nb & $\mathrm{nb}$ & nb & $n b$ & $n b$ & nb & $n b$ \\
\hline Th & {$[\mu \mathrm{g} / \mathrm{l}]$} & $<0,1$ & $<0,1$ & $<0,1$ & 2,8 & $<0,1$ & $<0,1$ & $<0,1$ & $<0,1$ \\
\hline $\mathbf{U}$ & {$[\mu \mathrm{g} / \mathrm{l}]$} & $<0,1$ & $<0,1$ & 0,33 & 28 & 3,7 & 11 & 17 & $<0,1$ \\
\hline
\end{tabular}


Tabelle A.1. (Forts.): Analysendaten der Porenwässer und Wasserproben aus Cospuden.

\begin{tabular}{|c|c|c|c|c|c|c|c|c|c|}
\hline & & RCO4 52 m & CML 21-22 m & CML $24 \mathrm{~m}$ & CML $26 \mathrm{~m}$ & CML $32 \mathrm{~m}$ & CML 36 m & CML $37.5 \mathrm{~m}$ & CML 39-40 m \\
\hline & & Porenlösung & Porenlösung & Porenlösung & Porenlösung & Porenlösung & Porenlösung & Porenlösung & Porenlösung \\
\hline & & Nov 1995 & \begin{tabular}{|l|} 
Aug 1998 \\
\end{tabular} & Aug 1998 & Aug 1998 & Aug 1998 & Aug 1998 & Aug 1998 & Aug 1998 \\
\hline $\mathbf{p H}$ & & 7,1 & 6,7 & 3,9 & 2,9 & 7,1 & 7,0 & 6,7 & 6,2 \\
\hline el Lf & {$[\mathrm{mS} / \mathrm{cm}]$} & 2,3 & 2,3 & 4,3 & 8,3 & 4,6 & 3,4 & 3,8 & 2,2 \\
\hline $\operatorname{Redox}$ & {$[\mathrm{mV}]$} & 220 & 240 & 390 & 470 & 293 & 268 & 293 & 261 \\
\hline Sulfat & {$[\mathrm{g} / \mathrm{l}]$} & 2,2 & 1,6 & $\mathrm{nb}$ & nb & $n b$ & $\mathrm{nb}$ & $\mathrm{nb}$ & 2,4 \\
\hline DOC & {$[\mathrm{mg} / \mathrm{l}]$} & 38,5 & 20,6 & 20,3 & $n b$ & 38,9 & 34,0 & 17,5 & 21,7 \\
\hline $\mathbf{L i}$ & {$[\mu \mathrm{g} / \mathrm{l}]$} & 57 & 86 & 670 & 1200 & 300 & 53 & 210 & 220 \\
\hline $\mathbf{B e}$ & {$[\mu \mathrm{g} / \mathrm{ll}]$} & $<0,1$ & $<0,1$ & 11 & 45 & 3,0 & 3,0 & 3,0 & 0,21 \\
\hline $\mathbf{N a}$ & {$[\mu \mathrm{g} / \mathrm{l}]$} & 110000 & 77000 & 49000 & 71000 & 120000 & 92000 & 88000 & 98000 \\
\hline $\mathrm{Mg}$ & {$[\mu \mathrm{g} / \mathrm{l}]$} & 210000 & 140000 & 370000 & 810000 & 730000 & 270000 & 300000 & 280000 \\
\hline Al & {$[\mu \mathrm{g} / \mathrm{l}]$} & $<1$ & $k 1$ & 29000 & 130000 & 710 & 270 & 1300 & 9,2 \\
\hline $\mathbf{K}$ & {$[\mu \mathrm{g} / \mathrm{I}]$} & 26000 & 17000 & 25000 & 66000 & 65000 & 36000 & 72000 & 34000 \\
\hline Ca & {$[\mu \mathrm{g} / \mathrm{l}]$} & 640000 & 600000 & 580000 & 220000 & 650000 & 740000 & 630000 & 660000 \\
\hline Sc & {$[\mu \mathrm{g} / \mathrm{l}]$} & $<0,1$ & 4,6 & 7,0 & 8,0 & 7,0 & 7,0 & 7,0 & $<0,1$ \\
\hline $\mathbf{T i}$ & {$[\mu \mathrm{g} / \mathrm{l}]$} & $<1$ & $<1$ & 76 & 14 & 19 & 10 & 48 & $<1$ \\
\hline $\mathbf{V}$ & {$[\mu \mathrm{g} / \mathrm{I}]$} & $<0,1$ & $<0,1$ & 66 & 21 & 10 & 13 & 16 & $<0,1$ \\
\hline $\mathrm{Cr}$ & {$[\mu \mathrm{g} / \mathrm{l}]$} & 1,7 & 4,2 & 45 & 15 & 50 & 40 & 48 & 2,8 \\
\hline Mn & {$[\mu \mathrm{g} / \mathrm{I}]$} & 6600 & 1900 & 17000 & nb & 8400 & 13000 & 11000 & 8100 \\
\hline $\mathbf{F e}$ & {$[\mu \mathrm{g} / \mathrm{l}]$} & 51 & 23 & 533985 & 417210 & 1443 & 183 & 29181 & 29 \\
\hline Co & {$[\mu \mathrm{g} / \mathrm{l}]$} & 29 & 5,0 & 210 & 1900 & 46 & 37 & 19 & 29 \\
\hline $\mathrm{Ni}$ & {$[\mu \mathrm{g} / \mathrm{l}]$} & 230 & 19 & 590 & 4800 & 190 & 170 & 40 & 93 \\
\hline $\mathbf{C u}$ & {$[\mu \mathrm{g} / \mathrm{I}]$} & 6,6 & 6,9 & 34 & 9,0 & 41 & 21 & 24 & 4,8 \\
\hline $\mathbf{Z n}$ & {$[\mu \mathrm{g} / \mathrm{l}]$} & 34 & 26 & 1700 & 11000 & 150 & 120 & 190 & 31 \\
\hline As & {$[\mu \mathrm{g} / \mathrm{I}]$} & 3,6 & 7,1 & 75 & 10 & 8,0 & 2,0 & 25 & $\begin{array}{l}<0,1 \\
\end{array}$ \\
\hline $\mathbf{R b}$ & {$[\mu \mathrm{g} / \mathrm{l}]$} & 10 & 17 & $\mathrm{nb}$ & $n b$ & $n b$ & $n b$ & 22 & 34 \\
\hline $\mathrm{Sr}$ & {$[\mu \mathrm{g} / \mathrm{ll}]$} & 8600 & 6500 & 5300 & 11000 & 7700 & 4800 & 4200 & 5600 \\
\hline $\mathbf{Y}$ & {$[\mu \mathrm{g} / \mathrm{l}]$} & 0,28 & 0,17 & 48 & 2700 & 7,0 & 6,0 & 2,0 & $\begin{array}{l}<0,1 \\
\end{array}$ \\
\hline $\mathbf{Z r}$ & {$[\mu \mathrm{g} / \mathrm{l}]$} & 0,28 & 2,3 & 49 & 9,4 & 7,0 & 7,0 & 2,0 & 2,3 \\
\hline $\mathbf{N b}$ & {$[\mu \mathrm{g} / \mathrm{l}]$} & 0,54 & 0,23 & $n b$ & $n b$ & nb & $\mathrm{nb}$ & 1,1 & 0,63 \\
\hline Mo & {$[\mu \mathrm{g} / \mathrm{l}]$} & 45 & 7,0 & $<0,1$ & 8,6 & 37 & 73 & 30 & 55 \\
\hline Cd & {$[\mu \mathrm{g} / \mathrm{l}]$} & 0,44 & 0,15 & $\mathrm{nb}$ & nb & $n b$ & $\mathrm{nb}$ & nb & 0,42 \\
\hline Sn & {$[\mu \mathrm{g} / \mathrm{l}]$} & $<0,1$ & 0,12 & $\mathrm{nb}$ & nb & $\mathrm{nb}$ & $\mathrm{nb}$ & 0,60 & 0,20 \\
\hline $\mathbf{S b}$ & {$[\mu \mathrm{g} / \mathrm{l}]$} & 3,2 & 0,81 & $\mathrm{nb}$ & nb & $\mathrm{nb}$ & $\mathrm{nb}$ & 0,40 & 0,55 \\
\hline Cs & {$[\mu \mathrm{g} / \mathrm{l}]$} & $<0,1$ & 0,13 & $\mathrm{nb}$ & nb & $\mathrm{nb}$ & $\mathrm{nb}$ & 0,60 & 0,31 \\
\hline Ba & {$[\mu \mathrm{g} / \mathrm{l}]$} & 31 & 74 & 73 & 14 & 62 & 68 & 130 & 27 \\
\hline La & {$[\mu \mathrm{g} / \mathrm{l}]$} & 0,15 & 0,12 & 85 & 2200 & 2,0 & \begin{tabular}{|l|}
1,0 \\
\end{tabular} & 5,0 & 1,9 \\
\hline $\mathrm{Ce}$ & {$[\mu \mathrm{g} / \mathrm{I}]$} & 0,18 & 0,18 & $n b$ & nb & $n b$ & nb & 11 & 2,3 \\
\hline Pr & {$[\mu \mathrm{g} / \mathrm{I}]$} & $<0,1$ & $k 0,1$ & $\mathrm{nb}$ & nb & nb & $\mathrm{nb}$ & 1,2 & 0,21 \\
\hline Nd & {$[\mu \mathrm{g} / \mathrm{l}]$} & 0,10 & $<0,1$ & $\mathrm{nb}$ & nb & $n b$ & nb & 4,8 & 0,78 \\
\hline Sm & {$[\mu \mathrm{g} / \mathrm{I}]$} & $<0,1$ & $<0,1$ & $n b$ & nb & $n b$ & nb & 0,93 & 0,14 \\
\hline $\mathbf{E u}$ & {$[\mu \mathrm{g} / \mathrm{I}]$} & $\mid<0,1$ & $<0,1$ & $n \mathrm{nb}$ & nb & $n b$ & $n \mathrm{nb}$ & 0,26 & $<0,1$ \\
\hline Gd & {$[\mu \mathrm{g} / \mathrm{I}]$} & $\mid<0,1$ & $<0,1$ & $n b$ & nb & $n b$ & $n b$ & 0,76 & 0,13 \\
\hline Tb & {$[\mu \mathrm{g} / \mathrm{l}]$} & $<0,1$ & $<0,1$ & $n b$ & nb & $n b$ & $n b$ & 0,13 & $<0,1$ \\
\hline Dy & {$[\mu \mathrm{g} / \mathrm{I}]$} & $\mid<0,1$ & $\mid<0,1$ & $n b$ & $n b$ & $n b$ & $n b$ & 0,66 & 0,13 \\
\hline Ho & {$[\mu \mathrm{g} / \mathrm{l}]$} & $<0,1$ & $<0,1$ & $\mathrm{nb}$ & nb & nb & $\mathrm{nb}$ & 0,13 & $<0,1$ \\
\hline $\mathbf{E r}$ & {$[\mu \mathrm{g} / \mathrm{I}]$} & $<0,1$ & $<0,1$ & $\mathrm{nb}$ & nb & nb & $\mathrm{nb}$ & 0,33 & $<0,1$ \\
\hline Tm & {$[\mu \mathrm{g} / \mathrm{I}]$} & $<0,1$ & $<0,1$ & $\mathrm{nb}$ & nb & nb & $\mathrm{nb}$ & $<0,1$ & $<0,1$ \\
\hline $\mathbf{Y b}$ & {$[\mu \mathrm{g} / \mathrm{I}]$} & $<0,1$ & $<0,1$ & $\mathrm{nb}$ & nb & nb & $\mathrm{nb}$ & 0,25 & $<0,1$ \\
\hline Lu & {$[\mu \mathrm{g} / \mathrm{I}]$} & $<0,1$ & $<0,1$ & $\mathrm{nb}$ & nb & nb & $\mathrm{nb}$ & $<0,1$ & $<0,1$ \\
\hline Hf & {$[\mu \mathrm{g} / \mathrm{l}]$} & $<0,1$ & $<0,1$ & $\mathrm{nb}$ & nb & $n b$ & $\mathrm{nb}$ & $<0,1$ & $<0,1$ \\
\hline Ta & {$[\mu \mathrm{g} / \mathrm{l}]$} & 0,23 & 0,13 & $\mathrm{nb}$ & nb & $n b$ & $\mathrm{nb}$ & 0,44 & 1,1 \\
\hline $\bar{W}$ & {$[\mu \mathrm{g} / \mathrm{l}]$} & 0,66 & 28 & $\mathrm{nb}$ & nb & $\mathrm{nb}$ & $\mathrm{nb}$ & 0,16 & 0,78 \\
\hline $\mathrm{Hg}$ & {$[\mu \mathrm{g} / \mathrm{l}]$} & nb & nb & $\mathrm{nb}$ & nb & $n b$ & $\mathrm{nb}$ & nb & nb \\
\hline Tl & {$[\mu \mathrm{g} / \mathrm{l}]$} & 0,24 & 0,16 & $\mathrm{nb}$ & $n b$ & $n b$ & $\mathrm{nb}$ & $<0,1$ & 0,38 \\
\hline $\mathbf{P b}$ & {$[\mu \mathrm{g} / \mathrm{I}]$} & 0,50 & 1,0 & $\mathrm{nb}$ & nb & $\mathrm{nb}$ & $\mathrm{nb}$ & 7,7 & \begin{tabular}{|l|}
0,83 \\
\end{tabular} \\
\hline $\mathrm{Bi}$ & {$[\mu \mathrm{g} / \mathrm{l}]$} & nb & nb & $\mathrm{nb}$ & nb & nb & $\mathrm{nb}$ & 0,10 & nb \\
\hline Th & {$[\mu \mathrm{g} / \mathrm{I}]$} & 0,14 & $<0,1$ & $\mathrm{nb}$ & nb & $n b$ & nb & 1,8 & $<0,1$ \\
\hline $\mathbf{U}$ & {$[\mu \mathrm{g} / \mathrm{l}]$} & 14 & 2,2 & $\operatorname{nb}$ & nb & $n b$ & $\mathrm{nb}$ & 18 & 3,5 \\
\hline
\end{tabular}


Tabelle A.1. (Forts.): Analysendaten der Porenwässer und Wasserproben aus Cospuden.

\begin{tabular}{|c|c|c|c|c|c|c|c|c|c|}
\hline & & RCO4 & $\widehat{R C O 10 A}$ & RCO12 & RCO13 & RCO14 & Bohrung 1m & Bohrung 2m & Bohrung 3m \\
\hline & & Wasserprobe & Wasserprobe & Wasserprob & eWasserprobe & Wasserprobe & Porenlösung & Porenlösung & Porenlösung \\
\hline & & Okt 1998 & Okt 1998 & Okt 1998 & Okt 1998 & Okt 1998 & \begin{tabular}{|l|} 
Nov 1998 \\
\end{tabular} & Nov 1998 & \begin{tabular}{|l|} 
Nov 1998 \\
\end{tabular} \\
\hline pH & & 6,2 & 6,9 & 6,0 & 7,1 & 6,4 & 3,5 & 4,9 & 4,3 \\
\hline el Lf & {$[\mathrm{mS} / \mathrm{cm}]$} & 3,1 & 1,3 & 3,4 & 1,1 & 1,1 & 3,2 & 1,7 & 1,9 \\
\hline Redox & {$[\mathrm{mV}]$} & 279 & 232 & 280 & 220 & 269 & 362 & nb & nb \\
\hline Sulfat & {$[\mathrm{g} / \mathrm{l}]$} & 4,2 & 1,4 & 5,0 & 1,0 & 0,6 & 1,8 & 2,1 & 2,1 \\
\hline DOC & {$[\mathrm{mg} / \mathrm{l}]$} & 101,2 & 68,9 & 79,2 & 36,1 & 31,0 & nb & 16,8 & nb \\
\hline $\mathbf{L i}$ & {$[\mu \mathrm{g} / \mathrm{l}]$} & 490 & 200 & 180 & 91 & 14 & 370 & 390 & 120 \\
\hline Be & {$[\mu \mathrm{g} / \mathrm{l}]$} & $<0,1$ & $<0,1$ & $<0,1$ & $<0,1$ & $<0,1$ & 23 & 0,19 & 0,77 \\
\hline $\mathbf{N a}$ & {$[\mu \mathrm{g} / \mathrm{I}]$} & 120000 & 58000 & 85000 & 33000 & 61000 & 5700 & 52000 & 11000 \\
\hline Mg & {$[\mu \mathrm{g} / \mathrm{I}]$} & 320000 & 51000 & 290000 & 45000 & 39000 & 50000 & 260000 & 160000 \\
\hline Al & {$[\mu \mathrm{g} / \mathrm{I}]$} & $<1$ & $k 1$ & $k 1$ & $<1$ & $<1$ & 7800 & 47 & 410 \\
\hline $\mathbf{K}$ & {$[\mu \mathrm{g} / \mathrm{I}]$} & 21000 & 6900 & 21000 & 5000 & 8600 & 18000 & 19000 & 20000 \\
\hline $\mathbf{C a}$ & {$[\mu \mathrm{g} / \mathrm{l}]$} & 570000 & 180000 & 630000 & 130000 & 100000 & 540000 & 580000 & 600000 \\
\hline Sc & {$[\mu \mathrm{g} / \mathrm{I}]$} & 3,3 & 4,7 & 8,2 & 5,9 & 1,8 & 54 & 7,6 & 6,1 \\
\hline $\mathbf{T i}$ & {$[\mu \mathrm{g} / \mathrm{I}]$} & $<1$ & $<1$ & $<1$ & $<1$ & $<1$ & $<1$ & $k 1$ & $<1$ \\
\hline $\mathbf{V}$ & {$[\mu \mathrm{g} / \mathrm{l}]$} & 0,22 & 0,26 & 0,65 & 0,45 & 0,22 & 0,51 & 0,64 & 0,60 \\
\hline $\mathrm{Cr}$ & {$[\mu \mathrm{g} / \mathrm{I}]$} & 0,52 & 0,36 & 0,37 & 11 & 8,6 & 1,2 & 0,58 & 0,74 \\
\hline Mn & {$[\mu \mathrm{g} / \mathrm{l}]$} & 2600 & 200 & 5200 & 460 & 420 & 4200 & 1200 & 5600 \\
\hline $\mathbf{F e}$ & {$[\mu \mathrm{g} / \mathrm{I}]$} & 82500 & 6,8 & 22600 & 7,6 & 13 & 213 & 57 & 1794 \\
\hline Co & {$[\mu \mathrm{g} / \mathrm{l}]$} & 6,1 & 6,1 & 17 & 6,3 & 10 & 280 & 7,1 & 97 \\
\hline $\mathbf{N i}$ & {$[\mu \mathrm{g} / \mathrm{l}]$} & 9,8 & 12 & 26 & 13 & 18 & 400 & 95 & 210 \\
\hline $\mathbf{C u}$ & {$[\mu \mathrm{g} / \mathrm{l}]$} & 13 & 15 & 17 & 12 & 11 & 210 & 15 & 220 \\
\hline $\mathbf{Z n}$ & {$[\mu \mathrm{g} / \mathrm{l}]$} & 82 & 30 & 53 & 16 & 59 & 420 & 46 & 0,82 \\
\hline \begin{tabular}{|l|}
$\mathbf{A s}$ \\
\end{tabular} & {$[\mu \mathrm{g} / \mathrm{l}]$} & $<0,1$ & $<0,1$ & $<0,1$ & $<0,1$ & $<0,1$ & 3,8 & 0,64 & 3,1 \\
\hline $\mathbf{R b}$ & {$[\mu \mathrm{g} / \mathrm{l}]$} & 7,3 & 3,9 & 11,4 & 3,9 & 0,71 & 3,4 & 4,6 & 2,4 \\
\hline $\mathbf{S r}$ & {$[\mu \mathrm{g} / \mathrm{l}]$} & 4500 & 1900 & 5100 & 1300 & 470 & 4000 & 8800 & 3200 \\
\hline $\mathbf{Y}$ & {$[\mu \mathrm{g} / \mathrm{l}]$} & 5,7 & 0,10 & 0,22 & $<0,1$ & $<0,1$ & 45 & 0,50 & 6,7 \\
\hline $\mathbf{Z r}$ & {$[\mu \mathrm{g} / \mathrm{l}]$} & 11 & 6,7 & 7,9 & 6,6 & 6,7 & 1,3 & 0,15 & 0,82 \\
\hline $\mathbf{N b}$ & {$[\mu \mathrm{g} / \mathrm{l}]$} & 0,31 & 0,12 & 0,24 & 0,08 & 0,10 & $<0,1$ & $<0,1$ & $<0,1$ \\
\hline Mo & {$[\mu \mathrm{g} / \mathrm{l}]$} & 0,48 & 0,19 & 1,5 & 0,30 & 0,34 & 0,21 & 0,66 & 2,0 \\
\hline Cd & {$[\mu \mathrm{g} / \mathrm{l}]$} & $\mathrm{nb}$ & nb & $\mathrm{nb}$ & $\mathrm{nb}$ & nb & 14 & 1,1 & 1,5 \\
\hline Sn & {$[\mu \mathrm{g} / \mathrm{I}]$} & 0,16 & 0,20 & 0,31 & $<0,1$ & 0,10 & 0,18 & 0,15 & 0,17 \\
\hline Sb & {$[\mu \mathrm{g} / \mathrm{I}]$} & $<0,1$ & $<0,1$ & $<0,1$ & $<0,1$ & $<0,1$ & 0,14 & 0,24 & 0,40 \\
\hline Cs & {$[\mu \mathrm{g} / \mathrm{I}]$} & $<0,1$ & $<0,1$ & $<0,1$ & $<0,1$ & $<0,1$ & 0,41 & 0,36 & 0,13 \\
\hline Ba & {$[\mu \mathrm{g} / \mathrm{l}]$} & 15 & 49 & 19 & 75 & 97 & 25 & 44 & 45 \\
\hline La & {$[\mu \mathrm{g} / \mathrm{I}]$} & 0,17 & $<0,1$ & 0,46 & $<0,1$ & $<0,1$ & 77 & 1,2 & 6,8 \\
\hline $\mathrm{Ce}$ & {$[\mu \mathrm{g} / \mathrm{l}]$} & $<0,1$ & $<0,1$ & $<0,1$ & $<0,1$ & $<0,1$ & 26 & 0,24 & 2,2 \\
\hline $\mathbf{P r}$ & {$[\mu \mathrm{g} / \mathrm{l}]$} & $<0,1$ & $<0,1$ & $<0,1$ & $<0,1$ & $<0,1$ & 13 & 0,12 & 1,1 \\
\hline Nd & {$[\mu \mathrm{g} / \mathrm{I}]$} & $<0,1$ & $<0,1$ & $<0,1$ & $<0,1$ & $<0,1$ & 46 & 0,33 & 4,5 \\
\hline Sm & {$[\mu \mathrm{g} / \mathrm{I}]$} & $<0,1$ & $<0,1$ & $<0,1$ & $<0,1$ & $<0,1$ & 8,9 & 0,22 & 1 \\
\hline Eu & {$[\mu \mathrm{g} / \mathrm{l}]$} & $<0,1$ & $<0,1$ & $<0,1$ & $<0,1$ & $<0,1$ & 2,1 & $<0,1$ & 0,27 \\
\hline Gd & {$[\mu \mathrm{g} / \mathrm{I}]$} & $<0,1$ & $<0,1$ & $<0,1$ & $<0,1$ & $<0,1$ & 13 & 0,12 & 1,1 \\
\hline Tb & {$[\mu \mathrm{g} / \mathrm{I}]$} & $<0,1$ & $<0,1$ & $<0,1$ & $<0,1$ & $<0,1$ & 1,8 & $<0,1$ & 0,15 \\
\hline Dy & {$[\mu \mathrm{g} / \mathrm{l}]$} & $<0,1$ & $<0,1$ & $<0,1$ & $<0,1$ & $<0,1$ & 10 & 0,12 & 0,84 \\
\hline Ho & {$[\mu \mathrm{g} / \mathrm{I}]$} & $<0,1$ & $<0,1$ & $<0,1$ & $<0,1$ & $<0,1$ & 2,1 & $<0,1$ & 0,84 \\
\hline $\mathbf{E r}$ & {$[\mu \mathrm{g} / \mathrm{l}]$} & $<0,1$ & $<0,1$ & $<0,1$ & $<0,1$ & $<0,1$ & 5,3 & $<0,1$ & 0,39 \\
\hline Tm & {$[\mu \mathrm{g} / \mathrm{l}]$} & $<0,1$ & $<0,1$ & $<0,1$ & $<0,1$ & $<0,1$ & 0,62 & 0,10 & $<0,1$ \\
\hline $\mathbf{Y b}$ & {$[\mu \mathrm{g} / \mathrm{l}]$} & $<0,1$ & $<0,1$ & $<0,1$ & $<0,1$ & $<0,1$ & 3,1 & $<0,1$ & 0,30 \\
\hline Lu & {$[\mu \mathrm{g} / \mathrm{I}]$} & $<0,1$ & $<0,1$ & $<0,1$ & $<0,1$ & $<0,1$ & 0,48 & $<0,1$ & $<0,1$ \\
\hline Hf & {$[\mu \mathrm{g} / \mathrm{I}]$} & $<0,1$ & $<0,1$ & $<0,1$ & $<0,1$ & $<0,1$ & $<0,1$ & $<0,1$ & $<0,1$ \\
\hline Ta & {$[\mu \mathrm{g} / \mathrm{I}]$} & 0,16 & $<0,1$ & 0,20 & $<0,1$ & $<0,1$ & $<0,1$ & $k 0,1$ & $<0,1$ \\
\hline $\mathbf{W}$ & {$[\mu \mathrm{g} / \mathrm{I}]$} & $<0,1$ & 0,16 & 0,28 & 0,11 & 0,11 & 0,69 & 1,7 & 0,14 \\
\hline $\mathrm{Hg}$ & {$[\mu \mathrm{g} / \mathrm{I}]$} & $n b$ & & & & & 0,17 & 0,23 & 0,14 \\
\hline TI & {$[\mu \mathrm{g} / \mathrm{I}]$} & $<0,1$ & $<0,1$ & 0,01 & $<0,1$ & $<0,1$ & 4,0 & 0,19 & 0,64 \\
\hline $\mathbf{P b}$ & {$[\mu \mathrm{g} / \mathbf{I}]$} & $<0,1$ & 0,27 & 0,16 & 0,10 & $<0,1$ & 9,3 & 0,47 & 1,3 \\
\hline $\mathbf{B i}$ & {$[\mu \mathrm{g} / \mathrm{l}]$} & $<0,1$ & $<0,1$ & $<0,1$ & $<0,1$ & $<0,1$ & $<0,1$ & $<0,1$ & $<0,1$ \\
\hline Th & {$[\mu \mathrm{g} / \mathrm{l}]$} & $<0,1$ & $<0,1$ & $<0,1$ & $<0,1$ & $<0,1$ & $<0,1$ & $<0,1$ & $<0,1$ \\
\hline $\mathbf{U}$ & {$[\mu \mathrm{g} / \mathrm{l}]$} & 8,1 & $\mid<0,1$ & 6,4 & 0,23 & $<0,1$ & 0,15 & $<0,1$ & $<0,1$ \\
\hline
\end{tabular}


Tabelle A.1. (Forts.): Analysendaten der Porenwässer und Wasserproben aus Cospuden.

\begin{tabular}{|c|c|c|c|c|c|c|c|c|c|}
\hline & & See & CML $20 \mathrm{~m}$ & CML 27,5 m & CML 31,5 m & CML 39,5 m & CML 43,5 m & CML 20 m & CML 27,5 m \\
\hline & & Wasserprobe & Wasserprobe & Wasserprobe & eWasserprobe & eWasserprobe & eWasserprobe & eWasserprobe & Wasserprobe \\
\hline & & Nov 1998 & Jan 1999 & Jan 1999 & Jan 1999 & Jan 1999 & Jan 1999 & Aug 1999 & Aug 1999 \\
\hline pH & & 7,0 & 3,5 & 7,7 & 6,2 & 7,5 & 7,4 & $\mathrm{nb}$ & 6,0 \\
\hline el Lf & {$[\mathrm{mS} / \mathrm{cm}]$} & 2,0 & 1,7 & 2,3 & 3,2 & 2,2 & 2,1 & 1,9 & 4,0 \\
\hline Redox & {$[\mathrm{mV}]$} & 240 & 202 & 203 & 274 & 202 & 204 & $146^{*}$ & $190^{*}$ \\
\hline Sulfat & {$[\mathrm{g} / \mathrm{l}]$} & 3,2 & 1,0 & 1,3 & 2,6 & 1,2 & 0,9 & 1,5 & 4,2 \\
\hline DOC & {$[\mathrm{mg} / \mathrm{l}]$} & 15,7 & 9,9 & 12,3 & 8,1 & 47,9 & 62,5 & 33,4 & 14,0 \\
\hline $\mathbf{L i}$ & {$[\mu \mathrm{g} / \mathrm{l}]$} & 100 & 9,3 & 160 & 210 & 120 & 170 & 24 & 270 \\
\hline $\mathbf{B e}$ & {$[\mu \mathrm{g} / \mathrm{l}]$} & $<0,1$ & $<0,1$ & $<0,1$ & $<0,1$ & $<0,1$ & $<0,1$ & $<0,1$ & 1,6 \\
\hline $\mathrm{Na}$ & {$[\mu \mathrm{g} / \mathrm{l}]$} & 51000 & 65000 & 62000 & 31000 & 88000 & 78000 & 60000 & 40000 \\
\hline $\mathbf{M g}$ & {$[\mu \mathrm{g} / \mathrm{l}]$} & 79000 & 6200 & 21000 & 330000 & 140000 & 100000 & 43000 & 550000 \\
\hline Al & {$[\mu \mathrm{g} / \mathrm{l}]$} & 42 & $<1$ & $<1$ & $<1$ & $<1$ & $<1$ & 130 & 2500 \\
\hline $\mathbf{K}$ & {$[\mu \mathrm{g} / \mathrm{l}]$} & 7800 & 35000 & 59000 & 12000 & 23000 & 14000 & 30000 & 23000 \\
\hline Ca & {$[\mu \mathrm{g} / \mathrm{l}]$} & 290000 & 320000 & 420000 & 220000 & 360000 & 280000 & 560000 & 410000 \\
\hline Sc & {$[\mu \mathrm{g} / \mathrm{l}]$} & 5,6 & $<0,1$ & $<0,1$ & $<0,1$ & $<0,1$ & $<0,1$ & 8,1 & 24 \\
\hline $\mathbf{T i}$ & {$[\mu \mathrm{g} / \mathrm{l}]$} & $<1$ & $k 1$ & $<1$ & $<1$ & $<1$ & $<1$ & $<1$ & $<1$ \\
\hline $\mathbf{V}$ & {$[\mu \mathrm{g} / \mathrm{l}]$} & 0,33 & 12 & $<0,1$ & 8,5 & $<0,1$ & $<0,1$ & 3,3 & 16 \\
\hline $\mathrm{Cr}$ & {$[\mu \mathrm{g} / \mathrm{l}]$} & 14 & 11,3 & 11 & 37 & 9,6 & 3,6 & 1,5 & 0,96 \\
\hline Mn & {$[\mu \mathrm{g} / \mathrm{l}]$} & 1700 & $<1$ & 530 & 7300 & 1500 & 880 & 520 & 12000 \\
\hline $\mathbf{F e}$ & {$[\mu \mathrm{g} / \mathrm{l}]$} & 7 & $<5$ & $<5$ & 523821 & 16 & 8,4 & 333 & 755180 \\
\hline Co & {$[\mu \mathrm{g} / \mathrm{l}]$} & 39 & 3,2 & 3,8 & 61 & 1,6 & 1,9 & 5,6 & 87 \\
\hline $\mathrm{Ni}$ & {$[\mu \mathrm{g} / \mathrm{l}]$} & 99 & 5,6 & 35 & 320 & $<0,1$ & $\mathrm{nq}$ & 20 & 490 \\
\hline $\mathbf{C u}$ & {$[\mu \mathrm{g} / \mathrm{l}]$} & 12 & 3,1 & 3,2 & 4,6 & 3,4 & 3,8 & 11 & 6,3 \\
\hline $\mathbf{Z n}$ & {$[\mu \mathrm{g} / \mathrm{l}]$} & 82 & $<0,1$ & 20 & 230 & 4,5 & 9,6 & 8,0 & 960 \\
\hline As & {$[\mu \mathrm{g} / \mathrm{l}]$} & $<0,1$ & $<0,1$ & $<0,1$ & $<0,1$ & $<0,1$ & $<0,1$ & 0,82 & 1,1 \\
\hline $\mathbf{R b}$ & {$[\mu \mathrm{g} / \mathrm{l}]$} & 7,6 & 3,5 & 7,0 & 3,8 & 1,7 & 1,6 & 11 & 30 \\
\hline $\mathbf{S r}$ & {$[\mu \mathrm{g} / \mathrm{l}]$} & 1600 & 3000 & 2900 & 3700 & 4000 & 3700 & 5900 & 4900 \\
\hline $\mathbf{Y}$ & {$[\mu \mathrm{g} / \mathrm{l}]$} & 5,9 & 0,10 & $<0,1$ & $<0,1$ & 0,29 & $<0,1$ & 0,29 & 990 \\
\hline $\mathbf{Z r}$ & {$[\mu \mathrm{g} / \mathrm{l}]$} & 6,5 & $<0,1$ & $<0,1$ & 22 & 0,18 & $<0,1$ & 0,62 & 40 \\
\hline $\mathbf{N b}$ & {$[\mu \mathrm{g} / \mathrm{l}]$} & 0,10 & $<0,1$ & 0,11 & 0,11 & $<0,1$ & $<0,1$ & 2,3 & 2,1 \\
\hline Mo & {$[\mu \mathrm{g} / \mathrm{l}]$} & 0,30 & 5,6 & 4,3 & 0,42 & 8,7 & 14 & 9,2 & 0,27 \\
\hline Cd & {$[\mu \mathrm{g} / \mathrm{l}]$} & nb & 0,23 & 0,21 & $\mathrm{nb}$ & 0,15 & 0,28 & $<0,1$ & $<0,1$ \\
\hline Sn & {$[\mu \mathrm{g} / \mathrm{l}]$} & 0,10 & $<0,1$ & 0,11 & $<0,1$ & $<0,1$ & $<0,1$ & 0,42 & 0,30 \\
\hline $\mathbf{S b}$ & {$[\mu \mathrm{g} / \mathrm{l}]$} & $<0,1$ & 0,10 & $<0,1$ & $<0,1$ & $<0,1$ & $<0,1$ & 0,1 & $<0,1$ \\
\hline Cs & {$[\mu \mathrm{g} / \mathrm{l}]$} & 0,12 & 0,25 & 0,23 & 0,30 & 0,20 & 0,10 & $<0,1$ & 0,16 \\
\hline $\mathbf{B a}$ & {$[\mu \mathrm{g} / \mathrm{l}]$} & 30 & 66 & 35 & 11 & 19 & 24 & 120 & 21 \\
\hline La & {$[\mu \mathrm{g} / \mathrm{l}]$} & 0,10 & $<0,1$ & $<0,1$ & $<0,1$ & $<0,1$ & $<0,1$ & 0,23 & 200 \\
\hline $\mathrm{Ce}$ & {$[\mu \mathrm{g} / \mathrm{l}]$} & $<0,1$ & $<0,1$ & $<0,1$ & $<0,1$ & $<0,1$ & $<0,1$ & 0,25 & 200 \\
\hline Pr & {$[\mu \mathrm{g} / \mathrm{l}]$} & $<0,1$ & $<0,1$ & $<0,1$ & $<0,1$ & $<0,1$ & $<0,1$ & $<0,1$ & 15 \\
\hline Nd & {$[\mu \mathrm{g} / \mathrm{l}]$} & $<0,1$ & 0,12 & $<0,1$ & $<0,1$ & $<0,1$ & $<0,1$ & 0,17 & 40 \\
\hline Sm & {$[\mu \mathrm{g} / \mathrm{l}]$} & $<0,1$ & $<0,1$ & $<0,1$ & $<0,1$ & $<0,1$ & $<0,1$ & $<0,1$ & 2,3 \\
\hline $\mathbf{E u}$ & {$[\mu \mathrm{g} / \mathrm{l}]$} & $<0,1$ & $<0,1$ & $<0,1$ & $<0,1$ & $<0,1$ & $<0,1$ & $<0,1$ & 0,76 \\
\hline Gd & {$[\mu \mathrm{g} / \mathrm{l}]$} & $<0,1$ & $<0,1$ & $<0,1$ & $<0,1$ & $<0,1$ & $<0,1$ & $<0,1$ & 7,9 \\
\hline Tb & {$[\mu \mathrm{g} / \mathrm{l}]$} & $<0,1$ & $<0,1$ & $<0,1$ & $<0,1$ & $<0,1$ & $<0,1$ & $<0,1$ & 1,3 \\
\hline Dy & {$[\mu \mathrm{g} / \mathrm{l}]$} & $<0,1$ & $<0,1$ & $<0,1$ & $<0,1$ & $<0,1$ & $<0,1$ & $<0,1$ & 5,2 \\
\hline Ho & {$[\mu \mathrm{g} / \mathrm{l}]$} & $<0,1$ & $<0,1$ & $<0,1$ & $<0,1$ & $<0,1$ & $<0,1$ & $<0,1$ & 1,7 \\
\hline $\mathbf{E r}$ & {$[\mu \mathrm{g} / \mathrm{l}]$} & $<0,1$ & $<0,1$ & $<0,1$ & $<0,1$ & $<0,1$ & $<0,1$ & $<0,1$ & 3,1 \\
\hline Tm & {$[\mu \mathrm{g} / \mathrm{l}]$} & $<0,1$ & $<0,1$ & $<0,1$ & $<0,1$ & $<0,1$ & $<0,1$ & $<0,1$ & 0,30 \\
\hline Yb & {$[\mu \mathrm{g} / \mathrm{l}]$} & $<0,1$ & $<0,1$ & $<0,1$ & $<0,1$ & $<0,1$ & $<0,1$ & $<0,1$ & 0,79 \\
\hline $\mathbf{L u}$ & {$[\mu \mathrm{g} / \mathrm{l}]$} & $<0,1$ & $<0,1$ & $<0,1$ & $<0,1$ & $<0,1$ & $<0,1$ & $<0,1$ & 0,20 \\
\hline Hf & {$[\mu \mathrm{g} / \mathrm{l}]$} & $<0,1$ & $<0,1$ & $<0,1$ & $<0,1$ & $<0,1$ & $<0,1$ & $<0,1$ & $<0,1$ \\
\hline Ta & {$[\mu \mathrm{g} / \mathrm{l}]$} & $<0,1$ & nb & nb & nb & nb & nb & 1,1 & 1,5 \\
\hline $\mathbf{W}$ & {$[\mu \mathrm{g} / \mathrm{l}]$} & $<0,1$ & 3,0 & 0,36 & 0,13 & $<0,1$ & 0,38 & 0,38 & 0,15 \\
\hline $\mathrm{Hg}$ & {$[\mu \mathrm{g} / \mathrm{l}]$} & nb & 0,2 & 0,13 & 0,18 & 0,18 & 0,20 & 0,22 & 0,23 \\
\hline TI & {$[\mu \mathrm{g} / \mathrm{l}]$} & $<0,1$ & $<0,1$ & $<0,1$ & $<0,1$ & 0,10 & $<0,1$ & $<0,1$ & $<0,1$ \\
\hline $\mathbf{P b}$ & {$[\mu \mathrm{g} / \mathrm{I}]$} & $<0,1$ & 0,58 & 0,31 & 0,11 & 0,14 & 0,13 & 0,17 & 0,33 \\
\hline $\mathbf{B i}$ & {$[\mu \mathrm{g} / \mathrm{l}]$} & $<0,1$ & $<0,1$ & $<0,1$ & $<0,1$ & $<0,1$ & $<0,1$ & $<0,1$ & $<0,1$ \\
\hline Th & {$[\mu \mathrm{g} / \mathrm{l}]$} & $<0,1$ & $<0,1$ & $<0,1$ & $<0,1$ & $<0,1$ & $<0,1$ & 0,11 & $<0,1$ \\
\hline $\mathbf{U}$ & {$[\mu \mathrm{g} / \mathrm{l}]$} & 0,45 & $<0,1$ & 0,22 & 1,2 & 29 & 12 & 3,5 & 3,9 \\
\hline
\end{tabular}


Tabelle A.1. (Forts.): Analysendaten der Porenwässer und Wasserproben aus Cospuden.

\begin{tabular}{|c|c|c|c|c|c|c|c|c|c|}
\hline & & CML 31,5 m & CML 39,5 m & CML 43,5 m & RCO3 & RCO4 & RCO12 & RCO15 & RCO17 \\
\hline & & Wasserprobe & Wasserprobe & Wasserprobe & eWasserprobe & Wasserprob & eWasserprob & Wasserprobe & eWasserprobe \\
\hline & & Aug 1999 & Aug 1999 & Aug 1999 & Aug 1999 & Aug 1999 & Aug 1999 & Aug 1999 & Aug 1999 \\
\hline pH & & 5,9 & 6,8 & 6,0 & 5,3 & 6,3 & 6,1 & 6,5 & 6,5 \\
\hline el Lf & {$[\mathrm{mS} / \mathrm{cm}]$} & 4,6 & 2,4 & 1,9 & 2,8 & 2,7 & 2,8 & 1,5 & 1,7 \\
\hline Redox & {$[\mathrm{mV}]$} & $193^{*}$ & $126^{*}$ & $24^{*}$ & $186^{*}$ & $147^{*}$ & $143^{*}$ & $269^{*}$ & $270^{*}$ \\
\hline Sulfat & {$[\mathrm{g} / \mathrm{l}]$} & 4,3 & 1,4 & 1,0 & 3,0 & 2,2 & 2,0 & 0,9 & 1,4 \\
\hline DOC & {$[\mathrm{mg} / \mathrm{l}]$} & 28,6 & nb & 71,8 & 16,3 & 60,0 & 83,1 & 30,6 & 19,5 \\
\hline $\mathbf{L i}$ & {$[\mu \mathrm{g} / \mathrm{l}]$} & 340 & 260 & 270 & 360 & 480 & 170 & 57 & 76 \\
\hline $\mathbf{B e}$ & {$[\mu \mathrm{g} / \mathrm{l}]$} & 3,2 & $<0,1$ & $<0,1$ & 0,58 & $<0,1$ & $<0,1$ & $<0,1$ & 0,67 \\
\hline $\mathbf{N a}$ & {$[\mu \mathrm{g} / \mathrm{l}]$} & 64000 & 99000 & 86000 & 80000 & 140000 & 110000 & 52000 & 59000 \\
\hline Mg & {$[\mu \mathrm{g} / \mathrm{l}]$} & 620000 & 200000 & 140000 & 300000 & 310000 & 250000 & 67000 & 91000 \\
\hline Al & {$[\mu \mathrm{g} / \mathrm{l}]$} & 6200 & 6,9 & 17 & 1100 & 29 & 48 & 49 & 61 \\
\hline $\mathbf{K}$ & {$[\mu \mathrm{g} / \mathrm{l}]$} & 33000 & 20000 & 17000 & 36000 & 24000 & 23000 & 5700 & 6300 \\
\hline $\mathbf{C a}$ & {$[\mu \mathrm{g} / \mathrm{l}]$} & 440000 & 470000 & 380000 & 480000 & 620000 & 600000 & 350000 & 410000 \\
\hline Sc & {$[\mu \mathrm{g} / \mathrm{l}]$} & 45 & 4,6 & 3,3 & 18 & 19 & 7,9 & $<0,1$ & $<0,1$ \\
\hline $\mathrm{Ti}$ & {$[\mu \mathrm{g} / \mathrm{l}]$} & $<1$ & $<1$ & $<1$ & $<1$ & $<1$ & $<1$ & $<1$ & $<1$ \\
\hline $\mathbf{V}$ & {$[\mu \mathrm{g} / \mathrm{l}]$} & 21 & 0,69 & 0,67 & 9,9 & 14 & 0,22 & $<0,1$ & 0,64 \\
\hline $\mathrm{Cr}$ & {$[\mu \mathrm{g} / \mathrm{l}]$} & 5,4 & 1,0 & 1,1 & 14 & 2,4 & 7,3 & 3,0 & 2,4 \\
\hline Mn & {$[\mu \mathrm{g} / \mathrm{l}]$} & 15000 & 1900 & 1300 & 9600 & 3000 & 5100 & 1000 & 1000 \\
\hline $\mathbf{F e}$ & {$[\mu \mathrm{g} / \mathrm{l}]$} & 1240170 & 20990 & 1560 & 584050 & 116440 & 50920 & 58 & 71 \\
\hline Co & {$[\mu \mathrm{g} / \mathrm{l}]$} & 110 & 3,4 & 3,4 & 3,8 & 6,8 & 12 & 1,9 & 1,2 \\
\hline $\mathrm{Ni}$ & {$[\mu \mathrm{g} / \mathrm{l}]$} & 690 & 7,5 & 17 & $<0,1$ & $<0,1$ & 27 & 5,1 & 8,7 \\
\hline $\mathrm{Cu}$ & {$[\mu \mathrm{g} / \mathrm{l}]$} & 18 & 2,0 & 9,0 & 6,6 & 13 & 12 & 2,5 & 2,1 \\
\hline $\mathbf{Z n}$ & {$[\mu \mathrm{g} / \mathrm{l}]$} & 910 & 28 & 29 & 170 & 42 & 19 & 2,6 & 2,7 \\
\hline As & {$[\mu \mathrm{g} / \mathrm{l}]$} & 27 & 1,5 & 1,5 & 0,92 & 14 & 8,9 & 0,19 & 0,24 \\
\hline $\mathbf{R b}$ & {$[\mu \mathrm{g} / \mathrm{l}]$} & 42 & 11 & 2,9 & 79 & 27 & 19 & 4,8 & 6,3 \\
\hline $\mathbf{S r}$ & {$[\mu \mathrm{g} / \mathrm{l}]$} & 9300 & 5700 & 4700 & 8000 & 10000 & 10000 & 3300 & 5200 \\
\hline $\mathbf{Y}$ & {$[\mu \mathrm{g} / \mathrm{l}]$} & 46 & 0,91 & 7,7 & 4,2 & 0,40 & 0,21 & 0,21 & 0,21 \\
\hline $\mathbf{Z r}$ & {$[\mu \mathrm{g} / \mathrm{l}]$} & 99 & 2,9 & 1,4 & 50 & 6,7 & 1,9 & $<0,1$ & $<0,1$ \\
\hline $\mathbf{N b}$ & {$[\mu \mathrm{g} / \mathrm{l}]$} & 2,3 & $<0,1$ & $<0,1$ & $<0,1$ & 0,50 & $<0,1$ & 0,67 & 0,15 \\
\hline Mo & {$[\mu \mathrm{g} / \mathrm{l}]$} & 4,4 & 5,5 & 3,6 & 0,69 & 7,6 & 1,2 & 0,27 & 13 \\
\hline Cd & {$[\mu \mathrm{g} / \mathrm{l}]$} & 0,14 & $<0,1$ & $<0,1$ & $<0,1$ & $<0,1$ & $<0,1$ & $<0,1$ & $<0,1$ \\
\hline Sn & {$[\mu \mathrm{g} / \mathrm{l}]$} & 0,25 & $<0,1$ & $<0,1$ & 0,22 & 1,2 & 1,2 & $<0,1$ & $<0,1$ \\
\hline Sb & {$[\mu \mathrm{g} / \mathrm{l}]$} & $<0,1$ & $<0,1$ & $<0,1$ & $<0,1$ & $<0,1$ & 0,10 & $<0,1$ & $<0,1$ \\
\hline Cs & {$[\mu \mathrm{g} / \mathrm{l}]$} & 1,2 & $<0,1$ & $<0,1$ & 0,80 & 0,12 & $<0,1$ & $<0,1$ & $<0,1$ \\
\hline $\mathbf{B a}$ & {$[\mu \mathrm{g} / \mathrm{l}]$} & 35 & 2,5 & 3,1 & 24 & 26 & 31 & 5,6 & 24 \\
\hline La & {$[\mu \mathrm{g} / \mathrm{l}]$} & 32 & $<0,1$ & 0,20 & 7,4 & 0,27 & 0,21 & $<0,1$ & $<0,1$ \\
\hline $\mathrm{Ce}$ & {$[\mu \mathrm{g} / \mathrm{l}]$} & 52 & $<0,1$ & 0,10 & 2,1 & 0,24 & $<0,1$ & $<0,1$ & 0,15 \\
\hline Pr & {$[\mu \mathrm{g} / \mathrm{l}]$} & 4,3 & $<0,1$ & $<0,1$ & 0,69 & $<0,1$ & $<0,1$ & $<0,1$ & $<0,1$ \\
\hline Nd & {$[\mu \mathrm{g} / \mathrm{l}]$} & 13 & 0,18 & 0,39 & 2,2 & 0,22 & 0,25 & $<0,1$ & $<0,1$ \\
\hline $\mathrm{Sm}$ & {$[\mu \mathrm{g} / \mathrm{l}]$} & 1,4 & 0,15 & 0,15 & 0,55 & 0,14 & 0,27 & $<0,1$ & $<0,1$ \\
\hline Eu & {$[\mu \mathrm{g} / \mathrm{l}]$} & 0,43 & $<0,1$ & $<0,1$ & 0,15 & $<0,1$ & 0,10 & $<0,1$ & $<0,1$ \\
\hline Gd & {$[\mu \mathrm{g} / \mathrm{l}]$} & 2,9 & $<0,1$ & 0,11 & 0,50 & $<0,1$ & $<0,1$ & $<0,1$ & $<0,1$ \\
\hline $\mathbf{T b}$ & {$[\mu \mathrm{g} / \mathrm{l}]$} & 0,53 & $<0,1$ & $<0,1$ & $<0,1$ & $<0,1$ & $<0,1$ & $<0,1$ & $<0,1$ \\
\hline Dy & {$[\mu \mathrm{g} / \mathrm{l}]$} & 1,5 & $<0,1$ & 0,13 & 0,44 & $<0,1$ & 0,10 & $<0,1$ & $<0,1$ \\
\hline Ho & {$[\mu \mathrm{g} / \mathrm{l}]$} & 0,78 & $<0,1$ & $<0,1$ & $<0,1$ & $<0,1$ & $<0,1$ & $<0,1$ & $<0,1$ \\
\hline $\mathbf{E r}$ & {$[\mu \mathrm{g} / \mathrm{l}]$} & 1,4 & $<0,1$ & $<0,1$ & 0,25 & $<0,1$ & $<0,1$ & $<0,1$ & $<0,1$ \\
\hline Tm & {$[\mu \mathrm{g} / \mathrm{l}]$} & 0,18 & $<0,1$ & $<0,1$ & $<0,1$ & $<0,1$ & $<0,1$ & $<0,1$ & $<0,1$ \\
\hline Yb & {$[\mu \mathrm{g} / \mathrm{l}]$} & 0,74 & $<0,1$ & 0,10 & 0,17 & $<0,1$ & $<0,1$ & $<0,1$ & $<0,1$ \\
\hline Lu & {$[\mu \mathrm{g} / \mathrm{l}]$} & 0,18 & $<0,1$ & $<0,1$ & $<0,1$ & $<0,1$ & $<0,1$ & $<0,1$ & $<0,1$ \\
\hline Hf & {$[\mu \mathrm{g} / \mathrm{l}]$} & $<0,1$ & $<0,1$ & $<0,1$ & $<0,1$ & $<0,1$ & $<0,1$ & $<0,1$ & $<0,1$ \\
\hline Ta & {$[\mu \mathrm{g} / \mathrm{l}]$} & 0,25 & 0,14 & $<0,1$ & $<0,1$ & 0,34 & $<0,1$ & 0,31 & $<0,1$ \\
\hline $\mathbf{W}$ & {$[\mu \mathrm{g} / \mathrm{l}]$} & 0,13 & $<0,1$ & 0,24 & $<0,1$ & 0,17 & $<0,1$ & $<0,1$ & 0,15 \\
\hline $\mathrm{Hg}$ & {$[\mu \mathrm{g} / \mathrm{l}]$} & 0,31 & 0,28 & 0,22 & 0,12 & 0,41 & 0,15 & 0,16 & 0,12 \\
\hline TI & {$[\mu \mathrm{g} / \mathrm{l}]$} & 0,17 & $<0,1$ & $<0,1$ & $<0,1$ & $<0,1$ & $<0,1$ & $<0,1$ & $<0,1$ \\
\hline $\mathbf{P b}$ & {$[\mu \mathrm{g} / \mathrm{l}]$} & 0,53 & 0,12 & 0,14 & 0,28 & 0,28 & 0,37 & 0,20 & 0,16 \\
\hline $\mathbf{B i}$ & {$[\mu \mathrm{g} / \mathrm{l}]$} & $<0,1$ & $<0,1$ & $<0,1$ & $<0,1$ & $<0,1$ & $<0,1$ & $<0,1$ & $<0,1$ \\
\hline Th & {$[\mu \mathrm{g} / \mathrm{l}]$} & 0,16 & $<0,1$ & $<0,1$ & $<0,1$ & $<0,1$ & $<0,1$ & $<0,1$ & $<0,1$ \\
\hline $\mathbf{U}$ & {$[\mu \mathrm{g} / \mathrm{l}]$} & 1,3 & 15 & 13 & $<0,1$ & 20 & 4,2 & 1,3 & 0,12 \\
\hline
\end{tabular}


Tabelle A.1. (Forts.): Analysendaten der Porenwässer und Wasserproben aus Cospuden.

\begin{tabular}{|c|c|c|c|c|c|c|c|c|c|}
\hline & & RCO18 & See & SeeSed & Sedgepr & CML $20 \mathrm{~m}$ & CML 27,5 m & CML 31,5 & \begin{tabular}{|l|l} 
& CML,5 m \\
\end{tabular} \\
\hline & & Wasserprobe & Wasserprobe & eWasserprob & ePorenlösung & Wasserprobe & Wasserprobe & Wasserpr & eWasserprobe \\
\hline & & Aug 1999 & Aug 1999 & Aug 1999 & Aug 1999 & Jan 2000 & Jan 2000 & Jan 2000 & Jan 2000 \\
\hline $\mathbf{p H}$ & & 6,4 & 6,9 & 6,7 & 6,6 & 6,1 & 3,4 & 3,2 & 7,4 \\
\hline el Lf & {$[\mathrm{mS} / \mathrm{cm}]$} & 2,0 & 1,1 & $\mathrm{nb}$ & $\mathrm{nb}$ & 1,7 & 3,3 & 3,5 & 1,9 \\
\hline Redox & {$[\mathrm{mV}]$} & $163^{*}$ & nb & $\mathrm{nb}$ & $\mathrm{nb}$ & 215 & -2 & 445 & 205 \\
\hline Sulfat & {$[\mathrm{g} / \mathrm{l}]$} & 1,4 & 1,0 & 1,0 & 0,8 & 1,6 & 4,2 & 4,2 & 1,5 \\
\hline DOC & {$[\mathrm{mg} / \mathrm{l}]$} & 58,6 & 12,9 & 32,9 & 74,2 & 103,5 & 91,7 & 31,7 & 80,7 \\
\hline $\mathbf{L i}$ & {$[\mu \mathrm{g} / \mathrm{l}]$} & 280 & 73 & 20 & 9,2 & 33 & 420 & 390 & 610 \\
\hline $\mathrm{Be}$ & {$[\mu \mathrm{g} / \mathrm{ll}]$} & $<0,1$ & $k 0,1$ & $<0,1$ & $<0,1$ & $k 0,1$ & 0,73 & 1,3 & $<0,1$ \\
\hline $\mathbf{N a}$ & {$[\mu \mathrm{g} / \mathrm{ll}]$} & 48000 & 39000 & 48000 & 41000 & 56000 & 49000 & 38000 & 83000 \\
\hline $\mathbf{M g}$ & {$[\mu \mathrm{g} / \mathrm{ll}]$} & 130000 & 70000 & 71000 & 60000 & 46000 & 840000 & 840000 & 260000 \\
\hline $\mid \overline{A l}$ & {$[\mu \mathrm{g} / \mathrm{ll}]$} & 17 & 50 & $<1$ & 18 & $<1$ & 620 & 2700 & $<1$ \\
\hline $\mathbf{K}$ & {$[\mu \mathrm{g} / \mathrm{ll}]$} & 16000 & 7700 & 9100 & 7600 & 27000 & 26000 & 33000 & 22000 \\
\hline $\mathbf{C a}$ & {$[\mu \mathrm{g} / \mathrm{ll}]$} & 560000 & 330000 & 340000 & 270000 & 700000 & 500000 & 390000 & 530000 \\
\hline Sc & {$[\mu \mathrm{g} / \mathrm{l}]$} & 3,1 & 1,9 & $<0,1$ & 7,0 & $<0,1$ & 0,19 & 0,27 & $<0,1$ \\
\hline Ti & {$[\mu \mathrm{g} / \mathrm{I}]$} & $<1$ & $k 1$ & $k 1$ & $<1$ & 0,22 & 14 & $k 1$ & $<1$ \\
\hline $\mathbf{V}$ & {$[\mu \mathrm{g} / \mathrm{I}]$} & 0,24 & 0,33 & 4,2 & 3,6 & 0,94 & 6,2 & 8,8 & 0,65 \\
\hline $\mathrm{Cr}$ & {$[\mu \mathrm{g} / \mathrm{I}]$} & 1,1 & 0,21 & 2,1 & 47 & 6,3 & $<0.1$ & $k 1$ & 1,1 \\
\hline $\mathbf{M n}$ & {$[\mu \mathrm{g} / \mathrm{I}]$} & 1300 & 750 & $<0,1$ & 3,8 & 570 & 7400 & 7500 & 1500 \\
\hline $\mathrm{Fe}$ & {$[\mu \mathrm{g} / \mathrm{I}]$} & 3230 & 57 & 92 & 48 & 150 & 393620 & 613040 & 28 \\
\hline Co & {$[\mu \mathrm{g} / \mathrm{l}]$} & 1,2 & 15 & 2,4 & 1,1 & $<0,1$ & 49 & 57 & $<0,1$ \\
\hline $\mathbf{N i}$ & {$[\mu \mathrm{g} / \mathrm{I}]$} & 17 & 52 & 17 & 30 & $<0,1$ & 370 & 450 & $<0,1$ \\
\hline $\mathbf{C u}$ & {$[\mu \mathrm{g} / \mathrm{l}]$} & 5,1 & 2,2 & 5,7 & 11 & 2,5 & 5,1 & 5,5 & 1,8 \\
\hline $\mathbf{Z n}$ & {$[\mu \mathrm{g} / \mathrm{l}]$} & 17 & 10 & 14 & 33 & 4,6 & 240 & 460 & 3,6 \\
\hline As & {$[\mu \mathrm{g} / \mathrm{l}]$} & 4,5 & 0,58 & 0,79 & 1,2 & $<0,1$ & 0,23 & 1,7 & 0,40 \\
\hline $\mathbf{R b}$ & {$[\mu \mathrm{g} / \mathrm{l}]$} & 3,8 & 2,5 & 7,3 & 12 & 0,13 & 27 & 9,4 & 9,6 \\
\hline $\mathbf{S r}$ & {$[\mu \mathrm{g} / \mathrm{I}]$} & 7500 & 2600 & 2200 & 1400 & 4200 & 6000 & 6500 & 7000 \\
\hline $\mathbf{Y}$ & {$[\mu \mathrm{g} / \mathrm{l}]$} & 0,15 & $<0,1$ & 0,14 & 0,24 & 0,23 & 51 & 34 & $<0,1$ \\
\hline $\mathbf{Z r}$ & {$[\mu \mathrm{g} / \mathrm{l}]$} & 1,4 & 1,4 & 0,19 & 0,55 & 4,0 & 18 & 27 & 3,2 \\
\hline $\mathbf{N b}$ & {$[\mu \mathrm{g} / \mathrm{l}]$} & 0,44 & 0,36 & 1,3 & 0,35 & $<0,1$ & $<0,1$ & $<0,1$ & 0,11 \\
\hline Mo & {$[\mu \mathrm{g} / \mathrm{I}]$} & 0,78 & 0,28 & 1,3 & 2,2 & 0,43 & 0,15 & 2,9 & 4,4 \\
\hline Cd & {$[\mu \mathrm{g} / \mathrm{l}]$} & $<0,1$ & 0,24 & $<0,1$ & 0,31 & $<0,1$ & $<0,1$ & 0,12 & 0,18 \\
\hline Sn & {$[\mu \mathrm{g} / \mathrm{ll}]$} & $<0,1$ & 0,25 & $<0,1$ & $<0,1$ & 0,20 & 0,39 & 0,30 & 2,0 \\
\hline Sb & {$[\mu \mathrm{g} / \mathrm{ll}]$} & $<0,1$ & $<0,1$ & 0,11 & 0,35 & $<0,1$ & $<0,1$ & 0,13 & 0,10 \\
\hline Cs & {$[\mu \mathrm{g} / \mathrm{l}]$} & $<0,1$ & $<0,1$ & $<0,1$ & $<0,1$ & $<0,1$ & 0,78 & 0,78 & $<0,1$ \\
\hline Ba & {$[\mu \mathrm{g} / \mathrm{l}]$} & 1,8 & 3,3 & 25 & 58 & 25 & 9,2 & 21 & 14 \\
\hline La & {$[\mu \mathrm{g} / \mathrm{I}]$} & $<0,1$ & $<0,1$ & 0,36 & 0,31 & $<0,1$ & 75 & $<0,1$ & $<0,1$ \\
\hline Ce & {$[\mu \mathrm{g} / \mathrm{l}]$} & $<0,1$ & $<0,1$ & $<0,1$ & 0,17 & $<0,1$ & 69 & $<0,1$ & $<0,1$ \\
\hline $\mathbf{P r}$ & {$[\mu \mathrm{g} / \mathrm{I}]$} & $<0,1$ & $<0,1$ & $<0,1$ & $<0,1$ & $<0,1$ & 4,7 & $<0,1$ & $<0,1$ \\
\hline Nd & {$[\mu \mathrm{g} / \mathrm{I}]$} & 0,17 & 0,17 & $<0,1$ & 0,14 & $<0,1$ & 15 & 0,14 & 0,19 \\
\hline Sm & {$[\mu \mathrm{g} / \mathrm{I}]$} & 0,14 & 0,12 & $<0,1$ & $<0,1$ & 0.26 & 1,1 & 0,16 & 0,16 \\
\hline Eu & {$[\mu \mathrm{g} / \mathrm{I}]$} & $<0,1$ & $<0,1$ & $<0,1$ & $<0,1$ & $<0,1$ & 0,28 & $<0,1$ & $<0,1$ \\
\hline Gd & {$[\mu \mathrm{g} / \mathrm{I}]$} & $<0,1$ & $<0,1$ & $<0,1$ & $<0,1$ & $<0,1$ & 2,9 & $<0,1$ & $<0,1$ \\
\hline Tb & {$[\mu \mathrm{g} / \mathrm{I}]$} & $<0,1$ & $<0,1$ & $<0,1$ & $<0,1$ & $<0,1$ & 0,50 & $<0,1$ & $<0,1$ \\
\hline Dy & {$[\mu \mathrm{g} / \mathrm{I}]$} & $<0,1$ & $<0,1$ & $<0,1$ & $<0,1$ & $<0,1$ & 2,2 & $<0,1$ & 0,10 \\
\hline Ho & {$[\mu \mathrm{g} / \mathrm{I}]$} & $<0,1$ & $<0,1$ & $<0,1$ & $<0,1$ & $<0,1$ & 0,78 & $<0,1$ & $<0,1$ \\
\hline $\mathbf{E r}$ & {$[\mu \mathrm{g} / \mathrm{l}]$} & $<0,1$ & $<0,1$ & $<0,1$ & $<0,1$ & $<0,1$ & 1,4 & $<0,1$ & $<0,1$ \\
\hline Tm & {$[\mu \mathrm{g} / \mathrm{I}]$} & $<0,1$ & $<0,1$ & $<0,1$ & $<0,1$ & $<0,1$ & 0,17 & $<0,1$ & $<0,1$ \\
\hline $\mathbf{Y b}$ & {$[\mu \mathrm{g} / \mathrm{l}]$} & $<0,1$ & $<0,1$ & $<0,1$ & $<0,1$ & $<0,1$ & 0,45 & $<0,1$ & $<0,1$ \\
\hline Lu & {$[\mu \mathrm{g} / \mathrm{I}]$} & $<0,1$ & $<0,1$ & $<0,1$ & $<0,1$ & $<0,1$ & 0,11 & $<0,1$ & $<0,1$ \\
\hline Hf & {$[\mu \mathrm{g} / \mathrm{I}]$} & $<0,1$ & $<0,1$ & $<0,1$ & $<0,1$ & $<0,1$ & $<0,1$ & $<0,1$ & $<0,1$ \\
\hline Ta & {$[\mu \mathrm{g} / \mathrm{I}]$} & 0,15 & 0,77 & 0,61 & 0,23 & 0,13 & $<0,1$ & $<0,1$ & 0,24 \\
\hline $\mathbf{W}$ & {$[\mu \mathrm{g} / \mathrm{I}]$} & $<0,1$ & $<0,1$ & 0,10 & 0,12 & $<0,1$ & $<0,1$ & 0,15 & 0,12 \\
\hline $\mathrm{Hg}$ & {$[\mu \mathrm{g} / \mathrm{I}]$} & 0,20 & 0,16 & 0,22 & 0,26 & 0,44 & 0,17 & 0,36 & 0,10 \\
\hline TI & {$[\mu \mathrm{g} / \mathrm{I}]$} & $<0,1$ & 0,12 & $<0,1$ & 0,13 & $<0,1$ & $<0,1$ & $<0,1$ & 0,10 \\
\hline $\mathbf{P b}$ & {$[\mu \mathrm{g} / \mathrm{I}]$} & 0,13 & 0,30 & 0,37 & 0,34 & $<0,1$ & 0,41 & 0,20 & 0,13 \\
\hline $\mathrm{Bi}$ & {$[\mu \mathrm{g} / \mathrm{l}]$} & $<0,1$ & $<0,1$ & $<0,1$ & $<0,1$ & $<0,1$ & $<0,1$ & $<0,1$ & $<0,1$ \\
\hline Th & {$[\mu \mathrm{g} / \mathrm{I}]$} & $<0,1$ & $<0,1$ & $<0,1$ & 0,10 & $<0,1$ & $<0,1$ & $<0,1$ & $<0,1$ \\
\hline $\mathbf{U}$ & {$[\mu \mathrm{g} / \mathrm{I}]$} & 1,1 & 0,90 & 0,89 & 4,0 & $<0,1$ & 4,8 & 0,14 & 11 \\
\hline
\end{tabular}


Tabelle A.1. (Forts.): Analysendaten der Porenwässer und Wasserproben aus Cospuden.

\begin{tabular}{|c|c|c|c|c|c|c|c|c|}
\hline & & CML 43,5 m & RCO3 & RCO4 & RCO12 & RCO15 & RCO17 & RCO18 \\
\hline & & Wasserprobe & Wasserprobe & Wasserprobe & Wasserprobe & Wasserprobe & Wasserprobe & Wasserprobe \\
\hline & & Jan 2000 & Jan 2000 & Jan 2000 & Jan 2000 & Jan 2000 & Jan 2000 & Jan 2000 \\
\hline $\mathbf{p H}$ & & 7,0 & 2,5 & 6,7 & 7,0 & 6,9 & 6,4 & 7,2 \\
\hline el Lf & {$[\mathrm{mS} / \mathrm{cm}]$} & 1,7 & 2,8 & 1,7 & 2,2 & 2,2 & 1,2 & 1,8 \\
\hline Redox & {$[\mathrm{mV}]$} & 226 & 467 & 226 & 205 & 204 & 205 & 207 \\
\hline Sulfat & {$[\mathrm{g} / \mathrm{l}]$} & 1,1 & 3,1 & 2,6 & 3,7 & 1,2 & 1,6 & 1,4 \\
\hline DOC & {$[\mathrm{mg} / \mathrm{ll}$} & 115,0 & 7,8 & 36,3 & 43,4 & 53,1 & 13,3 & 41,8 \\
\hline $\mathbf{L i}$ & {$[\mu \mathrm{g} / \mathrm{l}]$} & 480 & 640 & 640 & 43 & 180 & 170 & 450 \\
\hline $\mathrm{Be}$ & {$[\mu \mathrm{g} / \mathrm{I}]$} & $<0,1$ & 0,31 & $<0,1$ & 9,4 & $4<0,1$ & $<0,1$ & $<0,1$ \\
\hline $\mathbf{N a}$ & {$[\mu \mathrm{g} / \mathrm{ll}]$} & 70000 & 63000 & 88000 & 12000 & 65000 & 53000 & 43000 \\
\hline $\mathbf{M g}$ & {$[\mu \mathrm{g} / \mathrm{ll}]$} & 170000 & 370000 & 400000 & 67000 & 150000 & 130000 & 130000 \\
\hline Al & {$[\mu \mathrm{g} / \mathrm{I}]$} & 3,9 & 1500 & 5,1 & 22 & $2<1$ & 24 & $4<1$ \\
\hline $\mathbf{K}$ & {$[\mu \mathrm{g} / \mathrm{ll}]$} & 15000 & 34000 & 18300 & 1700 & 12000 & 12000 & 14000 \\
\hline $\mathbf{C a}$ & {$[\mu \mathrm{g} / \mathrm{l}]$} & 410000 & 530000 & 610000 & 150000 & 490000 & 540000 & 540000 \\
\hline Sc & {$[\mu \mathrm{g} / \mathrm{l}]$} & 0,12 & $<0,1$ & $<0,1$ & $<0,1$ & $<0,1$ & 0,16 & 60,1 \\
\hline Ti & {$[\mu \mathrm{g} / \mathrm{l}]$} & $<1$ & $<1$ & $<1$ & $<1$ & $<1$ & $<1$ & $<1$ \\
\hline $\mathbf{V}$ & {$[\mu \mathrm{g} / \mathrm{l}]$} & 0,75 & 4,7 & 0,33 & 0,12 & 0,59 & 0,71 & 0,94 \\
\hline $\mathrm{Cr}$ & {$[\mu \mathrm{g} / \mathrm{I}]$} & $<0,1$ & 2,8 & 1,3 & 1,3 & 1,3 & 1,7 & 0,48 \\
\hline Mn & {$[\mu \mathrm{g} / \mathrm{I}]$} & 920 & 9100 & 4300 & 790 & 1400 & 8,3 & 1000 \\
\hline $\mathrm{Fe}$ & {$[\mu \mathrm{g} / \mathrm{I}]$} & 25 & 290070 & & $0<5$ & 10 & 126 & 25 \\
\hline Co & {$[\mu \mathrm{g} / \mathrm{I}]$} & $<0,1$ & $k 0,1$ & & $1<0,1$ & 0,55 & $<0,1$ & $k 0,1$ \\
\hline $\mathbf{N i}$ & {$[\mu \mathrm{g} / \mathrm{I}]$} & $<0,1$ & $<0,1$ & & $4<0,1$ & 2,0 & $<0,1$ & $k 0,1$ \\
\hline $\mathbf{C u}$ & {$[\mu \mathrm{g} / \mathrm{I}]$} & 1,8 & 4,5 & 2,3 & 0,61 & 20 & 2,6 & 1,5 \\
\hline $\mathbf{Z n}$ & {$[\mu \mathrm{g} / \mathrm{I}]$} & 3,8 & 110 & 3,6 & 7,8 & 12 & 8,2 & 5,0 \\
\hline As & {$[\mu \mathrm{g} / \mathrm{l}]$} & $<0,1$ & 4,1 & 0,27 & 0,75 & 1,6 & 0,69 & 水 $<0,1$ \\
\hline $\mathbf{R b}$ & {$[\mu \mathrm{g} / \mathrm{l}]$} & 10 & 26 & 18 & 8,9 & 22 & 28 & 0,19 \\
\hline $\mathbf{S r}$ & {$[\mu \mathrm{g} / \mathrm{l}]$} & 5100 & 7200 & 7000 & 5600 & 5100 & 7100 & 6900 \\
\hline $\mathbf{Y}$ & {$[\mu \mathrm{g} / \mathrm{l}]$} & $<0,1$ & 3,1 & $<0,1$ & $<0,1$ & $<0,1$ & 0,49 & 水 0,1 \\
\hline $\mathbf{Z r}$ & {$[\mu \mathrm{g} / \mathrm{l}]$} & 3,1 & 15 & 3,3 & 6,6 & 3,4 & 6,8 & 3,2 \\
\hline $\mathbf{N b}$ & {$[\mu \mathrm{g} / \mathrm{l}]$} & 0,24 & 0,17 & 0,18 & 0,16 & 水 $<0,1$ & 0,64 & $4<0,1$ \\
\hline Mo & {$[\mu \mathrm{g} / \mathrm{l}]$} & 5,2 & 0,20 & 0,43 & 1,0 & 0,69 & 1,5 & 0,25 \\
\hline Cd & {$[\mu \mathrm{g} / \mathrm{I}]$} & 0,29 & 0,16 & 0,12 & $2<0,1$ & $<0,1$ & 0,29 & 水 0,1 \\
\hline Sn & {$[\mu \mathrm{g} / \mathrm{l}]$} & 3,7 & 0,22 & 1,1 & 0,47 & 0,36 & 1,8 & 0,26 \\
\hline Sb & {$[\mu \mathrm{g} / \mathrm{l}]$} & 0,26 & $6<0,1$ & $<0,1$ & $<0,1$ & $<0,1$ & 0,39 & 水 $<0,1$ \\
\hline Cs & {$[\mu \mathrm{g} / \mathrm{l}]$} & 0,11 & 0,44 & 0,11 & $\mid<0,1$ & $<0,1$ & 1,2 & $2<0,1$ \\
\hline $\mathrm{Ba}$ & {$[\mu \mathrm{g} / \mathrm{l}]$} & 8,3 & 25 & 6,1 & 2,6 & 16 & 20 & 17 \\
\hline La & {$[\mu \mathrm{g} / \mathrm{l}]$} & 0,28 & 46 & 0,15 & $5<0,1$ & $<0,1$ & 1,6 & $6<0,1$ \\
\hline Ce & {$[\mu \mathrm{g} / \mathrm{l}]$} & 0,25 & 50 & 0,11 & $1<0,1$ & 0,30 & 1,3 & $3<0,1$ \\
\hline $\mathbf{P r}$ & {$[\mu \mathrm{g} / \mathrm{I}]$} & 0,10 & 4,3 & $3<0,1$ & $<0,1$ & $k 0,1$ & 0,54 & $4<0,1$ \\
\hline Nd & {$[\mu \mathrm{g} / \mathrm{l}]$} & 1,7 & 15 & 0,34 & $4<0,1$ & 0,13 & 5,5 & 0,18 \\
\hline Sm & {$[\mu \mathrm{g} / \mathrm{I}]$} & 0,78 & 1,7 & 0,5 & 0,13 & 0,18 & 2,7 & 0,18 \\
\hline Eu & {$[\mu \mathrm{g} / \mathrm{l}]$} & 0,29 & 0,40 & $<<0,1$ & $<0,1$ & $<0,1$ & 0,96 & $6<0,1$ \\
\hline Gd & {$[\mu \mathrm{g} / \mathrm{l}]$} & 0,73 & 3,2 & 0,18 & $3<0,1$ & $<0,1$ & 1,4 & $4<0,1$ \\
\hline Tb & {$[\mu \mathrm{g} / \mathrm{l}]$} & 0,14 & 0,59 & $<<0,1$ & $<0,1$ & $<0,1$ & 0,34 & $4<0,1$ \\
\hline Dy & {$[\mu \mathrm{g} / \mathrm{l}]$} & 0,42 & 2,3 & 0,15 & $5<0,1$ & $\mid<0,1$ & 2,3 & $3<0,1$ \\
\hline Ho & {$[\mu \mathrm{g} / \mathrm{I}]$} & 0,14 & 0,78 & 0,11 & $1<0,1$ & $<0,1$ & 0,72 & $2<0,1$ \\
\hline $\mathbf{E r}$ & {$[\mu \mathrm{g} / \mathrm{l}]$} & 0,33 & 1,4 & $4<0,1$ & $<0,1$ & $<0,1$ & 0,91 & $1<0,1$ \\
\hline Tm & {$[\mu \mathrm{g} / \mathrm{I}]$} & $<0,1$ & 0,18 & $3<0,1$ & $<0,1$ & $<0,1$ & 0,28 & $3<0,1$ \\
\hline $\mathbf{Y b}$ & {$[\mu \mathrm{g} / \mathrm{l}]$} & 0,31 & 0,61 & 0,23 & $3<0,1$ & $<0,1$ & 0,57 & $7<0,1$ \\
\hline $\mathbf{L u}$ & {$[\mu \mathrm{g} / \mathrm{l}]$} & $<0,1$ & 0,14 & $4<0,1$ & $<0,1$ & $<0,1$ & 0,26 & $6<0,1$ \\
\hline Hf & {$[\mu \mathrm{g} / \mathrm{I}]$} & 0,34 & $4<0,1$ & 0,15 & $5<0,1$ & $<0,1$ & 0,48 & $3<0,1$ \\
\hline Ta & {$[\mu \mathrm{g} / \mathrm{l}]$} & 0,44 & $<<0,1$ & 0,42 & 0,38 & $3<0,1$ & 1,8 & 0,15 \\
\hline $\mathbf{W}$ & {$[\mu \mathrm{g} / \mathrm{l}]$} & 0,27 & 0,45 & 0,21 & $1<0,1$ & $<0,1$ & 0,69 & 0,10 \\
\hline $\mathrm{Hg}$ & {$[\mu \mathrm{g} / \mathrm{l}]$} & 0,14 & 0,29 & 0,23 & 0,11 & 0,29 & 0,22 & 0,36 \\
\hline TI & {$[\mu \mathrm{g} / \mathrm{I}]$} & 0,38 & $3<0,1$ & 0,39 & $8<0,1$ & $<0,1$ & 2,4 & 0,11 \\
\hline $\mathbf{P b}$ & {$[\mu \mathrm{g} / \mathrm{I}]$} & 0,19 & 0,32 & 0,31 & 0,16 & 0,23 & 1,0 & $\vec{b}<0,1$ \\
\hline $\mathbf{B i}$ & {$[\mu \mathrm{g} / \mathrm{I}]$} & $<0,1$ & $k 0,1$ & $k 0,1$ & $<0,1$ & $k 0,1$ & 0,18 & $8<0,1$ \\
\hline Th & {$[\mu \mathrm{g} / \mathrm{I}]$} & 0,35 & $\overrightarrow{5}<0,1$ & $<0,1$ & $<0,1$ & $<0,1$ & 0,97 & $7<0,1$ \\
\hline $\mathbf{U}$ & {$[\mu \mathrm{g} / \mathrm{l}]$} & 7,5 & $5<0,1$ & 2,5 & 3,8 & 0,79 & 0,63 & $3<0,1$ \\
\hline
\end{tabular}


Tabelle A.2.: Analysendaten der Porenwässer aus der Lausitz

\begin{tabular}{|c|c|c|c|c|c|c|c|c|}
\hline & & \multicolumn{2}{|c|}{ Lausitz 5,00 mLausitz 5,21 m } & \multicolumn{2}{|c|}{\begin{tabular}{|l|l|} 
Lausitz 5,30 m & Lausitz 5,50 m \\
\end{tabular}} & \multicolumn{3}{|c|}{\begin{tabular}{|l|l|l|} 
Lausitz 5,60 m & Lausitz 5,75 m & Lausitz 5,93 m \\
\end{tabular}} \\
\hline & & Porenlösung & Porenlösung & Porenlösung & Porenlösung & Porenlösung & Porenlösung & Porenlösung \\
\hline & & Okt 1999 & Okt 1999 & Okt 1999 & Okt 1999 & Okt 1999 & Okt 1999 & Okt 1999 \\
\hline $\mathrm{pH}$ & & 3,4 & 3,4 & 3,5 & 3,5 & 3,3 & 3,3 & 3,4 \\
\hline el Lf & {$[\mathrm{mS} / \mathrm{cm}]$} & 2,9 & $3,0 \mathrm{r}$ & $n \mathrm{nb}$ & 2,4 & 2,8 & 2,9 & 2,4 \\
\hline Redox & {$[\mathrm{mV}]$} & 432 & 431 & 423 & 425 & 433 & 434 & 429 \\
\hline Sulfat & {$[\mathrm{g} / \mathrm{l}]$} & 3,4 & 3,4 & 3,1 & 3,3 & 3,7 & 3,4 & 3,2 \\
\hline DOC & {$[\mathrm{mg} / \mathrm{ll}]$} & 22,3 & & $\mathrm{nb}$ & 30,5 & 32,9 & 42,6 & $6 \operatorname{lnb}$ \\
\hline $\mathbf{L i}$ & {$[\mu \mathrm{g} / \mathrm{l}]$} & 430 & 440 & 400 & 380 & 410 & 400 & 320 \\
\hline $\mathrm{Be}$ & {$[\mu \mathrm{g} / \mathrm{l}]$} & 120 & 120 & 90 & 80 & 90 & 90 & 50 \\
\hline $\mathrm{Na}$ & {$[\mu \mathrm{g} / \mathrm{l}]$} & 27000 & 27000 & 25000 & 23000 & 26000 & 25000 & 23000 \\
\hline $\mathbf{M g}$ & {$[\mu \mathrm{g} / \mathrm{l}]$} & 120000 & 110000 & 110000 & 110000 & 120000 & 120000 & 110000 \\
\hline$\overline{A l}$ & {$[\mu \mathrm{g} / \mathrm{l}]$} & 370000 & 360000 & 270000 & 270000 & 330000 & 340000 & 190000 \\
\hline $\mathbf{K}$ & {$[\mu \mathrm{g} / \mathrm{l}]$} & 32000 & 32000 & 32000 & 32000 & 32000 & 34000 & 34000 \\
\hline Ca & {$[\mu \mathrm{g} / \mathrm{ll}]$} & 520000 & 560000 & 510000 & 470000 & 520000 & 480000 & 430000 \\
\hline Sc & {$[\mu \mathrm{g} / \mathrm{l}]$} & 2,7 & 3,1 & 1,3 & 1,3 & 3,3 & 11 & 4,3 \\
\hline$T \mathbf{T i}$ & {$[\mu \mathrm{g} / \mathrm{ll}]$} & 9,4 & 8,9 & 4,7 & 8,4 & 7,1 & 9,2 & 9,5 \\
\hline $\mathbf{V}$ & {$[\mu \mathrm{g} / \mathrm{ll}]$} & 27 & 23 & 66 & 40 & 110 & 130 & 220 \\
\hline $\mathbf{C r}$ & {$[\mu \mathrm{g} / \mathrm{ll}]$} & 17 & 26 & 17 & 17 & 21 & 46 & 17 \\
\hline Mn & {$[\mu \mathrm{g} / \mathrm{l}]$} & 8400 & 8700 & 8100 & 8400 & 9000 & 9300 & 8100 \\
\hline $\mathbf{F e}$ & {$[\mu \mathrm{g} / \mathrm{l}]$} & 51420 & 53490 & 155030 & 247430 & 219430 & 104520 & 468990 \\
\hline Co & {$[\mu \mathrm{g} / \mathrm{l}]$} & 730 & 670 & 850 & 990 & 3000 & 1400 & 1400 \\
\hline $\mathbf{N i}$ & {$[\mu \mathrm{g} / \mathrm{l}]$} & 980 & 1100 & 1200 & 1600 & 3700 & 1500 & 1800 \\
\hline $\mathbf{C u}$ & {$[\mu \mathrm{g} / \mathrm{l}]$} & 35 & 83 & 50 & 61 & 17 & 97 & 17 \\
\hline $\mathbf{Z n}$ & {$[\mu \mathrm{g} / \mathrm{l}]$} & 5100 & 5800 & 7100 & 9100 & 4500 & 9300 & 5000 \\
\hline As & {$[\mu \mathrm{g} / \mathrm{l}]$} & 18 & 28 & 35 & 30 & 96 & 55 & 590 \\
\hline $\mathbf{R b}$ & {$[\mu \mathrm{g} / \mathrm{l}]$} & 510 & 480 & 560 & 520 & 590 & 480 & 380 \\
\hline $\mathbf{S r}$ & {$[\mu \mathrm{g} / \mathrm{l}]$} & 6100 & 6500 & 5800 & 5800 & 6500 & 4500 & 4100 \\
\hline $\mathbf{Y}$ & {$[\mu \mathrm{g} / \mathrm{l}]$} & 400 & 340 & 320 & 340 & 420 & 440 & 300 \\
\hline $\mathbf{Z r}$ & {$[\mu \mathrm{g} / \mathrm{ll}]$} & 8,2 & 8,2 & 16 & 19 & 20 & 15 & 38 \\
\hline $\mathbf{N b}$ & {$[\mu \mathrm{g} / \mathrm{l}]$} & 0,19 & 0,16 & 16 & 5,6 & 1,2 & 0,23 & 0,40 \\
\hline Mo & {$[\mu \mathrm{g} / \mathrm{l}]$} & 0,19 & 2,1 & 8,0 & 0,36 & 0,37 & 0,27 & 0,58 \\
\hline Cd & {$[\mu \mathrm{g} / \mathrm{l}]$} & 16 & 21 & 20 & 29 & 13 & 12 & 11 \\
\hline Sn & {$[\mu \mathrm{g} / \mathrm{l}]$} & $<0,1$ & $\begin{array}{ll}0,13 \\
\end{array}$ & 0,16 & 0,16 & 0,16 & 0,16 & 0,15 \\
\hline $\mathbf{S b}$ & {$[\mu \mathrm{g} / \mathrm{ll}]$} & $<0,1$ & 0,18 & 0,18 & $\begin{array}{l}0,17 \\
\end{array}$ & 0,19 & 0,15 & 0,37 \\
\hline Cs & {$[\mu \mathrm{g} / \mathrm{ll}]$} & 3,9 & 3,1 & 3,7 & 3,5 & 3,3 & 2,0 & 2,2 \\
\hline $\mathbf{B a}$ & {$[\mu \mathrm{g} / \mathrm{l}]$} & 38 & 36 & 32 & 40 & 41 & 32 & 48 \\
\hline La & {$[\mu \mathrm{g} / \mathrm{ll}]$} & 510 & 520 & 520 & 540 & 540 & 370 & 280 \\
\hline $\mathbf{C e}$ & {$[\mu \mathrm{g} / \mathrm{l}]$} & 940 & 910 & 1000 & 1100 & 1200 & 920 & 650 \\
\hline $\mathbf{P r}$ & {$[\mu \mathrm{g} / \mathrm{l}]$} & 79 & 78 & 83 & 84 & 100 & 83 & 59 \\
\hline Nd & {$[\mu \mathrm{g} / \mathrm{l}]$} & 270 & 260 & 280 & 290 & 340 & 280 & 200 \\
\hline Sm & {$[\mu \mathrm{g} / \mathrm{l}]$} & 47 & 46 & 44 & 44 & 51 & 46 & 32 \\
\hline Eu & {$[\mu \mathrm{g} / \mathrm{l}]$} & 12 & 11 & 11 & 11 & 13 & 12 & 8,3 \\
\hline Gd & {$[\mu \mathrm{g} / \mathrm{l}]$} & 46 & 44 & 43 & 42 & 49 & 49 & 35 \\
\hline Tb & {$[\mu \mathrm{g} / \mathrm{l}]$} & 8,9 & 8,3 & 8,4 & 8,4 & 10 & 9,6 & 6,9 \\
\hline Dy & {$[\mu \mathrm{g} / \mathrm{l}]$} & 37 & 35 & 35 & 34 & 41 & 42 & 30 \\
\hline Ho & {$[\mu \mathrm{g} / \mathrm{l}]$} & 9,5 & 8,6 & 8,5 & 8,4 & 10 & 11 & 7,5 \\
\hline Er & {$[\mu \mathrm{g} / \mathrm{l}]$} & 19 & 17 & 17 & 16 & 20 & 21 & 15 \\
\hline Tm & {$[\mu \mathrm{g} / \mathrm{l}]$} & 3,0 & 2,8 & 2,7 & 2,6 & 3,1 & 3,4 & 2,3 \\
\hline $\mathbf{Y b}$ & {$[\mu \mathrm{g} / \mathrm{l}]$} & 13 & 13 & 11 & 11 & 12 & 14 & 9,4 \\
\hline $\mathbf{L u}$ & {$[\mu \mathrm{g} / \mathrm{l}]$} & 2,2 & 2,1 & 1,9 & 1,8 & 2,1 & 2,4 & 1,6 \\
\hline Hf & {$[\mu \mathrm{g} / \mathrm{l}]$} & 0,11 & 0,13 & 0,14 & 0,12 & 0,21 & 0,15 & 0,11 \\
\hline Ta & {$[\mu \mathrm{g} / \mathrm{ll}]$} & 0,46 & 0,31 & 19 & 6,5 & 1,8 & 0,73 & 0,92 \\
\hline $\mathbf{W}$ & {$[\mu \mathrm{g} / \mathrm{ll}]$} & 0,23 & 0,25 & 0,39 & 0,37 & 0,42 & 0,25 & 1,4 \\
\hline $\mathrm{Hg}$ & {$[\mu \mathrm{g} / \mathrm{l}]$} & $\mathrm{nb}$ & nb & nb & nb & $n$ & nb & $n b$ \\
\hline Tl & {$[\mu \mathrm{g} / \mathrm{l}]$} & 6,7 & 4,8 & 3,7 & 3,3 & 2,1 & 8,1 & 0,92 \\
\hline $\mathbf{P b}$ & {$[\mu \mathrm{g} / \mathrm{l}]$} & 57 & 67 & 65 & 81 & 88 & 160 & 160 \\
\hline $\mathbf{B i}$ & {$[\mu \mathrm{g} / \mathrm{l}]$} & $\mathrm{nb}$ & nb & $n b$ & $n b$ & $n b$ & nb & $\mathrm{nb}$ \\
\hline Th & {$[\mu \mathrm{g} / \mathrm{I}]$} & 0,82 & 1,8 & 1,1 & 0,68 & 1,4 & 2,7 & 1,3 \\
\hline$\overline{\mathbf{U}}$ & {$[\mu \mathrm{g} / \mathrm{l}]$} & 1,1 & 1,9 & 1,4 & 1,4 & 2,2 & \begin{tabular}{|l|}
5,8 \\
\end{tabular} & 2,5 \\
\hline
\end{tabular}


Tabelle A.2. (Forts.): Analysendaten der Porenwässer aus der Lausitz.

\begin{tabular}{|c|c|c|c|c|c|c|c|}
\hline & & Lausitz 21,07 m I & Lausitz 21,13 m & 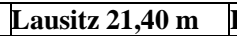 & Lausitz 21,70 m & Lausitz 21,80 m & Lausitz 21,90 m \\
\hline & & Porenlösung & Porenlösung & Porenlösung & Porenlösung & Porenlösung & Porenlösung \\
\hline & & Okt 1999 & Okt 1999 & Okt 1999 & Okt 1999 & Okt 1999 & Okt 1999 \\
\hline pH & & 3,9 & 3,8 & 3,7 & 3,9 & 3,8 & 2,7 \\
\hline el Lf & {$[\mathrm{mS} / \mathrm{cm}]$} & 2,1 & 1,8 & 2,1 & 2,2 & 2,3 & 2,7 \\
\hline Redox & {$[\mathrm{mV}]$} & 401 & 409 & 403 & 405 & 410 & 471 \\
\hline Sulfat & {$[\mathrm{g} / \mathrm{l}]$} & 2,5 & 2,9 & 2,4 & 2,4 & 2,2 & 2,5 \\
\hline DOC & {$[\mathrm{mg} / \mathrm{l}]$} & 14,3 & 14,6 & 28,8 & 15,3 & 19,1 & 8,0 \\
\hline $\mathbf{L i}$ & {$[\mu \mathrm{g} / \mathrm{l}]$} & 180 & 240 & 200 & 190 & 170 & 200 \\
\hline Be & {$[\mu \mathrm{g} / \mathrm{l}]$} & 10 & 20 & 20 & 9,5 & 13 & 15 \\
\hline $\mathbf{N a}$ & {$[\mu \mathrm{g} / \mathrm{I}]$} & 39000 & 50000 & 42000 & 27000 & 26000 & 40000 \\
\hline Mg & {$[\mu \mathrm{g} / \mathrm{l}]$} & 80000 & 110000 & 120000 & 91000 & 87000 & 110000 \\
\hline Al & {$[\mu \mathrm{g} / \mathrm{I}]$} & 23000 & 35000 & 54000 & 20000 & 30000 & 56000 \\
\hline $\mathbf{K}$ & {$[\mu \mathrm{g} / \mathrm{I}]$} & 24000 & 44000 & 64000 & 25000 & 24000 & 36000 \\
\hline $\mathbf{C a}$ & {$[\mu \mathrm{g} / \mathrm{I}]$} & 570000 & 760000 & 660000 & 560000 & 530000 & 630000 \\
\hline Sc & {$[\mu \mathrm{g} / \mathrm{l}]$} & $k 0,1$ & $<0,1$ & $<0,1$ & 0,30 & $<0,1$ & 0,59 \\
\hline Ti & {$[\mu \mathrm{g} / \mathrm{l}]$} & $<1$ & $<1$ & $<1$ & 0,55 & & $2<1$ \\
\hline $\mathbf{V}$ & {$[\mu \mathrm{g} / \mathrm{I}]$} & 6,8 & $<0,1$ & $<0,1$ & 4,7 & 6,9 & 0,81 \\
\hline $\mathrm{Cr}$ & {$[\mu \mathrm{g} / \mathrm{I}]$} & 7,8 & 7,6 & 4,3 & 1,0 & 0,70 & 4,3 \\
\hline Mn & {$[\mu \mathrm{g} / \mathrm{I}]$} & 8300 & 10000 & 7500 & 6900 & 6500 & 8600 \\
\hline Fe & {$[\mu \mathrm{g} / \mathrm{I}]$} & 294650 & 122020 & 97170 & 228890 & 163860 & 68260 \\
\hline Co & {$[\mu \mathrm{g} / \mathrm{l}]$} & 220 & 560 & 530 & 210 & 480 & 390 \\
\hline $\mathbf{N i}$ & {$[\mu \mathrm{g} / \mathrm{l}]$} & 480 & 930 & 670 & 330 & 370 & 550 \\
\hline $\mathbf{C u}$ & {$[\mu \mathrm{g} / \mathrm{l}]$} & 5,7 & 46 & 31 & $<0,1$ & $<0,1$ & 23 \\
\hline $\mathbf{Z n}$ & {$[\mu \mathrm{g} / \mathrm{l}]$} & 4500 & 5600 & 5400 & 2600 & 2300 & 4300 \\
\hline As & {$[\mu \mathrm{g} / \mathrm{ll}]$} & 120 & 19 & 14 & 52 & 32 & 0,95 \\
\hline $\mathbf{R b}$ & {$[\mu \mathrm{g} / \mathrm{l}]$} & 150 & 140 & 150 & 140 & 140 & 150 \\
\hline $\mathbf{S r}$ & {$[\mu \mathrm{g} / \mathrm{l}]$} & 4900 & 5600 & 5000 & 3500 & 3300 & 3800 \\
\hline $\mathbf{Y}$ & {$[\mu \mathrm{g} / \mathrm{l}]$} & 70 & 150 & 140 & 71 & 83 & 110 \\
\hline $\mathbf{Z r}$ & {$[\mu \mathrm{g} / \mathrm{l}]$} & 16 & 2,0 & 8,5 & 13 & 8,1 & 5,9 \\
\hline Nb & {$[\mu \mathrm{g} / \mathrm{I}]$} & 0,17 & 0,22 & 0,23 & 0,19 & 0,17 & 2,4 \\
\hline Mo & {$[\mu \mathrm{g} / \mathrm{l}]$} & 14 & 16 & 0,57 & 6,0 & & $<0,1$ \\
\hline Cd & {$[\mu \mathrm{g} / \mathrm{I}]$} & 2,2 & 5,1 & 4,2 & 0,90 & 2,0 & 4,9 \\
\hline Sn & {$[\mu \mathrm{g} / \mathrm{l}]$} & $<0,1$ & $<0,1$ & $<0,1$ & 0,12 & $<0,1$ & 0,16 \\
\hline Sb & {$[\mu \mathrm{g} / \mathrm{l}]$} & $<0,1$ & 0,17 & 0,15 & $<0,1$ & $<0,1$ & $<0,1$ \\
\hline Cs & {$[\mu \mathrm{g} / \mathrm{l}]$} & 0,98 & 1,6 & 2,2 & 1,2 & 1,2 & 1,2 \\
\hline $\mathbf{B a}$ & {$[\mu \mathrm{g} / \mathrm{l}]$} & 60 & 160 & 93 & 54 & 51 & 72 \\
\hline La & {$[\mu \mathrm{g} / \mathrm{l}]$} & 80 & 170 & 230 & 89 & 87 & 110 \\
\hline $\mathrm{Ce}$ & {$[\mu \mathrm{g} / \mathrm{l}]$} & 130 & 310 & 500 & 220 & 320 & 210 \\
\hline Pr & {$[\mu \mathrm{g} / \mathrm{I}]$} & 11 & 29 & 46 & 19 & 29 & 17 \\
\hline Nd & {$[\mu \mathrm{g} / \mathrm{I}]$} & 41 & 110 & 200 & 71 & 107 & 72 \\
\hline $\mathbf{S m}$ & {$[\mu \mathrm{g} / \mathrm{l}]$} & 5,4 & 17,8 & 34 & 10,2 & 14 & 12,5 \\
\hline $\mathbf{E u}$ & {$[\mu \mathrm{g} / \mathrm{I}]$} & 1,5 & 5,1 & 9,4 & 2,6 & 3,7 & 3,2 \\
\hline Gd & {$[\mu \mathrm{g} / \mathrm{l}]$} & 6,8 & 22 & 39 & 12 & 19 & 12 \\
\hline Tb & {$[\mu \mathrm{g} / \mathrm{l}]$} & 0,97 & 2,9 & 5,5 & 1,7 & 2,5 & 2,3 \\
\hline Dy & {$[\mu \mathrm{g} / \mathrm{l}]$} & 5,4 & 18 & 33 & 9,3 & 15 & 10 \\
\hline Ho & {$[\mu \mathrm{g} / \mathrm{l}]$} & 1,2 & 3,6 & 6,8 & 2,0 & 3,1 & 2,6 \\
\hline $\mathbf{E r}$ & {$[\mu \mathrm{g} / \mathrm{l}]$} & 3,0 & 9,8 & 18 & 5,0 & 8,6 & 5,3 \\
\hline Tm & {$[\mu \mathrm{g} / \mathrm{I}]$} & 0,33 & 1,2 & 2,1 & 0,55 & 0,91 & 0,94 \\
\hline Yb & {$[\mu \mathrm{g} / \mathrm{I}]$} & 2,3 & 7,2 & 13 & 3,6 & 5,5 & 4,1 \\
\hline Lu & {$[\mu \mathrm{g} / \mathrm{I}]$} & 0,24 & 0,80 & 1,4 & 0,44 & 0,51 & 0,74 \\
\hline Hf & {$[\mu \mathrm{g} / \mathrm{I}]$} & $<0,1$ & $<0,1$ & $<0,1$ & $<0,1$ & $k 0,1$ & $<0,1$ \\
\hline Ta & {$[\mu \mathrm{g} / \mathrm{I}]$} & $<0,1$ & 0,16 & 0,18 & $<0,1$ & $<0,1$ & 3,6 \\
\hline$\overline{\mathbf{W}}$ & {$[\mu \mathrm{g} / \mathrm{I}]$} & 0,93 & 0,18 & 0,27 & 0,30 & 0,14 & 0,14 \\
\hline $\mathrm{Hg}$ & {$[\mu \mathrm{g} / \mathrm{l}]$} & $<0,1$ & 0,13 & $3<0,1$ & $<0,1$ & $<0,1$ & $\mathrm{nb}$ \\
\hline TI & {$[\mu \mathrm{g} / \mathrm{l}]$} & 0,53 & 2,9 & 7,3 & 1,2 & 0,95 & 1,7 \\
\hline $\mathbf{P b}$ & {$[\mu \mathrm{g} / \mathrm{l}]$} & 9,4 & 36 & 35 & 5,3 & 11 & 22 \\
\hline $\mathrm{Bi}$ & {$[\mu \mathrm{g} / \mathrm{I}]$} & $<0,1$ & $<0,1$ & $<0,1$ & $<0,1$ & $<0,1$ & nb \\
\hline Th & {$[\mu \mathrm{g} / \mathrm{I}]$} & $<0,1$ & 0,18 & 0,30 & $<0,1$ & 0,13 & 0,34 \\
\hline$\overline{\mathbf{U}}$ & {$[\mu \mathrm{g} / \mathrm{I}]$} & 0,43 & 1,2 & 2,6 & 1,2 & 1,1 & 1,4 \\
\hline
\end{tabular}


Tabelle A.3.: Ergebnisse der Ultrafiltration für Porenwässer und Wasserproben aus Cospuden; Konzentrationen in \% im Durchlauf bezogen auf die Ausgangskonzentrationen (Tab. A.1.) in Ultrafiltraten (1 kD) von Porenlösungen und Wasserproben aus Cospuden.

\begin{tabular}{|c|c|c|c|c|c|c|c|c|c|c|}
\hline & & RCO4 36 m & CML 24 m & CML 32 m & RCO4 & RCO10A & RCO12 & RCO13 & RCO14 & Bohrung $1 \mathrm{~m}$ \\
\hline & & Porenlösung & Porenlösung & Porenlösung & Wasserprobe & Wasserprobe & Wasserprobe & Wasserprobe & Wasserprobe & Porenlösung \\
\hline & & Nov 1995 & Aug 1998 & Aug 1998 & Okt 1998 & Okt 1998 & Okt 1998 & Okt 1998 & Okt 1998 & Nov 1998 \\
\hline Sulfat & $\%$ & 97 & $7 \mathrm{nb}$ & $\mathrm{nb}$ & $\mathrm{nb}$ & 371 & $n b$ & & $n b$ & 96 \\
\hline DOC & $\%$ & $n b$ & & $n b$ & 69 & 82 & 105 & 85 & & $n b$ \\
\hline $\mathbf{L i}$ & $\%$ & 90 & 101 & 91 & 97 & 104 & 95 & 88 & 93 & 79 \\
\hline $\mathbf{B e}$ & $\%$ & $\mathrm{nq}$ & 100 & 100 & $\mathrm{nq}$ & nq & nq & nq & nq & 77 \\
\hline $\mathbf{N a}$ & $\%$ & 88 & 100 & 93 & 97 & 106 & 97 & 89 & 91 & 76 \\
\hline Mg & $\%$ & 96 & 100 & 99 & 102 & 108 & 95 & 86 & 4 & 75 \\
\hline Al & $\%$ & nq & 96 & 62 & $\mathrm{nq}$ & nq & nq & nq & nq & 73 \\
\hline $\mathbf{K}$ & $\%$ & 103 & 47 & 55 & 99 & 107 & 96 & 86 & 104 & 76 \\
\hline $\mathbf{C a}$ & $\%$ & 96 & 97 & 97 & 98 & -9 & 92 & 84 & 86 & 74 \\
\hline Sc & $\%$ & 82 & 114 & 100 & $\mathrm{nq}$ & $\mathrm{nq}$ & $\mathrm{nq}$ & & $\mathrm{nq}$ & 91 \\
\hline $\mathrm{Ti}$ & $\%$ & $\mathrm{nq}$ & 7 & 21 & $\operatorname{lnq}$ & $\mathrm{nq}$ & $\mathrm{nq}$ & $\mathrm{nq}$ & $\mathrm{nq}$ & $\mathrm{nq}$ \\
\hline $\mathbf{V}$ & $\%$ & 73 & 92 & 100 & $n$ & $\mathrm{nq}$ & $\mathrm{nq}$ & nq & 99 & 74 \\
\hline $\mathrm{Cr}$ & $\%$ & $\mathrm{nq}$ & 96 & 120 & 105 & -6 & 105 & 100 & 98 & 87 \\
\hline Mn & $\%$ & 92 & 100 & 103 & 97 & 104 & 93 & 88 & 2 & 70 \\
\hline $\mathbf{F e}$ & $\%$ & $\mathrm{nq}$ & 106 & 18 & 67 & 1 & 90 & 18 & 5 & 100 \\
\hline Co & $\%$ & 110 & 100 & 109 & 119 & 0 & 100 & 95 & 96 & 75 \\
\hline $\mathbf{N i}$ & $\%$ & $\mathrm{nq}$ & & $\mathrm{nq}$ & 87 & 106 & 91 & 106 & 98 & 76 \\
\hline $\mathbf{C u}$ & $\%$ & nq & 103 & 95 & 105 & 0 & 85 & 104 & 100 & 100 \\
\hline $\mathbf{Z n}$ & $\%$ & $\mathrm{nq}$ & & $\mathrm{nq}$ & 106 & 117 & 94 & 138 & 80 & 87 \\
\hline As & $\%$ & 82 & 89 & 25 & $\mathrm{nq}$ & $\mathrm{nq}$ & $\mathrm{nq}$ & $\mathrm{nq}$ & $\mathrm{nq}$ & 82 \\
\hline $\mathbf{R b}$ & $\%$ & & $n \mathrm{nb}$ & nb & 105 & 97 & 101 & 100 & 117 & 81 \\
\hline $\mathbf{S r}$ & $\%$ & 99 & 95 & 95 & 99 & 110 & 92 & 83 & 88 & 75 \\
\hline $\mathbf{Y}$ & $\%$ & $\mathrm{nq}$ & 100 & 86 & 97 & 99 & 101 & 100 & 97 & 83 \\
\hline $\mathbf{Z r}$ & $\%$ & 98 & 82 & 100 & 83 & 100 & 101 & 61 & 70 & 73 \\
\hline $\mathbf{N b}$ & $\%$ & & $n \mathrm{nb}$ & nb & $\mathrm{nq}$ & & $\mathrm{nq}$ & nq & $\mathrm{nq}$ & $\mathrm{nq}$ \\
\hline Mo & $\%$ & & $\mathrm{nq}$ & $\mathrm{nq}$ & $\mathrm{nq}$ & & $\mathrm{nq}$ & 94 & 49 & 84 \\
\hline Cd & $\%$ & nq & nb & $\mathrm{nb}$ & nb & nb & $n b$ & nb & $n b$ & 79 \\
\hline Sn & $\%$ & $\mathrm{nq}$ & $n b$ & $\mathrm{nb}$ & $\mathrm{nq}$ & & $\mathrm{nq}$ & 74 & 63 & $\mathrm{nq}$ \\
\hline Sb & $\%$ & & $6 \mathrm{nb}$ & $n b$ & nq & $\mathrm{nq}$ & $\mathrm{nq}$ & $\mathrm{nq}$ & $\mathrm{nq}$ & 81 \\
\hline Cs & $\%$ & & $\mathrm{nb}$ & $\mathrm{nb}$ & $\mathrm{nq}$ & $\mathrm{nq}$ & $\mathrm{nq}$ & $\mathrm{nq}$ & $\mathrm{nq}$ & 82 \\
\hline $\mathbf{B a}$ & $\%$ & 95 & 37 & 97 & 72 & 0 & 91 & & $\mathrm{nq}$ & 84 \\
\hline La & $\%$ & $\mathrm{nq}$ & 96 & 50 & 40 & $\mathrm{nq}$ & & $\mathrm{nq}$ & $\mathrm{nq}$ & 75 \\
\hline $\mathrm{Ce}$ & $\%$ & nq & nb & nb & $\mathrm{nq}$ & $\mathrm{nq}$ & $\mathrm{nq}$ & nq & $\mathrm{nq}$ & 75 \\
\hline $\mathbf{P r}$ & $\%$ & $\mathrm{nq}$ & nb & $\mathrm{nb}$ & $\mathrm{nq}$ & $\mathrm{nq}$ & $\mathrm{nq}$ & nq & $\mathrm{nq}$ & 75 \\
\hline \begin{tabular}{|l|}
$\mathbf{N d}$ \\
\end{tabular} & $\%$ & $\mathrm{nq}$ & nb & nb & nq & $\mathrm{nq}$ & $\mathrm{nq}$ & $\mathrm{nq}$ & $\mathrm{nq}$ & 75 \\
\hline Sm & $\%$ & nq & nb & $\mathrm{nb}$ & $\mathrm{nq}$ & $\mathrm{nq}$ & $\mathrm{nq}$ & $\mathrm{nq}$ & $\mathrm{nq}$ & 77 \\
\hline Eu & $\%$ & $\mathrm{nq}$ & $\mathrm{nq}$ & nq & $\mathrm{nq}$ & $\mathrm{nq}$ & $\mathrm{nq}$ & nq & $\mathrm{nq}$ & 79 \\
\hline Gd & $\%$ & $\mathrm{nq}$ & $\mathrm{nb}$ & $\mathrm{nb}$ & $\mathrm{nq}$ & $\mathrm{nq}$ & $\mathrm{nq}$ & $\mathrm{nq}$ & $\mathrm{nq}$ & 75 \\
\hline Tb & $\%$ & nq & nb & nb & $\mathrm{nq}$ & $\mathrm{nq}$ & $\mathrm{nq}$ & nq & $\mathrm{nq}$ & 79 \\
\hline Dy & $\%$ & nq & nb & nb & $\mathrm{nq}$ & nq & nq & nq & nq & 76 \\
\hline Ho & $\%$ & nq & nb & nb & $\mathrm{nq}$ & $\mathrm{nq}$ & $\mathrm{nq}$ & $\mathrm{nq}$ & $\mathrm{nq}$ & 75 \\
\hline $\mathbf{E r}$ & $\%$ & $\mathrm{nq}$ & nb & $\mathrm{nb}$ & $\mathrm{nq}$ & $\mathrm{nq}$ & $\mathrm{nq}$ & $\mathrm{nq}$ & $\mathrm{nq}$ & 77 \\
\hline Tm & $\%$ & nq & nb & $\mathrm{nb}$ & $\mathrm{nq}$ & $\mathrm{nq}$ & $\mathrm{nq}$ & nq & $\mathrm{nq}$ & 78 \\
\hline $\mathbf{Y b}$ & $\%$ & $\mathrm{nq}$ & nb & $\mathrm{nb}$ & $\mathrm{nq}$ & $\mathrm{nq}$ & $\mathrm{nq}$ & $\mathrm{nq}$ & $\mathrm{nq}$ & 76 \\
\hline $\mathbf{L u}$ & $\%$ & nq & 100 & & $\mathrm{nq}$ & $\mathrm{nq}$ & $\mathrm{nq}$ & $\mathrm{nq}$ & $\mathrm{nq}$ & 77 \\
\hline Hf & $\%$ & $\mathrm{nq}$ & $\mathrm{nb}$ & $\mathrm{nb}$ & $\mathrm{nq}$ & $\mathrm{nq}$ & $\mathrm{nq}$ & nq & $\mathrm{nq}$ & 72 \\
\hline Ta & $\%$ & $\mathrm{nq}$ & nb & $\mathrm{nb}$ & 60 & $\mathrm{nq}$ & $\mathrm{nq}$ & $\mathrm{nq}$ & $\mathrm{nq}$ & 90 \\
\hline $\mathbf{W}$ & $\%$ & $\mathrm{nq}$ & $\mathrm{nb}$ & $n b$ & $\mathrm{nq}$ & $\mathrm{nq}$ & $\mathrm{nq}$ & $\mathrm{nq}$ & 35 & 47 \\
\hline $\mathrm{Hg}$ & $\%$ & $\mathrm{nb}$ & nb & $n b$ & $\mathrm{nb}$ & $\mathrm{nb}$ & $\mathrm{nb}$ & $\mathrm{nb}$ & $n b$ & 108 \\
\hline TI & $\%$ & $\mathrm{nq}$ & nb & $\mathrm{nb}$ & $\mathrm{nq}$ & $\mathrm{nq}$ & $\mathrm{nq}$ & $\mathrm{nq}$ & $\mathrm{nq}$ & 78 \\
\hline $\mathbf{P b}$ & $\%$ & $\mathrm{nq}$ & $\mathrm{nq}$ & $\mathrm{nq}$ & $\mathrm{nq}$ & $\mathrm{nq}$ & $\mathrm{nq}$ & $\mathrm{nq}$ & $\mathrm{nq}$ & 98 \\
\hline $\mathbf{B i}$ & $\%$ & $\mathrm{nb}$ & $\mathrm{nb}$ & $\mathrm{nb}$ & $\mathrm{nq}$ & $\mathrm{nq}$ & $\mathrm{nq}$ & nq & $\mathrm{nq}$ & 91 \\
\hline Th & $\%$ & $\mathrm{nq}$ & $\mathrm{nb}$ & $\mathrm{nb}$ & $\mathrm{nq}$ & $\mathrm{nq}$ & $\mathrm{nq}$ & $\mathrm{nq}$ & $\mathrm{nq}$ & $\mathrm{nq}$ \\
\hline $\mathbf{U}$ & $\%$ & $\mathrm{nq}$ & nb & nb & nq & $\mathrm{nq}$ & 90 & 82 & 113 & 89 \\
\hline
\end{tabular}


Tabelle A.3. (Forts.): Ergebnisse der Ultrafiltration für Porenwässer und Wasserproben aus Cospuden; Konzentrationen in \% im Durchlauf bezogen auf die Ausgangskonzentrationen (Tab. A.1.) in Ultrafiltraten (1 $k D)$ von Porenlösungen und Wasserproben aus Cospuden.

\begin{tabular}{|c|c|c|c|c|c|c|c|c|c|c|}
\hline & & Bohrung 2 n & n Bohrung 3 m & See & CML $20 \mathrm{~m}$ & CML 27,5 m & CML 31,5 m & CML 39,5 m & CML 43,5 m & CML $20 \mathrm{~m}$ \\
\hline & & Porenlösung & Porenlösung & Wasserprobe & Wasserprobe & Wasserprobe & Wasserprobe & Wasserprobe & Wasserprobe & Wasserprobe \\
\hline & & \begin{tabular}{|l|} 
Nov 1998 \\
\end{tabular} & Nov 1998 & \begin{tabular}{|l|} 
Nov 1998 \\
\end{tabular} & Jan 1999 & Jan 1999 & Jan 1999 & Jan 1999 & Jan 1999 & Aug 1999 \\
\hline Sulfat & $\%$ & 8 & 92 & 53 & 75 & 95 & 96 & 88 & 107 & 93 \\
\hline DOC & $\%$ & $n b$ & $\mathrm{nb}$ & 105 & 66 & 106 & $5 \mathrm{nq}$ & 116 & & $\mathrm{nb}$ \\
\hline $\mathbf{L i}$ & $\%$ & 8 & 91 & 98 & 95 & 99 & 96 & 94 & 110 & 72 \\
\hline $\mathbf{B e}$ & $\%$ & 6 & 83. & $3 \mathrm{nq}$ & nq & nq & nq & $\mathrm{nq}$ & nq & nq \\
\hline $\mathbf{N a}$ & $\%$ & 81 & 91 & 97 & 84 & 98 & 100 & 92 & 108 & 90 \\
\hline $\mathbf{M g}$ & $\%$ & 8 & 91 & 97 & 76 & 95 & 96 & 91 & 109 & 89 \\
\hline Al & $\%$ & 6 & 82 & $2 n q$ & nq & $\operatorname{nq}$ & $\mathrm{nq}$ & nq & $\operatorname{nq}$ & 31 \\
\hline $\mathbf{K}$ & $\%$ & 8 & 89 & 98 & 80 & 95 & 97 & 92 & 110 & 88 \\
\hline Ca & $\%$ & 81 & 91 & 95 & 83 & 96 & 96 & 90 & 107 & 89 \\
\hline $\mathbf{S c}$ & $\%$ & 88 & 95 & $5 \mathrm{nq}$ & $n q$ & $n q$ & $\mathrm{nq}$ & $\mathrm{nq}$ & $\mathrm{nq}$ & $\mathrm{nq}$ \\
\hline $\mathbf{T i}$ & $\%$ & nq & nq & nq & nq & nq & nq & nq & nq & nq \\
\hline $\mathbf{V}$ & $\%$ & 7 & 53 & 14 & & nq & & $5 \mathrm{nq}$ & nq & 3 \\
\hline $\mathrm{Cr}$ & $\%$ & 7 & 81 & 99 & 62 & 65 & 58 & 120 & $\mathrm{nq}$ & 100 \\
\hline Mn & $\%$ & 8 & 92 & & $5 \mathrm{nq}$ & 94 & 97 & 91 & 106 & 88 \\
\hline $\mathbf{F e}$ & $\%$ & 88 & 88 & & $3 n q$ & $\operatorname{nq}$ & 96 & & $\operatorname{lnq}$ & 110 \\
\hline Co & $\%$ & 78 & 93 & 94 & 59 & 97 & 64 & 88 & & $5 \mathrm{nq}$ \\
\hline $\mathbf{N i}$ & $\%$ & 8 & 86 & & $3 \mathrm{nq}$ & 103 & 97 & 99 & 120 & 114 \\
\hline $\mathbf{C u}$ & $\%$ & $\mathrm{nq}$ & 92 & 104 & 81 & 69 & 100 & & $2 \mathrm{nq}$ & $\mathrm{nq}$ \\
\hline $\mathbf{Z n}$ & $\%$ & $\mathrm{nq}$ & 112 & & $\operatorname{lnq}$ & 75 & 101 & $\operatorname{lnq}$ & $\mathrm{nq}$ & $\mathrm{nq}$ \\
\hline As & $\%$ & 81 & 92 & $2 \operatorname{nq}$ & nq & $n q$ & $\mathrm{nq}$ & $\mathrm{nq}$ & nq & 81 \\
\hline $\mathbf{R b}$ & $\%$ & 7 & 117 & 10 & 60 & 33 & 100 & 76 & 100 & 107 \\
\hline $\mathbf{S r}$ & $\%$ & 7 & 91 & 95 & 76 & 93 & 97 & 89 & 111 & 90 \\
\hline $\mathbf{Y}$ & $\%$ & 8 & 88 & 98 & 60 & $\operatorname{ng}$ & $\mathrm{nq}$ & $\mathrm{nq}$ & $\mathrm{nq}$ & 13 \\
\hline $\mathbf{Z r}$ & $\%$ & & $1 \mathrm{nq}$ & & $5 \mathrm{nq}$ & nq & 94 & & $2 n q$ & 84 \\
\hline $\mathbf{N b}$ & $\%$ & $\mathrm{nq}$ & $\mathrm{nq}$ & & $\mathrm{nq}$ & $\mathrm{nq}$ & $\mathrm{nq}$ & $\mathrm{nq}$ & $\mathrm{nq}$ & 34 \\
\hline Mo & $\%$ & 7. & 81 & & $6 \mathrm{nq}$ & 114 & 93 & 87 & 102 & 100 \\
\hline $\mathbf{C d}$ & $\%$ & 8 & 85 & $5 \mathrm{nb}$ & 87 & & $7 \mathrm{nb}$ & & & $4 \mathrm{nq}$ \\
\hline Sn & $\%$ & $\mathrm{nq}$ & $\mathrm{nq}$ & $\mathrm{nq}$ & $\mathrm{nq}$ & 120 & $\mathrm{nq}$ & $\mathrm{nq}$ & $\mathrm{nq}$ & $\mathrm{nq}$ \\
\hline $\mathbf{S b}$ & $\%$ & 9 & 97 & $7 \mathrm{nq}$ & $\operatorname{nq}$ & $\operatorname{nq}$ & $\operatorname{nq}$ & $\mathrm{nq}$ & nq & nq \\
\hline Cs & $\%$ & 7 & 82 & $2 \operatorname{nq}$ & 60 & 104 & 10 & nnq & $\operatorname{nq}$ & nq \\
\hline $\mathrm{Ba}$ & $\%$ & 8 & 90 & 94 & 78 & 84 & 93 & 90 & 108 & 86 \\
\hline La & $\%$ & 8 & 93 & & $8 \mathrm{nq}$ & $\mathrm{nq}$ & $\mathrm{nq}$ & $\mathrm{nq}$ & $\mathrm{nq}$ & 115 \\
\hline $\mathrm{Ce}$ & $\%$ & 7. & 92 & $2 \mathrm{nq}$ & nq & nq & nq & nq & nq & 70 \\
\hline $\mathbf{P r}$ & $\%$ & 6 & 95 & $5 \mathrm{nq}$ & $\mathrm{nq}$ & $\operatorname{nq}$ & $\mathrm{nq}$ & $\mathrm{nq}$ & $\mathrm{nq}$ & $\mathrm{nq}$ \\
\hline $\mathbf{N d}$ & $\%$ & 6 & 92 & $2 \mathrm{nq}$ & nq & $\operatorname{lnq}$ & $\operatorname{nq}$ & nq & nq & 73 \\
\hline Sm & $\%$ & 8 & 95 & $5 \mathrm{nq}$ & $\mathrm{nq}$ & $\mathrm{nq}$ & $\mathrm{nq}$ & $\mathrm{nq}$ & $\mathrm{nq}$ & $\operatorname{nq}$ \\
\hline Eu & $\%$ & $\operatorname{nq}$ & 93 & $3 \mathrm{nq}$ & $\operatorname{nq}$ & $\operatorname{nq}$ & $\operatorname{nq}$ & $\mathrm{nq}$ & $\operatorname{nq}$ & nq \\
\hline Gd & $\%$ & 6 & 89 & $\mathrm{nq}$ & $\mathrm{nq}$ & $\mathrm{nq}$ & $\mathrm{nq}$ & $\mathrm{nq}$ & $\mathrm{nq}$ & $\mathrm{nq}$ \\
\hline Tb & $\%$ & nq & 90 & $\mathrm{nq}$ & $\mathrm{nq}$ & $\mathrm{nq}$ & $\mathrm{nq}$ & $\mathrm{nq}$ & $\mathrm{nq}$ & $\mathrm{nq}$ \\
\hline Dy & $\%$ & 6 & 94 & $\operatorname{lnq}$ & $\mathrm{nq}$ & $\mathrm{nq}$ & $\mathrm{nq}$ & $\mathrm{nq}$ & $\mathrm{nq}$ & $\mathrm{nq}$ \\
\hline Ho & $\%$ & $\mathrm{nq}$ & 88 & $\operatorname{lnq}$ & $\mathrm{nq}$ & $\mathrm{nq}$ & $\operatorname{nq}$ & $\mathrm{nq}$ & $\mathrm{nq}$ & $\mathrm{nq}$ \\
\hline $\mathbf{E r}$ & $\%$ & $\mathrm{nq}$ & 96 & $5 \mathrm{nq}$ & $\mathrm{nq}$ & $\mathrm{nq}$ & $\mathrm{nq}$ & $\mathrm{nq}$ & $\mathrm{nq}$ & $\mathrm{nq}$ \\
\hline $\mathbf{T m}$ & $\%$ & 79 & $9 \mathrm{nq}$ & $\mathrm{nq}$ & $\mathrm{nq}$ & $\mathrm{nq}$ & nq & $\mathrm{nq}$ & nq & $\mathrm{nq}$ \\
\hline $\mathbf{Y b}$ & $\%$ & $\operatorname{nq}$ & & $\operatorname{lnq}$ & nq & $\operatorname{nq}$ & $\operatorname{nq}$ & $\mathrm{nq}$ & $\operatorname{nq}$ & nq \\
\hline Lu & $\%$ & $\mathrm{nq}$ & $\mathrm{nq}$ & $\mathrm{nq}$ & $\mathrm{nq}$ & $\mathrm{nq}$ & $\mathrm{nq}$ & $\mathrm{nq}$ & $\mathrm{nq}$ & $\mathrm{nq}$ \\
\hline Hf & $\%$ & $\mathrm{nq}$ & $\mathrm{nq}$ & $\mathrm{nq}$ & $\mathrm{nq}$ & $\mathrm{nq}$ & $\mathrm{nq}$ & $\mathrm{nq}$ & $\mathrm{nq}$ & $\mathrm{nq}$ \\
\hline Ta & $\%$ & $\mathrm{nq}$ & $\mathrm{nq}$ & nb & nb & nb & nb & nb & nb & 35 \\
\hline $\mathbf{W}$ & $\%$ & 6 & & $\operatorname{lnq}$ & 47 & & $n$ & nq & $\operatorname{nq}$ & nq \\
\hline $\mathrm{Hg}$ & $\%$ & 6 & 91 & $\operatorname{lnq}$ & $n q$ & $\mathrm{nq}$ & $\mathrm{nq}$ & 100 & & $5 \mathrm{nq}$ \\
\hline TI & $\%$ & 9 & 89 & $\mathrm{nq}$ & nq & $\operatorname{nq}$ & $\operatorname{nq}$ & $\mathrm{nq}$ & $\operatorname{nq}$ & $\operatorname{nq}$ \\
\hline $\mathbf{P b}$ & $\%$ & 9 & 66 & $5 \mathrm{nq}$ & & nb & $\operatorname{nb}$ & & $6 \mathrm{nq}$ & 107 \\
\hline $\mathbf{B i}$ & $\%$ & nq & $\mathrm{nq}$ & $\mathrm{nq}$ & nb & nq & $\mathrm{nq}$ & $\mathrm{nq}$ & $\mathrm{nq}$ & $\mathrm{nq}$ \\
\hline Th & $\%$ & $\mathrm{nq}$ & $\mathrm{nq}$ & $\mathrm{nq}$ & $\mathrm{nq}$ & $\mathrm{nq}$ & $\mathrm{nq}$ & $\mathrm{nq}$ & $\mathrm{nq}$ & 29 \\
\hline $\mathbf{U}$ & $\%$ & $\operatorname{lnq}$ & $\mathrm{nq}$ & & $\operatorname{lnq}$ & 95 & 100 & 46 & & $6 \mathrm{nq}$ \\
\hline
\end{tabular}


Tabelle A.3. (Forts.): Ergebnisse der Ultrafiltration für Porenwässer und Wasserproben aus Cospuden; Konzentrationen in \% im Durchlauf bezogen auf die Ausgangskonzentrationen (Tab. A.1.) in Ultrafiltraten (1 kD) von Porenlösungen und Wasserproben aus Cospuden.

\begin{tabular}{|c|c|c|c|c|c|c|c|c|c|c|}
\hline & & CML 27,5 m & CML 31,5 m & CML 39,5 m & CML 43,5 m & RCO3 & RCO4 & RCO12 & RCO15 & RCO17 \\
\hline & & Wasserprobe & Wasserprobe & Wasserprobe & Wasserprobe & Wasserprobe & Wasserprobe & Wasserprobe & Wasserprobe & Wasserprobe \\
\hline & & Aug 1999 & Aug 1999 & Aug 1999 & Aug 1999 & Aug 1999 & Aug 1999 & Aug 1999 & Aug 1999 & Aug 1999 \\
\hline Sulfat & $\%$ & 92 & 84 & 104 & 112 & 103 & 83 & 92 & 92 & 95 \\
\hline DOC & $\%$ & nb & 49 & $n b$ & & nb & 111 & $n b$ & 70 & 86 \\
\hline $\mathbf{L i}$ & $\%$ & 93 & 86 & 92 & 106 & 90 & 75 & 92 & 95 & 98 \\
\hline $\mathbf{B e}$ & $\%$ & 119 & 54 & $\mathrm{nq}$ & $\mathrm{nq}$ & 64 & $\mathrm{nq}$ & $\mathrm{nq}$ & $\mathrm{nq}$ & 84 \\
\hline $\mathbf{N a}$ & $\%$ & 120 & 86 & 90 & 105 & 91 & 87 & 92 & 93 & 98 \\
\hline Mg & $\%$ & 92 & 90 & 91 & 106 & 88 & 76 & 92 & 94 & $\overline{96}$ \\
\hline Al & $\%$ & $\mathrm{nq}$ & 11 & nq & nq & 22 & $\mathrm{nq}$ & 58 & 99 & 91 \\
\hline $\mathbf{K}$ & $\%$ & 90 & 96 & 92 & 107 & 85 & 73 & 94 & 94 & 98 \\
\hline $\mathbf{C a}$ & $\%$ & 70 & 90 & 92 & 105 & 87 & 74 & 92 & 93 & 96 \\
\hline Sc & $\%$ & 117 & 74 & 90 & 103 & 81 & 92 & & $\mathrm{nq}$ & $\mathrm{nq}$ \\
\hline$\overline{T i}$ & $\%$ & $\mathrm{nq}$ & $\mathrm{nq}$ & $\mathrm{nq}$ & $\mathrm{nq}$ & $\mathrm{nq}$ & nq & $\mathrm{nq}$ & $\mathrm{nq}$ & $\mathrm{nq}$ \\
\hline $\mathbf{V}$ & $\%$ & $\mathrm{nq}$ & 77 & 40 & 75 & 82 & 47 & & $\mathrm{nq}$ & $\mathrm{nq}$ \\
\hline $\mathbf{C r}$ & $\%$ & nq & 36 & 66 & 71 & 34 & 14 & 51 & 100 & $\mathrm{nq}$ \\
\hline Mn & $\%$ & 93 & 89 & 91 & 106 & 88 & 77 & 91 & 93 & 96 \\
\hline $\mathrm{Fe}$ & $\%$ & $\mathrm{nq}$ & 89 & 49 & 89 & 90 & 62 & 61 & 73 & 50 \\
\hline \begin{tabular}{|l} 
Co \\
\end{tabular} & $\%$ & 87 & 83 & 100 & $\mathrm{nq}$ & 77 & 111 & 87 & 67 & $\mathrm{nq}$ \\
\hline $\mathbf{N i}$ & $\%$ & 100 & 88 & 102 & $\mathrm{nq}$ & $\mathrm{nq}$ & $\mathrm{nq}$ & $\mathrm{nq}$ & $\mathrm{nq}$ & 57 \\
\hline $\mathbf{C u}$ & $\%$ & & $\mathrm{nq}$ & $\mathrm{nq}$ & $\mathrm{nq}$ & $\mathrm{nq}$ & 100 & & & $n q$ \\
\hline $\mathbf{Z n}$ & $\%$ & 98 & 101 & & $\mathrm{nq}$ & $\mathrm{nq}$ & $\mathrm{nq}$ & $\mathrm{nq}$ & $\mathrm{nq}$ & $\mathrm{nq}$ \\
\hline As & $\%$ & $\mathrm{nq}$ & 42 & 66 & 49 & 87 & 45 & 43 & 104 & $n q$ \\
\hline$\overline{\mathbf{R b}}$ & $\%$ & 103 & 90 & 94 & $\mathrm{nq}$ & 87 & 100 & 94 & 79 & 105 \\
\hline $\mathbf{S r}$ & $\%$ & 88 & 91 & 92 & 104 & 87 & 71 & 91 & 93 & 98 \\
\hline $\mathbf{Y}$ & $\%$ & 114 & 5 & 72 & 83 & 41 & 100 & 86 & 80 & 91 \\
\hline $\mathbf{Z r}$ & $\%$ & nq & 85 & 57 & 103 & & $\mathrm{nq}$ & & $\mathrm{nq}$ & $\mathrm{nq}$ \\
\hline $\mathbf{N b}$ & $\%$ & 71 & 49 & $\mathrm{nq}$ & $\mathrm{nq}$ & $\mathrm{nq}$ & & $\mathrm{nq}$ & 26 & 60 \\
\hline \begin{tabular}{|l|} 
Mo \\
\end{tabular} & $\%$ & $\mathrm{nq}$ & 75 & 87 & 75 & 102 & 94 & 89 & 93 & 120 \\
\hline $\begin{array}{ll}\mathbf{C d} \\
\end{array}$ & $\%$ & nq & $\mathrm{nq}$ & nq & $\mathrm{nq}$ & $\mathrm{nq}$ & $\mathrm{nq}$ & nq & $\mathrm{nq}$ & $\mathrm{nq}$ \\
\hline Sn & $\%$ & 97 & 103 & & $\mathrm{nq}$ & 89 & 104 & $\mathrm{nq}$ & $\mathrm{nq}$ & nq \\
\hline $\mathbf{S b}$ & $\%$ & $\mathrm{nq}$ & $\mathrm{nq}$ & $\mathrm{nq}$ & $\mathrm{nq}$ & $\mathrm{nq}$ & $\mathrm{nq}$ & $120 \mathrm{n}$ & $\mathrm{nq}$ & $\mathrm{nq}$ \\
\hline \begin{tabular}{|l} 
Cs \\
\end{tabular} & $\%$ & 119 & 88 & $\mathrm{nq}$ & nq & 98 & & $\mathrm{nq}$ & nq & nq \\
\hline Ba & $\%$ & 12 & 90 & 86 & 105 & 89 & 64 & 94 & 76 & 92 \\
\hline La & $\%$ & 120 & & $\mathrm{nq}$ & 73 & 61 & & $\mathrm{nq}$ & $\mathrm{nq}$ & $\mathrm{nq}$ \\
\hline $\begin{array}{ll}\mathrm{Ce} \\
\end{array}$ & $\%$ & $\mathrm{nq}$ & & nq & 69 & 48 & 41 & $\mathrm{nq}$ & $\mathrm{nq}$ & 44 \\
\hline $\mathbf{P r}$ & $\%$ & nq & & $\mathrm{nq}$ & $\mathrm{nq}$ & 44 & $\mathrm{nq}$ & $\mathrm{nq}$ & $\mathrm{nq}$ & $\mathrm{nq}$ \\
\hline $\mathbf{N d}$ & $\%$ & nq & 15 & 92 & 71 & 47 & 69 & $110 \mathrm{n}$ & $\mathrm{nq}$ & $\mathrm{nq}$ \\
\hline Sm & $\%$ & $\mathrm{nq}$ & 2 & 96 & 98 & & $\mathrm{nq}$ & 103 & $\mathrm{nq}$ & $\mathrm{nq}$ \\
\hline Eu & $\%$ & $\mathrm{nq}$ & 30 & $\mathrm{nq}$ & $\mathrm{nq}$ & & $\mathrm{nq}$ & & $n q$ & $\mathrm{nq}$ \\
\hline Gd & $\%$ & $\mathrm{nq}$ & & $\mathrm{nq}$ & 68 & & $\mathrm{nq}$ & $\mathrm{nq}$ & $\mathrm{nq}$ & $\mathrm{nq}$ \\
\hline Tb & $\%$ & nq & & $\mathrm{nq}$ & $\mathrm{nq}$ & $\mathrm{nq}$ & $\mathrm{nq}$ & $\mathrm{nq}$ & $\mathrm{nq}$ & $\mathrm{nq}$ \\
\hline \begin{tabular}{|l|}
$\mathbf{D y}$ \\
\end{tabular} & $\%$ & nq & 10 & $\mathrm{nq}$ & 73 & & $\mathrm{nq}$ & & $\mathrm{nq}$ & nq \\
\hline Ho & $\%$ & $\mathrm{nq}$ & 12 & $\mathrm{nq}$ & $\mathrm{nq}$ & $\mathrm{nq}$ & $\mathrm{nq}$ & $\mathrm{nq}$ & $\mathrm{nq}$ & $\mathrm{nq}$ \\
\hline $\mathbf{E r}$ & $\%$ & nq & 11 & $\mathrm{nq}$ & $\mathrm{nq}$ & & $\mathrm{nq}$ & nq & $\mathrm{nq}$ & nq \\
\hline Tm & $\%$ & nq & & $\mathrm{nq}$ & $\mathrm{nq}$ & $\mathrm{nq}$ & $\mathrm{nq}$ & $\mathrm{nq}$ & $\mathrm{nq}$ & nq \\
\hline $\mathbf{Y b}$ & $\%$ & $\mathrm{nq}$ & 22 & $\mathrm{nq}$ & 81 & & $\mathrm{nq}$ & $\mathrm{nq}$ & $\mathrm{nq}$ & $\mathrm{nq}$ \\
\hline $\mathbf{L u}$ & $\%$ & $\mathrm{nq}$ & 24 & $\mathrm{nq}$ & $\mathrm{nq}$ & $\mathrm{nq}$ & $\mathrm{nq}$ & $\mathrm{nq}$ & $\mathrm{nq}$ & $\mathrm{nq}$ \\
\hline Hf & $\%$ & nq & nq & nq & $\mathrm{nq}$ & $\mathrm{nq}$ & $\mathrm{nq}$ & nq & $\mathrm{nq}$ & nq \\
\hline Ta & $\%$ & 31 & 99 & 46 & $\mathrm{nq}$ & $\mathrm{nq}$ & 41 & $\mathrm{nq}$ & 14 & nq \\
\hline $\mathbf{W}$ & $\%$ & 120 & & $\mathrm{nq}$ & 86 & $\mathrm{nq}$ & $n q$ & nq & $\mathrm{nq}$ & nq \\
\hline $\mathrm{Hg}$ & $\%$ & $\mathrm{nq}$ & nq & 66 & 106 & nq & nq & 57 & 28 & 33 \\
\hline TI & $\%$ & $\mathrm{nq}$ & & $\mathrm{nq}$ & $\mathrm{nq}$ & $\mathrm{nq}$ & $\mathrm{nq}$ & $\mathrm{nq}$ & $\mathrm{nq}$ & $\mathrm{nq}$ \\
\hline $\mathbf{P b}$ & $\%$ & 64 & & $\mathrm{nq}$ & $\mathrm{nq}$ & & $\mathrm{nq}$ & 87 & 103 & 65 \\
\hline$\overline{\mathbf{B i}}$ & $\%$ & nq & nq & nq & $\mathrm{nq}$ & $\mathrm{nq}$ & $\mathrm{nq}$ & nq & $\mathrm{nq}$ & 62 \\
\hline Th & $\%$ & $\mathrm{nq}$ & $\mathrm{nq}$ & $\mathrm{nq}$ & $\mathrm{nq}$ & $\mathrm{nq}$ & $\mathrm{nq}$ & $\mathrm{nq}$ & $\mathrm{nq}$ & $\mathrm{nq}$ \\
\hline $\mathbf{U}$ & $\%$ & 110 & 82 & 85 & & & 69 & 92 & 80 & 53 \\
\hline
\end{tabular}


Tabelle A.3. (Forts.): Ergebnisse der Ultrafiltration für Porenwässer und Wasserproben aus Cospuden; Konzentrationen in \% im Durchlauf bezogen auf die Ausgangskonzentrationen (Tab. A.1.) in Ultrafiltraten (1 $k D)$ von Porenlösungen und Wasserproben aus Cospuden.

\begin{tabular}{|c|c|c|c|c|c|c|c|c|c|c|}
\hline & & RCO18 & See & SeeSed & Sedgepr & CML $20 \mathrm{~m}$ & CML 27,5 m & CML 31,5 m & CML 39,5 m & CML 43,5 m \\
\hline & & Wasserprob & Wasserprobe & Wasserprobe & Porenlösung & Wasserprobe & Wasserprobe & Wasserprobe & Wasserprobe & Wasserprobe \\
\hline & & Aug 1999 & Aug 1999 & Aug 1999 & Aug 1999 & Jan 2000 & Jan 2000 & Jan 2000 & Jan 2000 & Jan 2000 \\
\hline Sulfat & $\%$ & 8 & 86 & 88 & 85 & 98 & 109 & 101 & 79 & 92 \\
\hline DOC & $\%$ & $n b$ & 105 & $79 n$ & $\mathrm{nq}$ & 62 & 66 & 66 & 94 & 72 \\
\hline $\mathbf{L i}$ & $\%$ & 9 & 90 & 89 & 90 & 97 & 113 & 102 & 67 & 94 \\
\hline $\mathbf{B e}$ & $\%$ & nq & nq & nq & nq & nq & 109 & & 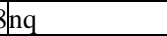 & nq \\
\hline $\mathbf{N a}$ & $\%$ & 8. & 90 & 90 & 87 & 97 & 111 & 107 & 74 & 95 \\
\hline $\mathbf{M g}$ & $\%$ & 9 & 90 & 85 & 84 & 98 & 105 & 100 & 77 & 94 \\
\hline Al & $\%$ & 12 & 90 & $n$ & nq & $\operatorname{nq}$ & 116 & 106 & $6 \mathrm{nq}$ & nq \\
\hline $\mathbf{K}$ & $\%$ & 9 & 90 & 79 & 78 & 97 & 108 & 101 & 70 & 93 \\
\hline Ca & $\%$ & 88 & 89 & 83 & 80 & 97 & 107 & 100 & 82 & 90 \\
\hline $\mathbf{S c}$ & $\%$ & 7 & $83_{1}$ & $3 \mathrm{nq}$ & & $5 \mathrm{nq}$ & 102 & 110 & $\operatorname{nq}$ & $\mathrm{nq}$ \\
\hline $\mathbf{T i}$ & $\%$ & nq & nq & nq & nq & 0 & 118 & & nq & nq \\
\hline $\mathbf{V}$ & $\%$ & 6 & 80 & 83 & 92 & 106 & 111 & inq & nq & nq \\
\hline $\mathrm{Cr}$ & $\%$ & 7 & 100 & 83 & 97 & & $7 \mathrm{nq}$ & nq & & $\mathrm{nq}$ \\
\hline Mn & $\%$ & 9 & 85 & $5 \mathrm{nq}$ & 55 & 100 & 105 & 101 & 76 & 92 \\
\hline $\mathbf{F e}$ & $\%$ & 5 & 43 & $100 \mathrm{n}$ & $n q$ & 25 & 104 & 99 & 40 & 76 \\
\hline Co & $\%$ & 8 & 73 & 96 & 100 & $n q$ & 107 & & $n$ & $\mathrm{nq}$ \\
\hline $\mathbf{N i}$ & $\%$ & 8 & 100 & 87 & & $n q$ & 110 & 113 & $3 \mathrm{nq}$ & $n \mathrm{qq}$ \\
\hline $\mathbf{C u}$ & $\%$ & nq & $\mathrm{nq}$ & $120 \mathrm{n}$ & & $\mathrm{nq}$ & $\mathrm{nq}$ & $\mathrm{nq}$ & & $n \mathrm{nq}$ \\
\hline $\mathbf{Z n}$ & $\%$ & $\mathrm{nq}$ & $\mathrm{nq}$ & $\mathrm{nq}$ & $\mathrm{nq}$ & $\mathrm{nq}$ & 117 & & $8 \mathrm{nq}$ & $\mathrm{nq}$ \\
\hline As & $\%$ & 6 & 81 & 91 & $100_{1}$ & $n q$ & 103 & 55 & & $n \mathrm{nq}$ \\
\hline $\mathbf{R b}$ & $\%$ & 8 & 73 & 60 & & $n$ & & $n \mathrm{nq}$ & $\mathrm{nq}$ & 80 \\
\hline $\mathbf{S r}$ & $\%$ & 88 & 89 & 84 & 79 & 96 & 109 & 98 & 70 & 92 \\
\hline $\mathbf{Y}$ & $\%$ & & $6 \mathrm{nq}$ & \begin{tabular}{l|l|}
82 \\
\end{tabular} & 76 & 0 & 110 & & $n$ & $\mathrm{nq}$ \\
\hline $\mathbf{Z r}$ & $\%$ & 88 & 811 & $\operatorname{lnq}$ & 90 & 86 & 109 & 110 & 100 & 109 \\
\hline $\mathbf{N b}$ & $\%$ & 6 & 22 & 42 & & $5 \mathrm{nq}$ & $\mathrm{nq}$ & $\mathrm{nq}$ & $\mathrm{nq}$ & 105 \\
\hline Mo & $\%$ & 88 & 821 & $2 n q$ & $n q$ & $\mathrm{nq}$ & $\mathrm{nq}$ & 13 & 118 & 104 \\
\hline $\mathbf{C d}$ & $\%$ & nq & 97 & $\mathrm{nq}$ & $100_{1}$ & $n \mathrm{nq}$ & $n q$ & 70 & 84 & 74 \\
\hline Sn & $\%$ & $\mathrm{nq}$ & $\mathrm{nq}$ & $\mathrm{nq}$ & $\mathrm{nq}$ & $\mathrm{nq}$ & $\mathrm{nq}$ & $\mathrm{nq}$ & 84 & 63 \\
\hline $\mathbf{S b}$ & $\%$ & nq & $\mathrm{nq}$ & 109 & & $n q$ & $\operatorname{nq}$ & 22 & 73 & 38 \\
\hline Cs & $\%$ & $\mathrm{nq}$ & $\mathrm{nq}$ & $\mathrm{nq}$ & $n q$ & $\mathrm{nq}$ & 108 & & $\operatorname{nq}$ & $n q$ \\
\hline $\mathrm{Ba}$ & $\%$ & 9. & 92 & 91 & 73 & 95 & 115 & 114 & 76 & 97 \\
\hline La & $\%$ & $n q$ & $\mathrm{nq}$ & nq & $n q$ & nq & 120 & & $n q$ & 47 \\
\hline $\mathrm{Ce}$ & $\%$ & nq & $\mathrm{nq}$ & $\mathrm{nq}$ & & $n \mathrm{nq}$ & 119 & $9 n q$ & nq & 62 \\
\hline Pr & $\%$ & $\mathrm{nq}$ & $\mathrm{nq}$ & $\mathrm{nq}$ & $\mathrm{nq}$ & $\mathrm{nq}$ & $\mathrm{nq}$ & $\mathrm{nq}$ & $\mathrm{nq}$ & $\mathrm{nq}$ \\
\hline Nd & $\%$ & 7 & 38 & 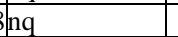 & & $n q$ & nq & nq & nq & 35 \\
\hline Sm & $\%$ & $\operatorname{nq}$ & & $n q$ & $n q$ & 106 & $5 \mathrm{nq}$ & $\mathrm{nq}$ & 92 & 107 \\
\hline Eu & $\%$ & & $0 \mathrm{nq}$ & $\mathrm{nq}$ & $\mathrm{nq}$ & $\mathrm{nq}$ & $\mathrm{nq}$ & $\mathrm{nq}$ & $\mathrm{nq}$ & 70 \\
\hline Gd & $\%$ & $\mathrm{nq}$ & $\mathrm{nq}$ & $\mathrm{nq}$ & $\mathrm{nq}$ & $\mathrm{nq}$ & $\mathrm{nq}$ & $\mathrm{nq}$ & $\mathrm{nq}$ & $\mathrm{nq}$ \\
\hline Tb & $\%$ & nq & $\mathrm{nq}$ & $\mathrm{nq}$ & $\mathrm{nq}$ & $\mathrm{nq}$ & $\mathrm{nq}$ & $\mathrm{nq}$ & $\mathrm{nq}$ & 66 \\
\hline Dy & $\%$ & $\mathrm{nq}$ & $\mathrm{nq}$ & $\mathrm{nq}$ & $n q$ & $\mathrm{nq}$ & $\mathrm{nq}$ & $\mathrm{nq}$ & $\mathrm{nq}$ & $\mathrm{nq}$ \\
\hline Ho & $\%$ & $\mathrm{nq}$ & $\mathrm{nq}$ & $\mathrm{nq}$ & $\mathrm{nq}$ & $\mathrm{nq}$ & $\mathrm{nq}$ & $\mathrm{nq}$ & $\mathrm{nq}$ & $\mathrm{nq}$ \\
\hline Er & $\%$ & $\mathrm{nq}$ & nq & $\mathrm{nq}$ & $\mathrm{nq}$ & $\mathrm{nq}$ & $\mathrm{nq}$ & $\mathrm{nq}$ & $\mathrm{nq}$ & $\mathrm{nq}$ \\
\hline $\mathbf{T m}$ & $\%$ & $\mathrm{nq}$ & $\mathrm{nq}$ & $\mathrm{nq}$ & $\mathrm{nq}$ & $\mathrm{nq}$ & 119 & & $\mathrm{nq}$ & $\mathrm{nq}$ \\
\hline $\mathbf{Y b}$ & $\%$ & $\operatorname{nq}$ & $\mathrm{nq}$ & $\mathrm{nq}$ & nq & $\mathrm{nq}$ & 120 & & $\mathrm{nq}$ & nq \\
\hline $\mathbf{L u}$ & $\%$ & $\mathrm{nq}$ & $\mathrm{nq}$ & $\mathrm{nq}$ & $\mathrm{nq}$ & $\mathrm{nq}$ & $\mathrm{nq}$ & $\mathrm{nq}$ & $\mathrm{nq}$ & $\mathrm{nq}$ \\
\hline Hf & $\%$ & $\mathrm{nq}$ & nq & $\mathrm{nq}$ & $\mathrm{nq}$ & nq & nq & $\mathrm{nq}$ & $\mathrm{nq}$ & 57 \\
\hline Ta & $\%$ & 7 & 27 & 27 & 70 & 106 & $5 \mathrm{nq}$ & $\mathrm{nq}$ & $\mathrm{nq}$ & 75 \\
\hline $\mathbf{W}$ & $\%$ & nq & $\mathrm{nq}$ & $n q$ & $n q$ & nq & nq & & $6 \mathrm{nq}$ & 50 \\
\hline $\mathbf{H g}$ & $\%$ & 6 & 97 & 97 & 79 & 98 & 102 & & $6 \mathrm{nq}$ & 66 \\
\hline TI & $\%$ & $\mathrm{nq}$ & 80 & $80 \mathrm{n}$ & $n q$ & $\mathrm{nq}$ & $\mathrm{nq}$ & $\mathrm{nq}$ & $\mathrm{nq}$ & 97 \\
\hline $\mathbf{P b}$ & $\%$ & nq & $\mathrm{nq}$ & $\mathrm{nq}$ & 105 & $5 \mathrm{nq}$ & $\mathrm{nq}$ & $\mathrm{nq}$ & $\mathrm{nq}$ & $\mathrm{nq}$ \\
\hline $\mathbf{B i}$ & $\%$ & nq & $\mathrm{nq}$ & nq & $n q$ & nq & $\mathrm{nq}$ & $\mathrm{nq}$ & $\mathrm{nq}$ & $\mathrm{nq}$ \\
\hline Th & $\%$ & $\mathrm{nq}$ & $\mathrm{nq}$ & $\mathrm{nq}$ & & nq & $\mathrm{nq}$ & $\mathrm{nq}$ & $\mathrm{nq}$ & 32 \\
\hline $\mathbf{U}$ & $\%$ & 8 & 75 & 75 & 107 & & $\operatorname{nq}$ & nq & $\operatorname{nq}$ & 87 \\
\hline
\end{tabular}


Tabelle A.3. (Forts.): Ergebnisse der Ultrafiltration für Porenwässer und Wasserproben aus Cospuden; Konzentrationen in \% im Durchlauf bezogen auf die Ausgangskonzentrationen (Tab. A.1.) in Ultrafiltraten (1 kD) von Porenlösungen und Wasserproben aus Cospuden.

\begin{tabular}{|c|c|c|c|c|c|c|c|}
\hline & & RCO3 & RCO4 & RCO12 & RCO15 & RCO17 & RCO18 \\
\hline & & Wasserprobe & Wasserprobe & Wasserprobe & Wasserprobe & Wasserprobe & Wasserprobe \\
\hline & & Jan 2000 & Jan 2000 & Jan 2000 & Jan 2000 & Jan 2000 & Jan 2000 \\
\hline Sulfat & $\%$ & 96 & 114 & $\mathrm{nq}$ & 102 & 109 & 111 \\
\hline DOC & $\%$ & 80 & 77 & & $\mathrm{nq}$ & 70 & 83 \\
\hline $\mathbf{L i}$ & $\%$ & 92 & 104 & 28 & 114 & 115 & $\mathrm{nq}$ \\
\hline $\mathbf{B e}$ & $\%$ & 109 & $\mathrm{nq}$ & nq & nq & $\mathrm{nq}$ & $<0.1$ \\
\hline $\mathbf{N a}$ & $\%$ & 95 & 106 & 39 & 104 & 112 & 117 \\
\hline $\mathbf{M g}$ & $\%$ & 95 & 105 & 16 & 107 & 110 & 112 \\
\hline Al & $\%$ & 98 & $\mathrm{nq}$ & nq & nq & nq & $<1$ \\
\hline $\mathbf{K}$ & $\%$ & 94 & 101 & 69 & 112 & 114 & nq \\
\hline $\mathbf{C a}$ & $\%$ & 94 & 101 & 58 & 108 & 109 & 111 \\
\hline Sc & $\%$ & $\mathrm{nq}$ & $\mathrm{nq}$ & $\mathrm{nq}$ & nq & & $n q$ \\
\hline $\mathbf{T i}$ & $\%$ & $\mathrm{nq}$ & $\mathrm{nq}$ & $\mathrm{nq}$ & nq & $\mathrm{nq}$ & $\mathrm{nq}$ \\
\hline $\mathbf{V}$ & $\%$ & 110 & $\mathrm{nq}$ & & $\mathrm{nq}$ & nq & 109 \\
\hline $\mathrm{Cr}$ & $\%$ & 87 & $\mathrm{nq}$ & & $\mathrm{nq}$ & & $n q$ \\
\hline Mn & $\%$ & 95 & 103 & 8 & 105 & $\mathrm{nq}$ & 113 \\
\hline $\mathbf{F e}$ & $\%$ & 94 & $\mathrm{nq}$ & $\mathrm{nq}$ & 88 & & $\mathrm{nq}$ \\
\hline \begin{tabular}{|l} 
Co \\
\end{tabular} & $\%$ & $\mathrm{nq}$ & $\mathrm{nq}$ & $\mathrm{nq}$ & $\mathrm{nq}$ & $\mathrm{nq}$ & $\mathrm{nq}$ \\
\hline $\mathbf{N i}$ & $\%$ & $\mathrm{nq}$ & $\mathrm{nq}$ & $\mathrm{nq}$ & nq & $\mathrm{nq}$ & $\mathrm{nq}$ \\
\hline $\mathbf{C u}$ & $\%$ & $\mathrm{nq}$ & $\mathrm{nq}$ & 0 & 481 & $\mathrm{nq}$ & nq \\
\hline $\mathbf{Z n}$ & $\%$ & $\mathrm{nq}$ & $\mathrm{nq}$ & $\mathrm{nq}$ & nq & $\mathrm{nq}$ & $\mathrm{nq}$ \\
\hline \begin{tabular}{|l|}
$\mathbf{A s}$ \\
\end{tabular} & $\%$ & 4 & 55 & & $\mathrm{nq}$ & & $\mathrm{nq}$ \\
\hline $\mathbf{R b}$ & $\%$ & 5 & 108 & $\mathrm{nq}$ & 74 & & $\mathrm{nq}$ \\
\hline $\mathbf{S r}$ & $\%$ & 94 & 100 & 86 & 110 & 113 & $\mathrm{nq}$ \\
\hline $\mathbf{Y}$ & $\%$ & 101 & $\mathrm{nq}$ & $\mathrm{nq}$ & $\mathrm{nq}$ & & $\mathrm{nq}$ \\
\hline $\mathbf{Z r}$ & $\%$ & 98 & 106 & & nq & 59 & 101 \\
\hline \begin{tabular}{|l|}
$\mathbf{N b}$ \\
\end{tabular} & $\%$ & 19 & 58 & $\mathrm{nq}$ & nq & & $n q$ \\
\hline Mo & $\%$ & $\mathrm{nq}$ & 96 & & $\mathrm{nq}$ & & $\mathrm{nq}$ \\
\hline \begin{tabular}{|l|} 
Cd \\
\end{tabular} & $\%$ & 22 & $\mathrm{nq}$ & $\mathrm{nq}$ & $\mathrm{nq}$ & nq & $\mathrm{nq}$ \\
\hline Sn & $\%$ & 29 & $\mathrm{nq}$ & 72 & 1011 & $\mathrm{nq}$ & $\mathrm{nq}$ \\
\hline $\mathbf{S b}$ & $\%$ & $\mathrm{nq}$ & & $\mathrm{nq}$ & nq & & $\mathrm{nq}$ \\
\hline \begin{tabular}{|l|} 
Cs \\
\end{tabular} & $\%$ & 3 & 91 & $\mathrm{nq}$ & nq & & $\mathrm{nq}$ \\
\hline $\mathbf{B a}$ & $\%$ & 99 & $\mathrm{nq}$ & 70 & 100 & 106 & 114 \\
\hline \begin{tabular}{|l|}
$\mathbf{L a}$ \\
\end{tabular} & $\%$ & 0 & 109 & $\mathrm{nq}$ & nq & & $\mathrm{nq}$ \\
\hline $\mathrm{Ce}$ & $\%$ & & $\mathrm{nq}$ & $\mathrm{nq}$ & 13 & & $\mathrm{nq}$ \\
\hline $\mathbf{P r}$ & $\%$ & 2 & $\mathrm{nq}$ & $\mathrm{nq}$ & nq & & $\mathrm{nq}$ \\
\hline Nd & $\%$ & 21 & $\mathrm{nq}$ & $\mathrm{nq}$ & 113 & 35 & $\mathrm{nq}$ \\
\hline $\mathbf{S m}$ & $\%$ & 7 & 68 & $\mathrm{nq}$ & 102 & 67 & $\mathrm{nq}$ \\
\hline \begin{tabular}{|l}
$\mathbf{E u}$ \\
\end{tabular} & $\%$ & & $\mathrm{nq}$ & $\mathrm{nq}$ & $\mathrm{nq}$ & $\mathrm{nq}$ & $\mathrm{nq}$ \\
\hline \begin{tabular}{|l|} 
Gd \\
\end{tabular} & $\%$ & 1 & & $\mathrm{nq}$ & nq & $\mathrm{nq}$ & $\mathrm{nq}$ \\
\hline Tb & $\%$ & & $\mathrm{nq}$ & $\mathrm{nq}$ & nq & & $\mathrm{nq}$ \\
\hline Dy & $\%$ & 2 & & $\mathrm{nq}$ & nq & $\mathrm{nq}$ & nq \\
\hline Ho & $\%$ & 3 & 79 & $\mathrm{nq}$ & nq & & $\mathrm{nq}$ \\
\hline Er & $\%$ & & $\mathrm{nq}$ & nq & nq & & $\mathrm{nq}$ \\
\hline Tm & $\%$ & & $\mathrm{nq}$ & $\mathrm{nq}$ & nq & & $\mathrm{nq}$ \\
\hline $\mathbf{Y b}$ & $\%$ & & $\mathrm{nq}$ & $\mathrm{nq}$ & nq & $\mathrm{nq}$ & $\mathrm{nq}$ \\
\hline \begin{tabular}{|l|}
$\mathbf{L u}$ \\
\end{tabular} & $\%$ & & $\mathrm{nq}$ & $\mathrm{nq}$ & $\mathrm{nq}$ & 113 & $\mathrm{nq}$ \\
\hline Hf & $\%$ & $\mathrm{nq}$ & 33 & $\mathrm{nq}$ & $\mathrm{nq}$ & 45 & $\mathrm{nq}$ \\
\hline $\mathbf{T a}$ & $\%$ & $\mathrm{nq}$ & 78 & 102 & $\mathrm{nq}$ & 69 & 109 \\
\hline $\mathbf{W}$ & $\%$ & 8 & 63 & $\mathrm{nq}$ & nq & 57 & $\mathrm{nq}$ \\
\hline $\mathrm{Hg}$ & $\%$ & 38 & 56 & 93 & 119 & 61 & $\mathrm{nq}$ \\
\hline TI & $\%$ & $\mathrm{nq}$ & 41 & $\mathrm{nq}$ & nq & 51 & 119 \\
\hline $\mathbf{P b}$ & $\%$ & 27 & 91 & $\mathrm{nq}$ & 71 & 106 & $\mathrm{nq}$ \\
\hline $\mathbf{B i}$ & $\%$ & $\mathrm{nq}$ & $\mathrm{nq}$ & $\mathrm{nq}$ & nq & 77 & $\mathrm{nq}$ \\
\hline Th & $\%$ & $\mathrm{nq}$ & $\mathrm{nq}$ & $\mathrm{nq}$ & nq & 39 & $\mathrm{nq}$ \\
\hline $\mathbf{U}$ & $\%$ & $\mathrm{nq}$ & 94 & 111 & $\mathrm{nq}$ & 35 & $\mathrm{nq}$ \\
\hline
\end{tabular}


Tab. A.4.: Ergebnisse der Ultrafiltration für Porenwässer und Wasserproben aus der Lausitz; Konzentrationen in \% im Durchlauf bezogen auf die Ausgangskonzentrationen (Tab. A.2.) in Ultrafiltraten (1 kD) von Porenlösungen und Wasserproben aus der Lausitz.

\begin{tabular}{|c|c|c|c|c|c|}
\hline & & Lausitz 5,00 m & Lausitz 5,60 m & Lausitz 5,75 m & Lausitz 5,93 m \\
\hline & & Porenlösung & Porenlösung & Porenlösung & Porenlösung \\
\hline & & Okt 1999 & Okt 1999 & Okt 1999 & Okt 1999 \\
\hline Sulfat & $\%$ & 87 & 84 & 97 & 90 \\
\hline DOC & $\%$ & 105 & 65 & & $6 \mathrm{nb}$ \\
\hline $\mathbf{L i}$ & $\%$ & 90 & 83 & 98 & 87 \\
\hline $\mathbf{B e}$ & $\%$ & 88 & 85 & 97 & 89 \\
\hline $\mathbf{N a}$ & $\%$ & 91 & 89 & 97 & 89 \\
\hline $\mathbf{M g}$ & $\%$ & 87 & 84 & 98 & 88 \\
\hline Al & $\%$ & 85 & 81 & 96 & 87 \\
\hline $\mathbf{K}$ & $\%$ & 87 & 83 & 97 & 67 \\
\hline $\mathbf{C a}$ & $\%$ & 86 & 82 & 97 & 88 \\
\hline Sc & $\%$ & 78 & 77 & 95 & 87 \\
\hline $\mathbf{T i}$ & $\%$ & 80 & 85 & 90 & 57 \\
\hline $\mathbf{V}$ & $\%$ & 22 & 83 & 96 & 86 \\
\hline $\mathrm{Cr}$ & $\%$ & 80 & 74 & 97 & 82 \\
\hline $\mathbf{M n}$ & $\%$ & 88 & 84 & 98 & 90 \\
\hline $\mathbf{F e}$ & $\%$ & 87 & 85 & 98 & 90 \\
\hline \begin{tabular}{|l|} 
Co \\
\end{tabular} & $\%$ & 87 & 84 & 97 & 90 \\
\hline $\mathbf{N i}$ & $\%$ & 86 & 82 & 96 & 88 \\
\hline $\mathbf{C u}$ & $\%$ & 104 & $4 \mathrm{nq}$ & 101 & $\mathrm{nq}$ \\
\hline $\mathbf{Z n}$ & $\%$ & 88 & 85 & 98 & 91 \\
\hline As & $\%$ & 88 & 93 & 101 & 102 \\
\hline $\mathbf{R b}$ & $\%$ & 84 & 81 & 101 & 95 \\
\hline $\mathbf{S r}$ & $\%$ & 83 & 79 & 95 & 85 \\
\hline $\mathbf{Y}$ & $\%$ & 85 & 82 & 96 & 88 \\
\hline $\mathbf{Z r}$ & $\%$ & 62 & 77 & 100 & 88 \\
\hline \begin{tabular}{|l|}
$\mathbf{N b}$ \\
\end{tabular} & $\%$ & 56 & 42 & 63 & 42 \\
\hline \begin{tabular}{|l|} 
Mo \\
\end{tabular} & $\%$ & 99 & 90 & 100 & 77 \\
\hline Cd & $\%$ & 85 & 88 & 99 & 92 \\
\hline Sn & $\%$ & $\mathrm{nq}$ & $\mathrm{nq}$ & $\mathrm{nq}$ & $\mathrm{nq}$ \\
\hline $\mathbf{S b}$ & $\%$ & $\mathrm{nq}$ & 83 & 101 & 89 \\
\hline Cs & $\%$ & 87 & 82 & 96 & 93 \\
\hline $\mathbf{B a}$ & $\%$ & 84 & 80 & 94 & 88 \\
\hline La & $\%$ & 84 & 77 & 96 & 92 \\
\hline $\mathrm{Ce}$ & $\%$ & 83 & 77 & 96 & 89 \\
\hline $\operatorname{Pr}$ & $\%$ & 84 & 80 & 96 & 89 \\
\hline \begin{tabular}{|l|}
$\mathbf{N d}$ \\
\end{tabular} & $\%$ & 83 & 80 & 96 & 90 \\
\hline $\mathrm{Sm}$ & $\%$ & 82 & 80 & 97 & 90 \\
\hline Eu & $\%$ & 80 & 80 & 97 & 90 \\
\hline \begin{tabular}{|l} 
Gd \\
\end{tabular} & $\%$ & 82 & 81 & 98 & 90 \\
\hline \begin{tabular}{|l}
$\mathbf{T b}$ \\
\end{tabular} & $\%$ & 83 & 83 & 99 & 89 \\
\hline Dy & $\%$ & 84 & 82 & 99 & 89 \\
\hline Ho & $\%$ & 84 & 83 & 98 & 90 \\
\hline $\mathbf{E r}$ & $\%$ & 85 & 82 & 97 & 90 \\
\hline Tm & $\%$ & 85 & 81 & 98 & 90 \\
\hline $\mathbf{Y b}$ & $\%$ & 85 & 83 & 98 & 89 \\
\hline $\mathbf{L u}$ & $\%$ & 83 & 83 & 96 & 90 \\
\hline Hf & $\%$ & $<83$ & 77 & $\mathrm{nq}$ & $<86$ \\
\hline Ta & $\%$ & 60 & 54 & 73 & 38 \\
\hline $\mathbf{W}$ & $\%$ & 88 & 77 & 92 & 68 \\
\hline $\mathrm{Hg}$ & $\%$ & $n b$ & $n b$ & $n b$ & $n b$ \\
\hline Tl & $\%$ & 91 & 94 & 97 & 92 \\
\hline $\mathbf{P b}$ & $\%$ & 86 & 81 & 95 & 89 \\
\hline $\mathbf{B i}$ & $\%$ & $n b$ & $n b$ & $n b$ & $n b$ \\
\hline Th & $\%$ & 64 & 51 & 85 & 66 \\
\hline $\mathbf{U}$ & $\%$ & 85 & 82 & 97 & 85 \\
\hline
\end{tabular}


Tab. A.4.: Ergebnisse der Ultrafiltration für Porenwässer und Wasserproben aus der Lausitz; Konzentrationen in \% im Durchlauf bezogen auf die Ausgangskonzentrationen (Tab. A.2.) in Ultrafiltraten (1 kD) von Porenlösungen und Wasserproben aus der Lausitz.

\begin{tabular}{|c|c|c|c|c|c|c|c|}
\hline & & Lausitz 21,07 m & Lausitz 21,13 m & Lausitz 21,40 m & Lausitz 21,70 m & Lausitz $21,80 \mathrm{~m}$ & Lausitz 21,90 m \\
\hline & & Porenlösung & Porenlösung & Porenlösung & Porenlösung & Porenlösung & Porenlösung \\
\hline & & Okt 1999 & Okt 1999 & Okt 1999 & Okt 1999 & Okt 1999 & Okt 1999 \\
\hline Sulfat & $\%$ & 99 & 79 & 38 & 85 & 87 & 89 \\
\hline DOC & $\%$ & nq & 92 & 39 & 70 & & $n \mathrm{nb}$ \\
\hline $\mathbf{L i}$ & $\%$ & 96 & 85 & 105 & 82 & 92 & 99 \\
\hline Be & $\%$ & 100 & 80 & nq & 87 & 85 & 89 \\
\hline $\mathbf{N a}$ & $\%$ & 96 & 84 & nq & 87 & 85 & 89 \\
\hline $\mathbf{M g}$ & $\%$ & 100 & 76 & 93 & 82 & 88 & 87 \\
\hline Al & $\%$ & 99 & 77 & 100 & 79 & 84 & 80 \\
\hline $\mathbf{K}$ & $\%$ & 106 & 81 & 64 & 81 & 90 & 86 \\
\hline $\mathbf{C a}$ & $\%$ & 104 & 81 & 102 & 81 & 86 & 87 \\
\hline Sc & $\%$ & $\mathrm{nq}$ & $\mathrm{nq}$ & 89 & & $7 \mathrm{nq}$ & 90 \\
\hline $\mathbf{T i}$ & $\%$ & $\mathrm{nq}$ & $\mathrm{nq}$ & 108 & $\mathrm{nq}$ & & $\mathrm{nq}$ \\
\hline $\mathbf{V}$ & $\%$ & 47 & $7 \mathrm{nq}$ & 77 & 63 & 71 & 80 \\
\hline $\mathbf{C r}$ & $\%$ & 70 & 75 & 84 & 17 & 100 & 100 \\
\hline Mn & $\%$ & 104 & 84 & $4 \mathrm{nq}$ & 83 & 86 & 88 \\
\hline $\mathbf{F e}$ & $\%$ & 103 & 81 & 105 & 84 & 87 & 86 \\
\hline Co & $\%$ & 93 & 62 & $2 n q$ & 84 & 88 & 91 \\
\hline$\overline{\mathbf{N i}}$ & $\%$ & 99 & 63 & $3 \mathrm{nq}$ & 83 & 87 & 90 \\
\hline$\overline{\mathbf{C u}}$ & $\%$ & $\mathrm{nq}$ & 74 & 113 & $3 \mathrm{nq}$ & $\mathrm{nq}$ & $\mathrm{nq}$ \\
\hline $\mathbf{Z n}$ & $\%$ & 100 & 82 & $2 \mathrm{nq}$ & 86 & 89 & 89 \\
\hline As & $\%$ & $\mathrm{nq}$ & 64 & 55 & 92 & 106 & 67 \\
\hline $\mathbf{R b}$ & $\%$ & 17 & 51 & 118 & 105 & 97 & 105 \\
\hline $\mathbf{S r}$ & $\%$ & 101 & 87 & 112 & 81 & 86 & 84 \\
\hline $\mathbf{Y}$ & $\%$ & 98 & 76 & $6 \mathrm{nq}$ & 81 & 86 & 84 \\
\hline $\mathbf{Z r}$ & $\%$ & 85 & 0 & 42 & 81 & 89 & 101 \\
\hline $\mathbf{N b}$ & $\%$ & 0 & 82 & & $\mathrm{nq}$ & 43 & 15 \\
\hline Mo & $\%$ & 80 & 83 & $3 \mathrm{nq}$ & $\mathrm{nq}$ & 64 & $\mathrm{nq}$ \\
\hline$\overline{C d}$ & $\%$ & 26 & 51 & 97 & 93 & 86 & 104 \\
\hline Sn & $\%$ & $\mathrm{nq}$ & $\mathrm{nq}$ & $\mathrm{nq}$ & $\mathrm{nq}$ & $\mathrm{nq}$ & $\mathrm{nq}$ \\
\hline Sb & $\%$ & $\mathrm{nq}$ & 105 & $5 \mathrm{nq}$ & $\mathrm{nq}$ & $\mathrm{nq}$ & $\mathrm{nq}$ \\
\hline \begin{tabular}{|l} 
Cs \\
\end{tabular} & $\%$ & 2 & 73 & 101 & 105 & 83 & 106 \\
\hline $\mathbf{B a}$ & $\%$ & 96 & 80 & $\mathrm{nq}$ & 82 & 89 & 87 \\
\hline La & $\%$ & $\mathrm{nq}$ & 85 & $\mathrm{nq}$ & 82 & 87 & 99 \\
\hline $\mathrm{Ce}$ & $\%$ & 0 & 0 & 110 & 86 & 63 & 98 \\
\hline Pr & $\%$ & 0 & f & 110 & 85 & 62 & 97 \\
\hline $\mathbf{N d}$ & $\%$ & 0 & 0 & 110 & 85 & 61 & 96 \\
\hline Sm & $\%$ & 0 & 0 & $3 \mathrm{nq}$ & 83 & 65 & 93 \\
\hline Eu & $\%$ & 0 & 5 & $4 \mathrm{ng}$ & 87 & 63 & 96 \\
\hline Gd & $\%$ & 0 & 5 & 109 & 85 & 63 & 96 \\
\hline Tb & $\%$ & 3 & 3 & 118 & 86 & 67 & 97 \\
\hline Dy & $\%$ & 0 & 5 & 116 & 89 & 64 & 98 \\
\hline Но & $\%$ & 0 & 5 & 117 & 86 & 66 & 99 \\
\hline $\mathbf{E r}$ & $\%$ & 0 & 5 & 118 & 87 & 64 & 97 \\
\hline Tm & $\%$ & 0 & 5 & $4 \mathrm{nq}$ & 99 & 67 & 98 \\
\hline $\mathbf{Y b}$ & $\%$ & 0 & 0 & $4 \mathrm{nq}$ & 86 & 62 & 97 \\
\hline $\mathbf{L u}$ & $\%$ & 1 & 1 & 118 & 81 & 72 & 98 \\
\hline Hf & $\%$ & $\mathrm{nq}$ & $\mathrm{nq}$ & $\mathrm{nq}$ & $\mathrm{nq}$ & $\mathrm{nq}$ & $\mathrm{nq}$ \\
\hline Ta & $\%$ & $\mathrm{nq}$ & 41 & 53 & $\mathrm{nq}$ & $\mathrm{nq}$ & 15 \\
\hline $\mathbf{W}$ & $\%$ & 10 & 63 & 83 & 35 & & $n q$ \\
\hline $\mathrm{Hg}$ & $\%$ & nq & 47 & $7 \mathrm{nq}$ & $\mathrm{nq}$ & $\mathrm{nq}$ & nb \\
\hline TI & $\%$ & 3 & 69 & $9 \mathrm{nq}$ & 102 & 76 & 113 \\
\hline $\mathbf{P b}$ & $\%$ & 1 & 20 & $\mathrm{nq}$ & 105 & 79 & 93 \\
\hline $\mathbf{B i}$ & $\%$ & nq & $\mathrm{nq}$ & $\mathrm{nq}$ & $\mathrm{nq}$ & $\mathrm{nq}$ & nb \\
\hline Th & $\%$ & $\mathrm{nq}$ & 87 & $7 \mathrm{nq}$ & nq & 58 & 63 \\
\hline $\mathbf{U}$ & $\%$ & 3 & 80 & nq & 84 & 76 & 104 \\
\hline
\end{tabular}


Tab. A.5.: Ergebnisse des Kationenaustausches für Porenwässer und Wasserproben aus Cospuden; Konzentrationen in Lösungen nach dem Kationenaustausch in \% bezogen auf die Ausgangskonzentrationen (Tab. A.1.) von Porenlösungen und Wasserproben aus Cospuden.

\begin{tabular}{|c|c|c|c|c|c|c|c|c|c|}
\hline & & RCO4 $17 \mathrm{~m}$ & RCO4 20 m & RCO4 $22 \mathrm{~m}$ & RCO4 26 m & RCO436 m & RCO4 44 m & RCO4 $52 \mathrm{~m}$ & CML 21-22 m \\
\hline & & Porenlösung & Porenlösung & Porenlösung I & Porenlösung & Porenlösung & Porenlösung & Porenlösung & Porenlösung \\
\hline & & Nov 1995 & Nov 1995 & Nov 1995 & Nov 1995 & Nov 1995 & Nov 1995 & Nov 1995 & Aug 1998 \\
\hline Sulfat & $\%$ & 59 & 84 & 80 & 95 & 54 & 83 & 89 & 83 \\
\hline DOC & $\%$ & nq & 34,9 & & 93 & 75 & $\mathrm{nq}$ & 72 & 80 \\
\hline $\mathbf{L i}$ & $\%$ & 1 & 59 & 17 & 97 & 88 & 52 & 68 & 31 \\
\hline $\mathbf{B e}$ & $\%$ & nq & $\mathrm{nq}$ & nq & 881 & $\mathrm{nq}$ & nq & $\mathrm{nq}$ & nq \\
\hline $\mathbf{N a}$ & $\%$ & 0 & 47 & & nq & 91 & 43 & 60 & 20 \\
\hline Mg & $\%$ & 0 & 32 & 3 & 95 & 84 & 27 & 44 & 11 \\
\hline Al & $\%$ & nq & $\mathrm{nq}$ & 80 & 90 & $\mathrm{nq}$ & nq & $\mathrm{nq}$ & nq \\
\hline $\mathbf{K}$ & $\%$ & 31 & 39 & & $6 \mathrm{nq}$ & 78 & 91 & 68 & 71 \\
\hline $\mathbf{C a}$ & $\%$ & 0 & 16 & 1 & 119 & 86 & 11 & 26 & 4 \\
\hline Sc & $\%$ & $\mathrm{nq}$ & $\mathrm{nq}$ & 59 & 98 & 80 & & $8 \mathrm{nq}$ & 71 \\
\hline Ti & $\%$ & $\mathrm{nq}$ & $\mathrm{nq}$ & nq & 891 & $\mathrm{nq}$ & $\mathrm{nq}$ & $\mathrm{nq}$ & $\mathrm{nq}$ \\
\hline $\mathbf{V}$ & $\%$ & & $\mathrm{nq}$ & 0 & 89 & $\mathrm{nq}$ & $\mathrm{nq}$ & $\mathrm{nq}$ & $\mathrm{nq}$ \\
\hline $\mathbf{C r}$ & $\%$ & 0 & $n$ & nq & 117 & $\mathrm{nq}$ & $\mathrm{nq}$ & & nq \\
\hline Mn & $\%$ & 0 & 32 & 2 & 88 & 86 & 27 & 45 & 11 \\
\hline $\mathbf{F e}$ & $\%$ & $<87$ & $\mathrm{nq}$ & 100 & 87 & 47 & $7 \mathrm{nq}$ & $\mathrm{nq}$ & nq \\
\hline Co & $\%$ & 0 & 11 & $\mathrm{nq}$ & 95 & 72 & 20 & 46 & 0 \\
\hline $\mathbf{N i}$ & $\%$ & 1 & 48 & 0 & 87 & 80 & 42 & 49 & 31 \\
\hline $\mathbf{C u}$ & $\%$ & $<16$ & 36 & 33 & 99 & 80 & $n q$ & 57 & 14 \\
\hline $\mathbf{Z n}$ & $\%$ & 4 & 37 & 35 & 85 & 27 & 18 & 49 & 14 \\
\hline As & $\%$ & $\mathrm{nq}$ & & $n q$ & 100 & $\mathrm{nq}$ & 42 & 67 & 63 \\
\hline $\mathbf{R b}$ & $\%$ & 32 & 34 & 12 & 94 & 87 & 28 & 47 & 21 \\
\hline \begin{tabular}{|l|} 
Sr \\
\end{tabular} & $\%$ & 0 & 5 & & $1 \mathrm{nq}$ & 78 & 3 & 15 & 2 \\
\hline $\mathbf{Y}$ & $\%$ & 30 & $<<77$ & 20 & 85 & 56 & $5<10$ & 88 & $<<45$ \\
\hline $\mathbf{Z r}$ & $\%$ & 43 & 103 & 112 & 99 & 91 & 107 & 107 & $\mathrm{nq}$ \\
\hline $\mathbf{N b}$ & $\%$ & 13 & 33 & $\mathrm{nq}$ & 79 & 45 & 24 & 22 & 47 \\
\hline Mo & $\%$ & 0 & 57 & & $2 n q$ & 7 & 38 & 69 & 40 \\
\hline Cd & $\%$ & 50 & $<<6$ & 27 & & $\mathrm{nq}$ & 25 & 55 & 33 \\
\hline Sn & $\%$ & $\mathrm{nq}$ & $\mathrm{nq}$ & nq & $\mathrm{nq}$ & $\mathrm{nq}$ & $\mathrm{nq}$ & $\mathrm{nq}$ & nq \\
\hline $\mathbf{S b}$ & $\%$ & 74 & 55 & $\begin{array}{l}55 \\
\end{array}$ & 96 & 98 & 53 & 76 & 78 \\
\hline \begin{tabular}{|l|} 
Cs \\
\end{tabular} & $\%$ & 42 & 31 & 14 & 90 & 86 & & $6 \mathrm{nq}$ & $<20$ \\
\hline $\mathbf{B a}$ & $\%$ & 0 & 0 & & nq & 79 & 0 & 0 & 0 \\
\hline La & $\%$ & 20 & $<<10$ & 33 & 66 & 33 & 13 & 34 & $4<71$ \\
\hline Ce & $\%$ & 29 & $<<12$ & 34 & 64 & 37 & 20 & 53 & 81 \\
\hline Pr & $\%$ & $\mathrm{nq}$ & $\mathrm{nq}$ & nq & & $\mathrm{nq}$ & $\mathrm{nq}$ & $\mathrm{nq}$ & $\mathrm{nq}$ \\
\hline $\mathbf{N d}$ & $\%$ & $<27$ & $<21$ & $\mathrm{nq}$ & 67 & 60 & $<32$ & $\mathrm{nq}$ & $\mathrm{nq}$ \\
\hline Sm & $\%$ & $\mathrm{nq}$ & $\mathrm{nq}$ & nq & & $\mathrm{nq}$ & nq & $\mathrm{nq}$ & nq \\
\hline Eu & $\%$ & $\mathrm{nq}$ & $\mathrm{nq}$ & $\mathrm{nq}$ & & $\mathrm{nq}$ & $\mathrm{nq}$ & $\mathrm{nq}$ & nq \\
\hline Gd & $\%$ & $\mathrm{nq}$ & $\mathrm{nq}$ & nq & & nq & $\mathrm{nq}$ & $\mathrm{nq}$ & $\mathrm{nq}$ \\
\hline Tb & $\%$ & $\mathrm{nq}$ & nq & nq & 741 & $n q$ & nq & $\mathrm{nq}$ & nq \\
\hline Dy & $\%$ & $\mathrm{nq}$ & $\mathrm{nq}$ & nq & 751 & $\mathrm{nq}$ & $\mathrm{nq}$ & $\mathrm{nq}$ & $\mathrm{nq}$ \\
\hline Ho & $\%$ & $\mathrm{nq}$ & $\mathrm{nq}$ & nq & 751 & $\mathrm{nq}$ & $\mathrm{nq}$ & $\mathrm{nq}$ & $\mathrm{nq}$ \\
\hline $\mathbf{E r}$ & $\%$ & $\mathrm{nq}$ & $\mathrm{nq}$ & $\mathrm{nq}$ & 74 & nq & nq & $\mathrm{nq}$ & nq \\
\hline Tm & $\%$ & $\mathrm{nq}$ & $\mathrm{nq}$ & nq & 731 & $\mathrm{nq}$ & $\mathrm{nq}$ & $\mathrm{nq}$ & $\mathrm{nq}$ \\
\hline Yb & $\%$ & $\mathrm{nq}$ & $\mathrm{nq}$ & nq & $71_{1}$ & $\mathrm{nq}$ & $\mathrm{nq}$ & $\mathrm{nq}$ & $\mathrm{nq}$ \\
\hline \begin{tabular}{|l|}
$\mathbf{L u}$ \\
\end{tabular} & $\%$ & $\mathrm{nq}$ & $\mathrm{nq}$ & nq & 711 & $n q$ & nq & $\mathrm{nq}$ & nq \\
\hline Hf & $\%$ & $\mathrm{nq}$ & $\mathrm{nq}$ & nq & 821 & $n q$ & nq & $\mathrm{nq}$ & nq \\
\hline Ta & $\%$ & $\mathrm{nq}$ & $\mathrm{nq}$ & nq & 791 & $\mathrm{nq}$ & $<13$ & 12 & $<<49$ \\
\hline $\mathbf{W}$ & $\%$ & $<6$ & 58 & 52 & 75 & 48 & 78 & 71 & 55 \\
\hline $\mathrm{Hg}$ & $\%$ & $\operatorname{lnb}$ & nb & $n b$ & $\mathrm{nb}$ & $\mathrm{nb}$ & $n b$ & nb & $n b$ \\
\hline TI & $\%$ & 95 & $5<31$ & 22 & 69 & 92 & 47 & $7<<38$ & $<48$ \\
\hline$\overline{\mathbf{P b}}$ & $\%$ & $\mathrm{nq}$ & 57 & 39 & 65 & 70 & 52 & 61 & 25 \\
\hline Bi & $\%$ & $\operatorname{lnb}$ & nb & nb & $n b$ & $n b$ & $n b$ & nb & $n b$ \\
\hline Th & $\%$ & $\mathrm{nq}$ & $\mathrm{nq}$ & $\mathrm{nq}$ & 80 & $\mathrm{nq}$ & $\mathrm{nq}$ & 15 & $\mathrm{nq}$ \\
\hline $\mathbf{U}$ & $\%$ & $\mathrm{nq}$ & $\mathrm{nq}$ & 6 & 85 & 4 & 16 & 38 & 19 \\
\hline
\end{tabular}


Tab. A.5. (Forts.): Ergebnisse des Kationenaustausches für Porenwässer und Wasserproben aus Cospuden; Konzentrationen in Lösungen nach dem Kationenaustausch in \% bezogen auf die Ausgangskonzentrationen (Tab. A.1.) von Porenlösungen und Wasserproben aus Cospuden.

\begin{tabular}{|c|c|c|c|c|c|c|c|c|c|}
\hline & & CML 24 m & CML 26 m & CML 32 m & CML 36 m & CML 37,5 m & CML 39-40 m & RCO4 & RCO10 \\
\hline & & Porenlösung & Porenlösung & Porenlösung & Porenlösung & Porenlösung & Porenlösung & Wasserprobe & Wasserprobe \\
\hline & & Aug 1998 & Aug 1998 & Aug 1998 & Aug 1998 & Aug 1998 & Aug 1998 & Okt 1998 & Okt 1998 \\
\hline Sulfat & $\%$ & $\mathrm{nb}$ & $\mathrm{nb}$ & nb & nb & $\mathrm{nb}$ & 109 & & $6 n b$ \\
\hline DOC & $\%$ & nb & nb & nb & $\mathrm{nb}$ & nb & & 50 & 32 \\
\hline $\mathbf{L i}$ & $\%$ & 55 & 60 & 53 & 23 & 56 & 100 & 2 & 1 \\
\hline $\mathbf{B e}$ & $\%$ & 64 & 71 & 67 & 67 & 67 & & $7 \mathrm{nq}$ & $\mathrm{nq}$ \\
\hline $\mathbf{N a}$ & $\%$ & 56 & 50 & 54 & 23 & 55 & 101 & 2 & 2 \\
\hline Mg & $\%$ & 33 & 48 & 38 & 9 & 32 & 53 & 0 & 0 \\
\hline Al & $\%$ & 16 & 56 & 25 & 30 & & nq & $\mathrm{nq}$ & $\mathrm{nq}$ \\
\hline $\mathbf{K}$ & $\%$ & 117 & 44 & 28 & 119 & 27 & 77 & 0 & 0 \\
\hline $\mathbf{C a}$ & $\%$ & 15 & 38 & 20 & 4 & 15 & 75 & 7 & 6 \\
\hline Sc & $\%$ & 100 & $\mathrm{nq}$ & 100 & 100 & 100 & $\mathrm{nq}$ & & $8 \mathrm{nq}$ \\
\hline Ti & $\%$ & 5 & 82 & 16 & 20 & & $6 \mathrm{nq}$ & $\mathrm{nq}$ & $\mathrm{nq}$ \\
\hline $\mathbf{V}$ & $\%$ & 45 & $5 \mathrm{nq}$ & 80 & 69 & & $\mathrm{nq}$ & & $1 \mathrm{nq}$ \\
\hline $\mathbf{C r}$ & $\%$ & 69 & $\mathrm{nq}$ & 82 & 118 & 54 & 101 & 0 & 0 \\
\hline Mn & $\%$ & 34 & $4 \mathrm{nq}$ & 37 & 10 & 34 & 37 & 3 & 90 \\
\hline $\mathbf{F e}$ & $\%$ & 33 & 51 & 4 & 78 & 12 & 55 & 11 & 96 \\
\hline Co & $\%$ & 36 & 54 & 41 & 22 & 47 & & $9 \mathrm{nq}$ & 0 \\
\hline $\mathbf{N i}$ & $\%$ & 34 & 53 & 39 & 17 & 33 & 46 & 0 & 13 \\
\hline $\mathbf{C u}$ & $\%$ & 135 & $\mathrm{nq}$ & 54 & 62 & 58 & 67 & 0 & 0 \\
\hline $\mathbf{Z n}$ & $\%$ & 35 & 63 & 87 & 52 & 37 & 53 & 0 & 19 \\
\hline \begin{tabular}{|l|}
$\mathbf{A s}$ \\
\end{tabular} & $\%$ & 59 & $\mathrm{nq}$ & & $0 \mathrm{nq}$ & & $\mathrm{nq}$ & $\mathrm{nq}$ & $\mathrm{nq}$ \\
\hline $\mathbf{R b}$ & $\%$ & $n b$ & $n b$ & $\mathrm{nb}$ & $\mathrm{nb}$ & $n b$ & 57 & 70 & 0 \\
\hline \begin{tabular}{|l|} 
Sr \\
\end{tabular} & $\%$ & 6 & 26 & 11 & 2 & 7 & 77 & 8 & 5 \\
\hline $\mathbf{Y}$ & $\%$ & 17 & 38 & 86 & 100 & & $\mathrm{nq}$ & 1 & 1 \\
\hline $\mathbf{Z r}$ & $\%$ & 43 & $\sqrt[n q]{ }$ & 100 & 100 & 70 & 102 & 92 & \\
\hline $\mathbf{N b}$ & $\%$ & nb & nb & $\mathrm{nb}$ & $\mathrm{nb}$ & $\mathrm{nb}$ & 37 & & $0 \mathrm{nq}$ \\
\hline Mo & $\%$ & $\mathrm{nq}$ & 35 & 65 & 29 & 53 & 57 & 69 & 68 \\
\hline Cd & $\%$ & nb & $\mathrm{nb}$ & $\mathrm{nb}$ & $\mathrm{nb}$ & & & $5 \mathrm{nb}$ & $\mathrm{nb}$ \\
\hline Sn & $\%$ & nb & nb & $\mathrm{nb}$ & $\mathrm{nb}$ & $n b$ & & $4 \mathrm{nq}$ & 0 \\
\hline $\mathbf{S b}$ & $\%$ & nb & nb & $\mathrm{nb}$ & $\mathrm{nb}$ & $\mathrm{nb}$ & $\mathrm{nq}$ & $\mathrm{nq}$ & $\mathrm{nq}$ \\
\hline \begin{tabular}{|l|} 
Cs \\
\end{tabular} & $\%$ & nb & nb & $\mathrm{nb}$ & $\mathrm{nb}$ & $n b$ & & $7 \mathrm{nq}$ & $\mathrm{nq}$ \\
\hline $\mathbf{B a}$ & $\%$ & 3 & 85 & 5 & 1 & 1 & 55 & 35 & 0 \\
\hline La & $\%$ & 0 & 27 & 7 & 0 & 0 & 14 & 93 & $3 \mathrm{nq}$ \\
\hline Ce & $\%$ & nb & nb & nb & $\mathrm{nb}$ & $n b$ & & $6 \mathrm{nq}$ & $\mathrm{nq}$ \\
\hline Pr & $\%$ & nb & $n b$ & $\mathrm{nb}$ & $\mathrm{nb}$ & $n b$ & & $5 \mathrm{nq}$ & $\mathrm{nq}$ \\
\hline $\mathbf{N d}$ & $\%$ & nb & nb & $\mathrm{nb}$ & $\mathrm{nb}$ & $\mathrm{nb}$ & & $0 \mathrm{nq}$ & $\mathrm{nq}$ \\
\hline Sm & $\%$ & nb & nb & $\mathrm{nb}$ & $\mathrm{nb}$ & $n b$ & & $3 \mathrm{nq}$ & $\mathrm{nq}$ \\
\hline Eu & $\%$ & $\mathrm{nq}$ & & $8 \mathrm{nq}$ & $\mathrm{nq}$ & $\mathrm{nq}$ & nq & $\mathrm{nq}$ & $\mathrm{nq}$ \\
\hline Gd & $\%$ & nb & nb & $\mathrm{nb}$ & $\mathrm{nb}$ & $\mathrm{nb}$ & $<26$ & $\mathrm{nq}$ & $\mathrm{nq}$ \\
\hline Tb & $\%$ & nb & nb & $\mathrm{nb}$ & $\mathrm{nb}$ & $n b$ & nq & $\mathrm{nq}$ & $\mathrm{nq}$ \\
\hline Dy & $\%$ & nb & nb & $\mathrm{nb}$ & $\mathrm{nb}$ & $\mathrm{nb}$ & $<31$ & $\mathrm{nq}$ & $\mathrm{nq}$ \\
\hline Ho & $\%$ & nb & nb & $\mathrm{nb}$ & $\mathrm{nb}$ & $n b$ & $\mathrm{nq}$ & $\mathrm{nq}$ & $\mathrm{nq}$ \\
\hline $\mathbf{E r}$ & $\%$ & nb & nb & $\mathrm{nb}$ & $\mathrm{nb}$ & $n b$ & nq & $\mathrm{nq}$ & $\mathrm{nq}$ \\
\hline Tm & $\%$ & nb & nb & $\mathrm{nb}$ & $\mathrm{nb}$ & $n b$ & $\mathrm{nq}$ & $\mathrm{nq}$ & $\mathrm{nq}$ \\
\hline Yb & $\%$ & nb & nb & $\mathrm{nb}$ & $\mathrm{nb}$ & $n b$ & $\mathrm{nq}$ & $\mathrm{nq}$ & $\mathrm{nq}$ \\
\hline \begin{tabular}{|l|}
$\mathbf{L u}$ \\
\end{tabular} & $\%$ & 27 & & $0 \mathrm{nq}$ & $\mathrm{nq}$ & & $\mathrm{nq}$ & $\mathrm{nq}$ & $\mathrm{nq}$ \\
\hline Hf & $\%$ & nb & nb & $\mathrm{nb}$ & $\mathrm{nb}$ & $n b$ & $\mathrm{nq}$ & $\mathrm{nq}$ & $\operatorname{nq}$ \\
\hline Ta & $\%$ & nb & $\mathrm{nb}$ & $\mathrm{nb}$ & $\mathrm{nb}$ & $\mathrm{nb}$ & 26 & 91 & $1 \mathrm{nq}$ \\
\hline $\mathbf{W}$ & $\%$ & nb & nb & $\mathrm{nb}$ & $\mathrm{nb}$ & $n b$ & & $7 \mathrm{nq}$ & $\mathrm{nq}$ \\
\hline $\mathrm{Hg}$ & $\%$ & nb & $\mathrm{nb}$ & $\mathrm{nb}$ & $\mathrm{nb}$ & $\mathrm{nq}$ & $\mathrm{nb}$ & $\mathrm{nb}$ & $\operatorname{lnb}$ \\
\hline Tl & $\%$ & $n b$ & nb & $\mathrm{nb}$ & $\mathrm{nb}$ & $n b$ & & $3 n q$ & $\mathrm{nq}$ \\
\hline $\mathbf{P b}$ & $\%$ & $\mathrm{nq}$ & $\mathrm{nq}$ & $\mathrm{nq}$ & $\mathrm{nq}$ & $\mathrm{nq}$ & & $3 \mathrm{nq}$ & $\mathrm{nq}$ \\
\hline $\mathrm{Bi}$ & $\%$ & $\mathrm{nb}$ & nb & $\mathrm{nb}$ & $\mathrm{nb}$ & $\mathrm{nb}$ & $n b$ & $\mathrm{nq}$ & $\mathrm{nq}$ \\
\hline Th & $\%$ & nb & nb & $\mathrm{nb}$ & $\mathrm{nb}$ & $\mathrm{nb}$ & $\mathrm{nq}$ & $\mathrm{nq}$ & $\mathrm{nq}$ \\
\hline $\mathbf{U}$ & $\%$ & nb & nb & $\mathrm{nb}$ & $\mathrm{nb}$ & $n b$ & 47 & & $9 \mathrm{nq}$ \\
\hline
\end{tabular}


Tab. A.5. (Forts.): Ergebnisse des Kationenaustausches für Porenwässer und Wasserproben aus Cospuden; Konzentrationen in Lösungen nach dem Kationenaustausch in \% bezogen auf die Ausgangskonzentrationen (Tab. A.1.) von Porenlösungen und Wasserproben aus Cospuden.

\begin{tabular}{|c|c|c|c|c|c|c|c|c|c|c|}
\hline & & RCO12 & RCO13 & RCO14 & Bohrung $1 \mathrm{~m}$ & Bohrung 2 m & Bohrung 3 m & See & CML $20 \mathrm{~m}$ & CML 27,5 m \\
\hline & & Wasserprobe & eWasserprobe & Wasserprobe & ePorenlösung & Porenlösung & Porenlösung & Wasserprobe & Wasserprobe & Wasserprobe \\
\hline & & Okt 1998 & Okt 1998 & Okt 1998 & Nov 1998 & Nov 1998 & Nov 1998 & Nov 1998 & Jan 1999 & Jan 1999 \\
\hline Sulfat & $\%$ & $n b$ & nb & 47 & 98 & 83 & 90 & 11 & 88 & 100 \\
\hline DOC & $\%$ & 73 & 26 & & $7 \mathrm{nb}$ & nb & nb & 100 & 81 & 90 \\
\hline $\mathbf{L i}$ & $\%$ & 21 & 0 & 0 & 48 & 60 & 57 & 75 & 84 & 113 \\
\hline $\mathbf{B e}$ & $\%$ & nq & $\mathrm{nq}$ & nq & 31 & 65 & $48 \mathrm{r}$ & $\mathrm{nq}$ & nq & nq \\
\hline $\mathbf{N a}$ & $\%$ & 19 & 14 & 2 & 8 & 32 & 36 & 68 & 72 & 108 \\
\hline Mg & $\%$ & 45 & 74 & 44 & 24 & 37 & 33 & 36 & 27 & 96 \\
\hline Al & $\%$ & nq & $\mathrm{nq}$ & nq & 6 & 104 & 56 & & nq & $\mathrm{nq}$ \\
\hline $\mathbf{K}$ & $\%$ & 0 & 0 & 6 & 32 & $2 \mathrm{nq}$ & 82 & 82 & 100 & 118 \\
\hline Ca & $\%$ & 63 & 92 & 58 & 12 & 24 & 21 & 17 & 12 & 82 \\
\hline Sc & $\%$ & 95 & & $\mathrm{nq}$ & 70 & 82 & & $\mathrm{nq}$ & $\mathrm{nq}$ & $\mathrm{nq}$ \\
\hline $\mathbf{T i}$ & $\%$ & $\mathrm{nq}$ & & $\mathrm{nq}$ & $\mathrm{nq}$ & nq & $\mathrm{nq}$ & $\mathrm{nq}$ & $\mathrm{nq}$ & $\mathrm{nq}$ \\
\hline $\mathbf{V}$ & $\%$ & 89 & 87 & 22 & 70 & 72 & 5 & 0 & 112 & $2 n q$ \\
\hline $\mathrm{Cr}$ & $\%$ & 0 & 1 & 0 & 53 & 881 & $\mathrm{nq}$ & 95 & 0 & 0 \\
\hline Mn & $\%$ & 46 & 5 & 44 & 24 & 39 & 35 & 36 & $\mathrm{nq}$ & 94 \\
\hline $\mathbf{F e}$ & $\%$ & 47 & 73 & 100 & & $2 \mathrm{nq}$ & 39 & 38 & $\mathrm{nq}$ & $\mathrm{nq}$ \\
\hline Co & $\%$ & 26 & 12 & 19 & 24 & 32 & 36 & 56 & 0 & 0 \\
\hline $\mathbf{N i}$ & $\%$ & 36 & 37 & 28 & 23 & 35 & 37 & 35 & 52 & 61 \\
\hline $\mathbf{C u}$ & $\%$ & 33 & 3 & 2 & 17 & 67 & 43 & 89 & 42 & $2 n q$ \\
\hline $\mathbf{Z n}$ & $\%$ & 38 & 52 & 0 & 24 & 44 & 54 & & $\mathrm{nq}$ & 77 \\
\hline As & $\%$ & $\mathrm{nq}$ & $\mathrm{nq}$ & $\mathrm{nq}$ & 18 & 73 & $79 / \mathrm{r}$ & $\mathrm{nq}$ & $\mathrm{nq}$ & $\mathrm{nq}$ \\
\hline $\mathbf{R b}$ & $\%$ & 3 & 38 & 9 & 34 & 73 & 74 & 0 & 54 & 113 \\
\hline $\mathbf{S r}$ & $\%$ & 74 & 99 & 68 & 6 & 13 & 12 & 6 & 2 & 63 \\
\hline $\mathbf{Y}$ & $\%$ & & $n q$ & $\mathrm{nq}$ & 0 & 12 & 2 & & $\mathrm{nq}$ & $\mathrm{nq}$ \\
\hline $\mathbf{Z r}$ & $\%$ & 8 & 76 & 38 & 88 & & $\mathrm{nq}$ & & $\mathrm{nq}$ & $\mathrm{nq}$ \\
\hline $\mathbf{N b}$ & $\%$ & $\mathrm{nq}$ & $\mathrm{nq}$ & & $\mathrm{nnq}$ & $\mathrm{nq}$ & $\mathrm{nq}$ & & $\mathrm{nq}$ & 100 \\
\hline Mo & $\%$ & 38 & & $\mathrm{nq}$ & 29 & 19 & 10 & 1 & 46 & 100 \\
\hline Cd & $\%$ & $n b$ & nb & nb & 25 & 43 & $43 \mathrm{r}$ & $n b$ & 70 & 81 \\
\hline Sn & $\%$ & $\mathrm{nq}$ & $\mathrm{nq}$ & $\mathrm{nq}$ & 47 & 54 & 84 & & $\mathrm{nq}$ & $\mathrm{nq}$ \\
\hline $\mathbf{S b}$ & $\%$ & nq & $\mathrm{nq}$ & $\mathrm{nq}$ & 61 & 94 & $89 \mathrm{r}_{\mathrm{r}}$ & $\mathrm{nq}$ & 100 & $\mathrm{nq}$ \\
\hline Cs & $\%$ & & nq & $\mathrm{nq}$ & 34 & 48 & $50 \mathrm{r}$ & $\mathrm{nq}$ & 60 & 100 \\
\hline $\mathbf{B a}$ & $\%$ & nq & 100 & 96 & 1 & 2 & 8 & 0 & 0 & 14 \\
\hline La & $\%$ & & $9 \mathrm{nq}$ & $\mathrm{nq}$ & 0 & 5 & & $\mathrm{nq}$ & $\mathrm{nq}$ & nq \\
\hline $\mathrm{Ce}$ & $\%$ & nq & $\mathrm{nq}$ & nq & 0 & 5 & & $\mathrm{nq}$ & nq & $\mathrm{nq}$ \\
\hline Pr & $\%$ & $\mathrm{nq}$ & $\mathrm{nq}$ & $\mathrm{nq}$ & 0 & 12 & & $\mathrm{nq}$ & $\mathrm{nq}$ & $\mathrm{nq}$ \\
\hline Nd & $\%$ & $\mathrm{nq}$ & $\mathrm{nq}$ & $\mathrm{nq}$ & 0 & 17 & & $\mathrm{nq}$ & $\mathrm{nq}$ & $\mathrm{nq}$ \\
\hline $\mathrm{Sm}$ & $\%$ & nq & $\mathrm{nq}$ & nq & 1 & 41 & & $\mathrm{nq}$ & nq & $\mathrm{nq}$ \\
\hline Eu & $\%$ & $\mathrm{nq}$ & $\mathrm{nq}$ & $\mathrm{nq}$ & & $\mathrm{nq}$ & & $\mathrm{nq}$ & nq & $\mathrm{nq}$ \\
\hline Gd & $\%$ & $\mathrm{nq}$ & $\mathrm{nq}$ & $\mathrm{nq}$ & 0 & 23 & & $\mathrm{nq}$ & $\mathrm{nq}$ & nq \\
\hline Tb & $\%$ & nq & $\mathrm{nq}$ & $\mathrm{nq}$ & & $\mathrm{nq}$ & & $\mathrm{nq}$ & $\mathrm{nq}$ & $\mathrm{nq}$ \\
\hline Dy & $\%$ & $\mathrm{nq}$ & $\mathrm{nq}$ & $\mathrm{nq}$ & 0 & 40 & & $\mathrm{nq}$ & $\mathrm{nq}$ & $\mathrm{nq}$ \\
\hline Ho & $\%$ & $\mathrm{nq}$ & $\mathrm{nq}$ & $\mathrm{nq}$ & & $\mathrm{nq}$ & & $\mathrm{nq}$ & $\mathrm{nq}$ & $\mathrm{nq}$ \\
\hline $\mathbf{E r}$ & $\%$ & $\mathrm{nq}$ & $\mathrm{nq}$ & $\mathrm{nq}$ & & $\mathrm{nq}$ & & $\mathrm{nq}$ & $\mathrm{nq}$ & $\mathrm{nq}$ \\
\hline Tm & $\%$ & $\mathrm{nq}$ & $\mathrm{nq}$ & $\mathrm{nq}$ & 1 & 58 & $\mathrm{nq}$ & $\mathrm{nq}$ & $\mathrm{nq}$ & $\mathrm{nq}$ \\
\hline $\mathbf{Y b}$ & $\%$ & $\mathrm{nq}$ & $\mathrm{nq}$ & $\mathrm{nq}$ & & $\mathrm{nq}$ & & $\mathrm{nq}$ & $\mathrm{nq}$ & $\mathrm{nq}$ \\
\hline Lu & $\%$ & $\mathrm{nq}$ & $\mathrm{nq}$ & $\mathrm{nq}$ & & $\operatorname{lnq}$ & $\mathrm{nq}$ & $\mathrm{nq}$ & nq & nq \\
\hline Hf & $\%$ & $\mathrm{nq}$ & $\mathrm{nq}$ & $\mathrm{nq}$ & & $\mathrm{nq}$ & $\mathrm{nq}$ & $\mathrm{nq}$ & $\mathrm{nq}$ & $\mathrm{nq}$ \\
\hline Ta & $\%$ & $\mathrm{nq}$ & $\mathrm{nq}$ & $\mathrm{nq}$ & & $7 \mathrm{nq}$ & $\mathrm{nq}$ & $\mathrm{nq}$ & $n b$ & $n b$ \\
\hline $\mathbf{W}$ & $\%$ & nq & 50 & 78 & 32 & 20 & $47 \mathrm{r}_{\mathrm{r}}$ & $\mathrm{nq}$ & 13 & 86 \\
\hline $\mathrm{Hg}$ & $\%$ & $n b$ & nb & nb & 72 & 47 & $55 \mathrm{r}$ & $n b$ & $\mathrm{nq}$ & 100 \\
\hline Tl & $\%$ & $\mathrm{nq}$ & $\mathrm{nq}$ & $\mathrm{nq}$ & 31 & 77 & $\left.41\right|_{r}$ & $\mathrm{nq}$ & $\mathrm{nq}$ & $\mathrm{nq}$ \\
\hline $\mathbf{P b}$ & $\%$ & $\mathrm{nq}$ & $\mathrm{nq}$ & $\mathrm{nq}$ & 4 & 66 & $63 \mathrm{r}$ & $\mathrm{nq}$ & 34 & 30 \\
\hline $\mathbf{B i}$ & $\%$ & nq & nq & nq & & nq & nq & $\mathrm{nq}$ & nq & nq \\
\hline Th & $\%$ & $\mathrm{nq}$ & $\mathrm{nq}$ & $\mathrm{nq}$ & & $\mathrm{nq}$ & $\mathrm{nq}$ & $\mathrm{nq}$ & $\mathrm{nq}$ & $\mathrm{nq}$ \\
\hline $\mathbf{U}$ & $\%$ & 24 & & $2 \mathrm{nq}$ & & $7 \mathrm{nq}$ & $\mathrm{nq}$ & & $\mathrm{nq}$ & 64 \\
\hline
\end{tabular}


Tab. A.5. (Forts.): Ergebnisse des Kationenaustausches für Porenwässer und Wasserproben aus Cospuden; Konzentrationen in Lösungen nach dem Kationenaustausch in \% bezogen auf die Ausgangskonzentrationen (Tab. A.1.) von Porenlösungen und Wasserproben aus Cospuden.

\begin{tabular}{|c|c|c|c|c|c|c|c|c|c|c|}
\hline & & \multicolumn{3}{|c|}{\begin{tabular}{|l|l|l|} 
CML 31,5 m & CML 39,5 m & CML 43,5 m \\
\end{tabular}} & CML 20 m & \multicolumn{2}{|c|}{ CML 27,5 m CML 31,5 m } & \multirow{2}{*}{\begin{tabular}{|l|} 
CML 39,5 m \\
Aug 1999 \\
\end{tabular}} & \multirow{2}{*}{\begin{tabular}{|l|} 
CML 43,5 m \\
Aug 1999 \\
\end{tabular}} & \multirow{2}{*}{\begin{tabular}{|l|} 
RCO3 \\
Aug 1999 \\
\end{tabular}} \\
\hline & & Wasserprobe & Wasserprobe & Jan 1999 & Aug 1999 & Aug 1999 & Aug 1999 & & & \\
\hline & & Jan 1999 & Jan 1999 & Wasserprobe & Wasserprobe & Wasserprobe & Wasserprobe & Wasserprobe & Wasserprobe & Wasserprobe \\
\hline Sulfat & $\%$ & 100 & 96 & 99 & 100 & 83 & 90 & 97 & 58 & 86 \\
\hline DOC & $\%$ & 59 & 27 & 25 & 64 & 83 & & nb & & $2 \mathrm{nq}$ \\
\hline $\mathbf{L i}$ & $\%$ & 105 & 120 & 104 & nq & 69 & 85 & 104 & 12 & 51 \\
\hline $\mathbf{B e}$ & $\%$ & $\mathrm{nq}$ & $\mathrm{nq}$ & $\mathrm{nq}$ & $\mathrm{nq}$ & 86 & 89 & $\mathrm{nq}$ & $\mathrm{nq}$ & 56 \\
\hline $\mathbf{N a}$ & $\%$ & 96 & 113 & 98 & 90 & 56 & 77 & 96 & 1 & 54 \\
\hline Mg & $\%$ & 93 & 100 & & $\mathrm{nq}$ & 87 & 79 & 78 & 3 & 34 \\
\hline Al & $\%$ & $\mathrm{nq}$ & $\mathrm{nq}$ & $\mathrm{nq}$ & nq & 93 & 72 & $\mathrm{nq}$ & 51 & 28 \\
\hline $\mathbf{K}$ & $\%$ & 111 & & 97 & 118 & $3 \mathrm{nq}$ & nq & 98 & 12 & 45 \\
\hline Ca & $\%$ & 82 & 69 & 83 & 44 & 45 & 71 & 73 & 1 & 19 \\
\hline Sc & $\%$ & $\mathrm{nq}$ & $\mathrm{nq}$ & $\mathrm{nq}$ & $\mathrm{nq}$ & nq & 85 & 88 & 63 & 61 \\
\hline $\mathbf{T i}$ & $\%$ & $\mathrm{nq}$ & nq & $\mathrm{nq}$ & nq & $\mathrm{nq}$ & $\mathrm{nq}$ & $\mathrm{nq}$ & $\mathrm{nq}$ & $\mathrm{nq}$ \\
\hline $\mathbf{V}$ & $\%$ & & nq & $\mathrm{nq}$ & 109 & 74 & 76 & 98 & 35 & 31 \\
\hline $\mathbf{C r}$ & $\%$ & 56 & $5 \mathrm{nq}$ & $\mathrm{nq}$ & 100 & $\mathrm{nq}$ & 77 & 111 & & $\mathrm{nq}$ \\
\hline Mn & $\%$ & 92 & 99 & & $\mathrm{nq}$ & 56 & 78 & 75 & 3 & 35 \\
\hline $\mathrm{Fe}$ & $\%$ & & $\mathrm{nq}$ & $\mathrm{nq}$ & nq & 82 & 84 & 19 & 3 & 38 \\
\hline Co & $\%$ & & $\mathrm{nq}$ & & $\mathrm{nq}$ & 51 & 60 & 89 & 34 & 62 \\
\hline $\mathrm{Ni}$ & $\%$ & & $n q$ & 120 & & 48 & 69 & 95 & & $\mathrm{nq}$ \\
\hline$\overline{\mathrm{Cu}}$ & $\%$ & 107 & & & nq & 86 & 76 & 98 & 35 & 73 \\
\hline $\mathbf{Z n}$ & $\%$ & & $\mathrm{nq}$ & $\mathrm{nq}$ & $\mathrm{nq}$ & 60 & 62 & 68 & 14 & 37 \\
\hline As & $\%$ & $\mathrm{nq}$ & $\mathrm{nq}$ & $\mathrm{nq}$ & nq & $\mathrm{nq}$ & 62 & 72 & 38 & $\mathrm{nq}$ \\
\hline $\mathbf{R b}$ & $\%$ & 118 & & & $n q$ & 83 & 87 & 102 & 16 & 66 \\
\hline \begin{tabular}{|l|}
$\mathrm{Sr}$ \\
\end{tabular} & $\%$ & 72 & 36 & 74 & 56 & 35 & 67 & 61 & 0 & 8 \\
\hline $\mathbf{Y}$ & $\%$ & $\mathrm{nq}$ & $83 \mathrm{r}$ & $\mathrm{nq}$ & $\mathrm{nq}$ & 49 & 48 & 97 & 1 & 0 \\
\hline $\mathbf{Z r}$ & $\%$ & & $\mathrm{nq}$ & $\mathrm{nq}$ & nq & 92 & 78 & 102 & 75 & 37 \\
\hline $\mathbf{N b}$ & $\%$ & $\mathrm{nq}$ & $\mathrm{nq}$ & $\mathrm{nq}$ & & nq & $\mathrm{nq}$ & $\mathrm{nq}$ & $\mathrm{nq}$ & $\mathrm{nq}$ \\
\hline Mo & $\%$ & & $1 \mathrm{nq}$ & 120 & & $4 \mathrm{nq}$ & 78 & 95 & 27 & 36 \\
\hline Cd & $\%$ & nb & $\mathrm{nq}$ & & $\mathrm{nq}$ & 72 & 106 & $\mathrm{nq}$ & $\mathrm{nq}$ & $\mathrm{nq}$ \\
\hline Sn & $\%$ & $\mathrm{nq}$ & $\mathrm{nq}$ & $\mathrm{nq}$ & $\mathrm{nq}$ & nq & $\mathrm{nq}$ & $\mathrm{nq}$ & $\mathrm{nq}$ & 71 \\
\hline $\mathbf{S b}$ & $\%$ & $\mathrm{nq}$ & $\mathrm{nq}$ & $\mathrm{nq}$ & $\mathrm{nq}$ & $\mathrm{nq}$ & $\mathrm{nq}$ & $\mathrm{nq}$ & nq & $\mathrm{nq}$ \\
\hline Cs & $\%$ & 120 & 501 & $\mathrm{nq}$ & nq & nq & 89 & $\mathrm{nq}$ & $\mathrm{nq}$ & 73 \\
\hline $\mathbf{B a}$ & $\%$ & 55 & 0 & 43 & 4 & 72 & 52 & 45 & 0 & 0 \\
\hline La & $\%$ & $\mathrm{nq}$ & $\mathrm{nq}$ & $\mathrm{nq}$ & nq & 30 & 35 & $\mathrm{nq}$ & 3 & 35 \\
\hline $\mathrm{Ce}$ & $\%$ & $\mathrm{nq}$ & $\mathrm{nq}$ & $\mathrm{nq}$ & nq & 34 & 35 & $\mathrm{nq}$ & 2 & 20 \\
\hline Pr & $\%$ & $\mathrm{nq}$ & $\mathrm{nq}$ & $\mathrm{nq}$ & nq & 36 & 32 & $n q$ & $\mathrm{nq}$ & 13 \\
\hline Nd & $\%$ & $\mathrm{nq}$ & $\mathrm{nq}$ & $\mathrm{nq}$ & $\mathrm{nq}$ & 38 & 33 & 89 & 11 & 20 \\
\hline $\mathbf{S m}$ & $\%$ & $\mathrm{nq}$ & $\mathrm{nq}$ & $\mathrm{nq}$ & nq & 41 & 51 & 101 & 39 & 50 \\
\hline Eu & $\%$ & $\mathrm{nq}$ & $\mathrm{nq}$ & $\mathrm{nq}$ & $\mathrm{nq}$ & 38 & & $\mathrm{nq}$ & $\mathrm{nq}$ & 60 \\
\hline Gd & $\%$ & $\mathrm{nq}$ & $\mathrm{nq}$ & $\mathrm{nq}$ & $\mathrm{nq}$ & 43 & & $n q$ & 14 & 26 \\
\hline $\mathbf{T b}$ & $\%$ & $\mathrm{nq}$ & $\mathrm{nq}$ & $\mathrm{nq}$ & nq & 47 & 56 & nq & $\mathrm{nq}$ & $\mathrm{nq}$ \\
\hline Dy & $\%$ & $\mathrm{nq}$ & $\mathrm{nq}$ & $\mathrm{nq}$ & $\mathrm{nq}$ & 44 & 39 & $\mathrm{nq}$ & 13 & 19 \\
\hline Ho & $\%$ & $\mathrm{nq}$ & $\mathrm{nq}$ & $\mathrm{nq}$ & $\mathrm{nq}$ & 53 & 54 & $\mathrm{nq}$ & $\mathrm{nq}$ & $\mathrm{nq}$ \\
\hline $\mathbf{E r}$ & $\%$ & $\mathrm{nq}$ & $\mathrm{nq}$ & $\mathrm{nq}$ & nq & 50 & 38 & $n q$ & $\mathrm{nq}$ & 26 \\
\hline Tm & $\%$ & $\mathrm{nq}$ & $\mathrm{nq}$ & $\mathrm{nq}$ & $\mathrm{nq}$ & 58 & 50 & $\mathrm{nq}$ & $\mathrm{nq}$ & $\mathrm{nq}$ \\
\hline Yb & $\%$ & $\mathrm{nq}$ & $\mathrm{nq}$ & $\mathrm{nq}$ & $\mathrm{nq}$ & $\mathrm{nq}$ & 57 & $7 \mathrm{nq}$ & 17 & 55 \\
\hline $\mathbf{L u}$ & $\%$ & $\mathrm{nq}$ & $\mathrm{nq}$ & $\mathrm{nq}$ & nq & nq & 75 & $\mathrm{nq}$ & nq & $\mathrm{nq}$ \\
\hline Hf & $\%$ & $\mathrm{nq}$ & $\mathrm{nq}$ & $\mathrm{nq}$ & nq & nq & $\mathrm{nq}$ & $\mathrm{nq}$ & $\mathrm{nq}$ & $\mathrm{nq}$ \\
\hline Ta & $\%$ & $\mathrm{nb}$ & nb & $\mathrm{nb}$ & 34 & & $\mathrm{nq}$ & 67 & $\mathrm{nq}$ & $\mathrm{nq}$ \\
\hline $\mathbf{W}$ & $\%$ & $\mathrm{nq}$ & $\mathrm{nq}$ & 79 & & $2 \mathrm{nq}$ & nq & $\mathrm{nq}$ & & $\mathrm{nq}$ \\
\hline $\mathrm{Hg}$ & $\%$ & 50 & 111 & 100 & $\mathrm{nq}$ & $\mathrm{nq}$ & 95 & 70 & & $\mathrm{nq}$ \\
\hline Tl & $\%$ & $\mathrm{nq}$ & $\mathrm{nq}$ & $\mathrm{nq}$ & $\mathrm{nq}$ & $\mathrm{nq}$ & $\mathrm{nq}$ & $\mathrm{nq}$ & $\mathrm{nq}$ & $\mathrm{nq}$ \\
\hline $\mathbf{P b}$ & $\%$ & $\mathrm{nq}$ & $\mathrm{nq}$ & $\mathrm{nq}$ & nq & nq & nq & $\mathrm{nq}$ & & $\mathrm{nq}$ \\
\hline $\mathrm{Bi}$ & $\%$ & $\mathrm{nq}$ & $\mathrm{nq}$ & $\mathrm{nq}$ & nq & $\mathrm{nq}$ & nq & $\mathrm{nq}$ & nq & $\mathrm{nq}$ \\
\hline Th & $\%$ & $\mathrm{nq}$ & $\mathrm{nq}$ & $\mathrm{nq}$ & $\mathrm{nq}$ & $\mathrm{nq}$ & $\mathrm{nq}$ & $\mathrm{nq}$ & $\mathrm{nq}$ & $\mathrm{nq}$ \\
\hline $\mathbf{U}$ & $\%$ & & $7 \mathrm{nq}$ & 103 & & 86 & 91 & 110 & & $5 \mathrm{nq}$ \\
\hline
\end{tabular}


Tab. A.5. (Forts.): Ergebnisse des Kationenaustausches für Porenwässer und Wasserproben aus Cospuden; Konzentrationen in Lösungen nach dem Kationenaustausch in \% bezogen auf die Ausgangskonzentrationen (Tab. A.1.) von Porenlösungen und Wasserproben aus Cospuden.

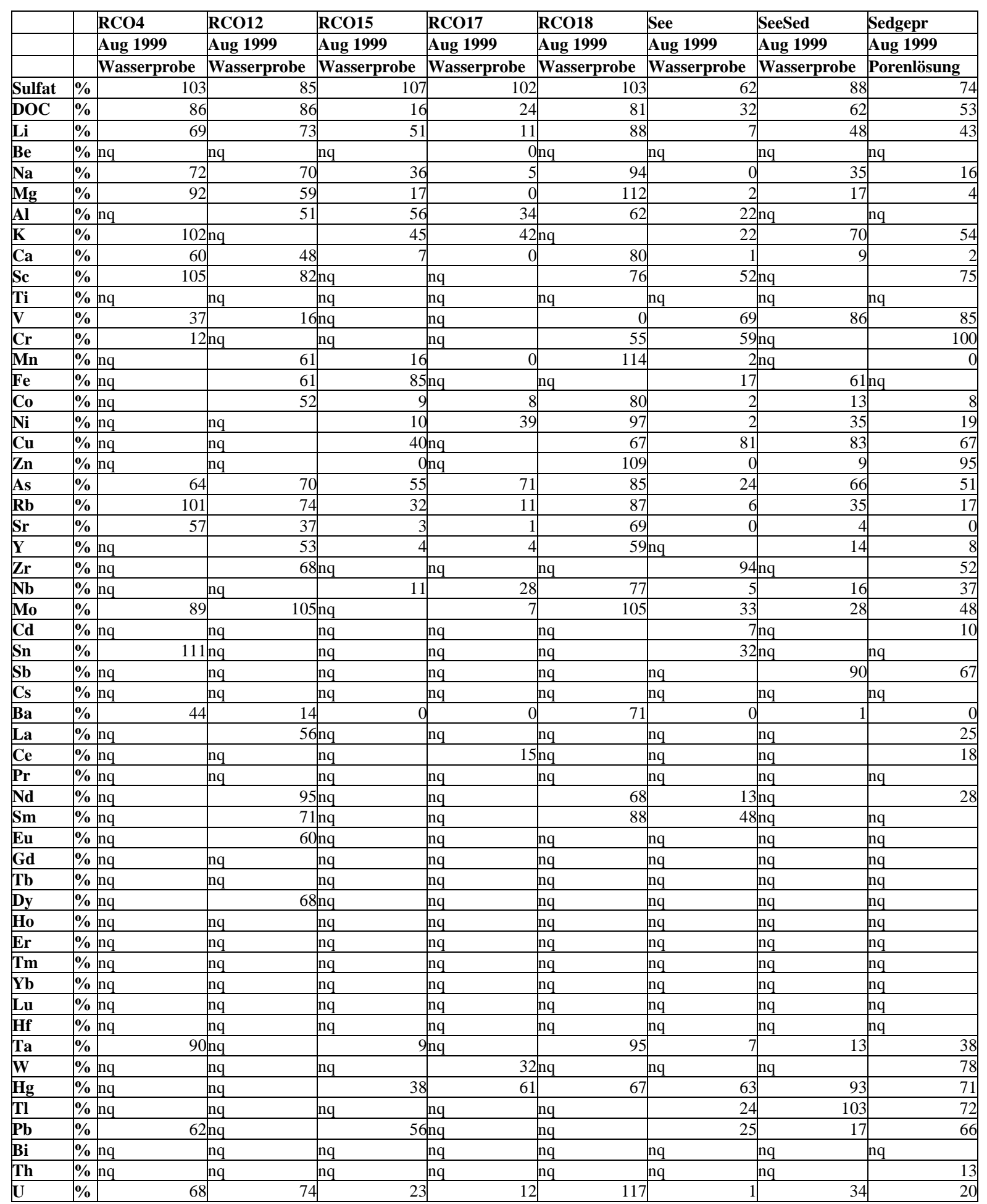


Tab. A.5. (Forts.): Ergebnisse des Kationenaustausches für Porenwässer und Wasserproben aus Cospuden; Konzentrationen in Lösungen nach dem Kationenaustausch in \% bezogen auf die Ausgangskonzentrationen (Tab. A.1.) von Porenlösungen und Wasserproben aus Cospuden.

\begin{tabular}{|c|c|c|c|c|c|c|}
\hline & & CML 20 m & CML 27,5 m & CML 31,5 m & CML 39,5 m & CML 43,5 m \\
\hline & & Jan 2000 & Jan 2000 & Jan 2000 & Jan 2000 & Jan 2000 \\
\hline & & Wasserprobe & Wasserprobe & Wasserprobe & Wasserprobe & Wasserprobe \\
\hline Sulfat & $\%$ & 91 & 99 & 107 & 70 & 106 \\
\hline DOC & $\%$ & 85 & 106 & 88 & 45 & 31 \\
\hline $\mathbf{L i}$ & $\%$ & 74 & 106 & 95 & 60 & 107 \\
\hline $\mathbf{B e}$ & $\%$ & $\mathrm{nq}$ & 82 & & $9 \mathrm{nq}$ & $\mathrm{nq}$ \\
\hline $\mathbf{N a}$ & $\%$ & 73 & 98 & 103 & 68 & 108 \\
\hline Mg & $\%$ & 55 & 99 & 88 & 63 & 96 \\
\hline Al & $\%$ & $\mathrm{nq}$ & 98 & 107 & $7 \mathrm{nq}$ & 0 \\
\hline $\mathbf{K}$ & $\%$ & 65 & 103 & 93 & 63 & 111 \\
\hline $\mathbf{C a}$ & $\%$ & 39 & 90 & 78 & 60 & 85 \\
\hline Sc & $\%$ & $\mathrm{nq}$ & 82 & & $4 \mathrm{nq}$ & 57 \\
\hline $\mathbf{T i}$ & $\%$ & 0 & 85 & $n$ & $\mathrm{nq}$ & $\mathrm{nq}$ \\
\hline $\mathbf{V}$ & $\%$ & $\mathrm{nq}$ & 89 & 91 & 120 & 71 \\
\hline $\mathbf{C r}$ & $\%$ & 119 & $\mathrm{nq}$ & nq & & $5 \mathrm{nq}$ \\
\hline Mn & $\%$ & 54 & 97 & 88 & 64 & 94 \\
\hline $\mathbf{F e}$ & $\%$ & 59 & 97 & & $5 \mathrm{nq}$ & $\mathrm{nq}$ \\
\hline Co & $\%$ & nq & 91 & & $7 \mathrm{nq}$ & $\mathrm{nq}$ \\
\hline $\mathrm{Ni}$ & $\%$ & $\mathrm{nq}$ & 93 & & nq & $\mathrm{nq}$ \\
\hline $\mathbf{C u}$ & $\%$ & 77 & 116 & 90 & & $8 \mathrm{nq}$ \\
\hline $\mathbf{Z n}$ & $\%$ & 112 & 93 & 85 & & $\mathrm{nq}$ \\
\hline As & $\%$ & $\mathrm{nq}$ & 64 & 4 & 64 & $4 \mathrm{nq}$ \\
\hline $\mathbf{R b}$ & $\%$ & $\mathrm{nq}$ & 89 & 0 & 99 & 98 \\
\hline \begin{tabular}{|l|}
$\mathrm{Sr}$ \\
\end{tabular} & $\%$ & 30 & 96 & 63 & 47 & 82 \\
\hline $\mathbf{Y}$ & $\%$ & 0 & 88 & & $1 \mathrm{nq}$ & $\mathrm{nq}$ \\
\hline $\mathbf{Z r}$ & $\%$ & 92 & 95 & 86 & 106 & 103 \\
\hline $\mathbf{N b}$ & $\%$ & $\mathrm{nq}$ & nq & $\mathrm{nq}$ & $\mathrm{nq}$ & 70 \\
\hline Mo & $\%$ & $\mathrm{nq}$ & 88 & 3 & 87 & $7 \mathrm{nq}$ \\
\hline Cd & $\%$ & $\mathrm{nq}$ & nq & 24 & 85 & 49 \\
\hline Sn & $\%$ & $\mathrm{nq}$ & 95 & 43 & 74 & 64 \\
\hline $\mathbf{S b}$ & $\%$ & $\mathrm{nq}$ & $\mathrm{nq}$ & 13 & 63 & 60 \\
\hline Cs & $\%$ & $\mathrm{nq}$ & 89 & & $2 n q$ & $\mathrm{nq}$ \\
\hline $\mathbf{B a}$ & $\%$ & 12 & 89 & 64 & 39 & 69 \\
\hline La & $\%$ & $\mathrm{nq}$ & 87 & $7 \mathrm{nq}$ & $\mathrm{nq}$ & 54 \\
\hline $\mathrm{Ce}$ & $\%$ & $\mathrm{nq}$ & 89 & $\mathrm{nq}$ & $\mathrm{nq}$ & 26 \\
\hline Pr & $\%$ & $\mathrm{nq}$ & 93 & $\mathrm{nq}$ & $\mathrm{nq}$ & $\mathrm{nq}$ \\
\hline Nd & $\%$ & $\mathrm{nq}$ & 96 & & $7 \mathrm{nq}$ & 17 \\
\hline Sm & $\%$ & 91 & 96 & 48 & & $8 \mathrm{nq}$ \\
\hline Eu & $\%$ & $\mathrm{nq}$ & 99 & $\mathrm{nq}$ & $\mathrm{nq}$ & 69 \\
\hline Gd & $\%$ & $\mathrm{nq}$ & 99 & $\mathrm{nq}$ & $\mathrm{nq}$ & 16 \\
\hline $\mathbf{T b}$ & $\%$ & $\mathrm{nq}$ & 104 & $n \mathrm{nq}$ & $\mathrm{nq}$ & 29 \\
\hline Dy & $\%$ & $\mathrm{nq}$ & 102 & $\mathrm{nq}$ & $\mathrm{nq}$ & 50 \\
\hline Ho & $\%$ & $\mathrm{nq}$ & 98 & $\mathrm{nq}$ & $\mathrm{nq}$ & $\mathrm{nq}$ \\
\hline Er & $\%$ & $\mathrm{nq}$ & 97 & $7 \mathrm{nq}$ & $\mathrm{nq}$ & 53 \\
\hline Tm & $\%$ & $\mathrm{nq}$ & 100 & $\mathrm{nq}$ & $\mathrm{nq}$ & $\mathrm{nq}$ \\
\hline $\mathbf{Y b}$ & $\%$ & $\mathrm{nq}$ & 105 & $\mathrm{nq}$ & $\mathrm{nq}$ & $\mathrm{nq}$ \\
\hline Lu & $\%$ & $\mathrm{nq}$ & 104 & $n \mathrm{nq}$ & $\mathrm{nq}$ & $\mathrm{nq}$ \\
\hline Hf & $\%$ & $\mathrm{nq}$ & nq & nq & $\mathrm{nq}$ & 44 \\
\hline Ta & $\%$ & $\mathrm{nq}$ & nq & $\mathrm{nq}$ & 111 & 68 \\
\hline $\mathbf{W}$ & $\%$ & $\mathrm{nq}$ & nq & 54 & & $8 \mathrm{nq}$ \\
\hline $\mathrm{Hg}$ & $\%$ & 103 & 112 & & $8 \mathrm{nq}$ & $\mathrm{nq}$ \\
\hline Tl & $\%$ & $\mathrm{nq}$ & nq & $\mathrm{nq}$ & $\mathrm{nq}$ & 31 \\
\hline $\mathbf{P b}$ & $\%$ & $\mathrm{nq}$ & 106 & & $6 \mathrm{nq}$ & 116 \\
\hline $\mathrm{Bi}$ & $\%$ & $\mathrm{nq}$ & nq & nq & $\mathrm{nq}$ & $\mathrm{nq}$ \\
\hline Th & $\%$ & $\mathrm{nq}$ & $\mathrm{nq}$ & nq & $\mathrm{nq}$ & 11 \\
\hline $\mathbf{U}$ & $\%$ & $\mathrm{nq}$ & 114 & 5 & 112 & $2 \mathrm{nq}$ \\
\hline
\end{tabular}


Tab. A.5. (Forts.): Ergebnisse des Kationenaustausches für Porenwässer und Wasserproben aus Cospuden; Konzentrationen in Lösungen nach dem Kationenaustausch in \% bezogen auf die Ausgangskonzentrationen (Tab. A.1.) von Porenlösungen und Wasserproben aus Cospuden.

\begin{tabular}{|c|c|c|c|c|c|c|c|}
\hline & & RCO3 & RCO4 & RCO12 & RCO15 & RCO17 & RCO18 \\
\hline & & Wasserprobe & Wasserprobe & Wasserprobe & Wasserprobe & Wasserprobe & Wasserprobe \\
\hline & & Jan 2000 & Jan 2000 & Jan 2000 & Jan 2000 & Jan 2000 & Jan 2000 \\
\hline Sulfat & $\%$ & 92 & $2 n q$ & 81 & 92 & 59 & 111 \\
\hline DOC & $\%$ & 109 & 28 & 51 & 114 & 28 & 66 \\
\hline $\mathbf{L i}$ & $\%$ & 81 & 111 & 12 & 91 & 102 & 98 \\
\hline $\mathbf{B e}$ & $\%$ & & $6 \mathrm{nq}$ & $\operatorname{lnq}$ & nq & $\mathrm{nq}$ & $\mathrm{nq}$ \\
\hline $\mathbf{N a}$ & $\%$ & 83 & 109 & 29 & 59 & 107 & 93 \\
\hline Mg & $\%$ & & $\mathrm{nq}$ & 27 & 61 & 102 & 70 \\
\hline Al & $\%$ & & $6 \mathrm{nq}$ & $\mathrm{nq}$ & $\mathrm{nq}$ & & $\mathrm{nq}$ \\
\hline $\mathbf{K}$ & $\%$ & $\mathrm{nq}$ & nq & 6 & 64 & $4 \mathrm{nq}$ & nq \\
\hline $\mathbf{C a}$ & $\%$ & 56 & 84 & 2 & 0 & 102 & 44 \\
\hline Sc & $\%$ & nq & $\mathrm{nq}$ & $\mathrm{nq}$ & $\mathrm{nq}$ & & $\mathrm{nq}$ \\
\hline $\mathbf{T i}$ & $\%$ & nq & $\mathrm{nq}$ & $\operatorname{nq}$ & $\mathrm{nq}$ & nq & $\mathrm{nq}$ \\
\hline $\mathbf{V}$ & $\%$ & & $6 \mathrm{nq}$ & & nq & $\mathrm{nq}$ & 96 \\
\hline $\mathrm{Cr}$ & $\%$ & nq & $\mathrm{nq}$ & 98 & $\mathrm{nq}$ & & nq \\
\hline Mn & $\%$ & & $\mathrm{nq}$ & 31 & 87 & 104 & 67 \\
\hline $\mathbf{F e}$ & $\%$ & 71 & $1 \mathrm{nq}$ & $\operatorname{nq}$ & $\mathrm{nq}$ & 57 & 95 \\
\hline Co & $\%$ & nq & $\mathrm{nq}$ & $\mathrm{nq}$ & $\mathrm{nq}$ & $\mathrm{nq}$ & $\mathrm{nq}$ \\
\hline $\mathbf{N i}$ & $\%$ & nq & $\mathrm{nq}$ & $\mathrm{nq}$ & 19 & $9 \mathrm{nq}$ & $\mathrm{nq}$ \\
\hline $\mathbf{C u}$ & $\%$ & 92 & 64 & 0 & 66 & 118 & 108 \\
\hline $\mathbf{Z n}$ & $\%$ & 77 & $7 \mathrm{nq}$ & $\operatorname{lnq}$ & $\mathrm{nq}$ & 63 & 63 \\
\hline As & $\%$ & 19 & 30 & 94 & 4 & & $\mathrm{nq}$ \\
\hline $\mathbf{R b}$ & $\%$ & $\operatorname{nq}$ & 51 & 106 & 0 & 63 & $\mathrm{nq}$ \\
\hline $\mathbf{S r}$ & $\%$ & 43 & 74 & 4 & 57 & 103 & 26 \\
\hline $\mathbf{Y}$ & $\%$ & & $6 \mathrm{nq}$ & $\mathrm{nq}$ & $\mathrm{nq}$ & & $\mathrm{nq}$ \\
\hline $\mathbf{Z r}$ & $\%$ & 81 & $1 \mathrm{nq}$ & & $4 \mathrm{nq}$ & 50 & 103 \\
\hline $\mathbf{N b}$ & $\%$ & 74 & 77 & $7 \mathrm{nq}$ & & $7 \mathrm{nq}$ & $\mathrm{nq}$ \\
\hline Mo & $\%$ & $\mathrm{nq}$ & 40 & 106 & $6 \mathrm{nq}$ & $\mathrm{nq}$ & nq \\
\hline Cd & $\%$ & 83 & $3 \mathrm{nq}$ & $\mathrm{nq}$ & 29 & $9 \mathrm{nq}$ & $\mathrm{nq}$ \\
\hline Sn & $\%$ & nq & 67 & & $4 \mathrm{nq}$ & & nq \\
\hline $\mathbf{S b}$ & $\%$ & $\mathrm{nq}$ & $\mathrm{nq}$ & $\mathrm{nq}$ & $\mathrm{nq}$ & & $\mathrm{nq}$ \\
\hline Cs & $\%$ & nq & 63 & $3 \mathrm{nq}$ & $\mathrm{nq}$ & & $\mathrm{nq}$ \\
\hline $\mathbf{B a}$ & $\%$ & & $4 \mathrm{nq}$ & 94 & 42 & 97 & 0 \\
\hline $\mathbf{L a}$ & $\%$ & 14 & $4 \mathrm{nq}$ & $\operatorname{nq}$ & $\mathrm{nq}$ & & $\mathrm{nq}$ \\
\hline $\mathrm{Ce}$ & $\%$ & 13 & $3 \mathrm{nq}$ & $\operatorname{nq}$ & nq & & $\mathrm{nq}$ \\
\hline $\mathbf{P r}$ & $\%$ & 13 & $3 \mathrm{nq}$ & $\operatorname{nq}$ & $\mathrm{nq}$ & $\mathrm{nq}$ & $\mathrm{nq}$ \\
\hline \begin{tabular}{|l}
$\mathbf{N d}$ \\
\end{tabular} & $\%$ & 14 & $4 \mathrm{nq}$ & $\mathrm{nq}$ & $\mathrm{nq}$ & $\mathrm{nq}$ & $\mathrm{nq}$ \\
\hline $\mathbf{S m}$ & $\%$ & 29 & 38 & 63 & $3 \mathrm{nq}$ & $\mathrm{nq}$ & $\mathrm{nq}$ \\
\hline $\mathbf{E u}$ & $\%$ & 32 & $2 n q$ & $\mathrm{nq}$ & $\mathrm{nq}$ & $\mathrm{nq}$ & $\mathrm{nq}$ \\
\hline Gd & $\%$ & 14 & 64 & $4 \mathrm{nq}$ & $\mathrm{nq}$ & 100 & $\mathrm{nq}$ \\
\hline \begin{tabular}{|l|}
$\mathrm{Tb}$ \\
\end{tabular} & $\%$ & 17 & $7 \mathrm{nq}$ & $\mathrm{nq}$ & $\mathrm{nq}$ & & $\mathrm{nq}$ \\
\hline Dy & $\%$ & 18 & $\mathrm{nq}$ & $\mathrm{nq}$ & $\mathrm{nq}$ & $\mathrm{nq}$ & $\mathrm{nq}$ \\
\hline Ho & $\%$ & & $9 \mathrm{nq}$ & $\mathrm{nq}$ & $\mathrm{nq}$ & & $\mathrm{nq}$ \\
\hline $\mathbf{E r}$ & $\%$ & & $\mathrm{nq}$ & $\mathrm{nq}$ & $\mathrm{nq}$ & & $\mathrm{nq}$ \\
\hline $\mathbf{T m}$ & $\%$ & & $7 \mathrm{nq}$ & $\mathrm{nq}$ & $\mathrm{nq}$ & 100 & $\mathrm{nq}$ \\
\hline $\mathbf{Y b}$ & $\%$ & 29 & $\mathrm{nq}$ & $\operatorname{nq}$ & $\mathrm{nq}$ & $\mathrm{nq}$ & $\mathrm{nq}$ \\
\hline $\mathbf{L u}$ & $\%$ & 31 & $1 \mathrm{nq}$ & $\mathrm{nq}$ & nq & $\mathrm{nq}$ & nq \\
\hline Hf & $\%$ & $\mathrm{nq}$ & 36 & $6 \mathrm{nq}$ & $\mathrm{nq}$ & $\mathrm{nq}$ & nq \\
\hline Ta & $\%$ & $\mathrm{nq}$ & 68 & 89 & $\mathrm{nq}$ & $\mathrm{nq}$ & 108 \\
\hline $\mathbf{W}$ & $\%$ & 19 & 42 & $2 n q$ & 74 & & $n q$ \\
\hline $\mathrm{Hg}$ & $\%$ & 117 & 37 & $7 \mathrm{nq}$ & $\mathrm{nq}$ & $\mathrm{nq}$ & $\mathrm{nq}$ \\
\hline \begin{tabular}{|l|}
$\mathbf{T l}$ \\
\end{tabular} & $\%$ & $\operatorname{nq}$ & 49 & $9 \mathrm{nq}$ & $\mathrm{nq}$ & & $\mathrm{nq}$ \\
\hline $\mathbf{P b}$ & $\%$ & $\mathrm{nq}$ & 84 & $4 \mathrm{nq}$ & $\mathrm{nq}$ & & $\mathrm{nq}$ \\
\hline $\mathbf{B i}$ & $\%$ & $\operatorname{nq}$ & $\mathrm{nq}$ & $\operatorname{nq}$ & nq & 111 & $\mathrm{nq}$ \\
\hline Th & $\%$ & $\mathrm{nq}$ & $\mathrm{nq}$ & $\mathrm{nq}$ & $\mathrm{nq}$ & & $4 \mathrm{nq}$ \\
\hline $\mathbf{U}$ & $\%$ & nq & 89 & 100 & & $1 \mathrm{nq}$ & $\mathrm{nq}$ \\
\hline
\end{tabular}


Tab. A.6.: Ergebnisse des Kationenaustausches für Porenwässer und Wasserproben aus der Lausitz;; Konzentrationen in Lösungen nach dem Kationenaustausch in \% bezogen auf die Ausgangskonzentrationen (Tab. A.2.) von Porenlösungen und Wasserproben aus der Lausitz.

\begin{tabular}{|c|c|c|c|c|c|c|c|}
\hline & & Lausitz 5,21m & Lausitz 5,30m & Lausitz 5,50m & Lausitz 5,60m & Lausitz 5,75 m & Lausitz 5,93 m \\
\hline & & Okt 1999 & Okt 1999 & Okt 1999 & Okt 1999 & Okt 1999 & Okt 1999 \\
\hline & & Porenlösung & Porenlösung & Porenlösung & Porenlösung & Porenlösung & Porenlösung \\
\hline Sulfat & $\%$ & 79 & 79 & 96 & 78 & 94 & 85 \\
\hline DOC & $\%$ & nb & nb & nq & 102 & & $n b$ \\
\hline $\mathbf{L i}$ & $\%$ & 46 & 51 & 104 & 58 & 89 & 64 \\
\hline $\mathbf{B e}$ & $\%$ & 53 & 42 & 84 & 48 & 86 & 53 \\
\hline $\mathbf{N a}$ & $\%$ & 37 & 42 & 89 & 44 & 82 & 46 \\
\hline Mg & $\%$ & 43 & 35 & 77 & 42 & 84 & 45 \\
\hline Al & $\%$ & 23 & 14 & 59 & 21 & 75 & 16 \\
\hline $\mathbf{K}$ & $\%$ & 67 & 75 & 81 & 42 & 81 & 37 \\
\hline $\mathbf{C a}$ & $\%$ & 30 & 20 & 66 & 28 & 79 & 26 \\
\hline Sc & $\%$ & 59 & 47 & 87 & 55 & 90 & 63 \\
\hline $\mathbf{T i}$ & $\%$ & 48 & 46 & 34 & 57 & 84 & 39 \\
\hline $\mathbf{V}$ & $\%$ & 46 & 39 & 81 & 47 & 81 & 52 \\
\hline $\mathbf{C r}$ & $\%$ & 93 & 56 & 89 & 47 & 83 & 51 \\
\hline Mn & $\%$ & 41 & 35 & 76 & 41 & 84 & 44 \\
\hline $\mathbf{F e}$ & $\%$ & 42 & 35 & 80 & 42 & 84 & 48 \\
\hline Co & $\%$ & 44 & 34 & 78 & 40 & 80 & 44 \\
\hline $\mathrm{Ni}$ & $\%$ & 45 & 34 & 77 & 39 & 78 & 43 \\
\hline $\mathbf{C u}$ & $\%$ & 41 & 33 & 79 & 30 & 76 & 33 \\
\hline $\mathbf{Z n}$ & $\%$ & 47 & 36 & 77 & 42 & 86 & 46 \\
\hline As & $\%$ & 67 & 65 & 81 & 72 & $\mathrm{nq}$ & 80 \\
\hline $\mathbf{R b}$ & $\%$ & 47 & 33 & 74 & 381 & $\mathrm{nq}$ & 53 \\
\hline \begin{tabular}{|l|}
$\mathrm{Sr}$ \\
\end{tabular} & $\%$ & 17 & 10 & 54 & 16 & 72 & 14 \\
\hline $\mathbf{Y}$ & $\%$ & 19 & 7 & 49 & 14 & 74 & 8 \\
\hline $\mathbf{Z r}$ & $\%$ & 95 & 58 & 83 & 53 & 99 & 51 \\
\hline $\mathbf{N b}$ & $\%$ & $\mathrm{nq}$ & 3 & 18 & & $\mathrm{nq}$ & 32 \\
\hline Mo & $\%$ & 21 & 63 & 82 & & $n q$ & 53 \\
\hline Cd & $\%$ & 55 & 39 & 79 & & $\mathrm{nq}$ & 48 \\
\hline Sn & $\%$ & $\mathrm{nq}$ & $\mathrm{nq}$ & nq & $\mathrm{nq}$ & $\mathrm{nq}$ & nq \\
\hline Sb & $\%$ & 93 & 73 & 98 & 751 & $\mathrm{nq}$ & 65 \\
\hline Cs & $\%$ & 51 & 35 & 73 & 381 & $\mathrm{nq}$ & 49 \\
\hline $\mathbf{B a}$ & $\%$ & 0 & 0 & 23 & 0 & 57 & 2 \\
\hline La & $\%$ & 4 & 0 & 19 & 2 & 65 & 0 \\
\hline $\mathrm{Ce}$ & $\%$ & 6 & 1 & 23 & & $n q$ & 1 \\
\hline Pr & $\%$ & 7 & 1 & 26 & & $\mathrm{nq}$ & 1 \\
\hline Nd & $\%$ & 9 & 1 & 28 & & $\mathrm{nq}$ & 1 \\
\hline Sm & $\%$ & 16 & 3 & 38 & & $\mathrm{nq}$ & 4 \\
\hline Eu & $\%$ & 30 & 5 & 42 & $10 \mathrm{r}$ & $\mathrm{nq}$ & 6 \\
\hline Gd & $\%$ & 19 & 5 & 42 & & $\mathrm{nq}$ & 6 \\
\hline $\mathbf{T b}$ & $\%$ & 24 & 8 & 49 & & $n q$ & 8 \\
\hline Dy & $\%$ & 26 & 8 & 50 & & $\mathrm{nq}$ & 9 \\
\hline Ho & $\%$ & 25 & 8 & 50 & & $\mathrm{nq}$ & 8 \\
\hline $\mathbf{E r}$ & $\%$ & 23 & 7 & 50 & & $\mathrm{nq}$ & 7 \\
\hline Tm & $\%$ & 22 & 6 & 48 & & $\mathrm{nq}$ & 6 \\
\hline $\mathbf{Y b}$ & $\%$ & 21 & 6 & 47 & & $\mathrm{nq}$ & 6 \\
\hline Lu & $\%$ & 20 & 6 & 46 & & $n q$ & 5 \\
\hline Hf & $\%$ & $<79$ & 44 & 70 & $394_{1}$ & $\mathrm{nq}$ & 51 \\
\hline Ta & $\%$ & nq & 4 & 21 & & nq & 11 \\
\hline $\mathbf{W}$ & $\%$ & 77 & 38 & 53 & & $\mathrm{nq}$ & 43 \\
\hline $\mathrm{Hg}$ & $\%$ & $n b$ & $n b$ & $n b$ & $n b$ & $\mathrm{nb}$ & $\mathrm{nb}$ \\
\hline Tl & $\%$ & 58 & 34 & 73 & 44 & $\mathrm{nq}$ & 43 \\
\hline $\mathbf{P b}$ & $\%$ & 17 & 6 & 45 & 11 & 62 & 9 \\
\hline $\mathrm{Bi}$ & $\%$ & $n b$ & $n b$ & $n b$ & $n b$ & $n b$ & $n b$ \\
\hline Th & $\%$ & 94 & 47 & 85 & & $\mathrm{nq}$ & 51 \\
\hline $\mathbf{U}$ & $\%$ & 75 & 45 & 86 & & $\mathrm{nq}$ & 55 \\
\hline
\end{tabular}


Tab. A.6. (Forts.): Ergebnisse des Kationenaustausches für Porenwässer und Wasserproben aus der Lausitz; Konzentrationen in Lösungen nach dem Kationenaustausch in \% bezogen auf die Ausgangskonzentrationen (Tab. A.2.) von Porenlösungen und Wasserproben aus der Lausitz.

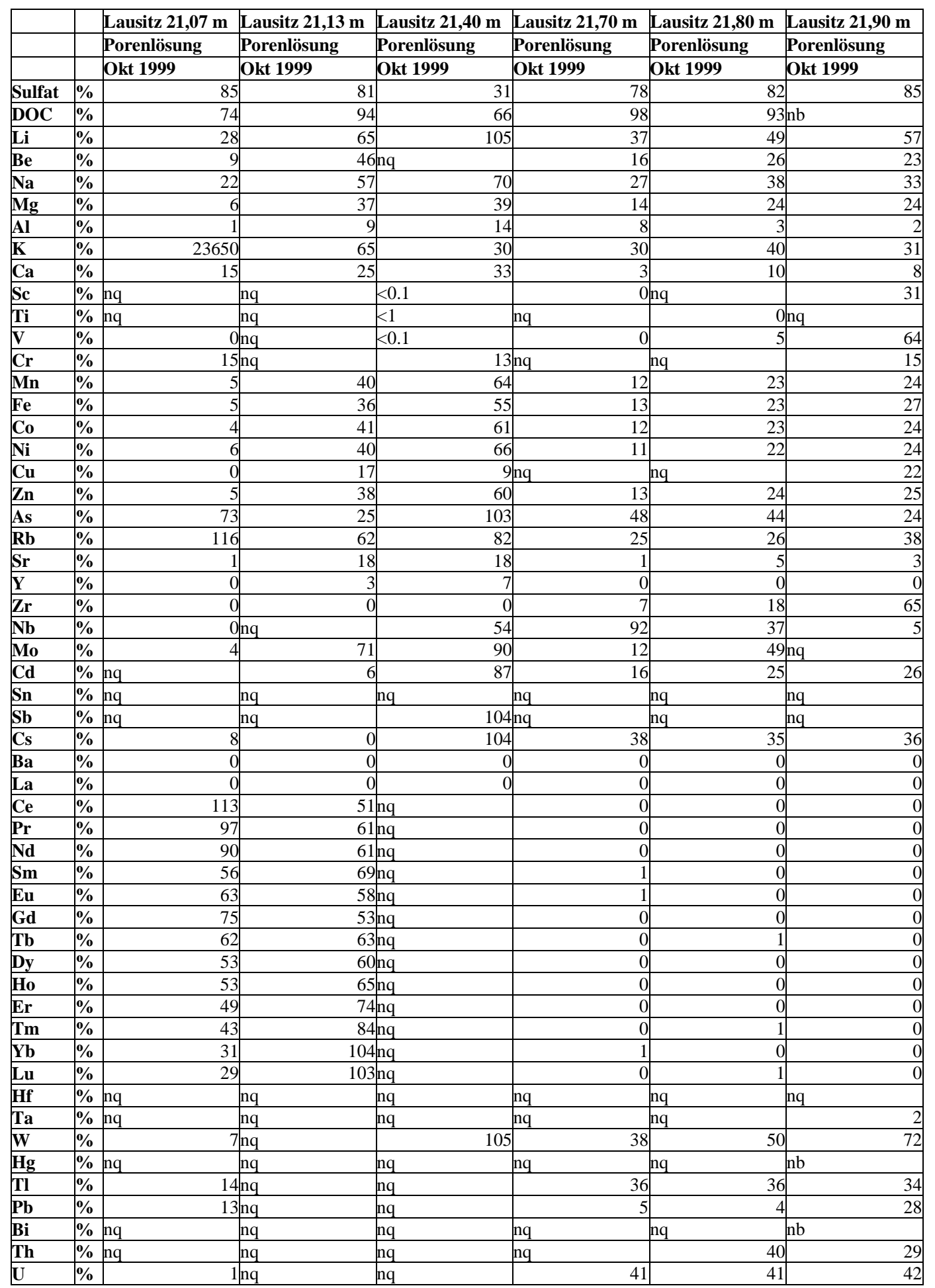


Tab. A.7.: Ergebnisse des Anionenaustausches für Porenwässer und Wasserproben aus Cospuden; Konzentrationen in Lösungen nach dem Anionenaustausch in \% bezogen auf die Ausgangskonzentrationen (Tab. A.1.) von Porenlösungen und Wasserproben aus Cospuden; AI = Austauschion.

\begin{tabular}{|c|c|c|c|c|c|c|c|c|c|}
\hline & & RCO4 $17 \mathrm{~m}$ & RCO4 20 m & RCO4 $22 \mathrm{~m}$ & RCO4 26 m & RCO436 m & RCO4 44 m & RCO4 $48 \mathrm{~m}$ & RCO4 $52 \mathrm{~m}$ \\
\hline \multirow[t]{3}{*}{$\mathbf{A I}$} & & $\mathrm{Cl}^{-}$ & $\mathrm{Cl}^{-}$ & $\mathrm{Cl}^{-}$ & $\mathrm{Cl}^{-}$ & $\mathrm{Cl}^{-}$ & $\mathrm{Cl}^{-}$ & $\mathrm{Cl}^{-}$ & $\mathrm{Cl}^{-}$ \\
\hline & & Porenlösung & Porenlösung & Porenlösung & Porenlösung & Porenlösung & Porenlösung & Porenlösung & Porenlösung \\
\hline & & Nov 1995 & Nov 1995 & Nov 1995 & Nov 1995 & Nov 1995 & Nov 1995 & Nov 1995 & Nov 1995 \\
\hline Sulfat & $\%$ & 14 & 23 & 23 & 98 & 87 & 31 & 26 & 33 \\
\hline DOC & $\%$ & nb & 115 & 66 & 84 & 68 & & 69 & 67 \\
\hline $\mathbf{L i}$ & $\%$ & 62 & 76 & 80 & 120 & 60 & 87 & 80 & 84 \\
\hline $\mathbf{B e}$ & $\%$ & nq & nq & nq & 105 & $\mathrm{nq}$ & nq & nq & nq \\
\hline $\mathbf{N a}$ & $\%$ & 75 & 96 & & $3 \mathrm{nq}$ & 54 & nq & 83 & 111 \\
\hline Mg & $\%$ & 72 & 83 & 84 & 105 & 51 & 85 & 83 & 86 \\
\hline Al & $\%$ & nq & $\mathrm{nq}$ & 92 & 113 & $\mathrm{nq}$ & nq & $\mathrm{nq}$ & nq \\
\hline $\mathbf{K}$ & $\%$ & nq & & $\mathrm{nq}$ & $\mathrm{nq}$ & 55 & $n$ & $\mathrm{nq}$ & 92 \\
\hline $\mathbf{C a}$ & $\%$ & 70 & 82 & 83 & 111 & 34 & 86 & 79 & 85 \\
\hline Sc & $\%$ & $\mathrm{nq}$ & $\mathrm{nq}$ & 66 & 104 & 73 & 43 & 61 & $\mathrm{nq}$ \\
\hline $\mathrm{Ti}$ & $\%$ & $\mathrm{nq}$ & $\mathrm{nq}$ & nq & 661 & $\mathrm{nq}$ & $\mathrm{nq}$ & $\mathrm{nq}$ & $\mathrm{nq}$ \\
\hline $\mathbf{V}$ & $\%$ & & $\mathrm{nq}$ & 0 & 100 & & $\mathrm{nq}$ & $\mathrm{nq}$ & $\mathrm{nq}$ \\
\hline $\mathbf{C r}$ & $\%$ & $\mathrm{nq}$ & 100 & & $2 n q$ & $\mathrm{nq}$ & $\mathrm{nq}$ & $\mathrm{nq}$ & 74 \\
\hline Mn & $\%$ & 71 & 77 & 83 & 104 & 52 & 61 & 80 & 85 \\
\hline $\mathbf{F e}$ & $\%$ & 120 & $\mathrm{nq}$ & 99 & 100 & 112 & 39 & 67 & 57 \\
\hline Co & $\%$ & 64 & & $n q$ & $\mathrm{nq}$ & 0 & & $7 \mathrm{nq}$ & 86 \\
\hline $\mathrm{Ni}$ & $\%$ & 70 & 79 & 82 & 107 & 70 & 61 & 87 & 82 \\
\hline $\mathbf{C u}$ & $\%$ & 31 & 89 & & $5 \mathrm{nq}$ & 51 & 55 & 19 & 50 \\
\hline $\mathbf{Z n}$ & $\%$ & 62 & 31 & 79 & 105 & 70 & 95 & 60 & 17 \\
\hline As & $\%$ & 82 & $\mathrm{nq}$ & $\mathrm{nq}$ & $\mathrm{nq}$ & 77 & $\mathrm{nq}$ & $\mathrm{nq}$ & $\mathrm{nq}$ \\
\hline $\mathbf{R b}$ & $\%$ & 2 & 58 & 73 & 100 & 66 & 70 & 80 & 69 \\
\hline $\mathbf{S r}$ & $\%$ & 67 & 81 & 81 & 110 & 18 & 86 & 78 & 84 \\
\hline $\mathbf{Y}$ & $\%$ & $<4$ & $<48$ & 73 & 110 & 35 & $5<28$ & 28 & 44 \\
\hline $\mathbf{Z r}$ & $\%$ & 90 & 10 & 98 & 88 & 86 & 97 & 92 & 29 \\
\hline $\mathbf{N b}$ & $\%$ & 8 & 38 & 89 & 24 & 38 & & $1 \mathrm{nq}$ & 26 \\
\hline Mo & $\%$ & 0 & 2 & 1 & $1 \mathrm{nq}$ & 70 & 0 & 9 & 1 \\
\hline Cd & $\%$ & 12 & $<<44$ & 40 & 94 & 100 & 31 & 70 & 37 \\
\hline Sn & $\%$ & $\mathrm{nq}$ & $\mathrm{nq}$ & nq & & $\mathrm{nq}$ & $\mathrm{nq}$ & $\mathrm{nq}$ & nq \\
\hline Sb & $\%$ & 60 & 50 & 55 & 36 & 89 & & $8 \mathrm{nq}$ & 76 \\
\hline Cs & $\%$ & $<3$ & 52 & 60 & 95 & 62 & $<<30$ & & nq \\
\hline $\mathbf{B a}$ & $\%$ & 70 & 80 & & $3 \mathrm{nq}$ & 0 & 83 & 78 & 85 \\
\hline La & $\%$ & $<5$ & $<15$ & 50 & 95 & 37 & $7<<24$ & 24 & $4<17$ \\
\hline $\mathrm{Ce}$ & $\%$ & $<5$ & $<18$ & 58 & 93 & 42 & $<<27$ & 34 & $4<14$ \\
\hline Pr & $\%$ & $\mathrm{nq}$ & $\mathrm{nq}$ & nq & $91_{1}$ & $\mathrm{nq}$ & $\mathrm{nq}$ & $\mathrm{nq}$ & nq \\
\hline Nd & $\%$ & $<8$ & $<32$ & nq & 91 & 44 & $4<22$ & 36 & $5<31$ \\
\hline Sm & $\%$ & $\mathrm{nq}$ & $\mathrm{nq}$ & nq & $90_{1}$ & $\mathrm{nq}$ & $\mathrm{nq}$ & $\mathrm{nq}$ & $\mathrm{nq}$ \\
\hline $\mathbf{E u}$ & $\%$ & $\mathrm{nq}$ & $\mathrm{nq}$ & nq & 89 & $\mathrm{nq}$ & $\mathrm{nq}$ & $\mathrm{nq}$ & $\mathrm{nq}$ \\
\hline Gd & $\%$ & $\mathrm{nq}$ & $\mathrm{nq}$ & nq & & $\mathrm{nq}$ & nq & $\mathrm{nq}$ & $\mathrm{nq}$ \\
\hline Tb & $\%$ & $\mathrm{nq}$ & $\mathrm{nq}$ & $\mathrm{nq}$ & $90_{1}$ & $\mathrm{nq}$ & $\mathrm{nq}$ & $\mathrm{nq}$ & $\mathrm{nq}$ \\
\hline Dy & $\%$ & $\mathrm{nq}$ & $\mathrm{nq}$ & $\mathrm{nq}$ & & $\mathrm{nq}$ & $\mathrm{nq}$ & $\mathrm{nq}$ & $\mathrm{nq}$ \\
\hline Ho & $\%$ & $\mathrm{nq}$ & $\mathrm{nq}$ & nq & $90_{1}$ & $\mathrm{nq}$ & $\mathrm{nq}$ & $\mathrm{nq}$ & $\mathrm{nq}$ \\
\hline $\mathbf{E r}$ & $\%$ & $\mathrm{nq}$ & $\mathrm{nq}$ & nq & $90_{1}$ & $\mathrm{nq}$ & $\mathrm{nq}$ & $\mathrm{nq}$ & nq \\
\hline Tm & $\%$ & $\mathrm{nq}$ & $\mathrm{nq}$ & nq & 89 & $\mathrm{nq}$ & $\mathrm{nq}$ & $\mathrm{nq}$ & nq \\
\hline Yb & $\%$ & $\mathrm{nq}$ & $\mathrm{nq}$ & nq & 881 & $\mathrm{nq}$ & $\mathrm{nq}$ & $\mathrm{nq}$ & nq \\
\hline Lu & $\%$ & $\mathrm{nq}$ & $\mathrm{nq}$ & nq & 88 & $\mathrm{nq}$ & nq & $\mathrm{nq}$ & $\mathrm{nq}$ \\
\hline Hf & $\%$ & $\mathrm{nq}$ & $\mathrm{nq}$ & nq & 461 & nq & $\mathrm{nq}$ & $\mathrm{nq}$ & $\mathrm{nq}$ \\
\hline Ta & $\%$ & $\mathrm{nq}$ & $\mathrm{nq}$ & nq & & $\mathrm{nq}$ & & $6 \mathrm{nq}$ & 7 \\
\hline $\mathbf{W}$ & $\%$ & 40 & $<<7$ & 15 & 77 & 112 & & $3 \mathrm{nq}$ & $<12$ \\
\hline $\mathrm{Hg}$ & $\%$ & $\operatorname{lnb}$ & nb & nb & $n b$ & $n b$ & nb & nb & nb \\
\hline Tl & $\%$ & 38 & $3<49$ & 103 & 82 & 94 & $4<58$ & $\mathrm{nq}$ & $<49$ \\
\hline $\mathbf{P b}$ & $\%$ & 53 & 76 & 64 & 95 & 82 & & $5 \mathrm{nq}$ & 40 \\
\hline $\mathbf{B i}$ & $\%$ & nb & nb & nb & $\mathrm{nb}$ & $n b$ & $\mathrm{nb}$ & nb & $\mathrm{nb}$ \\
\hline Th & $\%$ & $\mathrm{nq}$ & $\mathrm{nq}$ & $\mathrm{nq}$ & & $\mathrm{nq}$ & $\mathrm{nq}$ & $\mathrm{nq}$ & $<6$ \\
\hline $\mathbf{U}$ & $\%$ & $\mathrm{nq}$ & $\mathrm{nq}$ & 3 & 1 & 83 & & $0 \mathrm{nq}$ & 0 \\
\hline
\end{tabular}


Tab. A.7. (Forts.): Ergebnisse des Anionenaustausches für Porenwässer und Wasserproben aus Cospuden; Konzentrationen in Lösungen nach dem Anionenaustausch in \% bezogen auf die Ausgangskonzentrationen (Tab. A.1.) von Porenlösungen und Wasserproben aus Cospuden; AI = Austauschion.

\begin{tabular}{|c|c|c|c|c|c|c|c|c|c|}
\hline & & CML 21-22 m & CML 24 m & CML 26 m & CML 32 m & CML 36 m & CML 37,5 m & RCO4 & RCO10 \\
\hline \multirow[t]{3}{*}{$\mathbf{A I}$} & & $\mathbf{O H}^{-}$ & $\mathbf{O H}^{-}$ & $\mathbf{O H}^{-}$ & $\mathbf{O H}^{-}$ & $\mathbf{O H}^{-}$ & $\mathbf{O H}^{-}$ & $\mathbf{O H}^{-}$ & $\mathbf{O H}^{-}$ \\
\hline & & Porenlösung & Porenlösung & Porenlösung & Porenlösung & Porenlösung & Porenlösung & Wasserprobe & Wasserprobe \\
\hline & & Aug 1998 & Aug 1998 & Aug 1998 & Aug 1998 & Aug 1998 & Aug 1998 & Okt 1998 & Okt 1998 \\
\hline Sulfat & $\%$ & nb & $\mathrm{nb}$ & nb & nb & nb & nb & 75 & 16 \\
\hline DOC & $\%$ & $\operatorname{nb}$ & $\mathrm{nb}$ & $\mathrm{nb}$ & $\mathrm{nb}$ & $\operatorname{nb}$ & nb & 50 & 58 \\
\hline $\mathbf{L i}$ & $\%$ & 18 & 74 & 71 & 39 & 55 & 63 & 96 & 107 \\
\hline $\mathbf{B e}$ & $\%$ & $\mathrm{nq}$ & 36 & 9 & 100 & 100 & & $7 \mathrm{nq}$ & $\mathrm{nq}$ \\
\hline $\mathbf{N a}$ & $\%$ & 86 & 104 & 67 & 66 & 87 & 88 & 95 & 100 \\
\hline Mg & $\%$ & nq & 45 & 75 & 10 & 5 & 12 & 100 & 67 \\
\hline Al & $\%$ & 93 & 3 & 0 & 59 & 88 & & $7 \mathrm{nq}$ & $\operatorname{nq}$ \\
\hline $\mathbf{K}$ & $\%$ & & $6 \mathrm{nq}$ & 28 & & $5 \mathrm{nq}$ & 37 & 103 & 121 \\
\hline \begin{tabular}{|l|}
$\mathbf{C a}$ \\
\end{tabular} & $\%$ & $\mathrm{nq}$ & 72 & 71 & 48 & 59 & 66 & 101 & 48 \\
\hline Sc & $\%$ & $\mathrm{nq}$ & 100 & 89 & 100 & 100 & 100 & & $8 \mathrm{nq}$ \\
\hline $\mathbf{T i}$ & $\%$ & 83 & 4 & 24 & 16 & 40 & & $3 \mathrm{nq}$ & $\operatorname{lnq}$ \\
\hline $\mathbf{V}$ & $\%$ & 69 & 12 & 24 & 80 & 69 & 50 & & $0 \mathrm{nq}$ \\
\hline $\mathbf{C r}$ & $\%$ & $\mathrm{nq}$ & & $9 \mathrm{nq}$ & 36 & 42 & 42 & 101 & 10 \\
\hline Mn & $\%$ & $\mathrm{nq}$ & & $5 \mathrm{nq}$ & 0 & 0 & 0 & 115 & 68 \\
\hline $\mathrm{Fe}$ & $\%$ & 47 & 2 & 30 & 3 & 10 & 0 & 84 & 8 \\
\hline Co & $\%$ & 82 & 4 & 25 & 13 & 22 & 37 & 120 & 15 \\
\hline $\mathbf{N i}$ & $\%$ & $\mathrm{nq}$ & 5 & 26 & 9 & 10 & & $n q$ & 78 \\
\hline $\mathbf{C u}$ & $\%$ & 60 & & $4 \mathrm{nq}$ & 37 & 76 & 67 & 110 & 12 \\
\hline $\mathbf{Z n}$ & $\%$ & 66 & 6 & 5 & 34 & 54 & 44 & 119 & 101 \\
\hline As & $\%$ & 53 & ( & 45 & 12 & 0 & & nq & $\mathrm{nq}$ \\
\hline $\mathbf{R b}$ & $\%$ & & $9 \mathrm{nb}$ & $\mathrm{nb}$ & $\mathrm{nb}$ & $\mathrm{nb}$ & 41 & 39 & 98 \\
\hline $\mathbf{S r}$ & $\%$ & 89 & 79 & 73 & 51 & 66 & 100 & 97 & 38 \\
\hline $\mathbf{Y}$ & $\%$ & 90 & 12 & 0 & 86 & 100 & 8 & 100 & 100 \\
\hline $\mathbf{Z r}$ & $\%$ & 83 & & $4 \mathrm{nq}$ & 100 & 86 & 35 & 57 & 102 \\
\hline $\mathbf{N b}$ & $\%$ & & $7 \mathrm{nb}$ & nb & $\mathrm{nb}$ & $n b$ & 20 & & $3 \mathrm{nq}$ \\
\hline Mo & $\%$ & & $3 \mathrm{nq}$ & 39 & 25 & 15 & 2 & 40 & 79 \\
\hline Cd & $\%$ & & $2 \mathrm{nb}$ & $\mathrm{nb}$ & $\mathrm{nb}$ & $n b$ & $\mathrm{nb}$ & $\mathrm{nb}$ & $\mathrm{nb}$ \\
\hline Sn & $\%$ & & $1 \mathrm{nb}$ & $\mathrm{nb}$ & $\mathrm{nb}$ & $n b$ & 42 & & $9 \mathrm{nq}$ \\
\hline Sb & $\%$ & & $3 \mathrm{nb}$ & $\mathrm{nb}$ & $\mathrm{nb}$ & $n b$ & & $n q$ & $\mathrm{nq}$ \\
\hline Cs & $\%$ & $\mathrm{nq}$ & $\mathrm{nb}$ & $n b$ & $\mathrm{nb}$ & $n b$ & & $2 n q$ & $\mathrm{nq}$ \\
\hline $\mathbf{B a}$ & $\%$ & & $5 \mathrm{nq}$ & $\mathrm{nq}$ & $\mathrm{nq}$ & & $\mathrm{nq}$ & $\mathrm{nq}$ & 92 \\
\hline La & $\%$ & 75 & 5 & 0 & 1 & 0 & 6 & & $3 \mathrm{nq}$ \\
\hline Ce & $\%$ & & $2 n b$ & $\mathrm{nb}$ & $\mathrm{nb}$ & $n b$ & & $5 \mathrm{nq}$ & $\mathrm{nq}$ \\
\hline $\mathbf{P r}$ & $\%$ & 105 & $5 \mathrm{nb}$ & $\mathrm{nb}$ & $\mathrm{nb}$ & $n b$ & & $5 \mathrm{nq}$ & $\mathrm{nq}$ \\
\hline Nd & $\%$ & & $8 \mathrm{nb}$ & $\mathrm{nb}$ & $\mathrm{nb}$ & $n b$ & & $6 \mathrm{nq}$ & $\mathrm{nq}$ \\
\hline $\mathrm{Sm}$ & $\%$ & $\mathrm{nq}$ & $\mathrm{nb}$ & $n b$ & $\mathrm{nb}$ & $n b$ & & nq & $\mathrm{nq}$ \\
\hline $\mathbf{E u}$ & $\%$ & $\mathrm{nq}$ & $\operatorname{nq}$ & $\mathrm{nq}$ & $\mathrm{nq}$ & $\mathrm{nq}$ & & $3 \mathrm{nq}$ & $\operatorname{nq}$ \\
\hline Gd & $\%$ & $\mathrm{nq}$ & $\mathrm{nb}$ & $\mathrm{nb}$ & $\mathrm{nb}$ & $n b$ & & $7 \mathrm{nq}$ & $\mathrm{nq}$ \\
\hline Tb & $\%$ & $\mathrm{nq}$ & $\mathrm{nb}$ & $\mathrm{nb}$ & $\mathrm{nb}$ & $\mathrm{nb}$ & & $5 \mathrm{nq}$ & $\mathrm{nq}$ \\
\hline Dy & $\%$ & $\mathrm{nq}$ & $\mathrm{nb}$ & $\mathrm{nb}$ & $\mathrm{nb}$ & $n b$ & & $7 \mathrm{nq}$ & $\mathrm{nq}$ \\
\hline Нo & $\%$ & $\mathrm{nq}$ & $\mathrm{nb}$ & nb & $\mathrm{nb}$ & $n b$ & & $\mathrm{nq}$ & $\mathrm{nq}$ \\
\hline $\mathbf{E r}$ & $\%$ & $\mathrm{nq}$ & $\operatorname{lnb}$ & $\mathrm{nb}$ & $\mathrm{nb}$ & $n b$ & & $\mathrm{nq}$ & $\mathrm{nq}$ \\
\hline Tm & $\%$ & $\mathrm{nq}$ & $\operatorname{lnb}$ & $\mathrm{nb}$ & $\mathrm{nb}$ & $\mathrm{nb}$ & & $1 \mathrm{nq}$ & $\operatorname{nq}$ \\
\hline $\mathbf{Y b}$ & $\%$ & $\mathrm{nq}$ & $\mathrm{nb}$ & $n b$ & $\mathrm{nb}$ & $n b$ & & $4 \mathrm{nq}$ & $\mathrm{nq}$ \\
\hline $\mathbf{L u}$ & $\%$ & $\mathrm{nq}$ & $\operatorname{nq}$ & $\mathrm{nq}$ & $\mathrm{nq}$ & $\mathrm{nq}$ & nq & $\mathrm{nq}$ & $\operatorname{lnq}$ \\
\hline Hf & $\%$ & $\mathrm{nq}$ & $\mathrm{nb}$ & $\mathrm{nb}$ & $\mathrm{nb}$ & $\mathrm{nb}$ & $\mathrm{nq}$ & $\mathrm{nq}$ & $\mathrm{nq}$ \\
\hline Ta & $\%$ & $\mathrm{nq}$ & $\operatorname{lnb}$ & $n b$ & $\mathrm{nb}$ & $n b$ & 18 & & $9 \mathrm{nq}$ \\
\hline $\mathbf{W}$ & $\%$ & $\mathrm{nq}$ & $\operatorname{lnb}$ & $\mathrm{nb}$ & $\mathrm{nb}$ & $n b$ & & $6 \mathrm{nq}$ & $\mathrm{nq}$ \\
\hline $\mathrm{Hg}$ & $\%$ & & $5 \mathrm{nb}$ & $n b$ & $\mathrm{nb}$ & $n b$ & $n b$ & $n b$ & $\mathrm{nb}$ \\
\hline Tl & $\%$ & & $1 \mathrm{nb}$ & $\mathrm{nb}$ & $\mathrm{nb}$ & $n b$ & $\mathrm{nq}$ & $\mathrm{nq}$ & $\operatorname{nq}$ \\
\hline $\mathbf{P b}$ & $\%$ & $n b$ & $\operatorname{nq}$ & $\mathrm{nq}$ & $\mathrm{nq}$ & $\mathrm{nq}$ & & nq & $\operatorname{nq}$ \\
\hline $\mathbf{B i}$ & $\%$ & $\mathrm{nq}$ & $\mathrm{nb}$ & $\mathrm{nb}$ & $\mathrm{nb}$ & $\mathrm{nb}$ & $\mathrm{nq}$ & $\mathrm{nq}$ & $\mathrm{nq}$ \\
\hline Th & $\%$ & & $1 \mathrm{nb}$ & $\mathrm{nb}$ & $\mathrm{nb}$ & $n b$ & & $6 \mathrm{nq}$ & $\mathrm{nq}$ \\
\hline $\mathbf{U}$ & $\%$ & $\mathrm{nb}$ & $\operatorname{lnb}$ & $\mathrm{nb}$ & $\mathrm{nb}$ & $n b$ & 0 & & $0 \mathrm{nq}$ \\
\hline
\end{tabular}


Tab. A.7. (Forts.): Ergebnisse des Anionenaustausches für Porenwässer und Wasserproben aus Cospuden; Konzentrationen in Lösungen nach dem Anionenaustausch in \% bezogen auf die Ausgangskonzentrationen (Tab. A.1.) von Porenlösungen und Wasserproben aus Cospuden; AI = Austauschion.

\begin{tabular}{|c|c|c|c|c|c|c|c|c|c|c|}
\hline & & RCO12 & RCO13 & RCO13 & RCO14 & Bohrung $1 \mathrm{~m}$ & Bohrung 2 m| & Bohrung $3 \mathrm{~ms}$ & See & See \\
\hline & & $\mathrm{OH}^{-}$ & $\mathrm{OH}^{-}$ & $\mathrm{Cl}^{-}$ & $\mathbf{O H}^{-}$ & $\mathrm{Cl}^{-}$ & $\mathrm{Cl}^{-}$ & $\mathrm{Cl}^{-}$ & $\mathrm{OH}^{-}$ & $\mathrm{Cl}^{-}$ \\
\hline & & Wasserprob & eWasserprobe & eWasserprobe & Wasserprobe & Porenlösung & Porenlösung & Porenlösung & Wasserprobe & Wasserprobe \\
\hline & & Okt 1998 & Okt 1998 & Okt 1998 & Okt 1998 & Nov 1998 & Nov 1998 & Nov 1998 & Nov 1998 & Nov 1998 \\
\hline Sulfat & $\%$ & nb & 96 & & $5 \mathrm{nb}$ & 17 & 13 & 24 & 91 & 69 \\
\hline DOC & $\%$ & 7. & 26 & $6 \mathrm{nb}$ & & $n b$ & nb & $\mathrm{nb}$ & & $n b$ \\
\hline $\mathbf{L i}$ & $\%$ & 9 & 95 & 93 & 102 & 65 & 72 & 77 & 78 & 89 \\
\hline $\mathbf{B e}$ & $\%$ & $\mathrm{nq}$ & $\mathrm{nq}$ & $\mathrm{nq}$ & $\mathrm{nq}$ & 35 & 37 & & $\mathrm{nq}$ & $\mathrm{nq}$ \\
\hline $\mathbf{N a}$ & $\%$ & 9. & 97 & 91 & 102 & 67 & 100 & 83 & 86 & 90 \\
\hline Mg & $\%$ & 8 & 100 & 4 & 107 & 68 & 77 & 83 & 3 & 91 \\
\hline Al & $\%$ & $\mathrm{nq}$ & $\mathrm{nq}$ & $\mathrm{nq}$ & $\mathrm{nq}$ & 11 & 83 & 83 & 83 & 80 \\
\hline $\mathbf{K}$ & $\%$ & 9 & 112 & 104 & 109 & & $\mathrm{nq}$ & 77 & 80 & 92 \\
\hline Ca & $\%$ & 6 & 93 & 85 & 102 & 67 & 77 & \begin{tabular}{l|l|}
83 \\
\end{tabular} & 76 & 91 \\
\hline Sc & $\%$ & 7 & 80 & 41 & $1 \mathrm{nq}$ & 19 & 81 & 64 & 104 & 43 \\
\hline $\mathbf{T i}$ & $\%$ & $\mathrm{nq}$ & 94 & 27 & $7 \mathrm{nq}$ & $\mathrm{nq}$ & $\mathrm{nq}$ & $n q$ & $\mathrm{nq}$ & nq \\
\hline $\mathbf{V}$ & $\%$ & & $8 \mathrm{nq}$ & 51 & nq & & $\mathrm{nq}$ & & $\mathrm{nq}$ & 27 \\
\hline $\mathbf{C r}$ & $\%$ & 7 & 101 & 98 & 101 & & $\mathrm{nq}$ & $\mathrm{nq}$ & 98 & 102 \\
\hline Mn & $\%$ & 32 & 3 & 1 & 24 & 68 & 68 & 73 & 0 & 91 \\
\hline $\mathbf{F e}$ & $\%$ & ( & 12 & 5 & 1 & 62 & $n q$ & 20 & 0 & 16 \\
\hline Co & $\%$ & 6 & 104 & 97 & 63 & 66 & 65 & 44 & 20 & 65 \\
\hline $\mathrm{Ni}$ & $\%$ & 50 & 96 & 98 & 45 & 64 & 64 & 67 & 5 & 36 \\
\hline $\mathbf{C u}$ & $\%$ & 79 & 98 & 100 & 99 & 26 & 46 & 51 & 100 & 100 \\
\hline $\mathbf{Z n}$ & $\%$ & 70 & 64 & 80 & 18 & 61 & 39 & 77 & 7 & 25 \\
\hline As & $\% 1$ & $\mathrm{nq}$ & $\mathrm{nq}$ & $\mathrm{nq}$ & nq & & $n q$ & & $\mathrm{nq}$ & $n q$ \\
\hline Rb & $\%$ & 100 & 154 & 88 & $\mathrm{nq}$ & 70 & 104 I & $\mathrm{nq}$ & 56 & 48 \\
\hline $\mathbf{S r}$ & $\%$ & 2 & 93 & 88 & 105 & 66 & 77 & $81 \mid$ & 103 & 90 \\
\hline $\mathbf{Y}$ & $\%$ & & $0 \mathrm{nq}$ & $\mathrm{nq}$ & nq & 8 & 47 & 3 & 98 & 99 \\
\hline $\mathbf{Z r}$ & $\%$ & 92 & 117 & 16 & $\mathrm{nq}$ & 70 & 82 & $91 \mid$ & 44 & 19 \\
\hline $\mathbf{N b}$ & $\%$ & & $6 \mathrm{nq}$ & $\mathrm{nq}$ & & $\mathrm{nq}$ & $\mathrm{nq}$ & $\mathrm{nq}$ & 13 & 26 \\
\hline Mo & $\%$ & 20 & 50 & 32 & 22 & 47 & 10 & 5 & 41 & 28 \\
\hline Cd & $\%$ & nb & $\mathrm{nb}$ & nb & nb & 60 & 58 & & $n b$ & $\mathrm{nb}$ \\
\hline Sn & $\%$ & & $7 \mathrm{nq}$ & $\mathrm{nq}$ & 54 & 64 & 89 & & $\mathrm{nq}$ & $\mathrm{nq}$ \\
\hline $\mathbf{S b}$ & $\%$ & $\mathrm{nq}$ & $\mathrm{nq}$ & $\mathrm{nq}$ & nq & 63 & 54 & & $\mathrm{nq}$ & nq \\
\hline Cs & $\%$ & $\mathrm{nq}$ & $\mathrm{nq}$ & $\mathrm{nq}$ & nq & 69 & 75 & 108 & 59 & 98 \\
\hline $\mathbf{B a}$ & $\% 1$ & nq & & $2 \mathrm{nq}$ & $\mathrm{nq}$ & 2 & 95 & 118 & 378 & 449 \\
\hline La & $\%$ & & $8 \mathrm{nq}$ & $\mathrm{nq}$ & nq & 31 & 19 & 2 & 6 & 8 \\
\hline $\mathrm{Ce}$ & $\%$ & $\mathrm{nq}$ & $\mathrm{nq}$ & $\mathrm{nq}$ & nq & 31 & 17 & & $\mathrm{nq}$ & $n q$ \\
\hline $\mathbf{P r}$ & $\%$ & $\mathrm{nq}$ & $\mathrm{nq}$ & $\mathrm{nq}$ & nq & 30 & 24 & & $\mathrm{nq}$ & $\mathrm{nq}$ \\
\hline $\mathbf{N d}$ & $\% 1$ & $\mathrm{nq}$ & $\mathrm{nq}$ & $\mathrm{nq}$ & nq & 30 & 28 & & $\mathrm{nq}$ & $n q$ \\
\hline Sm & $\%$ & $\mathrm{nq}$ & $\mathrm{nq}$ & $\mathrm{nq}$ & nq & 30 & 77 & & $\mathrm{nq}$ & $\mathrm{nq}$ \\
\hline $\mathbf{E u}$ & $\%$ & $\mathrm{nq}$ & $\mathrm{nq}$ & $\mathrm{nq}$ & nq & & $\mathrm{nq}$ & & $\mathrm{nq}$ & $\mathrm{nq}$ \\
\hline Gd & $\% 1$ & $\mathrm{nq}$ & $\mathrm{nq}$ & $\mathrm{nq}$ & nq & 29 & 34 & & $\mathrm{nq}$ & $\mathrm{nq}$ \\
\hline $\mathbf{T b}$ & $\% 1$ & $\mathrm{nq}$ & $\mathrm{nq}$ & $\mathrm{nq}$ & nq & & $\mathrm{nq}$ & & $\mathrm{nq}$ & $\mathrm{nq}$ \\
\hline Dy & $\% \mathrm{n}$ & $\mathrm{nq}$ & $\mathrm{nq}$ & $\mathrm{nq}$ & nq & 28 & 60 & & $\mathrm{nq}$ & $\mathrm{nq}$ \\
\hline Ho & $\% n$ & nq & $\mathrm{nq}$ & nq & nq & 28 & $\mathrm{nq}$ & & $\mathrm{nq}$ & $n q$ \\
\hline Er & $\% \mathrm{n}$ & nq & $\mathrm{nq}$ & $\mathrm{nq}$ & $\mathrm{nq}$ & 27 & $\mathrm{nq}$ & & $\mathrm{nq}$ & $\mathrm{nq}$ \\
\hline $\mathbf{T m}$ & $\% \mathrm{n}$ & $\mathrm{nq}$ & $\mathrm{nq}$ & $\mathrm{nq}$ & $\mathrm{nq}$ & 27 & & $\mathrm{nq}$ & $\mathrm{nq}$ & $\mathrm{nq}$ \\
\hline $\mathbf{Y b}$ & $\%$ & $\mathrm{nq}$ & $\mathrm{nq}$ & $\mathrm{nq}$ & $\mathrm{nq}$ & 25 & $\mathrm{nq}$ & & $\mathrm{nq}$ & $\mathrm{nq}$ \\
\hline $\mathbf{L u}$ & $\%$ & nq & $\mathrm{nq}$ & nq & nq & 25 & $\mathrm{nq}$ & $\mathrm{nq}$ & $\mathrm{nq}$ & nq \\
\hline Hf & $\%$ & $\mathrm{nq}$ & $\mathrm{nq}$ & $\mathrm{nq}$ & $\mathrm{nq}$ & 43 & $\mathrm{nq}$ & $\mathrm{nq}$ & $\mathrm{nq}$ & $\mathrm{nq}$ \\
\hline Ta & $\%$ & nq & $\mathrm{nq}$ & $\mathrm{nq}$ & nq & 66 & $\mathrm{nq}$ & $\mathrm{nq}$ & $\mathrm{nq}$ & nq \\
\hline $\mathbf{W}$ & $\%$ & nq & 49 & 97 & 33 & 13 & 3 & & $\mathrm{nq}$ & $\mathrm{nq}$ \\
\hline $\mathrm{Hg}$ & $\%$ & $n b$ & nb & nb & $n b$ & $\mathrm{nq}$ & 71 & & $\mathrm{nb}$ & $\mathrm{nb}$ \\
\hline TI & $\%$ & $\mathrm{nq}$ & $\mathrm{nq}$ & $\mathrm{nq}$ & $\mathrm{nq}$ & 62 & $\mathrm{nq}$ & & $\mathrm{nq}$ & $\mathrm{nq}$ \\
\hline $\mathbf{P b}$ & $\%$ & $\mathrm{nq}$ & $\mathrm{nq}$ & $\mathrm{nq}$ & nq & 28 & 30 & $104 \mathrm{n}$ & $\mathrm{nq}$ & $n q$ \\
\hline $\mathbf{B i}$ & $\%$ & $\mathrm{nq}$ & $\mathrm{nq}$ & $\mathrm{nq}$ & $\mathrm{nq}$ & 74 & $\mathrm{nq}$ & $\mathrm{nq}$ & $\mathrm{nq}$ & $\mathrm{nq}$ \\
\hline Th & $\%$ & $\mathrm{nq}$ & $\mathrm{nq}$ & $\mathrm{nq}$ & $\mathrm{nq}$ & 281 & $\mathrm{nq}$ & $\mathrm{nq}$ & $\mathrm{nq}$ & $\mathrm{nq}$ \\
\hline $\mathbf{U}$ & $\%$ & 12 & 5 & 4 & $n q$ & & $\mathrm{nq}$ & $n q$ & 0 & \\
\hline
\end{tabular}


Tab. A.7. (Forts.): Ergebnisse des Anionenaustausches für Porenwässer und Wasserproben aus Cospuden; Konzentrationen in Lösungen nach dem Anionenaustausch in \% bezogen auf die Ausgangskonzentrationen (Tab. A.1.) von Porenlösungen und Wasserproben aus Cospuden; AI = Austauschion.

\begin{tabular}{|c|c|c|c|c|c|c|c|c|c|}
\hline & & CML $20 \mathrm{~m}$ & CML $20 \mathrm{~m}$ & CML 27,5 m & CML 27,5 m & CML 31,5 m & CML 31,5 m & CML 39,5 m & CML 39,5 m \\
\hline AI & & $\mathrm{OH}^{-}$ & $\mathrm{Cl}^{-}$ & $\mathrm{OH}^{-}$ & $\mathrm{Cl}^{-}$ & $\mathrm{OH}^{-}$ & $\mathrm{Cl}^{-}$ & $\mathbf{O H}^{-}$ & $\mathrm{Cl}^{-}$ \\
\hline & & Wasserprobe & Wasserprobe & Wasserprobe & Wasserprobe & \begin{tabular}{|l|} 
Wasserprobe \\
\end{tabular} & Wasserprobe & Wasserprobe & Wasserprobe \\
\hline & & Okt 1998 & Jan 1999 & Jan 1999 & Jan 1999 & Jan 1999 & Jan 1999 & \begin{tabular}{|l|} 
Jan 1999 \\
\end{tabular} & Jan 1999 \\
\hline Sulfat & $\%$ & 57 & 0 & 88 & 70 & 97 & 93 & 75 & 27 \\
\hline DOC & $\%$ & $\mathrm{nq}$ & 93 & 65 & & $n q$ & 77 & 10 & 38 \\
\hline $\mathbf{L i}$ & $\%$ & 63 & 80 & 101 & 101 & 101 & 100 & 99 & 87 \\
\hline$\overline{\mathrm{Be}}$ & $\%$ & $\operatorname{nq}$ & $\mathrm{nq}$ & $n q$ & $\mathrm{nq}$ & nq & $\mathrm{nq}$ & $n q$ & $n q$ \\
\hline $\mathbf{N a}$ & $\%$ & 93 & 86 & 99 & 120 & 101 & 104 & 94 & 88 \\
\hline $\mathbf{M g}$ & $\%$ & 15 & 86 & 78 & 101 & 108 & 100 & 119 & 90 \\
\hline Al & $\%$ & $\operatorname{nq}$ & nq & $n q$ & $\mathrm{nq}$ & $n q$ & $\mathrm{nq}$ & $\mathrm{nq}$ & $\mathrm{nq}$ \\
\hline $\mathbf{K}$ & $\%$ & 101 & 86 & 105 & 108 & 115 & 105 & 102 & 91 \\
\hline $\mathbf{C a}$ & $\%$ & 99 & 92 & 102 & 104 & 101 & 100 & 67 & 84 \\
\hline $\mathbf{S c}$ & $\%$ & $\mathrm{nq}$ & $\mathrm{nq}$ & nq & $\mathrm{nq}$ & nq & $\mathrm{nq}$ & $n q$ & $\mathrm{nq}$ \\
\hline $\mathbf{T i}$ & $\%$ & $\mathrm{nq}$ & $\mathrm{nq}$ & nq & $\mathrm{nq}$ & nq & $\mathrm{nq}$ & nq & $\mathrm{nq}$ \\
\hline $\mathbf{V}$ & $\%$ & 0 & $0 / 1$ & nq & $\mathrm{nq}$ & 79 & $94 \sqrt{n}$ & $n q$ & $\mathrm{nq}$ \\
\hline $\mathbf{C r}$ & $\%$ & 0 & 0 & 0 & 9 & 64 & $62 n$ & $n q$ & nq \\
\hline Mn & $\%$ & $\mathrm{ng}$ & $\mathrm{ng}$ & 0 & 12 & 118 & 100 & 12 & 21 \\
\hline $\mathbf{F e}$ & $\%$ & $\mathrm{nq}$ & $\mathrm{nq}$ & nq & $\mathrm{nq}$ & 87 & & $n q$ & $\mathrm{nq}$ \\
\hline \begin{tabular}{|l|} 
Co \\
\end{tabular} & $\%$ & 0 & 0 & 0 & 0 & 70 & 66 & 0 & 0 \\
\hline $\mathbf{N i}$ & $\%$ & 0 & 0 & 0 & 0 & 87 & 100 & $\mathrm{nq}$ & $\mathrm{nq}$ \\
\hline $\mathrm{Cu}$ & $\%$ & 42 & 97 & 97 & 91 & 59 & 76 & 62 & 85 \\
\hline $\mathbf{Z n}$ & $\%$ & $\operatorname{nq}$ & $\mathrm{nq}$ & 70 & 70 & 28 & & $\mathrm{nq}$ & $\mathrm{nq}$ \\
\hline \begin{tabular}{|l|}
$\mathbf{A s}$ \\
\end{tabular} & $\%$ & $\mathrm{nq}$ & $\mathrm{nq}$ & $\mathrm{nq}$ & $\mathrm{nq}$ & nq & $\mathrm{nq}$ & $\mathrm{nq}$ & $\mathrm{nq}$ \\
\hline $\mathbf{R b}$ & $\%$ & 69 & 63 & 103 & 104 & 111 & 111 & 100 & 88 \\
\hline $\mathbf{S r}$ & $\%$ & 93 & 87 & 100 & 102 & 101 & 100 & 72 & 86 \\
\hline $\mathbf{Y}$ & $\%$ & $\mathrm{nq}$ & $\mathrm{nq}$ & nq & $\mathrm{nq}$ & nq & $\mathrm{nq}$ & $\bar{c}$ & 0 \\
\hline $\mathbf{Z r}$ & $\%$ & $\mathrm{nq}$ & $\mathrm{nq}$ & $\mathrm{nq}$ & $\mathrm{nq}$ & 86 & \begin{tabular}{|c|}
93 \\
\end{tabular} & 0 & 0 \\
\hline $\mathbf{N b}$ & $\%$ & $\mathrm{nq}$ & $\mathrm{nq}$ & 64 & 100 & $\mathrm{nq}$ & $\mathrm{nq}$ & $\mathrm{nq}$ & $\mathrm{nq}$ \\
\hline Mo & $\%$ & 7 & 4 & 35 & 5 & 120 & 62 & 4 & 3 \\
\hline Cd & $\%$ & $\mathrm{nq}$ & $\mathrm{nq}$ & 71 & & $n b$ & nb & $n q$ & $\mathrm{nq}$ \\
\hline Sn & $\%$ & $\mathrm{nq}$ & $n q$ & $n q$ & $\mathrm{nq}$ & $n q$ & $\mathrm{nq}$ & nq & $\mathrm{nq}$ \\
\hline $\mathbf{S b}$ & $\%$ & $\mathrm{nq}$ & nq & nq & $\mathrm{nq}$ & nq & $\mathrm{nq}$ & $n q$ & $\mathrm{nq}$ \\
\hline Cs & $\%$ & 60 & 64 & 109 & 96 & 117 & 120 & 50 & 50 \\
\hline $\mathrm{Ba}$ & $\%$ & $\mathrm{ng}$ & 95 & 94 & & $\mathrm{nq}$ & 94 & 28 & 79 \\
\hline La & $\%$ & $\mathrm{nq}$ & nq & nq & $\mathrm{nq}$ & nq & $\mathrm{nq}$ & $\mathrm{nq}$ & $\mathrm{nq}$ \\
\hline $\mathrm{Ce}$ & $\%$ & $\mathrm{nq}$ & $\mathrm{nq}$ & nq & $\mathrm{nq}$ & nq & $\mathrm{nq}$ & nq & $\mathrm{nq}$ \\
\hline$\overline{P r}$ & $\%$ & $\mathrm{nq}$ & $\mathrm{nq}$ & nq & $\mathrm{nq}$ & nq & $\mathrm{nq}$ & $\mathrm{nq}$ & $\mathrm{nq}$ \\
\hline $\mathbf{N d}$ & $\%$ & $\mathrm{nq}$ & $n q$ & nq & $\mathrm{nq}$ & nq & $\mathrm{nq}$ & $\mathrm{nq}$ & $\mathrm{nq}$ \\
\hline $\mathbf{S m}$ & $\%$ & $\mathrm{nq}$ & $\mathrm{nq}$ & nq & $\mathrm{nq}$ & nq & $n q$ & $n q$ & $\mathrm{nq}$ \\
\hline $\mathbf{E u}$ & $\%$ & $\mathrm{nq}$ & nq & nq & $\mathrm{nq}$ & nq & $\mathrm{nq}$ & nq & $\mathrm{nq}$ \\
\hline Gd & $\%$ & $\mathrm{ng}$ & $\mathrm{nq}$ & nq & $\mathrm{nq}$ & nq & $\mathrm{ng}$ & $\mathrm{nq}$ & nq \\
\hline Tb & $\%$ & $\mathrm{nq}$ & $\mathrm{nq}$ & $\mathrm{nq}$ & $\mathrm{nq}$ & nq & $\mathrm{nq}$ & $\mathrm{nq}$ & $\mathrm{nq}$ \\
\hline Dy & $\%$ & $\mathrm{nq}$ & $\mathrm{nq}$ & nq & $\mathrm{nq}$ & nq & $\mathrm{nq}$ & $n q$ & $\mathrm{nq}$ \\
\hline Ho & $\%$ & $\mathrm{nq}$ & $\mathrm{nq}$ & $n q$ & $\mathrm{nq}$ & $\mathrm{nq}$ & $\mathrm{nq}$ & $\mathrm{nq}$ & $\mathrm{nq}$ \\
\hline $\mathbf{E r}$ & $\%$ & $\mathrm{nq}$ & nq & nq & $\mathrm{nq}$ & nq & $\operatorname{nq}$ & $n q$ & $\mathrm{nq}$ \\
\hline Tm & $\%$ & $\mathrm{nq}$ & $\mathrm{nq}$ & nq & $\mathrm{nq}$ & nq & $\mathrm{nq}$ & nq & nq \\
\hline $\mathbf{Y b}$ & $\%$ & $\mathrm{nq}$ & nq & nq & $\mathrm{nq}$ & nq & $\operatorname{nq}$ & $n q$ & $\mathrm{nq}$ \\
\hline $\mathbf{L u}$ & $\%$ & $\mathrm{nq}$ & $\mathrm{nq}$ & $\mathrm{nq}$ & $\mathrm{nq}$ & $\mathrm{nq}$ & $\mathrm{nq}$ & $\mathrm{nq}$ & $\mathrm{nq}$ \\
\hline Hf & $\%$ & $\mathrm{nq}$ & $\mathrm{nq}$ & nq & $\mathrm{nq}$ & nq & $\mathrm{nq}$ & $n q$ & $\mathrm{nq}$ \\
\hline Ta & $\%$ & $\mathrm{nb}$ & $\mathrm{nb}$ & $\mathrm{nb}$ & nb & $\mathrm{nb}$ & $\mathrm{nb}$ & $\mathrm{nb}$ & nb \\
\hline $\mathbf{W}$ & $\%$ & 7 & 10 & 83 & & $\mathrm{nq}$ & $\mathrm{nq}$ & $\mathrm{nq}$ & $\mathrm{nq}$ \\
\hline $\mathbf{H g}$ & $\%$ & 65 & 85 & 92 & & $\mathrm{nq}$ & & $\mathrm{nq}$ & $\mathrm{nq}$ \\
\hline $\mathbf{T I}$ & $\%$ & $\mathrm{nq}$ & $\mathrm{nq}$ & nq & $\mathrm{nq}$ & nq & $\mathrm{nq}$ & $n q$ & $\mathrm{nq}$ \\
\hline $\mathbf{P b}$ & $\%$ & & $0 \mathrm{nq}$ & 39 & 113 & $n q$ & $\operatorname{nq}$ & $n q$ & $\mathrm{nq}$ \\
\hline $\mathbf{B i}$ & $\%$ & $\mathrm{nq}$ & $\mathrm{nq}$ & $n q$ & $\mathrm{nq}$ & nq & $\mathrm{nq}$ & $\mathrm{nq}$ & $\mathrm{nq}$ \\
\hline Th & $\%$ & $\mathrm{nq}$ & $\mathrm{nq}$ & nq & $\mathrm{nq}$ & $\mathrm{nq}$ & $\mathrm{nq}$ & $n q$ & $\mathrm{nq}$ \\
\hline $\mathbf{U}$ & $\%$ & $\mathrm{nq}$ & $\mathrm{nq}$ & 10 & 10 & 2 & 3 & 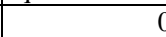 & 5 \\
\hline
\end{tabular}


Tab. A.7. (Forts.): Ergebnisse des Anionenaustausches für Porenwässer und Wasserproben aus Cospuden; Konzentrationen in Lösungen nach dem Anionenaustausch in \% bezogen auf die Ausgangskonzentrationen (Tab. A.1.) von Porenlösungen und Wasserproben aus Cospuden; AI = Austauschion.

\begin{tabular}{|c|c|c|c|c|c|c|c|c|c|c|}
\hline & & CML 43,5 m & CML 43,5 m & CML 20 m & CML 27;5 m & CML 31,5 m & CML 39,5 m & CML 43,5 m & RCO3 & \multirow{2}{*}{\begin{tabular}{|l|}
$\mathrm{RCO} 4$ \\
$\mathrm{Cl}^{-}$
\end{tabular}} \\
\hline \multirow[t]{3}{*}{ AI } & & $\mathrm{OH}^{-}$ & $\mathrm{Cl}^{-}$ & $\mathrm{Cl}^{-}$ & $\mathrm{Cl}^{-}$ & $\mathrm{Cl}^{-}$ & $\mathrm{Cl}^{-}$ & $\mathrm{Cl}^{-}$ & $\mathrm{Cl}^{-}$ & \\
\hline & & Wasserprobe & eWasserprobe & Wasserprobe & Wasserprobe & Wasserprobe & Wasserprobe & Wasserprobe & Wasserprobe & Wasserprobe \\
\hline & & Jan 1999 & Jan 1999 & Aug 1999 & Aug 1999 & Aug 1999 & Aug 1999 & Aug 1999 & Aug 1999 & Aug 1999 \\
\hline Sulfat & $\%$ & 77 & 65 & 100 & 93 & 63 & 106 & 0 & 42 & 98 \\
\hline DOC & $\%$ & 5 & 33 & $3 \mathrm{nb}$ & nb & & nb & & $\mathrm{nq}$ & 120 \\
\hline $\mathbf{L i}$ & $\%$ & 105 & 104 & $4 \mathrm{nq}$ & 93 & 82 & 96 & 77 & 69 & 68 \\
\hline Be & $\%$ & nq & $\mathrm{nq}$ & $\mathrm{nq}$ & nq & & $6 \mathrm{nq}$ & nq & & $\mathrm{nq}$ \\
\hline $\mathbf{N a}$ & $\%$ & 99 & 102 & 98 & 95 & 90 & 95 & 90 & 69 & 69 \\
\hline Mg & $\%$ & 120 & 103 & $3 \mathrm{nq}$ & 96 & 85 & 94 & 80 & 85 & 93 \\
\hline Al & $\%$ & nq & $\mathrm{nq}$ & $\mathrm{nq}$ & 110 & 35 & 101 & 65 & & nq \\
\hline $\mathbf{K}$ & $\%$ & 108 & 106 & 99 & 98 & 86 & 94 & 81 & 60 & 71 \\
\hline \begin{tabular}{|l|}
$\mathbf{C a}$ \\
\end{tabular} & $\%$ & 77 & 103 & 93 & 94 & 84 & 95 & 79 & 67 & 81 \\
\hline Sc & $\%$ & $\mathrm{nq}$ & $\mathrm{nq}$ & $\mathrm{nq}$ & nq & 81 & 92 & 87 & & nq \\
\hline Ti & $\%$ & $\mathrm{nq}$ & $\mathrm{nq}$ & $\mathrm{nq}$ & nq & $\mathrm{nq}$ & $\mathrm{nq}$ & $\mathrm{nq}$ & $\mathrm{nq}$ & nq \\
\hline $\mathbf{V}$ & $\%$ & $\mathrm{nq}$ & $\mathrm{nq}$ & & $\mathrm{nq}$ & 76 & 0 & 13 & 51 & 83 \\
\hline $\mathbf{C r}$ & $\%$ & $\mathrm{nq}$ & $\mathrm{nq}$ & $\mathrm{nq}$ & nq & nq & $\mathrm{nq}$ & 70 & 100 & 100 \\
\hline Mn & $\%$ & 16 & & $9 \mathrm{nq}$ & 93 & 88 & 93 & 80 & 74 & 108 \\
\hline $\mathbf{F e}$ & $\%$ & $\mathrm{nq}$ & $\mathrm{nq}$ & $\mathrm{nq}$ & 120 & 84 & 67 & 7 & 75 & 105 \\
\hline Co & $\%$ & 0 & 0 & $\mathrm{nq}$ & 87 & & $\mathrm{nq}$ & 64 & & $n q$ \\
\hline $\mathbf{N i}$ & $\%$ & $\mathrm{nq}$ & $\mathrm{nq}$ & $\mathrm{nq}$ & 84 & & $\mathrm{nq}$ & & $\mathrm{nq}$ & nq \\
\hline $\mathbf{C u}$ & $\%$ & 55 & & $8 n q$ & 91 & 81 & 881 & $\mathrm{nq}$ & $\mathrm{nq}$ & $\mathrm{nq}$ \\
\hline $\mathbf{Z n}$ & $\%$ & 79 & & $2 \mathrm{nq}$ & 102 & 81 & 84 & 67 & & $\mathrm{nq}$ \\
\hline As & $\%$ & $\mathrm{nq}$ & $\mathrm{nq}$ & $\mathrm{nq}$ & $\mathrm{nq}$ & 74 & 821 & $\mathrm{nq}$ & $\mathrm{nq}$ & 58 \\
\hline $\mathbf{R b}$ & $\%$ & 88 & & $1 \mathrm{nq}$ & 108 & 87 & 105 & $\mathrm{nq}$ & 66 & 96 \\
\hline $\mathbf{S r}$ & $\%$ & 81 & 103 & 100 & 94 & 83 & 95 & 78 & 65 & 72 \\
\hline $\mathbf{Y}$ & $\%$ & $\mathrm{nq}$ & $\mathrm{nq}$ & $\mathrm{nq}$ & 94 & & $\mathrm{nq}$ & 30 & 27 & $\mathrm{nq}$ \\
\hline $\mathbf{Z r}$ & $\%$ & $\mathrm{nq}$ & $\mathrm{nq}$ & $\mathrm{nq}$ & $\mathrm{nq}$ & 80 & 64 & 96 & 69 & 84 \\
\hline $\mathbf{N b}$ & $\%$ & nq & $\mathrm{nq}$ & 38 & 42 & & $\mathrm{nq}$ & nq & $\mathrm{nq}$ & 59 \\
\hline Mo & $\%$ & 2 & 1 & 9 & 58 & 23 & 23 & 10 & 98 & 95 \\
\hline Cd & $\%$ & 50 & & $4 \mathrm{nq}$ & $\mathrm{nq}$ & $\mathrm{nq}$ & $\mathrm{nq}$ & $\mathrm{nq}$ & $\mathrm{nq}$ & $\mathrm{nq}$ \\
\hline Sn & $\%$ & $\mathrm{nq}$ & $\mathrm{nq}$ & 4 & 55 & & $\mathrm{nq}$ & $\mathrm{nq}$ & 57 & 80 \\
\hline $\mathbf{S b}$ & $\%$ & $\mathrm{nq}$ & $\mathrm{nq}$ & & $\mathrm{nq}$ & nq & $\mathrm{nq}$ & $\mathrm{nq}$ & $\mathrm{nq}$ & nq \\
\hline Cs & $\%$ & $\mathrm{nq}$ & $\mathrm{nq}$ & $\mathrm{nq}$ & nq & 95 & $5 \mathrm{nq}$ & $\mathrm{nq}$ & 73 & $\mathrm{nq}$ \\
\hline $\mathbf{B a}$ & $\%$ & 43 & 102 & 83 & 120 & 100 & 100 & 81 & 100 & 70 \\
\hline La & $\%$ & $\mathrm{nq}$ & $\mathrm{nq}$ & $\mathrm{nq}$ & 103 & & $n \mathrm{nq}$ & 18 & & $\mathrm{nq}$ \\
\hline Ce & $\%$ & $\mathrm{nq}$ & $\mathrm{nq}$ & $\mathrm{nq}$ & 99 & & $\mathrm{nq}$ & 14 & & $\mathrm{nq}$ \\
\hline $\mathbf{P r}$ & $\%$ & $\mathrm{nq}$ & $\mathrm{nq}$ & $\mathrm{nq}$ & 95 & & $\mathrm{nq}$ & $\mathrm{nq}$ & & $\mathrm{nq}$ \\
\hline $\mathbf{N d}$ & $\%$ & $\mathrm{nq}$ & $\mathrm{nq}$ & $\mathrm{nq}$ & 98 & & $\mathrm{nq}$ & 25 & & $\mathrm{nq}$ \\
\hline Sm & $\%$ & $\mathrm{nq}$ & $\mathrm{nq}$ & $\mathrm{nq}$ & 80 & & $\mathrm{nq}$ & 88 & & $\mathrm{nq}$ \\
\hline $\mathbf{E u}$ & $\%$ & $\mathrm{nq}$ & $\mathrm{nq}$ & $\mathrm{nq}$ & 79 & & $2 \mathrm{nq}$ & $\mathrm{nq}$ & & $\mathrm{nq}$ \\
\hline Gd & $\%$ & $\mathrm{nq}$ & $\mathrm{nq}$ & $\mathrm{nq}$ & 94 & & $\mathrm{nq}$ & 33 & & $\mathrm{nq}$ \\
\hline Tb & $\%$ & $\mathrm{nq}$ & $\mathrm{nq}$ & $\mathrm{nq}$ & 93 & & $7 \mathrm{nq}$ & $\mathrm{nq}$ & $\mathrm{nq}$ & $\mathrm{nq}$ \\
\hline \begin{tabular}{|l|}
$\mathbf{D y}$ \\
\end{tabular} & $\%$ & $\mathrm{nq}$ & $\mathrm{nq}$ & $\mathrm{nq}$ & 87 & & $\mathrm{nq}$ & 38 & & $\mathrm{nq}$ \\
\hline Ho & $\%$ & $\mathrm{nq}$ & $\mathrm{nq}$ & $\mathrm{nq}$ & 92 & & $\mathrm{nq}$ & nq & $\mathrm{nq}$ & $\mathrm{nq}$ \\
\hline $\mathbf{E r}$ & $\%$ & $\mathrm{nq}$ & $\mathrm{nq}$ & $\mathrm{nq}$ & 91 & & $\mathrm{nq}$ & $\mathrm{nq}$ & & $\mathrm{nq}$ \\
\hline Tm & $\%$ & $\mathrm{nq}$ & $\mathrm{nq}$ & $\mathrm{nq}$ & 120 & & $5 \mathrm{nq}$ & $\mathrm{nq}$ & $\mathrm{nq}$ & nq \\
\hline $\mathbf{Y b}$ & $\%$ & $\mathrm{nq}$ & $\mathrm{nq}$ & $\mathrm{nq}$ & nq & & $\mathrm{nq}$ & 49 & 29 & $\mathrm{nq}$ \\
\hline Lu & $\%$ & $\mathrm{nq}$ & $\mathrm{nq}$ & $\mathrm{nq}$ & 87 & & $\mathrm{nq}$ & nq & $\mathrm{nq}$ & nq \\
\hline Hf & $\%$ & $\mathrm{nq}$ & $\mathrm{nq}$ & $\mathrm{nq}$ & $\mathrm{nq}$ & $\mathrm{nq}$ & nq & $\mathrm{nq}$ & $\mathrm{nq}$ & $\mathrm{nq}$ \\
\hline Ta & $\%$ & nb & $\mathrm{nb}$ & 40 & 33 & 102 & $\left.59\right|_{1}$ & $\mathrm{nq}$ & $\mathrm{nq}$ & 36 \\
\hline $\mathbf{W}$ & $\%$ & 68 & 82 & 78 & 105 & & $\mathrm{nq}$ & & $\mathrm{nq}$ & $\mathrm{nq}$ \\
\hline $\mathrm{Hg}$ & $\%$ & $\mathrm{nq}$ & $\mathrm{nq}$ & $\mathrm{nq}$ & $\mathrm{nq}$ & 93 & 69 & 117 & $\mathrm{nq}$ & 87 \\
\hline TI & $\%$ & $\mathrm{nq}$ & $\mathrm{nq}$ & $\mathrm{nq}$ & nq & nq & $\mathrm{nq}$ & $\mathrm{nq}$ & $\mathrm{nq}$ & $\mathrm{nq}$ \\
\hline $\mathbf{P b}$ & $\%$ & $\mathrm{nq}$ & $\mathrm{nq}$ & $\mathrm{nq}$ & & $\mathrm{nq}$ & 113 & $\mathrm{nq}$ & $\mathrm{nq}$ & 70 \\
\hline $\mathbf{B i}$ & $\%$ & $\mathrm{nq}$ & $\mathrm{nq}$ & $\mathrm{nq}$ & $\mathrm{nq}$ & $\mathrm{nq}$ & $\mathrm{nq}$ & $\mathrm{nq}$ & $\mathrm{nq}$ & $\mathrm{nq}$ \\
\hline Th & $\%$ & $\mathrm{nq}$ & $\mathrm{nq}$ & 41 & $\mathrm{nq}$ & $\mathrm{nq}$ & $\mathrm{nq}$ & $\mathrm{nq}$ & $\mathrm{nq}$ & nq \\
\hline $\mathbf{U}$ & $\%$ & 0 & 0 & 3 & 0 & 6 & 0 & & $\mathrm{nq}$ & 5 \\
\hline
\end{tabular}


Tab. A.7. (Forts.): Ergebnisse des Anionenaustausches für Porenwässer und Wasserproben aus Cospuden; Konzentrationen in Lösungen nach dem Anionenaustausch in \% bezogen auf die Ausgangskonzentrationen (Tab. A.1.) von Porenlösungen und Wasserproben aus Cospuden; AI = Austauschion.

\begin{tabular}{|c|c|c|c|c|c|c|c|c|c|c|}
\hline & & RCO12 & RCO15 & RCO17 & RCO18 & See & SeeSed & Sedgepr & CML 20 m & CML 27,5 m \\
\hline \multirow[t]{3}{*}{ AI } & & $\mathrm{Cl}^{-}$ & $\mathrm{Cl}^{-}$ & $\mathrm{Cl}^{-}$ & $\mathrm{Cl}^{-}$ & $\mathrm{Cl}^{-}$ & $\mathrm{Cl}^{-}$ & $\mathrm{Cl}^{-}$ & $\mathrm{Cl}^{-}$ & $\mathrm{Cl}^{-}$ \\
\hline & & Wasserprol & eWasserprobe & Wasserprobe & Wasserprobe & Wasserprobe & Wasserprobe & Porenlösung & Wasserprobe & Wasserprobe \\
\hline & & Aug 1999 & Aug 1999 & Aug 1999 & Aug 1999 & Aug 1999 & Aug 1999 & Aug 1999 & Jan 1999 & Jan 1999 \\
\hline Sulfat & $\%$ & & 59 & 42 & 103 & 3 & 14 & 0 & 89 & 97 \\
\hline DOC & $\%$ & & 55 & 60 & 90 & 108 & 63 & 40 & 90 & 95 \\
\hline $\mathbf{L i}$ & $\%$ & & 97 & 98 & 92 & 85 & 96 & 87 & 95 & 88 \\
\hline $\mathbf{B e}$ & $\%$ & nq & $\mathrm{nq}$ & 63 & $\mathrm{nq}$ & nq & $\mathrm{nq}$ & $\mathrm{nq}$ & nq & 112 \\
\hline $\mathbf{N a}$ & $\%$ & & 97 & 103 & 103 & 96 & 86 & 87 & 97 & 95 \\
\hline Mg & $\%$ & & 100 & 100 & 116 & 91 & 87 & 65 & 98 & 91 \\
\hline Al & $\%$ & & 94 & 94 & 44 & & $\mathrm{nq}$ & nq & nq & 102 \\
\hline $\mathbf{K}$ & $\%$ & & $3 \mathrm{nq}$ & 116 & 100 & 114 & $\mathrm{nq}$ & 65 & 94 & 89 \\
\hline Ca & $\%$ & & 100 & 100 & 103 & 89 & 82 & 62 & 96 & 92 \\
\hline Sc & $\%$ & & $1 \mathrm{nq}$ & $\mathrm{nq}$ & 88 & & $\mathrm{nq}$ & & $\mathrm{nq}$ & 58 \\
\hline $\mathbf{T i}$ & $\%$ & nq & $\mathrm{nq}$ & $\mathrm{nq}$ & nq & nq & nq & $\mathrm{nq}$ & 0 & 74 \\
\hline $\mathbf{V}$ & $\%$ & & $2 \mathrm{nq}$ & $\mathrm{nq}$ & 11 & & $\mathrm{nq}$ & 93 & 75 & 91 \\
\hline $\mathbf{C r}$ & $\%$ & & $0 \mathrm{nq}$ & $\mathrm{nq}$ & 100 & 85 & $100 \mathrm{n}$ & $\mathrm{nq}$ & 93 & $\mathrm{nq}$ \\
\hline Mn & $\%$ & & 96 & & $\mathrm{nq}$ & & $\mathrm{nq}$ & 28 & 98 & 90 \\
\hline $\mathbf{F e}$ & $\%$ & & 75 & 104 & $\mathrm{nq}$ & $\mathrm{nq}$ & & $\mathrm{nq}$ & 57 & 90 \\
\hline \begin{tabular}{|l|} 
Co \\
\end{tabular} & $\%$ & & 65 & 80 & $\mathrm{nq}$ & 63 & $119 \mathrm{r}$ & $\mathrm{nq}$ & $\mathrm{nq}$ & 95 \\
\hline $\mathrm{Ni}$ & $\%$ & 10 & 75 & 115 & 90 & 54 & 82 & & $\mathrm{nq}$ & 97 \\
\hline $\mathbf{C u}$ & $\%$ & $\mathrm{nq}$ & $66 \mathrm{r}$ & $\mathrm{nq}$ & 35 & $5 \mathrm{nq}$ & 120 & 79 & 92 & 90 \\
\hline $\mathbf{Z n}$ & $\%$ & 12 & 0 & 41 & 117 & 56 & & $\mathrm{nq}$ & 111 & 96 \\
\hline As & $\%$ & & $1 \mathrm{nq}$ & $\mathrm{nq}$ & 108 & $\mathrm{nq}$ & $\mathrm{nq}$ & $\mathrm{nq}$ & $\mathrm{nq}$ & 84 \\
\hline $\mathbf{R b}$ & $\%$ & & 82 & 115 & 99 & 50 & 71 & 38 & $\mathrm{nq}$ & 107 \\
\hline $\mathbf{S r}$ & $\%$ & & 100 & 100 & 100 & 88 & 76 & 60 & 95 & 89 \\
\hline $\mathbf{Y}$ & $\%$ & & 0 & 99 & & $\mathrm{nq}$ & 62 & & $\mathrm{nq}$ & 99 \\
\hline $\mathbf{Z r}$ & $\%$ & & $0 \mathrm{nq}$ & $\mathrm{nq}$ & $\mathrm{nq}$ & 105 & $\mathrm{nq}$ & 10 & 81 & 96 \\
\hline $\mathbf{N b}$ & $\%$ & $\mathrm{nq}$ & 15 & 53 & 75 & 2 & 9 & 42 & $\mathrm{nq}$ & $\mathrm{nq}$ \\
\hline Mo & $\%$ & & $3 \mathrm{nq}$ & $\mathrm{nq}$ & 96 & 111 & 79 & 95 & $\mathrm{nq}$ & 80 \\
\hline Cd & $\%$ & $\mathrm{nq}$ & $\mathrm{nq}$ & $\mathrm{nq}$ & $\mathrm{nq}$ & & $\mathrm{nq}$ & 33 & $\mathrm{nq}$ & $\mathrm{nq}$ \\
\hline Sn & $\%$ & & $3 \mathrm{nq}$ & $\mathrm{nq}$ & $\mathrm{nq}$ & & $\mathrm{nq}$ & $\mathrm{nq}$ & $\mathrm{nq}$ & 4 \\
\hline $\mathbf{S b}$ & $\%$ & & $4 n q$ & $\mathrm{nq}$ & nq & nq & 82 & 22 & $\mathrm{nq}$ & $n q$ \\
\hline Cs & $\%$ & $\mathrm{nq}$ & $\mathrm{nq}$ & $\mathrm{nq}$ & $\mathrm{nq}$ & $\mathrm{nq}$ & $\mathrm{nq}$ & $\mathrm{nq}$ & $\mathrm{nq}$ & 92 \\
\hline $\mathbf{B a}$ & $\%$ & & 96 & 99 & 110 & 88 & 73 & 69 & 101 & 97 \\
\hline La & $\%$ & & $7 \mathrm{nq}$ & $\mathrm{nq}$ & nq & nq & & $\mathrm{nq}$ & $\mathrm{nq}$ & 95 \\
\hline $\mathrm{Ce}$ & $\%$ & $\mathrm{nq}$ & $\mathrm{nq}$ & 38 & $\mathrm{nq}$ & nq & $\mathrm{nq}$ & 84 & $\mathrm{nq}$ & 97 \\
\hline $\mathbf{P r}$ & $\%$ & $\mathrm{nq}$ & $\mathrm{nq}$ & $\mathrm{nq}$ & $\mathrm{nq}$ & $\mathrm{nq}$ & $\mathrm{nq}$ & $\mathrm{nq}$ & $\mathrm{nq}$ & 99 \\
\hline Nd & $\%$ & & $8 \mathrm{nq}$ & $\mathrm{nq}$ & 72 & & $n q$ & 59 & $\mathrm{nq}$ & 100 \\
\hline Sm & $\%$ & & $0 \mathrm{nq}$ & $\mathrm{nq}$ & 84 & & $\mathrm{nq}$ & $\mathrm{nq}$ & $\mathrm{nq}$ & 103 \\
\hline $\mathbf{E u}$ & $\%$ & & $3 \mathrm{nq}$ & $\mathrm{nq}$ & nq & $\mathrm{nq}$ & $\mathrm{nq}$ & $\mathrm{nq}$ & $\mathrm{nq}$ & 101 \\
\hline Gd & $\%$ & $\mathrm{nq}$ & $\mathrm{nq}$ & $\mathrm{nq}$ & $\mathrm{nq}$ & $\mathrm{nq}$ & $\mathrm{nq}$ & $\mathrm{nq}$ & $\mathrm{nq}$ & 101 \\
\hline $\mathbf{T b}$ & $\%$ & $\mathrm{nq}$ & $\mathrm{nq}$ & $\mathrm{nq}$ & $\mathrm{nq}$ & $\mathrm{nq}$ & nq & $\mathrm{nq}$ & $\mathrm{nq}$ & 104 \\
\hline Dy & $\%$ & & $6 \mathrm{nq}$ & $\mathrm{nq}$ & $\mathrm{nq}$ & $\mathrm{nq}$ & $\mathrm{nq}$ & $\mathrm{nq}$ & $\mathrm{nq}$ & 102 \\
\hline Ho & $\%$ & nq & $\mathrm{nq}$ & $\mathrm{nq}$ & nq & $\mathrm{nq}$ & $\mathrm{nq}$ & $\mathrm{nq}$ & $\mathrm{nq}$ & 103 \\
\hline Er & $\%$ & nq & $\mathrm{nq}$ & $\mathrm{nq}$ & $\mathrm{nq}$ & $\mathrm{nq}$ & $\mathrm{nq}$ & $\mathrm{nq}$ & $\mathrm{nq}$ & 103 \\
\hline $\mathbf{T m}$ & $\%$ & $\mathrm{nq}$ & $\mathrm{nq}$ & $\mathrm{nq}$ & $\mathrm{nq}$ & $\mathrm{nq}$ & $\mathrm{nq}$ & $\mathrm{nq}$ & $\mathrm{nq}$ & 107 \\
\hline $\mathbf{Y b}$ & $\%$ & $\mathrm{nq}$ & $\mathrm{nq}$ & $\mathrm{nq}$ & $\mathrm{nq}$ & $\mathrm{nq}$ & $\mathrm{nq}$ & $\mathrm{nq}$ & $\mathrm{nq}$ & 100 \\
\hline $\mathbf{L u}$ & $\%$ & nq & $\mathrm{nq}$ & $\mathrm{nq}$ & nq & $\mathrm{nq}$ & $\mathrm{nq}$ & $\mathrm{nq}$ & $\mathrm{nq}$ & 106 \\
\hline Hf & $\%$ & $\mathrm{nq}$ & $\mathrm{nq}$ & $\mathrm{nq}$ & $\mathrm{nq}$ & $\mathrm{nq}$ & $\mathrm{nq}$ & $\mathrm{nq}$ & $\mathrm{nq}$ & $\mathrm{nq}$ \\
\hline Ta & $\%$ & nq & $11 \mathrm{n}$ & $\mathrm{nq}$ & 81 & 4 & 8 & 31 & $\mathrm{nq}$ & nq \\
\hline $\mathbf{W}$ & $\%$ & nq & $\mathrm{nq}$ & 63 & $\mathrm{nq}$ & $\mathrm{nq}$ & 79 & 105 & $\mathrm{nq}$ & nq \\
\hline $\mathrm{Hg}$ & $\%$ & & 56 & 104 & 103 & 79 & 84 & 65 & $\mathrm{nq}$ & 103 \\
\hline Tl & $\%$ & $\mathrm{nq}$ & $\mathrm{nq}$ & $\mathrm{nq}$ & $\mathrm{nq}$ & & $\mathrm{nq}$ & & $\mathrm{nq}$ & $\mathrm{nq}$ \\
\hline $\mathbf{P b}$ & $\%$ & & 80 & 58 & 72 & 22 & & $\mathrm{nq}$ & $\mathrm{nq}$ & 25 \\
\hline $\mathbf{B i}$ & $\%$ & $\mathrm{nq}$ & $\mathrm{nq}$ & $\mathrm{nq}$ & $\mathrm{nq}$ & $\mathrm{nq}$ & $\mathrm{nq}$ & $\mathrm{nq}$ & $\mathrm{nq}$ & $\mathrm{nq}$ \\
\hline Th & $\%$ & $\mathrm{nq}$ & $\mathrm{nq}$ & $\mathrm{nq}$ & $\mathrm{nq}$ & $\mathrm{nq}$ & $\mathrm{nq}$ & 25 & $\mathrm{nq}$ & $\mathrm{nq}$ \\
\hline $\mathbf{U}$ & $\%$ & & 1 & 20 & 4 & 0 & 1 & & $\mathrm{nq}$ & 0 \\
\hline
\end{tabular}


Tab. A.7. (Forts.): Ergebnisse des Anionenaustausches für Porenwässer und Wasserproben aus Cospuden; Konzentrationen in Lösungen nach dem Anionenaustausch in \% bezogen auf die Ausgangskonzentrationen (Tab. A.1.) von Porenlösungen und Wasserproben aus Cospuden; AI = Austauschion.

\begin{tabular}{|c|c|c|c|c|c|c|c|c|c|c|}
\hline & & CML 31,5 m C & CML 39,5 m & CML 43,5 m & RCO3 & RCO4 & RCO12 & RCO15 & RCO17 & RCO18 \\
\hline \multirow[t]{3}{*}{$\mathbf{A I}$} & & \begin{tabular}{l|l}
$\mathrm{Cl}^{-}$ & $\mathrm{C}$
\end{tabular} & $\mathrm{Cl}^{-}$ & $\mathrm{Cl}^{-}$ & $\mathrm{Cl}^{-}$ & $\mathrm{Cl}^{-}$ & $\mathrm{Cl}^{-}$ & $\mathrm{Cl}^{-}$ & $\mathrm{Cl}^{-}$ & $\mathrm{Cl}^{-}$ \\
\hline & & \multicolumn{3}{|c|}{ WasserprobeWasserprobe Wasserprobe } & \multicolumn{3}{|c|}{ WasserprobeWasserprobe Wasserprobe } & Wasserprobe & Wasserprobe & \\
\hline & & Jan 1999 & Jan 1999 & Jan 1999 & Jan 1999 & Jan 1999 & Jan 1999 & Jan 1999 & Jan 1999 & Jan 1999 \\
\hline Sulfat & $\%$ & 88 & 60 & 96 & 77 & & $7 \mathrm{nq}$ & nq & 90 & 101 \\
\hline DOC & $\%$ & 88 & 79 & 44 & 69 & & & $\mathrm{nq}$ & 44 & 107 \\
\hline $\mathbf{L i}$ & $\%$ & \begin{tabular}{l|l}
107 \\
\end{tabular} & 75 & 110 & 85 & & $2 \mathrm{nq}$ & 64 & 57 & 119 \\
\hline $\mathbf{B e}$ & $\%$ & $89 n$ & $\mathrm{nq}$ & $\mathrm{nq}$ & & $5 \mathrm{nq}$ & & $\mathrm{nq}$ & $\mathrm{nq}$ & $\mathrm{nq}$ \\
\hline $\mathbf{N a}$ & $\%$ & 104 & 83 & 112 & $\mathrm{nq}$ & & $5 \mathrm{nq}$ & 46 & 61 & 115 \\
\hline Mg & $\%$ & 106 & 83 & 108 & 90 & & $1 \mathrm{nq}$ & 49 & 35 & 113 \\
\hline Al & $\%$ & $97 \mathrm{n}$ & $\mathrm{nq}$ & 12 & & nq & & $\mathrm{nq}$ & $41_{11}$ & nq \\
\hline $\mathbf{K}$ & $\%$ & 106 & 77 & 112 & & $\mathrm{nq}$ & $\mathrm{nq}$ & 39 & 51 & 119 \\
\hline Ca & $\%$ & 103 & 83 & 106 & 88 & & $3 \mathrm{nq}$ & 102 & 14 & 115 \\
\hline Sc & $\%$ & $71 \mathrm{n}$ & $\mathrm{nq}$ & $89_{1}$ & $\mathrm{nq}$ & $\mathrm{nq}$ & $\mathrm{nq}$ & $\mathrm{nq}$ & & $\mathrm{nq}$ \\
\hline $\mathbf{T i}$ & $\%$ & $\mathrm{nq}$ & nq & $\mathrm{nq}$ & nq & $\mathrm{nq}$ & $\mathrm{nq}$ & nq & $\mathrm{nq}$ & nq \\
\hline $\mathbf{V}$ & $\%$ & 105 & 103 & 94 & & $7 \mathrm{nq}$ & $\mathrm{nq}$ & & $\mathrm{nq}$ & 83 \\
\hline $\mathrm{Cr}$ & $\%$ & $\mathrm{nq}$ & 99 & $\mathrm{nq}$ & $\mathrm{nq}$ & $\mathrm{nq}$ & & $\mathrm{nq}$ & & $\mathrm{nq}$ \\
\hline Mn & $\%$ & 107 & 86 & 108 & 83 & & $1 \mathrm{nq}$ & 46 & 20 & 111 \\
\hline $\mathbf{F e}$ & $\%$ & 104 & 104 & 39 & & $7 \mathrm{nq}$ & $\mathrm{nq}$ & $\mathrm{nq}$ & 32 & 78 \\
\hline Co & $\%$ & $102 \mathrm{n}$ & $\mathrm{nq}$ & $\mathrm{nq}$ & $\mathrm{nq}$ & & $0 \mathrm{nq}$ & nq & $\mathrm{nq}$ & $\mathrm{nq}$ \\
\hline $\mathrm{Ni}$ & $\%$ & $102 \mathrm{n}$ & $\mathrm{nq}$ & $\mathrm{nq}$ & nq & & $1 \mathrm{nq}$ & nq & nq & nq \\
\hline $\mathrm{Cu}$ & $\%$ & 90 & 51 & $74_{1}$ & $\mathrm{nq}$ & & $3 \mathrm{nq}$ & 3 & $1011_{1}$ & nq \\
\hline $\mathbf{Z n}$ & $\%$ & 97 & 54 & 15 & & $4 \mathrm{nq}$ & 20 & 75 & $40_{1}$ & $\mathrm{nq}$ \\
\hline As & $\%$ & $71 \mathrm{n}$ & nq & $\mathrm{nq}$ & & $\mathrm{nq}$ & $\mathrm{nq}$ & $\mathrm{nq}$ & $\mathrm{nq}$ & nq \\
\hline Rb & $\%$ & $\mathrm{nq}$ & 107 & $94_{1}$ & $\mathrm{nq}$ & & $1 \mathrm{nq}$ & 56 & 521 & $\mathrm{nq}$ \\
\hline $\mathbf{S r}$ & $\%$ & 107 & 77 & 110 & 87 & & $3 \mathrm{nq}$ & 41 & 4 & 118 \\
\hline $\mathbf{Y}$ & $\%$ & $98 \mathrm{n}$ & $\mathrm{nq}$ & $\mathrm{nq}$ & & $\mathrm{nq}$ & $\mathrm{nq}$ & $\mathrm{nq}$ & 171 & $\mathrm{nq}$ \\
\hline $\mathbf{Z r}$ & $\%$ & 97 & 99 & 96 & 81 & & & $7 \mathrm{nq}$ & 54 & 94 \\
\hline $\mathbf{N b}$ & $\%$ & $\mathrm{nq}$ & 98 & 44 & & $7 \mathrm{nq}$ & $\mathrm{nq}$ & 88 & $\mathrm{nq}$ & $\mathrm{nq}$ \\
\hline Mo & $\%$ & 8 & 8 & 17 & $\mathrm{nq}$ & & 24 & 15 & & $\mathrm{nq}$ \\
\hline Cd & $\%$ & 102 & 56 & 42 & & $3 \mathrm{nq}$ & $\mathrm{nq}$ & $\mathrm{nq}$ & $\mathrm{nq}$ & $\mathrm{nq}$ \\
\hline Sn & $\%$ & nq & 67 & 451 & $\mathrm{nq}$ & $\mathrm{nq}$ & 70 & 53 & $\mathrm{nq}$ & nq \\
\hline $\mathbf{S b}$ & $\%$ & & $\mathrm{nq}$ & 92 & $\mathrm{nq}$ & $\mathrm{nq}$ & $\mathrm{nq}$ & $\mathrm{nq}$ & $100_{1}$ & $\mathrm{nq}$ \\
\hline Cs & $\%$ & $56 \mathrm{n}$ & $\mathrm{nq}$ & $\mathrm{nq}$ & $\mathrm{nq}$ & $\mathrm{nq}$ & $\mathrm{nq}$ & $\mathrm{nq}$ & & $\mathrm{nq}$ \\
\hline $\mathbf{B a}$ & $\%$ & 106 & 82 & 113 & 87 & & $1 \mathrm{nq}$ & 113 & 9 & 115 \\
\hline La & $\%$ & $\mathrm{nq}$ & nq & 40 & & $\mathrm{nq}$ & $\mathrm{nq}$ & $\mathrm{nq}$ & & $\mathrm{nq}$ \\
\hline $\mathrm{Ce}$ & $\%$ & nq & nq & 39 & & $6 \mathrm{nq}$ & $\mathrm{nq}$ & 16 & 64 & $\mathrm{nq}$ \\
\hline $\mathbf{P r}$ & $\%$ & nq & $\mathrm{nq}$ & $\mathrm{nq}$ & & $7 \mathrm{nq}$ & $\mathrm{nq}$ & $\mathrm{nq}$ & & $\mathrm{nq}$ \\
\hline Nd & $\%$ & $\mathrm{nq}$ & nq & $\mathrm{nq}$ & & $\mathrm{nq}$ & $\mathrm{nq}$ & 41 & & $\mathrm{nq}$ \\
\hline Sm & $\%$ & nq & $\mathrm{nq}$ & $\mathrm{nq}$ & 24 & & 62 & & $\mathrm{nq}$ & $\mathrm{nq}$ \\
\hline Eu & $\%$ & $\mathrm{nq}$ & $\mathrm{nq}$ & 45 & & $4 \mathrm{nq}$ & $\mathrm{nq}$ & $\mathrm{nq}$ & 391 & $\mathrm{nq}$ \\
\hline Gd & $\%$ & $\mathrm{nq}$ & $\mathrm{nq}$ & $\mathrm{nq}$ & & $\mathrm{nq}$ & $\mathrm{nq}$ & $\mathrm{nq}$ & $\mathrm{nq}$ & $\mathrm{nq}$ \\
\hline $\mathbf{T b}$ & $\%$ & $\mathrm{nq}$ & nq & 37 & & $\mathrm{nq}$ & $\mathrm{nq}$ & nq & 99 1 & $\mathrm{nq}$ \\
\hline Dy & $\%$ & $\mathrm{nq}$ & $\mathrm{nq}$ & 56 & & $3 \mathrm{nq}$ & $\mathrm{nq}$ & $\mathrm{nq}$ & $\mathrm{nq}$ & $\mathrm{nq}$ \\
\hline Ho & $\%$ & $\mathrm{nq}$ & $\mathrm{nq}$ & 75 & & $\mathrm{nq}$ & $\mathrm{nq}$ & $\mathrm{nq}$ & 108 & $\mathrm{nq}$ \\
\hline $\mathbf{E r}$ & $\%$ & $\mathrm{nq}$ & $\mathrm{nq}$ & $\mathrm{nq}$ & & $3 \mathrm{nq}$ & $\mathrm{nq}$ & $\mathrm{nq}$ & $\mathrm{nq}$ & $\mathrm{nq}$ \\
\hline Tm & $\%$ & $\mathrm{nq}$ & $\mathrm{nq}$ & $\mathrm{nq}$ & & $7 \mathrm{nq}$ & $\mathrm{nq}$ & $\mathrm{nq}$ & & $\mathrm{nq}$ \\
\hline Yb & $\%$ & $\mathrm{nq}$ & $\mathrm{nq}$ & $\mathrm{nq}$ & & $\mathrm{nq}$ & $\mathrm{nq}$ & $\mathrm{nq}$ & $\mathrm{nq}$ & nq \\
\hline Lu & $\%$ & $\mathrm{nq}$ & $\mathrm{nq}$ & $\mathrm{nq}$ & & $\mathrm{nq}$ & $\mathrm{nq}$ & nq & $\mathrm{nq}$ & nq \\
\hline Hf & $\%$ & $\mathrm{nq}$ & $\mathrm{nq}$ & 561 & $\mathrm{nq}$ & $\mathrm{nq}$ & $\mathrm{nq}$ & $\mathrm{nq}$ & $\mathrm{nq}$ & $\mathrm{nq}$ \\
\hline Ta & $\%$ & nq & 63 & 103 & $\mathrm{nq}$ & & $4 \mathrm{nq}$ & $\mathrm{nq}$ & & $\mathrm{nq}$ \\
\hline $\mathbf{W}$ & $\%$ & 67 & & $\mathrm{nq}$ & 23 & & $8 \mathrm{nq}$ & $\mathrm{nq}$ & & $\mathrm{nq}$ \\
\hline $\mathrm{Hg}$ & $\%$ & & $\mathrm{nq}$ & 105 & & & $0 \mathrm{nq}$ & & $\mathrm{nq}$ & $\mathrm{nq}$ \\
\hline Tl & $\%$ & $\mathrm{nq}$ & $\mathrm{nq}$ & $60_{1}$ & $\mathrm{nq}$ & & $7 \mathrm{nq}$ & $\mathrm{nq}$ & & $\mathrm{nq}$ \\
\hline $\mathbf{P b}$ & $\%$ & $\mathrm{nq}$ & nq & 54 & $\mathrm{nq}$ & & $0 \mathrm{nq}$ & 65 & 100 & $\mathrm{nq}$ \\
\hline $\mathbf{B i}$ & $\%$ & $\mathrm{nq}$ & nq & $\mathrm{nq}$ & nq & $\mathrm{nq}$ & $\mathrm{nq}$ & $\mathrm{nq}$ & 119 & $\mathrm{nq}$ \\
\hline Th & $\%$ & $\mathrm{nq}$ & $\mathrm{nq}$ & & $\mathrm{nq}$ & $\mathrm{nq}$ & $\mathrm{nq}$ & $\mathrm{nq}$ & $799_{1}$ & $\mathrm{nq}$ \\
\hline $\mathbf{U}$ & $\%$ & nq & 4 & & $\mathrm{nq}$ & & 16 & & $\mathrm{nq}$ & $\mathrm{nq}$ \\
\hline
\end{tabular}


Tab. A.8.: Ergebnisse des Anionenaustausches für Porenwässer und Wasserproben aus der Lausitz; Konzentrationen in Lösungen nach dem Anionenaustausch in \% bezogen auf die Ausgangskonzentrationen (Tab. A.2.) von Porenlösungen und Wasserproben aus der Lausitz; AI = Austauschion.

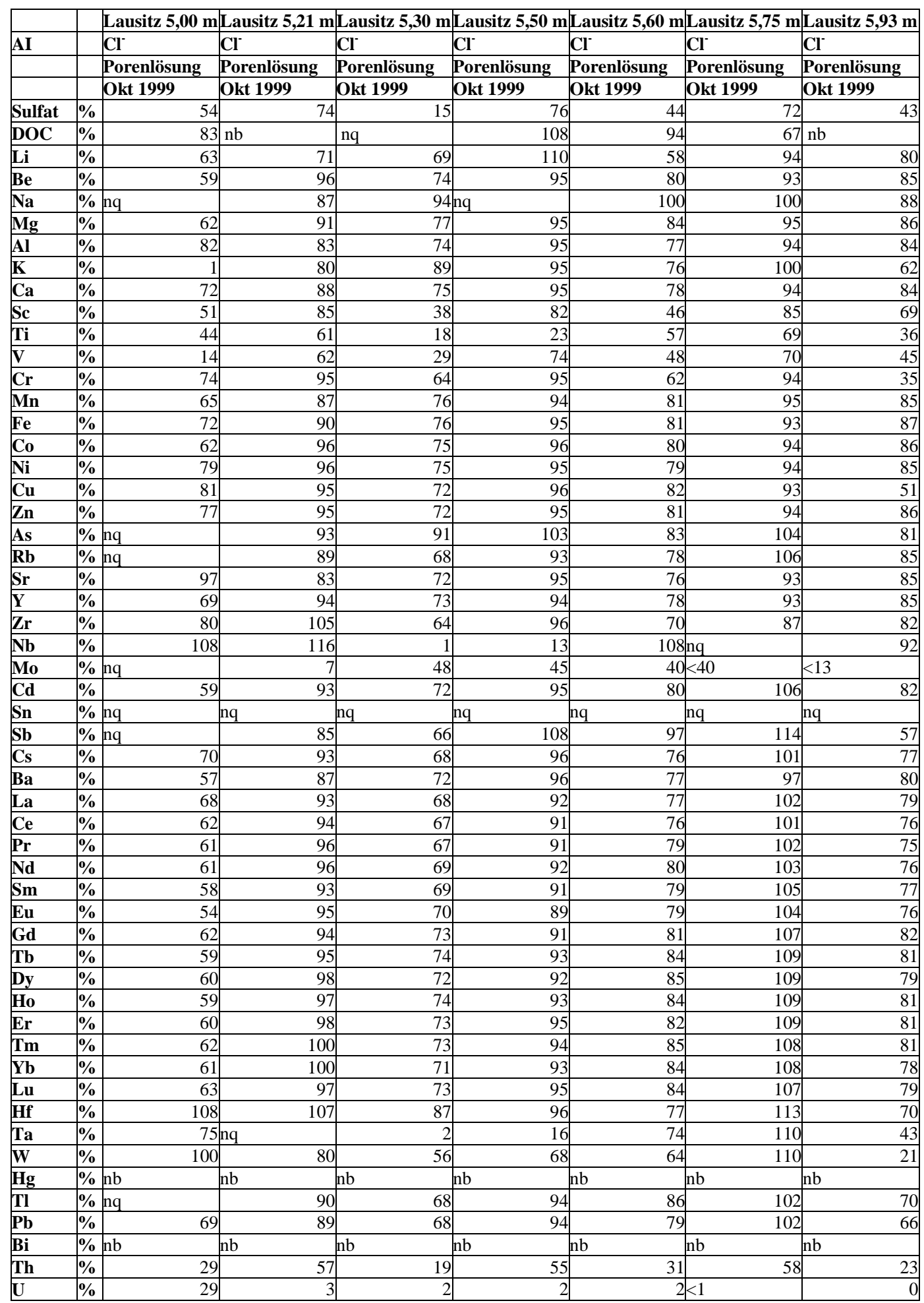


Tab. A.8. (Forts.): Ergebnisse des Anionenaustausches für Porenwässer und Wasserproben aus der Lausitz; Konzentrationen in Lösungen nach dem Anionenaustausch in \% bezogen auf die Ausgangskonzentrationen (Tab. A.2.) von Porenlösungen und Wasserproben aus der Lausitz; AI = Austauschion.

\begin{tabular}{|c|c|c|c|c|c|c|c|}
\hline & & Lausitz 21,07 & mLausitz 21,13 & Lausitz 21,40 m & Lausitz 21,70 m & \begin{tabular}{|l|} 
Lausitz 21,80 \\
\end{tabular} & Lausitz $21,90 \mathrm{~m}$ \\
\hline \multirow[t]{3}{*}{ AI } & & $\mathrm{Cl}^{-}$ & $\mathrm{Cl}^{-}$ & $\mathrm{Cl}^{-}$ & $\mathrm{Cl}^{-}$ & $\mathrm{Cl}^{-}$ & $\mathrm{Cl}^{-}$ \\
\hline & & Wasserprobe & Wasserprobe & Wasserprobe & Wasserprobe & Wasserprobe & Wasserprobe \\
\hline & & Okt 1999 & Okt 1999 & Okt 1999 & Okt 1999 & Okt 1999 & Okt 1999 \\
\hline Sulfat & $\%$ & & 22 & $1 \mathrm{nb}$ & 13 & 24 & 40 \\
\hline DOC & $\%$ & $\mathrm{nq}$ & $\mathrm{nq}$ & 67 & 96 & & $6 n b$ \\
\hline $\mathbf{L i}$ & $\%$ & & 73 & $2 \mathrm{nb}$ & 66 & 73 & 101 \\
\hline $\mathbf{B e}$ & $\%$ & & 50 & $4 n b$ & 50 & 48 & 86 \\
\hline $\mathbf{N a}$ & $\%$ & $\mathrm{nq}$ & & $3 n b$ & 85 & 87 & 106 \\
\hline Mg & $\%$ & & 30 & nb & 72 & 80 & 90 \\
\hline Al & $\%$ & & 22 & $7 \mathrm{nb}$ & 26 & 31 & 87 \\
\hline $\mathbf{K}$ & $\%$ & & $39 \mathrm{nq}$ & nb & 5 & 89 & 85 \\
\hline Ca & $\%$ & & 79 & $6 \mathrm{nb}$ & 71 & 78 & 89 \\
\hline Sc & $\%$ & nq & nq & $<0.1$ & & $\mathrm{nq}$ & 85 \\
\hline $\mathbf{T i}$ & $\%$ & $\mathrm{nq}$ & $\mathrm{nq}$ & $<1$ & 0 & & $0 \mathrm{nq}$ \\
\hline V & $\%$ & & $0 \mathrm{nq}$ & $<0.1$ & 0 & & $0 \mathrm{nq}$ \\
\hline $\mathrm{Cr}$ & $\%$ & & $9 \mathrm{nq}$ & nb & $\mathrm{nq}$ & 70 & $0 \mathrm{nq}$ \\
\hline Mn & $\%$ & & 79 & nb & 72 & 76 & 89 \\
\hline $\mathbf{F e}$ & $\%$ & & 79 & nb & 70 & 76 & 81 \\
\hline Co & $\%$ & & 71 & $3 \mathrm{nb}$ & 72 & 77 & 92 \\
\hline $\mathrm{Ni}$ & $\%$ & & 75 & $4 \mathrm{nb}$ & 71 & 76 & 93 \\
\hline $\mathrm{Cu}$ & $\%$ & & 0 & $\mathrm{nb}$ & $\mathrm{nq}$ & nq & $\mathrm{ng}$ \\
\hline $\mathbf{Z n}$ & $\%$ & & 75 & $2 n b$ & 64 & 73 & 83 \\
\hline As & $\%$ & & 55 & 115 & 52 & 70 & $\mathrm{nq}$ \\
\hline $\mathbf{R b}$ & $\%$ & & $0 \mathrm{nq}$ & 104 & 58 & 56 & 99 \\
\hline $\mathbf{S r}$ & $\%$ & & 79 & $9 \mathrm{nb}$ & 71 & 76 & 92 \\
\hline $\mathbf{Y}$ & $\%$ & & 55 & $2 n b$ & 59 & 60 & 85 \\
\hline $\mathbf{Z r}$ & $\%$ & & 56 & nb & 63 & 73 & 95 \\
\hline $\mathbf{N b}$ & $\%$ & & $0 \mathrm{nq}$ & 26 & 49 & 51 & 11 \\
\hline Mo & $\%$ & & 77 & $8 \mathrm{nb}$ & $\mathrm{nq}$ & 68 & $8 \mathrm{nq}$ \\
\hline Cd & $\%$ & & 1 & 85 & 91 & 66 & 88 \\
\hline Sn & $\%$ & $\mathrm{nq}$ & $\mathrm{nq}$ & $\mathrm{nq}$ & $\mathrm{nq}$ & $\mathrm{nq}$ & $\mathrm{nq}$ \\
\hline $\mathbf{S b}$ & $\%$ & nq & 1 & 63 & $\mathrm{nq}$ & $\mathrm{nq}$ & 65 \\
\hline Cs & $\%$ & & $0 \mathrm{nq}$ & 66 & 92 & 76 & 91 \\
\hline $\mathbf{B a}$ & $\%$ & & $76 \mathrm{nq}$ & nb & 71 & 78 & 97 \\
\hline La & $\%$ & & $53 \mathrm{nq}$ & nb & 63 & 65 & 91 \\
\hline $\mathrm{Ce}$ & $\%$ & & $0 \mathrm{nq}$ & 63 & 68 & 47 & 91 \\
\hline $\mathbf{P r}$ & $\%$ & & $0 \mathrm{nq}$ & 60 & 64 & 45 & 90 \\
\hline Nd & $\%$ & & $0 \mathrm{nq}$ & 57 & 65 & 45 & 90 \\
\hline Sm & $\%$ & & $0 \mathrm{nq}$ & 50 & 61 & 51 & 90 \\
\hline Eu & $\%$ & & $0 \mathrm{nq}$ & 45 & 67 & 56 & 89 \\
\hline Gd & $\%$ & & $0 \mathrm{nq}$ & 52 & 71 & 48 & 94 \\
\hline $\mathbf{T b}$ & $\%$ & & $0 \mathrm{nq}$ & 55 & 70 & 52 & 96 \\
\hline Dy & $\%$ & & $0 \mathrm{nq}$ & 50 & 69 & 49 & 94 \\
\hline Ho & $\%$ & & $0 \mathrm{nq}$ & 50 & 74 & 50 & 93 \\
\hline $\mathbf{E r}$ & $\%$ & & $0 \mathrm{nq}$ & 54 & 74 & 51 & 93 \\
\hline Tm & $\%$ & & $0 \mathrm{nq}$ & 53 & 87 & 57 & 94 \\
\hline $\mathbf{Y b}$ & $\%$ & & $0 \mathrm{nq}$ & 52 & 70 & 48 & 95 \\
\hline Lu & $\%$ & & $0 \mathrm{nq}$ & 56 & 64 & 69 & 96 \\
\hline Hf & $\%$ & $\mathrm{nq}$ & $\mathrm{nq}$ & $\mathrm{nq}$ & $\mathrm{nq}$ & $\mathrm{nq}$ & $\mathrm{nq}$ \\
\hline Ta & $\%$ & $\mathrm{nq}$ & $\mathrm{nq}$ & 381 & $n$ & $\mathrm{nq}$ & 8 \\
\hline $\mathbf{W}$ & $\%$ & & $2 \mathrm{nq}$ & $\mathrm{nq}$ & $\mathrm{nq}$ & nq & 84 \\
\hline $\mathrm{Hg}$ & $\%$ & $\mathrm{nq}$ & & $2 \mathrm{nq}$ & $\mathrm{nq}$ & $\mathrm{nq}$ & $\mathrm{nb}$ \\
\hline TI & $\%$ & & $0 \mathrm{nq}$ & 56 & 103 & 86 & 88 \\
\hline $\mathbf{P b}$ & $\%$ & & $0 \mathrm{nq}$ & 26 & 10 & 52 & 72 \\
\hline $\mathbf{B i}$ & $\%$ & $\mathrm{nq}$ & $\mathrm{nq}$ & $\mathrm{nq}$ & $\mathrm{nq}$ & $\mathrm{nq}$ & nb \\
\hline Th & $\%$ & nq & $\mathrm{nq}$ & & $\mathrm{nq}$ & 12 & 42 \\
\hline $\mathbf{U}$ & $\%$ & & $0 \mathrm{nq}$ & 1 & 2 & 4 & 3 \\
\hline
\end{tabular}


Tab. A.9.: Ultrafiltration der Referenzlösung und der Referenzlösung mit organischen Zusatzstoffen; je $30 \mathrm{~g} / \mathrm{l}$ organischer Zusatz; angegeben sind die Anteile der gelösten Elemente, die im Durchlauf wiedergefunden wurden.

\begin{tabular}{|c|c|c|c|c|c|c|}
\hline & Testlösung $[\mu \mathrm{g} / \mathrm{I}] \mathrm{R}$ & Referenzlösung & Alanin & Threonin & Harnstoff & Cystein \\
\hline $\mathbf{L i}$ & 100 & 97 & 99 & 97 & 93 & 98 \\
\hline $\mathbf{B e}$ & 100 & 98 & 100 & 101 & 94 & 100 \\
\hline $\mathrm{Na}$ & 10000 & 100 & 103 & 96 & 94 & 99 \\
\hline Mg & 5000 & 97 & 103 & 102 & 92 & 100 \\
\hline Al & 2000 & 99 & 101 & 101 & 95 & 99 \\
\hline $\mathbf{K}$ & 1000 & 98 & 104 & 99 & 99 & 103 \\
\hline $\mathbf{C a}$ & 5000 & 101 & 103 & 102 & 95 & 100 \\
\hline Sc & 100 & 100 & 100 & 100 & 94 & 98 \\
\hline $\mathbf{T i}$ & 100 & 100 & 92 & 100 & 96 & 98 \\
\hline $\mathbf{V}$ & 100 & 101 & 94 & 97 & 96 & 101 \\
\hline $\mathbf{C r}$ & 100 & 98 & 102 & 97 & 93 & 102 \\
\hline Mn & 200 & 100 & 101 & 97 & 94 & 100 \\
\hline $\mathbf{F e}$ & 2000 & 115 & 99 & 100 & 96 & 109 \\
\hline Co & 100 & 98 & 100 & 100 & 94 & 100 \\
\hline $\mathrm{Ni}$ & 200 & 99 & 101 & 76 & 93 & 100 \\
\hline $\mathbf{C u}$ & 100 & 102 & 102 & 96 & 99 & 39 \\
\hline $\mathbf{Z n}$ & 1000 & 98 & 104 & 102 & 96 & 101 \\
\hline As & 100 & 86 & 66 & 83 & 79 & 97 \\
\hline $\mathbf{R b}$ & $100 \mathrm{n}$ & & 102 & 116 & 60 & 89 \\
\hline $\mathbf{S r}$ & 500 & 98 & 100 & 101 & 94 & 99 \\
\hline $\mathbf{Y}$ & 20 & 98 & 102 & 105 & 95 & 100 \\
\hline $\mathbf{Z r}$ & 20 & 93 & 71 & 98 & 103 & 82 \\
\hline $\mathbf{N b}$ & 20 & 80 & 76 & 96 & 62 & 72 \\
\hline Mo & 100 & 93 & 76 & 80 & 95 & 87 \\
\hline Sn & 20 & 82 & 79 & 255 & 61 & 62 \\
\hline Sb & 20 & 96 & 96 & 94 & 61 & 58 \\
\hline Cs & $100 \mathrm{n}$ & & $\mathrm{nq}$ & 105 & 59 & 90 \\
\hline $\mathbf{B a}$ & 100 & 102 & 101 & 101 & 94 & 99 \\
\hline La & 100 & 101 & 102 & 102 & 94 & 100 \\
\hline Ce & 100 & 97 & 104 & 112 & 64 & 93 \\
\hline $\mathbf{P r}$ & 20 & 95 & 105 & 111 & 64 & 92 \\
\hline Nd & 100 & 95 & 106 & 114 & 64 & 91 \\
\hline Sm & 20 & 97 & 103 & 113 & 64 & 90 \\
\hline Eu & 20 & 104 & 102 & 100 & 94 & 99 \\
\hline Gd & 20 & 107 & 104 & 112 & 61 & 91 \\
\hline $\mathbf{T b}$ & 20 & 102 & 101 & 113 & 61 & 90 \\
\hline Dy & 20 & 98 & 104 & 113 & 62 & 92 \\
\hline Ho & 20 & 93 & 101 & 116 & 62 & 89 \\
\hline $\mathbf{E r}$ & 20 & 93 & 104 & 115 & 62 & 90 \\
\hline $\mathbf{T m}$ & 20 & 87 & 99 & 119 & 62 & 88 \\
\hline $\mathbf{Y b}$ & 20 & 97 & 102 & 114 & 60 & 91 \\
\hline $\mathbf{L u}$ & 20 & 97 & 103 & 104 & 94 & 100 \\
\hline Hf & 20 & 86 & 56 & 109 & 68 & 66 \\
\hline $\mathbf{T a}$ & 20 & 73 & 102 & 45 & 40 & 96 \\
\hline W & 20 & 65 & 68 & 86 & 49 & 61 \\
\hline Tl & 20 & 23 & 44 & 107 & 21 & 94 \\
\hline $\mathbf{P b}$ & 100 & 103 & 98 & 100 & 92 & 82 \\
\hline $\mathbf{B i}$ & 20 & 80 & $\begin{array}{l}97 \\
\end{array}$ & 107 & 56 & 32 \\
\hline Th & 20 & 100 & 91 & $114 \mathrm{n}$ & & 87 \\
\hline $\mathbf{U}$ & 20 & 100 & 99 & 113 & 49 & 95 \\
\hline
\end{tabular}


Tab. A.10.: Kationenaustausch der Referenzlösung und der Referenzlösung mit organischen Zusatzstoffen; je 30 g/l organischer Zusatz; angegeben sind die Anteile der gelösten Elemente in \%, die im Durchlauf wiedergefunden wurden.

\begin{tabular}{|c|c|c|c|c|c|c|}
\hline & Testlösung $[\mu \mathrm{g} / \mathrm{l}]$ & Referenzlösung & Alanin & Threonin & Harnstoff & Cystein \\
\hline $\mathbf{L i}$ & 100 & 22 & 22 & 13 & 18 & 17 \\
\hline $\mathbf{B e}$ & 100 & 3 & 9 & 5 & 3 & 5 \\
\hline $\mathbf{N a}$ & 10000 & 31 & 18 & 6 & 12 & 30 \\
\hline $\mathbf{M g}$ & 5000 & 0 & 0 & 0 & 0 & 0 \\
\hline Al & 2000 & 0 & 18 & 9 & 0 & 10 \\
\hline $\mathbf{K}$ & 1000 & $\mathrm{nq}$ & 72 & $\mathrm{nq}$ & $\mathrm{nq}$ & $\mathrm{nq}$ \\
\hline Ca & 5000 & 0 & 0 & 3 & 0 & 0 \\
\hline Sc & 100 & 7 & 7 & 8 & 7 & 7 \\
\hline Ti & 100 & 0 & 0 & 1 & 1 & 1 \\
\hline $\mathbf{V}$ & 100 & 15 & 31 & 18 & 14 & 10 \\
\hline $\mathbf{C r}$ & 100 & 3 & 0 & 4 & 3 & 0 \\
\hline Mn & 200 & 5 & 5 & 6 & 4 & 5 \\
\hline $\mathbf{F e}$ & 2000 & 0 & 1 & 1 & 0 & 1 \\
\hline Co & 100 & 5 & 0 & 6 & 5 & 5 \\
\hline $\mathrm{Ni}$ & 200 & 3 & 4 & 3 & 3 & 4 \\
\hline $\mathbf{C u}$ & 100 & 10 & 28 & 10 & 9 & 9 \\
\hline $\mathbf{Z n}$ & 1000 & 0 & 1 & 0 & 0 & 0 \\
\hline As & 100 & 88 & 84 & 79 & 80 & 29 \\
\hline $\mathbf{R b}$ & 100 & 12 & 6 & 7 & 1 & 6 \\
\hline $\mathbf{S r}$ & 500 & 0 & 0 & 0 & 0 & 0 \\
\hline $\mathbf{Y}$ & 20 & 0 & 0 & 0 & 0 & 0 \\
\hline $\mathbf{Z r}$ & 20 & 0 & 17 & 0 & 0 & 0 \\
\hline $\mathbf{N b}$ & 20 & 8 & 54 & 6 & 2 & 14 \\
\hline Mo & 100 & 53 & 77 & 107 & 44 & 47 \\
\hline Sn & 20 & 3 & 13 & 2 & 5 & 9 \\
\hline $\mathbf{S b}$ & 20 & 84 & 94 & 99 & 4 & 2 \\
\hline Cs & 100 & 7 & 3 & 0 & 1 & 0 \\
\hline $\mathbf{B a}$ & 100 & 0 & 0 & 0 & 0 & 0 \\
\hline $\mathbf{L a}$ & 100 & 0 & 0 & 0 & 0 & 0 \\
\hline $\mathrm{Ce}$ & 100 & 0 & 1 & 0 & 0 & 0 \\
\hline $\mathbf{P r}$ & 20 & 0 & 1 & 0 & 0 & 0 \\
\hline Nd & 100 & 0 & 1 & 0 & 0 & 0 \\
\hline Sm & 20 & 0 & 1 & 1 & 1 & 1 \\
\hline $\mathbf{E u}$ & 20 & 0 & 1 & 0 & 0 & 0 \\
\hline Gd & 20 & 0 & 1 & 1 & 0 & 0 \\
\hline Tb & 20 & 0 & 1 & 0 & 0 & 0 \\
\hline Dy & 20 & 0 & 1 & 0 & 0 & 0 \\
\hline Ho & 20 & 0 & 1 & 0 & 0 & 0 \\
\hline $\mathbf{E r}$ & 20 & 0 & 1 & 0 & 0 & 0 \\
\hline Tm & 20 & 0 & 1 & 0 & 0 & 0 \\
\hline $\mathbf{Y b}$ & 20 & 0 & 1 & 0 & 0 & 0 \\
\hline $\mathbf{L u}$ & 20 & 0 & 0 & 0 & 0 & 0 \\
\hline Hf & 20 & 2 & $\begin{array}{ll}19 \\
\end{array}$ & 2 & 1 & 6 \\
\hline $\mathbf{T a}$ & 20 & 12 & 18 & 16 & 16 & 20 \\
\hline $\mathbf{W}$ & 20 & 55 & $\begin{array}{l}59 \\
\end{array}$ & 34 & 7 & 14 \\
\hline TI & 20 & 70 & 1 & 0 & 9 & 9 \\
\hline $\mathbf{P b}$ & 100 & 0 & 0 & 0 & 0 & 0 \\
\hline $\mathbf{B i}$ & 20 & 2 & 3 & 1 & 1 & 1 \\
\hline Th & 20 & 1 & 3 & 1 & 0 & 0 \\
\hline $\mathbf{U}$ & 20 & 1 & 2 & 0 & 0 & 0 \\
\hline
\end{tabular}


Tab. A.11.: Anionenaustausch der Referenzlösung und der Referenzlösung mit organischen Zusatzstoffen gegen $\mathrm{OH}^{-}$; je $30 \mathrm{~g} / \mathrm{l}$ organischer Zusatz; angegeben sind die Anteile der gelösten Elemente in \%, die im Durchlauf wiedergefunden wurden.

\begin{tabular}{|c|c|c|c|c|c|c|}
\hline & Testlösung $[\mu \mathrm{g} / \mathrm{l}]$ & Referenzlösung & Alanin & Threonin & Harnstoff & Cystein \\
\hline $\mathbf{L i}$ & 100 & 91 & 77 & 84 & 88 & 93 \\
\hline $\mathbf{B e}$ & 100 & 74 & 10 & 70 & 84 & 45 \\
\hline $\mathbf{N a}$ & 10000 & $\mathrm{nq}$ & 90 & 101 & 102 & 101 \\
\hline $\mathrm{Mg}$ & 5000 & $\mathrm{nq}$ & 78 & 86 & 92 & 64 \\
\hline Al & 2000 & 97 & 7 & 51 & 40 & 43 \\
\hline $\mathbf{K}$ & 1000 & $\mathrm{nq}$ & $\mathrm{nq}$ & $\mathrm{nq}$ & $\mathrm{nq}$ & $\mathrm{nq}$ \\
\hline $\mathbf{C a}$ & 5000 & 92 & 93 & 86 & 91 & 96 \\
\hline Sc & 100 & 86 & 14 & 57 & 44 & 51 \\
\hline $\mathbf{T i}$ & 100 & 82 & 3 & 30 & 17 & 42 \\
\hline $\mathbf{V}$ & 100 & 72 & 9 & 37 & 36 & 31 \\
\hline $\mathbf{C r}$ & 100 & 58 & 81 & 73 & 77 & 50 \\
\hline Mn & 200 & $\mathrm{nq}$ & 58 & 90 & $\mathrm{nq}$ & 61 \\
\hline Fe & 2000 & $\mathrm{nq}$ & 5 & 44 & 45 & 44 \\
\hline Co & 100 & 93 & 23 & 58 & 30 & 45 \\
\hline $\mathrm{Ni}$ & 200 & 46 & 12 & 53 & 37 & 48 \\
\hline \begin{tabular}{|l|}
$\mathbf{C u}$ \\
\end{tabular} & 100 & 95 & 86 & 81 & 94 & 51 \\
\hline $\mathbf{Z n}$ & 1000 & 81 & 25 & 61 & 42 & 44 \\
\hline As & 100 & 77 & 15 & 44 & 89 & 18 \\
\hline $\mathbf{R b}$ & 100 & 105 & 100 & 206 & 70 & 27 \\
\hline $\mathbf{S r}$ & 500 & 93 & 95 & 89 & 93 & 80 \\
\hline $\mathbf{Y}$ & 20 & 95 & 35 & 80 & 84 & 63 \\
\hline $\mathbf{Z r}$ & 20 & 72 & 60 & 57 & 49 & 46 \\
\hline $\mathbf{N b}$ & 20 & 43 & 6 & 19 & 11 & 9 \\
\hline Mo & 100 & 38 & 32 & 29 & 49 & 28 \\
\hline Sn & 20 & 75 & 7 & 28 & 25 & 9 \\
\hline Sb & 20 & 68 & 4 & 39 & 46 & 6 \\
\hline Cs & 100 & 94 & 161 & 77 & 71 & 81 \\
\hline $\mathbf{B a}$ & 100 & $\mathrm{nq}$ & $\mathrm{nq}$ & $\mathrm{nq}$ & $\mathrm{nq}$ & $\mathrm{nq}$ \\
\hline La & 100 & 96 & 31 & 79 & 78 & 55 \\
\hline Ce & 100 & 93 & 22 & 68 & 60 & 16 \\
\hline Pr & 20 & 96 & 20 & 68 & 60 & 45 \\
\hline Nd & 100 & 96 & 19 & 69 & 59 & 44 \\
\hline Sm & 20 & 95 & 15 & 67 & 58 & 46 \\
\hline Eu & 20 & 90 & 15 & 74 & 74 & 46 \\
\hline Gd & 20 & 98 & 15 & 68 & 59 & 42 \\
\hline Tb & 20 & 96 & 13 & 68 & 60 & 50 \\
\hline Dy & 20 & 99 & 13 & 67 & 58 & 42 \\
\hline Ho & 20 & 100 & 13 & 67 & 59 & 49 \\
\hline Er & 20 & 99 & 13 & 66 & 58 & 47 \\
\hline Tm & 20 & 98 & 12 & 67 & 59 & 61 \\
\hline $\mathbf{Y b}$ & 20 & 94 & 12 & 66 & 57 & 53 \\
\hline $\mathbf{L u}$ & 20 & 87 & 12 & 72 & 72 & 47 \\
\hline Hf & 20 & 64 & 7 & 26 & 6 & 22 \\
\hline \begin{tabular}{|l|} 
Ta \\
\end{tabular} & 20 & 99 & 9 & 62 & 56 & 41 \\
\hline $\mathbf{W}$ & 20 & 5 & 3 & 4 & 3 & 3 \\
\hline TI & 20 & 44 & 88 & 76 & 63 & 101 \\
\hline $\mathbf{P b}$ & 100 & 85 & 0 & 76 & 60 & 38 \\
\hline $\mathbf{B i}$ & 20 & 33 & 7 & 25 & 499 & 11 \\
\hline \begin{tabular}{|l|} 
Th \\
\end{tabular} & 20 & 85 & 15 & 48 & 37 & 113 \\
\hline $\mathbf{U}$ & 20 & 75 & 8 & 37 & 27 & 45 \\
\hline
\end{tabular}


Tab. A.12.: Anionenaustausch der Referenzlösung und der Referenzlösung mit organischen Zusatzstoffen gegen $\mathrm{Cl}$; je $30 \mathrm{~g} / \mathrm{l}$ organischer Zusatz; angegeben sind die Anteile der gelösten Elemente in \%, die im Durchlauf wiedergefunden wurden.

\begin{tabular}{|c|c|c|c|c|c|c|}
\hline & Testlösung $[\mu \mathrm{g} / \mathrm{l}]$ & Referenzlösung & Alanin & Threonin & Harnstoff & Cystein \\
\hline $\mathbf{L i}$ & 100 & 85 & 86 & 88 & 81 & 79 \\
\hline $\mathbf{B e}$ & 100 & 85 & 84 & 88 & 95 & 78 \\
\hline $\mathbf{N a}$ & 10000 & 95 & 87 & 90 & $\mathrm{nq}$ & $\mathrm{nq}$ \\
\hline Mg & 5000 & 86 & 87 & 89 & 83 & 80 \\
\hline Al & 2000 & 86 & 86 & 90 & 80 & 78 \\
\hline $\mathbf{K}$ & 1000 & 100 & 97 & 94 & 99 & 89 \\
\hline $\mathbf{C a}$ & 5000 & $\mathrm{nq}$ & $\mathrm{nq}$ & $\mathrm{nq}$ & 113 & $\mathrm{nq}$ \\
\hline Sc & 100 & 86 & 85 & 90 & 83 & 79 \\
\hline Ti & 100 & 86 & 82 & 89 & 79 & 75 \\
\hline $\mathbf{V}$ & 100 & 81 & 29 & 65 & 74 & 35 \\
\hline $\mathbf{C r}$ & 100 & 81 & 28 & 56 & 57 & 77 \\
\hline Mn & 200 & 86 & 86 & 90 & 78 & 80 \\
\hline Fe & 2000 & 87 & $\mathrm{nq}$ & 90 & $\mathrm{nq}$ & 80 \\
\hline Co & 100 & 84 & 63 & 84 & 78 & 80 \\
\hline $\mathbf{N i}$ & 200 & 88 & 89 & 90 & 85 & 82 \\
\hline $\mathbf{C u}$ & 100 & 88 & 88 & 91 & 83 & 79 \\
\hline $\mathbf{Z n}$ & 1000 & 89 & 90 & 92 & 83 & $\mathrm{nq}$ \\
\hline As & 100 & 86 & 86 & 88 & 82 & 80 \\
\hline $\mathbf{R b}$ & 100 & 87 & 24 & 71 & 11 & 111 \\
\hline $\mathbf{S r}$ & 500 & 108 & 103 & 107 & 30 & 58 \\
\hline $\mathbf{Y}$ & 20 & 86 & 86 & 89 & 82 & 78 \\
\hline $\mathbf{Z r}$ & 20 & 89 & 87 & 93 & 85 & 81 \\
\hline $\mathbf{N b}$ & 20 & 67 & 64 & 57 & 45 & 49 \\
\hline Mo & 100 & 11 & 32 & 30 & 7 & 18 \\
\hline Sn & 20 & 32 & 26 & 28 & 31 & 65 \\
\hline Sb & 20 & 68 & 8 & 73 & 16 & 52 \\
\hline Cs & 100 & 60 & 12 & 76 & 3 & 90 \\
\hline $\mathbf{B a}$ & 100 & 83 & 97 & 102 & 89 & 62 \\
\hline La & 100 & 87 & 96 & 89 & 96 & 78 \\
\hline $\mathbf{C e}$ & 100 & 86 & 80 & 92 & 77 & 74 \\
\hline Pr & 20 & 82 & 93 & 103 & 34 & 58 \\
\hline Nd & 100 & 82 & 92 & 105 & 94 & 58 \\
\hline Sm & 20 & 85 & 93 & 109 & 94 & 57 \\
\hline Eu & 20 & 85 & 92 & 110 & 97 & 55 \\
\hline Gd & 20 & 84 & 91 & 91 & 77 & 73 \\
\hline Tb & 20 & 87 & 94 & 108 & 89 & 57 \\
\hline Dy & 20 & 85 & 88 & 106 & 108 & 60 \\
\hline Ho & 20 & 87 & 92 & 107 & 92 & 56 \\
\hline $\mathbf{E r}$ & 20 & 89 & 89 & 108 & 107 & 59 \\
\hline Tm & 20 & 83 & 91 & 109 & 103 & 58 \\
\hline $\mathbf{Y b}$ & 20 & 88 & 83 & 111 & $\mathrm{nq}$ & 62 \\
\hline $\mathbf{L u}$ & 20 & 89 & 89 & 109 & 106 & 57 \\
\hline Hf & 20 & 85 & 82 & 90 & 78 & 73 \\
\hline Ta & 20 & 63 & 36 & 27 & 35 & 7 \\
\hline $\mathbf{W}$ & 20 & 37 & 62 & 90 & 62 & 67 \\
\hline TI & 20 & 2 & 4 & 5 & 4 & 3 \\
\hline $\mathbf{P b}$ & 100 & 27 & 90 & 101 & 118 & 58 \\
\hline$\overline{\mathrm{Bi}}$ & 20 & 86 & 80 & 90 & 77 & $\mathrm{nq}$ \\
\hline Th & 20 & 2 & 22 & 56 & 3 & $\mathrm{nq}$ \\
\hline $\mathbf{U}$ & 20 & 77 & 52 & 106 & 149 & 33 \\
\hline
\end{tabular}


Tabelle A.13.: Hauptelementgehalte der Festphasen im Bohrkern des Grundwasserpegels RCO4.

\begin{tabular}{|c|c|c|c|c|c|c|c|}
\hline Tiefe [m] & $\%$ Carbonat-C & $\% \mathrm{CaO}$ & $\% \mathrm{MgO}$ & $\%$ Pyrit & $\%$ Sulfat-S & $\mathrm{SiO}_{2}$ & $\mathrm{TiO}_{2}$ \\
\hline 1 & 0,003 & 0,23 & 0,39 & 0 & 0,023 & 87,2 & 0,40 \\
\hline 2 & 0,002 & 0,20 & 0,37 & 0,07 & 0,023 & 86,5 & 0,45 \\
\hline 3 & 0,005 & 0,14 & 0,08 & 0,16 & 0,063 & 92,5 & 0,25 \\
\hline 5 & 0,009 & 0,43 & 0,11 & 1,0 & 0,26 & 81,8 & 0,35 \\
\hline 7 & 0,001 & 0,23 & 0,06 & 1,8 & 0,14 & 88,3 & 0,29 \\
\hline 8 & 0,021 & 0,28 & 0,18 & 0,35 & 0,085 & 84,0 & 0,56 \\
\hline 9 & 0,004 & 0,10 & 0,06 & 0,17 & 0,047 & 93,6 & 0,30 \\
\hline 10 & 0,006 & 0,11 & 0,07 & 0,22 & 0,053 & 93,1 & 0,32 \\
\hline 11 & 0,12 & 0,60 & 0,18 & 0,72 & 0,31 & 78,3 & 0,35 \\
\hline 13 & 0,004 & 0,20 & 0,07 & 0,62 & 0,12 & 90,9 & 0,24 \\
\hline 14 & 0,008 & 0,30 & 0,09 & 0,64 & 0,17 & 86,0 & 0,38 \\
\hline 18 & 0,18 & 1,1 & 0,25 & 0,11 & 0,18 & 82,1 & 0,41 \\
\hline 19 & 0,12 & 0,36 & 0,21 & 0,26 & 0,13 & 86,1 & 0,93 \\
\hline 22 & 0,14 & 1,2 & 0,30 & 0,94 & 0,43 & 69,5 & 0,69 \\
\hline 24 & 0,04 & 0,41 & 0,27 & 0,06 & 0,14 & 80,8 & 0,70 \\
\hline 26 & 0,02 & 0,88 & 0,73 & 0,51 & 0,59 & 77,1 & 0,71 \\
\hline 28 & 0,45 & 3,1 & 1,6 & 0,06 & 0,52 & 76,3 & 0,43 \\
\hline 30 & 0,003 & 0,34 & 0,56 & 2,2 & 0,30 & 82,7 & 0,52 \\
\hline 32 & 0,045 & 0,65 & 0,66 & 3,4 & 0,40 & 82,2 & 0,47 \\
\hline 34 & 0,051 & 0,70 & 0,65 & 3,6 & 0,38 & 80,8 & 0,47 \\
\hline 38 & 0,048 & 0,75 & 0,7 & 3,3 & 0,35 & 80,9 & 0,52 \\
\hline 42 & 0,46 & 2,1 & 1,2 & 0,71 & 0,23 & 79,8 & 0,45 \\
\hline 46 & 0,35 & 1,7 & 1,0 & 1,8 & 0,44 & 78,2 & 0,50 \\
\hline 56 & 0,64 & 2,9 & 1,4 & 1,1 & 0,31 & 77,0 & 0,45 \\
\hline 58 & 0,60 & 2,2 & 0,61 & 0,75 & 0,23 & 51,1 & 0,27 \\
\hline
\end{tabular}


Tab. A.13. (Forts.): Hauptelementgehalte der Festphasen im Bohrkern des Grundwasserpegels RCO4.

\begin{tabular}{|c|c|c|c|c|c|c|}
\hline Tiefe [m] & $\mathrm{Al}_{2} \mathrm{O}_{3}$ & $\mathrm{Fe}_{2} \mathrm{O}_{3}$ & MnO & $\mathrm{Na}_{2} \mathrm{O}$ & $\mathrm{K}_{2} \mathbf{O}_{3}$ & \\
\hline 1 & 5,6 & 1,3 & 0,022 & 0,58 & 1,5 & 0,06 \\
\hline 2 & 5,7 & 1,2 & 0,017 & 0,49 & 1,4 & 0,05 \\
\hline 3 & 2,3 & 0,31 & 0,005 & 0,25 & 0,43 & 0,022 \\
\hline 5 & 2,8 & 0,68 & 0,009 & 0,2 & 0,29 & 0,045 \\
\hline 7 & 2,2 & 0,96 & 0,006 & 0,21 & 0,24 & 0,026 \\
\hline 8 & 7 & 0,37 & 0,006 & 0,21 & 0,36 & 0,028 \\
\hline 9 & 2,2 & 0,22 & 0,006 & 0,2 & 0,27 & 0,021 \\
\hline 10 & 2,5 & 0,37 & 0,008 & 0,21 & 0,36 & 0,026 \\
\hline 11 & 3,2 & 0,66 & 0,017 & 0,27 & 0,55 & 0,045 \\
\hline 13 & 1,9 & 0,41 & 0,007 & 0,2 & 0,29 & 0,025 \\
\hline 14 & 3,2 & 0,44 & 0,008 & 0,21 & 0,37 & 0,027 \\
\hline 18 & 3,7 & 0,74 & 0,0018 & 0,34 & 0,74 & 0,037 \\
\hline 19 & 6,9 & 0,55 & 0,011 & 0,3 & 0,72 & 0,038 \\
\hline 22 & 5,3 & 1,0 & 0,017 & 0,3 & 0,8 & 0,054 \\
\hline 24 & 6,5 & 1,1 & 0,007 & 0,3 & 0,9 & 0,040 \\
\hline 26 & 6,7 & 2,9 & 0,011 & 0,4 & 1,7 & 0,061 \\
\hline 28 & 6,3 & 2,0 & 0,037 & 0,72 & 1,4 & 0,087 \\
\hline 30 & 5,1 & 2,3 & 0,011 & 0,32 & 1,2 & 0,081 \\
\hline 32 & 4,4 & 2,6 & 0,015 & 0,33 & 1,1 & 0,079 \\
\hline 34 & 4,4 & 3,0 & 0,02 & 0,3 & 1,1 & 0,103 \\
\hline 38 & 4,7 & 2,8 & 0,016 & 0,33 & 1,2 & 0,22 \\
\hline 42 & 6,1 & 2,0 & 0,04 & 0,76 & 1,5 & 0,093 \\
\hline 46 & 5,7 & 2,5 & 0,036 & 0,53 & 1,3 & 0,09 \\
\hline 56 & 6,3 & 2,3 & 0,042 & 0,73 & 1,4 & 0,104 \\
\hline 58 & 3,2 & 0,88 & 0,019 & 0,35 & 0,49 & 0,038 \\
\hline
\end{tabular}


Tab. A.14.: Spurenelementgehalte der Festphasen im Bohrkern des Grundwasserpegels RCO4.

\begin{tabular}{|c|c|c|c|c|c|c|c|}
\hline Tiefe [m] & & $\mathrm{Sr}$ & $\mathbf{R b}$ & $\mathbf{C u}$ & $\mathbf{Z n}$ & $\mathbf{P b}$ & $\mathbf{V}$ \\
\hline 1 & 170 & 54 & 66 & 9 & 29 & 14 & 47 \\
\hline 2 & 210 & 48 & 63 & 3 & 32 & 10 & 39 \\
\hline 3 & 210 & 23 & 23 & 3 & 6 & 6 & 6 \\
\hline 5 & 200 & 100 & 22 & 1 & 7 & 1 & 28 \\
\hline 7 & 230 & 52 & 18 & 1 & 9 & 1 & 18 \\
\hline 8 & 295 & 47 & 22 & 1 & 7 & 11 & 40 \\
\hline 9 & 280 & 21 & 15 & 1 & 4 & 4 & 15 \\
\hline 10 & 250 & 23 & 18 & 1 & 7 & 4 & 14 \\
\hline 11 & 250 & 77 & 27 & 1 & 11 & 1 & 22 \\
\hline 13 & 220 & 29 & 26 & 1 & 9 & 4 & 16 \\
\hline 14 & 300 & 42 & 27 & 1 & 11 & 1 & 23 \\
\hline 18 & 210 & 53 & 33 & 1 & 12 & 8 & 36 \\
\hline 19 & 320 & 63 & 36 & 1 & 7 & 15 & 56 \\
\hline 22 & 236 & 84 & 28 & 13 & 16 & 15 & 41 \\
\hline 24 & 297 & 66 & 37 & 1 & 16 & 14 & 62 \\
\hline 26 & 348 & 97 & 60 & 3 & 23 & 4,9 & 84 \\
\hline 28 & 180 & 95 & 63 & 6 & 37 & 7 & 57 \\
\hline 30 & 340 & 65 & 51 & 1 & 26 & 5 & 71 \\
\hline 32 & 300 & 84 & 45 & 1 & 22 & 1 & 77 \\
\hline 34 & 300 & 81 & 56 & 1 & 27 & 1 & 91 \\
\hline 38 & 330 & 100 & 50 & 1 & 30 & 3 & 88 \\
\hline 42 & 220 & 90 & 65 & 4 & 38 & 5 & 58 \\
\hline 46 & 270 & 97 & 57 & 5 & 46 & 5 & 74 \\
\hline 56 & 200 & 105 & 65 & 7 & 40 & 11 & 65 \\
\hline 58 & 152 & 150 & 27 & 1 & 16 & 1 & 29 \\
\hline
\end{tabular}


Tab. A.14. (Forts.): Spurenelementgehalte der Festphasen im Bohrkern des Grundwasserpegels RCO4.

\begin{tabular}{|c|c|c|c|c|c|c|c|}
\hline Tiefe [m] & $\mathrm{Cr}$ & Co & $\mathbf{N i}$ & $\mathbf{Y}$ & $\mathbf{N b}$ & $\mathbf{B a}$ & Sc \\
\hline 1 & 36 & 7 & 19 & 16 & 9 & 290 & 7 \\
\hline 2 & 37 & 6 & 19 & 16 & 10 & 290 & 7 \\
\hline 3 & 20 & 1 & 6 & 12 & 7 & 110 & 1 \\
\hline 5 & 19 & 5 & 11 & 11 & 9 & 97 & 4 \\
\hline 7 & 20 & 4 & 8 & 11 & 9 & 74 & 2 \\
\hline 8 & 35 & 4 & 12 & 16 & 16 & 430 & 1 \\
\hline 9 & 21 & 3 & 6 & 11 & 7 & 74 & 3 \\
\hline 10 & 18 & 1 & 10 & 13 & 10 & 98 & 4 \\
\hline 11 & 24 & 3 & 10 & 14 & 9 & 150 & 6 \\
\hline 13 & 20 & 5 & 7 & 12 & 8 & 73 & 2 \\
\hline 14 & 28 & 1 & 11 & 15 & 11 & 120 & 3 \\
\hline 18 & 28 & 2 & 9 & 12 & 11 & 155 & 5 \\
\hline 19 & 42 & 1 & 10 & 20 & 22 & 200 & 7 \\
\hline 22 & 49 & 1 & 7 & 12 & 15 & 160 & 7 \\
\hline 24 & 48 & 1 & 9 & 17 & 18 & 212 & 10 \\
\hline 26 & 70 & 2 & 10 & 15 & 18 & 359 & 5 \\
\hline 28 & 100 & 10 & 43 & 18 & 10 & 230 & 8 \\
\hline 30 & 59 & 4 & 16 & 16 & 17 & 210 & 4 \\
\hline 32 & 54 & 3 & 13 & 17 & 15 & 290 & 5 \\
\hline 34 & 61 & 5 & 14 & 16 & 16 & 260 & 7 \\
\hline 38 & 63 & 6 & 14 & 20 & 18 & 290 & 5 \\
\hline 42 & 110 & 11 & 37 & 17 & 11 & 280 & 7 \\
\hline 46 & 88 & 5 & 31 & 20 & 14 & 300 & 7 \\
\hline 56 & 120 & 7 & 39 & 18 & 12 & 280 & 7 \\
\hline 58 & 40 & 4 & 15 & 11 & 8 & 160 & 3 \\
\hline
\end{tabular}


Tab. A.15.: Modell der Elementgehalte in der quarzreichen Deckschicht, berechnet auf der Basis der Elementgehalte im Grauen Sand (Kittsteiner 1998); angenommene Zusammensetzung der Deckschicht aus den Größenfraktionen des Grauen Sandes und reinem Quarz:82 \% reines $\mathrm{SiO}_{2}$, 10,7 \% Fraktion > 63 $\mu \mathrm{m}, 6,3 \%$ Fraktion < $63 \mu \mathrm{m}, 1$ \% Pyrit; für Berechnung der Hauptelemente (außer Ti und Mn): SiO-Gehalt auf $95 \%$ gesetzt.

\begin{tabular}{|c|c|c|}
\hline Element & Einheit & $\begin{array}{l}\text { Elementgehalt in der quarzreichen } \\
\text { Deckschicht (Modell) }\end{array}$ \\
\hline $\mathrm{SiO}_{2}$ & Massen - \% & 95 \\
\hline $\mathrm{TiO}_{2}$ & Massen - \% & 0,095 \\
\hline $\mathrm{Al}_{2} \mathrm{O}_{3}$ & Massen - \% & 1,4 \\
\hline $\mathrm{FeS}_{2}$ & Massen - \% & 1,0 \\
\hline $\mathrm{Fe}_{2} \mathrm{O}_{3}$ & Massen - \% & 0,82 \\
\hline MnO & Massen - \% & 0,005 \\
\hline MgO & Massen - \% & 0,20 \\
\hline $\mathrm{CaO}$ & Massen - \% & 0,17 \\
\hline $\mathrm{Na}_{2} \mathrm{O}$ & Massen - \% & 0,09 \\
\hline $\mathrm{K}_{2} \mathrm{O}$ & Massen - \% & 0,37 \\
\hline $\mathbf{P}_{2} \mathbf{O}_{5}$ & Massen - \% & 0,03 \\
\hline Cges & Massen - \% & 0,16 \\
\hline Sulfat & Massen - \% & 0,00 \\
\hline $\mathrm{H}_{2} \mathrm{O}$ & Massen - \% & 0,77 \\
\hline $\mathbf{L i}$ & $\mathrm{mg} / \mathrm{kg}$ & 4,0 \\
\hline Sc & $\mathrm{mg} / \mathrm{kg}$ & 1,6 \\
\hline $\mathbf{V}$ & $\mathrm{mg} / \mathrm{kg}$ & 9,5 \\
\hline $\mathrm{Cr}$ & $\mathrm{mg} / \mathrm{kg}$ & 26 \\
\hline Co & $\mathrm{mg} / \mathrm{kg}$ & 2,0 \\
\hline $\mathbf{N i}$ & $\mathrm{mg} / \mathrm{kg}$ & 2,3 \\
\hline $\mathbf{C u}$ & $\mathrm{mg} / \mathrm{kg}$ & 1,6 \\
\hline Zn & mg/kg & 10 \\
\hline As & $\mathrm{mg} / \mathrm{kg}$ & 3,2 \\
\hline $\mathbf{R b}$ & $\mathrm{mg} / \mathrm{kg}$ & 9,6 \\
\hline $\mathbf{S r}$ & $\mathrm{mg} / \mathrm{kg}$ & 21 \\
\hline $\mathbf{Y}$ & $\mathrm{mg} / \mathrm{kg}$ & 2,5 \\
\hline $\mathbf{Z r}$ & $\mathrm{mg} / \mathrm{kg}$ & 38 \\
\hline Mo & $\mathrm{mg} / \mathrm{kg}$ & 1,4 \\
\hline Cd & $\mathrm{mg} / \mathrm{kg}$ & 0,02 \\
\hline Sb & $\mathrm{mg} / \mathrm{kg}$ & 0,16 \\
\hline Cs & $\mathrm{mg} / \mathrm{kg}$ & 0,42 \\
\hline $\mathbf{B a}$ & $\mathrm{mg} / \mathrm{kg}$ & 54 \\
\hline Hf & $\mathrm{mg} / \mathrm{kg}$ & 0,97 \\
\hline $\mathbf{P b}$ & $\mathrm{mg} / \mathrm{kg}$ & 1,7 \\
\hline $\mathbf{B i}$ & $\mathrm{mg} / \mathrm{kg}$ & 0,01 \\
\hline Th & $\mathrm{mg} / \mathrm{kg}$ & 1,3 \\
\hline $\mathbf{U}$ & $\mathrm{mg} / \mathrm{kg}$ & 0,81 \\
\hline
\end{tabular}


Tab. A.16.: Sättigungsindizes einiger wichtiger Festphasen in Proben aus Cospuden berechnet mit MINTEQA2; $A P=$ Proben aus der Arbeit von PLEßOW (1998), KB = Proben aus der vorliegenden Arbeit; PL = Porenlösung, WP $=$ Wasserprobe.

\begin{tabular}{|c|c|c|c|c|c|c|c|}
\hline & RCO4 5-8 m & RCO4 11-19 m & RCO417 m & RCO4 $20 \mathrm{~m}$ & RCO4 22 m & RCO4 26 m & RCO4 28 m \\
\hline & PL AP & PL AP & PL KB & PL KB & PL KB & PL KB & PL AP \\
\hline $\mathrm{Al}(\mathrm{OH})_{3} \mathrm{am}$ & -4.189 & -1.672 & -2.224 & & -0.774 & -5.335 & -1.057 \\
\hline Diaspor & -0.262 & 2.256 & 1.728 & & 3.154 & -1.406 & 2.895 \\
\hline Alunit & -25.714 & -29.229 & -33.234 & & -31.662 & -18.715 & -39.484 \\
\hline $\mathrm{Jurb}^{-\mathrm{AlOHSO}}{ }_{4}$ & -1.078 & -1.004 & -1.858 & & -1.309 & 0.32 & -2.611 \\
\hline $\mathrm{Al}_{4}(\mathrm{OH})_{10} \mathrm{SO}_{4}$ & -7.176 & 0.451 & -1.064 & & 2.84 & -9.214 & 1.684 \\
\hline Gibbsit & -1.68 & 0.837 & 0.332 & & 1.735 & -2.826 & 1.499 \\
\hline Ferrihydrit & 0.015 & & -0.703 & 0.386 & -0.224 & & 0.308 \\
\hline Goethit & 2.715 & & 2.035 & 3.124 & 2.476 & & 3.046 \\
\hline Hämatit & 7.831 & & 6.448 & 8.626 & 7.352 & & 8.47 \\
\hline Melanterit & -4.296 & & -6.628 & -7.097 & -7.006 & -2.324 & -6.743 \\
\hline K-Jarosit & 3.871 & & -2.802 & -1.994 & -2.841 & & -2.32 \\
\hline Magnetit & 7.428 & & 6.491 & 9.712 & 7.883 & & 10.319 \\
\hline Lepidocrocit & 1.835 & & 1.336 & 2.426 & 1.596 & & 2.347 \\
\hline $\mathrm{Cu}(\mathrm{OH}) 2$ & -7.065 & -5.137 & -4.865 & -3.342 & -3.789 & -8.701 & -2.381 \\
\hline Chalcanthit & -6.672 & -7.187 & -7.363 & -7.352 & -7.041 & -5.769 & -6.799 \\
\hline Tenorit & -6.034 & -4.106 & -3.86 & -2.337 & -2.758 & -7.67 & -1.376 \\
\hline Kupferferrit & 2.034 & & 2.777 & 6.478 & 4.831 & & 7.283 \\
\hline Cölestin & & & -0.21 & -0.402 & -0.573 & -0.242 & 0.132 \\
\hline $\mathrm{SbO}_{2}$ & 1.045 & -1.121 & 0.352 & -0.273 & 0.843 & 2.64 & 0.195 \\
\hline Gips & 0.025 & 0.06 & 0.134 & -0.072 & -0.234 & 0.026 & \\
\hline Baryt & 1.168 & 1.213 & 1.301 & 1.226 & 1.029 & 0.7 & 1.395 \\
\hline Pyrolusit & -13.213 & -8.98 & -13.61 & -12.29 & -13.052 & -17.319 & -12.669 \\
\hline Manganit & -8.994 & -5.961 & -7.523 & -6.249 & -7.66 & -11.984 & -5.787 \\
\hline $\mathrm{Cr}(\mathrm{OH})_{3}$ (am) & -3.917 & -1.365 & -1.669 & -0.227 & -0.786 & -6.918 & 1.404 \\
\hline $\mathrm{Cr}_{2} \mathrm{O}_{3}$ & -6.976 & -1.871 & -2.63 & 0.253 & -0.714 & -12.975 & 3.515 \\
\hline $\mathrm{Cr}(\mathrm{OH})_{3}$ & -6.003 & -3.451 & -3.843 & -2.402 & -2.872 & -9.004 & -0.771 \\
\hline $\mathrm{FeCr}_{2} \mathrm{O}_{4}$ & -12.116 & & -7.354 & -3.429 & -4.92 & -18.681 & 0.596 \\
\hline
\end{tabular}

\begin{tabular}{|c|c|c|c|c|c|c|c|}
\hline & RCO4 $30 \mathrm{~m}$ & RCO4 32 m & RCO4 $34 \mathrm{~m}$ & RCO4 $36 \mathrm{~m}$ & RCO4 $38 \mathrm{~m}$ & RCO4 $40 \mathrm{~m}$ & RCO4 $42 \mathrm{~m}$ \\
\hline & PL AP & PL AP & PL AP & PL KB & PL AP & PL KB & PL AP \\
\hline $\mathrm{Al}(\mathrm{OH})_{3}$ am & -0.486 & -1.275 & -0.351 & & -1.031 & 0.184 & -0.832 \\
\hline Diaspor & 3.466 & 2.653 & 3.576 & & 2.897 & 4.135 & 3.095 \\
\hline Alunit & -31.059 & -32.356 & -33.86 & & -40.682 & -33.374 & -38.68 \\
\hline Jurb-AlOHSO $_{4}$ & -0.645 & -1.564 & -1.552 & & -3.32 & -1.11 & -2.81 \\
\hline $\mathrm{Al}_{4}(\mathrm{OH})_{10} \mathrm{SO}_{4}$ & 5.363 & 1.082 & 3.864 & & 0.057 & 6.907 & 1.162 \\
\hline Gibbsit & 2.07 & 1.234 & 2.158 & & 1.478 & 2.74 & 1.677 \\
\hline Ferrihydrit & 1.407 & 1.132 & 2.538 & 0.883 & & 2.019 & \\
\hline Goethit & 4.146 & 3.833 & 5.239 & 3.583 & & 4.758 & \\
\hline Hämatit & 10.669 & 10.066 & 12.878 & 9.567 & & 11.892 & \\
\hline Melanterit & -8.003 & -7.399 & -7.574 & -7.014 & & -5.849 & \\
\hline K-Jarosit & 2.892 & 2.039 & 4.982 & -0.605 & & 2.804 & \\
\hline Magnetit & 9.861 & 9.96 & 13.508 & 11.13 & & 14.373 & \\
\hline Lepidocrocit & 3.447 & 2.953 & 4.359 & 2.703 & & 4.059 & \\
\hline $\mathrm{Cu}(\mathrm{OH}) 2$ & -3.997 & -4.292 & -3.039 & -2.778 & -2.347 & -2.838 & -2.584 \\
\hline Chalcanthit & -7.02 & -7.299 & -6.958 & -7.069 & -7.355 & -6.995 & -7.28 \\
\hline Tenorit & -2.992 & -3.261 & -2.009 & -1.748 & -1.317 & -1.833 & -1.554 \\
\hline Kupferferrit & 7.866 & 7.043 & 11.107 & 8.058 & & 10.248 & \\
\hline Cölestin & -0.023 & -0.316 & -0.138 & -0.096 & & -0.515 & -0.579 \\
\hline $\mathrm{SbO}_{2}$ & -3.011 & -1.806 & -3.119 & -1.045 & -4.228 & -0.779 & -0.666 \\
\hline Gips & -0.33 & 0.243 & -0.051 & 0.045 & 0.013 & -0.006 & -0.1 \\
\hline Baryt & 1.332 & 1.035 & 1.16 & 0.638 & 0.96 & 1.017 & 1.324 \\
\hline Pyrolusit & -6.519 & -8.698 & -6.144 & -11.35 & -2.751 & -11.16 & -8.644 \\
\hline Manganit & -3.394 & -5.3 & -3.415 & -6.033 & -1.333 & -5.357 & -4.203 \\
\hline $\mathrm{Cr}(\mathrm{OH})_{3}$ (am) & -0.109 & -1.015 & 0.33 & -0.322 & 1.045 & 0.165 & 0.72 \\
\hline $\mathrm{Cr}_{2} \mathrm{O}_{3}$ & 0.49 & -1.17 & 1.518 & 0.216 & 2.95 & 1.036 & 2.298 \\
\hline $\mathrm{Cr}(\mathrm{OH})_{3}$ & -2.283 & -3.1 & -1.756 & -2.407 & -1.04 & -2.01 & -1.366 \\
\hline $\mathrm{FeCr}_{2} \mathrm{O}_{4}$ & -5.085 & -6.014 & -2.589 & -2.959 & & -1.25 & \\
\hline
\end{tabular}


Tab. A.16. (Forts.): Sättigungsindizes einiger wichtiger Festphasen in Proben aus Cospuden berechnet mit MINTEQA2; AP = Proben aus der Arbeit von PLEßOW (1998), KB = Proben aus der vorliegenden Arbeit; PL = Porenlösung, WP = Wasserprobe.

\begin{tabular}{|c|c|c|c|c|c|c|c|}
\hline & RCO4 44 m & RCO4 46 m & RCO4 48 m & RCO4 52 m & RCO4 56 m & RCO4 58 m & RCO10 2 m \\
\hline & PL KB & PL AP & PL KB & PL KB & PL AP & PL AP & PL AP \\
\hline $\mathrm{Al}(\mathrm{OH})_{3} \mathrm{am}$ & & -0.606 & & & -1.152 & -0.995 & -1.204 \\
\hline Diaspor & & 3.322 & & & 2.776 & 2.933 & 2.747 \\
\hline Alunit & & -35.744 & & & -35.382 & $\mid-33.82$ & -47.112 \\
\hline Jurb-AIOHSO $_{4}$ & & -2.112 & & & -2.102 & \begin{tabular}{|l|}
-1.793 \\
\end{tabular} & -4.631 \\
\hline $\mathrm{Al}_{4}(\mathrm{OH})_{10} \mathrm{SO}_{4}$ & & 2.541 & & & 0.913 & $\begin{array}{l}1.693 \\
\end{array}$ & -0.778 \\
\hline Gibbsit & & 1.903 & & & 1.357 & 1.514 & 1.352 \\
\hline Ferrihydrit & 0.381 & & & 1.894 & 0.71 & 0.897 & \\
\hline Goethit & 3.12 & & & 4.595 & 3.411 & 3.597 & \\
\hline Hämatit & 8.618 & & & $\begin{array}{l}11.59 \\
\end{array}$ & 9.222 & 9.595 & \\
\hline Melanterit & -7.15 & & -3.935 & -6.842 & -8.459 & -8.463 & \\
\hline K-Jarosit & -2.251 & & & 1.008 & -0.223 & 0.228 & \\
\hline Magnetit & 9.696 & & & 14.153 & 8.715 & 8.935 & \\
\hline Lepidocrocit & 2.421 & & & 3.715 & 2.531 & 2.717 & \\
\hline $\mathrm{Cu}(\mathrm{OH}) 2$ & -3.536 & -3.22 & -4.263 & -2.093 & -3.396 & -3.884 & -0.815 \\
\hline Chalcanthit & -7.59 & -7.444 & -6.76 & -7.212 & -7.064 & -7.4 & -7.105 \\
\hline Tenorit & -2.531 & -2.19 & -3.258 & $\begin{array}{l}-1.062 \\
\end{array}$ & -2.366 & -2.853 & 0.189 \\
\hline Kupferferrit & 6.276 & & & 10.766 & 7.094 & 6.98 & \\
\hline Cölestin & & -0.35 & -0.377 & $\begin{array}{l}-0.165 \\
\end{array}$ & -0.145 & \begin{tabular}{|c|}
-0.078 \\
\end{tabular} & -1.636 \\
\hline $\mathrm{SbO}_{2}$ & -0.198 & -0.886 & & -0.613 & -1.876 & -2.137 & -5.019 \\
\hline Gips & -0.278 & 0.108 & 0.089 & 0.013 & -0.469 & 0.303 & -0.823 \\
\hline Baryt & 0.707 & 1.085 & 0.794 & 0.731 & 1.434 & 0.879 & 0.534 \\
\hline Pyrolusit & -11.896 & -9.066 & -13.79 & $\begin{array}{l}-10.162 \\
\end{array}$ & -7.386 & \begin{tabular}{|l|}
-7.977 \\
\end{tabular} & \begin{tabular}{|l|}
-3.871 \\
\end{tabular} \\
\hline Manganit & -5.859 & -4.662 & -7.617 & -4.857 & -3.966 & -4.898 & -1.633 \\
\hline $\mathrm{Cr}(\mathrm{OH})_{3}(\mathrm{am})$ & -0.738 & 0.485 & -1.659 & 0.179 & 0.078 & -0.05 & 1.419 \\
\hline $\mathrm{Cr}_{2} \mathrm{O}_{3}$ & -0.767 & 1.829 & -2.611 & 1.218 & 1.014 & 0.759 & 3.544 \\
\hline $\mathrm{Cr}(\mathrm{OH})_{3}$ & -2.913 & -1.6 & -3.834 & -1.906 & -2.008 & -2.136 & -0.756 \\
\hline $\mathrm{FeCr}_{2} \mathrm{O}_{4}$ & -4.456 & & -4.643 & -0.957 & -4.23 & -4.638 & 1.674 \\
\hline
\end{tabular}

\begin{tabular}{|c|c|c|c|c|c|c|c|}
\hline & $\begin{array}{ll}\operatorname{RCO10} & 18,5 \\
\mathrm{~m} & \\
\end{array}$ & RCO10 29 m & RCO13 15 m & RCO1330 m & RCO16 29 m & Fremdflutung & Sickerwasser \\
\hline & PL AP & PL AP & PL AP & PL AP & PL AP & WP AP & WP AP \\
\hline $\mathrm{Al}(\mathrm{OH})_{3}$ am & -1.144 & -1.487 & -0.929 & -1.286 & -0.954 & $\begin{array}{l}-1.729 \\
\end{array}$ & -1.595 \\
\hline Diaspor & 2.807 & 2.464 & 3.023 & 2.665 & 2.997 & 2.222 & 2.356 \\
\hline Alunit & -35.083 & -35.529 & -33.185 & -40.734 & \begin{tabular}{|l|}
-33.674 \\
\end{tabular} & -48.921 & -51.185 \\
\hline Jurb-AIOHSO $_{4}$ & -1.995 & -2.262 & -1.31 & -3.424 & -1.456 & -5.255 & -5.704 \\
\hline $\mathrm{Al}_{4}(\mathrm{OH})_{10} \mathrm{SO}_{4}$ & 2.037 & 0.741 & 3.369 & 0.182 & 3.146 & -2.977 & -3.023 \\
\hline Gibbsit & 1.411 & 1.068 & 1.627 & 1.269 & 1.601 & 0.827 & 0.961 \\
\hline Ferrihydrit & & & & 0.699 & 1.042 & & \\
\hline Goethit & & & & 3.437 & 3.781 & & \\
\hline Hämatit & & & & 9.251 & 9.938 & & \\
\hline Melanterit & -7.406 & & -7.056 & -7.85 & -7.004 & -7.061 & -7.052 \\
\hline K-Jarosit & & & & -2.906 & 1.188 & & \\
\hline Magnetit & & & & 10.573 & 10.47 & & \\
\hline Lepidocrocit & & & & 2.738 & 3.082 & & \\
\hline $\mathrm{Cu}(\mathrm{OH}) 2$ & -3.736 & -4.269 & -3.703 & -2.703 & -3.901 & -2.221 & -1.716 \\
\hline Chalcanthit & -7.448 & -7.906 & -6.948 & -7.702 & -7.265 & $\begin{array}{l}-8.609 \\
\end{array}$ & -8.687 \\
\hline Tenorit & -2.731 & -3.265 & -2.697 & -1.698 & -2.896 & -1.216 & -0.711 \\
\hline Kupferferrit & & & & 7.742 & 7.231 & & \\
\hline Cölestin & -0.513 & -0.627 & 0.327 & -0.835 & -0.024 & -1.081 & -1.069 \\
\hline $\mathrm{SbO}_{2}$ & -2.319 & -3.664 & 0.247 & -0.564 & -1.444 & \begin{tabular}{l|l|}
-4.702 \\
\end{tabular} & -5.039 \\
\hline Gips & -0.407 & -0.52 & 0.188 & -0.724 & -0.359 & -0.454 & -0.459 \\
\hline Baryt & 1.335 & 1.094 & 1.479 & 1.087 & 1.355 & 0.701 & 0.552 \\
\hline Pyrolusit & -8.33 & -6.388 & \begin{tabular}{|l|l|}
-13.449 \\
\end{tabular} & -12.208 & -10.101 & -5.704 & -6.569 \\
\hline Manganit & -4.703 & -4.088 & $\begin{array}{l}-7.07 \\
\end{array}$ & -6.244 & -5.271 & $\begin{array}{l}-1.709 \\
\end{array}$ & -2.874 \\
\hline $\mathrm{Cr}(\mathrm{OH})_{3}(\mathrm{am})$ & -0.51 & -0.321 & -0.31 & 1.027 & $\begin{array}{l}-0.122 \\
\end{array}$ & $\begin{array}{l}1.342 \\
\end{array}$ & 1.582 \\
\hline $\mathrm{Cr}_{2} \mathrm{O}_{3}$ & -0.314 & 0.064 & 0.087 & 2.76 & 0.462 & 3.39 & 3.87 \\
\hline $\mathrm{Cr}(\mathrm{OH})_{3}$ & -2.685 & -2.496 & -2.485 & -1.148 & -2.297 & -0.833 & -0.593 \\
\hline $\mathrm{FeCr}_{2} \mathrm{O}_{4}$ & -4.602 & & -4.317 & -0.686 & -3.773 & 2.122 & 3.139 \\
\hline
\end{tabular}


Tab. A.16. (Forts.): Sättigungsindizes einiger wichtiger Festphasen in Proben aus Cospuden berechnet mit MINTEQA2; AP = Proben aus der Arbeit von PLEßOW (1998), KB = Proben aus der vorliegenden Arbeit; PL = Porenlösung, WP = Wasserprobe.

\begin{tabular}{|c|c|c|c|c|c|c|c|}
\hline & Standgew. 1 & Standgew. 2 & Standgew. 2 & Standgew. 3 & Standgew. 3 & Kippe SW & Kippe W \\
\hline & WP AP & PL AP & WP AP & WP AP & PL AP & PL AP & PL AP \\
\hline $\mathrm{Al}(\mathrm{OH})_{3}$ am & -5.725 & -8.049 & -8.02 & -7.657 & \begin{tabular}{l|l|}
-7.127 \\
\end{tabular} & -7.956 & -5.917 \\
\hline Diaspor & -1.774 & -4.098 & -4.069 & -3.706 & -3.176 & -4.005 & -1.966 \\
\hline Alunit & -23.228 & -21.689 & -21.603 & $\begin{array}{l}-20.336 \\
\end{array}$ & $\mid-19.211$ & -20.49 & -21.863 \\
\hline Jurb-AIOHSO $_{4}$ & -0.744 & -1.04 & -1.026 & -0.481 & -0.215 & -0.587 & -0.407 \\
\hline $\mathrm{Al}_{4}(\mathrm{OH})_{10} \mathrm{SO}_{4}$ & -10.455 & $\begin{array}{l}-17.722 \\
\end{array}$ & -17.621 & -15.988 & \begin{tabular}{ll|}
-14.132 \\
\end{tabular} & -16.99 & $\begin{array}{l}-10.694 \\
\end{array}$ \\
\hline Gibbsit & -3.169 & -5.493 & -5.464 & -5.102 & -4.572 & -5.4 & \\
\hline \begin{tabular}{|l|} 
Ferrihydrit \\
\end{tabular} & -4.748 & & & & & & \\
\hline Goethit & -2.01 & & & & & & \\
\hline Hämatit & -1.643 & & & & & & \\
\hline Melanterit & -4.995 & -2.973 & -3.144 & -2.553 & -2.372 & -2.446 & -3.315 \\
\hline K-Jarosit & -8.825 & & & & & & \\
\hline \begin{tabular}{|l|} 
Magnetit \\
\end{tabular} & -4.585 & & & & & & \\
\hline \begin{tabular}{|l} 
Lepidocrocit \\
\end{tabular} & -2.709 & & & & & & \\
\hline $\mathrm{Cu}(\mathrm{OH}) 2$ & -8.98 & -10.223 & -10.346 & -10.18 & -10.296 & -10.28 & -9.313 \\
\hline Chalcanthit & -6.861 & -6.076 & -6.214 & -5.866 & -6.248 & -5.775 & -6.666 \\
\hline Tenorit & -7.975 & -9.218 & -9.341 & -9.174 & -9.29 & -9.275 & -8.308 \\
\hline Kupferferrit & -9.429 & & & & & & \\
\hline Cölestin & -0.767 & -1.085 & -1.089 & -1.088 & \begin{tabular}{|c|}
-0.96 \\
\end{tabular} & -1.029 & -0.551 \\
\hline $\mathrm{SbO}_{2}$ & & & & -8.642 & -8.263 & -8.743 & -8.343 \\
\hline Gips & \begin{tabular}{|c|}
-0.33 \\
\end{tabular} & -0.457 & -0.481 & -0.261 & -0.211 & -0.187 & -0.074 \\
\hline Baryt & 0.739 & 0.396 & 0.244 & 1.391 & 0.339 & 0.179 & 0.469 \\
\hline Pyrolusit & -20.448 & -26.555 & -26.558 & 3.721 & 3.815 & 3.805 & 4.077 \\
\hline Manganit & \begin{tabular}{|c|}
-13.3 \\
\end{tabular} & -17.36 & -17.363 & -2.164 & -1.969 & -2.209 & -0.943 \\
\hline $\mathrm{Cr}(\mathrm{OH})_{3}(\mathrm{am})$ & -6.337 & -8.155 & -8.328 & -7.84 & -8.252 & -8.04 & -6.648 \\
\hline $\mathrm{Cr}_{2} \mathrm{O}_{3}$ & $\begin{array}{l}-11.968 \\
\end{array}$ & -15.602 & $\begin{array}{l}-15.949 \\
\end{array}$ & $\begin{array}{l}-14.972 \\
\end{array}$ & -15.795 & $\begin{array}{l}-15.372 \\
\end{array}$ & -12.59 \\
\hline $\mathrm{Cr}(\mathrm{OH})_{3}$ & -8.512 & -10.329 & -10.502 & -10.014 & -10.426 & -12.214 & -8.823 \\
\hline $\mathrm{FeCr}_{2} \mathrm{O}_{4}$ & -19.677 & -23.318 & -23.82 & -22.433 & $\mid-22.808$ & -22.917 & -19.146 \\
\hline
\end{tabular}

\begin{tabular}{|c|c|c|c|c|c|c|c|}
\hline & See Südufer & See Südufer & SGM 48,5 m & SGM 24,5 m & CML 21-22 m & CML 24 m & CML 26 m \\
\hline & WP AP & PL AP & WP AP & WP AP & PL KB & PL KB & PL KB \\
\hline $\mathrm{Al}(\mathrm{OH})_{3}$ am & -3.336 & -5.368 & -3.305 & -3.104 & & -5.051 & -6.449 \\
\hline Diaspor & 0.615 & -1.417 & 0.647 & 0.847 & & -1.1 & -2.498 \\
\hline Alunit & & -23.25 & -22.396 & -21.658 & & -21.118 & -18.73 \\
\hline Jurb-AlOHSO $_{4}$ & 0.167 & -0.553 & 0.42 & 0.59 & & -0.203 & -0.294 \\
\hline $\mathrm{Al}_{4}(\mathrm{OH})_{10} \mathrm{SO}_{4}$ & -2.375 & -9.193 & -2.028 & -1.258 & & -7.893 & -12.177 \\
\hline Gibbsit & -0.78 & -2.813 & -0.749 & -0.549 & & -2.496 & -3.894 \\
\hline Ferrihydrit & -1.847 & & & & & & \\
\hline Goethit & 0.892 & & & & & & \\
\hline Hämatit & 4.16 & & & & & & \\
\hline Melanterit & -5.554 & -3.229 & -2.112 & -2.128 & & & \\
\hline \multicolumn{8}{|l|}{ K-Jarosit } \\
\hline Magnetit & 3.139 & & & & & & \\
\hline Lepidocrocit & 0.193 & & & & & & \\
\hline $\mathrm{Cu}(\mathrm{OH}) 2$ & -7.864 & -8.748 & -8.403 & -8.099 & & & \\
\hline Chalcanthit & -7.224 & -6.796 & -7.543 & -7.269 & & & \\
\hline Tenorit & -6.859 & -7.743 & -7.398 & -7.094 & & & \\
\hline Kupferferrit & -2.51 & & & & & & \\
\hline Cölestin & -0.47 & -0.432 & -0.734 & -0.776 & -0.319 & -0.499 & -0.316 \\
\hline $\mathrm{SbO}_{2}$ & 0.676 & 3.973 & 2.886 & 3.122 & -0.965 & & \\
\hline Gips & -0.037 & 0.011 & -0.158 & -0.2 & -0.046 & -0.15 & -0.678 \\
\hline Baryt & 0.613 & 0.744 & 0.709 & 0.681 & 1.115 & 0.977 & 0.12 \\
\hline Pyrolusit & -14.559 & -21.414 & -19.108 & -19.117 & -11.934 & -18.548 & \\
\hline Manganit & -9.392 & -13.419 & -11.713 & -11.722 & -5.976 & -11.96 & \\
\hline $\mathrm{Cr}(\mathrm{OH})_{3}(\mathrm{am})$ & -4.384 & -5.44 & -2.654 & -2.776 & 0.045 & -5.878 & -8.433 \\
\hline $\mathrm{Cr}_{2} \mathrm{O}_{3}$ & -8.061 & -10.172 & -4.6 & -4.845 & 0.796 & \begin{tabular}{|l|l|}
-11.048 \\
\end{tabular} & -16.159 \\
\hline $\mathrm{Cr}(\mathrm{OH})_{3}$ & -6.559 & -7.614 & -4.828 & -4.951 & -2.13 & -8.052 & -10.608 \\
\hline $\mathrm{FeCr}_{2} \mathrm{O}_{4}$ & -14.85 & -15.948 & -8.165 & -8.396 & -2.534 & -16.31 & -22.943 \\
\hline
\end{tabular}


Tab. A.16. (Forts.): Sättigungsindizes einiger wichtiger Festphasen in Proben aus Cospuden berechnet mit MINTEQA2; AP = Proben aus der Arbeit von PLEßOW (1998), KB = Proben aus der vorliegenden Arbeit; PL = Porenlösung, WP = Wasserprobe.

\begin{tabular}{|c|c|c|c|c|c|c|c|}
\hline & CML 32 m & CML 36 m & CML 37,5 m & CML 39-40 m & RCO4 Okt 98 & RCO4 Okt 98 & RC012 Okt 98 \\
\hline & PL KB & PL KB & PL KB & PL KB & WP KB & WP KB & WP KB \\
\hline $\mathrm{Al}(\mathrm{OH})_{3}$ am & 0.182 & -0.148 & 0.655 & -1.655 & & & \\
\hline Diaspor & 4.133 & 3.803 & 4.606 & 2.296 & & & \\
\hline Alunit & -37.056 & -37.011 & -31.677 & -34.162 & & & \\
\hline Jurb-AIOHSO $_{4}$ & -2.095 & -2.085 & & -1.89 & & & \\
\hline $\mathrm{Al}_{4}(\mathrm{OH})_{10} \mathrm{SO}_{4}$ & 5.915 & 4.935 & & 0.611 & & & \\
\hline Gibbsit & 2.737 & 2.407 & 3.211 & 0.901 & & & \\
\hline Ferrihydrit & 3.555 & 2.446 & 4.285 & -0.372 & 3.503 & 0.531 & 2.075 \\
\hline Goethit & 6.293 & 5.185 & 7.024 & 2.366 & 6.204 & 3.27 & 4.814 \\
\hline Hämatit & 14.964 & 12.747 & 16.424 & 7.109 & 14.809 & 1.497 & 12.005 \\
\hline Melanterit & -6.404 & -6.622 & -4.248 & -6.819 & -3.33 & -7.529 & -3.762 \\
\hline K-Jarosit & 6.255 & 3.245 & 9.944 & -2.643 & 8.987 & -2.729 & 5.725 \\
\hline Magnetit & 17.874 & 15.098 & 20.558 & 7.562 & 18.799 & 10.07 & 14.778 \\
\hline Lepidocrocit & 5.595 & 4.486 & 6.325 & 1.667 & 5.324 & 2.571 & 4.115 \\
\hline $\mathrm{Cu}(\mathrm{OH}) 2$ & -1.386 & -1.9 & -2.406 & -4.144 & -3.685 & -2.216 & -4.166 \\
\hline Chalcanthit & -6.527 & & -6.615 & & -6.719 & -6.727 & -6.527 \\
\hline Tenorit & -0.381 & -0.895 & -1.401 & -3.139 & -2.654 & -1.211 & -3.16 \\
\hline Kupferferrit & 14.772 & 12.041 & 15.213 & 4.159 & 12.392 & 7.894 & 9.033 \\
\hline Cölestin & -0.36 & -0.456 & -0.509 & -0.317 & -0.296 & -0.751 & -0.189 \\
\hline $\mathrm{SbO}_{2}$ & & & -2.06 & -0.439 & & & \\
\hline Gips & -0.119 & 0.039 & -0.025 & 0.055 & 0.102 & -0.47 & 0.201 \\
\hline Baryt & 0.882 & 1.067 & 1.36 & 0.764 & 0.653 & 1.097 & 0.899 \\
\hline Pyrolusit & -7.971 & -9.118 & -9.653 & -12.785 & -12.136 & -12.525 & -13.287 \\
\hline Manganit & -3.276 & -3.873 & -4.463 & -6.62 & -7.013 & -6.561 & -7.248 \\
\hline $\mathrm{Cr}(\mathrm{OH})_{3}$ (am) & 1.633 & 1.403 & 1.102 & -0.943 & -1.657 & -0.743 & -2.315 \\
\hline $\mathrm{Cr}_{2} \mathrm{O}_{3}$ & 3.973 & 3.514 & 2.912 & -1.179 & -2.454 & -0.779 & -3.922 \\
\hline $\mathrm{Cr}(\mathrm{OH})_{3}$ & -0.542 & -0.771 & -1.072 & -3.118 & -3.742 & -2.917 & -4.49 \\
\hline $\mathrm{FeCr}_{2} \mathrm{O}_{4}$ & 2.115 & 1.097 & 2.278 & -5.494 & -3.201 & -4.392 & -5.916 \\
\hline
\end{tabular}

\begin{tabular}{|c|c|c|c|c|c|c|c|}
\hline & RCO13 Okt 98 & RCO14 Okt 98 & $\begin{array}{|llll|}\text { Bohrung } & 1 & \text { m } \\
\text { Nov } 98 & & \\
\end{array}$ & \begin{tabular}{|l|l|l|} 
Bohrung & 2 & m \\
Nov 98 & & \\
\end{tabular} & \begin{tabular}{|l|l|l|l} 
Bohrung & 3 & m \\
Nov 98 & & \\
\end{tabular} & See Nov 98 & \begin{tabular}{|l|} 
CML 20 m \\
Jan 99
\end{tabular} \\
\hline & WP KB & WP KB & WP KB & WP KB & WP KB & WP KB & WP KB \\
\hline $\mathrm{Al}(\mathrm{OH})_{3}$ am & & & -5.917 & -3.951 & -4.815 & -1.146 & \\
\hline Diaspor & & & -1.966 & 0 & -0.864 & 2.855 & \\
\hline Alunit & & & -23.042 & -29.691 & -26.801 & -39.164 & \\
\hline Jurb-AlOHSO $_{4}$ & & & -0.722 & -1.626 & -1.266 & -2.011 & \\
\hline $\mathrm{Al}_{4}(\mathrm{OH})_{10} \mathrm{SO}_{4}$ & & & -11.008 & -6.015 & -8.248 & 4.11 & \\
\hline Gibbsit & & & -3.362 & -1.396 & -2.26 & 1.508 & \\
\hline Ferrihydrit & 0.948 & 0.147 & & -0.001 & & 0.225 & \\
\hline Goethit & 3.687 & 2.885 & & 2.738 & & 3.044 & \\
\hline Hämatit & 9.75 & 8.147 & & 7.853 & & 8.417 & \\
\hline Melanterit & -7.621 & -7.333 & -5.829 & & -5.002 & -7.274 & \\
\hline K-Jarosit & -2.436 & -2.834 & & 2.033 & & -2.789 & \\
\hline Magnetit & 11.327 & 8.78 & & & & 9.112 & \\
\hline Lepidocrocit & 2.988 & 2.186 & & 2.039 & & 2.727 & \\
\hline $\mathrm{Cu}(\mathrm{OH}) 2$ & -1.884 & -3.198 & -7.809 & -6.23 & -6.266 & -2.592 & \\
\hline Chalcanthit & -6.91 & -6.992 & -5.475 & -6.768 & -5.579 & -6.625 & \\
\hline Tenorit & -0.879 & -2.193 & -6.804 & -5.225 & -5.261 & -1.64 & \\
\hline Kupferferrit & 9.06 & 6.142 & & 2.817 & & 6.863 & \\
\hline Cölestin & -0.975 & -1.498 & -0.352 & -0.141 & -0.561 & -0.651 & -0.664 \\
\hline $\mathrm{SbO}_{2}$ & & & 2.664 & -8.823 & -1.159 & & 5.266 \\
\hline Gips & -0.675 & -0.846 & -0.921 & -0.014 & 0.018 & -0.108 & -0.331 \\
\hline Baryt & 1.201 & 1.173 & 0.915 & 0.951 & 0.996 & 1.236 & 1.046 \\
\hline Pyrolusit & -11.725 & -12.813 & -20.44 & 4.73 & -9.732 & -12.475 & \\
\hline Manganit & -5.755 & -6.985 & -13.427 & 0.437 & -7.062 & -5.182 & \\
\hline $\mathrm{Cr}(\mathrm{OH})_{3}(\mathrm{am})$ & 1.004 & -0.004 & -7.619 & -4.231 & -5.64 & 0.772 & -6.511 \\
\hline $\mathrm{Cr}_{2} \mathrm{O}_{3}$ & 2.715 & 0.698 & -14.531 & -7.756 & -10.573 & 1.933 & -12.316 \\
\hline $\mathrm{Cr}(\mathrm{OH})_{3}$ & -1.17 & -2.179 & -9.793 & -6.406 & -7.814 & -1.589 & -8.686 \\
\hline $\mathrm{FeCr}_{2} \mathrm{O}_{4}$ & -0.475 & -3.436 & -23.288 & & -16.856 & -2.203 & \\
\hline
\end{tabular}


Tab. A.16. (Forts.): Sättigungsindizes einiger wichtiger Festphasen in Proben aus Cospuden berechnet mit MINTEQA2; AP = Proben aus der Arbeit von PLEßOW (1998), KB = Proben aus der vorliegenden Arbeit; PL = Porenlösung, WP = Wasserprobe.

\begin{tabular}{|c|c|c|c|c|c|c|c|}
\hline & $\begin{array}{lll}\text { CML 27,5 m } \\
\text { Jan 99 }\end{array}$ & \begin{tabular}{|l} 
CML 31,5 m \\
Jan 99
\end{tabular} & $\begin{array}{l}\text { CML 39,5 m } \\
\text { Jan 99 }\end{array}$ & $\begin{array}{l}\text { CML 43,5 m } \\
\text { Jan 99 }\end{array}$ & $\begin{array}{l}\text { CML 27,5 m } \\
\text { Aug 99 }\end{array}$ & $\begin{array}{l}\text { CML 31,5 m } \\
\text { Aug 99 }\end{array}$ & $\begin{array}{l}\text { CML 39,5 m } \\
\text { Aug 99 }\end{array}$ \\
\hline & WP KB & WP KB & WP KB & WP KB & WP KB & WP KB & WP KB \\
\hline $\mathrm{Al}(\mathrm{OH})_{3}$ am & & & & & 0.459 & 0.862 & -1.625 \\
\hline Diaspor & & & & & 4.411 & 4.814 & 2.326 \\
\hline Alunit & & & & & -25.895 & -24.638 & -40.09 \\
\hline Jurb-AIOHSO $_{4}$ & & & & & 0.787 & 1.134 & -3.265 \\
\hline $\mathrm{Al}_{4}(\mathrm{OH})_{10} \mathrm{SO}_{4}$ & & & & & 9.63 & 11.186 & -0.675 \\
\hline Gibbsit & & & & & 3.015 & 3.418 & 0.931 \\
\hline Ferrihydrit & & 4.079 & 1.854 & 1.442 & & & \\
\hline Goethit & & 6.818 & 4.592 & 4.18 & & & \\
\hline Hämatit & & 16.013 & 11.561 & 10.738 & & & \\
\hline Melanterit & & -2.546 & -7.691 & -7.891 & & -2.146 & -4.049 \\
\hline K-Jarosit & & 10.336 & -0.435 & -1.678 & & & \\
\hline Magnetit & & 20.693 & 13.954 & 12.784 & & & \\
\hline Lepidocrocit & & 6.119 & 3.893 & 3.481 & & & \\
\hline $\mathrm{Cu}(\mathrm{OH}) 2$ & & & -1.724 & -1.825 & & -4.074 & -3.595 \\
\hline Chalcanthit & & -7.233 & -7.636 & -7.591 & & -6.667 & -8.098 \\
\hline Tenorit & & & -0.719 & -0.82 & & -3.068 & -2.59 \\
\hline Kupferferrit & & 13.027 & 11.031 & 10.106 & & & \\
\hline Cölestin & \begin{tabular}{l|l|}
-0.643 \\
\end{tabular} & -0.469 & -0.564 & -0.628 & -0.31 & -0.073 & -0.426 \\
\hline \multicolumn{8}{|l|}{$\mathrm{SbO}_{2}$} \\
\hline Gips & -0.17 & -0.403 & -0.302 & -0.431 & -0.079 & -0.099 & -0.204 \\
\hline Baryt & 0.834 & 0.426 & 0.473 & 0.529 & 0.764 & 0.914 & -0.431 \\
\hline Pyrolusit & -9.876 & -12.402 & -10.253 & -10.774 & -15.937 & -15.848 & -15.571 \\
\hline Manganit & $\mid-4.213$ & -6.46 & -4.373 & -4.828 & -8.351 & -8.262 & -7.685 \\
\hline $\mathrm{Cr}(\mathrm{OH})_{3}$ (am) & 1.618 & 0.164 & 1.359 & 0.84 & -1.836 & $\begin{array}{l}-1.082 \\
\end{array}$ & -0.432 \\
\hline $\mathrm{Cr}_{2} \mathrm{O}_{3}$ & 3.943 & 1.035 & 3.425 & 2.386 & -2.964 & -1.455 & -0.158 \\
\hline $\mathrm{Cr}(\mathrm{OH})_{3}$ & -0.556 & -2.01 & -0.815 & -1.335 & -4.011 & -3.256 & -2.607 \\
\hline $\mathrm{FeCr}_{2} \mathrm{O}_{4}$ & & 0.949 & 1.05 & -0.334 & -3.34 & -1.601 & -0.298 \\
\hline
\end{tabular}

\begin{tabular}{|c|c|c|c|c|c|c|c|}
\hline & \begin{tabular}{|l|} 
CML 43,5 m \\
Aug 99
\end{tabular} & RCO3 Aug 99 & RCO4 Aug 99 & \begin{tabular}{|l|} 
RCO12 \\
Aug 99 \\
\end{tabular} & \begin{tabular}{|l|} 
RCO15 \\
Aug 99 \\
\end{tabular} & \begin{tabular}{|l|} 
RCO17 \\
Aug 99 \\
\end{tabular} & \begin{tabular}{|l|} 
RCO18 \\
Aug 99 \\
\end{tabular} \\
\hline & WP KB & WP KB & WP KB & WP KB & WP KB & WP KB & WP KB \\
\hline $\mathrm{Al}(\mathrm{OH})_{3}$ am & -1.539 & -1.505 & -1.067 & -1.032 & -0.727 & -1.64 & -1.223 \\
\hline Diaspor & 2.412 & 2.446 & 2.884 & 2.919 & 3.223 & 2.311 & 2.728 \\
\hline Alunit & -32.893 & -25.477 & -33.585 & -31.709 & -35.369 & -37.803 & $\begin{array}{l}-35.332 \\
\end{array}$ \\
\hline Jurb-AIOHSO $_{4}$ & -1.669 & 0.118 & $\begin{array}{l}-1.572 \\
\end{array}$ & -1.139 & -1.835 & -2.611 & -2.034 \\
\hline $\mathrm{Al}_{4}(\mathrm{OH})_{10} \mathrm{SO}_{4}$ & 1.178 & 3.069 & 2.691 & 3.229 & 3.448 & -0.064 & 1.762 \\
\hline Gibbsit & 1.016 & 1.051 & 1.488 & 1.523 & 1.828 & 0.916 & 1.333 \\
\hline Ferrihydrit & & & 1.57 & & 1.015 & 1.068 & \\
\hline Goethit & & & 4.308 & & 3.753 & 3.806 & \\
\hline Hämatit & & & 10.994 & & 9.883 & 9.989 & \\
\hline Melanterit & \begin{tabular}{|c|}
-5.22 \\
\end{tabular} & -2.474 & -3.244 & -3.595 & -6.74 & -6.568 & -4.837 \\
\hline K-Jarosit & & & 2.599 & & -0.67 & -0.209 & \\
\hline Magnetit & & & 15.291 & & 11.284 & 11.426 & \\
\hline Lepidocrocit & & & 3.609 & & 3.054 & 3.107 & \\
\hline $\mathrm{Cu}(\mathrm{OH}) 2$ & -6.031 & -5.873 & -3.692 & -4.128 & -3.687 & -3.821 & -3.634 \\
\hline Chalcanthit & -9.023 & -7.114 & \begin{tabular}{|c|}
-7.06 \\
\end{tabular} & -7.098 & -7.656 & -7.654 & -7.307 \\
\hline Tenorit & -5.026 & -4.868 & \begin{tabular}{|l|}
-5.687 \\
\end{tabular} & \begin{tabular}{|l|}
-3.123 \\
\end{tabular} & -2.682 & -2.816 & -2.629 \\
\hline Kupferferrit & & & 8.496 & & 7.389 & 7.362 & \\
\hline Cölestin & -0.562 & -0.137 & -0.099 & & -0.682 & -0.401 & -0.284 \\
\hline $\mathrm{SbO}_{2}$ & & & & 1.038 & & & \\
\hline Gips & -0.338 & -0.062 & -0.019 & -0.022 & -0.337 & -0.193 & -0.104 \\
\hline Baryt & -0.398 & 0.75 & 0.675 & 0.763 & -0.096 & 0.646 & -0.539 \\
\hline Pyrolusit & -22.397 & -18.923 & -7.672 & -17.42 & -12.076 & -12.089 & -16.086 \\
\hline Manganit & $\mid-11.957$ & -10.568 & -8.744 & -9.126 & -6.348 & -6.378 & -8.436 \\
\hline $\mathrm{Cr}(\mathrm{OH})_{3}(\mathrm{am})$ & -1.603 & -2.035 & -1.828 & -0.68 & -0.344 & -0.469 & -0.965 \\
\hline $\mathrm{Cr}_{2} \mathrm{O}_{3}$ & -2.499 & -3.362 & -2.948 & -0.653 & 0.019 & -0.231 & -1.223 \\
\hline $\mathrm{Cr}(\mathrm{OH})_{3}$ & -3.777 & -4.209 & -4.002 & -2.855 & -2.518 & -2.643 & -3.14 \\
\hline $\mathrm{FeCr}_{2} \mathrm{O}_{4}$ & -5.322 & -5.189 & -3.419 & -1.872 & -3.347 & -3.561 & -2.981 \\
\hline
\end{tabular}


Tab. A.16. (Forts.): Sättigungsindizes einiger wichtiger Festphasen in Proben aus Cospuden berechnet mit MINTEQA2; AP = Proben aus der Arbeit von PLEßOW (1998), KB = Proben aus der vorliegenden Arbeit; PL = Porenlösung, WP = Wasserprobe.

\begin{tabular}{|c|c|c|c|c|c|c|c|}
\hline & See Aug 99 & $\begin{array}{l}\text { SeeSed } \\
\text { Aug } 99\end{array}$ & $\begin{array}{l}\text { Sedgepr } \\
\text { Aug } 99 \\
\end{array}$ & $\begin{array}{|ll|}\text { CML } 20 & \mathrm{~m} \\
\text { Jan 00 } & \\
\end{array}$ & $\begin{array}{l}\text { CML 27,5 m } \\
\text { Jan 00 }\end{array}$ & $\begin{array}{l}\text { CML 31,5 m } \\
\text { Jan 00 }\end{array}$ & $\begin{array}{l}\text { CML 39,5 m } \\
\text { Jan 00 }\end{array}$ \\
\hline & WP KB & WP KB & WP KB & WP KB & WP KB & WP KB & WP KB \\
\hline $\mathrm{Al}(\mathrm{OH})_{3}$ am & -0.801 & & -1.149 & & -7.513 & -7.47 & \\
\hline Diaspor & 3.15 & & 2.802 & & -3.561 & -3.518 & \\
\hline Alunit & -38.985 & & -37.45 & & -26.418 & -24.409 & \\
\hline Jurb-AIOHSO $_{4}$ & -2.671 & & -2.48 & & -2.018 & -1.59 & \\
\hline $\mathrm{Al}_{4}(\mathrm{OH})_{10} \mathrm{SO}_{4}$ & 2.392 & & 1.537 & & -17.093 & -16.535 & \\
\hline Gibbsit & 1.755 & & 1.406 & & -4.957 & -4.914 & \\
\hline Ferrihydrit & & & & & & & 1.936 \\
\hline Goethit & & & & & & & 4.675 \\
\hline Hämatit & & & & & & & 11.727 \\
\hline Melanterit & -6.599 & -6.405 & -6.703 & & & -2.445 & -7.332 \\
\hline K-Jarosit & & & & & & & 0.134 \\
\hline Magnetit & & & & & & & 14.25 \\
\hline Lepidocrocit & & & & & & & 3.976 \\
\hline $\mathrm{Cu}(\mathrm{OH}) 2$ & -2.974 & -2.943 & -2.825 & & & -10.162 & -2.205 \\
\hline Chalcanthit & -7.707 & -7.295 & -7.018 & & & & -7.887 \\
\hline Tenorit & -1.969 & -1.938 & -1.82 & & & -9.156 & -1.2 \\
\hline Kupferferrit & & & & & & & 10.716 \\
\hline Cölestin & -0.758 & -0.85 & -1.04 & -0.498 & -0.247 & -0.223 & -0.334 \\
\hline $\mathrm{SbO}_{2}$ & & -5.119 & -4.511 & & & & \\
\hline Gips & -0.338 & -0.339 & -0.452 & 0.032 & -0.024 & -0.145 & -0.149 \\
\hline Baryt & -0.288 & 0.571 & 0.914 & 0.66 & 0.361 & 0.703 & 0.329 \\
\hline Pyrolusit & -5.27 & & -8.14 & -15.856 & -33.152 & -18.573 & -10.587 \\
\hline Manganit & -2.613 & & -5.483 & -8.799 & -19.665 & -12.571 & -4.659 \\
\hline $\mathrm{Cr}(\mathrm{OH})_{3}$ (am) & -0.955 & -0.217 & 1.009 & -0.717 & & & 0.306 \\
\hline $\mathrm{Cr}_{2} \mathrm{O}_{3}$ & -1.203 & 0.272 & 2.725 & -0.727 & & & 1.319 \\
\hline $\mathrm{Cr}(\mathrm{OH})_{3}$ & -3.129 & -2.392 & -1.165 & -2.892 & & & -1.868 \\
\hline $\mathrm{FeCr}_{2} \mathrm{O}_{4}$ & -3.664 & -2.376 & -0.381 & -4.442 & & & -0.924 \\
\hline
\end{tabular}

\begin{tabular}{|c|c|c|c|c|c|c|c|}
\hline & \begin{tabular}{|l|} 
CML 43,5 m \\
Jan 00 \\
WP KB
\end{tabular} & \begin{tabular}{|l|} 
RCO3 Jan 00 \\
WP KB
\end{tabular} & \begin{tabular}{|l|} 
RCO4 Jan 00 \\
WP KB
\end{tabular} & \begin{tabular}{|l|} 
RCO12 \\
Jan 00 \\
WP KB
\end{tabular} & \begin{tabular}{|l|} 
RCO15 J \\
an 00 \\
WP KB
\end{tabular} & \begin{tabular}{|l|} 
RCO17 \\
Jan 00 \\
WP KB
\end{tabular} & \begin{tabular}{|l|}
$\begin{array}{l}\text { RCO18 } \\
\text { Jan 00 }\end{array}$ \\
WP KB
\end{tabular} \\
\hline $\mathrm{Al}(\mathrm{OH})_{3} \mathrm{am}$ & -1.974 & -9.749 & -1.737 & -1.237 & & -1.08 & \\
\hline Diaspor & 1.977 & -5.798 & 2.215 & 2.714 & & 2.871 & \\
\hline Alunit & -43.149 & -25.046 & -39.162 & -40.725 & & -34.932 & \\
\hline $\mathrm{Jurb}-\mathrm{AlOHSO}_{4}$ & -4.063 & -2.533 & -2.963 & -2.721 & & -1.839 & \\
\hline $\mathrm{Al}_{4}(\mathrm{OH})_{10} \mathrm{SO}_{4}$ & -2.52 & -24.315 & -0.708 & 1.033 & & 2.385 & \\
\hline Gibbsit & 0.582 & -7.193 & 0.819 & 1.318 & & 1.475 & \\
\hline Ferrihydrit & 1.303 & & & & 0.356 & & \\
\hline Goethit & 4.042 & & & & 3.095 & & \\
\hline Hämatit & 10.46 & & & & 8.566 & & \\
\hline Melanterit & -7.194 & -2.784 & -7.419 & & -7.446 & & -6.951 \\
\hline K-Jarosit & -0.845 & & & & -3.458 & & \\
\hline Magnetit & 12.39 & & & & 10.027 & & \\
\hline Lepidocrocit & 3.343 & & & & 2.396 & & \\
\hline $\mathrm{Cu}(\mathrm{OH}) 2$ & -2.899 & -11.6 & -3.486 & -3.637 & -2.067 & -3.966 & -2.635 \\
\hline Chalcanthit & -7.85 & -7.248 & -7.575 & -7.983 & -6.801 & -7.587 & -7.915 \\
\hline Tenorit & -1.894 & -10.595 & -2.481 & -2.632 & -1.062 & -2.961 & -1.63 \\
\hline Kupferferrit & 8.756 & & & & 7.694 & & \\
\hline Cölestin & -0.496 & -0.191 & -0.224 & -0.209 & -0.49 & -0.27 & -0.323 \\
\hline $\mathrm{SbO}_{2}$ & -1.741 & & & & & -0.008 & -0.121 \\
\hline Gips & -0.282 & -0.02 & 0.019 & -0.396 & -0.195 & -0.082 & \\
\hline Baryt & 0.06 & 0.757 & 0.116 & 0.088 & 0.355 & 0.556 & 0.437 \\
\hline Pyrolusit & -11.625 & -20.484 & -12.275 & -12.666 & -12.625 & -16.835 & -11.461 \\
\hline Manganit & -5.658 & -14.16 & -6.008 & -6.338 & -6.179 & -9.907 & -5.367 \\
\hline $\mathrm{Cr}(\mathrm{OH})_{3}(\mathrm{am})$ & & -10.382 & -0.507 & -0.143 & -0.193 & -0.785 & -0.265 \\
\hline $\mathrm{Cr}_{2} \mathrm{O}_{3}$ & & -20.057 & -0.306 & 0.422 & 0.321 & -0.862 & 0.176 \\
\hline $\mathrm{Cr}(\mathrm{OH})_{3}$ & & -12.557 & -2.681 & -2.317 & -2.367 & -2.959 & -2.44 \\
\hline $\mathrm{FeCr}_{2} \mathrm{O}_{4}$ & & -27.786 & -4.23 & & -2.985 & & -2.09 \\
\hline
\end{tabular}


Tab. A.17.: Sättigungsindizes einiger wichtiger Festphasen in Proben aus der Lausitz berechnet mit MINTEQA2; AP = Proben aus der Arbeit von PLEßOW (1998), KB = Proben aus der vorliegenden Arbeit; PL = Porenlösung, WP = Wasserprobe.

\begin{tabular}{|c|c|c|c|c|c|c|c|}
\hline & \begin{tabular}{|l|} 
Lausitz \\
$5,00 \mathrm{~m}$ \\
WP KB
\end{tabular} & \begin{tabular}{|l|} 
Lausitz \\
$5,21 \mathrm{~m}$ \\
WP KB
\end{tabular} & \begin{tabular}{|l|} 
Lausitz \\
$5,30 \mathrm{~m}$ \\
WP KB
\end{tabular} & \begin{tabular}{|l|} 
Lausitz \\
$5,50 \mathrm{~m}$ \\
WP KB
\end{tabular} & \begin{tabular}{|l|} 
Lausitz \\
$5,60 \mathrm{~m}$ \\
WP KB
\end{tabular} & \begin{tabular}{|l|} 
Lausitz \\
$5,75 \mathrm{~m}$ \\
WP KB
\end{tabular} & \begin{tabular}{|l|} 
Lausitz \\
$5,93 \mathrm{~m}$ \\
WP KB
\end{tabular} \\
\hline $\mathrm{Al}(\mathrm{OH})_{3} \mathrm{am}$ & \begin{tabular}{|l|} 
WP KB \\
-4.598
\end{tabular} & \begin{tabular}{|l|} 
WP KB \\
-4.328
\end{tabular} & \begin{tabular}{|l|} 
WP KB \\
-4.429
\end{tabular} & \begin{tabular}{|l|} 
WP KB \\
-4.454
\end{tabular} & $\begin{array}{l}\text { WP KB } \\
-4.982\end{array}$ & $\begin{array}{r}\text { WP KB } \\
-4.947\end{array}$ & \begin{tabular}{|l} 
WP KB \\
-4.918
\end{tabular} \\
\hline Diaspor & -0.647 & -0.401 & -0.478 & -0.502 & -1.03 & -0.996 & -0.967 \\
\hline Alunit & -17.793 & -17.395 & -18.192 & -18.216 & -17.973 & -17.886 & -18.648 \\
\hline $\mathrm{Jurb}^{\mathrm{AlOHSO}}{ }_{4}$ & 0.779 & 0.693 & 0.748 & 0.746 & 0.633 & 0.644 & 0.499 \\
\hline $\mathrm{Al}_{4}(\mathrm{OH})_{10} \mathrm{SO}_{4}$ & -5.551 & -5.821 & -5.075 & -5.15 & -6.847 & -6.733 & -6.789 \\
\hline Gibbsit & -2.043 & -1.819 & -1.874 & -1.898 & -2.426 & -2.392 & -2.362 \\
\hline \multicolumn{8}{|l|}{ Ferrihydrit } \\
\hline \multicolumn{8}{|l|}{ Goethit } \\
\hline \multicolumn{8}{|l|}{ Hämatit } \\
\hline Melanterit & -3.544 & & & -2.848 & -2.898 & -3.228 & -2.562 \\
\hline \multicolumn{8}{|l|}{ K-Jarosit } \\
\hline \multicolumn{8}{|l|}{ Magnetit } \\
\hline \multicolumn{8}{|l|}{ Lepidocrocit } \\
\hline $\mathrm{Cu}(\mathrm{OH}) 2$ & -8.88 & -8.338 & -8.523 & -8.447 & -9.409 & -8.643 & \\
\hline Chalcanthit & -6.366 & & -6.209 & -6.111 & -6.658 & -5.915 & -6.66 \\
\hline Tenorit & -7.875 & -7.307 & -7.518 & -7.442 & -8.403 & -7.637 & \\
\hline \multicolumn{8}{|l|}{ Kupferferrit } \\
\hline Cölestin & -0.276 & -0.277 & -0.291 & -0.283 & -0.227 & -0.393 & -0.424 \\
\hline $\mathrm{SbO}_{2}$ & & 1.881 & 1.696 & 1.66 & 1.92 & 1.906 & 2.105 \\
\hline Gips & -0.043 & -0.036 & -0.049 & -0.073 & -0.022 & -0.066 & -0.097 \\
\hline Baryt & 0.925 & 0.808 & 0.855 & 0.97 & 0.985 & 0.864 & 0.983 \\
\hline Pyrolusit & -18.096 & -17.553 & -18.02 & -17.944 & -18.45 & -10.288 & -18.23 \\
\hline Manganit & -12.071 & -12.199 & -11.94 & -11.898 & -12.342 & -12.295 & -12.153 \\
\hline $\mathrm{Cr}(\mathrm{OH})_{3}(\mathrm{am})$ & -6.87 & -6.357 & -6.58 & -6.589 & -7.107 & -6.742 & -6.91 \\
\hline $\mathrm{Cr}_{2} \mathrm{O}_{3}$ & -13.032 & -11.855 & -12.452 & -12.47 & -13.505 & -12.777 & -13.112 \\
\hline $\mathrm{Cr}(\mathrm{OH})_{3}$ & -9.044 & -8.442 & -8.754 & -8.763 & -9.281 & -8.917 & -9.084 \\
\hline $\mathrm{FeCr}_{2} \mathrm{O}_{4}$ & -19.684 & -18.222 & -18.422 & -18.248 & -19.747 & -19.327 & -18.821 \\
\hline
\end{tabular}

\begin{tabular}{|c|c|c|c|c|c|c|}
\hline & Lausitz 21,07 m & Lausitz 21,13 m & Lausitz 21,40 m & Lausitz 21,70 m & Lausitz 21,80 m & \begin{tabular}{|l|} 
Lausitz 21,90 m \\
WP KB
\end{tabular} \\
\hline $\mathrm{Al}(\mathrm{OH})_{3} \mathrm{am}$ & -4.347 & -4.594 & -4.658 & -4.498 & -4.521 & -7.493 \\
\hline Diaspor & -0.396 & -0.643 & -0.707 & -0.547 & -0.57 & -3.542 \\
\hline Alunit & -21.545 & -20.774 & -19.962 & -21.699 & -21.123 & -20.166 \\
\hline $\mathrm{Jurb}^{-\mathrm{AlOHSO}}{ }_{4}$ & 0.068 & 0.12 & 0.199 & -0.022 & 0.084 & -0.735 \\
\hline $\mathrm{Al}_{4}(\mathrm{OH})_{10} \mathrm{SO}_{4}$ & -5.509 & -6.198 & -6.309 & -6.052 & -6.015 & -15.75 \\
\hline Gibbsit & -1.792 & -2.039 & -2.102 & -1.943 & -1.966 & -4.938 \\
\hline \multicolumn{7}{|l|}{ Ferrihydrit } \\
\hline \multicolumn{7}{|l|}{ Goethit } \\
\hline \multicolumn{7}{|l|}{ Hämatit } \\
\hline Melanterit & -2.766 & -3.135 & -3.272 & -2.872 & -3.03 & -3.424 \\
\hline \multicolumn{7}{|l|}{ K-Jarosit } \\
\hline \multicolumn{7}{|l|}{ Magnetit } \\
\hline \multicolumn{7}{|l|}{ Lepidocrocit } \\
\hline $\mathrm{Cu}(\mathrm{OH}) 2$ & -8.686 & -8.064 & -8.418 & & & -10.447 \\
\hline Chalcanthit & -7.134 & -6.213 & -6.424 & & & -6.552 \\
\hline Tenorit & -7.681 & -7.059 & -7.413 & & & -9.441 \\
\hline \multicolumn{7}{|l|}{ Kupferferrit } \\
\hline Cölestin & -0.353 & -0.277 & -0.369 & -0.491 & -0.526 & -0.481 \\
\hline $\mathrm{SbO}_{2}$ & & 1.388 & & & & \\
\hline Gips & 0.023 & 0.159 & 0.061 & 0.013 & -0.022 & 0.043 \\
\hline Baryt & 1.156 & 1.602 & 1.306 & 1.116 & 1.069 & 1.198 \\
\hline Pyrolusit & -17.202 & -17.384 & -18.126 & -17.265 & -17.421 & -19.527 \\
\hline Manganit & -11.134 & -11.323 & -11.852 & -11.235 & -11.397 & -13.472 \\
\hline $\mathrm{Cr}(\mathrm{OH})_{3}(\mathrm{am})$ & -5.782 & -6.195 & -6.711 & -6.759 & -7.002 & -9.522 \\
\hline $\mathrm{Cr}_{2} \mathrm{O}_{3}$ & -10.858 & -11.682 & -12.715 & -12.811 & -13.296 & -18.336 \\
\hline $\mathrm{Cr}(\mathrm{OH})_{3}$ & -7.957 & -8.369 & -8.885 & -8.934 & -9.176 & -11.696 \\
\hline $\mathrm{FeCr}_{2} \mathrm{O}_{4}$ & -15.77 & -17.262 & -18.575 & -17.891 & -18.663 & -26.249 \\
\hline
\end{tabular}


Tab. A.18.: Statistische Auswertung der Ultrafiltration für Proben aus Cospuden; Probenanzahl mit Anteil (\% der Gesamtkonzentration) im Durchlauf.

\begin{tabular}{|c|c|c|c|c|c|c|c|c|c|c|c|}
\hline Element & Anzahl d & r Proben & mit Anteile & n (\%) im I & Durchlauf & von & & & & & $\Sigma$ Anzahl \\
\hline & $0-9$ & $10-19$ & $20-29$ & \begin{tabular}{|l|}
$30-39$ \\
\end{tabular} & \begin{tabular}{|l|}
$40-49$ \\
\end{tabular} & \begin{tabular}{|c|}
$50-59$ \\
\end{tabular} & $60-69$ & \begin{tabular}{|c|}
$70-79$ \\
\end{tabular} & \begin{tabular}{|l|}
$80-89$ \\
\end{tabular} & \begin{tabular}{|l|}
$90-100$ \\
\end{tabular} & \\
\hline Sulfat & 15 & 3 & 6 & 3 & 1 & 1 & 1 & 2 & 8 & 24 & 64 \\
\hline DOC & 6 & 6 & 3 & 4 & 4 & 2 & 6 & 8 & 7 & 8 & 54 \\
\hline $\mathbf{L i}$ & 15 & 11 & 3 & 0 & 0 & 0 & 1 & 4 & 4 & 32 & 70 \\
\hline $\mathbf{B e}$ & 7 & 7 & 0 & 1 & 0 & 2 & 3 & 1 & 2 & 6 & 29 \\
\hline $\mathrm{Na}$ & 23 & 5 & 1 & 1 & 0 & 0 & 0 & 2 & 8 & 31 & 71 \\
\hline Mg & 18 & 9 & 0 & 1 & 0 & 1 & 0 & 4 & 6 & 30 & 69 \\
\hline Al & 10 & 4 & 3 & 9 & 0 & 4 & 2 & 3 & 3 & 9 & 47 \\
\hline $\mathbf{K}$ & 19 & 8 & 1 & 0 & 1 & 1 & 1 & 5 & 6 & 28 & 70 \\
\hline $\mathbf{C a}$ & 22 & 2 & 0 & 2 & 0 & 2 & 0 & 3 & 11 & 26 & 68 \\
\hline Sc & 9 & 12 & 5 & 1 & 2 & 0 & 1 & 2 & 4 & 12 & 48 \\
\hline $\mathbf{T i}$ & 5 & 2 & 3 & 2 & 3 & 0 & 1 & 4 & 1 & 1 & 22 \\
\hline $\mathbf{V}$ & 13 & 12 & 4 & 0 & 3 & 2 & 1 & 7 & 3 & 9 & 54 \\
\hline $\mathrm{Cr}$ & 16 & 9 & 5 & 3 & 0 & 2 & 4 & 4 & 6 & 12 & 61 \\
\hline Mn & 18 & 5 & 1 & 2 & 0 & 3 & 0 & 3 & 6 & 28 & 66 \\
\hline $\mathbf{F e}$ & 9 & 5 & 3 & 3 & 7 & 4 & 5 & 3 & 7 & 12 & 58 \\
\hline Co & 12 & 4 & 9 & 2 & 0 & 2 & 3 & 4 & 5 & 18 & 59 \\
\hline $\mathbf{N i}$ & 12 & 6 & 5 & 2 & 1 & 1 & 2 & 1 & 6 & 17 & 53 \\
\hline $\mathbf{C u}$ & 9 & 11 & 8 & 0 & 3 & 1 & 1 & 0 & 3 & 14 & 50 \\
\hline $\mathrm{Zn}$ & 13 & 10 & 1 & 2 & 1 & 1 & 2 & 1 & 2 & 11 & 44 \\
\hline As & 15 & 10 & 4 & 1 & 4 & 3 & 2 & 0 & 7 & 7 & 53 \\
\hline $\mathbf{R b}$ & 6 & 19 & 0 & 1 & 1 & 1 & 3 & 5 & 5 & 17 & 58 \\
\hline $\mathbf{S r}$ & 17 & 6 & 1 & 1 & 0 & 1 & 0 & 6 & 9 & 26 & 67 \\
\hline $\mathbf{Y}$ & 10 & 9 & 10 & 3 & 2 & 0 & 1 & 3 & 8 & 13 & 59 \\
\hline $\mathbf{Z r}$ & 1 & 2 & 1 & 2 & 10 & 2 & 3 & 5 & 9 & 15 & 50 \\
\hline $\mathrm{Nb}$ & 16 & 2 & 2 & 1 & 4 & 2 & 3 & 1 & 1 & 1 & 33 \\
\hline Mo & 24 & 4 & 2 & 0 & 1 & 0 & 1 & 1 & 9 & 13 & 55 \\
\hline Cd & 10 & 6 & 7 & 4 & 0 & 1 & 0 & 3 & 4 & 5 & 40 \\
\hline Sn & 8 & 5 & 2 & 0 & 1 & 0 & 2 & 2 & 2 & 5 & 27 \\
\hline $\mathbf{S b}$ & 22 & 4 & 2 & 1 & 1 & 1 & 0 & 1 & 2 & 6 & 40 \\
\hline Cs & 6 & 10 & 0 & 1 & 0 & 0 & 2 & 1 & 5 & 6 & 31 \\
\hline $\mathbf{B a}$ & 17 & 10 & 3 & 1 & 0 & 1 & 1 & 6 & 7 & 24 & 70 \\
\hline La & 6 & 6 & 3 & 0 & 3 & 3 & 3 & 2 & 1 & 6 & 33 \\
\hline $\mathrm{Ce}$ & 7 & 8 & 1 & 2 & 3 & 0 & 2 & 3 & 0 & 3 & 29 \\
\hline Pr & 4 & 7 & 0 & 2 & 2 & 1 & 1 & 1 & 0 & 1 & 19 \\
\hline Nd & 6 & 6 & 1 & 4 & 1 & 1 & 3 & 4 & 0 & 4 & 30 \\
\hline Sm & 7 & 7 & 1 & 0 & 1 & 0 & 4 & 2 & 1 & 8 & 31 \\
\hline Eu & 6 & 7 & 1 & 1 & 1 & 0 & 2 & 3 & 0 & 2 & 23 \\
\hline Gd & 7 & 3 & 2 & 0 & 0 & 2 & 2 & 1 & 2 & 0 & 19 \\
\hline $\mathbf{T b}$ & 6 & 4 & 1 & 1 & 0 & 1 & 1 & 1 & 0 & 1 & 16 \\
\hline Dy & 6 & 9 & 1 & 1 & 0 & 3 & 2 & 2 & 0 & 1 & 25 \\
\hline Ho & 6 & 3 & 1 & 2 & 0 & 1 & 0 & 2 & 1 & 0 & 16 \\
\hline Er & 6 & 8 & 1 & 1 & 1 & 0 & 2 & 1 & 0 & 1 & 21 \\
\hline Tm & 11 & 0 & 2 & 1 & 0 & 0 & 0 & 2 & 1 & 1 & 18 \\
\hline $\mathbf{Y b}$ & 6 & 6 & 2 & 1 & 1 & 1 & 0 & 1 & 1 & 2 & 21 \\
\hline $\mathbf{L u}$ & 5 & 5 & 5 & 0 & 0 & 0 & 0 & 1 & 0 & 2 & 18 \\
\hline Hf & 12 & 2 & 0 & 1 & 1 & 1 & 0 & 1 & 0 & 0 & 18 \\
\hline Ta & 0 & 1 & 2 & 2 & 2 & 0 & 2 & 4 & 0 & 5 & 18 \\
\hline $\mathbf{W}$ & 1 & 0 & 0 & 1 & 2 & 2 & 2 & 0 & 2 & 3 & 13 \\
\hline $\mathrm{Hg}$ & 0 & 0 & 1 & 2 & 0 & 2 & 5 & 2 & 1 & 10 & 23 \\
\hline Tl & 6 & 0 & 0 & 0 & 1 & 1 & 1 & 1 & 3 & 3 & 16 \\
\hline $\mathbf{P b}$ & 10 & 6 & 3 & 2 & 1 & 0 & 4 & 3 & 2 & 8 & 39 \\
\hline $\mathbf{B i}$ & 0 & 0 & 0 & 0 & 0 & 0 & 1 & 1 & 0 & 1 & 3 \\
\hline Th & 3 & 1 & 9 & 4 & 3 & 5 & 1 & 1 & 2 & 1 & 30 \\
\hline $\mathbf{U}$ & 8 & 6 & 10 & 3 & 1 & 2 & 1 & 5 & 7 & 10 & 53 \\
\hline
\end{tabular}


Tab. A.19.: Statistische Auswertung der Ultrafiltration für Proben aus der Lausitz; Probenanzahl mit Anteil (\% der Gesamtkonzentration) im Durchlauf.

\begin{tabular}{|c|c|c|c|c|c|c|c|c|c|c|c|}
\hline Element & Anzahl d & r Proben & mit Anteile & n (\%) im I & Durchlauf & von & & & & & $\Sigma$ Anzahl \\
\hline & $0-9$ & $10-19$ & $20-29$ & \begin{tabular}{|l|}
$30-39$ \\
\end{tabular} & $40-49$ & \begin{tabular}{|c|}
$50-59$ \\
\end{tabular} & $60-69$ & \begin{tabular}{|c|}
$70-79$ \\
\end{tabular} & \begin{tabular}{|c|}
$80-89$ \\
\end{tabular} & \begin{tabular}{|l|}
$90-100$ \\
\end{tabular} & \\
\hline Sulfat & 0 & 0 & 0 & 1 & 0 & 0 & 0 & 1 & 5 & 3 & 10 \\
\hline DOC & 0 & 0 & 1 & 1 & 0 & 0 & 0 & 1 & 1 & 1 & 5 \\
\hline $\mathbf{L i}$ & 0 & 0 & 0 & 0 & 0 & 0 & 0 & 0 & 4 & 6 & 10 \\
\hline $\mathbf{B e}$ & 0 & 0 & 0 & 0 & 0 & 0 & 0 & 0 & 7 & 2 & 9 \\
\hline $\mathrm{Na}$ & 0 & 0 & 0 & 0 & 0 & 0 & 0 & 0 & 6 & 3 & 9 \\
\hline Mg & 0 & 0 & 0 & 0 & 0 & 0 & 0 & 1 & 6 & 3 & 10 \\
\hline Al & 0 & 0 & 0 & 0 & 0 & 0 & 0 & 2 & 5 & 3 & 10 \\
\hline $\mathbf{K}$ & 0 & 0 & 0 & 0 & 0 & 0 & 2 & 0 & 5 & 3 & 10 \\
\hline $\mathbf{C a}$ & 0 & 0 & 0 & 0 & 0 & 0 & 0 & 0 & 7 & 3 & 10 \\
\hline Sc & 0 & 0 & 0 & 0 & 0 & 1 & 0 & 2 & 2 & 2 & 7 \\
\hline $\mathbf{T i}$ & 0 & 0 & 0 & 0 & 0 & 1 & 0 & 0 & 2 & 1 & 4 \\
\hline V & 0 & 0 & 1 & 0 & 1 & 0 & 1 & 2 & 2 & 1 & 8 \\
\hline $\mathrm{Cr}$ & 0 & 1 & 0 & 0 & 0 & 0 & 0 & 3 & 3 & 3 & 10 \\
\hline Mn & 0 & 0 & 0 & 0 & 0 & 0 & 0 & 0 & 6 & 3 & 9 \\
\hline $\mathbf{F e}$ & 0 & 0 & 0 & 0 & 0 & 0 & 0 & 0 & 7 & 4 & 11 \\
\hline Co & 0 & 0 & 0 & 0 & 0 & 0 & 1 & 0 & 4 & 4 & 9 \\
\hline $\mathbf{N i}$ & 0 & 0 & 0 & 0 & 0 & 0 & 1 & 0 & 5 & 3 & 9 \\
\hline $\mathbf{C u}$ & 0 & 0 & 0 & 0 & 0 & 0 & 0 & 1 & 0 & 3 & 4 \\
\hline $\mathrm{Zn}$ & 0 & 0 & 0 & 0 & 0 & 0 & 0 & 0 & 5 & 3 & 8 \\
\hline As & 0 & 0 & 0 & 0 & 0 & 1 & 2 & 0 & 1 & 5 & 9 \\
\hline $\mathbf{R b}$ & 0 & 1 & 5 & 0 & 0 & 1 & 0 & 0 & 2 & 6 & 15 \\
\hline $\mathbf{S r}$ & 0 & 0 & 0 & 0 & 0 & 0 & 0 & 1 & 6 & 3 & 10 \\
\hline $\mathbf{Y}$ & 0 & 0 & 0 & 0 & 0 & 0 & 0 & 1 & 6 & 2 & 9 \\
\hline $\mathbf{Z r}$ & 1 & 0 & 0 & 0 & 1 & 0 & 1 & 1 & 4 & 2 & 10 \\
\hline $\mathrm{Nb}$ & 1 & 1 & 1 & 0 & 3 & 1 & 1 & 0 & 1 & 0 & 9 \\
\hline Mo & 0 & 0 & 0 & 0 & 0 & 0 & 1 & 1 & 2 & 3 & 7 \\
\hline Cd & 0 & 0 & 1 & 0 & 0 & 1 & 0 & 0 & 3 & 5 & 10 \\
\hline Sn & 0 & 0 & 0 & 0 & 0 & 0 & 0 & 0 & 0 & 0 & 0 \\
\hline $\mathbf{S b}$ & 0 & 0 & 0 & 0 & 0 & 0 & 0 & 0 & 2 & 2 & 4 \\
\hline Cs & 1 & 0 & 0 & 0 & 0 & 0 & 0 & 1 & 3 & 5 & 10 \\
\hline $\mathbf{B a}$ & 0 & 0 & 0 & 0 & 0 & 0 & 0 & 0 & 7 & 2 & 9 \\
\hline La & 0 & 0 & 0 & 0 & 0 & 0 & 0 & 1 & 4 & 3 & 8 \\
\hline $\mathrm{Ce}$ & 2 & 0 & 0 & 0 & 0 & 0 & 1 & 1 & 3 & 3 & 10 \\
\hline Pr & 1 & 0 & 0 & 0 & 0 & 0 & 1 & 0 & 4 & 3 & 9 \\
\hline Nd & 2 & 0 & 0 & 0 & 0 & 0 & 1 & 0 & 3 & 4 & 10 \\
\hline Sm & 2 & 0 & 0 & 0 & 0 & 0 & 1 & 0 & 3 & 3 & 9 \\
\hline Eu & 2 & 0 & 0 & 0 & 0 & 0 & 1 & 0 & 3 & 3 & 9 \\
\hline Gd & 2 & 0 & 0 & 0 & 0 & 0 & 1 & 0 & 3 & 4 & 10 \\
\hline $\mathbf{T b}$ & 2 & 0 & 0 & 0 & 0 & 0 & 1 & 0 & 4 & 3 & 10 \\
\hline Dy & 2 & 0 & 0 & 0 & 0 & 0 & 1 & 0 & 4 & 3 & 10 \\
\hline Ho & 2 & 0 & 0 & 0 & 0 & 0 & 1 & 0 & 3 & 4 & 10 \\
\hline Er & 2 & 0 & 0 & 0 & 0 & 0 & 1 & 0 & 3 & 4 & 10 \\
\hline Tm & 2 & 0 & 0 & 0 & 0 & 0 & 1 & 0 & 2 & 4 & 9 \\
\hline $\mathbf{Y b}$ & 2 & 0 & 0 & 0 & 0 & 0 & 1 & 0 & 4 & 2 & 9 \\
\hline $\mathbf{L u}$ & 2 & 0 & 0 & 0 & 0 & 0 & 0 & 1 & 3 & 3 & 9 \\
\hline Hf & 0 & 0 & 0 & 0 & 0 & 0 & 0 & 1 & 2 & 0 & 3 \\
\hline Ta & 0 & 1 & 0 & 1 & 1 & 2 & 1 & 1 & 0 & 0 & 7 \\
\hline $\mathbf{W}$ & 0 & 1 & 0 & 1 & 0 & 0 & 2 & 1 & 2 & 1 & 8 \\
\hline $\mathrm{Hg}$ & 0 & 0 & 0 & 0 & 1 & 0 & 0 & 0 & 0 & 0 & 1 \\
\hline Tl & 1 & 0 & 0 & 0 & 0 & 0 & 1 & 1 & 0 & 6 & 9 \\
\hline $\mathbf{P b}$ & 1 & 0 & 1 & 0 & 0 & 0 & 0 & 1 & 3 & 3 & 9 \\
\hline $\mathbf{B i}$ & 0 & 0 & 0 & 0 & 0 & 0 & 0 & 0 & 0 & 0 & 0 \\
\hline Th & 0 & 0 & 0 & 0 & 0 & 2 & 3 & 0 & 2 & 0 & 7 \\
\hline $\mathbf{U}$ & 1 & 0 & 0 & 0 & 0 & 0 & 0 & 2 & 5 & 2 & 10 \\
\hline
\end{tabular}


Tab. A.20.: Statistische Auswertung des Kationenaustausches für Proben aus Cospuden; Probenanzahl mit Anteil (\% der Gesamtkonzentration) im Durchlauf.

\begin{tabular}{|c|c|c|c|c|c|c|c|c|c|c|c|}
\hline Element & Anzahl d & r Proben & mit Anteile & n (\%) im I & Durchlauf & von & & & & & $\Sigma$ Anzahl \\
\hline & $0-9$ & $10-19$ & $20-29$ & \begin{tabular}{|l|}
$30-39$ \\
\end{tabular} & \begin{tabular}{|l|}
$40-49$ \\
\end{tabular} & \begin{tabular}{|c|}
$50-59$ \\
\end{tabular} & $60-69$ & \begin{tabular}{|c|}
$70-79$ \\
\end{tabular} & \begin{tabular}{|l|}
$80-89$ \\
\end{tabular} & \begin{tabular}{|l|}
$90-100$ \\
\end{tabular} & \\
\hline Sulfat & 14 & 3 & 7 & 4 & 2 & 6 & 1 & 2 & 12 & 22 & 73 \\
\hline DOC & 8 & 2 & 8 & 4 & 2 & 7 & 3 & 4 & 8 & 6 & 52 \\
\hline $\mathbf{L i}$ & 15 & 13 & 5 & 4 & 4 & 8 & 6 & 4 & 6 & 15 & 80 \\
\hline $\mathbf{B e}$ & 9 & 5 & 1 & 4 & 3 & 1 & 5 & 1 & 5 & 0 & 34 \\
\hline $\mathrm{Na}$ & 13 & 13 & 8 & 7 & 3 & 8 & 4 & 8 & 2 & 15 & 81 \\
\hline Mg & 11 & 9 & 8 & 12 & 9 & 4 & 2 & 8 & 4 & 12 & 79 \\
\hline Al & 6 & 5 & 6 & 5 & 3 & 10 & 3 & 7 & 4 & 5 & 54 \\
\hline $\mathbf{K}$ & 19 & 10 & 6 & 4 & 5 & 1 & 4 & 6 & 3 & 13 & 71 \\
\hline $\mathbf{C a}$ & 17 & 10 & 6 & 4 & 9 & 2 & 6 & 12 & 9 & 4 & 79 \\
\hline Sc & 6 & 4 & 5 & 10 & 2 & 7 & 2 & 4 & 8 & 9 & 57 \\
\hline $\mathbf{T i}$ & 7 & 5 & 1 & 2 & 4 & 2 & 0 & 2 & 5 & 1 & 29 \\
\hline $\mathbf{V}$ & 13 & 2 & 6 & 8 & 4 & 2 & 4 & 7 & 11 & 7 & 64 \\
\hline $\mathrm{Cr}$ & 20 & 5 & 4 & 2 & 5 & 7 & 3 & 2 & 2 & 9 & 59 \\
\hline Mn & 12 & 7 & 3 & 18 & 5 & 7 & 7 & 4 & 4 & 1 & 68 \\
\hline $\mathbf{F e}$ & 8 & 4 & 1 & 6 & 3 & 5 & 4 & 5 & 15 & 8 & 59 \\
\hline Co & 13 & 5 & 4 & 8 & 11 & 8 & 6 & 3 & 4 & 5 & 67 \\
\hline $\mathbf{N i}$ & 9 & 6 & 3 & 15 & 11 & 7 & 4 & 1 & 2 & 8 & 66 \\
\hline $\mathbf{C u}$ & 10 & 5 & 4 & 7 & 7 & 7 & 9 & 5 & 5 & 11 & 70 \\
\hline $\mathrm{Zn}$ & 10 & 7 & 4 & 11 & 5 & 11 & 7 & 5 & 3 & 8 & 71 \\
\hline As & 7 & 10 & 6 & 7 & 3 & 4 & 7 & 5 & 7 & 3 & 59 \\
\hline $\mathbf{R b}$ & 13 & 6 & 7 & 11 & 7 & 5 & 2 & 4 & 6 & 11 & 72 \\
\hline $\mathbf{S r}$ & 21 & 6 & 5 & 6 & 4 & 6 & 6 & 10 & 6 & 8 & 78 \\
\hline $\mathbf{Y}$ & 14 & 6 & 2 & 8 & 7 & 7 & 1 & 5 & 7 & 7 & 64 \\
\hline $\mathbf{Z r}$ & 6 & 4 & 1 & 4 & 6 & 5 & 2 & 4 & 5 & 18 & 55 \\
\hline $\mathrm{Nb}$ & 8 & 10 & 4 & 4 & 3 & 1 & 0 & 5 & 0 & 3 & 38 \\
\hline Mo & 13 & 7 & 10 & 7 & 7 & 4 & 7 & 3 & 5 & 6 & 69 \\
\hline Cd & 7 & 1 & 8 & 8 & 8 & 3 & 0 & 5 & 5 & 1 & 46 \\
\hline Sn & 9 & 7 & 0 & 1 & 2 & 3 & 3 & 3 & 1 & 2 & 31 \\
\hline $\mathbf{S b}$ & 11 & 4 & 8 & 4 & 0 & 5 & 4 & 3 & 2 & 5 & 46 \\
\hline Cs & 8 & 8 & 2 & 3 & 3 & 3 & 2 & 2 & 3 & 3 & 37 \\
\hline $\mathbf{B a}$ & 26 & 4 & 0 & 5 & 4 & 3 & 6 & 6 & 6 & 11 & 71 \\
\hline La & 9 & 3 & 4 & 7 & 2 & 3 & 5 & 1 & 1 & 6 & 41 \\
\hline $\mathrm{Ce}$ & 6 & 4 & 5 & 5 & 2 & 2 & 2 & 0 & 3 & 5 & 34 \\
\hline Pr & 3 & 3 & 1 & 5 & 1 & 1 & 1 & 0 & 0 & 6 & 21 \\
\hline Nd & 3 & 5 & 4 & 7 & 0 & 1 & 3 & 0 & 2 & 8 & 33 \\
\hline Sm & 1 & 2 & 4 & 3 & 6 & 4 & 2 & 3 & 2 & 8 & 35 \\
\hline Eu & 1 & 2 & 1 & 4 & 0 & 0 & 4 & 2 & 0 & 6 & 20 \\
\hline Gd & 3 & 3 & 6 & 0 & 1 & 0 & 2 & 1 & 1 & 7 & 24 \\
\hline $\mathbf{T b}$ & 3 & 1 & 3 & 0 & 1 & 1 & 1 & 1 & 1 & 6 & 18 \\
\hline Dy & 1 & 6 & 2 & 2 & 3 & 1 & 2 & 1 & 1 & 6 & 25 \\
\hline Ho & 2 & 4 & 1 & 0 & 0 & 3 & 0 & 1 & 1 & 6 & 18 \\
\hline Er & 2 & 2 & 3 & 1 & 0 & 4 & 1 & 1 & 1 & 7 & 22 \\
\hline Tm & 1 & 0 & 3 & 1 & 0 & 4 & 0 & 1 & 1 & 6 & 17 \\
\hline $\mathbf{Y b}$ & 1 & 4 & 2 & 2 & 0 & 2 & 0 & 1 & 0 & 5 & 17 \\
\hline $\mathbf{L u}$ & 2 & 2 & 3 & 2 & 1 & 0 & 0 & 2 & 0 & 1 & 13 \\
\hline Hf & 8 & 5 & 2 & 1 & 1 & 0 & 0 & 0 & 1 & 0 & 18 \\
\hline Ta & 2 & 3 & 1 & 3 & 1 & 1 & 3 & 1 & 1 & 5 & 21 \\
\hline $\mathbf{W}$ & 1 & 1 & 1 & 2 & 3 & 5 & 1 & 8 & 3 & 1 & 26 \\
\hline $\mathrm{Hg}$ & 0 & 0 & 0 & 2 & 1 & 4 & 3 & 3 & 0 & 8 & 21 \\
\hline Tl & 2 & 0 & 3 & 5 & 4 & 1 & 1 & 2 & 0 & 4 & 22 \\
\hline $\mathbf{P b}$ & 4 & 5 & 5 & 7 & 1 & 4 & 8 & 4 & 2 & 4 & 44 \\
\hline $\mathbf{B i}$ & 0 & 0 & 0 & 1 & 0 & 0 & 0 & 0 & 0 & 1 & 2 \\
\hline Th & 6 & 7 & 2 & 2 & 2 & 4 & 1 & 1 & 4 & 1 & 30 \\
\hline $\mathbf{U}$ & 10 & 9 & 6 & 8 & 3 & 2 & 4 & 1 & 3 & 9 & 55 \\
\hline
\end{tabular}


Tab. A.21.: Statistische Auswertung des Kationenaustausches für Proben aus der Lausitz; Probenanzahl mit Anteil (\% der Gesamtkonzentration) im Durchlauf.

\begin{tabular}{|c|c|c|c|c|c|c|c|c|c|c|c|}
\hline Element & Anzahl d & r Proben & nit Anteile & n (\%) im I & Durchlauf & von & & & & & E Anzahl \\
\hline & $0-9$ & $10-19$ & $20-29$ & \begin{tabular}{|l|}
$30-39$ \\
\end{tabular} & $40-49$ & \begin{tabular}{|c|}
$50-59$ \\
\end{tabular} & $60-69$ & \begin{tabular}{|c|}
$70-79$ \\
\end{tabular} & \begin{tabular}{|c|}
$80-89$ \\
\end{tabular} & \begin{tabular}{|l|}
$90-100$ \\
\end{tabular} & \\
\hline Sulfat & 0 & 0 & 0 & 1 & 0 & 0 & 0 & 4 & 5 & 2 & 12 \\
\hline DOC & 0 & 0 & 0 & 0 & 0 & 0 & 1 & 1 & 0 & 5 & 7 \\
\hline $\mathbf{L i}$ & 0 & 0 & 1 & 1 & 2 & 3 & 2 & 0 & 1 & 2 & 12 \\
\hline $\mathbf{B e}$ & 1 & 1 & 2 & 0 & 3 & 2 & 0 & 0 & 2 & 0 & 11 \\
\hline $\mathrm{Na}$ & 0 & 0 & 2 & 3 & 3 & 1 & 0 & 1 & 2 & 0 & 12 \\
\hline Mg & 1 & 1 & 2 & 3 & 3 & 0 & 0 & 1 & 1 & 0 & 12 \\
\hline Al & 5 & 3 & 2 & 0 & 0 & 1 & 0 & 1 & 0 & 0 & 12 \\
\hline $\mathbf{K}$ & 0 & 0 & 0 & 4 & 2 & 0 & 2 & 1 & 2 & 1 & 12 \\
\hline $\mathbf{C a}$ & 2 & 2 & 4 & 2 & 0 & 0 & 1 & 1 & 0 & 0 & 12 \\
\hline Sc & 1 & 0 & 0 & 1 & 1 & 2 & 1 & 0 & 1 & 1 & 8 \\
\hline $\mathbf{T i}$ & 1 & 0 & 0 & 2 & 3 & 1 & 0 & 0 & 1 & 0 & 8 \\
\hline $\mathbf{V}$ & 3 & 0 & 0 & 1 & 2 & 1 & 1 & 0 & 2 & 0 & 10 \\
\hline $\mathrm{Cr}$ & 0 & 3 & 0 & 0 & 1 & 2 & 0 & 0 & 2 & 1 & 9 \\
\hline Mn & 1 & 1 & 2 & 1 & 4 & 0 & 1 & 1 & 0 & 0 & 11 \\
\hline $\mathbf{F e}$ & 1 & 1 & 2 & 2 & 3 & 1 & 0 & 0 & 2 & 0 & 12 \\
\hline Co & 1 & 1 & 2 & 1 & 4 & 0 & 1 & 1 & 1 & 0 & 12 \\
\hline $\mathbf{N i}$ & 1 & 1 & 2 & 2 & 3 & 0 & 1 & 2 & 0 & 0 & 12 \\
\hline $\mathbf{C u}$ & 2 & 1 & 1 & 3 & 1 & 0 & 0 & 2 & 0 & 0 & 10 \\
\hline $\mathrm{Zn}$ & 1 & 1 & 2 & 2 & 3 & 0 & 1 & 1 & 1 & 0 & 12 \\
\hline As & 0 & 0 & 2 & 0 & 2 & 0 & 2 & 2 & 2 & 1 & 11 \\
\hline $\mathbf{R b}$ & 0 & 0 & 2 & 3 & 1 & 1 & 1 & 1 & 1 & 1 & 11 \\
\hline $\mathbf{S r}$ & 4 & 6 & 0 & 0 & 0 & 1 & 0 & 1 & 0 & 0 & 12 \\
\hline $\mathbf{Y}$ & 8 & 2 & 2 & 0 & 1 & 0 & 0 & 1 & 0 & 0 & 14 \\
\hline $\mathbf{Z r}$ & 4 & 1 & 0 & 0 & 0 & 3 & 1 & 0 & 1 & 0 & 10 \\
\hline $\mathrm{Nb}$ & 3 & 1 & 1 & 2 & 0 & 1 & 0 & 0 & 0 & 1 & 9 \\
\hline Mo & 1 & 1 & 1 & 0 & 1 & 2 & 1 & 1 & 1 & 1 & 10 \\
\hline Cd & 1 & 1 & 2 & 1 & 2 & 1 & 0 & 1 & 1 & 0 & 10 \\
\hline Sn & 0 & 0 & 0 & 0 & 0 & 0 & 0 & 0 & 0 & 0 & 0 \\
\hline $\mathbf{S b}$ & 0 & 0 & 0 & 0 & 0 & 0 & 1 & 2 & 0 & 3 & 6 \\
\hline Cs & 2 & 0 & 0 & 5 & 1 & 1 & 0 & 1 & 0 & 1 & 11 \\
\hline $\mathbf{B a}$ & 10 & 0 & 1 & 0 & 0 & 1 & 0 & 0 & 0 & 0 & 12 \\
\hline La & 10 & 1 & 0 & 0 & 0 & 0 & 1 & 0 & 0 & 0 & 12 \\
\hline $\mathrm{Ce}$ & 7 & 0 & 1 & 0 & 0 & 1 & 0 & 0 & 0 & 1 & 10 \\
\hline Pr & 7 & 0 & 1 & 0 & 0 & 0 & 1 & 0 & 0 & 1 & 10 \\
\hline Nd & 7 & 0 & 1 & 0 & 0 & 0 & 1 & 0 & 1 & 1 & 11 \\
\hline Sm & 6 & 1 & 0 & 1 & 0 & 1 & 1 & 0 & 0 & 0 & 10 \\
\hline Eu & 5 & 1 & 0 & 1 & 1 & 1 & 1 & 0 & 0 & 0 & 10 \\
\hline Gd & 5 & 2 & 0 & 0 & 1 & 1 & 0 & 1 & 0 & 0 & 10 \\
\hline $\mathbf{T b}$ & 5 & 1 & 1 & 0 & 1 & 0 & 2 & 0 & 0 & 0 & 10 \\
\hline Dy & 5 & 1 & 1 & 0 & 0 & 2 & 1 & 0 & 0 & 0 & 10 \\
\hline Ho & 5 & 1 & 1 & 0 & 0 & 2 & 1 & 0 & 0 & 0 & 10 \\
\hline Er & 5 & 1 & 1 & 0 & 1 & 1 & 0 & 1 & 0 & 0 & 10 \\
\hline Tm & 5 & 1 & 1 & 0 & 1 & 0 & 0 & 0 & 1 & 0 & 9 \\
\hline $\mathbf{Y b}$ & 5 & 1 & 1 & 1 & 1 & 0 & 0 & 0 & 0 & 1 & 10 \\
\hline $\mathbf{L u}$ & 5 & 1 & 2 & 0 & 1 & 0 & 0 & 0 & 0 & 1 & 10 \\
\hline Hf & 0 & 0 & 0 & 1 & 1 & 1 & 0 & 2 & 0 & 0 & 5 \\
\hline Ta & 2 & 1 & 2 & 0 & 0 & 0 & 0 & 0 & 0 & 0 & 5 \\
\hline $\mathbf{W}$ & 1 & 0 & 0 & 2 & 1 & 2 & 0 & 1 & 0 & 1 & 8 \\
\hline $\mathrm{Hg}$ & 0 & 0 & 0 & 0 & 0 & 0 & 0 & 0 & 0 & 0 & 0 \\
\hline Tl & 0 & 1 & 0 & 4 & 2 & 1 & 0 & 1 & 0 & 0 & 9 \\
\hline $\mathbf{P b}$ & 4 & 3 & 1 & 0 & 1 & 0 & 1 & 0 & 0 & 0 & 10 \\
\hline $\mathbf{B i}$ & 0 & 0 & 0 & 0 & 0 & 0 & 0 & 0 & 0 & 0 & 0 \\
\hline Th & 0 & 0 & 1 & 0 & 3 & 1 & 0 & 0 & 1 & 1 & 7 \\
\hline $\mathbf{U}$ & 1 & 0 & 0 & 0 & 4 & 2 & 0 & 1 & 1 & 0 & 9 \\
\hline
\end{tabular}


Tab. A.22.: Statistische Auswertung des Anionenaustausches für Proben aus Cospuden; Probenanzahl mit Anteil (\% der Gesamtkonzentration) im Durchlauf.

\begin{tabular}{|c|c|c|c|c|c|c|c|c|c|c|c|}
\hline Element & Anzahl d & r Proben & mit Anteile & n (\%) im I & Durchlauf & von & & & & & $\Sigma$ Anzahl \\
\hline & $0-9$ & $10-19$ & $20-29$ & \begin{tabular}{|l|}
$30-39$ \\
\end{tabular} & $40-49$ & \begin{tabular}{|c|}
$50-59$ \\
\end{tabular} & $60-69$ & \begin{tabular}{|c|}
$70-79$ \\
\end{tabular} & \begin{tabular}{|l|}
$80-89$ \\
\end{tabular} & \begin{tabular}{|l|}
$90-100$ \\
\end{tabular} & \\
\hline Sulfat & 5 & 10 & 8 & 4 & 6 & 4 & 8 & 5 & 6 & 14 & 70 \\
\hline DOC & 1 & 2 & 4 & 4 & 6 & 6 & 7 & 5 & 3 & 10 & 48 \\
\hline $\mathbf{L i}$ & 14 & 7 & 7 & 2 & 0 & 2 & 7 & 8 & 13 & 20 & 80 \\
\hline $\mathbf{B e}$ & 9 & 0 & 4 & 3 & 2 & 1 & 2 & 1 & 5 & 5 & 32 \\
\hline $\mathrm{Na}$ & 23 & 3 & 1 & 2 & 1 & 1 & 6 & 2 & 9 & 30 & 78 \\
\hline Mg & 9 & 5 & 1 & 5 & 6 & 3 & 4 & 4 & 16 & 26 & 79 \\
\hline Al & 8 & 3 & 2 & 6 & 8 & 4 & 4 & 1 & 7 & 13 & 56 \\
\hline $\mathbf{K}$ & 21 & 5 & 3 & 4 & 0 & 2 & 3 & 4 & 5 & 22 & 69 \\
\hline $\mathbf{C a}$ & 9 & 7 & 2 & 3 & 6 & 2 & 5 & 6 & 15 & 23 & 78 \\
\hline Sc & 3 & 4 & 1 & 2 & 7 & 10 & 12 & 7 & 7 & 6 & 59 \\
\hline $\mathbf{T i}$ & 3 & 2 & 5 & 1 & 1 & 0 & 1 & 7 & 2 & 5 & 27 \\
\hline $\mathbf{V}$ & 6 & 4 & 3 & 3 & 1 & 8 & 6 & 8 & 10 & 13 & 62 \\
\hline $\mathrm{Cr}$ & 9 & 1 & 3 & 7 & 9 & 5 & 3 & 5 & 6 & 15 & 63 \\
\hline Mn & 14 & 5 & 4 & 1 & 3 & 2 & 5 & 4 & 11 & 32 & 81 \\
\hline $\mathbf{F e}$ & 14 & 4 & 3 & 6 & 2 & 2 & 6 & 8 & 5 & 17 & 67 \\
\hline Co & 16 & 2 & 3 & 4 & 4 & 2 & 14 & 5 & 3 & 20 & 73 \\
\hline $\mathbf{N i}$ & 13 & 1 & 1 & 2 & 2 & 4 & 5 & 6 & 20 & 17 & 71 \\
\hline $\mathbf{C u}$ & 3 & 2 & 3 & 3 & 6 & 12 & 5 & 11 & 5 & 22 & 72 \\
\hline $\mathrm{Zn}$ & 8 & 4 & 3 & 4 & 4 & 4 & 7 & 10 & 8 & 28 & 80 \\
\hline As & 5 & 4 & 2 & 5 & 1 & 1 & 1 & 7 & 12 & 7 & 45 \\
\hline $\mathbf{R b}$ & 10 & 8 & 9 & 4 & 3 & 5 & 5 & 5 & 7 & 19 & 75 \\
\hline $\mathbf{S r}$ & 15 & 5 & 4 & 3 & 1 & 2 & 7 & 9 & 15 & 24 & 85 \\
\hline $\mathbf{Y}$ & 17 & 4 & 7 & 7 & 6 & 3 & 2 & 4 & 3 & 13 & 66 \\
\hline $\mathbf{Z r}$ & 2 & 5 & 3 & 2 & 4 & 5 & 7 & 1 & 12 & 19 & 60 \\
\hline $\mathrm{Nb}$ & 6 & 10 & 8 & 3 & 6 & 4 & 1 & 1 & 2 & 3 & 44 \\
\hline Mo & 21 & 6 & 10 & 5 & 4 & 7 & 3 & 3 & 4 & 18 & 81 \\
\hline Cd & 8 & 2 & 1 & 3 & 6 & 6 & 3 & 5 & 3 & 4 & 41 \\
\hline Sn & 12 & 6 & 1 & 0 & 3 & 3 & 2 & 3 & 2 & 1 & 33 \\
\hline $\mathbf{S b}$ & 4 & 1 & 8 & 9 & 5 & 8 & 5 & 2 & 2 & 3 & 47 \\
\hline Cs & 8 & 4 & 5 & 1 & 1 & 5 & 4 & 4 & 0 & 9 & 41 \\
\hline $\mathbf{B a}$ & 7 & 2 & 2 & 0 & 1 & 2 & 1 & 5 & 11 & 23 & 54 \\
\hline La & 13 & 7 & 3 & 5 & 1 & 4 & 2 & 0 & 0 & 8 & 43 \\
\hline $\mathrm{Ce}$ & 7 & 9 & 2 & 4 & 1 & 2 & 2 & 0 & 2 & 7 & 36 \\
\hline Pr & 6 & 4 & 1 & 4 & 0 & 1 & 0 & 0 & 1 & 4 & 21 \\
\hline Nd & 7 & 5 & 4 & 4 & 2 & 2 & 1 & 1 & 0 & 6 & 32 \\
\hline Sm & 5 & 3 & 1 & 3 & 0 & 5 & 2 & 2 & 4 & 6 & 31 \\
\hline Eu & 4 & 1 & 5 & 4 & 3 & 2 & 0 & 1 & 2 & 2 & 24 \\
\hline Gd & 5 & 4 & 2 & 3 & 1 & 0 & 0 & 0 & 0 & 5 & 20 \\
\hline $\mathbf{T b}$ & 7 & 1 & 3 & 1 & 0 & 0 & 1 & 0 & 0 & 5 & 18 \\
\hline Dy & 5 & 5 & 2 & 3 & 2 & 1 & 1 & 1 & 1 & 4 & 25 \\
\hline Ho & 5 & 3 & 3 & 0 & 1 & 0 & 0 & 1 & 0 & 5 & 18 \\
\hline Er & 5 & 4 & 3 & 0 & 2 & 1 & 0 & 0 & 0 & 4 & 19 \\
\hline Tm & 6 & 2 & 2 & 0 & 0 & 0 & 1 & 0 & 2 & 3 & 16 \\
\hline $\mathbf{Y b}$ & 4 & 3 & 4 & 1 & 1 & 0 & 1 & 0 & 1 & 2 & 17 \\
\hline $\mathbf{L u}$ & 6 & 0 & 2 & 2 & 1 & 0 & 0 & 0 & 2 & 1 & 14 \\
\hline Hf & 9 & 3 & 2 & 0 & 2 & 1 & 0 & 0 & 0 & 0 & 17 \\
\hline Ta & 6 & 2 & 0 & 4 & 1 & 1 & 2 & 0 & 3 & 3 & 22 \\
\hline $\mathbf{W}$ & 4 & 4 & 1 & 2 & 3 & 1 & 4 & 4 & 4 & 5 & 32 \\
\hline $\mathrm{Hg}$ & 0 & 0 & 0 & 0 & 1 & 2 & 5 & 4 & 3 & 8 & 23 \\
\hline Tl & 3 & 0 & 0 & 3 & 4 & 2 & 3 & 1 & 1 & 2 & 19 \\
\hline $\mathbf{P b}$ & 5 & 2 & 7 & 5 & 3 & 9 & 5 & 6 & 2 & 7 & 51 \\
\hline $\mathbf{B i}$ & 0 & 0 & 0 & 0 & 0 & 0 & 0 & 1 & 0 & 1 & 2 \\
\hline Th & 5 & 2 & 3 & 2 & 3 & 1 & 5 & 3 & 4 & 2 & 30 \\
\hline $\mathbf{U}$ & 33 & 5 & 3 & 0 & 0 & 0 & 0 & 1 & 2 & 13 & 57 \\
\hline
\end{tabular}


Tab. A.23.: Statistische Auswertung des Anionenaustausches für Proben aus der Lausitz; Probenanzahl mit Anteil (\% der Gesamtkonzentration) im Durchlauf.

\begin{tabular}{|c|c|c|c|c|c|c|c|c|c|c|c|}
\hline Element & Anzahl d & r Proben & mit Anteile & n (\%) im I & Durchlauf & von & & & & & E Anzahl \\
\hline & $0-9$ & $10-19$ & $20-29$ & \begin{tabular}{|l|}
$30-39$ \\
\end{tabular} & $40-49$ & \begin{tabular}{|c|}
$50-59$ \\
\end{tabular} & $60-69$ & \begin{tabular}{|c|}
$70-79$ \\
\end{tabular} & \begin{tabular}{|c|}
$80-89$ \\
\end{tabular} & \begin{tabular}{|l|}
$90-100$ \\
\end{tabular} & \\
\hline Sulfat & 0 & 2 & 2 & 1 & 3 & 1 & 0 & 2 & 0 & 0 & 11 \\
\hline DOC & 0 & 0 & 0 & 0 & 0 & 0 & 2 & 0 & 2 & 3 & 7 \\
\hline $\mathbf{L i}$ & 0 & 0 & 0 & 0 & 0 & 1 & 3 & 4 & 1 & 3 & 12 \\
\hline $\mathbf{B e}$ & 0 & 0 & 0 & 0 & 1 & 3 & 1 & 1 & 3 & 3 & 12 \\
\hline $\mathrm{Na}$ & 0 & 0 & 0 & 0 & 0 & 0 & 0 & 0 & 4 & 5 & 9 \\
\hline Mg & 0 & 0 & 0 & 0 & 0 & 0 & 1 & 3 & 4 & 4 & 12 \\
\hline Al & 0 & 0 & 2 & 1 & 1 & 0 & 0 & 2 & 4 & 2 & 12 \\
\hline $\mathbf{K}$ & 2 & 0 & 0 & 0 & 0 & 0 & 1 & 1 & 5 & 2 & 11 \\
\hline $\mathbf{C a}$ & 0 & 0 & 0 & 0 & 0 & 0 & 1 & 6 & 3 & 2 & 12 \\
\hline Sc & 1 & 0 & 0 & 1 & 1 & 1 & 1 & 0 & 4 & 0 & 9 \\
\hline $\mathbf{T i}$ & 2 & 1 & 1 & 1 & 1 & 1 & 2 & 0 & 0 & 0 & 9 \\
\hline $\mathbf{V}$ & 3 & 1 & 1 & 0 & 2 & 0 & 1 & 2 & 0 & 0 & 10 \\
\hline $\mathrm{Cr}$ & 1 & 0 & 0 & 1 & 0 & 0 & 2 & 2 & 0 & 3 & 9 \\
\hline Mn & 0 & 0 & 0 & 0 & 0 & 0 & 1 & 5 & 4 & 2 & 12 \\
\hline $\mathbf{F e}$ & 0 & 0 & 0 & 0 & 0 & 0 & 1 & 5 & 3 & 3 & 12 \\
\hline Co & 0 & 0 & 0 & 0 & 0 & 0 & 1 & 5 & 2 & 4 & 12 \\
\hline $\mathbf{N i}$ & 0 & 0 & 0 & 0 & 0 & 0 & 0 & 7 & 1 & 4 & 12 \\
\hline $\mathbf{C u}$ & 1 & 0 & 1 & 0 & 0 & 1 & 0 & 1 & 2 & 3 & 9 \\
\hline $\mathrm{Zn}$ & 0 & 0 & 0 & 0 & 0 & 0 & 2 & 4 & 3 & 3 & 12 \\
\hline As & 0 & 0 & 0 & 0 & 0 & 2 & 0 & 1 & 3 & 5 & 11 \\
\hline $\mathbf{R b}$ & 1 & 0 & 0 & 0 & 0 & 2 & 1 & 1 & 2 & 4 & 11 \\
\hline $\mathbf{S r}$ & 0 & 0 & 0 & 0 & 0 & 0 & 1 & 5 & 2 & 4 & 12 \\
\hline $\mathbf{Y}$ & 0 & 0 & 0 & 0 & 0 & 2 & 3 & 2 & 2 & 3 & 12 \\
\hline $\mathbf{Z r}$ & 1 & 0 & 0 & 0 & 0 & 1 & 2 & 2 & 3 & 3 & 12 \\
\hline $\mathrm{Nb}$ & 2 & 2 & 1 & 0 & 1 & 1 & 0 & 0 & 0 & 4 & 11 \\
\hline Mo & 1 & 1 & 0 & 1 & 3 & 0 & 1 & 1 & 0 & 1 & 9 \\
\hline Cd & 1 & 0 & 0 & 0 & 0 & 1 & 1 & 1 & 4 & 5 & 13 \\
\hline Sn & 0 & 0 & 0 & 0 & 0 & 0 & 0 & 0 & 0 & 0 & 0 \\
\hline $\mathbf{S b}$ & 0 & 0 & 0 & 0 & 0 & 1 & 3 & 0 & 1 & 4 & 9 \\
\hline Cs & 1 & 0 & 0 & 0 & 0 & 0 & 2 & 4 & 0 & 5 & 12 \\
\hline $\mathbf{B a}$ & 0 & 0 & 0 & 0 & 0 & 1 & 0 & 5 & 2 & 3 & 11 \\
\hline La & 0 & 0 & 0 & 0 & 0 & 0 & 5 & 2 & 0 & 4 & 11 \\
\hline $\mathrm{Ce}$ & 1 & 0 & 0 & 0 & 1 & 0 & 4 & 2 & 0 & 4 & 12 \\
\hline Pr & 1 & 0 & 0 & 0 & 1 & 0 & 4 & 2 & 0 & 4 & 12 \\
\hline Nd & 1 & 0 & 0 & 0 & 1 & 1 & 3 & 1 & 1 & 4 & 12 \\
\hline Sm & 1 & 0 & 0 & 0 & 0 & 3 & 2 & 2 & 0 & 4 & 12 \\
\hline Eu & 1 & 0 & 0 & 0 & 1 & 2 & 1 & 3 & 2 & 2 & 12 \\
\hline Gd & 1 & 0 & 0 & 0 & 1 & 1 & 1 & 2 & 2 & 4 & 12 \\
\hline $\mathbf{T b}$ & 1 & 0 & 0 & 0 & 0 & 3 & 0 & 2 & 2 & 4 & 12 \\
\hline Dy & 1 & 0 & 0 & 0 & 1 & 1 & 2 & 2 & 1 & 4 & 12 \\
\hline Ho & 1 & 0 & 0 & 0 & 0 & 3 & 0 & 2 & 2 & 4 & 12 \\
\hline Er & 1 & 0 & 0 & 0 & 0 & 2 & 1 & 2 & 2 & 4 & 12 \\
\hline Tm & 1 & 0 & 0 & 0 & 0 & 2 & 1 & 1 & 3 & 4 & 12 \\
\hline $\mathbf{Y b}$ & 1 & 0 & 0 & 0 & 1 & 1 & 1 & 3 & 1 & 4 & 12 \\
\hline $\mathbf{L u}$ & 1 & 0 & 0 & 0 & 0 & 1 & 3 & 2 & 1 & 4 & 12 \\
\hline Hf & 0 & 0 & 0 & 0 & 0 & 0 & 0 & 2 & 1 & 4 & 7 \\
\hline Ta & 1 & 1 & 0 & 1 & 1 & 0 & 0 & 2 & 0 & 1 & 7 \\
\hline $\mathbf{W}$ & 1 & 0 & 1 & 0 & 0 & 1 & 2 & 0 & 2 & 2 & 9 \\
\hline $\mathrm{Hg}$ & 0 & 0 & 0 & 0 & 0 & 0 & 0 & 0 & 0 & 1 & 1 \\
\hline Tl & 1 & 0 & 0 & 0 & 0 & 1 & 1 & 1 & 3 & 4 & 11 \\
\hline $\mathbf{P b}$ & 1 & 1 & 1 & 0 & 0 & 1 & 3 & 3 & 1 & 2 & 13 \\
\hline $\mathbf{B i}$ & 0 & 0 & 0 & 0 & 0 & 0 & 0 & 0 & 0 & 0 & 0 \\
\hline Th & 1 & 2 & 2 & 1 & 1 & 3 & 0 & 0 & 0 & 0 & 10 \\
\hline $\mathbf{U}$ & 11 & 0 & 1 & 0 & 0 & 0 & 0 & 0 & 0 & 0 & 12 \\
\hline
\end{tabular}


Tab. A.24.: Einordnung der Proben in Gruppen nach den Ergebnissen der Ultrafiltration; AP = Proben aus der Arbeit von PLEßOW (1998), KB = Proben aus der vorliegenden Arbeit; $P L=$ Porenlösung, WP $=$ Wasserprobe.

\begin{tabular}{|c|c|c|c|}
\hline Nrr & Probe & $\begin{array}{l}\text { Gruppe } \\
\text { nach } \\
\text { UF }\end{array}$ & Kriterien \\
\hline 1 & RCO4 5-8m PL AP & 3 & hohe UF-Raten für $\mathrm{Cu}, \mathrm{Zn}, \mathrm{Cd}, \mathrm{Ba}$ und $\mathrm{Pb}$ \\
\hline 2 & RCO4 11-19m PL AP & $3 /$ & geringer $\mathrm{Cu}$-Gehalt, hohe UF-Raten für $\mathrm{Ca}, \mathrm{Cu}, \mathrm{Zn}, \mathrm{Cd}$ und $\mathrm{Ba}$ \\
\hline 3 & RCO4 17m PL KB & $3 / 5 / 7$ & geringer $\mathrm{Cu}$ - und Cd-Gehalt; vgl. RCO4 11-19 m AP, wahrscheinlich Gruppe 3 \\
\hline 4 & RCO4 20m PL KB & $3 / 5 / 7$ & geringer Gehalt an $\mathrm{Co}, \mathrm{Cu}$ und $\mathrm{Zn}$, wahrscheinlich Gruppe 3 \\
\hline 5 & RCO4 22m PL KB & $3 / 5 / 7$ & geringer Co-, Cu-, Zn- und Cd-Gehalt; vgl. weitere Proben aus dem RCO4-Bohrkern \\
\hline 6 & RCO4 26m PL KB & $1 / 2 / 4 / 6$ & niedriger $\mathrm{pH}$-Wert $(3,2)$; sehr hohe Elementkonzentrationen \\
\hline 7 & RCO4 28m PL AP & 3 & $\begin{array}{l}\text { geringer } \mathrm{Cu} \text {-Gehalt, hohe UF-Raten für } \mathrm{Ca}, \mathrm{Cu}, \mathrm{Zn} \text {, und } \mathrm{Ba} \text {; } \\
\text { aber: sehr niedrige UF-Rate für } \mathrm{Cd} \text {, relativ hoher } \mathrm{Cd} \text {-Absolutgehalt in der Lösung }\end{array}$ \\
\hline 8 & RCO4 30m PL AP & 3 & geringer $\mathrm{Cu}$-Gehalt, hohe UF-Raten für $\mathrm{Ca}, \mathrm{Cu}, \mathrm{Zn}, \mathrm{Cd}$ und $\mathrm{Ba}$ \\
\hline 9 & RCO4 32m PL AP & 5 & $\begin{array}{l}\text { Elementverarmung nicht feststellbar; hohe UF-Raten für } \mathrm{Ca}, \mathrm{Cu}, \mathrm{Zn}, \mathrm{Cd}, \mathrm{Ba} \text { und } \mathrm{Pb} \text {, ohne } \\
\text { dass größere Mengen anorganischer Kolloide festgestellt wurden }\end{array}$ \\
\hline 10 & RCO4 34m PL AP & 3 & geringer $\mathrm{Cu}$-Gehalt, hohe UF-Raten für $\mathrm{Ca}, \mathrm{Cu}, \mathrm{Zn}, \mathrm{Cd}$ und $\mathrm{Ba}$ \\
\hline 11 & RCO4 36m PL KB & 7 & geringer Gehalt an $\mathrm{Co}, \mathrm{Cu}$ und $\mathrm{Zn}$; sehr geringe UF-Rate für $\mathrm{Ca}$ \\
\hline 12 & RCO4 38m PL AP & 5 & geringer $\mathrm{Cu}$-Gehalt, hohe UF-Raten für $\mathrm{Ca}, \mathrm{Cu}, \mathrm{Zn}, \mathrm{Cd}$ und $\mathrm{Ba}$ \\
\hline 13 & RCO4 40m PL KB & $3 / 5 / 7$ & zahlreiche Festphasen stark übersättigt \\
\hline 14 & RCO4 42m PL AP & 5 & geringer absoluter $\mathrm{Cu}$-Gehalt, hohe UF-Raten für $\mathrm{Ca}, \mathrm{Cu}, \mathrm{Zn}, \mathrm{Cd}$ und $\mathrm{Pb}$ \\
\hline 15 & RCO4 44m PL KB & $3 / 5 / 7$ & geringer absoluter $\mathrm{Cu}-\mathrm{Gehalt}$, hohe UF-Raten für $\mathrm{Ca}, \mathrm{Cu}, \mathrm{Zn}, \mathrm{Cd}$ und $\mathrm{Pb}$ \\
\hline 16 & RCO4 46m PL AP & 3 & geringer absoluter $\mathrm{Cu}$-Gehalt, hohe UF-Raten für $\mathrm{Ca}, \mathrm{Cu}, \mathrm{Zn}, \mathrm{Cd}$ und $\mathrm{Pb}$ \\
\hline 17 & RCO4 48m PL KB & wohl 7 & zu kleine Datenbasis \\
\hline 18 & RCO4 52m PL KB & 7 & hohe UF-Raten für Ca und Spurenelemente \\
\hline 19 & RCO4 56m PL AP & 3 & geringer absoluter $\mathrm{Cu}$-Gehalt, hohe UF-Raten für $\mathrm{Cu}, \mathrm{Zn}$ und $\mathrm{Cd}$ \\
\hline 20 & RCO4 58m PL AP & 3 & geringer absoluter $\mathrm{Cu}-\mathrm{Gehalt}$, hohe UF-Raten für $\mathrm{Ca}, \mathrm{Cu}, \mathrm{Zn}, \mathrm{Cd}$ und $\mathrm{Pb}$ \\
\hline 21 & RCO10 2m PL AP & 7 & einige Festphasen übersättigt \\
\hline 22 & RCO10 18.5m PL AP & 3 & geringer absoluter $\mathrm{Cu}-\mathrm{Gehalt}$, hohe UF-Raten für $\mathrm{Ca}, \mathrm{Cu}, \mathrm{Zn}, \mathrm{Cd}$ und $\mathrm{Pb}$ \\
\hline 23 & RCO10 29m PL AP & 3 & $\begin{array}{l}\text { geringer Gehalt an } \mathrm{Cu} \text {; hohe UF-Raten für } \mathrm{Ca}, \mathrm{Cu}, \mathrm{Zn}, \mathrm{Cd} \text { und } \mathrm{Pb} \\
\text { aber: SI für Mn-Minerale nicht relevant }\end{array}$ \\
\hline 24 & RCO13 15m PL AP & 3 & $\begin{array}{l}\text { geringer absoluter Cu-Gehalt, hohe UF-Raten für Ca und Cd; für } \mathrm{Cu} 58 \% \text {, für } \mathrm{Zn} 68 \% \\
\text { Rückhalterate bei der Ultrafiltration }\end{array}$ \\
\hline 25 & RCO13 30m PL AP & 5 & $\begin{array}{l}\text { geringe Gehalt an } \mathrm{Co} \text { und } \mathrm{Cu} \\
\text { aber: hohe UF-Raten für } \mathrm{Ca}, \mathrm{Cu}, \mathrm{Zn}, \mathrm{Cd}, \mathrm{Ba} \text { und } \mathrm{Pb} \text {, ohne dass große Mengen anorganischer } \\
\text { Kolloide feststellbar waren }\end{array}$ \\
\hline 26 & RCO16 29m PL AP & 3 & geringer absoluter $\mathrm{Cu}$-Gehalt, hohe UF-Raten für $\mathrm{Ca}, \mathrm{Cu}, \mathrm{Zn}, \mathrm{Cd}$ un $\mathrm{Pb}$ \\
\hline 27 & $\begin{array}{l}\text { Fremdflutung WP } \\
\text { AP }\end{array}$ & 5 & $\begin{array}{l}\text { geringe Konzentrationen von Spurenelementen, vollständige Abtrennung von Ca in der } \\
\text { Ultrafiltration, hohe UF-Raten bei Spurenelementen }\end{array}$ \\
\hline 28 & Sickerwasser WP AP & 5 & $\begin{array}{l}\text { geringe Konzentrationen von Spurenelementen, vollständige Abtrennung von } \mathrm{Ca} \text { in der } \\
\text { Ultrafiltration, hohe UF-Raten bei Spurenelementen }\end{array}$ \\
\hline 29 & $\begin{array}{l}\text { Standgewässer WP } \\
\text { AP }\end{array}$ & 5 & $\begin{array}{l}\text { niedriger pH-Wert, vollständige Abtrennung von } \mathrm{Ca} \text { bei der Ultrafiltration, hohe } \\
\text { Ultrafiltrationsrate bei } \mathrm{Cu}, \mathrm{Zn} \text { und } \mathrm{Ba}\end{array}$ \\
\hline 30 & $\begin{array}{ll}\text { Standgewässer2 } & \text { PL } \\
\text { AP }\end{array}$ & 1 & $\begin{array}{l}\text { hohe UF-Raten für } \mathrm{Cu}, \mathrm{Zn}, \mathrm{Cd} \text { und } \mathrm{Pb} \text {; REEs werden im Kationen. und Anionenaustuasch } \\
\text { nur wenig ausgetauscht }\end{array}$ \\
\hline 31 & $\begin{array}{l}\text { Standgewässer2 WP } \\
\text { AP }\end{array}$ & 1 & $\begin{array}{l}\text { hohe UF-Raten für } \mathrm{Cu}, \mathrm{Zn}, \mathrm{Cd} \text { und } \mathrm{Pb} \text {; V REEs werden im Kationen. und Anionenaustuasch } \\
\text { nur wenig ausgetauscht }\end{array}$ \\
\hline 32 & $\begin{array}{l}\text { Standgewässer3 WP } \\
\text { AP }\end{array}$ & 1 & hohe UF-Raten für $\mathrm{Cu}, \mathrm{Zn}, \mathrm{Cd}$ und $\mathrm{Pb}$ \\
\hline 33 & $\begin{array}{l}\text { Standgewässer3 PL } \\
\text { AP }\end{array}$ & 4 & $\begin{array}{l}\text { niedriger } \mathrm{pH}-\text { Wert }(2,3) \text {; hohe UF-Raten für } \mathrm{Cu}, \mathrm{Cd} \text { und } \mathrm{Pb} \\
\text { aber: niedrige UF-Rate für } \mathrm{Zn} \text { und } \mathrm{Ba}\end{array}$ \\
\hline 34 & Kippe SW PL AP & 4 & $\begin{array}{l}\text { niedriger } \mathrm{pH}-\text { Wert }(2,4) \text {; hohe UF-Raten für } \mathrm{Cu}, \mathrm{Cd}, \mathrm{Ba} \text { und } \mathrm{Pb} \\
\text { aber: niedrige UF-Rate für } \mathrm{Zn}\end{array}$ \\
\hline 35 & Kippe W PL AP & 2 & $\begin{array}{l}\text { niedriger pH-Wert, vollständige Abtrennung von } \mathrm{Ca} \text { bei der Ultrafiltration, hohe } \\
\text { Ultrafiltrationsrate bei } \mathrm{Cu}, \mathrm{Zn}, \mathrm{Ba} \text { und } \mathrm{Pb}\end{array}$ \\
\hline
\end{tabular}


Tab. A.24. (Forts.): Einordnung der Proben in Gruppen nach den Ergebnissen der Ultrafiltration; AP = Proben aus der Arbeit von PLEßOW (1998), KB = Proben aus der vorliegenden Arbeit; PL = Porenlösung, WP = Wasserprobe.

\begin{tabular}{|c|c|c|c|}
\hline Nr. & Probe & $\begin{array}{l}\text { Gruppe } \\
\text { nach } \\
\text { UF }\end{array}$ & Kriterien \\
\hline 36 & See Südufer WP AP & 5 & $\begin{array}{l}\text { niedriger Gehalt an } \mathrm{Cu} \text {, hohe } \mathrm{Rückhalterate} \text { für } \mathrm{Cu} \text { und } \mathrm{Pb} \\
\text { aber: niedriger } \mathrm{pH} \text {-Wert, außer } \mathrm{Cu} \text { hohe Spurenelementgehalte, mittlere bis geringe UF- } \\
\text { Raten }\end{array}$ \\
\hline 37 & See Südufer PL AP & 2 & $\begin{array}{l}\text { niedriger pH-Wert, vollständige Abtrennung von } \mathrm{Ca} \text { bei der Ultrafiltration, hohe } \\
\text { Ultrafiltrationsrate bei } \mathrm{Cu}, \mathrm{Zn}, \mathrm{Ba} \text { und } \mathrm{Pb}\end{array}$ \\
\hline 38 & SGM 48.5m WP AP & 1 & hohe UF-Raten für $\mathrm{Cu}, \mathrm{Zn}, \mathrm{Cd}$ und $\mathrm{Pb}$ \\
\hline 39 & SGM 24.5m WP AP & 6 & hohe UF-Raten für $\mathrm{Cu}, \mathrm{Zn}, \mathrm{Cd}$ und $\mathrm{Pb}$ \\
\hline 40 & CML 21-22m PL KB & 7 & niedrige Gehalte an $\mathrm{Co}, \mathrm{Cu}$ und $\mathrm{Cd}$ \\
\hline 41 & CML 24m PL KB & & geringe UF-Raten für $\mathrm{Cu}$ und $\mathrm{Zn}$ \\
\hline 42 & CML 26m PL KB & $\begin{array}{l}\text { zuwenig } \\
\text { Daten }\end{array}$ & keine drastischen Übersättigungen, aber SI von Gips nahe Null \\
\hline 43 & CML 32m PL KB & 7 & sehr geringe UF-Rate für $\mathrm{Cu}$ \\
\hline 44 & CML 36m PL KB & $\begin{array}{l}\text { zuwenig } \\
\text { Daten }\end{array}$ & zahlreiche Phasen, auch Gips, übersättigt \\
\hline 45 & CML 37.5m PL KB & \begin{tabular}{|l|} 
zuwenig \\
Daten
\end{tabular} & starke Übersättigung zahlreicher Eisenphasen \\
\hline 46 & CML 39-40m PL KB & $\begin{array}{l}\text { zuwenig } \\
\text { Daten }\end{array}$ & \\
\hline 47 & RCO 4 Okt 98PL KB & 6 & $\begin{array}{l}\text { geringe UF-Raten } \\
\text { aber: kein niedriger } \mathrm{pH}-\mathrm{Wert}(6,2) \text {, geringe Spurenelementgehalte }\end{array}$ \\
\hline 48 & $\begin{array}{l}\text { RCO 10A Okt 98 PL } \\
\text { KB }\end{array}$ & 5 & $\begin{array}{l}\text { geringer Gehalt an Co, geringe UF-Rate für } \mathrm{Zn} \\
\text { aber: sehr hohe UF-Rate für } \mathrm{Ca}, \mathrm{Cu}, \mathrm{Ba}\end{array}$ \\
\hline 49 & $\begin{array}{ll}\text { RCO } 12 \text { Okt } 98 \text { PL } \\
\text { KB }\end{array}$ & 6 & sehr geringe UF-Raten \\
\hline 50 & $\begin{array}{l}\text { RCO } 13 \text { Okt } 98 \text { PL } \\
\text { KB }\end{array}$ & 3 & $\begin{array}{l}\text { Gehalte an Spurenelementen nicht auffällig } \\
\text { aber: sehr geringe UF-Rate für } \mathrm{Cu}\end{array}$ \\
\hline 51 & $\begin{array}{l}\text { RCO } 14 \text { Okt } 98 \text { PL } \\
\text { KB }\end{array}$ & 5 & sehr geringe UF-Raten \\
\hline 52 & $\begin{array}{l}\text { Bohrung 1m Nov } 98 \\
\text { PL KB }\end{array}$ & 7 & niedriger $\mathrm{pH}$, hohe Spurenelementgehalte, niedrige UF-Raten \\
\hline 53 & $\begin{array}{l}\text { Bohrung 2m Nov } 98 \\
\text { PL KB }\end{array}$ & 7 & niedriger Gehalt an $\mathrm{Co}$; geringe UF-Raten für $\mathrm{Cd}, \mathrm{Ba}$ und $\mathrm{Pb}$ \\
\hline 54 & $\begin{array}{l}\text { Bohrung 3m Nov } 98 \\
\text { PL KB }\end{array}$ & 7 & $\begin{array}{l}\text { niedriger } \mathrm{pH}-\mathrm{Wert}(4,3) \text {; hoher abs. } \mathrm{Cu} \text {-Gehalt, niedrige UF-Raten für } \mathrm{Fe}, \mathrm{Mn} \text {, Sulfat und } \\
\text { Spurenelemente }\end{array}$ \\
\hline 55 & See Nov 98 WP KB & 7 & mittlere UF-Rate für $\mathrm{Zn}$; geringe UF-Raten für $\mathrm{Cu}$ und $\mathrm{Ba}$ \\
\hline 56 & $\begin{array}{l}\text { CML 20m Jan } 99 \\
\text { WP KB }\end{array}$ & 7 & niedrige Gehalte an $\mathrm{Co}, \mathrm{Ni}, \mathrm{Cu}$ und $\mathrm{Zn}$; geringe UF-Raten für $\mathrm{Cu}$ und $\mathrm{Cd}$ \\
\hline 57 & $\begin{array}{l}\text { CML 27.5m Jan } 99 \\
\text { WP KB }\end{array}$ & 7 & $\begin{array}{l}\text { geringe Gehalte an } \mathrm{Co}, \mathrm{Cu} \text { und } \mathrm{Zn} \text {; niedrige UF-Raten für } \mathrm{Zn} \text { und } \mathrm{Ba} \text {, mittlere UF-Raten für } \\
\mathrm{Cu} \text { und } \mathrm{Cd}\end{array}$ \\
\hline 58 & $\begin{array}{l}\text { CML 31.5m Jan } 99 \\
\text { WP KB }\end{array}$ & 7 & $\begin{array}{l}\text { pH nicht niedrig }(6,2) \text {; trotz hoher Fe-Gehalte geringe UF-Raten für Haupt- und } \\
\text { Spurenelemente }\end{array}$ \\
\hline 59 & $\begin{array}{l}\text { CML 39.5m Jan } 99 \\
\text { WP KB }\end{array}$ & 7 & geringer Gehalt an $\mathrm{Cu}$ und $\mathrm{Zn}$, aber sehr geringe UF-Raten für $\mathrm{Cu}$ und $\mathrm{Pb}$ \\
\hline 60 & $\begin{array}{l}\text { CML 43.7m Jan } 99 \\
\text { WP KB }\end{array}$ & 7 & geringe Gehalte an $\mathrm{Co}, \mathrm{Cu}$ und $\mathrm{Zn}$; UF-Rate für $\mathrm{Cd}$ und $\mathrm{Ba}$ nahe Null \\
\hline 61 & $\begin{array}{l}\text { CML 20m Aug } 99 \\
\text { WP KB }\end{array}$ & 7 & sehr geringe UF-Raten \\
\hline 62 & $\begin{array}{l}\text { CML 27.5m Aug } 99 \\
\text { WP KB }\end{array}$ & 6 & $\mathrm{pH}$ nicht niedrig $(6,0)$, niedrige UF-Raten für $\mathrm{Cu}$ und $\mathrm{Zn}$ \\
\hline 63 & $\begin{array}{l}\text { CML 31.5m Aug 99 } \\
\text { WP KB }\end{array}$ & 6 & $\begin{array}{l}\text { hohe Spurenelementgehalte, niedrige UF-Raten } \\
\text { aber: kein niedriger pH-Wert }(5,9), \text { Al-Festphasen deutlich übersättigt }\end{array}$ \\
\hline 64 & $\begin{array}{l}\text { CML 39.5m Aug } 99 \\
\text { WP KB } \\
\end{array}$ & 6 & $\begin{array}{l}\text { niedrige UF-Raten } \\
\text { aber: kein niedriger } \mathrm{pH} \text {-Wert }(6,8)\end{array}$ \\
\hline 65 & $\begin{array}{l}\text { CML 43.5m Aug 99 } \\
\text { WP KB }\end{array}$ & 7 & $\begin{array}{l}\text { niedrige UF-Raten } \\
\text { aber: kein niedriger } \mathrm{pH} \text {-Wert }(6,0)\end{array}$ \\
\hline
\end{tabular}


Tab. A.24. (Forts.): Einordnung der Proben in Gruppen nach den Ergebnissen der Ultrafiltration; AP = Proben aus der Arbeit von PLEßOW (1998), KB = Proben aus der vorliegenden Arbeit; PL = Porenlösung, WP = Wasserprobe.

\begin{tabular}{|c|c|c|c|}
\hline Nr. & Probe & $\begin{array}{l}\text { Gruppe } \\
\text { nach } \\
\text { UF }\end{array}$ & Kriterien \\
\hline 66 & $\begin{array}{l}\text { RCO3 Aug } 99 \text { WP } \\
\text { KB }\end{array}$ & 6 & $\mathrm{pH}$ nicht sehr niedrig $(5,3)$, geringe UF-Raten \\
\hline 67 & $\begin{array}{l}\text { RCO4 Aug } 99 \text { WP } \\
\text { KB }\end{array}$ & 6 & $\mathrm{pH}$ nicht niedrig $(6,3)$ \\
\hline 68 & $\begin{array}{l}\text { RCO12 Aug } 99 \text { WP } \\
\text { KB }\end{array}$ & 6 & $\begin{array}{l}\text { niedrige UF-Raten; aber: kein niedriger } \mathrm{pH} \text {-Wert }(6,1) \text {, zahlreiche Festphasen } \\
\text { deutlich übersättigt }\end{array}$ \\
\hline 69 & $\begin{array}{l}\text { RCO15 Aug } 99 \text { WP } \\
\text { KB }\end{array}$ & 7 & geringe Gehalte an $\mathrm{Co}, \mathrm{Ni}, \mathrm{Cu}$ und $\mathrm{Zn}$; geringe UF-Raten für $\mathrm{Cu}, \mathrm{Ba}$ und $\mathrm{Pb}$ \\
\hline 70 & $\begin{array}{l}\text { RCO17 Aug } 99 \text { WP } \\
\text { KB }\end{array}$ & 7 & $\begin{array}{l}\text { geringe Gehalte an } \mathrm{Co}, \mathrm{Ni}, \mathrm{Cu} \text { und } \mathrm{Zn} \\
\text { aber: UF-Rate für } \mathrm{Pb} 35 \%\end{array}$ \\
\hline 71 & $\begin{array}{l}\text { RCO18 Aug } 99 \text { WP } \\
\text { KB }\end{array}$ & 7 & kein niedriger pH-Wert $(6,4)$; zahlreiche Festphasen übersättigt \\
\hline 72 & See Aug 99 WP KB & 7 & geringe Konzentrationen für $\mathrm{Cu}$ und $\mathrm{Zn}$; geringe UF-Rate für $\mathrm{Cd}$ und $\mathrm{Ba}$ \\
\hline 73 & $\begin{array}{l}\text { See Sediment Aug } 99 \\
\text { WP KB }\end{array}$ & 7 & geringe Gehalte für $\mathrm{Co}, \mathrm{Cu}$ und $\mathrm{Zn}$; geringe Uf-Rate für $\mathrm{Cu}$ und $\mathrm{Ba}$ \\
\hline 74 & $\begin{array}{l}\text { See Sediment Aug } 99 \\
\text { PL KB }\end{array}$ & 7 & geringer Gehalt für $\mathrm{Co}$; geringe UF-Raten für $\mathrm{Cd}, \mathrm{Ba}$ und $\mathrm{Pb}$ \\
\hline 75 & $\begin{array}{l}\text { CML 20m } \\
\text { Jan 00 WP KB }\end{array}$ & 7 & geringe Gehalte von $\mathrm{Co}, \mathrm{Ni}, \mathrm{Cu}$ und $\mathrm{Zn}$ \\
\hline 76 & $\begin{array}{l}\text { CML 27.5m } \\
\text { Jan 00 WP KB }\end{array}$ & 6 & niedriger $\mathrm{pH}$-Wert $(3,4)$, niedrige UF-Raten für $\mathrm{Ca}, \mathrm{Zn}$ und $\mathrm{Ba}$ \\
\hline 77 & $\begin{array}{l}\text { CML 31.5m } \\
\text { Jan 00 WP KB }\end{array}$ & 7 & niedriger $\mathrm{pH}$-Wert $(3,42$, niedrige UF-Raten für $\mathrm{Ca}, \mathrm{Zn}, \mathrm{Cd}$ und $\mathrm{Ba}$ \\
\hline 78 & $\begin{array}{l}\text { CML 39.5m Jan 00 } \\
\text { WP KB }\end{array}$ & 7 & $\begin{array}{l}\text { geringe Gehalte an Spurenelementen wie } \mathrm{Co}, \mathrm{Ni}, \mathrm{Cu}, \mathrm{Zn} \text {; geringe UF-Raten } \\
\text { für Haupt- und Spurenelemente }\end{array}$ \\
\hline 79 & $\begin{array}{l}\text { CML 43.5m Jan 00 } \\
\text { WP KB }\end{array}$ & 7 & $\begin{array}{l}\text { geringe Gehalte an Spurenelementen wie } \mathrm{Co}, \mathrm{Ni}, \mathrm{Cu}, \mathrm{Zn} \text {; geringe UF-Raten } \\
\text { für Haupt- und Spurenelemente }\end{array}$ \\
\hline 80 & \begin{tabular}{llll|} 
RCO3 Jan & 00 & WP \\
KB & & &
\end{tabular} & 6 & $\begin{array}{l}\text { niedriger } \mathrm{pH}-\text { Wert }(2,5) \text {, hohe Gehalt an } \mathrm{Al}, \mathrm{Mn} \text { und } \mathrm{Fe} \text {, geringe UF-Raten für } \\
\mathrm{Ca} \text { und } \mathrm{Ba} \\
\text { aber: hohe UF-Raten für } \mathrm{Cd} \text { un } \mathrm{Pb}\end{array}$ \\
\hline 81 & $\begin{array}{l}\text { RCO4 Jan } 00 \text { WP } \\
\text { KB }\end{array}$ & 7 & geringe Gehalte an $\mathrm{Cu}$ und $\mathrm{Zn}$; geringe UF-Raten für $\mathrm{Ca}$ und $\mathrm{Pb}$ \\
\hline 82 & $\begin{array}{l}\text { RCO12 Jan } 00 \text { WP } \\
\text { KB }\end{array}$ & 5 & $\begin{array}{l}\text { geringe Gehalte an } \mathrm{Co}, \mathrm{Ni}, \mathrm{Cu} \text { und } \mathrm{Zn} \text {; hohe UF-Rate für } \mathrm{Cu} \text {, mittlere UF- } \\
\text { Rate für } \mathrm{Ca}\end{array}$ \\
\hline 83 & $\begin{array}{l}\text { RCO15 Jan 00 WP } \\
\text { KB }\end{array}$ & 7 & $\begin{array}{l}\text { geringer Gehalt an Co und } \mathrm{Zn} \text {; geringe UF-Raten für } \mathrm{Ca}, \mathrm{Ba} \text { und } \mathrm{Pb} \\
\text { aber: relativ hoher Gehalt }(20 \mu \mathrm{g} / \mathrm{l}) \text { an } \mathrm{Cu}\end{array}$ \\
\hline 84 & \begin{tabular}{llll|} 
RCO17 Jan 00 & WP \\
KB
\end{tabular} & 7 & geringer Gehalt an $\mathrm{Co}, \mathrm{Ni}, \mathrm{Cu}$ und $\mathrm{Zn}$; geringe UF-Raten für $\mathrm{Ca}, \mathrm{Ba}$ und $\mathrm{Pb}$ \\
\hline 85 & $\begin{array}{l}\text { RCO18 Jan 00 WP } \\
\text { KB }\end{array}$ & 7 & geringer Gehalt an $\mathrm{Co}, \mathrm{Ni}, \mathrm{Cu}$ und $\mathrm{Zn}$; geringe UF-Raten für $\mathrm{Ca}$ und $\mathrm{Ba}$ \\
\hline
\end{tabular}


Tab. A.24. (Forts.): Einordnung der Proben in Gruppen nach den Ergebnissen der Ultrafiltration; AP = Proben aus der Arbeit von PLEßOW (1998), KB = Proben aus der vorliegenden Arbeit; PL = Porenlösung, WP = Wasserprobe.

\begin{tabular}{|c|c|c|c|}
\hline Nr. & Probe & $\begin{array}{l}\text { Gruppe } \\
\text { nach } \\
\text { UF }\end{array}$ & Kriterien \\
\hline 86 & $\begin{array}{|lll|}\text { Lausitz } & 5,00 m & \text { WP } \\
\text { KB } & & \\
\end{array}$ & 6 & niedriger $\mathrm{pH}$-Wert $(3,4)$; sehr geringe UF-Raten \\
\hline 87 & $\begin{array}{|lll|}\text { Lausitz } & 5,21 \mathrm{~m} & \text { WP } \\
\text { KB }\end{array}$ & 7 & niedriger $\mathrm{pH}-$ Wert $(3,4)$ \\
\hline 88 & $\begin{array}{|lll|}\text { Lausitz } & 5,30 m & \text { WP } \\
\text { KB } & & \\
\end{array}$ & 7 & niedriger $\mathrm{pH}-$ Wert $(3,5)$ \\
\hline 89 & $\begin{array}{|lll|}\text { Lausitz } & 5,50 m & \text { WP } \\
\text { KB } & & \\
\end{array}$ & 7 & niedriger $\mathrm{pH}-\mathrm{Wert}(3,5)$ \\
\hline 90 & $\begin{array}{|lll|}\text { Lausitz } & 5,60 m & \text { WP } \\
\text { KB } & & \\
\end{array}$ & 6 & $\begin{array}{l}\text { niedriger } \mathrm{pH}-\text { Wert }(3,3) \text {, hohe Elementkonzentrationen, sehr geringe } \\
\text { Rückhalteraten von } \mathrm{Ca}, \mathrm{Fe}, \mathrm{Mn} \text {, Sulfat und Spurenelementen in der } \\
\text { Ultrafiltration }\end{array}$ \\
\hline 91 & $\begin{array}{lll}\text { Lausitz } & 5,75 m & \text { WP } \\
\text { KB } & & \\
\end{array}$ & 6 & $\begin{array}{l}\text { niedriger } \mathrm{pH}-\text { Wert }(3,3) \text {, hohe Elementkonzentrationen, sehr geringe } \\
\text { Rückhalteraten von } \mathrm{Ca}, \mathrm{Fe}, \mathrm{Mn} \text {, Sulfat und Spurenelementen in der } \\
\text { Ultrafiltration }\end{array}$ \\
\hline 92 & $\begin{array}{|lll|}\text { Lausitz } & \mathbf{5 , 9 3 m} & \text { WP } \\
\text { KB } & & \\
\end{array}$ & 6 & $\begin{array}{l}\text { niedriger } \mathrm{pH}-\text { Wert }(3,4) \text {, hohe Elementkonzentrationen, sehr geringe } \\
\text { Rückhalteraten von } \mathrm{Ca}, \mathrm{Fe}, \mathrm{Mn} \text {, Sulfat und Spurenelementen in der } \\
\text { Ultrafiltration }\end{array}$ \\
\hline 93 & $\begin{array}{l}\text { Lausitz 21,07m WP } \\
\text { KB }\end{array}$ & 6 & niedriger $\mathrm{pH}$-Wert $(3,9)$; Ca wird bei UF gar nicht abgetrennt \\
\hline 94 & $\begin{array}{l}\text { Lausitz 21,13m WP } \\
\text { KB }\end{array}$ & 6 & $\begin{array}{l}\text { niedriger } \mathrm{pH}-\text { Wert }(3,8) \text {, hohe Elementkonzentrationen, sehr geringe } \\
\text { Rückhalteraten von } \mathrm{Ca}, \mathrm{Fe}, \mathrm{Mn} \text {, Sulfat und Spurenelementen in der } \\
\text { Ultrafiltration }\end{array}$ \\
\hline 95 & $\begin{array}{l}\text { Lausitz 21,40m WP } \\
\text { KB }\end{array}$ & 4 & niedriger $\mathrm{pH}$, niedrige UF-Raten \\
\hline 96 & $\begin{array}{l}\text { Lausitz 21,70m WP } \\
\text { KB }\end{array}$ & $6 a$ & $\begin{array}{l}\text { niedriger } \mathrm{pH}-\text { Wert }(3,9) \text {, hohe Elementkonzentrationen, sehr geringe } \\
\text { Rückhalteraten von } \mathrm{Ca}, \mathrm{Fe}, \mathrm{Mn} \text {, Sulfat und Spurenelementen in der } \\
\text { Ultrafiltration }\end{array}$ \\
\hline 97 & $\begin{array}{l}\text { Lausitz 21,80m WP } \\
\text { KB }\end{array}$ & 6 & $\begin{array}{l}\text { niedriger } \mathrm{pH}-\text { Wert }(3,8), \text { hohe Elementkonzentrationen, sehr geringe } \\
\text { Rückhalteraten von } \mathrm{Ca}, \mathrm{Fe}, \mathrm{Mn} \text {, Sulfat und Spurenelementen in der } \\
\text { Ultrafiltration }\end{array}$ \\
\hline 98 & $\begin{array}{l}\text { Lausitz 21,90m WP } \\
\text { KB }\end{array}$ & 6 & $\begin{array}{l}\text { niedriger } \mathrm{pH}-\text { Wert }(2,7) \text {, hohe Elementkonzentrationen, sehr geringe } \\
\text { Rückhalteraten von } \mathrm{Ca}, \mathrm{Fe}, \mathrm{Mn} \text {, Sulfat und Spurenelementen in der } \\
\text { Ultrafiltration }\end{array}$ \\
\hline
\end{tabular}


Tab. A.25.: Ergebnisse der Speziationsberechnung mit MINTEQA2.

\begin{tabular}{|c|c|c|c|c|c|c|c|c|c|c|}
\hline & $\begin{array}{l}\text { RCO4 } \\
5-8 \text { m } \\
\text { PL AP }\end{array}$ & $\begin{array}{l}\text { RCO4 } \\
11-19 \text { m } \\
\text { PL AP }\end{array}$ & $\begin{array}{l}\text { RCO4 } \\
17 \mathrm{~m} \\
\text { PL KB }\end{array}$ & \begin{tabular}{|l}
$\mathrm{RCO4}$ \\
$20 \mathrm{~m}$ \\
$\mathrm{PL} \mathrm{KB}$ \\
\end{tabular} & \begin{tabular}{|l}
$\mathrm{RCO4}$ \\
$22 \mathrm{~m}$ \\
PL KB
\end{tabular} & \begin{tabular}{|l} 
RCO4 \\
26 m \\
PL KB
\end{tabular} & $\begin{array}{l}\text { RCO4 } \\
28 \text { m } \\
\text { PL AP }\end{array}$ & \begin{tabular}{|l|}
$\mathrm{RCO4}$ \\
$30 \mathrm{~m}$ \\
$\mathrm{PL}$ AP
\end{tabular} & $\begin{array}{l}\mathrm{RCO4} \\
32 \mathrm{~m} \\
\mathrm{PL} \text { AP }\end{array}$ & $\begin{array}{l}\text { RCO4 } \\
34 \text { m } \\
\text { PL AP }\end{array}$ \\
\hline Spezies & $\%$ & $\%$ & $\%$ & $\%$ & $\%$ & $\%$ & $\%$ & $\%$ & $\%$ & $\%$ \\
\hline $\mathbf{L i}^{+}$ & 96,8 & 97,1 & 97,3 & 97,9 & 98,2 & 95,6 & 92,7 & 95,1 & 96,1 & 96,1 \\
\hline $\mathrm{LiSO}_{4}^{-}$ & 3,2 & 2,9 & 2,7 & 2,1 & 1,8 & 5,3 & 7,3 & 4,9 & 3,2 & 3,9 \\
\hline $\mathrm{Be}^{2+}$ & 64,7 & 43,3 & $\mathrm{nq}$ & $\mathrm{nq}$ & $\mathrm{nq}$ & 64,4 & $\mathrm{nq}$ & $\mathrm{nq}$ & $\mathrm{nq}$ & $\mathrm{nq}$ \\
\hline $\mathrm{BeOH}^{+}$ & 3,3 & 36,4 & $\mathrm{nq}$ & $\mathrm{nq}$ & $\mathrm{nq}$ & 0,0 & $\mathrm{nq}$ & $\mathrm{nq}$ & $\mathrm{nq}$ & $\mathrm{nq}$ \\
\hline $\mathrm{BeSO}_{4}(\mathrm{aq})$ & 30,5 & 19,3 & $\mathrm{nq}$ & $\mathrm{nq}$ & $\mathrm{nq}$ & 32,6 & $\mathrm{nq}$ & $\mathrm{nq}$ & $\mathrm{nq}$ & $\mathrm{nq}$ \\
\hline $\mathrm{Be}\left(\mathrm{SO}_{4}\right)_{2}{ }^{2-}$ & 1,5 & 0,0 & $\mathrm{nq}$ & $\mathrm{nq}$ & $\mathrm{nq}$ & 2,8 & $\mathrm{nq}$ & $\mathrm{nq}$ & $\mathrm{nq}$ & $\mathrm{nq}$ \\
\hline $\mathrm{Na}^{+}$ & 96,1 & 96,4 & 96,8 & 97,5 & 97,7 & 94,7 & 91,2 & 94,0 & 96,1 & 95,2 \\
\hline $\mathrm{NaSO}_{4}^{-}$ & 3,9 & 3,6 & 3,2 & 2,5 & 2,3 & 5,3 & 8,8 & 6,0 & 3,9 & 4,8 \\
\hline $\mathrm{Mg}^{2+}$ & 64,3 & 65,6 & 68,2 & 71,6 & 72,8 & 62,7 & 46,7 & 53,4 & 64,8 & 58,8 \\
\hline $\mathrm{MgSO}_{4}$ (aq) & 35,7 & 34,4 & 31,8 & 28,4 & 27,2 & 37,3 & 53,3 & 46,6 & 35,2 & 41,2 \\
\hline $\mathbf{A l}^{3+}$ & 8,5 & 6,5 & 4,2 & $\mathrm{nq}$ & 1,6 & 9,5 & 0,0 & 0,0 & 2,1 & 0,0 \\
\hline $\mathrm{AlOH}^{2+}$ & 0,0 & 9,0 & 11,1 & $\mathrm{nq}$ & 8,2 & 0,0 & 0,0 & 4,4 & 8,4 & 1,0 \\
\hline $\mathrm{Al}(\mathrm{OH})_{2}{ }^{+}$ & 0,0 & 15,1 & 36,7 & $\mathrm{nq}$ & 47,8 & 0,0 & 2,3 & 35,8 & 42,6 & 17,3 \\
\hline $\mathrm{Al}(\mathrm{OH})_{3}(\mathrm{aq})$ & 0,0 & 0,0 & 4,6 & $\mathrm{nq}$ & 10,0 & 0,0 & 4,4 & 11,3 & 8,4 & 10,9 \\
\hline $\mathrm{Al}(\mathrm{OH})_{4}{ }^{-}$ & 0,0 & 0,0 & 5,9 & $\mathrm{nq}$ & 19,3 & 0,0 & 93,2 & 36,2 & 17,2 & 69,6 \\
\hline $\mathrm{AlSO}_{4}{ }^{+}$ & 83,9 & 63,2 & 35,1 & $\mathrm{nq}$ & 12,5 & 81,3 & 0,0 & 10,3 & 19,7 & 0,0 \\
\hline $\mathrm{Al}\left(\mathrm{SO}_{4}\right)_{2}^{-}$ & 6,8 & 4,6 & 2,4 & $\mathrm{nq}$ & 0,0 & 9,2 & 0,0 & 1,3 & 1,6 & 0,0 \\
\hline $\mathbf{K}^{+}$ & 94,9 & 95,4 & 95,8 & 96,7 & 97,1 & 93,1 & 88,7 & 92,3 & 94,9 & 93,9 \\
\hline $\mathrm{KSO}_{4}^{-}$ & 5,1 & 4,6 & 4,2 & 3,3 & 2,9 & 6,9 & 11,3 & 7,7 & 5,1 & 6,1 \\
\hline $\mathrm{Ca}^{2+}$ & 58,9 & 60,2 & 63,0 & 66,7 & 68,0 & 57,2 & 41,0 & 47,6 & 59,3 & 53,1 \\
\hline $\mathrm{CaSO}_{4}(\mathrm{aq})$ & 41,1 & 39,8 & 37,0 & 33,3 & 32,0 & 42,8 & 59,0 & 52,4 & 40,7 & 46,9 \\
\hline $\mathrm{VO}^{2+}$ & 14,4 & 0,0 & 0,0 & $\mathrm{nq}$ & 0,0 & 0,0 & 0,0 & 0,0 & 0,0 & 0,0 \\
\hline $\mathrm{V}(\mathrm{OH})_{3}{ }^{+}$ & 0,4 & 0,0 & 0,0 & $\mathrm{nq}$ & 0,0 & 0,0 & 0,0 & 0,0 & 0,0 & 0,0 \\
\hline $\mathrm{VOSO}_{4}(\mathrm{aq})$ & 12,1 & 0,0 & 0,0 & $\mathrm{nq}$ & 0,0 & 0,0 & 0,0 & 0,0 & 0,0 & 0,0 \\
\hline $\mathrm{V}(\mathrm{OH})_{3}(\mathrm{aq})$ & 2,2 & 0,0 & 21,5 & $\mathrm{nq}$ & 100,0 & 100,0 & 100,0 & 0,0 & 0,0 & 0,0 \\
\hline $\mathrm{VO}_{2}{ }^{+}$ & 1,6 & 0,0 & 0,0 & $\mathrm{nq}$ & 0,0 & 0,0 & 0,0 & 0,0 & 0,0 & 0,0 \\
\hline $\mathrm{HVO}_{4}{ }^{2-}$ & 0,0 & 0,0 & 0,0 & $\mathrm{nq}$ & 0,0 & 0,0 & 0,0 & 0,0 & 0,0 & 1,9 \\
\hline $\mathrm{H}_{2} \mathrm{VO}_{4}^{-}$ & 48,1 & 95,1 & 76,9 & $\mathrm{nq}$ & 0,0 & 0,0 & 0,0 & 97,5 & 98,7 & 97,4 \\
\hline $\mathrm{H}_{3} \mathrm{VO}_{4}(\mathrm{aq})$ & 15,1 & 1,9 & 0,0 & $\mathrm{nq}$ & 0,0 & 0,0 & 0,0 & 0,0 & 0,0 & 0,0 \\
\hline $\mathbf{H}_{3} \mathbf{V}_{2} \mathbf{O}_{7}^{-}$ & 5,9 & 2,7 & 0,0 & $\mathrm{nq}$ & 0,0 & 0,0 & 0,0 & 1,2 & 0,0 & 0,0 \\
\hline $\mathrm{Cr}^{3+}$ & 14,1 & 1,6 & 0,0 & 0,0 & 0,0 & 26,9 & 0,0 & 0,0 & 0,0 & 0,0 \\
\hline $\mathrm{Cr}(\mathrm{OH})_{2}{ }^{+}$ & 0,0 & 12,4 & 23,2 & 63,4 & 36,8 & 0,0 & 72,1 & 36,5 & 30,7 & 56,6 \\
\hline $\mathrm{CrOH}^{2+}$ & 25,4 & 48,3 & 46,0 & 23,5 & 41,5 & 2,3 & 10,1 & 29,0 & 39,5 & 22,2 \\
\hline $\mathrm{CrSO}_{4}^{+}$ & 41,7 & 4,7 & 2,0 & 0,0 & 0,0 & 69,0 & 0,0 & 0,0 & 1,3 & 0,0 \\
\hline $\mathrm{Cr}(\mathrm{OH})_{3}(\mathrm{aq})$ & 0,0 & 0,0 & 0,0 & 0,0 & 0,0 & 0,0 & 2,6 & 0,0 & 0,0 & 0,0 \\
\hline $\mathrm{CrOHSO}_{4}(\mathrm{aq})$ & 18,4 & 33,0 & 28,0 & 12,1 & 20,2 & 1,8 & 15,1 & 33,1 & 28,0 & 20,3 \\
\hline $\mathrm{Mn}^{2+}$ & 64,8 & 66,1 & 68,7 & 72,1 & 73,2 & 63,2 & 47,2 & 53,9 & 65,3 & 59,3 \\
\hline $\mathrm{MnSO}_{4}(\mathrm{aq})$ & 35,2 & 33,9 & 31,3 & 27,9 & 26,8 & 36,8 & 52,7 & 46,1 & 34,7 & 40,7 \\
\hline $\mathrm{Fe}^{2+}$ & 57,2 & 0,0 & 59,7 & 54,6 & 63,2 & 55,5 & 37,3 & 1,0 & 11,9 & 0,8 \\
\hline $\mathrm{FeSO}_{4}(\mathrm{aq})$ & 42,8 & 0,0 & 37,5 & 29,2 & 31,9 & 44,5 & 57,6 & 1,1 & 8,8 & 0,7 \\
\hline $\mathrm{FeOH}^{2+}$ & 0,0 & 0,0 & 0,0 & 0,0 & 0,0 & 0,0 & 0,0 & 0,0 & 0,0 & 0,0 \\
\hline $\mathrm{Fe}(\mathrm{OH})_{2}{ }^{+}$ & 1,2 & 0,0 & 2,8 & 15,6 & 4,9 & 0,0 & 4,3 & 96,2 & 78,4 & 95,0 \\
\hline $\mathrm{Fe}(\mathrm{OH})_{3}$ & 0,0 & 0,0 & 0,0 & 0,6 & 0,1 & 0,0 & 0,4 & 1,7 & 0,9 & 3,2 \\
\hline $\mathrm{Co}^{2+}$ & 62,2 & 63,5 & 66,1 & 69,7 & 0,0 & 60,5 & 44,4 & 51,1 & 62,6 & 56,5 \\
\hline $\mathrm{CoSO}_{4}(\mathrm{aq})$ & 37,8 & 36,5 & 33,8 & 30,3 & 0,0 & 39,5 & 55,6 & 48,9 & 37,4 & 43,4 \\
\hline $\mathrm{Ni}^{2+}$ & 62,2 & 63,5 & 66,1 & 69,7 & 70,9 & 60,5 & 44,3 & 51,0 & 62,6 & 56,5 \\
\hline $\mathrm{NiSO}_{4}(\mathrm{aq})$ & 37,8 & 36,5 & 33,8 & 30,3 & 29,1 & 39,5 & 55,5 & 48,9 & 37,4 & 43,4 \\
\hline $\mathrm{Cu}^{+}$ & 0,0 & 0,0 & 0,3 & 1,2 & 0,5 & 0,0 & 11,5 & 0,0 & 0,0 & 0,0 \\
\hline $\mathrm{Cu}^{2+}$ & 58,9 & 60,0 & 62,3 & 62,9 & 66,6 & \begin{tabular}{l|l}
57,2 \\
\end{tabular} & 33,7 & 46,9 & 58,6 & 51,3 \\
\hline $\mathrm{Cu}(\mathrm{OH})^{+}$ & 0,0 & 0,0 & 0,0 & 4,4 & 1,5 & 0,0 & 6,2 & 1,5 & 1,2 & 3,4 \\
\hline $\mathrm{CuSO}_{4}(\mathrm{aq})$ & 41,1 & 39,6 & 36,6 & 31,4 & 31,3 & 42,8 & 48,4 & 51,6 & 40,2 & 45,3 \\
\hline
\end{tabular}


Tab. A.25. (Forts.): Ergebnisse der Speziationsberechnung mit MINTEQA2.

\begin{tabular}{|c|c|c|c|c|c|c|c|c|c|c|}
\hline & \begin{tabular}{|l} 
RCO4 \\
$5-8$ m \\
PL AP
\end{tabular} & $\begin{array}{l}\text { RCO4 } \\
11-19 \text { m } \\
\text { PL AP }\end{array}$ & \begin{tabular}{|l} 
RCO4 \\
17 m \\
PL KB
\end{tabular} & \begin{tabular}{|l} 
RCO4 \\
20 m \\
PL KB
\end{tabular} & \begin{tabular}{|l} 
RCO4 \\
22 m \\
PL KB
\end{tabular} & \begin{tabular}{|l} 
RCO4 \\
$26 \mathrm{~m}$ \\
PL KB
\end{tabular} & \begin{tabular}{|l} 
RCO4 \\
28 m \\
PL AP
\end{tabular} & $\begin{array}{l}\mathrm{RCO4} \\
\mathbf{3 0} \mathrm{m} \\
\mathrm{PL} \text { AP }\end{array}$ & \begin{tabular}{|l} 
RCO4 \\
$32 \mathrm{~m}$ \\
PL AP
\end{tabular} & $\begin{array}{l}\text { RCO4 } \\
\text { 34 m } \\
\text { PL AP }\end{array}$ \\
\hline \begin{tabular}{|l|} 
Spezies \\
\end{tabular} & $\%$ & $\%$ & $\%$ & $\%$ & $\%$ & $\%$ & $\%$ & $\%$ & $\%$ & $\%$ \\
\hline $\mathrm{Zn}+{ }^{2}$ & 56,3 & 58,1 & 61,1 & 65,7 & 67,3 & 51,9 & 33,3 & 43,1 & 56,7 & 49,8 \\
\hline $\mathrm{ZnOH}^{+}$ & 0,0 & 0,0 & 0,0 & 0,0 & 0,0 & 0,0 & 0,0 & 0,0 & 0,0 & 0,0 \\
\hline $\mathrm{ZnSO}_{4}(\mathrm{aq})$ & 37,5 & 36,6 & 34,3 & 31,3 & 30,3 & 37,1 & 45,8 & 45,3 & 37,1 & 41,9 \\
\hline $\mathrm{Zn}\left(\mathrm{SO}_{4}\right)_{2}{ }^{2-}$ & 6,2 & 5,2 & 4,5 & 2,9 & 2,4 & 10,9 & 20,7 & 11,5 & 6,2 & 8,2 \\
\hline $\mathrm{HAsO}_{4}{ }^{2-}$ & 0,0 & 7,6 & 14,4 & 44,0 & 19,3 & 0,0 & 74,9 & 29,9 & 21,5 & 0,0 \\
\hline $\mathrm{H}_{2} \mathrm{AsO}_{4}^{-}$ & 98,9 & 92,3 & 85,6 & 56,0 & 80,7 & 92,6 & 25,1 & 70,1 & 78,5 & 54,5 \\
\hline $\mathrm{H}_{3} \mathrm{AsO}_{4}$ & 0,0 & 0,0 & 0,0 & 0,0 & 0,0 & 7,4 & 0,0 & 0,0 & 0,0 & 45,5 \\
\hline $\mathrm{H}_{3} \mathrm{AsO}_{3}$ & 0,0 & 0,0 & 0,0 & 0,0 & 0,0 & 0,0 & 0,0 & 0,0 & 0,0 & 0,0 \\
\hline $\mathrm{Sr}^{2+}$ & nq. & nq. & 66,2 & 69,7 & 70,9 & 60,5 & 44,4 & 51,1 & 62,6 & 56,5 \\
\hline $\mathrm{SrSO}_{4}(\mathrm{aq})$ & nq. & nq. & 33,8 & 30,3 & 29,1 & 39,5 & 55,6 & 48,9 & 37,4 & 43,5 \\
\hline $\mathrm{MoO}_{4}{ }^{2-}$ & 66,8 & 97,4 & 98,7 & 99,7 & 99,1 & 4,1 & 99,9 & 99,5 & 99,2 & 99,7 \\
\hline $\mathrm{HMoO}_{4}^{-}$ & 27,0 & 2,6 & 1,3 & 0,0 & 0,0 & 22,0 & 0,0 & 0,0 & 0,0 & 0,0 \\
\hline $\mathrm{H}_{2} \mathrm{MoO}_{4}$ (aq) & 6,2 & 0,0 & 0,0 & 0,0 & 0,0 & 73,9 & 0,0 & 0,0 & 0,0 & 0,0 \\
\hline $\mathrm{Cd}^{2+}$ & 52,7 & 54,8 & 58,0 & 63,1 & 64,9 & 47,2 & 28,5 & 38,9 & 53,1 & 45,9 \\
\hline $\mathrm{CdSO}_{4}(\mathrm{aq})$ & 37,7 & 37,0 & 34,9 & 32,2 & 31,3 & 36,2 & 42,0 & 43,8 & 37,2 & 41,5 \\
\hline $\mathrm{Cd}\left(\mathrm{SO}_{4}\right)_{2}{ }^{2-}$ & 9,6 & 8,2 & 7,2 & 4,7 & 3,8 & 16,5 & 29,4 & 17,3 & 9,7 & 12,6 \\
\hline $\operatorname{Sn}(\mathrm{OH})_{6}^{-}$ & 99,4 & 99,4 & 0,0 & 0,0 & 99,4 & 100,0 & $\mathrm{nq}$ & $\mathrm{nq}$ & $\mathrm{nq}$ & $\mathrm{nq}$ \\
\hline $\mathrm{SbO}_{3}^{-}$ & 99,9 & 99,9 & 99,9 & 99,9 & 99,9 & 99,9 & 99,9 & 99,9 & 99,9 & 99,9 \\
\hline $\mathrm{Sb}(\mathrm{OH})_{3}$ & 0,0 & 0,0 & 0,0 & 0,0 & 0,0 & 0,0 & 0,0 & 0,0 & 0,0 & 0,0 \\
\hline $\mathrm{Sb}(\mathrm{OH})_{2}{ }^{+}$ & 0,0 & 0,0 & 0,0 & 0,0 & 0,0 & 0,0 & 0,0 & 0,0 & 0,0 & 0,0 \\
\hline $\mathrm{HSbO}_{2}$ & 0,0 & 0,0 & 0,0 & 0,0 & 0,0 & 0,0 & 0,0 & 0,0 & 0,0 & 0,0 \\
\hline $\mathrm{SbO}^{+}$ & 0,0 & 0,0 & 0,0 & 0,0 & 0,0 & 0,0 & 0,0 & 0,0 & 0,0 & 0,0 \\
\hline $\mathrm{Ba}^{2+}$ & 100,0 & 100,0 & 100,0 & 100,0 & 100,0 & 100,0 & 100,0 & 100,0 & 100,0 & 100,0 \\
\hline $\mathrm{TI}^{+}$ & 84,9 & 86,1 & 87,2 & 89,8 & 90,8 & 80,2 & $\mathrm{nq}$ & $\mathrm{nq}$ & $\mathrm{nq}$ & $\mathrm{nq}$ \\
\hline $\mathrm{TISO}_{4}^{-}$ & 15,1 & 13,9 & 12,8 & 10,2 & 9,2 & 19,8 & $\mathrm{nq}$ & $\mathrm{nq}$ & $\mathrm{nq}$ & $\mathrm{nq}$ \\
\hline $\mathbf{P b}^{2+}$ & 37,5 & 39,1 & 42,0 & 45,7 & 48,1 & 34,1 & 19,3 & 26,4 & $\mathrm{nq}$ & 31,3 \\
\hline $\mathrm{PbOH}^{+}$ & 0,0 & 0,0 & 0,0 & 2,5 & 0,0 & 0,0 & 2,8 & 0,0 & $\mathrm{nq}$ & 1,6 \\
\hline $\mathrm{Pb}(\mathrm{OH})_{2}(\mathrm{aq})$ & 0,0 & 0,0 & 0,0 & 0,0 & 0,0 & 0,0 & 0,0 & 0,0 & $\mathrm{nq}$ & 0,0 \\
\hline $\mathrm{PbSO}_{4}$ (aq) & 56,1 & 55,2 & 52,7 & 48,6 & 48,4 & 54,7 & 59,3 & 62,0 & $\mathrm{nq}$ & 59,1 \\
\hline $\mathrm{Pb}\left(\mathrm{SO}_{4}\right)_{2}{ }^{2-}$ & 6,4 & 5,4 & 4,8 & 3,1 & 2,6 & 11,2 & 18,6 & 10,9 & $\mathrm{nq}$ & 8,0 \\
\hline $\mathrm{UO}_{2}{ }^{+}$ & $\mathrm{nq}$ & 0,0 & $\mathrm{nq}$ & $\mathrm{nq}$ & 0,0 & 0,0 & 0,0 & 0,0 & 0,0 & 0,0 \\
\hline $\mathrm{U}(\mathrm{OH})_{2}{ }^{2+}$ & $\mathrm{nq}$ & 0,0 & $\mathrm{nq}$ & $\mathrm{nq}$ & 0,0 & 0,0 & 0,0 & 0,0 & 0,0 & 0,0 \\
\hline $\mathrm{U}(\mathrm{OH})_{3}{ }^{+}$ & $\mathrm{nq}$ & 0,0 & $\mathrm{nq}$ & $\mathrm{nq}$ & 0,0 & 0,0 & 0,0 & 0,0 & 0,0 & 0,0 \\
\hline $\mathrm{USO}_{4}{ }^{2+}$ & $\mathrm{nq}$ & 0,0 & $\mathrm{nq}$ & $\mathrm{nq}$ & 0,0 & 0,0 & 0,0 & 0,0 & 0,0 & 0,0 \\
\hline $\mathrm{U}\left(\mathrm{SO}_{4}\right)_{2}(\mathrm{aq})$ & $\mathrm{nq}$ & 0,0 & $\mathrm{nq}$ & $\mathrm{nq}$ & 0,0 & 0,0 & 0,0 & 0,0 & 0,0 & 0,0 \\
\hline $\mathrm{UO}_{2}{ }^{2+}$ & $n q$ & 28,3 & $\mathrm{nq}$ & $\mathrm{nq}$ & 30,3 & 22,3 & 1,6 & 14,2 & 23,2 & 14,2 \\
\hline $\mathrm{UO}_{2} \mathrm{OH}^{+}$ & $\mathrm{nq}$ & 7,5 & $\mathrm{nq}$ & $\mathrm{nq}$ & 27,9 & 0,0 & 11,6 & 18,4 & 18,7 & 37,4 \\
\hline$\left(\mathrm{UO}_{2}\right)_{2}(\mathrm{OH})_{2}{ }^{2+}$ & $\mathrm{nq}$ & 0,0 & $\mathrm{nq}$ & $\mathrm{nq}$ & 0,0 & 0,0 & 0,0 & 0,0 & 0,0 & 0,0 \\
\hline$\left(\mathrm{UO}_{2}\right)_{3}(\mathrm{OH})_{5}{ }^{+}$ & $\mathrm{nq}$ & 0,0 & $\mathrm{nq}$ & $\mathrm{nq}$ & 0,0 & 0,0 & 72,7 & 0,0 & 0,0 & 0,0 \\
\hline $\mathrm{UO}_{2} \mathrm{SO}_{4}(\mathrm{aq})$ & $\mathrm{nq}$ & 42,9 & $\mathrm{nq}$ & $\mathrm{nq}$ & 32,7 & 38,4 & 5,2 & 35,8 & 36,6 & 28,8 \\
\hline $\mathrm{UO}_{2}\left(\mathrm{SO}_{4}\right)_{2}{ }^{2-}$ & $\mathrm{nq}$ & 21,2 & $\mathrm{nq}$ & $\mathrm{nq}$ & 8,9 & 39,2 & 8,1 & 31,6 & 21,3 & 19,5 \\
\hline $\mathrm{SO}_{4}{ }^{2-}$ & 63,5 & 64,3 & 60,3 & 63,0 & 66,0 & 44,2 & 86,3 & 83,5 & 60,3 & 73,0 \\
\hline $\mathrm{HSO}_{4}^{-}$ & 0,0 & 0,0 & 0,0 & 0,0 & 0,0 & 1,2 & 0,0 & 0,0 & 0,0 & 0,0 \\
\hline $\mathrm{AlSO}_{4}{ }^{+}$ & 0,0 & 0,0 & 0,0 & 0,0 & 0,0 & 1,9 & 0,0 & 0,0 & 0,0 & 0,0 \\
\hline $\mathrm{Al}\left(\mathrm{SO}_{4}\right)_{2}^{-}$ & 0,0 & 0,0 & 0,0 & 0,0 & 0,0 & 2,5 & 0,0 & 0,0 & 0,0 & 0,0 \\
\hline $\mathrm{FeSO}_{4}(\mathrm{aq})$ & 0,0 & 0,0 & 0,0 & 0,0 & 0,0 & 9,6 & 0,0 & 0,0 & 0,0 & 0,0 \\
\hline $\mathrm{MgSO}_{4}(\mathrm{aq})$ & 15,1 & 9,6 & 9,6 & 9,0 & 9,2 & 23,0 & 7,9 & 8,4 & 8,4 & 10,1 \\
\hline CaSO4 (aq) & 20,1 & 25,4 & 29,2 & 27,1 & 24,0 & 7,8 & 5,0 & 7,2 & 30,9 & 16,3 \\
\hline
\end{tabular}


Tab. A.25. (Forts.): Ergebnisse der Speziationsberechnung mit MINTEQA2.

\begin{tabular}{|c|c|c|c|c|c|c|c|c|c|c|}
\hline & $\begin{array}{l}\text { RCO4 } \\
30 \text { m } \\
\text { PL AP }\end{array}$ & $\begin{array}{l}\mathrm{RCO4} \\
32 \mathrm{~m} \\
\text { PL AP } \\
\end{array}$ & $\begin{array}{l}\text { RCO4 } \\
34 \text { m } \\
\text { PL AP }\end{array}$ & $\begin{array}{l}\mathrm{RCO4} \\
36 \mathrm{~m} \\
\mathrm{PL} \mathrm{KB}\end{array}$ & $\begin{array}{l}\text { RCO4 } \\
38 \text { m } \\
\text { PL AP }\end{array}$ & $\begin{array}{l}\text { RCO4 } \\
40 \mathrm{~m} \\
\text { PL KB }\end{array}$ & $\begin{array}{l}\mathrm{RCO4} \\
42 \mathrm{~m} \\
\mathrm{PL} \text { AP }\end{array}$ & \begin{tabular}{|l} 
RCO4 \\
$44 \mathrm{~m}$ \\
PL KB
\end{tabular} & $\begin{array}{l}\mathrm{RCO4} \\
46 \mathrm{~m} \\
\mathrm{PL} \text { AP }\end{array}$ & $\begin{array}{l}\text { RCO4 } \\
48 \mathrm{~m} \\
\mathrm{PL} \mathrm{KB}\end{array}$ \\
\hline Spezies & $\%$ & $\%$ & $\%$ & $\%$ & $\%$ & $\%$ & $\%$ & $\%$ & $\%$ & $\%$ \\
\hline $\mathrm{Li}^{+}$ & 95,1 & 96,1 & 96,1 & 97,3 & 96,8 & 97,7 & 97,4 & 98,8 & 96,9 & 97,3 \\
\hline $\mathrm{LiSO}_{4}^{-}$ & 4,9 & 3,2 & 3,9 & 2,7 & 3,2 & 2,3 & 2,6 & 1,2 & 3,1 & 2,7 \\
\hline $\mathrm{Be}^{2+}$ & $\mathrm{nq}$ & $\mathrm{nq}$ & $\mathrm{nq}$ & $\mathrm{nq}$ & 3,5 & $\mathrm{nq}$ & 5,1 & $\mathrm{nq}$ & 8,4 & $\mathrm{nq}$ \\
\hline $\mathrm{BeOH}^{+}$ & $\mathrm{nq}$ & $\mathrm{nq}$ & $\mathrm{nq}$ & $\mathrm{nq}$ & 89,2 & $\mathrm{nq}$ & 89,0 & $\mathrm{nq}$ & 85,5 & $\mathrm{nq}$ \\
\hline $\mathrm{Be}(\mathrm{OH})_{2}$ & $\mathrm{nq}$ & $\mathrm{nq}$ & $\mathrm{nq}$ & $\mathrm{nq}$ & 5,6 & $\mathrm{nq}$ & 3,6 & $\mathrm{nq}$ & 2,1 & $\mathrm{nq}$ \\
\hline $\mathrm{BeSO}_{4}(\mathrm{aq})$ & $\mathrm{nq}$ & $\mathrm{nq}$ & $\mathrm{nq}$ & $\mathrm{nq}$ & 1,6 & $\mathrm{nq}$ & 2,2 & $\mathrm{nq}$ & 3,8 & $\mathrm{nq}$ \\
\hline $\mathrm{Na}^{+}$ & 94,0 & 96,1 & 95,2 & 96,7 & 96,1 & 97,2 & 96,8 & 98,5 & 96,2 & 96,7 \\
\hline $\mathrm{NaSO}_{4}^{-}$ & 6,0 & 3,9 & 4,8 & 3,3 & 3,9 & 2,8 & 3,2 & 1,5 & 3,8 & 3,3 \\
\hline $\mathrm{Mg}^{2+}$ & 53,4 & 64,8 & 58,8 & 69,2 & 64,8 & 70,3 & 66,9 & 86,5 & 65,4 & 68,5 \\
\hline $\mathrm{MgSO}_{4}(\mathrm{aq})$ & 46,6 & 35,2 & 41,2 & 30,8 & 35,2 & 29,7 & 33,1 & 13,5 & 34,6 & 31,5 \\
\hline $\mathrm{Al}^{3+}$ & 0,0 & 2,1 & 0,0 & $\mathrm{nq}$ & 0,0 & 12,1 & 0,0 & $\mathrm{nq}$ & 0,0 & 0,0 \\
\hline $\mathrm{AlOH}^{2+}$ & 4,4 & 8,4 & 1,0 & $\mathrm{nq}$ & 0,0 & 0,0 & 0,0 & $\mathrm{nq}$ & 0,0 & 0,0 \\
\hline $\mathrm{Al}(\mathrm{OH})_{2}{ }^{+}$ & 35,8 & 42,6 & 17,3 & $\mathrm{nq}$ & 2,3 & 0,0 & 5,4 & $\mathrm{nq}$ & 12,1 & 0,0 \\
\hline $\mathrm{Al}(\mathrm{OH})_{3}(\mathrm{aq})$ & 11,3 & 8,4 & 10,9 & $\mathrm{nq}$ & 4,6 & 9,7 & 7,0 & $\mathrm{nq}$ & 9,6 & 0,0 \\
\hline $\mathrm{Al}(\mathrm{OH})_{4}^{-}$ & 36,2 & 17,2 & 69,6 & $\mathrm{nq}$ & 93,1 & 77,3 & 87,3 & $\mathrm{nq}$ & 77,3 & 0,0 \\
\hline $\mathrm{AlSO}_{4}{ }^{+}$ & 10,3 & 19,7 & 0,0 & $\mathrm{nq}$ & 0,0 & 0,0 & 0,0 & $\mathrm{nq}$ & 0,0 & 0,0 \\
\hline $\mathrm{Al}\left(\mathrm{SO}_{4}\right)_{2}^{-}$ & 1,3 & 1,6 & 0,0 & nq & 0,0 & 0,0 & 0,0 & $\mathrm{nq}$ & 0,0 & 0,0 \\
\hline $\mathbf{K}^{+}$ & 92,3 & 94,9 & 93,9 & 95,8 & 94,9 & 96,3 & 95,8 & 98,0 & 95,1 & 95,8 \\
\hline $\mathrm{KSO}_{4}^{-}$ & 7,7 & 5,1 & 6,1 & 4,2 & 5,1 & 3,7 & 4,2 & 2,0 & 4,9 & 4,2 \\
\hline $\mathrm{Ca}^{2+}$ & 47,6 & 59,3 & 53,1 & 64,1 & 59,4 & 65,3 & 61,6 & 83,6 & 60,1 & 63,3 \\
\hline $\mathrm{CaSO}_{4}(\mathrm{aq})$ & 52,4 & 40,7 & 46,9 & 35,9 & 40,6 & 34,7 & 38,4 & 16,4 & 39,9 & 36,7 \\
\hline $\mathrm{V}(\mathrm{OH})_{3}(\mathrm{aq})$ & 0,0 & 0,0 & 0,0 & 3,4 & 0,0 & 1,1 & 0,0 & 4,5 & 100,0 & 28,6 \\
\hline $\mathrm{HVO}_{4}{ }^{2-}$ & 0,0 & 0,0 & 1,9 & 2,3 & 5,9 & 2,3 & 3,5 & 1,8 & 0,0 & 0,0 \\
\hline $\mathrm{H}_{2} \mathrm{VO}_{4}{ }^{-}$ & 97,5 & 98,7 & 97,4 & 94,0 & 93,9 & 96,1 & 96,3 & 93,3 & 0,0 & 69,9 \\
\hline $\mathrm{H}_{3} \mathrm{VO}_{4}(\mathrm{aq})$ & 0,0 & 0,0 & 0,0 & 0,0 & 0,0 & 0,0 & 0,0 & 0,0 & 0,0 & 0,0 \\
\hline $\mathbf{H}_{3} \mathbf{V}_{2} \mathbf{O}_{7}^{-}$ & 1,2 & 0,0 & 0,0 & 0,0 & 0,0 & 0,0 & 0,0 & 0,0 & 0,0 & 0,0 \\
\hline $\mathrm{Cr}^{3+}$ & 0,0 & 0,0 & 0,0 & 0,0 & 0,0 & 0,0 & 0,0 & 0,0 & 0,0 & 0,0 \\
\hline $\mathrm{Cr}(\mathrm{OH})_{2}^{+}$ & 36,5 & 30,7 & 56,6 & 65,3 & 78,6 & 67,0 & 74,8 & 56,9 & 64,1 & 23,1 \\
\hline $\mathrm{CrOH}^{2+}$ & 29,0 & 39,5 & 22,2 & 21,2 & 10,1 & 20,5 & 14,1 & 35,1 & 20,6 & 46,3 \\
\hline $\mathrm{CrSO}_{4}{ }^{+}$ & 0,0 & 1,3 & 0,0 & 0,0 & 0,0 & 0,0 & 0,0 & 0,0 & 0,0 & 2,0 \\
\hline $\mathrm{Cr}(\mathrm{OH})_{3}(\mathrm{aq})$ & 0,0 & 0,0 & 0,0 & 0,0 & 3,0 & 1,0 & 1,8 & 0,0 & 0,0 & 0,0 \\
\hline $\mathrm{CrOHSO}_{4}(\mathrm{aq})$ & 33,1 & 28,0 & 20,3 & 12,3 & 7,2 & 11,3 & 9,1 & 7,1 & 14,2 & 27,8 \\
\hline $\mathrm{CrO}_{2}^{-}$ & 0,0 & 0,0 & 0,0 & 0,0 & 0,0 & 0,0 & 0,0 & 0,0 & 0,0 & 0,0 \\
\hline $\mathrm{CrO}_{4}^{2-}$ & 0,0 & 0,0 & 0,0 & 0,0 & 1,0 & 0,0 & 0,0 & 0,0 & 0,0 & 0,0 \\
\hline $\mathrm{HCrO}_{4}^{-}$ & 0,0 & 0,0 & 0,0 & 0,0 & 0,1 & 0,0 & 0,0 & 0,0 & 0,0 & 0,0 \\
\hline $\mathrm{Mn}^{2+}$ & 53,9 & 65,3 & 59,3 & 69,7 & 65,3 & 70,8 & 67,4 & 86,8 & 66,0 & 69,0 \\
\hline $\mathrm{MnSO}_{4}(\mathrm{aq})$ & 46,1 & 34,7 & 40,7 & 30,3 & 34,7 & 29,2 & 32,6 & 13,2 & 34,0 & 31,0 \\
\hline $\mathrm{Fe}^{2+}$ & 1,0 & 11,9 & 0,8 & 51,9 & $\mathrm{nq}$ & 45,6 & $\mathrm{nq}$ & $\mathrm{nq}$ & $\mathrm{nq}$ & 60,3 \\
\hline $\mathrm{FeSO}_{4}(\mathrm{aq})$ & 1,1 & 8,8 & 0,7 & 31,2 & $\mathrm{nq}$ & 26,0 & $\mathrm{nq}$ & $\mathrm{nq}$ & $\mathrm{nq}$ & 37,4 \\
\hline $\mathrm{FeOH}^{2+}$ & 0,0 & 0,0 & 0,0 & 0,0 & $\mathrm{nq}$ & 0,0 & $\mathrm{nq}$ & $\mathrm{nq}$ & $\mathrm{nq}$ & 0,0 \\
\hline $\mathrm{Fe}(\mathrm{OH})_{2}{ }^{+}$ & 96,2 & 78,4 & 95,0 & 16,2 & $\mathrm{nq}$ & 27,2 & $\mathrm{nq}$ & $\mathrm{nq}$ & $\mathrm{nq}$ & 2,3 \\
\hline $\mathrm{Fe}(\mathrm{OH})_{3}$ & 1,7 & 0,9 & 3,2 & 0,7 & $\mathrm{nq}$ & 1,2 & $\mathrm{nq}$ & $\mathrm{nq}$ & $\mathrm{nq}$ & 0,0 \\
\hline $\mathrm{Co}^{2+}$ & 51,1 & 62,6 & 56,5 & 67,2 & 62,6 & 68,3 & 64,8 & 85,4 & 63,3 & 66,4 \\
\hline $\mathrm{CoSO}_{4}(\mathrm{aq})$ & 48,9 & 37,4 & 43,4 & 32,8 & 37,3 & 31,6 & 35,1 & 14,6 & 36,7 & 33,6 \\
\hline $\mathrm{Ni}^{2+}$ & 51,0 & 62,6 & 56,5 & 67,2 & 62,6 & 68,3 & 64,8 & 85,4 & 63,3 & 66,4 \\
\hline $\mathrm{NiSO}_{4}$ (aq) & 48,9 & 37,4 & 43,4 & 32,8 & 37,3 & 31,6 & 35,1 & 14,6 & 36,6 & 33,6 \\
\hline $\mathrm{Cu}^{+}$ & 0,0 & 0,0 & 0,0 & 0,0 & 0,0 & 0,0 & 0,0 & 0,9 & 0,0 & 0,0 \\
\hline $\mathrm{Cu}^{2+}$ & 46,9 & 58,6 & 51,3 & 61,0 & 52,9 & 61,8 & 56,8 & 80,0 & 57,3 & 62,8 \\
\hline $\mathrm{Cu}(\mathrm{OH})^{+}$ & 1,5 & 1,2 & 3,4 & 4,9 & 10,7 & 5,2 & 7,8 & 3,4 & 4,6 & 0,0 \\
\hline $\mathrm{Cu}(\mathrm{OH})_{2}$ & 0,0 & 0,0 & 0,0 & 0,0 & 0,0 & 0,0 & 0,0 & 0,0 & 0,0 & 0,0 \\
\hline $\mathrm{CuSO}_{4}(\mathrm{aq})$ & 51,6 & 40,2 & 45,3 & 34,1 & 36,2 & 32,9 & 35,3 & 15,8 & 38,1 & 36,4 \\
\hline
\end{tabular}


Tab. A.25. (Forts.): Ergebnisse der Speziationsberechnung mit MINTEQA2.

\begin{tabular}{|c|c|c|c|c|c|c|c|c|c|c|}
\hline & $\begin{array}{l}\text { RCO4 } \\
30 \text { m } \\
\text { PL AP }\end{array}$ & $\begin{array}{l}\text { RCO4 } \\
32 \text { m } \\
\text { PL AP }\end{array}$ & $\begin{array}{l}\text { RCO4 } \\
34 \text { m } \\
\text { PL AP }\end{array}$ & $\begin{array}{l}\text { RCO4 } \\
36 \text { m } \\
\text { PL KB }\end{array}$ & $\begin{array}{l}\text { RCO4 } \\
38 \text { m } \\
\text { PL AP }\end{array}$ & $\begin{array}{l}\text { RCO4 } \\
40 \mathrm{~m} \\
\text { PL KB }\end{array}$ & \begin{tabular}{|l}
$\mathrm{RCO4}$ \\
$42 \mathrm{~m}$ \\
$\mathrm{PL} \mathrm{AP}$
\end{tabular} & $\begin{array}{l}\text { RCO4 } \\
44 \mathrm{~m} \\
\text { PL KB }\end{array}$ & $\begin{array}{l}\text { RCO4 } \\
46 \mathrm{~m} \\
\text { PL AP }\end{array}$ & \begin{tabular}{|l}
$\mathrm{RCO4}$ \\
$48 \mathrm{~m}$ \\
$\mathrm{PL} \mathrm{KB}$
\end{tabular} \\
\hline Spezies & $\%$ & $\%$ & $\%$ & $\%$ & $\%$ & $\%$ & $\%$ & $\%$ & $\%$ & $\%$ \\
\hline $\mathrm{Zn}+{ }^{2}$ & 43,1 & 56,7 & 49,8 & 62,0 & 56,5 & 63,8 & 59,8 & 83,0 & 57,5 & 61,4 \\
\hline $\mathrm{ZnOH}^{+}$ & 0,0 & 0,0 & 0,0 & 0,0 & 0,0 & 0,0 & 0,0 & 0,0 & 0,0 & 0,0 \\
\hline $\mathrm{ZnSO}_{4}(\mathrm{aq})$ & 45,3 & 37,1 & 41,9 & 33,2 & 36,9 & 32,4 & 53,6 & 15,6 & 36,5 & 34,0 \\
\hline $\mathrm{Zn}\left(\mathrm{SO}_{4}\right)_{2}{ }^{2-}$ & 11,5 & 6,2 & 8,2 & 4,6 & 6,2 & 3,6 & 4,4 & 1,3 & 5,8 & 4,6 \\
\hline $\mathrm{HAsO}_{4}{ }^{2-}$ & 29,9 & 21,5 & 0,0 & 52,3 & 73,2 & 50,8 & 61,5 & 45,3 & 52,0 & 14,5 \\
\hline $\mathrm{H}_{2} \mathrm{AsO}_{4}^{-}$ & 70,1 & 78,5 & 54,5 & 47,7 & 26,7 & 49,2 & 38,5 & 54,7 & 48,0 & 85,5 \\
\hline $\mathrm{H}_{3} \mathrm{AsO}_{4}$ & 0,0 & 0,0 & 45,5 & 0,0 & 0,0 & 0,0 & 0,0 & 0,0 & 0,0 & 0,0 \\
\hline $\mathrm{H}_{3} \mathrm{AsO}_{3}$ & 0,0 & 0,0 & 0,0 & 0,0 & 0,0 & 0,0 & 0,0 & 0,0 & 0,0 & 0,0 \\
\hline $\mathrm{Sr}^{2+}$ & 51,1 & 62,6 & 56,5 & 67,2 & $\mathrm{nq}$ & 68,3 & 64,9 & 85,4 & 63,3 & 66,4 \\
\hline $\mathrm{SrSO}_{4}(\mathrm{aq})$ & 48,9 & 37,4 & 43,5 & 32,8 & $\mathrm{nq}$ & 1,7 & 35,1 & 14,6 & 36,7 & 33,6 \\
\hline $\mathrm{MoO}_{4}{ }^{2-}$ & 99,5 & 99,2 & 99,7 & 99,8 & 99,9 & 99,8 & 99,9 & 99,7 & 99,8 & 98,7 \\
\hline $\mathrm{HMoO}_{4}^{-}$ & 0,0 & 0,0 & 0,0 & 0,0 & 0,0 & 0,0 & 0,0 & 0,0 & 0,0 & 1,3 \\
\hline $\mathrm{H}_{2} \mathrm{MoO}_{4}(\mathrm{aq})$ & 0,0 & 0,0 & 0,0 & 0,0 & 0,0 & 0,0 & 0,0 & 0,0 & 0,0 & 0,0 \\
\hline $\mathrm{Cd}^{2+}$ & 38,9 & 53,1 & 45,9 & 58,9 & 53,1 & 61,0 & 56,9 & 81,5 & 54,1 & 58,2 \\
\hline $\mathrm{CdSO}_{4}(\mathrm{aq})$ & 43,8 & 37,2 & 41,5 & 33,8 & 37,2 & 33,2 & 36,2 & 16,4 & 36,8 & 34,6 \\
\hline $\mathrm{Cd}\left(\mathrm{SO}_{4}\right)_{2}{ }^{2-}$ & 17,3 & 9,7 & 12,6 & 7,3 & 9,6 & 5,7 & 6,9 & 2,1 & 9,1 & 7,2 \\
\hline $\mathrm{Sn}(\mathrm{OH})_{6}^{-}$ & 0,0 & & 0,0 & 99,4 & 99,4 & 99,4 & & 0,0 & 99,4 & 0,0 \\
\hline $\mathrm{SbO}_{3}^{-}$ & 99,9 & 99,9 & 99,9 & 99,9 & 99,9 & 99,9 & 99,9 & 99,9 & 99,9 & $\mathrm{nq}$ \\
\hline $\mathrm{Sb}(\mathrm{OH})_{3}$ & 0,0 & 0,0 & 0,0 & 0,0 & 0,0 & 0,0 & 0,0 & 0,0 & 0,0 & $\mathrm{nq}$ \\
\hline $\mathrm{Sb}(\mathrm{OH})_{2}{ }^{+}$ & 0,0 & 0,0 & 0,0 & 0,0 & 0,0 & 0,0 & 0,0 & 0,0 & 0,0 & $\mathrm{nq}$ \\
\hline $\mathrm{HSbO}_{2}$ & 0,0 & 0,0 & 0,0 & 0,0 & 0,0 & 0,0 & 0,0 & 0,0 & 0,0 & $\mathrm{nq}$ \\
\hline $\mathrm{SbO}^{+}$ & 0,0 & 0,0 & 0,0 & 0,0 & 0,0 & 0,0 & 0,0 & 0,0 & 0,0 & $\mathrm{nq}$ \\
\hline $\mathrm{Ba}^{2+}$ & 100,0 & 100,0 & 100,0 & 100,0 & 100,0 & 100,0 & 100,0 & 100,0 & 100,0 & 100,0 \\
\hline $\mathrm{Tl}^{+}$ & $\mathrm{nq}$ & $\mathrm{nq}$ & $\mathrm{nq}$ & 87,2 & $\mathrm{nq}$ & 88,7 & $\mathrm{nq}$ & $\mathrm{nq}$ & $\mathrm{nq}$ & 87,2 \\
\hline $\mathrm{TISO}_{4}^{-}$ & $\mathrm{nq}$ & $\mathrm{nq}$ & $\mathrm{nq}$ & 12,8 & $\mathrm{nq}$ & 11,3 & $\mathrm{nq}$ & $\mathrm{nq}$ & $\mathrm{nq}$ & 12,8 \\
\hline $\mathbf{P b}^{2+}$ & 26,4 & $\mathrm{nq}$ & 31,3 & 42,1 & 35,8 & 43,6 & 39,2 & 67,7 & 37,8 & 42,3 \\
\hline $\mathbf{P b O H}^{+}$ & 0,0 & $\mathrm{nq}$ & 1,6 & 2,7 & 5,7 & 2,9 & 4,3 & 2,3 & 2,4 & 0,0 \\
\hline $\mathrm{Pb}(\mathrm{OH})_{2}(\mathrm{aq})$ & 0,0 & $\mathrm{nq}$ & 0,0 & 0,0 & 0,0 & 0,0 & 0,0 & 0,0 & 0,0 & 0,0 \\
\hline $\mathrm{PbSO}_{4}(\mathrm{aq})$ & 62,0 & $\mathrm{nq}$ & 59,1 & 50,4 & 52,4 & 49,6 & 52,1 & 28,4 & 53,8 & 52,4 \\
\hline $\mathrm{Pb}\left(\mathrm{SO}_{4}\right)_{2}{ }^{2-}$ & 10,9 & $\mathrm{nq}$ & 8,0 & 4,9 & 6,1 & 3,8 & 4,4 & 1,6 & 5,9 & 4,9 \\
\hline $\mathrm{UO}_{2}{ }^{+}$ & 0,0 & 0,0 & 0,0 & 0,0 & 0,0 & $\mathrm{nq}$ & 0,0 & 0,4 & 0,0 & 0,0 \\
\hline $\mathrm{U}(\mathrm{OH})_{2}{ }^{2+}$ & 0,0 & 0,0 & 0,0 & 0,0 & 0,0 & $\mathrm{nq}$ & 0,0 & 0,0 & 0,0 & 0,0 \\
\hline $\mathrm{U}(\mathrm{OH})_{3}{ }^{+}$ & 0,0 & 0,0 & 0,0 & 0,0 & 0,0 & $\mathrm{nq}$ & 0,0 & 0,0 & 0,0 & 0,0 \\
\hline $\mathrm{U}(\mathrm{OH})_{4}(\mathrm{aq})$ & 0,0 & 0,0 & 0,0 & 0,0 & 0,0 & $\mathrm{nq}$ & 0,0 & 0,0 & 0,0 & 0,0 \\
\hline $\mathrm{USO}_{4}{ }^{2+}$ & 0,0 & 0,0 & 0,0 & 0,0 & 0,0 & $\mathrm{nq}$ & 0,0 & 0,0 & 0,0 & 0,0 \\
\hline $\mathrm{U}\left(\mathrm{SO}_{4}\right)_{2}$ (aq) & 0,0 & 0,0 & 0,0 & 0,0 & 0,0 & $\mathrm{nq}$ & 0,0 & 0,0 & 0,0 & 0,0 \\
\hline $\mathrm{UO}_{2}{ }^{2+}$ & 14,2 & 23,2 & 14,2 & 14,0 & 2,6 & nq & 4,5 & 21,6 & 7,2 & 0,0 \\
\hline $\mathrm{UO}_{2} \mathbf{O H}^{+}$ & 18,4 & 18,7 & 37,4 & 44,6 & 20,7 & $\mathrm{nq}$ & 24,5 & 36,2 & 23,3 & 0,0 \\
\hline$\left(\mathrm{UO}_{2}\right)_{2}(\mathrm{OH})_{2}{ }^{2+}$ & 0,0 & 0,0 & 0,0 & 1,6 & 1,0 & $\mathrm{nq}$ & 1,5 & 3,9 & 2,3 & 0,0 \\
\hline$\left(\mathrm{UO}_{2}\right)_{3}(\mathrm{OH})_{5}{ }^{+}$ & 0,0 & 0,0 & 0,0 & 13,1 & 69,3 & $\mathrm{nq}$ & 60,4 & 25,7 & 50,0 & 0,0 \\
\hline $\mathrm{UO}_{2} \mathrm{SO}_{4}(\mathrm{aq})$ & 35,8 & 36,6 & 28,8 & 18,0 & 4,1 & $\mathrm{nq}$ & 6,4 & 9,7 & 11,1 & 0,0 \\
\hline $\mathrm{UO}_{2}\left(\mathrm{SO}_{4}\right)_{2}{ }^{2-}$ & 31,6 & 21,3 & 19,5 & 8,7 & 2,4 & 0,0 & 2,7 & 2,8 & 6,1 & 0,0 \\
\hline $\mathrm{SO}_{4}{ }^{2-}$ & 83,5 & 60,3 & 73,0 & 58,2 & 62,3 & 61,2 & 66,7 & 39,9 & 61,6 & 59,9 \\
\hline $\mathrm{HSO}_{4}^{-}$ & 0,0 & 0,0 & 0,0 & 0,0 & 0,0 & 0,0 & 0,0 & 0,0 & 0,0 & 0,0 \\
\hline $\mathrm{AlSO}_{4}{ }^{+}$ & 0,0 & 0,0 & 0,0 & 0,0 & 0,0 & 0,0 & 0,0 & 0,0 & 0,0 & 0,0 \\
\hline $\mathrm{Al}\left(\mathrm{SO}_{4}\right)_{2}{ }^{-}$ & 0,0 & 0,0 & 0,0 & 0,0 & 0,0 & 0,0 & 0,0 & 0,0 & 0,0 & 0,0 \\
\hline $\mathrm{FeSO}_{4}(\mathrm{aq})$ & 0,0 & 0,0 & 0,0 & 0,0 & 0,0 & 0,0 & 0,0 & 0,0 & 0,0 & 0,0 \\
\hline $\mathrm{MgSO}_{4}(\mathrm{aq})$ & 8,4 & 8,4 & 10,1 & 18,0 & 18,2 & 12,3 & 11,1 & 51,3 & 13,2 & 12,9 \\
\hline CaSO4 (aq) & \begin{tabular}{ll|}
7,2 & \\
\end{tabular} & 30,9 & 16,3 & 22,7 & 18,8 & 25,6 & 21,1 & 8,3 & 24,3 & 25,7 \\
\hline
\end{tabular}


Tab. A.25. (Forts.): Ergebnisse der Speziationsberechnung mit MINTEQA2.

\begin{tabular}{|c|c|c|c|c|c|c|c|c|c|c|}
\hline & \begin{tabular}{|l} 
RCO4 \\
$52 \mathrm{~m}$ \\
$\mathrm{PL}$ KB
\end{tabular} & \begin{tabular}{|l} 
RCO4 \\
$56 \mathrm{~m}$ \\
PL AP
\end{tabular} & $\begin{array}{l}\text { RCO4 } \\
58 \mathrm{~m} \\
\text { PL AP }\end{array}$ & $\begin{array}{l}\text { RCO10 } \\
2 \mathrm{~m} \\
\text { PL AP }\end{array}$ & $\begin{array}{l}\text { RCO10 } \\
18,5 \mathrm{~m} \\
\mathrm{PL} \text { AP }\end{array}$ & \begin{tabular}{|l} 
RCO10 \\
29 m \\
PL AP
\end{tabular} & $\begin{array}{l}\text { RCO13 } \\
15 \mathrm{~m} \\
\text { PL AP }\end{array}$ & $\begin{array}{l}\text { RCO13 } \\
30 \text { m } \\
\text { PL AP }\end{array}$ & $\begin{array}{l}\text { RCO16 } \\
29 \text { m } \\
\text { PL AP }\end{array}$ & $\begin{array}{l}\text { Fremd- } \\
\text { flutung } \\
\text { WP AP }\end{array}$ \\
\hline \begin{tabular}{|l|} 
Spezies \\
\end{tabular} & $\%$ & $\%$ & $\%$ & $\%$ & $\%$ & $\%$ & $\%$ & $\%$ & $\%$ & $\%$ \\
\hline $\mathbf{L i}^{+}$ & 97,5 & 95,6 & 97,5 & 98,9 & 98,3 & 98,7 & 95,3 & 99,1 & 96,4 & 98,6 \\
\hline $\mathrm{LiSO}_{4}^{-}$ & 2,5 & 4,4 & 2,5 & 1,1 & 1,7 & 1,3 & 4,7 & 0,0 & 3,6 & 1,4 \\
\hline $\mathrm{Be}^{2+}$ & $\mathrm{nq}$ & $\mathrm{nq}$ & $\mathrm{nq}$ & $\mathrm{nq}$ & $\mathrm{nq}$ & $\mathrm{nq}$ & $\mathrm{nq}$ & $\mathrm{nq}$ & 13,9 & $\mathrm{nq}$ \\
\hline $\mathrm{BeOH}^{+}$ & $\mathrm{nq}$ & $\mathrm{nq}$ & $\mathrm{nq}$ & $\mathrm{nq}$ & $\mathrm{nq}$ & $\mathrm{nq}$ & $\mathrm{nq}$ & $\mathrm{nq}$ & 77,8 & $\mathrm{nq}$ \\
\hline$\overline{\mathrm{Be}(\mathrm{OH})_{2}}$ & $\mathrm{nq}$ & $\mathrm{nq}$ & nq & $\mathrm{nq}$ & $\mathrm{nq}$ & $\mathrm{nq}$ & $\mathrm{nq}$ & $\mathrm{nq}$ & 1,0 & $\mathrm{nq}$ \\
\hline $\mathrm{BeSO}_{4}$ (aq) & $\mathrm{nq}$ & $\mathrm{nq}$ & $\mathrm{nq}$ & $\mathrm{nq}$ & $\mathrm{nq}$ & $\mathrm{nq}$ & $\mathrm{nq}$ & $\mathrm{nq}$ & 6,9 & $\mathrm{nq}$ \\
\hline $\mathrm{Na}^{+}$ & 96,9 & 94,7 & 96,9 & 98,6 & 98,0 & 98,5 & 94,3 & 98,9 & 95,6 & 98,3 \\
\hline $\mathrm{NaSO}_{4}^{-}$ & 3,1 & 5,3 & 3,1 & 1,4 & 2,0 & 1,5 & 5,7 & 1,1 & 4,4 & 1,7 \\
\hline $\mathrm{Mg}^{2+}$ & 69,1 & 55,5 & 70,8 & 79,3 & 73,8 & 78,4 & 56,8 & 83,3 & 59,3 & 76,6 \\
\hline $\mathrm{MgSO}_{4}$ (aq) & 30,9 & 44,5 & 29,2 & 20,7 & 26,2 & 21,6 & 43,2 & 16,7 & 40,7 & 23,4 \\
\hline $\mathbf{A l}^{3+}$ & $\mathrm{nq}$ & 0,0 & 0,0 & 0,0 & 0,0 & 0,0 & 0,0 & 0,0 & 0,0 & 0,0 \\
\hline $\mathrm{AlOH}^{2+}$ & $\mathrm{nq}$ & 1,8 & 4,8 & 0,0 & 2,8 & 4,0 & 3,4 & 0,0 & 3,1 & 0,0 \\
\hline $\mathrm{Al}(\mathrm{OH})_{2}{ }^{+}$ & $\mathrm{nq}$ & 23,6 & 38,1 & 0,0 & 49,2 & 55,8 & 47,0 & 13,7 & 47,8 & 0,0 \\
\hline $\mathrm{Al}(\mathrm{OH})_{3}(\mathrm{aq})$ & $\mathrm{nq}$ & 11,9 & 11,8 & 5,3 & 21,0 & 19,1 & 18,4 & 19,0 & 19,6 & 4,2 \\
\hline $\mathrm{Al}(\mathrm{OH})_{4}^{-}$ & $\mathrm{nq}$ & 59,9 & 38,6 & 93,9 & 24,0 & 17,2 & 22,9 & 67,0 & 23,3 & 95,3 \\
\hline $\mathrm{AlSO}_{4}{ }^{+}$ & $\mathrm{nq}$ & 2,4 & 5,5 & 0,0 & 2,4 & 3,2 & 6,8 & 0,0 & 5,2 & 0,0 \\
\hline $\mathbf{K}^{+}$ & 96,0 & 93,2 & 96,0 & 98,4 & 97,6 & 98,2 & 93,2 & 98,7 & 94,7 & 98,0 \\
\hline $\mathrm{KSO}_{4}^{-}$ & 4,0 & 6,8 & 4,0 & 1,6 & 2,4 & 1,8 & 6,8 & 1,3 & 5,3 & 2,0 \\
\hline $\mathrm{Ca}^{2+}$ & 64,0 & 49,7 & 65,9 & 75,4 & 69,3 & 74,4 & 51,3 & 80,0 & 53,9 & 72,4 \\
\hline $\begin{array}{l}\mathrm{CaSO}_{4}(\mathrm{aq}) \\
\end{array}$ & 36,0 & 50,3 & 34,1 & 24,6 & 30,7 & 25,6 & 48,7 & 20,0 & 46,1 & 27,6 \\
\hline $\mathrm{VO}^{2+}$ & $\mathrm{nq}$ & 0,0 & 0,0 & 0,0 & 21,4 & 0,0 & 0,0 & 0,0 & 0,0 & 0,0 \\
\hline$\overline{\mathrm{V}(\mathrm{OH})_{3}{ }^{+}}$ & $\mathrm{nq}$ & 0,0 & 0,0 & 0,0 & 66,8 & 0,0 & 0,0 & 0,0 & 0,0 & 0,0 \\
\hline $\mathrm{H}_{2} \mathrm{~V}_{2} \mathrm{O}_{4}{ }^{2+}$ & $\mathrm{nq}$ & 0,0 & 0,0 & 0,0 & 1,3 & 0,0 & 0,0 & 0,0 & 0,0 & 0,0 \\
\hline $\mathrm{VOSO}_{4}(\mathrm{aq})$ & $\mathrm{nq}$ & 0,0 & 0,0 & 0,0 & 10,6 & 0,0 & 0,0 & 0,0 & 0,0 & 0,0 \\
\hline $\mathrm{V}(\mathrm{OH})_{3}(\mathbf{a q})$ & $\mathrm{nq}$ & 100,0 & 0,0 & 0,0 & 0,0 & 0,0 & 34,1 & 100,0 & 0,0 & 0,0 \\
\hline $\mathrm{VO}_{2}{ }^{+}$ & $\mathrm{nq}$ & 0,0 & 0,0 & 0,0 & 0,0 & 95,9 & 0,0 & 0,0 & 0,0 & 0,0 \\
\hline $\mathrm{HVO}_{4}{ }^{2-}$ & $\mathrm{nq}$ & 0,0 & 0,0 & 13,0 & 0,0 & 0,0 & 0,9 & 0,0 & 1,1 & 16,6 \\
\hline $\mathrm{H}_{2} \mathrm{VO}_{4}^{-}$ & $\mathrm{nq}$ & 0,0 & 98,1 & 86,9 & 0,0 & 0,0 & 64,8 & 0,0 & 97,7 & 83,3 \\
\hline $\mathbf{H}_{3} \mathbf{V}_{2} \mathbf{O}_{7}^{-}$ & $\mathrm{nq}$ & 0,0 & 0,0 & & 0,0 & 3,0 & 0,0 & 0,0 & 0,0 & 0,0 \\
\hline$\overline{\mathrm{Cr}(\mathrm{OH})_{2}{ }^{+}}$ & 81,0 & 49,5 & 43,5 & 74,4 & 46,0 & 42,2 & 35,0 & 74,9 & 38,8 & 81,6 \\
\hline $\mathrm{CrOH}^{2+}$ & 10,0 & 24,2 & 36,1 & 3,9 & 41,3 & 46,5 & 39,5 & 19,9 & 38,7 & 3,6 \\
\hline $\mathrm{CrSO}_{4}{ }^{+}$ & 0,0 & 0,0 & 0,0 & 0,0 & 0,0 & 0,0 & 1,1 & 0,0 & 0,0 & 0,0 \\
\hline $\mathrm{Cr}(\mathrm{OH})_{3}(\mathrm{aq})$ & 3,1 & 0,0 & 0,0 & 9,7 & 0,0 & 0,0 & 0,0 & 2,0 & 0,0 & 13,2 \\
\hline $\mathrm{CrOHSO}_{4}(\mathrm{aq})$ & 5,8 & 25,3 & 19,4 & 0,0 & 11,6 & 10,2 & 23,8 & 3,2 & 21,0 & 0,0 \\
\hline $\mathrm{CrO}_{4}{ }^{2-}$ & 0,0 & 0,0 & 0,0 & 10,2 & 0,0 & 0,0 & 0,0 & 0,0 & 0,0 & 0,0 \\
\hline $\mathrm{HCrO}_{4}^{-}$ & 0,0 & 0,0 & 0,0 & 0,5 & 0,0 & 0,0 & 0,0 & 0,0 & 0,0 & 0,0 \\
\hline $\mathrm{Mn}^{2+}$ & 69,6 & 56,0 & 71,3 & 80,0 & 74,6 & 79,1 & 57,9 & 83,9 & 60,4 & 77,3 \\
\hline $\mathrm{MnSO}_{4}$ (aq) & 30,4 & 44,0 & 28,7 & 20,0 & 25,4 & 20,9 & 42,1 & 16,1 & 39,6 & 22,6 \\
\hline $\mathrm{Fe}^{2+}$ & 39,2 & 5,3 & 60,0 & 73,9 & 68,0 & $\mathrm{nq}$ & 49,8 & 49,7 & 22,0 & 70,7 \\
\hline $\mathrm{FeSO}_{4}(\mathrm{aq})$ & 23,6 & 5,7 & 33,3 & 25,6 & 32,0 & $\mathrm{nq}$ & 50,2 & 13,2 & 20,0 & 28,7 \\
\hline $\mathrm{FeOH}^{2+}$ & 0,0 & 0,0 & 0,0 & 0,0 & 0,0 & $\mathrm{nq}$ & 0,0 & 0,0 & 0,0 & 0,0 \\
\hline $\mathrm{Fe}(\mathrm{OH})_{2}{ }^{+}$ & 33,3 & 86,6 & 6,6 & 0,0 & 0,0 & $\mathrm{nq}$ & 0,0 & 35,7 & 57,3 & 0,0 \\
\hline $\mathrm{Fe}(\mathrm{OH})_{3}$ & 3,6 & 2,4 & 0,1 & 0,0 & 0,0 & $\mathrm{nq}$ & 0,0 & 1,3 & 0,6 & 0,0 \\
\hline $\mathrm{Co}^{2+}$ & 67,1 & 53,2 & 68,9 & 77,5 & 72,0 & 76,8 & 54,6 & 81,9 & 57,2 & 74,6 \\
\hline $\mathrm{CoSO}_{4}(\mathrm{aq})$ & 32,9 & 46,8 & 31,1 & 22,1 & 27,9 & 23,2 & 45,4 & 18,0 & 42,8 & 24,9 \\
\hline $\mathrm{Ni}^{2+}$ & 67,1 & 53,2 & 68,9 & 77,6 & 72,0 & 76,8 & 54,5 & 81,9 & 57,1 & 74,7 \\
\hline $\mathrm{NiSO}_{4}(\mathrm{aq})$ & 32,9 & 46,8 & 31,1 & 22,2 & 28,0 & 23,2 & 45,4 & 18,0 & 42,9 & 25,0 \\
\hline $\mathbf{C u}^{+}$ & 3,3 & 0,0 & 0,0 & 0,0 & 0,0 & 0,0 & 0,0 & 2,2 & 0,0 & 0,0 \\
\hline $\mathrm{Cu}^{2+}$ & 54,3 & 48,4 & 64,5 & 48,9 & 67,7 & 72,9 & 50,8 & 70,8 & 53,2 & 44,4 \\
\hline${\mathrm{Cu}(\mathrm{OH})^{+}}^{+}$ & 11,5 & 2,6 & 2,0 & 32,3 & 2,6 & 2,3 & 1,6 & 9,3 & 1,9 & 34,9 \\
\hline $\mathrm{Cu}(\mathrm{OH})_{2}$ & 0,0 & 0,0 & 0,0 & 2,9 & 0,0 & 0,0 & 0,0 & 0,0 & 0,0 & 3,8 \\
\hline $\mathrm{CuSO}_{4}(\mathrm{aq})$ & 30,6 & 49,0 & 33,4 & 15,7 & 29,6 & 24,8 & 47,6 & 17,5 & 45,0 & 16,8 \\
\hline
\end{tabular}


Tab. A.25. (Forts.): Ergebnisse der Speziationsberechnung mit MINTEQA2.

\begin{tabular}{|c|c|c|c|c|c|c|c|c|c|c|}
\hline & \begin{tabular}{|l} 
RCO4 \\
52 m \\
PL KB
\end{tabular} & \begin{tabular}{|l} 
RCO4 \\
$56 \mathrm{~m}$ \\
$\mathrm{PL}$ AP
\end{tabular} & \begin{tabular}{|l} 
RCO4 \\
$58 \mathrm{~m}$ \\
$\mathrm{PL}$ AP
\end{tabular} & $\begin{array}{l}\text { RCO10 } \\
2 \mathrm{~m} \\
\text { PL AP }\end{array}$ & $\begin{array}{l}\text { RCO10 } \\
18,5 \text { m } \\
\text { PL AP }\end{array}$ & $\begin{array}{l}\text { RCO10 } \\
29 \text { m } \\
\text { PL AP }\end{array}$ & $\begin{array}{l}\text { RCO13 } \\
15 \mathrm{~m} \\
\text { PL AP }\end{array}$ & $\begin{array}{l}\text { RCO13 } \\
\text { 30 m } \\
\text { PL AP }\end{array}$ & \begin{tabular}{|l} 
RCO16 \\
29 m \\
PL AP
\end{tabular} & $\begin{array}{l}\text { Fremd- } \\
\text { flutung } \\
\text { WP AP }\end{array}$ \\
\hline Spezies & $\%$ & $\%$ & $\%$ & $\%$ & $\%$ & $\%$ & $\%$ & $\%$ & $\%$ & $\%$ \\
\hline $\mathrm{Zn}+{ }^{2}$ & 62,1 & 46,0 & 64,0 & 74,3 & 68,7 & 74,2 & 46,3 & 79,9 & 50,9 & 70,9 \\
\hline $\mathrm{ZnOH}^{+}$ & 0,0 & 0,0 & 0,0 & 1,4 & 0,0 & 0,0 & 0,0 & 0,0 & 0,0 & 1,5 \\
\hline $\mathrm{ZnSO}_{4}(\mathrm{aq})$ & 33,4 & 44,4 & 31,7 & 23,3 & 29,2 & 24,6 & \begin{tabular}{l|l}
42,2 \\
\end{tabular} & 19,2 & 41,8 & 26,0 \\
\hline $\mathrm{Zn}\left(\mathrm{SO}_{4}\right)_{2}{ }^{2-}$ & 4,1 & 9,6 & 4,3 & 0,0 & 2,0 & 1,2 & 11,4 & 0,0 & 7,2 & 1,4 \\
\hline $\mathrm{HAsO}_{4}{ }^{2-}$ & 72,4 & 39,6 & 30,7 & 86,5 & 30,3 & 25,2 & 35,4 & 56,3 & 32,6 & 89,5 \\
\hline $\mathrm{H}_{2} \mathrm{AsO}_{4}^{-}$ & 27,6 & 60,4 & 69,3 & 13,5 & 69,7 & 74,8 & 64,6 & 43,7 & 67,4 & 10,5 \\
\hline $\mathrm{H}_{3} \mathrm{AsO}_{4}$ & 0,0 & 0,0 & 0,0 & 0,0 & 0,0 & 0,0 & 0,0 & 0,0 & 0,0 & 0,0 \\
\hline $\mathrm{H}_{3} \mathrm{AsO}_{3}$ & 0,0 & 0,0 & 0,0 & 0,0 & 0,0 & 0,0 & 0,0 & 0,0 & 0,0 & 0,0 \\
\hline $\mathbf{S r}^{2+}$ & 67,1 & 53,2 & 68,9 & 78,0 & 72,3 & 77,0 & 54,9 & 82,2 & 57,5 & 75,2 \\
\hline $\mathrm{SrSO}_{4}$ (aq) & 32,9 & 46,8 & 31,1 & 22,0 & 27,7 & 23,0 & 45,1 & 17,8 & 42,5 & 24,8 \\
\hline $\mathrm{MoO}_{4}{ }^{2-}$ & 99,9 & 99,7 & 99,5 & 100,0 & 99,6 & 99,4 & 99,7 & 99,9 & 99,6 & 100,0 \\
\hline $\mathrm{HMoO}_{4}{ }^{-}$ & 0,0 & 0,0 & 0,0 & 0,0 & 0,0 & 0,0 & 0,0 & 0,0 & 0,0 & 0,0 \\
\hline $\mathrm{H}_{2} \mathrm{MoO}_{4}(\mathrm{aq})$ & 0,0 & 0,0 & 0,0 & 0,0 & 0,0 & 0,0 & 0,0 & 0,0 & 0,0 & 0,0 \\
\hline $\mathbf{C d}^{2+}$ & 59,4 & 42,0 & 61,0 & 74,0 & 66,9 & 72,7 & \begin{tabular}{l|l}
42,2 \\
\end{tabular} & 79,0 & 47,6 & 70,4 \\
\hline $\mathrm{CdSO}_{4}(\mathrm{aq})$ & 34,2 & 43,5 & 32,3 & 24,4 & 29,9 & 25,3 & 40,5 & 20,0 & 41,2 & 27,2 \\
\hline $\mathrm{Cd}\left(\mathrm{SO}_{4}\right)_{2}{ }^{2-}$ & 6,4 & 14,5 & 6,7 & 1,5 & 3,2 & 1,9 & 17,3 & 0,0 & 11,2 & 2,4 \\
\hline $\mathrm{SbO}_{3}{ }^{-}$ & 99,9 & 99,9 & 99,9 & 100,0 & 99,9 & 99,9 & 99,9 & 99,9 & 99,9 & 99,9 \\
\hline $\mathrm{Sb}(\mathrm{OH})_{3}$ & 0,0 & 0,0 & 0,0 & 0,0 & 0,0 & 0,0 & 0,0 & 0,0 & 0,0 & 0,0 \\
\hline $\mathrm{Sb}(\mathrm{OH})_{2}{ }^{+}$ & 0,0 & 0,0 & 0,0 & 0,0 & 0,0 & 0,0 & 0,0 & 0,0 & 0,0 & 0,0 \\
\hline $\mathrm{HSbO}_{2}$ & 0,0 & 0,0 & 0,0 & 0,0 & 0,0 & 0,0 & 0,0 & 0,0 & 0,0 & 0,0 \\
\hline $\mathrm{SbO}^{+}$ & 0,0 & 0,0 & 0,0 & 0,0 & 0,0 & 0,0 & 0,0 & 0,0 & 0,0 & 0,0 \\
\hline $\mathrm{Ba}^{2+}$ & 100,0 & 100,0 & 100,0 & 100,0 & 100,0 & 100,0 & 100,0 & 100,0 & 100,0 & 100,0 \\
\hline $\mathbf{T l}^{+}$ & 87,9 & $\mathrm{nq}$ & $\mathrm{nq}$ & $\mathrm{nq}$ & $\mathrm{nq}$ & $\mathrm{nq}$ & $\mathrm{nq}$ & $\mathrm{nq}$ & $\mathrm{nq}$ & $\mathrm{nq}$ \\
\hline TISO $_{4}^{-}$ & 12,1 & $\mathrm{nq}$ & $\mathrm{nq}$ & $\mathrm{nq}$ & $\mathrm{nq}$ & $\mathrm{nq}$ & $\mathrm{nq}$ & $\mathrm{nq}$ & $\mathrm{nq}$ & $\mathrm{nq}$ \\
\hline $\mathbf{P b}^{2+}$ & 40,5 & 28,4 & 0,0 & 41,2 & 48,2 & 54,6 & 28,2 & 58,6 & 31,5 & 37,1 \\
\hline $\mathrm{PbOH}^{+}$ & 6,8 & 1,2 & 0,0 & 27,6 & 1,9 & 1,8 & 0,0 & 7,8 & 1,1 & 29,6 \\
\hline $\mathrm{Pb}(\mathrm{OH})_{2}(\mathrm{aq})$ & 0,0 & 0,0 & 0,0 & 0,0 & 0,0 & 0,0 & 0,0 & 0,0 & 0,0 & 0,0 \\
\hline $\mathrm{PbSO}_{4}$ (aq) & 48,6 & 61,3 & 0,0 & 30,1 & 47,8 & 42,2 & 60,1 & 32,9 & 60,4 & 31,7 \\
\hline $\mathrm{Pb}\left(\mathrm{SO}_{4}\right)_{2}{ }^{2-}$ & 4,1 & 9,1 & $\mathrm{nq}$ & $\mathrm{nq}$ & 2,1 & 1,4 & 10,8 & 0,0 & 6,9 & 1,2 \\
\hline $\mathrm{UO}_{2}{ }^{+}$ & 0,0 & 0,0 & $\mathrm{nq}$ & $\mathrm{nq}$ & 0,0 & 0,0 & 0,0 & 0,0 & 0,0 & 0,0 \\
\hline $\mathrm{U}(\mathrm{OH})_{2}{ }^{2+}$ & 0,0 & 0,0 & $\mathrm{nq}$ & $\mathrm{nq}$ & 0,0 & 0,0 & 0,0 & 0,0 & 0,0 & 0,0 \\
\hline $\mathrm{U}(\mathbf{O H})_{3}{ }^{+}$ & 0,0 & 0,0 & $\mathrm{nq}$ & $\mathrm{nq}$ & 0,0 & 0,0 & 0,0 & 0,0 & 0,0 & 0,0 \\
\hline $\mathrm{U}(\mathrm{OH})_{4}(\mathrm{aq})$ & 0,0 & 0,0 & $\mathrm{nq}$ & $\mathrm{nq}$ & 0,0 & 0,0 & 0,0 & 0,0 & 0,0 & 0,0 \\
\hline $\mathrm{USO}_{4}{ }^{2+}$ & 0,0 & 0,0 & $\mathrm{nq}$ & $\mathrm{nq}$ & 0,0 & 0,0 & 0,0 & 0,0 & 0,0 & 0,0 \\
\hline $\mathrm{U}\left(\mathrm{SO}_{4}\right)_{2}(\mathrm{aq})$ & 0,0 & 0,0 & $\mathrm{nq}$ & $\mathrm{nq}$ & 0,0 & 0,0 & 0,0 & 0,0 & 0,0 & 0,0 \\
\hline $\mathrm{UO}_{2}{ }^{2+}$ & 2,1 & 13,9 & $\mathrm{nq}$ & $\mathrm{nq}$ & 27,3 & 33,3 & 14,3 & 15,3 & 19,7 & 1,5 \\
\hline $\mathrm{UO}_{2} \mathbf{O H}^{+}$ & 18,0 & 29,4 & $\mathrm{nq}$ & $\mathrm{nq}$ & 38,7 & 38,5 & 16,1 & 73,5 & 25,2 & 43,8 \\
\hline$\left(\mathrm{UO}_{2}\right)_{2}(\mathrm{OH})_{2}{ }^{2+}$ & 0,0 & 0,0 & $\mathrm{nq}$ & $\mathrm{nq}$ & 1,2 & 0,0 & 2,6 & 0,0 & 0,0 & 0,0 \\
\hline$\left(\mathbf{U O}_{2}\right)_{3}(\mathbf{O H})_{5}{ }^{+}$ & 75,0 & 0,0 & $\mathrm{nq}$ & $\mathrm{nq}$ & 1,9 & 0,0 & 13,7 & 1,8 & 0,0 & 52,9 \\
\hline $\mathrm{UO}_{2} \mathrm{SO}_{4}(\mathrm{aq})$ & 2,8 & 32,2 & $\mathrm{nq}$ & $\mathrm{nq}$ & 25,3 & 24,0 & 28,4 & 0,0 & 35,3 & 1,2 \\
\hline $\mathrm{UO}_{2}\left(\mathrm{SO}_{4}\right)_{2}{ }^{2-}$ & 1,2 & 24,0 & $\mathrm{nq}$ & $\mathrm{nq}$ & 5,5 & 3,8 & 24,8 & 8,0 & 19,8 & 0,0 \\
\hline $\mathrm{SO}_{4}{ }^{2-}$ & 61,4 & 83,4 & 53,5 & 75,8 & 72,4 & 70,9 & 71,5 & 70,6 & 82,0 & 71,2 \\
\hline $\mathrm{HSO}_{4}^{-}$ & 0,0 & 0,0 & 0,0 & 0,0 & 0,0 & 0,0 & 0,0 & 0,0 & 0,0 & 0,0 \\
\hline $\mathrm{AlSO}_{4}{ }^{+}$ & 0,0 & 0,0 & 0,0 & 0,0 & 0,0 & 0,0 & 0,0 & 0,0 & 0,0 & 0,0 \\
\hline $\mathrm{Al}\left(\mathrm{SO}_{4}\right)_{2}{ }^{-}$ & 0,0 & 0,0 & 0,0 & 0,0 & 0,0 & 0,0 & 0,0 & 0,0 & 0,0 & 0,0 \\
\hline $\mathrm{FeSO}_{4}$ (aq) & 0,0 & 0,0 & 0,0 & 0,0 & 0,0 & 0,0 & 0,0 & 0,0 & 0,0 & 0,0 \\
\hline $\mathrm{MgSO}_{4}(\mathrm{aq})$ & 12,0 & 8,8 & 6,4 & 10,1 & 6,4 & 7,0 & 7,4 & 8,4 & 7,2 & 6,1 \\
\hline $\begin{array}{l}\text { CaSO4 (aq) } \\
\end{array}$ & 25,5 & 6,5 & 39,4 & 13,7 & 20,5 & 21,3 & 19,7 & 20,4 & 10,0 & 22,1 \\
\hline
\end{tabular}


Tab. A.25. (Forts.): Ergebnisse der Speziationsberechnung mit MINTEQA2.

\begin{tabular}{|c|c|c|c|c|c|c|c|c|c|c|}
\hline & $\begin{array}{l}\text { Sicker- } \\
\text { wasser } \\
\text { WP AP }\end{array}$ & \begin{tabular}{|l|} 
Stand- \\
gewässer \\
WP AP \\
\end{tabular} & \begin{tabular}{|l|} 
Stand- \\
gewässer 2 \\
PL AP
\end{tabular} & \begin{tabular}{|l|} 
Stand- \\
gewässer 2 \\
WP AP \\
\end{tabular} & \begin{tabular}{|l|} 
Stand- \\
gewässer 3 \\
WP AP
\end{tabular} & \begin{tabular}{|l|} 
Stand- \\
gewässer 3 \\
PL AP
\end{tabular} & \begin{tabular}{|l|l} 
Kippe \\
Süd-West \\
PL AP
\end{tabular} & $\begin{array}{l}\text { Kippe } \\
\text { West } \\
\text { PL AP }\end{array}$ & $\begin{array}{l}\text { See } \\
1997 \\
\text { WP AP }\end{array}$ & \begin{tabular}{|l} 
See \\
1997 \\
PL AP
\end{tabular} \\
\hline Spezies & $\%$ & $\%$ & $\%$ & $\%$ & $\%$ & $\%$ & $\%$ & $\%$ & $\%$ & $\%$ \\
\hline $\mathbf{L i}^{+}$ & 98,5 & 98,2 & 98,1 & 98,2 & 97,2 & 98,5 & 97,3 & 97,6 & 97,7 & 97,0 \\
\hline $\mathrm{LiSO}_{4}^{-}$ & 1,5 & 1,8 & 1,9 & 1,8 & 2,8 & 1,5 & 2,7 & 2,4 & 2,3 & 3,0 \\
\hline $\mathrm{Be}^{2+}$ & $\mathrm{nq}$ & 77,7 & 78,0 & 78,2 & 73,6 & 86,0 & 74,6 & 75,9 & 73,0 & 70,6 \\
\hline $\mathrm{BeOH}^{+}$ & $\mathrm{nq}$ & 0,0 & 0,0 & 0,0 & 0,0 & 0,0 & 0,0 & 0,0 & 3,2 & 0,0 \\
\hline $\mathrm{BeSO}_{4}(\mathrm{aq})$ & $\mathrm{nq}$ & 21,2 & 21,4 & 21,1 & 25,1 & 13,5 & 24,2 & 22,8 & 22,9 & 27,3 \\
\hline $\mathrm{Be}\left(\mathrm{SO}_{4}\right)_{2}{ }^{2-}$ & $\mathrm{nq}$ & 0,0 & 0,0 & 0,0 & 1,2 & 0,0 & 1,2 & 0,0 & 0,0 & 1,4 \\
\hline $\mathrm{Na}^{+}$ & 98,2 & 97,8 & 97,7 & 97,8 & 96,6 & 98,1 & 96,7 & 97,1 & 97,2 & 96,3 \\
\hline $\mathrm{NaSO}_{4}^{-}$ & 1,8 & 2,2 & 2,3 & 2,2 & 3,4 & 1,9 & 3,3 & 2,9 & 2,8 & 3,7 \\
\hline $\mathrm{Mg}^{2+}$ & 76,0 & 72,7 & 72,6 & 72,9 & 68,0 & 82,2 & 69,1 & 70,7 & 69,7 & 65,3 \\
\hline $\mathrm{MgSO}_{4}(\mathrm{aq})$ & 24,0 & 27,3 & 27,4 & 27,1 & 32,0 & 17,8 & 30,9 & 29,3 & 30,3 & 34,7 \\
\hline $\mathbf{A l}^{3+}$ & 0,0 & 11,6 & 12,2 & 12,1 & 11,2 & 24,8 & 12,1 & 12,4 & 11,3 & 9,7 \\
\hline $\mathrm{Al}(\mathrm{OH})_{3}(\mathrm{aq})$ & 2,2 & 0,0 & 0,0 & 0,0 & 0,0 & 0,0 & 0,0 & 0,0 & 0,0 & 0,0 \\
\hline $\mathrm{Al}(\mathrm{OH})_{4}^{-}$ & 97,7 & 0,0 & 0,0 & 0,0 & 0,0 & 0,0 & 0,0 & 0,0 & 0,0 & 0,0 \\
\hline $\mathrm{AlSO}_{4}{ }^{+}$ & 0,0 & 84,1 & 83,4 & 83,6 & 82,4 & 72,1 & 81,7 & 82,0 & 82,6 & 83,1 \\
\hline $\mathrm{Al}\left(\mathrm{SO}_{4}\right)_{2}^{-}$ & 0,0 & 4,2 & 4,4 & 4,3 & 6,4 & 3,1 & 6,3 & 5,5 & 5,4 & 7,1 \\
\hline $\mathbf{K}^{+}$ & 97,9 & 97,4 & 97,2 & 97,3 & 96,0 & 97,8 & 96,1 & 96,5 & 96,6 & 95,6 \\
\hline $\mathrm{KSO}_{4}^{-}$ & 2,1 & 2,6 & 2,8 & 2,7 & 4,0 & 2,2 & 3,9 & 3,5 & 3,4 & 4,4 \\
\hline $\mathrm{Ca}^{2+}$ & 71,7 & 68,1 & 68,0 & 68,3 & 63,0 & 78,8 & 64,2 & 65,9 & 64,9 & 60,1 \\
\hline $\mathrm{CaSO}_{4}(\mathrm{aq})$ & 28,3 & 31,9 & 32,0 & 31,7 & 37,0 & 21,2 & 35,8 & 34,1 & 35,1 & 39,9 \\
\hline $\mathrm{VO}^{2+}$ & 0,0 & $\mathrm{nq}$ & 50,9 & 50,8 & 0,0 & 0,0 & 0,0 & 0,0 & 38,2 & 0,0 \\
\hline $\mathrm{V}(\mathrm{OH})_{3}{ }^{+}$ & 0,0 & $\mathrm{nq}$ & 0,0 & 0,0 & 0,0 & 0,0 & 0,0 & 0,0 & 0,9 & 0,0 \\
\hline $\mathrm{VOSO}_{4}(\mathrm{aq})$ & 0,0 & $\mathrm{nq}$ & 26,9 & 26,5 & 0,0 & 0,0 & 0,0 & 0,0 & 23,2 & 0,0 \\
\hline $\mathrm{V}(\mathrm{OH})_{3}(\mathrm{aq})$ & 0,0 & $\mathrm{nq}$ & 22,2 & 22,7 & 0,0 & 0,0 & 0,0 & 0,0 & 5,8 & 100,0 \\
\hline $\mathrm{VO}_{2}{ }^{+}$ & 0,0 & $\mathrm{nq}$ & 0,0 & 0,0 & 77,6 & 83,6 & 78,6 & 45,1 & 1,1 & 0,0 \\
\hline $\mathrm{HVO}_{4}{ }^{2-}$ & 28,5 & $\mathrm{nq}$ & 0,0 & 0,0 & 0,0 & 0,0 & 0,0 & 0,0 & 0,0 & 0,0 \\
\hline $\mathrm{H}_{2} \mathrm{VO}_{4}{ }^{-}$ & 71,5 & $\mathrm{nq}$ & 0,0 & 0,0 & 0,0 & 0,0 & 0,0 & 9,0 & 22,0 & 0,0 \\
\hline $\mathrm{VO}_{2} \mathrm{SO}_{4}^{-}$ & 0,0 & $\mathrm{nq}$ & 0,0 & 0,0 & 12,1 & 7,1 & 12,0 & 6,0 & 0,0 & 0,0 \\
\hline $\mathrm{H}_{3} \mathrm{VO}_{4}(\mathrm{aq})$ & 0,0 & $\mathrm{nq}$ & 0,0 & 0,0 & 7,2 & 7,3 & 5,7 & 26,5 & 6,6 & 0,0 \\
\hline $\mathrm{H}_{3} \mathrm{~V}_{2} \mathrm{O}_{7}^{-}$ & 0,0 & $\mathrm{nq}$ & 0,0 & 0,0 & 2,7 & 1,6 & 3,5 & 13,4 & 2,0 & 0,0 \\
\hline $\mathrm{Cr}^{3+}$ & 0,0 & 29,3 & 32,9 & 32,7 & 31,4 & 53,4 & 33,2 & 32,4 & 21,2 & 25,6 \\
\hline $\mathrm{Cr}(\mathrm{OH})_{2}{ }^{+}$ & 72,3 & 0,0 & 0,0 & 0,0 & 0,0 & 0,0 & 0,0 & 0,0 & 0,0 & 0,0 \\
\hline $\mathrm{CrOH}^{2+}$ & 1,6 & 6,3 & 0,0 & 0,0 & 0,0 & 0,0 & 0,0 & 3,5 & 24,4 & 6,9 \\
\hline $\mathrm{CrSO}_{4}{ }^{+}$ & 0,0 & 62,5 & 66,3 & 66,4 & 67,9 & 45,8 & 66,2 & 63,0 & 45,8 & 64,5 \\
\hline $\mathrm{Cr}(\mathrm{OH})_{3}$ (aq) & 23,3 & 0,0 & 0,0 & 0,0 & 0,0 & 0,0 & 0,0 & 0,0 & 0,0 & 0,0 \\
\hline $\mathrm{CrOHSO}_{4}(\mathrm{aq})$ & 1,1 & 1,9 & 0,0 & 0,0 & 0,0 & 0,0 & 0,0 & 1,1 & 8,4 & 2,9 \\
\hline $\mathrm{CrO}_{2}^{-}$ & 1,3 & 0,0 & 0,0 & 0,0 & 0,0 & 0,0 & 0,0 & 0,0 & 0,0 & 0,0 \\
\hline $\mathrm{Mn}^{2+}$ & 76,7 & 73,5 & 73,4 & 73,7 & 68,9 & 82,9 & 70,0 & 71,6 & 70,6 & 66,2 \\
\hline $\mathrm{MnSO}_{4}(\mathrm{aq})$ & 23,2 & 26,5 & 26,6 & 26,3 & 31,1 & 17,1 & 30,0 & 28,4 & 29,4 & 33,8 \\
\hline $\mathrm{Fe}^{2+}$ & 69,6 & 66,7 & 66,6 & 66,9 & 61,5 & 77,7 & 62,8 & 64,5 & 63,4 & 58,6 \\
\hline $\mathrm{FeSO}_{4}(\mathrm{aq})$ & 29,6 & 33,3 & 33,4 & 33,1 & 38,5 & 22,3 & 37,2 & 35,5 & 36,6 & 41,4 \\
\hline $\mathrm{FeOH}^{2+}$ & 1,2 & 0,0 & 0,0 & 0,0 & 0,0 & 0,0 & 0,0 & 0,0 & 0,0 & 0,0 \\
\hline $\mathrm{Fe}(\mathrm{OH})_{2}{ }^{+}$ & 0,0 & 0,0 & 0,0 & 0,0 & 0,0 & 0,0 & 0,0 & 0,0 & 0,0 & 0,0 \\
\hline $\mathrm{Fe}(\mathrm{OH})_{3}$ & 0,0 & 0,0 & 0,0 & 0,0 & 0,0 & 0,0 & 0,0 & 0,0 & 0,0 & 0,0 \\
\hline $\mathrm{Co}^{2+}$ & 73,6 & 70,9 & 70,8 & 71,1 & 66,0 & 80,9 & 67,2 & 68,8 & 67,8 & 63,2 \\
\hline $\mathrm{CoSO}_{4}(\mathrm{aq})$ & 25,5 & 29,1 & 29,2 & 28,9 & 34,0 & 19,1 & 32,8 & 31,2 & 32,2 & 36,8 \\
\hline $\mathrm{Ni}^{2+}$ & 73,9 & 70,8 & 70,7 & 71,0 & 65,9 & 80,9 & 67,1 & 68,8 & 67,8 & 63,1 \\
\hline $\mathrm{NiSO}_{4}(\mathrm{aq})$ & 25,6 & 29,2 & 29,3 & 29,0 & 34,0 & 19,1 & 32,9 & 31,2 & 32,2 & 36,8 \\
\hline $\mathrm{Cu}^{2+}$ & 30,3 & 68,3 & 68,2 & 68,5 & 63,2 & 79,0 & 64,5 & 66,2 & 65,1 & 60,4 \\
\hline $\mathrm{Cu}(\mathrm{OH})^{+}$ & 47,4 & 0,0 & 0,0 & 0,0 & 0,0 & 0,0 & 0,0 & 0,0 & 0,0 & 0,0 \\
\hline $\mathrm{Cu}(\mathrm{OH})_{2}$ & 10,4 & 0,0 & 0,0 & 0,0 & 0,0 & 0,0 & 0,0 & 0,0 & 0,0 & 0,0 \\
\hline $\mathrm{CuSO}_{4}(\mathrm{aq})$ & 11,8 & 31,7 & 31,8 & 31,5 & 36,8 & 21,0 & 35,5 & 33,8 & 34,9 & 39,6 \\
\hline
\end{tabular}


Tab. A.25. (Forts.): Ergebnisse der Speziationsberechnung mit MINTEQA2.

\begin{tabular}{|c|c|c|c|c|c|c|c|c|c|c|}
\hline & $\begin{array}{l}\text { Sicker- } \\
\text { wasser } \\
\text { WP AP }\end{array}$ & \begin{tabular}{|l} 
Stand- \\
gewässer \\
WP AP \\
\end{tabular} & $\begin{array}{l}\text { Stand- } \\
\text { gewässer } 2 \\
\text { PL AP }\end{array}$ & \begin{tabular}{|l|} 
Stand- \\
gewässer 2 \\
WP AP
\end{tabular} & \begin{tabular}{|l|} 
Stand- \\
gewässer 3 \\
WP AP
\end{tabular} & \begin{tabular}{|l|} 
Stand- \\
gewässer \\
PL AP
\end{tabular} & \begin{tabular}{|l|} 
Kippe \\
Süd-West \\
PL AP
\end{tabular} & $\begin{array}{l}\text { Kippe } \\
\text { West } \\
\text { PL AP }\end{array}$ & $\begin{array}{l}\text { See } \\
1997 \\
\text { WP AP }\end{array}$ & \begin{tabular}{|l} 
See \\
1997 \\
PL AP
\end{tabular} \\
\hline Spezies & $\%$ & $\%$ & $\%$ & $\%$ & $\%$ & $\%$ & $\%$ & $\%$ & $\%$ & $\%$ \\
\hline $\mathrm{Zn}^{2+}$ & 68,8 & 67,4 & 67,1 & 67,5 & 60,8 & 78,0 & 62,0 & 64,3 & 63,4 & 57,6 \\
\hline $\mathrm{ZnOH}^{+}$ & 3,0 & 0,0 & 0,0 & 0,0 & 0,0 & 0,0 & 0,0 & 0,0 & 0,0 & 0,0 \\
\hline $\mathrm{ZnSO}_{4}$ (aq) & 26,1 & 30,4 & 30,4 & 30,1 & 34,3 & 20,2 & 33,2 & 31,9 & 32,9 & 36,8 \\
\hline $\mathrm{Zn}\left(\mathrm{SO}_{4}\right)_{2}{ }^{2-}$ & 1,5 & 2,2 & 2,5 & 2,4 & 4,9 & 1,9 & 4,8 & 3,8 & 3,6 & 5,6 \\
\hline $\mathrm{HAsO}_{4}{ }^{2-}$ & 94,4 & 0,0 & 0,0 & 0,0 & 0,0 & 0,0 & 0,0 & 0,0 & 99,0 & 0,0 \\
\hline $\mathrm{H}_{2} \mathrm{AsO}_{4}^{-}$ & 5,5 & 92,8 & 0,0 & 0,0 & 70,7 & 71,7 & 65,9 & 93,8 & 0,0 & 50,4 \\
\hline $\mathrm{H}_{3} \mathrm{AsO}_{4}$ & 0,0 & 4,1 & 0,0 & 0,0 & 29,3 & 28,3 & 34,1 & 6,2 & 0,0 & 1,3 \\
\hline $\mathrm{H}_{3} \mathrm{AsO}_{3}$ & 0,0 & 3,0 & 99,8 & 99,8 & 0,0 & 0,0 & 0,0 & 0,0 & 0,0 & 48,2 \\
\hline $\mathbf{S r}^{2+}$ & 74,5 & 71,1 & 71,0 & 71,3 & 66,3 & 81,1 & 67,5 & 69,1 & 68,1 & 63,5 \\
\hline $\mathrm{SrSO}_{4}(\mathrm{aq})$ & 25,5 & 28,9 & 29,0 & 28,7 & 33,7 & 18,9 & 32,5 & 30,9 & 31,9 & 36,5 \\
\hline $\mathrm{MoO}_{4}{ }^{2-}$ & 100,0 & $\mathrm{nq}$ & 0,0 & 0,0 & 0,0 & 0,0 & 0,0 & 4,1 & 59,5 & 17,2 \\
\hline $\mathrm{HMoO}_{4}^{-}$ & 0,0 & $\mathrm{nq}$ & 3,6 & 3,6 & 3,8 & 4,0 & 3,1 & 19,1 & 28,7 & 31,8 \\
\hline $\mathrm{H}_{2} \mathrm{MoO}_{4}(\mathrm{aq})$ & 0,0 & $\mathrm{nq}$ & 96,2 & 96,3 & 96,1 & 95,8 & 96,8 & 76,8 & 11,8 & 51,0 \\
\hline $\mathrm{Cd}^{2+}$ & $\mathrm{nq}$ & 65,4 & 65,0 & 65,4 & 57,8 & 76,2 & 59,1 & 61,7 & 60,9 & 54,6 \\
\hline $\mathrm{CdSO}_{4}(\mathrm{aq})$ & $\mathrm{nq}$ & 31,1 & 31,0 & 30,8 & 34,4 & 20,8 & 33,3 & 32,2 & 33,3 & 36,7 \\
\hline $\mathrm{Cd}\left(\mathrm{SO}_{4}\right)_{2}{ }^{2-}$ & $\mathrm{nq}$ & 3,6 & 4,0 & 3,8 & 7,8 & 3,0 & 7,6 & 6,1 & 5,8 & 8,8 \\
\hline $\mathrm{Sn}(\mathrm{OH})_{2}$ & $\mathrm{nq}$ & $\mathrm{nq}$ & 0,0 & 0,0 & 0,0 & 0,0 & 0,0 & 0,0 & $\mathrm{nq}$ & 0,0 \\
\hline $\mathbf{S n}^{2+}$ & $\mathrm{nq}$ & $\mathrm{nq}$ & 0,0 & 0,0 & 0,0 & 0,0 & 0,0 & 0,0 & $\mathrm{nq}$ & 0,0 \\
\hline $\mathrm{SnOH}^{+}$ & $\mathrm{nq}$ & $\mathrm{nq}$ & 0,0 & 0,0 & 0,0 & 0,0 & 0,0 & 0,0 & $\mathrm{nq}$ & 0,0 \\
\hline $\mathbf{S n}^{4+}$ & $\mathrm{nq}$ & $\mathrm{nq}$ & 100,0 & 100,0 & 100,0 & 100,0 & 100,0 & 99,7 & $\mathrm{nq}$ & 56,2 \\
\hline $\operatorname{Sn}(\mathrm{OH})_{6}^{-}$ & $\mathrm{nq}$ & $\mathrm{nq}$ & 0,0 & 0,0 & 0,0 & 0,0 & 0,0 & 0,0 & 99,4 & 43,5 \\
\hline $\mathrm{SbO}_{3}^{-}$ & 99,9 & $\mathrm{nq}$ & 0,0 & 0,0 & 99,9 & 99,9 & 99,9 & 99,9 & 99,9 & 45,5 \\
\hline $\mathrm{Sb}(\mathrm{OH})_{3}$ & 0,0 & $\mathrm{nq}$ & 47,6 & 47,6 & 0,0 & 0,0 & 0,0 & 0,0 & 0,0 & 27,5 \\
\hline $\mathrm{Sb}(\mathrm{OH})_{2}{ }^{+}$ & 0,0 & $\mathrm{nq}$ & 4,4 & 4,4 & 0,0 & 0,0 & 0,0 & 0,0 & 0,0 & 0,0 \\
\hline $\mathrm{HSbO}_{2}$ & 0,0 & $\mathrm{nq}$ & 46,5 & 46,6 & 0,0 & 0,0 & 0,0 & 0,0 & 0,0 & 26,8 \\
\hline $\mathbf{S b O}^{+}$ & 0,0 & $\mathrm{nq}$ & 1,4 & 1,4 & 0,0 & 0,0 & 0,0 & 0,0 & 0,0 & 0,0 \\
\hline $\mathrm{Ba}^{2+}$ & 100,0 & 100,0 & 100,0 & 100,0 & 100,0 & 100,0 & 100,0 & 100,0 & 100,0 & 100,0 \\
\hline$\overline{\mathrm{Tl}^{+}}$ & $\mathrm{nq}$ & $\mathrm{nq}$ & $\mathrm{nq}$ & $\mathrm{nq}$ & $\mathrm{nq}$ & $\mathrm{nq}$ & $\mathrm{nq}$ & 88,3 & 88,5 & 85,6 \\
\hline TISO $_{4}^{-}$ & $\mathrm{nq}$ & $\mathrm{nq}$ & $\mathrm{nq}$ & $\mathrm{nq}$ & $\mathrm{nq}$ & $\mathrm{nq}$ & $\mathrm{nq}$ & 11,7 & 11,5 & 14,4 \\
\hline $\mathbf{P b}^{2+}$ & 28,1 & 47,5 & 47,3 & $\mathrm{nq}$ & 40,9 & 60,9 & 42,2 & 44,4 & 43,4 & 37,9 \\
\hline $\mathrm{PbOH}^{+}$ & 44,8 & 0,0 & 0,0 & $\mathrm{nq}$ & 0,0 & 0,0 & 0,0 & 0,0 & 0,0 & 0,0 \\
\hline $\mathrm{Pb}(\mathrm{OH})_{2}(\mathrm{aq})$ & \begin{tabular}{l|}
1,2 \\
\end{tabular} & 0,0 & 0,0 & $\mathrm{nq}$ & 0,0 & 0,0 & 0,0 & 0,0 & 0,0 & 0,0 \\
\hline $\mathrm{PbSO}_{4}$ (aq) & 24,9 & 50,1 & 50,0 & $\mathrm{nq}$ & 54,0 & 36,8 & 52,8 & 51,5 & 52,7 & 56,5 \\
\hline $\mathrm{Pb}\left(\mathrm{SO}_{4}\right)_{2}{ }^{2-}$ & 0,0 & 2,4 & 2,7 & $\mathrm{nq}$ & 5,1 & 2,3 & 5,1 & 4,1 & 3,9 & 5,7 \\
\hline $\mathrm{UO}_{2}{ }^{+}$ & 0,0 & 0,0 & 0,0 & 0,0 & 100,0 & 100,0 & 100,0 & 100,0 & 0,0 & 0,0 \\
\hline $\mathrm{UO}_{2}{ }^{2+}$ & 0,0 & 45,1 & 44,4 & 45,0 & 0,0 & 0,0 & 0,0 & 0,0 & 39,2 & 32,5 \\
\hline $\mathrm{UO}_{2} \mathbf{O H}^{+}$ & 18,7 & 0,0 & 0,0 & 0,0 & 0,0 & 0,0 & 0,0 & 0,0 & 0,0 & 0,0 \\
\hline$\left(\mathrm{UO}_{2}\right)_{2}(\mathrm{OH})_{2}{ }^{2+}$ & 0,0 & 0,0 & 0,0 & 0,0 & 0,0 & 0,0 & 0,0 & 0,0 & 0,0 & 0,0 \\
\hline$\left(\mathrm{UO}_{2}\right)_{3}(\mathrm{OH})_{5}{ }^{+}$ & 80,5 & 0,0 & 0,0 & 0,0 & 0,0 & 0,0 & 0,0 & 0,0 & 0,0 & 0,0 \\
\hline $\mathrm{UO}_{2} \mathrm{SO}_{4}(\mathrm{aq})$ & 0,0 & 44,4 & 43,9 & 43,9 & 0,0 & 0,0 & 0,0 & 0,0 & 44,5 & 45,3 \\
\hline $\mathrm{UO}_{2}\left(\mathrm{SO}_{4}\right)_{2}{ }^{2-}$ & 0,0 & 10,5 & 11,8 & 11,1 & 0,0 & 0,0 & 0,0 & 0,0 & 15,9 & 22,2 \\
\hline $\mathrm{SO}_{4}{ }^{2-}$ & 71,6 & 69,5 & 57,8 & 58,5 & 49,6 & 31,5 & 47,0 & 59,4 & 64,1 & 65,6 \\
\hline $\mathrm{HSO}_{4}^{-}$ & 0,0 & 1,1 & 9,1 & 9,3 & 6,9 & 3,9 & 8,1 & 1,3 & 0,0 & 0,0 \\
\hline $\mathrm{AlSO}_{4}{ }^{+}$ & 0,0 & 2,2 & 8,3 & 9,1 & 15,6 & 30,0 & 14,5 & 4,2 & 1,8 & 1,0 \\
\hline$\overline{\mathrm{Al}\left(\mathrm{SO}_{4}\right)_{2}{ }^{-}}$ & 0,0 & 0,0 & 0,0 & 0,0 & 2,4 & 2,5 & 2,2 & 0,0 & 0,0 & 0,0 \\
\hline $\mathrm{FeSO}_{4}(\mathrm{aq})$ & 0,0 & 0,0 & 8,4 & 6,1 & 11,0 & 16,6 & 13,2 & 2,7 & 0,0 & 2,9 \\
\hline $\mathrm{MgSO}_{4}(\mathrm{aq})$ & 7,0 & 4,9 & 3,5 & 3,8 & 5,1 & 5,1 & 4,6 & 9,9 & 7,3 & 7,4 \\
\hline $\begin{array}{l}\text { CaSO4 (aq) } \\
\end{array}$ & 21,0 & 21,6 & 11,8 & 11,9 & 9,2 & 10,2 & 10,2 & 20,2 & 25,7 & 21,5 \\
\hline
\end{tabular}


Tab. A.25. (Forts.): Ergebnisse der Speziationsberechnung mit MINTEQA2.

\begin{tabular}{|c|c|c|c|c|c|c|c|c|c|c|}
\hline & $\begin{array}{l}\text { SGM } \\
48,5 \mathrm{~m} \\
\text { WP AP }\end{array}$ & \begin{tabular}{|l} 
SGM \\
24,5 m \\
WP AP
\end{tabular} & $\begin{array}{l}\text { CML } \\
\text { 21-22 m } \\
\text { PL KB }\end{array}$ & \begin{tabular}{|l} 
CML \\
24m \\
PL KB
\end{tabular} & $\begin{array}{l}\text { CML } \\
26 \text { m } \\
\text { PL KB }\end{array}$ & \begin{tabular}{|l|} 
CML \\
32 m \\
PL KB
\end{tabular} & \begin{tabular}{|l} 
CML \\
$36 \mathrm{~m}$ \\
PL KB
\end{tabular} & \begin{tabular}{|l} 
CML \\
37 m \\
PL KB
\end{tabular} & \begin{tabular}{|l} 
CML \\
39 m \\
PL KB
\end{tabular} & $\begin{array}{l}\text { RCO4 } \\
\text { Okt } 98 \\
\text { WP KB }\end{array}$ \\
\hline Spezies & $\%$ & $\%$ & $\%$ & $\%$ & $\%$ & $\%$ & $\%$ & $\%$ & $\%$ & $\%$ \\
\hline $\mathrm{Li}^{+}$ & 96,2 & 96,4 & 98,0 & 98,3 & 98,7 & 98,3 & 97,9 & 97,8 & 97,3 & 95,3 \\
\hline $\mathrm{LiSO}_{4}^{-}$ & 3,8 & 3,6 & 2,0 & 1,7 & 1,3 & 1,7 & 2,1 & 2,2 & 2,7 & 4,7 \\
\hline $\mathrm{Be}^{2+}$ & 66,6 & 68,0 & $\mathrm{nq}$ & 82,5 & 87,1 & 3,5 & 4,3 & 8,2 & 21,5 & $\mathrm{nq}$ \\
\hline $\mathrm{BeOH}^{+}$ & 2,6 & 2,6 & $\mathrm{nq}$ & 0,0 & 0,0 & 90,0 & 90,0 & 87,3 & 70,5 & $\mathrm{nq}$ \\
\hline $\mathrm{Be}(\mathrm{OH})_{2}$ & 0,0 & 0,0 & $\mathrm{nq}$ & 0,0 & 0,0 & 5,8 & 4,5 & 2,3 & 0,0 & $\mathrm{nq}$ \\
\hline $\mathrm{BeSO}_{4}(\mathrm{aq})$ & 28,6 & 27,4 & $\mathrm{nq}$ & 16,3 & 12,4 & 0,0 & 1,1 & 2,2 & 7,1 & $\mathrm{nq}$ \\
\hline $\mathrm{Be}\left(\mathrm{SO}_{4}\right)_{2}{ }^{2-}$ & 2,2 & 2,0 & $\mathrm{nq}$ & 0,0 & 0,0 & 0,0 & 0,0 & 0,0 & 0,0 & $\mathrm{nq}$ \\
\hline $\mathrm{Na}^{+}$ & 95,3 & 95,6 & 97,5 & 97,9 & 98,4 & 97,9 & 97,4 & 97,3 & 96,7 & 94,3 \\
\hline $\mathrm{NaSO}_{4}^{-}$ & 4,7 & 4,4 & 2,5 & 2,1 & 1,6 & 2,1 & 2,6 & 2,7 & 3,3 & 5,7 \\
\hline $\mathrm{Mg}^{2+}$ & 62,8 & 64,2 & 72,8 & 78,6 & 83,6 & 79,5 & 73,4 & 72,9 & 68,8 & 56,7 \\
\hline $\mathrm{MgSO}_{4}(\mathrm{aq})$ & 37,2 & 35,8 & 27,2 & 21,4 & 16,4 & 20,5 & 26,6 & 27,1 & 31,2 & 43,3 \\
\hline $\mathrm{Al}^{3+}$ & 9,6 & 10,2 & $\mathrm{nq}$ & 19,2 & 26,1 & 0,0 & 0,0 & 0,0 & 1,6 & 0,0 \\
\hline $\mathrm{AlOH}^{2+}$ & 0,0 & 0,0 & $\mathrm{nq}$ & 0,0 & 0,0 & 0,0 & 0,0 & 0,0 & 6,1 & 0,0 \\
\hline $\mathrm{Al}(\mathrm{OH})_{2}{ }^{+}$ & 0,0 & 0,0 & $\mathrm{nq}$ & 0,0 & 0,0 & 6,1 & 9,7 & 25,7 & 55,5 & 0,0 \\
\hline $\mathrm{Al}(\mathrm{OH})_{3}(\mathrm{aq})$ & 0,0 & 0,0 & $\mathrm{nq}$ & 0,0 & 0,0 & 12,3 & 15,4 & 20,9 & 14,0 & 0,0 \\
\hline $\mathrm{Al}(\mathrm{OH})_{4}^{-}$ & 0,0 & 0,0 & $\mathrm{nq}$ & 0,0 & 0,0 & 81,5 & 74,7 & 52,1 & 10,8 & 0,0 \\
\hline $\mathrm{AlSO}_{4}{ }^{+}$ & 81,0 & 81,0 & $\mathrm{nq}$ & 77,0 & 71,2 & 0,0 & 0,0 & 0,0 & 11,2 & 0,0 \\
\hline $\mathrm{Al}\left(\mathrm{SO}_{4}\right)_{2}^{-}$ & 8,9 & 8,3 & $\mathrm{nq}$ & 3,7 & 2,7 & 0,0 & 0,0 & 0,0 & 0,0 & 0,0 \\
\hline $\mathbf{K}^{+}$ & 94,4 & 94,8 & 97,0 & 97,5 & 98,0 & 97,5 & 96,9 & 96,8 & 96,1 & 92,6 \\
\hline $\mathrm{KSO}_{4}^{-}$ & 5,6 & 5,2 & 3,0 & 2,5 & 2,0 & 2,5 & 3,1 & 3,2 & 3,9 & 7,4 \\
\hline $\mathrm{Ca}^{2+}$ & 57,5 & 59,0 & 68,2 & 74,6 & 80,3 & 75,7 & 68,9 & 68,3 & 63,9 & 51,0 \\
\hline $\mathrm{CaSO}_{4}(\mathrm{aq})$ & 42,5 & 41,0 & 31,8 & 25,4 & 19,7 & 24,3 & 31,1 & 31,7 & 36,1 & 49,0 \\
\hline $\mathrm{VO}^{2+}$ & 4,4 & 4,4 & $\mathrm{nq}$ & 66,8 & 78,4 & 0,0 & 0,0 & 0,0 & $\mathrm{nq}$ & 0,0 \\
\hline $\mathrm{V}(\mathrm{OH})_{3}{ }^{+}$ & 0,1 & 0,1 & $\mathrm{nq}$ & 0,0 & 0,0 & 0,0 & 0,0 & 0,0 & $\mathrm{nq}$ & 4,4 \\
\hline $\mathrm{VOSO}_{4}(\mathrm{aq})$ & 3,6 & 3,4 & $\mathrm{nq}$ & 25,5 & 21,6 & 0,0 & 0,0 & 0,0 & $\mathrm{nq}$ & 0,0 \\
\hline $\mathrm{V}(\mathrm{OH})_{3}(\mathrm{aq})$ & 91,9 & 92,1 & $\mathrm{nq}$ & 7,5 & 0,0 & 0,0 & 0,0 & 0,0 & $\mathrm{nq}$ & 0,0 \\
\hline $\mathrm{VO}_{2}{ }^{+}$ & 0,0 & 0,0 & $\mathrm{nq}$ & 0,0 & 0,0 & 0,0 & 0,0 & 0,0 & $\mathrm{nq}$ & 0,0 \\
\hline $\mathrm{HVO}_{4}{ }^{2-}$ & 0,0 & 0,0 & $\mathrm{nq}$ & 0,0 & 0,0 & 6,4 & 4,6 & 2,4 & $\mathrm{nq}$ & 0,0 \\
\hline $\mathrm{H}_{2} \mathrm{VO}_{4}^{-}$ & 0,0 & 0,0 & $\mathrm{nq}$ & 0,0 & 0,0 & 93,4 & 95,1 & 96,9 & $\mathrm{nq}$ & 94,4 \\
\hline $\mathrm{Cr}^{3+}$ & 20,7 & 21,4 & 0,0 & 41,7 & 54,4 & 0,0 & 0,0 & 0,0 & 0,0 & 0,0 \\
\hline $\mathrm{Cr}(\mathrm{OH})_{2}{ }^{+}$ & 0,0 & 0,0 & 59,7 & 0,0 & 0,0 & 76,7 & 72,6 & 58,9 & 29,5 & 31,4 \\
\hline $\mathrm{CrOH}^{2+}$ & 18,9 & 19,7 & 30,2 & 7,3 & 1,7 & 16,9 & 19,5 & 30,8 & 50,3 & 33,5 \\
\hline $\mathrm{CrSO}_{4}{ }^{+}$ & 51,3 & 50,0 & 0,0 & 49,4 & 43,6 & 0,0 & 0,0 & 0,0 & 1,3 & 1,2 \\
\hline $\mathrm{Cr}(\mathrm{OH})_{3}(\mathrm{aq})$ & 0,0 & 0,0 & 0,0 & 0,0 & 0,0 & 2,9 & 2,2 & 0,0 & 0,0 & 0,0 \\
\hline $\mathrm{CrOHSO}_{4}(\mathrm{aq})$ & 8,9 & 8,7 & 8,9 & 1,6 & 0,0 & 3,4 & 5,6 & 9,1 & 18,1 & 33,4 \\
\hline $\mathrm{Mn}^{2+}$ & 63,8 & 65,2 & 73,7 & 79,3 & $\mathrm{nq}$ & 80,2 & 74,3 & 73,7 & 69,7 & 57,3 \\
\hline $\mathrm{MnSO}_{4}$ (aq) & 36,2 & 34,8 & 26,3 & 20,7 & $\mathrm{nq}$ & 9,8 & 25,7 & 26,3 & 30,3 & 42,7 \\
\hline $\mathrm{Fe}^{2+}$ & 55,9 & 57,5 & 66,8 & 73,4 & 79,3 & 5,5 & 18,7 & 27,0 & 59,8 & 45,9 \\
\hline $\mathrm{FeSO}_{4}(\mathrm{aq})$ & 44,1 & 42,5 & 33,1 & 26,6 & 20,7 & 1,9 & 9,0 & 13,3 & 36,0 & 47,2 \\
\hline $\mathrm{FeOH}^{2+}$ & 0,0 & 0,0 & 0,0 & 0,0 & 0,0 & 0,0 & 0,0 & 0,0 & 0,0 & 0,0 \\
\hline $\mathrm{Fe}(\mathrm{OH})_{2}{ }^{+}$ & 0,0 & 0,0 & 0,0 & 0,0 & 0,0 & 87,8 & 69,3 & 58,0 & 4,2 & 6,8 \\
\hline $\mathrm{Fe}(\mathrm{OH})_{3}$ & 0,0 & 0,0 & 0,0 & 0,0 & 0,0 & 4,7 & 2,9 & 1,3 & 0,0 & 0,1 \\
\hline $\mathrm{Co}^{2+}$ & 60,7 & 62,2 & 71,0 & 77,0 & 82,3 & 78,0 & 71,6 & 71,1 & 66,9 & 54,4 \\
\hline $\mathrm{CoSO}_{4}(\mathrm{aq})$ & 39,3 & 37,8 & 29,0 & 23,0 & 17,7 & 21,9 & 28,3 & 28,9 & 33,1 & 45,6 \\
\hline $\mathrm{Ni}^{2+}$ & 60,6 & 62,1 & 71,0 & 77,0 & 82,3 & 78,0 & 71,6 & 71,0 & 66,8 & 54,4 \\
\hline $\mathrm{NiSO}_{4}(\mathrm{aq})$ & 39,4 & 37,9 & 29,0 & 23,0 & 17,7 & 22,0 & 28,4 & 28,9 & 33,2 & 45,5 \\
\hline $\mathrm{Cu}^{+}$ & 0,0 & 0,0 & 0,0 & 0,0 & 0,0 & 0,0 & 0,0 & 0,0 & 0,0 & 0,0 \\
\hline $\mathrm{Cu}^{2+}$ & 57,7 & 59,3 & 65,4 & 74,8 & 80,5 & 67,5 & 63,3 & 65,5 & 63,3 & 50,4 \\
\hline $\mathrm{Cu}(\mathrm{OH})^{+}$ & 0,0 & 0,0 & 4,5 & 0,0 & 0,0 & 10,7 & 8,2 & 4,4 & 1,3 & 1,2 \\
\hline $\mathrm{Cu}(\mathrm{OH})_{2}$ & 0,0 & 0,0 & 0,0 & 0,0 & 0,0 & 0,0 & 0,0 & 0,0 & 0,0 & 0,0 \\
\hline $\mathrm{CuSO}_{4}(\mathrm{aq})$ & 42,3 & 40,7 & 30,1 & 25,2 & 19,5 & 21,4 & 28,3 & 30,1 & 35,4 & 48,4 \\
\hline
\end{tabular}


Tab. A.25. (Forts.): Ergebnisse der Speziationsberechnung mit MINTEQA2.

\begin{tabular}{|c|c|c|c|c|c|c|c|c|c|c|}
\hline & \begin{tabular}{|l} 
SGM \\
48,5 m \\
WP AP
\end{tabular} & \begin{tabular}{|l} 
SGM \\
24,5 m \\
WP AP \\
\end{tabular} & \begin{tabular}{|l|} 
CML \\
21-22 m \\
PL KB \\
\end{tabular} & \begin{tabular}{|l|} 
CML \\
$24 m$ \\
PL KB \\
\end{tabular} & $\begin{array}{l}\text { CML } \\
26 \mathrm{~m} \\
\text { PL KB }\end{array}$ & \begin{tabular}{|l|} 
CML \\
$32 \mathrm{~m}$ \\
PL KB \\
\end{tabular} & \begin{tabular}{|l} 
CML \\
$36 \mathrm{~m}$ \\
PL KB \\
\end{tabular} & \begin{tabular}{|l} 
CML \\
37 m \\
PL KB \\
\end{tabular} & \begin{tabular}{|l} 
CML \\
39 m \\
PL KB \\
\end{tabular} & $\begin{array}{l}\text { RCO4 } \\
\text { Okt } 98 \\
\text { WP KB }\end{array}$ \\
\hline Spezies & $\%$ & $\%$ & $\%$ & $\%$ & $\%$ & $\%$ & $\%$ & $\%$ & $\%$ & $\%$ \\
\hline $\mathrm{Zn}^{2+}$ & 53,5 & 55,4 & 67,0 & 73,6 & 79,8 & 74,5 & 67,3 & 66,8 & 61,7 & 46,3 \\
\hline $\mathrm{ZnSO}_{4}$ (aq) & 38,0 & 36,9 & 30,0 & 24,1 & 18,8 & 23,0 & 29,2 & 29,7 & 33,5 & 42,4 \\
\hline $\mathrm{Zn}\left(\mathrm{SO}_{4}\right)_{2}{ }^{2-}$ & 8,5 & 7,7 & 2,9 & 2,3 & 1,5 & 2,3 & 3,2 & 3,4 & 4,7 & 11,3 \\
\hline $\mathrm{HAsO}_{4}{ }^{2-}$ & 0,0 & 0,0 & 49,4 & 0,0 & 0,0 & 74,6 & 67,4 & 51,4 & 0,0 & 0,0 \\
\hline $\mathrm{H}_{2} \mathrm{AsO}_{4}^{-}$ & 98,9 & 98,9 & 50,6 & 96,5 & 84,9 & 25,4 & 32,6 & 48,6 & 0,0 & 0,0 \\
\hline $\mathrm{H}_{3} \mathrm{AsO}_{4}$ & 0,0 & 0,0 & 0,0 & 3,4 & 15,1 & 0,0 & 0,0 & 0,0 & 0,0 & 0,0 \\
\hline $\mathrm{Sr}^{2+}$ & 61,0 & 62,5 & 71,3 & 77,2 & 82,5 & 78,3 & 71,9 & 71,4 & 67,1 & 54,4 \\
\hline $\mathrm{SrSO}_{4}(\mathrm{aq})$ & 39,0 & 37,5 & 28,7 & 22,8 & 17,5 & 21,7 & 28,1 & 28,6 & 32,9 & 45,6 \\
\hline $\mathrm{MoO}_{4}^{2-}$ & 63,2 & 63,1 & 99,8 & $\mathrm{nq}$ & 8,4 & 99,9 & $\mathrm{nq}$ & 99,8 & 99,4 & 99,4 \\
\hline $\mathrm{HMoO}_{4}^{-}$ & 26,5 & 26,6 & 0,0 & $\mathrm{nq}$ & 90,9 & 0,0 & $\mathrm{nq}$ & 0,0 & 0,0 & 0,0 \\
\hline $\mathrm{H}_{2} \mathrm{MoO}_{4}(\mathrm{aq})$ & 10,3 & 10,3 & 0,0 & $\mathrm{nq}$ & 0,0 & 0,0 & $\mathrm{nq}$ & 0,0 & 0,0 & 0,0 \\
\hline $\mathrm{Cd}^{2+}$ & 49,7 & 51,7 & 64,8 & $\mathrm{nq}$ & $\mathrm{nq}$ & $\mathrm{nq}$ & $\mathrm{nq}$ & $\mathrm{nq}$ & 58,8 & $\mathrm{nq}$ \\
\hline $\mathrm{CdSO}_{4}(\mathrm{aq})$ & 37,2 & 36,4 & 30,5 & $\mathrm{nq}$ & $\mathrm{nq}$ & $\mathrm{nq}$ & $\mathrm{nq}$ & $\mathrm{nq}$ & 33,7 & $\mathrm{nq}$ \\
\hline $\mathrm{Cd}\left(\mathrm{SO}_{4}\right)_{2}{ }^{2-}$ & 13,2 & 11,9 & 4,7 & $\mathrm{nq}$ & $\mathrm{nq}$ & $\mathrm{nq}$ & $\mathrm{nq}$ & $\mathrm{nq}$ & 7,5 & $\mathrm{nq}$ \\
\hline $\operatorname{Sn}(\mathrm{OH})_{6}^{-}$ & 99,3 & 99,3 & 99,4 & $\mathrm{nq}$ & $\mathrm{nq}$ & $\mathrm{nq}$ & $\mathrm{nq}$ & 99,4 & 99,4 & $\mathrm{nq}$ \\
\hline $\mathrm{SbO}_{3}^{-}$ & 99,9 & 99,9 & 99,9 & $\mathrm{nq}$ & $\mathrm{nq}$ & $\mathrm{nq}$ & $\mathrm{nq}$ & 99,9 & 99,9 & $\mathrm{nq}$ \\
\hline $\mathrm{Sb}(\mathrm{OH})_{3}$ & 0,0 & 0,0 & 0,0 & $\mathrm{nq}$ & $\mathrm{nq}$ & $\mathrm{nq}$ & $\mathrm{nq}$ & 0,0 & 0,0 & $\mathrm{nq}$ \\
\hline $\mathrm{Sb}(\mathrm{OH})_{2}{ }^{+}$ & 0,0 & 0,0 & 0,0 & $\mathrm{nq}$ & $\mathrm{nq}$ & $\mathrm{nq}$ & $\mathrm{nq}$ & 0,0 & 0,0 & $\mathrm{nq}$ \\
\hline $\mathrm{HSbO}_{2}$ & 0,0 & 0,0 & 0,0 & $\mathrm{nq}$ & $\mathrm{nq}$ & $\mathrm{nq}$ & $\mathrm{nq}$ & 0,0 & 0,0 & $\mathrm{nq}$ \\
\hline $\mathrm{SbO}^{+}$ & 0,0 & 0,0 & 0,0 & $\mathrm{nq}$ & $\mathrm{nq}$ & $\mathrm{nq}$ & $\mathrm{nq}$ & 0,0 & 0,0 & $\mathrm{nq}$ \\
\hline $\mathrm{Ba}^{2+}$ & 100,0 & 100,0 & 100,0 & 100,0 & 100,0 & 100,0 & 100,0 & 100,0 & 100,0 & 100,0 \\
\hline $\mathrm{Tl}^{+}$ & 82,3 & 83,3 & 89,9 & $\mathrm{nq}$ & $\mathrm{nq}$ & $\mathrm{nq}$ & $\mathrm{nq}$ & $\mathrm{nq}$ & 87,0 & $\mathrm{nq}$ \\
\hline $\mathrm{TISO}_{4}^{-}$ & 17,7 & 16,7 & 10,1 & $\mathrm{nq}$ & $\mathrm{nq}$ & $\mathrm{nq}$ & $\mathrm{nq}$ & $\mathrm{nq}$ & 13,0 & $\mathrm{nq}$ \\
\hline $\mathrm{Pb}^{2+}$ & 34,4 & 36,0 & 45,8 & $\mathrm{nq}$ & $\mathrm{nq}$ & $\mathrm{nq}$ & $\mathrm{nq}$ & 45,7 & 41,5 & $\mathrm{nq}$ \\
\hline $\mathrm{PbOH}^{+}$ & 0,0 & 0,0 & 3,2 & $\mathrm{nq}$ & $\mathrm{nq}$ & $\mathrm{nq}$ & $\mathrm{nq}$ & 3,1 & 0,0 & $\mathrm{nq}$ \\
\hline $\mathrm{Pb}(\mathrm{OH})_{2}(\mathrm{aq})$ & 0,0 & 0,0 & 0,0 & $\mathrm{nq}$ & $\mathrm{nq}$ & $\mathrm{nq}$ & $\mathrm{nq}$ & 0,0 & 0,0 & $\mathrm{nq}$ \\
\hline $\mathrm{PbSO}_{4}(\mathrm{aq})$ & 57,1 & 56,2 & 47,9 & $\mathrm{nq}$ & $\mathrm{nq}$ & $\mathrm{nq}$ & $\mathrm{nq}$ & 47,6 & 52,7 & $\mathrm{nq}$ \\
\hline $\mathrm{Pb}\left(\mathrm{SO}_{4}\right)_{2}{ }^{2-}$ & 8,5 & 7,7 & 3,1 & $\mathrm{nq}$ & $\mathrm{nq}$ & $\mathrm{nq}$ & $\mathrm{nq}$ & 3,6 & 4,9 & $\mathrm{nq}$ \\
\hline $\mathrm{UO}_{2}{ }^{+}$ & 0,0 & 0,0 & 0,0 & $\mathrm{nq}$ & $\mathrm{nq}$ & $\mathrm{nq}$ & $\mathrm{nq}$ & 0,0 & 0,0 & $\mathrm{nq}$ \\
\hline $\mathrm{U}(\mathrm{OH})_{2}{ }^{2+}$ & 0,0 & 0,0 & 0,0 & $\mathrm{nq}$ & $\mathrm{nq}$ & $\mathrm{nq}$ & $\mathrm{nq}$ & 0,0 & 0,0 & $\mathrm{nq}$ \\
\hline $\mathrm{U}(\mathbf{O H})_{3}{ }^{+}$ & 0,0 & 0,0 & 0,0 & $\mathrm{nq}$ & $\mathrm{nq}$ & $\mathrm{nq}$ & $\mathrm{nq}$ & 0,0 & 0,0 & $\mathrm{nq}$ \\
\hline $\mathrm{U}(\mathrm{OH})_{4}(\mathrm{aq})$ & 0,0 & 0,0 & 0,0 & $\mathrm{nq}$ & $\mathrm{nq}$ & $\mathrm{nq}$ & $\mathrm{nq}$ & 0,0 & 0,0 & $\mathrm{nq}$ \\
\hline $\mathrm{USO}_{4}{ }^{2+}$ & 0,0 & 0,0 & 0,0 & $\mathrm{nq}$ & $\mathrm{nq}$ & $\mathrm{nq}$ & $\mathrm{nq}$ & 0,0 & 0,0 & $\mathrm{nq}$ \\
\hline $\mathrm{U}\left(\mathrm{SO}_{4}\right)_{2}(\mathrm{aq})$ & 0,0 & 0,0 & 0,0 & $\mathrm{nq}$ & $\mathrm{nq}$ & $\mathrm{nq}$ & $\mathrm{nq}$ & 0,0 & 0,0 & $\mathrm{nq}$ \\
\hline $\mathrm{UO}_{2}{ }^{2+}$ & 27,1 & 29,0 & 18,9 & $\mathrm{nq}$ & $\mathrm{nq}$ & $\mathrm{nq}$ & $\mathrm{nq}$ & 19,7 & 28,6 & $\mathrm{nq}$ \\
\hline $\mathrm{UO}_{2} \mathbf{O H}^{+}$ & 0,0 & 0,0 & 47,7 & $\mathrm{nq}$ & $\mathrm{nq}$ & $\mathrm{nq}$ & $\mathrm{nq}$ & 47,9 & 21,3 & $\mathrm{nq}$ \\
\hline$\left(\mathrm{UO}_{2}\right)_{2}(\mathrm{OH})_{2}{ }^{2+}$ & 0,0 & 0,0 & 1,5 & $\mathrm{nq}$ & $\mathrm{nq}$ & $\mathrm{nq}$ & $\mathrm{nq}$ & 1,3 & 0,0 & $\mathrm{nq}$ \\
\hline$\left(\mathrm{UO}_{2}\right)_{3}(\mathrm{OH})_{5}{ }^{+}$ & 0,0 & 0,0 & 7,6 & $\mathrm{nq}$ & $\mathrm{nq}$ & $\mathrm{nq}$ & $\mathrm{nq}$ & 5,0 & 0,0 & $\mathrm{nq}$ \\
\hline $\mathrm{UO}_{2} \mathrm{SO}_{4}(\mathrm{aq})$ & 42,1 & 42,3 & 18,5 & $\mathrm{nq}$ & $\mathrm{nq}$ & $\mathrm{nq}$ & $\mathrm{nq}$ & 19,1 & 33,9 & $\mathrm{nq}$ \\
\hline $\mathrm{UO}_{2}\left(\mathrm{SO}_{4}\right)_{2}{ }^{2-}$ & 30,6 & 28,4 & 5,8 & $\mathrm{nq}$ & $\mathrm{nq}$ & $\mathrm{nq}$ & $\mathrm{nq}$ & 7,0 & 15,5 & $\mathrm{nq}$ \\
\hline $\mathrm{SO}_{4}{ }^{2-}$ & 57,7 & 56,3 & 62,1 & $\mathrm{nb}$ & 40,3 & 50,5 & 57,6 & 58,7 & 61,0 & 68,4 \\
\hline $\mathrm{HSO}_{4}^{-}$ & 0,0 & 0,0 & 0,0 & $\mathrm{nb}$ & 2,3 & 0,0 & 0,0 & 0,0 & 0,0 & 0,0 \\
\hline $\mathrm{AlSO}_{4}{ }^{+}$ & 1,5 & 2,4 & 0,0 & $\mathrm{nb}$ & 16,6 & 0,0 & 0,0 & 0,0 & 0,0 & 0,0 \\
\hline $\mathrm{Al}\left(\mathrm{SO}_{4}\right)_{2}^{-}$ & 0,0 & 0,0 & 0,0 & $\mathrm{nb}$ & 1,2 & 0,0 & 0,0 & 0,0 & 0,0 & 0,0 \\
\hline $\mathrm{FeSO}_{4}(\mathrm{aq})$ & 22,7 & 23,1 & 0,0 & $\mathrm{nb}$ & 7,4 & 0,0 & 0,0 & 0,0 & 0,0 & 1,6 \\
\hline $\mathrm{MgSO}_{4}(\mathrm{aq})$ & 8,5 & 8,9 & 9,3 & $\mathrm{nb}$ & 26,1 & 29,5 & 13,9 & 16,1 & 14,3 & 13,2 \\
\hline CaSO4 (aq) & 8,7 & 8,3 & 27,8 & $\mathrm{nb}$ & 5,2 & 19,0 & 27,5 & 23,7 & 23,8 & 16,0 \\
\hline
\end{tabular}


Tab. A.25. (Forts.): Ergebnisse der Speziationsberechnung mit MINTEQA2.

\begin{tabular}{|c|c|c|c|c|c|c|c|c|c|c|}
\hline & \begin{tabular}{|l|} 
RCO10 \\
Okt 98 \\
WP KB \\
\end{tabular} & $\begin{array}{l}\text { RCO12 } \\
\text { Okt } 98 \\
\text { WP KB }\end{array}$ & $\begin{array}{l}\text { RCO13 } \\
\text { Okt } 98 \\
\text { WP KB }\end{array}$ & $\begin{array}{l}\text { RCO14 } \\
\text { Okt } 98 \\
\text { WP KB }\end{array}$ & \begin{tabular}{|l|} 
Bohr. $1 \mathrm{~m}$ \\
Nov 98 \\
PL KB
\end{tabular} & \begin{tabular}{|l|} 
Bohr. 2 m \\
Nov 98 \\
PL KB
\end{tabular} & \begin{tabular}{|l|} 
Bohr. $3 \mathrm{~m}$ \\
Nov 98 \\
PL KB
\end{tabular} & $\begin{array}{l}\text { See } \\
\text { Nov } 98 \\
\text { WP KB }\end{array}$ & $\begin{array}{l}\text { CML } 20 \text { m } \\
\text { Jan } 99 \\
\text { WP KB }\end{array}$ & $\begin{array}{l}\text { CML 27,5 m } \\
\text { Jan 99 } \\
\text { WP KB }\end{array}$ \\
\hline Spezies & $\%$ & $\%$ & $\%$ & $\%$ & $\%$ & $\%$ & $\%$ & $\%$ & $\%$ & $\%$ \\
\hline $\mathrm{Li}^{+}$ & 97,4 & 94,3 & 98,0 & 98,6 & 96,5 & 97,5 & 97,4 & 95,1 & 98,3 & 98,0 \\
\hline $\mathrm{LiSO}_{4}^{-}$ & 2,6 & 5,7 & 2,0 & 1,4 & 3,5 & 2,5 & 2,6 & 4,9 & 1,7 & 2,0 \\
\hline $\mathrm{Be}^{2+}$ & $\mathrm{nq}$ & $\mathrm{nq}$ & $\mathrm{nq}$ & $\mathrm{nq}$ & 64,9 & 67,0 & 71,7 & $\mathrm{nq}$ & $\mathrm{nq}$ & $\mathrm{nq}$ \\
\hline $\mathrm{BeOH}^{+}$ & $\mathrm{nq}$ & $\mathrm{nq}$ & $\mathrm{nq}$ & $\mathrm{nq}$ & 0,0 & 11,3 & 3,1 & $\mathrm{nq}$ & $\mathrm{nq}$ & $\mathrm{nq}$ \\
\hline $\mathrm{BeSO}_{4}(\mathrm{aq})$ & $\mathrm{nq}$ & $\mathrm{nq}$ & $\mathrm{nq}$ & $\mathrm{nq}$ & 32,9 & 20,8 & 24,2 & $\mathrm{nq}$ & $\mathrm{nq}$ & $\mathrm{nq}$ \\
\hline $\mathrm{Be}\left(\mathrm{SO}_{4}\right)_{2}{ }^{2-}$ & $\mathrm{nq}$ & $\mathrm{nq}$ & $\mathrm{nq}$ & $\mathrm{nq}$ & 1,7 & 0,0 & 1,1 & $\mathrm{nq}$ & $\mathrm{nq}$ & $\mathrm{nq}$ \\
\hline $\mathrm{Na}^{+}$ & 96,8 & 93,1 & 97,5 & 98,3 & 95,8 & 97,0 & 96,8 & 94,1 & 98,0 & 97,5 \\
\hline $\mathrm{NaSO}_{4}^{-}$ & 3,2 & 6,9 & 2,5 & 1,7 & 4,2 & 3,0 & 3,2 & 5,9 & 2,0 & 2,5 \\
\hline $\mathrm{Mg}^{2+}$ & 64,8 & 53,5 & 68,9 & 75,3 & 58,9 & 70,0 & 68,2 & 55,7 & 73,7 & 71,0 \\
\hline $\mathrm{MgSO}_{4}(\mathrm{aq})$ & 35,2 & 46,5 & 31,1 & 24,7 & 41,1 & 30,0 & 31,8 & 44,3 & 26,3 & 29,0 \\
\hline $\mathbf{A l}^{3+}$ & $\mathrm{nq}$ & $\mathrm{nq}$ & $\mathrm{nq}$ & $\mathrm{nq}$ & 6,7 & 11,6 & 10,8 & 0,0 & $\mathrm{nq}$ & $\mathrm{nq}$ \\
\hline $\mathrm{AlOH}^{2+}$ & $\mathrm{nq}$ & $\mathrm{nq}$ & $\mathrm{nq}$ & $\mathrm{nq}$ & 0,0 & 2,3 & 0,0 & 0,0 & $\mathrm{nq}$ & $\mathrm{nq}$ \\
\hline $\mathrm{Al}(\mathrm{OH})_{2}{ }^{+}$ & $\mathrm{nq}$ & $\mathrm{nq}$ & $\mathrm{nq}$ & $\mathrm{nq}$ & 0,0 & 1,1 & 0,0 & 30,9 & $\mathrm{nq}$ & $\mathrm{nq}$ \\
\hline $\mathrm{Al}(\mathrm{OH})_{3}(\mathrm{aq})$ & $\mathrm{nq}$ & $\mathrm{nq}$ & $\mathrm{nq}$ & $\mathrm{nq}$ & 0,0 & 0,0 & 0,0 & 49,2 & $\mathrm{nq}$ & $\mathrm{nq}$ \\
\hline $\mathrm{Al}(\mathrm{OH})_{4}^{-}$ & $\mathrm{nq}$ & $\mathrm{nq}$ & $\mathrm{nq}$ & $\mathrm{nq}$ & 0,0 & 0,0 & 0,0 & 19,4 & $\mathrm{nq}$ & $\mathrm{nq}$ \\
\hline $\mathrm{AlSO}_{4}{ }^{+}$ & $\mathrm{nq}$ & $\mathrm{nq}$ & $\mathrm{nq}$ & $\mathrm{nq}$ & 84,9 & 79,4 & 82,5 & 0,0 & $\mathrm{nq}$ & $\mathrm{nq}$ \\
\hline $\mathrm{Al}\left(\mathrm{SO}_{4}\right)_{2}^{-}$ & $\mathrm{nq}$ & $\mathrm{nq}$ & $\mathrm{nq}$ & $\mathrm{nq}$ & 8,3 & 5,5 & 6,0 & 0,0 & $\mathrm{nq}$ & $\mathrm{nq}$ \\
\hline $\mathbf{K}^{+}$ & 96,2 & 91,8 & 97,1 & 98,0 & 95,0 & 96,4 & 96,2 & 94,1 & 97,6 & 97,0 \\
\hline $\mathrm{KSO}_{4}^{-}$ & 3,8 & 8,2 & 2,9 & 2,0 & 5,0 & 3,6 & 3,8 & 5,9 & 2,4 & 3,0 \\
\hline $\mathrm{Ca}^{2+}$ & 59,6 & 48,0 & 64,0 & 71,0 & 53,4 & 65,2 & 63,3 & 50,6 & 69,2 & 66,2 \\
\hline $\mathrm{CaSO}_{4}(\mathrm{aq})$ & 40,4 & 52,0 & 36,0 & 29,0 & 46,6 & 34,8 & 36,7 & 49,4 & 30,8 & 33,8 \\
\hline $\mathrm{VO}^{2+}$ & 0,0 & 0,0 & 0,0 & 0,0 & 39,9 & 0,0 & 0,0 & 0,0 & 0,5 & $\mathrm{nq}$ \\
\hline $\mathrm{VOSO}_{4}(\mathrm{aq})$ & 0,0 & 0,0 & 0,0 & 0,0 & 39,1 & 0,0 & 0,0 & 0,0 & 0,3 & $\mathrm{nq}$ \\
\hline $\mathrm{V}(\mathrm{OH})_{3}(\mathbf{a q})$ & 2,5 & 21,2 & 1,7 & 4,4 & 20,9 & 0,0 & 0,0 & 0,0 & 99,3 & $\mathrm{nq}$ \\
\hline $\mathrm{VO}_{2}^{+}$ & 3,1 & 77,9 & 0,0 & 0,0 & 0,0 & 0,0 & 3,6 & 0,0 & 0,0 & $\mathrm{nq}$ \\
\hline $\mathrm{HVO}_{4}{ }^{2-}$ & 0,0 & 0,0 & 4,6 & 0,0 & 0,0 & 0,0 & 0,0 & 4,6 & 0,0 & $\mathrm{nq}$ \\
\hline $\mathrm{H}_{2} \mathrm{VO}_{4}^{-}$ & 94,3 & 0,0 & 93,6 & 94,5 & 0,0 & 91,5 & 71,5 & 95,4 & 0,0 & $\mathrm{nq}$ \\
\hline $\mathrm{VO}_{2} \mathrm{SO}_{4}^{-}$ & 0,0 & 0,0 & 0,0 & 0,0 & 0,0 & 0,0 & 0,0 & 0,0 & 0,0 & $\mathrm{nq}$ \\
\hline $\mathrm{H}_{3} \mathrm{VO}_{4}(\mathrm{aq})$ & 0,0 & 0,0 & 0,0 & 0,0 & 0,0 & 6,8 & 21,2 & 0,0 & 0,0 & $\mathrm{nq}$ \\
\hline $\mathbf{H}_{3} \mathbf{V}_{2} \mathbf{O}_{7}^{-}$ & 0,0 & 0,0 & 0,0 & 0,0 & 0,0 & 1,3 & 3,2 & 0,0 & 0,0 & $\mathrm{nq}$ \\
\hline $\mathrm{Cr}^{3+}$ & 0,0 & 1,1 & 0,0 & 0,0 & 19,7 & 11,3 & 21,0 & 0,0 & 29,9 & 0,0 \\
\hline $\mathrm{Cr}(\mathrm{OH})_{2}^{+}$ & 69,2 & 15,9 & 78,3 & 48,4 & 0,0 & 1,5 & 0,0 & 48,9 & 0,0 & 81,4 \\
\hline $\mathrm{CrOH}^{2+}$ & 20,2 & 46,9 & 13,6 & 40,1 & 4,3 & 48,1 & 23,2 & 40,2 & 6,6 & 3,8 \\
\hline $\mathrm{CrSO}_{4}^{+}$ & 0,0 & 3,8 & 0,0 & 0,0 & 73,6 & 22,7 & 47,1 & 0,0 & 61,6 & \\
\hline $\mathrm{Cr}(\mathrm{OH})_{3}(\mathrm{aq})$ & 1,7 & 0,0 & 3,2 & 0,0 & 0,0 & 0,0 & 0,0 & 1,5 & 0,0 & 12,9 \\
\hline $\mathrm{CrOHSO}_{4}(\mathrm{aq})$ & 8,7 & 32,4 & 4,9 & 10,4 & 2,4 & 16,4 & 8,6 & 8,9 & 1,9 & 1,2 \\
\hline $\mathrm{Mn}^{2+}$ & 65,8 & 54,6 & 69,8 & 76,1 & 59,9 & 70,9 & 69,2 & 57,8 & $\mathrm{nq}$ & 71,8 \\
\hline $\mathrm{MnSO}_{4}(\mathrm{aq})$ & 34,2 & 45,4 & 30,2 & 23,9 & 40,1 & 29,1 & 30,8 & 42,2 & $\mathrm{nq}$ & 28,1 \\
\hline $\mathrm{Fe}^{2+}$ & 41,6 & 45,2 & 36,4 & 57,0 & 51,8 & 0,0 & 61,8 & 33,9 & $\mathrm{nq}$ & $\mathrm{nq}$ \\
\hline $\mathrm{FeSO}_{4}(\mathrm{aq})$ & 30,1 & 52,3 & 21,8 & 24,8 & 48,2 & 0,0 & 38,2 & 34,6 & $\mathrm{nq}$ & $\mathrm{nq}$ \\
\hline $\mathrm{FeOH}^{2+}$ & 0,0 & 0,0 & 0,0 & 0,0 & 0,0 & 0,0 & 0,0 & 0,0 & $\mathrm{nq}$ & $\mathrm{nq}$ \\
\hline $\mathrm{Fe}(\mathrm{OH})_{2}{ }^{+}$ & 27,2 & 2,5 & 39,4 & 18,0 & 0,0 & 99,5 & 0,0 & 31,7 & $\mathrm{nq}$ & $\mathrm{nq}$ \\
\hline $\mathrm{Fe}(\mathrm{OH})_{3}$ & 1,0 & 0,0 & 2,3 & 0,2 & 0,0 & 0,0 & 0,0 & 0,0 & $\mathrm{nq}$ & $\mathrm{nq}$ \\
\hline $\mathrm{Co}^{2+}$ & 62,7 & 51,3 & 66,9 & 73,6 & 56,7 & 68,1 & 66,3 & 53,6 & 71,9 & 68,8 \\
\hline $\mathrm{CoSO}_{4}(\mathrm{aq})$ & 37,2 & 48,7 & 33,0 & 26,4 & 43,3 & 31,9 & 33,7 & 46,4 & 28,1 & 30,8 \\
\hline $\mathrm{Ni}^{2+}$ & 62,7 & 51,2 & 66,9 & 73,6 & 56,6 & 68,1 & 66,2 & 53,4 & 71,8 & 68,9 \\
\hline $\mathrm{NiSO}_{4}(\mathrm{aq})$ & 37,3 & 48,8 & 33,1 & 26,4 & 43,4 & 31,9 & 33,8 & 46,6 & 28,1 & 30,9 \\
\hline $\mathrm{Cu}^{+}$ & 0,0 & 0,0 & 2,7 & 0,0 & 0,0 & 0,0 & 0,0 & 0,0 & 6,4 & 3,7 \\
\hline $\mathrm{Cu}^{2+}$ & 55,8 & 48,0 & 55,2 & 69,1 & 53,7 & 65,4 & 63,5 & 49,5 & 65,0 & 41,4 \\
\hline $\mathrm{Cu}(\mathrm{OH})^{+}$ & 6,6 & 0,0 & 11,1 & 2,9 & 0,0 & 34,5 & 0,0 & 3,8 & 0,0 & 30,6 \\
\hline $\mathrm{Cu}(\mathrm{OH})_{2}$ & 0,0 & 0,0 & 0,0 & 0,0 & 0,0 & 0,0 & 0,0 & 0,0 & 0,0 & 3,3 \\
\hline $\mathrm{CuSO}_{4}(\mathrm{aq})$ & 37,4 & 51,5 & 30,7 & 27,9 & 46,3 & & 36,5 & 46,6 & 28,6 & 20,9 \\
\hline
\end{tabular}


Tab. A.25. (Forts.): Ergebnisse der Speziationsberechnung mit MINTEQA2.

\begin{tabular}{|c|c|c|c|c|c|c|c|c|c|c|}
\hline & \begin{tabular}{|l|} 
RCO10 \\
Okt 98 \\
WP KB \\
\end{tabular} & $\begin{array}{l}\text { RCO12 } \\
\text { Okt } 98 \\
\text { WP KB }\end{array}$ & $\begin{array}{l}\text { RCO13 } \\
\text { Okt } 98 \\
\text { WP KB }\end{array}$ & $\begin{array}{l}\text { RCO14 } \\
\text { Okt } 98 \\
\text { WP KB }\end{array}$ & \begin{tabular}{|l|} 
Bohr. 1 m \\
Nov 98 \\
PL KB
\end{tabular} & \begin{tabular}{|l|} 
Bohr. 2 m \\
Nov 98 \\
PL KB \\
\end{tabular} & \begin{tabular}{|l|} 
Bohr. $3 \mathrm{~m}$ \\
Nov 98 \\
PL KB
\end{tabular} & $\begin{array}{l}\text { See } \\
\text { Nov } 98 \\
\text { WP KB }\end{array}$ & \begin{tabular}{|l|} 
CML $20 \mathrm{~m}$ \\
Jan 99 \\
WP KB \\
\end{tabular} & $\begin{array}{l}\text { CML 27,5 m } \\
\text { Jan 99 } \\
\text { WP KB }\end{array}$ \\
\hline Spezies & $\%$ & $\%$ & $\%$ & $\%$ & $\%$ & $\%$ & $\%$ & $\%$ & $\%$ & $\%$ \\
\hline $\mathbf{Z n}^{2+}$ & 57,9 & 41,6 & 62,9 & 70,7 & 50,9 & 63,4 & 61,4 & 45,1 & $\mathrm{nq}$ & 64,3 \\
\hline $\mathrm{ZnOH}^{+}$ & 0,0 & 0,0 & 0,0 & 0,0 & 0,0 & 0,0 & 0,0 & 0,0 & $\mathrm{nq}$ & 1,3 \\
\hline $\mathrm{ZnSO}_{4}(\mathrm{aq})$ & 37,7 & 43,3 & 34,0 & 27,8 & 42,6 & 32,5 & 34,2 & 42,8 & $\mathrm{nq}$ & 31,5 \\
\hline $\mathrm{Zn}\left(\mathrm{SO}_{4}\right)_{2}{ }^{2-}$ & 4,2 & 15,1 & 2,7 & 1,4 & 6,5 & 4,0 & 4,4 & 12,1 & $\mathrm{nq}$ & 2,7 \\
\hline $\mathrm{HAsO}_{4}{ }^{2-}$ & $\mathrm{nq}$ & $\mathrm{nq}$ & $\mathrm{nq}$ & $\mathrm{nq}$ & 0,0 & 1,6 & 0,0 & $\mathrm{nq}$ & $\mathrm{nq}$ & $\mathrm{nq}$ \\
\hline $\mathrm{H}_{2} \mathrm{AsO}_{4}^{-}$ & $\mathrm{nq}$ & $\mathrm{nq}$ & $\mathrm{nq}$ & $\mathrm{nq}$ & 96,1 & 98,3 & 98,9 & $\mathrm{nq}$ & $\mathrm{nq}$ & $\mathrm{nq}$ \\
\hline $\mathrm{H}_{3} \mathrm{AsO}_{4}$ & $\mathrm{nq}$ & $\mathrm{nq}$ & $\mathrm{nq}$ & $\mathrm{nq}$ & 3,8 & 0,0 & 0,0 & $\mathrm{nq}$ & $\mathrm{nq}$ & $\mathrm{nq}$ \\
\hline $\mathrm{H}_{3} \mathrm{AsO}_{3}$ & $\mathrm{nq}$ & $\mathrm{nq}$ & $\mathrm{nq}$ & $\mathrm{nq}$ & 0,0 & 0,0 & 0,0 & $\mathrm{nq}$ & $\mathrm{nq}$ & $\mathrm{nq}$ \\
\hline $\mathrm{Sr}^{2+}$ & 63,0 & 51,6 & 67,2 & 73,9 & 57,0 & 68,4 & 66,6 & 54,6 & 72,2 & 69,4 \\
\hline $\mathrm{SrSO}_{4}$ (aq) & 37,0 & 48,4 & 32,8 & 26,1 & 43,0 & 31,6 & 33,4 & 45,4 & 27,8 & 30,6 \\
\hline $\mathrm{MoO}_{4}{ }^{2-}$ & 99,9 & 99,2 & 99,9 & $\mathrm{nq}$ & 9,1 & 88,7 & 60,2 & 99,9 & 7,3 & 100,0 \\
\hline $\mathrm{HMoO}_{4}^{-}$ & 0,0 & 0,0 & 0,0 & $\mathrm{nq}$ & 26,7 & 10,3 & 28,3 & 0,0 & 25,1 & 0,0 \\
\hline $\mathrm{H}_{2} \mathrm{MoO}_{4}(\mathrm{aq})$ & 0,0 & 0,0 & 0,0 & $\mathrm{nq}$ & 64,2 & 1,0 & 11,5 & 0,0 & 67,6 & 0,0 \\
\hline $\mathrm{Cd}^{2+}$ & $\mathrm{nq}$ & $\mathrm{nq}$ & $\mathrm{nq}$ & $\mathrm{nq}$ & 47,8 & 60,7 & 58,6 & nq & 66,7 & 62,9 \\
\hline $\mathrm{CdSO}_{4}(\mathrm{aq})$ & $\mathrm{nq}$ & $\mathrm{nq}$ & $\mathrm{nq}$ & $\mathrm{nq}$ & 42,1 & 32,8 & 34,4 & $\mathrm{nq}$ & 30,1 & 32,5 \\
\hline $\mathrm{Cd}\left(\mathrm{SO}_{4}\right)_{2}{ }^{2-}$ & $\mathrm{nq}$ & $\mathrm{nq}$ & $\mathrm{nq}$ & $\mathrm{nq}$ & 10,1 & 6,4 & 6,9 & $\mathrm{nq}$ & 3,2 & 4,5 \\
\hline $\mathrm{Sn}(\mathrm{OH})_{2}$ & 0,0 & 0,0 & $\mathrm{nq}$ & 0,0 & 0,0 & 0,0 & $\mathrm{nq}$ & 0,0 & $\mathrm{nq}$ & 0,0 \\
\hline $\mathbf{S n}^{2+}$ & 0,0 & 0,0 & $\mathrm{nq}$ & 0,0 & 0,0 & 0,0 & $\mathrm{nq}$ & 0,0 & $\mathrm{nq}$ & 0,0 \\
\hline $\mathrm{SnOH}^{+}$ & 0,0 & 0,0 & $\mathrm{nq}$ & 0,0 & 0,0 & 0,0 & $\mathrm{nq}$ & 0,0 & $\mathrm{nq}$ & 0,0 \\
\hline $\mathbf{S n}^{4+}$ & 0,0 & 0,0 & $\mathrm{nq}$ & 0,0 & 88,9 & 0,0 & $\mathrm{nq}$ & 0,0 & $\mathrm{nq}$ & 0,0 \\
\hline $\mathrm{Sn}(\mathrm{OH})_{6}^{-}$ & 99,4 & 99,4 & $\mathrm{nq}$ & 99,4 & 11,1 & 99,4 & $\mathrm{nq}$ & 99,4 & $\mathrm{nq}$ & 99,4 \\
\hline $\mathrm{SbO}_{3}^{-}$ & $\mathrm{nq}$ & $\mathrm{nq}$ & $\mathrm{nq}$ & $\mathrm{nq}$ & 94,7 & 99,9 & 99,9 & $\mathrm{nq}$ & 99,9 & $\mathrm{nq}$ \\
\hline $\mathrm{Sb}(\mathrm{OH})_{3}$ & $\mathrm{nq}$ & $\mathrm{nq}$ & $\mathrm{nq}$ & $\mathrm{nq}$ & 2,6 & 0,0 & 0,0 & $\mathrm{nq}$ & 0,0 & $\mathrm{nq}$ \\
\hline $\mathrm{Sb}(\mathrm{OH})_{2}{ }^{+}$ & $\mathrm{nq}$ & $\mathrm{nq}$ & $\mathrm{nq}$ & $\mathrm{nq}$ & 0,0 & 0,0 & 0,0 & $\mathrm{nq}$ & 0,0 & $\mathrm{nq}$ \\
\hline $\mathrm{HSbO}_{2}$ & $\mathrm{nq}$ & $\mathrm{nq}$ & $\mathrm{nq}$ & $\mathrm{nq}$ & 2,6 & 0,0 & 0,0 & $\mathrm{nq}$ & 0,0 & $\mathrm{nq}$ \\
\hline $\mathrm{SbO}^{+}$ & $\mathrm{nq}$ & $\mathrm{nq}$ & $\mathrm{nq}$ & $\mathrm{nq}$ & 0,0 & 0,0 & 0,0 & $\mathrm{nq}$ & 0,0 & $\mathrm{nq}$ \\
\hline $\mathbf{B a}^{2+}$ & 100,0 & 100,0 & 100,0 & 100,0 & 100,0 & 100,0 & 100,0 & 100,0 & 100,0 & 100,0 \\
\hline $\mathrm{Tl}^{+}$ & $\mathrm{nq}$ & $\mathrm{nq}$ & $\mathrm{nq}$ & $\mathrm{nq}$ & 83,8 & 88,0 & 87,4 & $\mathrm{nq}$ & $\mathrm{nq}$ & $\mathrm{nq}$ \\
\hline $\mathrm{TISO}_{4}^{-}$ & $\mathrm{nq}$ & $\mathrm{nq}$ & $\mathrm{nq}$ & $\mathrm{nq}$ & 16,2 & 12,0 & 12,6 & $\mathrm{nq}$ & $\mathrm{nq}$ & $\mathrm{nq}$ \\
\hline $\mathbf{P b}^{2+}$ & 36,3 & 24,9 & $\mathrm{nq}$ & $\mathrm{nq}$ & 31,7 & 43,5 & 41,4 & $\mathrm{nq}$ & 48,9 & 33,6 \\
\hline $\mathrm{PbOH}^{+}$ & 4,4 & 0,0 & $\mathrm{nq}$ & $\mathrm{nq}$ & 0,0 & 0,0 & 0,0 & $\mathrm{nq}$ & 0,0 & 25,3 \\
\hline $\mathrm{Pb}(\mathrm{OH})_{2}(\mathrm{aq})$ & 0,0 & 0,0 & $\mathrm{nq}$ & $\mathrm{nq}$ & 0,0 & 0,0 & 0,0 & $\mathrm{nq}$ & 0,0 & 0,0 \\
\hline $\mathrm{PbSO}_{4}(\mathrm{aq})$ & 55,2 & 60,7 & $\mathrm{nq}$ & $\mathrm{nq}$ & 62,0 & 52,1 & 54,0 & $\mathrm{nq}$ & 48,9 & 38,5 \\
\hline $\mathrm{Pb}\left(\mathrm{SO}_{4}\right)_{2}{ }^{2-}$ & 4,1 & 14,0 & $\mathrm{nq}$ & $\mathrm{nq}$ & 6,3 & 4,3 & 4,6 & $\mathrm{nq}$ & 2,2 & 2,2 \\
\hline $\mathrm{UO}_{2}{ }^{2+}$ & $\mathrm{nq}$ & 15,9 & 9,9 & $\mathrm{nq}$ & 26,8 & $\mathrm{nq}$ & $\mathrm{nq}$ & 14,9 & $\mathrm{nq}$ & 2,6 \\
\hline $\mathrm{UO}_{2} \mathbf{O H}^{+}$ & $\mathrm{nq}$ & 6,9 & 72,9 & $\mathrm{nq}$ & 0,0 & $\mathrm{nq}$ & $\mathrm{nq}$ & 35,7 & $\mathrm{nq}$ & 69,3 \\
\hline$\left(\mathrm{UO}_{2}\right)_{2}(\mathrm{OH})_{2}{ }^{2+}$ & $\mathrm{nq}$ & 0,0 & 0,0 & $\mathrm{nq}$ & 0,0 & $\mathrm{nq}$ & $\mathrm{nq}$ & 0,0 & $\mathrm{nq}$ & 0,0 \\
\hline$\left(\mathrm{UO}_{2}\right)_{3}(\mathrm{OH})_{5}{ }^{+}$ & $\mathrm{nq}$ & 0,0 & 2,1 & $\mathrm{nq}$ & 0,0 & $\mathrm{nq}$ & $\mathrm{nq}$ & 0,0 & $\mathrm{nq}$ & 24,4 \\
\hline $\mathrm{UO}_{2} \mathrm{SO}_{4}$ (aq) & $\mathrm{nq}$ & 36,3 & 11,7 & $\mathrm{nq}$ & 49,0 & $\mathrm{nq}$ & $\mathrm{nq}$ & 26,9 & $\mathrm{nq}$ & 2,7 \\
\hline $\mathrm{UO}_{2}\left(\mathrm{SO}_{4}\right)_{2}{ }^{2-}$ & $\mathrm{nq}$ & 40,8 & 3,0 & $\mathrm{nq}$ & 24,2 & $\mathrm{nq}$ & $\mathrm{nq}$ & 21,2 & $\mathrm{nq}$ & 0,0 \\
\hline $\mathrm{SO}_{4}^{2-}$ & 82,2 & 72,1 & 83,1 & 81,9 & 88,9 & 61,5 & 64,3 & 84,2 & 72,6 & 70,7 \\
\hline $\mathrm{HSO}_{4}^{-}$ & 0,0 & 0,0 & 0,0 & 0,0 & 1,3 & 0,0 & 0,0 & 0,0 & 1,2 & 0,0 \\
\hline $\mathrm{AlSO}_{4}{ }^{+}$ & 0,0 & 0,0 & 0,0 & 0,0 & 1,3 & 0,0 & 0,0 & 0,0 & 0,0 & 0,0 \\
\hline $\mathrm{Al}\left(\mathrm{SO}_{4}\right)_{2}^{-}$ & 0,0 & 0,0 & 0,0 & 0,0 & 0,0 & 0,0 & 0,0 & 0,0 & 0,0 & 0,0 \\
\hline $\mathrm{FeSO}_{4}(\mathrm{aq})$ & 0,0 & 0,0 & 0,0 & 0,0 & 0,0 & 0,0 & 0,0 & 0,0 & 0,0 & 0,0 \\
\hline $\mathrm{MgSO}_{4}(\mathrm{aq})$ & 5,0 & 10,8 & 5,6 & 6,0 & 4,5 & 14,7 & 9,5 & 4,4 & 0,0 & 1,9 \\
\hline CaSO4 (aq) & 12,2 & 16,0 & 10,8 & 11,3 & 3,4 & 23,2 & 25,3 & 10,9 & 24,7 & 26,5 \\
\hline
\end{tabular}


Tab. A.25. (Forts.): Ergebnisse der Speziationsberechnung mit MINTEQA2.

\begin{tabular}{|c|c|c|c|c|c|c|c|c|c|c|}
\hline & \begin{tabular}{|l|} 
CML 31m \\
Jan 99 \\
WP KB \\
\end{tabular} & \begin{tabular}{|l|} 
CML 39m \\
Jan 99 \\
WP KB
\end{tabular} & \begin{tabular}{|l|} 
CML 43,5m \\
Jan 99 \\
WP KB
\end{tabular} & \begin{tabular}{|l|} 
CML 20m \\
Aug 99 \\
WP KB \\
\end{tabular} & \begin{tabular}{|l|} 
CML 27,5m \\
Aug 99 \\
WP KB \\
\end{tabular} & \begin{tabular}{|l|} 
CML 31,5m \\
Aug 99 \\
WP KB
\end{tabular} & \begin{tabular}{|l|} 
CML39m \\
Aug 99 \\
WP KB \\
\end{tabular} & \begin{tabular}{|l|} 
CML 43,5m \\
Aug 99 \\
WP KB
\end{tabular} & $\begin{array}{l}\text { RCO3 } \\
\text { Aug 99 } \\
\text { WP KB }\end{array}$ & \begin{tabular}{|l|} 
RCO4 \\
Aug 99 \\
WP KB
\end{tabular} \\
\hline Spezies & $\%$ & $\%$ & $\%$ & $\%$ & $\%$ & $\%$ & $\%$ & $\%$ & $\%$ & $\%$ \\
\hline $\mathrm{Li}^{+}$ & 97,0 & 98,3 & 98,5 & 97,9 & 96,1 & 96,6 & 98,3 & 98,6 & 96,9 & 97,7 \\
\hline $\mathrm{LiSO}_{4}^{-}$ & 3,0 & 1,7 & 1,5 & 2,1 & 3,9 & 3,4 & 1,7 & 1,4 & 3,1 & 2,3 \\
\hline $\mathrm{Be}^{2+}$ & $\mathrm{nq}$ & $\mathrm{nq}$ & $\mathrm{nq}$ & $\mathrm{nq}$ & 29,9 & 31,1 & $\mathrm{nq}$ & $\mathrm{nq}$ & 56,3 & $\mathrm{nq}$ \\
\hline $\mathrm{BeOH}^{+}$ & $\mathrm{nq}$ & $\mathrm{nq}$ & $\mathrm{nq}$ & $\mathrm{nq}$ & 56,3 & 56,9 & $\mathrm{nq}$ & $\mathrm{nq}$ & 22,4 & $\mathrm{nq}$ \\
\hline $\mathrm{BeSO}_{4}(\mathrm{aq})$ & $\mathrm{nq}$ & $\mathrm{nq}$ & $\mathrm{nq}$ & $\mathrm{nq}$ & 12,5 & 10,9 & $\mathrm{nq}$ & $n q$ & 20,1 & $\mathrm{nq}$ \\
\hline $\mathrm{Be}\left(\mathrm{SO}_{4}\right)_{2}{ }^{2-}$ & $\mathrm{nq}$ & $\mathrm{nq}$ & $\mathrm{nq}$ & $\mathrm{nq}$ & 0,0 & 0,0 & $\mathrm{nq}$ & $\mathrm{nq}$ & 1,2 & $\mathrm{nq}$ \\
\hline $\mathrm{Na}^{+}$ & 96,4 & 98,0 & 98,2 & 97,5 & 95,3 & 95,9 & 97,9 & 98,3 & 96,3 & 97,2 \\
\hline $\mathrm{NaSO}_{4}^{-}$ & 3,6 & 2,0 & 1,8 & 2,5 & 4,7 & 4,1 & 2,1 & 1,7 & 3,7 & 2,8 \\
\hline $\mathrm{Mg}^{2+}$ & 66,6 & 75,3 & 76,6 & 71,7 & 63,5 & 67,3 & 76,1 & 78,5 & 67,1 & 72,3 \\
\hline $\mathrm{MgSO}_{4}(\mathrm{aq})$ & 33,4 & 24,7 & 23,4 & 28,3 & 36,5 & 32,7 & 23,9 & 21,5 & 32,9 & 27,7 \\
\hline $\mathbf{A l}^{3+}$ & $\mathrm{nq}$ & $\mathrm{nq}$ & $\mathrm{nq}$ & 6,8 & 4,0 & 4,5 & 0,0 & 3,0 & 10,2 & 0,0 \\
\hline $\mathrm{AlOH}^{2+}$ & $\mathrm{nq}$ & $\mathrm{nq}$ & $\mathrm{nq}$ & 9,7 & 8,2 & 8,8 & 0,0 & 9,0 & 4,6 & 4,8 \\
\hline $\mathrm{Al}(\mathrm{OH})_{2}{ }^{+}$ & $\mathrm{nq}$ & $\mathrm{nq}$ & $\mathrm{nq}$ & 30,8 & 42,9 & 44,8 & 19,5 & 58,3 & 5,1 & 54,2 \\
\hline $\mathrm{Al}(\mathrm{OH})_{3}(\mathbf{a q})$ & $\mathrm{nq}$ & $\mathrm{nq}$ & $\mathrm{nq}$ & 2,6 & 6,5 & 6,7 & 20,0 & 9,7 & 0,0 & 17,1 \\
\hline $\mathrm{Al}(\mathrm{OH})_{4}^{-}$ & $\mathrm{nq}$ & $\mathrm{nq}$ & $\mathrm{nq}$ & 0,0 & 3,3 & 3,5 & 59,8 & 4,5 & 0,0 & 16,7 \\
\hline $\mathrm{AlSO}_{4}{ }^{+}$ & $\mathrm{nq}$ & $\mathrm{nq}$ & $\mathrm{nq}$ & 46,9 & 31,5 & 28,9 & 0,0 & 14,9 & 73,6 & 5,9 \\
\hline $\mathrm{Al}\left(\mathrm{SO}_{4}\right)_{2}^{-}$ & $\mathrm{nq}$ & $\mathrm{nq}$ & $\mathrm{nq}$ & 2,7 & 3,5 & 2,8 & 0,0 & 0,0 & 6,4 & 0,0 \\
\hline $\mathbf{K}^{+}$ & 95,7 & 97,6 & 97,8 & 97,0 & 94,4 & 95,1 & 97,5 & 98,0 & 95,5 & 96,6 \\
\hline $\mathrm{KSO}_{4}^{-}$ & 4,3 & 2,4 & 2,2 & 3,0 & 5,6 & 4,9 & 2,5 & 2,0 & 4,5 & 3,4 \\
\hline $\mathrm{Ca}^{2+}$ & 61,6 & 71,0 & 72,4 & 67,0 & 58,2 & 62,3 & 71,9 & 74,5 & 62,0 & 67,7 \\
\hline $\mathrm{CaSO}_{4}(\mathrm{aq})$ & 38,4 & 29,0 & 27,6 & 33,0 & 41,8 & 37,7 & 28,1 & 25,5 & 38,0 & 32,3 \\
\hline $\mathrm{V}(\mathrm{OH})_{3}(\mathbf{a q})$ & 10,0 & $\mathrm{nq}$ & $\mathrm{nq}$ & 100,0 & 100,0 & 100,0 & 100,0 & 100,0 & 100,0 & 0,0 \\
\hline $\mathrm{VO}_{2}^{+}$ & 88,1 & $\mathrm{nq}$ & $\mathrm{nq}$ & 0,0 & 0,0 & 0,0 & 0,0 & 0,0 & 0,0 & 0,0 \\
\hline $\mathrm{H}_{2} \mathrm{VO}_{4}^{-}$ & 0,0 & $\mathrm{nq}$ & $\mathrm{nq}$ & 0,0 & 0,0 & 0,0 & 0,0 & 0,0 & 0,0 & 97,5 \\
\hline $\mathrm{H}_{3} \mathbf{V}_{2} \mathbf{O}_{7}^{-}$ & 0,0 & $\mathrm{nq}$ & $\mathrm{nq}$ & 0,0 & 0,0 & 0,0 & 0,0 & 0,0 & 0,0 & 1,3 \\
\hline $\mathrm{Cr}^{3+}$ & 0,0 & 0,0 & 0,0 & 2,0 & 1,2 & 1,3 & 0,0 & 0,0 & 5,8 & 0,0 \\
\hline $\mathrm{Cr}(\mathrm{OH})_{2}{ }^{+}$ & 28,9 & 83,5 & 83,6 & 12,7 & 18,0 & 18,4 & 65,9 & 25,0 & 4,0 & 35,5 \\
\hline $\mathrm{CrOH}^{2+}$ & 49,4 & 6,3 & 7,6 & 61,8 & 53,5 & 56,1 & 26,2 & 59,6 & 56,0 & 48,3 \\
\hline $\mathrm{CrSO}_{4}^{+}$ & 1,4 & 0,0 & 0,0 & 4,1 & 2,8 & 2,5 & 0,0 & 1,4 & 12,4 & 0,0 \\
\hline $\mathrm{Cr}(\mathrm{OH})_{3}(\mathrm{aq})$ & 0,0 & 8,3 & 6,7 & 0,0 & 0,0 & 0,0 & 1,3 & 0,0 & 0,0 & 0,0 \\
\hline $\mathrm{CrOHSO}_{4}(\mathrm{aq})$ & 19,6 & 1,6 & 1,9 & 19,4 & 24,4 & 21,6 & 6,5 & 13,0 & 21,8 & 14,7 \\
\hline $\mathrm{Mn}^{2+}$ & 67,6 & 76,1 & 77,3 & 72,6 & 64,5 & 68,3 & 76,9 & 79,2 & 68,0 & 73,1 \\
\hline $\mathrm{MnSO}_{4}(\mathrm{aq})$ & 32,4 & 23,9 & 22,7 & 27,4 & 35,5 & 31,7 & 23,1 & 20,8 & 32,0 & 26,9 \\
\hline $\mathrm{Fe}^{2+}$ & 56,1 & 20,2 & 26,0 & 65,6 & 56,7 & 60,8 & 70,5 & 73,3 & 60,5 & 66,3 \\
\hline $\mathrm{FeSO}_{4}(\mathrm{aq})$ & 37,3 & 8,8 & 10,6 & 34,4 & 43,3 & 39,2 & 29,4 & 26,7 & 39,5 & 33,7 \\
\hline $\mathrm{Fe}(\mathrm{OH})_{2}{ }^{+}$ & 6,6 & 61,8 & 56,6 & 0,0 & 0,0 & 0,0 & 0,0 & 0,0 & 0,0 & 0,0 \\
\hline $\mathrm{Fe}(\mathrm{OH})_{3}$ & 0,0 & 8,6 & 6,3 & 0,0 & 0,0 & 0,0 & 0,0 & 0,0 & 0,0 & 0,0 \\
\hline $\mathrm{Co}^{2+}$ & 64,6 & 73,4 & 74,8 & 69,8 & 61,4 & 65,3 & 74,4 & 76,9 & 65,1 & 70,5 \\
\hline $\mathrm{CoSO}_{4}(\mathrm{aq})$ & 35,4 & 26,3 & 25,0 & 30,2 & 38,6 & 34,7 & 25,5 & 23,0 & 34,9 & 29,5 \\
\hline $\mathrm{Ni}^{2+}$ & 64,6 & $\mathrm{nq}$ & $\mathrm{nq}$ & 69,8 & 61,3 & 65,3 & 74,4 & 76,9 & $\mathrm{nq}$ & $\mathrm{nq}$ \\
\hline $\mathrm{NiSO}_{4}(\mathbf{a q})$ & 35,4 & $\mathrm{nq}$ & $\mathrm{nq}$ & 30,2 & 38,6 & 34,7 & 25,6 & 23,1 & $\mathrm{nq}$ & $\mathrm{nq}$ \\
\hline
\end{tabular}


Tab. A.25. (Forts.): Ergebnisse der Speziationsberechnung mit MINTEQA2.

\begin{tabular}{|c|c|c|c|c|c|c|c|c|c|c|}
\hline & \begin{tabular}{|l|} 
CML 31m \\
Jan 99 \\
WP KB \\
\end{tabular} & \begin{tabular}{|l|} 
CML 39m \\
Jan 99 \\
WP KB
\end{tabular} & \begin{tabular}{|l|} 
CML 43,5m \\
Jan 99 \\
WP KB
\end{tabular} & \begin{tabular}{|l|} 
CML 20m \\
Aug 99 \\
WP KB
\end{tabular} & \begin{tabular}{|l|} 
CML 27,5m \\
Aug 99 \\
WP KB \\
\end{tabular} & \begin{tabular}{|l|} 
CML 31,5m \\
Aug 99 \\
WP KB
\end{tabular} & \begin{tabular}{|l|} 
CML39m \\
Aug 99 \\
WP KB \\
\end{tabular} & \begin{tabular}{|l|} 
CML 43,5m \\
Aug 99 \\
WP KB
\end{tabular} & $\begin{array}{l}\text { RCO3 } \\
\text { Aug 99 } \\
\text { WP KB }\end{array}$ & \begin{tabular}{|l|} 
RCO4 \\
Aug 99 \\
WP KB
\end{tabular} \\
\hline Spezies & $\%$ & $\%$ & $\%$ & $\%$ & $\%$ & $\%$ & $\%$ & $\%$ & $\%$ & $\%$ \\
\hline $\mathrm{Cu}^{+}$ & 0,0 & 4,6 & 4,7 & 35,6 & 6,5 & 6,7 & 54,6 & 100,0 & 8,4 & 65,6 \\
\hline $\mathrm{Cu}^{2+}$ & 61,1 & 50,4 & 53,6 & 43,1 & 54,3 & 57,9 & 30,8 & $\mathrm{nq}$ & 57,0 & 22,9 \\
\hline $\mathrm{Cu}(\mathrm{OH})^{+}$ & 1,2 & 23,1 & 20,4 & 0,0 & 0,0 & 0,0 & 2,7 & $\mathrm{nq}$ & 0,0 & 0,6 \\
\hline $\mathrm{Cu}(\mathrm{OH})_{2}$ & 0,0 & 1,5 & 1,1 & 0,0 & 0,0 & 0,0 & 0,0 & $\mathrm{nq}$ & 0,0 & 0,0 \\
\hline $\mathrm{CuSO}_{4}(\mathrm{aq})$ & 37,7 & 20,3 & 20,2 & 21,0 & 38,5 & 34,7 & 11,9 & $\mathrm{nq}$ & 34,5 & 10,9 \\
\hline $\mathrm{Zn}+{ }^{2}$ & 59,0 & $\mathrm{nq}$ & 71,4 & 65,9 & 54,0 & 58,6 & 71,0 & 74,1 & 59,2 & 66,0 \\
\hline $\mathrm{ZnOH}^{+}$ & 0,0 & $\mathrm{nq}$ & 0,0 & 0,0 & 0,0 & 0,0 & 0,0 & 0,0 & 0,0 & 0,0 \\
\hline $\mathrm{ZnSO}_{4}$ (aq) & 35,4 & $\mathrm{nq}$ & 26,2 & 31,2 & 37,2 & 34,1 & 26,7 & 24,4 & 34,8 & 30,3 \\
\hline $\mathrm{Zn}\left(\mathrm{SO}_{4}\right)_{2}{ }^{2-}$ & 5,6 & $\mathrm{nq}$ & 1,6 & 2,9 & 8,7 & 7,3 & 2,1 & 1,5 & 5,9 & 3,7 \\
\hline $\mathrm{HAsO}_{4}{ }^{2-}$ & $\mathrm{nq}$ & $\mathrm{nq}$ & $\mathrm{nq}$ & 7,3 & 18,6 & 19,0 & 54,9 & 0,0 & 0,4 & 29,4 \\
\hline $\mathrm{H}_{2} \mathrm{AsO}_{4}^{-}$ & $\mathrm{nq}$ & $\mathrm{nq}$ & $\mathrm{nq}$ & 77,7 & 81,4 & 81,0 & 45,1 & 0,0 & 10,1 & 70,6 \\
\hline $\mathrm{H}_{3} \mathrm{AsO}_{3}$ & $\mathrm{nq}$ & $\mathrm{nq}$ & $\mathrm{nq}$ & 15,0 & 0,0 & 0,0 & 0,0 & 99,9 & 89,5 & 0,0 \\
\hline $\mathbf{S r}^{2+}$ & 64,9 & 73,9 & 75,2 & 70,1 & 61,7 & 65,6 & 74,7 & 77,2 & 65,4 & 70,7 \\
\hline $\mathrm{SrSO}_{4}(\mathrm{aq})$ & 35,1 & 26,1 & 24,8 & 29,9 & 38,3 & 34,4 & 25,3 & 22,8 & 34,6 & 29,3 \\
\hline $\mathrm{MoO}_{4}{ }^{2-}$ & 99,4 & 100,0 & 100,0 & 98,0 & 99,2 & 99,2 & 99,8 & 99,0 & 95,7 & 99,6 \\
\hline $\mathrm{HMoO}_{4}^{-}$ & 0,0 & 0,0 & 0,0 & 1,9 & 0,0 & 0,0 & 0,0 & 1,0 & 4,1 & 0,0 \\
\hline $\mathbf{C d}^{2+}$ & $\mathrm{nq}$ & 68,4 & 70,2 & $\mathrm{nq}$ & $\mathrm{nq}$ & 54,9 & $\mathrm{nq}$ & $\mathrm{nq}$ & $\mathrm{nq}$ & $\mathrm{nq}$ \\
\hline $\mathrm{CdSO}_{4}$ (aq) & $\mathrm{nq}$ & 28,3 & 27,1 & $\mathrm{nq}$ & $\mathrm{nq}$ & 33,7 & $\mathrm{nq}$ & $\mathrm{nq}$ & $\mathrm{nq}$ & $\mathrm{nq}$ \\
\hline $\mathrm{Cd}\left(\mathrm{SO}_{4}\right)_{2}{ }^{2-}$ & $\mathrm{nq}$ & 3,3 & 2,6 & $\mathrm{nq}$ & $\mathrm{nq}$ & 11,4 & $\mathrm{nq}$ & $\mathrm{nq}$ & $\mathrm{nq}$ & $\mathrm{nq}$ \\
\hline $\mathrm{Sn}(\mathrm{OH})_{6}^{-}$ & $\mathrm{nq}$ & $\mathrm{nq}$ & $\mathrm{nq}$ & 99,4 & 99,4 & 99,4 & $\mathrm{nq}$ & $\mathrm{nq}$ & 99,4 & 99,4 \\
\hline $\mathrm{SbO}_{3}^{-}$ & $\mathrm{nq}$ & $\mathrm{nq}$ & $\mathrm{nq}$ & 99,9 & $\mathrm{nq}$ & $\mathrm{nq}$ & $\mathrm{nq}$ & $\mathrm{nq}$ & $\mathrm{nq}$ & $\mathrm{nq}$ \\
\hline $\mathrm{Ba}^{2+}$ & 100,0 & 100,0 & 100,0 & 100,0 & 100,0 & 100,0 & 100,0 & 100,0 & 100,0 & 100,0 \\
\hline $\mathrm{TI}^{+}$ & $\mathrm{nq}$ & 91,6 & $\mathrm{nq}$ & $\mathrm{nq}$ & $\mathrm{nq}$ & $\mathrm{nq}$ & $\mathrm{nq}$ & $\mathrm{nq}$ & $\mathrm{nq}$ & $\mathrm{nq}$ \\
\hline $\mathrm{TISO}_{4}^{-}$ & $\mathrm{nq}$ & 8,4 & $\mathrm{nq}$ & $\mathrm{nq}$ & $\mathrm{nq}$ & $n q$ & $\mathrm{nq}$ & $\mathrm{nq}$ & $\mathrm{nq}$ & $\mathrm{nq}$ \\
\hline $\mathbf{P b}^{2+}$ & 38,9 & 41,1 & 43,9 & 45,8 & 34,8 & 39,0 & 49,7 & 55,2 & 39,5 & 45,8 \\
\hline $\mathrm{PbOH}^{+}$ & 0,0 & 19,2 & 16,9 & 0,0 & 0,0 & 0,0 & 4,4 & 0,0 & 0,0 & 1,2 \\
\hline $\mathrm{Pb}(\mathrm{OH})_{2}(\mathrm{aq})$ & 0,0 & 0,0 & 0,0 & 0,0 & 0,0 & 0,0 & 0,0 & 0,0 & 0,0 & 0,0 \\
\hline $\mathrm{PbSO}_{4}(\mathrm{aq})$ & 54,6 & 37,7 & 37,6 & 50,7 & 56,1 & 53,0 & 43,6 & 42,3 & 54,3 & 49,1 \\
\hline $\mathrm{Pb}\left(\mathrm{SO}_{4}\right)_{2}{ }^{2-}$ & 5,7 & 1,8 & 1,5 & 3,1 & 8,7 & 7,5 & 2,3 & 1,7 & 6,1 & 4,0 \\
\hline $\mathrm{UO}_{2}^{+}$ & 0,0 & 0,0 & 0,0 & 31,3 & 0,0 & 0,0 & 37,9 & 91,7 & $\mathrm{nq}$ & 25,2 \\
\hline $\mathrm{U}(\mathrm{OH})_{4}(\mathrm{aq})$ & 0,0 & 0,0 & 0,0 & 0,0 & 0,0 & 0,0 & 0,0 & 0,3 & $\mathrm{nq}$ & 0,0 \\
\hline $\mathrm{U}(\mathrm{OH})_{5}^{-}$ & 0,0 & 0,0 & 0,0 & 0,0 & 0,0 & 0,0 & 0,0 & 8,0 & $\mathrm{nq}$ & 0,0 \\
\hline $\mathrm{UO}_{2}{ }^{2+}$ & 26,8 & 0,0 & 0,0 & 26,4 & 24,5 & 28,0 & 4,8 & 0,0 & $\mathrm{nq}$ & 22,4 \\
\hline $\mathrm{UO}_{2} \mathrm{OH}^{+}$ & 20,0 & 5,7 & 11,4 & 6,9 & 10,5 & 11,7 & 15,4 & 0,0 & $\mathrm{nq}$ & 20,9 \\
\hline$\left(\mathrm{UO}_{2}\right)_{2}(\mathrm{OH})_{2}{ }^{2+}$ & 0,0 & 0,0 & 0,0 & 0,0 & 0,0 & 0,0 & 1,4 & 0,0 & $\mathrm{nq}$ & 0,0 \\
\hline$\left(\mathrm{UO}_{2}\right)_{3}(\mathrm{OH})_{5}{ }^{+}$ & 0,0 & 93,3 & 86,6 & 0,0 & 0,0 & 0,0 & 35,5 & 0,0 & $\mathrm{nq}$ & 0,0 \\
\hline $\mathrm{UO}_{2} \mathrm{SO}_{4}(\mathrm{aq})$ & 35,2 & 0,0 & 0,0 & 27,3 & 36,9 & 35,6 & 4,0 & 0,0 & $\mathrm{nq}$ & 22,4 \\
\hline $\mathrm{UO}_{2}\left(\mathrm{SO}_{4}\right)_{2}{ }^{2-}$ & 17,8 & 0,0 & 0,0 & 8,2 & 27,9 & 24,7 & 1,0 & 0,0 & $\mathrm{nq}$ & 8,8 \\
\hline $\mathrm{SO}_{4}{ }^{2-}$ & 62,3 & 65,8 & 68,6 & 65,8 & 57,1 & 51,4 & 61,3 & 63,6 & 58,4 & 58,0 \\
\hline $\mathrm{HSO}_{4}^{-}$ & 0,0 & 0,0 & 0,0 & 0,0 & 0,0 & 0,0 & 0,0 & 0,0 & 0,0 & 0,0 \\
\hline $\mathrm{MgSO}_{4}(\mathrm{aq})$ & 16,8 & 11,5 & 10,3 & 3,3 & 19,0 & 18,9 & 13,6 & 11,9 & 12,9 & 15,7 \\
\hline CaSO4 (aq) & 7,6 & 21,8 & 20,2 & 30,2 & 9,9 & 9,2 & 23,4 & 23,5 & 14,4 & 22,2 \\
\hline
\end{tabular}


Tab. A.25. (Forts.): Ergebnisse der Speziationsberechnung mit MINTEQA2.

\begin{tabular}{|c|c|c|c|c|c|c|c|c|c|c|}
\hline & $\begin{array}{l}\text { RCO12 } \\
\text { Aug 99 } \\
\text { WP KB }\end{array}$ & $\begin{array}{l}\text { RCO15 } \\
\text { Aug } 99 \\
\text { WP KB }\end{array}$ & $\begin{array}{l}\text { RCO17 } \\
\text { Aug 99 } \\
\text { WP KB }\end{array}$ & $\begin{array}{l}\text { RCO18 } \\
\text { Aug 99 } \\
\text { WP KB } \\
\end{array}$ & \begin{tabular}{|l|} 
See \\
Aug 99 \\
WP KB \\
\end{tabular} & \begin{tabular}{|l} 
SeeSed \\
Aug 99 \\
PL KB
\end{tabular} & \begin{tabular}{|l|} 
SeeSed \\
Aug 99 \\
WP KB
\end{tabular} & \begin{tabular}{|l|} 
CML 20 m \\
Jan 00 \\
WP KB
\end{tabular} & \begin{tabular}{|l|} 
CML 27,5m \\
Jan 00 \\
WP KB \\
\end{tabular} & $\begin{array}{l}\text { CML 31,5m } \\
\text { Jan 00 } \\
\text { WP KB } \\
\end{array}$ \\
\hline Spezies & $\%$ & $\%$ & $\%$ & $\%$ & $\%$ & $\%$ & $\%$ & $\%$ & $\%$ & $\%$ \\
\hline $\mathrm{Li}^{+}$ & 97,7 & 98,5 & 98,0 & 98,2 & 98,4 & 98,6 & 98,5 & 97,9 & 96,4 & 96,5 \\
\hline $\mathrm{LiSO}_{4}^{-}$ & 2,3 & 1,5 & 2,0 & 1,8 & 1,6 & 1,4 & 1,5 & 2,1 & 3,6 & 3,5 \\
\hline $\mathrm{Be}^{2+}$ & $\mathrm{nq}$ & $\mathrm{nq}$ & 11,4 & $\mathrm{nq}$ & $\mathrm{nq}$ & $\mathrm{nq}$ & $\mathrm{nq}$ & $\mathrm{nq}$ & 71,0 & 71,8 \\
\hline $\mathrm{BeOH}^{+}$ & $\mathrm{nq}$ & $\mathrm{nq}$ & 83,9 & $\mathrm{nq}$ & $\mathrm{nq}$ & $\mathrm{nq}$ & $\mathrm{nq}$ & $\mathrm{nq}$ & 0,0 & 0,0 \\
\hline $\mathrm{Be}(\mathrm{OH})_{2}$ & $\mathrm{nq}$ & $\mathrm{nq}$ & 1,4 & $\mathrm{nq}$ & $\mathrm{nq}$ & $\mathrm{nq}$ & $\mathrm{nq}$ & $\mathrm{nq}$ & 0,0 & 0,0 \\
\hline $\mathrm{BeSO}_{4}(\mathrm{aq})$ & $\mathrm{nq}$ & $\mathrm{nq}$ & 3,2 & $\mathrm{nq}$ & $\mathrm{nq}$ & $\mathrm{nq}$ & $\mathrm{nq}$ & $\mathrm{nq}$ & 26,7 & 26,0 \\
\hline $\mathrm{Be}\left(\mathrm{SO}_{4}\right)_{2}{ }^{2-}$ & $\mathrm{nq}$ & $\mathrm{nq}$ & 0,0 & $\mathrm{nq}$ & $\mathrm{nq}$ & $\mathrm{nq}$ & $\mathrm{nq}$ & $\mathrm{nq}$ & 2,0 & 1,9 \\
\hline $\mathrm{Na}^{+}$ & 97,2 & 98,2 & 97,6 & 97,8 & 98,1 & 98,3 & 98,2 & 97,5 & 95,6 & 95,8 \\
\hline $\mathrm{NaSO}_{4}^{-}$ & 2,8 & 1,8 & 2,4 & 2,2 & 1,9 & 1,7 & 1,8 & 2,5 & 4,4 & 4,2 \\
\hline $\mathrm{Mg}^{2+}$ & 71,7 & 76,6 & 71,9 & 74,6 & 75,1 & 76,8 & 75,9 & 72,1 & 65,9 & 66,7 \\
\hline $\mathrm{MgSO}_{4}(\mathrm{aq})$ & 28,3 & 23,4 & 28,1 & 25,4 & 24,9 & 23,2 & 24,1 & 27,9 & 34,1 & 33,3 \\
\hline $\mathrm{Al}^{3+}$ & 2,4 & 0,0 & 0,0 & 0,0 & 0,0 & 0,0 & $\mathrm{nq}$ & $\mathrm{nq}$ & 11,5 & 12,0 \\
\hline $\mathrm{AlOH}^{2+}$ & 7,6 & 2,0 & 2,0 & 3,1 & 13,8 & 1,2 & $\mathrm{nq}$ & $\mathrm{nq}$ & 0,0 & 0,0 \\
\hline $\mathrm{Al}(\mathrm{OH})_{2}{ }^{+}$ & 55,9 & 41,9 & 41,8 & 49,3 & 0,0 & 33,8 & $\mathrm{nq}$ & $\mathrm{nq}$ & 0,0 & 0,0 \\
\hline $\mathrm{Al}(\mathrm{OH})_{3}(\mathrm{aq})$ & 11,2 & 22,4 & 22,0 & 20,3 & 18,6 & 23,0 & $\mathrm{nq}$ & $\mathrm{nq}$ & 0,0 & 0,0 \\
\hline $\mathrm{Al}(\mathrm{OH})_{4}^{-}$ & 6,8 & 32,4 & 32,3 & 24,0 & 67,3 & 41,4 & $\mathrm{nq}$ & $\mathrm{nq}$ & 0,0 & 0,0 \\
\hline $\mathrm{AlSO}_{4}^{+}$ & 15,1 & 1,1 & 1,5 & 2,6 & 0,0 & 0,0 & $\mathrm{nq}$ & $\mathrm{nq}$ & 80,2 & 80,1 \\
\hline $\mathrm{Al}\left(\mathrm{SO}_{4}\right)_{2}^{-}$ & 0,0 & 0,0 & 0,0 & 0,0 & 0,0 & 0,0 & $\mathrm{nq}$ & $\mathrm{nq}$ & 8,2 & 7,9 \\
\hline $\mathbf{K}^{+}$ & 96,7 & 97,9 & 97,1 & 97,4 & 97,7 & 98,0 & 97,8 & 97,0 & 94,8 & 95,0 \\
\hline $\mathrm{KSO}_{4}^{-}$ & 3,3 & 2,1 & 2,9 & 2,6 & 2,3 & 2,0 & 2,2 & 3,0 & 5,2 & 5,0 \\
\hline $\mathrm{Ca}^{2+}$ & 67,0 & 72,4 & 67,2 & 70,2 & 70,7 & 72,7 & 71,7 & 67,4 & 60,7 & 61,6 \\
\hline $\mathrm{CaSO}_{4}(\mathrm{aq})$ & 33,0 & 27,6 & 32,8 & 29,8 & 29,3 & 27,3 & 28,3 & 32,6 & 39,3 & 38,4 \\
\hline $\mathrm{VO}^{2+}$ & 0,0 & $\mathrm{nq}$ & 0,0 & 0,0 & 0,0 & 0,0 & 0,0 & 96,1 & 0,0 & 58,8 \\
\hline $\mathrm{VOSO}_{4}$ (aq) & 0,0 & $\mathrm{nq}$ & 0,0 & 0,0 & 0,0 & 0,0 & 0,0 & 0,0 & 0,0 & 41,1 \\
\hline $\mathrm{V}(\mathrm{OH})_{3}(\mathrm{aq})$ & 100,0 & $\mathrm{nq}$ & 0,0 & 100,0 & 0,0 & 0,0 & 0,0 & 0,0 & 100,0 & 0,0 \\
\hline $\mathrm{HVO}_{4}{ }^{2-}$ & 0,0 & $\mathrm{nq}$ & 1,3 & 0,0 & 3,2 & 1,6 & 2,0 & 0,0 & 0,0 & 0,0 \\
\hline $\mathrm{H}_{2} \mathrm{VO}_{4}^{-}$ & 0,0 & $\mathrm{nq}$ & 98,4 & 0,0 & 96,8 & 98,1 & 97,7 & 3,9 & 0,0 & 0,0 \\
\hline $\mathrm{Cr}(\mathrm{OH})_{2}{ }^{+}$ & 25,9 & 52,1 & 49,6 & 43,9 & 71,9 & 58,5 & 62,8 & 27,2 & $\mathrm{nq}$ & $\mathrm{nq}$ \\
\hline $\mathrm{CrOH}^{2+}$ & 54,6 & 37,7 & 37,6 & 43,3 & 20,7 & 32,6 & 28,7 & 54,0 & $\mathrm{nq}$ & $\mathrm{nq}$ \\
\hline $\mathrm{CrSO}_{4}^{+}$ & 1,5 & 0,0 & 0,0 & 0,0 & 0,0 & 0,0 & 0,0 & 1,4 & $\mathrm{nq}$ & $\mathrm{nq}$ \\
\hline $\mathrm{Cr}(\mathrm{OH})_{3}(\mathrm{aq})$ & 0,0 & 0,0 & 0,0 & 0,0 & 1,8 & 0,0 & 1,0 & 0,0 & $\mathrm{nq}$ & $\mathrm{nq}$ \\
\hline $\mathrm{CrOHSO}_{4}(\mathrm{aq})$ & 17,1 & 9,1 & 11,7 & 11,7 & 5,5 & 7,8 & 7,2 & 16,6 & $\mathrm{nq}$ & $\mathrm{nq}$ \\
\hline $\mathrm{Mn}^{2+}$ & 72,6 & 77,4 & 72,7 & 75,4 & 75,9 & 77,6 & 0,0 & 73,0 & 66,8 & 67,7 \\
\hline $\mathrm{MnSO}_{4}(\mathrm{aq})$ & 27,4 & 22,6 & 27,3 & 24,6 & 24,1 & 22,4 & 0,0 & 27,0 & 33,2 & 32,3 \\
\hline $\mathrm{Fe}^{2+}$ & 65,6 & 53,6 & 50,6 & 68,9 & 69,3 & 71,4 & 70,3 & 66,0 & 59,2 & 60,1 \\
\hline $\mathrm{FeSO}_{4}(\mathrm{aq})$ & 34,4 & 21,7 & 26,3 & 31,1 & 30,6 & 28,6 & 29,6 & 34,0 & 40,8 & 39,9 \\
\hline $\mathrm{Fe}(\mathrm{OH})_{2}{ }^{+}$ & 0,0 & 24,3 & 22,8 & 0,0 & 0,0 & 0,0 & 0,0 & 0,0 & 0,0 & 0,0 \\
\hline $\mathrm{Co}^{2+}$ & 69,9 & 75,0 & 70,0 & 72,9 & 73,3 & 75,2 & 74,2 & 0,0 & 63,8 & 64,7 \\
\hline $\mathrm{CoSO}_{4}(\mathrm{aq})$ & 30,1 & 25,0 & 30,0 & 27,1 & 26,6 & 24,8 & 25,7 & 0,0 & 36,2 & 35,3 \\
\hline $\mathrm{Ni}^{2+}$ & 69,8 & 74,9 & 70,0 & 72,8 & 73,3 & 75,2 & 74,2 & 0,0 & 63,8 & 64,6 \\
\hline $\mathrm{NiSO}_{4}(\mathrm{aq})$ & 30,2 & 25,1 & 30,0 & 27,1 & 26,7 & 24,8 & 25,8 & 0,0 & 36,2 & 35,4 \\
\hline $\mathrm{Cu}^{+}$ & 36,3 & 0,0 & 0,0 & 0,0 & 0,0 & 0,0 & 0,0 & 3,4 & 100,0 & 0,0 \\
\hline $\mathrm{Cu}^{2+}$ & 42,4 & 70,2 & 65,4 & 68,7 & 65,3 & 69,7 & 68,1 & 64,6 & 0,0 & 61,9 \\
\hline $\mathrm{Cu}(\mathrm{OH})^{+}$ & 0,7 & 3,4 & 3,0 & 2,4 & 7,9 & 4,3 & 5,2 & 1,2 & 0,0 & 0,0 \\
\hline $\mathrm{CuSO}_{4}(\mathrm{aq})$ & 20,6 & 26,4 & 31,6 & 28,8 & 26,7 & 25,9 & 26,6 & 30,8 & 0,0 & 38,1 \\
\hline
\end{tabular}


Tab. A.25. (Forts.): Ergebnisse der Speziationsberechnung mit MINTEQA2.

\begin{tabular}{|c|c|c|c|c|c|c|c|c|c|c|}
\hline & \begin{tabular}{|l|} 
RCO12 \\
Aug 99 \\
WP KB
\end{tabular} & \begin{tabular}{|l|} 
RCO15 \\
Aug 99 \\
WP KB \\
\end{tabular} & $\begin{array}{l}\text { RCO17 } \\
\text { Aug 99 } \\
\text { WP KB } \\
\end{array}$ & \begin{tabular}{|l|} 
RCO18 \\
Aug 99 \\
WP KB \\
\end{tabular} & \begin{tabular}{|l|l} 
See & 5 \\
Aug 99 & \\
WP KB & I
\end{tabular} & \begin{tabular}{|l|} 
SeeSed \\
Aug 99 \\
PL KB
\end{tabular} & \begin{tabular}{|l|} 
SeeSed \\
Aug 99 \\
WP KB \\
\end{tabular} & \begin{tabular}{|l|} 
CML 20 m \\
Jan 00 \\
WP KB \\
\end{tabular} & \begin{tabular}{|l|} 
CML 27,5m \\
Jan 00 \\
WP KB \\
\end{tabular} & $\begin{array}{l}\text { CML 31,5m } \\
\text { Jan 00 } \\
\text { WP KB }\end{array}$ \\
\hline Spezies & $\%$ & $\%$ & $\%$ & $\%$ & $\%$ & $\%$ & $\%$ & $\%$ & $\%$ & $\%$ \\
\hline $\mathbf{Z n}^{2+}$ & 65,4 & 72,0 & 66,2 & 69,3 & 70,1 & 72,3 & 71,1 & 66,2 & 56,8 & 57,9 \\
\hline $\mathrm{ZnSO}_{4}$ (aq) & 30,9 & 26,3 & 31,0 & 28,3 & 27,9 & 26,1 & 27,0 & 30,7 & 35,3 & 34,6 \\
\hline $\mathrm{Zn}\left(\mathrm{SO}_{4}\right)_{2}{ }^{2-}$ & 3,6 & 1,6 & 2,7 & 2,4 & 1,8 & 1,4 & 1,7 & 3,0 & 7,9 & 7,5 \\
\hline $\mathrm{HAsO}_{4}{ }^{2-}$ & 20,4 & 35,8 & 36,8 & 32,4 & 58,3 & 40,5 & 46,9 & $\mathrm{nq}$ & 0,0 & 0,0 \\
\hline $\mathrm{H}_{2} \mathrm{AsO}_{4}^{-}$ & 79,6 & 64,2 & 63,2 & 67,6 & 41,7 & 59,5 & 53,1 & $\mathrm{nq}$ & 95,2 & 92,7 \\
\hline $\mathrm{H}_{3} \mathrm{AsO}_{4}$ & 0,0 & 0,0 & 0,0 & 0,0 & 0,0 & 0,0 & 0,0 & $\mathrm{nq}$ & 4,7 & 7,3 \\
\hline $\mathrm{Sr}^{2+}$ & 70,1 & 75,2 & 70,3 & 73,1 & 73,6 & $\mathrm{nq}$ & 74,5 & 70,5 & 64,1 & 65,0 \\
\hline $\mathrm{SrSO}_{4}(\mathrm{aq})$ & 29,9 & 24,8 & 29,7 & 26,9 & 26,4 & $\mathrm{nq}$ & 25,5 & 29,5 & 35,9 & 35,0 \\
\hline $\mathrm{MoO}_{4}{ }^{2-}$ & 99,3 & 99,7 & 99,7 & 99,6 & 99,9 & 99,7 & 99,8 & 99,2 & 7,3 & 3,3 \\
\hline $\mathrm{HMoO}_{4}^{-}$ & 0,0 & 0,0 & 0,0 & 0,0 & 0,0 & 0,0 & 0,0 & 0,0 & 23,1 & 16,8 \\
\hline $\mathrm{H}_{2} \mathrm{MoO}_{4}(\mathrm{aq})$ & 0,0 & 0,0 & 0,0 & 0,0 & 0,0 & 0,0 & 0,0 & 0,0 & 69,9 & 79,9 \\
\hline $\mathbf{C d}^{2+}$ & $\mathrm{nq}$ & $\mathrm{nq}$ & $\mathrm{nq}$ & $\mathrm{nq}$ & 68,4 & 70,8 & $\mathrm{nq}$ & $\mathrm{nq}$ & $\mathrm{nq}$ & $\mathrm{nq}$ \\
\hline $\mathrm{CdSO}_{4}(\mathrm{aq})$ & $\mathrm{nq}$ & $\mathrm{nq}$ & $\mathrm{nq}$ & $\mathrm{nq}$ & 28,7 & 26,9 & $\mathrm{nq}$ & $\mathrm{nq}$ & $\mathrm{nq}$ & $\mathrm{nq}$ \\
\hline $\mathrm{Cd}\left(\mathrm{SO}_{4}\right)_{2}{ }^{2-}$ & $\mathrm{nq}$ & $\mathrm{nq}$ & $\mathrm{nq}$ & $\mathrm{nq}$ & 2,9 & 2,3 & $\mathrm{nq}$ & $\mathrm{nq}$ & $\mathrm{nq}$ & $\mathrm{nq}$ \\
\hline $\mathrm{Sn}(\mathrm{OH})_{2}$ & 0,0 & $\mathrm{nq}$ & $\mathrm{nq}$ & $\mathrm{nq}$ & 0,0 & $\mathrm{nq}$ & $\mathrm{nq}$ & 0,0 & 10,7 & 0,0 \\
\hline $\mathbf{S n}^{2+}$ & 0,0 & $\mathrm{nq}$ & $\mathrm{nq}$ & $\mathrm{nq}$ & 0,0 & $\mathrm{nq}$ & $\mathrm{nq}$ & 0,0 & 61,0 & 0,0 \\
\hline $\mathrm{SnOH}^{+}$ & 0,0 & $\mathrm{nq}$ & $\mathrm{nq}$ & $\mathrm{nq}$ & 0,0 & $\mathrm{nq}$ & $\mathrm{nq}$ & 0,0 & 28,3 & 0,0 \\
\hline $\mathbf{S n}^{4+}$ & 0,0 & $\mathrm{nq}$ & $\mathrm{nq}$ & $\mathrm{nq}$ & 0,0 & $\mathrm{nq}$ & $\mathrm{nq}$ & 0,0 & 0,0 & 100,0 \\
\hline $\operatorname{Sn}(\mathrm{OH})_{6}^{-}$ & 99,4 & $\mathrm{nq}$ & $\mathrm{nq}$ & $\mathrm{nq}$ & 99,4 & $\mathrm{nq}$ & $\mathrm{nq}$ & 99,4 & 0,0 & 0,0 \\
\hline $\mathrm{SbO}_{3}^{-}$ & 97,8 & $\mathrm{nq}$ & $\mathrm{nq}$ & $\mathrm{nq}$ & $\mathrm{nq}$ & 99,9 & 99,9 & $\mathrm{nq}$ & $\mathrm{nq}$ & $\mathrm{nq}$ \\
\hline $\mathrm{Sb}(\mathrm{OH})_{3}$ & 1,1 & $\mathrm{nq}$ & $\mathrm{nq}$ & $\mathrm{nq}$ & $\mathrm{nq}$ & 0,0 & 0,0 & $\mathrm{nq}$ & $\mathrm{nq}$ & $\mathrm{nq}$ \\
\hline $\mathrm{HSbO}_{2}$ & 1,1 & $\mathrm{nq}$ & $\mathrm{nq}$ & $\mathrm{nq}$ & $\mathrm{nq}$ & 0,0 & 0,0 & $\mathrm{nq}$ & $\mathrm{nq}$ & $\mathrm{nq}$ \\
\hline $\mathbf{B a}^{2+}$ & 100,0 & 100,0 & 100,0 & 100,0 & 100,0 & 100,0 & 100,0 & 100,0 & 100,0 & 100,0 \\
\hline $\mathrm{Tl}^{+}$ & $\mathrm{nq}$ & $\mathrm{nq}$ & $\mathrm{nq}$ & $\mathrm{nq}$ & 92,0 & 93,0 & $\mathrm{nq}$ & $\mathrm{nq}$ & $\mathrm{nq}$ & $\mathrm{nq}$ \\
\hline $\mathrm{TISO}_{4}{ }^{-}$ & $\mathrm{nq}$ & $\mathrm{nq}$ & $\mathrm{nq}$ & $\mathrm{nq}$ & 8,0 & 7,0 & $\mathrm{nq}$ & $\mathrm{nq}$ & $\mathrm{nq}$ & $\mathrm{nq}$ \\
\hline $\mathbf{P b}^{2+}$ & 45,3 & 51,6 & 45,3 & 49,0 & 47,8 & 51,6 & 49,9 & $\mathrm{nq}$ & 37,5 & 38,5 \\
\hline $\mathrm{PbOH}^{+}$ & 0,0 & 2,5 & 2,1 & 1,8 & 5,9 & 3,3 & 3,9 & $\mathrm{nq}$ & 0,0 & 0,0 \\
\hline $\mathrm{Pb}(\mathrm{OH})_{2}(\mathbf{a q})$ & 0,0 & 0,0 & 0,0 & 0,0 & 0,0 & 0,0 & 0,0 & $\mathrm{nq}$ & 54,4 & 53,8 \\
\hline $\mathrm{PbSO}_{4}(\mathrm{aq})$ & 50,1 & 44,1 & 49,7 & 46,7 & 44,4 & 43,5 & 44,3 & $\mathrm{nq}$ & 8,1 & 7,7 \\
\hline $\mathrm{Pb}\left(\mathrm{SO}_{4}\right)_{2}{ }^{2-}$ & 3,9 & 1,8 & 2,9 & 2,6 & 1,9 & 1,6 & 1,8 & $\mathrm{nq}$ & 0,0 & 0,0 \\
\hline $\mathrm{UO}_{2}^{+}$ & 28,8 & 0,0 & 0,0 & 14,7 & 0,0 & 0,0 & 0,0 & $\mathrm{nq}$ & 77,3 & 0,0 \\
\hline $\mathrm{U}(\mathrm{OH})_{3}{ }^{+}$ & 0,0 & 0,0 & 0,0 & 0,0 & 0,0 & 0,0 & 0,0 & $\mathrm{nq}$ & 1,3 & 0,0 \\
\hline $\mathrm{USO}_{4}{ }^{2+}$ & 0,0 & 0,0 & 0,0 & 0,0 & 0,0 & 0,0 & 0,0 & $\mathrm{nq}$ & 1,0 & 0,0 \\
\hline $\mathrm{U}\left(\mathrm{SO}_{4}\right)_{2}(\mathbf{a q})$ & 0,0 & 0,0 & 0,0 & 0,0 & 0,0 & 0,0 & 0,0 & $\mathrm{nq}$ & 19,1 & 0,0 \\
\hline $\mathrm{UO}_{2}{ }^{2+}$ & 23,4 & 26,4 & 25,0 & 24,8 & 14,1 & 19,7 & 20,2 & $\mathrm{nq}$ & 0,0 & 31,0 \\
\hline $\mathrm{UO}_{2} \mathrm{OH}^{+}$ & 14,2 & 46,5 & 42,0 & 32,0 & 62,5 & 45,1 & 56,3 & $\mathrm{nq}$ & 0,0 & 0,0 \\
\hline$\left(\mathrm{UO}_{2}\right)_{2}(\mathrm{OH})_{2}{ }^{2+}$ & 0,0 & 0,0 & 0,0 & 0,0 & 0,0 & 2,2 & 0,0 & $\mathrm{nq}$ & 0,0 & 0,0 \\
\hline$\left(\mathbf{U O}_{2}\right)_{3}(\mathbf{O H})_{5}{ }^{+}$ & 0,0 & 1,1 & 0,0 & 0,0 & 7,5 & 14,6 & 2,4 & $\mathrm{nq}$ & 0,0 & 0,0 \\
\hline $\mathrm{UO}_{2} \mathrm{SO}_{4}(\mathrm{aq})$ & 24,2 & 21,1 & 25,6 & 22,1 & 12,3 & 15,6 & 16,8 & $\mathrm{nq}$ & 0,0 & 40,5 \\
\hline $\mathrm{UO}_{2}\left(\mathrm{SO}_{4}\right)_{2}{ }^{2-}$ & 9,1 & 4,1 & 7,3 & 6,0 & 2,6 & 2,7 & 3,4 & $\mathrm{nq}$ & 0,0 & 28,4 \\
\hline $\mathrm{SO}_{4}{ }^{2-}$ & 60,0 & 67,7 & 67,8 & 61,9 & 69,1 & 70,7 & 68,3 & 62,7 & 54,2 & 53,0 \\
\hline $\mathrm{HSO}_{4}^{-}$ & 0,0 & 0,0 & 0,0 & 0,0 & 0,0 & 0,0 & 0,0 & 0,0 & 0,0 & 1,3 \\
\hline $\mathrm{FeSO}_{4}(\mathrm{aq})$ & 1,5 & 0,0 & 0,0 & 0,0 & 0,0 & 0,0 & 0,0 & 0,0 & 6,5 & 10,1 \\
\hline $\mathrm{MgSO}_{4}(\mathbf{a q})$ & 13,9 & 6,6 & 7,5 & 9,2 & 6,9 & 6,8 & 7,1 & 3,2 & 26,8 & 26,5 \\
\hline CaSO4 (aq) & 23,7 & 25,1 & 24,0 & 28,2 & 23,5 & 22,1 & 24,2 & 33,5 & 11,1 & 8,5 \\
\hline
\end{tabular}


Tab. A.25. (Forts.): Ergebnisse der Speziationsberechnung mit MINTEQA2.

\begin{tabular}{|c|c|c|c|c|c|c|c|c|}
\hline & $\begin{array}{l}\text { CML 39,5m } \\
\text { Jan 00 } \\
\text { WP KB }\end{array}$ & \begin{tabular}{|l|} 
CML 43,5m \\
Jan 00 \\
WP KB
\end{tabular} & $\begin{array}{l}\text { RCO3 } \\
\text { Jan 00 } \\
\text { WP KB }\end{array}$ & $\begin{array}{l}\text { RCO4 } \\
\text { Jan 00 } \\
\text { WP KB }\end{array}$ & $\begin{array}{l}\text { RCO12 } \\
\text { Jan 00 } \\
\text { WP KB }\end{array}$ & $\begin{array}{l}\text { RCO15 } \\
\text { Jan 00 } \\
\text { WP KB }\end{array}$ & $\begin{array}{l}\text { RCO17 } \\
\text { Jan 00 } \\
\text { WP KB }\end{array}$ & $\begin{array}{l}\text { RCO18 } \\
\text { Jan 00 } \\
\text { WP KB }\end{array}$ \\
\hline Spezies & $\%$ & $\%$ & $\%$ & $\%$ & $\%$ & $\%$ & $\%$ & $\%$ \\
\hline $\mathbf{L i}^{+}$ & 98,2 & 98,5 & 97,0 & 97,3 & 94,1 & 98,4 & 98,0 & 98,2 \\
\hline $\mathrm{LiSO}_{4}^{-}$ & 1,8 & 1,5 & 3,0 & 2,7 & 5,9 & 1,6 & 2,0 & 1,8 \\
\hline $\mathrm{Be}^{2+}$ & $\mathrm{nq}$ & $\mathrm{nq}$ & 73,1 & $\mathrm{nq}$ & 4,3 & $\mathrm{nq}$ & $\mathrm{nq}$ & $\mathrm{nq}$ \\
\hline $\mathrm{BeOH}^{+}$ & $\mathrm{nq}$ & $\mathrm{nq}$ & 0,0 & $\mathrm{nq}$ & 87,8 & $\mathrm{nq}$ & $\mathrm{nq}$ & $\mathrm{nq}$ \\
\hline $\mathrm{Be}(\mathrm{OH})_{2}$ & $\mathrm{nq}$ & $\mathrm{nq}$ & 0,0 & $\mathrm{nq}$ & 4,4 & $\mathrm{nq}$ & $\mathrm{nq}$ & $\mathrm{nq}$ \\
\hline $\mathrm{BeSO}_{4}(\mathrm{aq})$ & $\mathrm{nq}$ & $\mathrm{nq}$ & 25,4 & $\mathrm{nq}$ & 3,1 & $\mathrm{nq}$ & $\mathrm{nq}$ & $\mathrm{nq}$ \\
\hline $\mathrm{Be}\left(\mathrm{SO}_{4}\right)_{2}{ }^{2-}$ & $\mathrm{nq}$ & $\mathrm{nq}$ & 1,5 & $\mathrm{nq}$ & 0,0 & $\mathrm{nq}$ & $\mathrm{nq}$ & $\mathrm{nq}$ \\
\hline $\mathrm{Na}^{+}$ & 97,8 & 98,1 & 96,3 & 96,7 & 92,9 & 98,1 & 97,5 & 97,8 \\
\hline $\mathrm{NaSO}_{4}^{-}$ & 2,2 & 1,9 & 3,7 & 3,3 & 7,1 & 1,9 & 2,5 & 2,2 \\
\hline $\mathrm{Mg}^{2+}$ & 75,9 & 77,3 & 67,6 & 69,0 & 50,3 & 76,9 & 72,4 & 74,8 \\
\hline $\mathrm{MgSO}_{4}$ (aq) & 24,1 & 22,7 & 32,4 & 31,0 & 49,7 & 23,1 & 27,6 & 25,2 \\
\hline $\mathbf{A l}^{3+}$ & $\mathrm{nq}$ & 0,0 & 11,6 & 0,0 & 9,7 & $\mathrm{nq}$ & 0,0 & $\mathrm{nq}$ \\
\hline $\mathrm{AlOH}^{2+}$ & $\mathrm{nq}$ & 0,0 & 0,0 & 0,0 & 0,0 & $\mathrm{nq}$ & 3,1 & $\mathrm{nq}$ \\
\hline $\mathrm{Al}(\mathrm{OH})_{2}{ }^{+}$ & $\mathrm{nq}$ & 9,6 & 0,0 & 26,4 & 0,0 & $\mathrm{nq}$ & 49,1 & $\mathrm{nq}$ \\
\hline $\mathbf{A l}(\mathbf{O H})_{3}(\mathbf{a q})$ & $\mathrm{nq}$ & 15,9 & 0,0 & 20,8 & 15,2 & $\mathrm{nq}$ & 20,2 & $\mathrm{nq}$ \\
\hline $\mathrm{Al}(\mathrm{OH})_{4}{ }^{-}$ & $\mathrm{nq}$ & 74,3 & 0,0 & 51,2 & 74,8 & $\mathrm{nq}$ & 23,9 & $\mathrm{nq}$ \\
\hline $\mathrm{AlSO}_{4}{ }^{+}$ & $\mathrm{nq}$ & 0,0 & 81,4 & 0,0 & 0,0 & $\mathrm{nq}$ & 3,0 & $\mathrm{nq}$ \\
\hline $\mathrm{Al}\left(\mathrm{SO}_{4}\right)_{2}^{-}$ & $\mathrm{nq}$ & 0,0 & 6,9 & 0,0 & 0,0 & $\mathrm{nq}$ & 0,0 & $\mathrm{nq}$ \\
\hline $\mathbf{K}^{+}$ & 97,4 & 97,8 & 95,6 & 96,0 & 91,6 & 97,7 & 97,0 & 97,4 \\
\hline $\mathrm{KSO}_{4}^{-}$ & 2,6 & 2,2 & 4,4 & 4,0 & 8,4 & 2,3 & 3,0 & 2,6 \\
\hline $\mathbf{C a}^{2+}$ & 71,6 & 73,2 & 62,6 & 64,1 & 44,7 & 72,8 & 67,8 & 70,4 \\
\hline $\mathrm{CaSO}_{4}(\mathrm{aq})$ & 28,4 & 26,8 & 37,4 & 35,9 & 55,3 & 27,2 & 32,2 & 29,6 \\
\hline $\mathrm{VO}^{2+}$ & 0,0 & 0,0 & 59,8 & 0,0 & 7,3 & 0,0 & 0,0 & 0,0 \\
\hline $\mathrm{V}(\mathrm{OH})_{3}{ }^{+}$ & 0,0 & 0,0 & 0,0 & 0,0 & 73,5 & 0,0 & 0,0 & 0,0 \\
\hline $\mathrm{H}_{2} \mathrm{~V}_{2} \mathrm{O}_{4}{ }^{2+}$ & 0,0 & 0,0 & 0,0 & 0,0 & 10,1 & 0,0 & 0,0 & 0,0 \\
\hline $\mathrm{VOSO}_{4}(\mathrm{aq})$ & 0,0 & 0,0 & 40,1 & 13,5 & 9,2 & 18,8 & 12,7 & 2,2 \\
\hline $\mathrm{V}(\mathrm{OH})_{3}(\mathrm{aq})$ & 0,0 & 2,0 & 0,0 & 0,0 & 0,0 & 0,0 & 0,0 & 0,0 \\
\hline $\mathrm{VO}_{2}^{+}$ & 0,0 & 0,0 & 0,0 & 2,1 & 0,0 & 2,8 & 6,9 & 6,5 \\
\hline $\mathrm{HVO}_{4}{ }^{2-}$ & 10,4 & 4,1 & 0,0 & 84,3 & 0,0 & 78,4 & 86,6 & 91,3 \\
\hline $\mathrm{H}_{2} \mathrm{VO}_{4}^{-}$ & 89,6 & 93,8 & 0,0 & 0,0 & 0,0 & 0,0 & 0,0 & 0,0 \\
\hline $\mathrm{VO}_{2} \mathrm{SO}_{4}^{-}$ & 0,0 & 0,0 & 0,0 & 0,0 & 0,0 & 0,0 & 0,0 & 0,0 \\
\hline $\mathrm{H}_{3} \mathrm{VO}_{4}(\mathrm{aq})$ & 0,0 & 0,0 & 0,0 & 0,0 & 0,0 & 0,0 & 0,0 & 0,0 \\
\hline $\mathrm{H}_{3} \mathrm{~V}_{2} \mathrm{O}_{7}^{-}$ & 0,0 & $\mathrm{nq}$ & 0,0 & 0,0 & 0,0 & 0,0 & 0,0 & 0,0 \\
\hline $\mathbf{C r}^{3+}$ & 0,0 & $\mathrm{nq}$ & 32,4 & 56,6 & 65,6 & 70,9 & 43,1 & 80,1 \\
\hline $\mathrm{Cr}(\mathrm{OH})_{2}{ }^{+}$ & 82,9 & $\mathrm{nq}$ & 0,0 & 31,1 & 18,1 & 21,9 & 42,8 & 12,5 \\
\hline $\mathrm{CrOH}^{2+}$ & 8,5 & $\mathrm{nq}$ & 0,0 & 0,0 & 0,0 & 0,0 & 0,0 & 0,0 \\
\hline $\mathrm{CrSO}_{4}{ }^{+}$ & 0,0 & $\mathrm{nq}$ & 66,9 & 0,0 & 1,9 & 1,7 & 0,0 & 3,9 \\
\hline $\mathrm{Cr}(\mathrm{OH})_{3}(\mathrm{aq})$ & 6,3 & $\mathrm{nq}$ & 0,0 & 11,1 & 14,2 & 5,2 & 12,9 & 3,3 \\
\hline $\mathrm{CrOHSO}_{4}(\mathrm{aq})$ & 2,1 & $\mathrm{nq}$ & 0,0 & 0,0 & 0,0 & 0,0 & 0,0 & 0,0 \\
\hline $\mathrm{CrO}_{2}^{-}$ & 0,0 & $\mathrm{nq}$ & 0,0 & 0,0 & 0,0 & 0,0 & 0,0 & 0,0 \\
\hline $\mathrm{CrO}_{4}{ }^{2-}$ & 0,0 & $\mathrm{nq}$ & 0,0 & 0,0 & 0,0 & 0,0 & 0,0 & 0,0 \\
\hline $\mathrm{HCrO}_{4}^{-}$ & 0,0 & $\mathrm{nq}$ & 0,0 & 0,0 & 0,0 & 0,0 & 0,0 & 0,0 \\
\hline $\mathrm{Mn}^{2+}$ & 76,7 & 78,1 & 68,6 & 64,1 & 51,3 & 77,7 & 73,3 & 75,6 \\
\hline $\mathrm{MnSO}_{4}(\mathrm{aq})$ & 23,3 & 21,9 & 31,4 & 35,9 & 48,7 & 22,3 & 26,7 & 24,4 \\
\hline $\mathrm{Fe}^{2+}$ & 27,2 & 45,4 & 61,1 & 62,6 & $\mathrm{nq}$ & 62,1 & $\mathrm{nq}$ & 68,9 \\
\hline $\mathrm{FeSO}_{4}(\mathrm{aq})$ & 11,5 & 17,7 & 38,9 & 37,4 & $\mathrm{nq}$ & 24,8 & $\mathrm{nq}$ & 30,9 \\
\hline $\mathrm{FeOH}^{2+}$ & 55,0 & 35,3 & 0,0 & 0,0 & $\mathrm{nq}$ & 12,6 & $\mathrm{nq}$ & 0,0 \\
\hline $\mathrm{Fe}(\mathrm{OH})_{2}{ }^{+}$ & 5,9 & 1,5 & 0,0 & 0,0 & $\mathrm{nq}$ & 0,4 & $\mathrm{nq}$ & 0,0 \\
\hline $\mathrm{Fe}(\mathrm{OH})_{3}$ & 0,0 & 0,0 & 0,0 & 67,0 & $\mathrm{nq}$ & 75,2 & $\mathrm{nq}$ & 0,0 \\
\hline $\mathrm{Co}^{2+}$ & $\mathrm{nq}$ & $\mathrm{nq}$ & $\mathrm{nq}$ & 33,0 & $\mathrm{nq}$ & 24,7 & $\mathrm{nq}$ & $\mathrm{nq}$ \\
\hline $\mathrm{CoSO}_{4}(\mathrm{aq})$ & $\mathrm{nq}$ & $\mathrm{nq}$ & $\mathrm{nq}$ & 66,9 & $\mathrm{nq}$ & 75,2 & $\mathrm{nq}$ & $\mathrm{nq}$ \\
\hline $\mathrm{Ni}^{2+}$ & $\mathrm{nq}$ & $\mathrm{nq}$ & $\mathrm{nq}$ & 33,0 & $\mathrm{nq}$ & 22,3 & $\mathrm{nq}$ & $\mathrm{nq}$ \\
\hline $\mathrm{NiSO}_{4}(\mathrm{aq})$ & $\mathrm{nq}$ & $\mathrm{nq}$ & $\mathrm{nq}$ & 0,0 & $\mathrm{nq}$ & 0,0 & $\mathrm{nq}$ & $\mathrm{nq}$ \\
\hline $\mathrm{Cu}^{+}$ & 4,2 & 2,3 & 0,0 & 0,0 & 2,9 & 5,3 & 0,0 & 4,2 \\
\hline $\mathrm{Cu}^{2+}$ & 54,8 & 64,8 & 62,9 & 61,8 & 41,4 & 63,8 & 63,1 & 58,2 \\
\hline $\mathrm{Cu}(\mathrm{OH})^{+}$ & 18,6 & 9,3 & 0,0 & 3,9 & 5,2 & 7,2 & 2,2 & 12,9 \\
\hline $\mathrm{Cu}(\mathrm{OH})_{2}$ & 1,0 & 0,0 & 0,0 & 0,0 & 0,0 & 0,0 & 0,0 & 0,0 \\
\hline $\mathrm{CuSO}_{4}$ (aq) & 21,5 & 23,4 & 37,1 & 34,3 & 50,5 & 23,6 & 29,7 & 24,2 \\
\hline
\end{tabular}


Tab. A.25. (Forts.): Ergebnisse der Speziationsberechnung mit MINTEQA2.

\begin{tabular}{|c|c|c|c|c|c|c|c|c|}
\hline & $\begin{array}{l}\text { CML 39,5m } \\
\text { Jan 00 } \\
\text { WP KB } \\
\end{array}$ & $\begin{array}{l}\text { CML 43,5m } \\
\text { Jan 00 } \\
\text { WP KB } \\
\end{array}$ & $\begin{array}{l}\text { RCO3 } \\
\text { Jan 00 } \\
\text { WP KB }\end{array}$ & $\begin{array}{l}\text { RCO4 } \\
\text { Jan 00 } \\
\text { WP KB }\end{array}$ & $\begin{array}{l}\text { RCO12 } \\
\text { Jan } 00 \\
\text { WP KB }\end{array}$ & $\begin{array}{l}\text { RCO15 } \\
\text { Jan 00 } \\
\text { WP KB }\end{array}$ & $\begin{array}{l}\text { RCO17 } \\
\text { Jan 00 } \\
\text { WP KB }\end{array}$ & $\begin{array}{l}\text { RCO18 } \\
\text { Jan 00 } \\
\text { WP KB }\end{array}$ \\
\hline Spezies & $\%$ & $\%$ & $\%$ & $\%$ & $\%$ & $\%$ & $\%$ & $\%$ \\
\hline $\mathbf{Z n}^{2+}$ & 70,3 & 72,5 & 59,9 & 61,7 & 38,8 & 72,0 & 66,6 & 69,3 \\
\hline $\mathrm{ZnOH}^{+}$ & 0,0 & 0,0 & 0,0 & 33,3 & 46,0 & 25,9 & 30,4 & 28,0 \\
\hline $\mathrm{ZnSO}_{4}$ (aq) & 26,7 & 25,5 & 34,3 & 4,9 & 0,0 & 1,9 & 2,9 & 2,3 \\
\hline $\mathrm{Zn}\left(\mathrm{SO}_{4}\right)_{2}{ }^{2-}$ & 2,3 & 1,7 & 5,8 & 0,0 & 0,0 & 0,0 & 0,0 & 0,0 \\
\hline $\mathrm{HAsO}_{4}{ }^{2-}$ & 83,2 & $\mathrm{nq}$ & 0,0 & 51,5 & 67,9 & 60,0 & 32,5 & $\mathrm{nq}$ \\
\hline $\mathrm{H}_{2} \mathrm{AsO}_{4}{ }^{-}$ & 16,8 & $\mathrm{nq}$ & 71,1 & 48,5 & 32,0 & 40,0 & 67,5 & $\mathrm{nq}$ \\
\hline $\mathrm{H}_{3} \mathrm{AsO}_{4}$ & 0,0 & $\mathrm{nq}$ & 28,9 & 0,0 & 0,0 & 0,0 & 0,0 & $\mathrm{nq}$ \\
\hline $\mathrm{H}_{3} \mathrm{AsO}_{3}$ & 0,0 & $\mathrm{nq}$ & 0,0 & 0,0 & 0,0 & 0,0 & 0,0 & $\mathrm{nq}$ \\
\hline $\mathbf{S r}^{2+}$ & 74,5 & 75,9 & 65,9 & 67,3 & 48,3 & 75,5 & 70,8 & 73,3 \\
\hline $\mathrm{SrSO}_{4}(\mathrm{aq})$ & 25,5 & 24,1 & 34,1 & 32,7 & 51,7 & 24,5 & 29,2 & 26,7 \\
\hline $\mathrm{MoO}_{4}{ }^{2-}$ & 100,0 & 99,9 & 0,0 & 99,8 & 99,9 & 99,9 & 99,6 & 99,9 \\
\hline $\mathrm{HMoO}_{4}^{-}$ & 0,0 & 0,0 & 3,9 & 0,0 & 0,0 & 0,0 & 0,0 & 0,0 \\
\hline $\mathrm{H}_{2} \mathrm{MoO}_{4}(\mathrm{aq})$ & 0,0 & 0,0 & 96,0 & 0,0 & 0,0 & 0,0 & 0,0 & 0,0 \\
\hline $\mathbf{C d}^{2+}$ & 68,7 & 70,9 & 56,7 & 58,8 & $\mathrm{nq}$ & $\mathrm{nq}$ & $\mathrm{nq}$ & $\mathrm{nq}$ \\
\hline $\mathrm{CdSO}_{4}$ (aq) & 27,5 & 26,3 & 34,2 & 33,4 & $\mathrm{nq}$ & $\mathrm{nq}$ & $\mathrm{nq}$ & $\mathrm{nq}$ \\
\hline $\mathrm{Cd}\left(\mathrm{SO}_{4}\right)_{2}{ }^{2-}$ & 3,7 & 2,8 & 9,1 & 7,8 & $\mathrm{nq}$ & $\mathrm{nq}$ & $\mathrm{nq}$ & $\mathrm{nq}$ \\
\hline $\mathrm{Sn}(\mathrm{OH})_{2}$ & 0,0 & 0,0 & 0,0 & 0,0 & 0,0 & 0,0 & 0,0 & 0,0 \\
\hline $\mathbf{S n}^{2+}$ & 0,0 & 0,0 & 0,0 & 0,0 & 0,0 & 0,0 & 0,0 & 0,0 \\
\hline $\mathrm{SnOH}^{+}$ & 0,0 & 0,0 & 0,0 & 0,0 & 0,0 & 0,0 & 0,0 & 0,0 \\
\hline $\mathbf{S n}^{4+}$ & 0,0 & 0,0 & 100,0 & 0,0 & 0,0 & 0,0 & 0,0 & 0,0 \\
\hline $\mathrm{Sn}(\mathrm{OH})_{6}^{-}$ & 99,4 & 99,4 & 0,0 & 99,4 & 99,4 & 99,4 & 99,4 & 99,4 \\
\hline $\mathrm{SbO}_{3}^{-}$ & $\mathrm{nq}$ & 99,9 & $\mathrm{nq}$ & $\mathrm{nq}$ & $\mathrm{nq}$ & $\mathrm{nq}$ & 99,9 & $\mathrm{nq}$ \\
\hline $\mathrm{Sb}(\mathrm{OH})_{3}$ & $\mathrm{nq}$ & 0,0 & $\mathrm{nq}$ & $\mathrm{nq}$ & $\mathrm{nq}$ & $\mathrm{nq}$ & 0,0 & $\mathrm{nq}$ \\
\hline $\mathrm{Sb}(\mathrm{OH})_{2}{ }^{+}$ & $\mathrm{nq}$ & 0,0 & $\mathrm{nq}$ & $\mathrm{nq}$ & $\mathrm{nq}$ & $\mathrm{nq}$ & 0,0 & $\mathrm{nq}$ \\
\hline $\mathrm{HSbO}_{2}$ & $\mathrm{nq}$ & 0,0 & $\mathrm{nq}$ & $\mathrm{nq}$ & $\mathrm{nq}$ & $\mathrm{nq}$ & 0,0 & $\mathrm{nq}$ \\
\hline $\mathrm{SbO}^{+}$ & $\mathrm{nq}$ & 0,0 & $\mathrm{nq}$ & $\mathrm{nq}$ & $\mathrm{nq}$ & $\mathrm{nq}$ & 0,0 & $\mathrm{nq}$ \\
\hline $\mathbf{B a}^{2+}$ & 100,0 & 100,0 & 100,0 & 100,0 & 100,0 & 100,0 & 100,0 & 100,0 \\
\hline $\mathbf{T I}^{+}$ & $\mathrm{nq}$ & 92,3 & $\mathrm{nq}$ & 86,8 & $\mathrm{nq}$ & $\mathrm{nq}$ & 89,9 & 91,0 \\
\hline TISO $_{4}^{-}$ & $\mathrm{nq}$ & 7,7 & $\mathrm{nq}$ & 13,2 & $\mathrm{nq}$ & $\mathrm{nq}$ & 10,1 & 9,0 \\
\hline $\mathbf{P b}^{2+}$ & 43,7 & 49,9 & 40,2 & 40,9 & 22,2 & 50,1 & 46,1 & $\mathrm{nq}$ \\
\hline $\mathrm{PbOH}^{+}$ & 15,1 & 7,3 & & 2,6 & 2,8 & 5,7 & 1,6 & $\mathrm{nq}$ \\
\hline $\mathrm{Pb}(\mathrm{OH})_{2}(\mathrm{aq})$ & 38,9 & 41,0 & 53,8 & 51,5 & 61,6 & 42,1 & 49,2 & $\mathrm{nq}$ \\
\hline $\mathrm{PbSO}_{4}$ (aq) & 2,2 & 1,8 & 6,0 & 5,1 & 13,3 & 2,0 & 3,1 & $\mathrm{nq}$ \\
\hline $\mathrm{Pb}\left(\mathrm{SO}_{4}\right)_{2}{ }^{2-}$ & 0,0 & 0,0 & 0,0 & 0,0 & 0,0 & 0,0 & 0,0 & $\mathrm{nq}$ \\
\hline $\mathrm{UO}_{2}{ }^{+}$ & 0,0 & 0,6 & $\mathrm{nq}$ & 0,0 & 0,0 & 0,0 & 0,0 & 0,0 \\
\hline $\mathrm{U}(\mathrm{OH})_{2}{ }^{2+}$ & 0,0 & 0,0 & $\mathrm{nq}$ & 0,0 & 0,0 & 0,0 & 0,0 & 0,0 \\
\hline $\mathrm{U}(\mathrm{OH})_{3}{ }^{+}$ & 0,0 & 0,0 & $\mathrm{nq}$ & 0,0 & 0,0 & 0,0 & 0,0 & 0,0 \\
\hline $\mathrm{U}(\mathrm{OH})_{4}(\mathrm{aq})$ & 0,0 & 0,0 & $\mathrm{nq}$ & 0,0 & 0,0 & 0,0 & 0,0 & 0,0 \\
\hline $\mathrm{USO}_{4}{ }^{2+}$ & 0,0 & 0,0 & $\mathrm{nq}$ & 0,0 & 0,0 & 0,0 & 0,0 & 0,0 \\
\hline $\mathrm{U}\left(\mathrm{SO}_{4}\right)_{2}(\mathrm{aq})$ & 0,0 & 0,0 & $\mathrm{nq}$ & 0,0 & 0,0 & 0,0 & 0,0 & 0,0 \\
\hline $\mathrm{UO}_{2}{ }^{2+}$ & 0,0 & 12,4 & $\mathrm{nq}$ & 18,2 & 6,8 & 15,3 & 26,9 & 0,0 \\
\hline $\mathrm{UO}_{2} \mathbf{O H}^{+}$ & 12,2 & 65,1 & $\mathrm{nq}$ & 42,2 & 31,4 & 63,1 & 34,5 & 0,0 \\
\hline$\left(\mathrm{UO}_{2}\right)_{2}(\mathrm{OH})_{2}{ }^{2+}$ & 0,0 & 0,0 & $\mathrm{nq}$ & 1,4 & 1,2 & & 1,3 & 0,0 \\
\hline$\left(\mathrm{UO}_{2}\right)_{3}(\mathrm{OH})_{5}{ }^{+}$ & 85,3 & 9,2 & $\mathrm{nq}$ & 6,4 & 24,5 & 5,8 & 2,2 & 0,0 \\
\hline $\mathrm{UO}_{2} \mathrm{SO}_{4}(\mathrm{aq})$ & 0,0 & 9,5 & $\mathrm{nq}$ & 21,5 & 17,6 & 12,0 & 26,8 & 0,0 \\
\hline $\mathrm{UO}_{2}\left(\mathrm{SO}_{4}\right)_{2}{ }^{2-}$ & 0,0 & 2,1 & $\mathrm{nq}$ & 10,3 & 18,6 & 2,8 & 8,2 & 0,0 \\
\hline $\mathrm{SO}_{4}{ }^{2-}$ & 59,4 & 62,8 & 55,3 & 59,6 & 90,9 & 61,5 & 63,5 & 62,1 \\
\hline $\mathrm{HSO}_{4}{ }^{-}$ & 0,0 & 0,0 & 7,3 & 0,0 & 0,0 & 0,0 & 0,0 & 0,0 \\
\hline $\mathrm{AlSO}_{4}{ }^{+}$ & 0,0 & 0,0 & 0,0 & 0,0 & 0,0 & 0,0 & 0,0 & 0,0 \\
\hline $\mathrm{Al}\left(\mathrm{SO}_{4}\right)_{2}{ }^{-}$ & 0,0 & 0,0 & 0,0 & 0,0 & 0,0 & 0,0 & 0,0 & 0,0 \\
\hline $\mathrm{FeSO}_{4}(\mathrm{aq})$ & 0,0 & 0,0 & 6,2 & 0,0 & 0,0 & 0,0 & 0,0 & 0,0 \\
\hline $\mathrm{MgSO}_{4}(\mathrm{aq})$ & 16,2 & 13,2 & 15,1 & 19,2 & 3,5 & 11,4 & 9,1 & 9,7 \\
\hline CaSO4 (aq) & 23,7 & 23,4 & 15,2 & 20,4 & 5,4 & 26,4 & 26,8 & 27,7 \\
\hline
\end{tabular}


Tab. A.25. (Forts.): Ergebnisse der Speziationsberechnung mit MINTEQA2.

\begin{tabular}{|c|c|c|c|c|c|c|c|}
\hline & $\begin{array}{l}\text { Lausitz 5,00 m } \\
\text { PL KB }\end{array}$ & $\begin{array}{l}\text { Lausitz 5,21 m } \\
\text { PL KB }\end{array}$ & \begin{tabular}{|l|} 
Lausitz 5,30 m \\
PL KB
\end{tabular} & \begin{tabular}{|l|} 
Lausitz 5,50 m \\
PL KB
\end{tabular} & $\begin{array}{l}\text { Lausitz 5,60 m } \\
\text { PL KB }\end{array}$ & $\begin{array}{l}\text { Lausitz 5,75 m } \\
\text { PL KB }\end{array}$ & $\begin{array}{l}\text { Lausitz 5,93 m } \\
\text { PL KB }\end{array}$ \\
\hline Spezies & $\%$ & $\%$ & $\%$ & $\%$ & $\%$ & $\%$ & $\%$ \\
\hline $\mathbf{L i}^{+}$ & 97,2 & 97,4 & 97,2 & 97,1 & 97,0 & 97,1 & 97,0 \\
\hline $\mathrm{LiSO}_{4}{ }^{-}$ & 2,8 & 2,6 & 2,8 & 2,9 & 3,0 & 2,9 & 3,0 \\
\hline $\mathrm{Be}^{2+}$ & 73,5 & 70,9 & 73,3 & 72,4 & 72,3 & 73,0 & 71,8 \\
\hline $\mathrm{BeOH}^{+}$ & 0,0 & 0,0 & 0,0 & 0,0 & 0,0 & 0,0 & 0,0 \\
\hline $\mathrm{Be}(\mathrm{OH})_{2}$ & 0,0 & 0,0 & 0,0 & 0,0 & 0,0 & 0,0 & 0,0 \\
\hline $\mathrm{BeSO}_{4}$ (aq) & 24,8 & 27,6 & 25,0 & 25,8 & 25,9 & 25,4 & 26,3 \\
\hline $\mathrm{Be}\left(\mathrm{SO}_{4}\right)_{2}{ }^{2-}$ & 1,2 & 1,1 & 1,2 & 1,3 & 1,4 & 1,3 & 1,4 \\
\hline $\mathrm{Na}^{+}$ & 96,6 & 96,8 & 96,6 & 96,5 & 96,3 & 96,5 & 96,3 \\
\hline $\mathrm{NaSO}_{4}{ }^{-}$ & 3,4 & 3,2 & 3,4 & 3,5 & 3,7 & 3,5 & 3,7 \\
\hline $\mathrm{Mg}^{2+}$ & 68,2 & 68,6 & 68,0 & 67,1 & 66,9 & 67,5 & 66,4 \\
\hline $\mathrm{MgSO}_{4}(\mathrm{aq})$ & 31,8 & 31,4 & 32,0 & 32,9 & 33,1 & 32,5 & 33,6 \\
\hline $\mathbf{A l ^ { 3 + }}$ & 11,4 & 10,2 & 11,2 & 10,9 & 11,0 & 11,1 & 10,6 \\
\hline $\mathrm{AlOH}^{2+}$ & 0,0 & 0,0 & 0,0 & 0,0 & 0,0 & 0,0 & 0,0 \\
\hline $\mathrm{Al}(\mathrm{OH})_{2}{ }^{+}$ & 0,0 & 0,0 & 0,0 & 0,0 & 0,0 & 0,0 & 0,0 \\
\hline $\mathrm{Al}(\mathrm{OH})_{3}(\mathrm{aq})$ & 0,0 & 0,0 & 0,0 & 0,0 & 0,0 & 0,0 & 0,0 \\
\hline $\mathrm{Al}(\mathrm{OH})_{4}^{-}$ & 0,0 & 0,0 & 0,0 & 0,0 & 0,0 & 0,0 & 0,0 \\
\hline $\mathrm{AlSO}_{4}{ }^{+}$ & 82,1 & 84,1 & 82,3 & 82,3 & 82,0 & 82,2 & 82,3 \\
\hline $\mathrm{Al}\left(\mathrm{SO}_{4}\right)_{2}{ }^{-}$ & 6,4 & 5,6 & 6,4 & 6,8 & 7,0 & 6,6 & 7,0 \\
\hline $\mathbf{K}^{+}$ & 96,0 & 95,8 & 96,0 & 95,8 & 95,6 & 95,8 & 95,6 \\
\hline $\mathrm{KSO}_{4}^{-}$ & 4,0 & 4,2 & 4,0 & 4,2 & 4,4 & 4,2 & 4,4 \\
\hline $\mathrm{Ca}^{2+}$ & 63,2 & 63,5 & 63,0 & 62,1 & 61,9 & 62,5 & 61,3 \\
\hline $\mathrm{CaSO}_{4}$ (aq) & 36,8 & 36,5 & 37,0 & 37,9 & 38,1 & 37,5 & 38,7 \\
\hline $\mathrm{VO}^{2+}$ & 60,4 & 58,2 & 60,1 & 59,2 & 59,0 & 59,7 & 58,4 \\
\hline $\mathrm{V}(\mathrm{OH})_{3}{ }^{+}$ & 0,0 & 0,0 & 0,0 & 0,0 & 0,0 & 0,0 & 0,0 \\
\hline $\mathrm{H}_{2} \mathrm{~V}_{2} \mathrm{O}_{4}{ }^{2+}$ & 0,0 & 0,0 & 0,0 & 0,0 & 0,0 & 0,0 & 0,0 \\
\hline $\mathrm{VOSO}_{4}(\mathrm{aq})$ & 39,4 & 40,3 & 39,7 & 40,6 & 40,9 & 40,2 & 41,4 \\
\hline $\mathrm{V}(\mathrm{OH})_{3}(\mathrm{aq})$ & 0,0 & 1,3 & 0,0 & 0,0 & 0,0 & 0,0 & 0,0 \\
\hline $\mathrm{Cr}^{3+}$ & 30,4 & 26,6 & 29,5 & 29,0 & 30,0 & 30,1 & 28,9 \\
\hline $\mathrm{Cr}(\mathrm{OH})_{2}{ }^{+}$ & 0,0 & 4,9 & 0,0 & 0,0 & 0,0 & 0,0 & 0,0 \\
\hline $\mathrm{CrOH}^{2+}$ & 3,9 & 0,0 & 4,8 & 4,7 & 3,0 & 3,1 & 3,7 \\
\hline $\mathrm{CrSO}_{4}{ }^{+}$ & 64,3 & 65,6 & 63,8 & 64,5 & 65,9 & 65,7 & 65,9 \\
\hline $\mathrm{Cr}(\mathrm{OH})_{3}(\mathrm{aq})$ & 0,0 & 0,0 & 0,0 & 0,0 & 0,0 & 0,0 & 0,0 \\
\hline $\mathrm{CrOHSO}_{4}(\mathrm{aq})$ & 1,4 & 2,9 & 1,8 & 1,8 & 1,2 & 1,2 & 1,5 \\
\hline $\mathrm{Mn}^{2+}$ & 69,2 & 69,1 & 68,9 & 68,0 & 67,9 & 68,5 & 67,4 \\
\hline $\mathrm{MnSO}_{4}(\mathrm{aq})$ & 30,8 & 30,9 & 31,1 & 32,0 & 32,1 & 31,5 & 32,6 \\
\hline $\mathbf{F e}^{2+}$ & 61,8 & 61,8 & 61,5 & 60,6 & 60,4 & 61,0 & 59,8 \\
\hline $\mathrm{FeSO}_{4}(\mathrm{aq})$ & 38,2 & 38,2 & 38,5 & 39,4 & 39,6 & 39,0 & 40,2 \\
\hline $\mathrm{FeOH}^{2+}$ & 0,0 & 0,0 & 0,0 & 0,0 & 0,0 & 0,0 & 0,0 \\
\hline $\mathrm{Fe}(\mathrm{OH})_{2}{ }^{+}$ & 0,0 & 0,0 & 0,0 & 0,0 & 0,0 & 0,0 & 0,0 \\
\hline $\mathrm{Fe}(\mathrm{OH})_{3}$ & 0,0 & 0,0 & 0,0 & 0,0 & 0,0 & 0,0 & 0,0 \\
\hline $\mathrm{Co}^{2+}$ & 66,3 & 66,6 & 66,0 & 65,1 & 64,9 & 65,6 & 64,4 \\
\hline $\mathrm{CoSO}_{4}$ (aq) & 33,7 & 33,4 & 34,0 & 34,9 & 35,1 & 34,4 & 35,6 \\
\hline $\mathrm{Ni}^{2+}$ & 66,2 & 66,6 & 65,9 & 65,0 & 64,8 & 65,5 & 64,3 \\
\hline $\mathrm{NiSO}_{4}(\mathrm{aq})$ & 33,8 & 33,4 & 34,0 & 34,9 & 35,1 & 34,5 & 35,7 \\
\hline $\mathrm{Cu}^{+}$ & 0,0 & 0,0 & 0,0 & 0,0 & 0,0 & 0,0 & 0,0 \\
\hline $\mathrm{Cu}^{2+}$ & 63,5 & 63,5 & 63,3 & 62,3 & 62,1 & 62,8 & 61,6 \\
\hline $\mathrm{Cu}(\mathrm{OH})^{+}$ & 0,0 & 0,0 & 0,0 & 0,0 & 0,0 & 0,0 & 0,0 \\
\hline $\mathrm{Cu}(\mathrm{OH})_{2}$ & 0,0 & 0,0 & 0,0 & 0,0 & 0,0 & 0,0 & 0,0 \\
\hline $\mathrm{CuSO}_{4}$ (aq) & 36,5 & 36,5 & 36,7 & 37,7 & 37,9 & 37,2 & 38,4 \\
\hline $\mathbf{Z n}^{2+}$ & 61,0 & 61,6 & 60,8 & 59,6 & 59,2 & 60,1 & 58,7 \\
\hline $\mathrm{ZnOH}^{+}$ & 0,0 & 0,0 & 0,0 & 0,0 & 0,0 & 0,0 & 0,0 \\
\hline $\mathrm{ZnSO}_{4}$ (aq) & 34,1 & 33,9 & 34,3 & 35,0 & 35,1 & 34,6 & 35,6 \\
\hline $\mathrm{Zn}\left(\mathrm{SO}_{4}\right)_{2}{ }^{2-}$ & 5,0 & 4,5 & 4,9 & 5,4 & 5,7 & 5,2 & 5,7 \\
\hline $\mathrm{HAsO}_{4}{ }^{2-}$ & 0,0 & 0,0 & 0,0 & 0,0 & 0,0 & 0,0 & 0,0 \\
\hline $\mathrm{H}_{2} \mathrm{AsO}_{4}^{-}$ & 95,0 & 94,8 & 96,0 & 96,0 & 93,9 & 93,8 & 95,0 \\
\hline $\mathrm{H}_{3} \mathrm{AsO}_{4}$ & 4,9 & 5,2 & 4,0 & 4,0 & 6,1 & 6,1 & 4,9 \\
\hline $\mathrm{H}_{3} \mathrm{AsO}_{3}$ & 0,0 & 0,0 & 0,0 & 0,0 & 0,0 & 0,0 & 0,0 \\
\hline $\mathbf{S r}^{2+}$ & 66,5 & 66,6 & 66,3 & 65,4 & 65,2 & 65,8 & 64,7 \\
\hline $\mathrm{SrSO}_{4}(\mathrm{aq})$ & 33,5 & 33,4 & 33,7 & 34,6 & 34,8 & 34,2 & 35,3 \\
\hline
\end{tabular}


Tab. A.25. (Forts.): Ergebnisse der Speziationsberechnung mit MINTEQA2.

\begin{tabular}{|c|c|c|c|c|c|c|c|}
\hline & $\begin{array}{l}\text { Lausitz 5,00 m } \\
\text { PL KB }\end{array}$ & $\begin{array}{l}\text { Lausitz 5,21 m } \\
\text { PL KB }\end{array}$ & \begin{tabular}{|l|} 
Lausitz 5,30 m \\
PL KB
\end{tabular} & $\begin{array}{l}\text { Lausitz 5,50 m } \\
\text { PL KB }\end{array}$ & \begin{tabular}{|l|} 
Lausitz 5,60 m \\
PL KB
\end{tabular} & \begin{tabular}{l|} 
Lausitz 5,75 m \\
PL KB
\end{tabular} & $\begin{array}{l}\text { Lausitz 5,93 m } \\
\text { PL KB } \\
\end{array}$ \\
\hline Spezies & $\%$ & $\%$ & $\%$ & $\%$ & $\%$ & $\%$ & $\%$ \\
\hline $\mathrm{MoO}_{4}{ }^{2-}$ & 6,3 & 6,9 & 9,1 & 9,1 & 4,4 & \begin{tabular}{l|}
4,3 \\
\end{tabular} & 6,4 \\
\hline $\mathrm{HMoO}_{4}^{-}$ & 22,5 & 28,2 & 25,9 & 25,9 & 19,3 & 19,2 & 22,6 \\
\hline $\mathrm{H}_{2} \mathrm{MoO}_{4}(\mathrm{aq})$ & 71,2 & 64,9 & 65,1 & 65,0 & 76,3 & 76,5 & 71,1 \\
\hline $\mathbf{C d}^{2+}$ & 58,0 & 58,5 & 57,8 & 56,6 & 56,1 & 57,1 & 55,6 \\
\hline $\mathrm{CdSO}_{4}(\mathrm{aq})$ & 34,1 & 34,5 & 34,4 & 35,0 & 35,0 & 34,7 & 35,5 \\
\hline $\mathrm{Cd}\left(\mathrm{SO}_{4}\right)_{2}{ }^{2-}$ & 7,8 & 7,0 & 7,8 & 8,4 & 8,9 & 8,2 & 9,0 \\
\hline $\mathrm{Sn}(\mathrm{OH})_{2}$ & $\mathrm{nq}$ & 0,0 & 0,0 & 0,0 & 0,0 & 0,0 & 0,0 \\
\hline $\mathbf{S n}^{2+}$ & $\mathrm{nq}$ & 0,0 & 0,0 & 0,0 & 0,0 & 0,0 & 0,0 \\
\hline $\mathrm{SnOH}^{+}$ & $\mathrm{nq}$ & 0,0 & 0,0 & 0,0 & 0,0 & 0,0 & 0,0 \\
\hline $\mathbf{S n}^{4+}$ & $\mathrm{nq}$ & 98,9 & 95,8 & 95,9 & 99,7 & 99,7 & 99,0 \\
\hline $\operatorname{Sn}(\mathrm{OH})_{6}^{-}$ & $\mathrm{nq}$ & 1,1 & 4,2 & 4,1 & 0,0 & 0,0 & 1,0 \\
\hline $\mathrm{SbO}_{3}^{-}$ & $\mathrm{nq}$ & 99,9 & 99,9 & 99,9 & 99,9 & 99,9 & 99,9 \\
\hline $\mathrm{Sb}(\mathrm{OH})_{3}$ & $\mathrm{nq}$ & 0,0 & 0,0 & 0,0 & 0,0 & 0,0 & 0,0 \\
\hline $\mathrm{Sb}(\mathrm{OH})_{2}{ }^{+}$ & $\mathrm{nq}$ & 0,0 & 0,0 & 0,0 & 0,0 & 0,0 & 0,0 \\
\hline $\mathrm{HSbO}_{2}$ & $\mathrm{nq}$ & 0,0 & 0,0 & 0,0 & 0,0 & 0,0 & 0,0 \\
\hline $\mathrm{SbO}^{+}$ & $\mathrm{nq}$ & 0,0 & 0,0 & 0,0 & 0,0 & 0,0 & 0,0 \\
\hline $\mathbf{B a}^{2+}$ & 100,0 & 100,0 & 100,0 & 100,0 & 100,0 & 100,0 & 100,0 \\
\hline $\mathbf{T I}^{+}$ & 86,7 & 87,4 & 86,7 & 86,1 & 85,7 & 86,3 & 85,6 \\
\hline $\mathrm{TISO}_{4}^{-}$ & 13,3 & 12,6 & 13,3 & 13,9 & 14,3 & 13,7 & 14,4 \\
\hline $\mathbf{P b}^{2+}$ & 41,1 & 42,7 & 40,9 & 39,8 & 39,5 & 40,3 & 39,0 \\
\hline $\mathrm{PbOH}^{+}$ & 0,0 & 0,0 & 0,0 & 0,0 & 0,0 & 0,0 & 0,0 \\
\hline $\mathrm{Pb}(\mathrm{OH})_{2}(\mathrm{aq})$ & 0,0 & 0,0 & 0,0 & 54,6 & 54,6 & 54,3 & 55,2 \\
\hline $\mathrm{PbSO}_{4}$ (aq) & 53,7 & 52,5 & 53,9 & 5,5 & 5,9 & 5,4 & 5,9 \\
\hline $\mathrm{Pb}\left(\mathrm{SO}_{4}\right)_{2}{ }^{2-}$ & 5,2 & 4,8 & 5,1 & 0,0 & 0,0 & 0,0 & 0,0 \\
\hline $\mathrm{UO}_{2}{ }^{+}$ & 0,0 & 0,0 & 0,0 & 0,0 & 0,0 & 0,0 & 0,0 \\
\hline $\mathrm{U}(\mathrm{OH})_{2}{ }^{2+}$ & 0,0 & 0,0 & 0,0 & 0,0 & 0,0 & 0,0 & 0,0 \\
\hline $\mathrm{U}(\mathrm{OH})_{3}{ }^{+}$ & 0,0 & 0,0 & 0,0 & 0,0 & 0,0 & 0,0 & 0,0 \\
\hline $\mathrm{U}(\mathrm{OH})_{4}(\mathrm{aq})$ & 0,0 & 0,0 & 0,0 & 0,0 & 0,0 & 0,0 & 0,0 \\
\hline $\mathrm{USO}_{4}{ }^{2+}$ & 0,0 & 0,0 & 0,0 & 0,0 & 0,0 & 0,0 & 0,0 \\
\hline $\mathrm{U}\left(\mathrm{SO}_{4}\right)_{2}(\mathrm{aq})$ & 0,0 & 0,0 & 0,0 & 0,0 & 0,0 & 0,0 & 0,0 \\
\hline $\mathrm{UO}_{2}{ }^{2+}$ & 35,8 & 34,1 & 35,6 & 34,3 & 33,6 & 34,9 & 33,2 \\
\hline $\mathrm{UO}_{2} \mathbf{O H}^{+}$ & 0,0 & 0,0 & 0,0 & 0,0 & 0,0 & 0,0 & 0,0 \\
\hline$\left(\mathrm{UO}_{2}\right)_{2}(\mathrm{OH})_{2}{ }^{2+}$ & 0,0 & 0,0 & 0,0 & 0,0 & 0,0 & 0,0 & 0,0 \\
\hline$\left(\mathrm{UO}_{2}\right)_{3}(\mathrm{OH})_{5}{ }^{+}$ & 0,0 & 0,0 & 0,0 & 0,0 & 0,0 & 0,0 & 0,0 \\
\hline $\mathrm{UO}_{2} \mathrm{SO}_{4}(\mathrm{aq})$ & 43,6 & 45,1 & 43,9 & 43,9 & 43,5 & 43,8 & 43,9 \\
\hline $\mathrm{UO}_{2}\left(\mathrm{SO}_{4}\right)_{2}{ }^{2-}$ & 20,5 & 20,7 & 20,4 & 21,8 & 22,8 & 21,3 & 22,8 \\
\hline $\mathrm{SO}_{4}{ }^{2-}$ & 43,9 & 42,6 & 47,5 & 48,2 & 45,8 & 45,5 & 51,3 \\
\hline $\mathrm{HSO}_{4}^{-}$ & 0,0 & 0,0 & 0,0 & 0,0 & 0,0 & 0,0 & 0,0 \\
\hline $\mathrm{AlSO}_{4}{ }^{+}$ & 31,4 & 32,0 & 25,3 & 24,1 & 26,6 & 29,0 & 17,5 \\
\hline $\mathrm{Al}\left(\mathrm{SO}_{4}\right)_{2}^{-}$ & 4,9 & 4,2 & 4,0 & 4,0 & 4,5 & 4,7 & 3,0 \\
\hline $\mathrm{FeSO}_{4}(\mathrm{aq})$ & 0,0 & 1,0 & 3,3 & 5,1 & 4,1 & 2,0 & 10,0 \\
\hline $\mathrm{MgSO}_{4}(\mathrm{aq})$ & 4,2 & 4,2 & 4,4 & 4,4 & 4,4 & 4,5 & 4,4 \\
\hline CaSO4 (aq) & 13,2 & 14,6 & 14,3 & 12,9 & 13,0 & 12,6 & 12,4 \\
\hline
\end{tabular}


Tab. A.25. (Forts.): Ergebnisse der Speziationsberechnung mit MINTEQA2.

\begin{tabular}{|c|c|c|c|c|c|c|}
\hline & Lausitz 21,07 m & Lausitz 21,13 m & Lausitz 21,40 m & Lausitz 21,70 m & Lausitz 21,80 m & Lausitz 21,90 m \\
\hline Spezies & $\%$ & $\%$ & $\%$ & $\%$ & $\%$ & $\%$ \\
\hline $\mathrm{Li}^{+}$ & 97,1 & 96,9 & 97,4 & 97,1 & 97,3 & 97,3 \\
\hline $\mathrm{LiSO}_{4}^{-}$ & 2,9 & 3,1 & 2,6 & 2,9 & 2,7 & 2,7 \\
\hline $\mathrm{Be}^{2+}$ & 71,4 & 70,5 & 73,7 & 71,2 & 72,4 & 74,0 \\
\hline $\mathrm{BeOH}^{+}$ & 1,2 & 0,0 & 0,0 & 1,1 & 0,0 & 0,0 \\
\hline $\mathrm{Be}(\mathrm{OH})_{2}$ & 0,0 & 0,0 & 0,0 & 0,0 & 0,0 & 0,0 \\
\hline $\mathrm{BeSO}_{4}(\mathrm{aq})$ & 26,1 & 27,1 & 24,5 & 26,4 & 25,5 & 24,8 \\
\hline $\mathrm{Be}\left(\mathrm{SO}_{4}\right)_{2}{ }^{2-}$ & 1,3 & 1,5 & 1,1 & 1,3 & 1,2 & 1,1 \\
\hline $\mathrm{Na}^{+}$ & 96,5 & 96,2 & 96,8 & 96,5 & 96,7 & 96,8 \\
\hline $\mathrm{NaSO}_{4}^{-}$ & 3,5 & 3,8 & 3,2 & 3,5 & 3,3 & 3,2 \\
\hline $\mathrm{Mg}^{2+}$ & 66,5 & 65,4 & 68,6 & 66,2 & 67,3 & 68,4 \\
\hline $\mathrm{MgSO}_{4}$ (aq) & 33,5 & 34,6 & 31,4 & 33,8 & 32,7 & 31,6 \\
\hline $\mathbf{A l}^{3+}$ & 10,3 & 10,1 & 11,3 & 10,1 & 10,4 & 11,2 \\
\hline $\mathrm{AlOH}^{2+}$ & 0,0 & 0,0 & 0,0 & 0,0 & 0,0 & 0,0 \\
\hline $\mathrm{Al}(\mathrm{OH})_{2}{ }^{+}$ & 0,0 & 0,0 & 0,0 & 0,0 & 0,0 & 0,0 \\
\hline $\mathrm{Al}(\mathrm{OH})_{3}(\mathbf{a q})$ & 0,0 & 0,0 & 0,0 & 0,0 & 0,0 & 0,0 \\
\hline $\mathrm{Al}(\mathrm{OH})_{4}{ }^{-}$ & 0,0 & 0,0 & 0,0 & 0,0 & 0,0 & 0,0 \\
\hline $\mathrm{AlSO}_{4}{ }^{+}$ & 82,7 & 82,4 & 82,4 & 82,9 & 83,1 & 82,6 \\
\hline $\mathrm{Al}\left(\mathrm{SO}_{4}\right)_{2}^{-}$ & 6,7 & 7,3 & 6,1 & 6,8 & 6,3 & 6,2 \\
\hline $\mathbf{K}^{+}$ & 95,8 & 95,4 & 96,1 & 95,8 & 96,1 & 96,1 \\
\hline $\mathrm{KSO}_{4}^{-}$ & 4,2 & 4,6 & 3,9 & 4,2 & 3,9 & 3,9 \\
\hline $\mathrm{Ca}^{2+}$ & 61,4 & 60,2 & 63,6 & 61,1 & 62,3 & 63,4 \\
\hline $\mathrm{CaSO}_{4}(\mathrm{aq})$ & 38,6 & 39,8 & 36,4 & 38,9 & 37,7 & 36,6 \\
\hline $\mathrm{VO}^{2+}$ & 53,1 & $\mathrm{nq}$ & $\mathrm{nq}$ & 49,7 & 59,3 & 60,7 \\
\hline $\mathrm{V}(\mathrm{OH})_{3}{ }^{+}$ & 0,0 & $\mathrm{nq}$ & $\mathrm{nq}$ & 0,0 & 0,0 & 0,0 \\
\hline $\mathrm{H}_{2} \mathrm{~V}_{2} \mathrm{O}_{4}{ }^{2+}$ & 0,0 & $\mathrm{nq}$ & $\mathrm{nq}$ & 0,0 & 0,0 & 0,0 \\
\hline $\mathrm{VOSO}_{4}(\mathrm{aq})$ & 37,4 & $\mathrm{nq}$ & $\mathrm{nq}$ & 38,4 & 40,3 & 39,3 \\
\hline $\mathrm{V}(\mathrm{OH})_{3}(\mathbf{a q})$ & 9,1 & $\mathrm{nq}$ & $\mathrm{nq}$ & 7,4 & 0,0 & 0,0 \\
\hline $\mathrm{VO}_{2}^{+}$ & 0,0 & $\mathrm{nq}$ & $\mathrm{nq}$ & 0,0 & 0,0 & 0,0 \\
\hline $\mathrm{HVO}_{4}{ }^{2-}$ & 0,0 & $\mathrm{nq}$ & $\mathrm{nq}$ & 0,0 & 0,0 & 0,0 \\
\hline $\mathrm{H}_{2} \mathrm{VO}_{4}^{-}$ & 0,0 & $\mathrm{nq}$ & $\mathrm{nq}$ & 0,0 & 0,0 & 0,0 \\
\hline $\mathrm{VO}_{2} \mathrm{SO}_{4}{ }^{-}$ & 0,0 & $\mathrm{nq}$ & $\mathrm{nq}$ & 0,0 & 0,0 & 0,0 \\
\hline $\mathrm{H}_{3} \mathrm{VO}_{4}(\mathrm{aq})$ & 0,0 & $\mathrm{nq}$ & $\mathrm{nq}$ & 0,0 & 0,0 & 0,0 \\
\hline $\mathrm{H}_{3} \mathrm{~V}_{2} \mathrm{O}_{7}^{-}$ & 0,0 & $\mathrm{nq}$ & $\mathrm{nq}$ & 0,0 & 0,0 & 0,0 \\
\hline $\mathbf{C r}^{3+}$ & 25,3 & 26,2 & 28,9 & 25,2 & 26,2 & 31,2 \\
\hline $\mathrm{Cr}(\mathrm{OH})_{2}{ }^{+}$ & 0,0 & 0,0 & 0,0 & 0,0 & 0,0 & 0,0 \\
\hline $\mathrm{CrOH}^{2+}$ & 10,5 & 7,7 & 6,9 & 9,9 & 8,8 & 0,0 \\
\hline $\mathrm{CrSO}_{4}{ }^{+}$ & 59,9 & 62,8 & 61,8 & 60,9 & 61,6 & 67,7 \\
\hline $\mathrm{Cr}(\mathrm{OH})_{3}(\mathrm{aq})$ & 0,0 & 0,0 & 0,0 & 0,0 & 0,0 & 0,0 \\
\hline $\mathrm{CrOHSO}_{4}(\mathrm{aq})$ & 4,2 & 3,2 & 2,5 & 4,0 & 3,4 & 0,0 \\
\hline $\mathrm{CrO}_{2}^{-}$ & 0,0 & 0,0 & 0,0 & 0,0 & 0,0 & 0,0 \\
\hline $\mathrm{CrO}_{4}{ }^{2-}$ & 0,0 & 0,0 & 0,0 & 0,0 & 0,0 & 0,0 \\
\hline $\mathrm{HCrO}_{4}^{-}$ & 0,0 & 0,0 & 0,0 & 0,0 & 0,0 & 0,0 \\
\hline $\mathrm{Mn}^{2+}$ & 67,5 & 66,4 & 69,5 & 67,2 & 68,3 & 69,3 \\
\hline $\mathrm{MnSO}_{4}$ (aq) & 32,5 & 33,6 & 30,5 & 32,8 & 31,7 & 30,7 \\
\hline $\mathrm{Fe}^{2+}$ & 59,9 & 58,7 & 62,2 & 59,6 & 60,8 & 62,0 \\
\hline $\mathrm{FeSO}_{4}(\mathrm{aq})$ & 40,1 & 41,3 & 37,8 & 40,4 & 39,2 & 38,0 \\
\hline $\mathrm{FeOH}^{2+}$ & 0,0 & 0,0 & 0,0 & 0,0 & 0,0 & 0,0 \\
\hline $\mathrm{Fe}(\mathrm{OH})_{2}{ }^{+}$ & 0,0 & 0,0 & 0,0 & 0,0 & 0,0 & 0,0 \\
\hline $\mathrm{Fe}(\mathrm{OH})_{3}$ & 0,0 & 0,0 & 0,0 & 0,0 & 0,0 & 0,0 \\
\hline $\mathrm{Co}^{2+}$ & 64,5 & 63,4 & 66,6 & 64,2 & 65,3 & 66,4 \\
\hline $\mathrm{CoSO}_{4}(\mathrm{aq})$ & 35,5 & 36,6 & 33,4 & 35,8 & 34,7 & 33,6 \\
\hline $\mathrm{Ni}^{2+}$ & 64,4 & 63,3 & 66,5 & 64,1 & 65,3 & 66,4 \\
\hline $\mathrm{NiSO}_{4}(\mathrm{aq})$ & 35,5 & 36,7 & 33,4 & 35,9 & 34,7 & 33,6 \\
\hline $\mathrm{Cu}^{+}$ & 0,0 & 0,0 & 0,0 & $\mathrm{nq}$ & $\mathrm{nq}$ & 0,0 \\
\hline $\mathrm{Cu}^{2+}$ & 61,7 & 60,5 & 63,9 & $\mathrm{nq}$ & $\mathrm{nq}$ & 63,7 \\
\hline $\mathrm{Cu}(\mathrm{OH})^{+}$ & 0,0 & 0,0 & 0,0 & $\mathrm{nq}$ & $\mathrm{nq}$ & 0,0 \\
\hline $\mathrm{Cu}(\mathrm{OH})_{2}$ & 0,0 & 0,0 & 0,0 & $\mathrm{nq}$ & $\mathrm{nq}$ & 0,0 \\
\hline $\mathrm{CuSO}_{4}(\mathrm{aq})$ & 38,3 & 39,5 & 36,1 & $\mathrm{nq}$ & $\mathrm{nq}$ & 36,3 \\
\hline $\mathbf{Z n}^{2+}$ & 59,1 & 57,5 & 61,6 & 58,8 & 60,3 & 61,4 \\
\hline $\mathrm{ZnOH}^{+}$ & 0,0 & 0,0 & 0,0 & 0,0 & 0,0 & 0,0 \\
\hline $\mathrm{ZnSO}_{4}$ (aq) & 35,7 & 36,5 & 33,8 & 36,0 & 35,1 & 34,0 \\
\hline $\mathrm{Zn}\left(\mathrm{SO}_{4}\right)_{2}{ }^{2-}$ & 5,2 & 6,1 & 4,6 & 5,2 & 4,6 & 4,6 \\
\hline
\end{tabular}


Tab. A.25. (Forts.): Ergebnisse der Speziationsberechnung mit MINTEQA2.

\begin{tabular}{|c|c|c|c|c|c|c|}
\hline & Lausitz 21,07 m & Lausitz 21,13 m & Lausitz 21,40 m & Lausitz 21,70 m & Lausitz 21,80 m & Lausitz 21,90 m \\
\hline Spezies & $\%$ & $\%$ & $\%$ & $\%$ & $\%$ & $\%$ \\
\hline $\mathrm{HAsO}_{4}{ }^{2-}$ & 0,0 & 0,0 & 0,0 & 0,0 & 0,0 & 0,0 \\
\hline $\mathrm{H}_{2} \mathrm{AsO}_{4}{ }^{-}$ & 98,2 & 97,7 & 97,0 & 98,1 & 97,7 & 79,1 \\
\hline $\mathrm{H}_{3} \mathrm{AsO}_{4}$ & 1,7 & 2,2 & 2,9 & 1,8 & 2,2 & 20,9 \\
\hline $\mathrm{H}_{3} \mathrm{AsO}_{3}$ & 0,0 & 0,0 & 0,0 & 0,0 & 0,0 & 0,0 \\
\hline $\mathbf{S r}^{2+}$ & 64,8 & 63,6 & 66,9 & 64,5 & 65,6 & 66,7 \\
\hline $\mathrm{SrSO}_{4}(\mathrm{aq})$ & 35,2 & 36,4 & 33,1 & 35,5 & 34,4 & 33,3 \\
\hline $\mathrm{MoO}_{4}{ }^{2-}$ & 29,3 & 21,3 & 14,9 & 27,0 & 21,5 & $\mathrm{nq}$ \\
\hline $\mathrm{HMoO}_{4}^{-}$ & 34,8 & 33,1 & 30,5 & 34,6 & 33,5 & $\mathrm{nq}$ \\
\hline $\mathrm{H}_{2} \mathrm{MoO}_{4}(\mathrm{aq})$ & 35,9 & 45,6 & 54,6 & 38,4 & 45,0 & $\mathrm{nq}$ \\
\hline $\mathrm{Cd}^{2+}$ & 56,1 & 54,3 & 58,8 & 55,8 & 57,5 & 58,6 \\
\hline $\mathrm{CdSO}_{4}(\mathrm{aq})$ & 35,7 & 36,2 & 34,0 & 36,0 & 35,2 & 34,2 \\
\hline $\mathrm{Cd}\left(\mathrm{SO}_{4}\right)_{2}{ }^{2-}$ & 8,2 & 9,5 & 7,2 & 8,2 & 7,3 & 7,2 \\
\hline $\mathrm{Sn}(\mathrm{OH})_{2}$ & $\mathrm{nq}$ & 0,0 & $\mathrm{nq}$ & 0,0 & $\mathrm{nq}$ & $\mathrm{nq}$ \\
\hline $\mathbf{S n}^{2+}$ & $\mathrm{nq}$ & 0,0 & $\mathrm{nq}$ & 0,0 & $\mathrm{nq}$ & $\mathrm{nq}$ \\
\hline $\mathrm{SnOH}^{+}$ & $\mathrm{nq}$ & 0,0 & $\mathrm{nq}$ & 0,0 & $\mathrm{nq}$ & $\mathrm{nq}$ \\
\hline $\mathbf{S n}^{4+}$ & $\mathrm{nq}$ & 0,0 & $\mathrm{nq}$ & 12,3 & $\mathrm{nq}$ & $\mathrm{nq}$ \\
\hline $\mathrm{Sn}(\mathrm{OH})_{6}^{-}$ & $\mathrm{nq}$ & 0,0 & $\mathrm{nq}$ & 97,1 & $\mathrm{nq}$ & $\mathrm{nq}$ \\
\hline $\mathrm{SbO}_{3}^{-}$ & $\mathrm{nq}$ & 99,9 & $\mathrm{nq}$ & $\mathrm{nq}$ & $\mathrm{nq}$ & $\mathrm{nq}$ \\
\hline $\mathrm{Sb}(\mathrm{OH})_{3}$ & $\mathrm{nq}$ & 0,0 & $\mathrm{nq}$ & $\mathrm{nq}$ & $\mathrm{nq}$ & $\mathrm{nq}$ \\
\hline $\mathrm{Sb}(\mathrm{OH})_{2}{ }^{+}$ & $\mathrm{nq}$ & 0,0 & $\mathrm{nq}$ & $\mathrm{nq}$ & $\mathrm{nq}$ & $\mathrm{nq}$ \\
\hline $\mathrm{HSbO}_{2}$ & $\mathrm{nq}$ & 0,0 & $\mathrm{nq}$ & $\mathrm{nq}$ & $\mathrm{nq}$ & $\mathrm{nq}$ \\
\hline $\mathrm{SbO}^{+}$ & $\mathrm{nq}$ & 0,0 & $\mathrm{nq}$ & $\mathrm{nq}$ & $\mathrm{nq}$ & $\mathrm{nq}$ \\
\hline $\mathrm{Ba}^{2+}$ & 100,0 & 100,0 & 100,0 & 100,0 & 100,0 & 100,0 \\
\hline $\mathrm{Tl}^{+}$ & 86,2 & 85,1 & 87,2 & 86,1 & 87,0 & 87,2 \\
\hline $\mathrm{TlSO}_{4}^{-}$ & 13,8 & 14,9 & 12,8 & 13,9 & 13,0 & 12,8 \\
\hline $\mathrm{Pb}^{2+}$ & 39,3 & 37,8 & 41,7 & 38,9 & 40,3 & 41,5 \\
\hline $\mathrm{PbOH}^{+}$ & 0,0 & 0,0 & 0,0 & 0,0 & 0,0 & 0,0 \\
\hline $\mathrm{Pb}(\mathrm{OH})_{2}(\mathrm{aq})$ & 0,0 & 0,0 & 0,0 & 0,0 & 0,0 & 0,0 \\
\hline $\mathrm{PbSO}_{4}(\mathrm{aq})$ & 55,3 & 56,0 & 53,5 & 55,7 & 54,9 & 53,7 \\
\hline $\mathrm{Pb}\left(\mathrm{SO}_{4}\right)_{2}{ }^{2-}$ & 5,4 & 6,2 & 4,8 & 5,4 & 4,8 & 4,8 \\
\hline $\mathrm{UO}_{2}^{+}$ & 0,0 & 0,0 & 0,0 & 0,0 & 0,0 & 0,0 \\
\hline $\mathrm{U}(\mathrm{OH})_{2}{ }^{2+}$ & 0,0 & 0,0 & 0,0 & 0,0 & 0,0 & 0,0 \\
\hline $\mathrm{U}(\mathbf{O H})_{3}{ }^{+}$ & 0,0 & 0,0 & 0,0 & 0,0 & 0,0 & 0,0 \\
\hline $\mathrm{U}(\mathrm{OH})_{4}(\mathbf{a q})$ & 0,0 & 0,0 & 0,0 & 0,0 & 0,0 & 0,0 \\
\hline $\mathrm{USO}_{4}{ }^{2+}$ & 0,0 & 0,0 & 0,0 & 0,0 & 0,0 & 0,0 \\
\hline $\mathrm{U}_{\left(\mathrm{SO}_{4}\right)_{2}(\mathrm{aq})}$ & 0,0 & 0,0 & 0,0 & 0,0 & 0,0 & 0,0 \\
\hline $\mathrm{UO}_{2}{ }^{2+}$ & 34,0 & 31,9 & 36,7 & 33,7 & 35,5 & 36,6 \\
\hline $\mathrm{UO}_{2} \mathbf{O H}^{+}$ & 0,0 & 0,0 & 0,0 & 0,0 & 0,0 & 0,0 \\
\hline$\left(\mathrm{UO}_{2}\right)_{2}(\mathrm{OH})_{2}{ }^{2+}$ & 0,0 & 0,0 & 0,0 & 0,0 & 0,0 & 0,0 \\
\hline$\left(\mathrm{UO}_{2}\right)_{3}(\mathrm{OH})_{5}{ }^{+}$ & 0,0 & 0,0 & 0,0 & 0,0 & 0,0 & 0,0 \\
\hline $\mathrm{UO}_{2} \mathrm{SO}_{4}(\mathrm{aq})$ & 44,7 & 44,2 & 44,0 & 45,0 & 45,1 & 44,2 \\
\hline $\mathrm{UO}_{2}\left(\mathrm{SO}_{4}\right)_{2}{ }^{2-}$ & 21,1 & 23,8 & 19,2 & 21,2 & 19,3 & 19,2 \\
\hline $\mathrm{SO}_{4}{ }^{2-}$ & 61,6 & 60,4 & 58,0 & 62,9 & 62,4 & 56,6 \\
\hline $\mathrm{HSO}_{4}{ }^{-}$ & 0,0 & 0,0 & 0,0 & 0,0 & 0,0 & 5,1 \\
\hline $\mathrm{AlSO}_{4}{ }^{+}$ & 2,7 & 3,6 & 6,6 & 2,4 & 4,1 & 6,7 \\
\hline $\mathrm{Al}\left(\mathrm{SO}_{4}\right)_{2}^{-}$ & 0,0 & 0,0 & 0,0 & 0,0 & 0,0 & 0,0 \\
\hline $\mathrm{FeSO}_{4}(\mathrm{aq})$ & 8,3 & 3,0 & 2,6 & 6,6 & 5,0 & 1,8 \\
\hline $\mathrm{MgSO}_{4}(\mathrm{aq})$ & 4,3 & 5,5 & 6,3 & 5,1 & 5,1 & 5,5 \\
\hline CaSO4 (aq) & 21,5 & 25,4 & 24,0 & 21,6 & 21,8 & 22,6 \\
\hline
\end{tabular}




\section{Danksagung}

Ganz herzlichen Dank all denen, die in vielfältiger Weise am Gelingen dieser Arbeit beteiligt waren! Besonders danken möchte ich

Herrn Prof. Dr. Heinrichs für die Überlassung des Themas, die große Freiheit, die er mir bei der Ausgestaltung gelassen hat, die stete Bereitschaft zur Diskussion und die vielen nützlichen Hinweise.

- Herrn Dr. Pleßow für die Einarbeitung in das Thema und Herrn Dipl.-Min. Brinkmann für die Unterstützung bei Probennahmen und -bearbeitung.

- Herrn Dr. K. Simon für die Betreuung bei der Massenspektrometrie.

- Herrn Skrandies, Herrn Schiffczyk und Herrn Herborg für die immer freundliche, prompte und einfallsreiche Hilfe bei technischen Problemen.

Den Mitarbeitern des UFZ Halle-Leipzig GmbH, insbesondere Herrn Gläser, Herrn Richert und Frau Dr. Bozau für die Bereitstellung von Probenmaterial, die Unterstützung bei den Probennahmen und die hilfreichen Diskussionen.

- Frau Dr. Schlabach für Hilfestellung an der ICP-OES und die Untersuchung der Festphasen des Bohrkerns RCO4.

Allen nicht genannten Mitarbeitern des Geochemischen Instituts für Rat und Tat und die sehr angenehme Atmosphäre.

- Herrn Dipl.-Phys. Ulrich Köhler für das Korrekturlesen dieser Arbeit.

- Herrn Prof. Dr. J. Hoefs für die Übernahme des Korreferats.

Allen Freunden in- und außerhalb der StMV Blaue Sänger und des Sondershäuser Verbandes dafür, dass Ihr mich immer daran erinnert habt, dass es ein Leben neben der Arbeit gibt.

- Meinen Eltern für ihre stete Unterstützung.

- Maxi für seine Geduld, fürs Korrekturlesen, für den Schokopudding, für moralischen Aufbau und Ablenkung und für sein großes Verständnis. 


\section{Lebenslauf}

Name: Kathrin Ulrike Brüschke

geboren am 05.06.1973

in Heidelberg

$1979-1983$

$1983-1992$

1992

WS 1992/93 - WS 1994/95

Februar 1995

SS 1995 - WS 1996/97

Oktober 1996 - Februar 1997

Februar 1997

Mai 1997 - Februar 1998

SS 1998

Oktober 1998 - März 2001

seit Oktober 1998
Besuch der Schillerschule Nußloch

Besuch des Kurfürst-Friedrich-Gymnasiums Heidelberg Abitur

Studium der Chemie an der Georg-August-Universität Göttingen

Vordiplom

Studium der Chemie an der Universität Leipzig

Diplomarbeit im Arbeitskreis von Prof. Dr. P. Welzel am Institut für Organische Chemie der Universität Leipzig; Thema: "Ein gluco-Disacchardid-Strukturanalogon des Antibiotikums Moenomycin A als Syntheseziel"

Diplom

Arbeiten zu einer Dissertation im Arbeitskreis von Prof. Dr. R. Brückner am Institut für Organische Chemie der GeorgAugust-Universität Göttingen (aus gesundheitlichen Gründen abgebrochen)

Beginn des Studiums der Publizistik und Kommunikationswissenschaft an der Georg-AugustUniversität Göttingen

wissenschaftl. Mitarbeiterin am Geochemischen Institut der Georg-August-Universität Göttingen

Arbeiten zur Dissertation im Arbeitskreis von Prof. Dr. H. Heinrichs am Geochemischen Institut der Georg-AugustUniversität Göttingen 